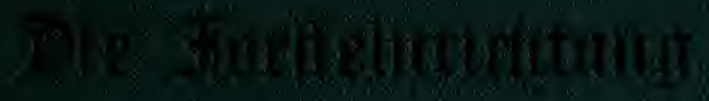

$$
\begin{aligned}
& \text { Sim }
\end{aligned}
$$

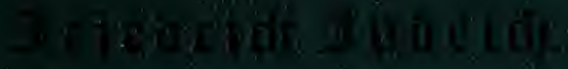

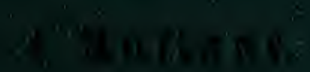

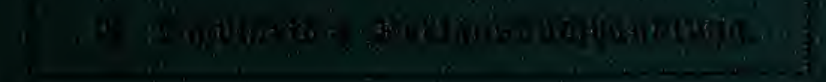

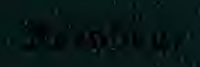


conte th 
THIS BOOK BELONGED TO WILFRIDE. HILEY 
Digitized by the Internet Archive in 2009 with funding from NCSU Libraries 


\section{Mit}

\section{forfteiltidtul}

$30 n$

Dr. Ericorid) Illorid,

fgl. jädj. Gehcimen Sberforjtrath, Director ber Foritatademie zu Tharant.

Bicrte, vermehrte und verbelicrte Pülage.

Mit einer Sarte in jarbentond.

จreson.

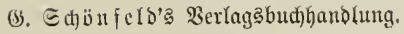

1885. 
Stle Fechte worbehalten. 


\section{Eortuort ;}

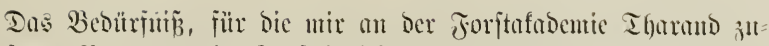

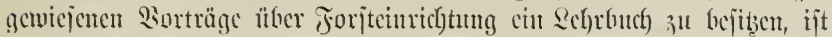

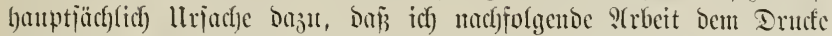

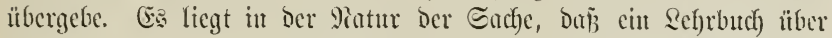

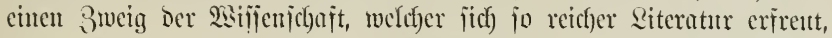

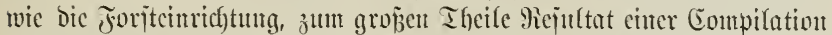

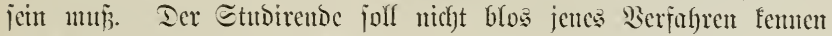
fermen, weldyes ber sierfajier für bas richtigite hält, jontoern audd bie

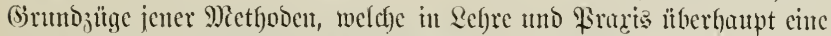
Bebentung erfangt faben. Borzugazeije aber joll er mit ben allge=

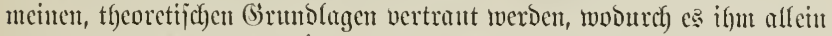

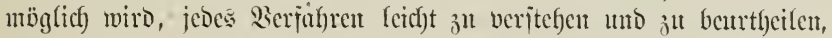
ment bafjelfe andf) nicht bejonders erörtert ober errwähnt wurbe.

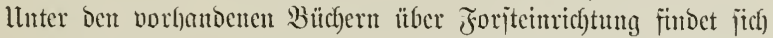

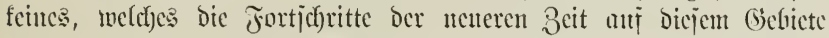

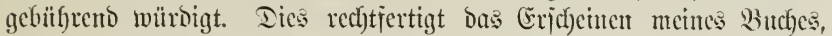

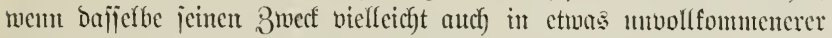

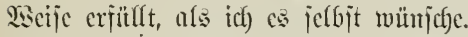

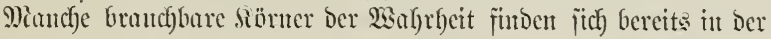
älteren Siteratur, man hat ifunen jeond) nicht bie verdiente Beadftung

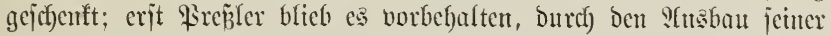

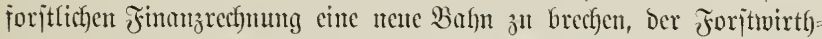
ichaft richtig rechnen zu lefgren. Die 3ufunft wiro bies noch mefor anerfenten, als bie Begenwart. Dem Ëinflltije ber in neterer Beit

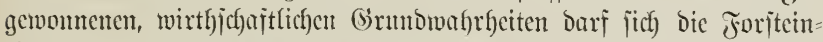

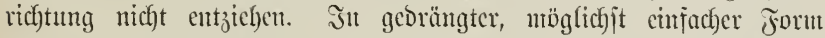

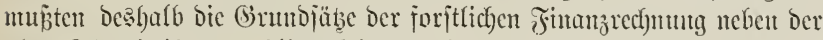

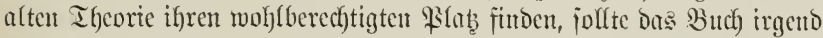

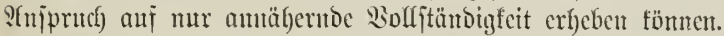

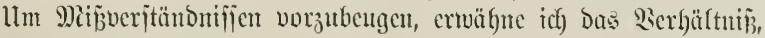

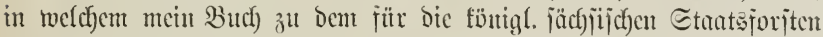

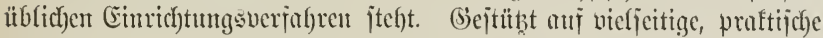

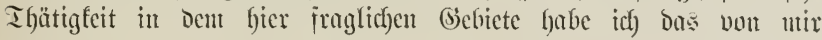


empfohlene Berfahren dem in Sachjen gegennärtig üblichen jebr mafge

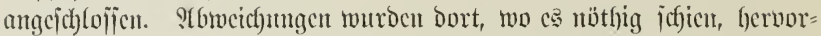
gethoben. Eine bis in Das Eingelne gehende Injtruction mit jtrengem Fejthalten alfer 2iorzüge mb afler Miängel cintes bejtchentoen 2icr=

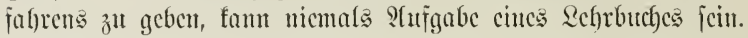

Bercits vor cinigen Jafren war bas Mamujeript fajt vollendet,

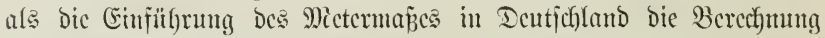
volljtändig neuter Beijpicle zur (Erläuterming nöthig machte. Ilm aurd) für bie Geloredfmung cin Decimaljojtem anzumenben, wählte ich)

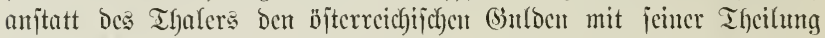
iil 100 Siremzer.

If) a $\mathfrak{x}$ a d, im Samar 1871.

\section{Der Derfafler.}

\section{Jorwort zant viceten Aluflagre.}

Mancferlei Berbejienungen und nothyembige Erweiterungen haben beu llmfang meincr "Foritcinrichtung" lciber abermals ctwas ver= größjert, obgleich ich licber éntige fïtzungen vorgenommen fätte.

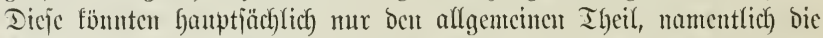

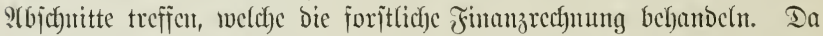

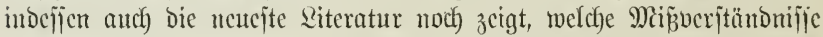

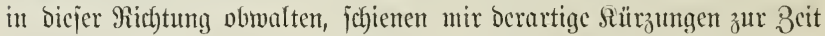

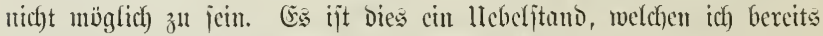
im Siorworte zur 3. Sufflage bes Buches betfagte.

Jch) bin weit entfernt Davon, Das jecil ber Forjtwifijenjchaft über= faupt ober das ber Forjteinrichtung im Bejonderen in mathematifecten Formelı zu crblicten, allein critens fömen bicjelben niclyt cripart werden, went man ben leitenden Girundgedanfen cinen fnoppen, fluren

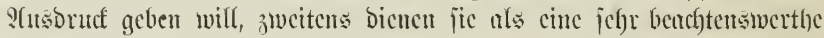

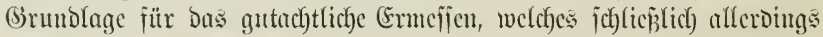

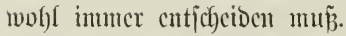

Dic Beigabe Der fleincu, in Farbenorudf antsgejütyrten Bcitands = furte biurfte woh)l Biclen wilffommen jein.

Iharand, im Mai 1885 .

ซ. Jutocid). 


\section{Intlịalt.}

\section{(E) i I $\mathfrak{c}^{\mathrm{t}} \mathrm{t} \mathfrak{\mathrm { H }}$.}

5.

1. Borbentertung.

2. Ier Ertrag .

$\therefore$ Ter Machbaltabctricb

4. Stugabe uno Begrifi Der Joriteinridtung . . . . . . 5

5. Eittheilung Der Qehre Der joriteiuridytung . . . . . . 6

\section{Bud).}

Yllgeueinc Sirund lagen.

\section{I. :(bjd)ıitt.}

6. Girnnobingutgen Des liormalnaldes

\section{II. ?(bid)uitt.}

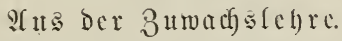

7. Tie veridicdenen Prten bes 3umadies

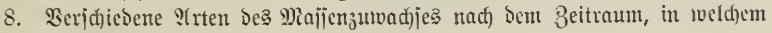
er erjolgt

9. Gang ১cs Majienzuwadję . . . . . . . . . . 11

10. Majienzutwat) Procent . . . . . . . . . 22

11. Qehrbeijpiel für ben Majienzumadis . . . . . . 27

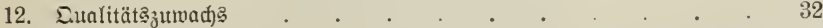

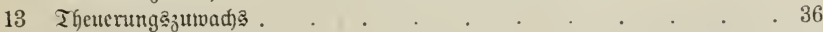

14. Eummiruny Der 3uwad) Eprocente . . . . . . . 37

15. Elemente DeẼ :

16. Beredung dę \$iseijerprocentes. . . . . . . . 47

1) Eer fouptbejtano . . . . . . . 47

2) Der 3 wijaenbejtand . . . . . 54 


\section{2(bjidnitt.}

Der ll mtricb.

§. Seite.

17. Begrifi . . . . . . . . . . . . 56

18. Der phyitidide llmtricb . . . . . . . . . . . 57

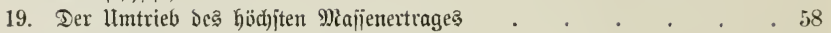

20. Der ted)nifidje llmtricb . . . . . . . . 58

21. Der llmtrieb ber hödjten Waldrente . . . . . . . 59

22. Der finanzielle llmtrieb . . . . . . . . . . 62

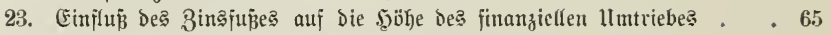

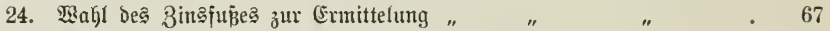

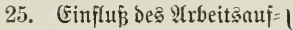
wandes u. Der Steuern 1 auf b. Söke " " . " 71

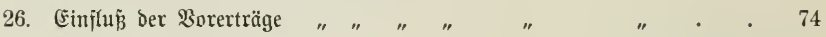

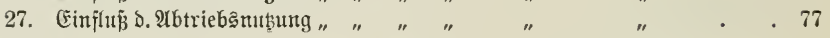

\section{2(bjd)uitt.}

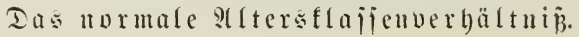

28. Der Jahre:̧đjlag . . . . . . . . . . . . 83

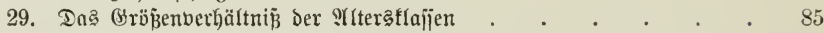

30. Dic Bertheilung Der MUterß̈flajen . . . . . . . . . . . 99

\section{V. $\mathfrak{A b j}$ d)}

Der Rormalvorrath.

A. Bom ङtandpunfte ber Mafienertragsiegelung.

31. Bedeutung des Pormalvorrathes . . . . . . . 103

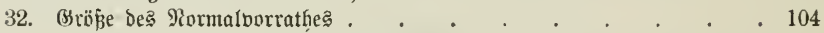

B. כom Stanopunfte ১er Finanzrednung.

33. Bedeutung bes finanziellen Normalvorrathes . . . . . 118

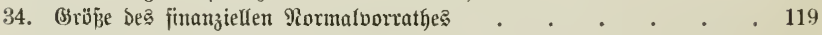

VI. Ibjiduttt.

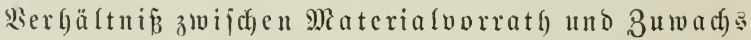
im Siormalwalde.

35. Bertfcilung Des 3uwadjes auf Den alten uno neuen Borruth wägreno Der Berjüngungesbauer eine $\mathfrak{B}$ ejtandes. . . . . .

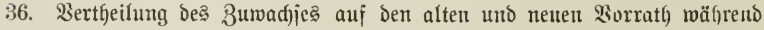
ber Ulmtriebszzeit einç ganzen $\mathfrak{W a l b e s ~ . ~ . ~ . ~ . ~ . ~ . ~} 136$

37. Beredfunt oer 3uwad) Reiträแแแแน, alą Der Untrieb 


\section{M(bjd)}

\section{Der normale Sicbsjab.}

\$.
A. Bou Etandpunte Der Mafienertragąregelung.

38. Begrifï .

39. Brö̧̈e deß normalen biebșațes

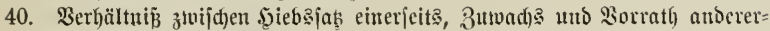
jeits in Mormalwalbe

B. Bom Stanopunfe Der Finanzed)ung.

41. Begrifi uno Bebeutung

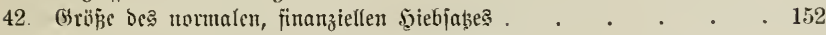

\section{2(bjd)uitt.}

\section{Betricbsflajien. (WEirtfjedaftsflajien.)}

43. Begrifi uno Bedeutung ber Betriebsflafien . . . . . . 153

44. Ilrjad)en, weldhe Die Bildung von Betriebätlaijen besingen . . 154

\section{2(bja)uitt.}

Der mirflidye $2 \mathfrak{s a l d}$.

45. Der abnorme 3uitano . . . . . . . . . . 155

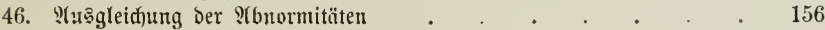

\section{Budj.}

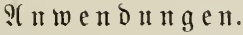

\section{A. Borarhrifen.}

47. 3meat uno Eintheilung ber Sorarbeiten

\section{2(bja)nitt.}

\section{For jt vermej门u}

(Geometrifale Sorarbeiten.)

48. Yufgabe ber Foritbermejïtng

49. Die Forjtbermejīng vorbereitende Srbeiten . . . . . 161

50. Sermeijung unь 9tujnafme fel6jt . . . . . . . . 162

51. Fläd)enbered)mung . . . . . . . . . . 163

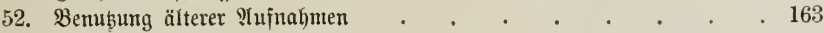




\section{2(bja)}

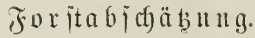

(Taratoriface Sorarbeiten.)

s.

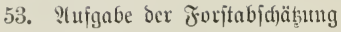

\section{Ermittelung der Standortsuerbältuij门c.}

54. Buvect

55. Standortabejdreibung

56. B̉onitätsmañe

57. Ermittelung der @tandortsgïte.

58. \$erth der Standorts=Bonitirung

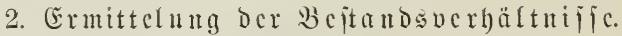

59. 3rvet uno Eintheilung Der 2ufgabe . . . . . . . 176

60. Wabl ber Metfod . . . . . . . . . . . 177

A. Borbereitungarbeiten

61. Эon ben Ertragstafeln überfaupt . . . . . . . . 178

62. Jnthalt der Ertraģtafeln . . . . . . . . . 180

63. Ertragstajeln der verjofiedenen Betriebajt)iteme . . . . . . 187

64. Werth und Bedeutung Der SAfgemeinen oder Normal= und Der Socal= Ertragitajeln . . . . . . . . . . 188

65 Bej̣timmung Der Majiengehalte der ortsitblidben Jiaummaj̃e. . . 190

B. Bejtandsbejdicibung.

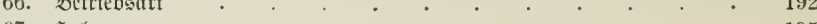

67. 5olzart . . . . . . . . . . . . . . . 195

68. Beptodungggrad . . . . . . . . . . . . . . . 196

69. Bejtandsalter. . . . . . . . . . . . . 199

70. Entitehung . . . . . . . . . . . . 202

71. Sol 3majie . . . . . . . . . . . . . 202

72. 3umadis . . . . . . . . . . . . . 205

73. Sorratbglapital . . . . . . . . . . . . . . 209

74. Grundfapital . . . . . . . . . . 210

75. Dą Ieijerprocent. . . . . . . . . . . 212

76 Bcitandsbonitirung . . . . . . . . . . 214

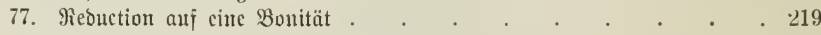

78. Motizen ïber Die fünftige Bemirtbjd)nftuny . . . . . 223

3. Ermittelü ber bisherigeu Erträge ud Siditen.

79. Bejtimmung der ?tujgabe ïberbaupt . . . . . . . 226;

80. Ier Materialertrag Der bauptmuţungen . . . . . . 226

81. Ter Belbertrag Der Souptnuţungen . . . . . . . 228

82 Dic Rebenmuţungen . . . . . . . . . . . . 229

83. Die אojten . . . . . . . . . . 230

84. Seitere Jolgerungen . . . . . . . . . . 231 


\section{III. :(bid) $)$ itt.}

Ermittelug Der allgemeincn uno äuñeren Forituerbältuijic.

$\S$

85. 3wed unb Eintheilung Der YIugabe . . . . . . . . 233

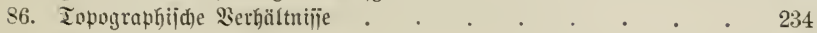

87. (3) iffichte . . . . . . . . . . . . 234

88. Eigentfututäverfältniije . . . . . . . . . 234

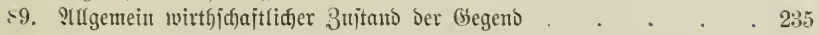

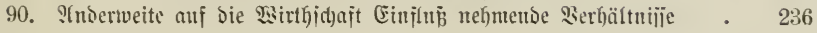

\section{2(bja)uitt.}

fiaten un Echriften.

91. 3ivecf

1. Dic fiaten.

92. Sartent ̈̈berfautpt

93. Tie Epecialtarte . . . . . . . . . . . . 238

94. Die Bejtandsfarte . . . . . . . . . 241

95. Die Terrainfarte . . . . . . . . . 244

96. Tie Bodenfarte . . . . . . . . . . 245

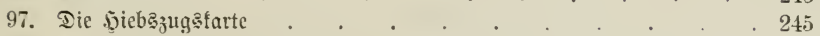

98. Die Peşfarte . . . . . . . . . . 246

2. Dic Echriften.

99. Sdifriften ïberfaupt. . . . . . . . . . 247

100. Ia Tarationşmanual . . . . . . . . . 247

101. Tie Bejtande-Rlaijentahelle . . . . . . . . 250

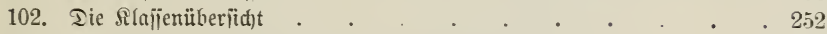

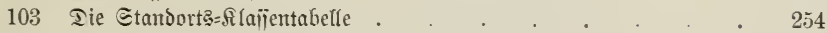

104. Die $\mathfrak{A}$ butsugatabelfe . . . . . . . . . . 256

105 Iaßs brrenzregifter. . . . . . . . . . 258

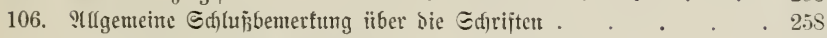

\section{B. It)aloxinflycilunț.}

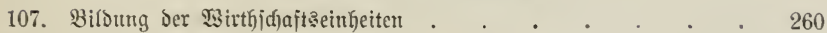

108. Bilbung und Begremzung Der Betriebąfaijen . . . . . 261

109. Bildung von biebşż̈gen . . . . . . . . . 263

110. Bildurtg ber 2fbtheiluntgen . . . . . . . . . . 269

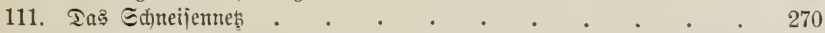

112. Sidferbeitß̄teine . . . . . . . . . . . 277

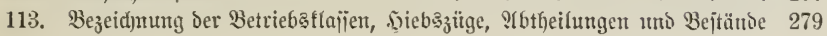


\$. Seite.

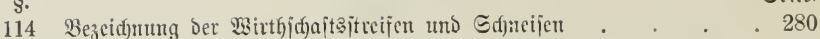

115. @ostiebe . . . . . . . . . . 280

116. Rejerben . . . . . . . . . . . . 284

117. Michtgolźboden und beїен Bezeichnung . . . . . . 286

\section{Exfraṇtheffimm}

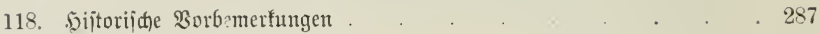

119. Die Edflageintbeilung . . . . . . . . 312

120. Tie Fad)wer烼metfoden ïberfant . . . . . . . . 316

121. Ias Jläd)enfad)wert . . . . . . . . . . 318

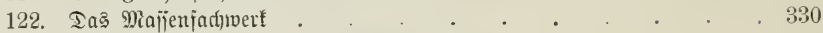

123. Tą combintrte Facfiwert . . . . . . . . . . 337

124. Cäd)iitches Berfahren bis zur Mitte ber 1860er Jahre . . . 341

125. Die Rormalborrattgemethoden überhaupt . . . . . . 349

126. Die אameraltąe . . . . . . . . . . 350

127. 5undeăhagen'? Beriafren . . . . . . . . . 357

128. Cart bener's Berrahren . . . . . . . . . . 363

129. Rarl's Berfafren. . . . . . . . . . . 382

130. Brenntant's 'Serfahren . . . . . . . . . 388

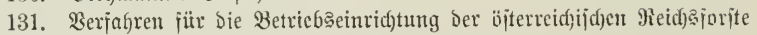

1 Siad) Der Jnjtutution bon 1856 . . . . 390

$2 . "$ " " 1878 . . . . 393

132. Werfafren ber Bejtanbsuirthjchajt . . . . . . . 399

A. Ter allgentine $\mathfrak{s i r t h j d ) a j t a p l a n ~ . ~ . ~} 400$

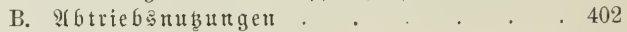

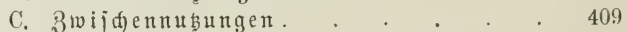

D. 巨todfolz . . . . . . 414

139. Yntwendung ber im \$ 132 geichilberten Methode auf andere betrieba: arter, ala auf ben jollagiveijen şod)waldobetrieb . . . . 415
A, Piebertwald. . . . . . . . 415
B. Mitteltuald . . . . . . . . . 415
C. Flenteriolo . . . 418
D. Ilmwandungen . . . . . . . 420

134. Buitav Sisagener's \$erfafren . . . . . . . . 422

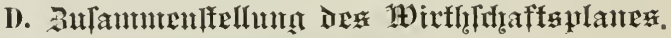

135. Ier פsirtficlafteplan ïberffanpt . . . . . . . 427

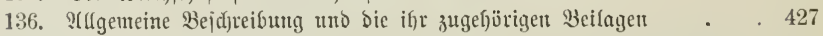

137. Epecielle Bejodreibung . . . . . . . . . 430

138. Der fpecielle 5aumngplan . . . . . . . . . 432

139. Der ppecille §ultulplan . . . . . . . . . 444

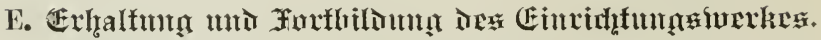

140. YUlgemeine? . 


\section{I. :(bjd)uitt.}

\section{노ำ}

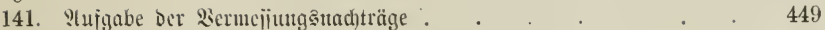

142. Ią̧ Piotizentutc) . . . . . . . . . . . 452

143. Daริ श(ad)trag引bud) . . . . . . . . . . 454

\section{II. :(bjd)}

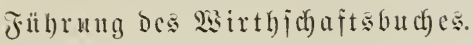

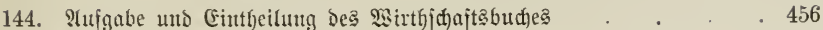

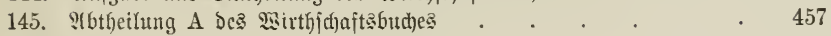

146. " B " . . . . . 458

147. " C . . . . . . . . 460

148. . . . . . . . . 462

149. " $"$ E " . . . . . . . . . . . . . 464

150. " $"$ F" . . . . . . . . . . . 466

151. :nbentungen für eine fpeciellere Budfiugrung. . . . . 475

\section{III. :(bjd)uitt.}

Iic Revijionen.

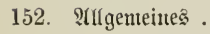

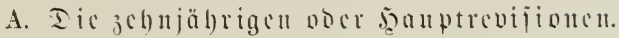

153. शैufgabe ber ફauptrebifionen

1) Ilnterjưngen barüber, wie bie Bejtimungen bes abgelaufenen Branes bejolgt wurden, ob und welde Girüne etwaige St bueidungen red)tfertigen

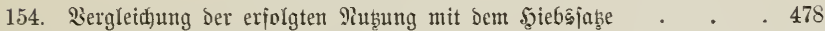

155. Bujammenitellung Der plantorigen Şaunigen . . . . 480

156. 3ujammenitellung Der Durdforitungen . . . . . . . 480

157 Betradtung ber übrigen 3wijd)entuthungen . . . . . 482

158. Die Rebcrmuthungen . . . . . . . . 484

159. Joritvirbeijerungen . . . . . . . . . . 485

2) Interiudungen barüber, wie jid) bie Bejtinumgen Des Pilanes bewährt haben.

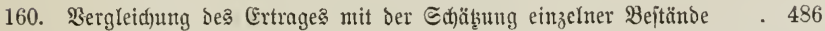

161. Die 3 wijd)ennutsungen . . . . . . . . . 486

162. Die fiebsfolge . . . . . . . . . . . . . . 487

163. Tie Rebcrtutsungen . . . . . . . . . 487

164. Forjtberbcijerugen . . . . . . . . . . . 488 
3) QHiftellung des neuen \$lancs.

$\S$.

Scite.

165. 9llgemeines . . . . . . . . . . 488

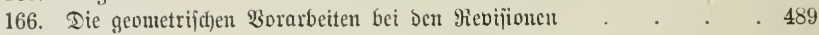

167. Die taratorijchen Borarbeiten bei ben Mevifionen . . . . 493

168. Dic für dic Revifion nöthigen Mamuale . . . . . . . 494

168. Der $\mathfrak{B}$ irtbidjajtaplan . . . . . . . . . 497

B. Dic fünfiälrigen oder Buijchentevijionen.

170. Sicjen und :Hafgabe Derfelben . . . . . . . . 499

171. Bebeutung Der Bwijđ)enrevijionen . . . . . . 505

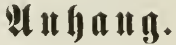

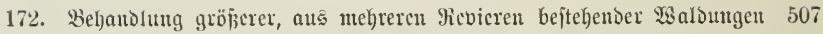

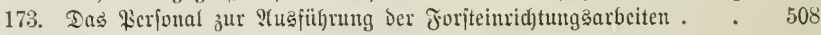

Beritjtigu ugen:

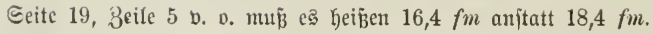

Seite 400, Beile 13 v. D. fehlt vor Der Ueberjd)rift ein A.

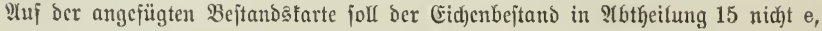
jondern c heiß̄en. 


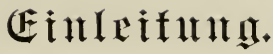

\section{$\S 1$.}

\section{Dorbemexkatg.}

Der 3 wed ber Joritwirthjodjat ijt bie möglichjt vor= theilfafte Benubung IIID Bodents.

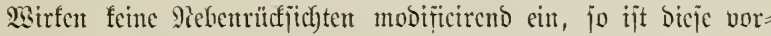

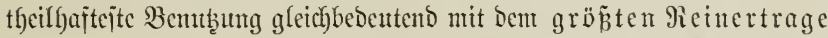
ober Der höchjten Reroinjung aller in ber $23 i r t b j$ chajt thätigen fapitale, oder autch mit Dem höchjten llutentelyergetwinn.

Diejem nächjtfiegenden 3wecfe Des fjöchjten Reintertrages entzichen

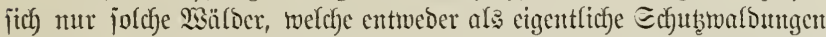

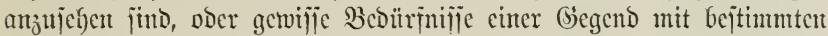
Eortimenten volfswirtbjefaftlich) nachweisbar befiriebigen miijfen, ober

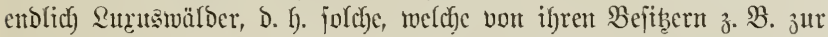
Berjedjönerung Der Bécgend als grożartige, natürliche Parfanlagen an= gejeben werben, welche als Ifiergärten bienen jollen $\mathfrak{i}$.. iv.

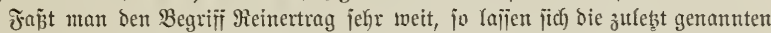

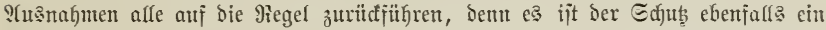
Ertrag, ebenjo die Befriebigung eines gewijien Syolabedarfes, ebenjo endich die (Sewährung periönlichen \$̧ergntitgen?.

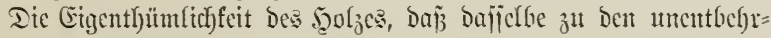

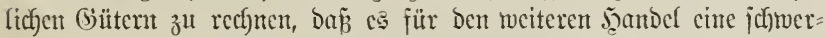

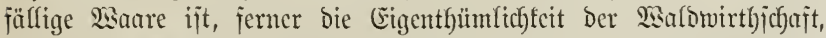

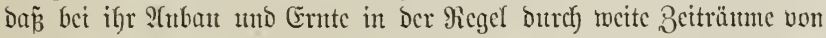
einander getresunt liegen, liȩ̄en Gald bie Ěrmittelung bes möglichen

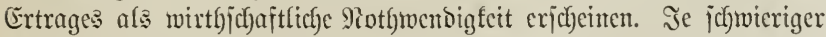
bie Rojjung biejer P(ujgabe zu jein jefien voer mirfficl) autch war, bejto

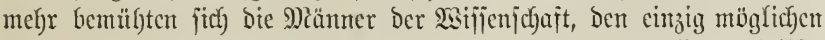

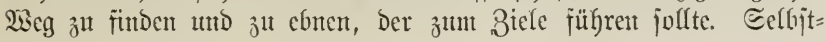


veritändich fonnte da: leitende \$rinzip nicht aus bem wirren Buitande

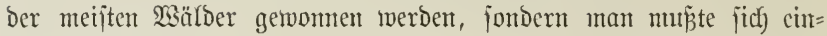

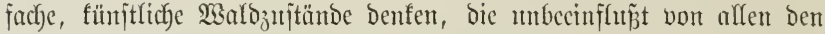

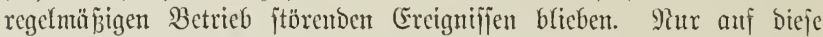

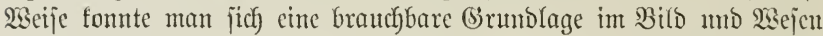

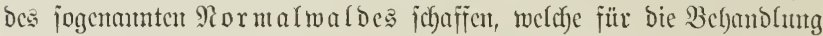

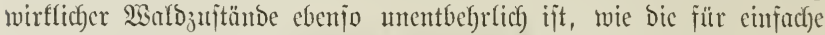

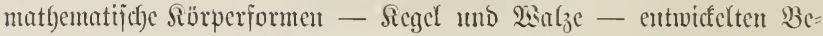

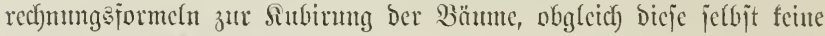

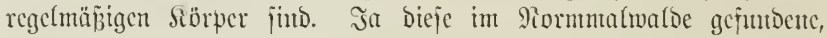

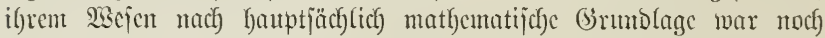
mehr, fie bilocte das Biel, anf weldbes man mebr ober weniger birect ben vorfantocnen, abutormen 3ujtano bes 2 aldos fiuzulenten jutchte,

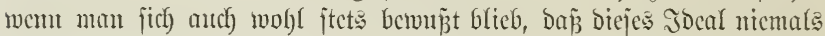
gouz zut exreichen jei.

\section{§ 2.}

\section{Dex Erfrag.}

Der Ertrag ift Folge Der in ber 2 irthjefaft thätigen Factoren Sibeit un Sapital. (Unter Yeșterem Den Grumb und Soden

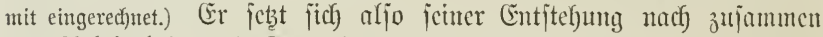
aแล S(rbcitslohnt und Sapitalzins.

Robertrag ift bie Summe alfer währent eincs getwifien 3cit=

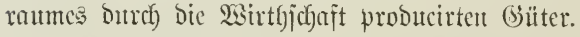

Befreit man Den Rohertrag Des Waldes von Don jälurfich 3n

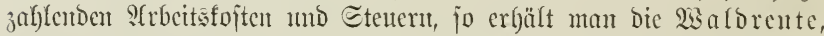

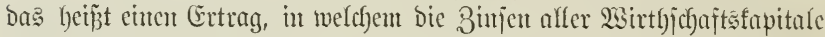
nod) mit inbegriffen fino.

Bringt mant von Dem Rohertrage jänmeliche ßroductionsfojten,

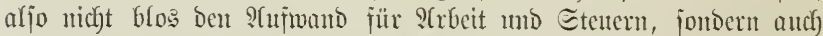

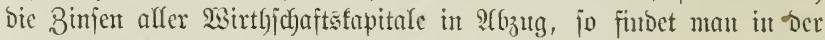
Differcuz ben llnteruehmergervintu.

Scheidet mat den Girmb und 20 ben ans Der Eumme ber in ber Wirthjchaft thätigen Sapitalc anß, jo erbält man in ber Different

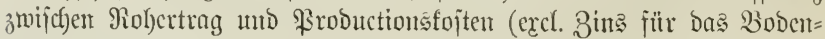
fapital) Die Bodenrente, Den Bodenreinertrag.

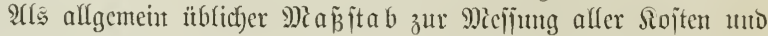
Frträge dient dą Gicld. 


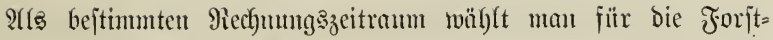
wirth)ichaft alfgemein bas Jaf)r.

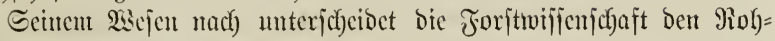
ertrag eines 2araloce it

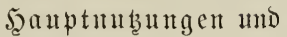 \\ Rebenumbugen.}

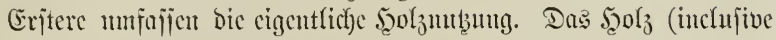

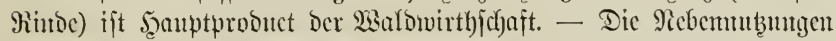
bejteben aus allen übrigen 2 aloprobucten: Streu, (3ras, Frïchten,

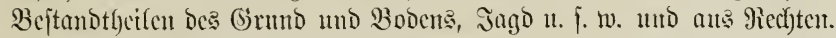

Dic şauptunbutugen zerfallem wicoserum in

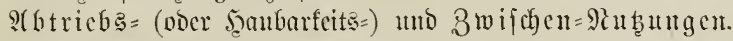

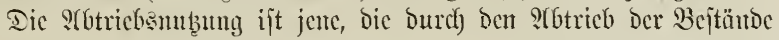
erfolgt, wobei unter Sorausjebung einer fortzujetsenden Wolswirtl)= j(d)aft bic Begrünoung neuer Bejtänoc notfywendig wiro. Die Bwijchen=

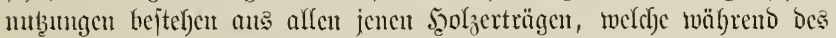

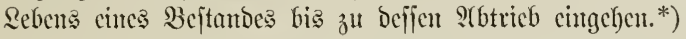

\section{$\S 3$.}

\section{Dex I2authrgalfutufriefr.}

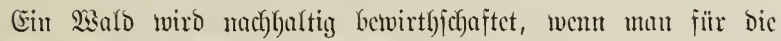
Wiederverjüngumg aller abgetriebenen Bejtände jorgt, jo bar baburch) Der Boben Der Seoljzutcht getwiomet bleibt.

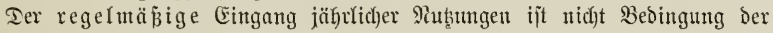
Piad)haltigfeit.

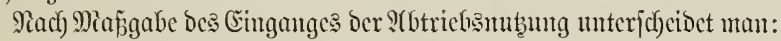

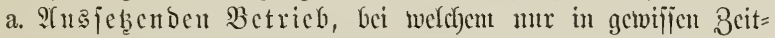
räımen, aber nicht alljäbrlich cine atbtricbsmbung erfolgt;

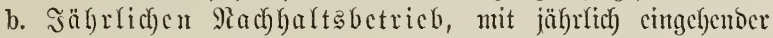
?(btricbsmubung.

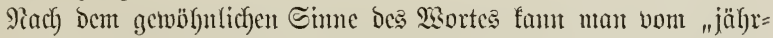

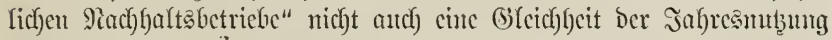

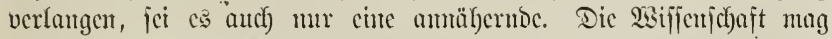

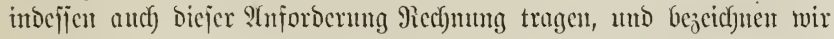

*) In Eachjen murde Durdy die, Injtruction in Bezug auf die Jorittaratione:

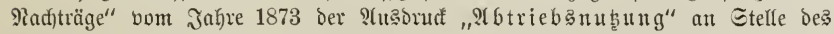

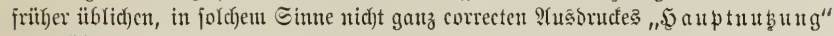
eingeriitgrt. 


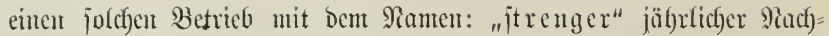
baltabctrieb.*)

(Fine für jich bejtelende, ijolirte Saldoparedle, Deren Bejtand jtets im uten \&ebensjahre abgetricben wird, liefert alfe a Jaljre cime 9 (b=

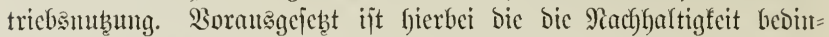
gende 2 icoerverjüngung. Es ijt bies bie cinjacbite Form bes aus= jeßzenden Betricbes.

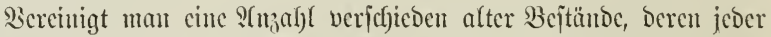

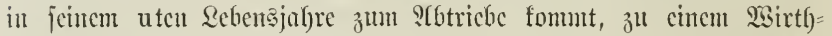

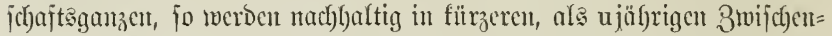

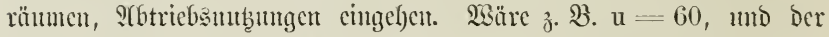

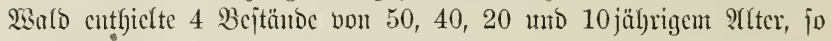

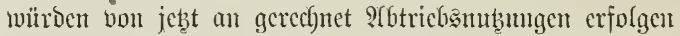

$$
\begin{aligned}
& \text { nack } 10,20,40 \text { แmb } 50 \text {, } \\
& \text { " 70, 80, 100, " 110, } \\
& \text { " } 130,140,160 \quad \text { " } 170 \text { Эnfren }
\end{aligned}
$$

น. โ. w.

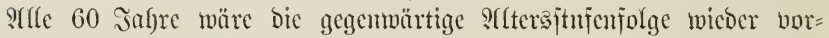

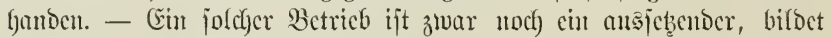

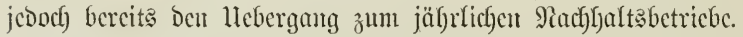

Bereinigt cine Wirtfjefaft Dagegen u Derartige Bejtëmbe in einer

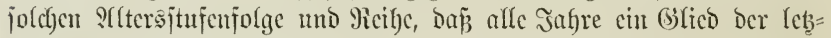
teren abgetrichen werden fantu, jo giebt jic uns bas $\mathfrak{B}$ ild liçen Plachbalts̄betriebes.

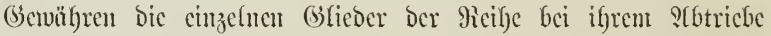

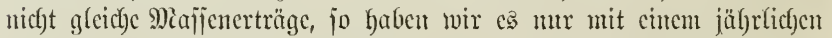

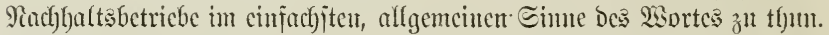
Dieje Serjebiedentyeit Der Erträge famt, abgejefon von ben imuner แn= vermeidlichen Etörungen bcs Bctriebes, als bleibende bebingt merben burch) verjeficdene, mit ber Etandortšbonität micht im Sicrljältmific

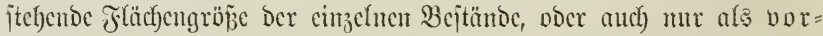

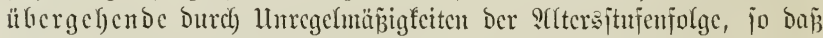
zum Beippiel an Etelle von 10 in regelmäß̈iger 2 (bjtufung 20 bi: 29 jäfrigeli Bejtünden 10 gleicfalterige vorfanden twären, twoburch Deren nïcbjte şbtriebsalter abutorm würbc.

*) C. 5eyer unterjobet in Diejem Einne „itrengeren" und "itrengiten"

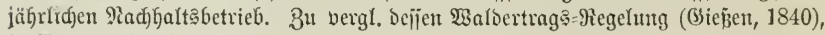

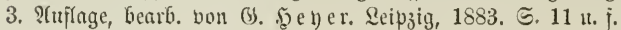




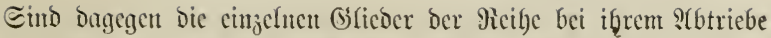
gleich majjenbaltig, ijt aljo bic seltersitufenfolge ber o bis u-1 ober

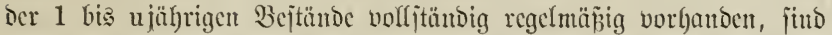

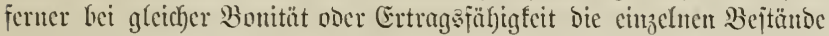

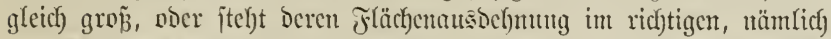

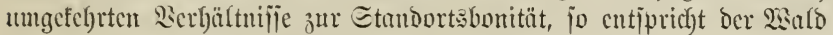
Der Form bes itrengen jâfhrlichen Pach)laltsbetricbes.

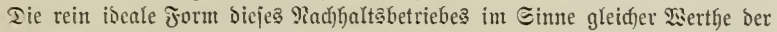

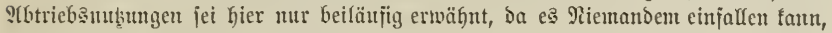

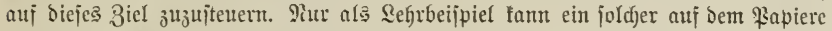
entworfener জals

Ilnter allen llmjtäubeu ijt im Suge zu begalten, da

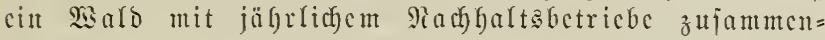
gejebt ijt aus cinzelnen Bejtändeu oder Bejtandesgruppen, bic für jid betradbet im ansjebenden Betricbe bewirth= jefajtet werden.

\section{$\S 4$.}

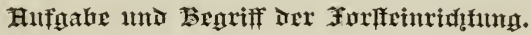

Die Sufgabe ber Forjeinrichtung ijt bic, Den gejammten

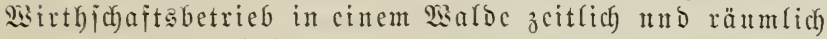
jo ju oronen, ba f ber 3 wed ber sirthjchaft möglichjt cr= reicht iverde.

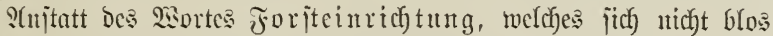
in Eacfjen, jondern auch anderwärts fijtorijche Berechtigutg crmorben,

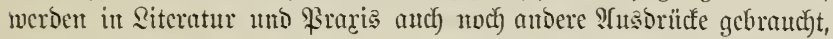
namentlicy: Betriebsregulirung, Forjtaxation, Forjujemi= jirung (in Scitereich). Dic Waldertragseregelung betrachten wix nicht als junoulym mit Forjteinricftung, fondern als cinen wejentficjent Theil Derjelbent.

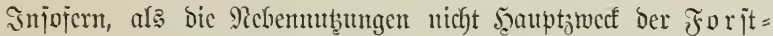

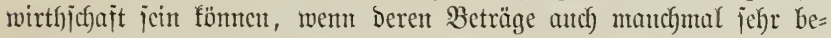

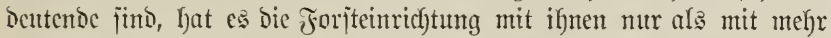
ober mentiger modificirend ciumirfenden Factoren zu thum.

Jür jebr fleine, im cinfachjten, antsjebenden Betriebe zu berwirtf)=

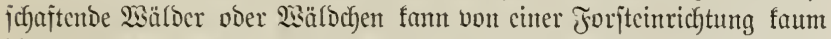

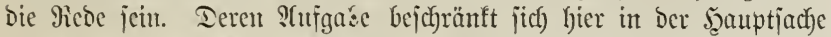

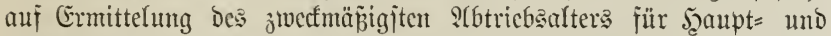

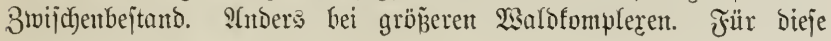


madyen bie im $\$ 1$ antgedenteten Eigentfïmlichfeiten ber Foritwirtb=

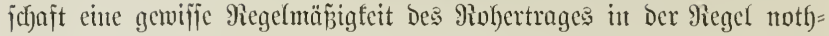

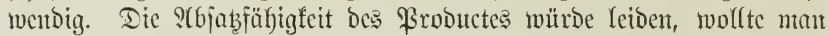

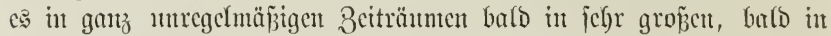

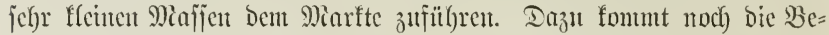

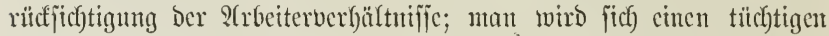

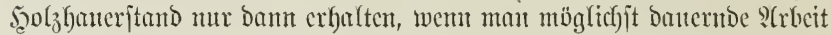

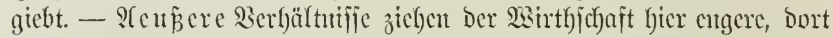
weitere Brenzen, ittocm fic mebr ober wentiger Die Giegelmäp̈tgfeit Der

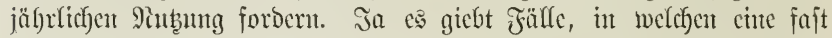

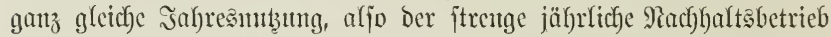
nöthig ober wïnjchenswertl) ijt; 子. 3. gejeb̨lidfe Bejchränfungen Der

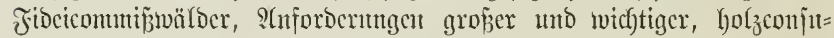

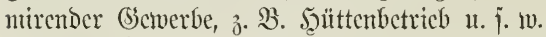

Eine innere Potfmendigfeit Der Waldwirthjechaft ijt ber itrentge

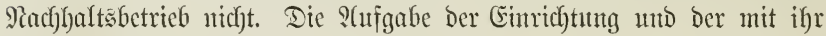

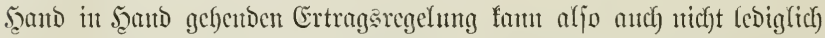

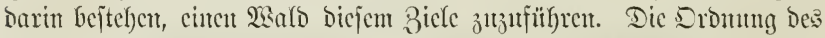

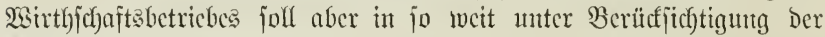

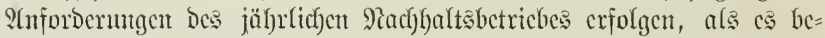

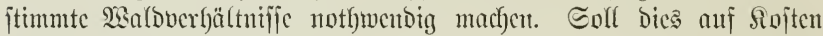

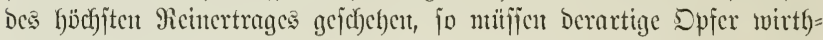
jefjaftlich gerechtfertigt werben fönnen.

\section{$\S 5$.}

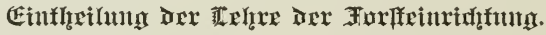

Sie bat zu belambelut:

1) Die allgenteinen theoretijchen (Srumblagen, anf weldye fich) bie Einricftung jtübç nut

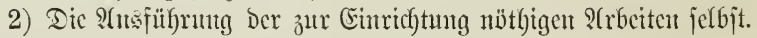
Sebtere zerfallen ifrer Patur nach) in:

A. Boxarbeiten.

B. Waldeintbeilung.

C. Ertragsbejtimmung.

D. Bufammenftelfung Des Winthjchaftsplanes.

E. Erbaltung und Fortbiloung Des Eimrichtungswertes. 


\section{Butht}

\section{Atgemeine grunofagen.}

\section{AGrrfinntrf.}

\section{Orunbbedingutgen bez Mormaltwalbes.}

$\S 6$.

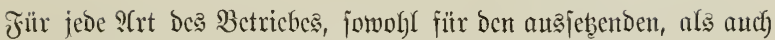

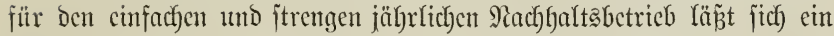

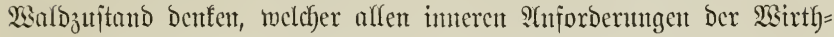

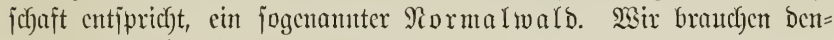

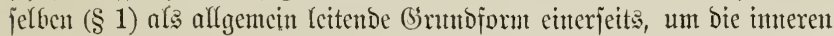

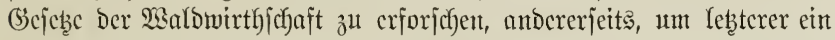
idealce 3 iel 3 ut jteffen.

๔eb̧t mant bie ben gegebenen Berfältuififen entiprect)ende 5 golzart

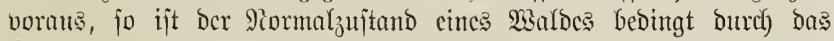
Sortgandenjein

1) Des normalcn 3 uwadje马,

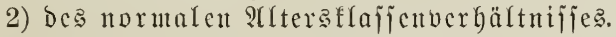

2(la ummittelbare Folge Der Errfüllung bicjer betben Bedittgungen ericheint

3) Der normale Şolguorath.

3"1 1. Inter normalem Buwachje verjtelyt man im Sinte der

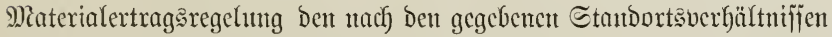

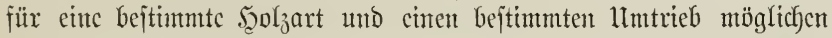

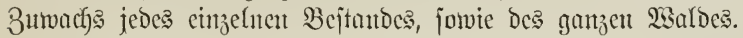

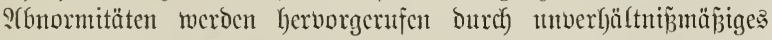

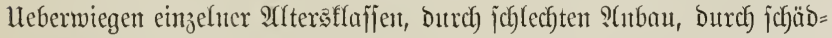
liche. Elementarercignifife u. j. w.

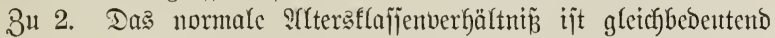

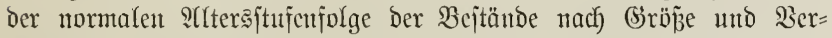
theilung. Das heiất, bie einzelnen beitände mülīen jo gruppint jein, 


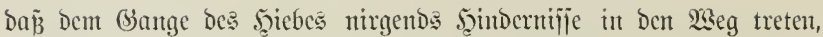

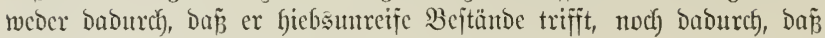
er biebsureife Srte nicht rect)tzeitig erreichen fumt.

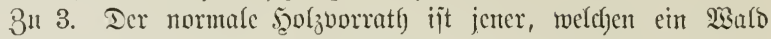

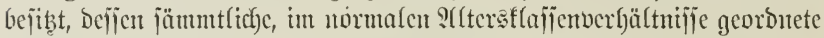
Bejtänto normalen (laujentoen uno Durchjefhnittlichen) Bunachs haben. Siscroen bie SAforderntugen unter 1 und 2 befriebigt, jo iteflt jidf) afjo Der Pormalvorratf) won jelbjt her. Dodf fan er and bei cincm $a b=$

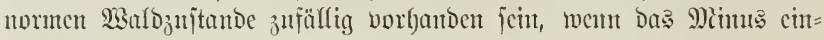
jeluer Bejtände burch Das $\mathfrak{B}$ Gus antocrer übertragen wird. -

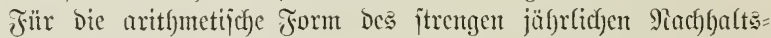
betriches wäre aljo Der 3ujtand bes 2ialdes normal, went lebterer

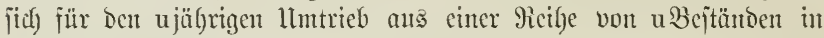

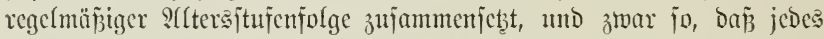

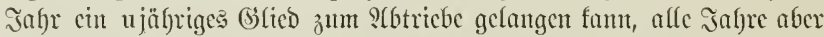
Dericlbe Daterialertrag erjolgt. Jede Störuntg des normalen 3uwad)jes

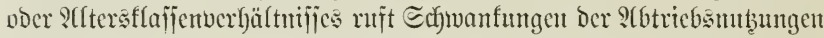
lervor.

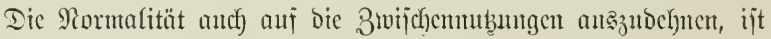

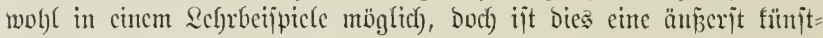
liche Mafaregel bon mu untergcorbuctem, wijjenjchaftlichem mo praf=

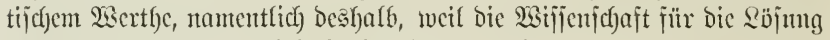
biejer Frage noch) zu vicle Sücfen bejib̨t, weld)e autd) Die 3ufunft vor= autsjichtlick nich)t gentïgend ansfüllent wird.

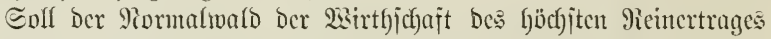

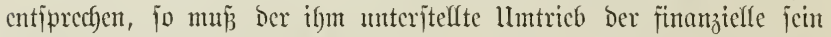

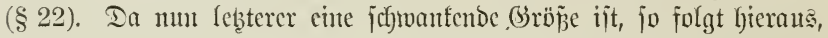

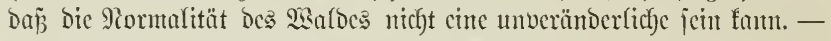

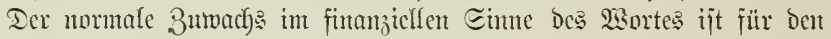
ganzen Wald Damt vorlfanden, wemt meder cin . gaupt= noch ein 3wijajenbejtand jich Darin findet, Dejjen $\mathfrak{2 e c i j e r p r o c e n t ~}(\$ 15.16)$ bei

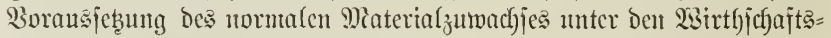
zinsfup gejunfen ijt. 


\title{
II. AGritrutitf.
}

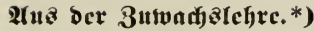

\author{
$\S 7$.
}

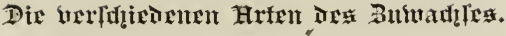

SIn jebem Baum ober Bejtand erfolgt:

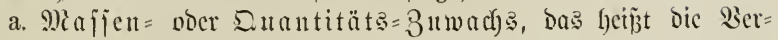

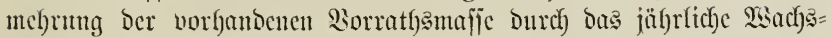
thum bes Baumes vocr Bejtandes. Er wirb gemeffen Durdf bic Maffen= cintheit, Das fubifmeter.**)

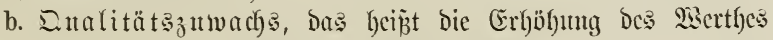

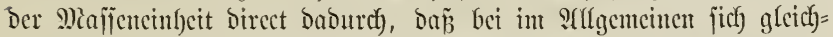

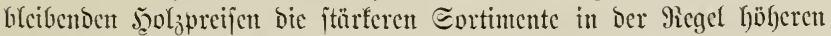

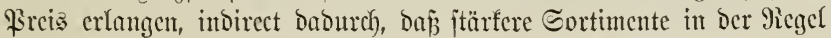
geringere Erntefojten werurjachen. Er wirb gemefien ourch Den 14 bic Erntefojten verminderten Preis ber verjejtedenen Sortimente zt berfelfen Beit.

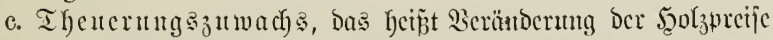
überbaupt. Er wirb gemefijen onth) Den Prets Derjelben Sortimente ju verjefjedenen 3eiten.

Beijpiel. CFin gegentwättig 50 jähriger Bejtant entfält anf bem b̧eftar $200 \mathrm{fm}$, nach 10 Jafren wiro er voraus fict)tlich) $240 \mathrm{fm}$ entfalten, fo beträgt jein

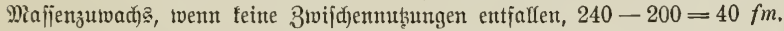

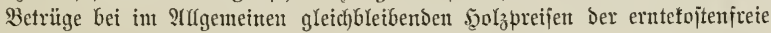

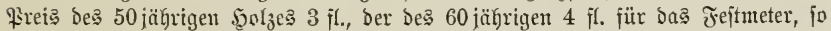

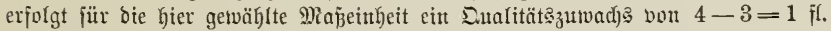

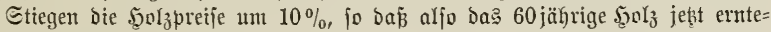

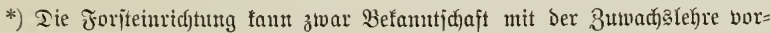

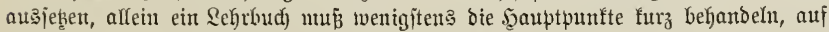

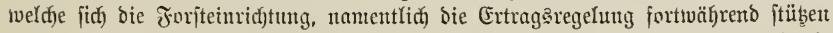

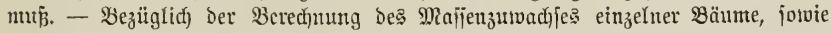

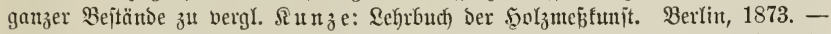

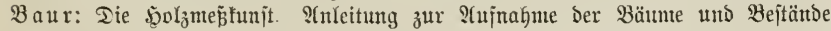

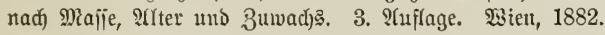

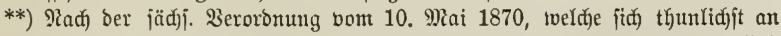
bie betreffenden preusifichen Bejtimmungen vom 30. Dltober 1869 anjdjliešt, fint

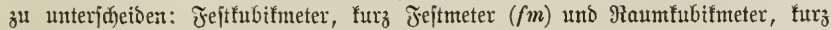

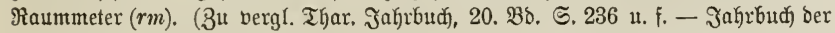

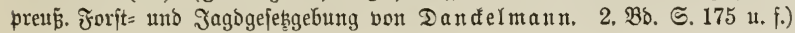




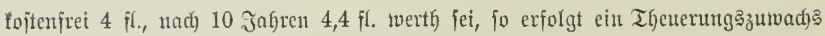
von 0,4 fl. für Dą Jejtmeter.

Der Bejamutzuwarfas bes ganzen Bejtandes witrde fid) in beldeșerth be= red)nen auf

$$
240 \times 4,4-200 \times 3=456 \mathrm{fr} .
$$

Denfelben Betrag erfalten wir burd) Eunminung ber einzelnen Fojten, wie folgt:

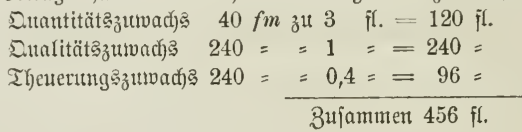

$\S 8$.

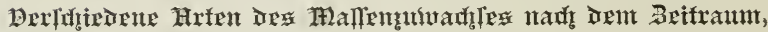

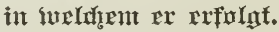

P(ut Batm ober Bejtanto erfolgt:

1) sährficher (faufento jüfrlicher, einjäfriger) Buwarbs it cinem Jabre.

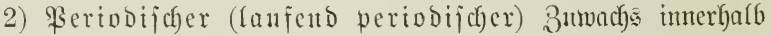
cutes gemäh) ten, mefrjäl)rigen 3citabjchuttes.

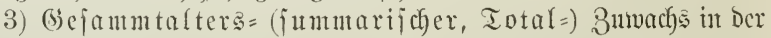
3eit wou der Entitchung Des Bauntes oder Bejtutbes bis ju jeitem gegenvärtigen ?(íter.

Mian bejicht biejen Buwards and anf bic Beit von Der Ent= ftefung des Baumes oder Bejtundes bis zum P(btriebe.

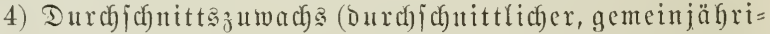
ger). Er ift Der Duntient ans Der Bafl ber Jabre eines unteritellten 3eitraumes in Den wäl)reno bes leb̧teren crffolgten 3utuach šbetrag. Da=

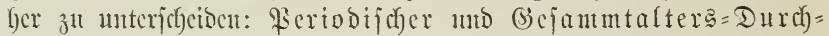

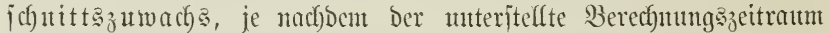

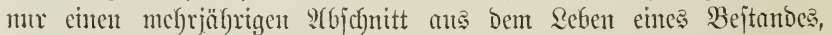

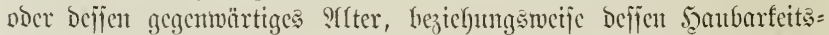

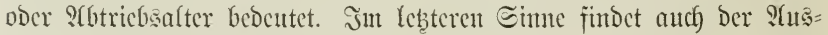

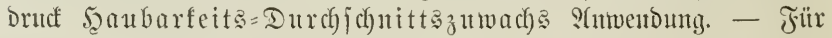

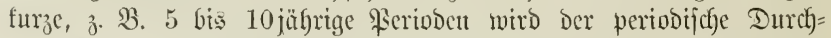

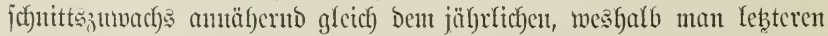
Durch Berechunng bes exiteren ant bejten findet.

Die Buwachsermittelungen fam man entweder uur auf bie Mafje Des prädomintrenden, bes 5̧auptbejtandes, oder nur auf ben 3 wi jichenbefand oder auf bie Summe von beiden bezichen. 
Beifpiel (nad) § 11). Fïr Den şantptbejtanto allein: Ter 60 jährige Beitant enthält 354 , Der 65 jährige $394 \mathrm{fm}$, jo ijt ber periodija) 3uwadj3 40 ; Der periobijche

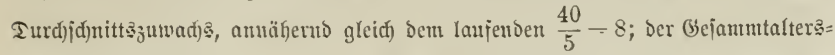

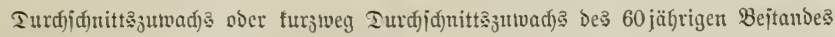
$\frac{354}{60}=5,90$, Der Deş 65 jährigen $\frac{394}{65}=6,06$. Jür Die Summe Des şaupt $=$ แno 3wija)enbejtandes beträgt der periobijaje Buwad) $40+15=55$; Der periobijaje

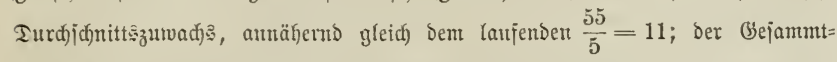

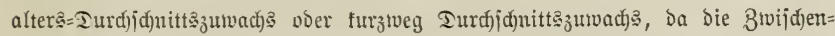

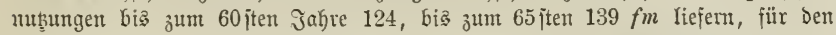
60 jäfrigen Bejtan๖ $\frac{354+124}{60}=7,97$, jür den 65 jäfrigen $\frac{394+139}{65}=8,20$.

\section{$\S 9$.}

\section{(Gang Des H2allenutuadțtes.}

1. : un eiuzefuen Baume.

Der Bmuachs erfolgt ant cutzelnen Baune nad) Der \&änge an

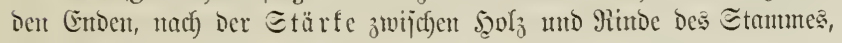
Der 3weige uto Der $23 u r j e l t$.

Ier Etamm noer Echajt ijt in Den meijten Fällen Der wichtigite Theil bes Banmes, Deshalb unterjcheidet man allgenteit gemb̈hnlich

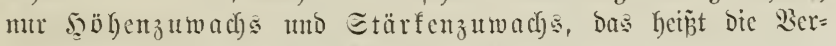
mef)rung Der \&änge und Der હtürfe Des હtammes.

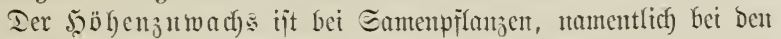

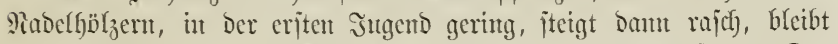

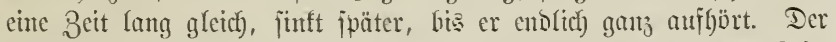
Gang ijt nach solzart tmo Etandort ein jebr verjefiedener. Ier 3 cit= punft Des jtärfiten Einfens ijt jener, mo Dic ?fbüblbuntg Der firone

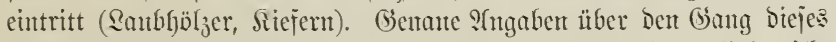

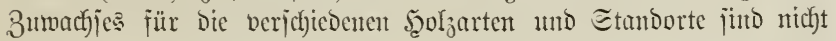
mïglich).

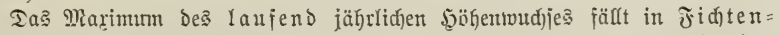

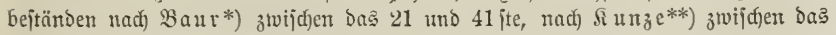

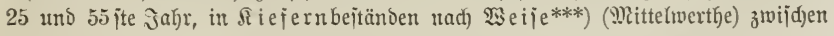

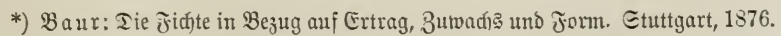

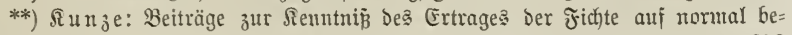
jtodten Fläden. Eupplemente z. Tharamber forjtlid)en Jahrbuche. I. Band, 1878 und III. Band, 1. ছeft, 1883.

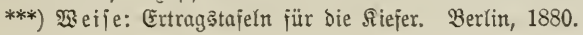




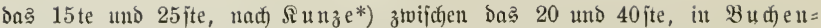

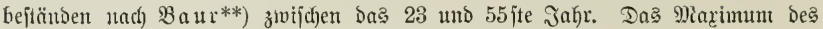

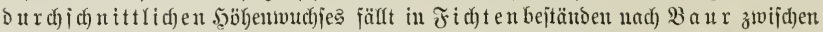

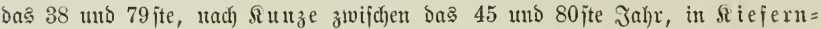

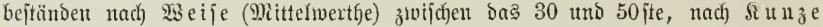

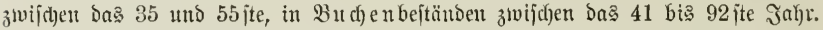

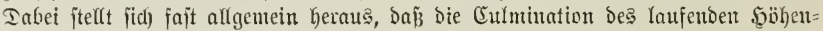
zuwadjfe bei ben bejeren Bonitäten frïher eintritt, als bei Den fchlechteren.

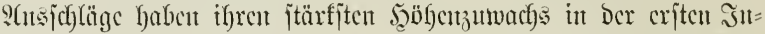

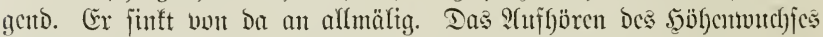

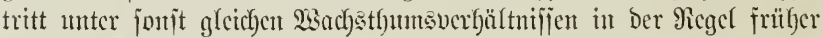
cin, als bci Sierntpflanjen.

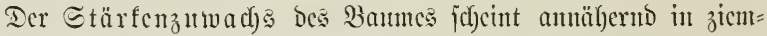

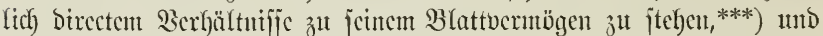

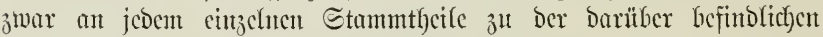

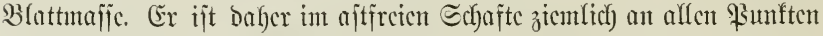

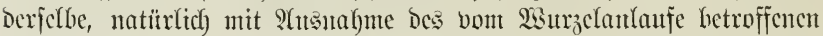
Ibciles, jutft bagegen in ber Sirone rajdy und olien lyin. Es tjt bies nicht für dic abjolute Brette ber cüjelnen Salyrestinge, fontocru für

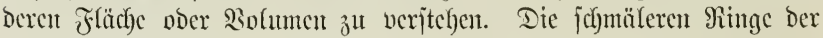
unteren Edyaftbcile bilben cinen grö̈̈eren firets, als bic breiteren Ringe des obcren Echaftes, bcibe fünten bufber jelbjt bei grofjen Diffe=

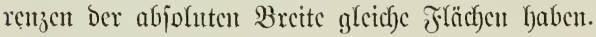

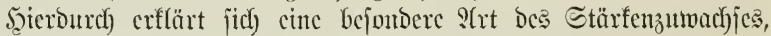

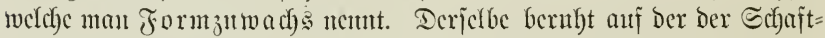
form günftigen Beränderung Der Differenzen zwoifclyen Dem Etärfent=

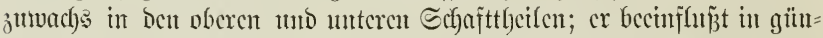

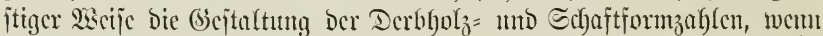
Der Sironenanfab hinaufritut.

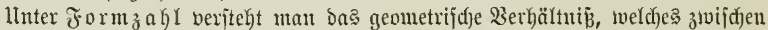

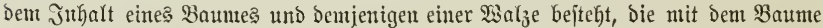

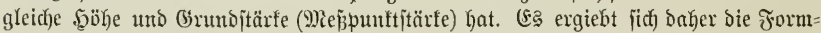
zahl eines Bauntes, wenn nan ben Inljalt befjelbent gennu ermittelt und Durch ben Inthalt ber zugehörigen Joealmalze Dibidirt. $\dagger$ )

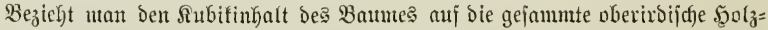

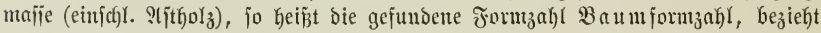

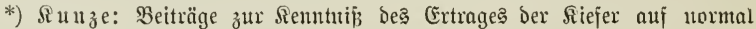
bejtodten Jläd)ent. Supplemente zum Tharander forjtl. Jahrb. III. BD., 2. Şeft, 1884.

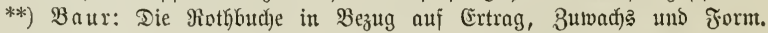
Berlit, 1881.

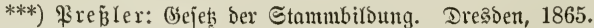

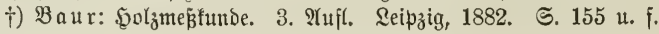




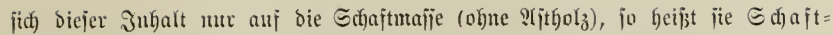
formzahl, bejieht fid) Diejer Jnthalt mux auj bas Serbholz, D. h. auf alles ïber

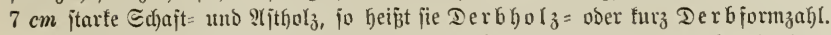

Mian unterjobibet norma le voer ä d)te Formzablen, zu Deren Ermittelung

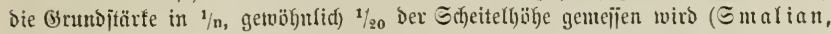
Fre B̆ler), uno unäd)te oder Brufthöbenformzahlen, zu deren Ermittelung die

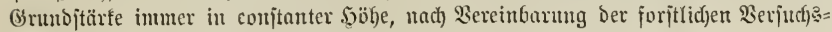
amjtalten $1,3 \mathrm{~m}$, über Dem Boben abgegrifien wirb.

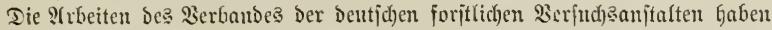

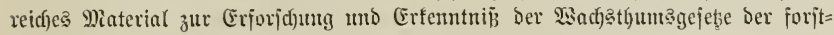
(id)en Bäunte uno Beịtänbe aud in Diejer Ric(jtung gebradft. *)

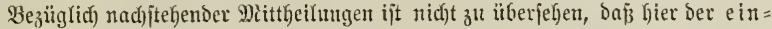
zelne Baum mur als Ślied eines ganzen Bejtandes zu betrad)ten ijt, ba jelbjt=

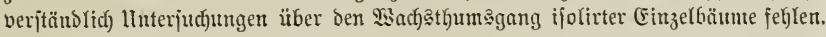

a) Sechte ober Mormalformaglent.

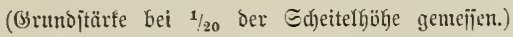

firfnfe. Sn Turdjidnitt aller Gitteflaijen.

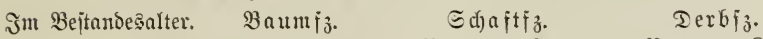

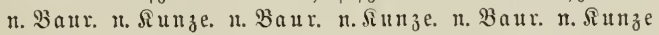

(äItere Mitth.)

$\begin{array}{rccccccc}21-40 \text { Jafre } & 0,698 & 0,671 & 0,439 & 0,487 & 0,298 & 0,201 \\ 41-60 \text { " } & 0,675 & 0,641 & 0,517 & 0,511 & 0,359 & 0,403 \\ 61-80 \text { " } & 0,596 & 0,601 & 0,500 & 0,521 & 0,438 & 0,507 \\ 81 \text { u. m. " } & 0,562 & - & 0,487 & - & 0,479 & - \\ 81-100 \text { " } & - & 0,591 & - & 0,521 & - & 0,519 \\ 101 \text { น. แ. " } & - & 0,573 & - & 0,515 & - & 0,508\end{array}$

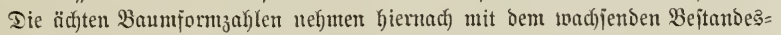
alter ab. Innerbalb gleidyer S(ltersflajien madjjen jie ïbrigens mit abnefymenter Bonität.

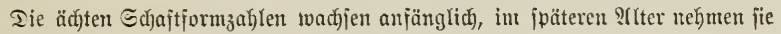

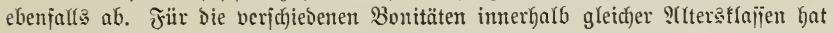
jid) ein bejtimmtes (sejêl nod) nicht ergebert.

Tie äd)ten Derbformzahlen müj|jen natïrlid) in Der eriten Jugend an fleinjten

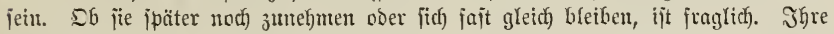

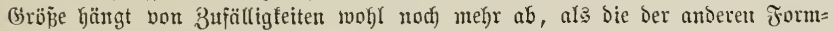
zaflen, audf fino fie itreng genommen nid)t ganz "äd)t", weil ber entideidente

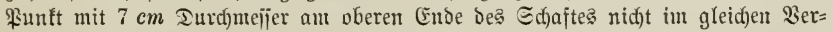

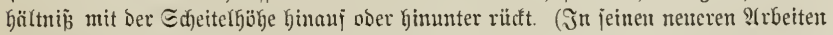
hat Dešbalb §umze bie äd)ten Derbjommahlen weggelajien.)

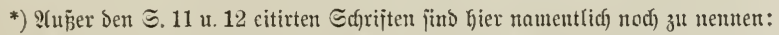
Sunze: Lie Formzahlen Der gemeinen ßiejer; ferner: Die Formzallen ber Fidjte.

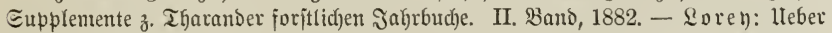
Baummajientajeln mit Beziegung auf bie Itnterjuchungen ber tönigl. Wisurttem= bergijden forjtlichen sierindysitation. Tübingen, 1882. 
Litefer nad) $\Omega$ ut ze.

\begin{tabular}{|c|c|c|c|}
\hline Jill Beitan & esalter. & $\mathfrak{B} a \mathfrak{u} \mathrm{m} f$. & (c) aftif \\
\hline $21-40$ & sabre & 0,509 & 0,412 \\
\hline $41-60$ & " & 0,486 & 0,418 \\
\hline $61-80$ & " & 0,484 & 0,434 \\
\hline $81-100$ & " & 0,474 & 0,428 \\
\hline $101-120$ & " & 0,537 & 0,455 \\
\hline $121-140$ & " & 0,517 & 0,456 \\
\hline
\end{tabular}

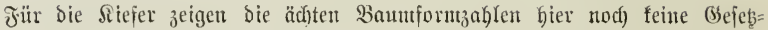

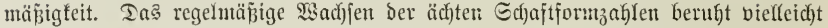

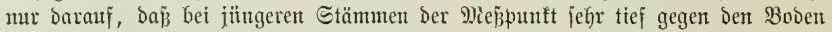

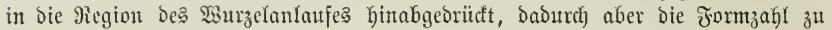

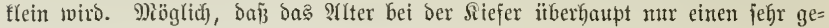
ringen Cinfluti auf bie Formzahl atşitbt.

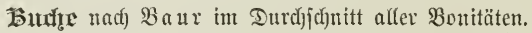

\begin{tabular}{|c|c|c|c|}
\hline Im Bejtan & eşalter. & $\mathfrak{B a u m} \mathfrak{f}_{z}$. & Derbiz. \\
\hline $21-40$ & jagre & 0,574 & 0,250 \\
\hline $41-60$ & $"$ & 0,562 & 0,347 \\
\hline $61-80$ & " & 0,558 & 0,424 \\
\hline $81-100$ & $"$ & 0,568 & 0,472 \\
\hline $101-135$ & $"$ & 0,581 & 0,499 \\
\hline
\end{tabular}

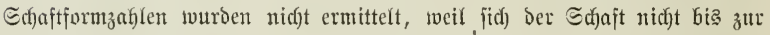
Epiţ̧e berfolgen läp̃t.

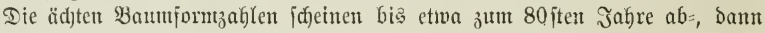

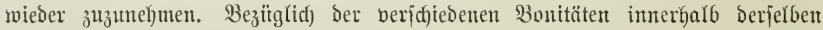

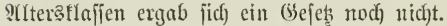

Die ächten Derbformzahlen wachjen mit bem Bejtandeşalter. Bei gleidyem YYter negmen fie mit abnégmentor Bontiät ab.

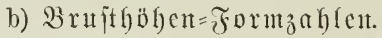

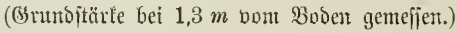
wertfe:

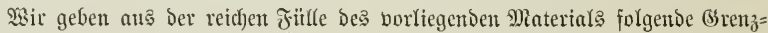

Fưf̆fe แad) B̧aนtr.

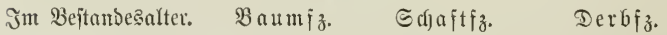

\begin{tabular}{|c|c|c|c|c|c|c|c|c|}
\hline $21-$ & & $\begin{array}{l}5-7 \mathrm{~m} \\
0,919\end{array}$ & $\begin{array}{c}17-19 m \\
0,580\end{array}$ & $\begin{array}{l}5-7 \mathrm{~m} \\
0,580\end{array}$ & $\begin{array}{c}17-19 n \\
0,500\end{array}$ & $\begin{array}{l}5-7 m \\
0,208\end{array}$ & $\begin{array}{c}17-19 m \\
0,470\end{array}$ & itelgäbre \\
\hline & " & $\begin{array}{c}8-10 \mathrm{~m} \\
0,760\end{array}$ & $\begin{array}{c}23-25 m \\
0,605\end{array}$ & $\begin{array}{c}8-10 \mathrm{~m} \\
0,579\end{array}$ & $\begin{array}{c}23-25 m \\
0,485\end{array}$ & $\begin{array}{c}8-1 \| m \\
0,240\end{array}$ & $\begin{array}{c}23-25 m \\
0,479\end{array}$ & " \\
\hline $61-80$ & " & $\begin{array}{c}11-13 m \\
0,687\end{array}$ & $\begin{array}{c}29-31 \mathrm{~m} \\
0,539\end{array}$ & $\begin{array}{c}11-13 m \\
0,567\end{array}$ & $\begin{array}{c}29-31 \mathrm{~m} \\
0,464\end{array}$ & $\begin{array}{c}11-13 m \\
0,434\end{array}$ & $\begin{array}{c}29-31 \mathrm{~m} \\
0,481\end{array}$ & " \\
\hline-1 & " & $\begin{array}{c}14-16 \mathrm{~m} \\
0,587\end{array}$ & $\begin{array}{c}32-34 m \\
0.516\end{array}$ & $\begin{array}{r}14-16 \mathrm{~m} \\
0,503\end{array}$ & $\begin{array}{r}32-34 m \\
0,445\end{array}$ & $\begin{array}{c}14-16 m \\
0,461\end{array}$ & $\begin{array}{c}32-34 \mathrm{~m} \\
0,445\end{array}$ & " \\
\hline
\end{tabular}




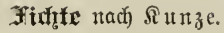

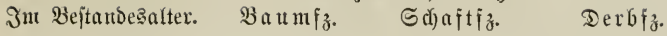

\begin{tabular}{|c|c|c|c|c|c|c|c|c|}
\hline $21-40$ & & $\begin{array}{l}5-\gamma \mathrm{m} \\
0,891\end{array}$ & $\begin{array}{c}17-19 m \\
0,655\end{array}$ & $\begin{array}{l}5-7 \mathrm{~m} \\
0,611\end{array}$ & $\begin{array}{c}17-19 m \\
0,512\end{array}$ & $\begin{array}{r}5-7 m \\
0,049\end{array}$ & $\begin{array}{c}17-19 m \\
0,498\end{array}$ & \\
\hline & " & & $\begin{array}{c}23-25 \mathrm{~m} \\
0,600\end{array}$ & $\begin{array}{c}8-10 m \\
0,591\end{array}$ & $\begin{array}{c}23-25 m \\
0,505\end{array}$ & $\begin{array}{c}8-10 \mathrm{~m} \\
0,350\end{array}$ & $\begin{array}{c}23-25, \mathrm{~m} \\
0,501\end{array}$ & " \\
\hline & $"$ & $\begin{array}{c}11-13 m \\
0,667\end{array}$ & $\begin{array}{c}29-31 \text { m } \\
0,559\end{array}$ & $\begin{array}{c}11-13 m \\
0,577\end{array}$ & $\begin{array}{c}29-31 \mathrm{~m} \\
0,490\end{array}$ & $\begin{array}{c}11-13 m \\
0,493\end{array}$ & $\begin{array}{c}29-31 \mathrm{~m} \\
0,488\end{array}$ & " \\
\hline $1-100$ & " & $\begin{array}{c}14-16 m \\
0,657\end{array}$ & $\begin{array}{c}32-34 m \\
0,534\end{array}$ & $\begin{array}{c}14-16 \mathrm{~m} \\
0,542\end{array}$ & $\begin{array}{c}32-34 m \\
0,480\end{array}$ & $\begin{array}{c}14-16 m \\
0,523\end{array}$ & $\begin{array}{c}32-34 m \\
0,478\end{array}$ & " \\
\hline & 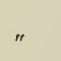 & $\begin{array}{c}17-19 m \\
0,599\end{array}$ & $\begin{array}{c}35-57 m \\
0,519\end{array}$ & $\begin{array}{c}17-19 m \\
0,525\end{array}$ & $\begin{array}{c}35-37 m \\
0,473\end{array}$ & $\begin{array}{c}17-19 m \\
0,517\end{array}$ & $\begin{array}{c}35-37 m \\
0,472\end{array}$ & " \\
\hline & , & $\begin{array}{c}20-22 m \\
0,580\end{array}$ & $\begin{array}{c}38-40 \mathrm{~m} \\
0,522\end{array}$ & $\begin{array}{c}20-22 m \\
0,513\end{array}$ & $\begin{array}{c}38-40 m \\
0,475\end{array}$ & $\begin{array}{c}20-22 \mathrm{~m} \\
0,508\end{array}$ & $\begin{array}{c}38-40 \mathrm{~m} \\
0,474\end{array}$ & $"$ \\
\hline
\end{tabular}

Mitifer nad $\Omega$ unze.

Im Bejtandesalter. Baumiz. Sdaftiz. Derbiz.

\begin{tabular}{|c|c|c|c|c|c|c|c|c|}
\hline $21-40$ & & $\begin{array}{l}5-7 \mathrm{~m} \\
0,846\end{array}$ & $\begin{array}{c}17-19 m \\
0,563\end{array}$ & $\begin{array}{l}5-7 \mathrm{~m} \\
0,660\end{array}$ & $\begin{array}{c}17-19 m \\
0,483\end{array}$ & $\begin{array}{r}5-7 m \\
0,122\end{array}$ & $\begin{array}{c}17-19 m \\
0,469\end{array}$ & \\
\hline & " & $\begin{array}{c}8-10 m \\
0,688\end{array}$ & $\begin{array}{c}20-22 \mathrm{~m} \\
0,516\end{array}$ & $\begin{array}{r}8-10 m \\
0,548\end{array}$ & $\begin{array}{c}20-22 \mathrm{~m} \\
0,461\end{array}$ & $\begin{array}{r}8-10 m \\
0,400\end{array}$ & $\begin{array}{c}20-22 m \\
0,455\end{array}$ & $"$ \\
\hline & " & $\begin{array}{c}11-13 m \\
0,597\end{array}$ & $\begin{array}{c}23-25 m \\
0,495\end{array}$ & $\begin{array}{c}11-13 \text { ж } \\
0,529\end{array}$ & $\begin{array}{c}23-25 m \\
0,453\end{array}$ & $\begin{array}{c}11-13 m \\
0,446\end{array}$ & $\begin{array}{c}23-25 m \\
0,451\end{array}$ & " \\
\hline-1 & $"$ & $\begin{array}{c}14-16 m \\
0,577\end{array}$ & $\begin{array}{c}26-28 m \\
0,472\end{array}$ & $\begin{array}{c}14-16 m \\
0,504\end{array}$ & $\begin{array}{c}26-28 m \\
0,440\end{array}$ & $\begin{array}{c}14-16 m \\
0,487\end{array}$ & $\begin{array}{c}26-28 m \\
0,439\end{array}$ & " \\
\hline & " & & $\begin{array}{c}29-31 m \\
0,469\end{array}$ & $\begin{array}{c}17-19 m \\
0,480\end{array}$ & $\begin{array}{c}29-31 m \\
0,430\end{array}$ & $\begin{array}{c}17-19 m \\
0,476\end{array}$ & $\begin{array}{c}29-31 \mathrm{~m} \\
0,432\end{array}$ & $"$ \\
\hline-14 & & $\begin{array}{c}17-19 m \\
0,557\end{array}$ & $\begin{array}{c}29-31 m \\
0,489\end{array}$ & $\begin{array}{c}17-19 m \\
0,486\end{array}$ & $\begin{array}{c}29-31 m \\
0,447\end{array}$ & $\begin{array}{c}17-19 m \\
0,487\end{array}$ & $\begin{array}{c}29-31 m \\
0,451\end{array}$ & $"$ \\
\hline
\end{tabular}

bituffe rnd) $b$ a ur.

IIn Bejtandeșalter. baum $\mathfrak{i}$.

\begin{tabular}{|c|c|c|c|c|c|c|}
\hline $21-40^{\circ}$ & Gre & $\begin{array}{l}6-8 m \\
0,742\end{array}$ & $\begin{array}{c}18-20 m \\
0,579\end{array}$ & $\begin{array}{l}7-9 m \\
0,191\end{array}$ & $\begin{array}{c}19-21 \mathrm{~m} \\
0,447\end{array}$ & Sctgeitelböbe \\
\hline $41-6$ & " & $\begin{array}{r}9-11 m \\
0,652\end{array}$ & $\begin{array}{c}21-23 m \\
0,562\end{array}$ & $\begin{array}{c}10-12 m \\
0,263\end{array}$ & $\begin{array}{c}22-24 m \\
0,468\end{array}$ & $"$ \\
\hline $61-80$ & $"$ & $\begin{array}{c}12-14 m \\
0,620\end{array}$ & $\begin{array}{c}24-26 \mathrm{~m} \\
0,557\end{array}$ & $\begin{array}{c}13-15 m \\
0,397\end{array}$ & $\begin{array}{c}25-27 \mathrm{~m} \\
0,480\end{array}$ & " \\
\hline $81-100$ & $"$ & $\begin{array}{c}15-17 m \\
0,571\end{array}$ & $\begin{array}{c}27-29 m \\
0,560\end{array}$ & $\begin{array}{c}16-18 m \\
0,440\end{array}$ & $\begin{array}{c}28-30 \mathrm{~m} \\
0,480\end{array}$ & $"$ \\
\hline 1 นt. $n$ & " & $\begin{array}{c}18-20 \mathrm{~m} \\
0,610\end{array}$ & $\begin{array}{c}30-32 m \\
0,613\end{array}$ & $\begin{array}{c}19-21 m \\
0,474\end{array}$ & $\begin{array}{c}31-33 \mathrm{~m} \\
0,541\end{array}$ & $n$ \\
\hline
\end{tabular}

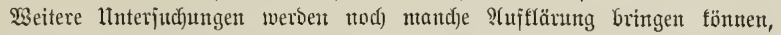

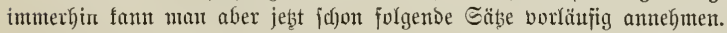

Die Baumformzaflen nefmen mit bem 9yter unt mit wadjentoer Sdfeitel=

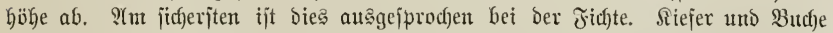

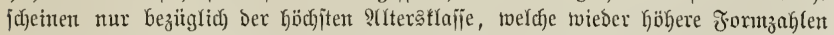

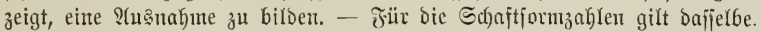

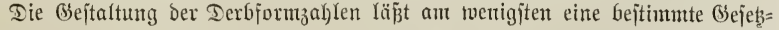

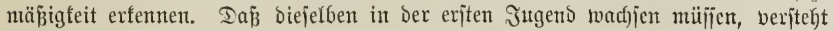

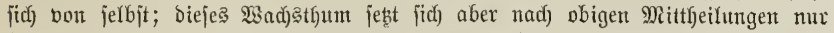

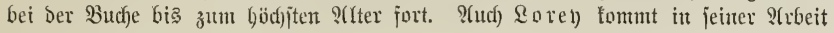




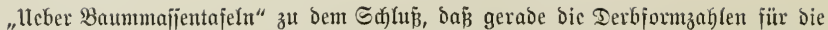

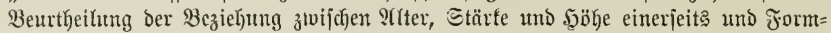

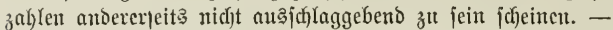

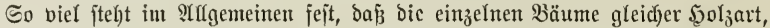

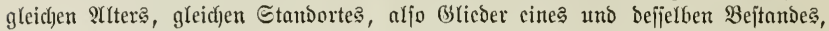

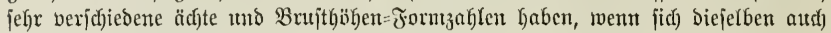

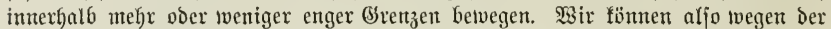
großen, oft unerffärten intoibioueflen Edgwanfungen nicht bom Einzelbaum auf Den

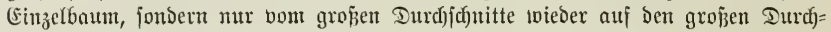

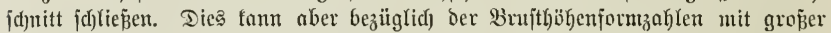

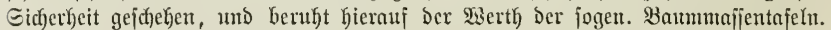

Da Der ijolirte Baum cinc viel tiefer Gerabgehende firvue bebält, als ber Baum int Bejtande, jo wiro jein Echaft jefor abformig und äjtig, baf)er für bic meijten 3wecke tec(fuifd reniger braudbbar. Ecin

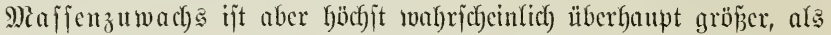
Der bes Bauntes in gejulofifenen Bejtande. Ileber Dic Beit ber Cul= mination bes Majjenzumadjes an ifolirten Banm ijt nichts befannt.

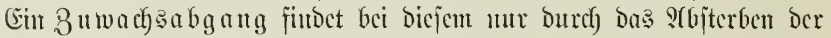
unteren S.ejte itatt, ijt baher nicht bead)tentşwerth).

2. STl Bejtandc.

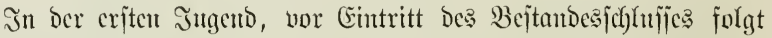

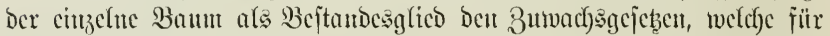
Den ijofirten Baum gelten. Durch Den Ech)lü wird jpäter bic jeit=

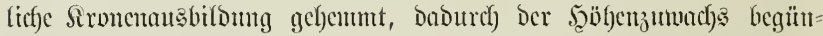

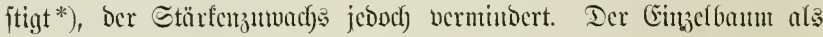

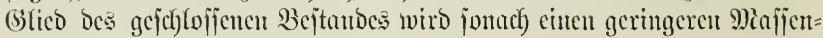
zuwarbs überbaupt haben, als ment er unter joujt glcidyen Bebiu= gungen ixei ftättoc. Sein Blattvermögen ijt geringer. Da fich in=

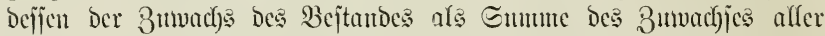
iu ifm enthaltenen Bammindividuen ober als \$irobuct bes 3utwachjes

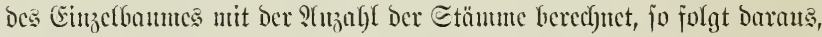

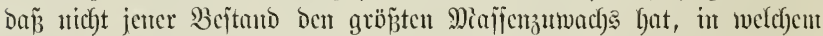
ib) ber Eimzelbaum bejizgt. Der Factor Der Etammzahd ijt mit in アectumutg zu jteflen.

Beifpiel. Ein Bejtand cnthalte 100 Bäınte, beren jeber $0,02 \mathrm{fm}$ jälyrlid zuwäd)it, burd) eine ftarfe Durchjorjtung werbe bie Stammzahl auj 60 reducirt, Der Buwad) bes (Einzelbaumes bagegen auf 0,025 gefoben, fo würoe Daburd) Der $B \mathfrak{B}=$ jtandeszzunadją bon $0,02 \times 100=2$ auf $0,025 \times 60=1,5 \mathrm{fm}$ jinfen.

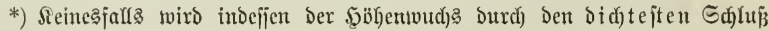

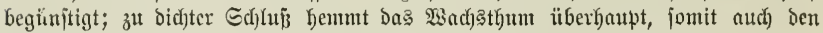

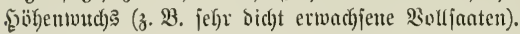




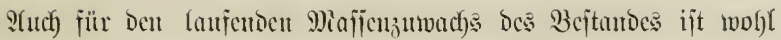
Defien BłLattucrmögen majzgebeno, welches nidft blos von bem Blatt=

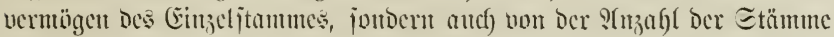

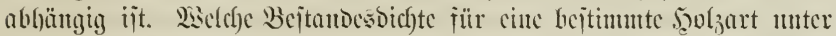

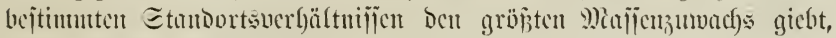
ijt cin ungelojites Problem.

Ier $\Xi$ d)

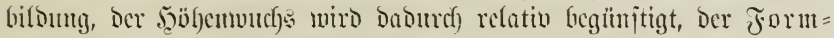
juwad) gefördert, bic Bammjefäfte merben vollfolziger, länger, ajt=

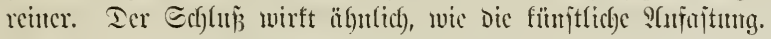

Ier 3 wad) sabgang ijt im Bejtanbe weit gröper, als beim

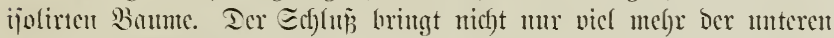

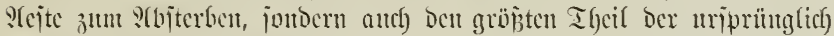

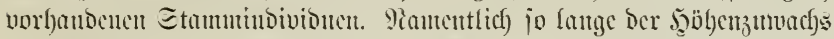

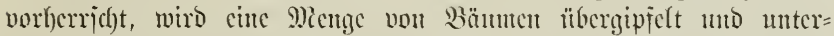

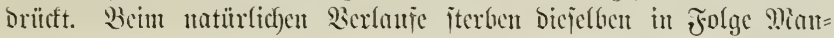

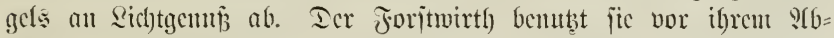

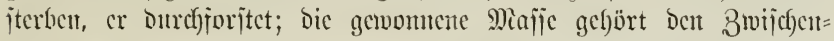
mtııungen ant.

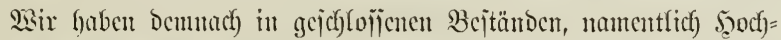

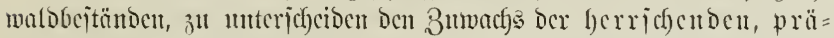
Dominirenden Etammflajje von dem ber unterdrütten, mit

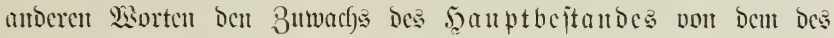
3mijchenbejtandes.

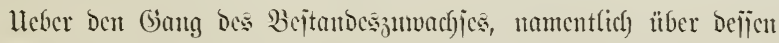

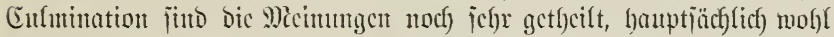

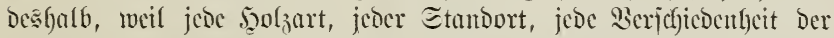
foritficfen Befandung Des Bejtandes vericficdente Rejultate hervor= ruten mü.

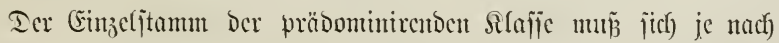

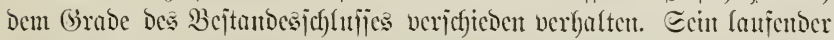

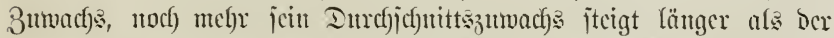

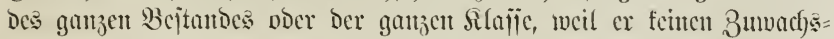

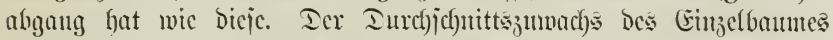

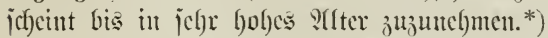

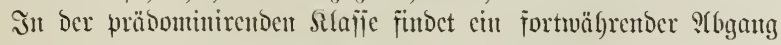

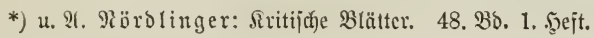




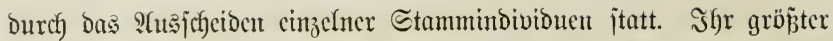

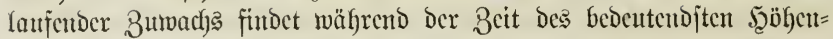

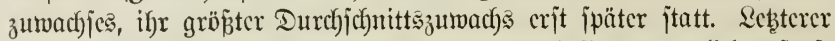
blcibt bann eine Beit lang zicmlich conjtant und jinft natürlich äuñerjt langjam.

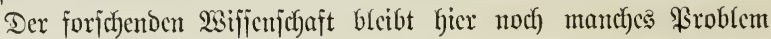

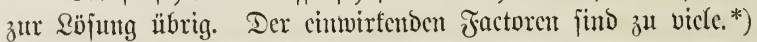

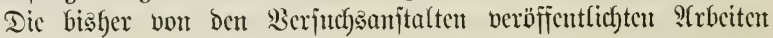

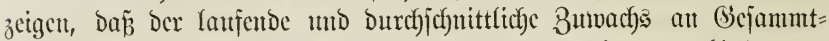

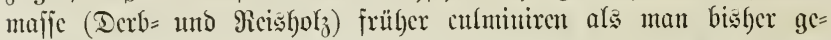

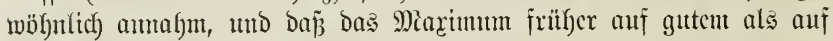
jeblechtem Etumbort cintritt. Für bas Derbholz allein fällft bas Mlari

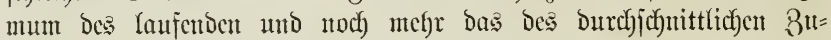

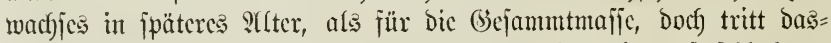
jelbe cbenfalls auf gutem Etanborte früber cin, als auf jeblect)tem.

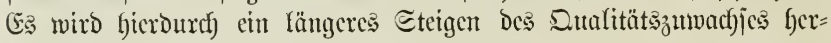
vorgerufet.

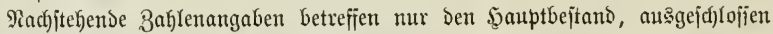

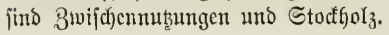

Finflife nad) $B a u r$.

Marimum bes laufenden,

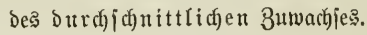

(5) ejammtmajfe.

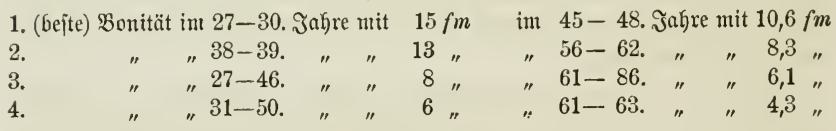

Derbjolz.

1. (Geftc) Bonität im $38-40$. Jafre mit $15 \mathrm{fm}$

$2 . "$ " $41-43$. " " 11 "

3. " " $57-60$. " 9 "

4. " " $" 55-60$. " 6 "

im $55-73$. Jahre mit $8,7 \mathrm{fm}$

"78-91. " " 7,0"

" 94-104. " " 5,2 "

" $103-113$. " " 3,4 "

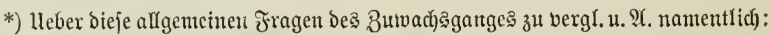

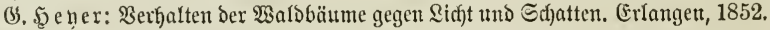

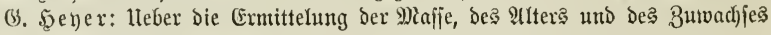
Der Sovzbejtände. Defịa, 1852.

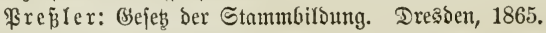

Baur: Die Şolzmeṕftude. 3. Itufl. WBien, 1882.

תй

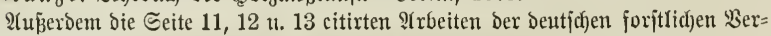

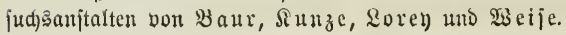


Fidnte nad) $\Re$ น

Max̧imum des raujenden,

Des ourdjidnittlidjen 3uwadjes.

(B) ja m m mafje.

1. (befte) Bunität im 30-35. Jahre nit $20,0 \mathrm{fm}$ im

2.

3.

4.
"

" " 8,3 " 65

" $60-75$. " 6,0 "

Derbfolz.

1. (Gejte) Bonität im 30-35. Jafre mit $18,4 \mathrm{fm}$

2.

3.

$$
\begin{array}{llll}
\text { " } & 35-40 . & \text { " } & 16,4 \text { " } \\
\text { " } 40-45 . & \text { " } & 13,6 \text { " } & 130-55 .
\end{array}
$$

4.

im

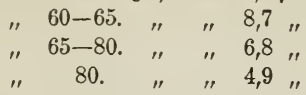

Iitefer nady $\Re$ un $\mathfrak{z}$ e.

Darimum des $\mathfrak{l a}$ ajenden,

Des ourdjidnittrinen Buwadjes.

(b) ejamm tmajje.

1. (Gejte) Bonität im $25-30$. Jahre mit $18,0 \mathrm{fm}$ im

2.

3.

4.

5.
35. Jahre mit 10,9 fm

40. " " 8,3 "

45. " " 6,2 "

50.4 " " 4,4 "

$55-65$. " " 2,7 "

\section{Derbjolz.}

1. (Gejte) Bonität im 30-35. Safre mit 17,8 fm

2.

3.

4.

5.

$$
\begin{array}{llll}
\text { " } 35-40 . & \text { " } & 12,4 & \text { " } \\
\text { " } 40-45 . & \text { " } & 8,8 \text { " } \\
\text { " } 45-50 . & \text { " } & 5,8 \text { " } \\
\text { " } 45-50 . & \text { " } & 3,2 \text { " }
\end{array}
$$

im

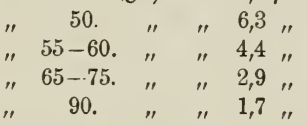

Firefer nad) Ş Marimum des raufenden, Des burdjidnittridgen Buwadjes. (G) ejammtmafie.

1. (bejte) Bonitüt im

2.

3.

4.

5.
30. Jahre mit $13,6 \mathrm{fm}$

30. " "10,6 "

25. " " 7,8 "

20. " " 7,0"

" 15-30. " " " 5,0 " im $30-35$. Jabre mit $11,9 \mathrm{fm}$

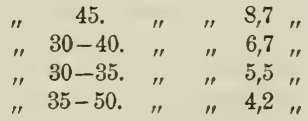

Derbholz.

1. (6ejte) Bonität im $25-30$. Jahre mit 18,0 fm

2.

3.

4.

5.
" 25-30. " " 13,8 "

" $30-40$. " " 8,6 " "

"30-40. " " 7,2 "

" 30-35. " " 5,6 " im 40-45. Jafre mit $10,2 \mathrm{fm}$

" $50-55$. " " 7,3 "

" $60-65$. " " 5,1 "

" 60-65. " " 4,0 "

" $60-70$. " " 3,0 " 
bututhe nach baur.

Marimum des laufenden,

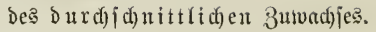

(íejammtmajje.

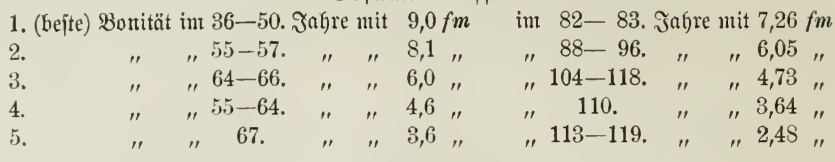

acrbigla

1. (bejte) Sonität in 51-52. Sahre mit $13,0 \mathrm{fm}$

im 75. כahre mit $6,16 \mathrm{fm}$

2. " "46-49. " " 8,9 "

$3 . \quad$ " $48-51$, " 7,5,

4.

" " $54-57$. " " 5,1 ,

" 94-113. " " 5,08 "

" 99-103. " " 4,16 "

|117-120. " "3,17"

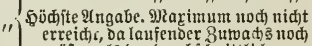

5.

4,0,

$111-115$. Jahre mit $2,16 \mathrm{fm}$

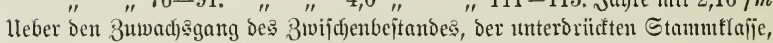

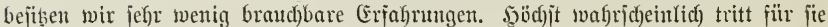

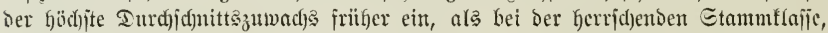

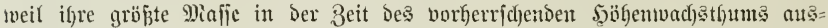

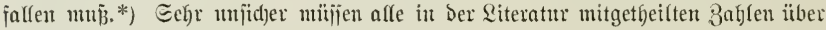

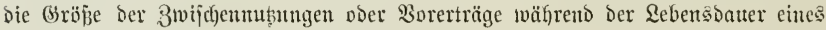

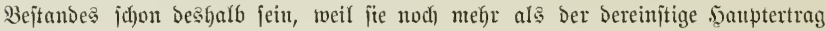

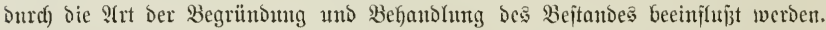

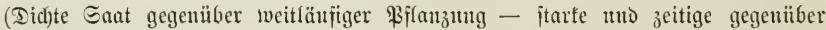

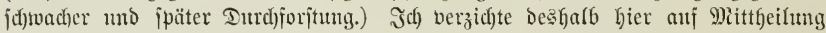

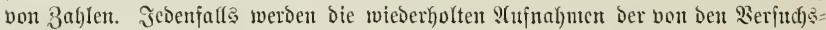

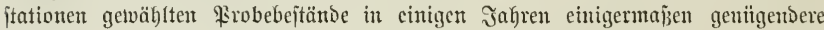
Angaben bringent.*) Die im \&efrbeifpiel, \& 11 mitgetheilten S(ngaben über bie

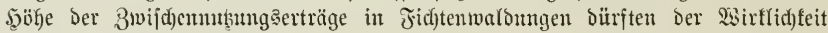
ziemlid) stahe fonmen.

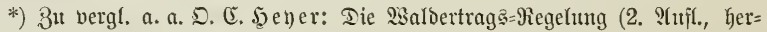

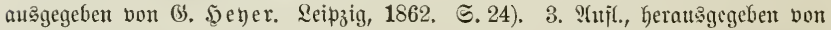

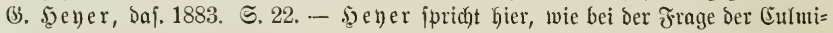
nation des 3uwadjes überbaupt, vou 3eitpunft der Mannbarfeit. Id) habe biejen

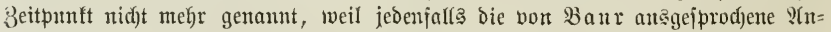

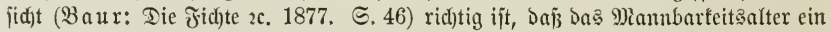

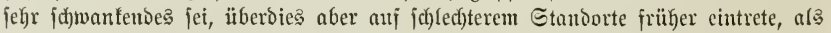

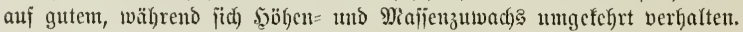

**) 2 tus neucrer Beit jeien hier erwähnt:

W3allmann: Sorertragstajel im Forit- und Jagdtalcnder. (Scheint Dod).

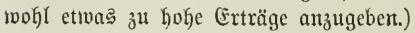

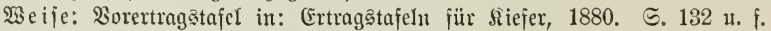

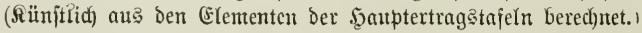

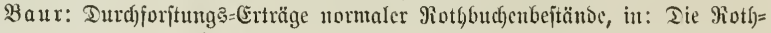




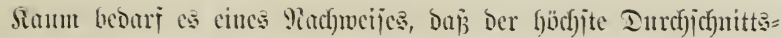

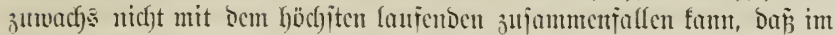
Begentheife lebzterer icf)on längere 3cit jüft, mäfrend ber crjtere nocf)

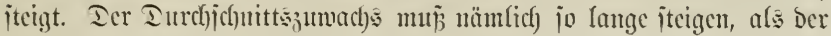

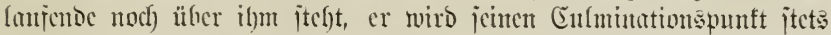
Dann erit erreichen, wenn er gleich bem (aufenben Buwadje wird.*)

Es gilt Dics chenjo für ben Ertrag bes 5autptbejtandes allein,

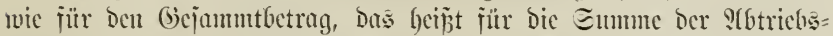

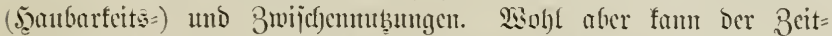

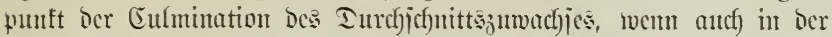
Fiegel mur unthedentend, ein anderer jein, je nachbem wir bic 3rwijchen=

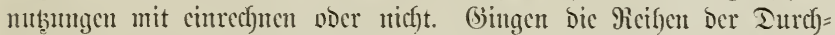

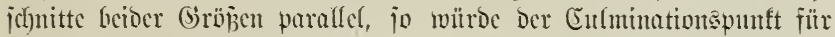
Stbtricbs = mo jür Gejammtertrag in bajlelbe Jafre falfen. Fiele Der

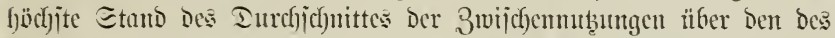

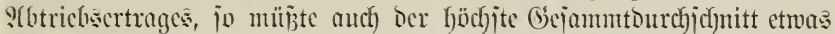

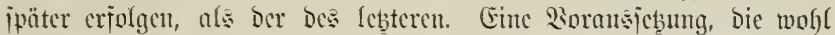

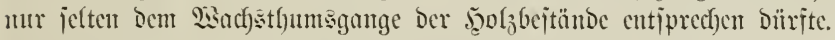

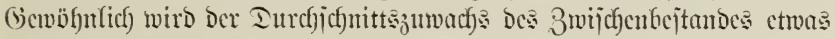
cher jeinen höbjiten Etand erreichen, afs Der Des Szanptbejtandes, Den

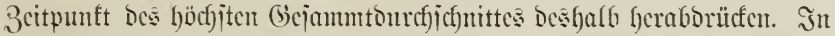

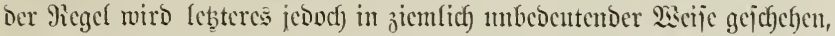

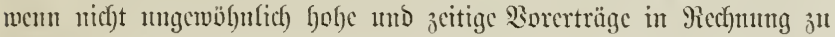
itellen jund.**)

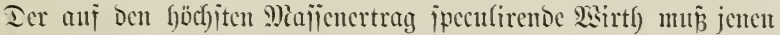

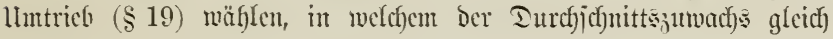

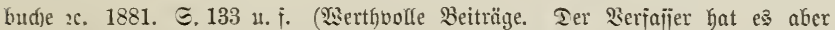
jebr rid)tig unterfaijen, biejelben ju "Borentragstafeln" zu verarbeiten, well jie mur Die eritmaligen Iurd)foritungserträgc enthalten, währeno erjt wicberfolte Iurch)= foritungen (Erträge liejern, meld)e als amüherno normale betradtet werden fömnen.)

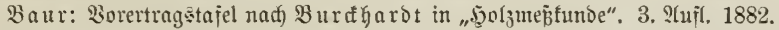
Seite 390 .

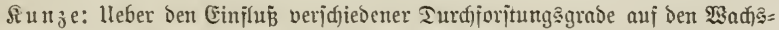

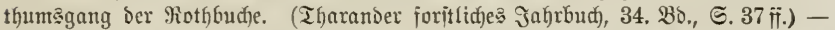

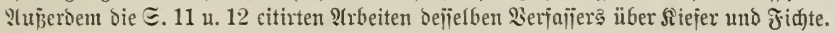

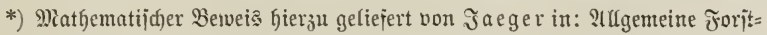
unb Jagbzeitung. 1841. ๔. 177. Tieier Berweis aud) mitgetfeilt in Baur: 5oolz=

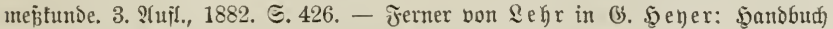
Der pritlichen Etatif. నeipzig, 1871. ङ. 126.

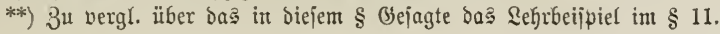




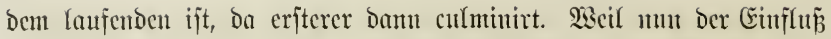

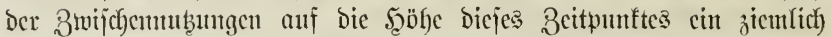
unbedeutentocr ijt, jo fann man fich babci ofne wejentlicten Irrtlyum

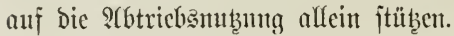

\section{$\S 10$.}

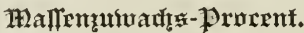

Drïcfen wir Den $\mathfrak{B a c h s t f y u m s g a n g ~ D e r ~ B a ̈ u m e ~ u n d ~ B e j t a ̈ n d e ~ i n ~}$

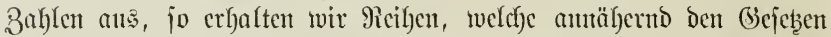
cinter aritfymetijchen Jicife entiprect)en, Deren Differenzen nicht völlig gleiche find. WBill man baher für irgend welchen 3wed ber blof̧en Maffen=Ertragsegchung bic \$rocentrect)mung anwenten, jo ijt es afler=

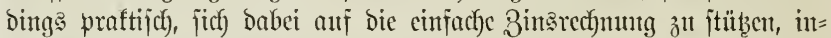

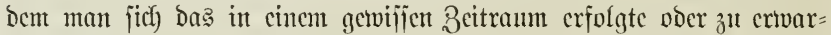

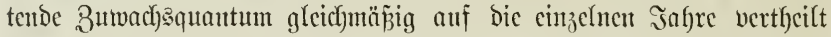
denft. Ie fitryer ber fragliche Beitraum ift, bejto geringeren Jrr= tfümern jebt man jich Dabei aus. Sndejjen ijt für berartige Siect)=

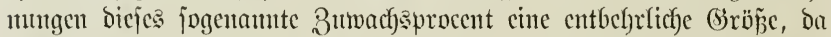

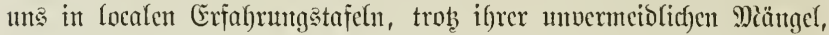

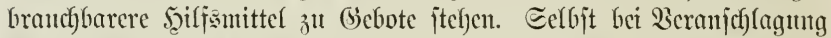

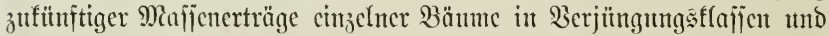
Dergleicf)en foumt man leichter jum Bicle, twem man cinfach vom Bumachşquantun ber jüngiten Bergangenkeit aũ bas ber nächjten Bufunft joh)liểt.

Das Procent Graudjen wir jedoct) zu cinem anderen Brvecfe,

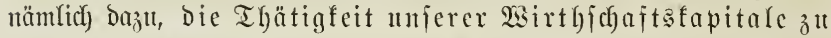
mcjicn. Dicje Miefjung wäre principiell cine unrichtige, mollten wir

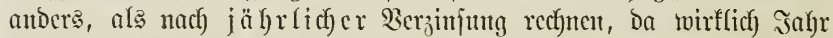

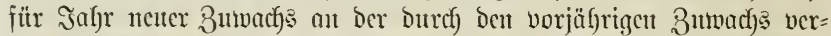
meforten Mafie erfolgt. Der cimzelute Baum, ber ciuzelue Bejtund ijt ebent weiter nichts, als cin in ber Foritwirthjofjajt thätiges Siapital. -

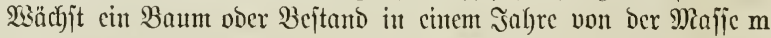
auf bie Miciffe $\mathrm{M}$, jo ift jeit 3uwad)s $\mathrm{z}=\mathrm{M}-\mathrm{m}$, und cs fintoct fict) das Buwact)procent $p$ nad) Der \$roportion

Gicrats

$$
\mathrm{m}:(\mathrm{M}-\mathrm{m})=100: \mathrm{p} \text {; }
$$

$$
\mathrm{p}=\frac{\mathrm{M}-\mathrm{m}}{\mathrm{m}} \times 100=\frac{100 \mathrm{z}}{\mathrm{m}} .
$$


Denjelben 2iserth crhält man, twem man $M$ als den cinjäbrigen Raclbuertly wou $\mathrm{m}$ anficht, alfo:

lierats

$$
\mathrm{M}=\mathrm{m} \cdot 1, \mathrm{pp}
$$

$$
\mathrm{p}=\left(\frac{\mathrm{M}}{\mathrm{m}}-1\right) 100=\frac{\mathrm{M}-\mathrm{m}}{\mathrm{m}} \times 100=\frac{100 \mathrm{z}}{\mathrm{m}} \text {. }
$$

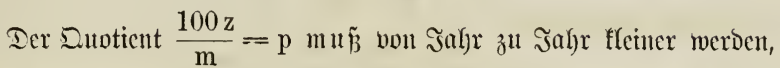

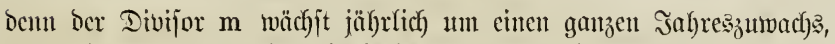
wälyrent z jtets nutr den lanfenden 31twachs eines Safres bedeutet,

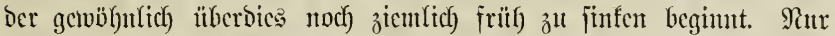
Majregefu Der Bejtambespflege, wie rechtzeitig cingelegte Dutrchforjt= ungen, vermïgen unter jefrr güujtigen llmp̈tänocn bas Buwach sprocent

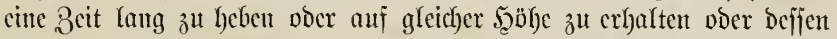
Sinten zu verlangianten.

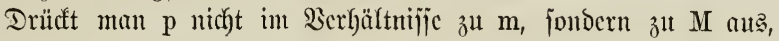
jo erbält man gewölunlich das für bie nächjtfolgende Beit höchjte \$ro= ccnt, näıltich):

$$
\mathrm{p}=\frac{\mathrm{M}-\mathrm{m}}{\mathrm{M}} \times 100=\frac{100 \mathrm{z}}{\mathrm{M}} .
$$

Betrad)tet man läıgere Beitränme als cinjälyrigc, twie es in ber

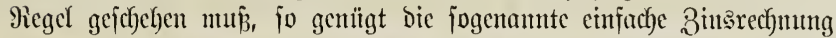

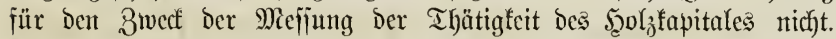
M ijt Der njäfrige Pactbwerth won $\mathrm{m}$, und bas entjprectjende $\mathrm{p}$ wiro nach) folgender (ślecichung gejumben:

baraus

$$
\mathrm{M}=\mathrm{m} \cdot 1,0 \mathrm{p}^{\mathrm{n}}
$$

$$
1,0 \mathrm{p}=\sqrt{\frac{\mathrm{n}}{\mathrm{m}}} \text { uno } \mathrm{p}=100\left(\sqrt{\frac{\mathrm{M}}{\mathrm{m}}}-1\right) .
$$

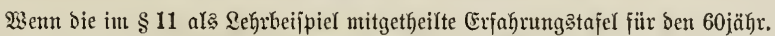
Bejtand 354, für ben 80 jäfr. $509 \mathrm{fm}$ nadjweijt, jo ijt bas Zutwad)spprocent nidjt

jonbern

$$
\frac{(509-354) \times 100}{354 \times 20}=2,189,
$$

$$
100\left(\sqrt{\frac{509}{354}}-1\right)=1,832 \text {. }
$$

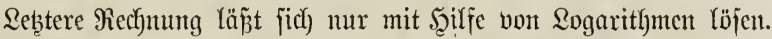

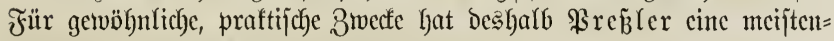

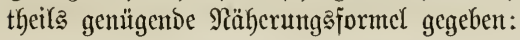


Bejicht man p weocr anf m, nodf anf $M$, jondern auf bas aritf)=

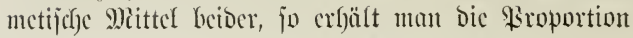

Daxaแล

$$
\frac{M+m}{2}: \frac{M-m}{n}=100: p
$$

Für vbiges Baflenbeippicl:

$$
\mathrm{p}=\frac{\mathrm{M}-\mathrm{m}}{\mathrm{M}+\mathrm{m}} \times \frac{200}{\mathrm{n}} .
$$

$$
\mathrm{p}=\frac{509-354}{509+354} \times \frac{200}{20}=1,796
$$

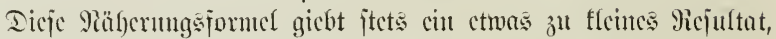

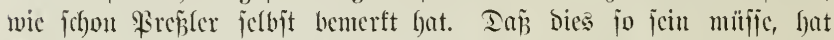

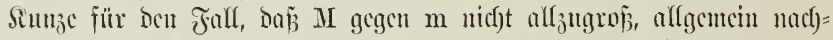

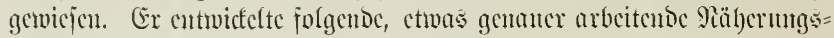
formel:*)

$$
\mathrm{p}=\frac{\mathrm{M}-\mathrm{m}}{\mathrm{M}(\mathrm{n}-1)+\mathrm{m}(\mathrm{n}+1)} \times 200 .
$$

ซึ̈̈r obiges 3ahlenbeipipicl wirb hiernad)

$$
\mathrm{p}=\frac{509-354}{509(20-1)+354(20+1)} \times 200=1,812 .
$$

S)at man cs mit nicht jul Langen Beitrüumen uno cinem itberhaupt

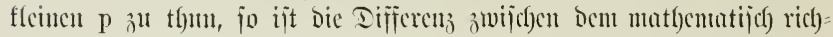

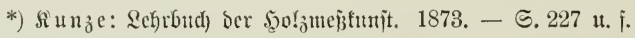

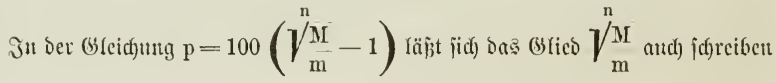

$$
\sqrt[n]{\frac{\mathrm{m}+\mathrm{M}-\mathrm{m}}{\mathrm{m}}}=\sqrt{1+\frac{\mathrm{n}}{\mathrm{m}-\mathrm{m}}} \text {. }
$$

Sit nun $\mathrm{M}-\mathrm{m}<\mathrm{m}$, jo ijt $\frac{\mathrm{I}-\mathrm{m}}{\mathrm{m}}<1$, umb Die (Sröbe $\sqrt[n]{1+\frac{\mathrm{M}-\mathrm{m}}{\mathrm{m}}}$ Darf

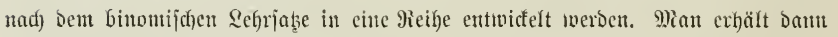

$$
\sqrt[n]{1+\frac{M-m}{m}}=1+\frac{1}{n} \frac{M-m}{m}-\frac{n-1}{2 n^{2}}\left(\frac{M-m}{m}\right)^{2}+\ldots .
$$

Multipficirt man beibe Seiten biejer Bleidnung mit $1+\frac{\mathrm{n}-1}{2 \mathrm{n}} \frac{\mathrm{M}-\mathrm{m}}{\mathrm{m}}$, jo wirb $\sqrt[n]{1+\frac{\mathrm{M}-\mathrm{m}}{\mathrm{m}}}\left(1+\frac{\mathrm{n}-1}{2 \mathrm{n}} \frac{\mathrm{M}-\mathrm{m}}{\mathrm{m}}\right)=1+\frac{\mathrm{n}-1 \mathrm{M}-\mathrm{m}}{2 \mathrm{n}}+\frac{1}{\mathrm{~m}} \frac{\mathrm{M}-\mathrm{m}}{\mathrm{m}}+$ $\frac{\mathrm{n}-1}{2 \mathrm{n}^{2}}\left(\frac{\mathrm{M}-\mathrm{m}}{\mathrm{m}}\right)^{2}-\frac{\mathrm{n}-1}{2 \mathrm{n}^{2}}\left(\frac{\mathrm{MI}-\mathrm{m}}{\mathrm{m}}\right)^{2}-\frac{(\mathrm{n}-1)^{2}}{4 \mathrm{n}^{3}}\left(\frac{\mathrm{M}-\mathrm{m}}{\mathrm{m}}\right)^{3}+\ldots$

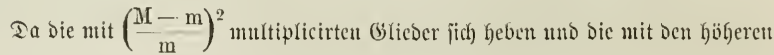




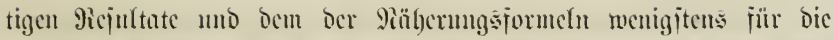

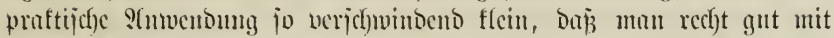

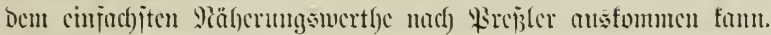

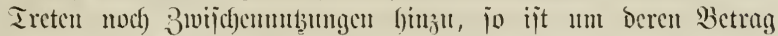

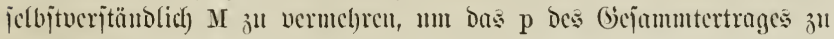
beredfuten.

In unjerem Beipipiele entjallen in ber Beit vom 60 iten bis 80 jten Jafre $46 \mathrm{fm}$

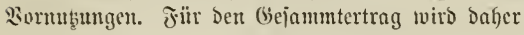

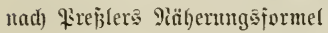

$$
\mathrm{p}=100\left(\sqrt{\frac{\mathrm{n}}{509+46}}_{354}-1\right)=2,274,
$$

$$
\mathrm{p}=\frac{509+46-354}{509+46+354} \times \frac{200}{20}=2,211,
$$

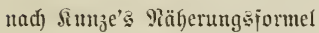

$$
\mathrm{p}=\frac{509+46-354}{(509+46)(20-1)+354(20+1)} \times 200=2,236 .
$$

Dieje qiocente, 1,83 für Den Jauptertrag allein und 2,27 jür ben bejaumnt=

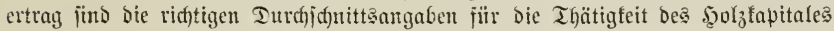
it bem gewö̈lten, 20 jäfrigen 3 eitraume, wenn man bajielbe jïr jid) allein be=

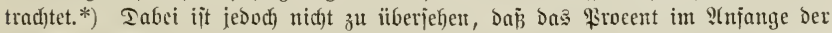

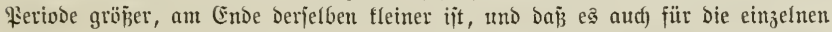

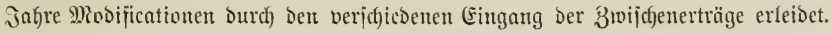

Rotenzen Diejer Grö̈p̈e behafteten vernach(äifitgt werben fömen, fo bleibt nad) einer leidjten Miedution

நุicraus folgt

$$
{V^{\prime}}^{\prime}+\frac{M-m}{m}\left(1+\frac{n-1}{2 n} \frac{M-m}{m}\right)=1+\frac{n+1}{2 n} \frac{M-m}{m} .
$$

$$
\sqrt[n]{1+\frac{\mathrm{n}-\mathrm{m}}{\mathrm{m}}}=\frac{1+\frac{\mathrm{n}+1}{2 \mathrm{n}} \frac{\mathrm{M}-\mathrm{m}}{\mathrm{m}}}{1+\frac{\mathrm{n}-1}{2 \mathrm{n}} \frac{\mathrm{M}-\mathrm{m}}{\mathrm{m}}} .
$$

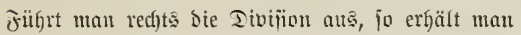

ober

$$
1+\frac{\frac{1}{n} \frac{M-m}{m}}{1+\frac{n-1}{2 n} \frac{M-m}{m}}
$$

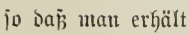

$$
1+\frac{2(\mathrm{M}-\mathrm{m})}{\mathrm{M}(\mathrm{n}-1)+\mathrm{m}(\mathrm{n}+1)^{\prime}}
$$

$$
\mathrm{p}=\left(1+\frac{2(\mathrm{M}-\mathrm{m})}{\mathrm{M}(\mathrm{n}-1)+\mathrm{m}(\mathrm{n}+1)}-1\right) 100=\frac{\mathrm{M}-\mathrm{m}}{\mathrm{M}(\mathrm{n}-1)+\mathrm{m}(\mathrm{n}+1)} \times 200 .
$$

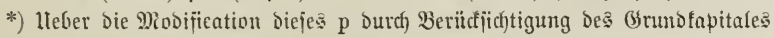
วu bergł. § 15 น. กิ. w. 
Cin für bic Ertragstegelung eben fo widhtiges, als cinfacfes (bese

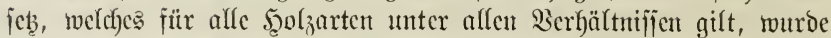

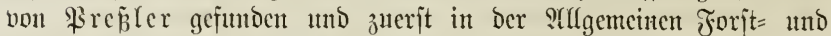
sago $=3$ citung 1860 veröffentlicht. CFe lautet:

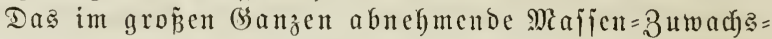

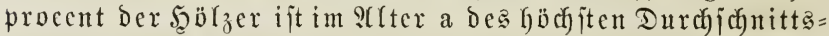
ertrages auf cinen Werth berabgejuten, ber fich genau

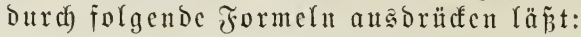

1) fïr ben $\mathfrak{S} \mathfrak{a} u p t e r t r a g$ allein $p=\frac{100}{a}$;

2) für den (Scjammtertrag $p^{\prime}=\frac{100+v}{a^{\prime}}$.

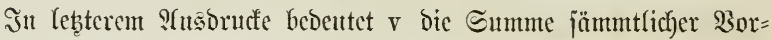
แub̧utgen im \$rocentjabe Des .̧auptertrages.

Beweis. Das Buwadjaprocent ift, wie wir jafen, gleid dem 100 fadjen Quotienten $\frac{\mathrm{z}}{\mathrm{m}}$; aljo $\quad \mathrm{p}=\frac{\mathrm{z}}{\mathrm{m}} \times 100$;

Gieraus

$$
z=\frac{m p}{100} .
$$

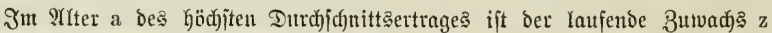
gleid) bem burdbidnnittlidien, alyo

folglich autd)

$$
\mathrm{z}=\frac{\mathrm{m}}{\mathrm{a}}
$$

แnเ hieraus

$$
\frac{\mathrm{mp}}{100}=\frac{\mathrm{m}}{\mathrm{a}}
$$

$$
\mathrm{p}=\frac{\mathrm{m}}{\mathrm{a}} \times \frac{100}{\mathrm{~m}}=\frac{100}{\mathrm{a}} .
$$

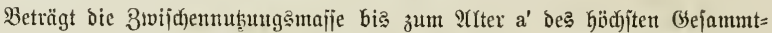

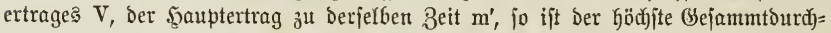

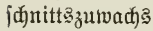

wetn $\mathrm{p}^{\prime}$ bas Getreffende 3uwad)

$$
\frac{\mathrm{m}^{\prime}+\mathrm{V}}{\mathrm{a}^{\prime}}=\frac{\mathrm{m}^{\prime} \mathrm{p}^{\prime}}{100}
$$

f̧ieraus folgt

$$
\mathrm{p}^{\prime}=\frac{\mathrm{m}^{\prime}+\mathrm{V}}{\mathrm{a}^{\prime}} \times \frac{100}{\mathrm{~m}^{\prime}}=\frac{100}{\mathrm{a}^{\prime}}\left(1+\frac{\mathrm{V}}{\mathrm{m}^{\prime}}\right) .
$$

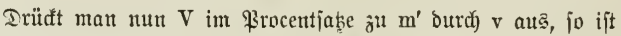

$$
\begin{aligned}
& \mathrm{v}=\frac{\mathrm{v} \cdot 100}{\mathrm{~m}^{\prime}} \text { uns } \\
& \mathrm{v}=\frac{\mathrm{vm}^{\prime}}{100^{\circ}} .
\end{aligned}
$$

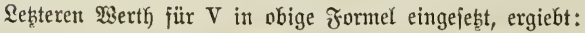

$$
\mathrm{p}^{\prime}=\frac{100}{\mathrm{a}^{\prime}}\left(1+\frac{\mathrm{v} \mathrm{m}^{\prime}}{100 \cdot \mathrm{m}^{\prime}}\right)=\frac{100+\mathrm{v}}{\mathrm{a}^{\prime}} \text {. }
$$




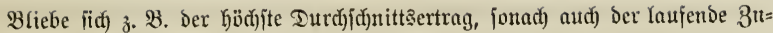

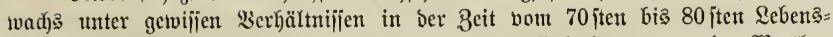

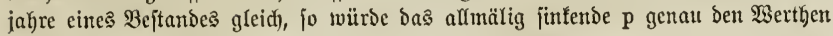
$\frac{100}{70}, \frac{100}{71}$ u. โ. w. bis $\frac{100}{80}$ entipredjen.

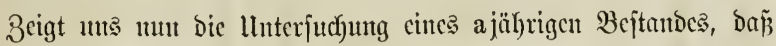

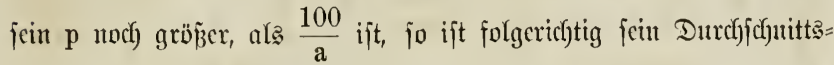

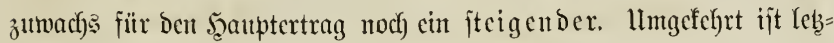
terer bercits im Sinfeu, fobald bes Bejtandes p fleiner als $\frac{100}{a}$. Daffelbe gilt natïrlich) für ben (Bsjammtertrag.

\section{$\S 11$.}

\section{Ixhrueilpiel.}

In Der unitehenden, ala 2 ch)rbeippicl*) conjtruirten Erfaljrung:

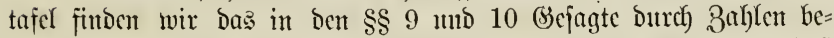

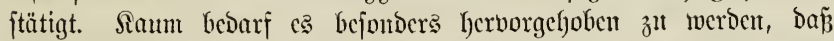

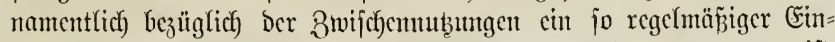

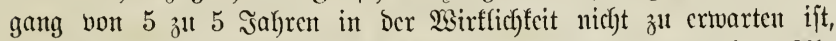

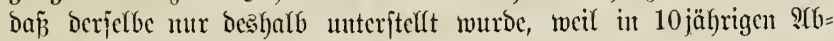
\{tufuugen jene Momente, auf weldfe es anfommt, nicht beutlich) gemug

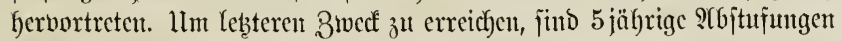
wohl bic längiten, dic man wählen barf.

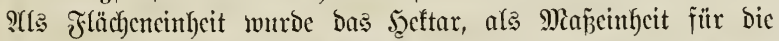

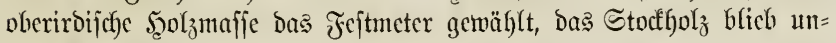
berücffict)tigt.

Wir heben aus ber Tafel folgento Şauptpunfte hervor:

1) Dic Zcit bes höchjten Durchjechnittsertrages ijt jowoh) für bic

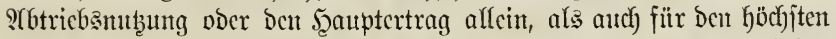

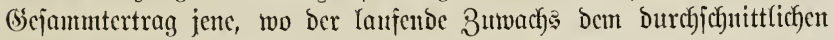
gleich) jtebt. Dabei tritt Dicfer Zcitpunft für ben (Scfammtertrag etwaß früber ein, als für Den J̧auptertrag, für crfteren im 80 ften Jabre mit 8,4875 , für leb̧teren im 85 jten Sahre mit $6,4 \mathrm{fm}$.

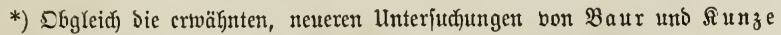

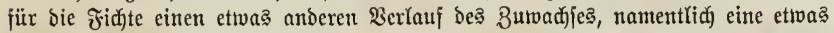

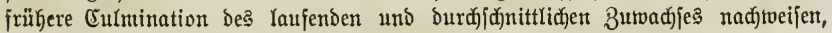

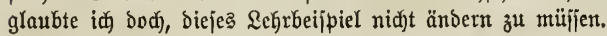




\begin{tabular}{|c|c|c|c|c|c|c|c|c|c|c|}
\hline a & b) & c & d & e & $f$ & 5 & h & i & k & 1 \\
\hline \multirow{4}{*}{ } & \multicolumn{5}{|c|}{ Dauptbeitaud. } & \multicolumn{5}{|c|}{ 3wifduenbeitand. } \\
\hline & & & & Itwad) & & & & 3uwa & d) 8 . & \\
\hline & 芯 & 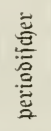 & 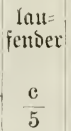 & $\begin{array}{c}\text { idhnitt= } \\
\text { licf)er } \\
\frac{b}{a}\end{array}$ & \multirow[t]{2}{*}{$\begin{array}{l}\text { Pro: } \\
\text { cent. }\end{array}$} & 芯 & 苛 & $\begin{array}{c}\text { laut } \\
\text { fenter } \\
\frac{\mathrm{h}}{5}\end{array}$ & $\begin{array}{l}\text { Durd) }= \\
\text { id(bnitt- } \\
\text { lidber } \\
\frac{1}{a}\end{array}$ & 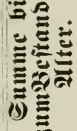 \\
\hline & \multicolumn{4}{|c|}{ Feitmeter. } & & \multicolumn{5}{|c|}{ Feitueter. } \\
\hline 10 & 20 & & & 2,0000 & & - & & & - & 一 \\
\hline 15 & 40 & 20 & & 2,6667 & 14,87 & - & - & 10 & & \\
\hline 20 & 65 & 25 & 5,0 & 3,2500 & 10,20 & 6 & 6 & & 0,3000 & 6 \\
\hline 25 & 96 & 31 & 6,2 & 3,8400 & 8,11 & 10 & 10 & 2,0 & 0,6400 & 16 \\
\hline 30 & 129 & 33 & 6,6 & 4,3000 & 6,09 & 12 & 12 & 2,4 & 0,9333 & 28 \\
\hline 35 & 164 & 30 & 8,0 & 4,6857 & 4,9 & 14 & 14 &, 8 & 1,2000 & 42 \\
\hline 40 & 200 & 30 & 7, & 5,0000 & 4,05 & 15 & 15 & 3,0 & 1,4250 & 57 \\
\hline 45 & 237 & 37 & 7,4 & 5,2667 & 3,45 & 16 & 16 & 3,2 & 1,6222 & 73 \\
\hline 50 & 275 & 38 & 6,6 & 5,5000 & 3,02 & 17 & 17 & 3,4 & 1,8000 & 90 \\
\hline 55 & 314 & 39 & 1,8 & 5,7091 & 2, & 17 & 17 & & 1,9455 & 107 \\
\hline 60 & 354 & 40 & 8, & 5,9000 & 2, & 17 & 18 & & 2,0667 & 124 \\
\hline 65 & 394 & 40 & 8,0 & 6,0615 & 2,16 & 15 & 15 & 3,0 & 2,1385 & 139 \\
\hline 70 & 433 & & 6,8 & 6,1857 & 1,9 & 13 & 13 & 2 & 2,1714 & 152 \\
\hline 75 & 472 & 39 & 7,8 & 6,2933 & 1,74 & 10 & 10 & 2,0 & 2,1600 & 162 \\
\hline 80 & 509 & 3 & 7,4 & 6,3625 & 1,52 & 8 & 8 & 1,6 & 2,1250 & 170 \\
\hline 85 & 544 & & & 6,4000 & & 6 & 6 & & 2,0706 & 176 \\
\hline 90 & 575 & 31 & & 6,3889 & 1,11 & 4 & 4 & 0,8 & 2,0000 & 180 \\
\hline 95 & 604 & & 5,8 & 6,3579 & 0,9 & - & - & & 1,8947 & 180 \\
\hline 00 & 630 & 20 & 2 & 6,3000 & $0,0 \pm 0$ & & 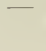 & - & 1,8000 & 180 \\
\hline
\end{tabular}




\begin{tabular}{|c|c|c|c|c|c|c|}
\hline $\mathbf{m}$ & $\mathbf{n}$ & 0 & $\mathrm{p}$ & $q$ & $\mathbf{r}$ & a \\
\hline \multicolumn{6}{|c|}{ Gcfainmtbetrag. } & \multirow{4}{*}{ 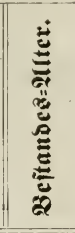 } \\
\hline Mir & fife. & & $3 u$ & & & \\
\hline $\mathrm{b}+\mathrm{g}$ & $b+1$ & $\begin{array}{l}\text { periobijater } \\
\qquad \mathrm{c}+\mathrm{h}\end{array}$ & $\begin{array}{l}\text { laufentoer } \\
\frac{0}{5}=\mathrm{d}+\mathrm{i}\end{array}$ & $\begin{array}{c}\text { Durdf) } \\
\text { f(d)uittlidfer } \\
\frac{\mathrm{n}}{\mathrm{a}}=\mathrm{e}+\mathrm{k}\end{array}$ & \multirow[t]{2}{*}{ Procent } & \\
\hline \multicolumn{5}{|c|}{ Feitmeter. } & & \\
\hline 20 & 20 & 20 & 4,0 & 2,0000 & 14,87 & 10 \\
\hline 40 & 40 & 31 & 6,2 & 2,6667 & 12,16 & 15 \\
\hline 71 & 71 & 41 & 8,2 & 3,5500 & 10,28 & 20 \\
\hline 106 & 112 & 45 & 9,0 & 4,4800 & 7,99 & 25 \\
\hline 141 & 157 & 49 & 9,8 & 5,2333 & 6,65 & 30 \\
\hline 178 & 206 & 51 & 10,2 . & 5,8857 & 5,56 & 35 \\
\hline 215 & 257 & 53 & 10,6 & 6,4250 & 4,81 & 40 \\
\hline 253 & 310 & 55 & 11,0 & 6,8889 & 4,26 & 45 \\
\hline 292 & 365 & 56 & 11,2 & 7,3000 & 3,78 & 50 \\
\hline 331 & 421 & 57 & 11,4 & 7,6545 & 3,39 & 55 \\
\hline 371 & 478 & 55 & 11,0 & 7,9667 & 2,93 & 60 \\
\hline 409 & 533 & 52 & 10,4 & 8,2000 & 2,51 & 65 \\
\hline 446 & 585 & 49 & 9,8 & 8,3571 & 2,17 & 70 \\
\hline 482 & 634 & 45 & 9,0 & 8,4533 & 1,84 & 75 \\
\hline 517 & 679 & 41 & 8,2 & 8,4875 & 1,56 & 80 \\
\hline 550 & 720 & 35 & 7,0 & 8,4706 & 1,25 & 85 \\
\hline 579 & 755 & 29 & 5,8 & 8,3889 & 2,989 & 90 \\
\hline 604 & 784 & 26 & 52 & 8,2526 & 0.846 & 95 \\
\hline 630 & 810 & & & 8,1000 & & 100 \\
\hline
\end{tabular}




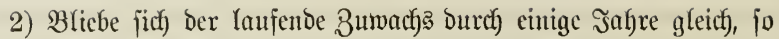

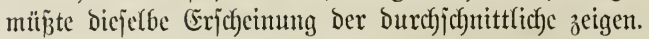

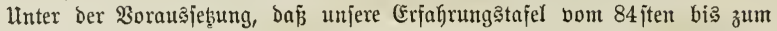
90 iten Эahre folgendermaßen laute, tritt bię̧ beutlich herbor.

\begin{tabular}{c|c||c|c}
\hline \multirow{2}{*}{ Jagr } & Majfe & \multicolumn{2}{|c}{ 3uwad) } \\
\cline { 2 - 3 } 83 & 531 & & $\frac{531}{83}=6,3976$ \\
84 & $531+6,6=537,6$ & 6,6 & $\frac{537,6}{84}=6,4$ \\
85 & $537,6+6,4=544$ & 6,4 & $\frac{544}{85}=6,4$ \\
86 & $544+6,4=550,4$ & 6,4 & $\frac{550,4}{86}=6,4$ \\
87 & $550,4+6,4=556,8$ & 6,4 & $\frac{556,8}{87}=6,4$ \\
88 & $556,8+6,3=563,1$ & 6,3 & $\frac{563,1}{88}=6,3989$ \\
89 & $563,1+6,1=569,2$ & 6,1 & $\frac{569,2}{89}=6,3955$ \\
90 & $569,2+5,8=575$ & 5,8 & $\frac{575}{90}=6,3889$
\end{tabular}

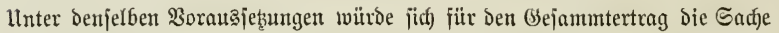
ebenjo gejtalten, wie hier fïr ben ફ̧auptertrag, nur da a andere Bahlettwerthe in

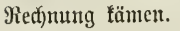

Son Dem Beitpuntte an, wo Der laufende Bmwachs fleiner als ber

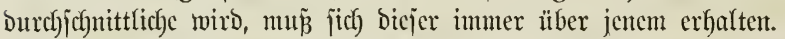

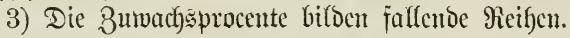

Bu bergleidfen bie Epalten $f$ uno $r$.

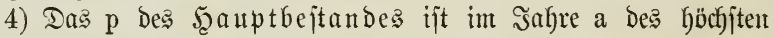
Durdjjeffnittsertrages, im 85 jten, gleich $\frac{100}{a}=\frac{100}{85}=1,1765$.

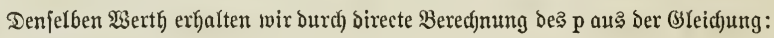
$\mathrm{p}=\frac{\mathrm{z} 100}{\mathrm{~m}}$, nämfiç)

$$
\frac{6,4 \times 100}{544}=1,1765
$$

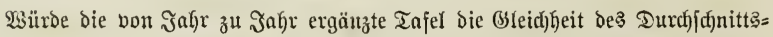
zuvadjes mit bem laufenden für bie Jahre 84,85 unb 86 ergeben, jo toären bie 3uwachaprocente in biejer 3eit genau $\frac{100}{84}, \frac{100}{85}$ und $\frac{100}{86}$. 
5) Der hörfjte Durchjochnitt Des (bejammtertrages fällt in bas

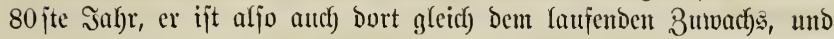

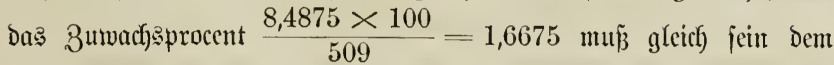
Serthe $\frac{100+v}{80}$.

$$
\mathrm{v}=\frac{\mathrm{V} \times 100}{509}=\frac{170 \times 100}{509}=33,399 .
$$

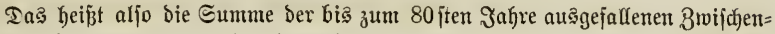
nuţungen beträgt $33,399 \%$ bcr Şauptbejtandeâmaije in biejem Jahre.

$$
\mathrm{p}^{\prime}+\frac{100+\mathrm{v}}{80}=\frac{133,399}{80}=1,6675 .
$$

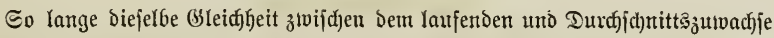
bejteht, wirb aud immer $p^{\prime}=\frac{100+v}{a^{\prime}}$ jein. Wäre beijpielsmeije ber Bejammt=

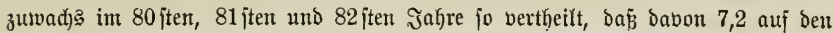

\begin{tabular}{|c|c|c|c|}
\hline $\begin{array}{l}\text { Im } 3 a \text { ahre: } \\
80\end{array}$ & $\begin{array}{l}\text { \$auptertrag: } \\
509\end{array}$ & $\begin{array}{l}\text { Gejammtertrag: } \\
679\end{array}$ & 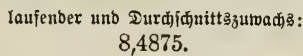 \\
\hline 81 & 516,2 & 687,4875 & 8,4875 . \\
\hline 0 & 523,4 & 695,9750 & 8,4875 . \\
\hline
\end{tabular}

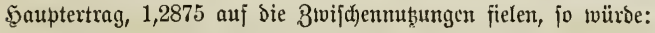

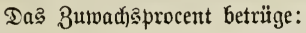

im §ałte:

80

81

82

$$
\begin{aligned}
& \frac{8,4875 \times 100}{509}=\frac{100+33,399}{80}=1,6675 . \\
& \frac{8,4875 \times 100}{516,2}=\frac{100+33,1824}{81}=1,6442 . \\
& \frac{8,4875 \times 100}{523,4}=\frac{100+32,9719}{82}=1,6216 .
\end{aligned}
$$

6) Eo lange ber Durchjefnittşuwachs noch im Steigen begriffen ijt, ift jowobl für 5̧aupt= als (5cjammtertrag ber Duotient aus 100 ober $100+v$ burch bas betreffende alter fleiner, als Das wirf $=$ liche $\mathrm{p}$ und $\mathrm{p}^{\prime}$, umgefefort größ̄er.

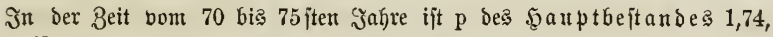
bagegent ijt

$$
\frac{100}{70}=1,43 \text { นกь } \frac{100}{75}=1,33 ;
$$

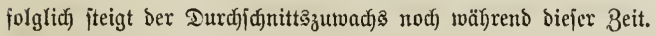

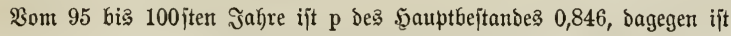

$$
\frac{100}{95}=1,05 \text { und } \frac{100}{100}=1 ;
$$

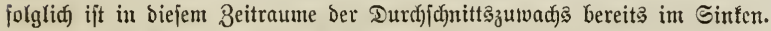

2(nalog berbält jidc) bie Gadje natïrlich audf mit bem \$rocente bes \&se= fammtertrages: 


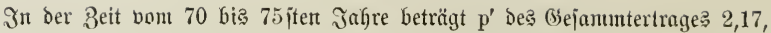
Dagegen iit

$$
\frac{100+35,104}{50}=1,93 \text { und } \frac{100+34,322}{75}=1,79 ;
$$

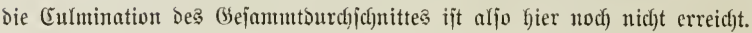

Som 85 bis 90 jten Jafre ift $\mathrm{p}^{\prime}$ bes (bejammtertrages 1,25 , Dagegen iit

$$
\frac{100+32,353}{85}=1,56 \mathrm{unD} \frac{100+31,304}{90}=1,46 ;
$$

folglidy) ijt in leșterer Beit Der Durd)j(d) in Sinfen.

\section{$\S 12$.}

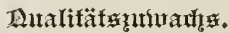

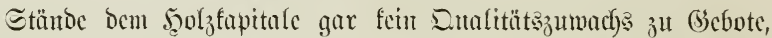
jo wären für bie Sect)walomirtbjechajt mur jebre nicorige llmtricbe finan= jicll möglicf).

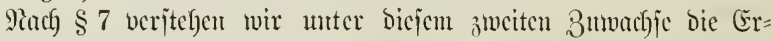

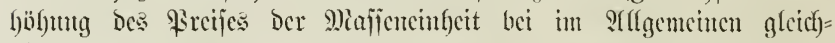

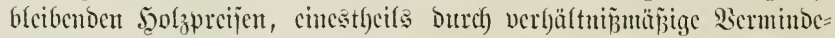

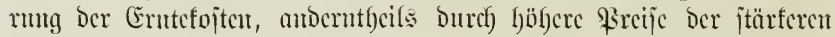
Eortimente gegenïber ben jefwächeren.

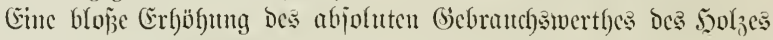
mit ber 3unalyme bes ?(lters, ber technijechen Picife, blcibt fïr ben Weirtl) jo lange bedentungslos, als jic nicht anth) cinte Erbjöfung bes Sircijes zur Folge hat oocr crwarten läpt.*)

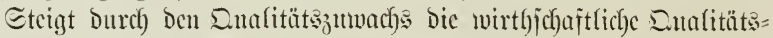
ziffer entes Bantmes oder Bejtandes in $\mathrm{n}$ Jaf)ren vou $q$ mif $Q$, jo ijt Deficn Duantum Q-q imb das betreffende 3uwacheprocent

$$
100\left(\sqrt{\frac{n}{q}}=1\right) \text {, }
$$

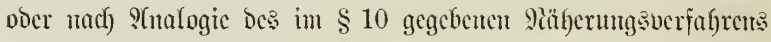

$$
\mathrm{Q}-\frac{\mathrm{q}}{\mathrm{q}} \times \frac{200}{\mathrm{n}} \text {. }
$$

Beifpiel. In einem Rebiere betrage erfafrungşmäpig bie Taxe ober Der.

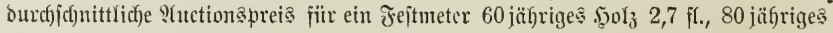

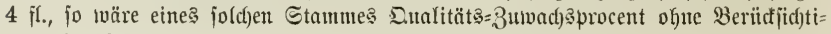
gung ber Erntefojten

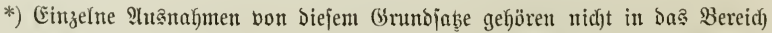
biejer Betrad)tungen, fo 3. 2. Die oft mit gropen Spjern verfnüpite Beriisfficdtigung

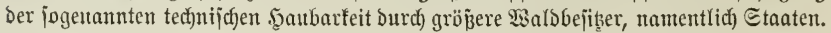


ober genauer

$$
\frac{4-2,7}{4+2,7} \times \frac{200}{20}=1,940
$$

$$
100\left(\sqrt{\frac{20}{2,7}}-1\right)=1,985 \text {. }
$$

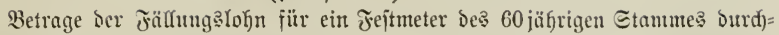

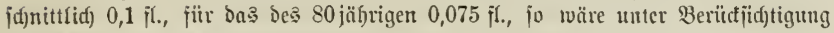
Diejer fiojten Das Donnfitüts= 3 uwad) sprocent:

ober genaner

$$
\frac{(4-0,075)-(2,7-0,1)}{(4-0,075)+(2,7-0,1)} \times \frac{200}{20}=2,031,
$$

$$
100\left(\sqrt{\frac{10}{2,7-0,0,1}}-1\right)=2,081 .
$$

Die Ficdunung wiro itets vercinfad)t, wenn ntan ben Einfeitspreis eunte= fojtenfrei it ?Inja bringt.

Beträje am erwähuten Etamune inteijen bas Sut. \% von 2,081 nur Den ala Siuțhol zu verwerthenden Theil. Siejer betrage $80 \%$ Des gegenwärtigen Baum=

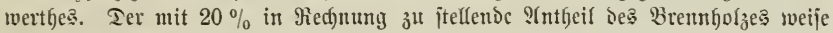
mux $0,25 \%$ Suafität rebuciten auf

$$
\frac{80 \times 2,081+20 \times 0,25}{100}=1,715 \% \text {. }
$$

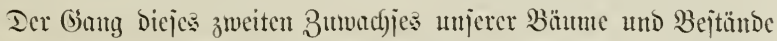

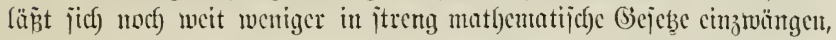

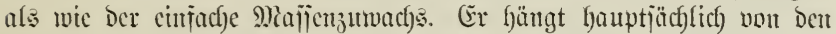

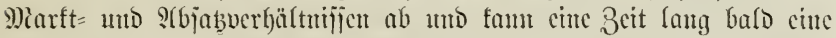

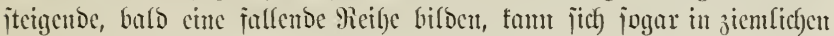
Eprüugen bewegen, vorjügfich) Danu, wem bie Bejtüntoe cinem S(lter

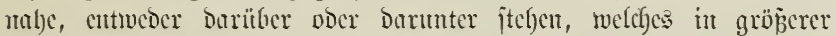
Mienge bejontors jtarf gejucfle utto beshalf gat bejaflte Eortimente

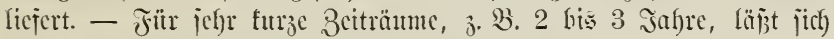
Der Eutulitätỡ

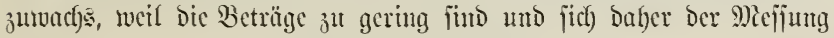
cuttjichent.

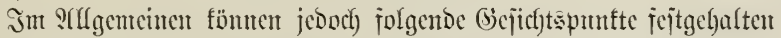
luerbert:

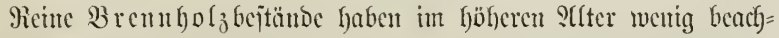
tems̄werthen Euafitätš̉ S(lters und ber Dafuci crajielten, gewifijen, auf Dem Miarfte belicbten

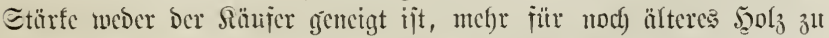

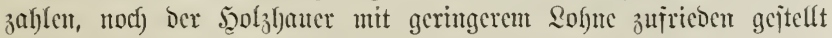

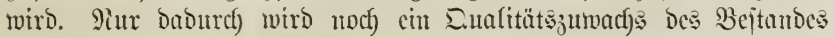




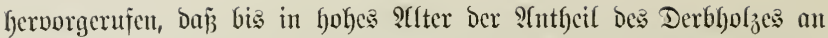
Der (Scjammtmafje toächjt. Bcijpielssweije entbält cin Fischtenbejtand

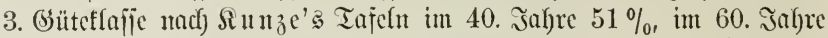
$81 \%$, im 80. Jafure $85 \%$, im 100. Jafre $86 \%$, im 120. Jafje $87 \%$

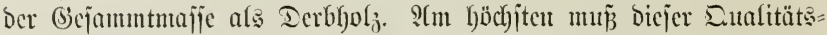
zuwad) in jentem jugendlichen ?ater jein, wo bie Bejtünde aufangen,

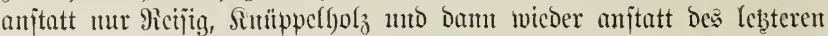

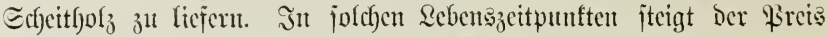
für bie (Ënl)eit menter gleich)zeitigem Sinfen Der (Erntefojten an meijten.

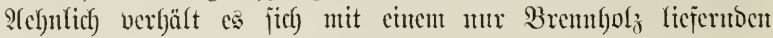
Ricbermalbe.

Wect bedentungsoveller bis in höhere Bejtundesalter ijt ber Sutult=

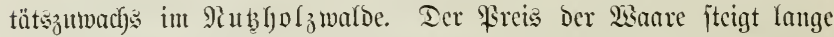

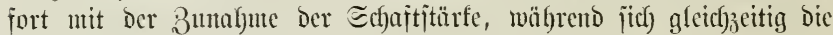
Erntefojten etwas vermindern. Ferner fommt $D a_{3} u$, Daj bis zu ciner

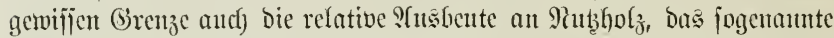

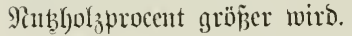

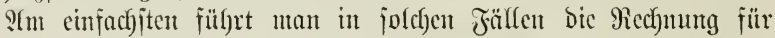
Den Bejtand jo ans, daj man bie ciujeluen Eortimente in Procenten

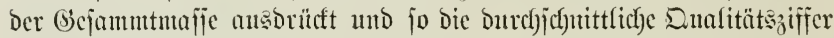
ermittelt.

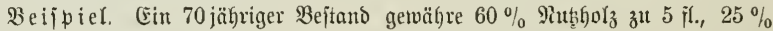

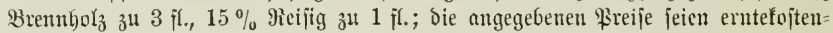

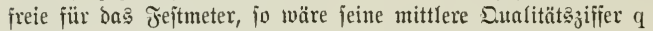

$$
\frac{60 \times 5+25 \times 3+15 \times 1}{100}=3,9 .
$$

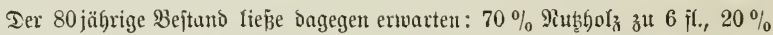

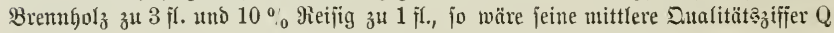

$$
\frac{70 \times 6+20 \times 3+10 \times 1}{100}=4,9 .
$$

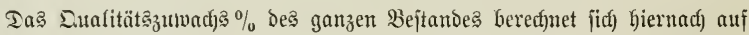

ober genauter

$$
\frac{4,9-3,9}{4,9+3,9} \times \frac{200}{10}=2,27,
$$

$$
100\left(\sqrt{\frac{10}{3,9}}-1\right)=2,31 .
$$

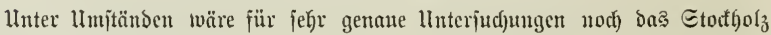
mit zu veranjojlagent.

Der Dic Bornubungen liejernde Bwijebenbejtand ijt getrennt vom Şauptbejtand ebenjo zn bejragen. Berabe bei ifm faun nament=

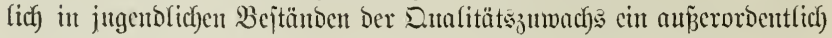




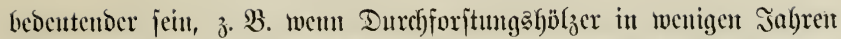
zll werthuollen, 5 bis $6 \mathrm{~cm}$ jtarfen 5̧opfenjtantgen werben, während

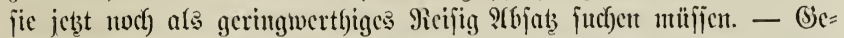

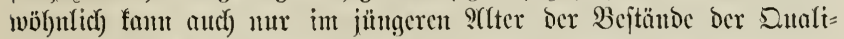

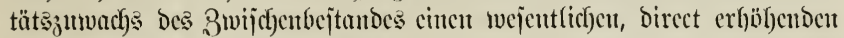

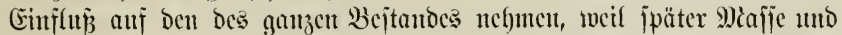

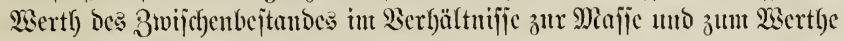
bes jeautbejtandes ju unbedeutend fint.

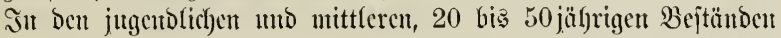

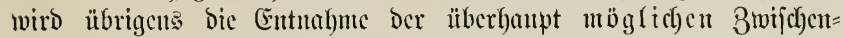

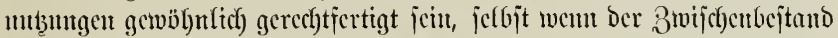

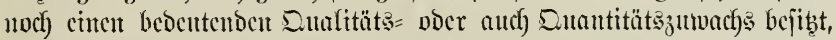

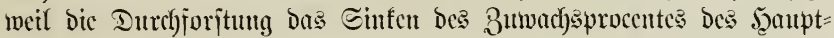

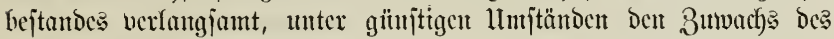
leb̧teren mogl aud erlyöl)t.*)

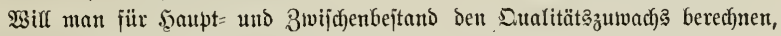
fo ijt folgende Red)untgeform auzuwenden:

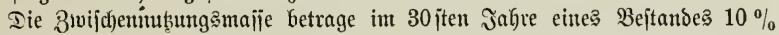

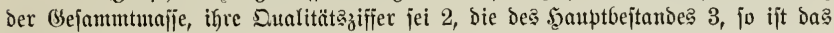
mittlere q des ganzen Bejtandes

$$
\frac{90 \times 3+10 \times 2}{100}=2,9 .
$$

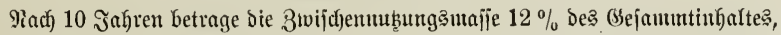

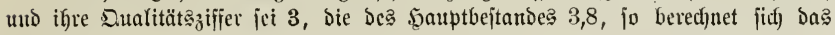
mittlere $Q$ auf

$$
\frac{88 \times 3,8+12 \times 3}{100}=3,704 \text {. }
$$

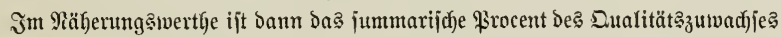

$$
\frac{3,704-2,9}{3,704+2,9} \times \frac{200}{10}=2,43 \text {. }
$$

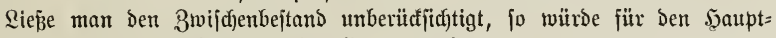

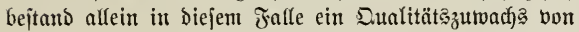

rejultiren.

$$
\frac{3,8-3}{3,8+3} \times \frac{200}{10}=2,35 \%
$$

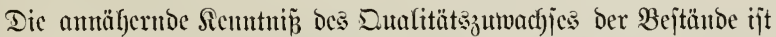

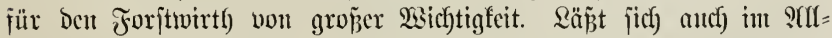
gemeinen faum mef)r Bejtimmtes itber bicjen zrociten 3uwachs jagen,

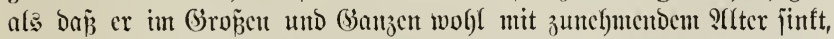

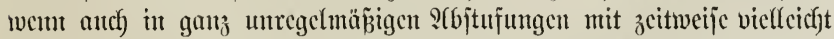
jogar bebeutendem Steigen und Dann wicber bedeutendem Fallen, jo

*) 8 u vergl. $\$ 16$. 
ift es bock ber localen Praris oft möglicf), genügende Erfabrungen

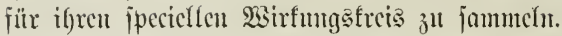

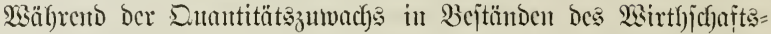

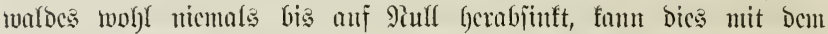

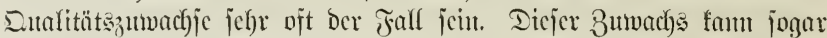

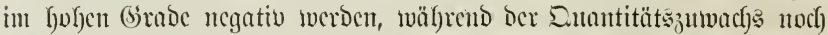

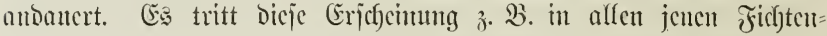

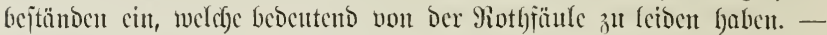

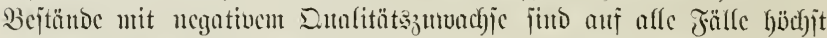
abtricbsbediurtig.

\section{$\S 13$.}

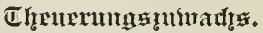

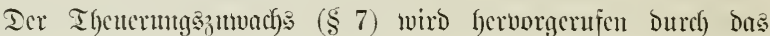

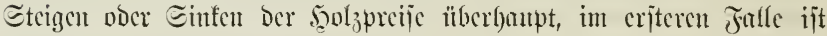
cr pojitiv, int zweiter negativ.

In wir ben Preis in Geld ansubritden pflegen, und da bas

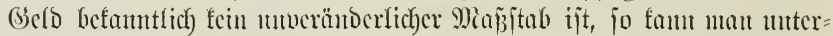

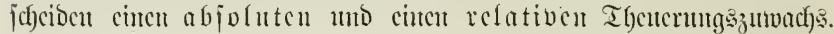

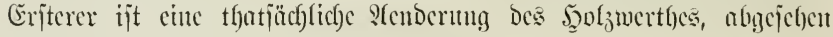

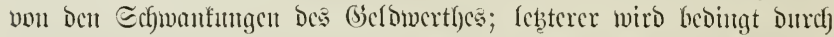

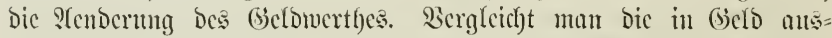

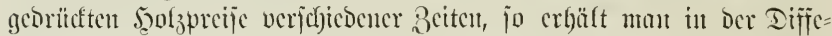

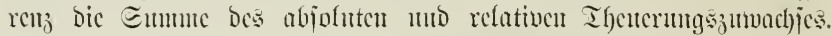

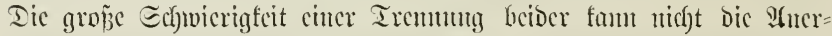
femuntg ber Ifatjache bicjes llnterifficoses verfuntorn. Etreng ge=

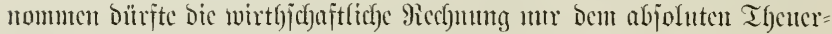

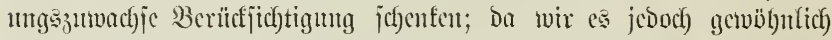

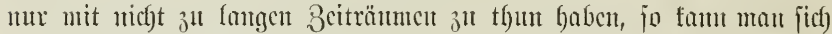

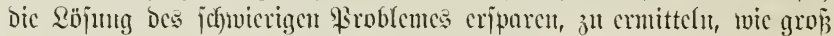
Der abjolute uto wie groj ber relative Ifenterungszmwady jei.

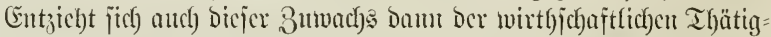
feit Des Forjtmannes, johald berjelbe fein Mittel utchr in Der jaand hat, Den Miarft zu werbejfern, jo verbient er gewie alle Beachtung bei

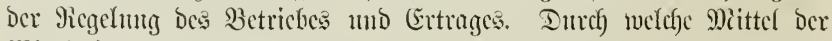

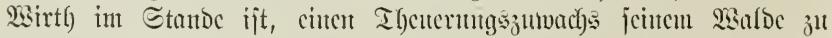

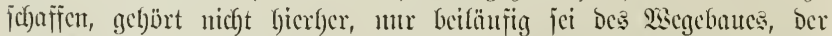

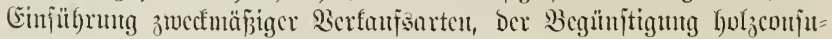

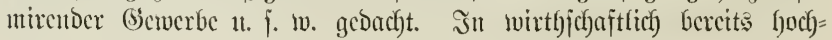




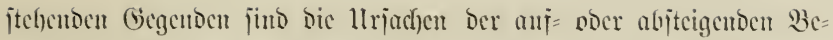

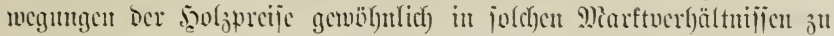

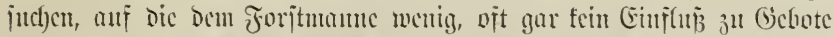

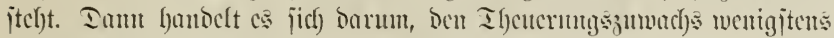

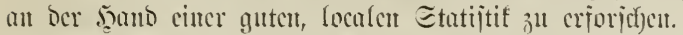

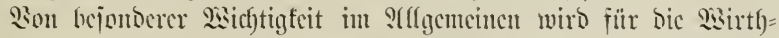

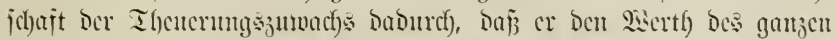

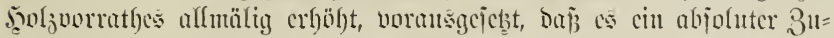
mad)

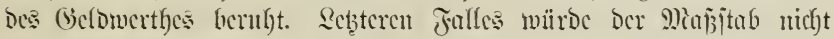
mehr pajicn; Demt bas Einfen Des Geldmerthes in jo allgencincu Fällen

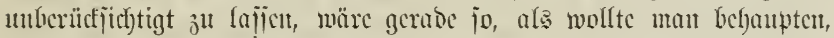
cin Baum jei n̈ber Siacht gemachjen, mentm man ify am anderen Tage mit fleincrem Miaje min̈t.

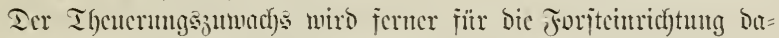

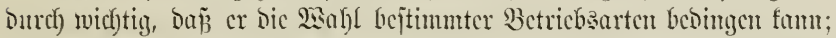
fïr bic fpecielle Ertragseregelung Daburef), baj cr auj bic finamjelle

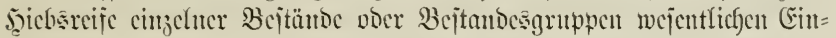

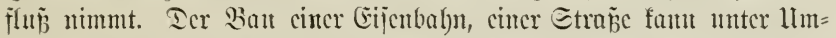

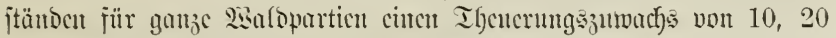
und noch melys \&irocenten hervorrufen.

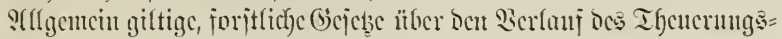

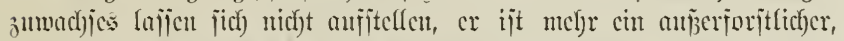
Der chenjomol)h alle Eortimente ïberfant, als and mur bejtimmte cin= jelute Eortimente treffen funtr.

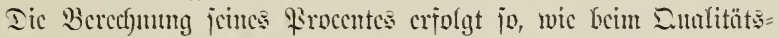
3uwad)s. Etcigt ber Preis Der Einfeit in $\mathrm{n}$ Jafren vout $\mathrm{t}$ anf $\mathrm{T}$, jo fintoct cin Thencrungsumady vou

$$
\mathrm{T}-\mathrm{t}+\mathrm{t} \times \frac{200}{\mathrm{n}}
$$

bocr gentuer vou

$$
100\left(\sqrt{\frac{\mathrm{T}}{\mathrm{T}}}-1\right)
$$

Firocuten jtatt.

$$
\text { S } 14 \text {. }
$$

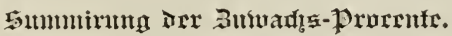

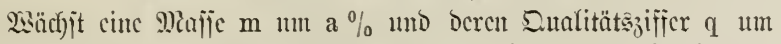
$\mathrm{b} \%$, jo get)t in $\mathrm{n}$ Jaffren ifre gegenwärtiger $\mathfrak{W e r t h} \mathrm{mq}$ üfber it 
hicraus

$$
\mathrm{W}=\mathrm{mq}(1,0 \mathrm{a})^{\mathrm{n}} \cdot(1,0 \mathrm{~b})^{\mathrm{n}} ;
$$

uni

$$
\sqrt{\frac{\mathrm{W}}{\mathrm{W}}}_{\mathrm{mq}}^{\mathrm{n}}=\left(1+\frac{\mathrm{a}}{100}\right)\left(1+\frac{\mathrm{b}}{100}\right)
$$

$$
100\left(V_{\frac{\mathrm{n}}{\mathrm{mq}}}^{\mathrm{m}}-1\right)=\mathrm{a}+\mathrm{b}+\frac{\mathrm{ab}}{100} \text {. }
$$

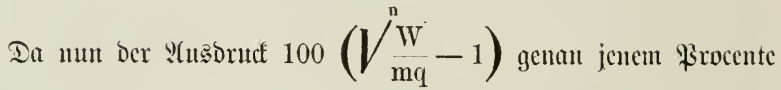

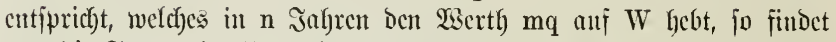
man bie Summe bes Duantität

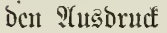

$$
a+b+\frac{a b}{100} .
$$

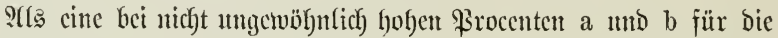

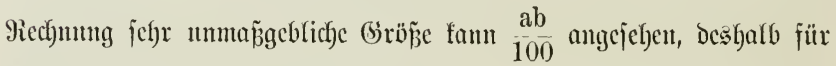

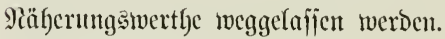

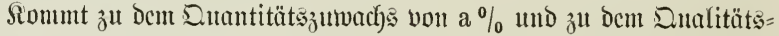

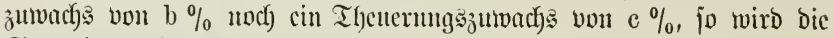
ธımmirungsformel:

licraus

$$
W=m q\left(1+\frac{a}{100}\right)^{n}\left(1+\frac{b}{100}\right)^{n}\left(1+\frac{c}{100}\right)^{n}
$$

IIII

$$
V^{\mathrm{n}} \frac{\mathrm{W}}{\mathrm{mq}}=\left(1+\frac{\mathrm{a}}{100}\right)\left(1+\frac{\mathrm{b}}{100}\right)\left(1+\frac{\mathrm{c}}{100}\right)
$$

$$
100\left(\sqrt{\frac{w}{m q}}-1\right)=a+b+c+\frac{a b+a c+b c}{100}+\frac{a b c}{100^{2}} .
$$

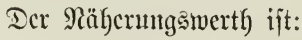

$$
a+b+c \text {. }
$$

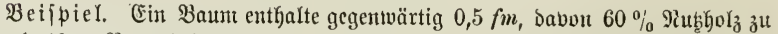

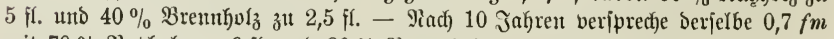

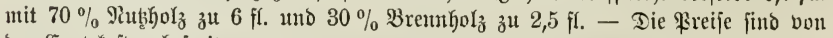
der Erntetopten befreit.

1) Duautität $\mathfrak{z} \mathfrak{u} \mathfrak{w a c b} \mathfrak{g}=\mathfrak{g}$ roceut a.

$$
\frac{0,7-0,5}{0,7+0,5} \times \frac{200}{10}=3,333
$$


wer gentuer

$$
\left(\sqrt{0,5}^{\frac{10}{0,5}}-1\right)=3,422 .
$$

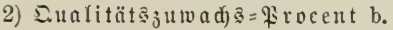

Jeţige Suttalität

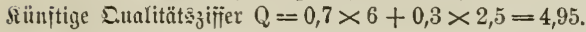

Procent b

noer gentauer

$$
\frac{4,95-4}{4,95+4} \times \frac{200}{10}=2,123
$$

$$
100\left(\sqrt{\frac{10}{4}-95}-1\right)=2,154 \text {. }
$$

Mard Der gormel $a+b+\frac{a b}{100}$ herechntet jiff bie Eumme ber burd bie

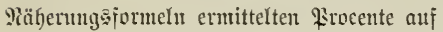

$$
3,333+2,123+\frac{3,333 \times 2,123}{100}=5,456+0,071=5,527 .
$$

Ceķcn wir in bie Mähferungşontel bie Baummerthe, fo erfalten wir:

$$
\frac{(0,7 \times 4,95)-(0,5 \times 4)}{(0,7 \times 4,95)+(0,5 \times 4)} \times \frac{200}{10}=5,361 .
$$

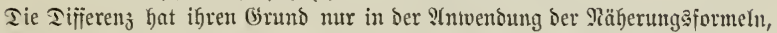
jie entfällt, wem wir bie mathematija) genauten siserthe in Red)unty itellen:

$$
3,422+2,154+\frac{3,422 \times 2,154}{100}=5,649 .
$$

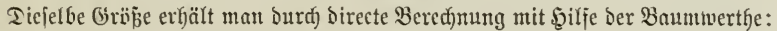

$$
100\left(\sqrt{\frac{10}{0,7 \times 4,95}}-1\right)=5,649 .
$$

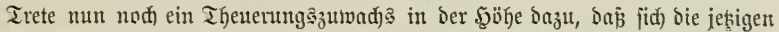

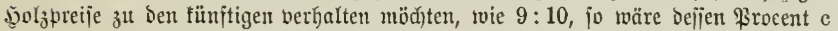

voer genauer

$$
\frac{10-9}{10+9} \times \frac{200}{10}=1,053
$$

$$
100\left(\sqrt[10]{\frac{10}{9}}-1\right)=1,059
$$

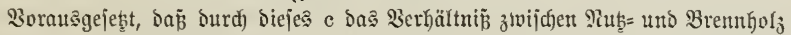

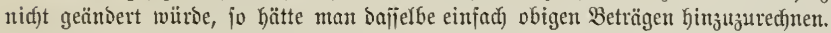
betragen:

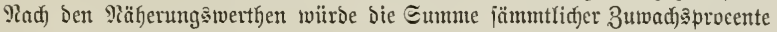

$$
a+b+c=3,333+2,123+1,053=6,509
$$

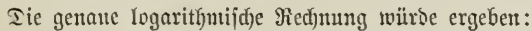

$$
\begin{aligned}
& a+b+c=6,635 \\
& \frac{a b+a c+b c}{100}=0,1328 \\
& \frac{a b c}{100^{2}}=0,0008 \\
& \text { Eumme } 6,7686 .
\end{aligned}
$$




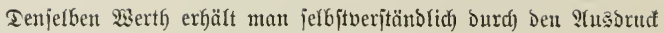

$$
{ }_{100}\left(\sqrt{\frac{10}{0,7 \times 4,95 \times \frac{10}{9}}}-1_{0,5 \times 4}\right)=6,7686 .
$$

(E⿱

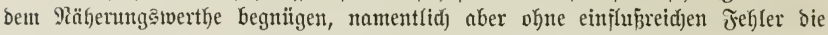
Orö̈̄̈e $\frac{a b+a c+b c}{100}+\frac{a b c}{100^{2}}$ weglafien fantu, weldee im vorliegenden Beifpiefe mur 0,1336 beträgt.

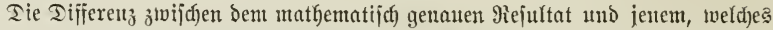
man aus Durd)gängiger S\{nvenoung Der Piäherungâwerthe erhält, ijt fier 6,7686-

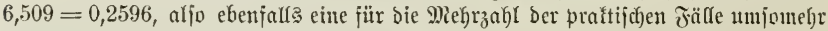

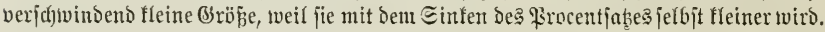

\section{$\S 15$. \\ (Elemente Des Wrifexprorentes.}

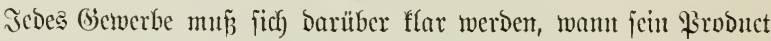
fertig, wann es reif zu Ernte ift. In Dem Eimne, wie ber Epinmer

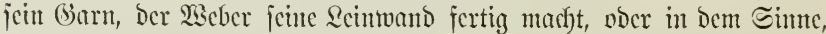
wie ber anowirth jein Gictreide reif nemten fann, fömen wir bei

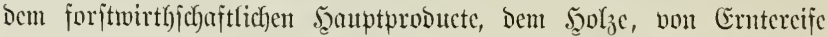
nicht reben. Ilno boch fann cine vernünftige $23 i r t b j$ dyaft ofne wentig= jtens principiclle Enticheidung bicjer Frage gar nicht gedacht tocrben. Gielativ crfolgt cin jolches Fertigmerden bes frolzes, wemn wir bejtimmte

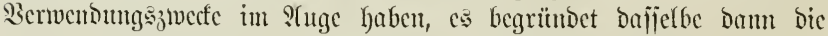

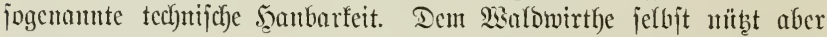

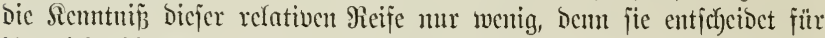
if)n nicht bic Frage, ob cr nicht bejier thäte, bic Bäume noch Yänger iteben zu Yafien doer früber abzutreiben. - Eine phyjifiche 9icife des Seolzes faum jajt gar nicht in Betracht fommen.

llm S(ntwort auf bie wichtige Jrage zu fimben, wann unjere Bejtände reif zux Erute jeien, mitijen wir un aljo nach andoren

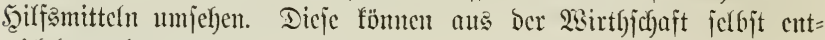
wicfelt werden.

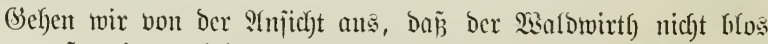

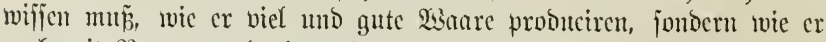

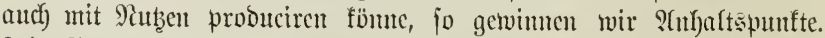

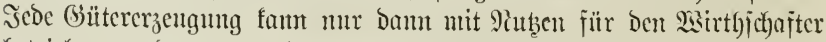
betrieben werden, went Der \&reis bes crjeugten Gutes mubejtens bic

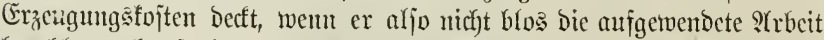
bezahlt madyt, jondern andy bie Butjen bes gebraudften, jtehenden Siapi= 
tales, bejichungsmeije mit ?(mortijation, bie Bimjen bes verbuntd)ten, mmfaufenton Sapitales unb bicjes jelbijt gewïh)rt. Eo ijt es auff) bcim 5ूolzc.

Die Ermittelung แnto Eummirung ber in Den vorigen ş be= iprochenen Frocente a, b und c genitgt bafer nicht, Im bic wirtl)= idfaftlidje Bedeutung bes Bumadjes zu mejien, Da bicjem nicht blos

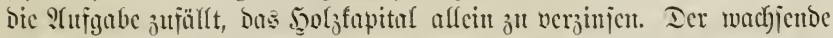

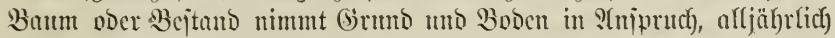

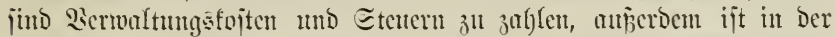

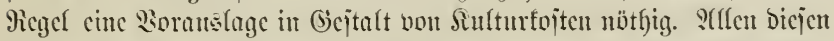

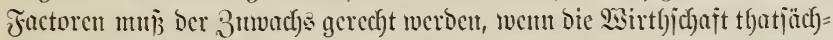

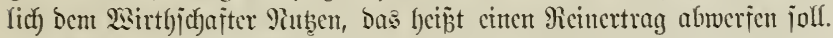

Sisir baben es aljo aujer mit bem 5ooljfapitale noch gu thum mit Berwaltungatoiten, Eteuern, Bobentapital un Sittur= fojten.

Sences Procent nun, weldyes ben 3ruvadys des̃ Bejtandes in $\mathfrak{B e}=$

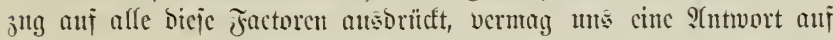
Dic Frage ber Erntercife Des Bejtandes ju gefort. Etcht erjteres noch

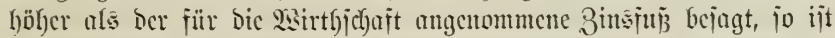

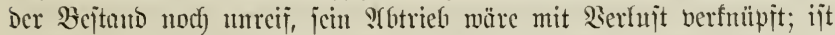

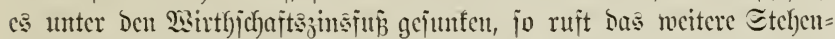
lafjen bes Bejtandes wirthjchajtlidfe Berlujte hervor. Bwijchen beiden

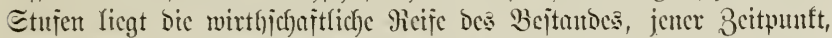
in weldbent jragliches Pirocent gleidh Dem 2istrtbjchajtsinsijuje miro.

In Der Sefre won Der Entereife Der Beftünde in joldyem Sinte

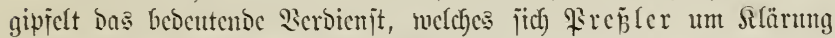
Der joritlicfen 2isijenjchajt und 2isirtfjidfajt erworben. Ias jragliche Procent nante cr Das 2 scijerprocent, weil ce un auj bicje Ernte= reife binmeijt.*)

Dic Cintüfruntg Der oben genannten Factoren in bie Fecfuntung bictet, ment jic mathematijef genan jein joll, groje Schwierigfeiten

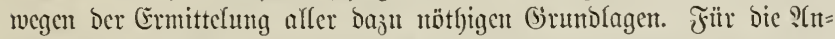

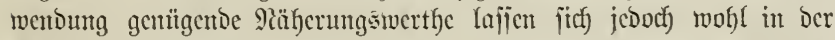
अegel finden.

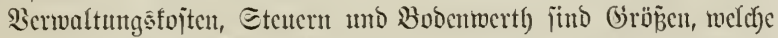

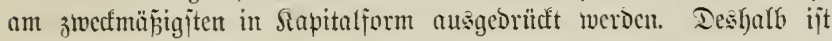

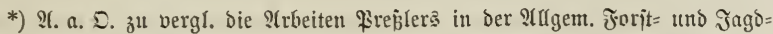

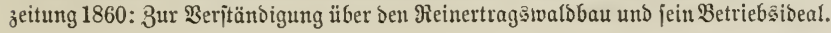




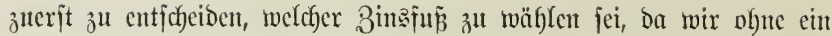
im Soraus für bie 2 Sirtbjeffaft angenommenes Procent (p) überbaupt

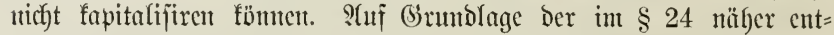
wicfelten Sarfichten biurften für bas forftliche Berwerbe etrva $3 \%$ in Rectunng zu jtellen feint.

1) Die Berwaltungatojten.

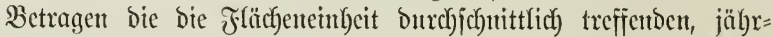

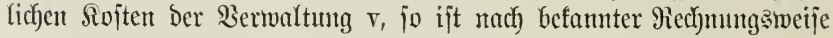
Das ßerwaltungsfapital $\mathrm{V}=\frac{\mathrm{V}}{0,0 \mathrm{p}}$.

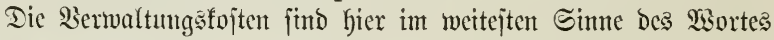

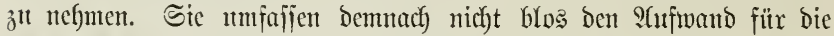
eigentliche Berwaltung, jondern aud ben fütr Echutb. Injpection, Direc=

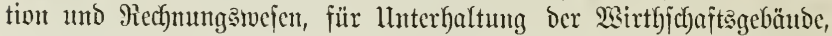

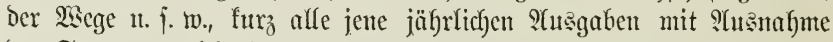

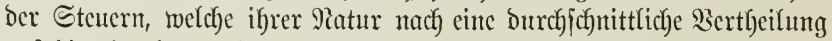
auf bie euzzelnen Fläclyeneintheiten eintes ganzen $\mathfrak{E a l b e}$ gcitatten.

Inmerfung. *) (Es fann fraglich erj(f)einen, ob ganz ridytig gerechnet wirb,

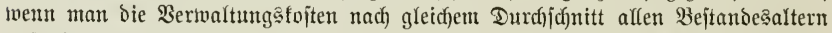

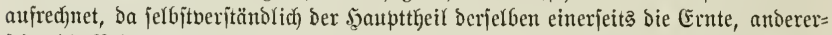
feits bie Bejtanbesgründung trifft. Correcter wäre eş baher Belajtung ber verichieben alten Bejtänoe anzujeţen. Berabe ber Unjitano indelien,

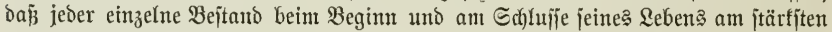

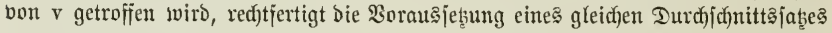

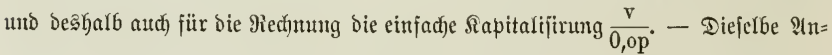
ichaunng gilt für Berecfunng ber Sojten= uno (Erwartungäwerthe ber Bejtände.

\section{2) Die Eteuern.}

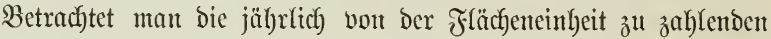
Eteuern s als reiutc Birunditeuern, näntich als joldbe, weldbe von ber Bobentente ju erheben jimb, jo berect)ntet jich) ttach SAnalogie bes Borigen bas biejer Sisjtenrente entiprectende Siapital $S=\frac{\mathrm{S}}{0,0 \mathrm{p}}$.

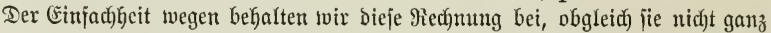

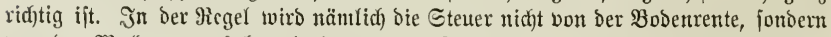

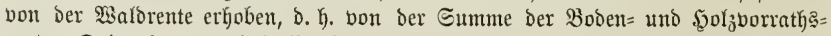
rente. Taburd) verwantbelt fid) bie jogenante (orrunsiteuer in eine Ertragsilteuer,

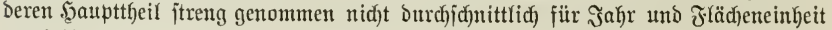
bertheilt werben fam, fonbern unmittelbar von Dem Bruttoertrage, wie bie Ernte=

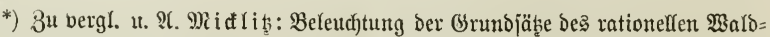
wirthes 2c. Dlmuth, 1861. 
fojten, in $\mathfrak{S E}_{3}$ ug (Eintoumenjtener. *)
3) Der Bodeutwerth.

Dic Berect)mung Des Bobenfapitales (B) funn in breifuct)er 9 trt erfolgen.

2iir unterjajeiden**)

a) Den Serfunfestoerth),

b) Den Rojtenuerth,

c) Den Erwartungsiverth) Des Bodens.

a) Der Berfanfawerth bes Bobens ijt jener, melden diejer

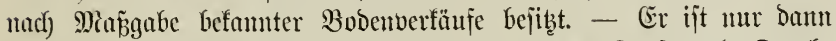

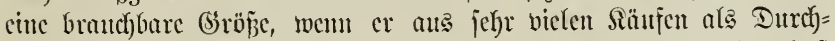

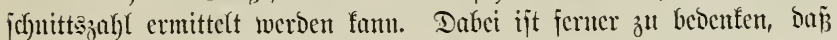

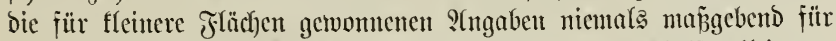

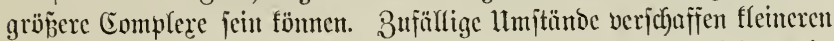

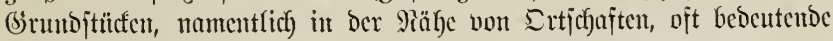
S(f̈ectionsiverthe.

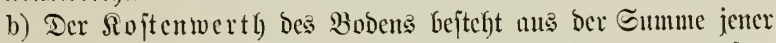

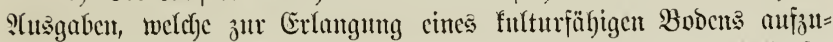

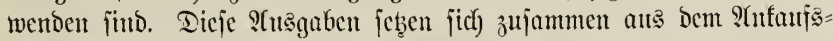

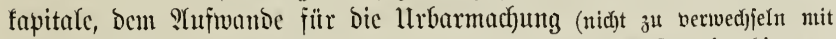

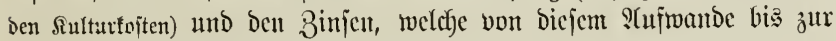
Beit Der Siulturfülgigfeit erwachjen.

Beifpiel. Ěin Sgeftar berifuntpter Sarbboden tojte 20 fl., für bie Ent=

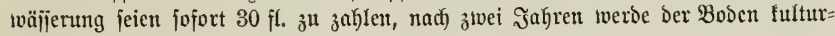

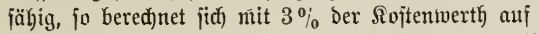

$$
(20+30) 1,03^{2}=53 \mathrm{fl} \text {. }
$$

PHef Dex Sojtenverth) Des Bobens funt unter llmitänoen jo be=

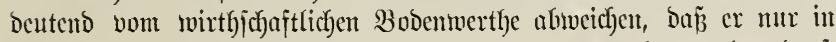

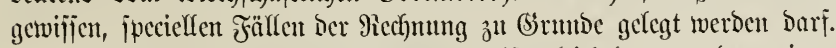

c) Der Bobenerwartungawerth ift gleich Dem ats ber reinen Bodenrente (r) gefmidenen Sapitule $\frac{\mathrm{r}}{0,0 \mathrm{p}}$. Berechnet man ihn mit

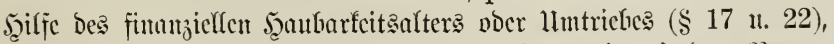
jo erbült man Dejïen Marimum $\left(B_{a}\right)$, weldes wir mit bem Mamen bes wirthichaftlidfen Bodentwertbes bejecifyen.

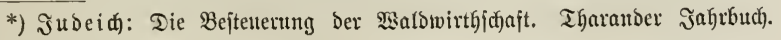
27. BD. 1877. ธ. 53 น. $\mathfrak{~}$.

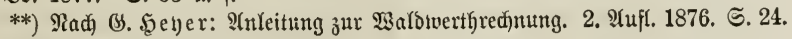

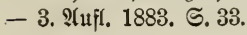




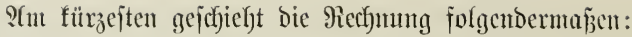

Dic Erträge cines Bcjtandes jeben fick zujammen ants Der S(btricbs:

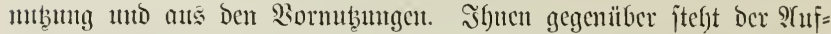
mand fïr Siultur, Ernte, Berwaltung umb Etentert. SUlfe Siojten mo Erträge müfje anf einen 3citpunft gebrad)t werben, um fic junntren

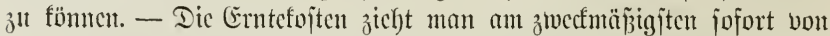
Den betreffenden Erträgen ab, jtellt bicje ponach) crutefojtenfrei in Picclunutit.

Bezcichnen wir ben erntefojtenfreien sfbtricbs= ober Şaubarfeits= crtrag im Sabre u mit $H_{u}$, bie im aten, bten . i. w. Sabre cint=

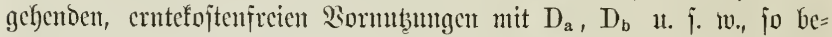
trägt bie auf Das Jahr u gebrachte Eumme ber Erträge

$$
\mathrm{H}_{\mathrm{u}}+\mathrm{D}_{\mathrm{a}} 1,0 \mathrm{p}^{\mathrm{n}-\mathrm{a}}+\mathrm{D}_{\mathrm{b}} 1,0 \mathrm{p}^{\mathrm{u}-\mathrm{b}} \ldots \ldots
$$

Der 2 sertl) ber Sitturfojten $k$ ijt im Sabre $u=k 1,0 p^{n}$.

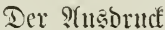

$$
\mathrm{H}_{\mathrm{u}}+\mathrm{D}_{\mathrm{a}} 1,0 \mathrm{p}^{\mathrm{u}-\mathrm{a}}+\mathrm{D}_{\mathrm{b}} 1,0 \mathrm{p}^{\mathrm{u}-\mathrm{b}} \ldots \ldots-\mathrm{k} 1,0 \mathrm{p}^{\mathrm{u}}
$$

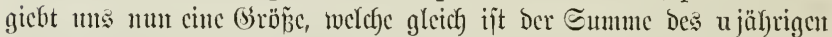

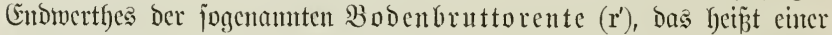
Rente, bic noch) mit Den Berwaltungsfojten (v) und Etcucru (s) be= lajtet ijt. Durch Divijion bicjes Endwertfes mit $\frac{1,0 p^{u}-1}{0,0 p}$ erfyalten

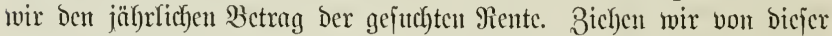

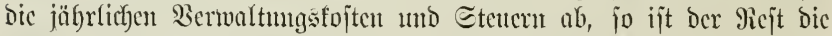
Bodenttorente oder Bodenrente (r), Deren Siapitalịïnung Den

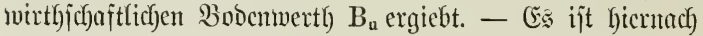

$$
\begin{gathered}
\mathrm{r}=\frac{\mathrm{H}_{\mathrm{u}}+\mathrm{D}_{\mathrm{a}} 1,0 \mathrm{p}^{\mathrm{u}-\mathrm{a}}+\mathrm{D}_{\mathrm{b}} 1,0 \mathrm{p}^{\mathrm{u}-\mathrm{b}} \ldots \ldots-\mathrm{k} 1,0 \mathrm{p}^{\mathrm{u}}}{\mathrm{lmo}}-(\mathrm{r}+\mathrm{s}) \\
\frac{1,0 \mathrm{p}^{\mathrm{u}}-1}{0,0 \mathrm{p}} \\
\mathrm{B}_{\mathrm{u}}=\frac{\mathrm{r}}{0,0 \mathrm{p}} .
\end{gathered}
$$

Durch Divifion Des allgencinen STtsorucfes für $\mathrm{r}$ mit $0,0 \mathrm{p}$ findot ntun Direct

$$
\left.\mathrm{B}_{\mathrm{u}}=\frac{\mathrm{H}_{\mathrm{u}}+\mathrm{D}_{\mathrm{a}} 1,0 \mathrm{p}^{\mathrm{u}-\mathrm{a}}+\mathrm{D}_{\mathrm{b}} 1,0 \mathrm{p}^{\mathrm{u}-\mathrm{b}} \ldots \ldots-\mathrm{k} 1,0 \mathrm{p}^{\mathrm{u}}}{1,0 \mathrm{p}^{\mathrm{u}}-1}-(\mathrm{V}+\mathrm{S})^{*}\right)
$$

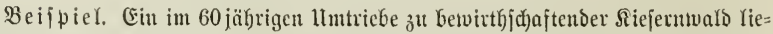

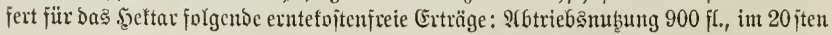

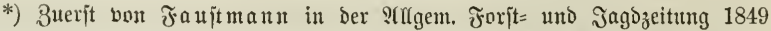

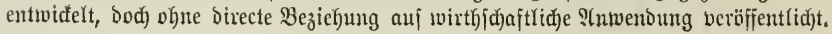




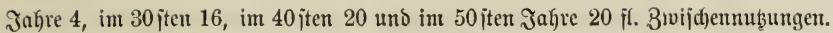

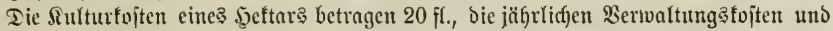

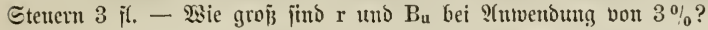

$$
\begin{gathered}
\mathrm{r}=\frac{900+4 \cdot 1,03^{40}+16 \cdot 1,03^{30}+20 \cdot 1,03^{20}+20 \cdot 1,03^{10}-20 \cdot 1,03^{60}}{\frac{1,03^{60}-1}{0,03}}-3=2,5016 \tilde{\mathrm{f}} . \\
\mathrm{B}_{\mathrm{u}}=\frac{2,5016}{0,03}=83,39 \tilde{\mathrm{\kappa l}} .
\end{gathered}
$$

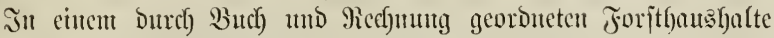

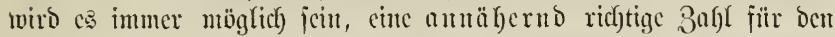

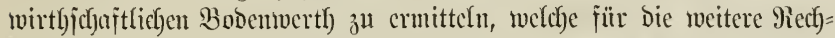

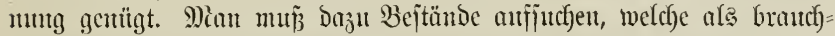

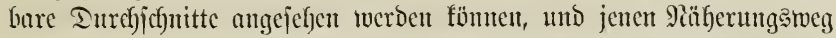

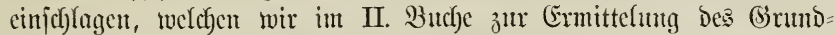
fapitales überfantpt in Sorjchlag gefracht baben. Man betrachtet ben

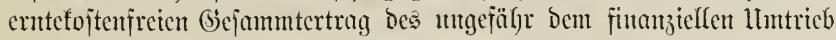

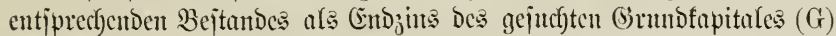
und berechnet Dajielbe ars ben Dutoticnten ans $1,0 \mathrm{p}^{\mathrm{u}}-1$ in bicjen (B)joumntertrag. Es ijt Dam $\mathrm{G}-(\mathrm{V}+\mathrm{S}+\mathrm{C})=\mathrm{B}_{\mathrm{u}}$. -

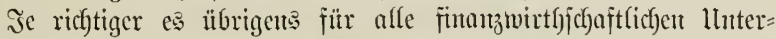

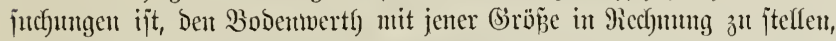

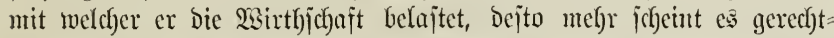

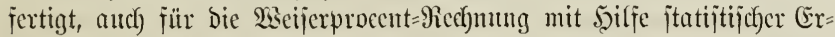

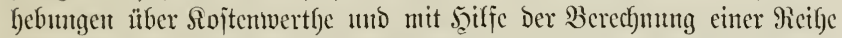

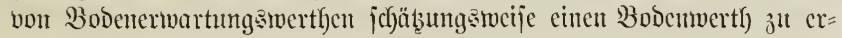
mittefu, benjefben ban aber nicht als eine und) ben verïnderlichen

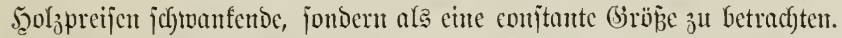

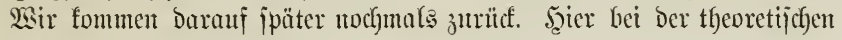

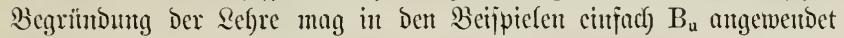
werdent.

\section{4) Die Sitturfoiten.}

Die Sulturfojten (k) jür bie Fläcl)eneinfleit fömen nicht twie bie Berwalturgsfojten und Etenten als cmige Rente fapitalifitrt merben, ment man Den einzelnen Bejtand für jich betrachtet. Entrueder funto jie mur als cimmalige stustage zu behandeln, dies cbenjo wie bei ber Ermittefung Der Bobcurente und bei Der Siojtenwertf)= Berect)mung cintes

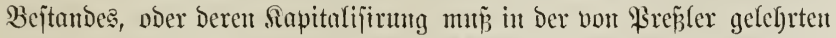
Weije erfolgen.*) Diejer jeţt bas sulturfapital (C) gleich Der

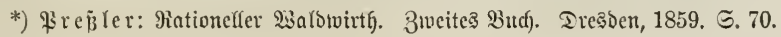


Summe aus ber eimmaligen P(uslage $\mathrm{k}$ und einem fiapitale, weld)es

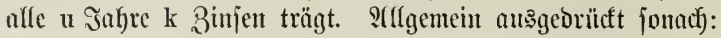

$$
\mathrm{C}=\mathrm{k}+\frac{\mathrm{k}}{1,0 \mathrm{p}^{\mathrm{u}}-1}=\frac{\mathrm{k} 1,0 \mathrm{p}^{\mathrm{u}}}{1,0 \mathrm{p}^{\mathrm{u}}-1} .
$$

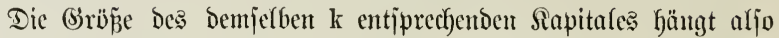
nicht blos ab vou ber şölye des Zinsfunges, fonbern auch won ber bes

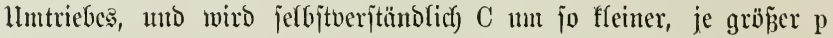
und u, da bicje Factoren mur Den Penter bes Bruches in Der Formel $\mathrm{k}+\frac{\mathrm{k}}{1,0 \mathrm{p}^{\mathrm{u}}-1}$ berüf)ren. jo wirb $\mathrm{C}$

Beifpiel. Ein Bejtand verurjache für bie Frädfeneinheit 20 fl. Sulturfojten,

für Den 60 jährigen Umtrieb:

$$
\begin{aligned}
\text { mit } 3 \% & =20+\frac{20}{1,03^{60}-1}=24,11, \\
\text { " } 4 \% & =20+\frac{20}{1,04^{60}-1}=22,10 .
\end{aligned}
$$

F̈ür Den 100 jäbrigen llmtrieb:

$$
\text { mit } \begin{aligned}
3 \% & =20+\frac{20}{1,03^{100}-1}=21,10, \\
\text { " } 4 \% & =20+\frac{20}{1,04^{100}-1}=20,40 .
\end{aligned}
$$

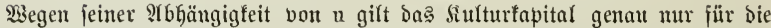

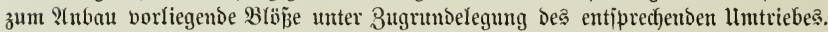
Dá geht z. 3. aut folgenden Betrad,tutgen hervor:

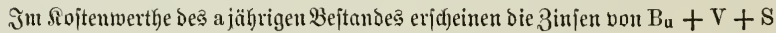
in ber form $\left(B_{u}+V+S\right)\left(1,0 p^{a}-1\right)$, bie Sulturfoiten $k$ jedod) als a jägriger

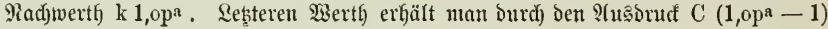

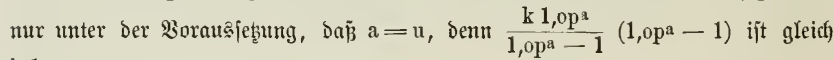
$\mathrm{k} 1, \mathrm{opa}$.

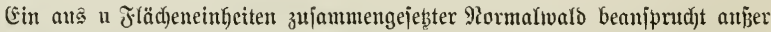
Dem $\mathfrak{5 g o l z}$ fapitale nicf)t u $\left(B_{\mathfrak{u}}+V+S+C\right)$, jondern u $\left(B_{u}+V+S\right)+\frac{k}{0,0 p} \Re a=$

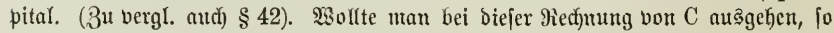

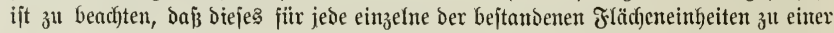

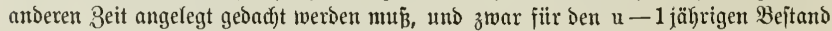
nad) einem Jahre, für ben $u-2$ jährigen nad) 2 u. f. w., für ben 1 jägrigen nad)

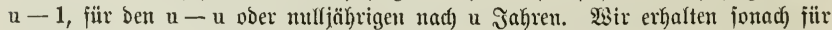

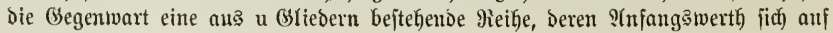
$\mathrm{C} \times \frac{1, \mathrm{pp}^{\mathrm{u}}-1}{1, \mathrm{op} \mathrm{p}, 0, \mathrm{op}}$ berectinnet. 
Diejer $\mathfrak{B}$ erth) (ijit fich auf in $\frac{\mathrm{k}}{0, o \mathrm{p}^{\prime}}$, Dent

uno

$$
\mathrm{C}=\frac{\mathrm{k} 1, \mathrm{op}}{1,0 \mathrm{p}^{\mathrm{u}}-1}
$$

$$
\begin{gathered}
\frac{\mathrm{k} 1, \mathrm{op} \mathrm{u}}{1, \mathrm{pp}^{\mathrm{u}}-1} \times \frac{1, \mathrm{op}-1}{1,0 \mathrm{p}^{\mathrm{u}} \cdot 0, \mathrm{op}}=\frac{\mathrm{k}}{0, \mathrm{op}} . \\
\text { § } 16 .
\end{gathered}
$$

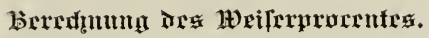

1) Der F̧anptbeftand.

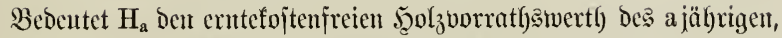
$\mathrm{H}_{\mathrm{a}+\mathrm{n}}$ den bes älteren, $\mathrm{a}+\mathrm{njührigen} \mathrm{Bejtandes,} \mathrm{jo} \mathrm{beträgt} \mathrm{bas}$

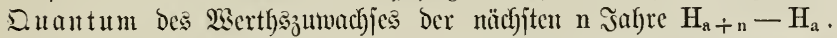
(bichen während bicjes Beitrantes Bornub̧utgen ein, jo jut bieje int entiprechenden Sachwertbe zuzurect)ment. (Fine Durchjorjtung in $\mathrm{m}$ ten Safre, beren ertutefojtenfreier Werth gleich $\mathrm{D}_{\mathrm{m}}$, müroc obigen Wertl) erböben auf

$$
\mathrm{H}_{\mathrm{a}+\mathrm{n}}+\mathrm{D}_{\mathrm{m}} 1,0 \mathrm{p}^{\mathrm{a}} \mathrm{n}-\mathrm{m}-\mathrm{H}_{\mathrm{a}},
$$

wenn ber angenommente $\mathfrak{B i r t l}$ )

Diejes Buwachşquntum ijt eine Durch) bie vorangegangene

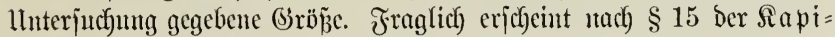

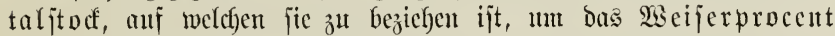

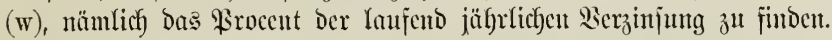

Bezeid)net man dic Summe der in vorigen $\$$ entwicfelten brei

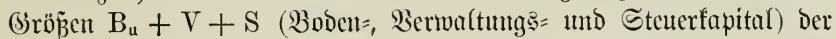
Sïrze wegen mit $\mathrm{g}$, jo repräjentirt bicjes g cinen Sapitalwerth), Dejjen

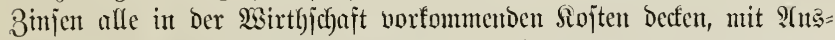

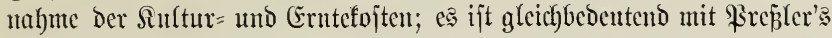
jogenantem Bobenbruttofapitale. - Diejes g min unter allen llmptünoen ben cinen Igeil bes fraglicben Sapitaljtodes bilden. $\mathfrak{B e}=$ züglich des anderen Theiles faun man von verjebiebenen (Sejichts: pututen ausgeben.

A. Will man alle Siojten und Erträge Der Sergangentleit in Siechnung jtellen, jo hat man Dem Bobenbruttofapitale g ben Siojten= werth Des ajüfrigen Bejtundes $\left({ }^{\mathrm{k}} \mathrm{H}_{\mathrm{a}}\right)$ jujurect)nen. Das 2 seijerprocent fintoct jich) Durd bic (sileichung

$$
\mathrm{H}_{\mathrm{a}+\mathrm{n}}+\mathrm{D}_{\mathrm{m}} 1,0 \mathrm{p}^{\mathrm{a}}+\mathrm{n}-\mathrm{m}-\mathrm{H}_{\mathrm{a}}=\left({ }^{\mathrm{k}} \mathrm{H}_{\mathrm{a}}+\mathrm{g}\right)\left(1,0 \mathrm{w}^{\mathrm{n}}-1\right),
$$

bieraนร 


$$
\begin{aligned}
& 1,0 w^{n}=\frac{H_{a+n}+D_{m} 1,0 p^{a+n-m}+{ }^{k} H_{a}-H_{a}+g}{{ }^{k} H_{a}+g} ;
\end{aligned}
$$

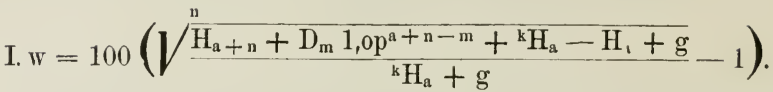

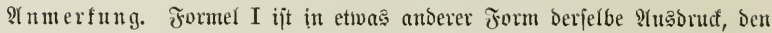

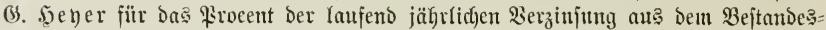

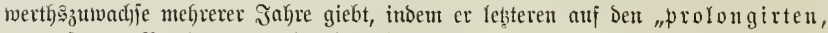
entlajteten Froductionsfonds" beziegt. *)

llm Den Betrag bes Productionsaufwandes zu 2 ufang bes Jafres a zu ermitteln, prolongirt man den im Эafre o vorkandenen \$roductionsfonds $\mathrm{B}+\mathrm{V}+\mathrm{k}$ bis zum Jahre a und zieht von biejem Piadjuerthe bie gleidffalls auf bas Jahr a

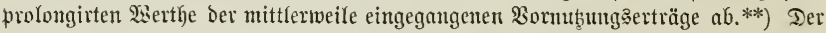
profongirte, entfajtete \$sroductions fonds im Jafre a ijt Giernad)

$$
(B+V+k) 1, o p^{a}-\left(D_{b} 1, o p^{a}-b+D_{q} 1, o p^{a}-q+\ldots .\right) .
$$

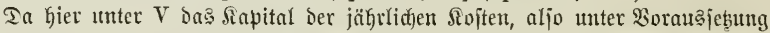
von jährlichen Etenern unjer $\mathrm{V}+\mathrm{S}$ ju verfteben ijt, ba ferner zum 3wecfe ber

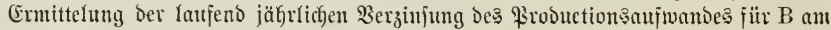

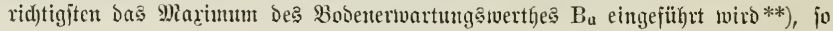
ijt $\mathrm{B}+\mathrm{V}$ gleidf) Dem Bobenbruttofapitale $\mathrm{g}$, weldyen Butdjtaben wir ber Sï̈rze wegen beibehalten.

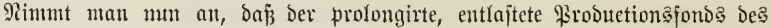

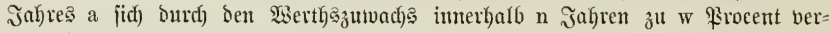
zinit, fo erbält man bie (bleid)ung:

$$
\begin{aligned}
& \mathrm{H}_{\mathrm{a}}+\mathrm{n}+\mathrm{D}_{\mathrm{m}} 1,0 \mathrm{p}^{\mathrm{a}}+\mathrm{n}-\mathrm{m}-\mathrm{H}_{\mathrm{a}}=\left[(\mathrm{g}+\mathrm{k}) 1,0 \mathrm{p}^{\mathrm{a}}-\left(\mathrm{D}_{\mathrm{b}} 1, \mathrm{op}^{\mathrm{a}-\mathrm{b}}+\right.\right. \\
& \left.\left.\mathrm{D}_{\mathrm{q}} 1, \mathrm{op} \mathrm{p}-\mathrm{q}+\ldots .\right)\right]\left(1,0 \mathrm{w}^{\mathrm{a}}-1\right) .
\end{aligned}
$$

Transformirt man bie zweite Ceite ber Bleidjung auf Den Bejtandeșfojten= wertf $\mathrm{kH}_{\mathrm{a}}$, jo erfält man, ba Giera!

$$
{ }^{\mathrm{k}} \mathrm{H}_{\mathrm{a}}=\mathrm{g}\left(1,0 \mathrm{p}^{\mathrm{a}}-1\right)+\mathrm{k} 1,0 \mathrm{p}^{\mathrm{a}}-\left(\mathrm{D}_{\mathrm{b}} 1,0 \mathrm{p}^{\mathrm{a}}-\mathrm{b}+\mathrm{D}_{\mathrm{q}} 1,0 \mathrm{p}^{\mathrm{a}}-\mathrm{q}+\ldots\right),
$$

$$
\begin{gathered}
\mathrm{kH}_{\mathrm{a}}=(\mathrm{g}+\mathrm{k}) 1, \mathrm{op} \mathrm{a}-\left(\mathrm{D}_{\mathrm{b}} 1,0 \mathrm{p}^{\mathrm{a}}-\mathrm{b}+\mathrm{D}_{\mathrm{q}} 1,0 \mathrm{p}^{\mathrm{a}}-\mathrm{q}+\ldots . . \mathrm{)}-\mathrm{g},\right. \\
\mathrm{H}_{\mathrm{a}}+\mathrm{n}+\mathrm{D}_{\mathrm{n}} 1,0 \mathrm{p}^{\mathrm{a}}+\mathrm{n}-\mathrm{m}-\mathrm{H}_{\mathrm{a}}=\left(\mathrm{kH}_{\mathrm{a}}+\mathrm{g}\right)\left(1,0 w^{\mathrm{a}}-1\right) .
\end{gathered}
$$

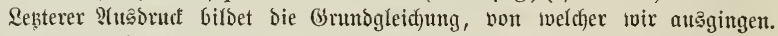
(3). Şet)er entwiffelt daraus***) bie formeln

$$
\begin{gathered}
1,0 w^{n}=\frac{H_{a}+n+\frac{D_{m} 1, o p^{a}+n-m}{k H_{a}+g}+\ldots-H_{a}}{k}+1 ; \\
w=100\left[\left(\frac{H_{a}+n+D_{m} 1, o p^{a}+n-m+\ldots-H_{a}}{k H_{a}+g}+1\right)^{1 / n}-1\right],
\end{gathered}
$$

weldye fid() leidd)t tranşormiren läp̈t in

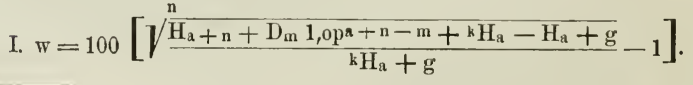

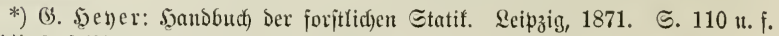

**) Dajerbjt a a. D. S. 16. 24. แ. 34.

***) Dajelbjt S. 112. 


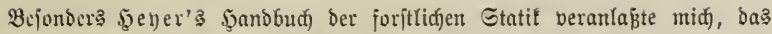
Siseijerprocent anders als in ber 1. Afuflage meiner forjteinrifftung zu befandeln.

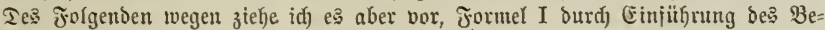
itandstojtenvertfes, anjtatt burdh bie des prolongirten, entlajteten Productions= jonbs zu entwidelur.

B. Berüffïcftigt man bic Siojten unb Erträge Der Bergangenfeit mur in jo weit, als bies zur Berechumg bes eincu wejentlidfen SYn= theil wong hildenden $B_{u}$ notfyendig ijt, fo fat man zutr Entmicfelumg von $w$ in bie Girunogleicfung anjtatt Des Sojtentwerthes Den gegen=

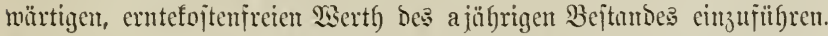

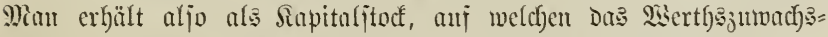
quantum $j^{\text {ul }}$ bejieben ijt, anjtatt ${ }^{\mathrm{k}} \mathrm{H}_{\mathrm{a}}+\mathrm{g}$ den : (jirunogleid)ung:

Gieraus

$$
\mathrm{H}_{\mathrm{a}+\mathrm{n}}+\mathrm{D}_{\mathrm{m}} 1,0 \mathrm{p}^{\mathrm{a}} \mathrm{n}-\mathrm{m}-\mathrm{H}_{\mathrm{a}}=\left(\mathrm{H}_{\mathrm{a}}+\mathrm{g}\right)\left(10 \mathrm{w}^{\mathrm{n}}-1\right),
$$

$$
1,0 w^{n}=\frac{H_{a}+n+D_{m} 1,0 p^{a+n-m}+g}{H_{a}+g}
$$

IIID

$$
\text { II. } w=100\left[\sqrt{\frac{n}{\frac{\mathrm{H}_{a}+n}{}+D_{m} 1,0 p^{a+n-m}+g}}-1\right] \text {. }
$$

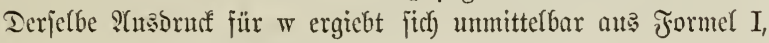
wenn man $\mathrm{H}_{\mathrm{a}}$ für ${ }^{\mathrm{k}} \mathrm{H}_{\mathrm{a}}$ jubjtituirt.

Bur Bejtimmung ber Jiebsreife eines Bejtandes verdient nicht blos aนs Dem Grumbe ber gröperen (Ëmfach)heit Formel II Den Sorzug

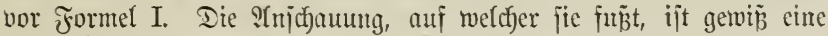

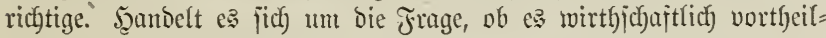
Gajt jei, einen ajüfrigen Bejtand jeb̧t zu fällen, ober benjelben noch n Safyre rachjen zu lajjen, jo giebt bie Stntwort folgende Errögung.

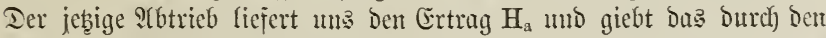
Bejtand gefefielte Bobenfapital für weitere \$roouction frei. Sajjen wir Den Bejtand nod) $n$ ज̌ahre jteben, jo liejert er ben Ertrag $\mathrm{H}_{a}+n$,

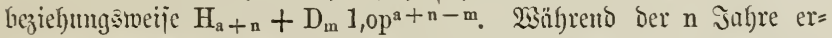

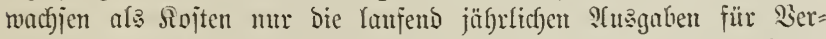
waltung mb Etcuern mo bie nicht bezogene Bobenrente, mit anderen Morten nux bie Bimjen beß Bobenbruttofapitales g. Bermag แm ber

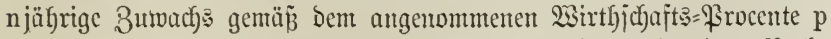

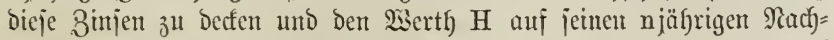
werth zu heben, jo gewährt Der Sabtrieb von $\mathrm{H}_{\mathrm{a}+\mathrm{n}}$ weder Sortheil noc) Nachtheil gegentüber Dem von $\mathrm{H}_{\mathrm{a}}$. Erfolgt Dagegen wäfremb Der

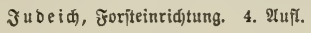


n Safre bic Scrzinjuntg non $\mathrm{g}$ und $\mathrm{H}_{\mathrm{a}}$ z̆ cinem anderen \$rocente $\mathrm{w}$, jo wirb ber âbtrieb bes Bejtandes $\mathrm{H}_{\mathrm{a}}$ gerectiffertigt, fobald $\mathrm{w}<\mathrm{p}$, jo erjecheint bagegen bas weitere Etebenlafjen Des ajährigen Bejtandes finanzmirtbjefaftlich vortbeilfaft, went $w>$ p. - 1 m bic bor bem

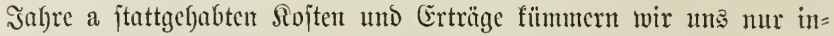
jofern, als twir fie zutr Berectumng von g brauchen. Der $\mathfrak{W e r t h} \mathrm{H}_{\mathrm{a}}$

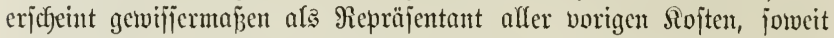

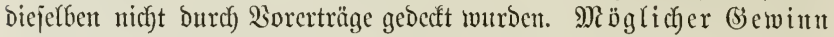
und Berlujt ber bergangenfeit gefören biejer an, werben nicht a uf bic 3 ufunft übertragen.*)

Formel II hat aber gegentiber I nod) Dent weiterent, für Den 3rwedf

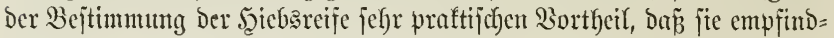
licher ijt. Da mit ?tusnafme bes Sahres ber finanjiellen Reife, in welchem $\mathrm{H}_{\mathrm{u}}={ }^{\mathrm{k}} \mathrm{H}_{\mathrm{u}}$, jtets $\mathrm{H}_{\mathrm{a}}<{ }^{\mathrm{k}} \mathrm{H}_{\mathrm{a}}$, mag $\mathrm{a} \leqslant \mathrm{u}$ jein, gleicfuiel, jo

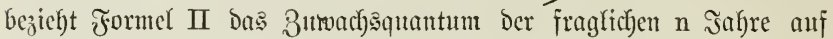
cincen flemeren Sapitaljtocf, als Formel I; fie wird alfo vor und nach) Dem Jafre u bas Procent w itets höber angeben, umb zwar um jo

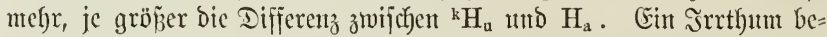
jüglich Der Beftimmung ber Sctebsercife cinte Bejtandes fann Daran nicht exfolgen, weil nach) Dem Jahre u bas nach Formel II berechnete w immerfin bie Gröjpe $p$ nidyt erreicht, wie b. Sectendorff nady)= gewiejen hat.**)

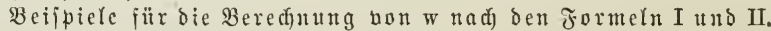

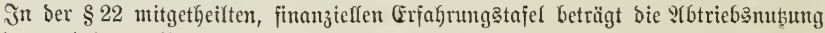
Deక 60 jäbrigen Bejtandes 955,8 fl., Die bes 70 jührigen 1428,9. Jn 65 . Jahre ent=

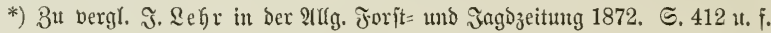
Iort Keibit e? (ธ. 414):

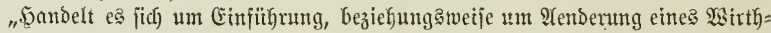

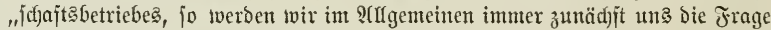
"borlegen, welche Esimahmen werben twit bon jeşt an beziefen und weldhe "Soiten werden wir bon demjelben Moment an atfjuvenden baben. Die

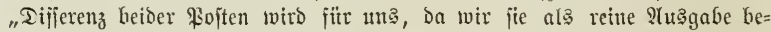
"traçten, ntä́gebend fein. Diejer Differenz gegentïber werben wir Erträge

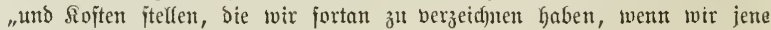

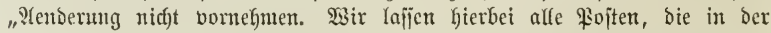
„Bergangenfeit eingegangen fins, voer verauşgabt wurben, ganz unberüct= "jichtigt, und zwar bies mit giedjt."

Dieje ?(nichaumg ijt entichieden bie rixutige.

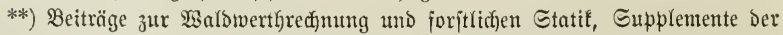

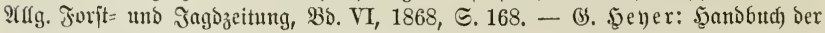
foritticfen Etatif, Qeipzig, 1871, S. 36 uno 124. 


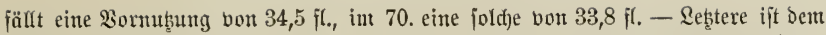

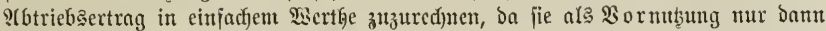

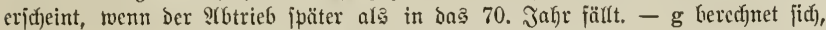
wenn Das barin enthaltene B bem Marimun des Bobentwertfes entipredicn joll, aljo

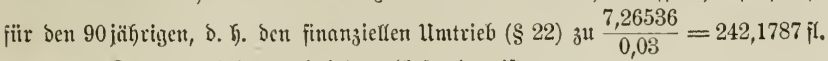

Der Sojtenwerth Des 60 jäfrigen Bejtandes iit

$$
\mathrm{bH}_{\mathrm{j} 0}=242,1787\left(1,03^{60}-1\right)+30 \times 1,03^{60}-223,5792=1137,8097 \text {. }
$$

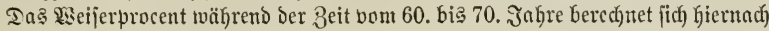
mit Fonel I:

$$
w=100\left[\sqrt{\frac{1428,9+33,8+34,5 \times 1,03^{5}+1137,8097-955,8+242,1787}{1137,8097+242,1787}-1}-1\right]=
$$

mit Jormel II:

$$
\mathrm{w}=100\left[\sqrt{\frac{10}{1428,9+33,8+34,5 \times 1,03^{5}+242,1787}}-1\right]=3,832 .
$$

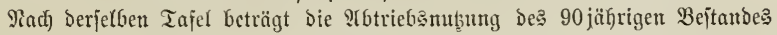
2875 fl., Die bes 100 jäfrigen 3780 fl. Sorerträge fallen in Der Beit vom 90 . biß 100. Jahre nid)t mebr aน3. Das g bleibt wie im vorigen beippiele 242,1787. Ia Der fittanzielle lumtrieb in Das 90. Jahr fälft, fo entipridyt Der Y(btriebsertrag

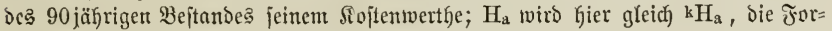
metr I und II werben baher gleichlautend.

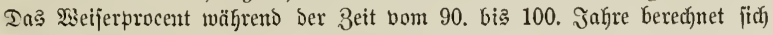
Giernad):

$$
\mathrm{w}=100\left[\sqrt{\frac{10}{2880+242,1787}}-1\right]=2,582 .
$$

Bur Erjparung Der logaritfmijichen Siecfunnt, namentlicf) aber audf) Deshalb, um bas פeijerprocent Yeicht ans jeinen Eementen be=

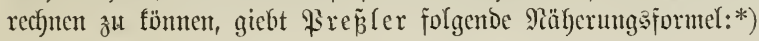

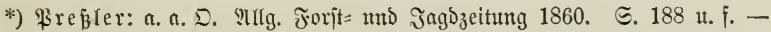

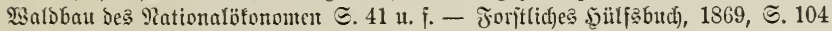

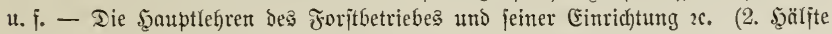

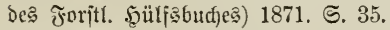

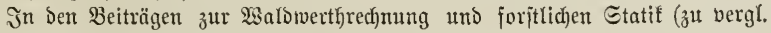

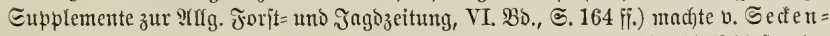

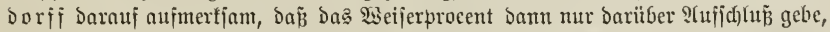

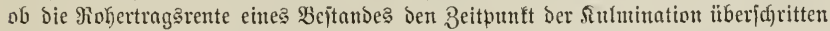
ober Denfelben noch nidjt erreid)t fabe, wenn man nid)t $B_{u}$, fondern einten ver=

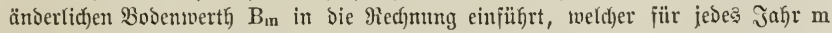

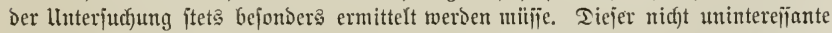

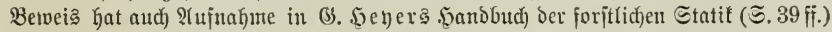

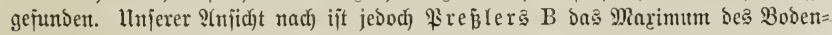
erwartuntgemertfes, aljo nidfts ?(nderes als unjer $B_{u}$. - Eeţit man nun in ber

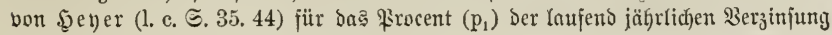




\section{III. $\quad \mathrm{w}=(\mathrm{a}+\mathrm{b} \pm \mathrm{c}) \frac{\mathrm{H}}{\mathrm{H}+\mathrm{G}}$.}

(5) bedeuten fier $a, b$ und $\mathrm{c}$ bie befannten Buwadjeprocente (§§ 10-14), H Den mittleren Bejtandswerth), arjo $\frac{H_{a}+n+H_{a}}{2}$, Doer unter Boransjeţung von ßoverträgen $\mathrm{D}_{\mathrm{m}}$ im Jahre $\mathrm{m}$ während bes nächjten njäbrigen Beitraumes $\frac{\mathrm{H}_{\mathrm{a}+\mathrm{n}}+\mathrm{D}_{\mathrm{m}} 1,0 \mathrm{p}^{\mathrm{a}+\mathrm{n}-\mathrm{m}}+\mathrm{H}_{\mathrm{a}}}{2}$, culo=

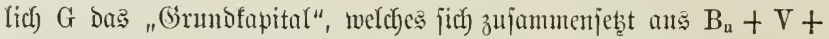
$\mathrm{S}+\mathrm{C}$ (§ 15: 1., 2., 3. и. 4.).

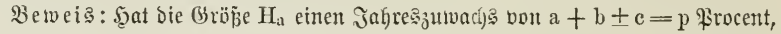

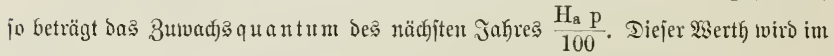

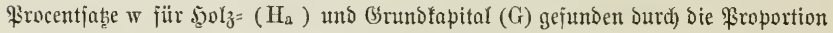

Gieraus

$$
\left(\mathrm{H}_{\mathrm{a}}+\mathrm{G}\right): 100=\frac{\mathrm{H}_{\mathrm{a}} \mathrm{p}}{100}: \mathrm{w},
$$

$$
\mathrm{w}=\mathrm{p} \frac{\mathrm{H}_{\mathrm{a}}}{\mathrm{H}_{\mathrm{a}}+\mathrm{G}} .
$$

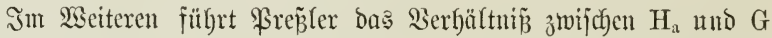

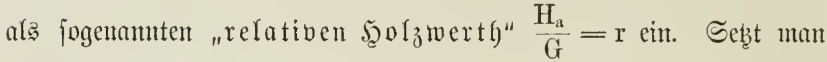
Giernach $\mathrm{H}_{\mathrm{a}}=\mathrm{rG}$, jo berwandelt fich obiger ?nesorutef in

$$
w=p \frac{r}{r+1} .
$$

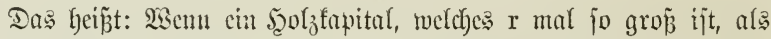

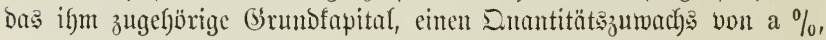

Des Froductionsanfwantos gegebenen Jorntel anjtatt bes fioitentwerthes des $\mathfrak{B}=$

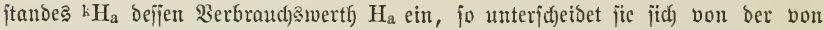

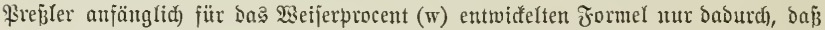

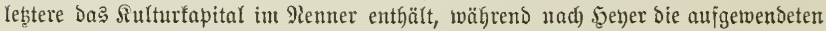
Siulturfojten als in Den 3 ç̄tandşwerth übergegangen gedad)t werden.

Ceţt man ben in Frage fommenden Beitraum $\mathrm{n}=1$, fo lautet

$$
\text { 5eyer's formel: } \quad \mathrm{pl}_{\mathrm{L}}=\frac{\left(\mathrm{H}_{\mathrm{a}}+1-\mathrm{H}_{\mathrm{a}}\right) 100}{\mathrm{H}_{\mathrm{a}}+\mathrm{B}_{\mathrm{u}}+\mathrm{V}} \text {, }
$$

Preṕler's Formel: $w=100\left(\frac{H_{a}+1+G}{H_{a}+G}-1\right)=\frac{\left(H_{a}+1-H_{a}\right) 100}{H_{a}+G}$.

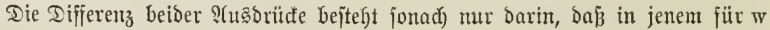
ber Sienter um Den Betrag des fiulturfapitales C größzer ijt, als in Dem für p1,

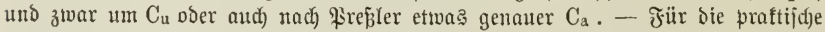
Anwendung ijt Dieje Frage ganz ofue Bebeutung, bom Bejichtspunfte Der Sijien: idchaft berbient fie jebod) Beachtung. 


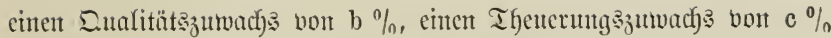

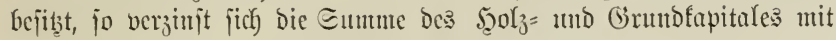
einem Procentiabe von $(a+b \pm c) \frac{r}{r+1}$.

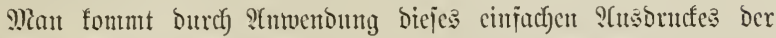

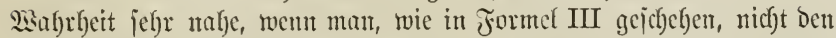

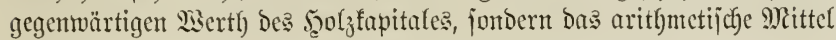
an Diejem uno bem fünftigen Bejtandswerth einjest, aljo $\frac{\mathrm{H}_{\mathrm{a}}+\mathrm{n}+\mathrm{H}_{\mathrm{a}}}{2}$,

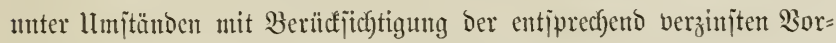
erträge. Der relative jeoljwertf) $r$ wird baum nicht $\frac{H_{a}}{G}$, jondern

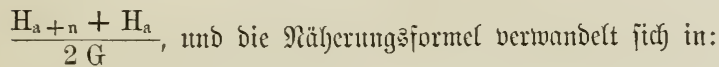

$$
\mathrm{w}=(\mathrm{a}+\mathrm{b} \pm \mathrm{c}) \frac{\mathrm{H}_{\mathrm{a}+\mathrm{n}}+\mathrm{H}_{\mathrm{a}}}{\mathrm{H}_{\mathrm{a}+\mathrm{n}}+\mathrm{H}_{\mathrm{a}}+2 \mathrm{G}}
$$

ober, went wir ben mittleren 50 ofjwerth mit $\mathrm{H}$ bezeichnen:

$$
\text { III. } \quad \mathrm{w}=(\mathrm{a}+\mathrm{b} \pm \mathrm{c}) \frac{\mathrm{H}}{\mathrm{H}+\mathrm{G}} \text {. }
$$

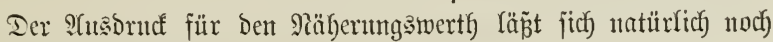
it verichiedenten Formen gebent.

Эe unbedcutender, wenigitens beim sechwaldobetriebe mit werth=

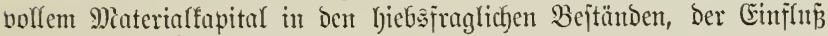
bes (S) jummarijchen ?ä̈)erungşwertbe bafür begnügen. Dicjer wirb am ein= fachjten gefunden, went man Den erntefoitenjreicn Gicjummtertrag bes ungçäl)r Dem fintanjiellen Untrieb entiprechenden Bejtandos als Enó zins Des gejuchten (Sinntofapitales betrachtet, biejes fonad) berectunet als Den Duptienten aus $1,0 \mathrm{p}^{\mathrm{u}}-1$ in Den Bejammtertrag.

Taล $w$ im obigen Bahlenbeifpiele für ben 60 jägrigen Bejtand bejtimmt fiđ nad) Frormel III in folgender (śröß̈e:

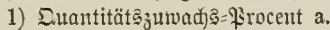

$$
a=\frac{433+13+15-354}{433+13+15+354} \times \frac{200}{10}=2,63 .
$$

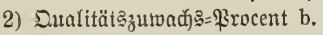

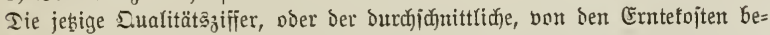
freite Cintheitąpreis für ben 60 jährigen $\mathfrak{B}$ ejtand beträgt 2,7 †l.

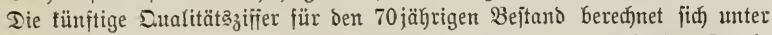

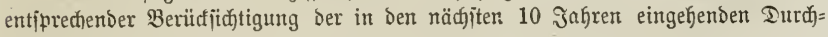
foritungäerträge auf

$$
\frac{443 \times 3,3+13 \times 2,6+15 \times 2,3 \times 1,03^{5}}{433+13+15}=3,26 ;
$$


hiernach

$$
\mathrm{b}=\frac{3,26-2,7}{3,26+2,7} \times \frac{200}{10}=1,88 .
$$

3) Das dom fintanzieffen ltmtrieb entfpredgende Ostundfapital $G_{90}$ ijt

$$
\frac{3650,1038}{1,03^{90}-1}=274,43 \text {. }
$$

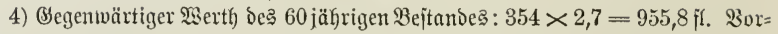

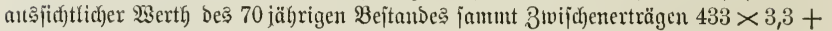
$13 \times 2,6+15 \times 2,3 \times 1,03^{5}=1502,7 \mathrm{fl}$.

(onad) $\mathrm{H}=\frac{955,8+1502,7}{2}=1229,25 \mathrm{fl}$.

5) Şieraug গ্Seijerprocent:

$$
\mathrm{w}=(2,63+1,88) \frac{1229,25}{1229,25+274,43}=4,51 \times 0,82=3,698 .
$$

Für dic getwöbulich vorfommenoc ?tnwenoung genügt offenbar Formel III. Fïn längcre als 10 jüfrrige Beiträume, ntumentlich beim

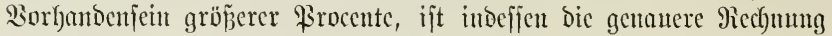
nach Formel II vorzuzichen. Sebetere wurbe auth in ber als $\Omega(f) r=$ beifpiel cutwicfelten finanziellen Iafel $(\$ 22)$ angewendet. Formel I bat nur Bedoutung für manche recin wifjenjobaftliche llnterjudyungen.

\section{2) Der 3wijedenbejtand.}

Der Bwifchenbejtand für fich betrad)tet verbält fich als wirtf)=

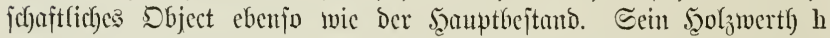
wächjt z" nach) Dutantität mo Dualität, aljo mit sincm a uno b, unter Umitünocn auch mit cinem e \$rocent. Dicje \$rocente fömen ganz

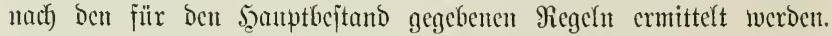
Eine andere Jrage ijt bie nach feinem 2 Seijerprocente w.

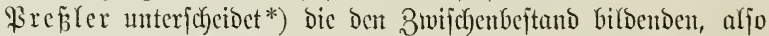

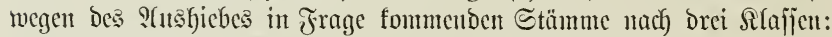

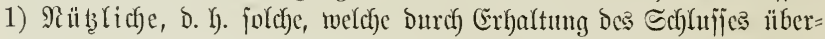

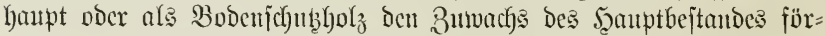
bern. 2) Bifeidggiltige Bäume, Deren Stebenlajien zumächjt ebenjo

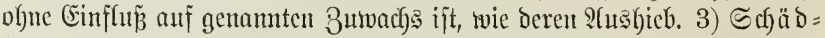
riche Bäume, weldye den Zuwachs des Scauptbejtandes benadytheifigen.

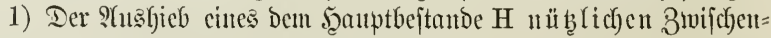
bejtandes h wird fajt nie in Frage fommen fömten, ba felbjt ein fleiner

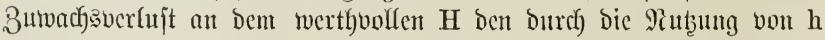
ju erziclenden Bortheil jtets ïberwiegt.

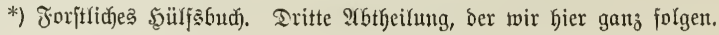




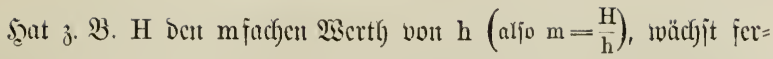

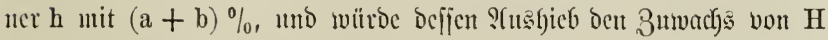

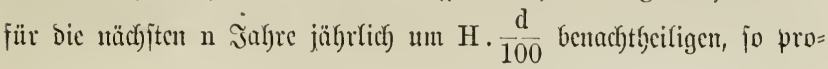
Ducirt bas itchenbleibento h jäbrfich h $\frac{a+b}{100}+H \cdot \frac{d}{100}$. Das $2 s c c=$ jerprocent $w$ für h fintoct jich) mun ans ber (b́ceidfung

bicratts

$$
\text { h. } 0,0 \mathrm{w}=\mathrm{h} \frac{\mathrm{a}+\mathrm{b}}{100}+\mathrm{H} \cdot \frac{\mathrm{d}}{100} \text {; }
$$

voer nutd)

$$
\begin{gathered}
w=(a+b)+\frac{H}{h} \cdot d, \\
w=(a+b)+d m .
\end{gathered}
$$

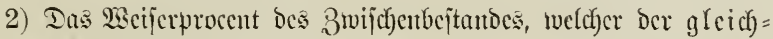
giltigen Silajie autgchört, ijt gleich feinen $a+b$, benn inbem er fein d Gewirft, wirb für ifn biejes $d=0$. Demnact)

$$
\mathrm{w}=\mathrm{a}+\mathrm{b} .
$$

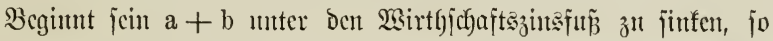
ijt cr um jo entichicbencr hicbsreif, als erjtens bicjes Sinfen gcwöbnt=

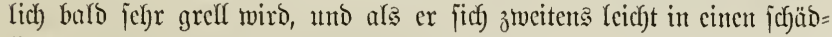
lidfen 3rwijchenbeitano verwandelt.

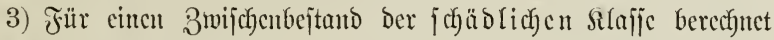

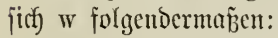

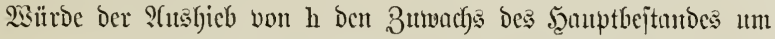

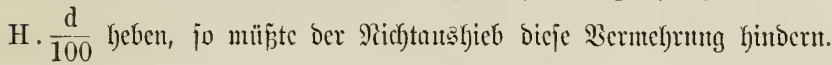

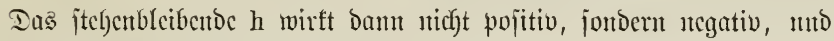
jein w fintoet fich atts ber (bileichung

Gictans

$$
\mathrm{h} \cdot 0, \text { ow }=\mathrm{h} \cdot \frac{\mathrm{a}+\mathrm{b}}{100}-\mathrm{H} \cdot \frac{\mathrm{d}}{100} \text {; }
$$

voer audf)

$$
w=(a+b)-\frac{H}{h} \cdot d,
$$

$$
w=(a+b)-d m \text {. }
$$

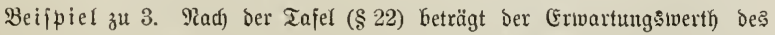
30 jäfrigen Şauptbejtandes in runder $3 a h \mathfrak{l} 400$ fl. Die Entnahue bes vorhandenen, 7,2 Fl, wserth Kaltenden 3 wij

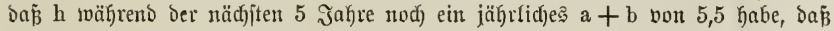

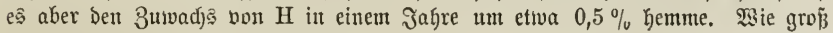
ijt bas seijerprocent bes h? 


$$
\mathrm{w}=\mathrm{a}+\mathrm{b}-\frac{\mathrm{H}}{\mathrm{h}} \cdot \mathrm{d}=5,5-\frac{400}{7,2} \cdot 0,5=-22 \% .
$$

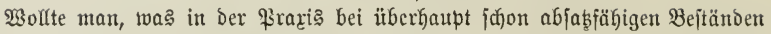

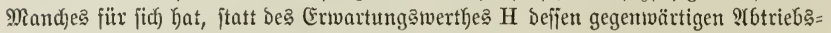

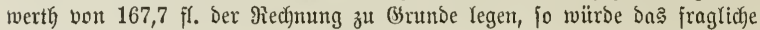

$$
\mathrm{w}=5,5-\frac{167,7}{7,2} \cdot 0,5=-7 \% \text {. }
$$

Die Durdfforftung toäre in foldeem Falfe natürlid) bringent geredtfertigt.

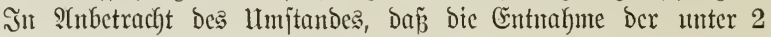

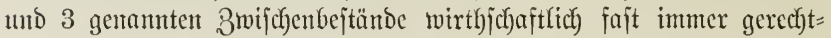

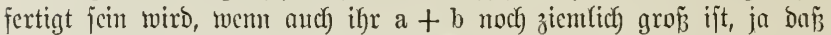
für bie britte, juäblidye Slafje Der SAtsficb als Sultumajregel oft

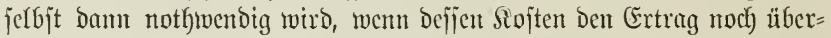
jchreiten, glauben wir uns bier mit biejen Slndeutungen begnügen zu

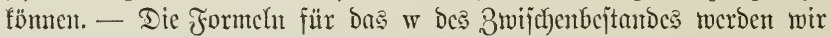

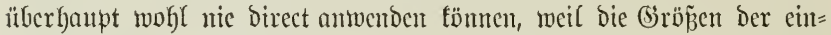
zelnen Factoren, namentlich bie bes d, nicht bejtimmbar finto; immerfin

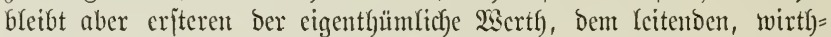

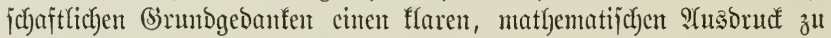
gebent.

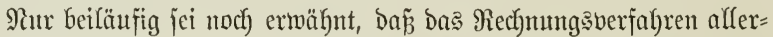

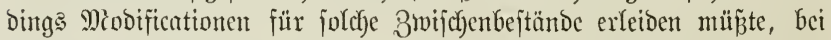
welchen bejondere Siojten zum Zwecte ifrer Erzichung aufgemendet

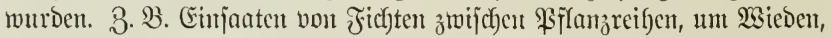

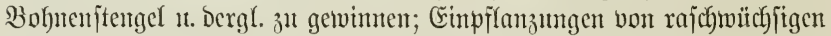
Birfen, Rärchen, lebiglich zum 3wecte zeitigen Sorertrages u. j. tw. In foldhen Fällen repräjentirt ber Bwijenentocitand cute fleine, fajt jelbjt=

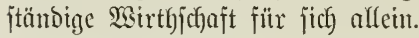

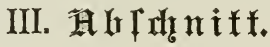

\section{Der Intricb.}

\section{$\S 17$.}

\section{Begriff.}

Ilnter IImtriebajeit, furz llmtrieb (Turnus), verjtelyt muแ jencn 3citraum, weldyer von ber Begründung eines Bejtandes bis zu

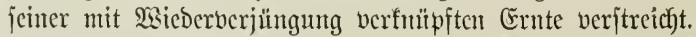

Das Ende diejes Zeitraumes, aljo bas Ilter des Bejtandes bei jeirtem

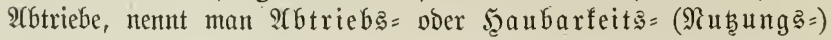


Scrter. Stimmt bicjes mit jencm überein, welches für bie Bmecfe der Wirthjochaft als bas vortbeilfaftejte erfannt worben ijt, jo ijt es ein normales, bagegen eit abnormes stbtricbs= ober 5̧aubarfeitsalter,

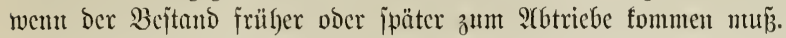

Q(bnormitätu treten ein burd) ftörende EYementarereignifije, Jnjectenfraß̄,

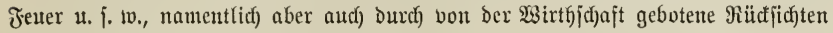
anf bie Şiebsfolge.

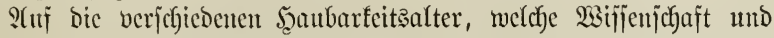
Praris unterjcheiden, ftüben jich verjecjiedene Umtriebc.

Die Şanbarfeitsalter find:

1) Das phyjifdxe,

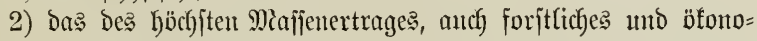
mijches genaunt,

3) bas techniijche,

4) Das ber lörchiten Maldorente,

5) Das finanjielle.

Sebem biejer Scaubarfeitsalter entipricht ein bejtimmter Untrieb.

\section{$\S 18$.}

\section{Dex phruglifrte Hmfrich.}

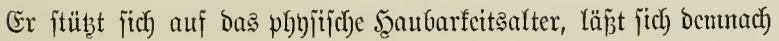
in ziwei Itnterarten jheiden:

a) Derjenige llmtrieb, weldfer für bie untürliche $\mathfrak{X}$ icderverjüng= ung einer \$̧olzart unter gewififen Standortsocrbältnifjen und für bejtummte. Betriebsjojteme geeignet ijt.

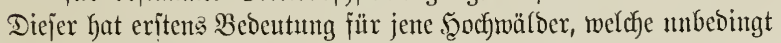
mit natürlicher Borverjüngung befandelt merben müjīen, weil ex nicht nieoriger gewäblt werben barf̆, als biş die Bäume guten unto reichlichen 巨amen zut tragen im Gtande jund. - Se mefrr wir im Wirtfjjchaftęmalde ïberfautpt zur fünjtlichen Serjüntgutg unjere $8 \mathfrak{u}=$

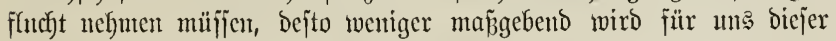
llmtrieb jein fönten.

3meitens fommt er in Betracht für ben 2iederwald, jo wie für

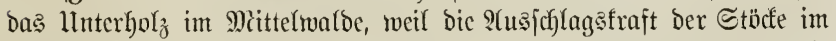
höheren Sflter geringer wirb, und wir bei bicjen Betriebsarten auf bie

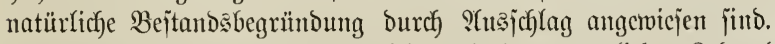

b) Derjenige llmtrieb, weldfer mit ber natürlichen Rebentsouter Der Bäume zujammenfällt. 


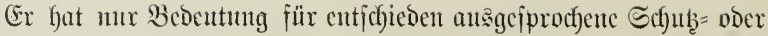

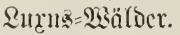

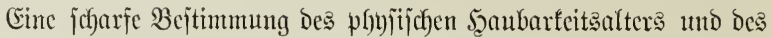
anf ifym berufenden llmtriebes ift nicht möglich).

\section{$\S 19$.}

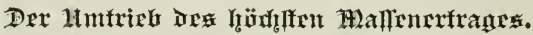

(Er ijt jener, weldfer jidc) anf Das forjtfiche oder öfonomijache Şau=

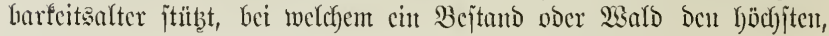

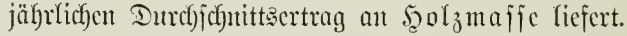

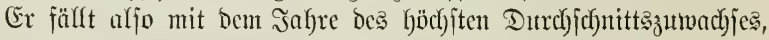

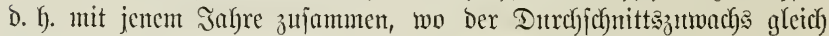
bem lanfenton wird, uto fant um cintige Juhgre bifferiren, je nad jocm mant mut Den Scauptertrag oder Den Sejammtertrag Der Siechnung jut Girunto legt.

Diejem-Ilmtrieb ijt lange 3 cit won Den meijten Forjtlenten ein bejonderer 23erth beigelegt worden, weil man yon Dem Brumbjabe ansiging, ę jei nothwendig, auf ber fleinjten Fläche Den möglichjt

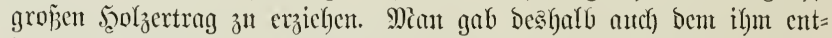

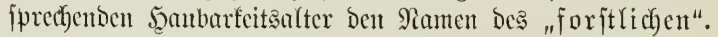

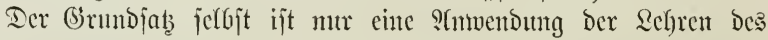

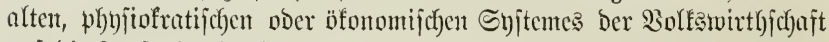

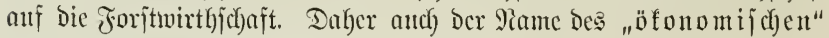
şanbarfeitsalters.

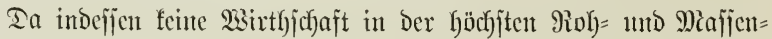

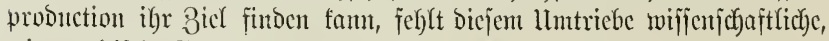
wic praftifche Beredftigutng. Er ift ciut Simb veralteter Ifycorien.

Mit Scilfe guter, loculer Erfalgruttgstafeln läß̈t fitch berjelbe für

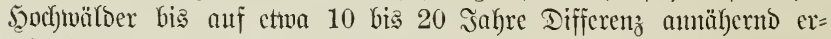

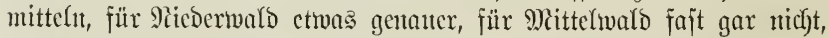

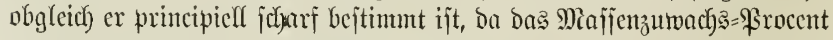
im Jafre bicjes llmtriches gentau anf $\frac{100}{\mathrm{u}}$ oder $\frac{100+v}{\mathrm{u}}$ herabgejun= fen ijt. (§ 10). - Er ijt von alfent llmtricben ber ant wenigften veründerlicbc.

\section{$\S 20$.}

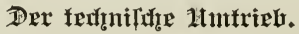

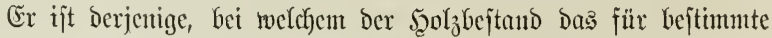
3ructe Der Berwentoung gecignetite Material licfert. 


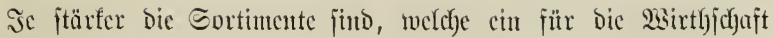

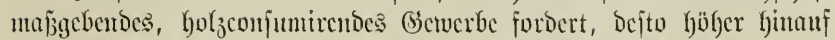

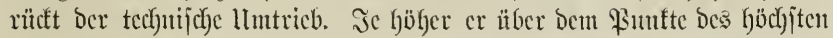

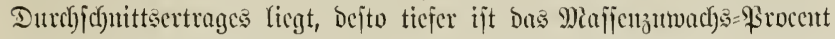

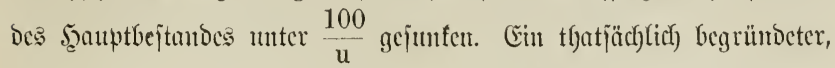

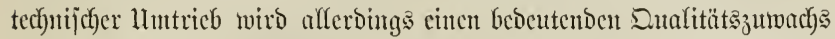

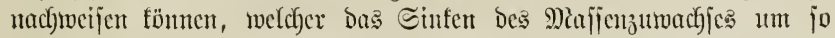

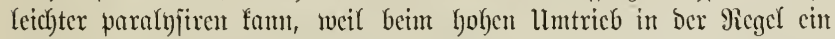

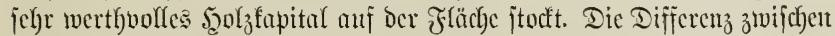

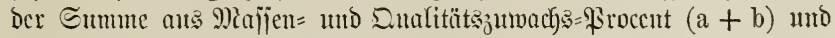

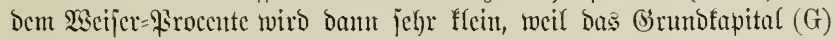

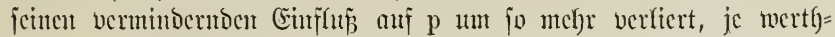
voller das $\mathfrak{S c}_{\text {olz }}$ fapital ijt.

Der teclunijede llmtrieb ijt forjtlicl) volljtämbig gerect)tfertigt, jobald

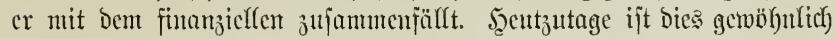

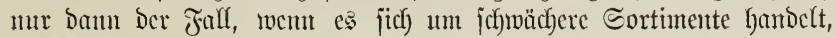

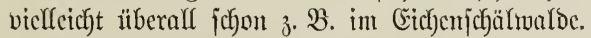

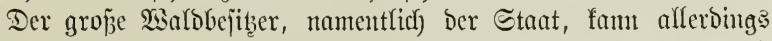

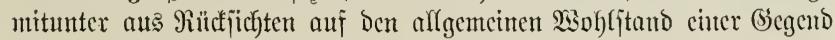
einen joldjen tedfnijchen limtrieb nod) fejtfalten milfien, welder mur

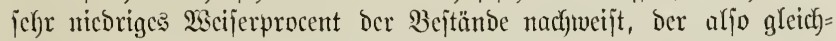

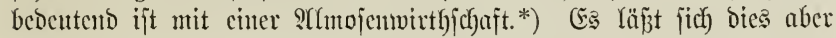

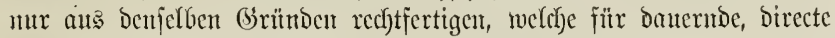

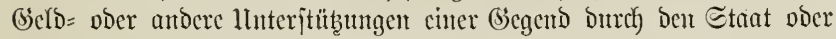

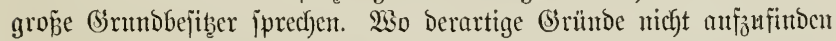
find, fechlt bicjent technijichen IIntricbe bic wirthjedfaftlicbe Beredjtigung.

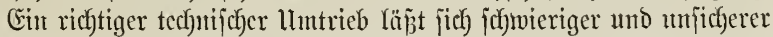
crmitteln, als Der bes hödjiten Mafjencrtrages. Dicje Echlvierigfeit

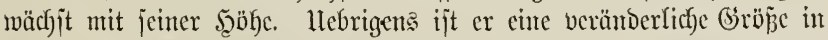

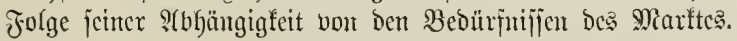

\section{$\S 21$.

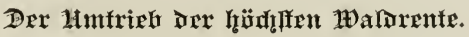

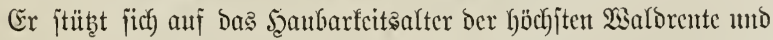
bedeutet jenen, bei weldyem der Bejtand poer $\mathfrak{B a l d}$ ben nach arity= metijchem Durchjechnitte berechneten, Göchjten Beldertrag licfert.

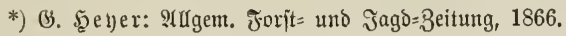




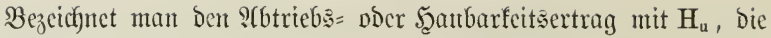
im aten, bten u. j. w. Jahre eingehentoen 3wijchentutbungen mit $\mathrm{D}_{\mathrm{a}}$,

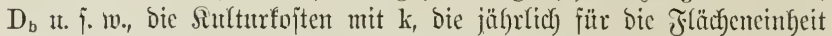
zu zalflenden Berwaltungsfojten und Eteutern mit $\mathrm{v}$ und $\mathrm{s}$, Den $\mathfrak{l} \mathrm{m}=$ tricb mit u, jo bercchnet jich Der jäbrliche, aritgmetijche Dutrefjichnitte= ertrag bes Bejtandes Durch) Den ?Atsorud

$$
\frac{\mathrm{H}_{\mathrm{u}}+\mathrm{D}_{\mathrm{a}}+\mathrm{D}_{\mathrm{b}} \ldots \ldots-\mathrm{k}-(\mathrm{v}+\mathrm{s}) \mathrm{u}}{\mathrm{u}} .
$$

Denft man fich cinen normalen $\mathfrak{B a l d}$ im ftrengen Nachfalts:

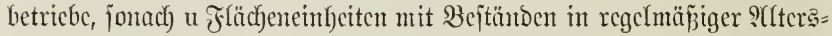

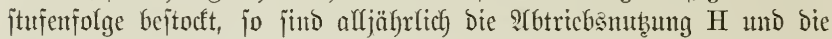
betreffenden 3wijchennubungen fällig, alljälyrlich ijt ber cinmalige $\mathfrak{B} e=$ trag Der Sulturfoiten, Der Serwaltungšfojten und Steuern zu zahlen,

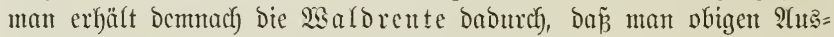

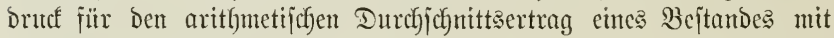
u multiplicirt, folglich

$$
\mathfrak{W a l b r e n t e}=\mathrm{H}_{\mathrm{u}}+\mathrm{D}_{\mathrm{a}}+\mathrm{D}_{\mathrm{b}} \ldots-\mathrm{k}-(\mathrm{v}+\mathrm{s}) \mathrm{u} .
$$

Sener IImtrieb Daher, bei weldyem ber Durdjichnittsertrag bes

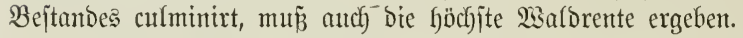

Beifpiel: (Fin Bejtand erfordent 30 fl. Sutturfojten, jähryich 3 fl. Berival=

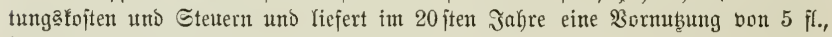
int 40 ften eine foldhe von 20 fl., in 60 jten einen 2 btriebsertrag bon 800 fl., fo ijt jein jägrliçer, jogenaunter Iutrd)idnnittäertrag:

$$
\frac{5+20+800-30-(3 \times 60)}{60}=10,25 \mathrm{fl} \text {. }
$$

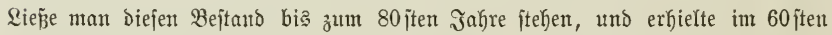

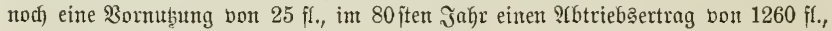

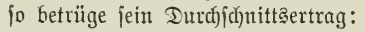

$$
\frac{5+20+25+1260-30-(3 \times 80)}{80}=13 \mathrm{fr}
$$

Ter 80 jährige 4 matrieb wäre Giernad Der vortheilfhaftere.

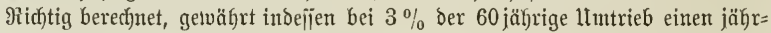
lidgen Bodenreinertrag (Bobenrente) von:

Der 80 jährige nur

$$
\frac{5 \times 1,03^{60-20}+20 \times 1,03^{60-40}+800-30 \times 1,03^{60}}{\frac{1,03^{60}-1}{0,03}}-3=1,1439 \text { fl., }
$$

$\frac{5 \times 1,03^{80-20}+20 \times 1,03^{80-40}+25 \times 1,03^{80-60}+1260-30 \times 1,03^{90}}{\frac{1,03^{80}-1}{0,03}}-3=0,3626$. 
Mit anberen Sisorten, bem 60 jährigen 1 mtrieb entjpridgt ein forjtwirthjdaft= Iicher ober Bodenerwartungşuerth von $\frac{1,1439}{0,03}=38,13$ fl., bem 80 jäfrigen bagegen mtre ein foldjer von $\frac{0,3626}{0,03}=12,09 \mathrm{fl}$. Die Sache verbält fict demunady ganz anderz, als bie Pied)unng nad) Dem aritfmetijchen Dutdjichnitte Yeyrt.

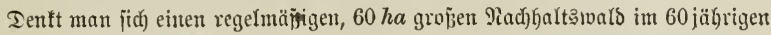

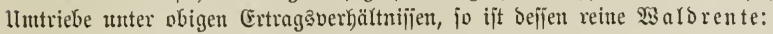

$$
5+20+800-30-(3 \times 60)=615 \mathrm{fl}_{1,}
$$

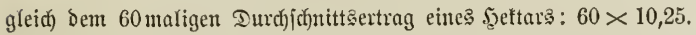

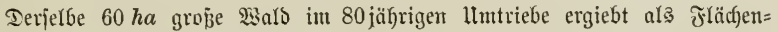
cinheit für einen Bejtanto $0,76 h a$, es entfalfen bager jührlida) 0,75 ber oben aut= gegebenten $\mathfrak{i o j t e n ~ u n b ~ E r t r a ̈ g e : ~}$

$$
\begin{aligned}
& \text { כägrlidce crtträge: }(5+20+25+1260) 0,75=982,5 \text { ¡l. } \\
& \text { Jährliche Riojter: }(30+3 \times 80) 0,75=202,5 \text { " } \\
& \text { Maforente: } \quad=780,0 \mathrm{fi} \text {. }
\end{aligned}
$$

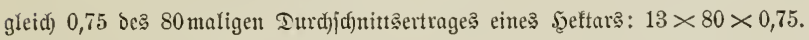

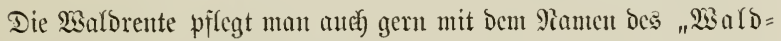
reincrtrages" zu bezcidfuten, weil fie gleich jencr Größze ijt, bie

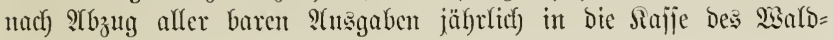
bcütberes fließ̃t.

Die Differenz obiger Aicjultate, weldye für Den 80 jäfrigen, gegen=

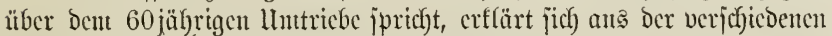

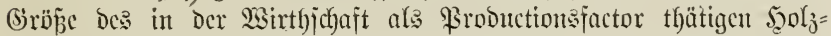

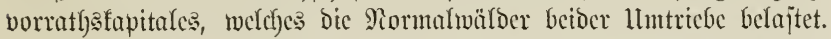

Cinto alle baren ?ltelagen vom Bruttocrtage des MBaldes in P(bred)untg gebrad)t, twie es bei Ermittedung ber Waldorente gejchicht, jo fan jich bicjelbe unr aus ben Binjen bes Boben= und Borratges= fapitales znjaumenjeben, went man cinen bejonderen llnternelymer=

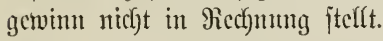

Die Differenz zwijhyen $\mathfrak{B a l d}=$ und Bodemente mun aljo gleid) jeit Den Bins Des jeolzoorrathsfapitales.

Fitr ben 60 jäfrigen ltmtrieb unjeres Sehrbeijpieles:

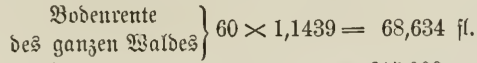

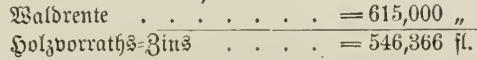

$$
\begin{aligned}
& \text { Şol } \left.3 \text { vortattą-Siapital } \frac{546,366}{0,03}\right\}=18212 \text { " }
\end{aligned}
$$




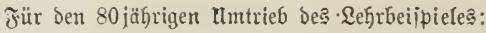

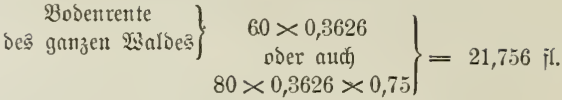

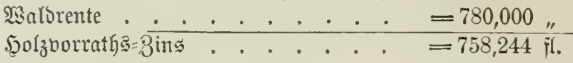

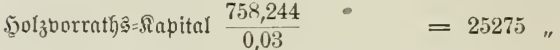

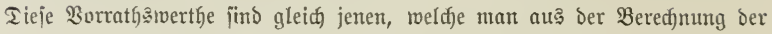
Siojten= und Errvartungşwertfe jänmtlidfer Beitänoc ergält (\$ 34).

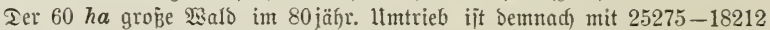

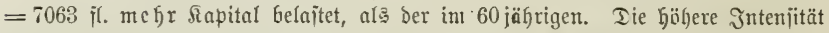
Der Wirtfjidajt des eriteren rujt zmar einen größ̈eren Gejamntertrag Gerbor, ber= mindert bagegen Den twirffidfen Rientertrag.

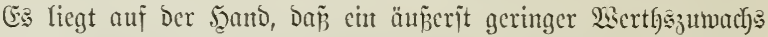

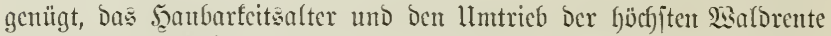
jefr hoch Ginauj zu jchieben, wem Der Majien=Durchjichnittšjuwachs nicht jefon jtarf im Einfen iit.

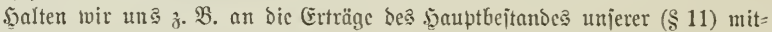

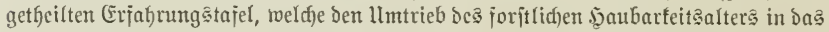

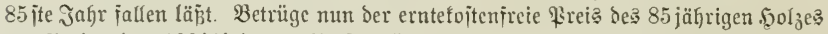

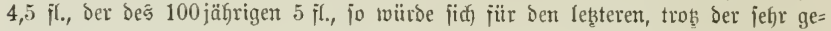

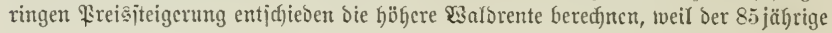

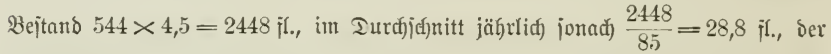

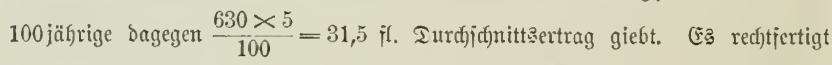

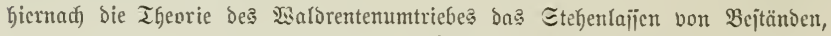

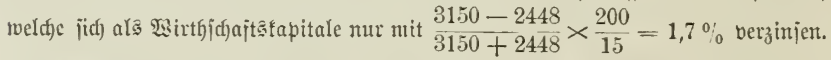

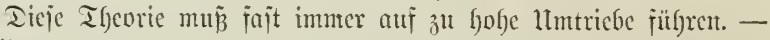

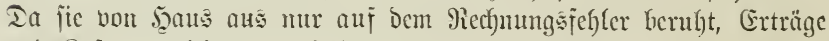
und Siojten, welche zu verjujiedenen Beiten jüllig jünd, cinfach) zu jum=

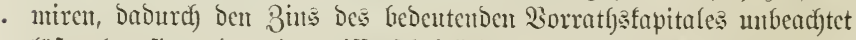

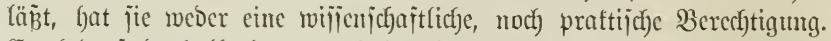

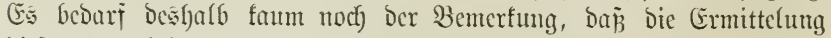

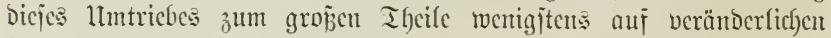
Factoren, nämlich) ben Şolzpreijen beruft, bafer er jelbjt eine ver= änocrliche, übrigen nicht jicher zu bejtimmende (şrößje ijt.

\section{$\S 22$.}

\section{Der funantielle Hutrich.}

llnter füanjicllem limtriebe verjteben wir jencu, bei weldfem ber 


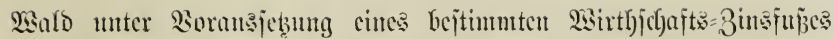

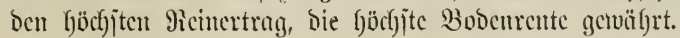

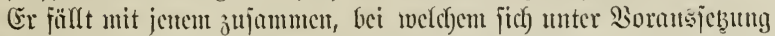

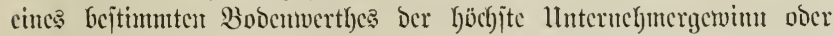

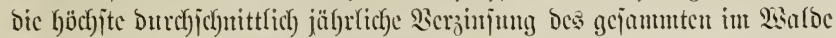
thätigen Probuctionsfonds bered)net.*)

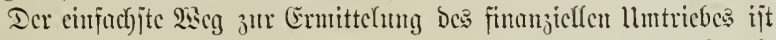

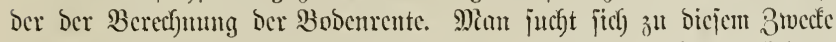

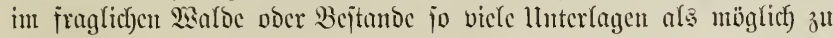
verjefaffen, unb heredfnet bum bic Bobenrente jür verjeficbente llmtricbe.

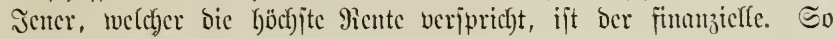

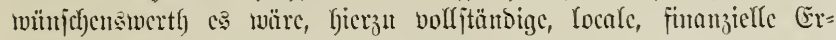
falyrungstajeln zu bejizen, jo ijt bies boch namentlicf) für höbere llm= tricbe bei ber Berünocrlichfeit ber Factoren nicht möylich. Dic 9 (n=

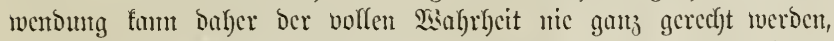

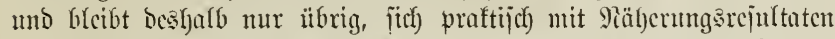

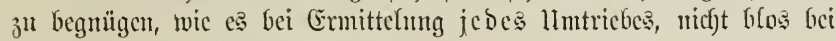
Dex Des finatjicllen, gcj(d)

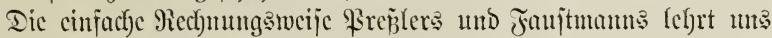
dic Bobentente $r$ Durch) Den ?(mosond füben:

$\mathrm{r}=\left(\mathrm{H}_{\mathrm{u}}+\mathrm{D}_{\mathrm{a}} 1,0 \mathrm{p}^{\mathrm{u}-\mathrm{a}}+\mathrm{D}_{\mathrm{b}} 1,0 \mathrm{p}^{\mathrm{u}-\mathrm{b}} \ldots \ldots . . \mathrm{k} 1,0 \mathrm{p}^{\mathrm{n}}\right): \frac{1, o \mathrm{p}^{\mathrm{n}}-1}{0,0 \mathrm{p}}-(\mathrm{v}+\mathrm{s})$.

(Es bedenten Gier bie Sufhjtaben:

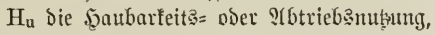

$\mathrm{D}_{\mathrm{a}}, \mathrm{D}_{\mathrm{b}}$ Borutķungen im aten, bten t. $\hat{j}$. w. Jafre,

- k Die Rulturtoiten,

$\checkmark$ bie jährlidjen Berwaltungšłojten,

$s$ bie jährlidjen Eteuern,

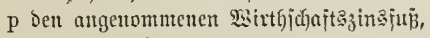

u den Ilmtrieb.

Errgänzen wir bic Ecite 28 als \&efyrbcipicl mitgetfjeilte Erfafrung: tajel zu einer futanjiellen burch Eimjeb̧ung ber betreffenton Siojten mo Erträge, und jwar jo, Daj bic 5 goljpreije Den crntefojtenfreicn Betrag im Iurchjefyntt aller Eortimente bedenter, jo crlfalten wix unter ber

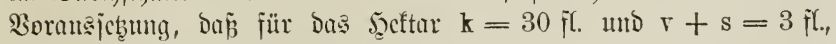
$p=3$ jei, folgento 9icjultate:

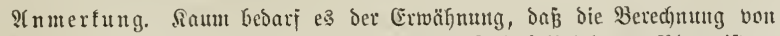

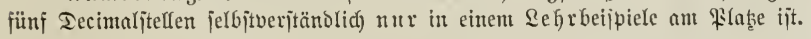

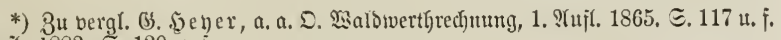

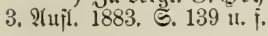









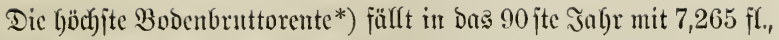
cbendahin bic hör(f)ite Bodenrente mit 7,265-3=4,265.

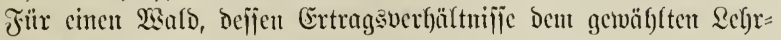

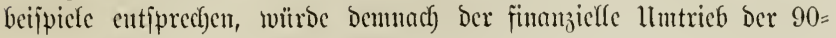
jülrige jeits.

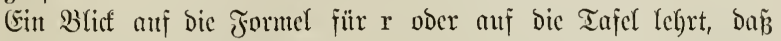

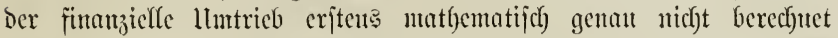

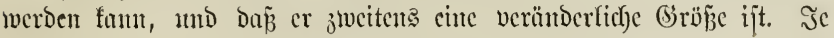

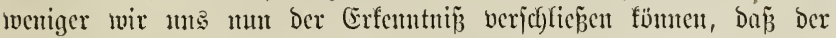

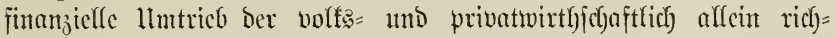

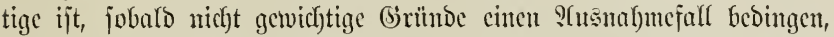

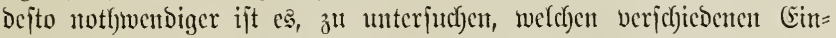
fluz auf bie Şöhe Diejes llumtricbes bic cinzelnen Factoren nefmen.**) Eeçtere (afjen jich) in jwei Şauptabtheilungen bringen:
1) Dic Sivitut,
2) Dic Crrträge.

Dic Soiten zerfallen ifyerjeits in zwei lluterabthethungen:

a) Siapitaljins,

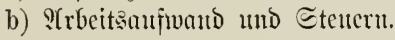

Dic Errträge thecilen fitch in:

a) Sorerträge,

b) STbtriebs= voer Şaubarfeitšertrïge.

$$
\S 23 .
$$

\section{Einfluf}

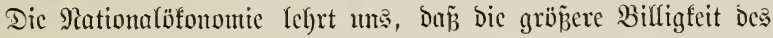

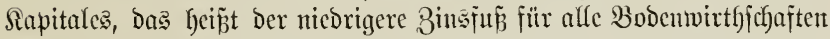
cine größ̄ere Intenjität bejügliç) Der Sapitalsantage crmöglicht, für bie

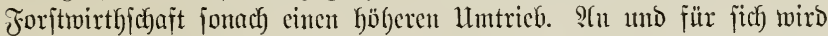
untür(ich) zuntächjt bie Bodentente burd) Unterftelfuttg cintes nicbrigeren

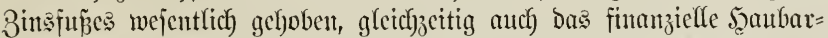

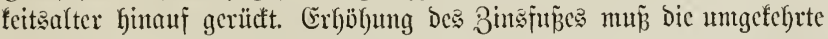

*) Ilnter "Bobenbuttorente" wird die Summe auts der reinten Bobentente uno den jäbrlicben Berwaltungsfoiten uns Eteutern, aljo $\mathrm{r}+\mathrm{v}+\mathrm{s}$ veritanden; wir bezeid)nen Diejelge im Folgenden jtets nit $r^{\prime}$.

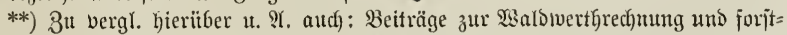
richen Etatif von $\{$. v. Sedfendorfi. Supplemente zur Alfgemeinen Jorit= uno

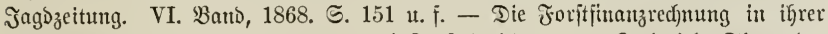

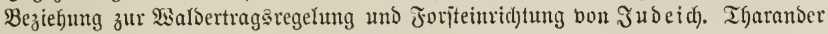
forjttidyes Jabrbud) (1866), 17. Bans. 5.3 u. f.

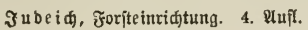




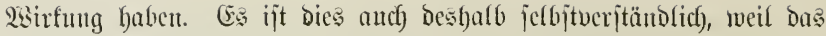
finanziclle Sanbarfeitsalter jenes ijt, in welchem bas \$scijerprocent

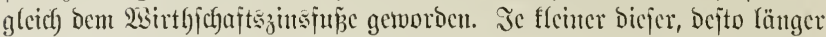
fant jich Das Weijerprocent l̈ber ifm crbalten. - Eimes birecten, mathematijech Beweifes bedarf es ficrzu faum.*)

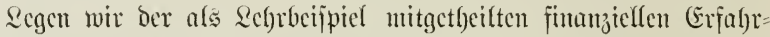

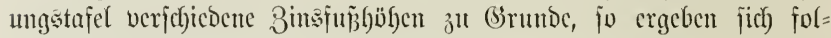
gende Bodenbruttorenten:

\begin{tabular}{r|c|c|c|c}
\hline AIter. & $\mathbf{4} \mathbf{0}_{\mathbf{0}}$ & $\mathbf{3 , 5} \%$ & $\mathbf{3} \%$ & $\mathbf{2} \mathbf{1} \mathbf{2} \%$ \\
\hline 65 & 3,893 & 5,116 & 6,488 & 8,188 \\
70 & $\mathbf{3 , 9 0 2}$ & 5,225 & 6,790 & 8,632 \\
75 & 3,827 & 5,225 & 6,909 & 8,926 \\
80 & 3,769 & 5,250 & 7,066 & 9,278 \\
85 & 3,721 & $\mathbf{5 , 2 8 7}$ & $\mathbf{7 , 2 4 3}$ & 9,671 \\
90 & 3,585 & 5,205 & $\mathbf{7 , 2 6 5}$ & 9,871 \\
95 & 3,392 & 5,083 & 7,170 & $\mathbf{9 , 9 1 7}$ \\
100 & 3,166 & 4,815 & 6,990 & 9,846
\end{tabular}

Dic Bobenbruttorente culminirt jonad) bei $4 \%$ im 70 iten, bei $3,5 \%$ int 85 jten, bci $3 \%$ int 90 jten, bei $2 \frac{1}{2} \%$ in 95 jten Jafyre. Dafjelbe gillt für bic reine Bobentente (\$25. a).

(Es vermag mithin, wie bic Differenz zivifach $4 \%$ mo $3 \%$ nach)=

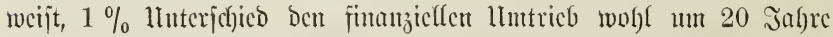
höber oder tiefer ju jtellen. Ein einfaches, matbematijches (sicjeb über

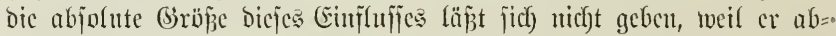

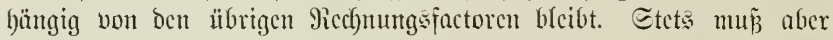

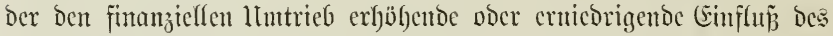
nicbrigeren ober höheren Bursfunes ein um jo bebentenderer jein, je geringer bic Differenzen in ben Panteffecten ber verjeficdenen, fitch nabe

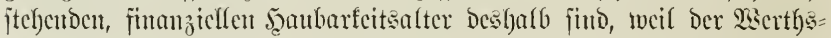

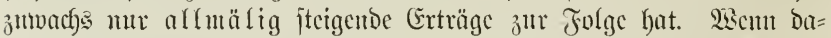
gegen in Folge bejtimmter, vom Marft cuergijof begef)rter Etärfen ge= wifïcr Eortimente cine bedentende \$sreisjtcigerung für cinen cngeren

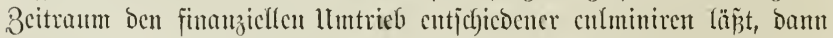
vermag anch $1 \%$ Differen im Binsfuje ben Eulminationspunft Der Siente nicht 1 m 10 oder unch mehr Jafyre zu verändoru.

*) Diejer Betweis unurde gefüfrt von I. v. Secfendorff l. c. S. 160 uno

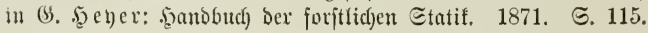


Mill man in Gier gegebenen Faffe bered)nen, wie hod) fidf Der Preis bes 90 jüfrigen şulzes ftelfen milije, mu bei einem Binsjuje von $4 \%$ biejelbe Boben= rente zแ getuähren, wie ber 70 jügrige llutrieb, jo fann bics auj jweiertei Wseije gejdeken:

1) Ier Ribtriebsertray Des 90 jäbrigen Beitandes muí baun gleid jein ber Eunune aus Dem 90 jährigen Endzinje Des Bodenbruttofapitales (g) und Dem $90=$

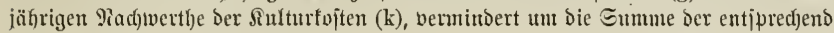
bernad)wertfeten \$ormuthingen:

$$
\begin{aligned}
\mathrm{g}\left(1,04^{90}-1\right)+30 & \times 1,04^{90}-\left(1,8.1,04^{70}+4.1,04^{65}+7,2.1,04^{60} \ldots \ldots+11,2\right) \\
& =\mathrm{g}\left(1,04^{90}-1\right)+30 \times 1,04^{90}-1116,798 .
\end{aligned}
$$

Jïr Den 70 jährigen llmtrieb ijt $B_{u}=\frac{3,90233-3}{0,04}=22,5583$.

Berwaltungs $=$ und Etenterfapital $\mathrm{V}+\mathrm{S}=\frac{3}{0,04}=75$. Taher

Tieje :erthe eingejeșt, ergiefot:

$$
\mathrm{g}=22,5583+75=97,5583
$$

$97,5583\left(1,04^{90}-1\right)+30 \times 1,04^{90}-1116,798=3137,847$ fl.,

als nothiwendigen \$serth bes 90 jährigen Bejtandes, bant ber biejem entipredfende llntrieb biejelbe Rente gewähre, wie ber 70 jäbrige.

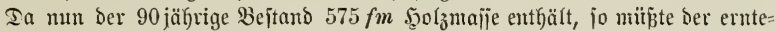
fojtenfreie Einfeitäpreis ber Yeţteren $\frac{3137,847}{575}=5,457 \mathrm{jl}$. Getragen, um bie gejtellte Bebingung zu erfïlfen. Es bebürf̈te aljo nur einer Erböhung Dex \&reije bes $90=$

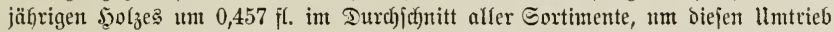

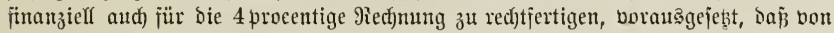
biejer \&reisjteigerung bie jüngeren Eortimente unberïfrt gelajīen wïtrden.

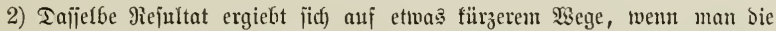
Ermme aus dem $90-70$, alpo 20 jührigen Endzinje Des Sapitales g uno bem 20=

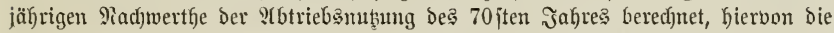

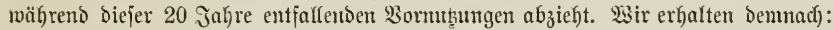
$97,5583\left(1,04^{20}-1\right)+1428,9 \times 1,04^{20}-\left(26.1,04^{15}+20,8 \cdot 1,04^{10}+16,8 \cdot 1,04^{5}+11,2\right)$ $=3247,095-109,253=3137,842$.

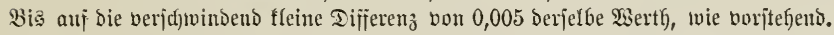

\section{$\S 24$.}

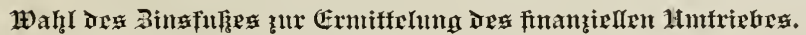

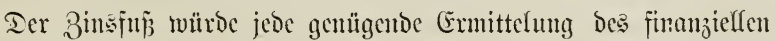

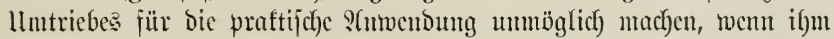

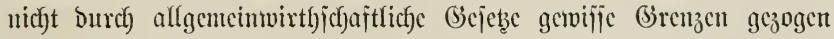
wören, inmergalg deren ex jidg berwegt.

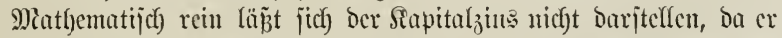

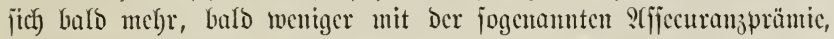
mit Dem Ilnternebmergenuine, ja jelbjt mit cincm ?fmortijationsquantum verbindet. 
2t( Preis Der Rapitalmubung richtet fich Der Rapitalzins, wic

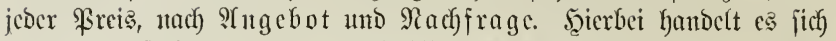
un bie Gejtaltung bes Berbältniffes beider Factoren zut cinander.

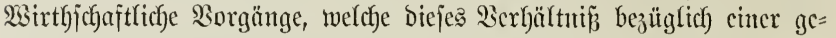
wifijcu Siapitalanlage äntorn, ändern aurd) Den für bicjes Sapital zu

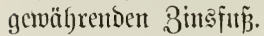

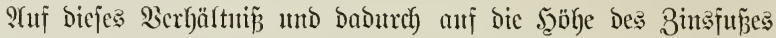

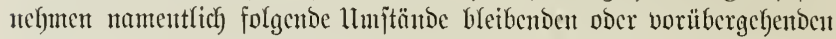

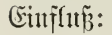

1) Sicherbeit Der Sapitalantage. Die Sapitale fitreben nicht

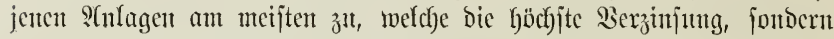
jenen, meldye bie ficherjte ?(nlage gewälyren. Sebstere vermefhrt jonach

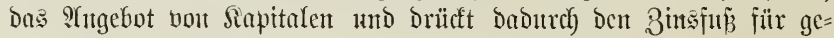
twijic SAltlagen berub.

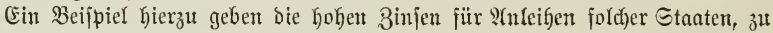
denen man fein Bertranen hat, man fordert Der ltnjicherheit wegen eine hohe Sijje=

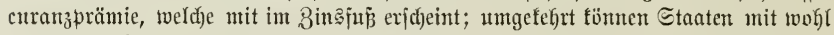

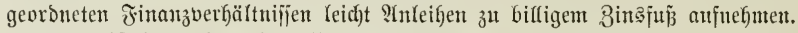

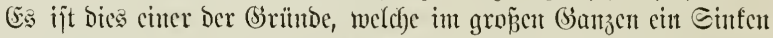
Des Ziusfupes mit Dem Stcigen Der Siultur bewirfen, benn mit bicjen Steigen wäd)jt bic allgemeine Pied)tsficherfect.

2) S(nuchmlidffeit Der Sapitalanlage. Se angenebmer ben Siapi= tulijten bie Form der SUtlage ift, je wentger Mithe Der Buijenbeztug veranlajpt, bejto melgr werben bei gleicher Sidferbeit bie Siapitale joldyen

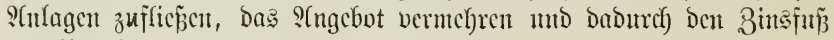
beruborücten.

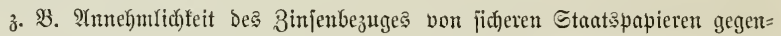
ïber ben solpothefen.

3) Siealifirbarfeit bes Sapitales. İ leidfter cs möglich ijt,

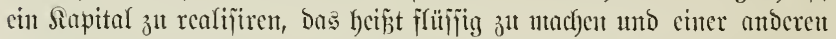

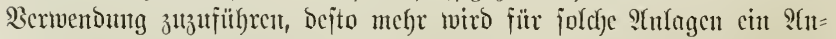
gebot namentficf) fleincrer Sapitale unter joujt gleichen Berbältnifjen ftuttfintoen, umgefelyrt Dejto weniger.

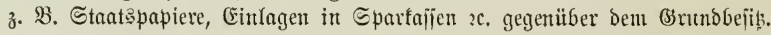

4) Beränderfichfeit des Siapitalwerthes. Gewifje Siapital=

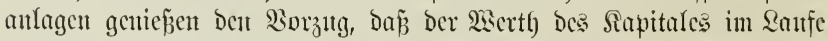
ber Beit jteigt, bei anocren berbält es jich amtgefefort. Llnter foujt

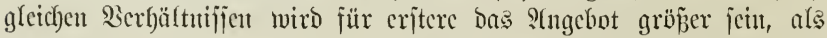
für leţtere; Daber müjjen und fönnen erjtere mit ciner niebrigerent ३erzinjunt zufrieden jein. 


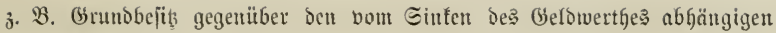
Staatspapieren oder (j)potheften.

5) Reigung Der Sintalijten ju gemij̈en Sapitulanlagen. Dic Mecinntgen über bie unter 1 bis 4 gentunten Bebingungen ber Sinpital= antagen fä̈ngen jefre von jubjectiven ?(njchaunngen Der Sapitalijten ab,

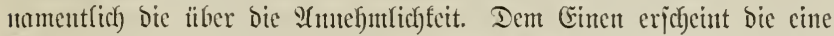

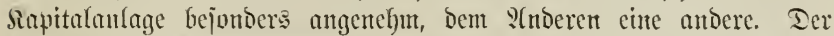
Siapitalijt, welcher cine bejondere Bovrliche jür cine bejtimnte Sapital=

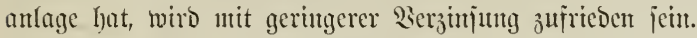

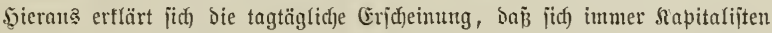

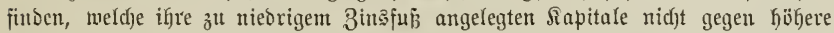

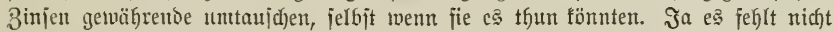
an jolchen Sapitarijten, wefdre mit Borficbe wenigitens einen Theil ifrer Siapitale

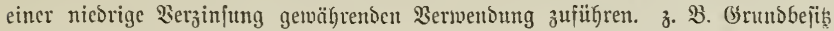

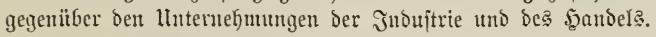

Dicje unter 1 bis 5 genaunten llmjtände wirfen in ben einzelnen F̈̈llen chter Sapitulantage neben und gegen einander; fie rufen bie

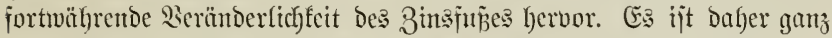
wergebliches Bemülyen, fïr bejtimmte Saptalanlagen cinen beftimmten

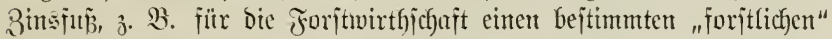

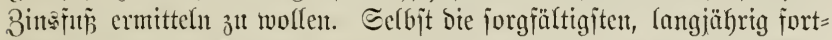

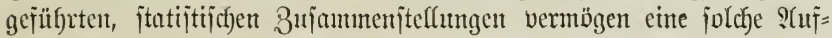
gabe nicht ju löjen.

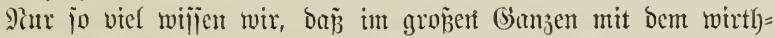

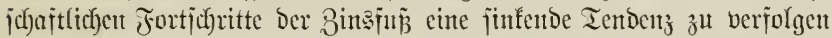
jucheint, jobald man jum Bmecfe ber Unterjuchung lange Beiträımte it

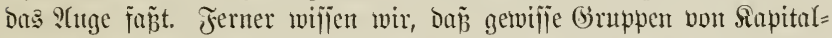
antagen in berjelfan Beit uno Begento eine niebere ober cinte bögere Beräinjung getwäbren, jenadfoem bei ifuen bie bas STgebot von oder bie bie Saachfrage nach Sapital becinflufjenden llmitünde ïberwiegent.

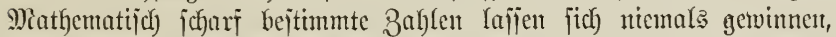

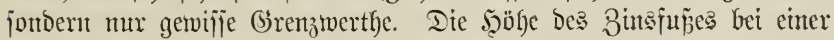
Sapitalanlage ijt uticht maj̃gebend für die bei ciner anderen.

Dic oben unter 1 bis 5 genannten Unjitände erflären $e_{3}$, warum

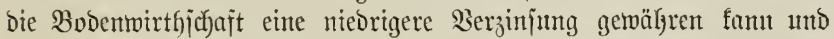

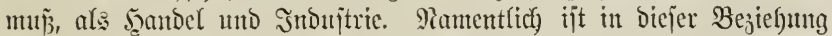
(S)emicf)t

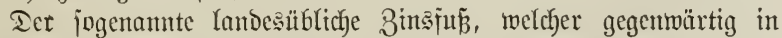
Ieutichland auf 4 bis 5 lautet, gilt als Durchjochnittsgröpe in ber Seauptjache nur für bie leicht flüifïgen bieldfapitale, fam aljo nicht 


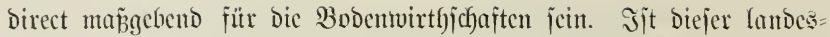

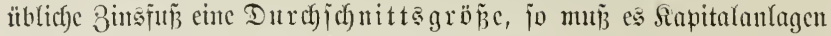

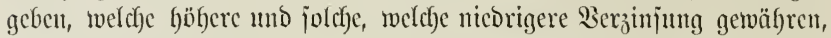
als 4 bie $5 \%$.

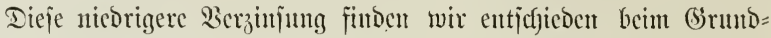
bejibze, namentlich bei der Foritwirtbjeffaft aus folgenden Gründen.

Erjtens. Dic Eicherheit Der forjtlichen Sapitalanlage ij̈t cine jebr

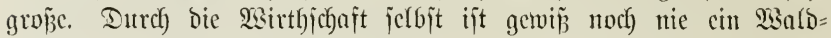

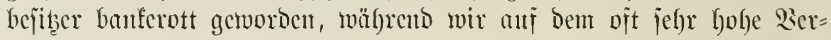

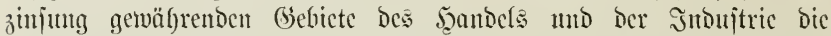

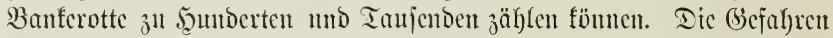

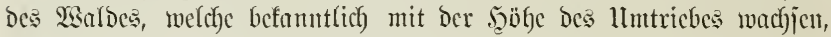

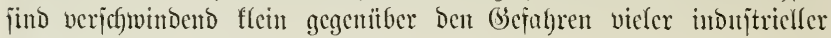
Unternefmungen.

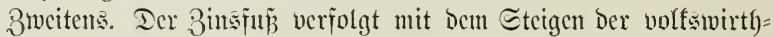

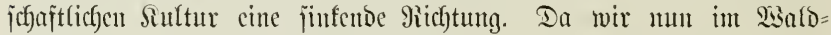
gemerbe in Der Ricgel auf längere Bcit hinaus rechnen, wenn es jich

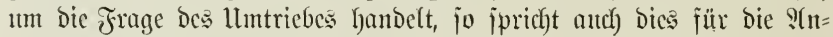

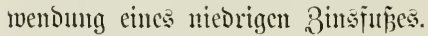

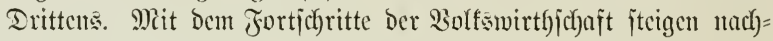
wcisbar Grmo und Boben, jowie Defien Producte int Siertfe nicht blos gegentïber Dem (Sclbe, jondern gegentüber ben meijten anderen

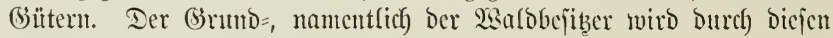

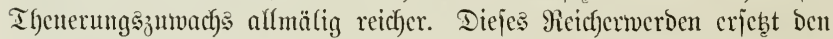

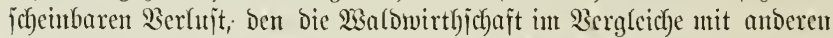
(Sewerben mit fich bringt, indem jic ifre Sapitale nicbriger ver jinjt; cin Theil ber nicht bezogenen Binjen legt jich getvifjermafien am ur= iprünglich vorbandenen Sappital ant.

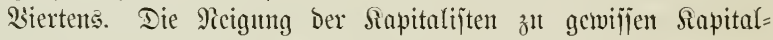

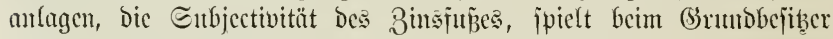
bejonders beim $\mathfrak{B a l b g e m e r b e ~ c i n t ~ s v i c h t i g e ~ P i o l l e . ~ W i r ~ f i n d e n ~ i m m e r ~}$

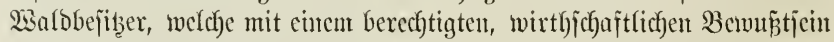
nutr niedrige Serzinjung ifres Sapitales beamprutden, wocil für jic

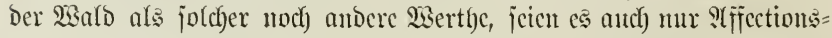
wertfe, bejibet, welche fich in ben Biffern ber Walbente nicht ants=

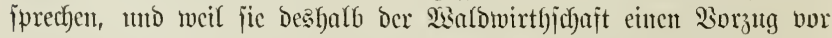
andocen Sapitalanlagen cintäumen, twent bicje and höl)ere Becrjimjung gerväbren. SIm meijten gilt bies für ben Staat, Dem alle die alfge=

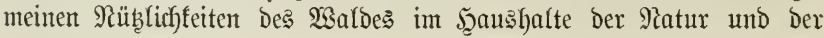




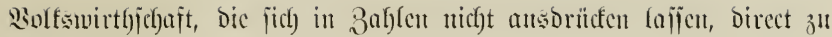
(B)utc fomment.

Rach) allen Errwägungen dïnftu gegentwärtig ungefül)r $3 \%$ Dic

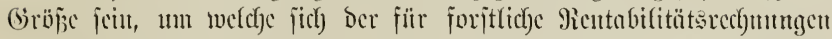

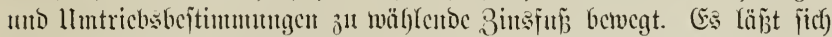

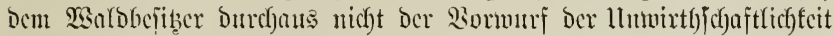

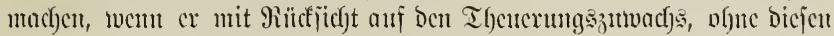

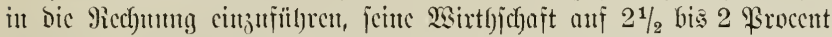

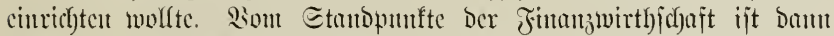

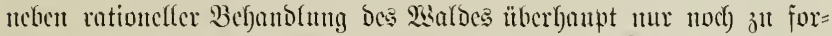

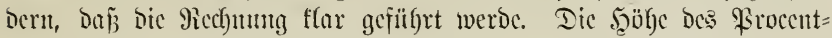

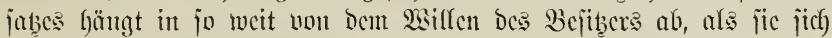

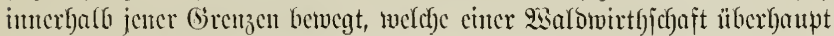
won Patur gezogen j̈mb.

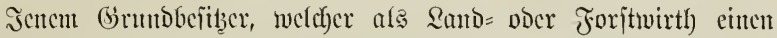

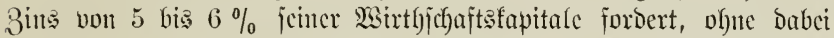

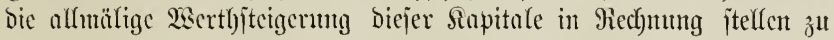

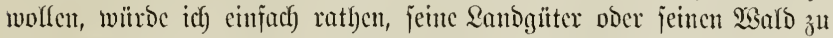

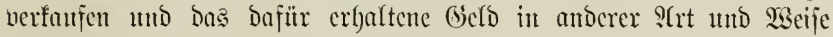
anjulegen.*)

\section{§ 25.}

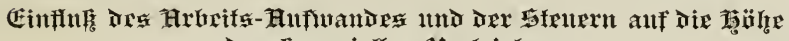
Des futantiellen 1tmfriebrex.

$3 \mathfrak{i}$ unterifbciocn find:
a) jälorliche,
b) periodijefe Sojten.

a) Die jährlidgen $\Re$ ojten.

SAl jolche fund in Der Siegel nutr Berwaltungsfojten und Steucut**) anzujelyen. Sionmen anbere vor, jo fallen fie als Rectumngajactoren in biejclbe Sategoric.

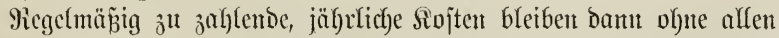

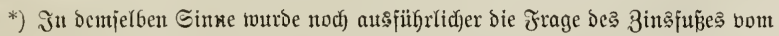

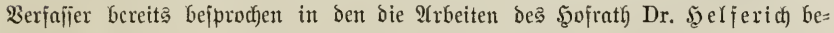

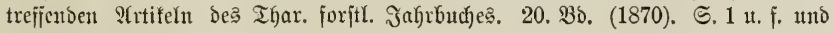
22. 3. (1872). S. 132 u. f.

**) $3 \mathfrak{u}$ vergl. § 15 , ङ. 42 . 


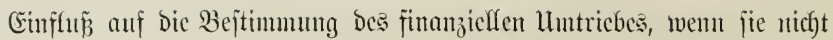
it Folge vou Umtricbsocränberungen fefbjt Senderutgen crleiden.

In jeltenen Fällen bïrfte bies thatjächlich vorfommen.

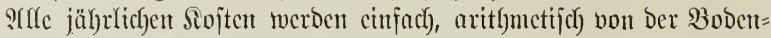

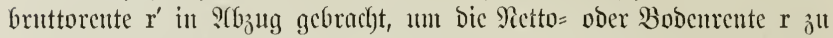
bejtimmen. - 2ebetere wiro natürlich Durch Seränderungen biejer Siojten

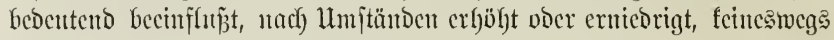

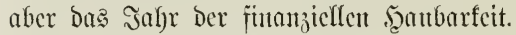

Beippiel. Eeben wir ben Fulf, daj für Den Wald unferer Er= tragstafer jtatt 3 fl., jälgrlich 5 fl., oder mur 1 fl. Bertwaltungsfojten

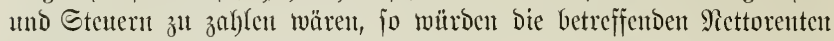
betragen:

\begin{tabular}{r||c||c|c|c}
\hline \multirow{2}{*}{ Sabr. } & $\begin{array}{c}\text { Brutto: } \\
\text { rente. }\end{array}$ & \multicolumn{3}{|c}{ शettorenten. } \\
\hline \hline & $\mathrm{v}+\mathrm{s}=1$ & $\mathrm{v}+\mathrm{s}=3$ & $\mathrm{v}+\mathrm{s}=\mathbf{5}$ \\
\hline 80 & 7,066 & 6,066 & 4,066 & 2,066 \\
85 & 7,243 & 6,243 & 4,243 & 2,243 \\
90 & $\mathbf{7 , 2 6 5}$ & $\mathbf{6 , 2 6 5}$ & $\mathbf{4 , 2 6 5}$ & $\mathbf{2 , 2 6 5}$ \\
95 & 7,170 & 6,170 & 4,170 & $\mathbf{2 , 1 7 0}$ \\
100 & 6,990 & 5,990 & 3,990 & 1,990
\end{tabular}

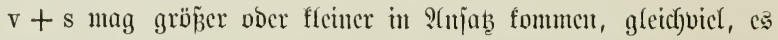

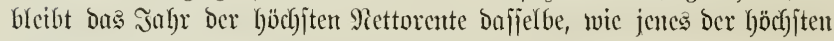
Bruttorente, fjicr das 90 jte.

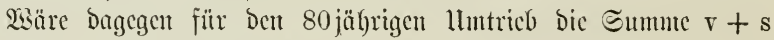
fleinter, als fütr Den 90 jälyrigen, $\mathfrak{z}$. $\mathfrak{B}$. für criteren 2,5 , für leşteren 3 , fo würbe dic Bodenrente im 80 jten Jafyre mit $7,066-2,5=4,566$ catminiren uto Dorthju der finanjielle limtricb fallen.

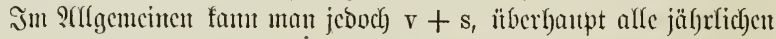

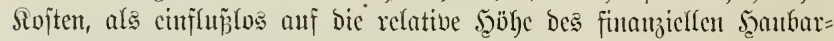
feitsalters und llmtriebes anjeben.

b) Periodijche Siojten.

Sic zerfalfen in Ernte= und Sulturfojten.

Dic Erntefojten werben in ber Regel in fo fern cintel etwas cr=

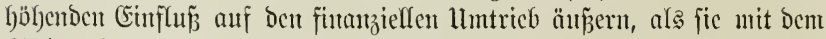

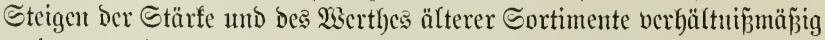
geringer weroen. Sie tragen Dadurch) zum Sualitätşumad) $(\S 7$ u. 12) 
bei. - 3ur Ermittelung des 5̧aubarfeitanalters weroen fie an ein=

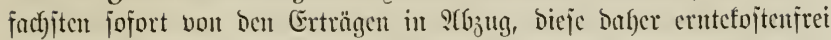

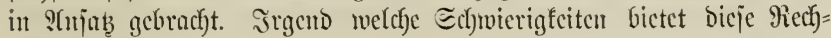
mungsoperation nidft.

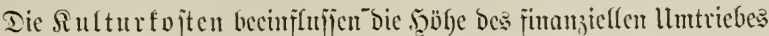

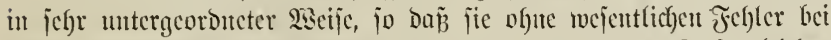

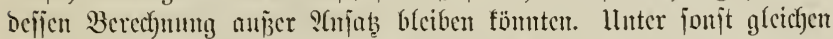

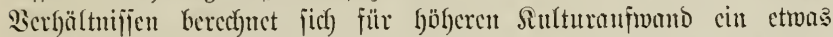

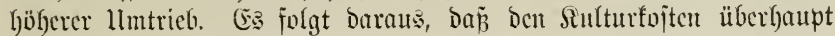

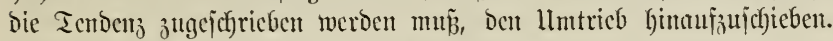
Se niebriger ber leb̧tere, bejto öfterer felfen fie wieder.

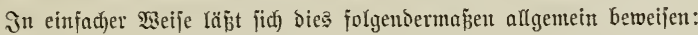

Die Sitturfoiten k erjdeinen in Der Rentenformel negatio in ber Form

$$
\mathrm{k} 1, \mathrm{op} \mathrm{p}^{\mathrm{n}}:\left(\frac{1, \mathrm{op} \mathrm{p}-1}{0, \mathrm{op}}\right)=\frac{\mathrm{k} 1, \mathrm{op} \mathrm{p}^{\mathrm{u}} \times 0, \mathrm{op}}{1, \mathrm{op} \mathrm{p}^{\mathrm{u}}-1} .
$$

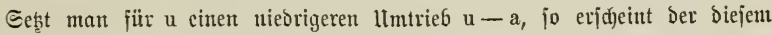

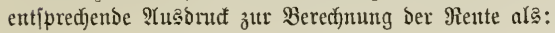

$$
\frac{\mathrm{k} 1, \mathrm{op} u-\mathrm{a} \times 0, \mathrm{op}}{1,0 \mathrm{p}^{\mathrm{u}}-\mathrm{a}-1} .
$$

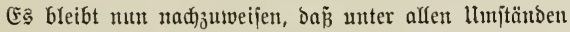

$$
\frac{\mathrm{k} 1, o p^{u-a} \times 0,0 p}{1,0 p^{u-a}-1}>\frac{\mathrm{k} 1, o p^{u} \times 0, o p}{1, o p^{u}-1} .
$$

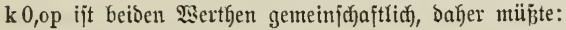

$$
\frac{1,0 p^{\mathrm{u}-\mathrm{a}}}{1,0 \mathrm{p}^{\mathrm{u}-\mathrm{a}-1}}>\frac{1,0 \mathrm{p}^{\mathrm{u}}}{1,0 \mathrm{p}^{\mathrm{u}}-1} \text {. }
$$

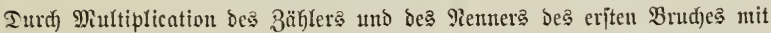

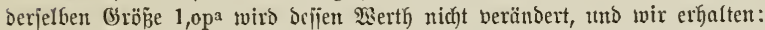

$$
\frac{1,0 \mathrm{p}^{\mathrm{u}}}{1, \mathrm{op} \mathrm{p}^{\mathrm{u}}-1, \mathrm{op} \mathrm{p}^{\mathrm{a}}}>\frac{1, \mathrm{op} \mathrm{u}}{1,0 \mathrm{p}^{\mathrm{u}}-1} \text {. }
$$

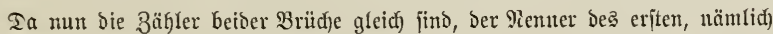

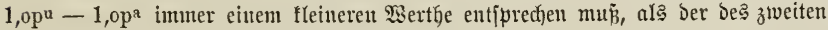

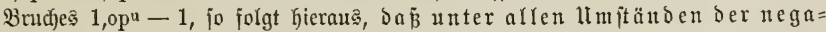
tibe Einflúb ber Sulturfoiten auf bie Bobenrente um folleiner jein mú, je Göger ber llmtrieb ijt.

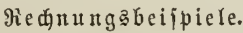

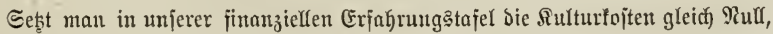
jo bercefinet iid) mit $3 \%$ bie $B$ odcubruttorente für ben

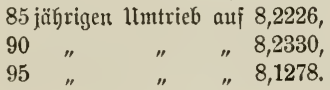

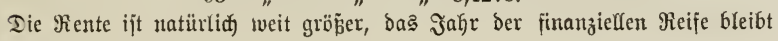


indeîen fajt bajietbe, wir mögen 30 ff. Sulturfviten bered)nen ober nidjt. Der jefr

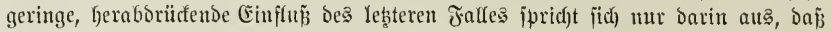
hier die Differenz Der Renten Des 85 und 90 jährigen ltutriebes etwas geringer ijt.

Sebst man $\mathrm{k}=100$, jo ergiebt fich bei $3 \%$ eime Bodenbruttorente jür Den

85 jübrigen llutrieb vou 4,9579,
$90 "$ "
$95 \quad$ "

Jhr Betrag ift natïrlich weit geringer; Dagegen bleibt bas finangielle Şaubar= leitsalter immer nod) im 90 jten Jahre; Der jehr geringe, hebende Einflup Der ver:

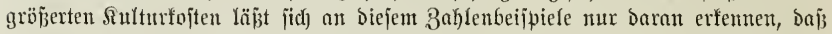

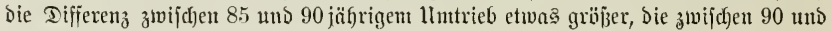
95 jührigem etıon fleiner ijt, als frïher.

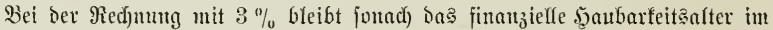
90 iten Jahre, wir mögen gar feine, 30 , voer 100 fl. Siufturfojten anfeben, gleid)viel.

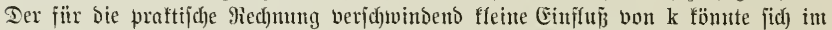
vorliegenden Beifpiefe nur Dann Deutfich zeigen, wenn man bie Rerfunng nid)t in 5 jäbriger 9fbjtıfung, foutbern für bie einzelnen Эabre geben würde.

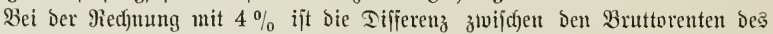

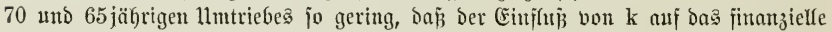

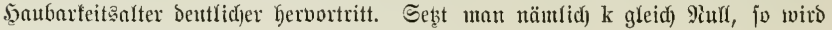
Die Bobenturuttorente Deڤ

65 jäbrigen llutriebe 5,1943 ,

70 " $\quad 5,1847$.

$\S 26$.

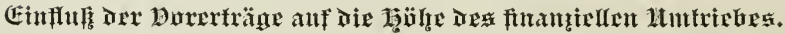

Daj̉ bic Borerträge vocr Sornubungen cuten jefre bedentenoen

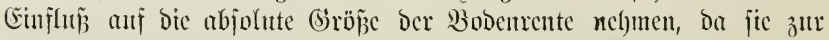

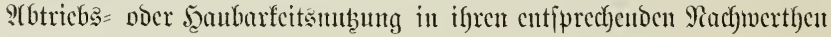

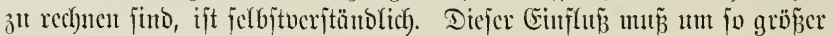
feit, je zcitiger und in je höberen Beträgen fic cingeben.

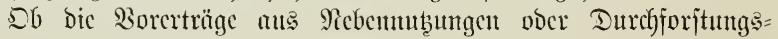

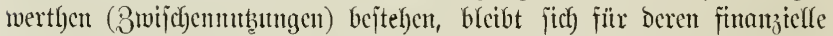

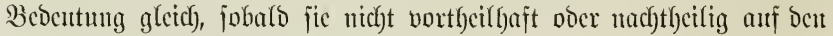
3utwadjsgang ocs Sauntbejtantocs cinwirfent.

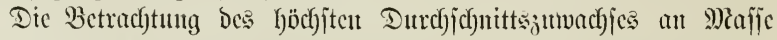

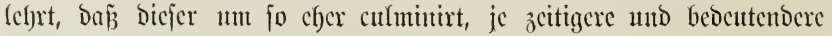

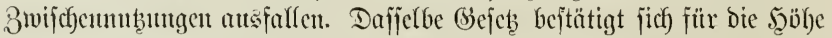
des fintuniclfen llntricbes.

Dic Bermedruntg ber 2ormubungen wiro in ber 9iegel, jeder zei= tigere Ëingang berjelben ftets bas funanjelle Scaubarfeitsalter etmas heraborüctent. 
Ëmen in biejen Sinne crhähenden Ëinfluz form und wirb cinte

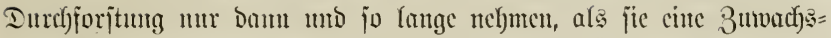
vermebrung Des Sauntbejtandes zur Folge hat. Duburd) fum unter

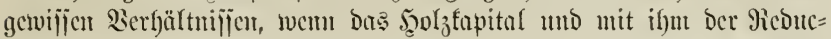

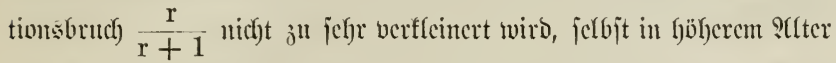

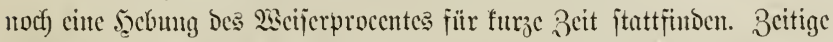

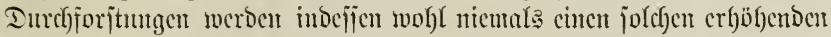

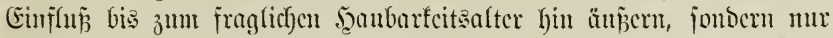
joldec, weldye in Bejtünoen vorgenommen werden, bic bicjem S(lter jobon nube jtelden.

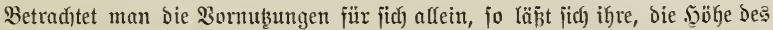

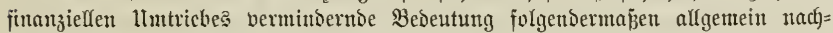
sweijen:

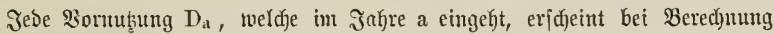
ber Bobentente als pofitibe Gröbe in ber Form:

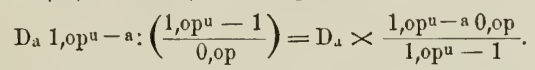

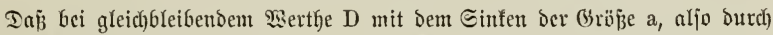

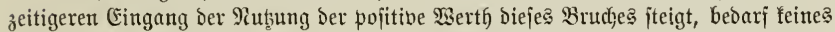
weiteren Beweijes, ba ber Dibijor berjelbe bleibt, ber Dibidento dagegen um fo größ̈er

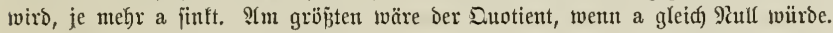

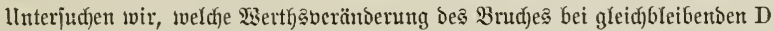

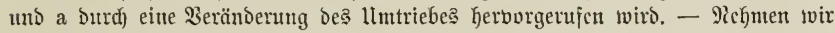

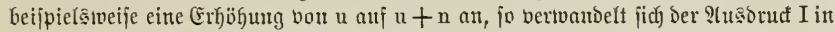

$$
\mathrm{D}_{\mathrm{a}} \times \frac{1, \mathrm{op} \mathrm{p}-\mathrm{a}+\mathrm{n} 0, \mathrm{op}}{1, \mathrm{op} u+\mathrm{u}-1} .
$$

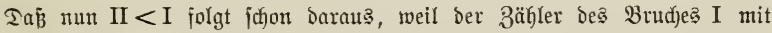

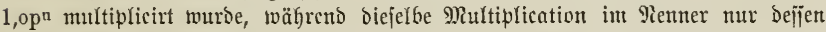

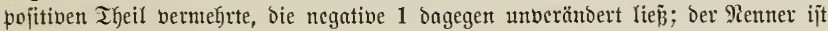

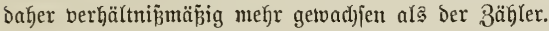

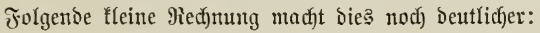

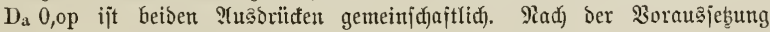

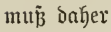

$$
\frac{1, o p^{u}-\mathrm{a}+\mathrm{n}}{1,0 \mathrm{p}^{\mathrm{u}}+\mathrm{n}-1}<\frac{1, \mathrm{op} \mathrm{p}-\mathrm{a}}{1,0 \mathrm{p}^{\mathrm{u}}-1}
$$

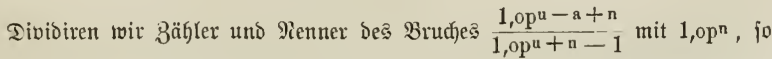
wiro befien

$$
\frac{1, o p^{u}-\mathrm{a}}{1, o p^{\mathrm{u}}-\frac{1}{1, o \mathrm{p}^{\mathrm{n}}}}<\frac{1, \mathrm{op} \mathrm{u}-\mathrm{a}}{1, \mathrm{op} \mathrm{p}^{\mathrm{u}}-1} .
$$


Dieje Ungleid)ung ijt jesenfalla rid)tig, benn bie Bäfler beiber Brüche fint

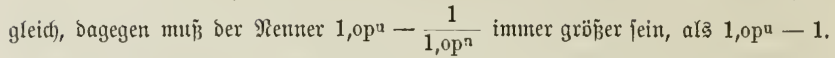

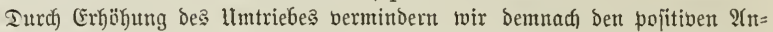
theil Der Sornutsungen an ber Bodenrente. Ilmgefehrt würbe biejer 2(ntheil burd)

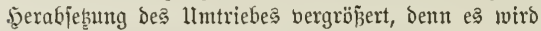

$$
\mathrm{D}_{\mathrm{a}} \times \frac{1, \mathrm{op} \mathrm{p}-\mathrm{a}-\mathrm{n} 0, \mathrm{op}}{1, \mathrm{op}-\mathrm{n}-1}>\mathrm{D}_{\mathrm{a}} \times \frac{1, \mathrm{op} \mathrm{p}^{\mathrm{u}}-\mathrm{a} 0, \mathrm{op}}{1, \mathrm{op}-1} .
$$

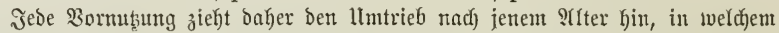
fie erforgt, je nicoriger ber ltmtrieb, bejto öfter fehrt fie wieder.

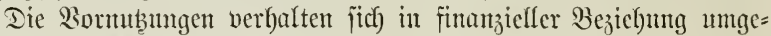
fefrt, wie bie Sulturtojten, was auch jefbjtucrjtändfich), da leb̧tere als negative Erträge angciehen werben fömten.

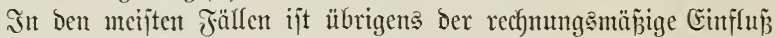

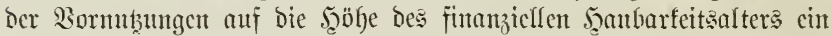
äuperjt geringer, fajt verichwimbento fleinter, fofrafd fie nicht ungemöhn= lich) groje Beträge nachiweijen, went auch bic afjolute (Sirößje ber Yiente

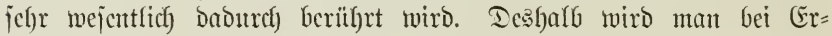
mittchung bes fütanziellen Şaubarfcitsalters nic jebr weit irren, wem

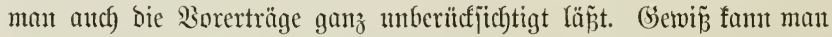

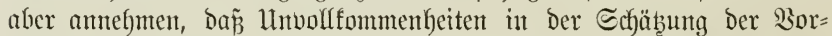

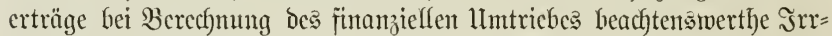
tfïnter nicht jux Folge haben fönnen. Scierbei ift allerbings voraus=

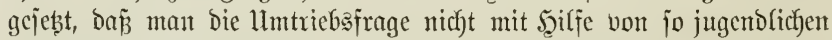
Bejtämben zu löjen jutcht, sveldye derartige Durchforitungen noch er=

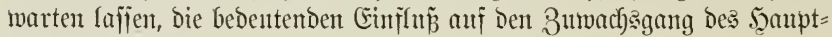
bcjtandos nefment. Bum 3wecfe Der linterjudfung wird man viefmebr

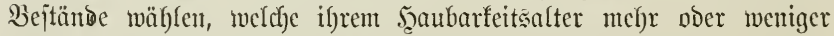

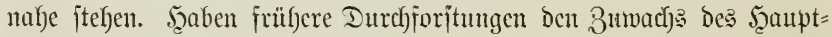
bejtandes günjtig becinfluñt, jo jpricht jich dicjer Einflup im gegen= wärtigen Bujtande jolcher Bejtände aus. $\$ \mathfrak{b}$ aber bie Durchforjtungen vicl oder wenig Ertrag gemälyten, bas bleibt jich ficr für bas prat= tijche Biel ber Piectumutg gleidf, Da cine beachtensmertbe S(enderung Der

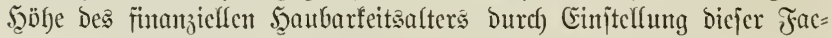
toren in bie Rectunung nicht bemirft wiro.

\section{Beifpiele.}

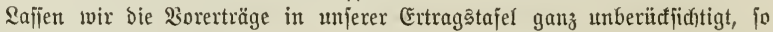

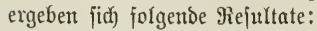

Redumung mit $3 \%$.

85 jähriger Umtrieb, Bodenbruttorente 5,4992 , 


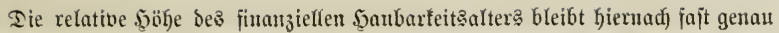

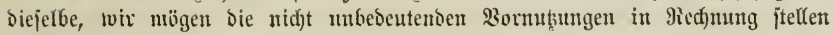
ober nicjt.

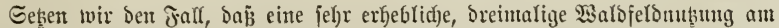
Ed)luije bes britten Эahres 100 fl, werth jei, bie übrigen Bormub̧ungen, jovie bie

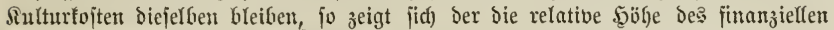

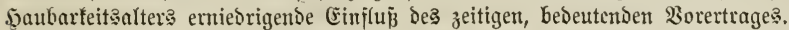

80 jähriger LImtrieb, Bodenbruttorente 10,0960,

85

$\begin{array}{llll}85 & \text { " } & \text { " } & 10,2308, \\ 90 & \text { " } & 10,2172, \\ 95 & \text { " } & 10,0917 .\end{array}$

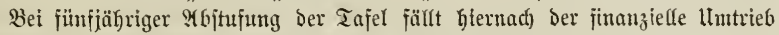

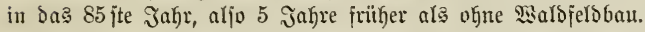

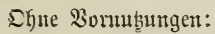

Miectyung mit $4 \%$.

\begin{tabular}{|c|c|c|}
\hline jühriger & $\mathbb{U I}$ & 3lodent \\
\hline 35 " & " & " \\
\hline$"$ & " & $"$ \\
\hline
\end{tabular}

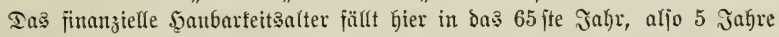

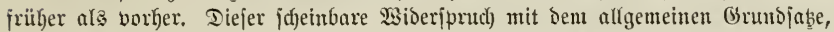

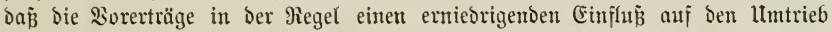

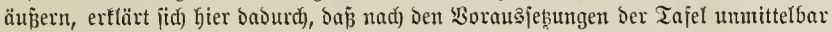

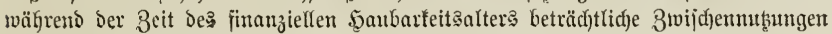
entfallen.

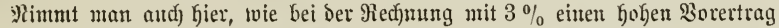

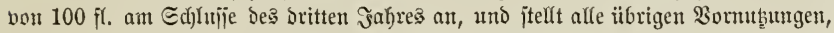

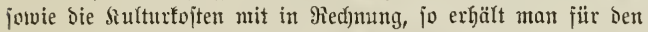
60 jäfrigen Lmtrieb, Bosenbruttorente 7,7098,

$\begin{array}{llll}65 & \text { " } & & 7,7500, \\ 70 & " & 7,7024, \\ 75 & " & 7,5798 .\end{array}$

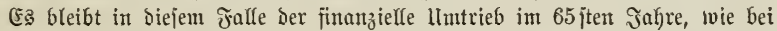

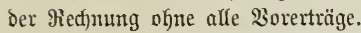

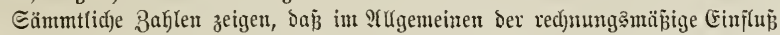

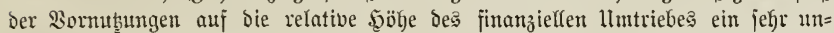
bedeutender iit.

\section{$\S 27$.}

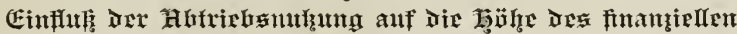 Hintriches.}

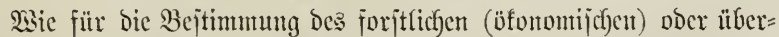

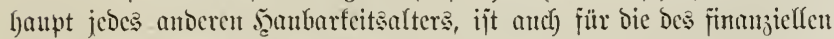

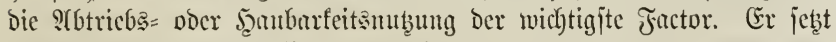
fich zujammen aus Mafife und \$reis.

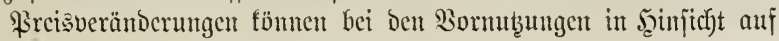


Ermittefung Des llmtriebes unberïffichtigt bleiben, weil bicje ofnefju

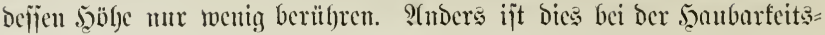
mubutg.

Dic Beränderung Des̃ in Gelo antsgedorüften Preijes, mag fic im Einten bejtehen ober im Steigen, fann zweierlei llrjachen baben: Ent= weder ijt jic Folge einer STenderumg bes Gelowerthes, ober Folge ciner

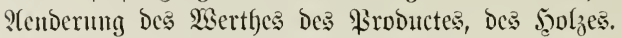

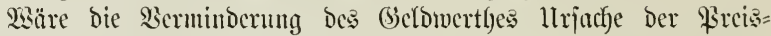

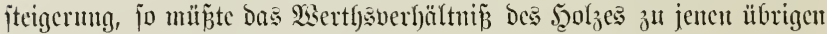
(Sittern, welche an fich feine $23 e r t$ säntorung crlitten, Dafjelbe geblicben jein. (5s müp̈te it biejem Falle ferner, worauf Gcjonderes (scmicht zu legen, bie \$rcisjteigerming arle Eortimente und Siojten gleidgmä̈̈ig treffen. Dic in Beld ausgebrïfte Sodennettorente wïroe Daburch jwar

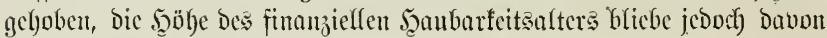
gaıtz umberülyt.

Sticgen in Folge finfenden Belowerthes alle Sojten und Erträge auf ben mfachen Betrag, jo würoe bic Bobenuttorente $\mathrm{r}$ chenfalls auf m r jich beben. Pauch Ecite 63 ijt

$$
\mathrm{r}=\left[\mathrm{H}_{\mathrm{u}}+\mathrm{D}_{\mathrm{a}} 1,0 \mathrm{p}^{\mathrm{u}-\mathrm{a}}-\mathrm{k} 1,0 \mathrm{p}^{\mathrm{u}}\right]: \frac{1,0 \mathrm{p}^{\mathrm{u}}-1}{0,0 \mathrm{p}}-(\mathrm{v}+\mathrm{s}) .
$$

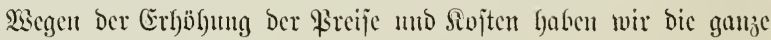
(S)lciclung mit m ju multipliciten und crforlten

$$
\mathrm{mr}=\mathrm{m}\left[\mathrm{H}_{\mathrm{u}}+\mathrm{D}_{\mathrm{a}} 1,0 \mathrm{p}^{\mathrm{u}-\mathrm{a}}-\mathrm{k} 1,0 \mathrm{p}^{\mathrm{u}}\right]: \frac{1,0 \mathrm{p}^{\mathrm{n}}-1}{0,0 \mathrm{p}}-\mathrm{m}(\mathrm{v}+\mathrm{s}) .
$$

Da nu burefy cine joldfe Steigerung aller pojitiven uno negativen

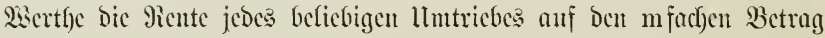

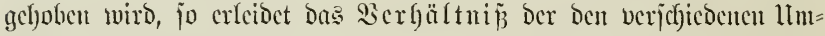
trieben entiprechenden Menten, jonad) aud bas finanjielfe saubarfeits = alter feine S(cnderung.

Beifpiel. खäre $\mathrm{m}=1,5$, fo ütroen bie Bobennettorenten bes gewählten Beijpiels mit $3 \%$ für ben

80 jäbrigen llmtriéb $4,0658 \times 1,5=6,0987$,

$\begin{array}{llll}85 & \quad & & 4,2432 \times 1,5=6,3648, \\ 90 \quad " \quad & \quad & 4,2654 \times 1,5=6,3981, \\ 95 \quad \prime & \quad & 4,1701 \times 1,5=6,2551 .\end{array}$

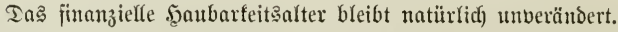

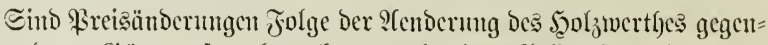

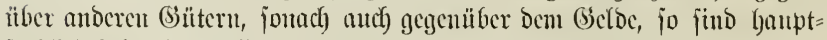
jächliç) folgende F̈̈lle ju beachten: 
A. Senocrumg ber Preije alfer Eortimente bei unuer= änderteu oder wenig veränderten Siviten.

Piefmen wir an, Daj bie \$ireije aller Eortimente (erntefojtenjeci) anj ben mfachen Betrug iteigen, bie Siviten bugegen unverünocrt blciben,

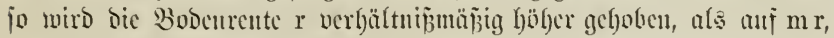
das finaujiclle jaubarfeitsulter in jef)r unbedentender siscije ferab= gedrüctt.

Bezcicfunen wir bic größjere Fiente mit $\mathrm{R}$, jo wiro, weun ber 5ुan=

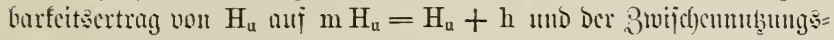
crtrag im Jubre a vou $\mathrm{D}_{\mathrm{a}}$ auj $\mathrm{m} \mathrm{D}_{\mathrm{a}}=\mathrm{D}_{\mathrm{a}}+\mathrm{d}_{\mathrm{a}}$ itcigen:

$$
\begin{gathered}
\mathrm{R}=\left[\mathrm{H}_{\mathrm{u}}+\mathrm{h}+\left(\mathrm{D}_{\mathrm{a}}+\mathrm{d}_{\mathrm{a}}\right) 1,0 \mathrm{p}^{\mathrm{u}-\mathrm{a}}-\mathrm{k} 1,0 \mathrm{p}^{\mathrm{u}}\right]: \frac{1,0 \mathrm{p}^{\mathrm{u}}-1}{0,0 \mathrm{p}}-(\mathrm{v}+\mathrm{s}) ; \\
\mathrm{R}=\left[\mathrm{H}_{\mathrm{u}}+\mathrm{D}_{\mathrm{a}} 1,0 \mathrm{p}^{\mathrm{u}-\mathrm{a}}-\mathrm{k} 1,0 \mathrm{p}^{\mathrm{u}}\right]: \frac{1,0 \mathrm{p}^{\mathrm{u}}-1}{0,0 \mathrm{p}}-(\mathrm{v}+\mathrm{s}) \\
+\left[\mathrm{h}+\mathrm{d}_{\mathrm{a}} 1,0 \mathrm{p}^{\mathrm{u}-\mathrm{a}}\right]: \frac{1,0 \mathrm{p}^{\mathrm{u}}-1}{0,0 \mathrm{p}} .
\end{gathered}
$$

Iก แแก

$$
\left[\mathrm{H}_{\mathrm{u}}+\mathrm{D}_{\mathrm{a}} 1,0 \mathrm{p}^{\mathrm{u}-\mathrm{a}}-\mathrm{k} 1,0 \mathrm{p}^{\mathrm{u}}\right]: \frac{1, o \mathrm{p}^{\mathrm{u}}-1}{0, \mathrm{op}}-(\mathrm{v}+\mathrm{s})=\mathrm{r},
$$

jo ijt

$$
\mathrm{R}=\mathrm{r}+\left[\mathrm{h}+\mathrm{d}_{\mathrm{a}} 1,0 \mathrm{p}^{\mathrm{u}-\mathrm{a}}\right]: \frac{1,0 \mathrm{p}^{\mathrm{u}}-1}{0, \mathrm{op}} .
$$

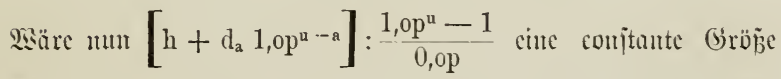
oder cinte foldye, weldye mit ber Bodentente $\mathrm{r}$ ober ber Bobenbrutto=

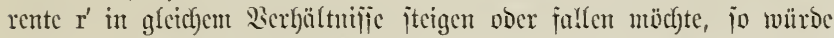

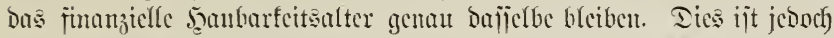
nicf)t ber Fall, und fömte mur bam cintreten, weun bic Sulturfojten $k$

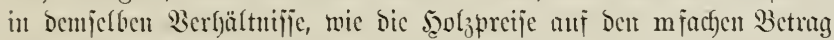
gejtiegen wären. Ia wir mun gejeben haben (ङ. 73), Daj cine Err= höbung vou $k$ bas finanjiclle Sgaubarfeitšlter, wem auch) mux jefrr

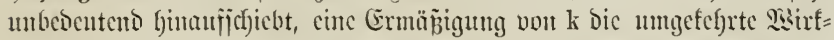
ung Gat, jo mui Der Enoticut $\left[\mathrm{h}+\mathrm{d}_{\mathrm{a}} 1,0 \mathrm{p}^{\mathrm{u}-\mathrm{a}}\right]: \frac{1,0 \mathrm{p}^{\mathrm{u}}-1}{0,0 \mathrm{p}}$ bei cincut ctmas flemeren u culminiren, als bic frübere 30 ocmente $r$, weil it if)m teine Siulturfojten entbalten j̈mb. 


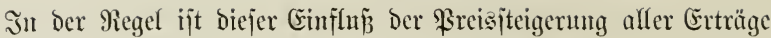

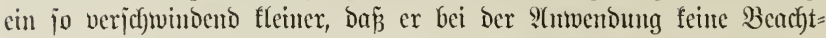
uttg verbient.

Beifpiel. Qafjen wir in unjerer Ertragätafel bei unberänderten Sojten bie Prreife auf bas Ioppulte jteigen, jo ergeben fidf folgende Rettorenten:

Recf)nung mit $3 \%$.

Für ben 80 jährigen lintrieb $\mathrm{R}=12,1250$,

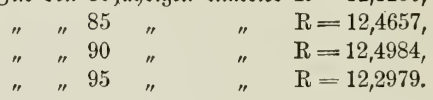

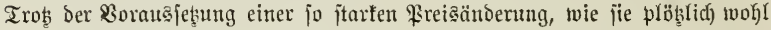

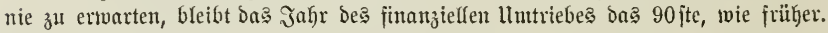

Rechnung mit $4 \%$.

Fürr ben 60 jäfrigen llutrieb $\mathrm{R}=5,8866$,

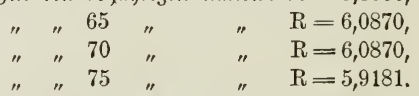

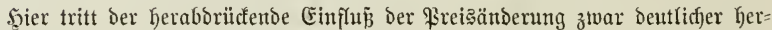

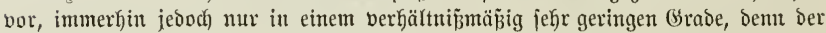

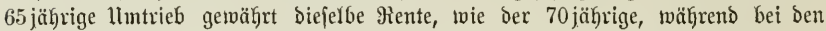

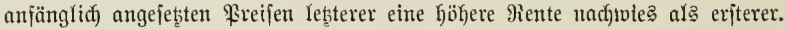

Eine \$reisverminderung aller Eortintente bei gleid)bleibenden

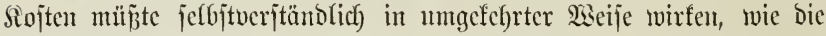

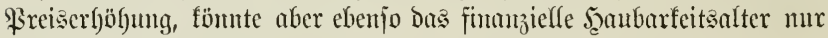
äuß̌perit twenig alteriren.

Wair find bientach berecftigt, folgenden Gab anzunefumen:

\{rle Beränberungen der Şolzpreije, weldye Die Erträge

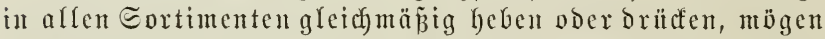
die Rojten bavon berübrt werben ober nidgt, faben entreder feinen, voer höbjtens eiten verjebindend fleiten Einfluf

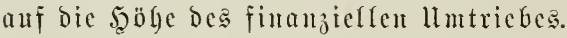

B. Nenderung ber ßreije bejtimmter Sortinente bei gar nicht oder wenig veränderten Sojten.

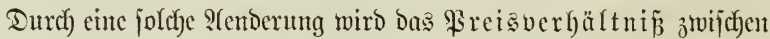

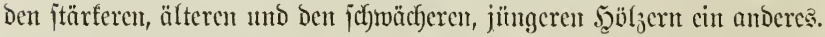

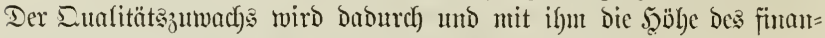
ziellen Љ̧aubarfeitsalter mejentlich) berüf)rt.

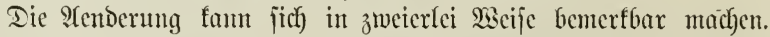

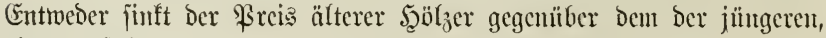
ober er jteigt. 


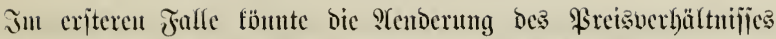

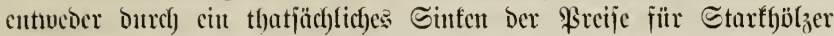
hervorgertfen werben, wäl)rent bic jef)wachen Sortimente ifren \$reis

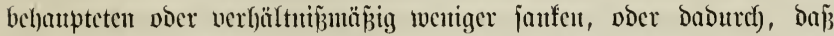

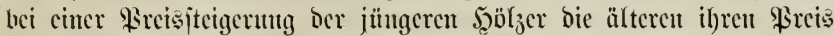

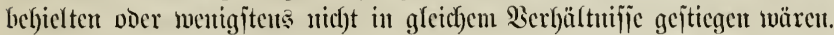
Der Fall ijt Denfbar, gebürt aher offentbar ju Den Ecltenlbeiten.

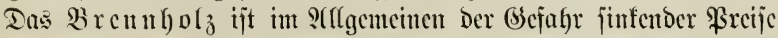

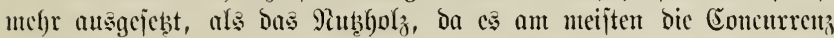
müchtiger Eurrogate z"l befümpfen hat. Eeben wir in Folge deffen

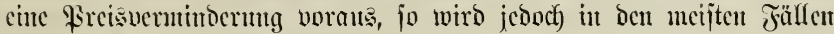

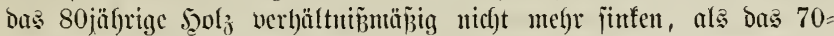

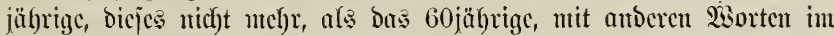

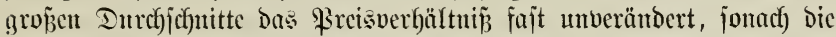
Seble des fütanziellen llutriebes Diejelbe bleibent.

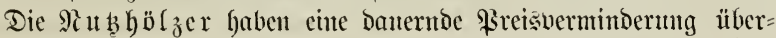

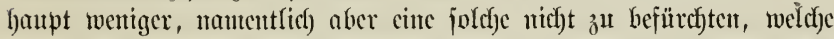

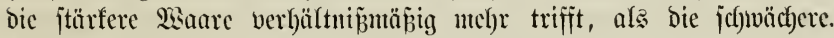
Derartige Fälle fint in ber Begel am vorübergelento, locale Itrjachen

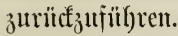

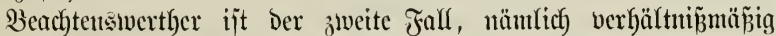

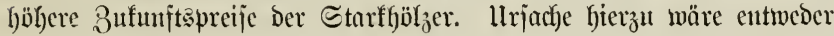

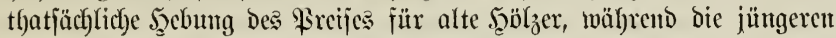
nticht ober weniger an Der Preisjteigerung theilnefymen, ober cin Sinfent

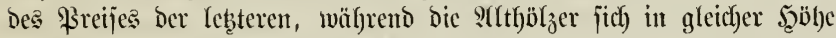
erbielten ober weniger jutfert.

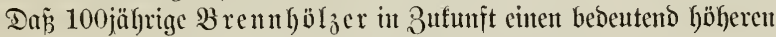
Preis erlangen jolften, als 80 voer 70 jährige, wäre einte nach) alfent

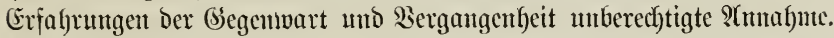

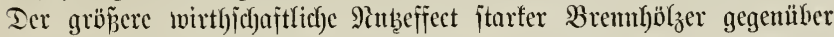
Den f(f)wachen bejteljt ja hauptjächlicf) mur in ber ziemlicf) unbebententocu Erjparnug an Erntefojten, jobals wir nicht für bicjen Fall antpraftijche

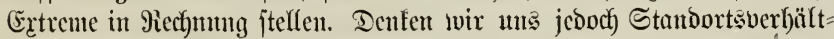

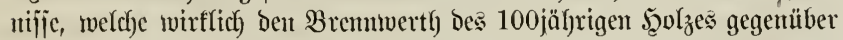

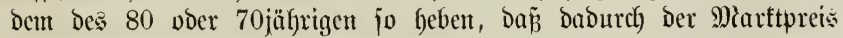

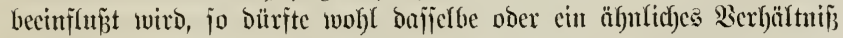
anch) fünftig jtattfinden. Snt S(llgemeinen fönten unb bürfen wir von

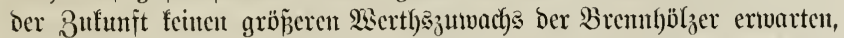

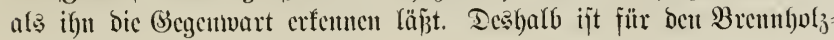

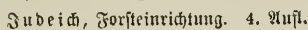




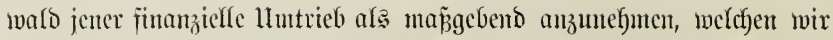

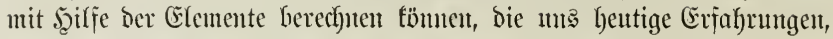
(begenwart und Bergangenteit an die 5eand gebent.

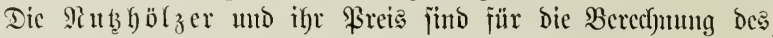

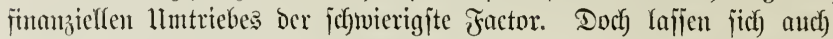
fïr bicjen alfgemeit wirtlfichaftliche (sejichtspunfte gemimnen, weld)e STnfalt gemïhrest.

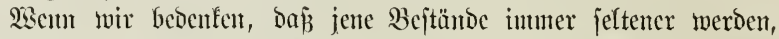
wodche uns bie cigentfich) jturfen Eortimente liejern, ba bieje jicf) nicht cimmal mit ber Ifycoric des föbchiten Mafienertrages recht vertragen

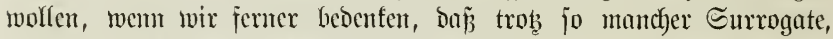

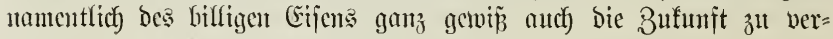
jejiedenen Zwedten itarfe Sortimente wird haben müfien, fo ipricht

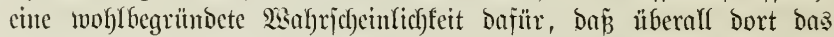
S(ngebot ber älteren jtärferen Waare ctwas melyr jinfen bïrfte, als

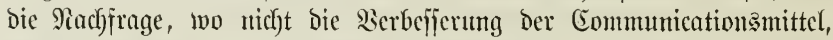

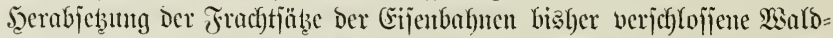
ungen zutgänglicf) macken. Dic natürliche Folge bes junfenden Ŝngebotes

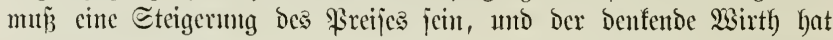

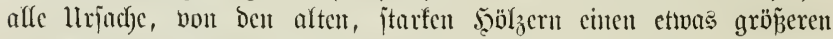

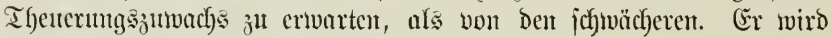

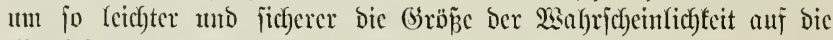

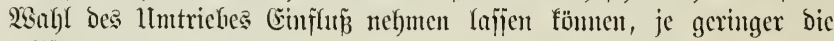

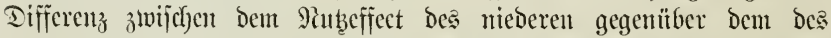

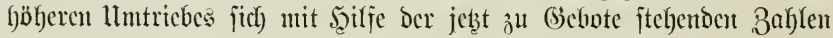

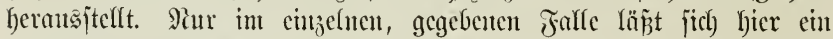

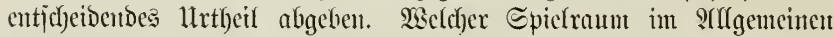
fier Der Epceulation zu gejtatten jei, läp̈t jich) nicht bejtimment. (3se= rabe hierin liegt an meipten bie Beränberfichfeeit bes funangieflen llu= triches begrüutoct.

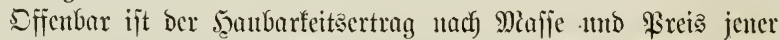
Factor, won welchem bie relative Şb̈be des fünuziellen llmtricbes am meiften abbängig blcibt. Slym ijt beshalb auch worzugsweije bei Er= mittclung bes Iebsteren porgfältigite Bead)tutg zu jochenten. Pach bent

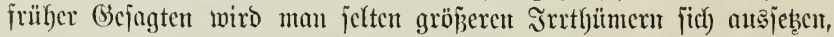
jelbijt wenn man für bicje Ermittelung nur ocn stbtricbsertrag in

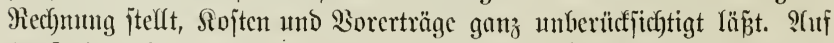

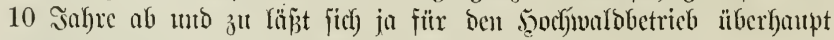
gar feit llutrieb genau bejtinment. 


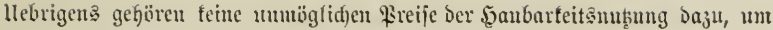
ziemlid) hobe, finanjielle llutriebe ju rechtfertigen. Sollte z. 3. bei $3 \%$ Der 120 jährige llutrieb biejelbe Bobennettorente getwähren, wie jeß̨t Der 90jährige, jo

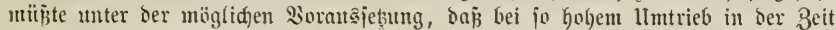

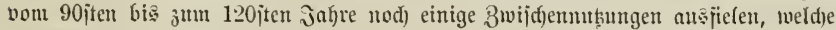
an Ed)lujije bes lețteren 30 fi. werth frien, Der 120 jähr. Bejtand folgenden ernte= fujtenjecen Rireis haben:

$$
\frac{\mathrm{r}^{\prime}\left(1,03^{30}-1\right)}{0,03}+\mathrm{H}_{90} \times 1,03^{30}-30=7294 .
$$

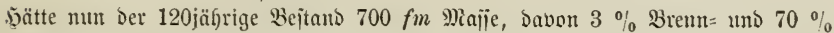
Yutşbol

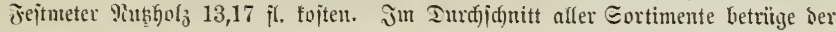

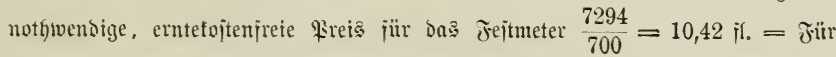
jo fojtbare Siaare fein unerid)winglidfer Betrag. Freilid ijt babei vorauggejestit,

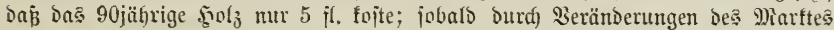

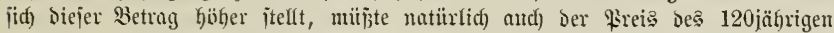

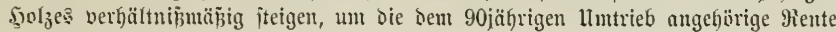
3น getvähren.

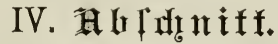

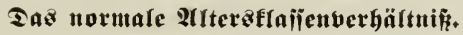

$\$ 28$.

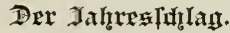

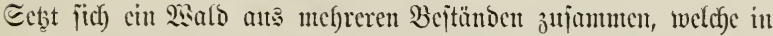

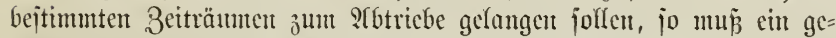

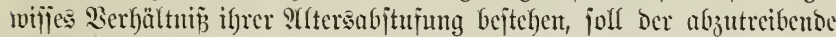
bejtand itets bas normale sanbarfeitsalter erreichen.

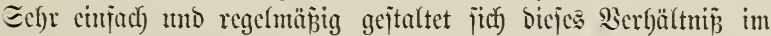

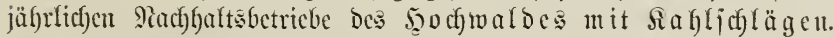

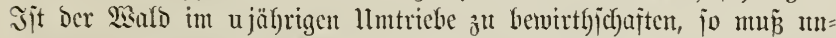
mittelfar nach bem in Winter erfolgenden ?(btrieb eine Bejtunbesreige

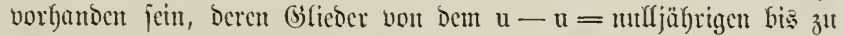
bem $n-1$ jüfrigen bejtande volljtändig vertreten füto. Llmmittelfar

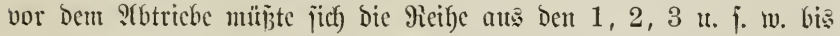

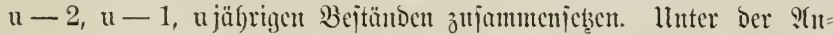

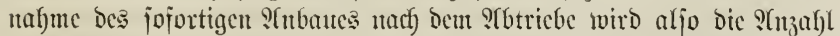

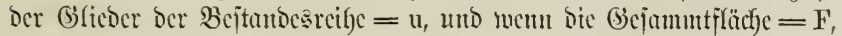

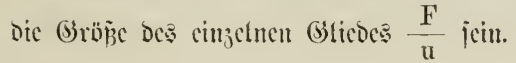


Ia nu beim jüfrlicfen Machbaltäbetrieb alle Jabre cin Bsfied

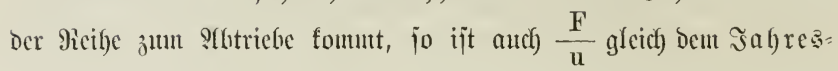
joflage i.

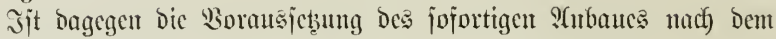

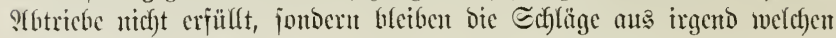

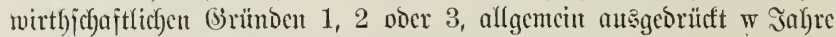

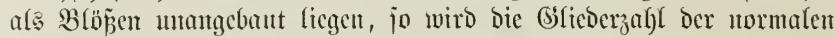

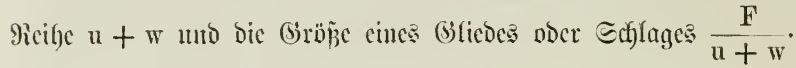

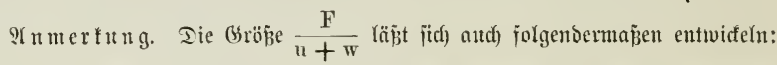

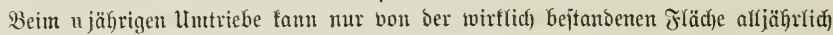

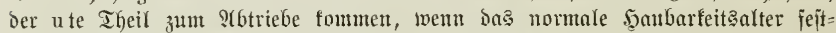

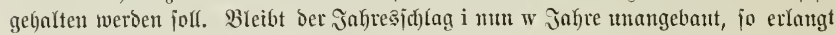
er mur sie Griȫe von $i=\frac{F-w i}{u}=\frac{F}{u+w}$.

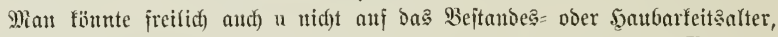

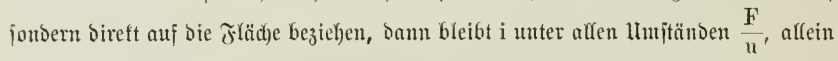

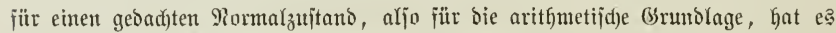

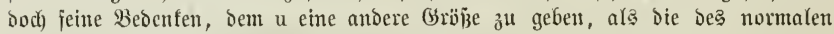

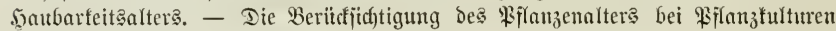

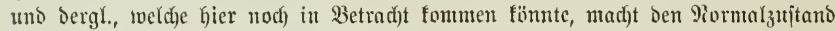
complicitter, ars nötthig ijt.

Im sochiolde mit qifenteridglaghetricb, wo aljo fünit= fiche voer natürfiche Sorverjüngung erfolgt, weroen mefrere Safores=

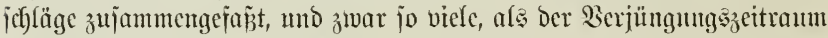

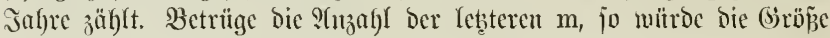

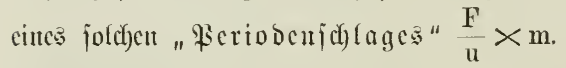

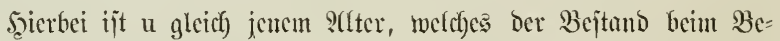

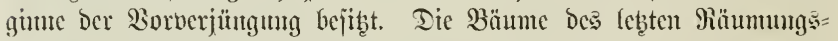
ichfages würden $u+m$ jäfrig.

3. B. Cin 2 Saro von $120 \mathrm{ha}$ im 100jäbrigen Itmtriebe mit 10jährigem $3 \mathrm{er}=$

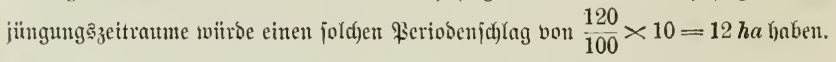

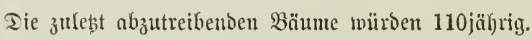

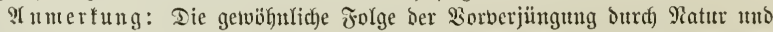

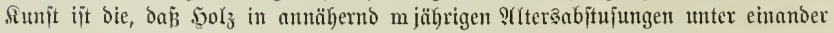

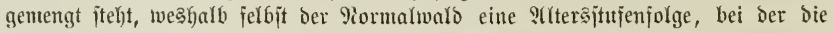

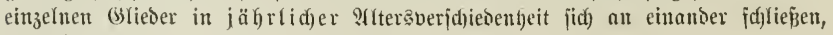
nicht bejigt. 


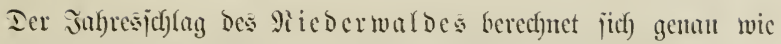

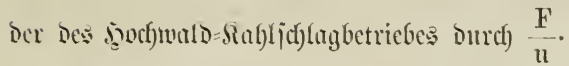

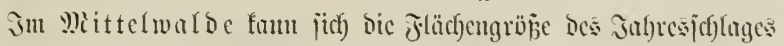

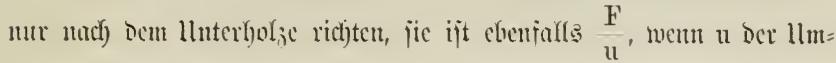
trich bes leţteren.

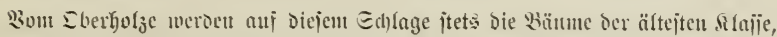

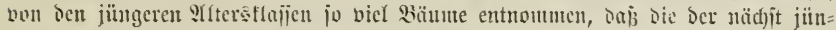

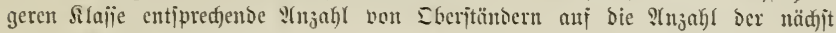
älteren Slajīe rebucirt wiro.

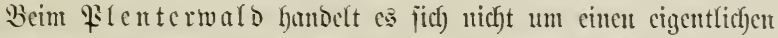

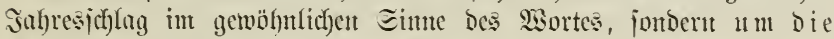

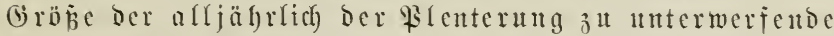

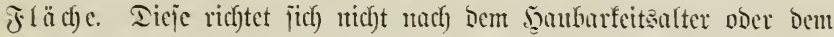

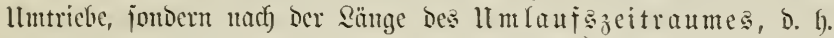

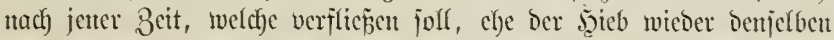
gisaldotheil trifft. - Bejecchnen wir biejen 3eitraum mit $l$, jo ijt bie

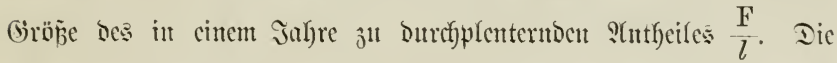

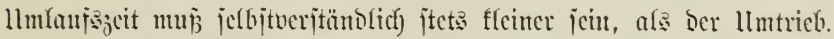

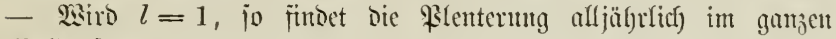
Sisuldo jtutt.

\section{$\$ 29$.}

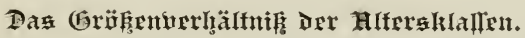

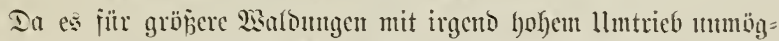

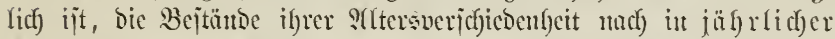

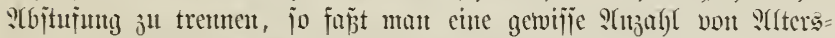

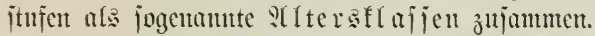

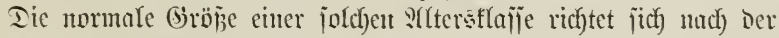

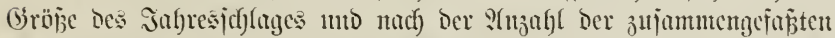

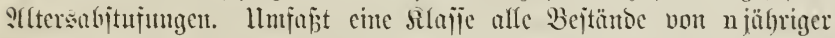

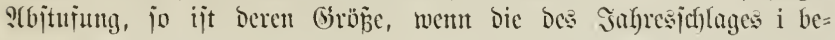
trägt, $\mathrm{n}$ i.

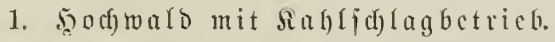

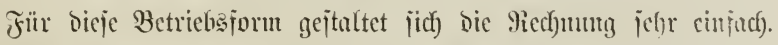
— Eine s(ltersflajie A ijt gleid) $n$ i, aljo $=n \frac{F}{u}$ ooer $n \frac{F}{u+1}$ ooer 


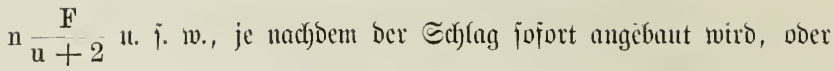
1,2 นt. j. w. Jahre liegen bleibt.

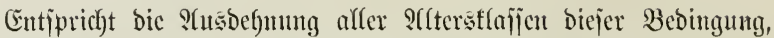

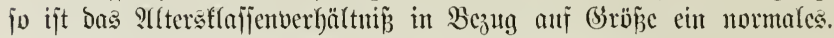

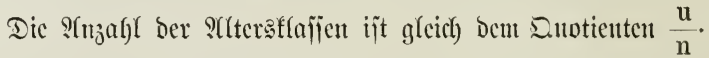

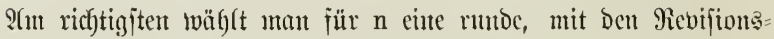
zeiträumen wenigitents in jo roeit übereinjtimmende 3 ahbl, baj fic cint Bielfaches biejer Beitrüume ijt.

S(nfä̈tglich) lag wol)l meijt bie Joec vor, ben llmfang ber :(lters:

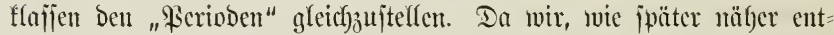

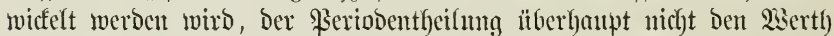

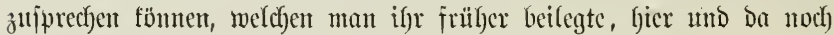
beilegt, jo vermögen wir and nicht biejer soce juzujtimment. - In cinigen 23 irthichaften, 3. 2 . in Bayern, bejtebt noch hente ber (Ge=

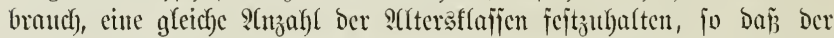

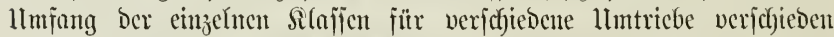

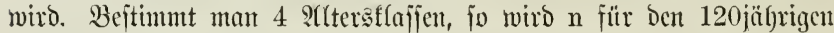
IImtrieb gleich) 30 , fütr Den 60jührigen gleidh 15 แ. j. เo. Diejes 23er= falyren madyt jebe flare lleberficht über bas wirffiche s(Ytersflajjen=

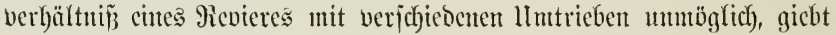

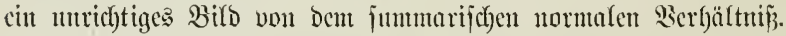

Scben wir im Folgentoen $n=20$, jo foll Damit utidft ats=

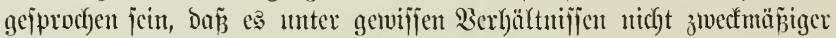

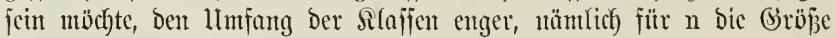
10 zll wäl)(en. *)

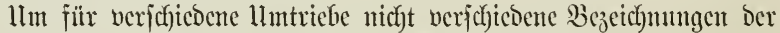

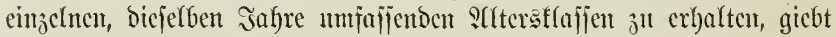

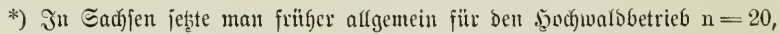

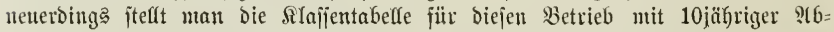

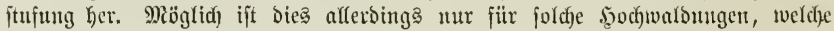

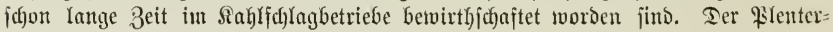
jdylagbetrieb mit natürlidber ober fünfllicher Borberjüngung erzengt Bejtänbe, Deten

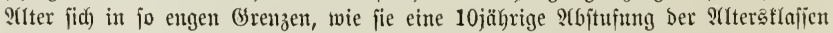

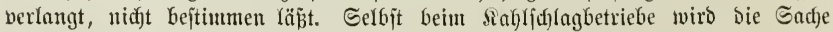

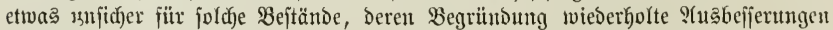
nöthig machte. Jmmerhin bleiben aber bie möglidger rseife babei vorfommenden Srrthümer ohne ftörende Bebeutmg für bie praftifd)e S(utwendung. 


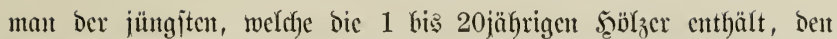
Sianten ber erjten, 1. j. w.

Siv veriteben aljo unter

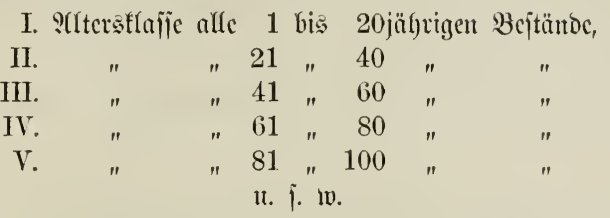

1. Beifpier. Čin 1200 ha grojerer $\mathfrak{B a r b}$ forf in 100jägrigem llmtriebe bewirthidfaftet werben. Die Sdbläge foument jofort zum Shubau, fo wirb eine 2rterstlafie $\mathrm{A}=\mathrm{n} \frac{\mathrm{F}}{\mathrm{u}}=20 \times \frac{1200}{100}=240 \mathrm{ha}$.

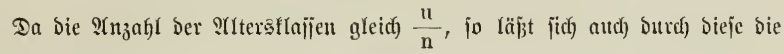
fraglicje Größe finten:

$$
\frac{\mathrm{u}}{\mathrm{n}}=\frac{100}{20}=5 \text { und } \frac{1200}{5}=240 \mathrm{ha} .
$$

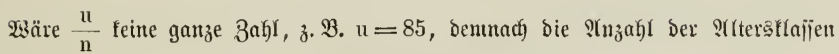

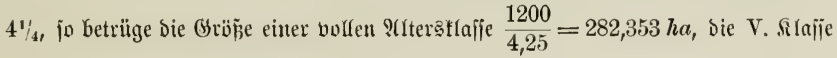
wäre nur mit $1 / 4$ ber vollen Fläcje, aljo mit 70,588 vertreten. - 3 u bemjelben

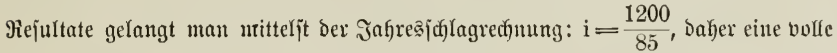

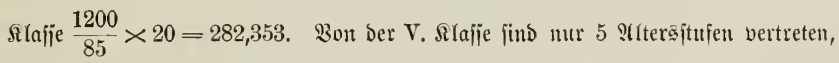
Destn 86 biß 100 jäfriges şorz foumt nidgt bor, Dafer

$$
\frac{1200}{85} \times \frac{n}{4}=\frac{1200}{85} \times 5=70,588 .
$$

2. Beifpier. Ein $\mathfrak{3 a l b}$ von $1164 h a$ joll im 95jäfrigen ltmtriebe bewittf= inaftet werben, bie Sdyläge b(eiben nad) Dem S(btriebe żwei Jahre unangebaut

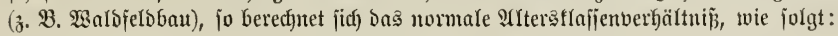

$$
\mathrm{i}=\frac{1164}{95+2}=12 h a \text {. }
$$

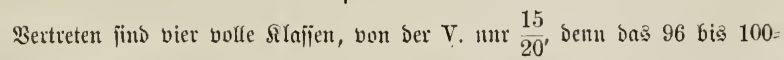

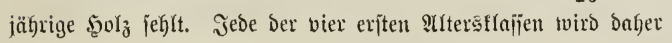

$$
20 \times 12=240 h a,
$$

bie ältejte, V. Dagegen mur

$$
15 \times 12=180 h a
$$

umfajien. 


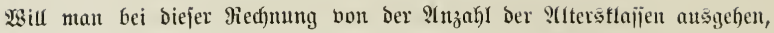

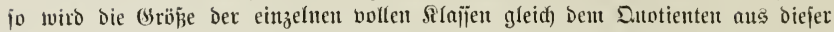

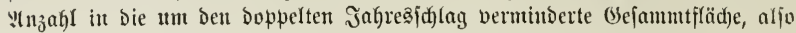

$$
\frac{\mathrm{F}-2 \mathrm{i}}{\frac{\mathrm{u}}{\mathrm{n}}}=\frac{1140}{4,75}=240 .
$$

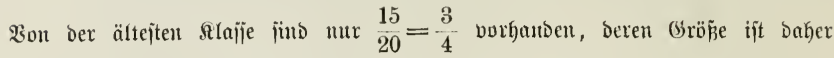
$240 \times \frac{3}{4}=180$.

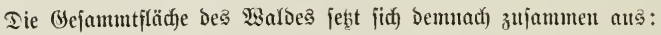

$$
\begin{aligned}
& \text { I. + II. + III. + IV. z’l } 240=4 \times 240=960 h n \text {, } \\
& \text { v. }=180 \text { " }
\end{aligned}
$$

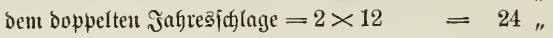

$$
\begin{aligned}
& \mathrm{F}=1164 h a \text {. }
\end{aligned}
$$

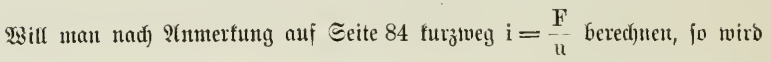

$$
\mathrm{i}=\frac{1164}{95}=12,2527 .
$$

eine bolle P(Iterăllaije Demnad)

$$
12,2527 \times 20=245,05 .
$$

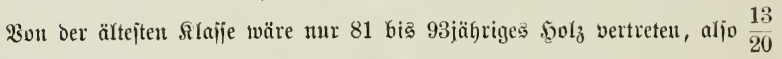

$$
12,2527 \times 13=159,3 \text {. }
$$

Siernad):

$$
\begin{aligned}
& \text { I. + II. + III. + IV. }=245,05 \times 4=980,2 \mathrm{ha}, \\
& \text { V. }=159,3 \text { " }
\end{aligned}
$$

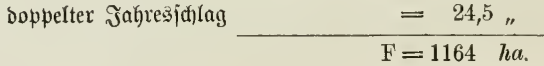

\section{Plenterjdylagbetrieb.}

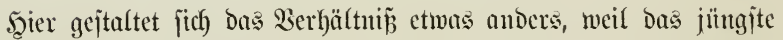
und ältejte $\mathfrak{S}_{2} \mathfrak{d}$ z

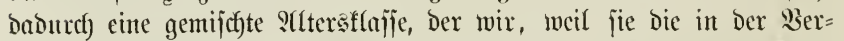

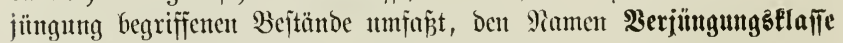
beilegen.*)

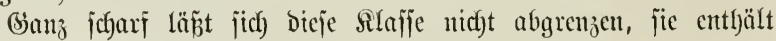

*) In Sadjfen nannte man jrïfer bie ßerjïngungăfajie "Betriebsflafie",

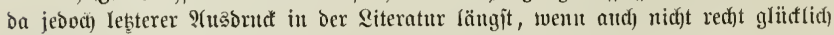
gewuablt, an einen anderen Begriff vergeben ift, jo wax es nütfig, ein anderes Wort einzuführen. 


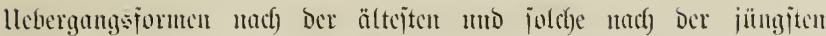

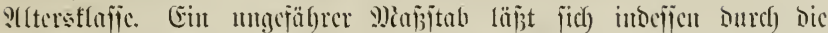

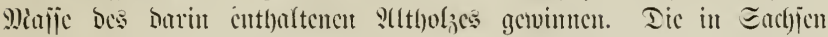

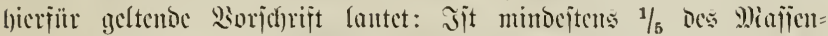

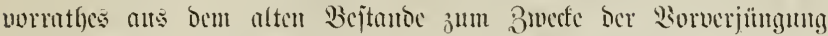

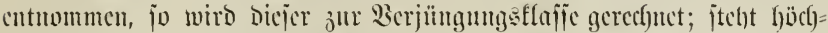

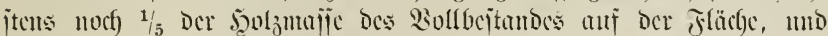

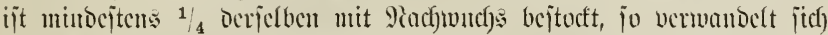
bic Sierjünguntystlajic in bic jüngjte Sflterstajie.

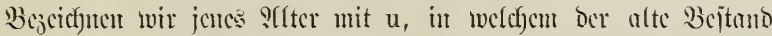

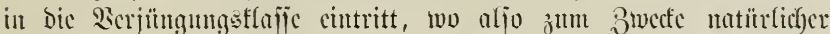

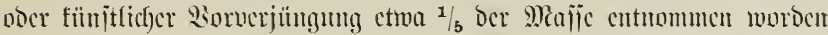

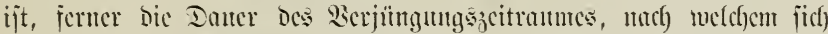
bie Serjüngungsflafje in bic jüngite ?flersflajic verwantodt, mit $\mathrm{m}$, bic Beriüngungstafic mit $A_{v}, F$ uno $n$ befalten bic angenommente Bebeututut.

a. Eebt man voras, Dañ mit bem eriten Eintritte bes alten Bejtandes in bic Beriüngungsfafic $\left(A_{v}\right)$ Dic Borveriünyung in ber Seatptiache volfenoct jei, jo baj ber weitere Eleritand un unch bic Bescututg von Echubbäumen foabe, jo bered)ret fich bas normale

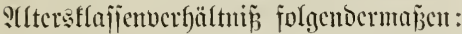

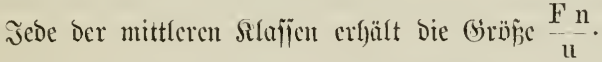

Die Scrjüngutgastlajic $A_{v}$ miro $\frac{\mathrm{Fm}}{\mathrm{u}}$.

Die jüngite, crite ?llersflajie ijt zum Theil in Der Qseriüngungs= flafic enthalten, jie eridfeint, went $\mathrm{m}<\mathrm{n}$, in ber stusdefuming vout

$$
\mathrm{I}=\frac{\mathrm{F}(\mathrm{n}-\mathrm{m})}{\mathrm{u}} \text {. }
$$

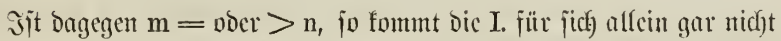

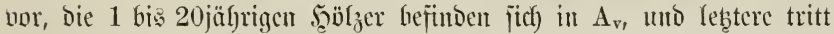

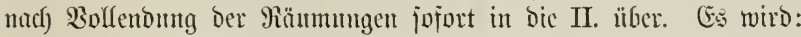

$$
\begin{aligned}
\mathrm{I} . & =\Re \mathfrak{U} \text { U, } \\
\mathrm{II} . & =\frac{\mathrm{F}(2 \mathrm{n}-\mathrm{m})}{\mathrm{u}} .
\end{aligned}
$$

Beifpiel: Fïr einen sials bon 1200 ha fei $u=120, \mathrm{~m}=10$, fo find bie

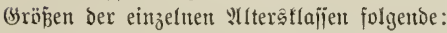




$$
\begin{aligned}
& \text { I. }=\frac{1200}{120} \times(20-10)=100 h a, \\
& \text { II. }=\frac{1200}{120} \times 20=200 \text { " } \\
& \text { III. + IV. + V. + VI. }=\frac{1200}{120} \times 20 \times 4=800 \text { " } \\
& A_{\mathrm{v}}=\frac{1200}{120} \times 10=100 \text { ， } \\
& \text { Ģäre } m=20 \text {, fo wïrbe } \\
& \mathrm{F}=1200 h a \text {. }
\end{aligned}
$$

$$
\begin{aligned}
& \text { I. }=\frac{1200}{120} \times(20-20)=0 \mathrm{ha}, \\
& \text { II. + III. + IV. + V. + VI. }=5 \times 200 \quad 1000 \text { " } \\
& \mathrm{A}_{\mathrm{v}}=\frac{1200}{120} \times 20 \quad=200 ，
\end{aligned}
$$

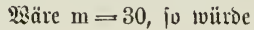

$$
\begin{aligned}
& \mathrm{F}=1200 h a \text {. } \\
& \begin{aligned}
\text { I. } & =0 \mathrm{ha}, \\
\text { II. }=\frac{1200}{120} \times(2 \times 20-30) & =100 "
\end{aligned} \\
& \text { III. + IV. + V. + VI. }=4 \times 200=800 \text { " }
\end{aligned}
$$

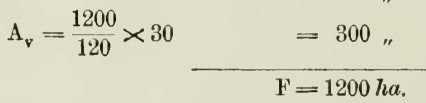

b. Siummt man an, Daj narfy dem llebertritte Des alten Bejtandes in bie Berjüngungstlajie nodh w Sahre vergehen, ehe bie Begründung

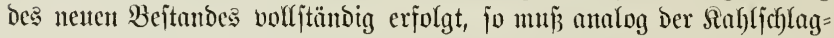
rect)mung, wemt ber Eablag w Sahre liegen bleibt, biefer nicht mit $\frac{\mathrm{F}}{\mathrm{u}}$, fondern mit $\frac{F}{u+w}$ in Rechnung geitellt weroen. Die 2erjüngung

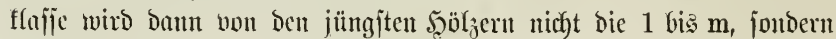
nur die 1 bis $\mathrm{m}$ - wjübrigen enthalten.

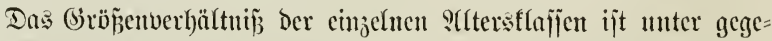
bener Sorausjeţung folgentes:

$$
\mathrm{I} .=\frac{\mathrm{F}}{\mathrm{u}+\mathrm{w}} \times(\mathrm{n}-(\mathrm{m}-\mathrm{w})),
$$

II., III. u. j. w. $=\frac{\mathrm{F}}{\mathrm{u}+\mathrm{w}} \times \mathrm{n}$,

$$
A_{v}=\frac{F}{u+w} \times m ;
$$


Docr fïr Delt Fall, Daj;

$$
\begin{aligned}
(\mathrm{m}-\mathrm{w}) & =\text { voer }>\mathrm{n}, \\
\text { I. } & =9 \text { iull, } \\
\text { II. } & =\frac{\mathrm{F}}{\mathrm{u}+\mathrm{w}} \times(2 \mathrm{n}-(\mathrm{m}-\mathrm{w})),
\end{aligned}
$$

III. u. j. w. $=\frac{\mathrm{F}}{\mathrm{u}+\mathrm{w}} \times \mathrm{n}$,

$$
A_{v}=\frac{F}{u+w} \times m,
$$

Beifpiel. Für cinen 1200 ha groben sisalo fei $\mathrm{u}=120, \mathrm{~m}=20$ uno $w=5:$

$$
\begin{aligned}
\text { I. } & =\frac{1200}{120+5} \times(20-(20-5))=48 h a, \\
\text { II. u. Ғ. } & =\frac{1200}{120+5} \times 20=192, \text { Dafcr }
\end{aligned}
$$

$$
\begin{aligned}
& \text { II. + III. + IV. + V. + VI. }=\quad 5 \times 192=960 "
\end{aligned}
$$

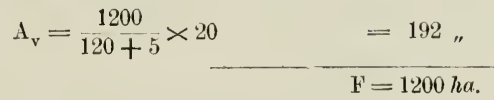

ख3äre $\mathrm{m}=30$ unt $\mathrm{w}=5$, fo wito

$$
\begin{aligned}
& \text { I. }=0 \text { ha, } \\
& \text { II. }=9,6 \times(2 \times 20-(30-5))=144 "
\end{aligned}
$$

III. u. f. $=9,6 \times 20=192$, onfer

$$
\begin{aligned}
\text { III. + IV. + V. + VI. } & =4 \times 192 \\
A_{\mathrm{v}} & =9,6 \times 30 \quad=768 " \\
& =288 " \\
\quad \mathrm{~F} & =1200 \mathrm{ha} .
\end{aligned}
$$

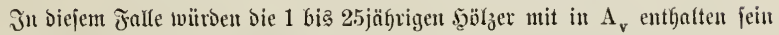

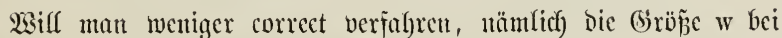
Beredynung bes Schlages unberïdjichtigt lajien, bicje aljo anjtatt mit

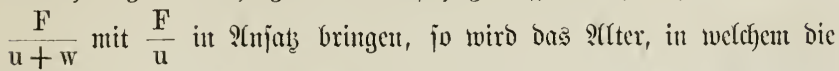
Bejtünde in bie 2ierjüngungsflafje treten, um joviel fleiner als $u$, ala w Salyre bebentet.

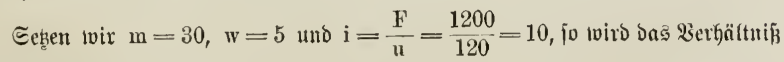
Der YItergła fajen: 


$$
\begin{aligned}
& \begin{aligned}
\text { I. }= & =0 \mathrm{ha}, \\
\text { II. }=10 \times(2 \times 20-(30-5)) & =150 ",
\end{aligned} \\
& \text { III. }+\mathrm{IV}+\mathrm{V} .=3 \times 200 \quad=600 \text { " } \\
& \text { VI. }=10 \times(20-5) \quad=150 \text { " } \\
& A_{\mathrm{v}}=10 \times 30 \quad=300 " \text { " } \\
& \mathrm{F}=1200 \mathrm{ha} .
\end{aligned}
$$

II. enthält die 26 bis 40jägrigen, VI. Die 101 bis 115jägrigen Bejtände.

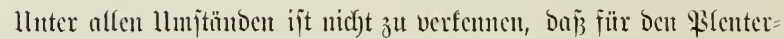
ichlagbetrieb, namentlich) fïr bent mit natürlicker Borverjüngung, Der

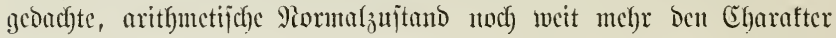
Des soealen trägt, als fïr ben Siaflid)lagbetrieb. Plan wiro baber

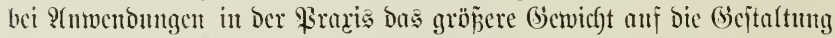
Der mittleren altersflafjen ju legen haben. Dic Berjüngutgâflaj̣e, bic ältejte uno jüngite, bei jebr langent Berjüngungsjoctraum ant bic

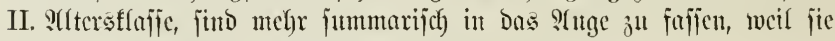

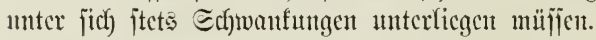

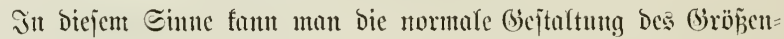

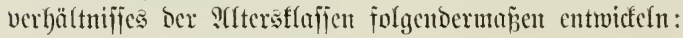

a) Ilnter Der Soransjeb̧ung, baj bie Serjüngung fofort mit bem Eintritte des âthoulges in Dic Werjüngungsflaffe gelingt, mo muter

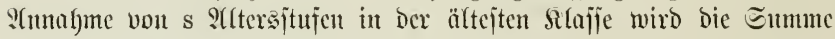

$$
\begin{aligned}
\text { Aältejte }+A_{v}+I .= & \frac{F}{u} \times s+\frac{F}{u} \times m+\frac{F}{u}(n-m) \\
& =\frac{F}{u}(s+n) .
\end{aligned}
$$

Dicje Eumme ijt aljo glcich) Der Eumme aus ber ältejten mo ciucr bollen slltersflafje.

2aill man bei langent Serjünguntgscitraunte nod) bie II mit cin= rechnen, fo erbält man bie Eumme ans ber ältejten unb zwei vollen Sutersflafjen:

$$
\text { Aältẹte }+A_{v}+I .+I I .=\frac{F}{u}(s+2 n)
$$

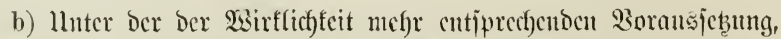

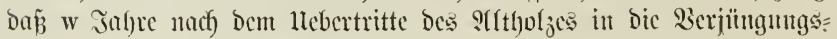
flajje verflię̃en, che bic Berjüngung erfolgt, wird bic Summe 


$$
\begin{gathered}
\text { Aültejte }+A_{v}+I . \\
=\frac{F}{u+w} \times s+\frac{F}{u+w} \times m+\frac{F}{u+w}(u-(m-w)) \\
=\frac{F}{u+w}(s+w+n) .
\end{gathered}
$$

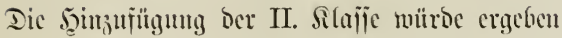

$$
\frac{\mathrm{F}}{\mathrm{u}+\mathrm{w}}(\mathrm{s}+\mathrm{w}+2 \mathrm{n}) \text {. }
$$

Beipiel. $\mathrm{F}=1102, \mathrm{~m}=24, \mathrm{w}=6, \mathrm{u}=110$, fo wirs

$$
\frac{\mathrm{F}}{\mathrm{u}+\mathrm{w}}=\frac{1102}{110+6}=9,5 \mathrm{ha} \text {. }
$$

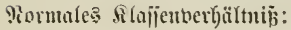

$$
\begin{aligned}
& \text { VI. }+A_{v}+I .=9,5(10+6+20)=342 h a, \\
& \text { II. }=9,5 \times 20=190 \text { " } \\
& \text { III. }=9,5 \times 20 \quad=190 \text { " } \\
& \text { IV. }=9,5 \times 20 \quad=190 ， \\
& \mathrm{~V} .=9,5 \times 20 \quad=190 \text {, } \\
& \mathrm{F}=1102 h a \text {. }
\end{aligned}
$$

Da jiff) mun bie Formeln unter b von jelbjt auj bic Soraus=

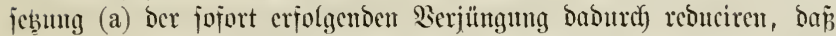

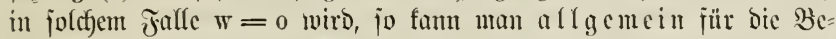
rech)mutg jolgende ?4:

Juhalt ciner vollen : Sltersflafie: $\frac{\mathrm{F}}{\mathrm{u}+\mathrm{w}} \times \mathrm{n}$.

$$
\begin{aligned}
& A_{\text {ältejte }}+A_{v}+I .=\frac{F}{u+w}(s+w+n) . \\
& A_{\text {älteìte }}+A_{v}+I .+I I=\frac{F}{u+w}(s+w+2 n) .
\end{aligned}
$$

3. Picderivald.

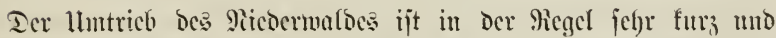

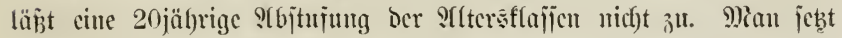

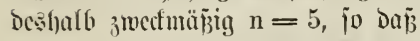

bic I. SAltersflajie dic 1 bis 5jäbrigen,

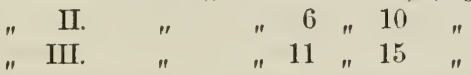

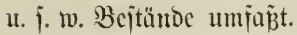




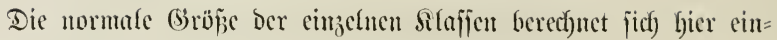
fach) Durch $\frac{\mathrm{Fn}}{\mathrm{ul}}$.

Beifpier. CEin Rieberivalo von 180 ha im 18 jägrigen llmtriebe mïrbe nor= mal bejtodt jein, wenn

$$
\begin{array}{r}
\text { I. }=\frac{180}{18} \times 5=50 \mathrm{ha} . \\
\text { II. }=\frac{180}{18} \times 5=50 \quad \\
\text { III. }=\frac{180}{18} \times 5 \times 50 " \\
\text { IV. }=\frac{180}{18} \times 3=30 " \\
\text { F }=180 \mathrm{ha} .
\end{array}
$$

\section{Mittelwald.}

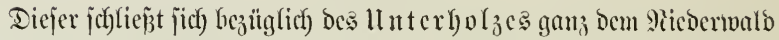

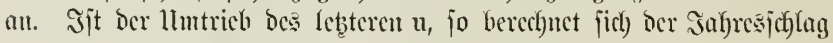

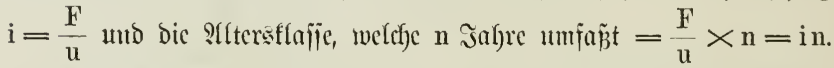

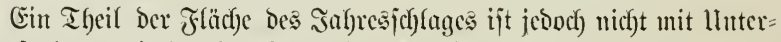

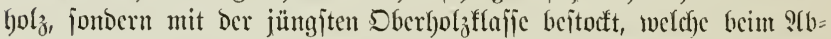
tricbe bes lluterlyolzes ühergebalten wird. Sit ber llutricb Des Sber=

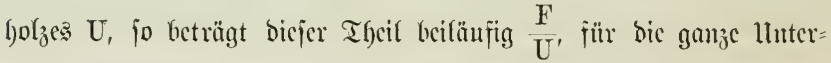

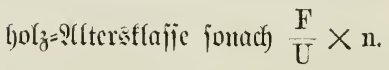

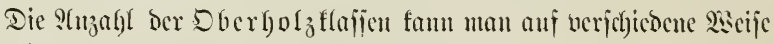
crmitteln.

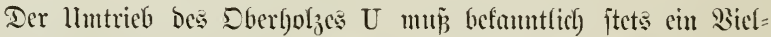

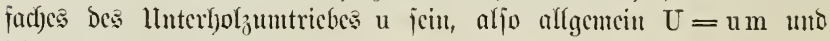
$\frac{\mathrm{U}}{\mathrm{u}}=\mathrm{m}$. Redunct man um die jüngite Sitafie bes Socrgor zes zum

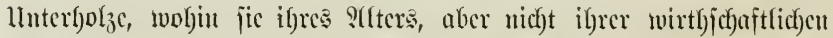
Bcbcutung wegen geflört, jo crgicbt jich) für bic ujäl)rig abgejtuften

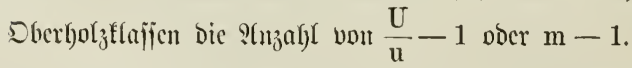

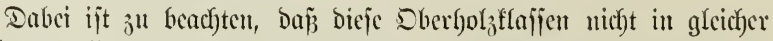

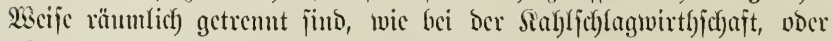

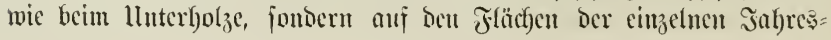




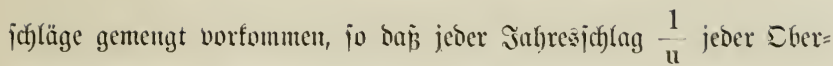
holzftajie cuttyält.

3eifpiel. Ein 120 ha groper Mittelwals, Deijen $\mathrm{U}=60$, dejien $\mathrm{u}=15$,

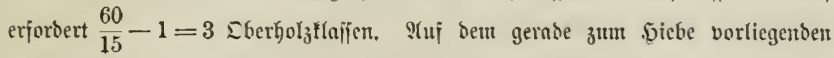

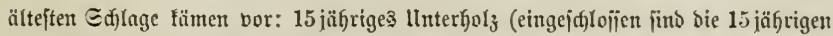

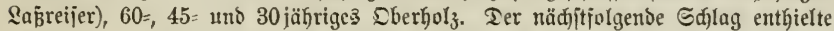

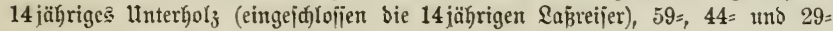
iähriges Sberholz; u. i. w.

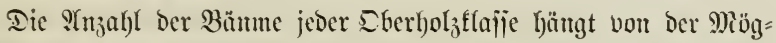

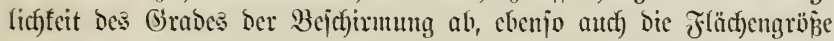

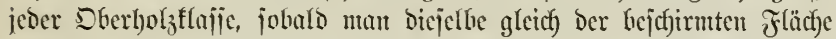
jeţen will.

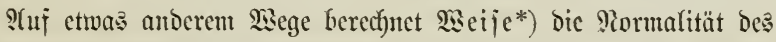

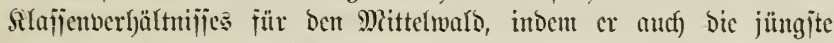

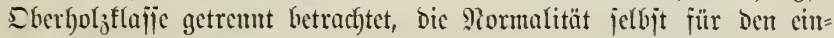
zelnen Echlag aufitellt.

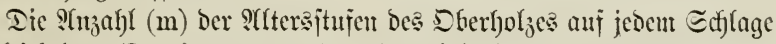
ijt gleich bem Dutotienten aus Dem ltmtriebe Des lluterfolzes in Den Des Dherholzes:

$$
\left.\frac{\mathrm{U}}{\mathrm{u}} \text {, ober wemu } \mathrm{U}=\mathrm{mu} \text {, aucf }\right)=\mathrm{m} \text {. }
$$

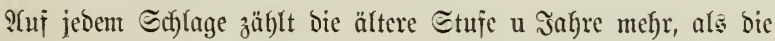

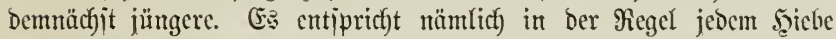
cine \$̉erjüntuntg.

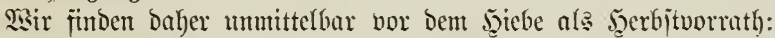

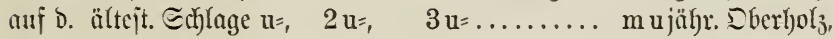
" " jüngit. " $1=, \mathrm{u}+1=, 2 \mathrm{u}+1=\ldots(\mathrm{m}-1) \mathrm{u}+1$ "

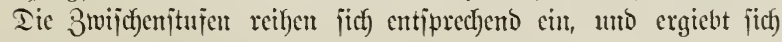

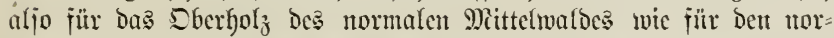

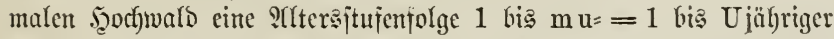

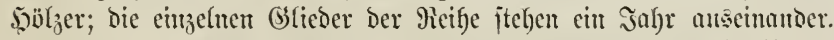

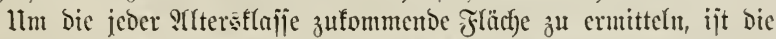

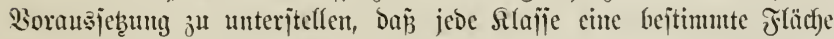

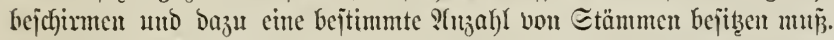
Die jüngite Silajie sviro aljo bie meijten, bie ältejte bie menigiten

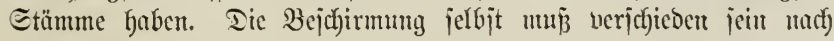

*) Wr. Weife: Die Taration Des Mittelwalbes. Berlin, 1878. 


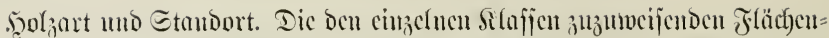

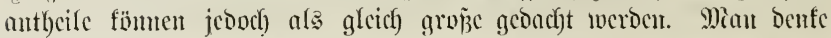

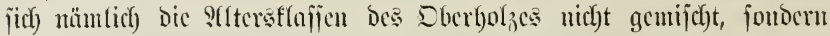

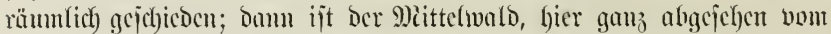

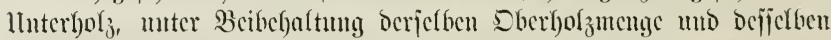

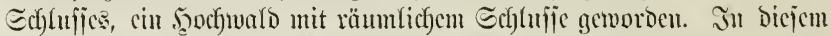

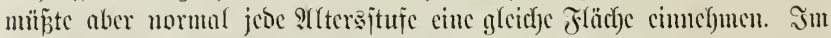

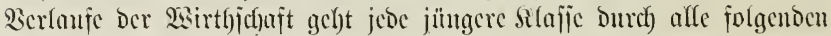
S(ltersjtupen hindurch bis jic als ältejte abgetrichen wiro, fie muis aljo

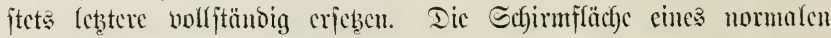
Sauptbauntes bleibt bicjelbe, ebenjo Der normale Edj)

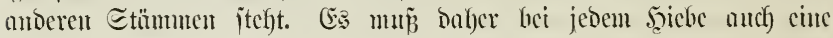

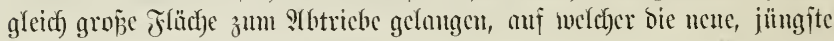

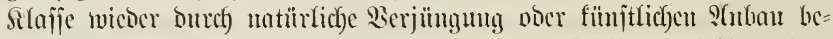

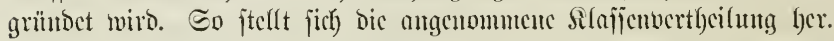

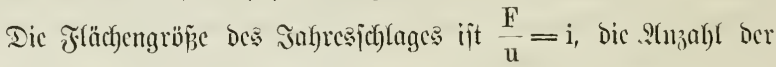

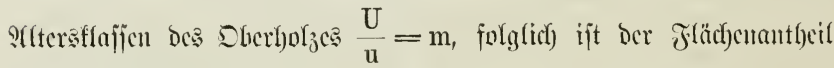

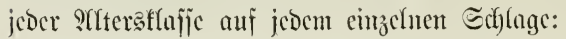

$$
\left.\frac{\mathrm{F}}{\mathrm{mu}}=\frac{\mathrm{F}}{\mathrm{U}}=\frac{\mathrm{i}}{\mathrm{m}} \cdot *\right)
$$

Da ntm ber gamze 2Salo aus u Schlägen bejtelt, fo witroc jebe

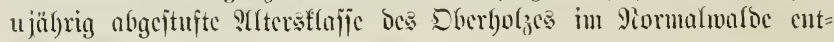

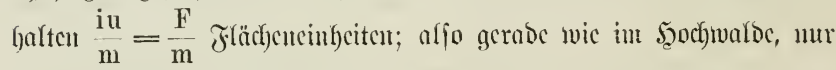

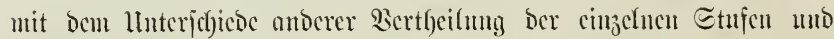

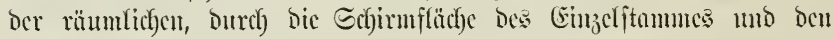
Echlın bebiugten Etcllung.

Bcijpiel. Der 120 ha grope Mittefwals, sejēen $U=60$, Dejen $n=15$,

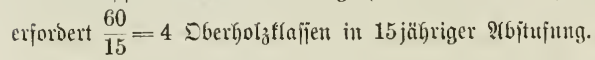

Ter normale Jahresjid)lag i ijt gleid) $\frac{120}{15}=8 \mathrm{ha}$.

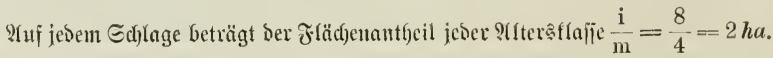

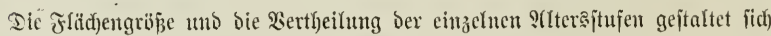
แนи jolgendermapen:

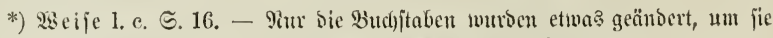
mit ben bon mir ïberbaupt angewenseten in Einflang zu bringen. 
Seltejter,

1. Ed)lag: llnterfyol $3: 8$ ha 15 jährig,

Piäd)itfolgender, 2. " Ilnterłolz: 8 ha 14 jäbrig,

Eberbol 2 : 2 ha 14, 2 ha 29=, 2 ha 44, 2 ha 59 jährig.

3. " Iluterfor

Eberfor $f_{3}: 2$ ha 13, 2 ha $28=, 2$ ha 43, 2 ha 58 jägrig, 4. $\{$. เv.

Sïngiter, 15. " llnterfyol $: 8$ ha 1 jägrig,

Ebergols: 2 ha 1=, 2 ha 16=, 2 ha $31=, 2$ ha 46 jährig.

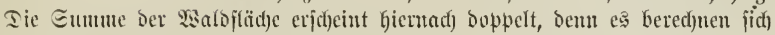
$15 \times 8=120$ ha fïr ธaร Untergol un $15 \times(2+2+2+2)=120$ ha für ธaล

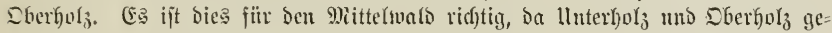
mengt auf berfelben Fläche vorfommen, und zivar bas lluterfolz and unter bem

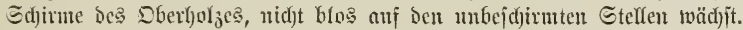

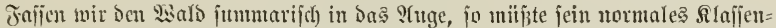

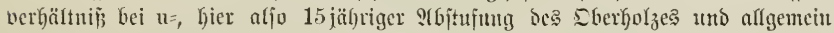

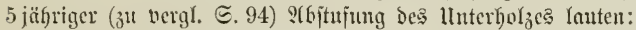

lluterfolz:

I. Sînafle 1 bis 5 jährig $40 h a$,

II. " 6 " 10 " 40 "

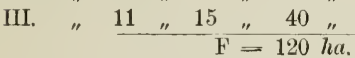

Dberifor

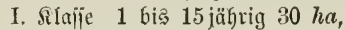

II. " 16 " 30 " 30 "

III. " 31 " 45 " 30 "

IV. " $\frac{46 \text { " } 60 \text { " } 30 \text { " }}{\mathrm{F}=120 \mathrm{ha} .}$

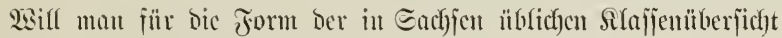

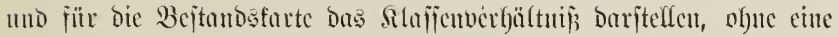

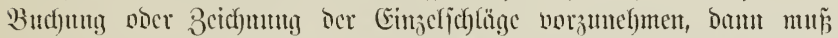

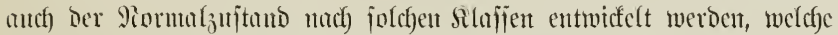

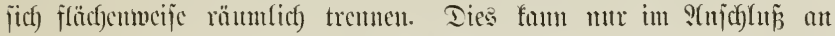

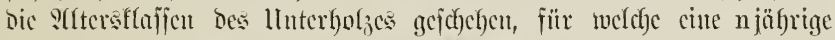

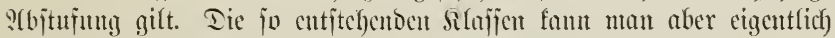

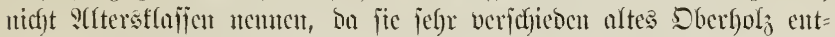

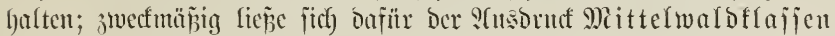
เväl)lent.

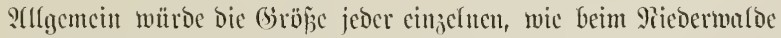

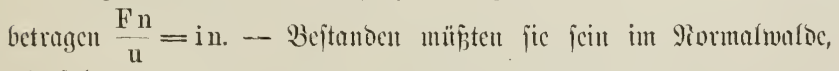
uvie folgt:
I. Mittelmalofl. 1luterl):
1 bis (jïngite)
Ebcrl).:
1
$u+1$
njälrig, in $h a$,

" $\quad$ "

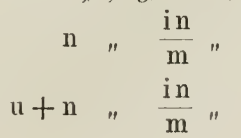

3ubeid, Foriteinridtumg. 4. 2uft. 
I. Mittefmaloff. Sberf): $\quad 2 u+1$ bis $\quad 2 u+n j a ̈ g r i g, \frac{\text { in }}{m} h a$, 11. j. w.

$$
\text { " }(\mathrm{m}-1) \mathrm{u}+1,(\mathrm{~m}-1) \mathrm{u}+\mathrm{n} \quad \text { " } \frac{\mathrm{in}}{\mathrm{m}} "
$$

II. Mittefwaloff. Iluterl): $\quad n+1 \quad$ bis

$$
\begin{aligned}
& \text { Sterf).: } n+1 \quad \text { " } n \quad \frac{\text { in }}{m} "
\end{aligned}
$$

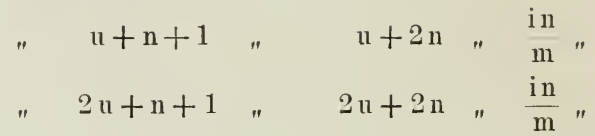

11. i. w.

$$
\text { " }(\mathrm{m}-1) \mathrm{u}+\mathrm{n}+1,(\mathrm{~m}-1) \mathrm{u}+2 \mathrm{n}, \quad \frac{\mathrm{in}}{\mathrm{m}} "
$$

III. Mrittelwaldfl. lluterh): $\quad 2 n+1 \quad$ bis

$$
\begin{aligned}
& \text { Dberf.: } 2 \mathrm{n}+1 \quad \text { " } \mathrm{n}, \frac{\mathrm{in}}{\mathrm{m}} \text { " }
\end{aligned}
$$

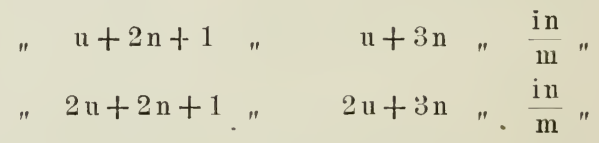

1t. i. w.

$$
\text { " }(\mathrm{m}-1) \mathrm{u}+2 \mathrm{n}+1,(\mathrm{~m}-1) \mathrm{u}+3 \mathrm{n}, \frac{\mathrm{in}}{\mathrm{m}} \text { " }
$$

\begin{tabular}{|c|c|c|c|}
\hline Dberyolz: & 1 & " 5 & \\
\hline " & 16 & " 20 & " \\
\hline " & 31 & " 35 & " \\
\hline$"$ & 46 & " 50 & $"$ \\
\hline
\end{tabular}

it. $\tilde{j}$. w.

Beifpiel. Fïr obigen Mittelwald ergeben fid) Gientad folgende Zahlen:

I. Mittelwaloflaife. Ilnterfol (jüngite)

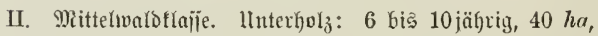

$$
\begin{aligned}
& \text { Dberfoly : } 6 \text { " } 10 \text { " } 10 \mathrm{ha} \text {, } \\
& \text { " } 21 \text { " } 25 \text { " } 10 \text { " } \\
& \text { " } 36 \text { " } 40 \text { " } 10 \text { " }
\end{aligned}
$$

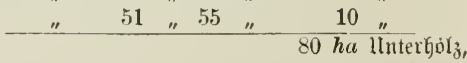

$$
\begin{aligned}
& 80 \text { ha } \text { ¿berfolla. }
\end{aligned}
$$


llebertrag: 80 ha lluterhol

80 ha Ekerfolz.

III. Mittelwalsflajic. Ilnterfold $: 11$ bis 15 jäfrig, $40 \mathrm{ha}$,

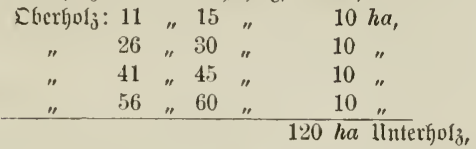

120 ha Eberfol?.

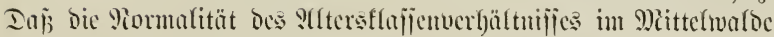
jebr idealer siatur ift, verjteft jich) won jelbjt. Smmerbiu fömnen mix

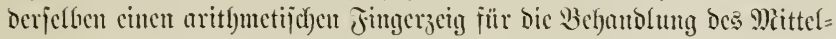
maldes entuchumen.

\section{Rilenterivald.}

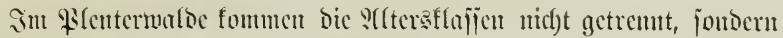
untereinmor gemenyt vor. Die s(bjtufung ber cinzelnen wälglt man am bejten gleich) ber llmfanjszeit vocr wenigjtens als cinen Duptienten ans Der letsterent.

Sit bic llmlaufsjeit $l$, ber llmtricb u, jo wäre im criten Falle die 3ahl ocr slltersflafīen $\frac{u}{l}$, im jweiten Falle, wenn cine Silaffe untr $\frac{l}{\mathrm{~m}}$ sah)re mminjīen jollte, $\frac{\mathrm{mu}}{l}$.

3 cifpiel. Eit 600 ha grojer \$lenterwalo im 120 jübrigen llmtriebe, mit $40=$

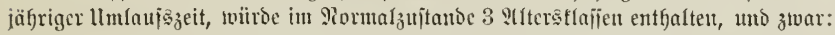
I. 1 bis 40 jähriges $\$ g o l$ z $\frac{600 \times 40}{120}=200 \mathrm{ha}$,

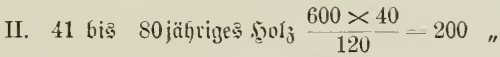
III. 81 bi 120 jäfriges . gold $\frac{600 \times 40}{120}=200$ "

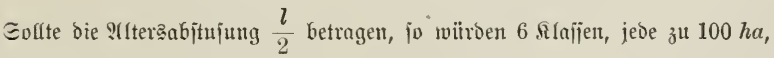
vorkander jein miijien.

\section{$\$ 30$.}

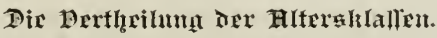

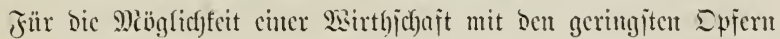

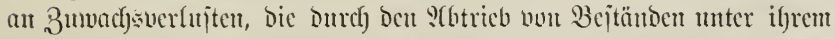
Southarfeitsalter oder Durd) Das Etehenlafien vou Beftäntoen weit über

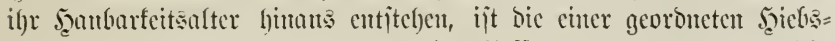

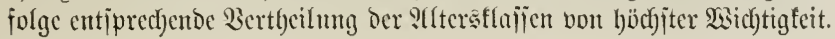




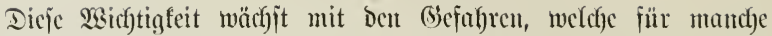

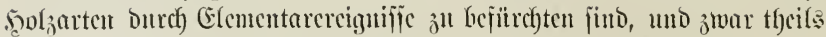

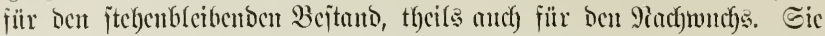

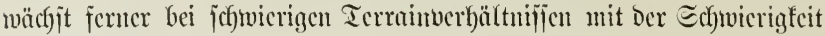
DCe 50 ol 3 tram sportes.

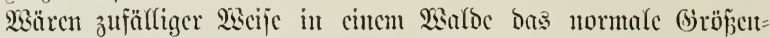

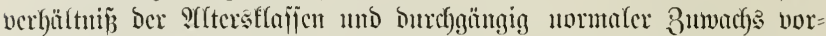

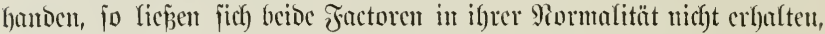

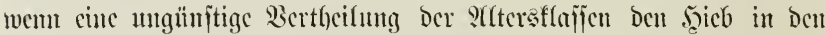
ältejten, bicbsreifen Bcitünden ummöglich madyt. - Durch abnorme

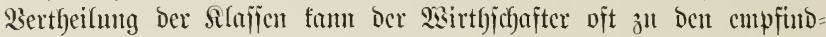
fichjten, funaubielfen spyern gejwungen werbent.

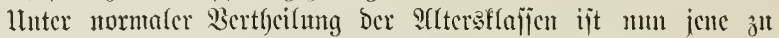
verjtelfen, weldac allen ?njorbernutgen einer richtigen Sgiebsjolge ent= ipricht.

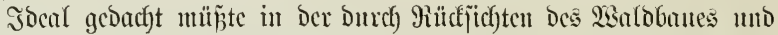

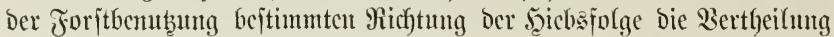

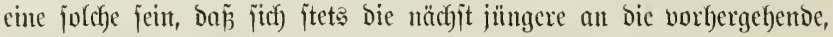

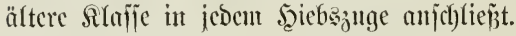

Beim Salgljoblagbetriebe mit 80 jäbrigen llmtricbe $\mathfrak{o}$. B.:

\section{\begin{tabular}{|l|l|}
\hline I. II. III. IV. \\
\hline
\end{tabular}}

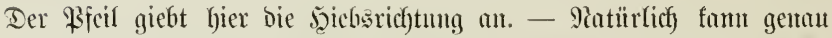

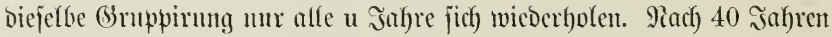
wïrde bic normale Sertl)eilung folgento jein:

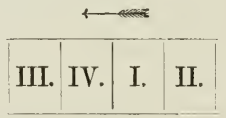

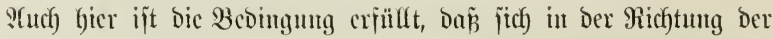
Seicbsjolge bie näedjt jüngere an bie vorbergebento ältere Silaffe ant= joblicint.

Unter S(unabme eincs ein= unto mel)rjührigen Echlages als ciner

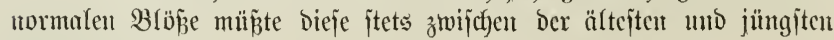
Slaffe licgen. 


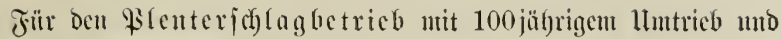

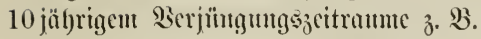

\begin{tabular}{|l|l|l|l|l|l|}
\hline I. & II. & III. & IV. & V. & $A_{\mathrm{v}}$ \\
\hline
\end{tabular}

Mach) 20 Sal)ren würbe folgende Figur Dem Mormalzuitand ent= iprecten:

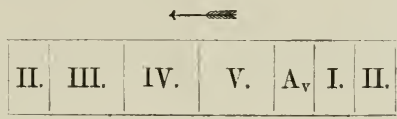

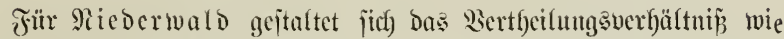

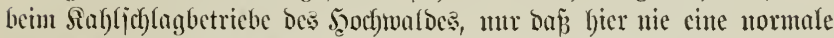

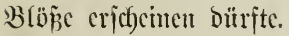

Für Mittelwạlo gilt bajielfe, mur bleibt bier hervorzuheben,

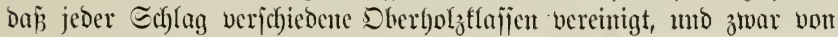

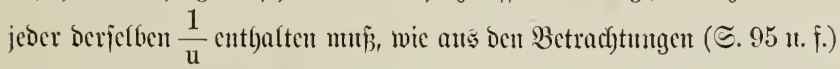

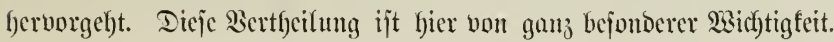

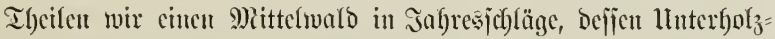
umtricb u, bejīen Dberfgolzamtricb 4 u ijt, jo crfalten wir folgende $\mathfrak{B} e r=$ theifung, wenn ber gerabe zum Syiebe vorliegende Eshlag mit 1, bie folgendon mit 2,3 it. j. w. u bezeidfnet werben.

u. $\mathrm{u}-1$.

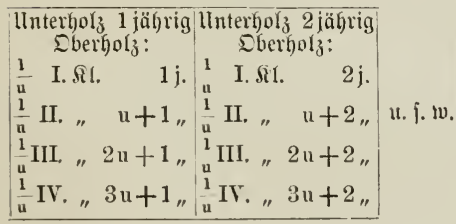

jüngiter Srflag.
2.

1.

Lnterf. u-1 jähr.|Unterhol Dberhorl 3 : Dbergorls:

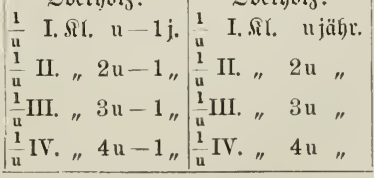

ältẹter Edylag.

Dic Schläge 1 bis 5 mürden ciute "Mittefmaldoflajfe" bilden, cbenjo Die Єajläge 6 bis 10 und 11 bis 15 . 
Ein normaler \$Slenterwald*) mit 120 jäfrigem Imtriebe und

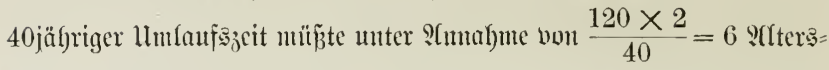
flafien unmittelbar vor Begim bes Şiebes folgente Slafjenvertbeilung nadyrocijent :

\begin{tabular}{|c|c|c|c|}
\hline \multicolumn{4}{|c|}{$\longleftarrow$} \\
\hline d. & c. & b. & a. \\
\hline $\begin{array}{l}\frac{1}{2} \text { V. } 81-90 . \\
\frac{1}{2} \text { III. } 41-50 . \\
\frac{1}{2} \text { I. } 1-10 .\end{array}$ & $\begin{array}{l}\frac{1}{2} \quad \text { V. } 91-100 . \\
\frac{1}{2} \text { III. } 51-60 . \\
\frac{1}{2} \quad \text { I. } 11-20 .\end{array}$ & $\begin{array}{l}\frac{1}{2} \text { VI. } 101-110 . \\
\frac{1}{2} \text { IV. } 61-70 . \\
\frac{1}{2} \text { II. } 21-30 .\end{array}$ & $\begin{array}{l}\frac{1}{2} \text { VI. } 111-120 . \\
\frac{1}{2} \text { IV. } 71-80 . \\
\frac{1}{2} \text { II. } 31-40 .\end{array}$ \\
\hline
\end{tabular}

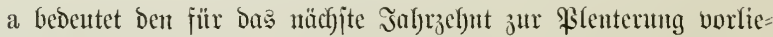
genden Theil, b Den für Das żweite, e Den für Das britte, d Den für

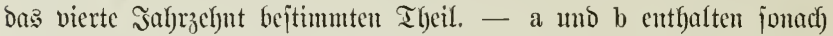
II., IV. Int VI., c uno d bic I., III. Into V. Slafie unter cintuber

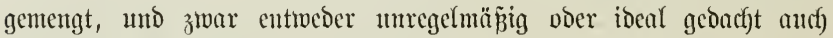
borjt $=$ oder itreifentecije.

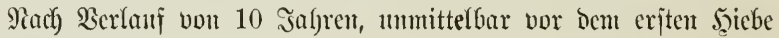

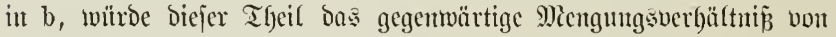
a zeigen, c bas vou b 1 . j. w.

Wäre ber ganje Warb 600 ha grof, a jonad) 150 , jo würoen in 10 Jafren 50, in cittem Jafje 5 ha horjtweije heransgefonten.

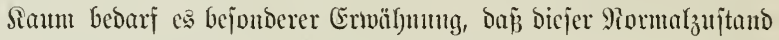

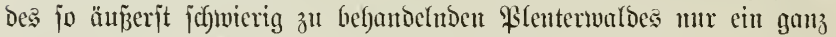

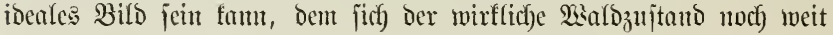

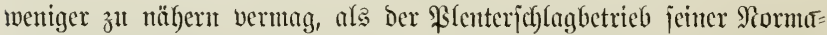

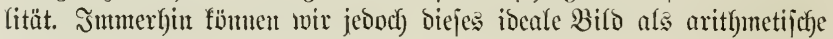

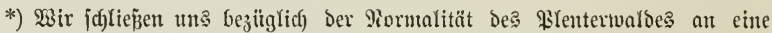

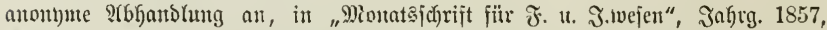

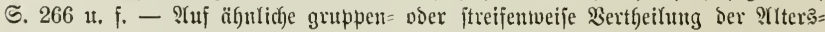

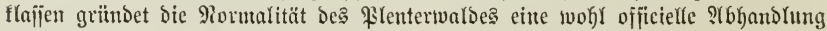

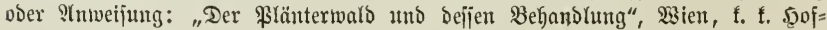
u. Staatsoructerei 1878.

Fin anderes, bead)tenswerthes Berfabren theilt Berenger im 25. Bande

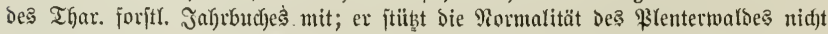

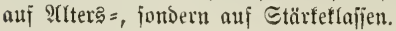




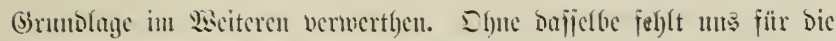
Foritcimrichtmuly Der leitentoc (jedanfe.

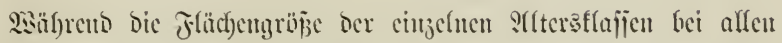

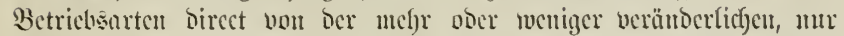

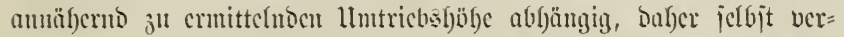

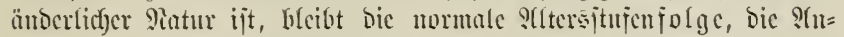

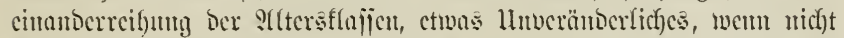

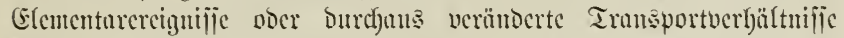

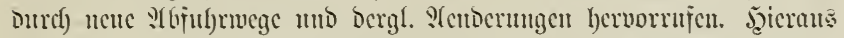

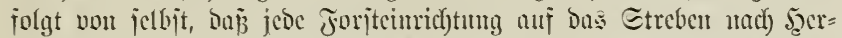

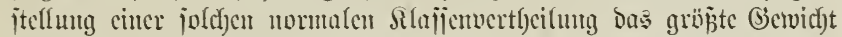
legen fam แmD

Snmerfung. Pid)t blos für Den jäbrlichen, jtrengen Padjhaltsbetrieb, jon:

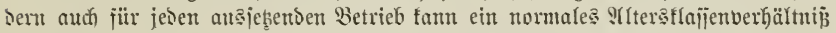

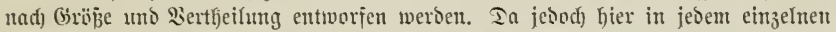
Jalle anters zu verjafren ijt, jo baj jid etwas sulfgemeines barïber nid)t aufitellen

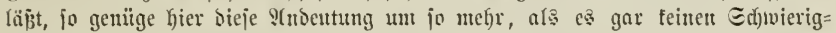
feiten unterliegt, Die Piormalität bes jüfrlidjen Pad)haltsbetriebes auj Den gegebe= nen einzelnen jall bes atsjeşenden ju übertragen.

\section{Alritgniff.}

\section{Scr Rorualuorath.}

A. Vou Stanopunkte der Jiaffenertragstegefung.

$$
\S 31 \text {. }
$$

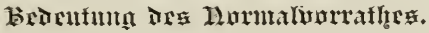

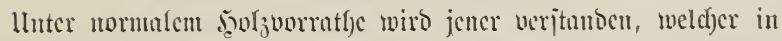

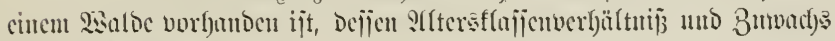
normul bejchaffen jitno.

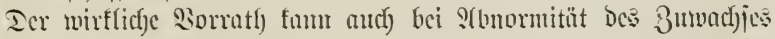

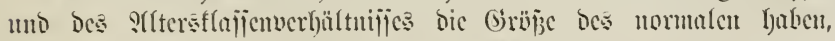

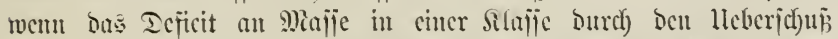

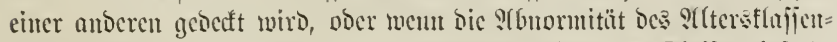

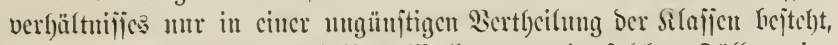

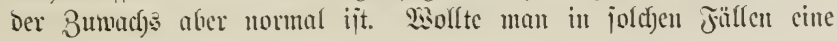


jäfrtich) gleidfe Şolzmajje joflagen, jo fömute bies mur mit ben bebeu=

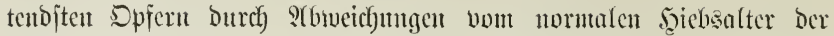
Bẹtänto gejchchent.

Bejtünde z. B. Der 2 sald ans ciner cinzigen slltersflajic nit nor

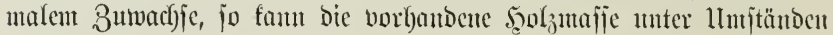

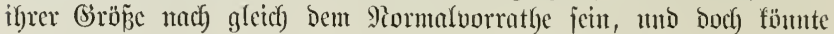

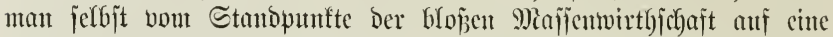

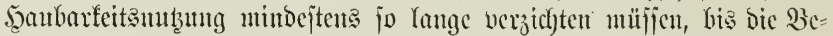
ftände abjab̧fäliges Material Yiejern. Dics jogar bant, went man

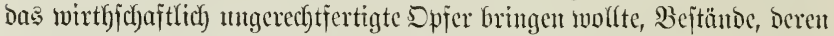

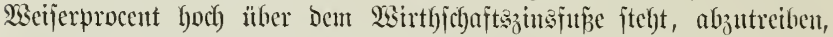

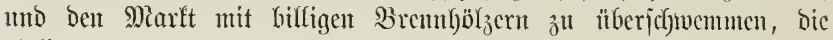

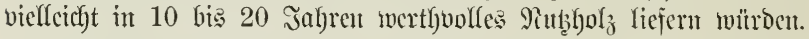

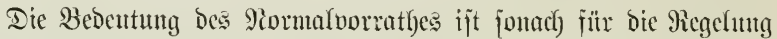
des Ertrages eine mur untergeorducte. Mrjacke des Pormaljujtandes überbaupt funt er nientals jein.

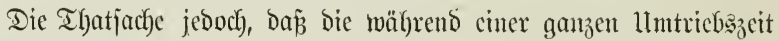

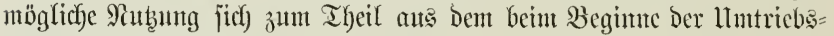

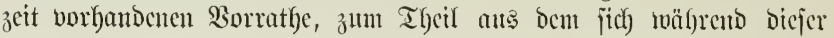

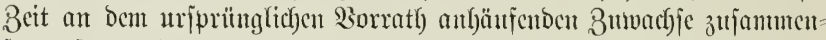
jeb̧t, ferner ber llutitand, baj einige Methoden ber Ertragsregefung bem Rormalvorrath eiue groje Bebentung beilegen, erfordert fier cin= gebende Betradytung Dejielben.

\section{$\S 32$. \\ (G)}

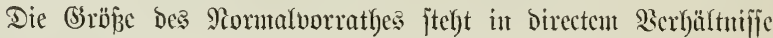
zu Dent llmtriebe. Š höber Diejer, Dejto gröper ber ßorrath).

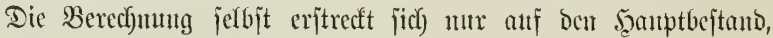

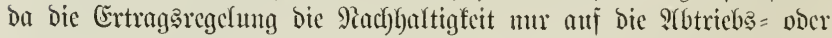

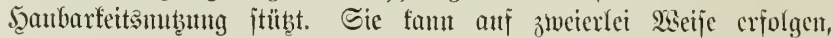

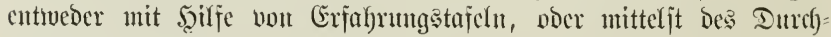

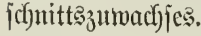

\section{1. $\Re \mathfrak{a} \mathfrak{b} \mathfrak{l} \mathfrak{d} \mathfrak{l} \mathfrak{l} \mathfrak{a} \mathfrak{b} \mathfrak{c} t \mathfrak{i} \mathfrak{i c b}$.}

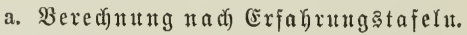

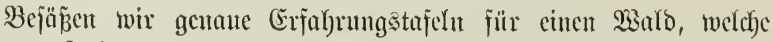

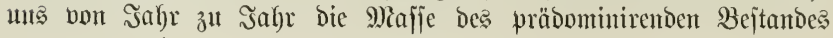


angeben, jo wïroc jich ocr Pormafvorrath als Summe fümuntlicher

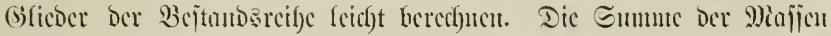

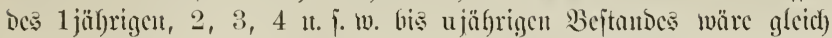

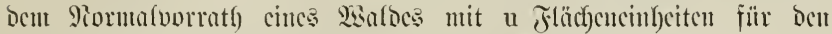

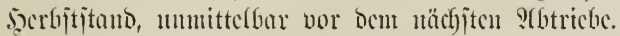

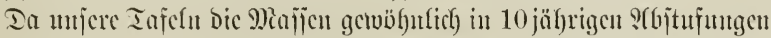

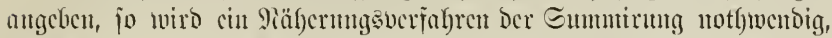

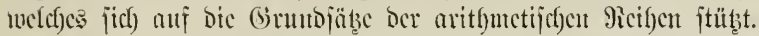

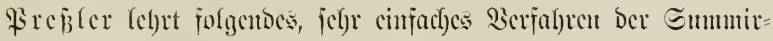
III!̣ cince Eertragstofel.

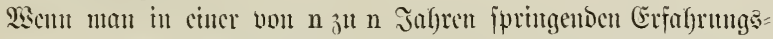
tujel bic ben cinzelute Jaf)ren jugeförigen Bejtandsmajien nach)

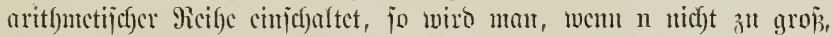

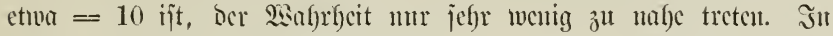

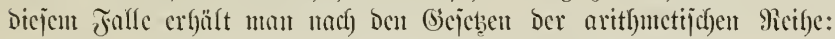

siter Miaje Miajie aller QYrtersitujen von

$$
\begin{aligned}
& \text { 0. } 0\} . .0 \text { bis crel. a }(0+a) \frac{n+1}{2}-a
\end{aligned}
$$

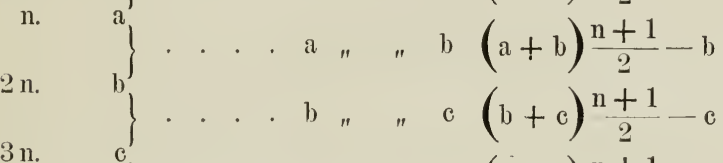

$$
\begin{aligned}
& \left.{ }_{d}^{c}\right\} \cdot \text {. . c n incl. d }(c+d) \frac{n+1}{2} \\
& \text { Simm }=\frac{n+1}{2}(0+2 a+2 b+2 c+d)-(a+b+c) \\
& =(n+1)\left(a+b+c+\frac{d}{2}\right)-(a+b+c) \\
& =n\left(a+b+c+\frac{d}{2}\right)+\frac{d}{2} \text {. }
\end{aligned}
$$

Diejer Sorrath gilt als normaler im Secrljte, munittelbar vor

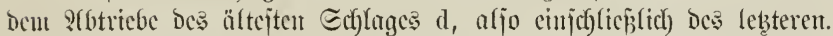
Int Jrüfjaf)re, nach bem S(Gtriebe vou d, wiltoc bic Eumme bes ?ormalvorratbes, aljo crelujuce d

$$
\begin{gathered}
\frac{n+1}{2}(0+2 a+2 b+2 c+d)-(a+b+c+d) \\
=n\left(a+b+c+\frac{d}{2}\right)-\frac{d}{2} .
\end{gathered}
$$




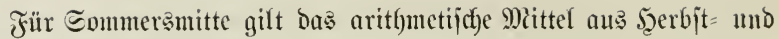
Frübjafrsvorratf), näm(ich)

$$
n\left(a+b+c+\frac{d}{2}\right) \text {. }
$$

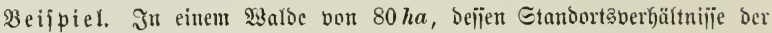
$\S 11$ angegebenen Ertragstajel entiprechen, beträgt bei 80 jäfrigem Ilntriebe ber Formalvorrath:

1) Šü Den Sorbjţtanopunft

$$
\begin{gathered}
10\left(20+65+129+200+275+354+433+\frac{509}{2}\right)+\frac{509}{2} \\
=10 \times 1730,5+254,5=17559,5 \mathrm{fm} .
\end{gathered}
$$

2) Fïr Den Frühjahrą̧tano

$$
10 \times 1730,5-254,5=17050,5,
$$

3) รีür Sommersmitte

$$
10 \times 1730,5=17305,
$$

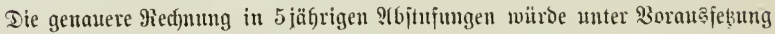
won $6 \mathrm{fm}$ für ben 5 jährigen Beitanı ergeben:

$5 \times 3453,5+254,5=17522 \quad \mathrm{fm}$,
$5 \times 3453,5-254,5=17013 \quad "$
$5 \times 3453,5=17267,5 "$

Die fleine Differenz von 37,5 für jämuntlidje Sorräthe ertläst fïc) Daburth),

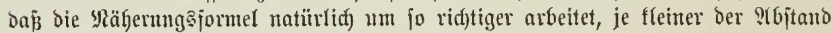
der (乡lieder ift.

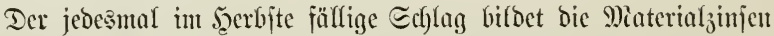

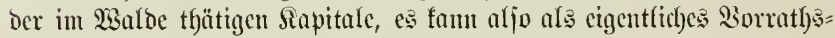
fapital nux ber Frübjahrsoverrath angejeben werden.

Snmerfung. Şat man für einen beítinuten lluntrieb u ben Rormal=

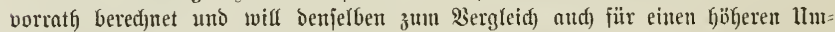
trieb $u^{\prime}$ juchen, jo empfieh(t jid) folgendes furze Berfagren, um bas fehlente Etüd z" finden:

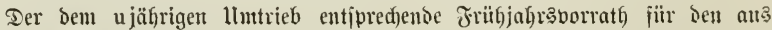
u Flächeneingeiten bejtehenden $\mathfrak{S}_{\text {Sals beträgt }}$

$$
n\left(a+b+c+\ldots+\frac{d}{2}\right)-\frac{d}{2},
$$

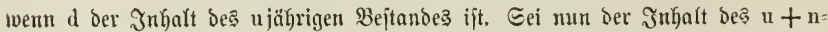

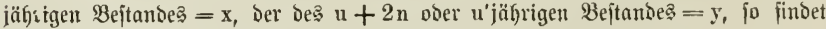

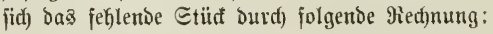




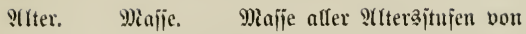

$$
\begin{array}{r}
u^{\prime}=u+2 n \sum_{y}^{u} u+n \ldots u^{\prime}=(x+y) \frac{n+1}{2}-y . \\
\text { summe }=\frac{n+1}{2}(d+2 x+y)-(x+y) \\
=n\left(\frac{d}{2}+x+\frac{y}{2}\right)+\frac{d}{2}-\frac{y}{2} .
\end{array}
$$

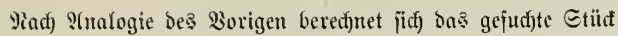
für Den serbititanopuntt zu $\mathrm{n}\left(\frac{\mathrm{d}}{2}+\mathrm{x}+\frac{\mathrm{y}}{2}\right)-\frac{\mathrm{d}}{2}+\frac{\mathrm{y}}{2}$,

$$
\text { Eonmersmitte "n }\left(\frac{d}{2}+x+\frac{y}{2}\right) \text {. }
$$

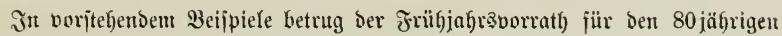
Ilmtrieb nad) 5 jübriger 2 (bjtujung bered)net

$$
5\left(6+20+40+\ldots \ldots+472+\frac{509}{2}\right)-\frac{509}{2}=17013 .
$$

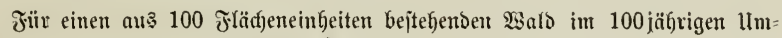

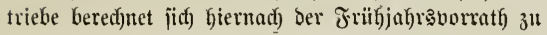

$$
17013+5\left(\frac{509}{2}+544+575+604+\frac{630}{2}\right)+\frac{509}{2}-\frac{630}{2}=28415
$$

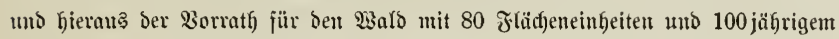
Untriebe: $28415 \times \frac{80}{100}=22732$.

b. Berednung mit Dilfe bes Durdjđdnitt

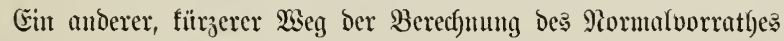
betrad)tet Den laujenden Buwad) in affen Rebensaltern Der Bejtände als einen gleidfen, uto zwar als einen joldfen, ber gleidf ijt dem

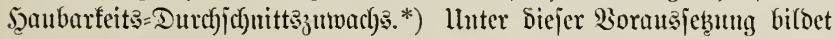
Der Mafjengebalt aller normal bejtandenen Salläge vom jüngjten bis

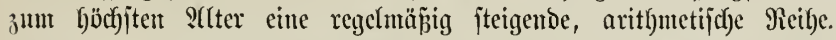
Das erite (Ślieb a biejer Rieife ijt gleich ibrer. Differenz, nümlich) gleich) bem an jedent einzelnen Bejtande jälyrlich erfolgenden Buwachje. Das leb̧te ujährige (ślico t ijt gleid) bem \$robucte bes eimjübrigen 3 umachjes cines Edflages mit ber lintriebszeit $u$, es ijt aber aud gleich Der Eumme bes jäbrlich ant alfen Sdblägen erfolgenden $8 u=$

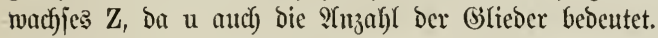

*) Dejterreidjijufe Siameraltace. Carl şeyer. 
Demutach) iijt:

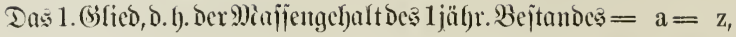

$$
\begin{aligned}
& \text { "2. " " " " " " " " } \quad=2 \mathrm{a}=2 \mathrm{z} \text {, } \\
& \text { "3. " " " " " } 3 \text { " " }=3 \mathrm{a}=3 \mathrm{z} \text {, } \\
& \text { U. }\{\text {. w. }
\end{aligned}
$$

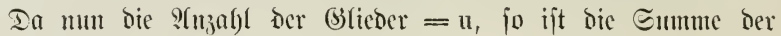
ganzen Reife

$$
\begin{gathered}
(a+t) \frac{u}{2}=\frac{u a}{2}+\frac{u t}{2} ; \\
\text { un๖ ьа иа }=t,
\end{gathered}
$$

Pormalvorrath für Den Seerbititandpunft:

$$
\frac{u t}{2}+\frac{t}{2}
$$

Das heip̈t ber Rormafvorratly bes aus u Beitämben beitefenden Likaldes ijt ummitteffor vor bem ?fbtriebe bes ältejten Eef)lages gleicf) Dem Pro= bucte aus ber Eumune bes jüngiten und älteiten (S)liedes ber Beptands: reife mit ber halben llmtriebzzeit.

Im Früljjalgre, nach bem scbtriebe bes lețteren ift ber $u-1=$

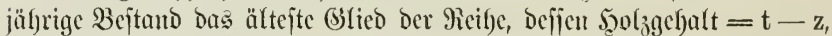

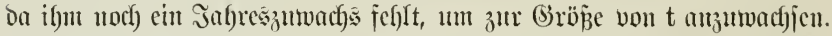

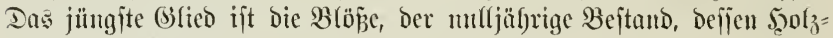

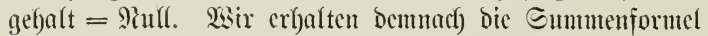

Sormalvorratl) fïr bas Früfjabr

$$
[0+(t-z)] \frac{u}{2}=\frac{u t}{2}-\frac{u z}{2} ;
$$

$$
\frac{\mathrm{ut}}{2}-\frac{\mathrm{t}}{2} \text {. }
$$

Für Commersmitte gilt das aritgmetifafe Mittel aus dem Frïl)jal)rs: und seerbituorratl), aljo $\frac{\mathrm{ut}}{2}$.

Denjefben Betrag findet man birect, wenn man bebenft, da $\tilde{\beta}$ in Soumersmitte ber jüngite Edflag cinen ballon Zumad)s befibet, bem

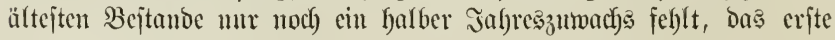
(Glied der Reibe fontad) $=\frac{z}{2}$, das lebete $t-\frac{z}{2}$ ijt.

$$
\text { Eumme: }\left[\frac{\mathrm{z}}{2}+\left(\mathrm{t}-\frac{\mathrm{z}}{2}\right)\right] \frac{\mathrm{u}}{2}=\frac{\mathrm{ut}}{2} \text {. }
$$


Dem cigentficfen Winterialfupitul entiptidyt and) bier itreng ge=

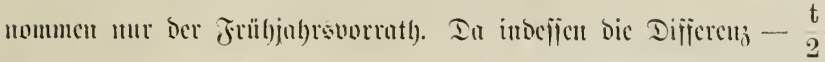

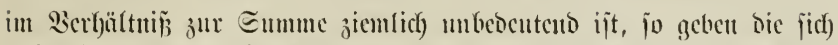

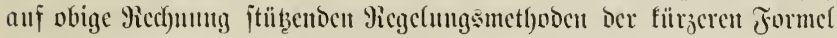

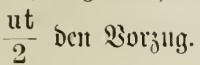

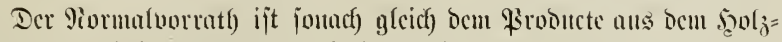

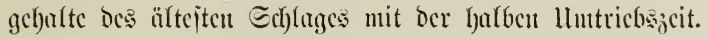

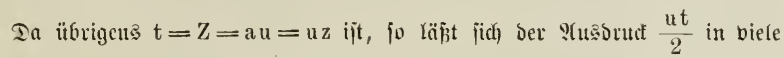

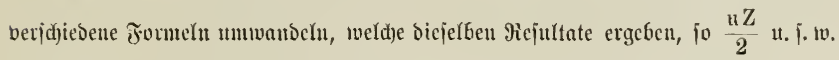

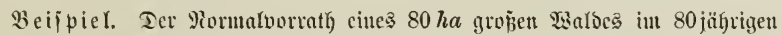

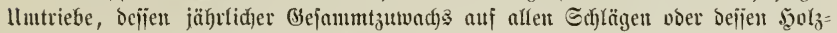
gefalt bes ältệten Sdhlage $509 \mathrm{fm}$ Geträgt, ijt

$$
\begin{aligned}
& \text { im Jrühjahr } \frac{80 \times 509}{2}-\frac{509}{2}=20105,5 \mathrm{fm}, \\
& \text { im Soumer } \frac{80 \times 509}{2}=20360, \\
& \text { im şerbft } \frac{80 \times 509}{2}+\frac{509}{2}=20614,5,
\end{aligned}
$$

Gegen bie Dutrd) Sumunirung der 10 jäfrig abgejtuften Ertragştnfel gefun=

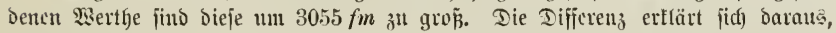

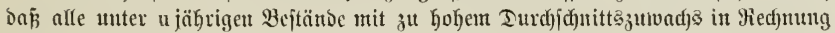
geitellt wutbent.*)

*) 2 nuerfungen. 1. Llu bicjen Fefler fïr Dic Rejultate ber Ertragä=

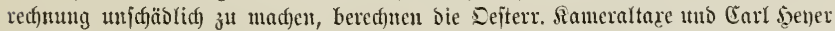

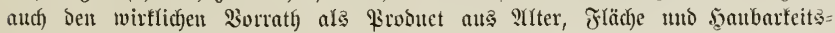

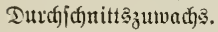

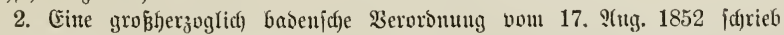
Deşhalb zur Bered)ung des Yormalvorrnthes bie formel 0,45 u $Z$ vor. Mian

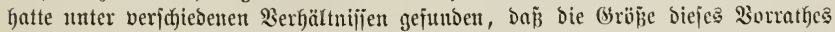
nid)t 0,5 , fondern nur 0,44 bis 0,46 a Z antäberno betrage. Sđlferbings fann

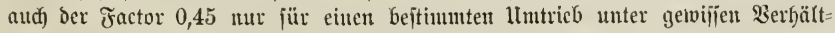

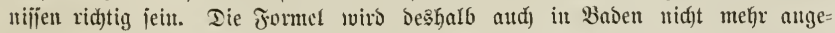

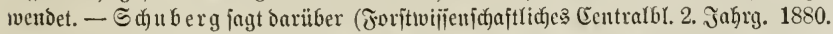

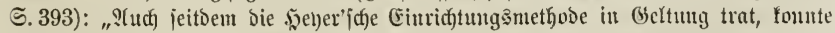
bie 3nhl 0,45 an ber Etelle von 0,50 bie lluridgtigteit bes Mormalvortathes ans

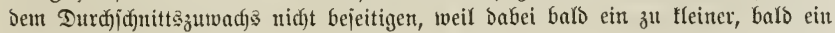

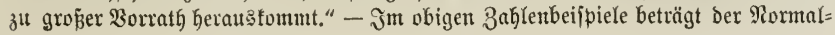


2. Fleuterid)lagbetrieb.

a. Bered)ung uad) Eriagrungata jeln.

Der Mormalworrath wirb am richtigiten gefmroen, wem man bie

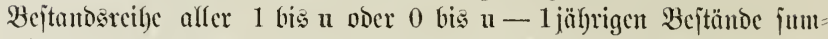
mirt und bieje Summe nod) 1 m den alten Borrath Der Berjüngungs=

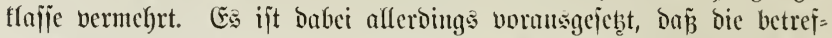

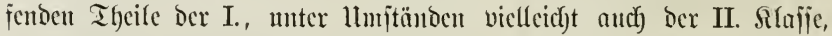

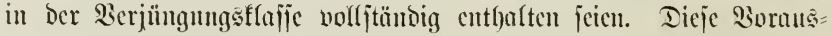
febung fun beshalb nur cinen jebr fleinen Jehler ergeben, weil bic

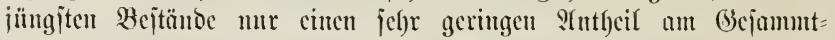
vorratbe ilberbant halen. Der mbebentenoe Fefler wächjt mit ber

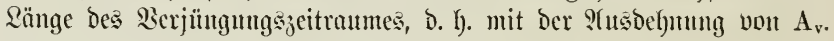

Beipiel. Fïr cinen $\mathfrak{B a l}_{\text {ald }}$ von 80 ha gelte bie Erfahrungstafel (\$ 11) in

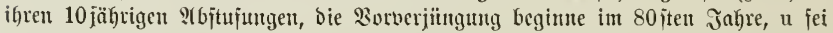
aljo $=80$, Der Serjüngungazeitraum umjajie 10 Jahre. Der Sorntalvorrath für 80 ha beredfutet fid) Giernad, menn wir Den Borrath bon $\mathrm{A}_{\mathrm{v}}$ mit $\mathrm{V}$ bejeidfun:

a) Für Den şerbititanto:

$$
\begin{gathered}
10\left(20+65+129+200+275+354+433+\frac{509}{2}\right)+\frac{509}{2}+\mathrm{V} \\
=10 \times 1730,5+254,5+\mathrm{V}=17559,5+\mathrm{V} .
\end{gathered}
$$

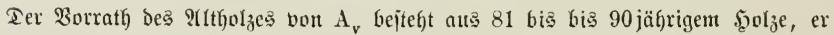

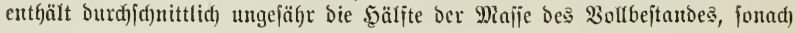

$$
\frac{\frac{10}{2}(516+575)}{2}=2727,5 \mathrm{fm},
$$

Der gatze Mormalvorrath Demuad)

$$
17559,5+2727,5=2028 \mathrm{i} \mathrm{fm} .
$$

b) F̈̈r Sommersెmitte:

$$
10 \times 1730,5+\mathrm{V}
$$

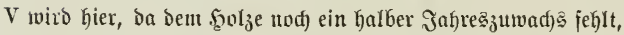

$$
\frac{10}{2}\left(\frac{512,5+571,9}{2}\right)=2711,
$$

Der Mormalvorrath Demnad)

$$
17305+2711=20016 \mathrm{fm} .
$$

norrath fiernad) $0,45 \times 80 \times 509=18320 \mathrm{fm}$, aljo sur 1019 megr, als ber Som= merborrath nad) ber (Erjahrungatafel nit 10 jähriger 2(bjtufung.

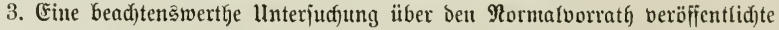

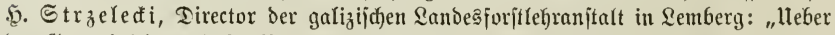

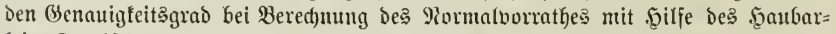

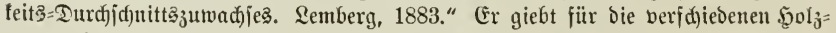

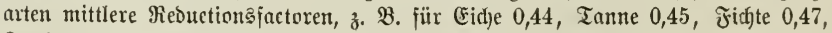
Lärche 0,51 u. f. w. 


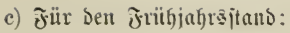

$$
10 \times 1730,5-254,5+\mathrm{V} .
$$

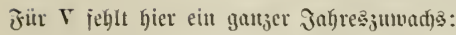

$$
\mathrm{V}=\frac{10}{2}\left(\frac{509+568,8}{2}\right)=2694,5,
$$

Der Siormalborratf Demuad)

$$
17050,5+2694,5=19745 \mathrm{fm} .
$$

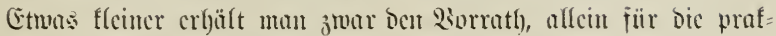

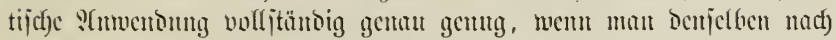

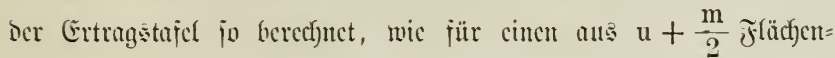

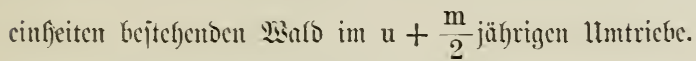

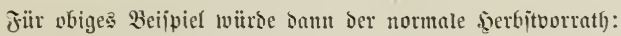

$$
10 \times 1730,5+254,5+\frac{5}{2}(516+544)=20: 209,5 \mathrm{fm} .
$$

Der Eommeritano ergiebt:

$$
17305+\frac{5}{2}(512,5+540,5)=19937,5 \mathrm{fm} .
$$

Ier Erïhjahrs̄itant:

$$
17050,5+\frac{5}{2}(509+537)=19665,5 \mathrm{fm} .
$$

Bei Snuvendung ber 5 jäfrig afgejtujten Tajel mürbe man, wie oben, gegen= iiber Der 10 jährigen ein um 37,5 fleineres Rejultat jür alle Borrätge erbalten:

serbjtoortatf:

Eonmerborrath:

$$
5\left(6+20+40+65+\ldots \ldots+433+509+\frac{544}{2}\right)+\frac{544}{2}=20172 \mathrm{fm},
$$

$5 \times 3980=$

19900 ＂

Jrühjahrs̄borrath:

$$
5 \times 3980-\frac{544}{2}=\quad 19628,
$$

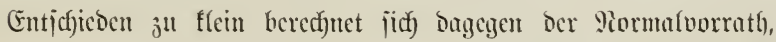
went man bic für $u+\frac{m}{2}$ Fläd)encinlyeiten gejunocnen 2isertbe anj u Einfeiten Dnrch) Mcultiplicution mit $\frac{u}{u+\frac{m}{2}}$ reduciren mollte.

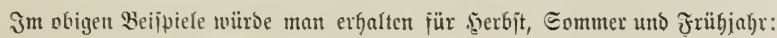
19021, 18765 unt $18509 \mathrm{fm}$. 


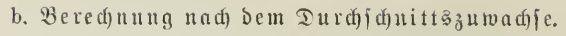

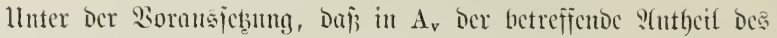

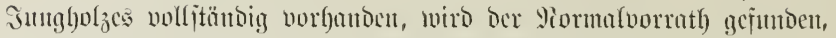
ittocm man Den Bettag Der vollen Bejtatbsreife noch sm Den alten Bortath in $A_{v}$ vermelfit.

Fïfren wir bic Rechnung für ben Etantopunft in Eommersmittc

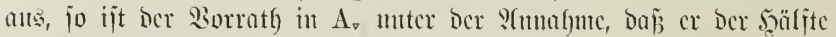
bes betreffentoen Bofl bejtumbes entipricht:

$$
\frac{\frac{m}{2}\left[\left(u+\frac{1}{2}\right) z+\left(u+m-\begin{array}{l}
1 \\
2
\end{array}\right) z\right]}{2} .
$$

Şierun, wenn $\mathrm{uz}=\mathrm{Z}$,

$$
\mathrm{m}_{2}^{\mathrm{m}}\left(\mathrm{z}+\frac{\mathrm{mz}}{2}\right)
$$

Der gejammte Normafoorratl) wäre benutach)

ober audf)

$$
\frac{u z}{2}+\frac{m}{2}\left(z+\frac{m z}{2}\right)
$$

$$
z\left(\frac{u+m}{2}\right)+\frac{m^{2} z}{4} .
$$

Beifpier. Ebiger warts, beîen $\mathrm{F}=80, \mathrm{u}=80$ und $\mathrm{m}=10$, fabe einen

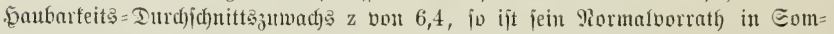
mers̄mitte:

$$
\frac{80 \times 512}{2}+\frac{10}{2}\left(512+\frac{10 \times 6,4}{2}\right)=20480+2720-23200 \mathrm{fm} .
$$

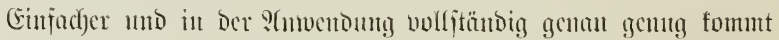

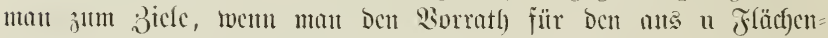

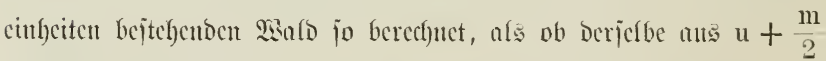

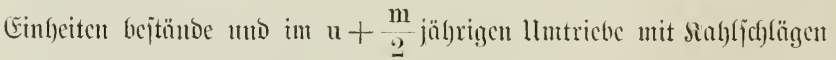
bewirtbjechajtet wilroce.

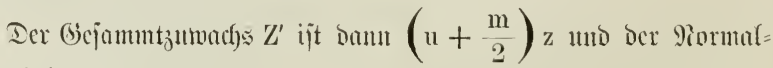
vorrath) in Sommersmitte:

$$
\frac{\left(u+\frac{m}{2}\right)\left(u+\frac{m}{2}\right) z}{2}=\frac{Z^{\prime}\left(u+\frac{m}{2}\right)}{2} .
$$


Beijpiel. Fïr denfelben :

$$
Z^{\prime}=\left(80+\frac{10}{2}\right) 6,4=544 .
$$

Rormalovitath:

$$
\frac{85 \times 544}{2}=23120 \mathrm{fm} \text {. }
$$

Die Iifjeren mit Dem vorigen Mejultate beträgt, ba fid) ber \&usbrud $\frac{\left(\mathrm{u}+\frac{\mathrm{m}}{2}\right)\left(\mathrm{u}+\frac{\mathrm{m}}{2}\right) \mathrm{z}}{2}$ umuandeln läß̈t in $\mathrm{z}\left(\frac{\mathrm{u}+\mathrm{m}}{2}\right)+\frac{\mathrm{m}^{2} \mathrm{z}}{8}$, mur $\frac{\mathrm{m}^{2} \mathrm{z}}{8}$, fier gleid) 80. - rasute man Dagegen Den 3uwad) ber $A_{v}$ mu mit beu einfachen Betrage, aljo Z bes Lsalbes gleid) uz bered)nen, jebod) cinen $u+\frac{m}{2}$ jägrigen Un= trieb auternten, jo wiro Der Sorrath in Sommer $\frac{\left(u+\frac{m}{2}\right) Z}{2}$. Jn obigen Beifpiele $\frac{512 \times 85}{2}=21760 \mathrm{fm}$.

\section{Tiebermalbetrich.}

Für ben Sicoermals berect)net fidf) Der Miormafvorrath genau fo,

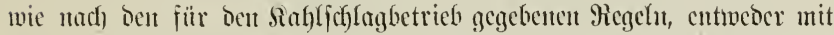

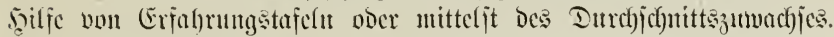

\section{Mittelwalofetrich.}

Eoll fïr bicje Betrichant cin Mormafoorrath) berecfynet merben,

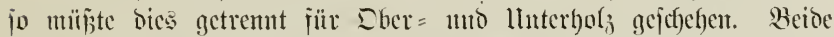

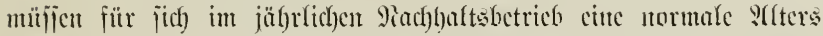

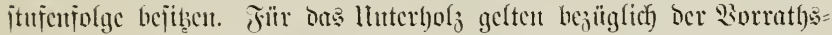

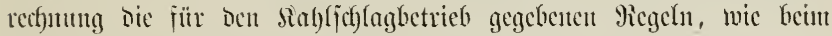

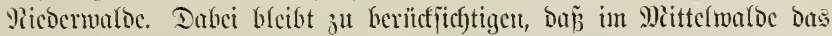

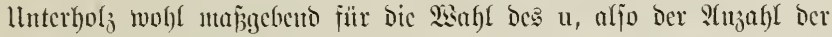

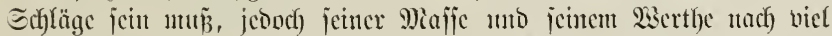

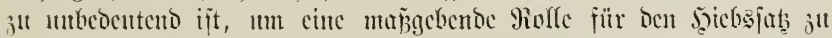

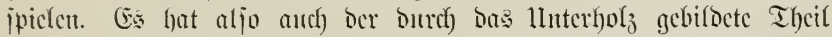

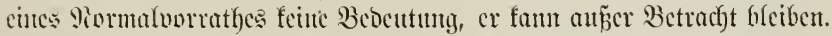

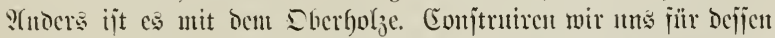

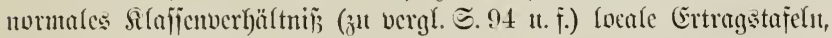
jo faut berent Eummirtung eben fo lcetght antogejülfyt weroen, wic für

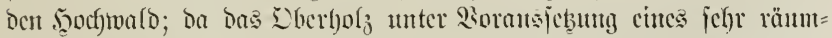

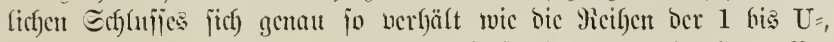

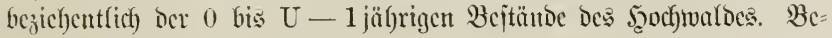




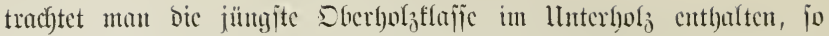

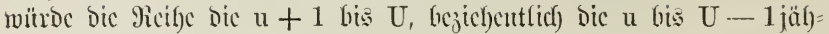

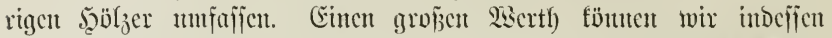
cinter jolchen Mechnmeng nicht beilcgen, Da critents bie Maffe Des Dber=

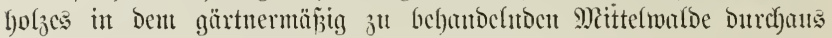

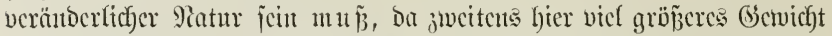

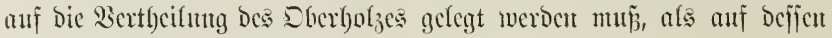

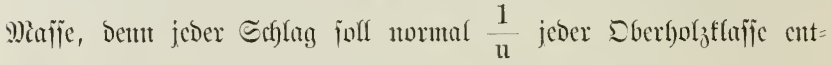

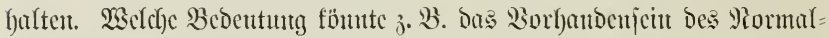

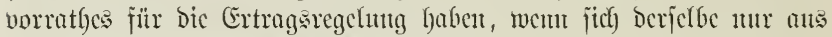

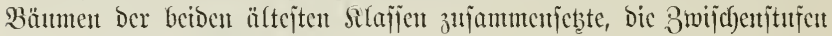

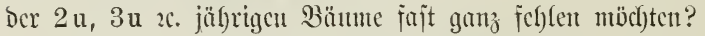

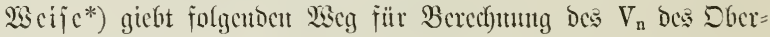
holzes im Mittclwaloc:

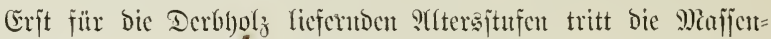
ermittelıtg eiu, bic jüngeren werbent mu nit Fläcl)e ansgejtattet.

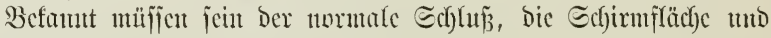

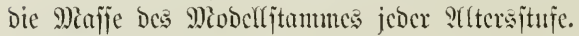

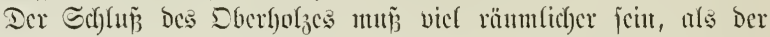

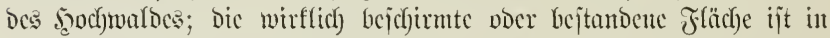

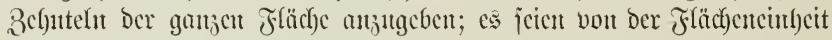
$0, \mathrm{~S}$ bejtandon.

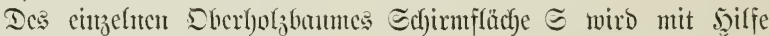

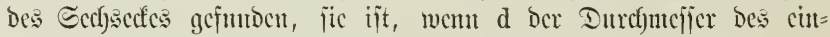
gejdricbenen - Sicijes, $\frac{1}{2} \mathrm{~d}^{2} \sqrt{3}$ Entubratmeter.

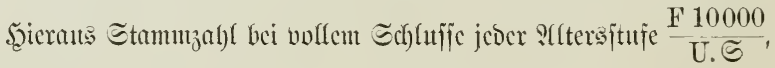

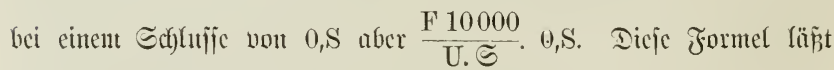
ïc) untwantolut it $\frac{\mathrm{F} 10000}{\mathrm{U}}: \frac{10 \Xi}{\mathrm{S}}$.

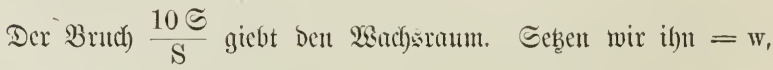

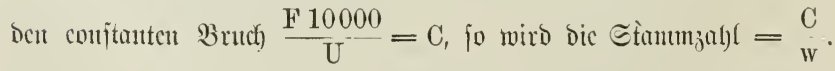

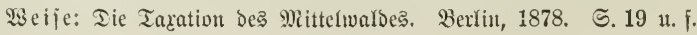




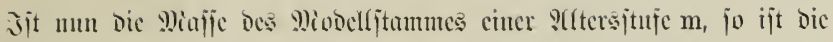

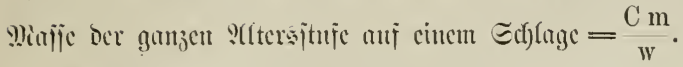

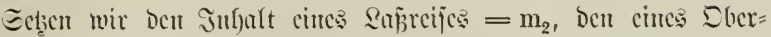

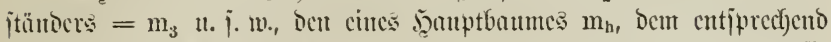
bic Nisact) jeser seltersituje $=\mathrm{C} \frac{\mathrm{m}_{2}}{w_{2}}, \mathrm{C} \frac{\mathrm{m}_{3}}{w_{3}}$ n.j. w. $\mathrm{C} \frac{\mathrm{m}_{\mathrm{h}-1}}{\mathrm{w}_{\mathrm{h}-1}}, \mathrm{C} \frac{\mathrm{m}_{\mathrm{h}}}{\mathrm{w}_{\mathrm{h}}}$.

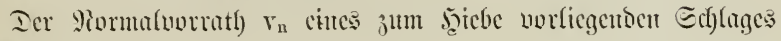
ijt Demuncf)

$$
r_{n}=C\left(\frac{m_{2}}{w_{2}}+\frac{m_{3}}{w_{3}}+\ldots .+\frac{m_{h-1}}{w_{h-1}}+\frac{m_{h}}{w_{h}}\right) .
$$

llm ben Piormalvortuth) $V_{n}$ fïț ben ganzen Lisalo ju herechnen, beoürfen wir noch bes 3nwardjes. Bejeicfnen wir ben cinjäfrigen

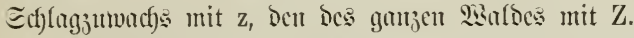
Etmum

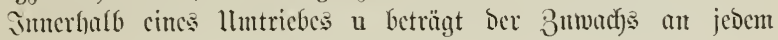

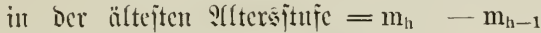

$$
\begin{aligned}
& \text { " " joveitültejten " } \quad=\mathrm{m}_{\mathrm{h}-1}-\mathrm{m}_{\mathrm{h}-2}
\end{aligned}
$$

II. ก. II.

bei ben \&abreijern

$$
=\mathrm{m}_{2} \quad-\mathrm{m}_{1}
$$

mithin fïr affe Etümme Des Echlages, indocm wir bicje Gröben mit Der Etummagl multipliziren:

$$
\begin{gathered}
\frac{C}{w_{h}}\left(m_{h}-m_{h-1}\right)+\frac{C}{w_{h-1}}\left(m_{h-1}-m_{h-2}\right)+\ldots .+\frac{C}{w_{3}}\left(m_{3}-m_{2}\right) \\
+\frac{C}{w_{2}}\left(m_{2}-m_{1}\right) .
\end{gathered}
$$

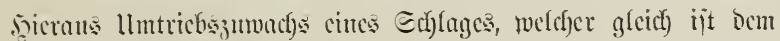
cinjübrigen bes ganjen $23 a$ loce $Z$,

$$
Z=C\left(\frac{m_{2}-m_{1}}{w_{2}}+\frac{m_{3}-m_{3}}{w_{3}}+\ldots .+\frac{m_{h-1}-m_{h-2}}{w_{h-1}}+\frac{m_{h}-m_{h-1}}{w_{h}}\right) .
$$

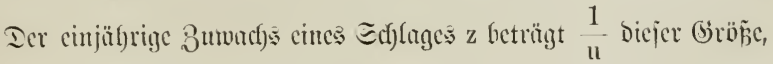
aljo $u z=Z$.

Ier Sorratl) jedes jüngeren Edylages muj um z geringer jein, als Der bes nü̈bjit älteren. Sit ber Sorrath) bes ujül)rigen Ect)lages $=v_{n}$, jo ijt Der Des $u-1$ jülfrigen $=v_{n}-z$, Der Des $\left.u-2 j a ̈ \mathfrak{f}\right)=$ rigen $=\mathrm{v}_{\mathrm{n}}-2 \mathrm{z}$ u.j.m., ber bes jüngiten, cinjährigen Edhlages 
$\mathrm{v}_{\mathrm{n}}-(\mathrm{u}-1)$ z. Mit Scilfe ber arithmetijefen Beifen herechnet, fteben jonach) auf allen Exhlägcu:

$$
\left.\mathrm{V}_{\mathrm{n}}=\mathrm{u}\left(\mathrm{v}_{\mathrm{n}}-\frac{\mathrm{u}-1}{2} \mathrm{z}\right) \text { ober aud }\right)=\mathrm{u} \nabla_{\mathrm{n}}-\frac{\mathrm{u}-1}{2} \mathrm{Z} \text {. }
$$

Beifpiel. F̈̈r Den 120 ha gropen Mittelwalb, Delien $\mathrm{u}=15$, Defïen $\mathrm{U}=60$

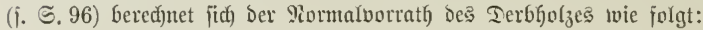

a) Bereduung bes ältejten Edilages furz vor bem Siebe. ( $v_{n}$.

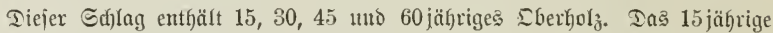

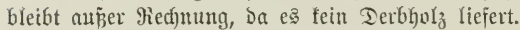

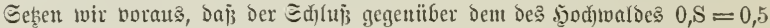
betrage unt

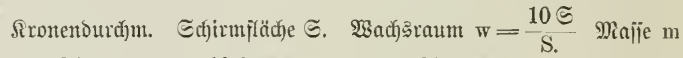

\begin{tabular}{|c|c|c|c|}
\hline 30 ․ B̧auแ & $3,5 \mathrm{~m}$ & $10,6 \mathrm{qm}$ & $21,2 \mathrm{gm}$ \\
\hline " 45 " & $5 \quad "$ & 21,7 & 43,4 " \\
\hline " 60" & " & 31,2, & 62,4 " \\
\hline
\end{tabular}

Der conjtante Brud) $\frac{\mathrm{F} 10000}{\mathrm{U}^{-}}=\mathrm{C}$ iit $\frac{120 \times 10000}{60}=20000$.

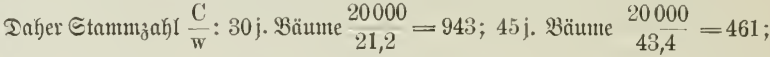

$$
60 \text { j. Bäนmt } \frac{20000}{62,4}=321 .
$$

Ies ältejten Єdylages $v_{n}=C\left(\frac{m_{2}}{w_{2}}+\frac{m_{3}}{w_{3}}+\frac{m_{4}}{w_{4}}\right)$,

$$
\begin{gathered}
\mathrm{v}_{\mathrm{n}}=20000\left(\frac{0,02}{21,2}+\frac{0,26}{43,4}+\frac{0,50}{62,4}\right)=299 \mathrm{fm}, \\
\text { Dder ald } \\
\mathrm{v}_{\mathrm{n}}=943 \times 0,02+461 \times 0,26+321 \times 0,50=299 \mathrm{fm} .
\end{gathered}
$$

b) Beredunng Des 3 uwadjles am ältejten Sd)lage wägrend einer Itmtriebazeit. (Z.)

$$
\begin{gathered}
\mathrm{Z}=\mathrm{C}\left(\frac{\mathrm{m}_{2}-\mathrm{m}_{1}}{\mathrm{w}_{2}}+\frac{\mathrm{m}_{3}-\mathrm{m}_{2}}{\mathrm{w}_{3}}+\frac{\mathrm{m}_{4}-\mathrm{m}_{3}}{\mathrm{~m}_{4}}\right), \\
\mathrm{Z}=20000\left(\frac{0,02-0}{21,2}+\frac{0,26-0,02}{43,4}+\frac{0,50-0,26}{62,4}\right)=206 \mathrm{fm}, \\
\text { ober aud }
\end{gathered}
$$

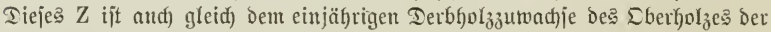
älteiten 3 Silaijen im ganżen

c) Beredung bes Sberfol $=$ Rormaloorrathez fïr bell ganzen

$$
\begin{gathered}
\mathrm{V}_{\mathrm{n}}=\mathrm{uv}_{\mathrm{n}}-\frac{\mathrm{u}-1}{2} \mathrm{Z}, \\
\mathrm{V}_{\mathrm{n}}=15 \times 299-\frac{15-1}{2} \times 206=3043 \mathrm{fm} \mathfrak{T e t b h o l} .
\end{gathered}
$$




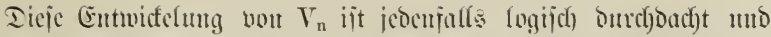

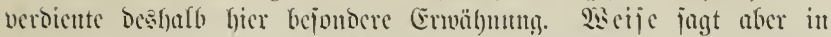

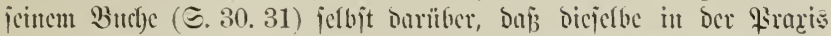

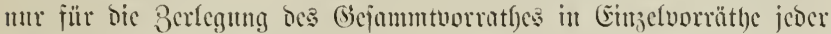
S(ftersflajic in modificitter form zat vernenten jei. Den Plormal=

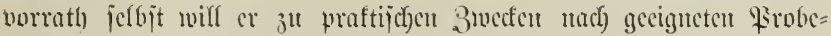

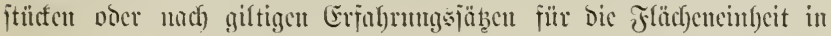

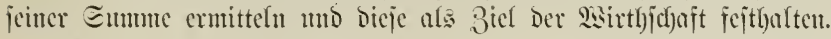

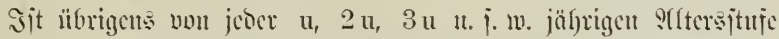

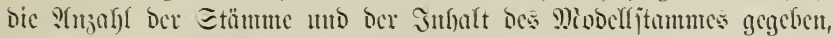

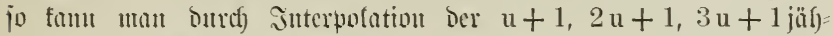

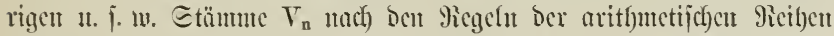

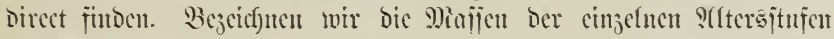
mit II, jo miro

$$
\mathrm{V}_{\mathrm{n}}=\frac{\mathrm{u}}{2}\left(\mathrm{M}_{\mathrm{u}+1}+\mathrm{MI}_{2 \mathrm{u}}+\mathrm{MI}_{2 \mathrm{u}+1}+\mathrm{MI}_{3 \mathrm{u}}+\mathrm{M}_{3 \mathrm{n}+1}+\mathrm{MI}_{4} \mathrm{u}\right) .
$$

Ebige siserthe eingejeşt, ergiebt:

Yinzahi ber

Infalt des

Etämme.

Mobelffammes.

\begin{tabular}{|c|c|c|c|c|c|c|c|c|}
\hline $\mathbf{M}_{60}$ & $=$ & 321 & $x$ & 0,50 & $=$ & 160,5 & $f m$ & Terbfol 3 , \\
\hline $\mathrm{M}_{46}$ & $=$ & 321 & $\times$ & 0,276 & $=$ & 88,6 & " & " \\
\hline $\mathrm{M}_{45}$ & $=$ & 461 & $\times$ & 0,26 & $=$ & 119,9 & $"$ & " \\
\hline $\mathrm{MI}_{31}$ & $=$ & 461 & $\times$ & 0,036 & $=$ & 16,6 & $"$ & " \\
\hline $\mathrm{MI}_{30}$ & $=$ & 943 & $\times$ & 0,02 & $=$ & 18,9 & " & " \\
\hline $\mathrm{M}_{16}$ & $=$ & 943 & $x$ & 0,0013 & $=$ & 1,2 & " & " \\
\hline
\end{tabular}

நieran:

$$
\mathrm{V}_{\mathrm{n}}=\frac{15}{2}(1,2+18,9+16,6+.119,9+88,6+160,5)=3043 \mathrm{fm} .
$$

5. Blenterbetricb.

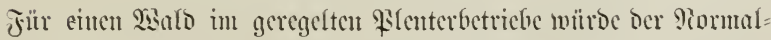

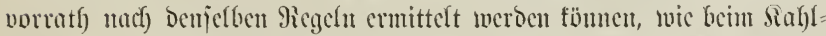

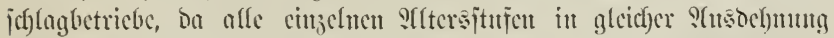

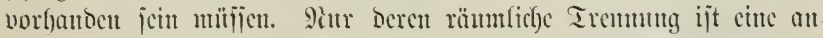

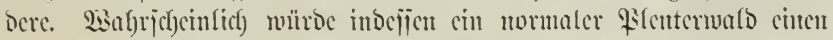

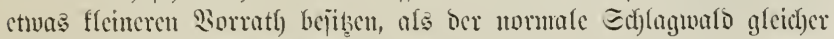

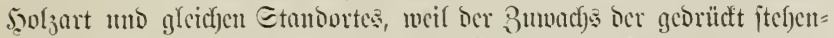

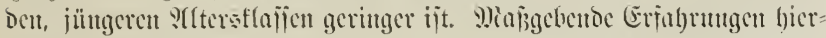
über fehlen jutr Beit nodl). 


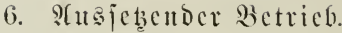

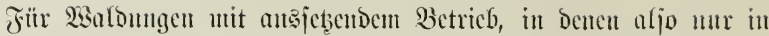

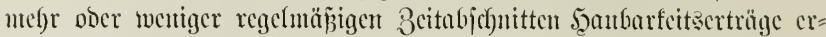

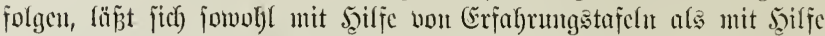

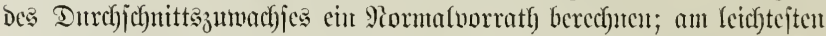

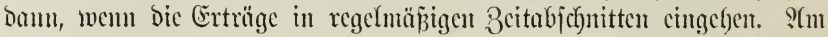

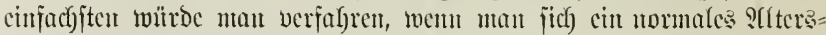

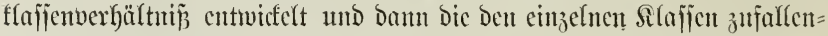
Den Sorräthe fummirt. Eclbjtuerftündficf) fund hier bic Differenjen,

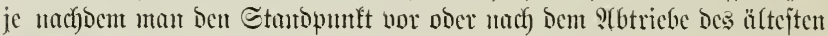

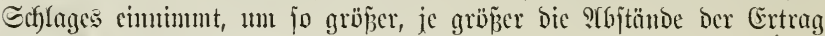
licfernden Jafyre.

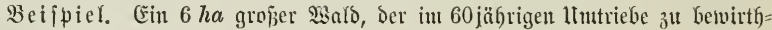

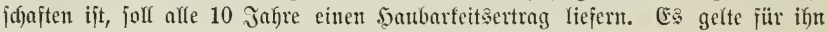
bie Ertragstafel \& 11.

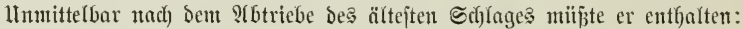

Einen 1 ha gropen, 10 jäfrigen Bejtand utit $20 \mathrm{fm}$,

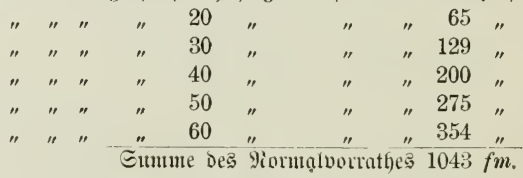

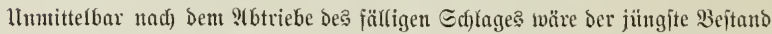

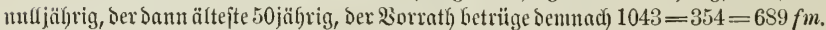

B. Voin Standpunkite der finanzmirtb $\{(f j a f t$. $\S 33$.

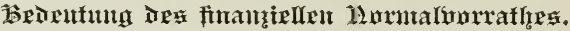

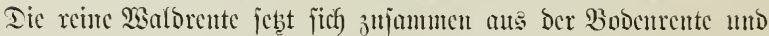

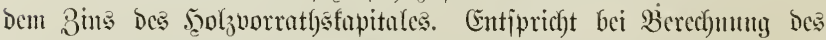

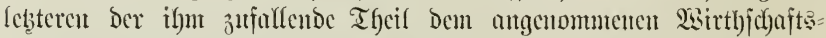
jutsfunce, jo ijt bie Gröbe biejes Borrathes eine nomale.

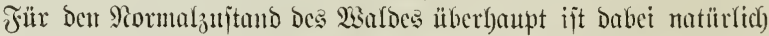

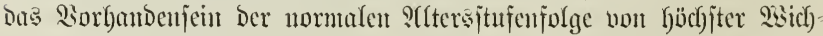

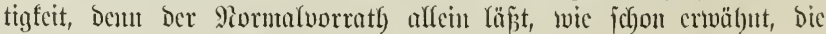
Epfer abnormer J̧anbarfeitsalter micft verneciben.

Dic Bebcutung Des fintanjicllen Pormalvorratfes für bic Sojiung Der SUfgaben Der Ertrugeregelung ift cine mur untergeorontete. Das

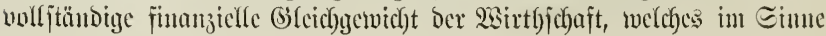




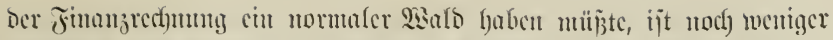
crrcicthar mo haftbar, als ber bloje shaterial=9iormaljuftumb.

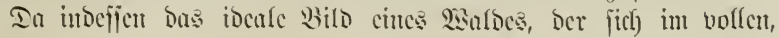

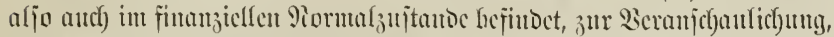

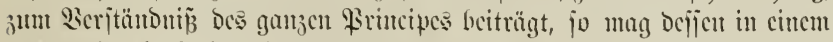

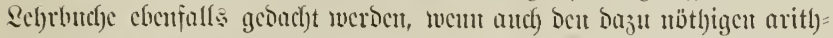

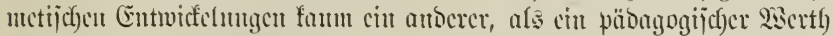
jugciprudben iverden fum. *)

\section{s. 34 .}

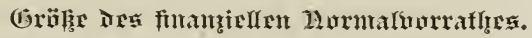

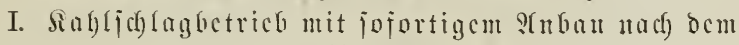
Sobtricbe.

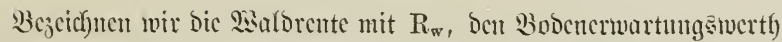

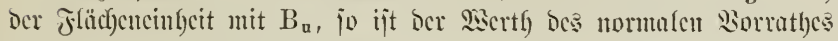
für bcu Jrithjafrsitanopunft $\mathrm{N}_{\mathrm{f}}=\frac{\mathrm{R}_{\mathrm{w}}}{0, \mathrm{pp}}-\mathrm{uB}_{\mathrm{u}}$.

Dic 2isuldorente $R_{w}$ ijt glecich ber Differenj jivijechen alfen birecten

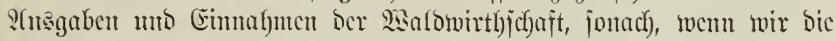

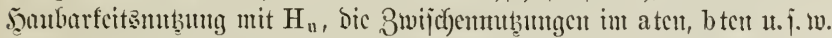

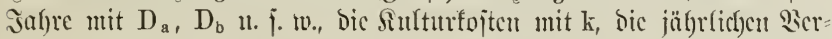

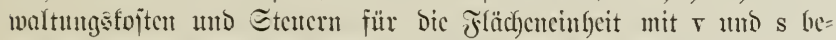
зciffucu, unter $\mathrm{H}$ uto $\mathrm{D}$ aber crntefojtenfrece Erträge veriteben:

$$
\mathrm{R}_{\mathrm{w}}=\mathrm{H}_{\mathrm{a}}+\mathrm{D}_{\mathrm{a}}+\mathrm{D}_{\mathrm{b}} \ldots-\mathrm{k}-\mathrm{u}(\mathrm{r}+\mathrm{s}) \text {; }
$$

Der 2Ecrtly bes mormalen Borrathes:

$$
\mathrm{N}_{\mathrm{f}}=\frac{\mathrm{H}_{\mathrm{u}}+\mathrm{D}_{\mathrm{a}}+\mathrm{D}_{\mathrm{b}} \ldots-\mathrm{k}-\mathrm{u}(\mathrm{r}+\mathrm{s})}{0,0 \mathrm{p}}-\mathrm{u} \mathrm{B}_{\mathrm{u}} \quad \mathrm{I} .
$$

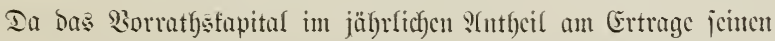

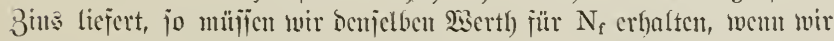

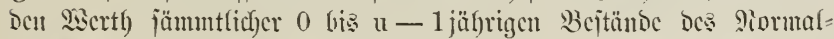

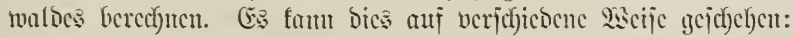

1. Durch Berecfynuy ber Eumme ber Siojtenuertfe aller Bejtänoc.

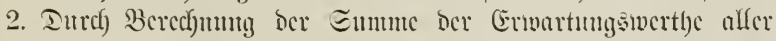
Bcitüntioc.

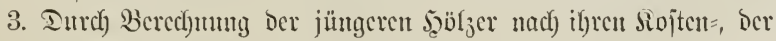
älteren nach) ibren (Erwartmisnerthen.

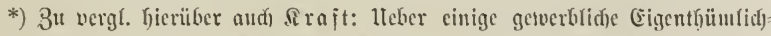

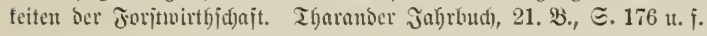




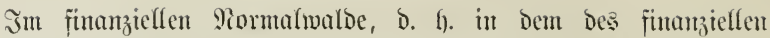

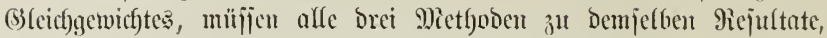

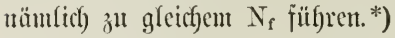

\section{Beredung Der Summe ber Sojtenwerthe aller $\mathfrak{B}$ ejtän oc.}

Der Sojtentwertf). ${ }^{\mathrm{k}} \mathrm{H}_{\mathrm{m}}$ bes mjüfrigen Bejtantoes ijt gleicf) ber Eumme aus Dem mjäfrigen (Enomertbe Der Bobenbruttorente $r^{\prime}$ und Dem mjährigen Sarfjwerthe Der Sulturfoiten $k$, verminbert

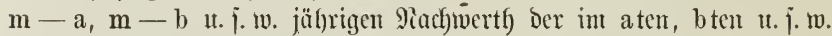

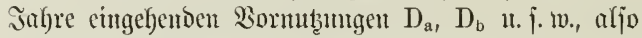

$$
{ }^{\mathrm{k}} \mathrm{H}_{\mathrm{m}}=\mathrm{r}^{\prime}\left(\frac{1,0 \mathrm{p}^{\mathrm{m}}-1}{0,0 \mathrm{p}}\right)+\mathrm{k} 1,0 \mathrm{p}^{\mathrm{m}}-\mathrm{D}_{\mathrm{a}} 1,0 \mathrm{p}^{\mathrm{m}-\mathrm{a}}-\mathrm{D}_{\mathrm{b}} 1,0 \mathrm{p}^{\mathrm{m}-\mathrm{b}} \ldots
$$

Da unu $\frac{r^{\prime}}{0,0 p}$ gleicf) Dem Bodenbruttofapitule, D. h). gleich) der Eumme aนร Boben=, Bermalturgs: und Steuerfapital, gleid) $B_{u}+V+S$ iit, jo ijt aud

$$
{ }^{k} H_{m}=\left(B_{u}+V+S\right)\left(1,0 p^{m}-1\right)+k 1,0 p^{m}-D_{a} 1,0 p^{m-a}-D_{b} 1,0 p^{m-b} .
$$

Sede andere Siormbung im eten, dten Jahre น. j. w. erjefeint unter berjelben Form bes Getreffenden Parfwertfes. a, b, с и. ई. w.

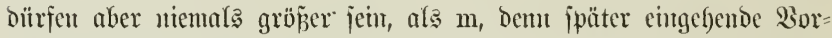
ertrüge, z. $\mathfrak{B}$. im $\mathrm{m}+\mathrm{nten}$ Jafre, föm jübrigen Bejtandes nicht berïf)rent.

In Der Sunme fümmtlicher Siojtentwertbe ber 0 bis $u-1$ jät)= rigen Bejtände erfalten wir mu Den Werth) Des fünuziellen Piormal=

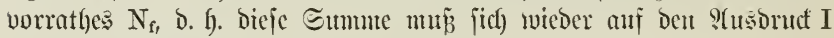
reduciren.

${ }^{\mathrm{k}} \mathrm{H}$ des $0 \mathrm{jüfr}$ r. Bejtandes $=\mathrm{r}^{\prime}\left(\frac{1,0 \mathrm{p}^{0}-1}{0,0 \mathrm{p}}\right)+\mathrm{k} 1,0 \mathrm{p}^{0}$.

$\begin{array}{ll}{ }^{\mathrm{k}} \mathrm{H}, 1 " \quad & =\mathrm{r}^{\prime}\left(\frac{1,0 \mathrm{p}^{1}-1}{0,0 \mathrm{p}}\right)+\mathrm{k} 1,0 \mathrm{p}^{1} . \\ { }^{\mathrm{k}} \mathrm{H}, 2, \quad & =\mathrm{r}^{\prime}\left(\frac{1,0 \mathrm{p}^{2}-1}{0,0 \mathrm{p}}\right)+\mathrm{k} 1,0 \mathrm{p}^{2} .\end{array}$

II. $\tilde{\text {. }}$ w.

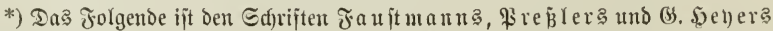

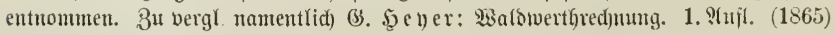

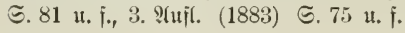


${ }^{k} \mathrm{H}$ bes a jüf)rigen $\mathrm{bie}^{\mathrm{C}}=$

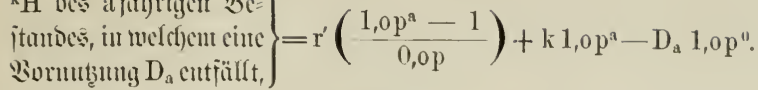
${ }^{\mathrm{k}} \mathrm{H}$ Des $\mathrm{a}+1 \mathrm{j} \cdot$ Becjtundes $=\mathrm{r}^{\prime}\left(\frac{1,0 \mathrm{p}^{\mathrm{a}+1}-1}{0,0 \mathrm{p}}\right)+\mathrm{k} 1,0 \mathrm{p}^{\mathrm{a}+1}-\mathrm{D}_{\mathrm{a}} 1,0 \mathrm{p}^{1}$. ${ }^{\mathrm{k}} \mathrm{H}, \mathrm{a}+2 \mathrm{j} . \quad, \quad=\mathrm{r}^{\prime}\left(\frac{1,0 \mathrm{p}^{\mathrm{q}+2}-1}{0,0 \mathrm{p}}\right)+\mathrm{k} 1,0 \mathrm{p}^{\mathrm{a}+2}-\mathrm{D}_{\mathrm{a}} 1,0 \mathrm{p}^{2}$.

u. i. m.

${ }^{\mathrm{k}} \mathrm{H}, \mathrm{u}-1 \mathrm{j} . \quad$ " $\quad=\mathrm{r}^{\prime}\left(\frac{1,0 \mathrm{p}^{\mathrm{u}-1}-1}{0,0 \mathrm{p}}\right)+\mathrm{k} 1,0 \mathrm{p}^{\mathrm{u}-1}-\mathrm{D}_{\mathrm{a}} 1,0 \mathrm{p}^{\mathrm{u}-1-\mathrm{l}}$.

J̧iermus bic Eumme:

$$
\begin{aligned}
& \text { 1. Für bie Bobenbruttorente r. } \\
& \mathrm{r}^{\prime}\left(\frac{1,0 \mathrm{p}^{0}-1}{0,0 \mathrm{p}}+\frac{1, \mathrm{op} \mathrm{p}^{1}-1}{0,0 \mathrm{p}}+\frac{1,0 \mathrm{p}^{2}-1}{0,0 \mathrm{p}}+\ldots+\frac{1,0 \mathrm{p}^{\mathrm{u}-1}-1}{0,0 \mathrm{p}}\right) \\
& =\frac{\mathrm{r}^{\prime}}{0,0 \mathrm{p}}\left(1,0 \mathrm{p}^{0}+1,0 \mathrm{p}^{1}+1,0 \mathrm{p}^{2}+\ldots+1,0 \mathrm{p}^{\mathrm{n}-1}\right)-\frac{\mathrm{ur}}{0,0 \mathrm{p}} \\
& =\frac{\mathrm{r}^{\prime}}{0, \mathrm{op}}\left(\frac{1,0 \mathrm{p}^{\mathrm{n}}-1}{0, \mathrm{op}}\right)-\frac{\mathrm{ur}^{\prime}}{0, \mathrm{op}}=\frac{\mathrm{r}^{\prime}}{0,0 \mathrm{p}}\left(\frac{1,0 \mathrm{p}^{\mathrm{n}}-1}{0,0 \mathrm{p}}-\mathrm{u}\right) \text {; } \\
& \text { mo Da } \frac{\mathrm{r}^{\prime}}{0, \mathrm{op}}=\mathrm{B}_{\mathrm{u}}+\mathrm{r}+\mathrm{S} \text {, }
\end{aligned}
$$

こunแe jümmtlicfer Bobenbruttorenten:

$$
\begin{aligned}
& \left(B_{u}+V+S\right) \frac{1,0 p^{u}-1}{0,0 p}-u\left(B_{u}+V+S\right) . \\
& \mathrm{k}\left(1,0 \mathrm{p}^{0}+1,0 \mathrm{p}^{1}+1,0 \mathrm{p}^{2}+\ldots+1,0 \mathrm{p}^{\mathrm{u}-1}\right) \\
& =\mathrm{k}\left(\frac{1,0 \mathrm{p}^{\mathrm{u}}-1}{0,0 \mathrm{p}}\right)=\mathrm{ujäfriger} \mathrm{(Enobmerth} \mathrm{ber} \mathrm{\Re ente} \mathrm{k} \text {. }
\end{aligned}
$$

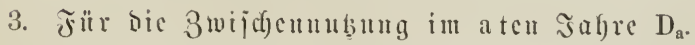

$$
\begin{aligned}
& \mathrm{D}_{\mathrm{a}}\left(1,0 \mathrm{p}^{0}+1,0 \mathrm{p}^{1}+1,0 \mathrm{p}^{2}+\ldots \ldots+1,0 \mathrm{p}^{\mathrm{n}-\mathrm{a}-1}\right) \\
= & \mathrm{D}_{\mathrm{a}}\left(\frac{1,0 \mathrm{p}^{\mathrm{u}-\mathrm{a}}-1}{0,0 \mathrm{p}}\right)=\mathrm{n}-\mathrm{a} \text { jäfriger Entrowertf eitrer タente } \mathrm{D}_{\mathrm{a}} .
\end{aligned}
$$


Die ganje Eumme für $\mathrm{N}_{\mathrm{f}}$ (autet jouad):*)

$$
\begin{aligned}
& N_{\mathrm{f}}=\frac{\mathrm{r}^{\prime}}{0,0 \mathrm{p}} \frac{\left(1,0 \mathrm{p}^{\mathrm{u}}-1\right)-\mathrm{ur}+\mathrm{k}\left(1,0 \mathrm{p}^{\mathrm{u}}-1\right)-\mathrm{D}_{a}\left(1,0 \mathrm{p}^{\mathrm{u}-\mathrm{a}}-1\right)}{0,0 \mathrm{p}} ;
\end{aligned}
$$

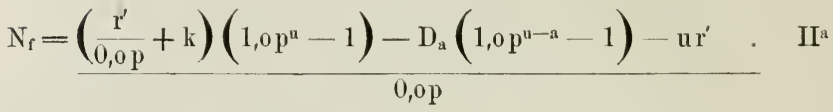

$$
\begin{aligned}
& \mathrm{N}_{\mathrm{f}}=\frac{\left(\mathrm{B}_{\mathrm{u}}+\mathrm{V}+\mathrm{S}+\mathrm{k}\right)\left(1,0 \mathrm{p}^{\mathrm{u}}-1\right)-\mathrm{D}_{\mathrm{a}}\left(1,0 \mathrm{p}^{\mathrm{u}-\mathrm{a}}-1\right)}{0,0 \mathrm{p}} \\
& -u\left(B_{u}+V+S\right) \text {. }
\end{aligned}
$$

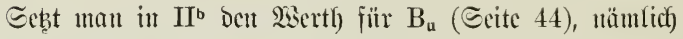

$$
\frac{\mathrm{H}_{\mathrm{u}}+\mathrm{D}_{\mathrm{a}} 1,0 \mathrm{p}^{\mathrm{n}-\mathrm{a}}-\mathrm{k} 1,0 \mathrm{p}^{\mathrm{n}}}{1,0 \mathrm{p}^{\mathrm{n}}-1}-(\mathrm{V}+\mathrm{S}),
$$

fo reoucirt jidf) $\mathrm{I}^{\mathrm{b}}$ auf $\mathrm{I}$ :

$$
\begin{aligned}
\mathrm{N}_{\mathrm{f}}= & \frac{\left[\frac{\mathrm{H}_{\mathrm{u}}+\mathrm{D}_{\mathrm{a}} 1,0 \mathrm{pou}^{\mathrm{u}-\mathrm{a}}-\mathrm{k} 1,0 \mathrm{p}^{\mathrm{u}}}{1,0 \mathrm{p}^{\mathrm{u}}-1}-(\mathrm{V}+\mathrm{S})+\mathrm{V}+\mathrm{S}+\mathrm{k}\right]\left(1,0 \mathrm{p}^{\mathrm{u}}-1\right)-\mathrm{D}_{\mathrm{a}}\left(1,0 \mathrm{pp}^{\mathrm{u}-\mathrm{a}-1)}\right.}{0,0 \mathrm{p}} \\
& -\mathrm{u}\left(\mathrm{B}_{\mathrm{u}}+\mathrm{V}+\mathrm{s}\right) .
\end{aligned}
$$

Scicrans:

$$
N_{f}=\frac{H_{u}+D_{a}-k}{0,0 p}-u\left(B_{u}+V+S\right) \text {. }
$$

Da $m \mathrm{~m} \mathrm{~V}+\mathrm{S}=\frac{\mathrm{v}+\mathrm{s}}{0,0 \mathrm{p}}$,

$$
\mathrm{N}_{\mathrm{f}}=\frac{\mathrm{H}_{\mathrm{u}}+\mathrm{D}_{\mathrm{a}}-\mathrm{k}-\mathrm{u}(\mathrm{v}+\mathrm{s})}{0,0 \mathrm{p}}-\mathrm{uB}_{\mathrm{u}} \text {. }
$$

2. B̧eredfung $\delta$ er Summe Der Ewartungaberthe fämutliçer bejtänoe.

Der (Erwartutugswertl) ${ }^{-} \mathrm{H}_{\mathrm{m}}$ Des mjäf)rigen Beftandes ijt gleidf) der Summe ans bem $\mathfrak{u}-$ mjäfrigen Bortwertbe ber Scantourfeits=

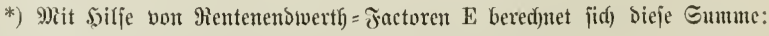

$$
\frac{\mathrm{r}^{\prime}}{0,0 \mathrm{p}} \times\left(\mathrm{E}_{\mathrm{u}}-\mathrm{u}\right)+\mathrm{k} \times \mathrm{E}_{\mathrm{u}}-\mathrm{D}_{\mathrm{a}} \times \mathrm{E}_{\mathrm{u}-\mathrm{a}} .
$$




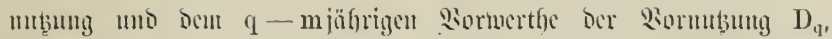

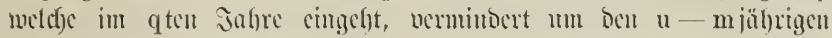
?(nfangsinertf) Der Boocubruttorente r'.

$$
\begin{gathered}
{ }^{e} H_{\mathrm{m}}=\frac{\mathrm{H}_{\mathrm{u}}}{1,0 \mathrm{p}^{\mathrm{u}-\mathrm{m}}}+\frac{\mathrm{D}_{\mathrm{q}}}{1,0 \mathrm{p}^{\mathrm{q}} \mathrm{m}} \ldots .-\mathrm{r}^{\prime}\left(\frac{1,0 \mathrm{p}^{\mathrm{n}-\mathrm{m}}-1}{0,0 \mathrm{p} \times 1,0 \mathrm{p}^{\mathrm{u}-\mathrm{m}}}\right), \\
\text { ober audf) } \\
{ }^{e} \mathrm{H}_{\mathrm{u}}=\frac{\mathrm{H}_{\mathrm{u}}}{1,0 \mathrm{p}^{\mathrm{n}-\mathrm{u}}}+\frac{\mathrm{D}_{\mathrm{q}}}{1,0 \mathrm{p}^{\mathrm{q}-\mathrm{m}}} \ldots-\frac{\left(\mathrm{B}_{\mathrm{u}}+\mathrm{V}+\mathrm{S}\right)\left(1,0 \mathrm{p}^{\mathrm{u}-\mathrm{m}}-1\right)}{1,0 \mathrm{p}^{\mathrm{u}-\mathrm{m}}} .
\end{gathered}
$$

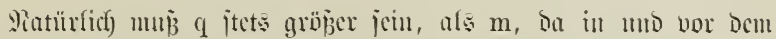

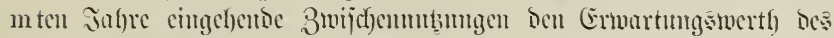
mjäfrigen Bejtandes nicft berïgren.

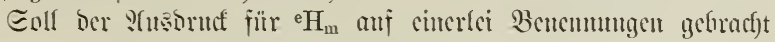

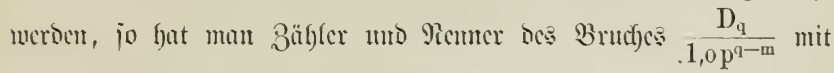
$1,0 p^{\mathrm{u}-\mathrm{q}}$. oll multipliciren mo crfjält

$$
{ }^{\mathrm{e}} \mathrm{H}_{\mathrm{m}}=\frac{\mathrm{H}_{\mathrm{u}}+\mathrm{D}_{\mathrm{q}} 1,0 \mathrm{p}^{\mathrm{u}-\mathrm{q}}-\left(\mathrm{B}_{\mathrm{u}}+\mathrm{V}+\mathrm{S}\right)\left(1,0 \mathrm{p}^{\mathrm{n}-\mathrm{m}}-1\right)}{1,0 \mathrm{p}^{\mathrm{u}-\mathrm{m}}} .
$$

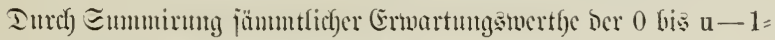
jǘrigen Bejtämbe crbält mun แแแ $\mathrm{N}_{\mathrm{f}}$ :

${ }^{\mathrm{e} H}$ Des $\mathrm{u}-1 \mathrm{j}$. Bejtundes $=\mathrm{H}_{\mathrm{u}} \times \frac{1}{1,0 \mathrm{p}^{1}}-\mathrm{r}^{\prime}\left(\frac{1,0 \mathrm{p}^{1}-1}{1,0 \mathrm{p}^{1} \cdot 0,0 \mathrm{p}}\right)$.

$$
{ }^{\mathrm{e}} \mathrm{H} \quad \mathrm{u}-2 \mathrm{j} . \quad \quad \quad=\mathrm{H}_{\mathrm{u}} \times \frac{1}{1,0 \mathrm{p}^{2}}-\mathrm{r}^{\prime}\left(\frac{1,0 \mathrm{p}^{2}-1}{1,0 \mathrm{p}^{2} \cdot 0,0 \mathrm{p}}\right) .
$$

11. โ. It.

eH ocs qjfrr. Bejtantes,

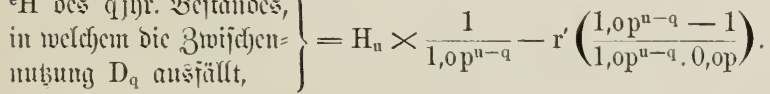

${ }^{\mathrm{e}} \mathrm{H}$ Всs $q-1$ j. Bejtumoç $=\mathrm{H}_{\mathrm{u}} \times \frac{1}{1,0 \mathrm{p}^{\mathrm{u}-(\mathrm{q}-1)}}-\mathrm{r}^{\prime}\left(\frac{1,0 \mathrm{p}^{\mathrm{u}-(\mathrm{q}-1)}-1}{1,0 \mathrm{p}^{\mathrm{u}-(\mathrm{q}-1)} \cdot 0,0 \mathrm{p}}\right)$

$$
+\mathrm{D}_{\mathrm{q}} \times \frac{1}{1,0 \mathrm{p}^{1}} \text {. }
$$

$$
\begin{aligned}
&{ }^{e} \mathrm{H} . " q-2 \mathrm{j} . \quad=\quad=\mathrm{H}_{\mathrm{u}} \times \frac{1}{1,0 \mathrm{p}^{\mathrm{n}-(\mathrm{q}-2)}}-\mathrm{r}^{\prime}\left(\frac{1,0 \mathrm{p}^{\mathrm{u}-(\mathrm{q}-2)}-1}{1,0 \mathrm{p}^{\mathrm{u}-(\mathrm{q}-2)} \cdot 0,0 \mathrm{p}}\right) \\
&+\mathrm{D}_{\mathrm{q}} \times \frac{1}{1,0 \mathrm{p}^{2}} .
\end{aligned}
$$

II. ¡. เพ. 
eH Des 0 jüfrr. Bejtandes $=H_{\mathrm{u}} \times \frac{1}{1,0 \mathrm{p}^{\mathrm{n}-0}}-\mathrm{r}^{\prime}\left(\frac{1,0 \mathrm{p}^{\mathrm{u}-\mathrm{o}}-1}{1,0 \mathrm{p}^{\mathrm{u}-0} \cdot 0,0 \mathrm{p}}\right)$

Jicraus dic Eumme:

$$
+\mathrm{D}_{\mathrm{q}} \times \frac{1}{1,0 \mathrm{p}^{\mathrm{q}}} \text {. }
$$

1. Für bic 5anbarfeitanแbug $H_{u}$.

$$
\mathrm{H}_{\mathrm{u}}\left(\frac{1}{1,0 \mathrm{p}}+\frac{1}{1,0 \mathrm{p}^{2}}+\ldots+\frac{1}{1,0 \mathrm{p}^{\mathrm{u}}}\right)
$$

(uad) Der Cunmentiormel für faflende gieifen $\frac{a\left(1-q^{n}\right)}{1-q}$ )

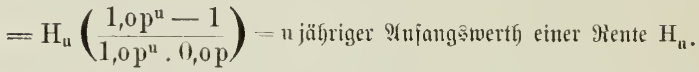

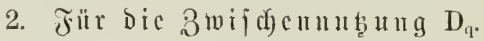

$$
\mathrm{D}_{\mathrm{q}}\left(\frac{1}{1,0 \mathrm{p}}+\frac{1}{1,0 \mathrm{p}^{2}}+\ldots+\frac{1}{1,0 \mathrm{p}^{\mathrm{q}}}\right)
$$

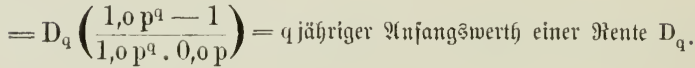

3. Für bie Bobenbruttoreute r'.

$$
\begin{aligned}
& \mathrm{r}^{\prime} \quad\left(\frac{1,0 \mathrm{p}-1}{1,0 \mathrm{p} \cdot 0,0 \mathrm{p}}+\frac{1,0 \mathrm{p}^{2}-1}{1,0 \mathrm{p}^{2} \cdot 0,0 \mathrm{p}}+\ldots+\frac{1,0 \mathrm{p}^{\mathrm{u}}-1}{1,0 \mathrm{p}^{\mathrm{u}} \cdot 0,0 \mathrm{p}}\right) \\
= & \frac{\mathrm{r}^{\prime}}{0,0 \mathrm{p}}\left(1-\frac{1}{1,0 \mathrm{p}}+1-\frac{1}{1,0 \mathrm{p}^{2}}+\ldots+1-\frac{1}{1,0 \mathrm{p}^{\mathrm{u}}}\right) \\
= & \frac{\mathrm{r}^{\prime}}{0,0 \mathrm{p}}\left[\mathrm{u}-\left(\frac{1}{1,0 \mathrm{p}}+\frac{1}{1,0 \mathrm{p}^{2}}+\ldots+\frac{1}{1,0 \mathrm{p}^{\mathrm{u}}}\right)\right] \\
= & \frac{\mathrm{r}^{\prime}}{0,0 \mathrm{p}}\left(\mathrm{u}-\frac{1,0 \mathrm{p}^{\mathrm{u}}-1}{1,0 \mathrm{p}^{\mathrm{u}} \cdot 0,0 \mathrm{p}}\right) .
\end{aligned}
$$

Die ganze Sunme für $\mathrm{N}_{\mathrm{f}}$ (antet jonac):*)

$$
\begin{array}{r}
\mathrm{N}_{\mathrm{f}}=\mathrm{H}_{\mathrm{u}}\left(\frac{1,0 \mathrm{p}^{\mathrm{u}}-1}{1,0 \mathrm{p}^{\mathrm{u}} \cdot 0,0 \mathrm{p}}\right)+\mathrm{D}_{q}\left(\frac{1,0 \mathrm{p}^{q}-1}{1,0 \mathrm{p}^{q} \cdot 0,0 \mathrm{p}}\right) \\
-\frac{\mathrm{r}^{\prime}}{0,0 \mathrm{p}}\left(\mathrm{u}-\frac{1,0 \mathrm{p}^{\mathrm{u}}-1}{1,0 \mathrm{p}^{\mathrm{u}} \cdot 0,0 \mathrm{p}}\right) .
\end{array}
$$

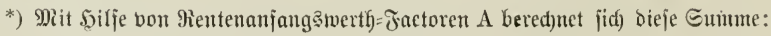

$$
H_{u} \times A_{u}+D_{q} \times A_{q}-\frac{r^{\prime}}{0, o p}\left(u-A_{u}\right) .
$$




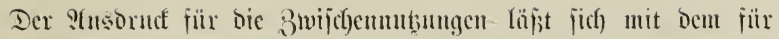

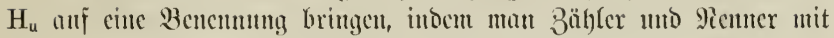
$1,0 \mathrm{p}^{\mathrm{u}-\mathrm{q}}$ multiplicirt, man erbäft bam!:

$$
\mathrm{D}_{\mathrm{q}} \frac{1,0 \mathrm{p}^{\mathrm{u}-\mathrm{q}}\left(1,0 \mathrm{p}^{\mathrm{q}}-1\right)}{1,0 \mathrm{p}^{\mathrm{u}} \cdot 0,0 \mathrm{p}} \text {. }
$$

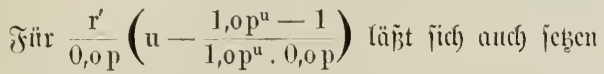

$$
\left(B_{u}+V+S\right) u-\left(B_{u}+V+S\right) \frac{1, o p^{u}-1}{1, o p^{u} \cdot 0,0 p} .
$$

Die 23 erthe $†$ unt \pm in Formed $\mathrm{III}^{\mathrm{a}}$ cingejebt, crgicbt:

$$
\mathrm{N}_{\mathrm{f}}=\frac{\left(\mathrm{H}_{\mathrm{u}}+\mathrm{B}_{\mathrm{u}}+\mathrm{V}+\mathrm{s}\right)\left(1,0 \mathrm{p}^{\mathrm{u}}-1\right)+\mathrm{D}_{\mathrm{q}} 1,0 \mathrm{p}^{\mathrm{u}-\mathrm{q}}\left(1,0 \mathrm{p}^{\mathrm{q}}-1\right)}{1,0 \mathrm{p}^{\mathrm{u}} \cdot 0,0 \mathrm{p}}
$$

Eebet man mun in III $^{\mathrm{b}}$ wiederum Den 2 ererth) für $\mathrm{B}_{\mathrm{u}}$ cin, fo er= hält mant:

$$
\begin{aligned}
\frac{\left[\mathrm{H}_{\mathrm{u}}\right.}{+}+\frac{\left.\mathrm{H}_{\mathrm{u}}+\mathrm{D}_{\mathrm{q}} 1,0 \mathrm{p}^{\mathrm{u}-\mathrm{q}}-\mathrm{k} 1,0 \mathrm{p}^{\mathrm{u}}-(\mathrm{V}+\mathrm{S})+\mathrm{V}+\mathrm{S}\right]\left(1,0 \mathrm{p}^{\mathrm{u}}-1\right)}{1,0 \mathrm{p}^{\mathrm{u}}-1} & 1,0 \mathrm{p}^{\mathrm{u}} \cdot 0,0 \mathrm{p} . \\
& +\frac{\mathrm{D}_{\mathrm{q}} 1,0 \mathrm{p}^{\mathrm{u}-\mathrm{q}}\left(1,0 \mathrm{p}^{\mathrm{q}}-1\right)}{1,0 \mathrm{p}^{\mathrm{u}} \cdot 0,0 \mathrm{p}}-\mathrm{u}\left(\mathrm{B}_{\mathrm{u}}+\mathrm{V}+\mathrm{S}\right) \\
& =\frac{\mathrm{H}_{\mathrm{u}} 1,0 \mathrm{p}^{\mathrm{u}}-\mathrm{k} 1,0 \mathrm{p}^{\mathrm{u}}+\mathrm{D}_{\mathrm{q}} 1,0 \mathrm{p}^{\mathrm{u}}}{1,0 \mathrm{p}^{\mathrm{u}} \cdot 0,0 \mathrm{p}}-\mathrm{u}\left(\mathrm{B}_{\mathrm{u}}+\mathrm{V}+\mathrm{S}\right) \\
& =\frac{\mathrm{H}_{\mathrm{u}}+\mathrm{D}_{\mathrm{q}}-\mathrm{k}-\mathrm{u}(\mathrm{v}+\mathrm{s})}{0,0 \mathrm{p}}-\mathrm{u} \mathrm{B}_{\mathrm{u}} .
\end{aligned}
$$

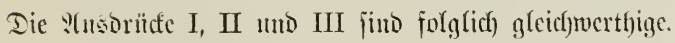

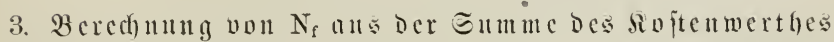
Der i üngeren und Des Ermartungsuerthes ber ältereu Bệtäude.

a. Soitenwerth ber jüngeren, 0 bis $n-1$ jögrigen $B$ ejtände.

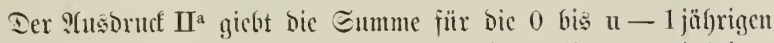

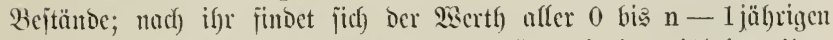
Srte, inbem wir uns dicje Theile des Waldes als im njäl)rigen $11 \mathrm{~m}=$ 
triche ftebeno Denfen, in Formel $I^{a}$ baber anjtutt $\mathrm{u}$ ïherall $\mathrm{n}$ jebeen. Lisir crlyulten Demundf):

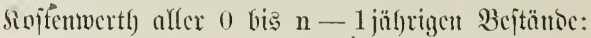

$$
\begin{aligned}
& \left(\frac{\mathrm{r}^{\prime}}{0, \mathrm{op}}-\mathrm{k}\right)\left(1,0 \mathrm{p}^{\mathrm{n}}-1\right)-\mathrm{D}_{\mathrm{a}}\left(1,0 \mathrm{p}^{\mathrm{n}-\mathrm{a}}-1\right)-\mathrm{n \textrm {r } ^ { \prime }} \quad \text { IV. } \\
& 0,0 \mathrm{p}
\end{aligned}
$$

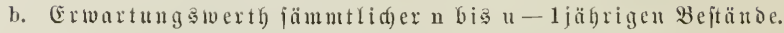

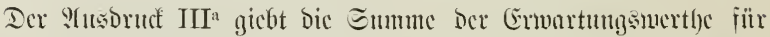
die 0 bis $u-1$ jübrigen Bejtämbc. Dic bei ber betreffenden Ent=

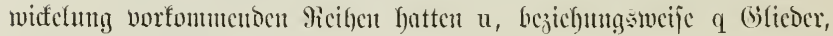

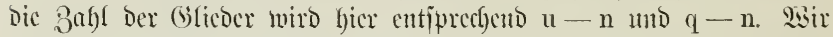
crbalten bemmady bic gejutffe Eumme, indocm wir in Formel $\mathrm{II}^{\mathrm{a}}$ anjtatt

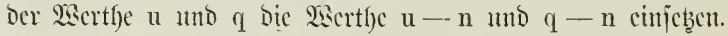

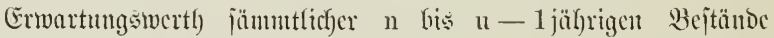
Demnady

$$
\begin{aligned}
& \mathrm{H}_{\mathrm{u}} \frac{1,0 \mathrm{p}^{\mathrm{n}-\mathrm{n}}-1}{1,0 \mathrm{p}^{\mathrm{u}-\mathrm{n}} \cdot 0,0 \mathrm{p}}+\mathrm{D}_{\mathrm{q}} \frac{1,0 \mathrm{p}^{\mathrm{q}-\mathrm{n}}-1}{1,0 \mathrm{p}^{\mathrm{q}-\mathrm{n}} \cdot 0,0 \mathrm{p}}+\ldots \\
& -\frac{\mathrm{r}^{\prime}}{0,0 \mathrm{p}}\left(\mathrm{u}-\mathrm{n}-\frac{1,0 \mathrm{p}^{\mathrm{n}-\mathrm{n}}-1}{1,0 \mathrm{p}^{\mathrm{n}-\mathrm{n}} \cdot 0,0 \mathrm{p}}\right) .
\end{aligned}
$$

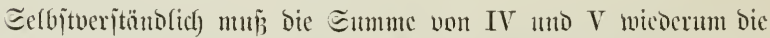

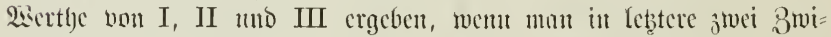

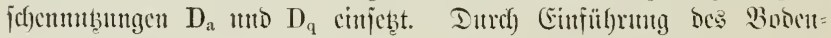

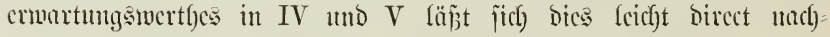
ncijent.

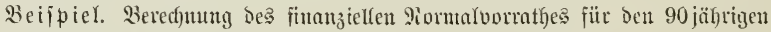
llutrief nach) Der Erfafrungstafel (Seite 64) aus Dem Soitenwerthe der 0 bis $40=$ jährigen un๖ Dem Erwartungąwerthe Der 41 bis 89 jäfrigen Bejtünde.

\section{a. Softenuerth Dex 0 bis 40 jährigen beitände.}

Miad) Formel IV. - Da biefe für die 0 bis $\mathrm{n}-1$ jäfrigen Beftüntoe $\mathrm{n}$ als Expontenten von 1,op gat, jo nuz̃ Derjelbe für bie 0 bis 40 jäfrigen Bejtä̈toc $40+1=41$ jein.

$$
\begin{gathered}
{\left[\left(\frac{7,26.536}{0,03}+30\right)\left(1,03^{41}-1\right)-\left(1,8\left(1,03^{41-20}-1\right)+4\left(1,03^{41-25}-1\right)+7,2\left(1,03^{41-30}-1\right)\right.\right.} \\
\left.\left.+11,2\left(1,03^{41-35}-1\right)+15\left(1,03^{41-40}-1\right)+41 \times 7,26536\right)\right]: 0,03 .
\end{gathered}
$$


ж) Beredunung ber neyativen Siertfe:

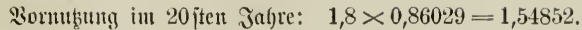

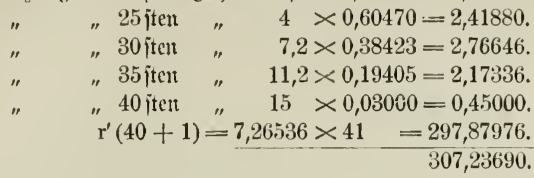

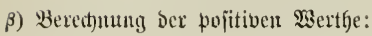

$$
\begin{gathered}
\left(\frac{7,26536}{0,03}+30\right)\left(1,03^{41}-1\right)=272,17867 \times 2,35990=642,31444 . \\
\text { Sunume } \\
\frac{642,31444-307,23690}{0,03}=11169,251 \mathrm{fl} .
\end{gathered}
$$

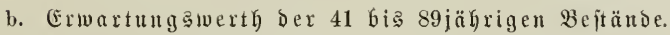

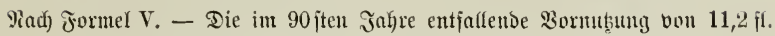

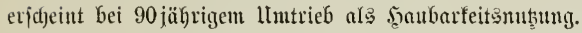

$$
\begin{gathered}
(2875+11,2) \frac{1,03^{90-41}-1}{1,03^{90-41} \cdot 0,03}+16,8 \times \frac{1,03^{85-41}-1}{1,03^{85-41} \cdot 0,03}+20,8 \times \frac{1,03^{80-41}-1}{1,03^{80-41} \cdot 0,03}+\ldots \\
+20,8 \times \frac{1,03^{45-41}-1}{1,03^{45-41} \cdot 0,03}-\frac{7,26536}{0,03}\left(90-41-\frac{1,03^{90-41}-1}{1,03^{90-41} \cdot 0,03}\right) .
\end{gathered}
$$

ж) Beredunuty ber pojitiven : Berthe:

j̧anbarfeits=

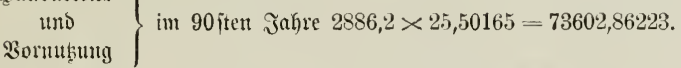

$$
\begin{aligned}
& \text { Bormubutg " } 85 \text { îten " } 16,8 \times 24,25427=407,47174 \text {. } \\
& \text { " } 80 \text { itent " } 20,8 \times 22,80821=474,41077 \text {. } \\
& \text { "75iten " } 26 \times 21,13183=549,42758 \text {. } \\
& \text { " } 70 \text { ïtell " } 33,8 \times 19,18845=648,56961 \text {. } \\
& \text { " } 65 \text { jten " } 34,5 \times 16,93554=584,27613 \text {. } \\
& \text { "60iten " } 34 \times 14,32380=487,00920 \text {. } \\
& \text { " } 55 \text { iten " } 30,6 \times 11,29607=345,65974 \text {. } \\
& \text { " } 50 \text { iten " } 27,2 \times 7,78611=211,78219 \text {. }
\end{aligned}
$$

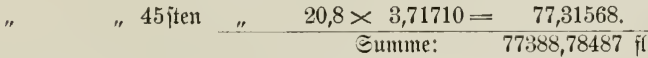

ß) Bered)nung ber negativen Werthe:

$$
\begin{aligned}
& 242,17866(49-25,50165)=5690,79891 \mathrm{fl} \text {. } \\
& \text { Summe der Erwwartutgsmertbe: } 71697,98596 \text { fl. }
\end{aligned}
$$

Werth Des normalen jolzolorrathes $\mathrm{N}_{\mathrm{f}}$ :

a) Summe der Sojtenverthe fämmtlicher 0 bis 40 jägrigen Beptände $=11169,251$ ¡l.

b) " "Evwartung?werthe " 41 " $89 "$ " $=71697,986$, Rormaler solzporrath $\mathrm{N}_{\mathrm{f}}=82867,237 \mathrm{fi}$. 


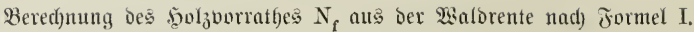

$$
\begin{gathered}
\frac{2875+11,2+16,8+20,8+26+33,8+34,5+34+30,6+27,2+20,8+15}{0,03} \\
+\frac{11,2+7,2+4+1,8-30-90 \times 3}{0,03}-90 \times 142,17867 \\
=\frac{3169,9-300}{0,03}-12796,080=82867,253 \mathrm{fl} .
\end{gathered}
$$

Tie 2sertbe 82867,237 un 82867,253 fl. fimo als gleidye anzuleben, oa die

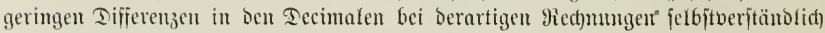
unto berjanimbento flein fint.

4. Bered)ung Des normaren Riorutbstapitales für Deu sccubititandount $\mathrm{N}_{\mathrm{h}}$.

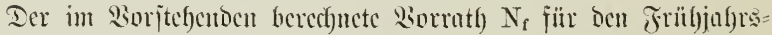

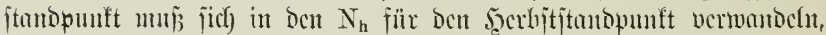

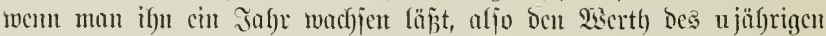
Bejtandes dajutref)net, ben ocs o jührigen bawon abjicht.

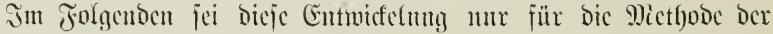
soitcmwertbsred)mung gegehen. Es joll aljo jein:

$$
\mathrm{N}_{\mathrm{h}}=\mathrm{N}_{\mathrm{f}}+{ }^{\mathrm{k}} \mathrm{H}_{\mathrm{u}}-{ }^{\mathrm{k}} \mathrm{H}_{0} \text {. }
$$

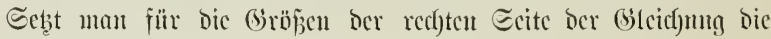
Liscrtbe eil, jo crbält man:

$$
\begin{gathered}
\mathrm{N}_{\mathrm{h}}=\frac{\mathrm{r}^{\prime}}{0,0 \mathrm{p}}\left(\mathrm{E}_{\mathrm{u}}-\mathrm{u}\right)+\mathrm{k} \mathrm{E}_{\mathrm{u}}-\mathrm{D}_{\mathrm{a}} \mathrm{E}_{\mathrm{u}-\mathrm{a}}+\mathrm{r}^{\prime} \mathrm{E}_{\mathrm{u}}+\mathrm{k} 1,0 \mathrm{p}^{\mathrm{u}} \\
-\mathrm{D}_{\mathrm{a}} 1,0 \mathrm{p}^{\mathrm{u}-\mathrm{a}}-\mathrm{k} .
\end{gathered}
$$

Dicjc Formel läßjt jicly umwantolı in

$$
\mathrm{N}_{\mathrm{h}}=\frac{\mathrm{r}^{\prime}}{0,0 \mathrm{p}}\left(\mathrm{E}_{\mathrm{u}} 1,0 \mathrm{p}-\mathrm{u}\right)+\mathrm{k} 1,0 \mathrm{p} \mathrm{E} \mathrm{u}-\mathrm{D}_{\mathrm{a}} \mathrm{E}_{\mathrm{u}+1-\mathrm{a}}
$$

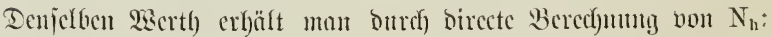
Sojtenmerth) Des 1 jülurigen Bcitundes $\mathrm{r}^{\prime} \mathrm{E}_{1}+\mathrm{k} 1,0 \mathrm{p}^{\mathbf{1}}$

11. $\lceil$. I1).

$$
\text { " } \quad \mathrm{r}^{\prime} \mathrm{E}_{\mathrm{a}}+\mathrm{k} 1,0 \mathrm{p}^{\mathrm{a}}-\mathrm{D}_{\mathrm{a}} 1,0 \mathrm{p}^{0}
$$

II. $\tilde{\jmath}$. Iv.

$$
\text { " " } \quad \text { ". " } \quad \mathrm{r}^{\prime} \mathrm{E}_{\mathrm{u}}+\mathrm{k} 1,0 \mathrm{p}^{\mathrm{u}}-\mathrm{D}_{\mathrm{a}} 1,0 \mathrm{p}^{\mathrm{u}-\mathrm{a}} \text {. }
$$

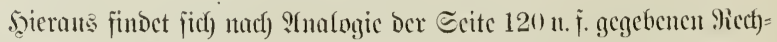
nung bie Summe

$$
\mathrm{N}_{\mathrm{h}}=\frac{\mathrm{r}^{\prime}}{0,0 \mathrm{p}}\left(\mathrm{E}_{\mathrm{u}} 1,0 \mathrm{p}-\mathrm{u}\right)+\mathrm{k} 1,0 \mathrm{p} \mathrm{E} \mathrm{E}_{\mathrm{u}}-\mathrm{D}_{\mathrm{a}} \mathrm{E}_{\mathrm{u}+1-\mathrm{a}}
$$


Beifpiel. Bered)ung bon $\mathrm{N}_{\mathrm{h}}$ fïr ben 90 jährigen llutrieb nad) Der (Ev-

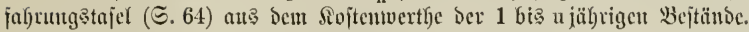

$$
\begin{gathered}
\mathrm{N}_{\mathrm{l}}=\frac{7,26536}{0,03}\left[\frac{1,03^{90}-1}{0,03} \cdot 1,03-90\right]+30 \cdot \frac{1,03^{90}-1}{0,03} \cdot 1,03-\left[1,8 \cdot \frac{1,03^{71}-1}{0,03}\right. \\
\left.\quad+4 \cdot \frac{1,03^{66}-1}{0,03}+\ldots \ldots+11,2 \cdot \frac{1,03-1}{0,03}\right] . \\
\mathrm{N}_{\mathrm{h}}=88794,667+13699,469-16781,875=85712,261 .
\end{gathered}
$$

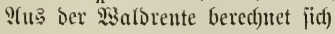

$$
\begin{gathered}
\mathrm{N}_{\mathrm{h}}=\mathrm{N}_{\mathrm{f}}+{ }^{\mathrm{k}} \mathrm{H}_{\mathrm{u}}-{ }^{\mathrm{k}} \mathrm{H}_{\mathrm{o}} \\
=82867,253+2875-30=85712,253 .
\end{gathered}
$$

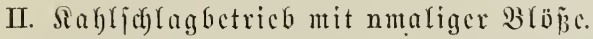

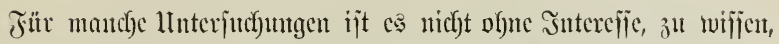

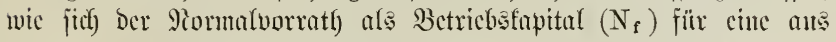

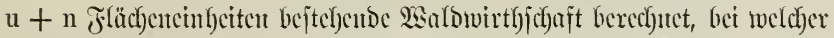

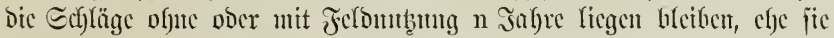
wicber angebant werden. Dafiei faut es fraglich erfecheinen, of mant

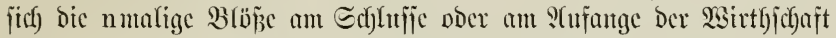

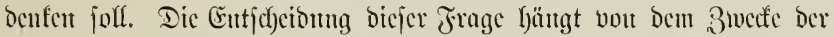

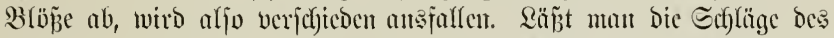
Rüffelfäfers wegen oder itberlyaupt jut Erziclung gïujtigerer Siultur

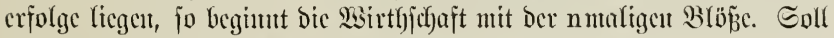

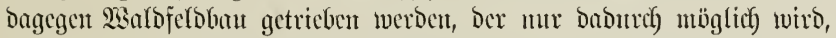

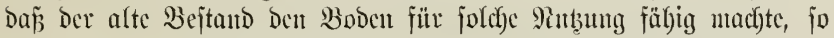

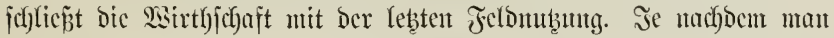

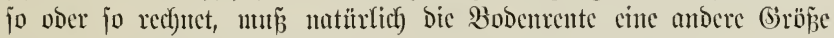

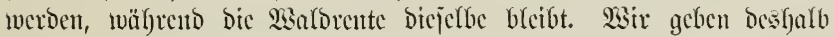

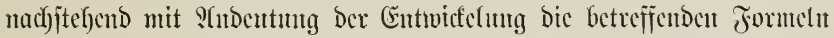

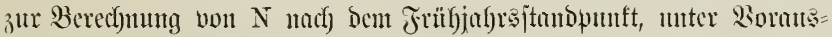

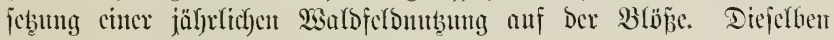

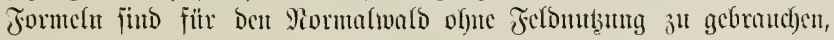

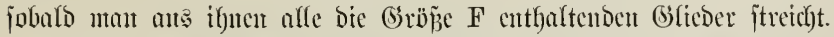

Dic Bezeidfumugen bleiben bicjelben, wic vorber: $\mathrm{E}=$ Fienten

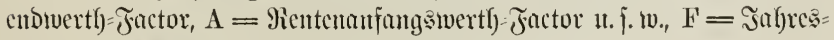
ertrag ber Flächenciull)eit Durth) Feloutbutg.

\section{A. Methobe ber Roitenwerthe zur Beredjutug wou $\mathrm{N}_{f}$.}

1. Die

Dic Bodenloruttorente $r_{a}^{\prime}$ wiro ljicr

$$
=\frac{\mathrm{H}_{\mathrm{u}}+\mathrm{D}_{\mathrm{a}} 1,0 \mathrm{p}^{\mathrm{u}-\mathrm{a}}-\mathrm{k} 1,0 \mathrm{p}^{\mathrm{n}}+\mathrm{FE}_{\mathrm{n}} 1,0 \mathrm{p}^{\mathrm{u}}}{\mathrm{E}_{\mathrm{u}+\mathrm{n}}}
$$


Siojtemwertf) Des u-1jüfr. Sejtandes $=\mathrm{r}_{\mathrm{a}}^{\prime} \mathrm{E}_{\mathrm{u}-1+\mathrm{n}}-\mathrm{FE}_{\mathrm{a}} 1,0 p^{\mathrm{n}-1}$ $+\mathrm{k} 1,0 \mathrm{p}^{\mathrm{n}-1}-\mathrm{D}_{\mathrm{a}} 1,0 \mathrm{p}^{\mathrm{n}-1-\mathrm{a}}$

it. i. tw.

" " a jǘ)r. Bcjtundes $=\mathrm{r}_{\mathrm{a}}^{\prime} \mathrm{E}_{\mathrm{a}+\mathrm{n}}-\mathrm{FE}_{\mathrm{n}} 1,0 \mathrm{p}^{\mathrm{a}}+\mathrm{k} 1,0 \mathrm{p}^{\mathrm{a}}$ - $\mathrm{D}_{\mathrm{a}} 1,0 \mathrm{p}^{\circ}$.

II. i. Iv.

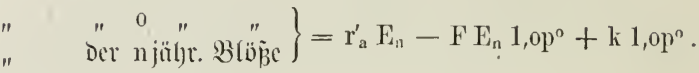

it. $i$. 10 .

" $\quad$ n-njühr. $\mathfrak{B}$ lö $\tilde{\beta} e=\mathrm{r}_{\mathrm{a}}^{\prime} \mathrm{E}_{0}-\mathrm{FE}_{0}$.

Jeteruns beredfut fict) bie Emmme:

$$
\begin{aligned}
\mathrm{N}_{\mathrm{f}}=\frac{\mathrm{r}_{\mathrm{a}}^{\prime}}{0,0 \mathrm{p}} & {\left[\mathrm{E}_{\mathrm{u}+\mathrm{n}}-(\mathrm{u}+\mathrm{n})\right]+\mathrm{k} \mathrm{E}_{\mathrm{u}}-\mathrm{D}_{\mathrm{a}} \mathrm{E}_{\mathrm{u}-\mathrm{a}} } \\
& -\mathrm{FE}_{\mathrm{n}} \mathrm{E}_{\mathrm{u}}-\frac{\mathrm{F}}{0,0 \mathrm{p}}\left[\mathrm{E}_{\mathrm{u}}-\mathrm{n}\right] .
\end{aligned}
$$

Dieje Summe jebst jich jujammen aus:

Dem Bejtandştitcf $=\frac{\mathrm{r}_{\mathrm{a}}^{\prime}}{0,0 \mathrm{p}}\left[\mathrm{E}_{\mathrm{u}} 1,0 \mathrm{p}^{\mathrm{n}}-\mathrm{u}\right]+\mathrm{k} \mathrm{E}_{\mathrm{u}}-\mathrm{D}_{\mathrm{a}} \mathrm{E}_{\mathrm{u}-\mathrm{a}}-\mathrm{FE}_{\mathrm{n}} \mathrm{E}_{\mathrm{u}}$

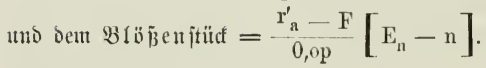

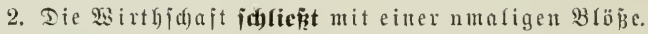

Die Bobenfruttorente $\mathrm{r}_{\mathrm{s}}$ wiro fier

$$
=\frac{\left(\mathrm{H}_{\mathrm{u}}+\mathrm{D}_{\mathrm{a}} \cdot 1,0 \mathrm{p}^{\mathrm{n}-\mathrm{a}}-\mathrm{k} 1,0 \mathrm{p}^{\mathrm{a}}\right) 1,0 \mathrm{p}^{\mathrm{n}}+\mathrm{FE}_{\mathrm{a}}}{\mathrm{E}_{\mathrm{a}+\mathrm{n}}} .
$$

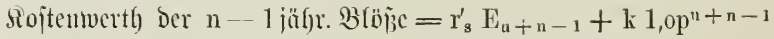

$$
-\mathrm{D}_{\mathrm{a}} 1,0 \mathrm{p}^{\mathrm{n}-\mathrm{a}+\mathrm{n}-\mathrm{t}}-\mathrm{H}_{\mathrm{u}} 1,0 \mathrm{p}^{\mathrm{n}-1}+\mathrm{FE}_{\mathrm{n}-1} \text {. }
$$

it. i. $\mathfrak{i v .}$

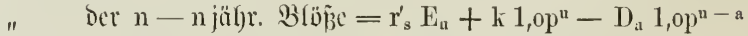

$$
-\mathrm{H}_{\mathrm{u}} 1,0 \mathrm{p}^{\mathrm{o}}-\mathrm{FE}_{\mathrm{o}} \text {. }
$$

" Des $\mathrm{u}-1 \mathrm{jühr}$. Bejtandes $=\mathrm{r}_{\mathrm{s}}^{\prime} \mathrm{E}_{\mathrm{u}-1}+\mathrm{k} 1,0 \mathrm{p}^{\mathrm{u}-1}$

$$
-D_{a} 1,0 p^{\mathrm{n}-\mathrm{a}-1} \text {. }
$$

It. f. w.

" " ajül)r. Bejtandes $=\mathrm{r}_{\mathrm{s}}^{\prime} \mathrm{E}_{\mathrm{a}}+\mathrm{k} 1,0 \mathrm{p}^{\mathrm{a}}-\mathrm{D}_{\mathrm{a}} 1,0 \mathrm{p}^{\circ}$.

11. i. 10.

" $\quad$ " $\mathrm{u}-\mathrm{u}=0$ jähr. Bejtanocs $=\mathrm{r}_{\mathrm{s}}^{\prime} \mathrm{E}_{0}+\mathrm{k} 1,0 \mathrm{p}^{\circ}$.

Jeicrans beredfut fith) bic Eumme: 


$$
\begin{gathered}
\mathrm{N}_{\mathrm{f}}=\frac{\mathrm{r}_{\mathrm{s}}^{\prime}}{0,0 \mathrm{p}}\left[\mathrm{E}_{\mathrm{u}+\mathrm{n}}-(\mathrm{u}+\mathrm{n})\right]+\mathrm{k} \mathrm{E}_{\mathrm{n}+\mathrm{n}}-\mathrm{D}_{\mathrm{a}} \mathrm{E}_{\mathrm{u}+\mathrm{n}-\mathrm{a}}-\mathrm{H}_{\mathrm{u}} \mathrm{E}_{\mathrm{u}} \\
-\frac{\mathrm{F}}{0,0 \mathrm{p}}\left[\mathrm{E}_{\mathrm{n}}-\mathrm{n}\right] .
\end{gathered}
$$

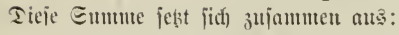

Sem Bejtandşititc $=\frac{r_{s}^{\prime}}{0,0 p}\left[E_{u}-u\right]+E_{a}-D_{a} E_{n-a}$

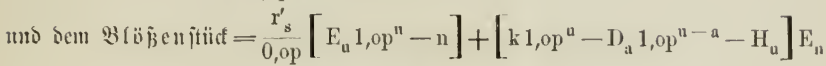

$$
\underset{0, \mathrm{op}}{\mathrm{k}}\left[\mathrm{E}_{\mathrm{u}}-\mathrm{n}\right] \text {. }
$$

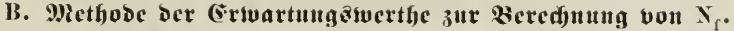

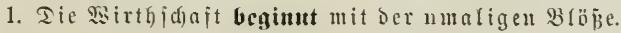

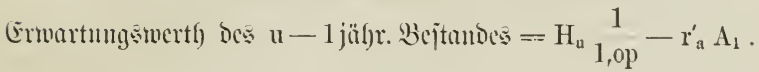

$$
\text { II. i. w. }
$$

" $a-1$ jübr. Bcitantocs $=H_{n} \frac{1}{1,0 p^{n-(a-1)}}$

$$
-r_{a}^{\prime} A_{u-(a-1)}+D_{a} \frac{1}{1,0 p} \text {. }
$$

11. $\tilde{i}$. เw.

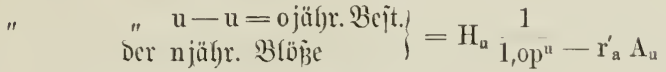

$$
+\mathrm{D}_{\mathrm{a}} \frac{1}{1,0 \mathrm{p}^{\mathrm{a}}} \text {. }
$$

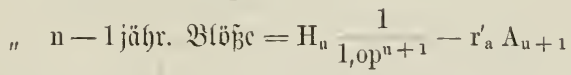

$$
+\mathrm{D}_{\mathrm{a}} \frac{1}{1,0 \mathrm{p}^{\mathrm{a}}+1}-\mathrm{k} \frac{1}{1,0 \mathrm{p}}+\mathrm{F} \mathbf{\Lambda}_{\mathrm{l}} \text {. }
$$

น. $\mathfrak{i}$. w.

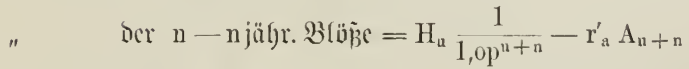

$$
+\mathrm{D}_{\mathrm{a}} \frac{1}{1,0 \mathrm{p}^{\mathrm{a}+\mathrm{n}}}-\mathrm{k} \frac{1}{1,0 \mathrm{p}^{\mathrm{n}}}+\mathrm{FA}_{\mathrm{n}} \text {. }
$$

Şicrams beredfutet fitc) bic Emmmte:

$$
\begin{gathered}
\mathrm{N}_{\mathrm{f}}=\mathrm{H}_{\mathrm{u}} \mathrm{A}_{\mathrm{u}+\mathrm{n}}+\mathrm{D}_{\mathrm{a}} \mathrm{A}_{\mathrm{a}+\mathrm{n}}-\mathrm{k} \mathrm{A}_{\mathrm{n}}-\frac{\mathrm{r}_{\mathrm{a}}^{\prime}}{0,0 \mathrm{p}}\left[\mathrm{u}+\mathrm{n}-\mathrm{A}_{\mathrm{u}+\mathrm{n}}\right] \\
+\underset{0, \mathrm{pp}}{\mathrm{F}}\left[\mathrm{n}-\mathrm{A}_{\mathrm{n}}\right] .
\end{gathered}
$$


Dieje Summe jeל̧t jid zujammen ans:

Deut Bejtanosftïct $=\mathrm{H}_{\mathrm{u}} \mathrm{A}_{\mathrm{u}}+\mathrm{D}_{\mathrm{a}} \mathrm{A}_{\mathrm{a}}-\frac{\mathrm{r}_{\mathrm{a}}^{\prime}}{0, \mathrm{op}}\left[\mathrm{u}-\mathrm{A}_{\mathrm{u}}\right]$

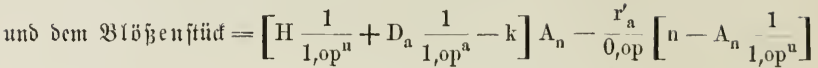

$$
+\frac{\mathrm{F}}{0, \mathrm{op}}\left[\mathrm{n}-\mathrm{A}_{\mathrm{n}}\right] \text {. }
$$

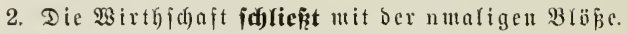

Errwartungstuertf) Der $n-1$ jül)r. $\mathfrak{B}\left(\ddot{0} \tilde{\beta} \mathrm{c}=\mathrm{FA}_{1}-\mathrm{r}_{\mathrm{s}}^{\prime} \mathrm{A}_{1}\right.$.

1t. i. iv.

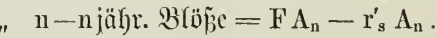

Des $\mathrm{u}-1 \quad$ "Bcitundes $=\mathrm{FA}_{\mathrm{n}} \frac{1}{1, \mathrm{op}}-\mathrm{r}_{\mathrm{s}}^{\prime} A_{\mathrm{n}+1}$

$+\mathrm{H}_{\mathrm{u}} \frac{1}{1,0 \mathrm{p}}$.

11. i. w.

" $\quad$ " a--1 jähr. Bej̣tmines $=\mathrm{FA}_{\mathrm{n}} \frac{1}{1,0 \mathrm{p}^{\mathrm{u}}-(\mathrm{a}-1)}$

$-r_{s}^{\prime} A_{u-(a-1)+n}+H_{u} \frac{1}{1,0 p^{u-(a-1)}}+D_{a} \frac{1}{1,0 p}$.

11. $\lceil$. tw.

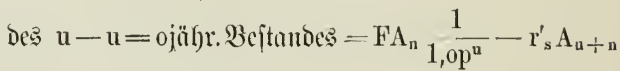

$+\mathrm{H}_{\mathrm{u}} \frac{1}{1,0 \mathrm{p}^{\mathrm{u}}}+\mathrm{D}_{\mathrm{a}} \frac{1}{1,0 \mathrm{p}^{\mathrm{a}}}$.

Şieraus beredfunt fidf bie Emmme:

$$
\begin{gathered}
\mathrm{N}_{\mathrm{f}}=\mathrm{H}_{\mathrm{u}} \mathrm{A}_{\mathrm{u}}+\mathrm{D}_{\mathrm{a}} \mathrm{A}_{\mathrm{a}}-\frac{\mathrm{r}_{\mathrm{s}}^{\prime}}{0, \mathrm{pp}}\left[\mathrm{u}+\mathrm{n}-\mathrm{A}_{\mathrm{n}+\mathrm{n}}\right]+\mathrm{FA}_{\mathrm{n}} A_{\mathrm{u}} \\
+\underset{0,0 \mathrm{p}}{\mathrm{F}}\left[\mathrm{n}-\mathrm{A}_{\mathrm{n}}\right] .
\end{gathered}
$$

Dieje Summe festt jït) zujammen aus:

Dem Beýtandştüd $=\mathrm{H}_{\mathrm{u}} \mathrm{A}_{\mathrm{u}}+\mathrm{I}_{\mathrm{a}} \mathrm{A}_{\mathrm{a}}-\frac{\mathrm{r}_{\mathrm{s}}^{\prime}}{0, \mathrm{op}}\left[\mathrm{u}-\mathrm{A}_{\mathrm{u}} \frac{1}{1,0 \mathrm{p}^{\mathrm{n}}}\right]+\mathrm{F} \mathrm{A}_{\mathrm{n}} \mathrm{A}_{\mathrm{u}}$

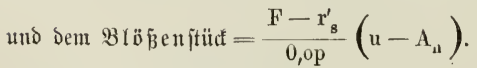




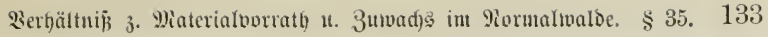

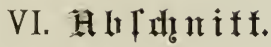

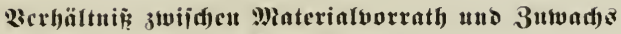 int Normalwalbe.}

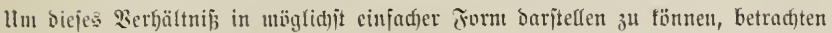

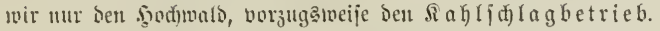

$$
\text { s } 35 .
$$

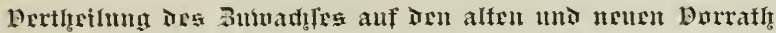

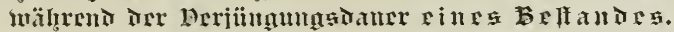

Tistro cin mjüf)riger Bejtund it $n$ gleichen Jaf)resichlägen ver= jï ngt, bas heipt abyetrichen uno jofort micner angebant, jo erfolgt cin Theil bes 3mmabjes Der nüchjten n sabre ant alten Borratbe $\left(V_{1}\right)$, cin moerer biffect ben utenen Bejtand ober Siorrath $\left(V_{2}\right)$ auf

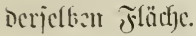

Echts man jum 3meffe Der Ertragsherectumutg Den laufenden

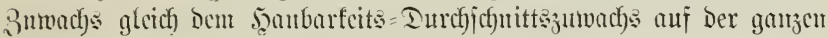

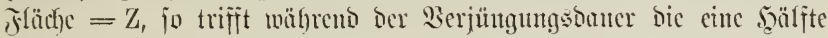

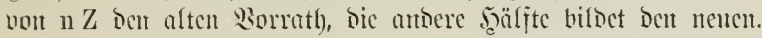

Beweis: Sälurlich wiro $\frac{1}{n}$ bes Bcjtandes genubt, fintet dieje Pubung jofort heim Beginte bes erjen Jafures ftatt, fo wiro im criten sabre nod) $\frac{n-1}{n}$, im jweiten $\frac{n-2}{n}$, im oritten $\frac{n-3}{n}$ n. i. m.,

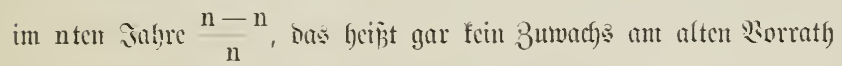

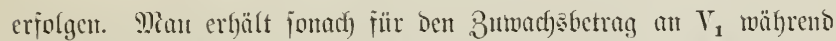
Der Berjüngumgszeit cinte fallende, ariffmetijache Ricibe, Deren Eumme

$$
\left.\mathrm{S}=\left(\frac{\mathrm{n}-1}{\mathrm{n}}+0\right) \frac{\mathrm{n}}{2}=\frac{\mathrm{n}-1}{2} \cdot *\right)
$$

Fintot ber erite Echlag ant Echluije ocs criten Jabres itatt, Der

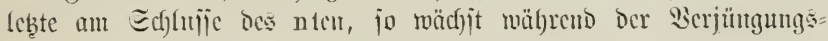
Dauer im criten Iaflece $\frac{n}{n}$, int zweiten $\frac{n-1}{n}$ u. i. w., im leşten $\frac{1}{n}$

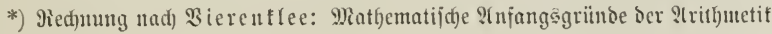
uno Beonetrie. Seipzig, 1767. ङ. 542 . 


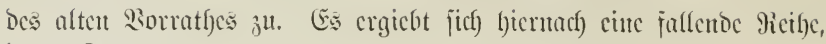
Deren Eummtc

$$
\left.\mathrm{S}=\left(\frac{\mathrm{n}}{\mathrm{n}}+\frac{1}{\mathrm{n}}\right) \frac{\mathrm{n}}{2}=\frac{\mathrm{n}+1}{2} *^{*}\right)
$$

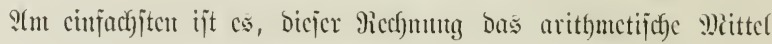

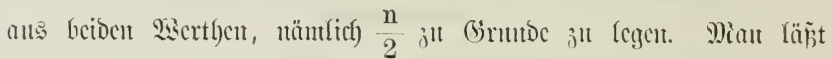

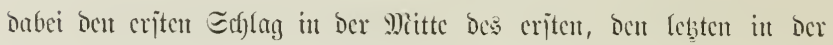

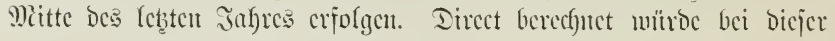

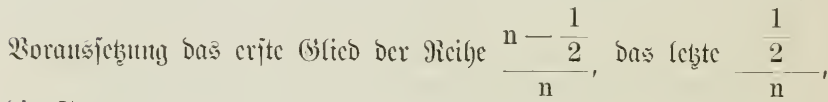
Die Eummic

$$
S=\left(\frac{n-\frac{1}{2}}{n}+\frac{\frac{1}{2}}{n}\right) \frac{n}{2}=\frac{n}{2} .
$$

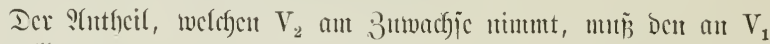

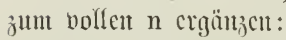

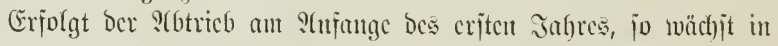

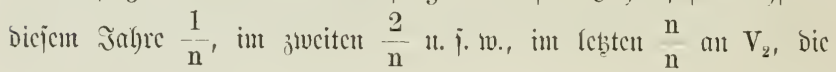
Enmute ber Micile wird Dafer

$$
\mathrm{S}=\left(\frac{1}{\mathrm{n}}+\frac{\mathrm{n}}{\mathrm{n}}\right)_{2}^{\mathrm{n}}=\frac{\mathrm{n}+1}{2} .
$$

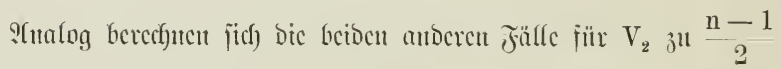
IInD $\frac{\mathrm{n}}{2}$.

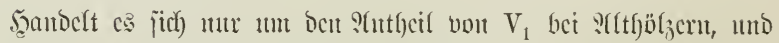

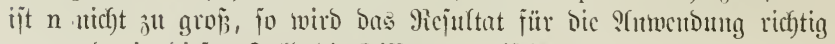

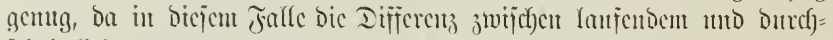

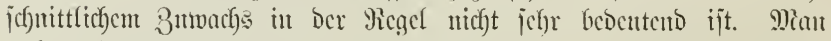

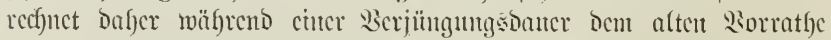

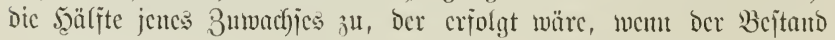

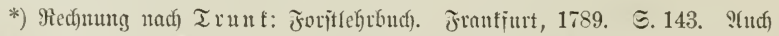

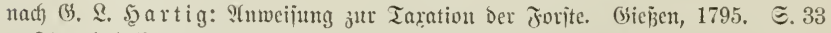
u. 34 und befien Jnitructionen fïr bie fgl. preuj. Forjttą̧atoren. Bertin, 1819. 2. 2ใuf̣. Berl. 1836. ङ. 18. 


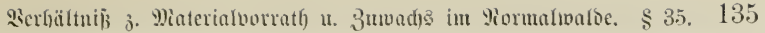

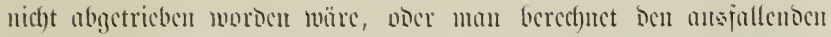

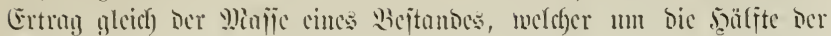

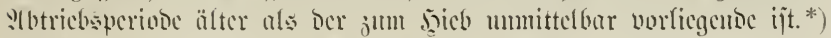

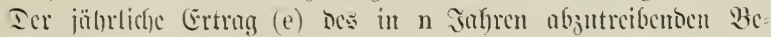

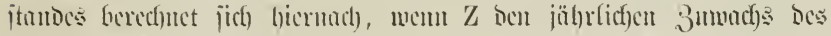

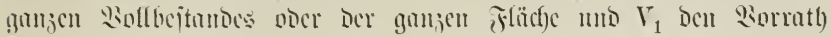
beocutet, mach ocr jormel:

$$
\mathrm{e}=\frac{\mathrm{V}_{1}+\frac{\mathrm{Z}}{2} \times \mathrm{n}}{\mathrm{n}}=\frac{\mathrm{V}_{1}}{\mathrm{n}}+\frac{\mathrm{Z}}{2} .
$$

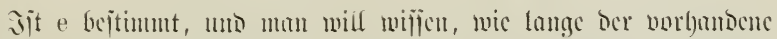

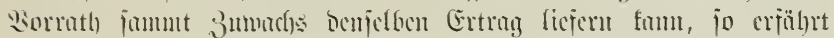

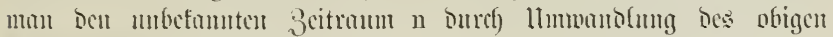
?tusoructeo in

$$
\mathrm{n}=\frac{\mathrm{T}_{1}}{\mathrm{e}-\frac{\mathrm{Z}}{2}} .
$$

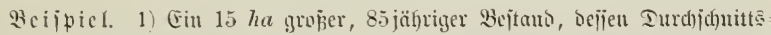
3)unact) voun boftar 6,4 fin beträgt, jofl in 10 Jafren abgetricben werbert. Wiste groj ijt ber Ertray bes Jabreşjhlages?

$$
\begin{aligned}
\mathrm{V}_{1} & =6,4 \times 85 \times 15=8160 \mathrm{fm}, \\
\mathrm{Z} & =6,4 \times 15=96 " \\
\mathrm{e} & =\frac{8160}{10}+\frac{96}{2}=864 " .
\end{aligned}
$$

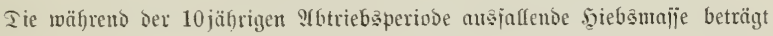
Semmad)

$$
864 \times 10=8640 \mathrm{fm} .
$$

Sujelben Betrag erbält man, venn man Dem gegenwärtigen Bejtandsåter

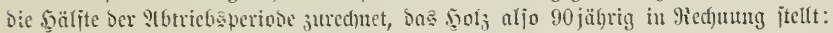

jägrlit()

$$
\begin{aligned}
90 \times 6,4 \times 15 & =8640 \mathrm{fm}, \\
8640 & =864 ， \\
10 & =864
\end{aligned}
$$

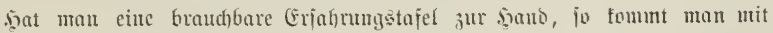

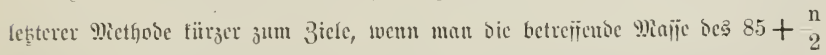

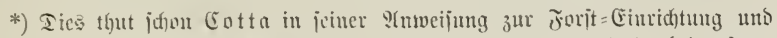

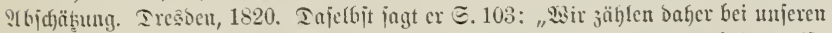

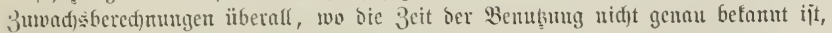

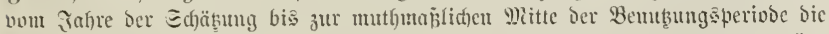

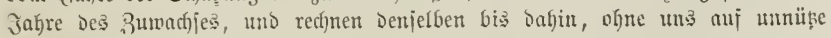
然eitlüufigleiten cinjulaj̄en." 
¡ägrigen Bejtanbeş abliçt. Mant vermeidet babei ben fleinen Fehler, Der möglid)er=

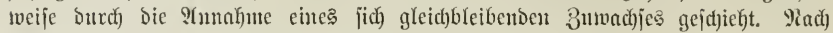

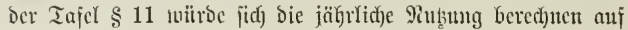

$$
\mathrm{e}=\frac{575 \times 15}{10}=862,5 \mathrm{fm} .
$$

2) SSie Ynuge wïrbe bicjer Bejtand einen jähryidjen (Ertrag von $1068 \mathrm{fm}$ Yięern fö̀nen?

$$
\mathrm{n}=\frac{8160}{1068-\frac{96}{2}}=8 \text { Эahre. }
$$

Der Bejtano tämte im Mittel $85+\frac{8}{2}$ jährig ว̆m şiebe, müroe aljo eine (bejaumutmajīe von $89 \times 6,4 \times 15=8544$, voer jä̆rrliç) $\frac{8544}{8}=1068 \mathrm{fm}$ gewäbren. (

3) Folgt Der Shbau bem S(btriebe jofort nad), jo ijt Der junge Bejtanto unmittel=

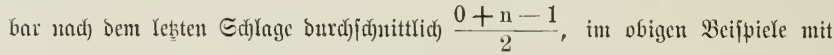

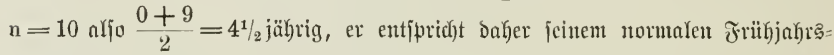

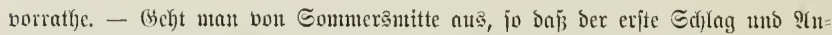

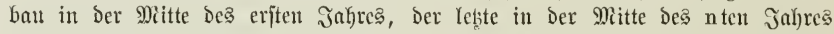

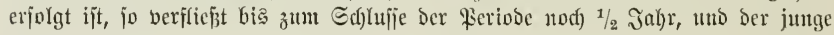

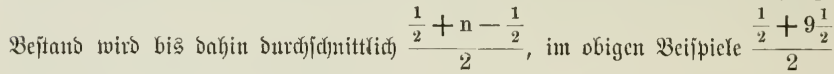
$=5$ jährig, enthä̆lt aljo jeinen mittleten Tormalvorratf́.

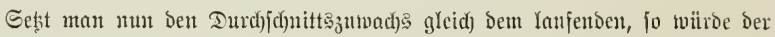
Borrath des jutgen Bejtandes im obigen Beijpiele am Edflujie der njäbrigen Ferivoe betragen

$$
6,4 \times 15 \times 5=480 \mathrm{fm} \text {. }
$$

Da bie ganze Flärhe bon 15 ha an altem แnto nettem Sorrathe jufammen in Derjelben Zeit

$$
6,4 \times 15 \times 10=960 \mathrm{fm}
$$

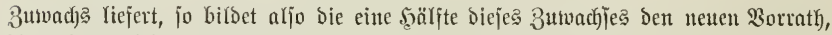
bie andere twirb allmälig mit bem alten Borratl) abgetriebent.

\section{$\S 36$.}

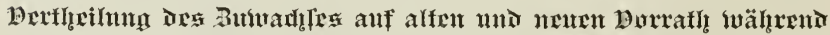

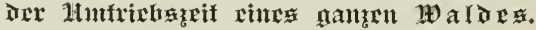

A. Rectinung mit gुiffe von Ertragstafeln.

S(chnlicf) wic mit bem einzelnen Bcjtande während cincr gewiffen

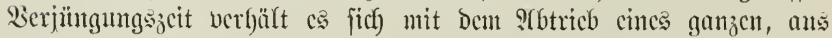
u Flächencinl)citen bejtel)enocn Pormalmalbes im ujäbrigen llmtriebe wäbrento einer ganzen limtriebszcit. 


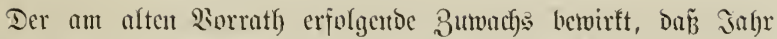

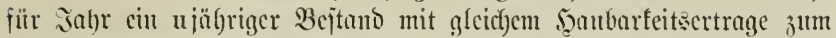

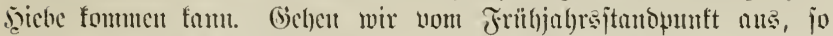

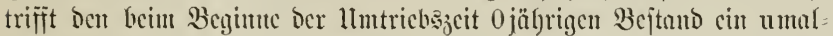
iger, Den 1 jü̈rrigen cin u -1 mnliger u. j. เo., Den u -1 jübrigen 8 cejtuto cetolich cit 1 muliger Jabreşumadys.

Der andere Theil Des am ganzen 2 Sald crfolgenden bejammt=

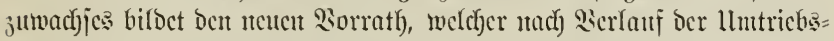

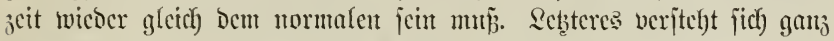
won jelbjt, da bei umaligent S(btricbe Des ftets ältejtet, ujä)rigen

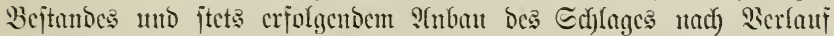

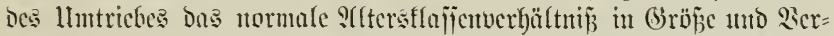

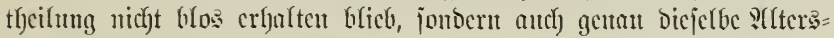
itufectfolge twieder cingetretent iit, bout ber man attoging.

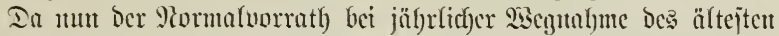

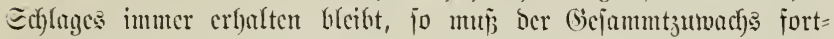

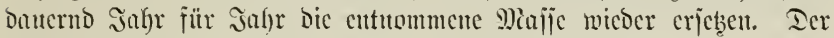

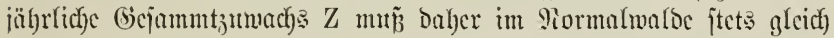

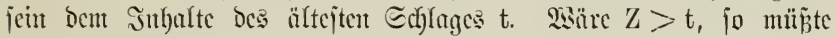

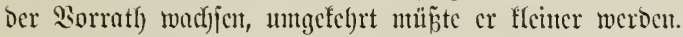

Beifpiele.

\section{Summarijo Bertheilung.}

Betrađten wir einen 60 ha gropen $\mathfrak{W a l b}$, bent bie Erfahrungatajel $\$ 11$

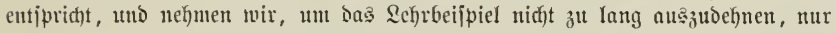

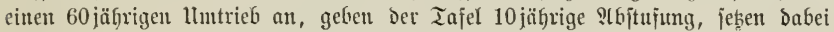

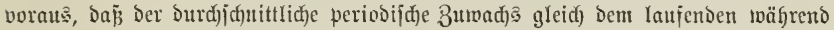

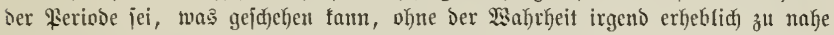
ju treten, fo ergiebt jid) folgende Red)nutg:

Pormalvorratf Durd) Eummirung Der Ertragstafel

$$
\begin{aligned}
& \text { für Den serbit: } 8837 \mathrm{fm} \text {, } \\
& \text { " Commer: } 8660 \text { " } \\
& \text { " Dกక Früfjałr: } 8483 \text { " }
\end{aligned}
$$

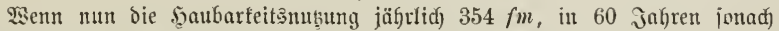
$354 \times 60=21240 \mathrm{fm}$ beträgt, babei aber fortwähreno, aljo autd) nad) 60 Jahren Der Rormalvorrath vorbanden ijt, jo mun bie Siertfeilung des in 60 Jahren $60 \times 10(2+4,5+6,4+7,1+7,5+7,9)$ ober fur $60 \times 354=21240 \mathrm{fm}$ be $=$ tragenden 3uwadjes an bem alten uno neten Borrathe folgente jein:

Sährento Der llmtriebszeit wiro oer ganze alte Borrath $\left(V_{1}\right)$ janunt bem

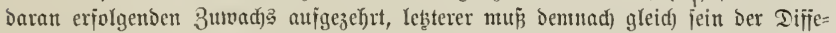

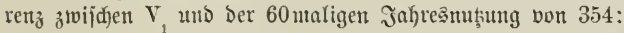

$$
\mathrm{V}_{1} \mathrm{Z} \text { all } \mathrm{V}_{1}
$$

(beht ntau bou serbjt aus, jonad) $60 \times 354-8837=12403$,

$$
\text { " " " Eommer " " " " " } \quad 60 \times 354-8660=12580,
$$




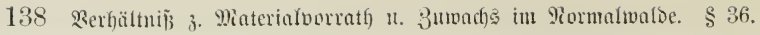

Tie Miaije des dru neuen Borrath $\mathrm{V}_{2}$ bildenden Bumachjes iit un gleid) den

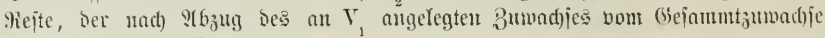
$=21240 \mathrm{fm}$ übrig bleibt.

$$
\begin{aligned}
& \text { Fïr Den .jerbititanopunft } \begin{array}{r}
\mathrm{Z} \text { an V, Z au V, } \\
21240-12403^{2}=88: 37^{2}
\end{array} \\
& \text { " " Eommeritanopmuft } 21240-12580=8660 \text {, } \\
& \text { " " సrïhjafrsitanoputft } 21240-12757=8483 \text {. }
\end{aligned}
$$

Siejer Rejt ijt jelbifweritämblich gleidh bem normales Borrathe.

2. Beredunug ber 3uwad)santheile an $V_{1}$ und $V_{z}$ wägrend der ll utriebsacit aus ben einjeluen Jahter.

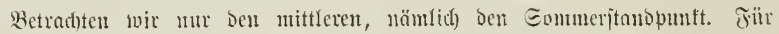

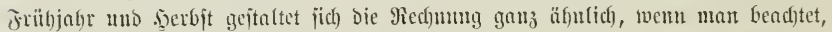

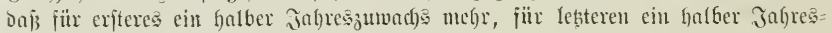

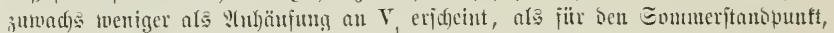

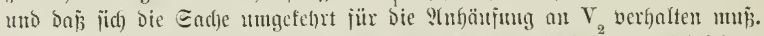

Ter lanfende 3 umad)s wirb, wie voritehend, gleid) bem periobijden (Iutd)

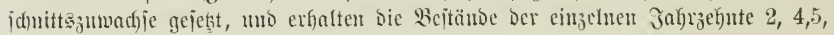
$6,4,7,1,7,5$ und 7,9 fïr Das Jafir und şeftar.

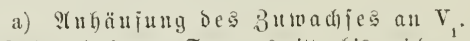

Jut eriten Jahre, D. h. von Eommersmitte bis wieder auf Eommersmitte, erfält an Buwach ơ der jeşt

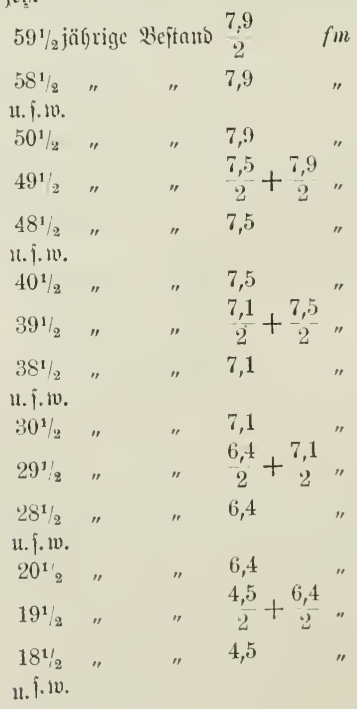

(er wird in serbite abgetrieben.) 


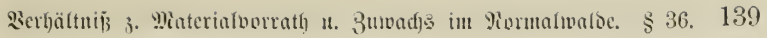

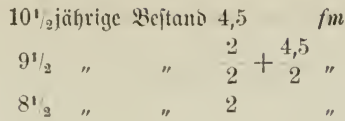

II. i. .w.

${ }^{12}$ " 2

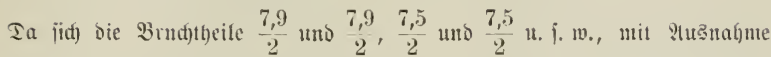

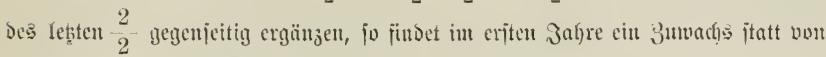

$$
10(7,9+7,5+7,1+6,4+4,5)+9,5 \times 2 .
$$

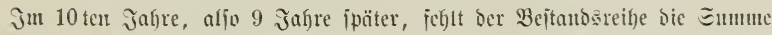

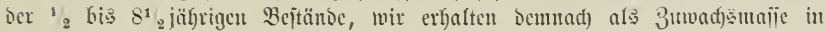
biejem Эafre

$$
10(7,9+7,5+7,1+6,4+4,5)+0,5 \times 2 .
$$

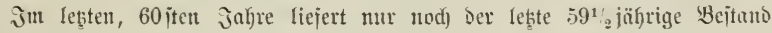

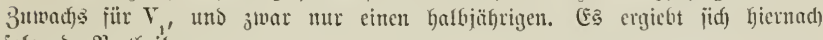
folgende Sertheilung:

Im 1. Jahre wadjien an $\mathrm{V}_{1}:(7,9+7,5+7,1+6,4+4,5) 10+2 \times 9,5=353$

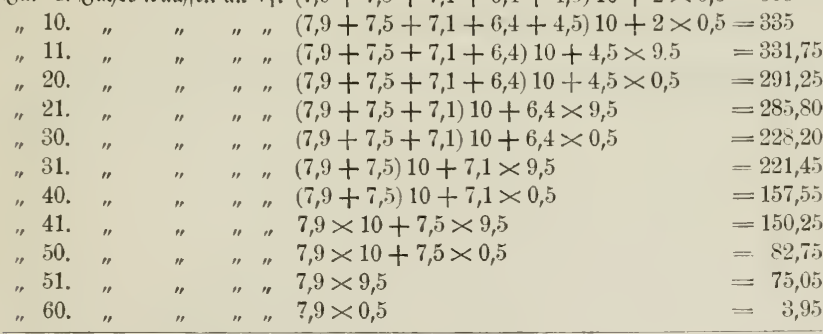

2516 .

In 60 Jafren ïbergaupt, on bie in bem 1 bis 10 ten, 11 bis 20 jten u. f. m.

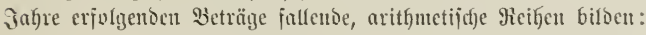

$$
\begin{gathered}
(353+335) \frac{10}{2}+\ldots \ldots \ldots+(75,05+3,95) \frac{10}{2} \\
=(353+335+331,75+\ldots \ldots+3,95) 5=2516 \times 5=12580 \mathrm{fm} .
\end{gathered}
$$

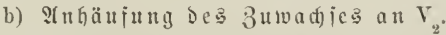

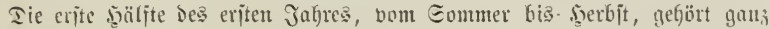

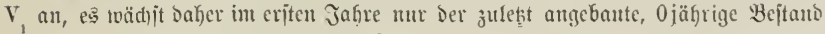

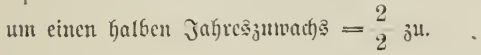


140 Berbältnī̄ 3. Materialvorrath u. 3uvad) im Mormalwalde. \$36.

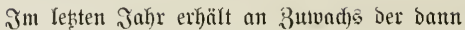

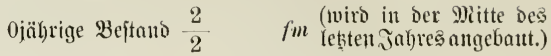

$$
\begin{aligned}
& \begin{array}{rlll}
1 / 2 & 2 & 2 & \\
1 / 2 & 2
\end{array} \\
& \text { แt. j. } 1 \text {. } \\
& 91 / 2 \text { " " } \frac{2}{2}+\frac{4,5}{2} \text { " } \\
& 181 / 2 \text { " " } 4,5 \text { " } \\
& 19 \% / 2 \text { " " } \frac{4,5}{2}+\frac{6,4}{2} \text { " } \\
& 28 \% / 2 \text { " " } 6,4 \\
& \text { 11. }\{.1 \mathrm{w} \text {. } \\
& 49^{1 / 2} \text { " " } \frac{7,5}{2}+\frac{7,9}{2} \text { " } \\
& 58^{1 / 2} \text { " " } 7,9
\end{aligned}
$$

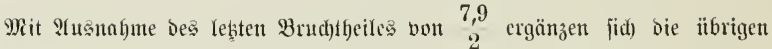

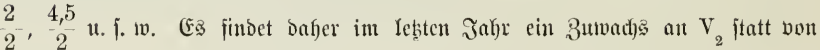

$$
10(2+4,5+6,4+7,1+7,5)+9,5 \times 7,9 .
$$

Wir erbalten bemnad) folgende Rertfeilung:

$$
\begin{aligned}
& \text { Im 1. Jahre 3muads an } \mathrm{V}_{2}: 2 \times 0,5 \\
& \text { "10. " " " " } 2 \times 9,5=19 \\
& \text { "11. " " " } 2 \times 10+4,5 \times 0,5=22,25 \\
& \text { "20. " " " " } " 2 \times 10+4,5 \times 9,5=62,75 \\
& \text { "21. " " " " } "(2+4,5) 10+6,4 \times 0,5=68,20 \\
& \text { "30. " " " " } "(2+4,5) 10+6,4 \times 9,5=125,80 \\
& \text { "31. " " " } "(2+4,5+6,4) 10+7,1 \times 0,5 \quad=132,55 \\
& \text { "40. " " " " } "(2+4,5+6,4) 10+7,1 \times 9,5 \quad=196,45 \\
& \text { "41. " " " " " } "(2+4,5+6,4+7,1) 10+7,5 \times 0,5=203,75 \\
& \text { " } 50 \text { " " " } "(2+4,5+6,4+7,1) 10+7,5 \times 9,5=271,25 \\
& \text { " 51. " " " } "(2+4,5+6,4+7,1+7,5) 10+7,9 \times 0,5=278,95 \\
& \text { " } 60 \text {. " " " } 12+4,5+6,4+7,1+7,5) 10+7,9 \times 9,5=350,05
\end{aligned}
$$

In 60 Эahren überhant:

$$
(1+19+22,25+\ldots \ldots+350,05) 5=1732 \times 5=8660 \mathrm{fm} .
$$

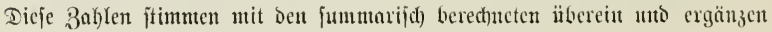
(iic) zน 1 Z, näm(ich:

Inbäufung des 3umachles an $\mathrm{V}_{2}=8660=$ normaler Soumervorrath,

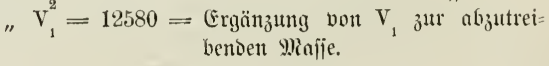


B. Rec(juung nit giffe des Durdffdjnittşuwadjfes.

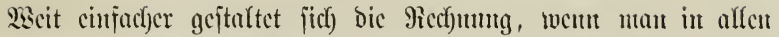

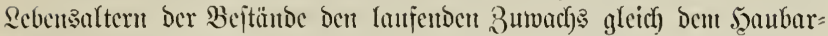

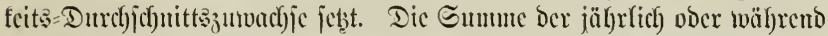

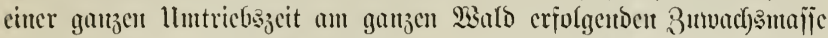
bleibt bicjelbe, me bic Bertbeilung wird cine anbere.

Bezeichnet t den ujübrigen Echlag, z ben 1 jäl)rigen Buwach?

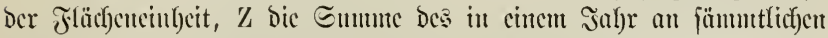

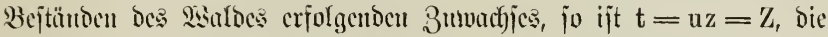
währeno ber ganjen llmtricbegzeit abjutreibende Miafic ift $=\mathrm{uZ}$. Da mแn währeno u jtets ber ganze, alte Bovrath aufgejchit wirb, jo

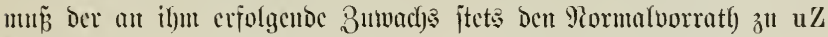
crgänǰn:

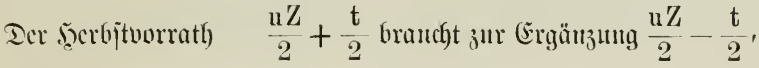

" Commerborraty $\frac{\mathrm{uZ}}{2} \quad$ " " $\quad \frac{\mathrm{uZ}}{2}$,

" Frïfjafravorrath) $\frac{\mathrm{uZ}}{2}-\frac{\mathrm{t}}{2} \quad$ " " $\quad \frac{\mathrm{uZ}}{2}+\frac{\mathrm{t}}{2}$.

(5. crgiebt fich licras wäbrento der llmtriebszeit folgenoc $3 \mathrm{u}=$

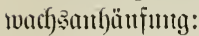

Für Den scerbititanopunft

$$
\text { unt } \left.\begin{array}{r}
\mathrm{V}_{1}=\frac{\mathrm{uZ}}{2}-\frac{\mathrm{t}}{2} \\
\mathrm{~V}_{2}=\frac{\mathrm{uZ}}{2}+\frac{\mathrm{t}}{2}
\end{array}\right\} \text { Eumme von beioen = uZ. }
$$

Für Den Eommeritantopunft

$$
\text { ant } \left.\begin{array}{rl}
V_{1} & =\frac{u Z}{2} \\
& V_{2}=\frac{u Z}{2}
\end{array}\right\} \text { Summe von beiden }=u Z
$$

Für Den Frübjabrsftanopunft

$$
\left.\begin{array}{l}
\text { an } V_{1}=\frac{\mathrm{uZ}}{2}+\frac{\mathrm{t}}{2} \\
\mathrm{~V}_{2}=\frac{\mathrm{uZ}}{2}-\frac{\mathrm{t}}{2}
\end{array}\right\} \text { Enmme von beioen }=\mathrm{uZ}
$$




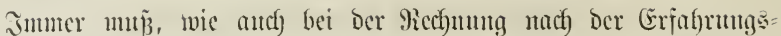

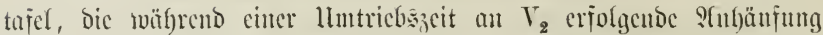
bes Bumadjes gleidg Dem entipredfenden Pormalvorratfe jeit.

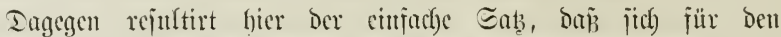
Eommerjtand won uZ genau bic şälite an $V_{1}$, bic andere şälite an $\mathrm{T}_{2}$ wertbeilt.

Beipiere

$$
\text { 1) Eumuarijge 3ertheilung. }
$$

Jür bas oben durdggejühorte 3 ahlenbeifpiel ijt $\mathrm{t}=\mathrm{Z}=354, \mathrm{u}=60, \mathrm{uZ}=21240$, แกเ $\mathrm{z}_{n}=\frac{354}{60}=5,9$.

(53 berech)net j̈̈(h) Giernad):

\begin{tabular}{|c|c|c|c|}
\hline & \multirow{2}{*}{$\begin{array}{l}\text { Normal } \\
\text { vorrath. }\end{array}$} & \multicolumn{2}{|c|}{ 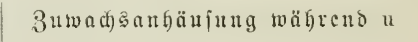 } \\
\hline & & an $V_{1}$ & an $\mathrm{V}_{2}$ \\
\hline Für Sen Serbjtitanopunft: & 10797 & $\frac{21240}{2}-\frac{354}{2}=10443$ & $\frac{21240}{2}+\frac{354}{2}=10797$ \\
\hline " " Eommeritanopmutt: & 10620 & $=10620$ & $\frac{21240}{2}$ \\
\hline " " Irübjahrsitanopunft: & 10443 & $\frac{21240}{2}+\frac{354}{2}=10797$ & $\frac{21240}{2}-\frac{354}{2}=10443$ \\
\hline
\end{tabular}

Ta bie betrefiensen Rethen mit greidhen Iifjerenzen jteigen ober fallen, jo be=

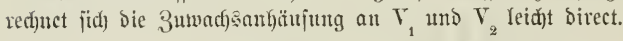

3. B. für ben Eommeriatanopunt:

a) ant $\mathrm{V}_{1}$.

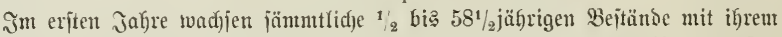

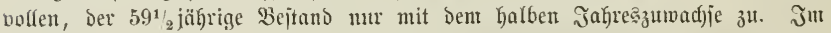

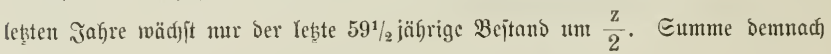

$$
\left(59 \times 5,9+\frac{5,9}{2}+\frac{5,9}{2}\right) \frac{60}{2}=10620 \mathrm{fm} .
$$

b) an $\mathrm{V}_{2}$.

Im eriten Эafure wächjt nut Der in Der Mitte biejes Gafres begrünbete 3ejtand mit einem halben, im reţten Iafre wadjen jämuntfiche $1 / 2$ bis $581 / 2$ jährigen Bejtänte mit ifrem ganzen, Der 0 jäfrige Bejtand mit bem halben z żu. Summe Demuad)

$$
\left(\frac{5,9}{2}+59 \times 5,9+\frac{5,9}{2}\right) \frac{60}{2}=10620 \mathrm{fm} .
$$




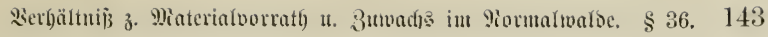

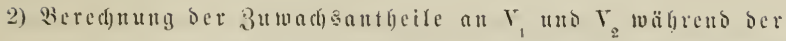

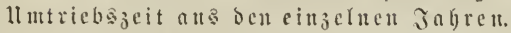

Eumutritanopuntt:

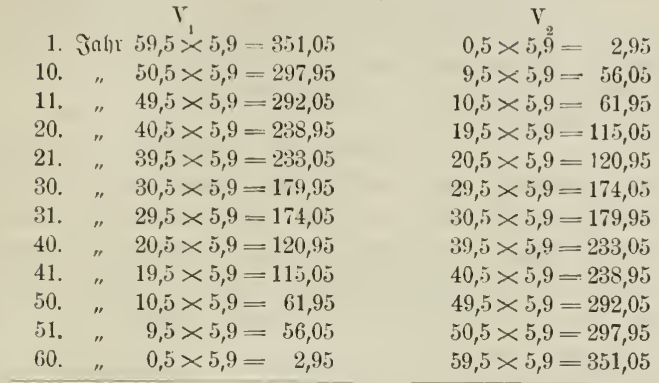

ङumute: $5 \times 2124=10620 \mathrm{fm}$.

Summe: $5 \times 2124=10620 \mathrm{fm}$.

\section{$\S 37$.}

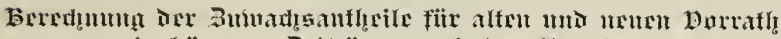

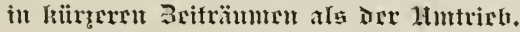

\section{A. Jlit gjiffe von Ertragstufefn.}

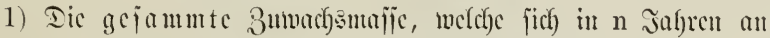
$V_{2}$ und $V_{1}$ angelegt hat, wird an cinfachiten gefumben, imbem man Den Sorratf) anf ber innerfarlb bicjer 3cit nerjüngten Fläche Dured)

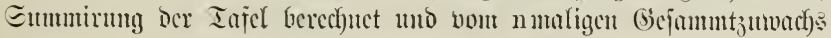
abjiclit.

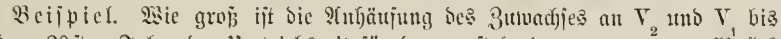

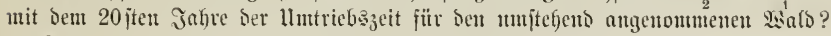
- Eonmeritanopuntt.

9t6getrieben wtrisen

$$
\begin{aligned}
& 20 \times \frac{60}{60}=20 \mathrm{ha}, \text { safer } \\
& \left(20+\frac{65}{2}\right) 10=525 \mathrm{fm}=\mathrm{V}_{2} .
\end{aligned}
$$

$354 \times 20-525=6555 \mathrm{fm}=\mathfrak{?}$ ?nłäufung an $\mathrm{V}_{1}$.

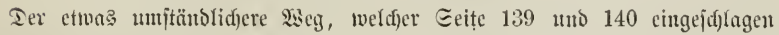

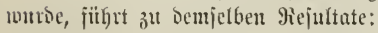

$$
\begin{aligned}
& 5(1+19+22,25+62,75)=525 \text { an } \mathrm{V}_{2^{*}} . \\
& 5(353+335+331,75+291,25)=6555 \text { an } \mathrm{V}_{1} .
\end{aligned}
$$

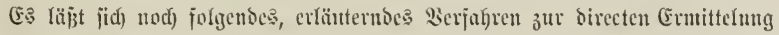

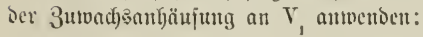


In Den 20 Jafhren wmitoen gefdjlagen alle bei Beginn von u $40 \% \frac{1}{2}$ bis $59 \frac{1}{2}=$ jührigen Bejtänoc,

$$
\text { Deren } \text { Borrath beträgt: } 5520 \mathrm{fm} \text {. }
$$

:(n Majie murben gejd)lagen $20 \times 354=7080$

$$
\text { (5. waren Daher Durd) Den Buwad) zu Decten } 1560 \mathrm{fm} \text {. }
$$

It Derfelben Beit berwandeln fid Die $1 / 2$ bis $39^{1} / 2$ jährigen Bejtänbe in $20^{1 / 2}$ bis $59^{1 / 2 j a ̈ b r i g e, ~ b e r e n ~ M a j i j e ~ j t i e g ~ D a b e r ~ v o n ~}$ 3140 aแf 8135 , bą heip̈t um 4995 3uwadys an $\mathrm{V}_{1}$ in 20 Jahren $6555 \mathrm{fm}$.

2) Dic Bumadjsbeträgc, weld ce fich in cincm beliebigen Jabre $n$ Der llmtriebsjeit an $\mathrm{V}_{1}$ und $\mathrm{V}_{2}$ anlegen, funbet man Durd Ermittelum

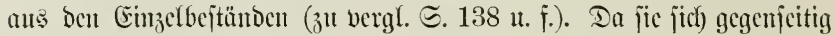

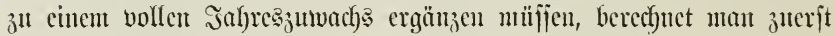

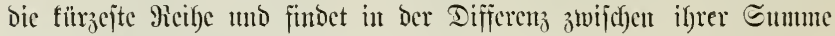
แmঠ Z dic Eumme der längeren.

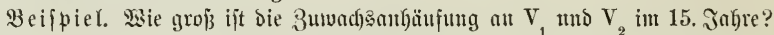
$\mathrm{V}_{2}$ bejteht an Sibluffe bes 14 ten oder ?tufaug Des 15 ten Jahbres aus einer

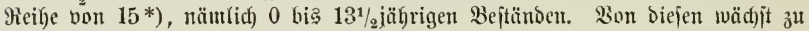

$$
\begin{aligned}
& \text { ser 0jäbrige Bejtand } 2 \text { (wirs in Der Mitte bes } \\
& \text { Der 0jäbrige Bejtand } \frac{2}{2} \quad 15 \text { ten Jabres angebaut.) } \\
& \text { " } 1 / 2, \quad 2 \\
& \text { " } 91 / 2 \text { " } \quad \frac{2}{2}+\frac{4,5}{2} \\
& \text { " } 10^{1 / 2} \text { " } \quad 4,5 \\
& \text { น.โ. เพ. } \\
& \text { " } 13 \frac{1}{2} \text { " " } 4,5 \\
& \text { Sumume: " } 10 \times 2+4,5 \times 4,5=40,25 \mathrm{fm} \text { fïr } \mathrm{V}_{2} \text {. } \\
& 354-40,25=313,75 " \text { " } \mathrm{V}_{1}^{2} \text {. }
\end{aligned}
$$

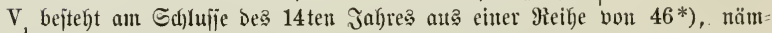

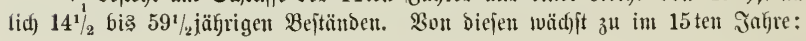
Der $14 \frac{1}{2}$ jährige Beitano 4,5

$$
\begin{aligned}
& \text { " } 19 \frac{1}{2} \text { " " } \frac{4,5}{2}+\frac{6,4}{2} \\
& \text { " } 29^{1} / 2 \quad \text { " } \quad \frac{6,4}{2}+\frac{7,1}{2} \\
& \text { " } 39 \% \text { " " } \frac{7,1}{2}+\frac{7,5}{2} \\
& \text { " } 49 \% \text { " " } \frac{7,5}{2}+\frac{7,9}{2} \\
& \text { " } 501 / 2 \text { " } 7,9 \\
& \text { " } 59 \mu_{2} \quad \text { " } \quad \frac{7,9}{2} \quad \begin{array}{c}
\text { (1virb in Der Mitte Deß } \\
\text { Jabre? abgetrieben.) }
\end{array}
\end{aligned}
$$

ธนแแนเ : $10(7,9+7,5+7,1+6,4)+5,5 \times 4,5=313,75 \mathrm{fm}$.

*) Der $j$ d) inbare Fefler, alg ob hiernad) Der Sals aus $15+46=61$

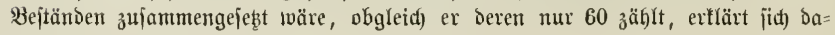




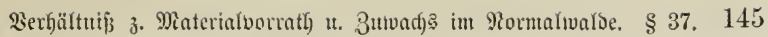

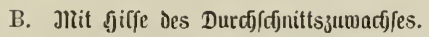

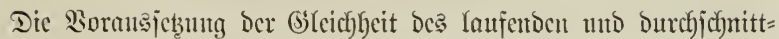

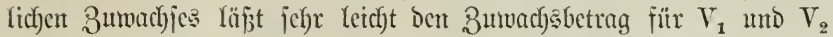
in jebem beliebigen S(bjefynitte ber Umtriebszeit funden, ebenjo ocjien

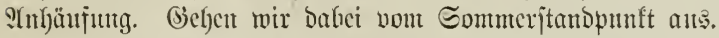

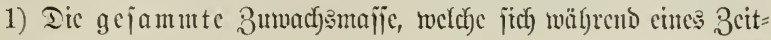
rauntes von $\mathrm{n}$ Jahrent an $\mathrm{V}_{1}$ und $\mathrm{V}_{2}$ angelegt hat, wird gefundent,

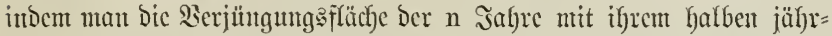

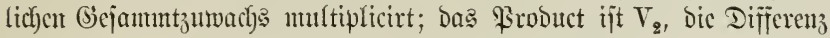
$\mathrm{nZ}-\mathrm{V}_{2}$ ijt gleich) bem an $V_{1}$ angelegten 3uwachje.

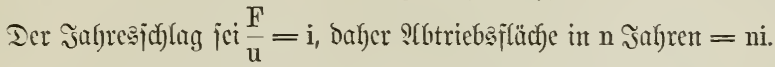

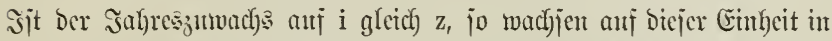

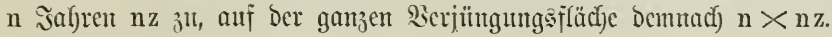

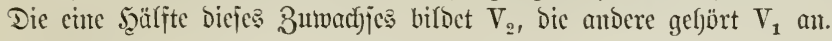
Daher

$$
\frac{\mathrm{n}^{2} \mathrm{z}}{2}=\mathrm{V}_{2}
$$

unD

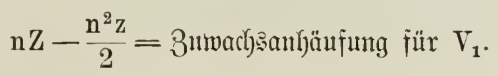

Beifpiel. Wie gró iit bie 3uwadjsanfäufung an $V_{2}$ und $V_{1}$ bis mit dem

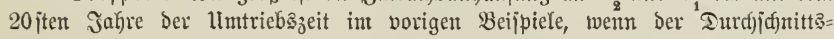
3utwad) 5,9?

$$
\begin{aligned}
& \frac{20^{2} \times 5,9}{2}=1180 \mathrm{fm}=\mathrm{V}_{2} .
\end{aligned}
$$

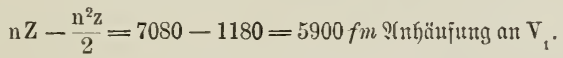

Die Directe 9ied)mung nad) Seite 143 jïfrt zu Denjelben giejultaten:

für $\mathrm{V}_{2}$ ergiebt jić) $5(2,95+56,05+61,95+115,05)=1180 \mathrm{fm}$,

" $\nabla_{1}^{2} "$ " $5(351,05+297,95+292,05+238,95)=5900$ "

2) Ien cimntaligen Bumachs an $V_{1}$ und $V_{2}$ int ntcu Jafre vou u fintoct man burch folgende Piecfunnt:

Der Butwadjs an $V_{2}$ ergiebt fich), tweun man für bas betref= fende, nte Jaf)r ben vollen Zutwaths ber bis zum ?trfange bicjes

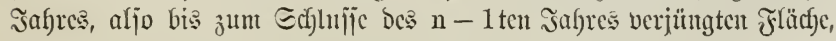

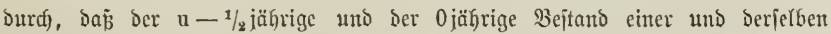
Fläd)eneinfheit angehören, fobalo man von Sommersmitte bis wieber auj Sonnters= mitte reduntet.

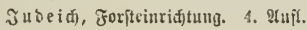




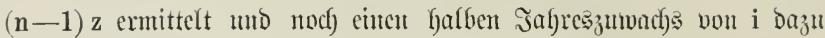
rect)net, aljo:

$$
\left(\mathrm{n}-1+\frac{1}{2}\right) \mathrm{z}=\mathrm{z}\left(\mathrm{n}-\frac{1}{2}\right) .
$$

Bieft man bicjen Betrag vou $\mathrm{uz}=\mathrm{Z}$ ab, jo erbält man ben be= treffenden sahreszutwachs an $V_{1}$, näm(ich:

$$
\mathrm{uz}-\mathrm{z}\left(\mathrm{n}-\frac{1}{2}\right)=\mathrm{z}\left(\mathrm{u}-\left(\mathrm{n}-\frac{1}{2}\right)\right) .
$$

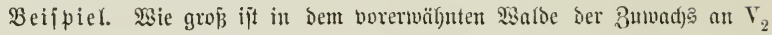
und $\mathrm{V}_{1}$ im 21 țen Iahre?

$$
\begin{aligned}
\text { In } \mathrm{V}_{2} & =5,9\left(21-\frac{1}{2}\right) \quad=120,95 \mathrm{fm}, \\
\prime \prime \mathrm{V}_{1} & =5,9\left(60-\left(21-\frac{1}{2}\right)\right)=233,05 \quad "
\end{aligned}
$$

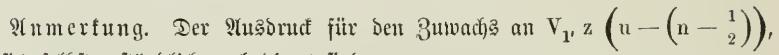

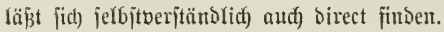

\section{Alrfrtgnniff.}

Der normale \$̧iebają̧.

A. Voin Stanipmurate der Mlaffenertragstegetung.

$\S 38$.

be $\mathfrak{x} \mathfrak{x}$ iff.

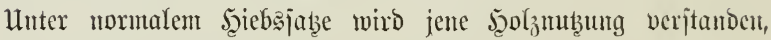
melche cin normal bejaffenter $\mathfrak{B a l d}$ nachbaltig liefern fann.

Derjelbe ijt entrober cin jäbrlicher ober cin ausjebender,

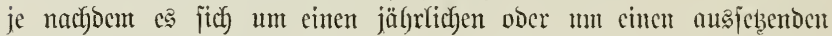
Pachlyaltabetrieb bandeli.

Unter peridoifdem Sicbajabe veriteht man dic Eumme des

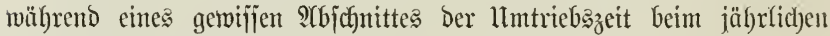
oder ausjebendon Betrieb erfolgenden Ertrages.

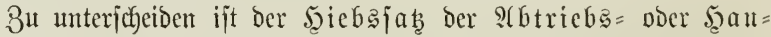
barfeit $\Xi=$ von Dem ber 3 woijdennubung. Die Materialcrtrage regelung ftüb̧t fitch hauptjäcblich auf Den erfteren, ba bic Bwijchen= mub̧ungen zu jeljr jofmanten.

Der Scolzertrag jebt jich aus verjoficonten Sortimenten zajammen, man faun für jedes berjelben euten bejondocren Szicbsjats berecthen. Man ipricht in bicjum Einne von einem Derbhol $z=$ Reishol $z=$

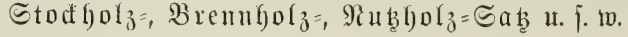


Die Sumue bes Ganzen wutrie jüther gewöfulid) in jogenannten Pormal=

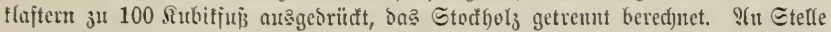

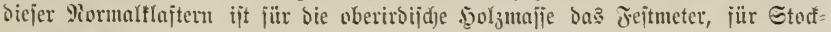

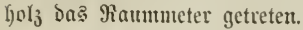

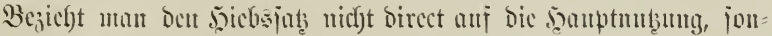

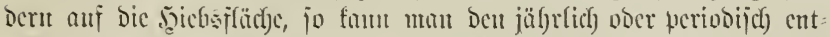

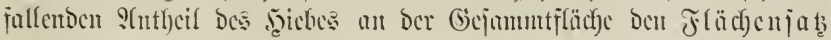
nemuen.

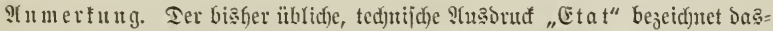

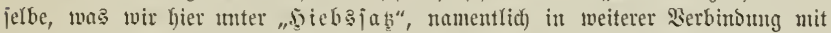

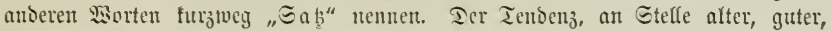

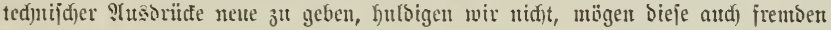
Eprachen entunumen jeir. Das 2 sort (Etat ijt jebod) nidjt blos ein jrembes, jon= bern bebeutet in ber franzijifiden Epradje burdjans utidjt einmal bas, was wir.

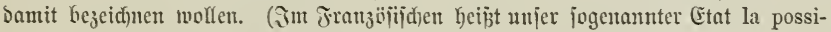

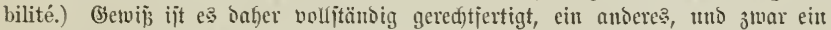

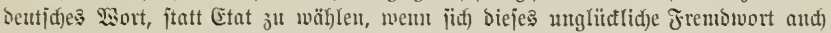

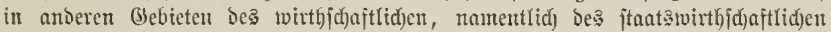

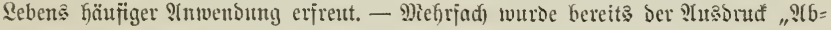

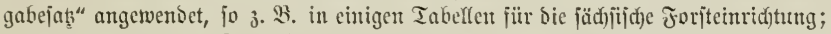

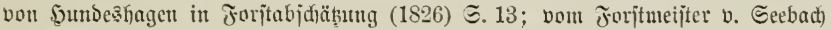

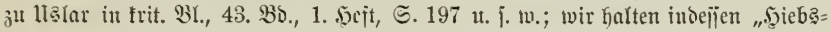

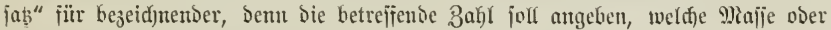

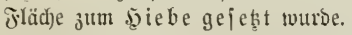

\section{$\S 39$.}

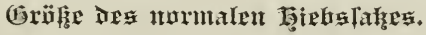

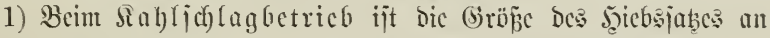

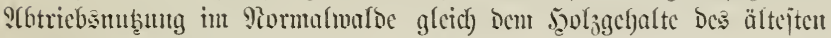
Jabresichlages $\left(\mathrm{H}_{\mathrm{u}}\right)$.

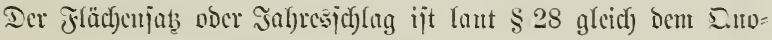
ticuten aus dem lluntricb in bic beitandenc Sicjammtfläche $\frac{F}{u}$ ober

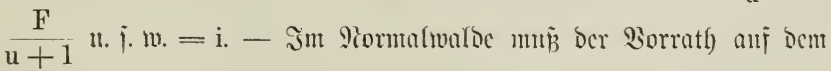

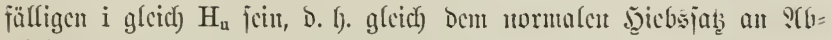
triebsuthtuty.

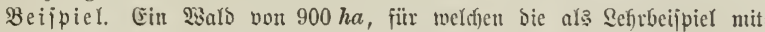
getfeilte Estragştajel gilt, wiro im 90 jährigen lluttricb cinten Fläđ)enfą̧ vou

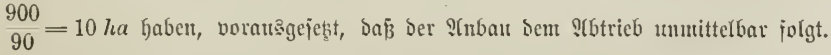

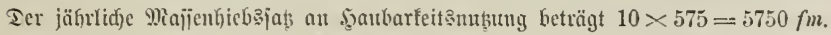

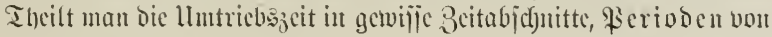


n Safren, jo wirb ber periobijefye Sciebsją̧ gleich bem mit n multi=

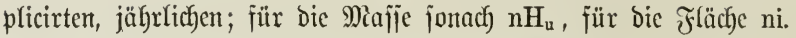

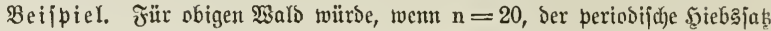
an Majie $20 \times 5750=115000 \mathrm{fm}$, an fläche $20 \times 10=200 \mathrm{ha}$.

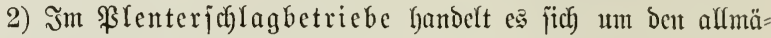
ligen $\mathfrak{A b t r i e b}$ ber Berjüngungaffafje $\left(A_{v}\right)$ und um allmälige Uebcr=

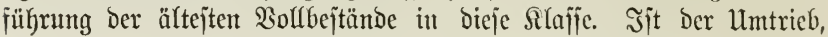

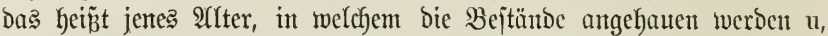
der $\mathfrak{B e r j u ̈ n g u n g s z z e i t r a u m ~} \mathrm{m}$, jo wiro jeder Bejtand Durchj(c)nittlich in

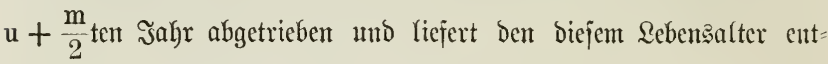
fprectendent Frtrag.

Beifpiel. Ein $\mathfrak{W a l b}$ von 800 ha entipred)e derjelten (Ertrags̆tafel, $u=80$

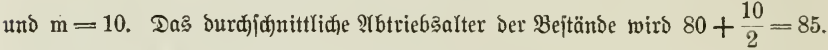

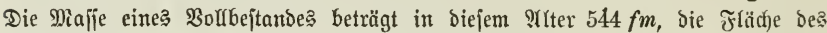

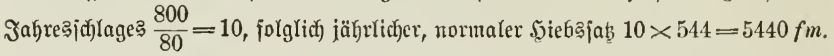

3) Im Riedermald ift ber normale ફ̧iebsjał ebenjo einjach,

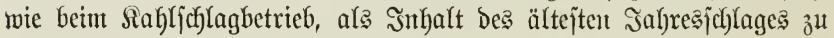
berechnen.

4) Sm Mittelmalde bejteht, wie beim Niebermalde, ber jälyrliche Flächenfał aus $\frac{F}{u}$, went u ben Umtrieb bes lluterfolzes bedeutet.

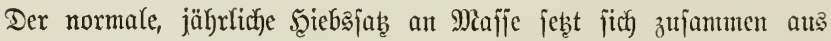

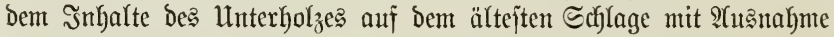

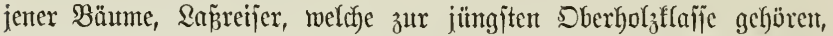

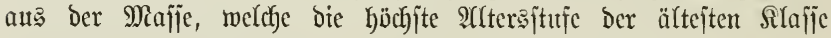

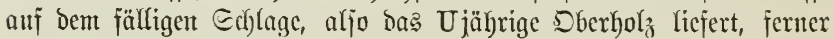

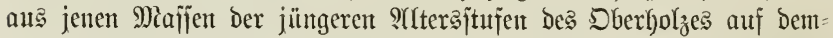
jelben Sablage, weldye entnommen twerben milfjen, um die Stummzahl

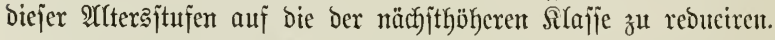

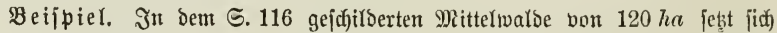

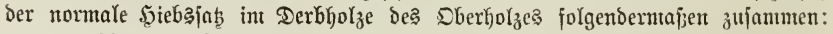

321 Etämme der 4. S(tterałłlajie, Ujäbrig, zł $0,50 \mathrm{fm}=160,5 \mathrm{fm}$

$$
\begin{aligned}
& 461-321=140 \text { " " } 3 \text {. " } " 3 \mathrm{u} " \text { " } 0,26=36,4 \text { " } \\
& 943-461=482 \quad \text { " "2. " } 2 u \text { " } 0,02 \text { Eumme } 206,5 \mathrm{fm} \text {. }
\end{aligned}
$$

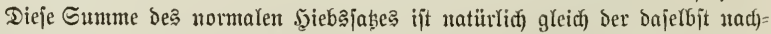
gewiejenten, Dort abgerunbeten Summe bes einjährigen Derbhol $=3$ utvadjes am ganzen $\mathfrak{g a r b e .}$ 


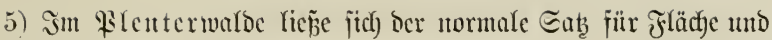

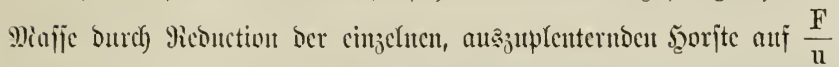

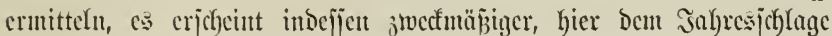

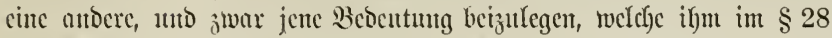

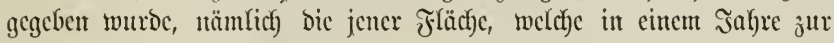
Flenteruntg gclangt: $\frac{F}{l}$. Der auf Dicjer Fläche Durch bic \$llenterung cutfallende, normale Ertrag ijt ber normale şicbsajab̨.

Beifpiel. EFin 600 ha groß̉er \$ilenterwals mit 120 jährigem llmtrieb uno

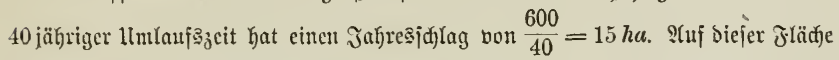

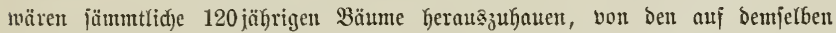

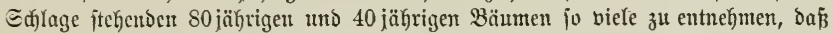

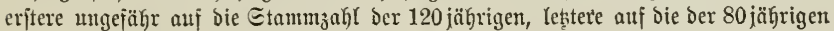
reducirt werben. Siejern bieje Fällungen, normal gedadyt, $3000 \mathrm{fm}$, fo wäre bię

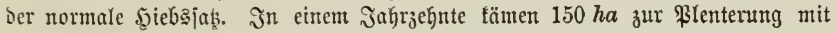
einem Miajienertrage von $30000 \mathrm{fm}$.

\section{$\S 40$.

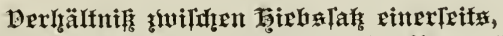

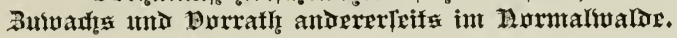

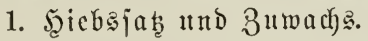

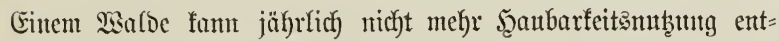
nommen werben, als ber Betrag des 3utwachjes, went der Pormal=

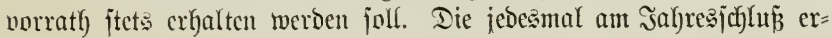

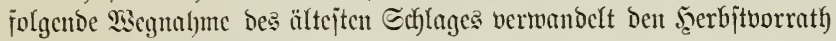
itt Den Des Jrïfjafyes.

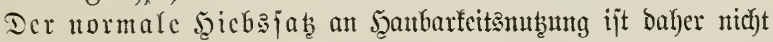
blos gleid) der Mi ajje bes älteften Jafresjulages, fondern aud

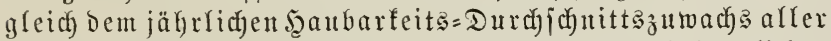
Bejtünde, und aud gleid) bem gejammten, Iaufend jährlichen 3uwad): aller Beftände.

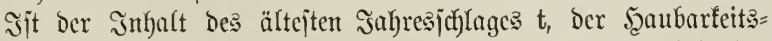

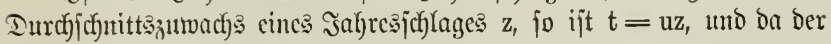
Şiebsiats gleich t, jo muti er aud gleich uz jein.

Die Eumme des laufenden 3umadjes am scauptbejtano aller altersitufen ijt ebcufalls gleich t, folglich auch gleich bem normalen

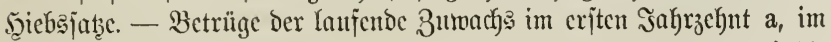
zweiten $b$ u. f. w., im lebeten $n$, jo ijt $t=(a+b+c+\ldots \ldots+n) 10$. 
Beifpiel. In dem Bahlenbeifpiele S. 137 ijt:

Dex normale ら̧iebşą̧ .

$t$

Die Summe des Iañ. Buwad) jes a ller Bełtände: $10(2+4,5+6,4+7,1+7,5+7,9)=354$

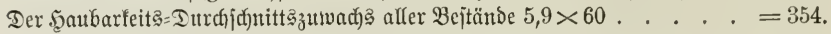

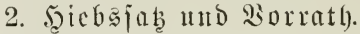

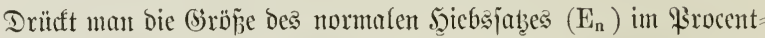

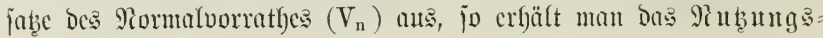
procent oder autd) Buwadysprocent des 2 aldes, es ijt bajfelbe $\frac{\mathrm{E}_{\mathrm{n}}}{\mathrm{V}_{\mathrm{n}}} 100$.

Mit Sumbeshagen verjteft man unter Sitbungsprocent in ber

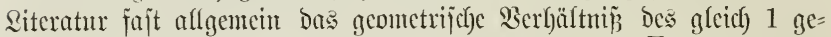
jeb̧ten Siormalvorratbes zum normalest Sciebsjaţ, aljo $\frac{\mathrm{E}_{\mathrm{n}}}{\mathrm{V}_{\mathrm{n}}}$. Jedenjalls

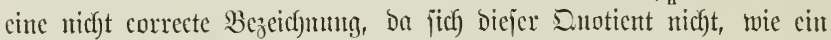
Frocent tyun joll, auf 100, jondern auf bie Einbeit bezicht.

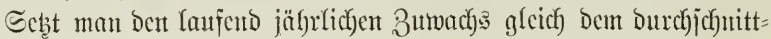

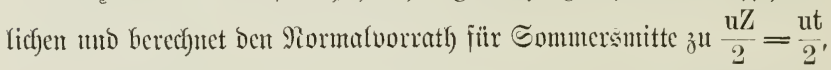

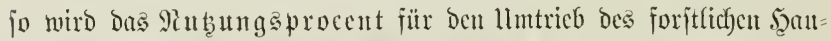

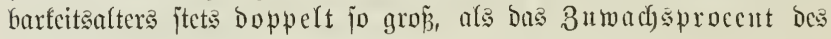
ältẹten Bejtandes jein.

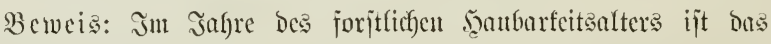

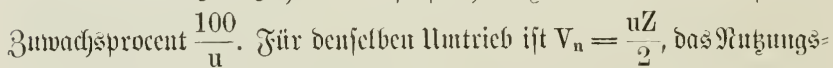
procent Daber $\frac{\mathrm{Z} 100}{\mathrm{u} \frac{\mathrm{Z}}{2}}=\frac{200 \mathrm{Z}}{\mathrm{uZ}}=\frac{200}{\mathrm{u}}$.

Da die Buwadfsprocente eine fallento Sicife bildon, jo müfjen dies

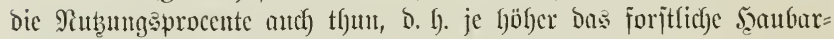
feitsalter liegt, bejto fleiner ijt bas Rubumysprocent.

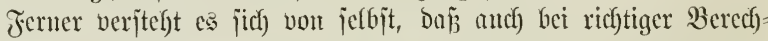

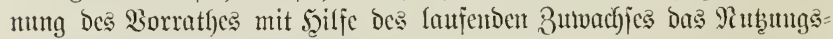

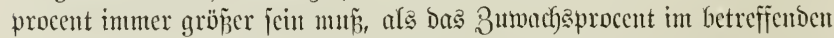

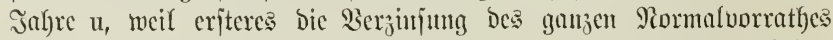

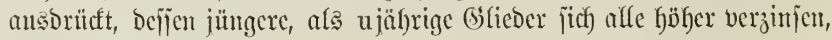
als das lebte.

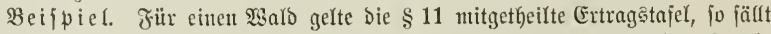

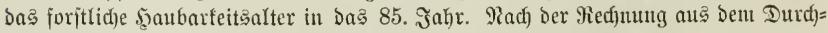

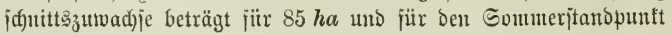


Pinkูug șprocent fiernad)

$$
\mathrm{V}_{\mathrm{n}}=\frac{85 \times 544}{2}=23120 \mathrm{fm} .
$$

$$
\frac{\mathrm{E}_{\mathrm{n}} 100}{\mathrm{~V}_{\mathrm{n}}}=\frac{54400}{23120}=\frac{200}{85}=2,35294 .
$$

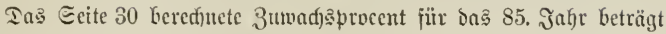

$$
\frac{6,4 \times 100}{544}=\frac{100}{u}=1,17647,
$$

jonad) gentu bic şälfte bes situsungąprocentes.

In ber Piormu(torrath), meld)en mat mad) Piedfunng Des Eommeritmopumftes nus ber Eummirung irgeno weld)er Ertragstufel crfält, fïr ben forjtficten llmtrich, aljo für jencu, melefer in bie 3eit bes

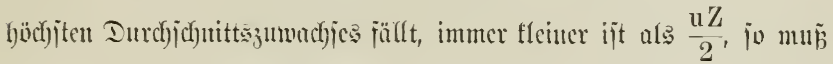

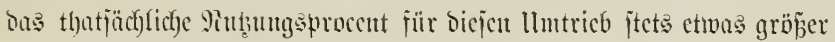
jein, $\mathrm{al}$ s $\frac{200}{\mathrm{u}}$.

Beippiel. Beredfnet mant burdy Summinutg ber Tafel ben Sonmerborrath jür obigen :alo uno llmtrieb, jo erfält man:

$$
\begin{aligned}
& \mathrm{V}_{\mathrm{u}}=5\left(6+20+40 \text { u. j.w. }+\frac{544}{2}\right)=19900 \mathrm{fm} . \\
& \text { Nututugsprocent }=\frac{544 \times 100}{19900}=2,78367>\frac{200}{85} .
\end{aligned}
$$

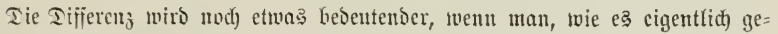

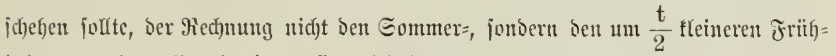
jafresuorrath 3" Grumbe legt. Es mito bam

$$
\text { Үuțungṣprocent }=\frac{544 \times 100}{19628}=2,77155 .
$$

B. Jiormafer giefisfał vom Stanopunfte Der finanjectinung.

$$
\text { s } 41 \text {. }
$$

\section{Brariff uni brorufung.}

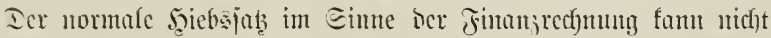
Durd) bas Miaterial allein ansgerieft, jontern mun mittelit bes biclo= maj̧ןtabes gemejien meroent.

Es ijt jener, meldyer alle in Der :

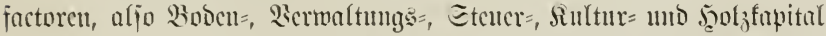

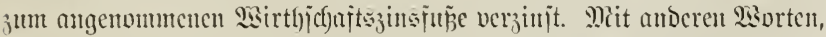

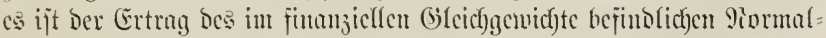
twalbcs, in melefem fein Bejtand unter tom ifju gebülyrenden Escijer= procentc arbeitet. 
P(ङ Factor Der Extrageregelutg felbjt, in äfulicber Weife, wic

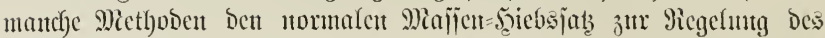
wirflichen verwenten, fant ex nicht bienen, jondern ex fan mtr Folge

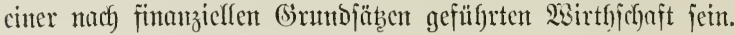

\section{$\S 42$.}

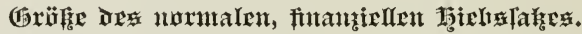

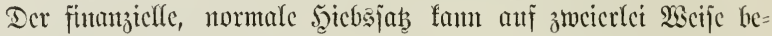
recfunet werden:

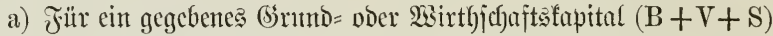
unb Den entfprechenden Siulturanfwand (k) ermittelt man ben Sioften=

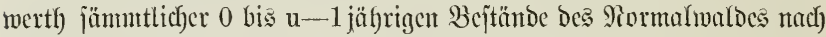
Formtel II (\$ 34). Dic Snmme aut Dicjem Sojtenucrth), aus B, V, S und $\frac{k}{0, o p}$ ift glcich eiutem Siapitale, Defien Bütjen ben normalen, fintan= ziellen Jeiebsjats bedenten.

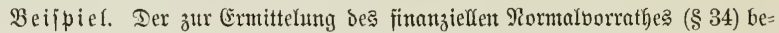

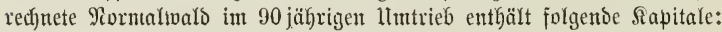

Bobenfapital $90 \times 142,17867=12796,08 \mathrm{fl}$.

$\left.\begin{array}{c}\text { Serwaltunga }=\mathfrak{u n} \delta \\ \text { Stcuerfapital }\end{array}\right\} 90 \times \frac{3}{0,03}=9000,00$ "

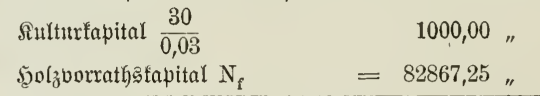

Summe bes ganzen, in fiapitalform

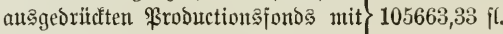

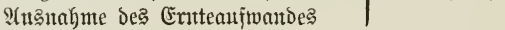

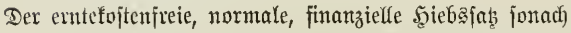
$105663,33 \times 0,03=3169,90 \mathrm{fl}$.

b) Einfacher fommt mall zum Bielc Durch Betrachtunt bes cin=

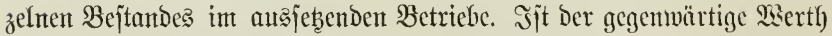
Des ujährigen Bejtandes in Folge Der Worantsfebung bes fintanjieflen

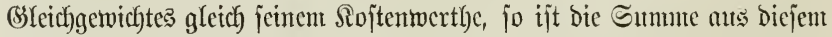
und Dem einfachen Betrage Der Sornubzungen gletid) Dem normalen,

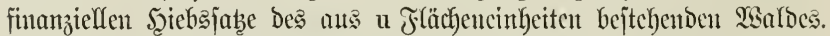

Beifpiel. Der Sojtentwerth des 90 jährigen Bejtandes, aljo im vorliegenden Falle ber $\mathfrak{B e r t h}$ jeintę 9(btriebsertrages ift:

$$
7,26536 \times \frac{1,03^{90}-1}{0,03}+30 \times 1,03^{90}-775,10=2875,0 \mathrm{fl} .
$$




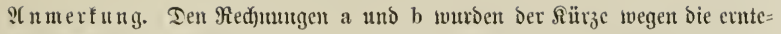
foîtenfreien Erträge der Tajel zu Brunbe gelegt. Wollte man baher den vollen

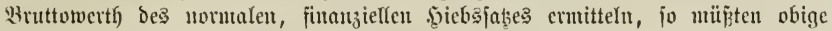

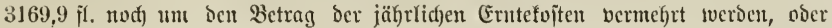

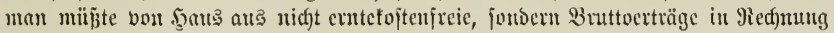
ftellen.

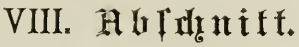 \\ Betricbałtafien. \\ (Wirtgf(x)aftskfaffen.)}

$\S 43$.

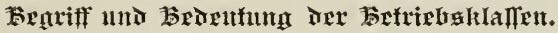

Für Den bisher betrachteten Pormalwalo muñten wir, abgejeben

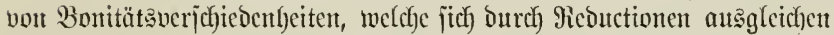

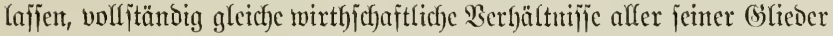

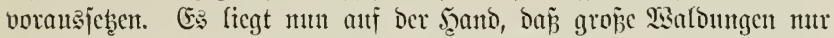

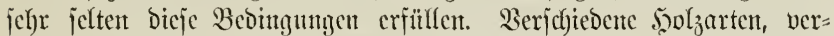
fefiedene llmtricbe und Betricbsarten bringen Factoren in bic Pormal= walbshedfunng, welde jidf) nidft vereinigen lajjen, jie bebingen bic Bil= Duntg felbitjtäntiger Theile, weldbe in gejontoerter Echlagorounng fïr jitch belyandelt jein wollen.

Einen joldjen Theil des waldes nentt man cine Betriebs= tlafje, werjtert aljo unter leşterer alle, eiucr und oerjelben

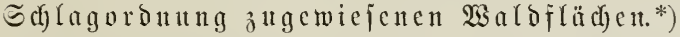

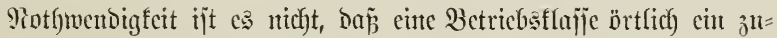
jammenfängendes Ganje bilbe. Erforberlich ijt es Dagegen, fïr jebe Betriebsflajie alle bic Ertragsregelutng Direct betreffentoen Yiect)mungen getrennt vorzunclymen.

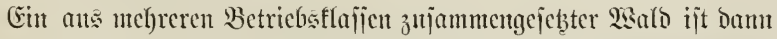

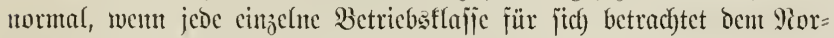
malzujtanto cutiprict)t.

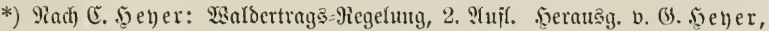

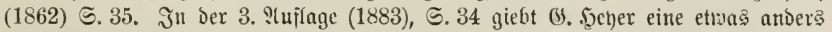

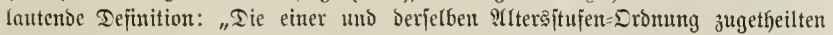
Walbflächen bilben eine Betriebstlalle". 


\section{$\S 44$.}

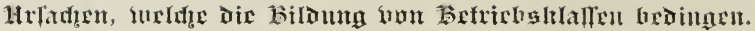
Deutungen:

こic folgen aus ben im vorigen Raragraplen gegebonen :fu=

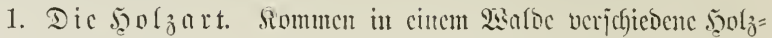
arten beitundsweife getrenut nor, jo wiro nanentlid bant cinc Iren=

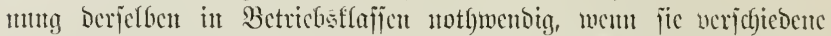

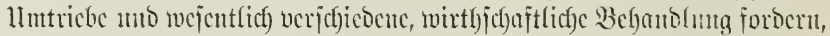

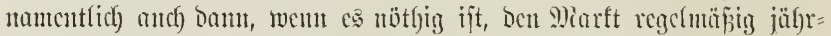

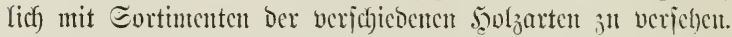

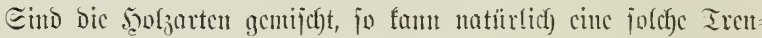
mutg uicl)t itattfintocn.

2. Die Betriebsart. S(lle bic möglidyen verjatedenen Betrebs:

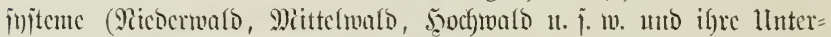
abtheilungen) fünncu bic Bifbung bejonberer Betrichsflafjen bedingen, bei mejentlicher Berjefjiconbeit mitfien jie cs.

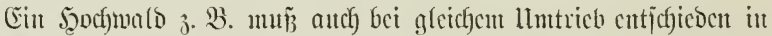
joci Silafien getfeilt werben, wenn es fith im Jeochgebirge voer an ber

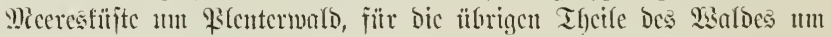
jeblaguecijen Betricb homoclt.

3. Der Il utrich. Eelbit Gei Derjelben Soljart, bemjelben be=

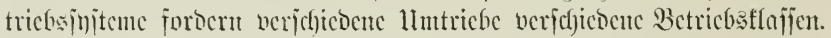

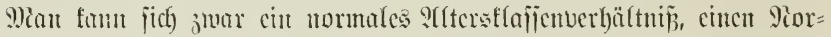

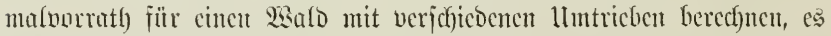

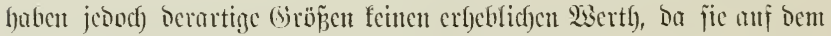

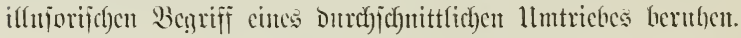

Serfpiel. In einem 23 albe von $3000 \mathrm{ha}$ jollen $360 \mathrm{ha}$ im 120 jährigen, 2400 ha im 80 jährigen uno 240 ha im 60 jäfrigen llmtriebe betwirtfjidgajtet merben.

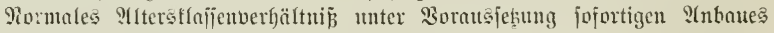
nad) Dem SItriteb, afjo ofne normale Bläpe:

YIteróflaijen:

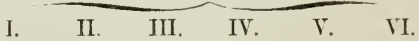

1. Betriebaflajie mit $\mathrm{u}=120$ : 60 ha 60 ha 60 ha 60 ha 60 ha 60 hu

2. " "

3. " " $"$ " $=60: 80$ " 80 " 80 " - " - - "

Summe: 740 ha 740 ha 740 ha 660 ha 60 ha 60 ha.

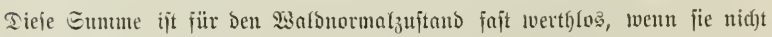

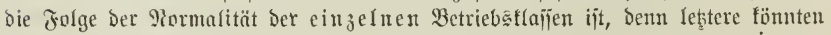
ganz abnorm bejaffen jein uno bod) eine normale Eumme liefern. 


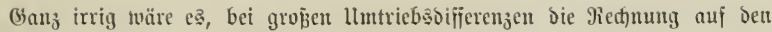

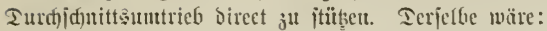

$$
3000:\left(\frac{360}{120}+\frac{2400}{80}+\frac{240}{60}\right)=\frac{3000}{37}=81 .
$$

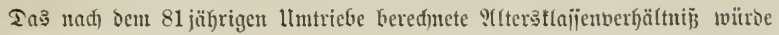
Iauten:

$$
\begin{aligned}
& \text { I. :uteratiaje }=740,8 \mathrm{ha} \text {. } \\
& \text { II. } \quad=740,7 \text {, } \\
& \text { III. " }=740,8 \text { " } \\
& \text { IV. " }=740,7 \text { " } \\
& \text { r. " = } 37 \text { " }
\end{aligned}
$$

Coldhe Bahlen juno für Die Ertragšregelung nid)t braudbar.

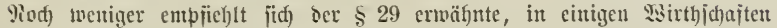

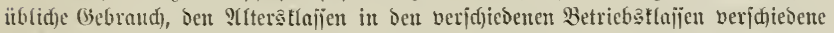

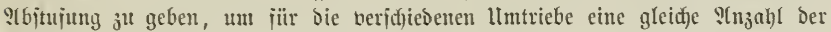

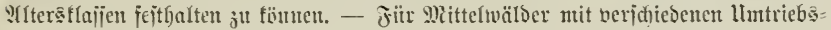

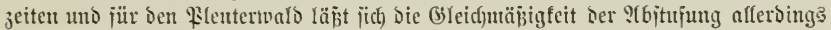
nid)t gut einjïbren.

4. Beaflajten fümen, menn jie bedentend fint, bic Bifloung veridjicoener Betricbasfojien nothwenbig machen.

5. Etandortsucrichicocubeiten weroen baut ltracte ber

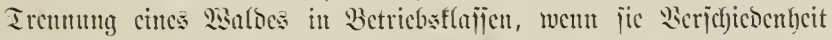
ber unter 1 bis 3 genunuten Serfä̈ltnifife beoingen.

शf nue rfung. Taj man zแm Bwerfe ber Ertragseregelung nid)t zu meit in

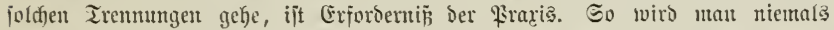

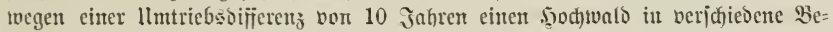
triebstlaijen theilen uno jï) Daburd) Das Yied)nungsmerf erjd)weten. Dies un jo

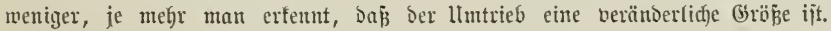

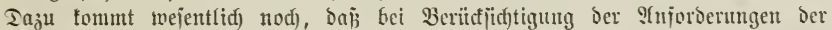
EFinzelbejtände ofnehin ber eine unter, Der anbere über Dent für bie Betriebsflajie

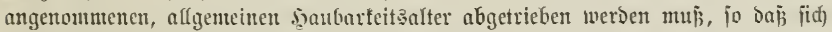
f̈̈r einzelne Bejtände ober Bejtandsyruppen vịt jefr bebeutende llmtriebsoijierenjen ergebent fömnen.

\title{
IX. H h f dig ift.
}

Icr wirflid)e $2 \mathfrak{z}$ alb.

\author{
$\$ 45$.

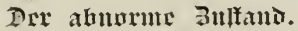

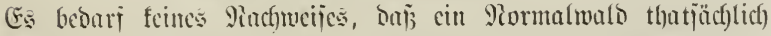
nirgents vorfommt, woh) and nic vorfomment fanth, namentlicly bamt 


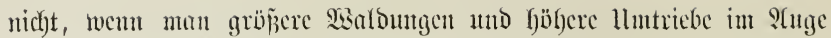
h)at mo nicht blos dic Erfüllutg Der Bebingungen bes normalen

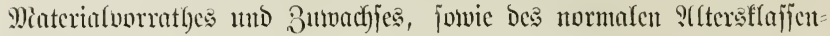

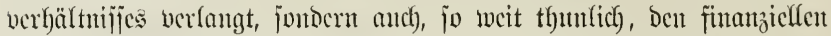
Tiormal $z_{j} u j$ tand crjtrebt.

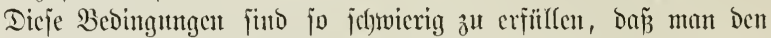
Pormatwald füglich and Socalwald nemen fiomute. Es faum, wic gejagt, bie Entwicfeluntg bes Rormalzujtantes, twie wir fie in ben vorigen S(bjefuntten Darlegten, mur Den Doppelten Bwedf haben, erjtens

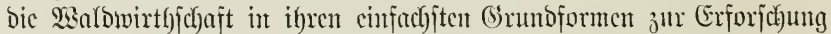

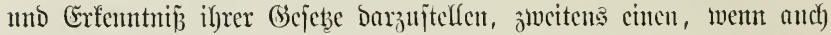

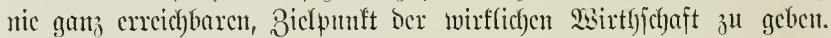

Die jablrecicfen, möglichen s(bnormitäten bes wirflicfen 2 albes laijen j̈ch) anf folgento Fälle reduciren:

1. Som Etamopuntte Der Materialertragaregelung allein fömten abnorm jein:

a) Der Butwad)s,

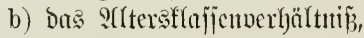

c) Der Sorrath.

Cntweber finto mun a, b mo e abnorm (woh) ber gemöhnticlec

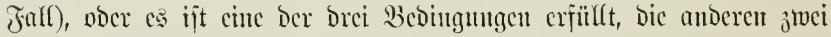
fino es micht, vocr cnolid), es futo jwei Bebingutngen crfüllt, bic Dritte ijt es nicht. Sebteren Falles ijt jull bead)ten, Daj bic Normalität vou a mub b bic von e wou jelbjt zux Folge bat.

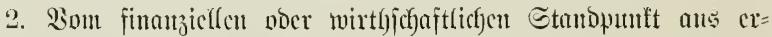

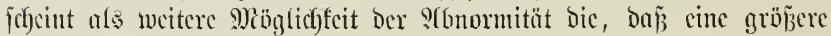

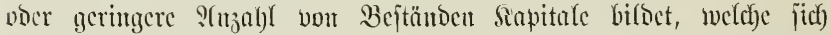

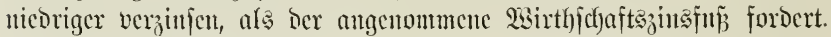

\section{$\$ 46$.}

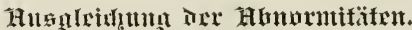

Dic Sufgabe ber Forjteinrichtung mo Ertrageregelung bejtebt

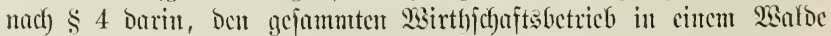

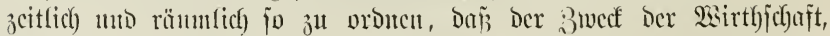

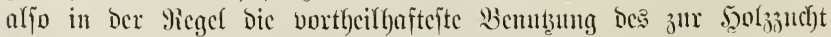

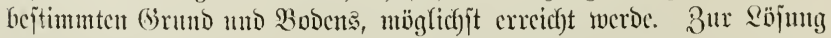

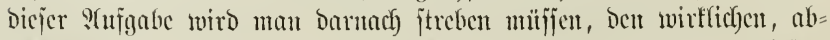

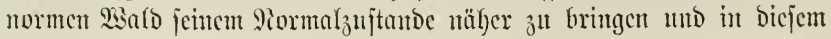




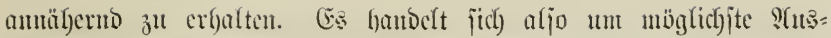
gleicl)uty ooer Bejeitigung ber ?(Gnormitïten.

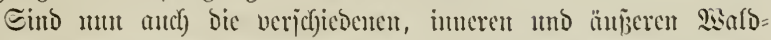

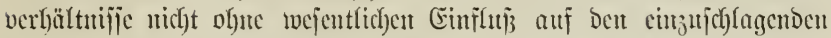

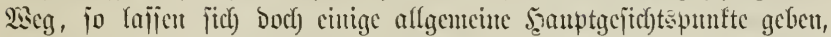

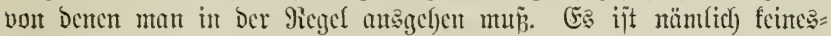

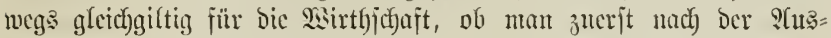
gleichung bicjer nocr nach ber jener ?(bnormität itrebt, ober anted nach)

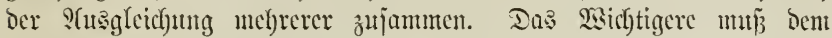
toeniger Wisteftigen vorangehen.

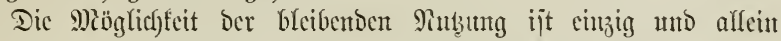

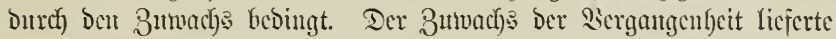

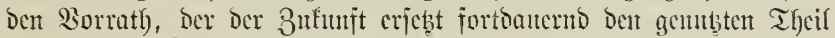

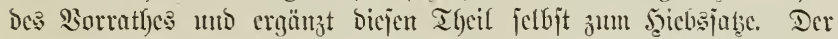

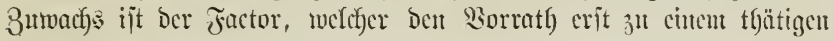
Sirtb)j(baftşapitare macht.

Dic \$iflege und Berbejierung Des 3mwachjes unţ aljo uächjt=

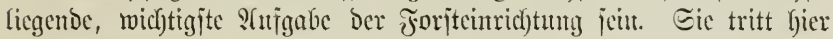
Dem 2Baloban, ๖. h. Der Bejtandsgründung und Bcjtundspflege er= gänzeno jutr Ecite, intocu fie erjtents fïr Den S(btricb zuvachsarnter Bejtänto, zweitens für bic Scerjtellung einer georonteten J̧icbajolge

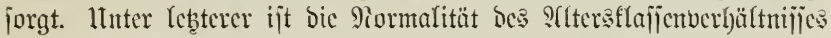

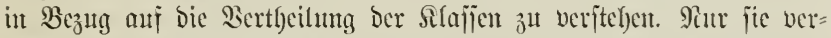

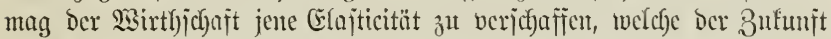

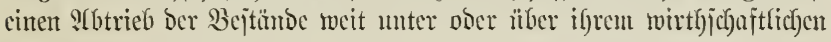
Şaubarfeitşalter nïgfić)jt cripart.

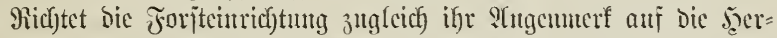

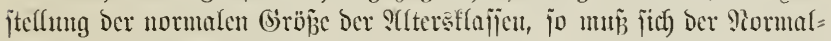

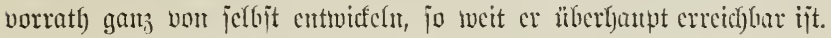
Diejem birect als erjten Biclpunfte jujujtreben, ijt baljer tein richtiger

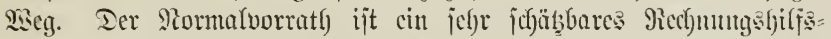
mittel, um Das saldowerntögen in Simte ber Materiafertragstegchutg

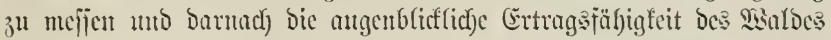
jummarijch ju ermittefu, jedoch fun bie birecte sereritelfung bicjes

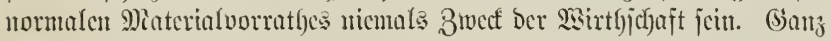
anders verbält es jich mit bem 3uwadjje.

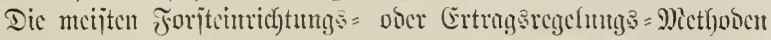
fajfen un ben gisulo voer aud) eine Betrichatlajic als cin untrent=

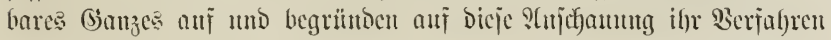




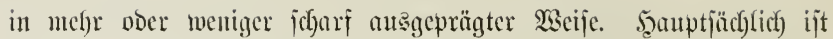

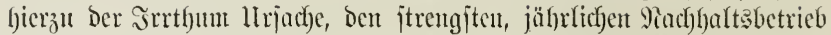

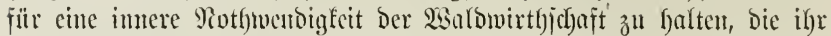
gleichjam angeboren jei. Das ijt aber thatjächlich nicht ber Fall.

23ie bie ansfülyrlichen Entwiefelungen uto Beifpicle ber vorigen Sapitel nadfweijen, ijt übrigens jelbjt ber normal gebad)te, jtrengjte

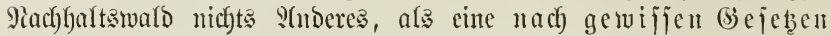

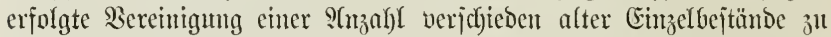

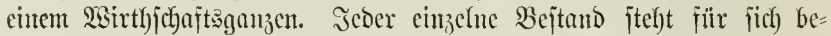
trad)tet im ansjebenden Betriebe. Der 5anbarteitsertrag jebes $2 \mathrm{C}=$

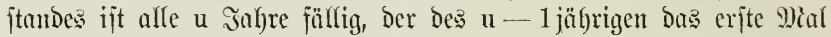
nad) einem Jafhre, Der bes u-2jührigen nad) zrwei Safren, Der bes $\mathrm{u}-\mathrm{u}$ voer 0 jäl)rigen nach) $\mathrm{u}$ Salyren. Seder Bejtand hat eine andere Begründungszeit. Sit bie s(tersitufenreife vollitünbig, fo entiteht ans ber Bereinigung von u Bejtänben ber Radjhaltswald bes jtrengiten jäbrlichen Betriebes im ujäbrigen llmtriebe. Eine imtere, wirtbjchaft=

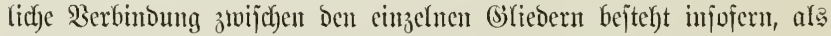

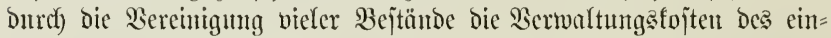

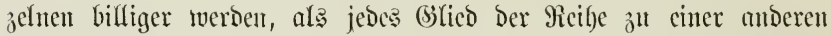
Beit, wie bas andere, angebant und genubt wirb, als endich) Dured)

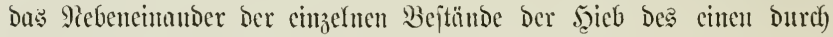

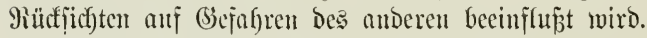

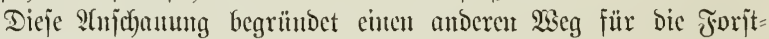
cinrictung umb Ertraggregelung. Man jerlegt ben $\mathfrak{W a l d}$ in jeine eimzeluen Bsfieder, Bejtünde oder Bejtandsgruppen, betrad)tet jedes

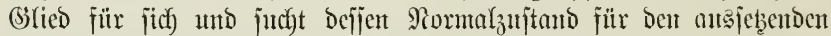
Betrieb herzujteffen. Mcobificirt wirb biejes Streben Durdh bie Mïtt= jicht auf Das Banze, indem man Dabei bie Bicjtaltung Des stlters=

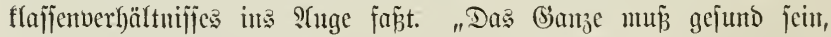

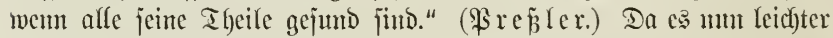
ijt, bie (Sejundheit Der cinzeluen Ihcife allmälig herjujtellen, anjtatt jofort anf bie bes (banzen birect Ginzuarbeiten, jo ijt jebenfalls biejer

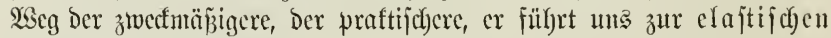

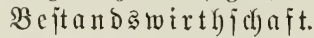

Sollen wir namentlich Den begründeten SUforberungen ber Finans= wirtljichajt im 2 alde jo weit Siedfunng tragen, als bies inberfanpt möglich ijt, jo bleibt mur biejer eine $\mathfrak{W} \mathrm{cg}$ ïbrig.

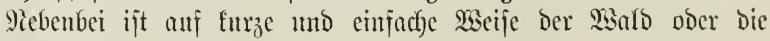
einzelne Betriebstlajie als Banzes aujzujafjen, um ber Paddyaltigfeit 


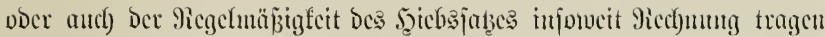

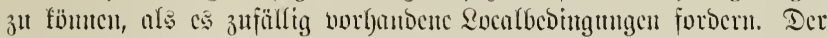

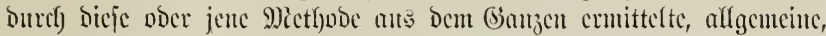

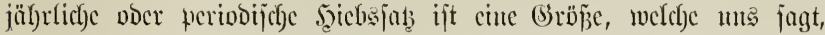

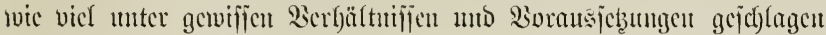

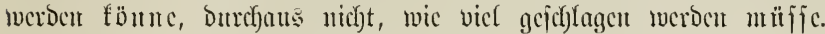

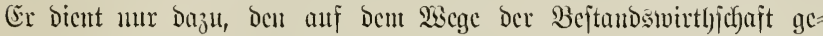

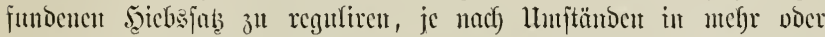
tweniger enge (Sirenzen zu bammen. Se freier Der Martt, Defto weiter

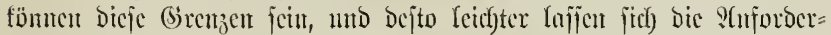

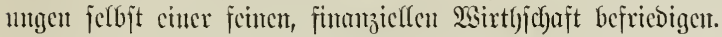

Peltere Mictgoden der Ertragsegelung modificirten

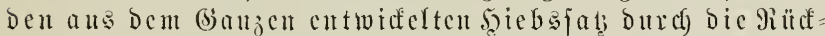
jicften anf Den cinzeluen Beftan. Wir wolfen bas llu= gefel)rte, näm(ich) Jiodificatioucn ber Bejtandswirthjuajt

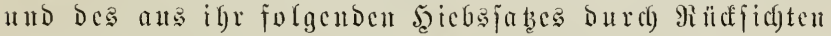
a $\Perp f \mathfrak{d} a \mathfrak{s}$ (janjc.

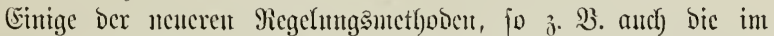

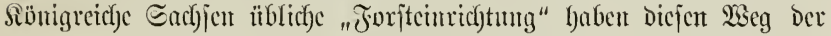
Sejtandswirtljichajt f(d)on vor längerer Bcit betreten, wenigitens autge=

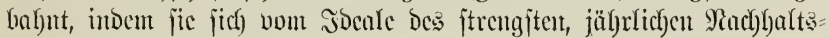
betricbes cmancipirtent.

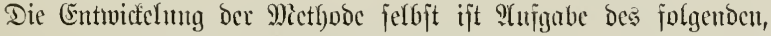

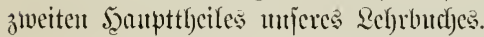




\section{Butft.}

\section{A n me n o แ g g n.}

\section{A. Borarbeiten.}

$\S 47$.

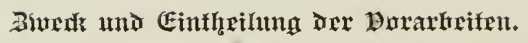

Die zux Forjteintrichtung und Ertrag haben ben Zwecf, den wirffichen Zujtand bes Saldes in allen Beziel)=

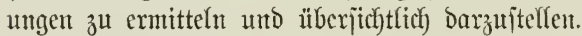

Eie zerfallen in folgende sfbjochnitte:

I. Forjtbermejiung.

II. Forjtabjääbung.

III. Ermittelung Der allgemeinen und äuf̧eren

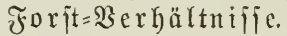

IV. Sartixug und Anfertigung Der Schriften.

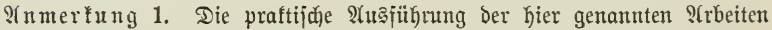
wirb żum Theil mit Der Waldeintheilung (Einrid)tung im engeren Einne) felbjt band in banto gehen, Durad) bieje wejentlid) unteritïb̨t werden. So z. B. gewährt

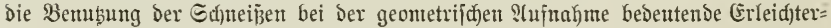

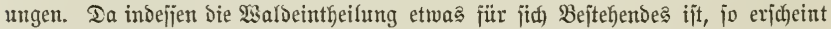
eine Trennung ber bieje betreffenden $\mathfrak{A}$ rbeiten bon ben 3orarbeiten hier jedenfalls geterftjertigt.

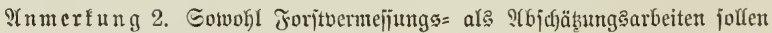

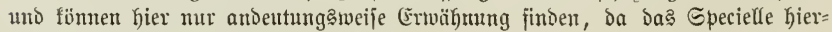
über als befant vorauş 


\section{I.}

\section{Foritueruteiiung.}

(geometrifaje Vorarfieiter.)

\section{$\leqq 48$. \\ Hufgahe ถ⿻上 Эurltuerntellutg.}

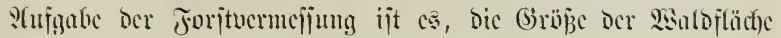
überfant, jowic bic ber cinzeluten, einer Eonberung hedürfenton Theile berjelben zu hejtumen und bie geometrije) Ilnterlagen $3 \|$ den Siarten und Echriften ju licfern.

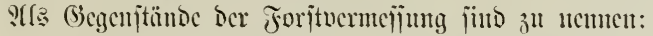

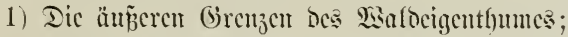

2) imucre Ecrvitutgrenjen;

3) Dic (Sirenzen Der 2Birtbjedajtseinfeiten (Beviere);

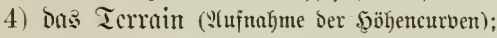

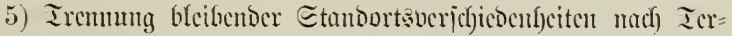
rain, হage uno Bodentejchaffentyeit;

6) Tremung bes jagly

7) Dic (Brentsen Der Betricheffajīen;

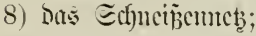

9) Tremmutg Der 5ob

10) alfe blcibenten 2isege, Eijenbahnen, Bäche, Flitile, Teiche, (3räben, Jefjen und dergleichen, jowte Bantuerte uto überhaupt alfe bejonders als Srientirumgspunte michtigen, cint= jelnen Gicgenjtänoc, j. B. Eignalpumfte sc.;

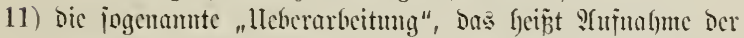

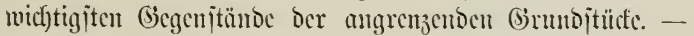

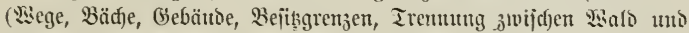
Ferto ut. j. m.)

\section{$\$ 49$.}

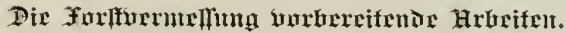

1) Miöglichjit vollitämbige Begelung Der Eigentfumsgrenzen Des LEaloes ijt bic wichtigite ber fier in Frage fommenden Borfereitungs:

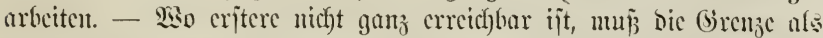
fragliche oder jtreitige aufgenommen mo an ber Sarte bejombers be= 3eicfurtet merden. 


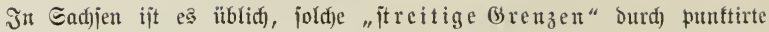
Qinten auf Der Siarte femutlid) z̆ madjen.

2) アegelung aller immeren Eervitutgrenzen.

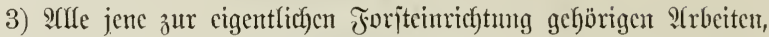

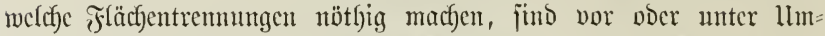

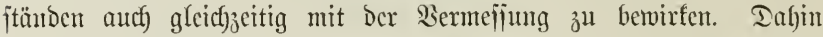

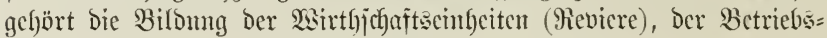
flajient :c.

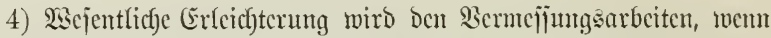
Das ganze Echneipenneb vorher Durchgefanen werden fann. Es ijt bies möglich bei regelmäñig rechtwinfeliger Eintbeilung und bei un= regelmäp̈iger bann, wenn alte, Daju Granthbare Sarten vorfanden jind. Sai alle Fälle hat jeboch das Iurchjtecfen ber Echneipen noch vor Der jogenannten Detail=23erme ïung o $\mathfrak{u}$ crjolgen.

5) Bejüglid) Der Beitandstrennungen bienen als bie Forjt vernejïung vorbereitenbe S(rbeiten jene Echäbungsurbeiten, welche die Iremung Der Bejtände bebingen. Bis zu welder Gröp̃e herab Be=

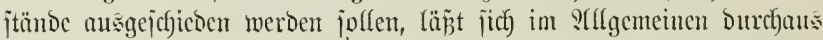
nicht bejtimmen, es hängt, bics ab von Der grö̈peren ober geringeren Feinfyeit Der (Ëmrichtungsarbeiten überfant. Inter eine fläche von 0,1 ha wird man menigitens in grïjperen Lialdungen jelten herab= geben, woem nicht ganj icfari ausgejpxochene, numentlidf bleibende Etandortsunterichicbe ober Bejtandsformen worbanden fimb, melche

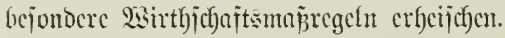

\section{$\S 50$.}

\section{Dexmellung unt Hufnalgme felglt.}

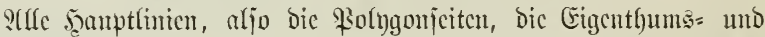

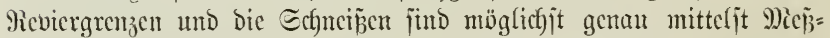
latten vocr Etaflbändern, und zwar Doppelt zu mej̄̄en. Qebeteres ijt

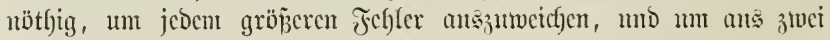

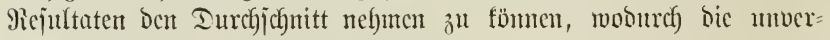
meidficfen Fehler jef)r vermindert meroen.

Dic P(ujnalyme jelbjt hat mit Dem Theodolit ju crjolgen. *)

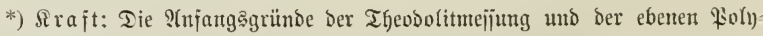
gorsmetric. Sannover, 1865.

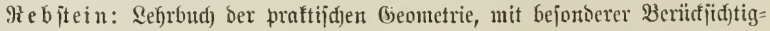
ung Der Theodolitmejïtng ı. f. tw. Frautenjels, 1868. 
3ur Shujnahme bes Details, numentlich ber Bejtandsgrenjen,

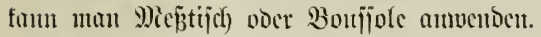

St umerfung. Die fönigl. jäd)j. Jorjtbermejïung arbeitete bisher fajt mur

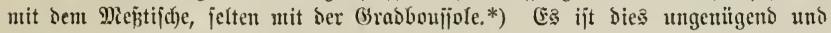
verbient un jo weniger Entpieflung, als Die Theodolit=?1ujnagne mit nur un=

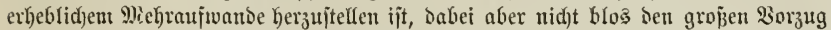
Der gräßjeren (Sentaugfeit, jontern aud) nod) Den hat, jïr alle Beit unberänderlidje

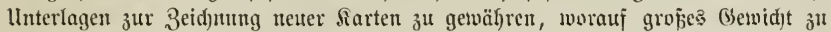

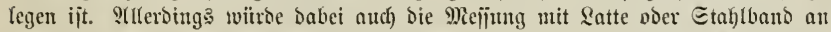

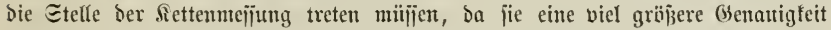

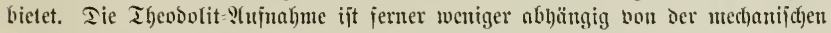

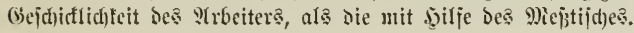

\section{$\S 51$.}

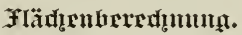

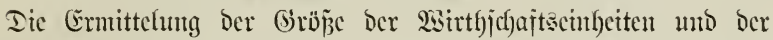
Şauptabtheilungent crjolgt ant Bejten mur Durch) Sioorbinatenberect)unty.

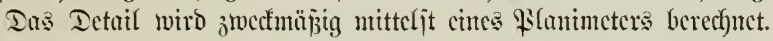

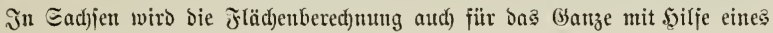
Eunbratuețes ausigejüfrt. Die Epecinlfarten find zu Diejem Brwecte in $1 \mathrm{ha}$

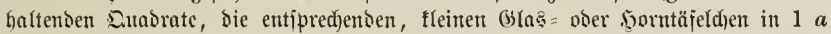
haltende Dutasrate eingetheilt.

\section{$\$ 52$.}

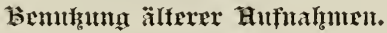

S6 unt in wie weit bercits vorhantocne, ältere Siermejïungäuerfe

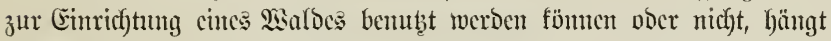
cinericits ab won Deren 3uberläjïtgfect, untorerjects von bem größzeren

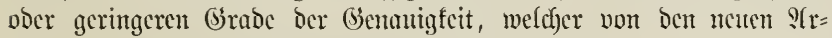
beiten jut verlangen ijt.

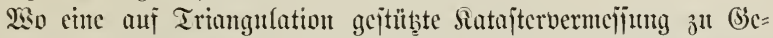

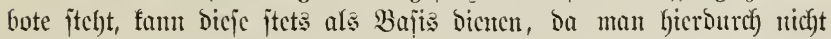
blos 3cit unto Sijten ipart, jontoern aud) Den möglicfjiten (brad ber

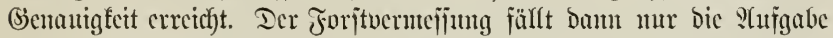

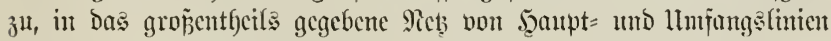
Das Detail rjemeinzuturbeiten.

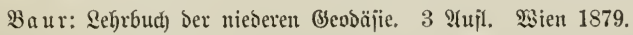

Borlaender: Snleitung zum Felomeijen. Bertin, 1871.

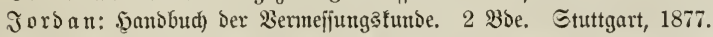

*) Injtruction vom 21. Ifpril 1841. Tharanber Jahrbud), 1. Bant. 1842. 


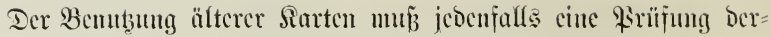
jelben botausgefen, welde burd) Micjumg ciniger soumptlimien und

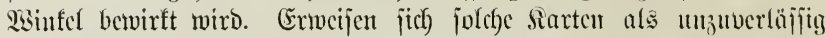

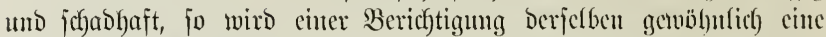

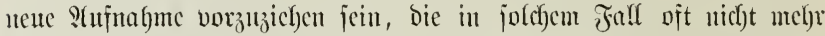
Siviten und Më̈lfe verurjacht, als jafflrciche Berichtigumgen. Bejüglict) Der Yebzteren ijt iumer jut bebenfeu, baj micht blos jene ?frbeiten in Betracht foumuen, welche jofort erlebigt werden muijfen, joutoern baj; man während ber ganjen 3eit, für weldbe Das Sermefijungswerf (Belt umg bebalten joll, bei Bejorgung Der Laufenden Rachtragsmejümugen mit mefre doer meniger ftötenden Differenzen zu fämpfen hat.

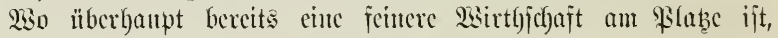

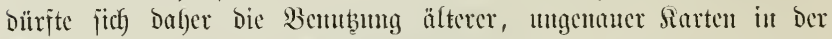

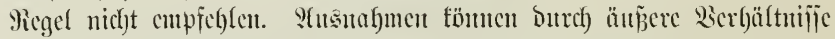

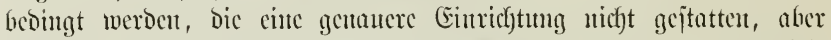
menigitens cinc oberflächliche Derartige s(rheit fordern, jo weit bicje

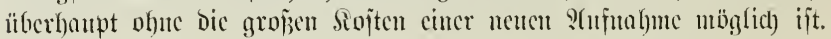

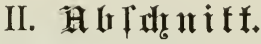

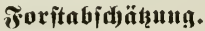

(Tap̧atorifffe Vorarfoiten.)

\section{$\S 53$.}

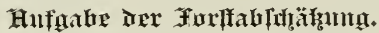

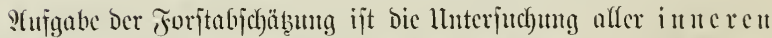

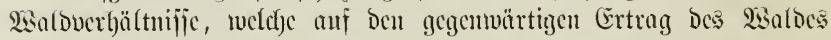

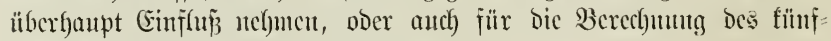
tigen Ertrages von 2isidytigfeit jüno.

Sie hat es bafor ju thum mit ber Ermittelung:

1) Der Standortsucrlältuific;

2) Der Bejtandoverbältuijic;

3) Der bisherigen Foritcrträgc แd Siojteu.

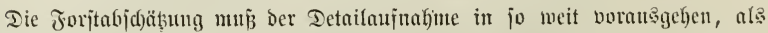

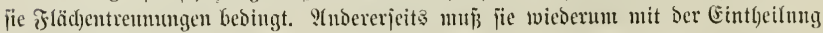

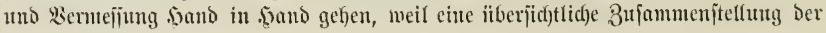




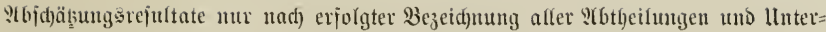
abtbeilungen mïglid) ijt, uno weil jie jelbjt bie Fläd)engröjsen braud)t.

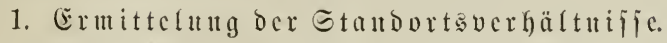

\section{$\$ 54$. \\ $3 \mathfrak{a} \mathfrak{x}$ th.}

Wion bem Etandorte bängen bic wejentlichjten Mionente ber 2 sald =

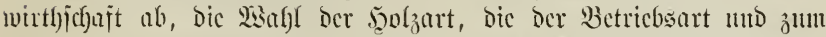

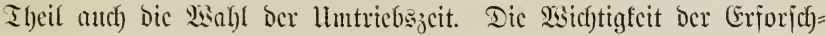
ung ber Standortafactoren ijt balger in bic ?atgen fpringent. Dic

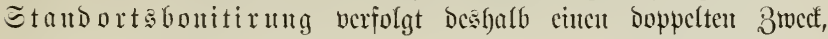
intocut jie lluterlagen licfert:

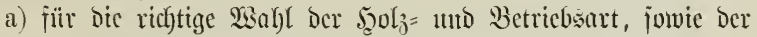
llmtricbsjeit,

b) für bic Berectumung der normalen Ertragefägigfeit jeder $\mathfrak{B} c=$ tricbstlaijc.

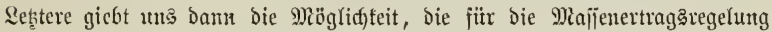

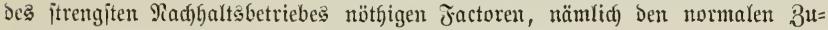
wad)

\section{$\S 55$.}

\section{5fanourfubefurfethung.}

Dic Etanovrts̆güte fängt ab vom Slima, vom Ierrait (\&agc), vom Boden.

Dicje Etantortsjactoren vermögen zrwar nicft cin in Baf)len ants=

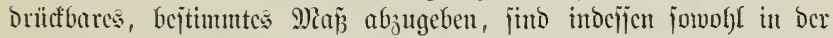

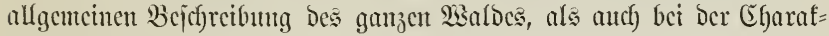
terijtif einjelner $2 \mathfrak{s} a$ lothcile zn erwähnent. ${ }^{*}$ )

*) Eotta: Grundriē der Foritwifïenduajt. 6. 2(ufl., herausgegeben von

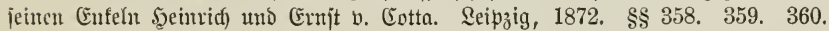

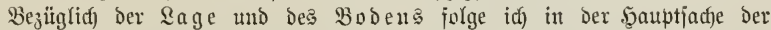

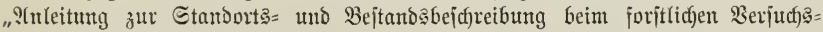
"vejen", weldje ber Berein Der Deutidjen forjtlidjen Berjudjanjtalten in Der Ber=

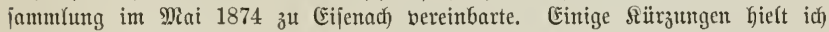

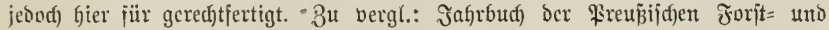
Jagogeieţgebung und Berwaltung. Şerauggegeben von Dan fïelmann. Berlin,

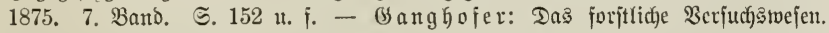
9ugşburg, 1877. I. BD. 1. Sit. S. 3 u. f.

De? $\Re$ Iima nad) Derjelben bei ber "örtlichen Rage" in Errwägung zu zieben jein. 


\section{A. Das Klima.}

Je nach ber tlimatijchen Bejchaffentyeit cintes Landes tömten für das örtliche Silima werichiedenc Ecalen entworfen merben. (5s lyat

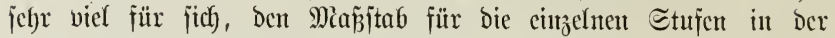

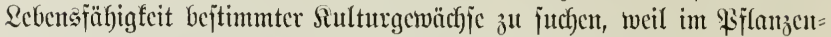

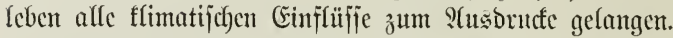

Fïr Sacbjen und älntich gelegene \&änder fann man folgcubc Ecala antendoct:

Gehr mild, wo ber 23 cin noch gut gedeifyt.

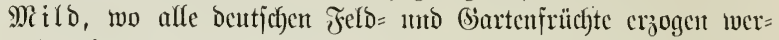
Dent fönuncrt.

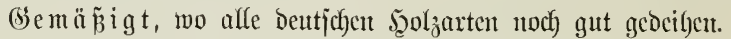

$\Re a \mathfrak{h}$, wo ber Dbjtbau nicht mehr anwenbbar ijt.

Sehr rauly, wo höchjtens mur noch Sartoffeln umb jeafer erhaut werben fümen, Itnd wo ber Jeofjamen mur jelten jur voll= fommenen Entwicfelung gelangt.

\section{B. Die $\leq a g e$.}

Es fommt in Bctrad)t bic allgemeine (geographiffe) und bie bejondere (örtlicbe) Sage.

I. Die allgemeine $2 a g e$ ijt nüber zu bejtinmen:

a) Durch Dic : Sngabe ber gcographijofhen Breite und Sänge.

b) Durd) Dic in Metern altsgedrüfte ?Angabe ber abjoluten $\mathrm{Er}_{\mathrm{r}}$ lyebung über Dem Mieeresjpicgel (Ditjec).

c) Dancben ift anjugeben, ob Das Revier angehört

a) Der Iicfebenc, insbejontore

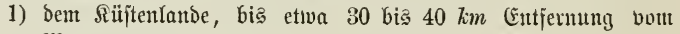
Meere,

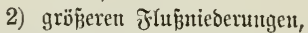

3) jonjtigent Tieflande;

$\boldsymbol{\beta})$ Der Şochebene;

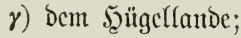

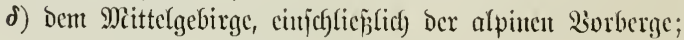

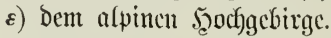

II. Dic bejondere (Brtlicke) Rage ift bebiugt:

a) Bon ber nachbarlichen llmgchung,

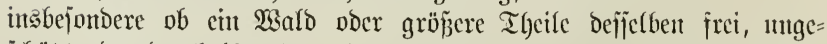

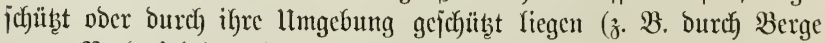
gegen Norbwinto), ob biejelfen gcjeloloffenen Dunjt = und fenthten 
Viebellagen angebören, ansfagernoen 2 intocn, bem Frojte, Duft= unD Echnceanthang crfabrutugsmäß̈ig crponirt jün.

b) Son Der Bodchansformung,

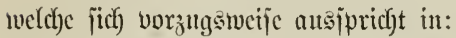

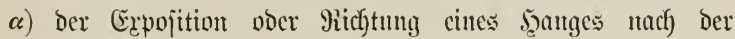
J̨imutsgegent;

$\boldsymbol{\beta})$ ber Bodeutucigutitg.

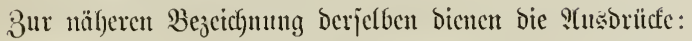
cben ober fajt eben bei cinter Bobenteigutg unter $5^{0}$, janft gencigt

lelgn (mäp̧ig jteil) iteil

ichroff

$\mathfrak{J} \mathfrak{e l} \mathfrak{s} \mathfrak{a} \mathfrak{b}$ jt $\mathfrak{u} \mathfrak{r} z$

\begin{tabular}{|c|c|c|c|}
\hline$"$ & " & $"$ & volt $5-10^{\circ}$, \\
\hline$"$ & " & $"$ & $11-20^{\circ}$, \\
\hline " & " & $"$ & $21-30^{\circ}$, \\
\hline & " & $"$ & $31-45$ \\
\hline & " & $"$ & über \\
\hline
\end{tabular}

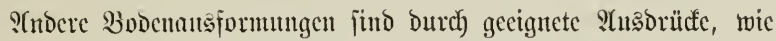

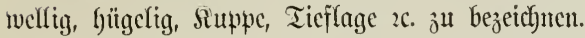

\section{Der Bodent.}

Der Boden ijt nach) dem (Grundgejteine, Den Bobenbejtandtheilen,

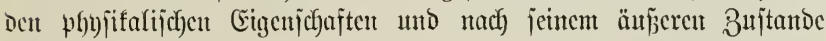
ıälycr ju bej d)reiben.

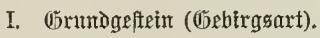

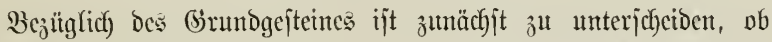
man es mit Śebirgs = ober Schroemmland, pocr mit anderen Worten,

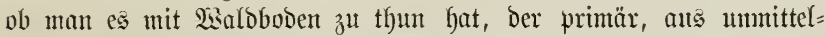
barer Berwitterung ber unterliegenden Giebirgsart, ober fecuntör aus Int = und Iuffichmemmung bervorgegangen ijt.

\section{Gebirgaland.}

Ileberall, wo Der Walbboden aue Der Berwitterung ber unter=

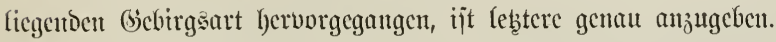

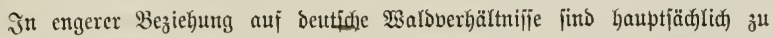
unterjoiben:

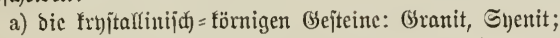

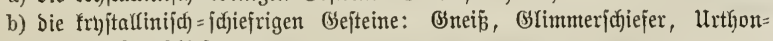
jujiejer, Taltjdjiefer 2 .;

c) bie \$orphyrc, 3. 3. Felïte, Duarz= \$orphyre $2 x$; 


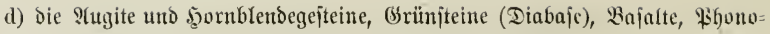
Yithe, Trady)te mit ifren Tuffen uno Conglomeraten;

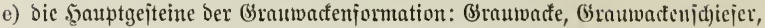
Ihonjdiefer in ihren verjdiedenten 9 (bönderungen;

f) Das Rothliegende mit jeinen Conglomeraten und Edjicfertfonen;

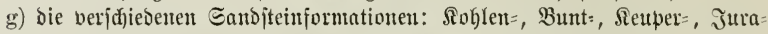

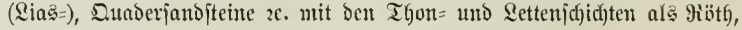
תeuperletten.

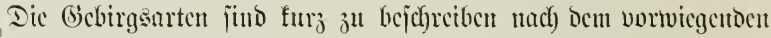

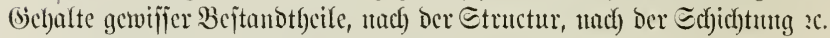

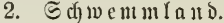

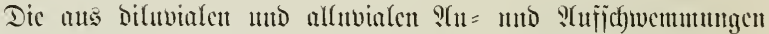

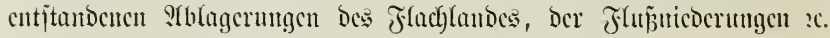
finto ljautptjüchliç):

\section{1) Bicrölle uno (Bicjochicbe (Ech)otter).}

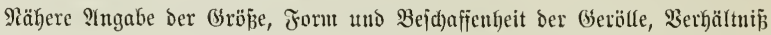
Derferben ătr beigementen Eroc.

2) Gandablagcrungen, แnto jwar:

a) falffrei, arm an Ferbjpath unb Silifaten;

b) falthaltig, jeloipatfreid);

c) Sgaibejant;

d) FItugiand in Bimtentante;

e) Dimenfant;

f) ₹̛lūị and.

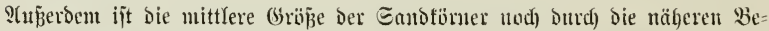
jeid)untren: grobförnig (ïber $0,5 \mathrm{~mm})$, mitteltörnig $(0,25$ bis $0,5 \mathrm{~mm})$ unb fein= förnig (unter $0,25 \mathrm{~mm}$ ) วu d)aratterifiiren.

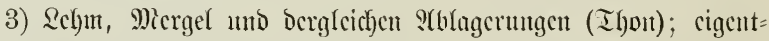

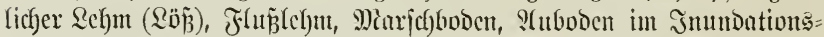
berciclye größ̄ercr f̧üjןc.

4) Mcoorboden.

II. Bodenbeftandtheile.

1. Mincralifde 3 ujammenfebaug.

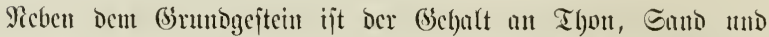

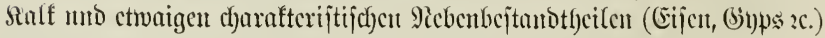
alyugcben:

ว. 3. Buntianditein, jandiger Thonboden; - voer Bajaltboden, thonig;

- ober Duaberjandjtein, weipjer, feintönuger Gans x. 


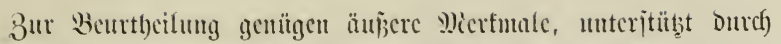

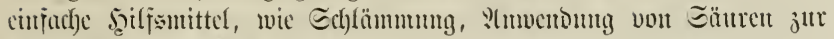
Ermittelunt Des Sialfes :c.

\section{Etcinbeimengung.}

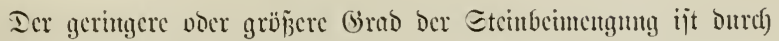
ctwas - jicmlid - ober jehr itciutig ausjutorütfen, wojern bic=

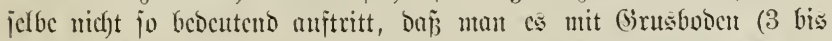

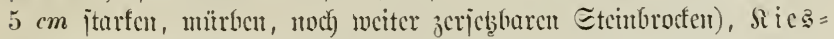

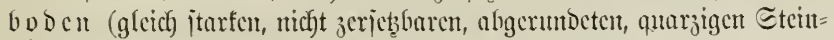

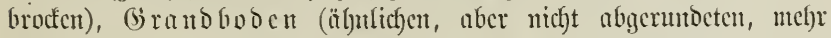
cefigen, vorrvicgeno quturzigen Miajien) ober mit (j) c

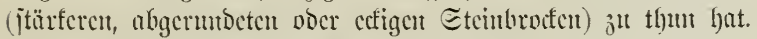

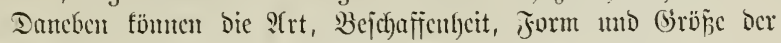
Etciutbcimengung näher angebeutct werben.

\section{3. รูน}

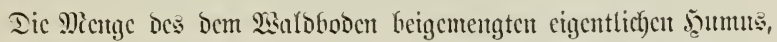

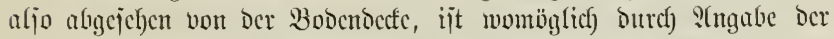

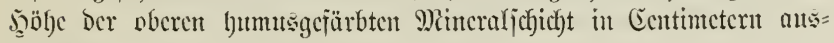
jubrüctctet.

III. Whyfitkaliridje Bodeneigenthaften.

1. Gơ $\mathrm{r}$ ün igfeit.

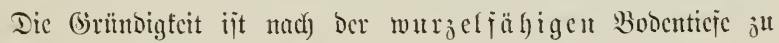

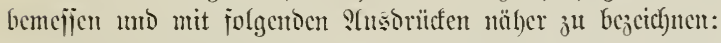

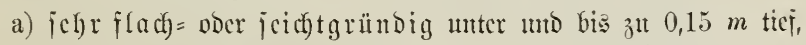

b) flach)= ober fcid)tgründig. . . . . 0,15 bis 0,3 " "

c) mittelticigründig . . . . . . . . "

d) tiefgründig . . . . . . . . " . " . " 0,6 "

e) ichrticfgründig. . . . . " " 1,2

Dabei jïnd jerner anz̆ugeben:

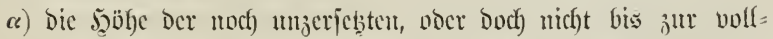

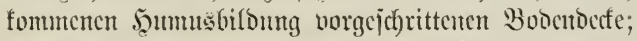

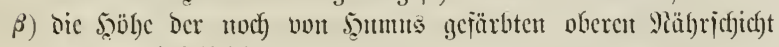
(Dummerocjeficft);

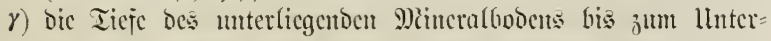
grmtoc, bejüglich) jowcit barit Dic Baumtwurzeln vorbringen; 
d) bejondere Bejchaffenfeit bes Ilntergrumbes, weldye cincu un=

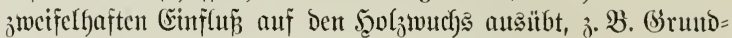
feudftigfeit, Fefsgrund, Drtftein, Ecfichtung bei Sdfiefer= geiteinen $2 c$.

\section{Bindigfeit.}

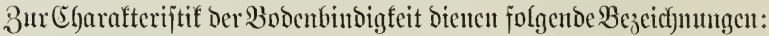

1) fejt, ein Boben, ber beim SUstrocfuen mit tief curbringendent,

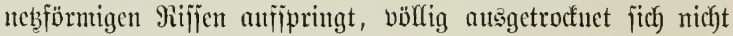
int fleine Stïdfe zerbrechen läß̈st;

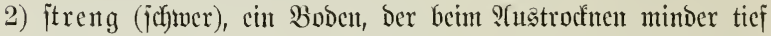
aufreipt, fich aber in fleine Stücfe zerbrect)en, went auch nicht jerreibent läp̈t;

3) mild (mürbe), ein Boden, ber jid) im trofenen Bujtande olye jonderlichen Wsiderjtand frümeln und in ein erbiges $\mathfrak{P u l v e r}$ zerreiben läßjt;

4) Yodfer, ein Boben, ber jich im feudyten 3ujtande jwar nod) haltbar balfen läp̈t, in trodenen Etücfen jebock viel Peigung zum Zerfallen zeigt;

5) Ioje, im trocfenen 3 ujtande völfig bindung

6) flitchtig, twent Der Boben vor Dem Wimbe weljt.

3. Frifde (Bobenfeudtigfeit).

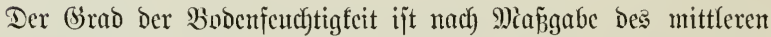

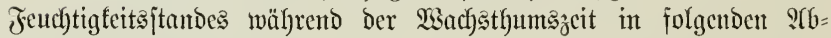
jtufungen anjujprect)en:

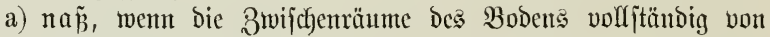

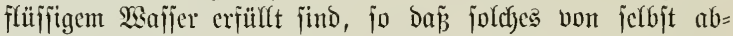
fliej̧t und jefbjt nach) längerer s(ntstroctuung nod) bis jutr Sberffächc jtaut;

b) feudft, wem ein Boden bcim Bujammentrefjen bas $23 a j j e r$ noch) tropfentweije abffiç̄en läß̈t;

c) Frifd), weun ein Boben Dem (Gefülyle nach von Feudftigfeit

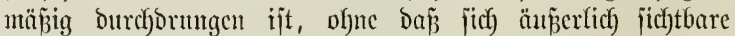
Epuren von tropffarem $\mathfrak{W a j f e r ~ b e i m ~} 311$ fammendrïfen zeigen;

d) troden, wenn es an Feudftigfeit merbr mangelt, und in Folge Deffen nad) erfolgter Durdfürjfung von Regen bie $\mathfrak{S a j j e r =}$ iputren jechon bimnen einigen Iagen jich verlieren;

e) Dürr, wenn aus den Sboden jede jidftbare Spur von Feud)= tigfeit ıad) furger (24jtündiger) $\mathfrak{Y}$ btrodinutg verjajwindet. 


\section{Farbe.}

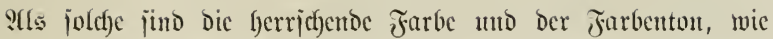
bicje iut trocfenen 3 utande Des ßobsens bervortreten, furz anjugeben.

\section{Aeuf̧ere Bodemututtünto.}

3u untericheiden jüno folgende 3 ujtände:

1) Difener (utater) Boben ijt free von jeder todten ober lebenden Bobendecfe; er erjecteint je nach) IImitünden flüchtig, mild, verfrujtet, aujgerififen, verbärtet, aušgchagert, aud burch vorangegangene lanomirtbjichaftlicke Bearbeitung anfgebrochen t. j. w.

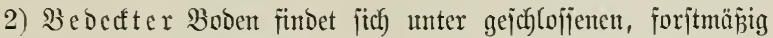
gefjaltenen 2ialdbejtämben und hat bie bem natürlicben $2 a u b=$ und

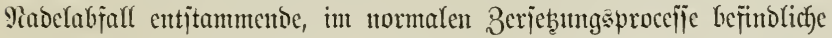
Bobeniocfe.

3) B̧enarbter (begrü ntcr) Boben ift mit eiuter, leb̧teren nicht

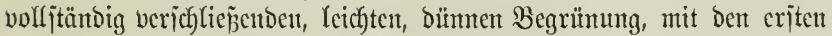

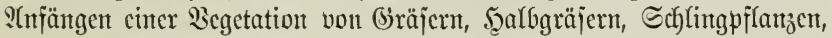

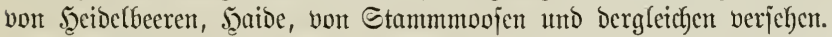

4) Berwilderter Boben zeigt eine ben Boben voll fä̈ndig ver=

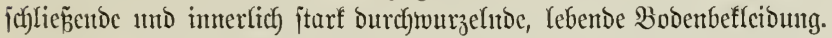
Se nach) Der STt ber leţteren ijt jut unterjcheiben:

a) Serangerung burd) mełr trodfene, ¡đuntalblätterige Sd(jmielengräjer ('Winto= halme, Edjmielen, einige Sd)wingelarten, Boritengraß, einige (Earerarten $2 c$. );

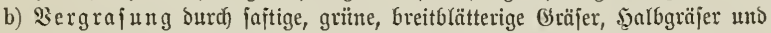
frautartige $\mathfrak{B}$ lattgewöad) ;

c) Seidelbeerïberzatg;

d) Sierhaioung;

e) Bermoojung, weldhe entweder als biffe, loctere Mloossecte von ben ver= äjtelten, wurzel= uno faftlojen Mioosagattuntgen (namentlid) Hypnum) auf = tritt, ober alę gejhlojijene, hajtende Decte von den jogenaunten Stamm= moojen (Polytrichum), ober alక geid)lojiente Decfe bon Den jogentanten siajiermoojen (Sphagnum).

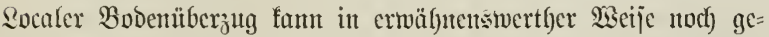

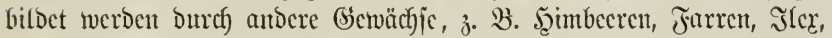
Wadylyolocr, Şungerflechten $2 c$.

5) Dic Bobcuvertourzelung tritt ale folgc ber boden=

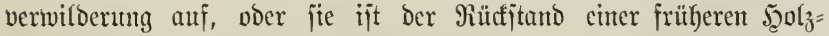

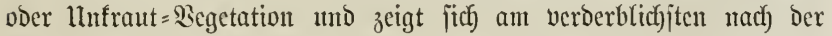

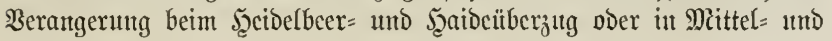




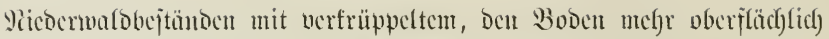
IIID jtart Durdjwurzelndem llnterfodze.

\section{$\$ 56$.}

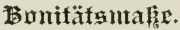

:Ule im vorigen \&aragraphen crmähnten Etandortsucrbältnijic

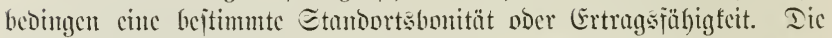

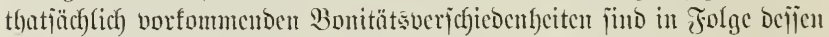

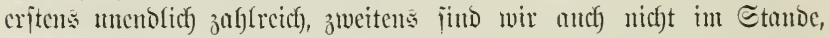

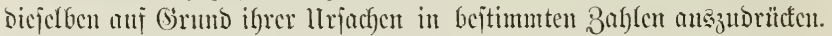

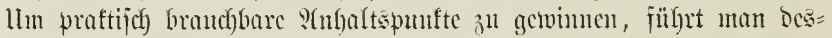

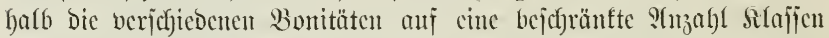

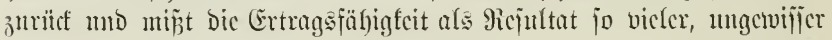

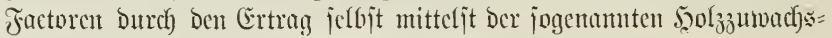
oder Ẽrtragstajeln.

Man fam hierbei ansgehen cntweder von bem cincm getvifïn

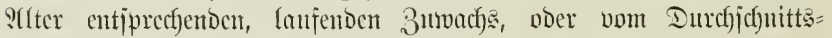

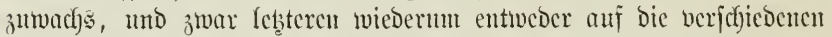

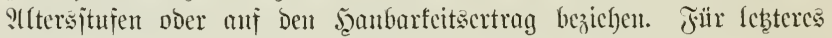

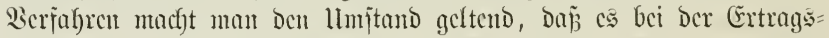

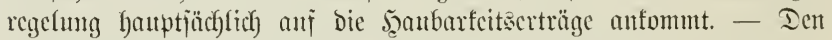
3umadys jelbjt brüdt man in Brudfthcilen bes Subifmeters ans.

3n unterjuciocn jims: normale und concrete Bontiät. Ilnter criterer beriteft man dic cinter getwijien Etandortsgüte für cinc ge=

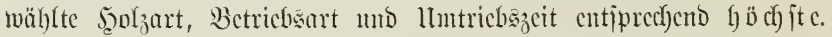
Iic concrete Bonität ijt jenc, wic jic ber Stundort in Folge ver= jeficoener anf ifn cintwirfender, mehr vocr tveniger vorübergefyender, änïerer Einflïjle zcigt. Eic fam mit ocr normalen übercinjtimmen ober nicht. Sm lebeteren Falle neunt man jie a butorme Bonität.

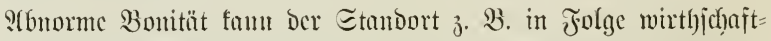
licf)er Fefhler (Streumubzung, Siablabtricbe mit vernachläjïigtent \$(nbau, Sorverjüngutg anf trocfencm Standorte sc.) DDer in Folgc ungünjtiger

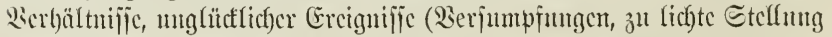

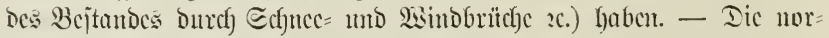
malc Bonität wiro audf idcale ober abjolnte genannt, lebztere ijt jic

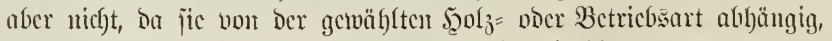
Daher cbenjo gut cinc relative Bonität ijt, wic bie conercte. 


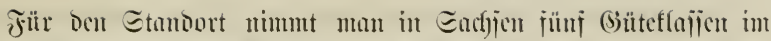

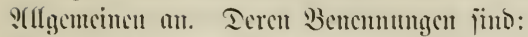

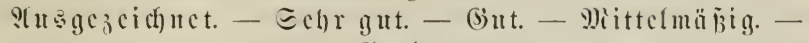
Gering.

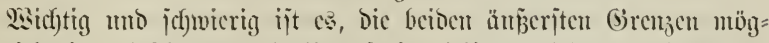

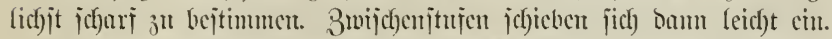

3 mectmäpig wird bic bejte Etambortsflajic $=1$ gejebst, mo twer=

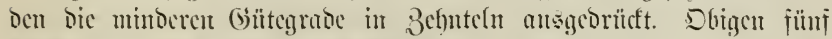
Silajīen müroc baum jolgembe s(bjtujumg cutjprechen:

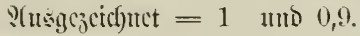

$$
\begin{aligned}
& \text { こelgr gut } \ldots=0,8,0,7 \text {. } \\
& \text { (Gist } \ldots \ldots=0,6 \text { " } 0,5 \text {. } \\
& \text { Mittcmmǟig. }=0,4,0,3 \text {. } \\
& \text { Gerring. . . }=0,2 \quad \text { " } 0,1 \text {. }
\end{aligned}
$$

Dicje Miethode hat Den Eorzhtg, Daj jie bie Bejtimmung ber

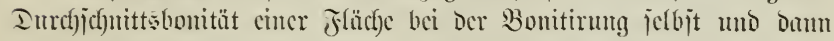

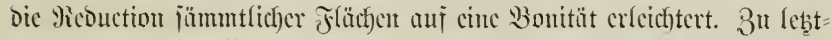

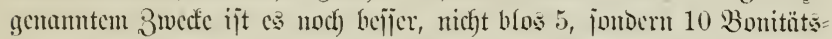

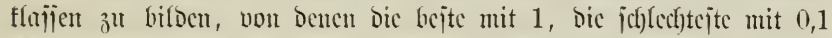
beseicfutet miro.

Y nmerfung. Gbiebt man Den eingelnen Bonitäten Bahlenbezeidnungen,

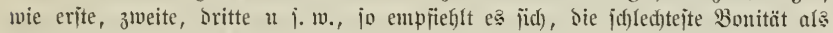
erite, bie bejte bei 5 sllajen als fünjte zu bezeidynen, Damit Der böchjten Ertrags:= fäbigfeit aud die bödjite 3ijier entiprid)t. In Der Fraxis hat bieje :(bjujumy

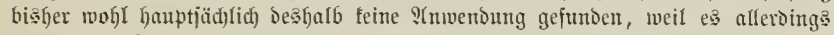
leidter ijt, 3ahlen für Das Wiaximum Der Ertragsiähtgfeit eines Etandortes zu

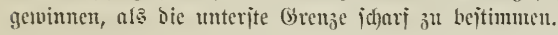

\section{$\$ 57$.}

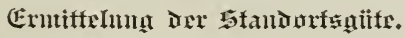

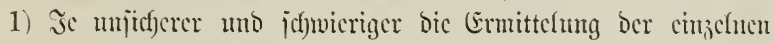
Jactoren ijt, wefche bis Etantoortsgüte bedingen, um jo melgr ijt man

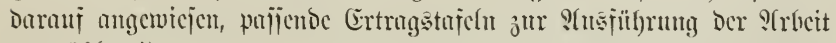

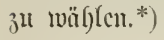

*) Ta wir Die Bedeutung Der Factoren ber Etandortsgüte mur in ihrer (Se=

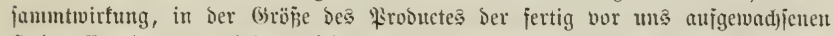

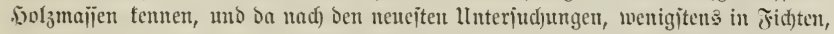

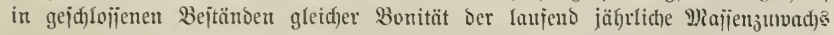

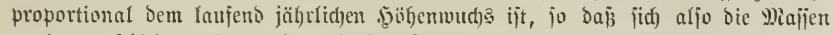

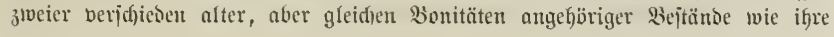




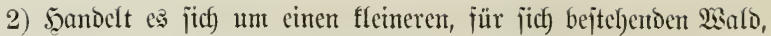
jo jind niöglichjt gute, locale Ertragstafeln zu entwerfen. Für grö=

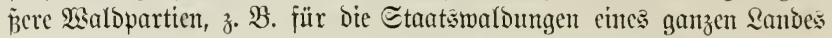

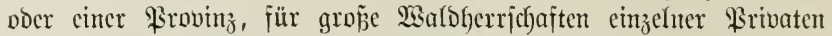

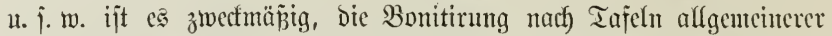

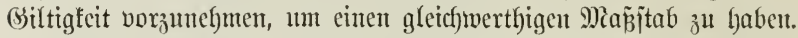

3) Dic Unterjudfung ciner $\mathfrak{S} \mathfrak{A}$ ah) vorfandener, älterer und mittel=

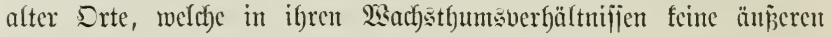
Störungen erlitten haben, nach Majie und 3umachs crgiebt Deren Bejtandśbnität $(\$ 76)$, bie wir als charafterijtijch für bie concrete

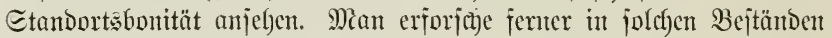
alle ङtandortşactoren (\$55), um Darnach) Die Bonität anderer Flächen,

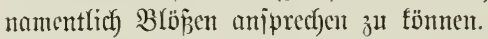

Junge, Der I. und unter llmitänden aud ber II. IIterîflajị angefjörende Bejtände beurtfeilt man an Bejtent im :Ifgemeinen nach)

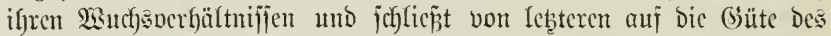
Etandortes. Eefjr gutes ânfalten gemährt hicr namentlicf bie $\mathfrak{B e}=$ achtung Des f̧öbemmuchjes. Will man jedoch möglichjt jicher Jrr=

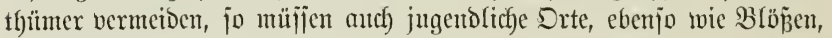

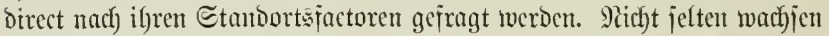
juntge Bejtände recht jreubig nur bis zu cinem gemifjen s(lter wegen Flact)gründigfeit Des Bobcuts, oder umgefefrt, jie haben eine Periode

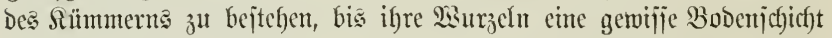
erreicht, oder ifre (bipfel bie froitregion liberjchritten haben.

4) Bur Prüfung Des Bobens mache man bis auj Den luntergrumb, bejichentlich) bis 2 Mieter tieje Ëinjchläge, und ztwar im Eommer, wo= mögfich jeboch nicht nach lang anduternocr, bejonders trocfenter ober

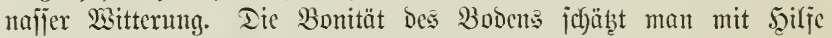
Der in Den unterjuçten Bejtänden gejumbenen Rejultate nad) Der ent= ipredfenden Slajje doer Etuje ein.

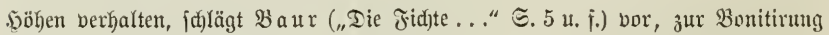

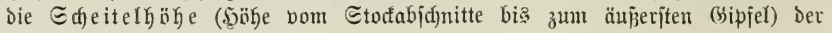
Bätme anzuwenden.

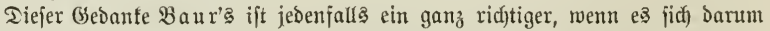
Gandelt, Bejtände einer bejtimmten Bonttätŝtlajīe zu孔weijen. Jmmerhin fönnen

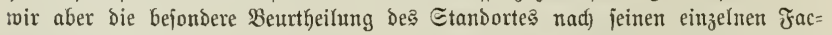
toren Daburd) nidft eriparen, jobald wir ben forjtlidjen Thatbejtand rid)tig ermitteln

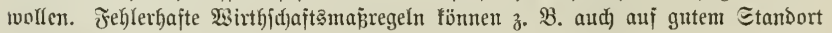
cinen jobledten Bejtano mit geringem Döbentudje herborrufen. 


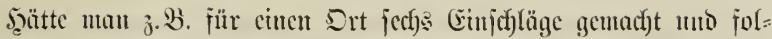
gende Ed)äbungşald)len gemonuen:

B o $1 \mathrm{i}$ t ä t.

E in $j$ d $\mathfrak{l}$ a g.

\begin{tabular}{l|l|l|l|l|l|l|l|l}
1. & - & - & - & 1 & - & - & - & - \\
2. & - & - & - & - & - & 1 & - & - \\
3. & - & - & 0,5 & 0,5 & - & - & - & - \\
4. & - & - & - & - & 1 & - & - & - \\
5. & - & - & 1 & - & - & - & - & - \\
6. & - & - & - & 0,6 & 0,4 & - & - & - \\
\hline & - & - & 1,5 & 2,1 & 1,4 & 1 & - & -
\end{tabular}

jo müroc dic Durchjechnittsbonität Dicjes Ertes

$$
\frac{1,5 \times 0,8+2,1 \times 0,7+1,4 \times 0,6+1 \times 0,5}{6}=0,67 .
$$

oder abgerumbet 0,7 betragen.

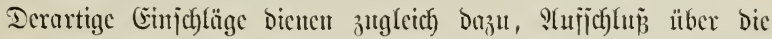
(5)ründigfeit Des Bobens (હ. 169) jall geben, unter llmitünden audf) Bodentrofile zu entmerfen.

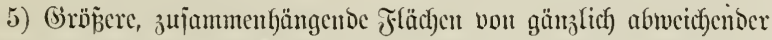

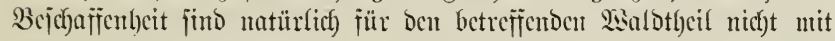

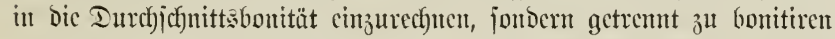
in Sarten und Echriften bejonders zu bezcichnen.

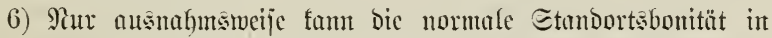
Fiechnung fommen, jobald ifre bie concrete nicht gleich iteft. In Der

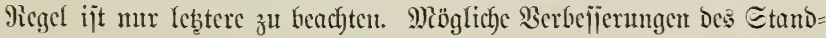
ortes gehen in Der 5̧auptjache jo fangiam vorwärts, ba $\tilde{\beta}$ cs ungerecht= fertigt erjebeint, biejelben jefyon oor bem wirflicfen Erfolge hei ber Bontitiung on berïffichtigen.

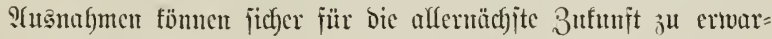

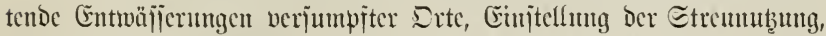

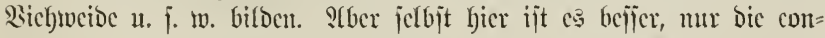
crete Bonität anzumenton, jo lange jie bejtegt, Dagegen aber in ber jpeciellen Bejdyrcibung Des Drtes Darauj hiuzuwcijen, Daßj cine Ber= befierung bes Etantortes in SAtsjicht genommen twerden finn.

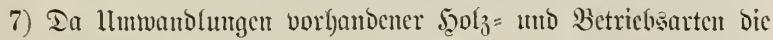
relative Sybike jeber Etandortsbonität verändern, jo fann man cut= 


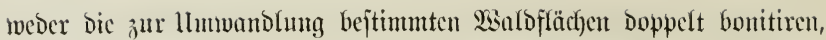

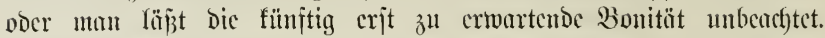
Rebterer Lieg ift Der cinfact)ere und gewöbulich auch ber richtigere. Piur Dom fömen wir cine joldfe jtets feldr unfichere Doppelbonitirung nicht gut entbebren, wem cs jich barmm handelt, erjt Enticheidung Darüber zu treffen, ob cine llumvandung erfolgen jolle ober nicht.

8) Dic Miconction jümmtlicf)er Flächen cincr Betricbsflajic oder cincs ganjen Mevieres anf cine Bonität crfolgt für Den Etandort in

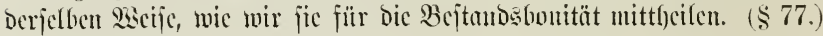

\section{$\$ 58$.}

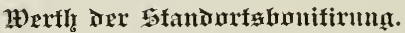

Dic Ermittelung Der Etombortsgitte mag moch jo genau und ge

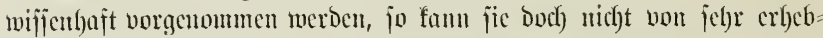
licfer, namentlich) nicht birecter Bebcutung für bic Ertragsegelung jelbit

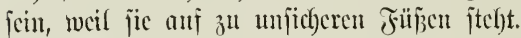

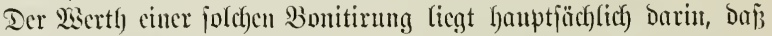
wir jie zur allgemeinen Bejchreibung vorliegender 2 şaloverfältmijice,

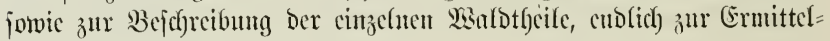
ung bes Grumbfapitales brauchen.

Ferner tönnen jene Ertragsregefungsmetf)oden, weldec Den von

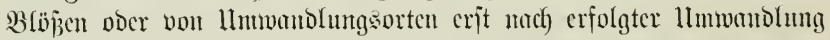

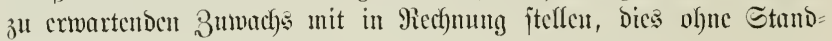
ortsbonitirung uicht thum.

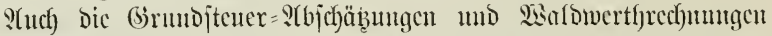
fömen lebtere nicht entbebren, Da bie (Ertragsfähigfeit des Bobens vou

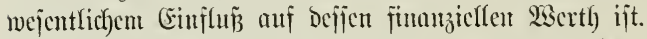

Dic Foritcinricftung mb Ertragsregelung alfein merben in Den meipten Füflen cinte in Das Detail gef)ende, jebr feiute Standorts= bonitirung nicht nöthig machen, fondern gröjereses (sictuicht auf bic Bonitirung Der vorbandenen Bejtünde legent.

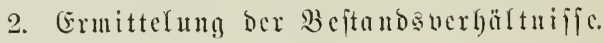

$\$ 59$.

3uxre und Eintheilung orr Hufiatux.

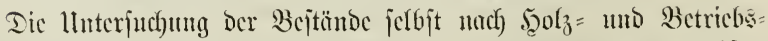

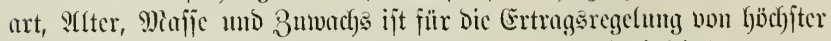

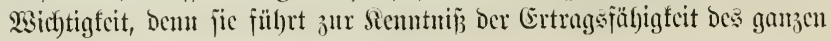




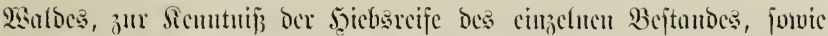

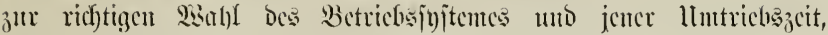

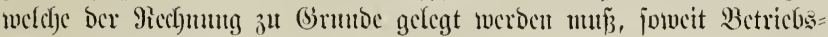

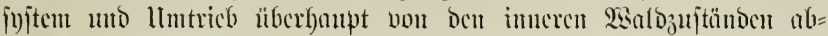
büitgen.

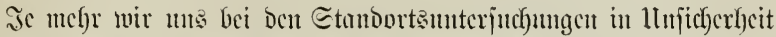

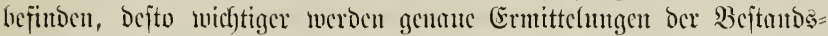

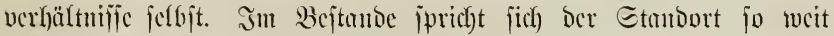

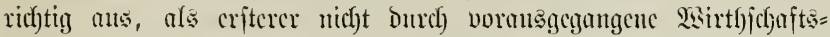

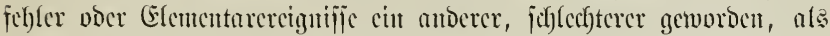
cr jein jollte.

Der ipecieflen bejtandsbejchrcibung müfjen ale Borbercitungs arbcitu vorauggel)en:

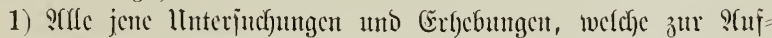
jtelfıug vou Erfabrungstrifeln nötbig fund,

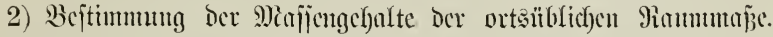

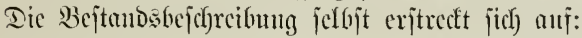

1) Betrichant,

2) รูolzart,

3) Bcitoctumg̣ggrab,

4) S(lter,

5) Eutitchutug,

6) Miafic,

7) Duantitïtşumach?

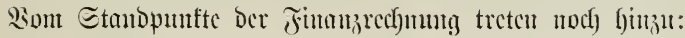

8) Dunalitïto =

9) Thencrungs = $\}$ Buwact)s,

10) Sorratf $s=(5,50)=$ Siapital,

11) Birumbtapital.

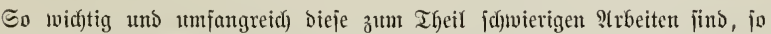

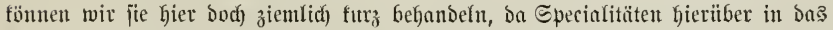

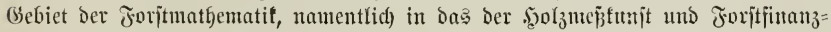

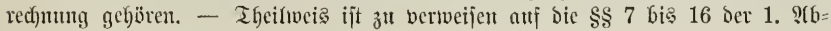
theilung unferes Qefrobuthes.

$$
\$ 60 \text {. }
$$

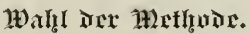

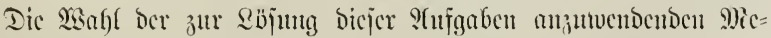

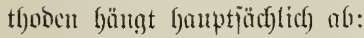

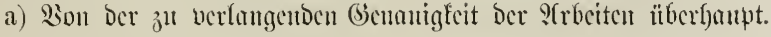




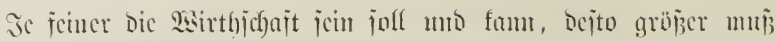

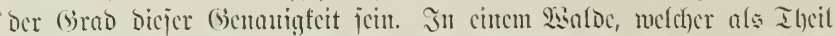

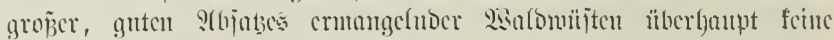

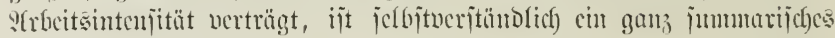
Ricriafyren am Pilabc.

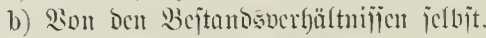

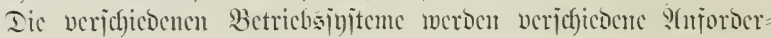

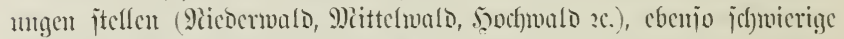
Terrain = uns complicirte Bejtandsocrbältnijic andere, als j. B. cin cimfïrmiger Sicjermmald ber Eambebenc.

c) $\$ 30 n$ bcm möglicfen Siojten= und 3citanjpande.

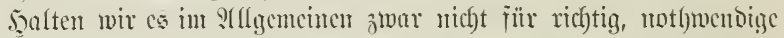

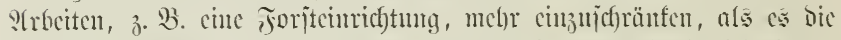
crjorberliche (benauigfeit berjelfen verträgt, jo treten boch nicht jelten

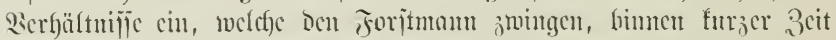
cincu vorlänfigen Betrichșlan jull cutwericn. Er wird baun gan

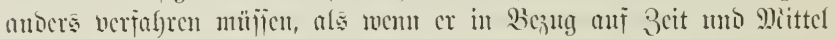
nicht (xcichränft ijt.

d) Qion bem Biloungsgrabe bes ju Gebote itehenden Perjonales.

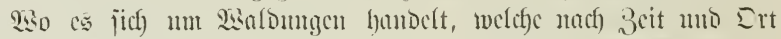

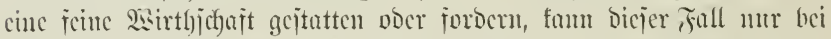

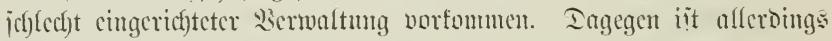
Der Foritcinrichter verpflichtet, anf ben nicorigeren Bildungsingo bes

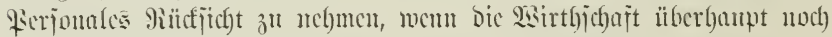

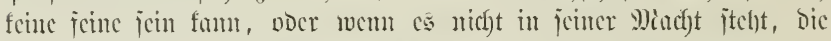
Iicnjteinricftumg jelbit ju werbejicru.

e) Sion Der Miethode Dor Ertragseregelung jelbjt.

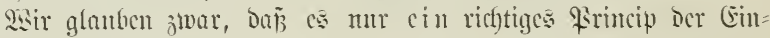

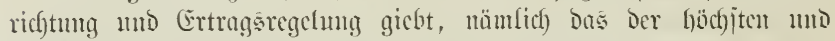

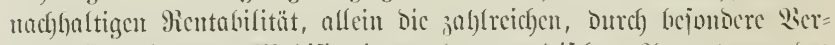

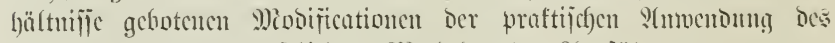

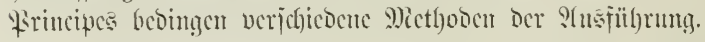

A. Vorbereitumģsarbeiten.

$$
\S 61 .
$$

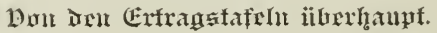

Dic Ertrags= (Erfal)rug

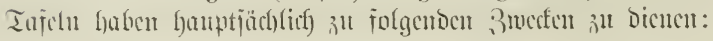


a) Bejtumbshonitirun!y,

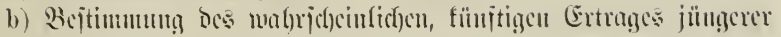
Bacitülloc,

c) Daritellung dos Bmmarbsganges,

d) Ermittelung Des Sionululvoratles,

e) Ermittelung ocr vortheill)ajtejten llutricbsisciten.

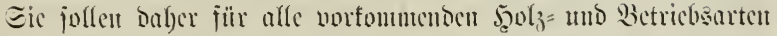

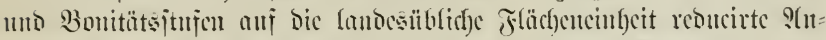

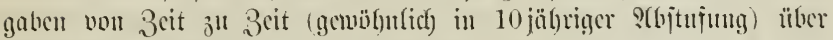

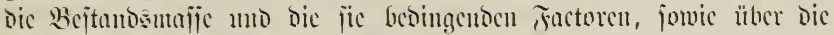
nerjobiedenen Eortimente enthalten.

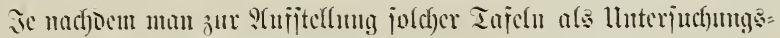
objecte normal crmadjicue, forjtmä̈̈ig bebandelte Bejtände verichiedener

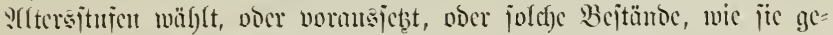

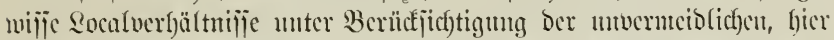

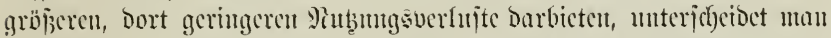
Viormal = uno Rocal=ertragstafelı. Ta critere unabräugiģ vou

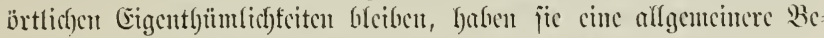

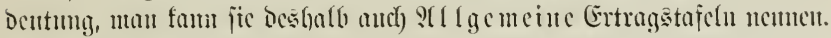
(3u vergleidgen $\$ 64$.)

Ier Infjalt beider, weust er gumb volljtämbig jein, namentlich)

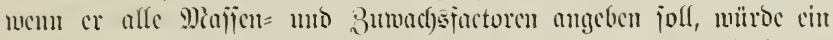

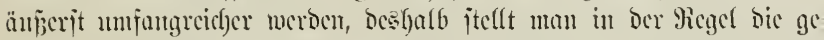
monucuen Rejultate in cinjad)eren Iajeln jujanmen. Es fömten unter jefficocu werden:

Jeantertragstafeln, weldye mu bic Mhaje bes mrïbomini renden oder nauptbejtandes nadyeijen.

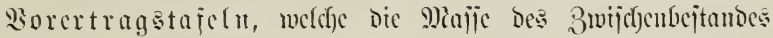
angeben.

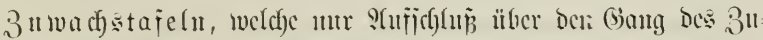

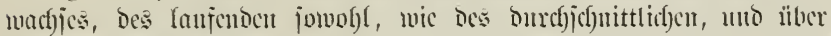
das 3umact)sprocent gemül)ren.

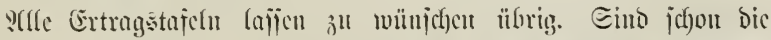
Rejultate, meldse man ans reinen Bejtünocn geminnt, feinc abjoluten

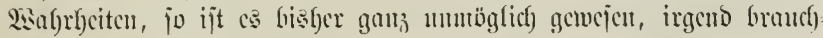

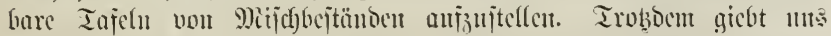

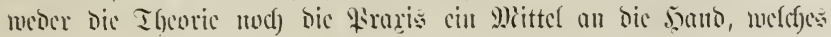


für jene Bmedfe, für weld)e wir überbaupt. Ertragstafeln brandfon, Dicje crjeben fönute. ${ }^{*}$ )

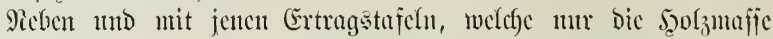
und igre Factoren berïcfjichtigen, wären, vom loücljiten, praf=

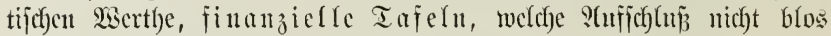

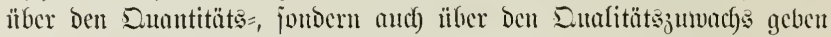

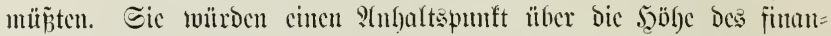
zielfen Şaubarfeitsalters und llmtriches getwäl)ren, jelbjtocrjtändlich)

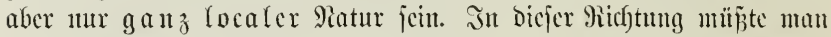
Deu Eefrwerpunft auj eine überjicftliche Darjtelfung ber Eortimente legen, weldye boch weniger weränberlich find, als ber Freis.

\section{$\$ 62$.

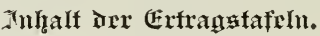

1) Sattersabjufung. Sift cs für mandbe mifjenjeffuftlicfec

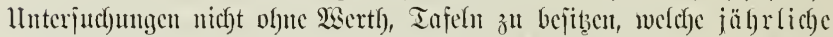

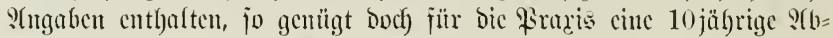
¡tufung, wie jic gewobntich gegeben wirb.

2) Bonitütsflajjen. WSie für bic Stamovrts= ijt es audf für

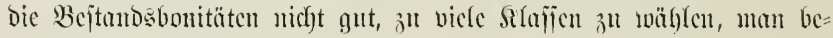

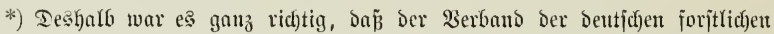

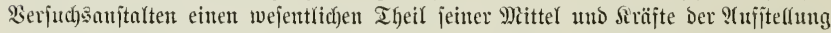

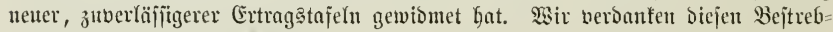
ungen bereite folgende ?hbeiten:

Baur: Die Fidte in Bejuly auf Ertrag, 3mwadjs uno Form. Stutt= gart, 1876.

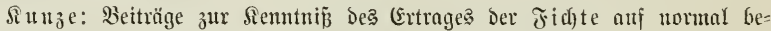
jtocften Flächent. Empplentente z. Thar. forjtl. Jahrbutche. I. Bant, 1878 uno

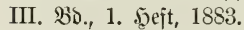

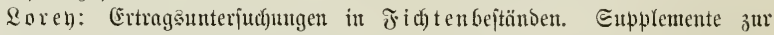

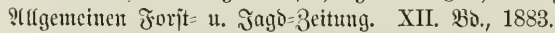

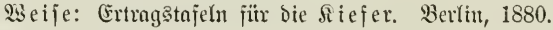

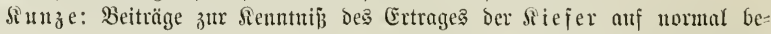

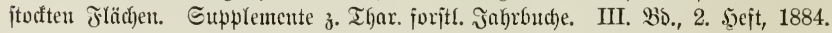

Baur: Die Rothbudse in Bezttg ati Ertrag, 3uwadis uns Jorut. Berlin, 1881.

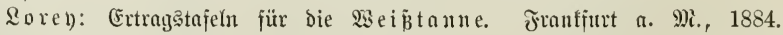

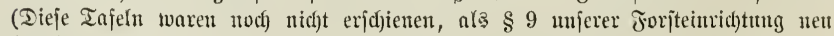
bearbeitet wurbe.)

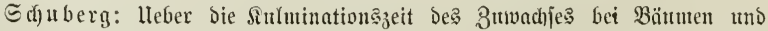

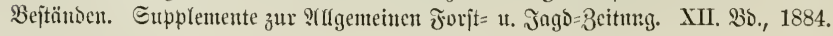


gutügt jid ber leidyteren lleberjicht wegen gewöbntid.), jo aud in Eachjen, mit füní.

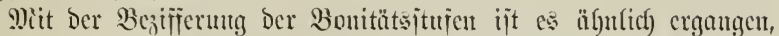

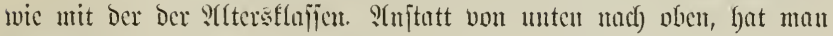

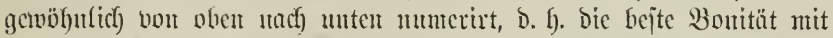

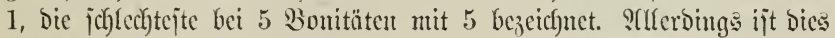
nur cine formprage, allein es handelt fidf) barum, nicht weldje form bie üblict)ere, jonbern weldye bie ridjtigere ijt. Wisir jtimmen in biejer

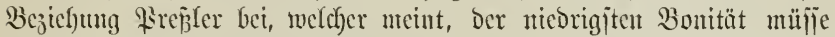
andf) Die uicorigite Bifjer entjpredfen. *)

Entuveder faum man mu für jäuntliçe Bounitütsflajīen alle S(ngabeu ber Erfafrumtgstafel getreunt anjül)ren, ober was namentlidf)

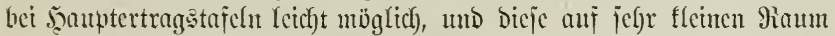
bejchräuft, mur für cine Bonitätsijtufe, bie anderen jedoch als Biel= faches berjelben betrachten. $\left.{ }^{*}\right)$ Mit leţterem Berfabren föntent wix

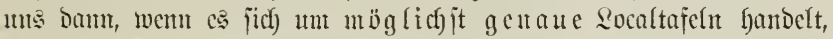
bcelfalb nicf)t gan einverītunden erflären, weil es jtreng genommen

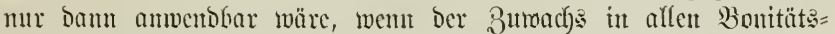
itufen parallel licje, was befountlich), namentlich in jüngeren Bejtänden, nicht Der Fall ijt. Dagegen läjpt es fich nicht verfenmen, baj jolche

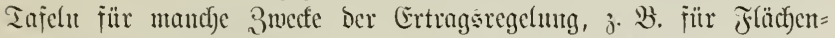

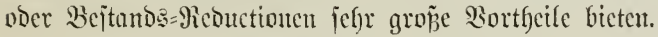

3) Dic $5 \mathfrak{c}$ oljmajic.

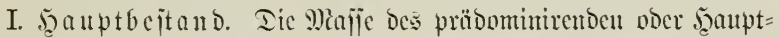

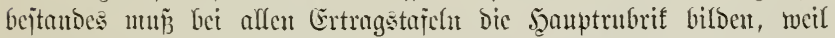

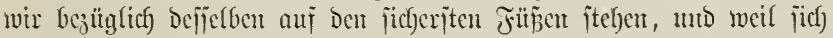

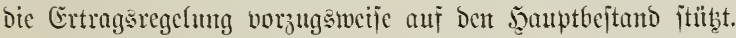

II. 3wijchenbejtand. Der Dic Siorerträge liejernde 3wijeden=

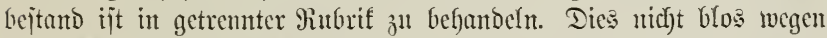

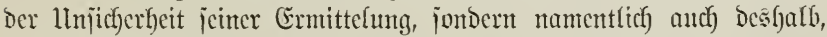
meif bic Sorerträge bei Den llnterjuf(futgen über Das vortheilfajtejte joubarfeitsalter antoren Red)mugsoperationen unterliegen, als ber 5ुauptertrag.

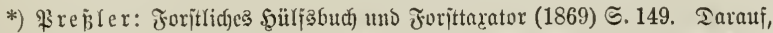

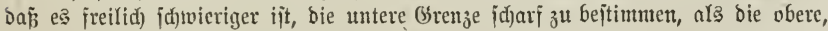
haben wir bereit: $\subseteq .173$ hingewiejen.

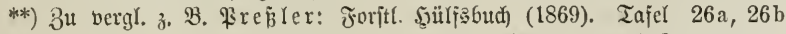

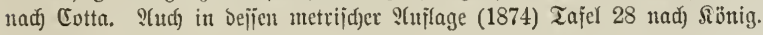


1II. Nia

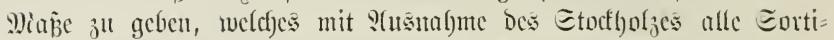
mente entljält. Es cmpfichlt jïch bazn das "Fejumeter".

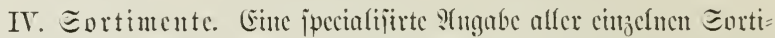

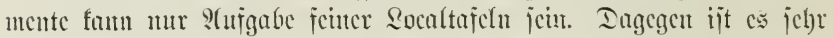

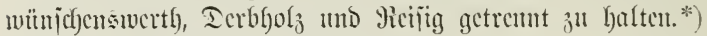

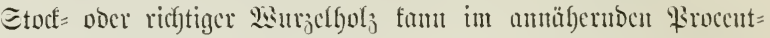
jabe nebentyer crwälnt werden.

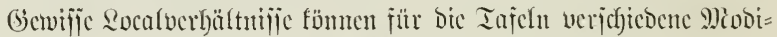

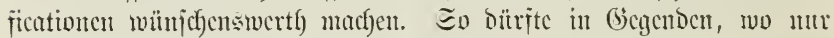
Das Ierbfols abjebbar ijt, andf un bicjes in bic Iafel anjouncfuten

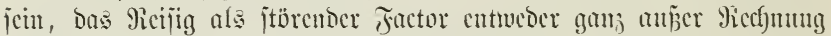

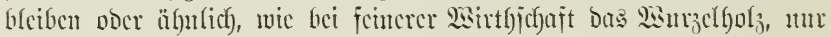
nebenbei crwäbut merocu.

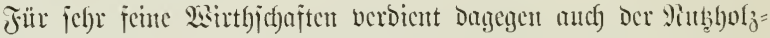
amijall Bcadftutig.

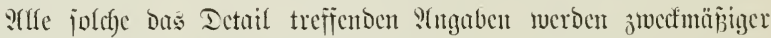
nticft ber cigentlichen Iajel jelbjt jugejügt, jontorn in ipecielle, bicjem 3 wede bejonders gerwiomete Tajeln verwicjen.

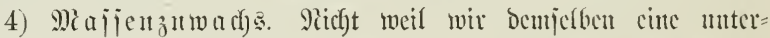
georbute Bcocutung jujpect)en, nenuen wir ben Miajen= ober Entut=

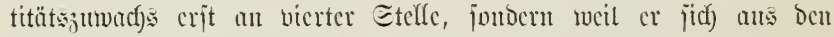

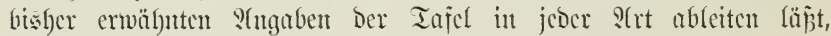

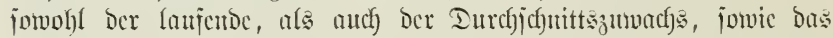

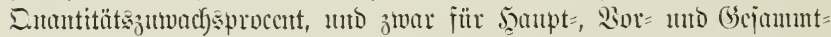
crtrag. Bei ber holjen Bebentung bes firocentes fïr bie 2isintbjefajt,

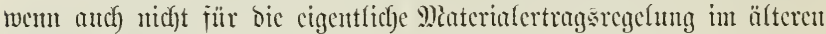
Eimne, falten wir es fïr jefrr juectmäpitg, menigitens bicjes in

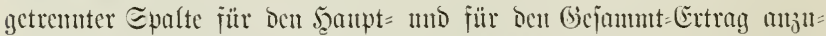

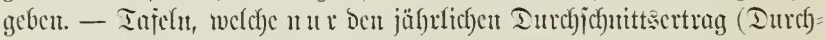

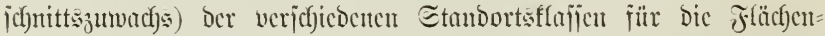

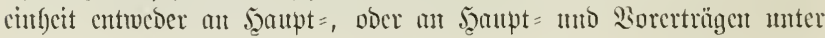

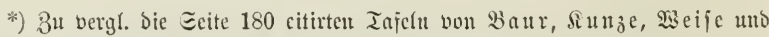
Qorey. - Dieje Trennung in Ierbfor

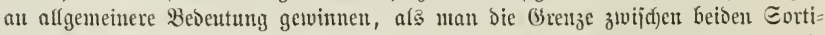

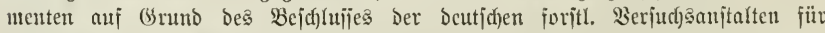

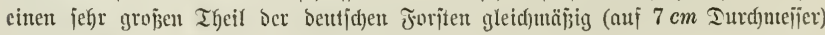
jeitgejęt Gatte. 


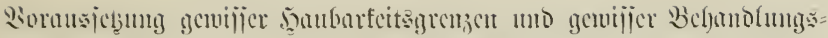

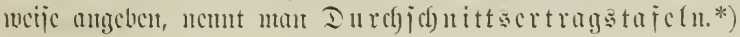

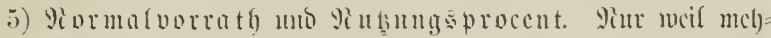

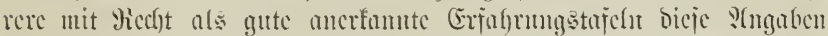

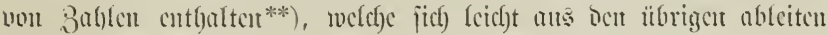

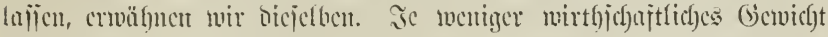

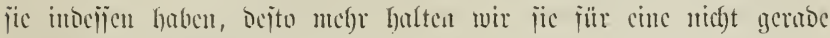
nothencubige Beigabe.

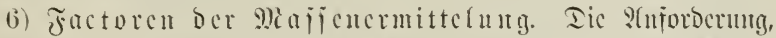

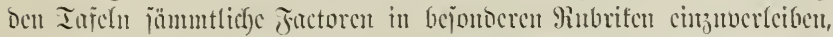

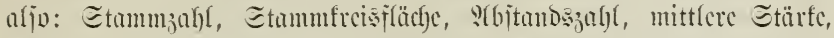

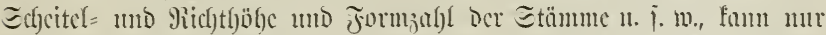

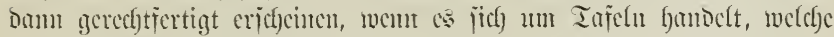

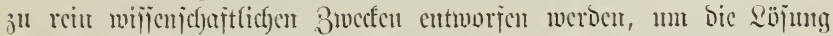

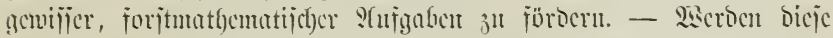

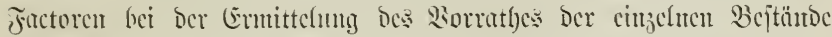

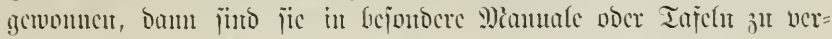

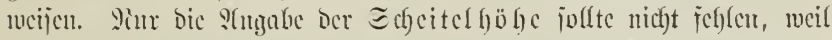

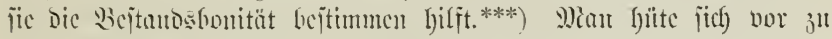

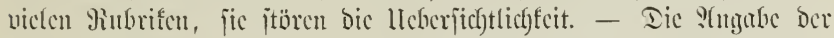

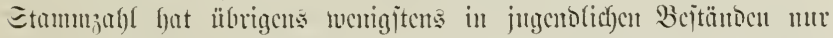

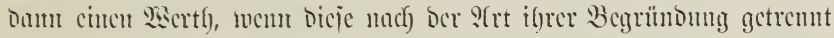

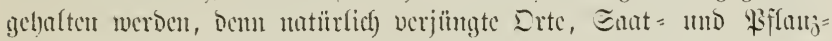

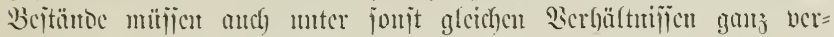

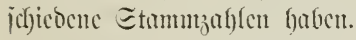

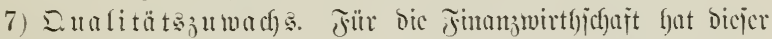

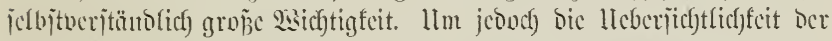

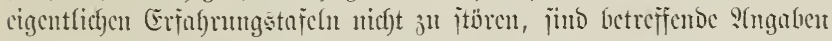

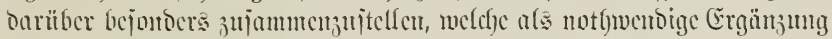

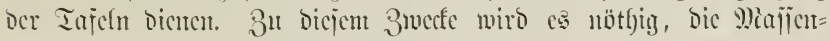
Iaj. VI.

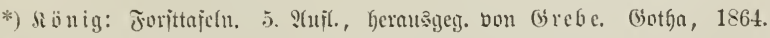

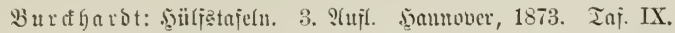

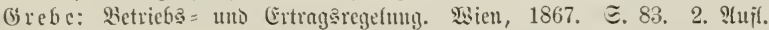
खitelt, 1879. こ. 104.

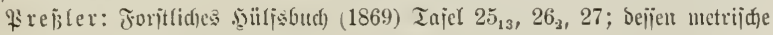
?turlage (1874), Tajel 28.

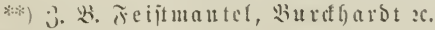

$\therefore$ *) 3 ucrgl. Wote ar 巨cite 173. 
crträge in if)re cinjelnen Eortinnente zu zerfällen, bamit ber crntes

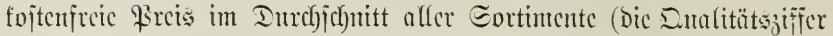

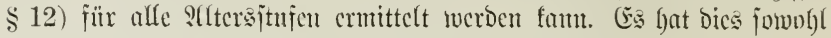
für bic Şaupt=, als für bic Sorerträge zn gej

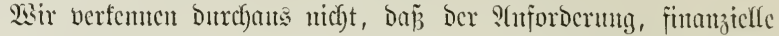

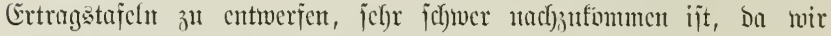

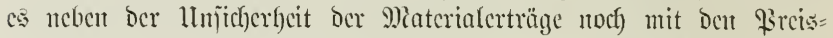

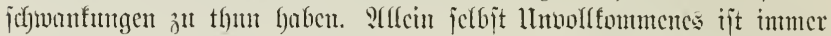

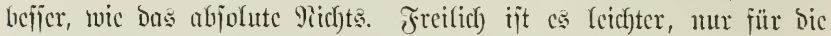

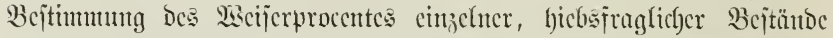

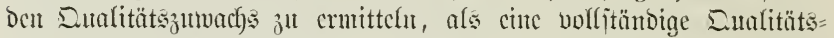

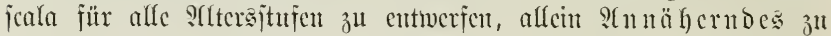

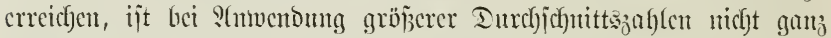

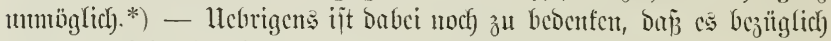

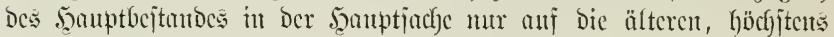
mittleren (s)ficoer ber Bejtandsreife anfonmen fant, wäf)reno bejüglich) Der Sorerträge vorjugstucije bie jüngeren und mittleren Gificder $b c=$ achtututg werdienctr.

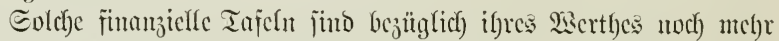

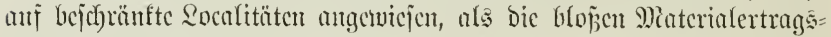

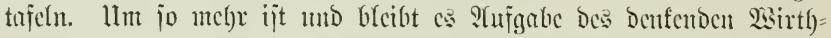

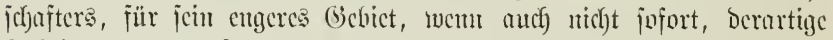

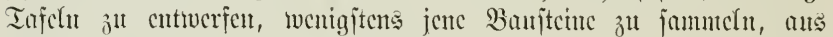

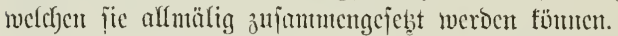

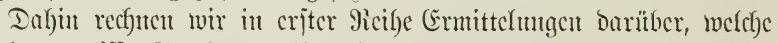

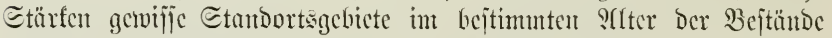

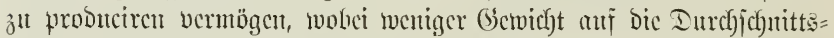
itärfe ber mittleren Etammflaije, mel)r anj bic jeder von Ecutimeter zut

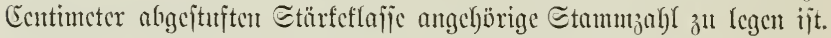

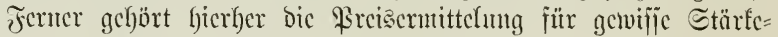

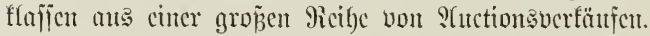

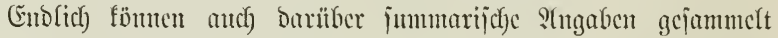

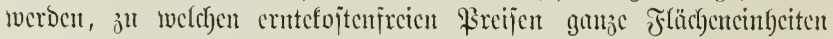

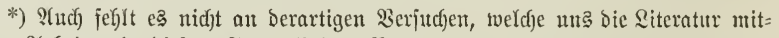
theilt. Qfrbeiten in biejem Simne jind z. $2 .:$

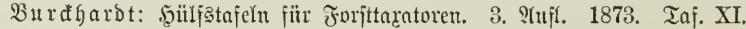

Srebe: Ter Budjenfodymalobetrieb. Eijenad), 1856.

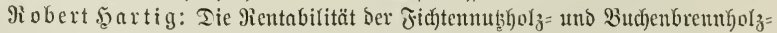

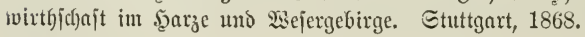




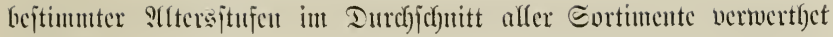
murben.

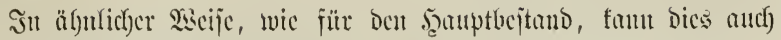
für ben Brvijd)enlejtand gejdychen.

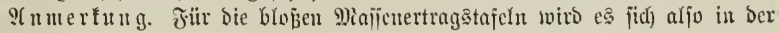

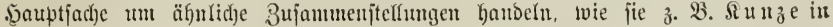

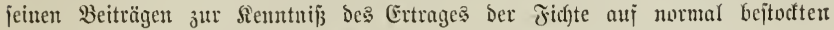

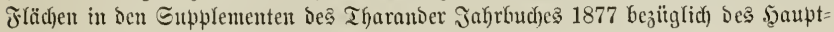
hejtandes gegeben. Beijpielsweije jei hier bie Iajel Der 3. Güiteflafie mitgetheilt:

\begin{tabular}{|c|c|c|c|c|c|c|c|c|c|c|}
\hline \multirow{3}{*}{$\begin{array}{c}\text { Sllter } \\
\text { đahre } \\
5\end{array}$} & \multicolumn{9}{|c|}{ Jicte. 3. (süteflafie. } & \multirow{3}{*}{$\frac{\mathfrak{3 l t e r}}{3 a \text { hre }}$} \\
\hline & \multicolumn{3}{|c|}{ 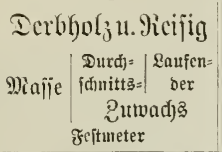 } & \multicolumn{3}{|c|}{ 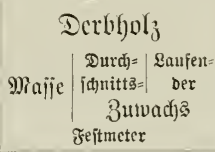 } & 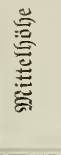 & \multicolumn{2}{|c|}{ 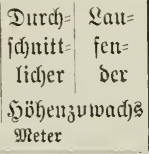 } & \\
\hline & 22 & 4,4 & & 0 & 0,0 & 0,0 & 0,9 & 0,18 & & \\
\hline 10 & 44 & 4,4 & 4, & 0 & 0,0 & & 1,8 & 0,18 & & 10 \\
\hline 15 & 68 & 4,5 & 4,8 & 0 & 0,0 & & 2,7 & 0,18 & 8 & 15 \\
\hline 20 & 94 & 4,7 & 5,2 & 0 & 0,0 & 0,0 & 3,7 & 0,19 & 20 & 20 \\
\hline 25 & 130 & 5,2 & 7,2 & 22 & 0,9 & & 4,8 & 0,19 & 2 & 25 \\
\hline 30 & 176 & 5,9 & 9,2 & 50 & 1,7 & 5, & 6,1 & 0,20 & 0,26 & 30 \\
\hline 35 & 230 & 6,6 & 10,8 & 92 & 2,6 & 8 & 7,6 & 0,22 & 30 & 35 \\
\hline 40 & 288 & 7,2 & 11,6 & 146 & 3,7 & 10, & 9,2 & 0,23 & 0,32 & 40 \\
\hline 45 & 347 & 7,7 & 11,8 & 214 & 4,8 & 13 & 10,9 & 0,24 & 03 & 45 \\
\hline 50 & 402 & 8,0 & 11,0 & 280 & 5,6 & 13 & 12,7 & 0,25 & 0,36 & 50 \\
\hline 55 & 452 & 8,2 & 10,0 & 345 & 6,3 & 15 & 14,4 & 0,26 & 0,34 & 55 \\
\hline 60 & 499 & 8,3 & 9,4 & 404 & 6,7 & 11 & 16,0 & 0,27 & 0,32 & 60 \\
\hline 65 & 535 & 8,3 & 7,2 & 441 & 6,8 & & 17,4 & 0,27 & 28 & 65 \\
\hline 70 & 568 & 8,1 & 6,6 & 478 & 6,8 & & 18,4 & 0,26 & 0,20 & 70 \\
\hline 75 & 601 & 8,0 & 6,6 & 511 & 6,8 & 6,6 & 19,3 & 0,26 & 0,18 & 75 \\
\hline 80 & 634 & 7,9 & 6,6 & 540 & 6,8 & & 20,2 & 0,25 & 0,18 & 80 \\
\hline 85 & 656 & 7,7 & 4,4 & 563 & 6,6 & 4,6 & 21,1 & 0,25 & 0,18 & 85 \\
\hline 90 & 676 & 7,5 & 4,0 & 582 & 6,5 & & 22,0 & 0,25 & 0,18 & 90 \\
\hline 95 & 693 & 7,3 & 3,4 & 597 & 6,3 & 3,0 & 22,9 & 0,24 & 0,18 & 95 \\
\hline 100 & 708 & 7,1 & 3,0 & 610 & 6,1 & 2,6 & 23,7 & 0,24 & 0,16 & 100 \\
\hline 105 & 723 & 6,9 & 3,0 & 623 & 5,9 & 0 & 24,5 & 0,23 & 0,16 & 105 \\
\hline 110 & 737 & 6,7 & 2,8 & 636 & 5,8 & 2, & 25,3 & 0,23 & 0,16 & 110 \\
\hline 115 & 751 & 6,5 & 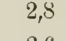 & 649 & 5,6 & 2,6 & 26,0 & 0,23 & 0,14 & 115 \\
\hline 120 & 764 & 6,4 & 2,6 & 662 & 5,5 & 2,6 & 26,7 & 0,22 & 0,14 & 120 \\
\hline
\end{tabular}




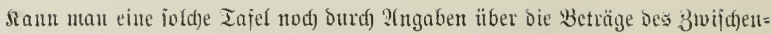

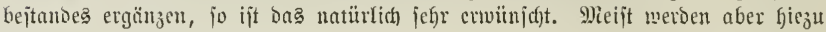
bie nïthigen Ilnterlagen fehlen.

Eollen bie Tajeln Gatptjächlich nur für bie Bejtandşbonititung biencn, bam fann wan fie nod) weit mefr bercinfad)en tno auf bie ?tngabe ber Mafjen bes

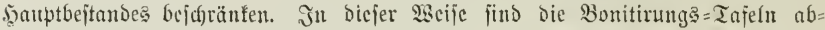
gejaj̧, weldac in Sadjen officiell angewendet werden. Tie bier z. 3 . füc dic Fi d te geltende ijt folgente:

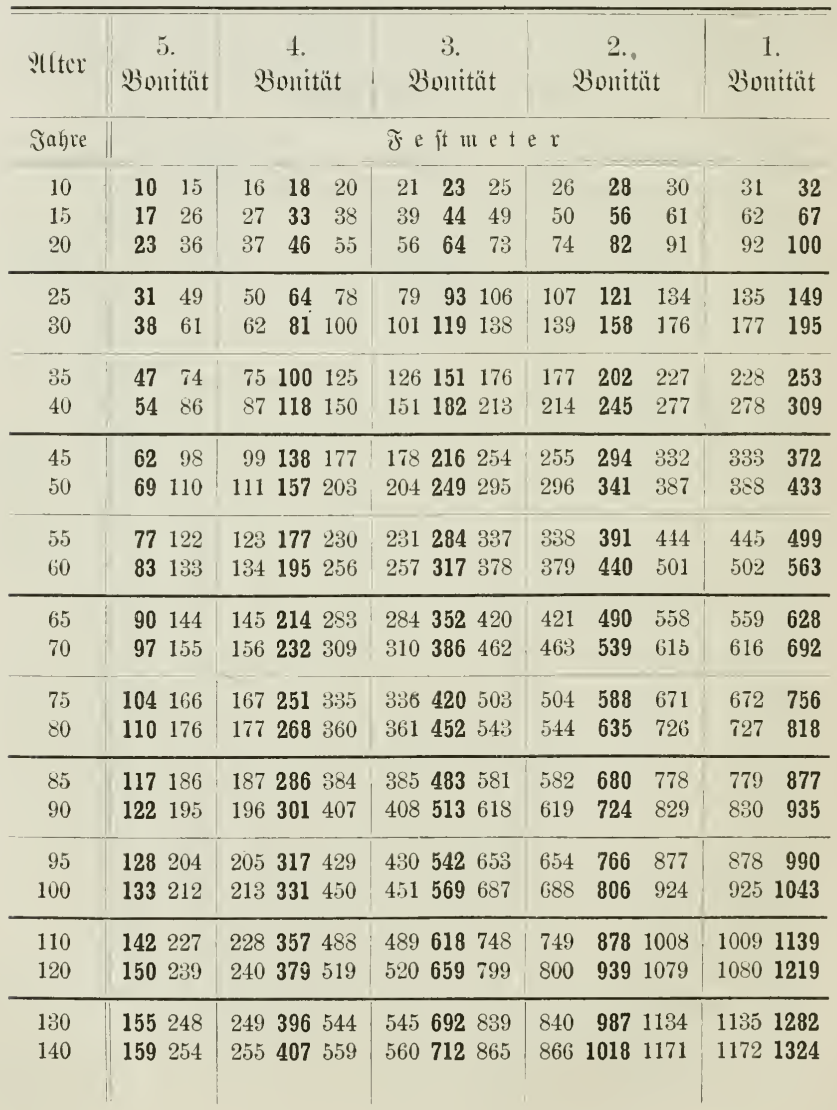




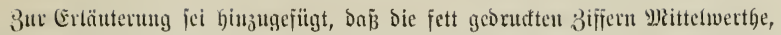

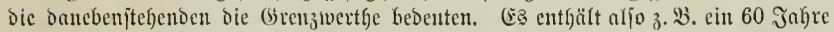

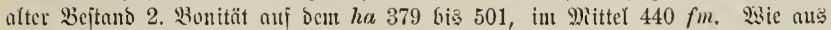

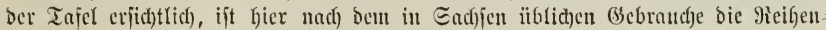

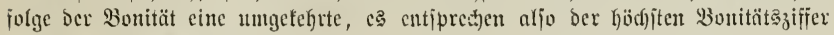
die nicorigiten Ertrïge.

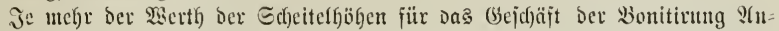

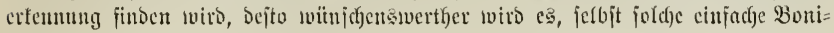

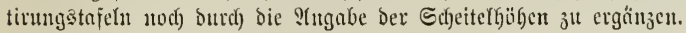

\section{$\$ 63$.}

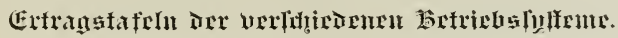

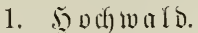

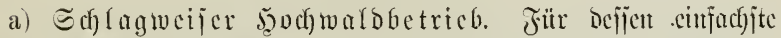

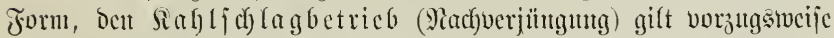

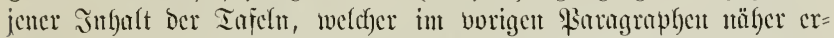

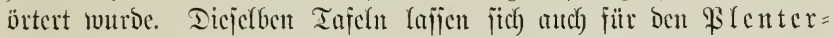
fdy lagbetrieb (Norverjü̈tgutg) entwerfen und anvenden. Der

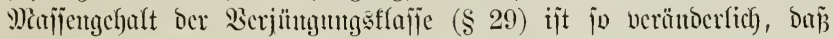

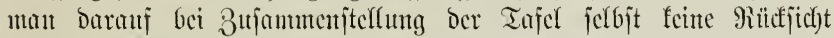

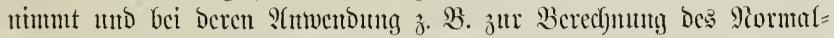
vorrathes, an bejten ben llmtricb gleich) jenem S(lter feb̧t, welches dic Bejtünoc in Der mittleren 3eit ber Berjüntyutgsonuer boben. (\$ 32. ๔. 112.)

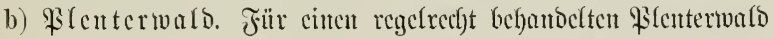

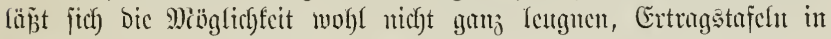

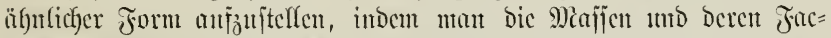

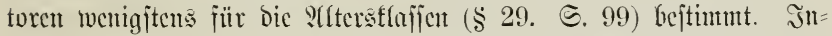

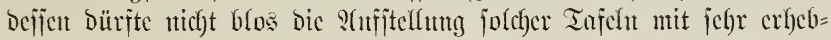
lichen Echwierigfeiten verfnïpft, jonbern aud Deren praftijher $23 \mathrm{erth}$ )

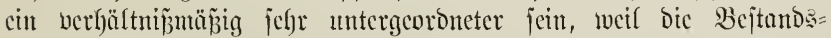
formen $\delta$ es Plenterwaldes zu wentig icharf charafterifitt find. -

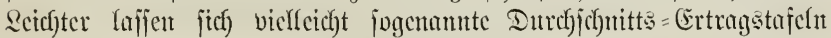

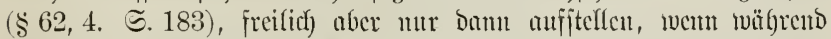

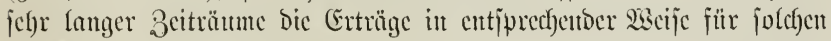
3wect gejanmelt toutrocu.

\section{Vitcderivald.}

Dic furjen llmtricbsjeiten Des 9iedermaldes madyen für bic Er: tragsregelutg Ertragstufelu numentlich Deswegen wentiger ntotfwentig, 


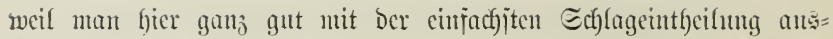

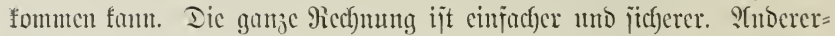
jeits erfeichtern es aber aucf bic furąen llmtriebs zeiten mejentlicf), brauchbare Iafeln nach wirflichen Şiebasergebnifjen ju entwerjen.

Dic S(ltersabjtujutg burf hier böchjtens cine jünjjäfrige jein, jefmjährige Beitrüume jimb für bie furzen llmtriebe bes Piedermaldes 3ll lang.

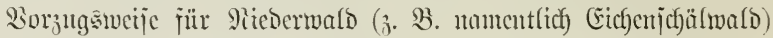
ijt es vou 2sichtigfeit und aud an leichtejten möglich), genïgende Eutalitütsjealen beizugeben.

\section{Mittelwald.}

Mefor in einer Ertragstajel geben zat wollen, als bie Erträge bes Ilnterboljes, ijt nicht gut möglich. - - Ier Miajjengehalt bes 5 ber=

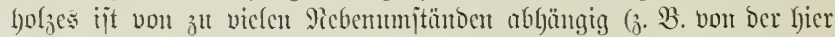

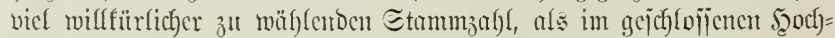

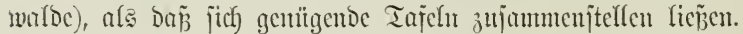

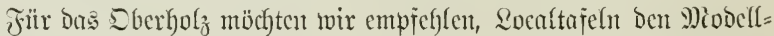

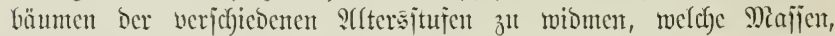

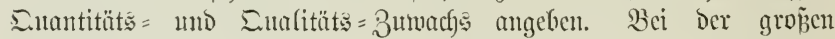
inbividuellen 2ierjefiedentfeit Der Sberitüntor Des Mittefwaldes

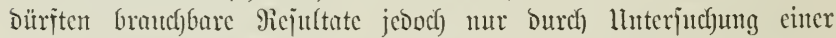

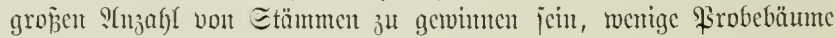
genügen nicht. *)

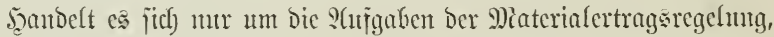
jo empjeflen jitch für Den Meittelwald, wie für bent \$ilentermald, Iutrdjichnittsertragstajelı.

\section{$\S 64$.}

\section{0) Luralertragstafelit.}

Der im \$62 angegebene Inljalt Der Ertragstajeln überfaupt fann jowohl für locale, als für allgemeine, normale Tafeln gelten.

*) 3u bergl. u. a. Qaupred)t: Borrathąermittelungen im Mittefmalde ou

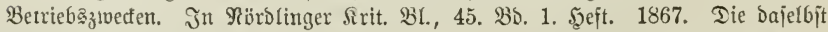

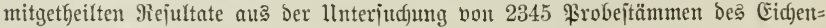

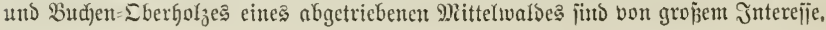




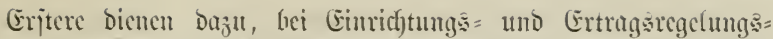

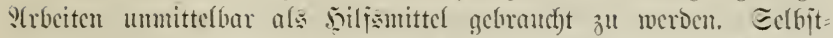

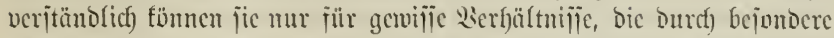
Etandortsbedingungen gegeben jimt, ?tnwentung finden. Eomeit bicje Bedingungen gleicf)artige ober unenigitens annäherno gleidfartige jüto,

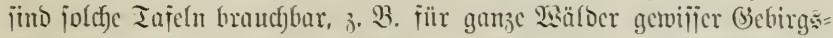

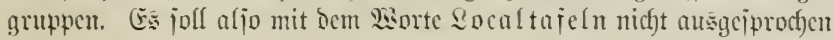

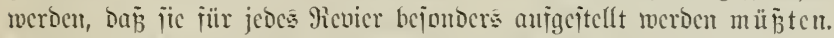

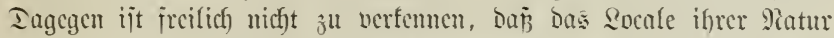

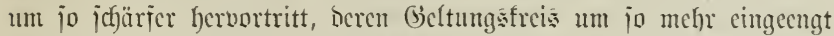
uvirb, je mefre wir bic von Den S(Gjaţverfältnifïen bebingten, f̈̈nan=

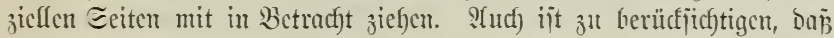

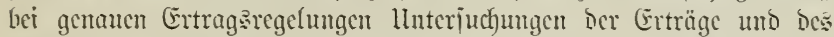

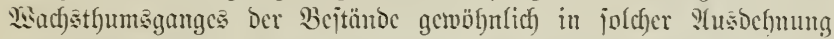

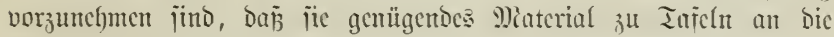

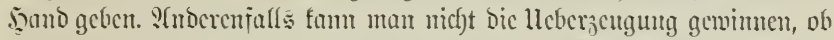

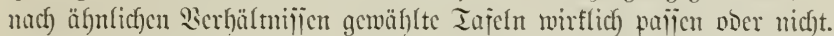

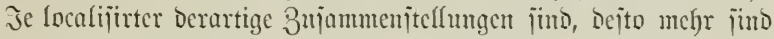

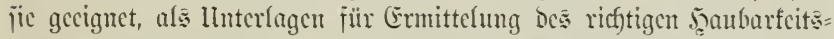

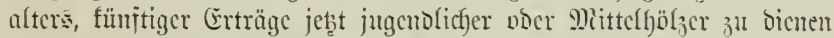

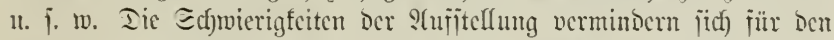

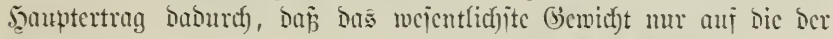

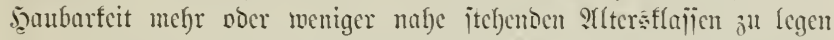

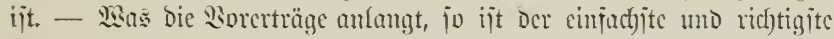
然eg für beren Ermittelung bic Bemubzung an Der Erfafrung u= mittelbar entnommener groß̄er Iurchjichnittšzaflen. Iicic gemäl)ren

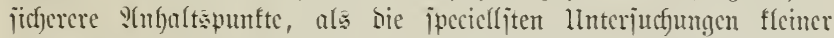

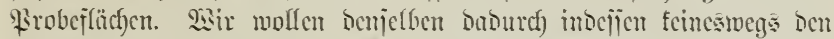

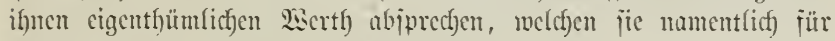

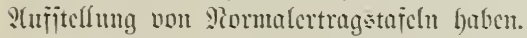

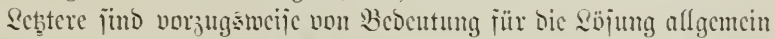

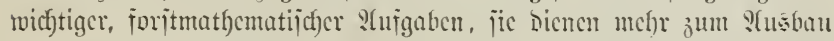
Der sisijenjchajt, als zur unmittef(baren praftijchen s(unendung. Segt man jolchen Iajeln nur Den 3wecf unter, als 2iorrathstajefn cinen

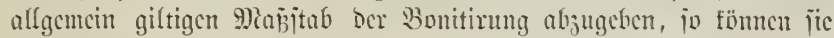

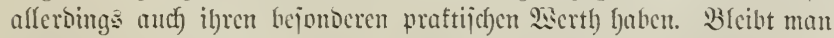

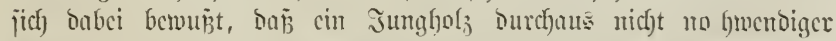

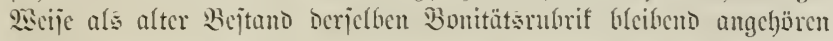

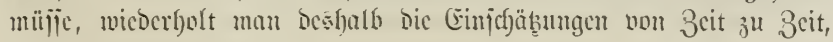




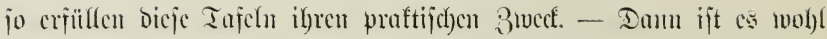

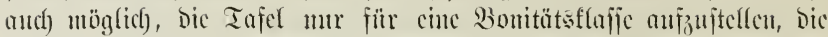

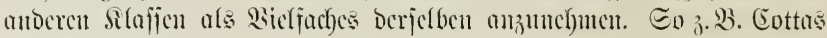
uno Siönigs Tafch.*)

Mit joldfen Ertragstafeln ijt es, wic (Eotta jeffr ricftig hervor hebt, wie mit Den siserfacugen ber Siüritter; wer cingeübt ift mo fic

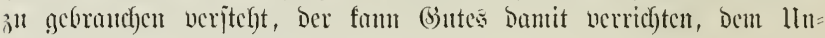
fumbigen nübęen fie nicht)

Eine bejombere 9let 9ormalertragstafel ijt bie bon (brebe**) nit

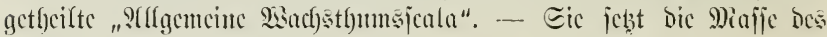

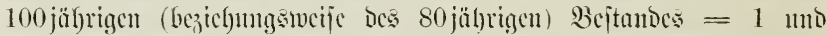
Drintfft bie alfer jüngeren und äfteren Bejtünoc in Decimalen ans.

\section{$\$ 65$.}

\section{beltimmแm}

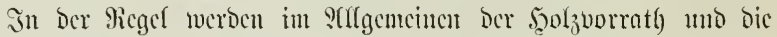

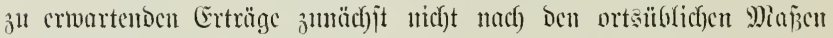
Der ueridbicbenen Eortintente, fonocm in Fejtmetern anzugeben fein,

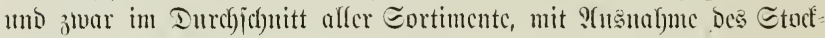

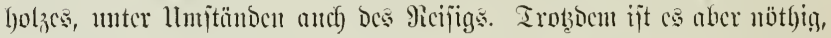

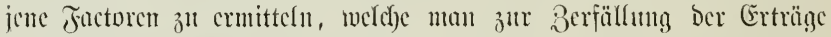

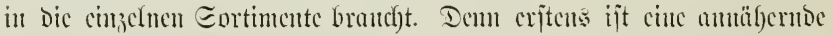

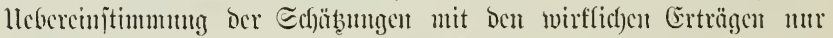

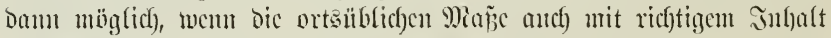

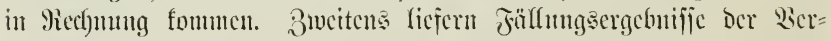

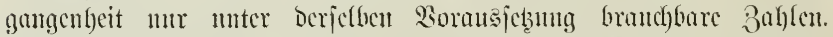

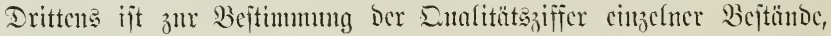
fowic ber ganzer Bejtandesgrnppen bicje Berfä̈llung unentbef)rlich).

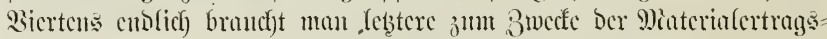

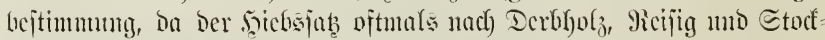

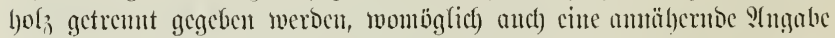

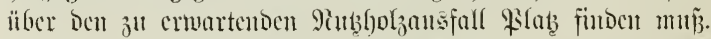

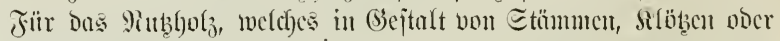

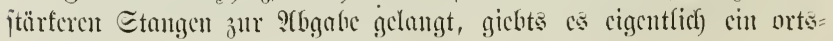

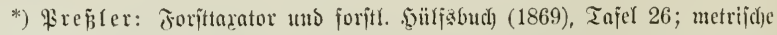
Y(uilage (1874), Tafel 28.

**) (5) rebe: Betriebs = uno Ertragstrgelung. 1867. S. 67. - 2. 9(ufilage

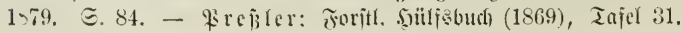




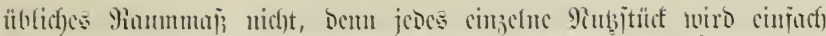

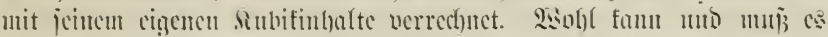

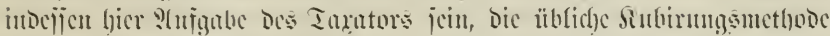

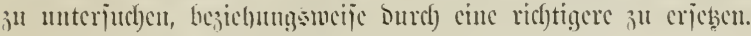

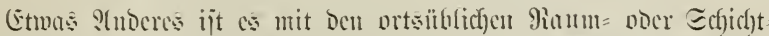

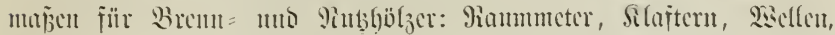

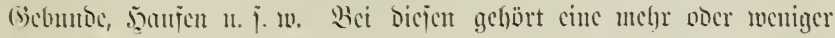

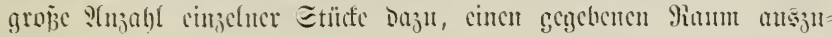

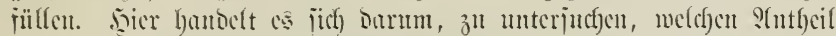

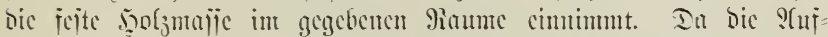

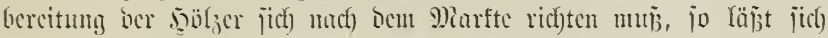

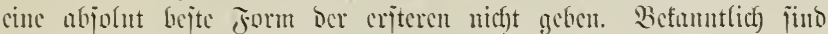

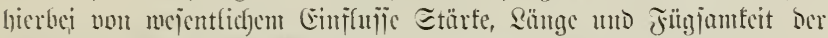

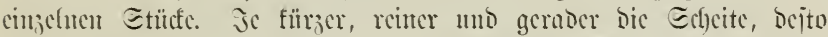

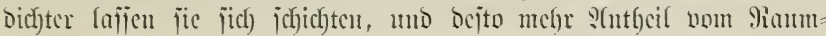

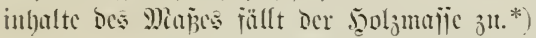

In georbneten Forjthanshalten mird man in Ser Regel bic nij=

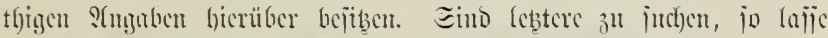

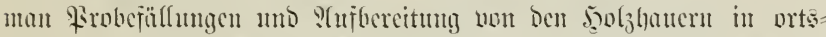

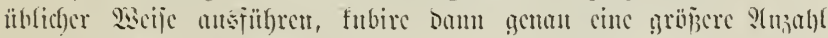
ocr werichicocuen ?imumaje. $\left.{ }^{* *}\right)$

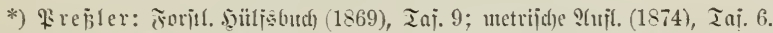

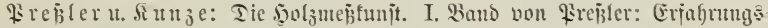

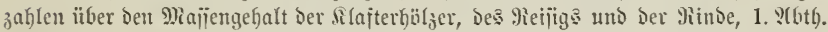

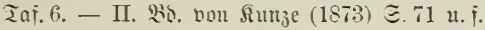

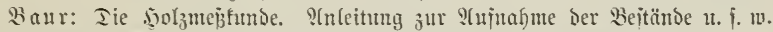

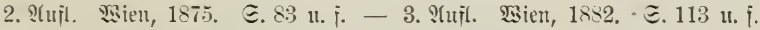

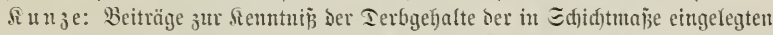
Solziortimente. Thar. Jahrbuth, 26. BD. (1876) ङ. 243 u. f.

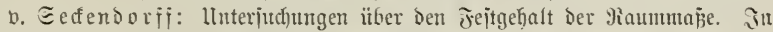

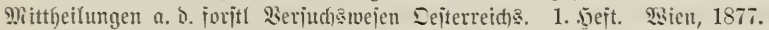

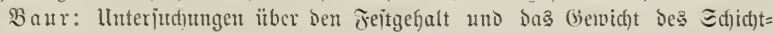

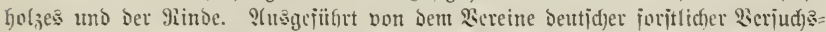

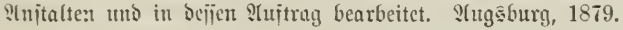

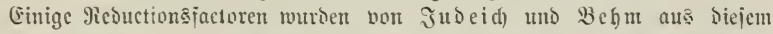
Durd) gewijienfajtejte llnterjuchung jef̣r reidhen Miateriales ausgezeidguetcu sierte fïr ben Jorit= uns Jagofalender, I. TGeil, entuommen.

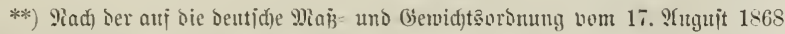

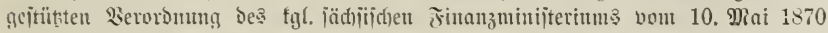
joll angenommen werden: 
Dubei ift Durchans nicht ausgejdyloffen, gelegentlict) bicjer

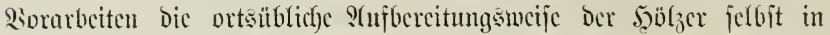

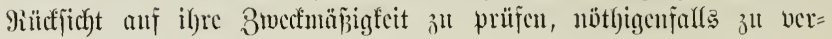
bcjferil.

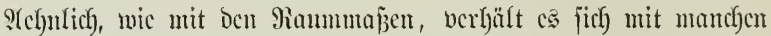

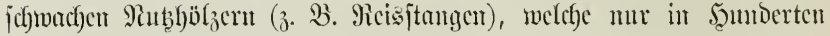

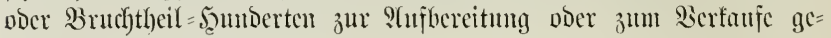
fantycit.

\section{B. ßeftandsbeffireifung. *)}

$\S 66$.

\section{Defriebrarf.}

Bei ben Borarbeiten hambelt es jich nicht Darum, zu bejtimmen,

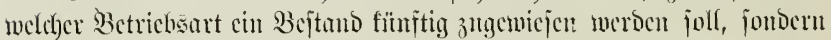
welcher er jeb̧t angehört.

Bu unterjed)cioen jind folgendo Betriebarten:

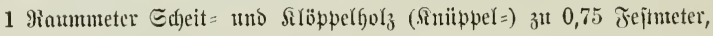

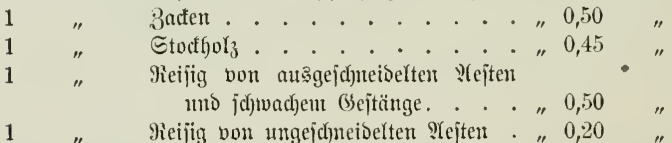

3u vergl. Tharander Jafrbud), 20. 23ธ. ङ. 236 u. f. - Wellenfunderte uno Sanghaujen werben in Eadjen in verjdjiebenen Dimenjionen aufbercitet, baher audf mit berjdjiedenem Jnthalte bered)nt. 3u vergl. Thar. Jafrbud), 22. 23D. ङ. 80 it. i.

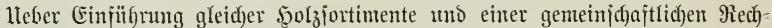

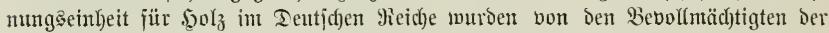
Regierungen von \$rtupen, Banern, Sïutemberg, Sadjen, Baben uns Eadjen= Bsotha am 23. T(ugujt 1875 Rejtimmungen vereinbart. Diejelben traten fïr die Etaatsporjtvermaltungen in \$reupen ourd) Beroronung vom 1. Setober 1875, mit geringet S(bueidjungen ober Ergänzungen in Eadjjen Durd) Beroromung bout 28. Decbr. 1875, in Sïrttemberg Durd) Beroromung bom 8. Eetbr. 1875, in Baben

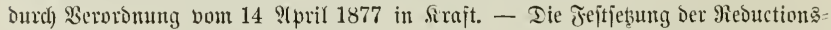

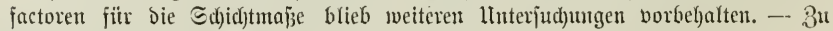

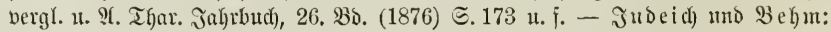
For $\tilde{t}=$ แก๊ Jagotalender, I. Theil.

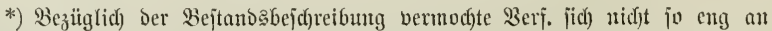

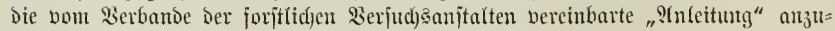

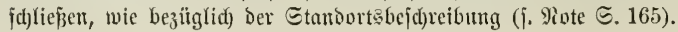




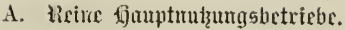

\section{I. Şodjwalbbetrieb. (Eanculoljbetrieb.)}

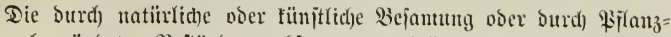

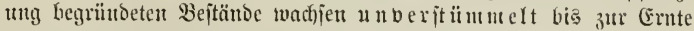
und weroen in gleicfyer Weije mieber berjüngt.

1. Rlenterbetrieh. (Jemelbetrich.)*)

Die jührlidhen Füllungen eritreden jid) über eine ganze Betriebs: flajie oser über grö̈̈ere Theile Derjefben derartig, baj man bie äfteren und jtärferen, fo wie bie jchashajten @täume bereingelt, Gorit weije ober itreifenweife anshaut ("autsplentert", "ausjemelt"), bie

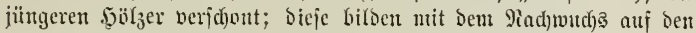
Etandräunt ber gefüllten Bäume fehr ungleichalterige Bejtände,

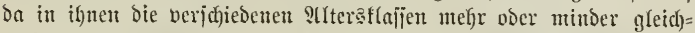

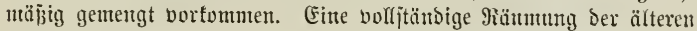
J̧ülzer erfolgt nie (i. ङ. 102).

2. Edylagweijer f̧ochtwalobetrieb.

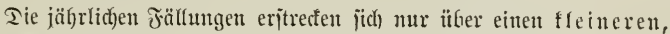

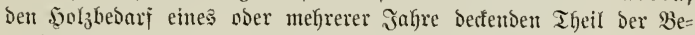
triebatlajie, uno wirb auf biejem Theil ein möglidj): gleidhalterige: Bejtand nad)gežngen.

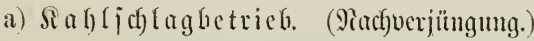

Die zu einer Jafresernte bentöthigte Edhfagfläd)e wirb anf eimmal rein abgefolzt. Die fïmiftlidje ober natïrlidge $23 e r=$

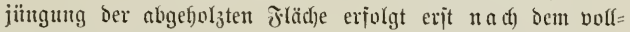
itünbigen ?totriebe.

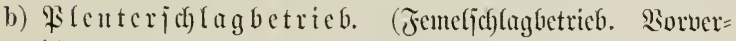
jïugumg. *)

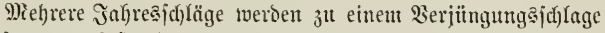

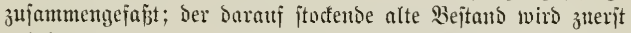
gefichtet und ban aflmälig abgetrieben Die fünjtliche voer natïrliche Serjüngung erfolgt nad) ber Sidjtung, aljo nod)

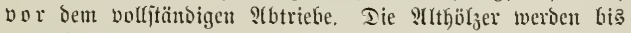

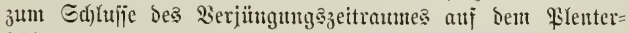
jơlage bollitänoig geräumt.

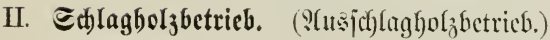

Es erfolgt cine periodijde צumung Der Edjäjte, Edyaftheile ober

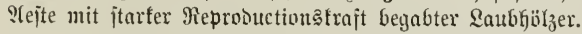

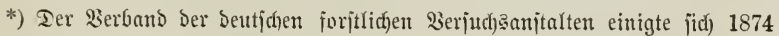

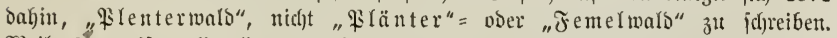

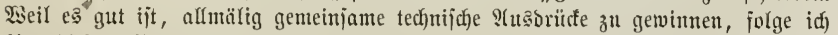

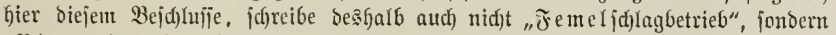
"Filenteriflaghetrieb".

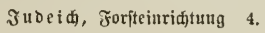


1. Niebcrualdbetrieb. (Etocfidglagbetricb.)

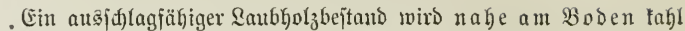

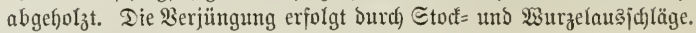

2. Siopflolzbetrieb.

Qaubgolźftämme werden in einer getwijien gä̆he ( bem Boben abgebauen ("geföpit"). Die Berjüngung erfolgt Durd)

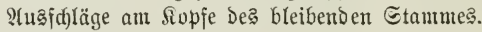

3. ङ(f)neibclholzbetrieb.

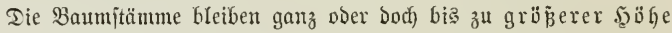

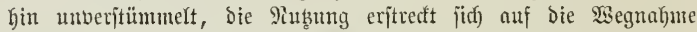

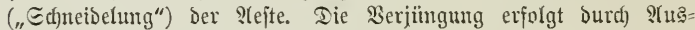

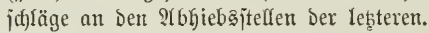

\section{Bujammengejef̧te (Compojitions $\Rightarrow$ ) Betriebe.}

1. Mittelwalobetrieb.

Berbindung Des Şodfwald = mit Dem Miebcrwalsbetrieb auf einer

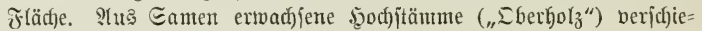

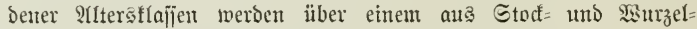

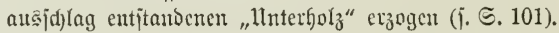

2. Qichtungabetrieb.

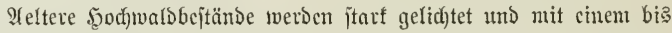

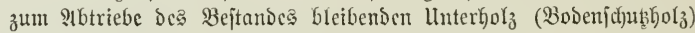

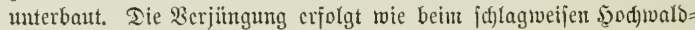
betrieb.

3. 5ुochmaldonjervationsbetrieb (J̧artig's Betrieb).

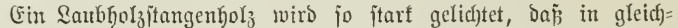
mäpiger Bertheilung nur jo biel Etangen jteben bleiben, als genïgen, um cinjt einen Eamenjollag jteflen ju fömen. Der bon Den Etö̈fen

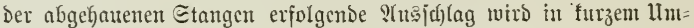
tricbe fo lange a( beim Pilenterfd)lagbetrieb.

B. fjaupt- แnd tlebemuntzunghbetriebe.

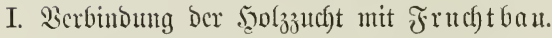

1. 5ุa

In einem Niebcrwalbe wirb unuttelbar nad) bejen jebesmmaligeut

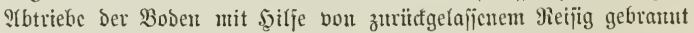
("gefaint"), Dan 1 bis 2 Jahre lang (betreibe jwifhen Den neuen Etoffloben gebaut.

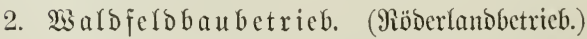

In einem fyod)walde wiro nad) Dem jedeşntaligen ?(btriebe bie

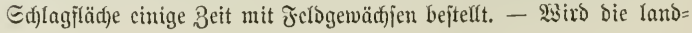




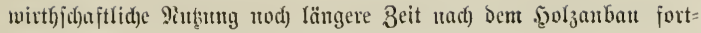

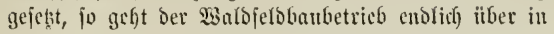

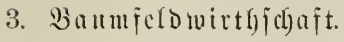

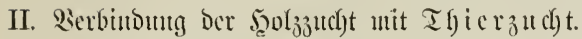

1. 2ialdweidebetrieb.

2. Thiergartenbetrieb.

ซitr bieje beiben Betriebe lajjen fich jehr berjhiebente Formen benfen; auf bie Forjteintrichtung nelgmen jie beseutenden (Einflaßj.

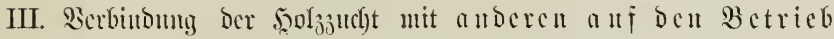
wejeutlidg (E)

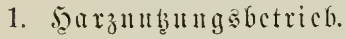

Sichtig 3. 23. fïr P. austriaca in Defterreich; P. maritima in Frantreidh, Epanien 2c.; socuiger jeb̨t nodh) für bie Fidate.

2. Etreutualobetricb.

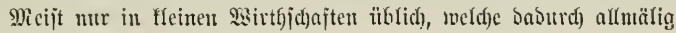
rutinit tuerber.

\section{$\$ 67$. \\ Lु $\mathfrak{x} \mathfrak{x} \mathfrak{a} \mathfrak{x} \mathfrak{t}$.}

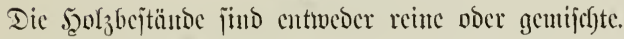

Fïr bic reinen Bejtände genügt bic cinfadfe Satgabe ber jic bilbenden \$̧olzarten.

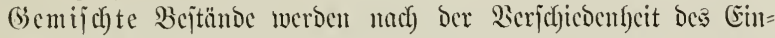

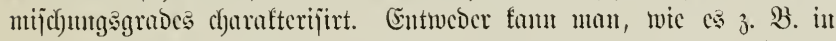

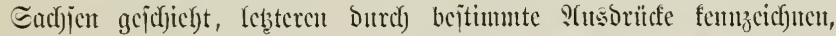

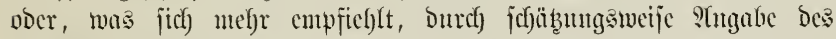

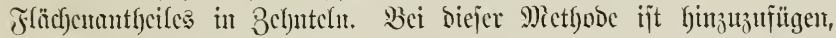

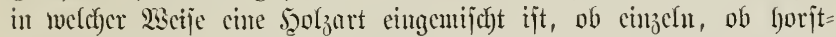
ober jtreifenweije. Dic Şanptgolzart ment man unter allen IInịtän= Den zuterit.

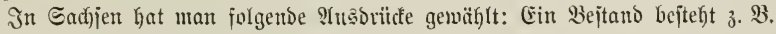
aus Fidjtelt ullo Tamnen, jo jagt man:

a) In Bezutg auf bie Mienge ber heigemifaten syolzart:

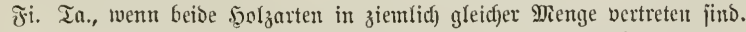

Fi. und Ta., wenn bie Jid)te Der Etanmzafl nad) etwas ïberwiegt.

Fi. mit Ta., meun bie Taunen in geringer Menge vertreten finto.

Şi. einige Ta., went nur wenige Tamten vorfandent.

b) Эn bezug auf bie bertheilung ber beigemifdeten bolzart:

Ji. einzelne Ta., getinge $3 \mathfrak{a b l}$ und zerftreuter Stand ber Tamen.

Ji. truppweife (horitweife) Ta., wem bie Tamen in flemeren ģorjten rein borfoumen. 
Ta bezüglidy biejer 2 usbrücfe eine jdarje (brenze nidgt gegeben ijt, jo er,

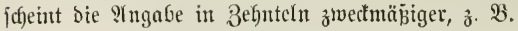

$$
\begin{aligned}
& \text { Etatt テi. Ta. } 0,5 \text { Ji., } 0,5 \text { Ta. }
\end{aligned}
$$

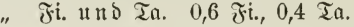

$$
\begin{aligned}
& \text { " Ji. mit Ta. } 0,7 \text { bis } 0,8 \text { テi., } 0,3 \text { biล } 0,2 \text { Ta. } \\
& \text { " Ji. einige Ta. über } 0,8 \text { Ji., unter } 0,2 \text { Ta. }
\end{aligned}
$$

Eebr geringe Ëinutiofungen f̈̈nnen entweber unberïdjichtigt bleiben ober,

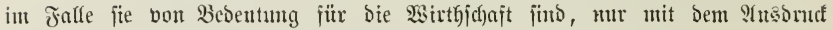
"einige" bezeichntet, ja unter limjtünden der Єtammzahl nach angegeben werber.

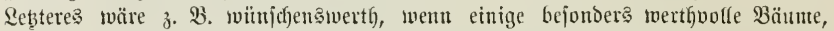
alte Eichen, ïbergebaltene Sisalored)ter unb Dergleichen vorfoumen.

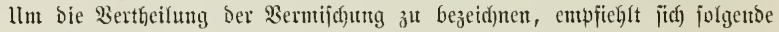
Form: 0,5 Эi., 0,3 Ta. einzeln uno truppweis; 0,2 Bu. einzeln.

(3" vergl. § 100, Daร Taçatiousmantul.)

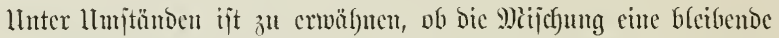

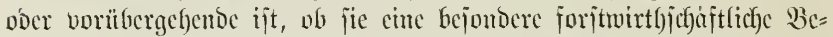

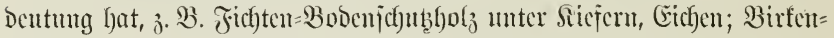

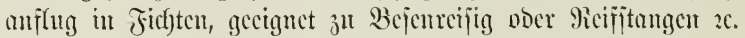

\section{$\$ 68$.}

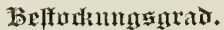

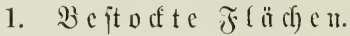

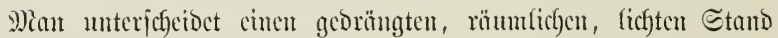

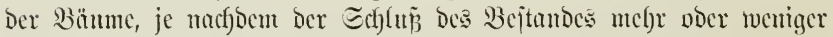

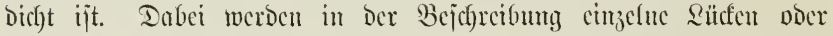

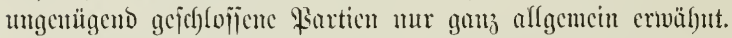

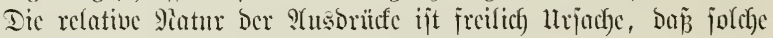

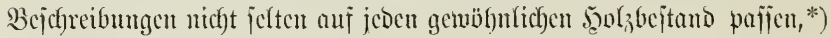
Dafer cin richtiges, flares Bild uicht gebent.

Mehr empfichlt co fich), Den Bejtodungsgrad in Bruchtycilen ber gleid) 1 gejebzten 3orfbejtofung amjuprect)en. Sebtere ijt jwar eben=

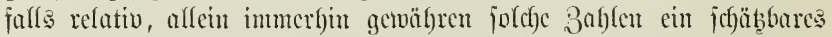

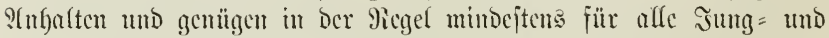

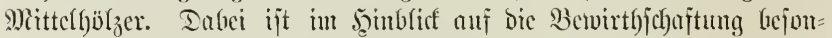

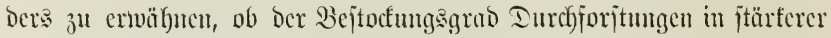
oder fatymächerer 2 secije nöthig ober möglich madyt.

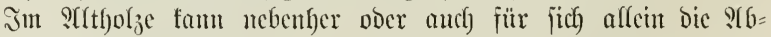

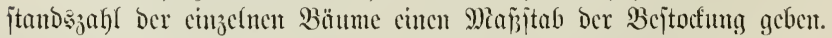

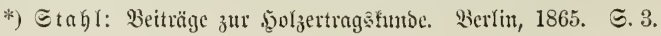




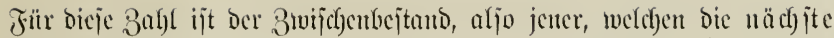

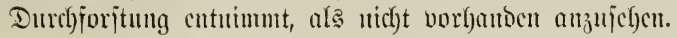

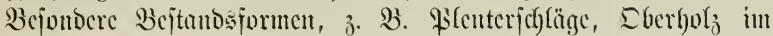

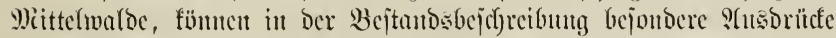
nothucndig machen.

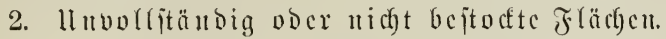

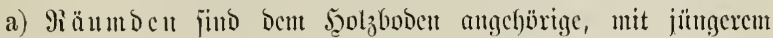

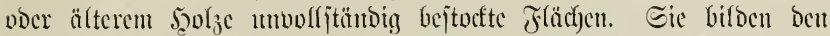

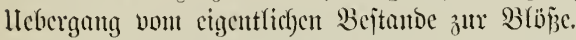

Bn untericheiden Gleibende und vorübergefende Räumben.

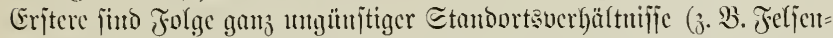

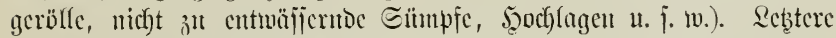

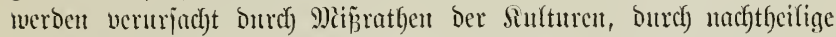

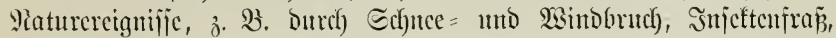
Feuter u.j. to.

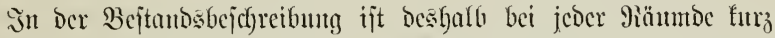

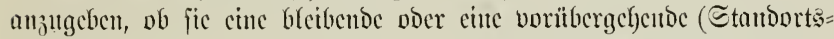

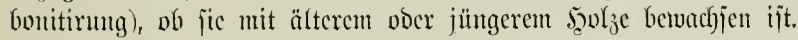

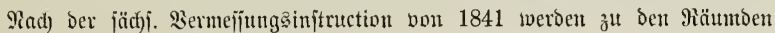
gezäglt:

a) "Diejenigen Drte, auj benen วิwar jüngeres, nicht über 40 Jafre altes,

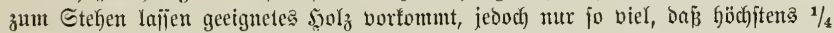

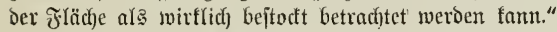

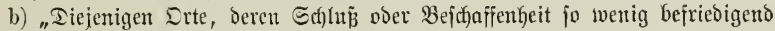

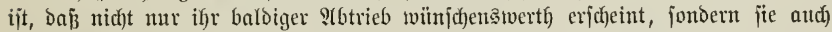

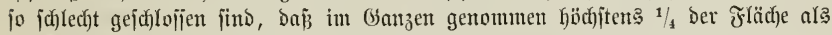
svirflich bejtofft betradjtet werben fann."

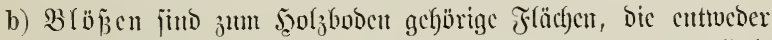

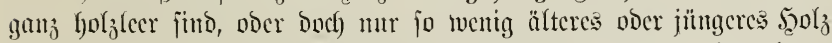

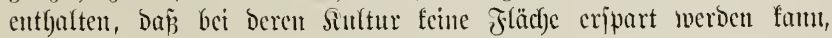
jontorn ein vollitündiger Peutanbau erfolgen un

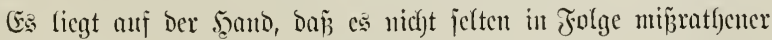

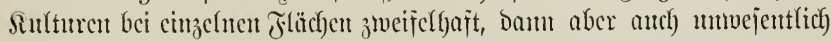

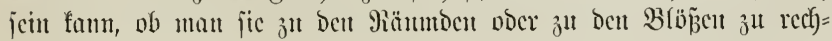
telt babe.

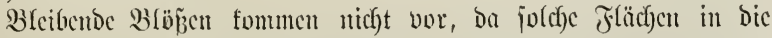

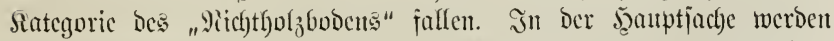

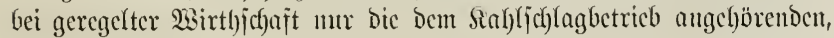

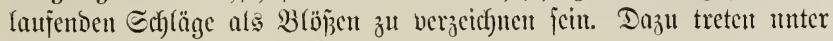




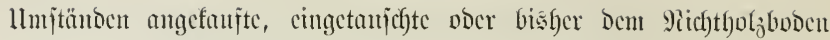

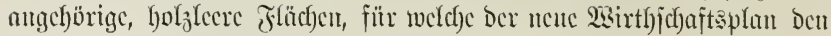
5ovlzanbau vorjadreibt.

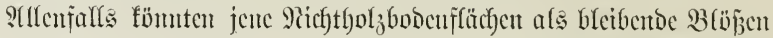

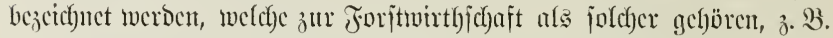

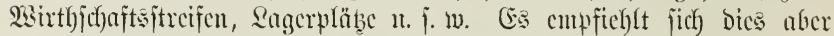

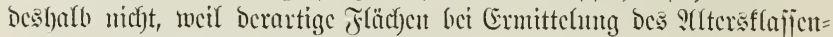

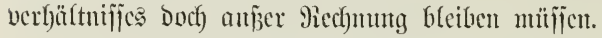

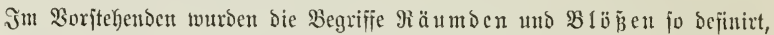

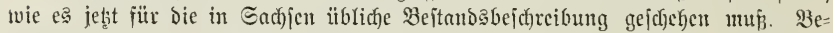

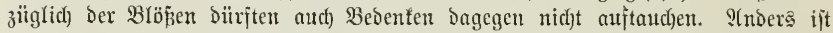

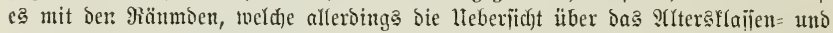

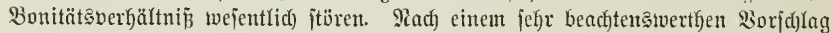

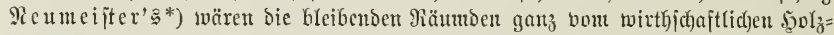

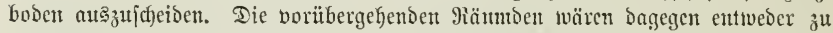

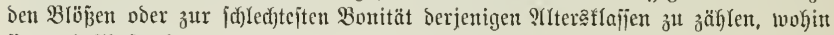

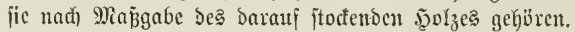

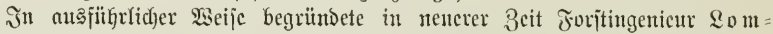

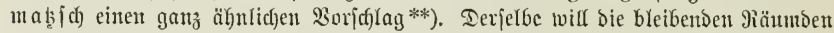

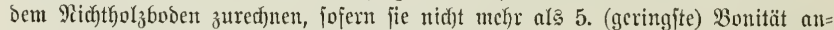
gejprodyen weroen fönten. Sit leșteres ber sall, fo werben fie bicjer Boutität jelbjtberitändlich) zugejd)rieben. Die vorüb rgehenoen giäumben wären entwveber

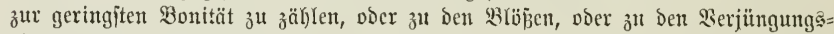

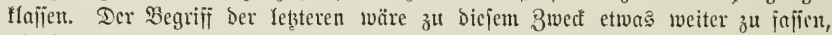
afs bisher gejdehen; man wïrbe unter Serjüngungeflajïen alle Drte jut veritehen Gaben, in welchen eine Entuthme von foldher Bebentung ftattgejunden hat, Daj

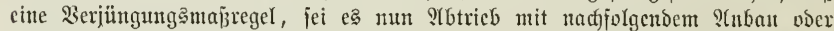

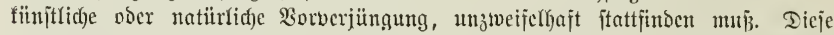
Entnahme fan entweder cine abjichtliche, freiwillige, ober eine mabjidftliche, burd) Brudd), Jnjeften, Jeuce voer Dergleid)en bebingte fein, gleidjoiel.

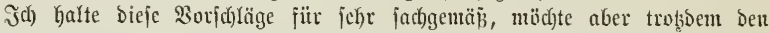
Begriff Räumben beibehalten, unt zivar nur für Die bleibenton Räumben, bicje

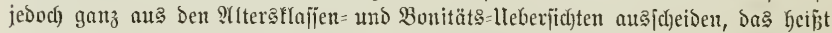

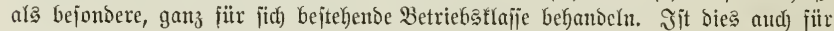

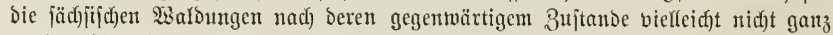

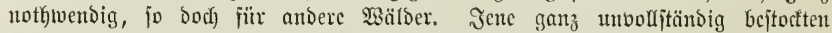

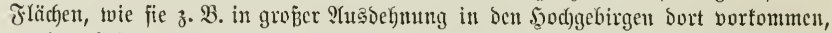
swo Der Utebergang won Der $\mathfrak{B}$ ald region zum walslojen Gébiet jtattfindet, werben

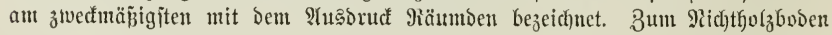

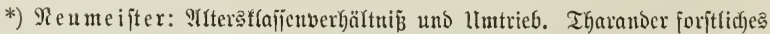

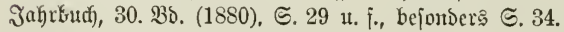

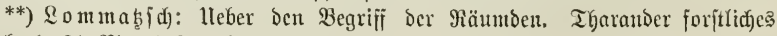
Jafhrbudy, 31. Bb. (1881), ธ. 222 u. f. 
fann man iie beşbalb nidgt rechnen, weil ite inmerbin noch cinigen govgertrag ge=

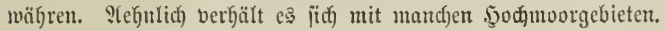

\section{s. 69.}

\section{Belfantialfex.}

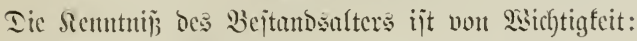

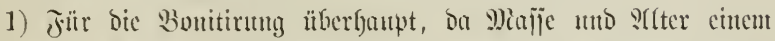
Bejtande bie Etelle in Der als Majijtab gewäblten Erjalyrungstafel antucijen.

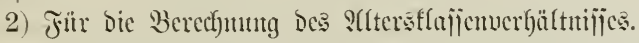

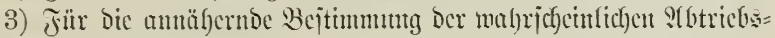
jeit cintes Bejtantoes.

Eine matfematfijcf) gentute S(ltersermittelutity ijt unt für jente

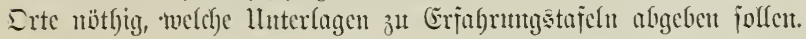

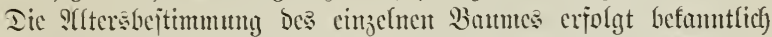

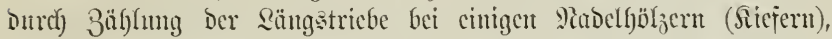
ober Der Jafresringe. Rebteres gejefielft ant bejten, wenn maut ben

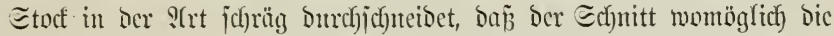

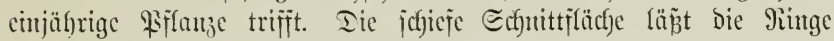

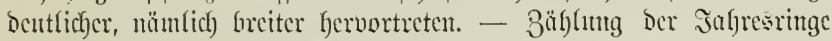

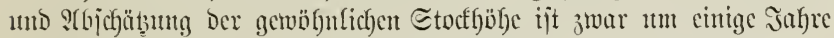
utjïcler, genïgt jedoch in Den meijten Fälleu.

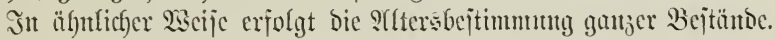

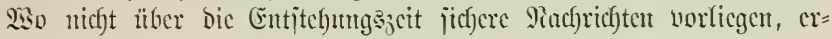
mittelt man bas s(lter an Probejtïmmen.

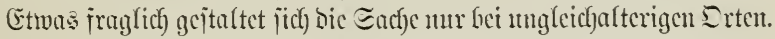

Bei Bejtünoen bcs ichlagmeijen Jondfwaldbetriebes genïgt cs,

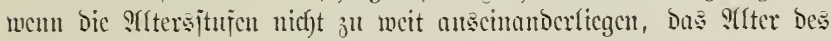
nad) Der Sircisfläd)e berechucten, aritbmetijefen Dittelitammes aus bem jounthejtande als Bejtundsalter antumefmen. Eind bic sflers: bifferenzen bedentend, io mitfien beren Grenzen in ber Bejefreibung antgegeben werden, unt ijt ber Bejtanto jener S(lftersflafje zuzutucijen,

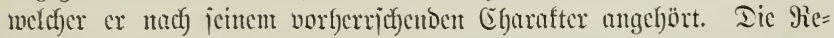

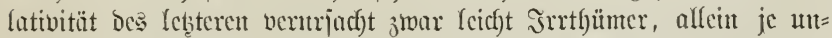
jicferer bie Bejtimmung an jitf) ijt, bejto weniger fonmt audf barmif

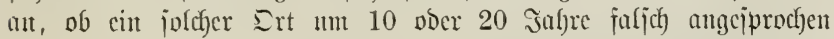
wiro. Eind cinzelte ältere ober jüngere fiorjte cingejprengt, weldje

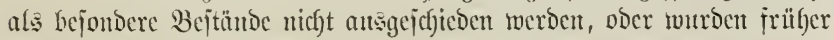

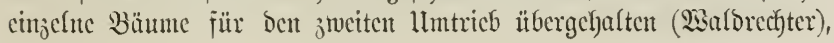


jo Gat man bics in Der Bcjchreibung entjprechend ju crmäfncu. Das

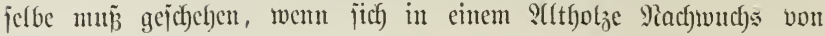

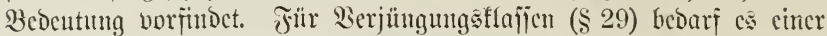

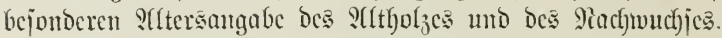

Dic Berectumng cincs mittleren Bejtandsalters, ober

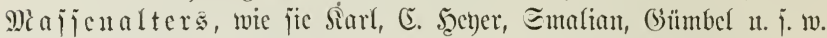

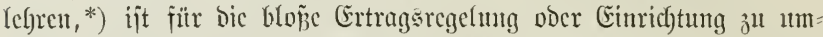

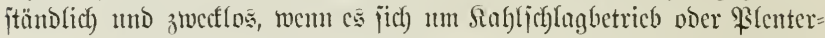

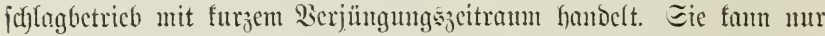
Dann nüthig werden, menn man entweder cinen Fienterichlagbetrich mit

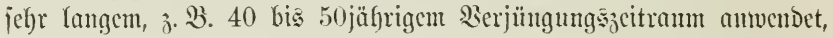

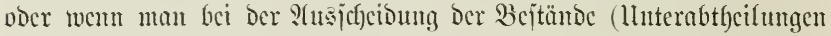

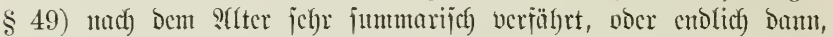

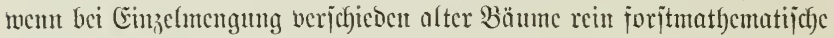
Tufgaben gelöft werben jollent.

Ias "Maffenalter" (wahres Mittelalter) eines ungleichalterigen Beitanoes

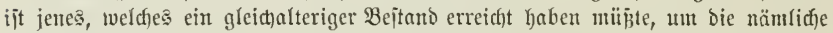

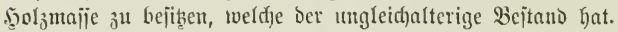

Die Ermittelung des Majientalters fam erfolgen:

\section{1) Mit bilie von Extragatafeln.}

Bejîț man eine bem jraglidjen, ungleidjalterigen Bejtande bejüglidg Der Etandortšverhältnijie entipred)ende Ertragştajel, fo Gat man nur bie präbominirende

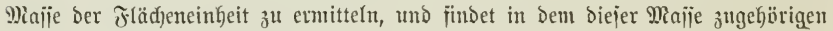
QIter ber Injel bas gejuchte Miajienalter.

Ein ungleidfalteriger, 10 ha großjer 2Bejtano enthalte $6000 \mathrm{fm}$, jo wäre et nad) unjerer Tnjel (ङ.28) im Mittel 95jäbrig.

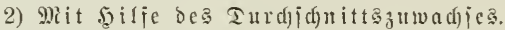

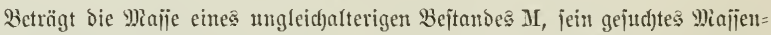
alter $\mathrm{A}$, jo ift Der Iurdjid)nitts̊zunad) $\mathrm{Z}=\frac{\mathrm{M}}{\mathrm{A}^{-}}$; hierans $\mathrm{A}=\frac{\mathrm{M}}{\mathrm{Z}}$.

Ier Bejtano enthalte heijpiclsmeife brei zu unterifeidende 9Itersijufen mit

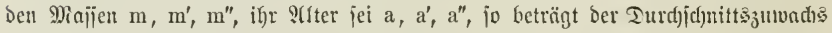

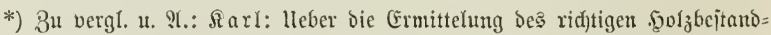

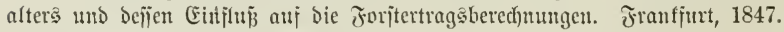

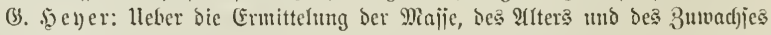
Det §olổcjtände. Teïan, 1852.

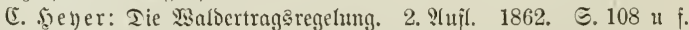

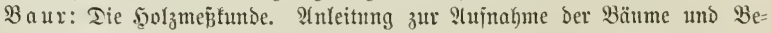

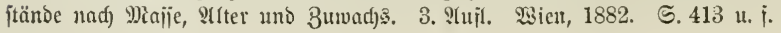


ieber cirjelnen $\frac{m}{a}, \frac{m^{\prime}}{a^{\prime}}, \frac{m^{\prime \prime}}{a^{\prime \prime}}=z, z^{\prime}, z^{\prime \prime}$. Tic Sunme $Z=z+z^{\prime}+z^{\prime \prime}$; Dic Summe $\mathrm{M}=\mathrm{m}+\mathrm{m}^{\prime}+\mathrm{m}^{\prime \prime}$; hiemad) $\mathrm{A}=\frac{\mathrm{m}+\mathrm{m}^{\prime}+\mathrm{m}^{\prime \prime}}{\mathrm{z}+\mathrm{z}^{\prime}+\mathrm{z}^{\prime \prime}}$.

Beifpiel. Ein Bejtand enthalte 2 SItersitujen, eine 80 jäfrige mit $2500 \mathrm{fm}$, eine 60 jǘrige mit $700 \mathrm{fm}$.

$$
A=\frac{2500+700}{\frac{2500}{80}+\frac{700}{60}}=74,4 \text { Эafre. }
$$

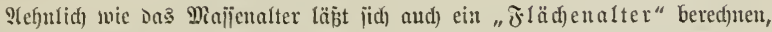

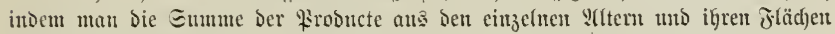
Durd) bic Flächenjumme bivibirt.

Beifpiel. Gin Bejtano bejtehe aus 2 ha $60 \mathrm{j}$., 3 ha $80 \mathrm{j}$., 4 ha 90 jährigem $\mathfrak{g o l} \mathfrak{l}_{\mathfrak{j}}$, jo ijt jein Flächenalter

$$
\frac{2 \cdot 60+3 \cdot 80+4 \cdot 90}{2+3+4}=80 \text { Эahre. }
$$

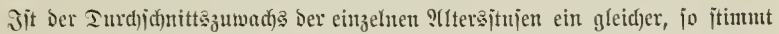

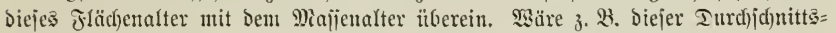
juwad) jiir $1 \mathrm{ha}=7$, jo betrïge Das Miajienalter $\frac{840+1680+2520}{14+21+28}=80$.

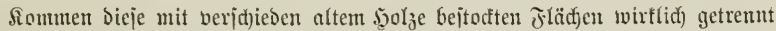
von cinander bor, fo verjährt man natïlich biel ridjtiger, memu man aus biejem einen bejtanbe 3 bilbet, wenigiten ben 60 jährigen bejonbers ansjd)eibet.

Im ungeregelten Pilenterwalo ijt bic Ermittelung eigentlicher Bejtunbsalter nicht möglich), ç genügt bie s(ngabe ber Grenjen Der

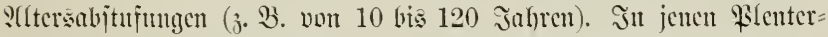

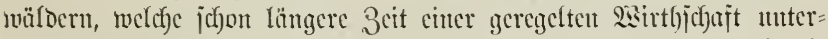
licyen, treten menigitens bic ?lltersflajicn jefärfer bervor, wie jie

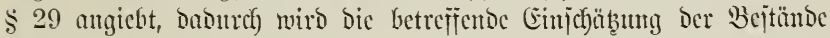
möglidf.

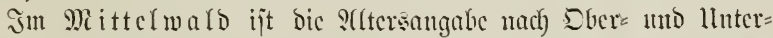

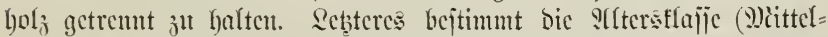

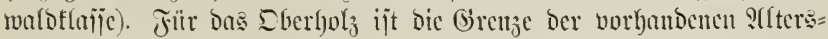
itufen anjugeben $(\$ 29,4)$.

:(m cinfachjten gejtaltet jitc) bic Frage beim 9iederwalde,

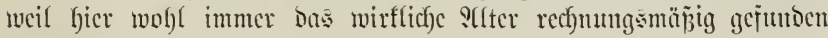
worben faum.

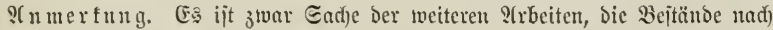

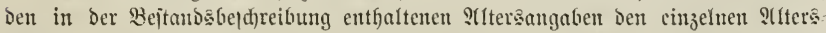
flaijen зuzufheilen; allein jebenfalls empïeglt es jid), bie Silajie ben einzelnen Beitänten im Mianuale jojort beizulifreiben. 


\section{$\S 70$.

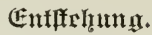

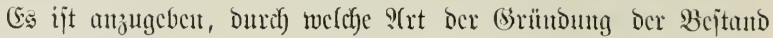

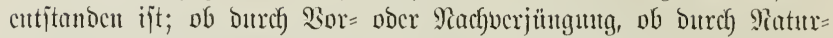

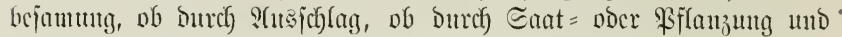

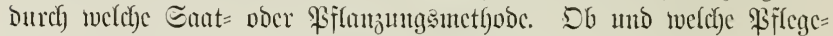
mâjregchn ober lluglücfs̈älfe Den Bcjtund getroffen babent.

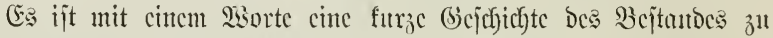
geben, joweit zuverläjïge ?ntgaben zu crlantgen fitto.

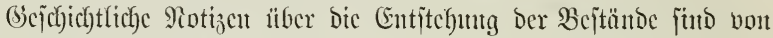

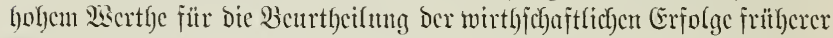

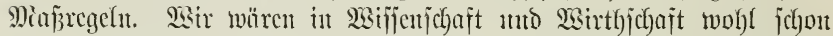

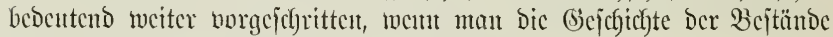

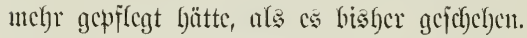

\section{$\S 71$.}

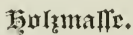

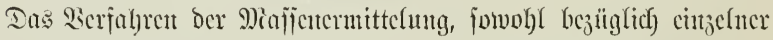

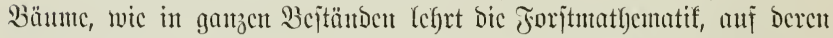

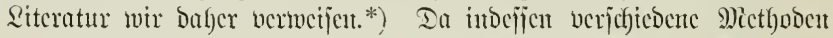

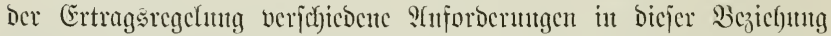

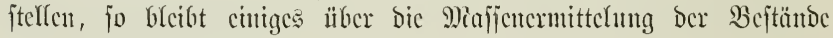

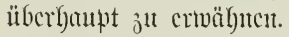

Fïr alle Ertragsecgefungsmetfyoden ift Der vorkantone Borratl) ciut wichtiger Factor. Dic größsere ober geringere Gsentunigfeit ber

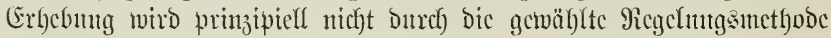

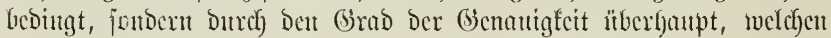

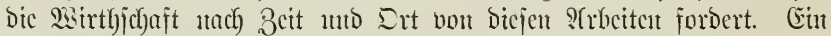

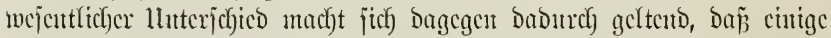

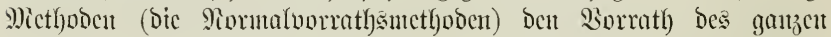

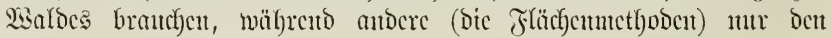

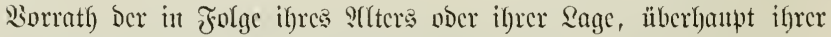

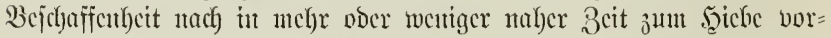

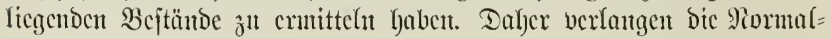

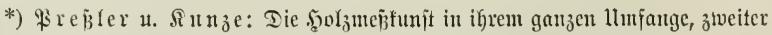

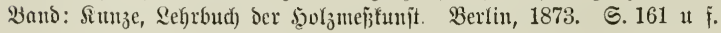

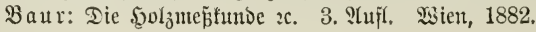




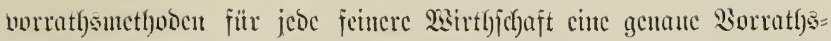

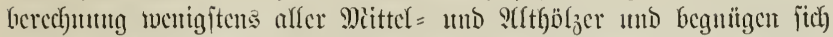

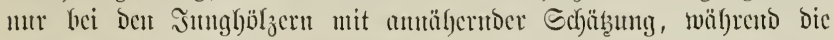
Flächermetfyoden weit geringere Shfordocrungen ftellen fömen. Fïr

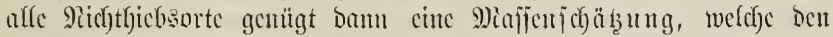
Bejtünocu ifrec Bonität zumeijt. Dabei ijt noch zu beachten, Daj

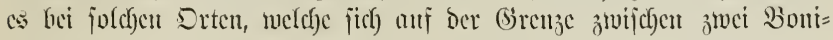

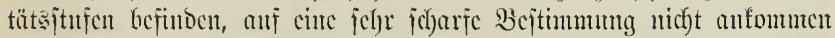

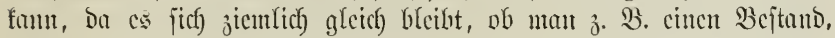

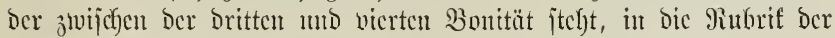
Dritten vocr in bic ber vierten jefreibt. Eefor ungleicfartige Bejtünde,

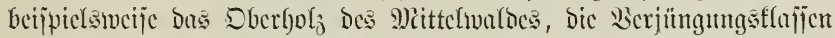

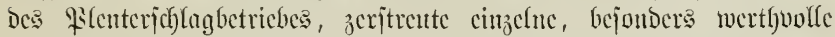

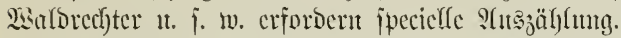

Soandelte cs fich bei ber Ertragsergefung mur Darum, ben an=

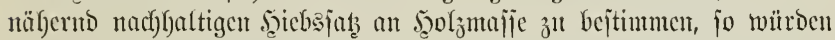

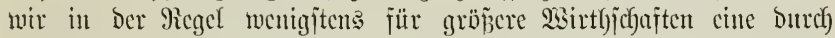

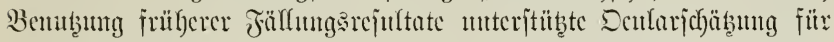

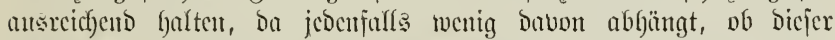

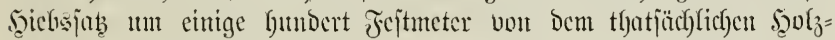
verifhlage bifferirt.

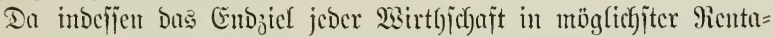
bilität gejuct)t, Da bicjes Biel aljo anch von ber Ertrageregelung im

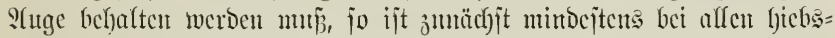

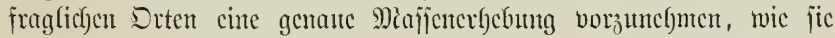
bic Berechnung bes ascijerprocentes für ben ganjen Bejtmo forbert.

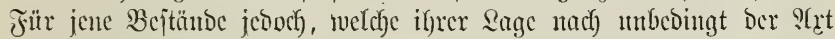

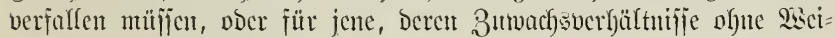

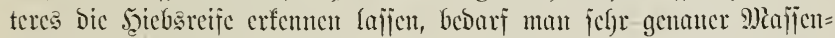
crutittefungen nicht.

Ferner erforden Dicjenigen Bejtünde ober Bejtandsproben genauc,

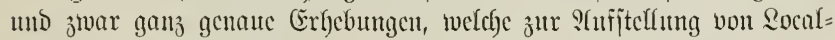

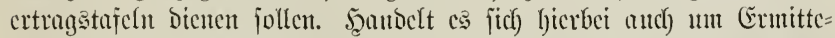
lung Der Sortimente, jo verbient bic Draubt jede Proportionalfälfungs=

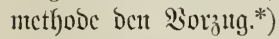

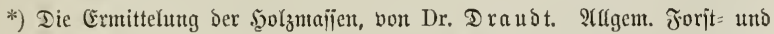

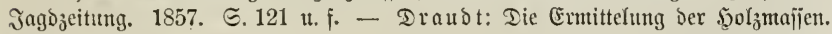

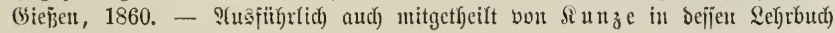
ธ. 191 น. $\mathrm{i}$. 
şalten wix aud zum alleinigen 3wecte ber Miaterialertrags=

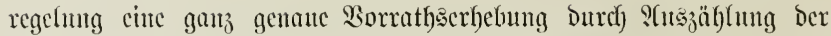

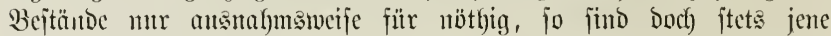
STbeiteu, welche als lluterlagen zu ben Rocalertragstafeln bicnen, আ jenc crwähnten genauten Ermittelungen in fraglicfen Şiebsosten von

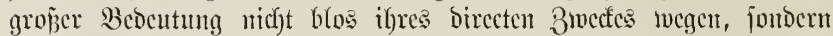

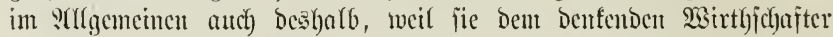

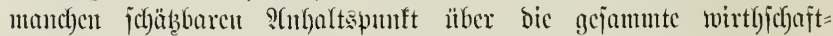
licke, D. b. funanjidfe Natur Des hetreffenden Socalforitbetriebes ge= wäloren.

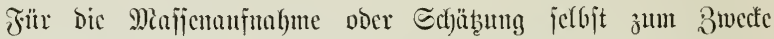

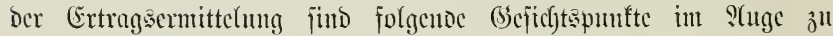
beljalten:

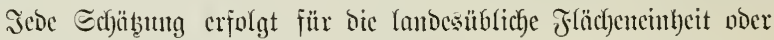
ijt anf bicje ju reduciren.

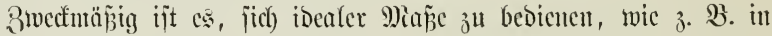

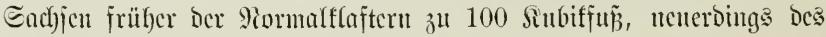

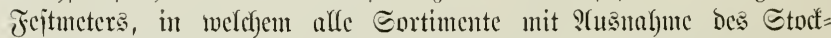

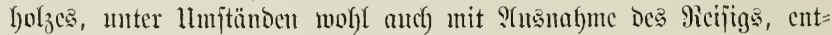

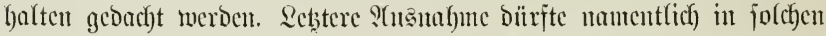

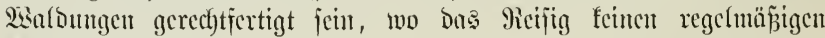
?lbjä fintoct.

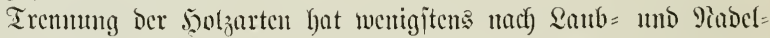

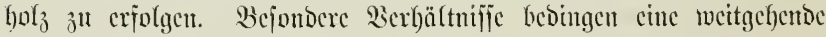

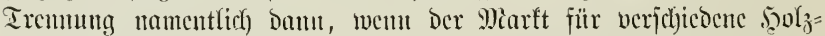

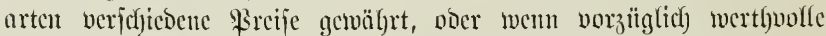

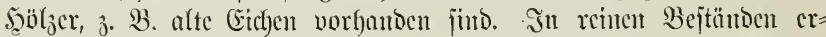
giebt fitch bicje Trenmung won jelbjt. $D$ b in gemijchten 2 bejtänden cin

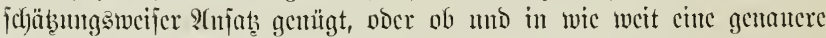

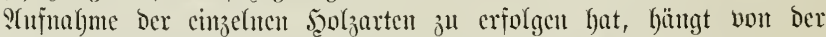
geforderten (benautigfeit ber Echäbungsarbeiten überbant ab.

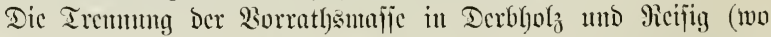

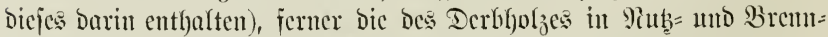
folz erfolgt nicht für jeben eiuzelnen Bejtand, fonderut erjt fïr ben bered)neten Jjicbsjab uach Yocalen Erfafrumgsprocenten, bic allerbings Durch wejentlich anderen (Efarafter Der nenen Jjiebsorte gegentiber bem Der alten, abgetricbenten, ober burd) wejentlicbe Beründerungen ber

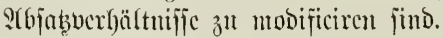

Ebenjo ijt bezüglich bes Stodtholjes ju verfaljern. 


\section{s 72.}

\section{3utuad!}

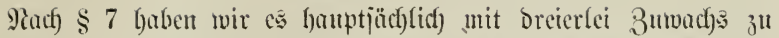

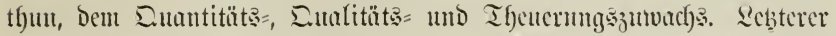

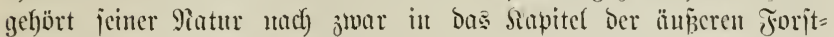

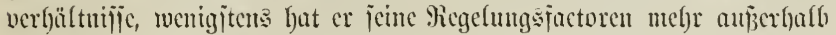

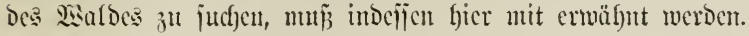

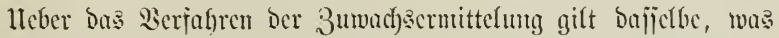

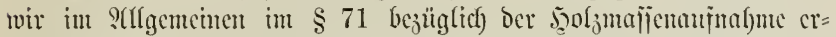

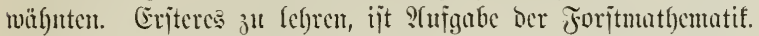

\section{Duantiätรวแmaç)}

Dic mefre oder weniger genaue Bejtimmunt bes Eutuntitüt:

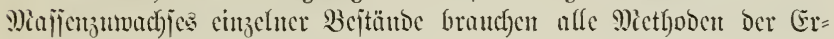
trugsiregelung.

Eelbit jences fombinirte Fad)werf, weldbes Den Şiebsjab nur fïr cine \$istiode, höchjtens für zwei Perioden beredfnet, funn wenigjtens bic 3umachserfebungen in Den wabricheinlichen beicbosten nicht ent=

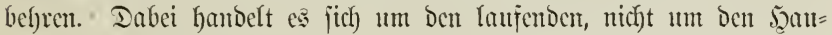
burfeits= Durd)id)nittš jumad)?.

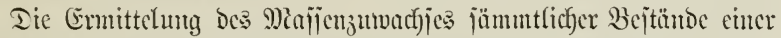

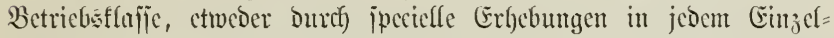

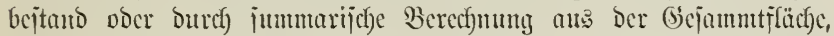

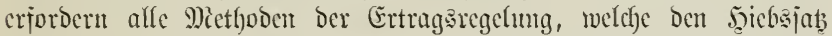
auj cine ganje llmtriebszeit berecfunen, mo jente, bei benen ber Ges=

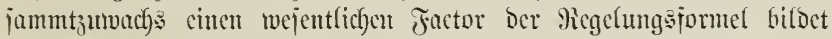

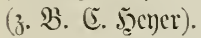

In cinfachjter ascije geben babei bic Sameralture und bie ifyr

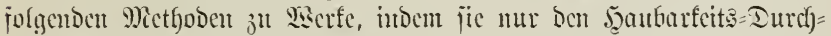

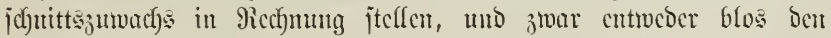

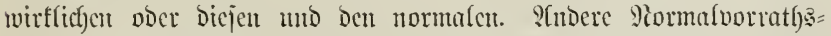
methoden (3. 3. Jjumbešlyagen) rechnen mit Dem laujenten 3uwachje,

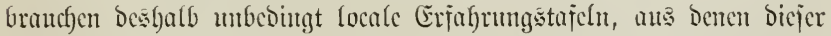
jicl) leidft herechnet, jobalo bic Bonitirmty ber Bejtände erjolgt ijt.

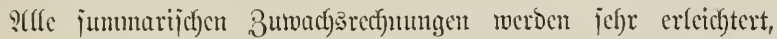

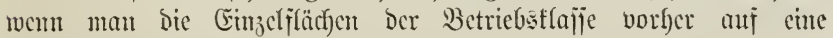
Boniät reducirt.

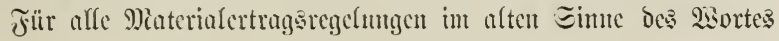




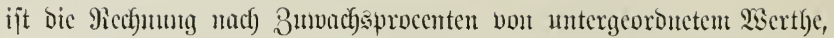
bem jic entpfichlt jich höchjtens für Sberjtünocr bes Mittelwaldes, für Camenbäume ber Derjüngung引flaffen, überfantpt jür wertfoolfere,

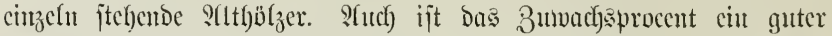

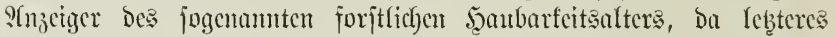
cingetreten, wenn erifteres für Den scautptbejtand auf $\frac{100}{\mathrm{u}}$ gejunfen ijt. (\$§ 10 แnเ 11.)

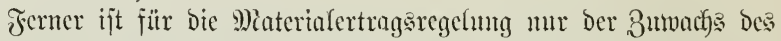

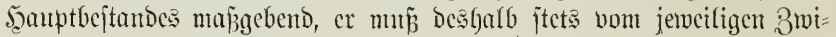

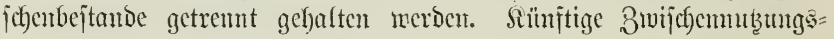

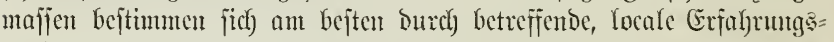

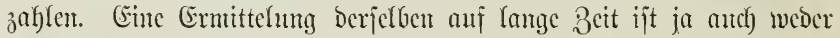

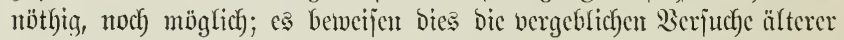
Mictgoder.

Bom Etandpunfte Der Fimanjrechmung ats gewiunt Das Miajice

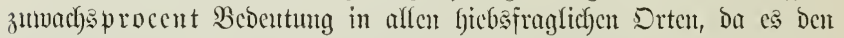

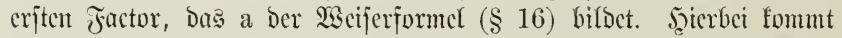

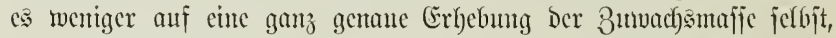

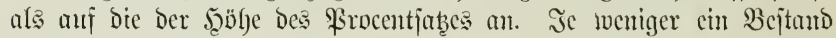

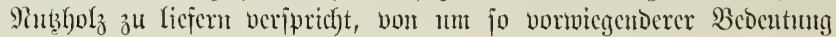

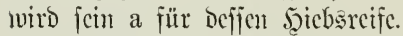

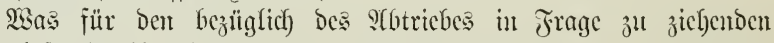

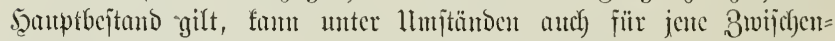
bejtänoe getten, Deren Durd)forjtungsweije Entnafynte in ber näddjten

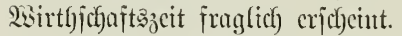

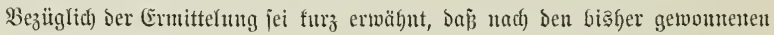

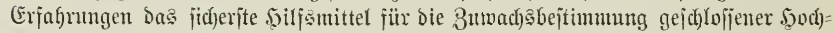
walsbejtänbe gute locale Ertragstajeln bieten. (Es ijt nämlich ein nod) ungelojites

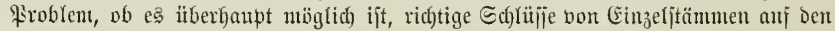

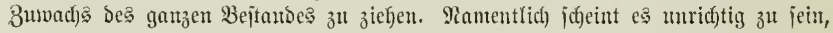

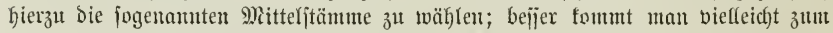
Biele, menn man bie \$robejtämme aus ber Silaj̄e ber jtärfiten Stämme svählt.

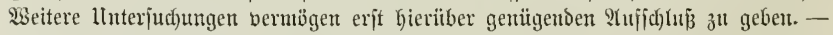

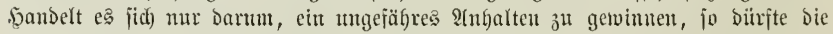

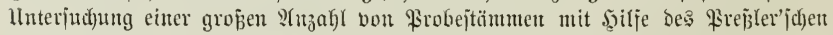

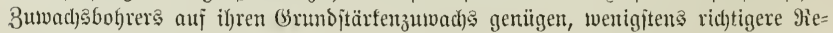
jultate gewähren, als wenn man 2 ober 3 Sirobejtämme jällt uno dieje mit alfen mathematij(ben Jeinfeiten bered)net.*)

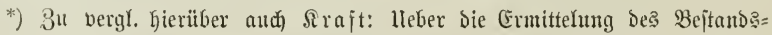

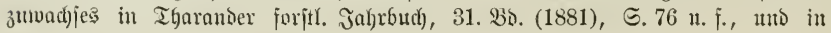




\section{Eualitätรzแmach)}

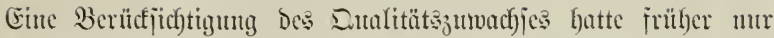

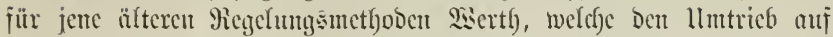

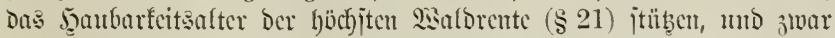
mur in jo meit, als cs fidf) um bic Ermittelmug bicjes llmtricbes bundelt.

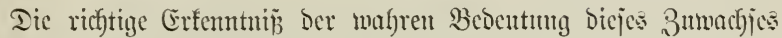

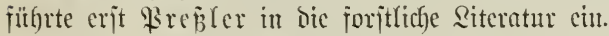

Fint bic Ertagsermittelung nach bem Principe ber Jinambrect)=

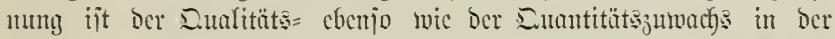

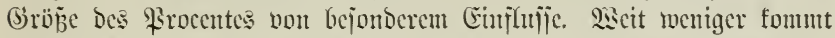

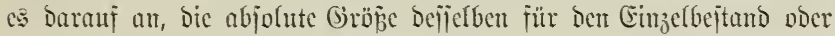
für ganze Bejtandsgruppen ju crmitteln.

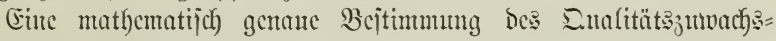
procentes b ijt noch meniger möglich, als cinte jolche bes a, ia es jich noch) tweniger regetmäp̈ig betwegt.

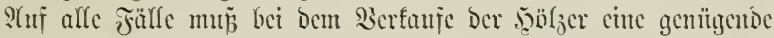
Eortirung ber Eortimente nudf) Etärfen ichon längere 3cit jtatt= gejumben fuben, wem dicjes b mit irgend anü̈formber Eicferfyeit bejtimunt werden joll. Inocilen ijt jefbjt in ?iubb)

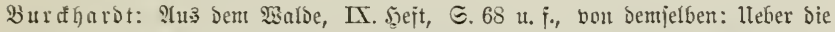

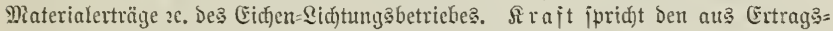

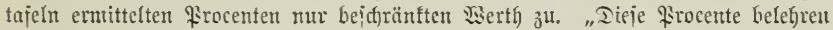

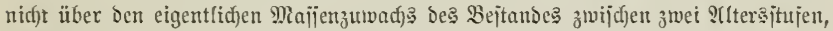
jonbern geben uts an, nad) weldjen Procsute Der souptbejtanto Der einen ?Iter: itufe jïf) auf Den ber andern gefoben habe. Die Ertragstajel= \$rocente tömen

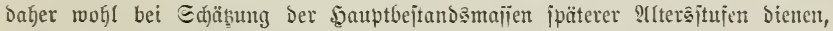
niemals aber aud) nur annähemb ïber bie Rentabilität ber Sisithj(hajt belefren;

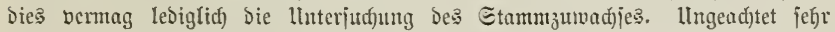

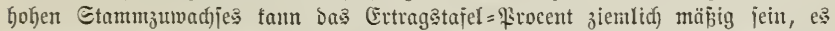

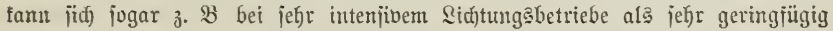
Gerausitellen, ja jelbjt gleich Plull, ober jogar negatib twerben." - Ëe ijt fier

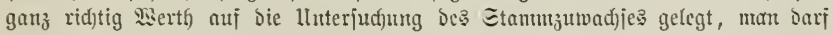
bei Diejen, Den (Ertragstajeln gentadten Sorwur aber nid)t ïberjefen, baj es

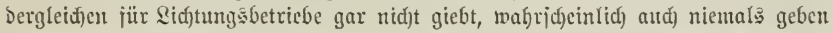
wirb, weil bie bleibenden Miajien bon bem grïberen ober geringeren Grabe ber

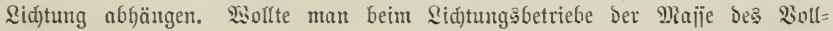
bejtandes Die Des älteren gelidjteten Bejtantbes gegen̈̈beritellen, un Das Bejtands=

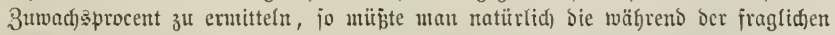

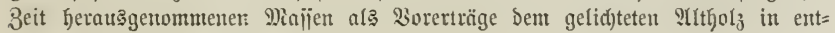
ipredjenter Siseije zuredjuen. 


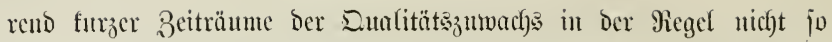

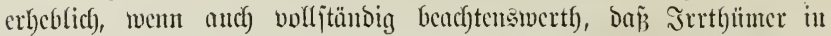

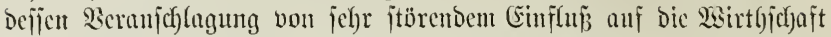
jeit fümuten. Bejondere S(usuafmen fommen jedoch bor, umb zmar überall Da, wo Der Marft Eortinente bejtimmter Etärfe vorwiegend brautcht umb bezalylt.

Eo weit cs irgend mïglich, gat ber Taxator bicjes b fïr bie Siebsorte zu bejtimment. Fïr bic Der Durdfforjtung anfeim falfenden

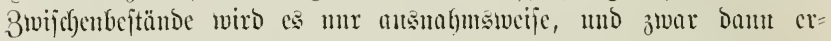
mittelt, wen beren Entualyme beshalb fraglich ericheint, weil jie mïglicher 2iseije werjpredyen, bald mertbodfere Eortimente zu liefern.

\section{Theucruggzumachs.}

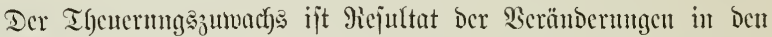

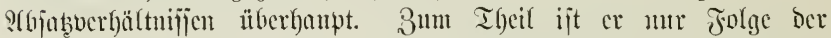
anperforjtlidjen S(enderungen bes Miarftes, D. l). Der Sachfrage nad)

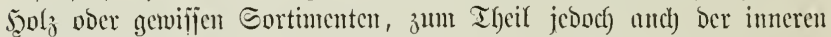

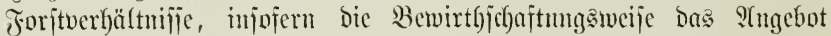

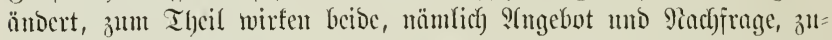

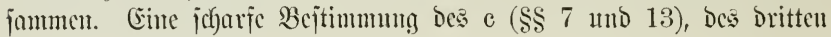

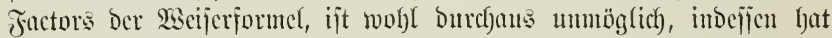

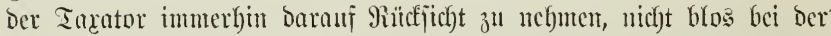

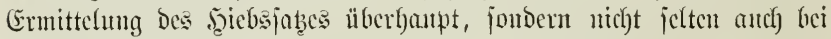
Der llnterijuffung Des cinzeluen Bejtandes. Rebeteren Falles ijt in Der

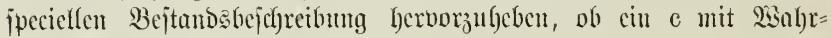

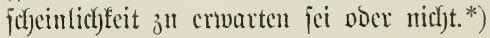

Der j̈ffer ju erwartende Ban ciner Brettjäge, einer Etraje,

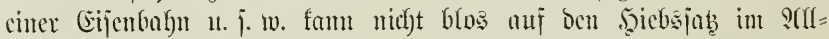

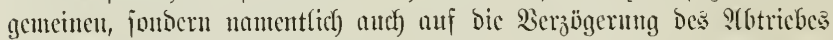

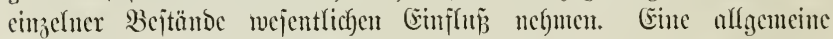
Eteigermng ber \$reije aller Eortimente änocrt żwar bic relative

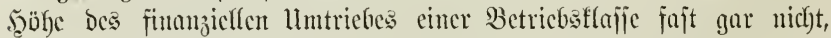
trobocm wird es abci jefre wirthjedfaftlich jeil, einige Bejtände und Bejtmosgruppen bis jum Eintritt cines folchen Beitpuntes aujan=

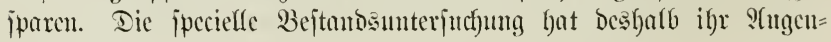

*) Ueber Die Bewegung Der \$golzpreije zแ vergl. $\mathfrak{L} \mathfrak{e b r : ~}$ Beiträge zur Statiftit

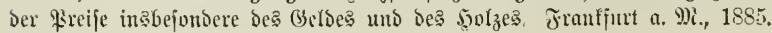




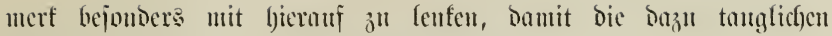
Bejtämo in simmale notirt merben fïnucu.

\section{$\leq 73$.}

\section{Burraflymapital.}

Ein weiterer Factor ber Siseijerformel ijt Der Riconctionsbruted) $\mathrm{H}$

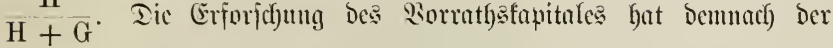

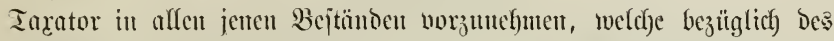

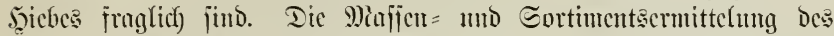
Bejtunbes gejtattet unttelit Der gegenmärtigen Freije bie Bejtimmung

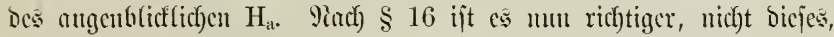

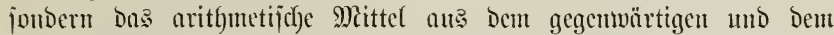

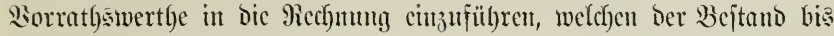
3u jener 3eit erlangen bïrfte, auj welche man itberfaupt bas 2 secijer= procent bejtimmen will. Der Tarator hat Demnad) nidfyt blos Das

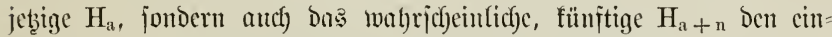
zelnen, ctwa fraglichen bejtünden im 9)ianuale beizujochreiben, nument= (ich) jo weit lestere gewijife (3ruppen dyarnfterifitren.

(Es jei lier nod) ein oft gefbirter (Eimwand erwähnt. Mian fagt nicht

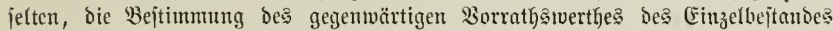

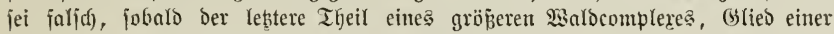

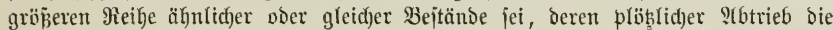
\$reife brïifen mixije. Diejer Einwatto ijt aber weder von ber rein theoretijchen,

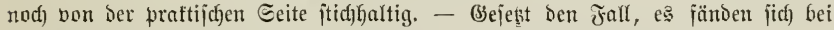
ber Einzelunterịtd)my jo viele finn

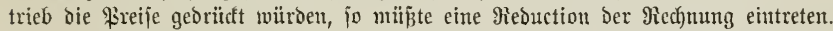

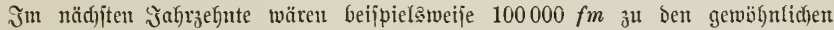

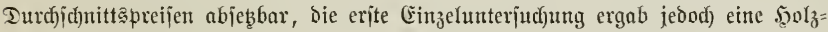

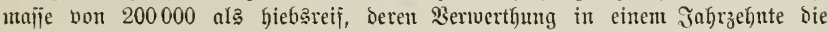

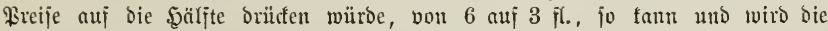

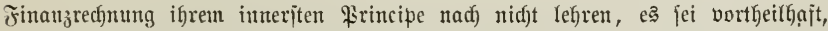
eine 20 jährige Bruttorente von 60000 fl. mit einer 10 jäfrigen dejijelben Betrage

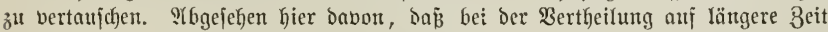

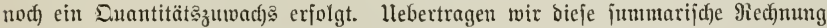
aนf zefnt aujzuparende biebsort allein ein $\mathrm{e}$ von reidf)lich 3 bis etwa 6 nad)=

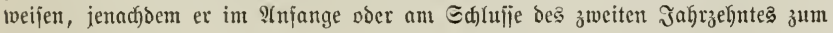
Silebe fommt.

Eo extreme 3ahfen fonmen freilidy nur in einem \&ehrbeifplele vor, praftijo werben bie Differenzen nie jo jharf herbortreten; Dagegen greift bie praftifdhe

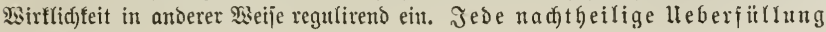


des Marfes rujt jofort bem $\mathfrak{S}$ irthidajter ein balt zu, taut aljo niemals ein jid) fortiesender Jegler werben.

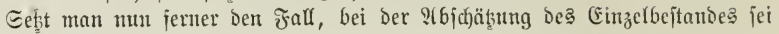
das $H$ etwas zu hod) gegrifien worden, jo wito bie umittelbare Folge in Der Re(f)nng nidft ein fleineres, fondern ein etwas, wenn aud) jehr unerbeblid) zu gro ถ̈e fïr j̧iebstcife iprechen.

\section{S 74 .}

\section{(jormokapifal.}

Echließ̧lich ijt noch das "Bodentoruttofupital" g ober bas "(Birund=

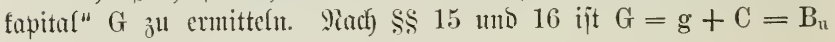
$+\mathrm{V}+\mathrm{S}+\mathrm{C}$.

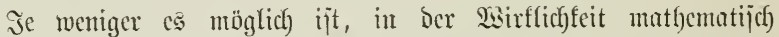

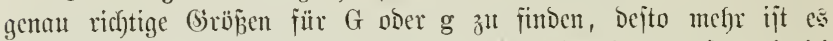

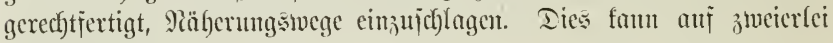
2isije gejchchen.

1. Summarifde Ermittelung von G.

Bercits $\odot .45$ und 53 Deuteten wir bicjes Berfafuren an. Mian

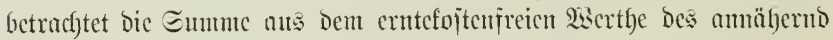
Dem* Finanjiclfen llmtricb entiprechenden 3 ejtundes und Dem jeiner anf Das Jufr u prolongirten Sornubungen als ben ujälyrigen Endzins

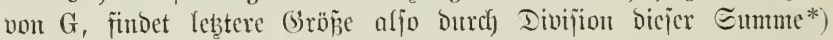
mit $1,0 p^{n}-1$.

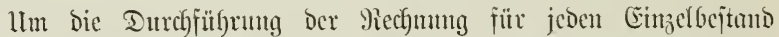
faum es jich rjerbei nicht handeln, jondern es genügt bic Ermittelung von G für gröjere Complexe, fïr cine gam

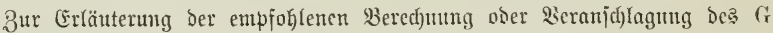
diene fulgendes Beijpiel:

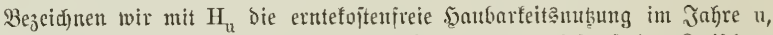
mit $\mathrm{D}_{\mathrm{s}}$ bie Eumure der auf Dajielbe Jafr gebradjten, erntefojtenjreien 31wijhen= mڤsungen, jo ijt

$$
\mathrm{G}=\frac{\mathrm{H}_{\mathrm{u}}+\mathrm{D}_{\mathrm{s}}}{1, \mathrm{op} \mathrm{p}^{\mathrm{u}}-1} .
$$

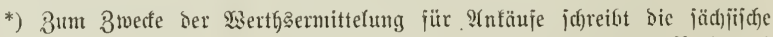

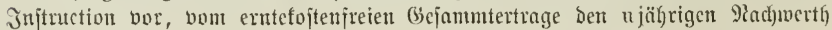
Der Sulturfoitcn abzuziełen, aljo nidjt G, jondern g zu bered)nen. Pad) unjwem

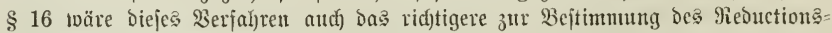

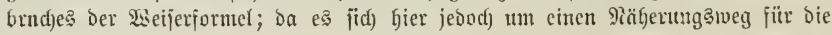




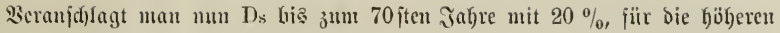

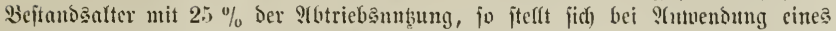

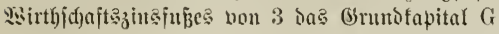

$$
\begin{aligned}
\text { fiir } \mathrm{u}=70 \text { ani } \frac{\mathrm{H}_{70}+0,2 \mathrm{H}_{70}}{1,03^{50}-1}=0,17 \mathrm{H}_{70,}, \\
" \mathrm{u}=80, \frac{\mathrm{H}_{80}+0,15 \mathrm{H}_{80}}{1,03^{80}-1}=0,13 \mathrm{H}_{80}, \\
" \mathrm{u}=90, \frac{\mathrm{H}_{90}+0,25 \mathrm{H}_{90}}{1,03^{90}-1}=0,09 \mathrm{H}_{90}, \\
" \mathrm{u}=100, \frac{\mathrm{H}_{100}+0,25 \mathrm{H}_{100}}{1,03^{100}-1}=0,07 \mathrm{H}_{100} .
\end{aligned}
$$

llnter $H_{z 0} \mathfrak{u}$ f. wo. Find Gier bie Sisertge Der $70=, 80=, 90=$ unt 100 jäbrigen

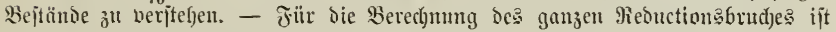

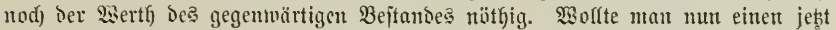
60 jäfrigen Bejtant, weldher ber Schlagreige Dę 70 jährigen ltutriebes angefört, auf fein siseiferprocent unterjuchen, uno fei befien Sorrattgeswerth $\mathrm{H}_{\text {so }}$, fo mürbe

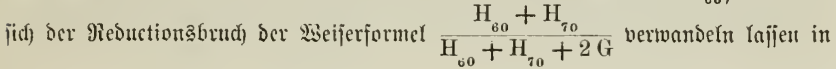

$$
\frac{\mathrm{H}_{60}+\mathrm{H}_{70}}{\mathrm{H}_{60}+\mathrm{H}_{70}+2 \times 0,17 \mathrm{H}_{70}}=\frac{\mathrm{H}_{60}+\mathrm{H}_{70}}{\mathrm{H}_{60}+1,34 \mathrm{H}_{70}} .
$$

Die cinfadjite Form, wenn man nämfid) nidjt baß aritgmetif(d)e Mittel ans

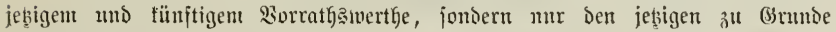
legt, märe:

$$
\frac{\mathrm{H}_{100}}{\mathrm{H}_{60}+0,17 \mathrm{H}_{70}},
$$

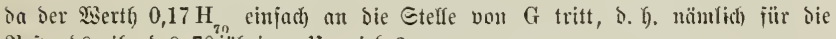
Sejtandasreige bes 70 jäbrigen llmtriefes.

2. B̧⿻上丨) $g$ ijt glecch $\mathrm{B}+\mathrm{V}+\mathrm{S}$. Dic mutälyemo richtige Bcjtimmung

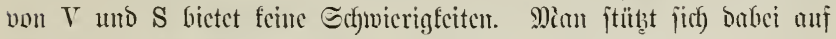
bie 3 ahlen ber Bisegenmart.

Der jefwicrige Factor bleibt B. Bercits @. 55 bouteten wir all,

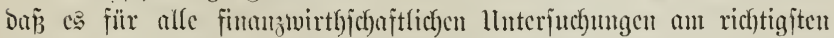

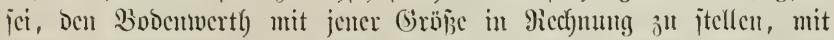

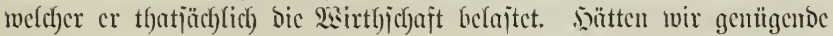

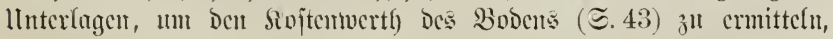

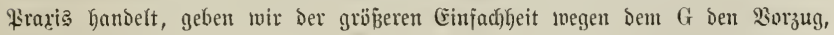

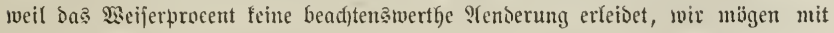
noer nfue Sitturfapital red)nen. 


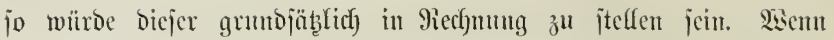
Jemanto ein Etüct Rano fauft und 2Esald darauj anlegt, jo ijt ber

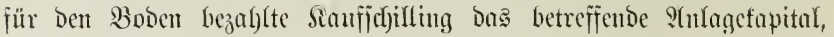
gleichuiel of $\mathrm{B}_{\mathrm{u}}$ fünftig höber noer niedriger lautet. ${ }^{*}$ ) Eo cirfacl)

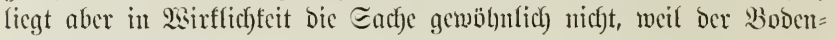
fojtentwertl) in uralten, gropesen 23 alogebieten gar nicht birect ermittelt werden faum. (Es bleibt aljo nidfts übrig, als für bie verjefiebenen Betriebs = und Etandortsflajjen cines Sicvieres unter 3ulfiffenalune

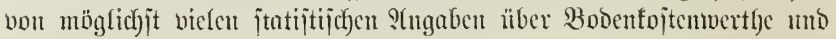
cincr Pieife von Bodenerwartungswerthen jebäbungšmeife cinen Boden= wertl) (B) jut ermittelı.

Die erjte Bejtimmunt cintes jolchen B ift nun alferbings um= jtänoficher als bie bes $B_{\text {n. }}$. Es wiro aber bicjes 2 erfahren jum cin= facferen, wohl andf jum richtigeren, twent wir bas eimmal gefundene

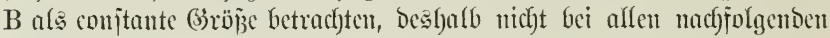
Sievijionen nen ermitteln, wie bies mit ber nach ben verändertict)en

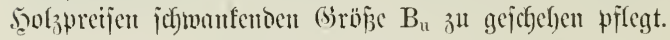

\section{$\$ 75$. \\ Das Heilexprorent.}

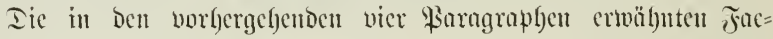
toren genügen, un bas 2seijerprocent $(\$ 16)$ Der cinjelnen Bejtünde วu bejtiument.

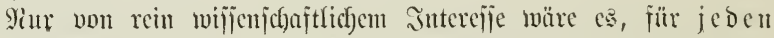
Bejtand des einzurichtenden 2saldes biejes Frocent ju ermitteln, aljo

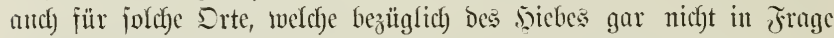
fommen fömmen, theils weil fie ifor nocf) bebentender Bumadfs als ljebsumreife, theils weil jie iffre sage inmerbalb antorer site ent=

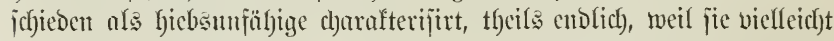

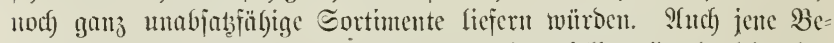

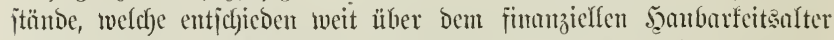

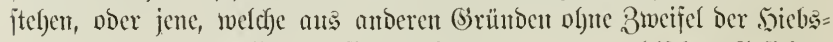
folge zum Dpfer fallen minijen, erforbern vou praftijchen (Bejichts: = punfte aus nicf)t Die ipeciefle Ermittelung bes SEcijerprocentes.**)

*) Die Frage Des Bobenwerthes wurbe ausjïhrlidjer vom Berfafier bejprodjen

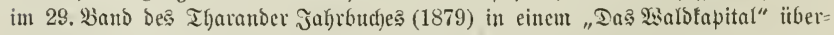
j(d)riebenen Sirtifel.

**) Tharander Jahtbud), 17. Bื. (1866). III. S. 46 ıt. f. 


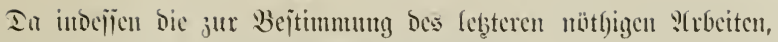

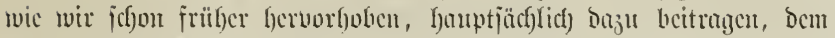

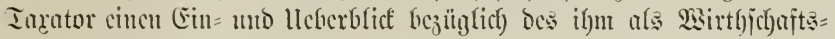

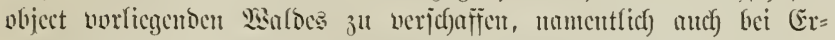

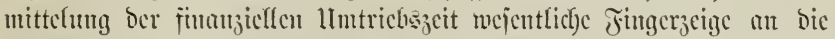

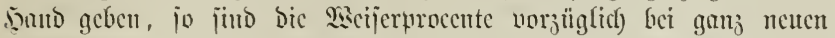

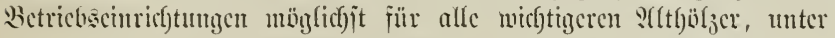

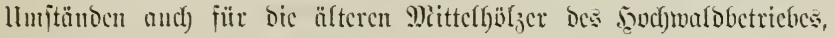

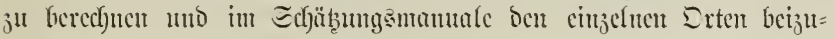
icf retilent.

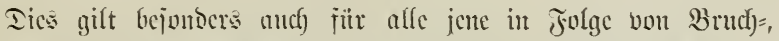

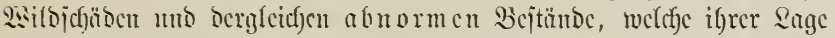

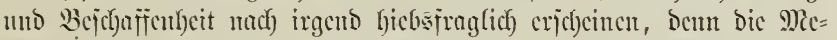

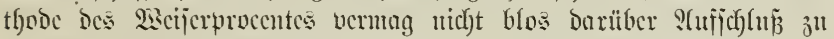

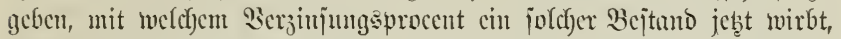

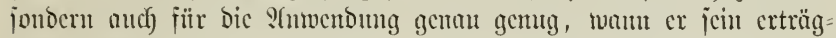

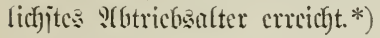

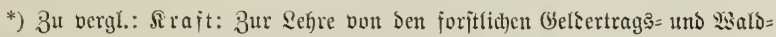

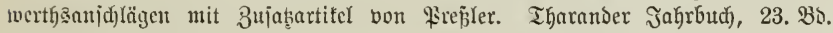
(1873). ङ. 137 น. ๆ.

In netejter 3cit murbe das siseiferprocent beiprodyen von 2 . Schiffel, jul bergl. Defien Edjrift: „3ur foritlid)en Ertragsregelung". Görz, 1884. Dieje

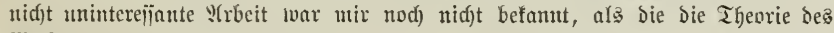

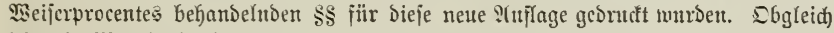
id) mit Wiandferlei barin nidjt einveritanden jein fann, ijt hier Dod) nidjt ber

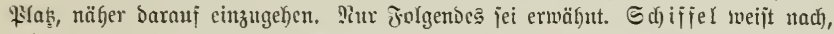

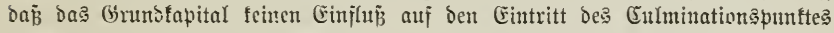

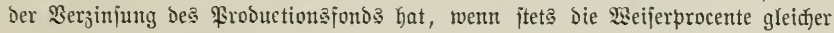

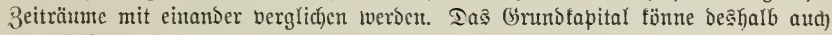

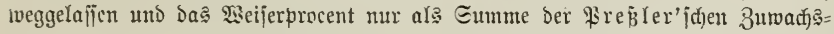
procentc $a+b \pm c$, aljo in Der form $w=100\left(\sqrt{ }^{\frac{n}{H_{a}+n}}-1\right)$, Stnwentung

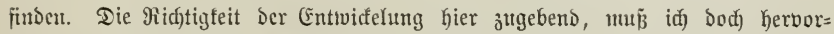

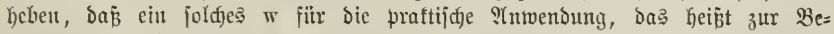
fimumung ber Erntereife bes Einjelbejtandes, und Daranf fomunt ç Dod) baupt=

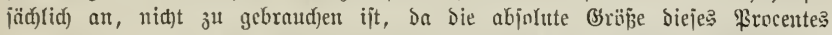

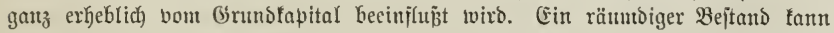

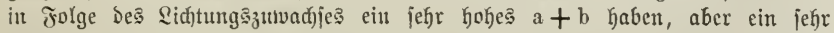

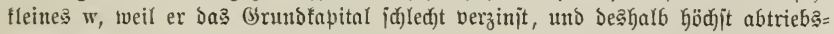
bebïrittig jeir. 


\section{$\$ 76$.}

\section{Belfantstonifimut}

a) Bcitandobonitirnng uad) Bonitätstlajicn.

Die in Den Ss 66 bis 75 Kejprochenten Factoren genïgen jumar, um, joweit bics überfaupt möglich), ein Bifo won bem wirtbj(f)aftlicfen

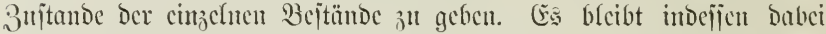

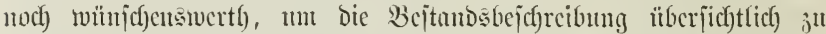
geitulten, ben alferbings nur relativ guten ober ichlecflen 3ujtand

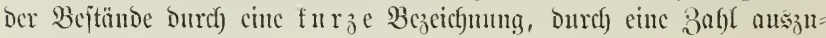
brücten.

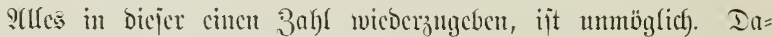
gegen bejib̧en wir in ber cinem bejtimmten Bejtundsalter entjpredgen-

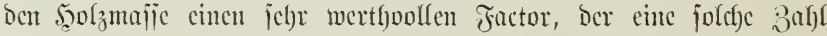

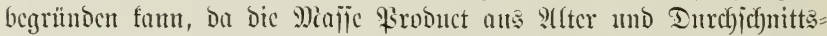
juvachs ijt, lcb̧terer aber wicberum abgängt vou Dem Etundort nuD ber früberen Befandungencije des Bejtundes jelbit. Penten wir dicje Zah) dic Bejtandsbonität.

Ecfon bei ber Bejprechuny der Etandortšbunität mujte biejer Bejtandšbonität geducht merben, weil leb̧tere in Den meijten Fällen

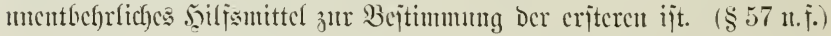

Sisic bei Der Bonitüt Des Etandortes, untericheidet mant and bei Der Des Beftandes cine normale mo eire coucrete.

Eritere ift dicjenige, welche cin Bejtand als dic jeincm

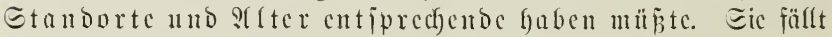
aljo mit Der für gegebene Betrichs= mo jeolzarts = Berbältuific vor= handencu, concreten Etanoortšbunität jujammen.*)

Die concrete Bonität cincs Bejtandes i jt bagegen

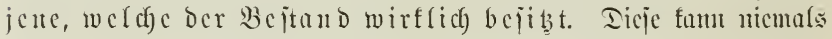
ïber, wirb aber bänfig unter Der normalen itchen. Sebsterer Fall

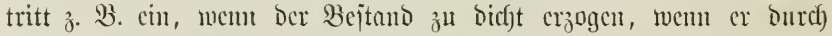
Echnccbruch, Injeften n.j. w. Gcjchäbigt murbc.

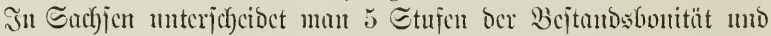
veriteft nuter ber 1. Dic heite, unter Der 5. Dic nicorigitc. $23 i r$ mifficu

*) Man föute unter Mormalbunität eintes Bej̃tandes aud) jente verjegen, weldje ber Bejtand der normalen oder gar idealen Etandortsbuntität gemäis al̨

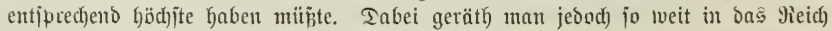

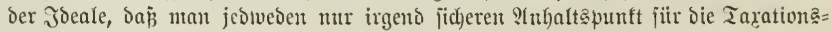
arbeiten berliert. 


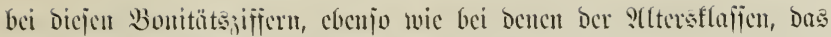

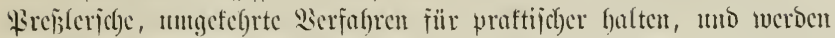
bafer mit 1. Bonität bic nicorigite, mit ben föberen Biffern bic

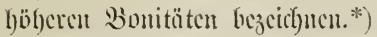

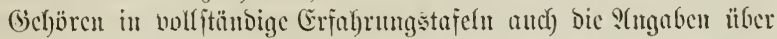

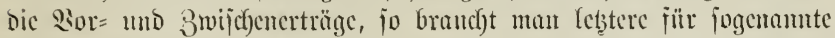

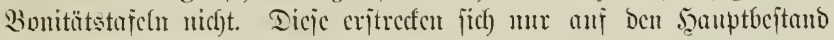

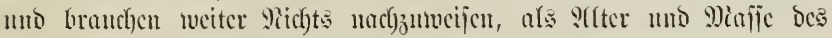

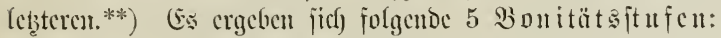

1) Gicrittg,

2) mittclmäzig,

3) $g u t$,

4) jefle gut,

5) allsgejeicfuct.

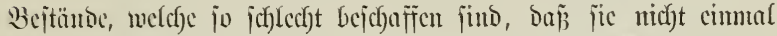
Der 1. Bontität cingercifyt weroen fömuen, falfen in bic Sintegoric ber

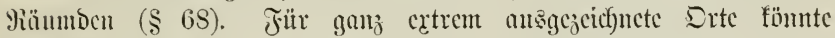

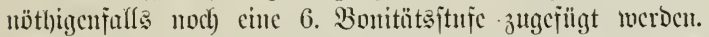

Braud)t man für gewijie Epccialzucde nod) feincre ?(bitufungen,

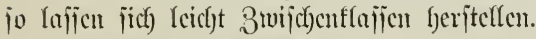

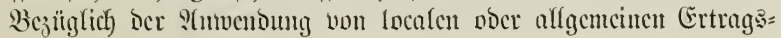
vocr Bonitätstufelu gilt and hicr, was wir jefon im $\$ 57$ bei ber

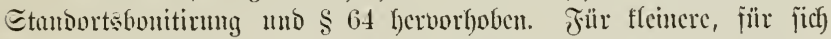

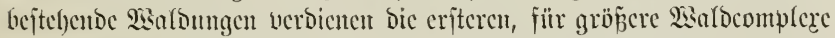
Die lebteren ben Boräıg.

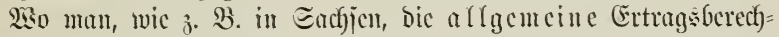

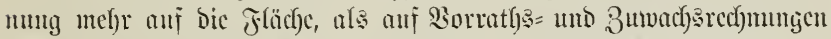

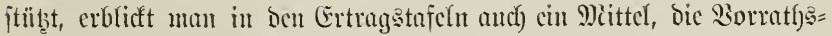

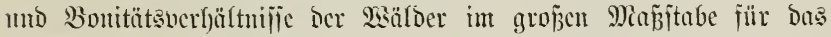

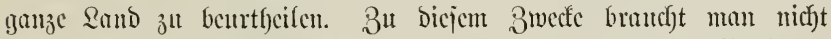
focale, jonderu alfgemeine Tafelu. Iabei gentïgen moh)l 5 Bonitäts= itufen, um joldje Iafeln audf) für andere Zwedfe ber localen Ertrags:

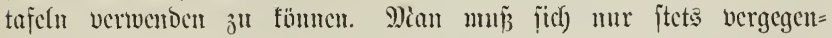

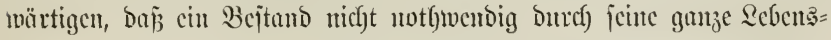

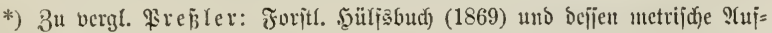
lage (1874), Tafel 25.

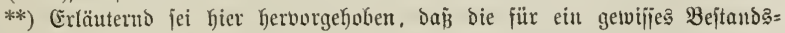

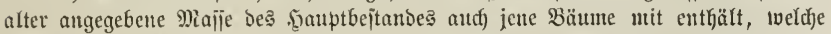

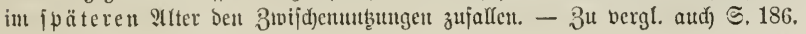




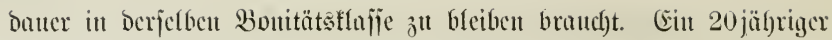

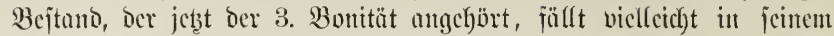

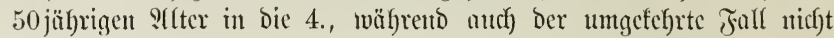
iclten ijt.

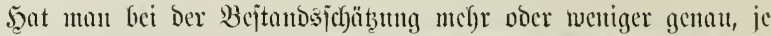

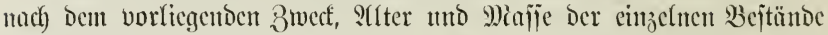

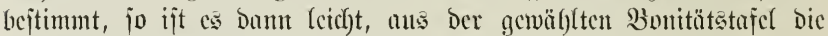
zugebörige Bonitätşiffer zuzujchreiben.

Beijpiel. Entipräche 3. B. unjere $\$ 11$ mitgetgeilte Ẽrtragstajel ber

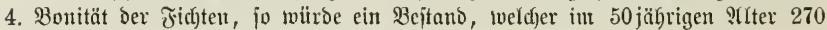
bis $280 \mathrm{fm}$ Maije entfält, biejer Bonitätęlajie angehören.

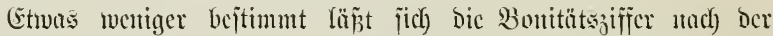
Diajje fïr jüngere Srte, an wenigiten für junge Sulturen ungeben.

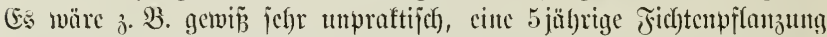

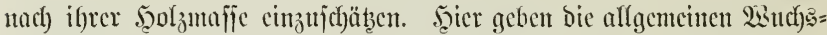

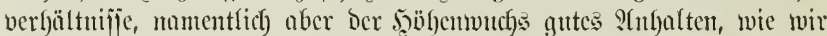
bereitś in $\$ 6$ bemerften. Etcllt man z. B. cinen 10 jäbrigcn Sicfernort in bic jucite Bonitätsflajice, jo gejoficht dies in Der 2orausjebung,

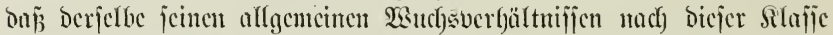

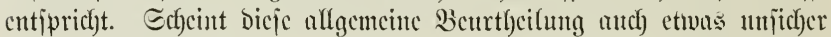

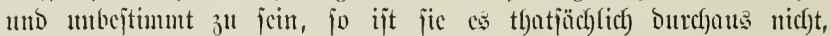
wenigitens nicft unbejtimmter, als bic Majicnermittelung jo jugend = lidfer Drte.

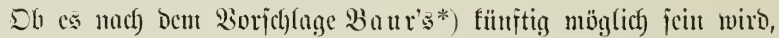

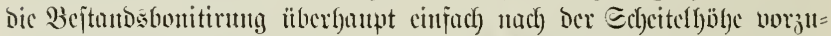
neffmen, aljo nicht blos bie ber jungen Sinfturen, fontorn and bic

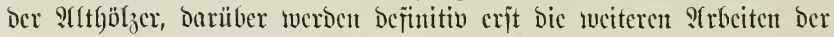

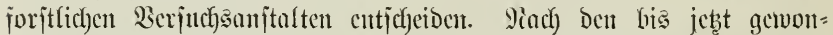

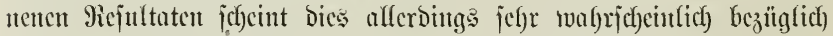
alfer $\mathfrak{j c o l z a r t e n ~} \mathfrak{j} \mathfrak{u}$ jein.

Das (sejchäft Der Bonitirung ijt für Bejtänoc Des Subljchlag= und Picdernaldbetricbes*) cin jefre cinfaches, jobald man jich cimmal

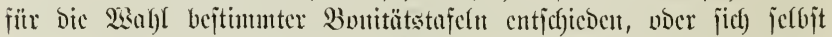

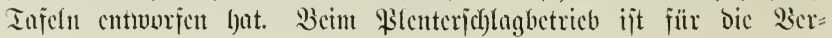

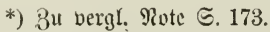

**) In Eadjen unterliegen weber ber Miebers, nod) Der Mittelwals, nod) bie

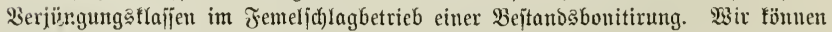

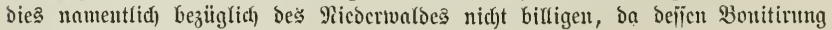
gar feine Ed)wicrigłeiten biefet. 


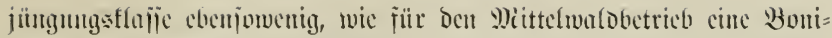
tirumy unch) ber Plajīe möglicf).

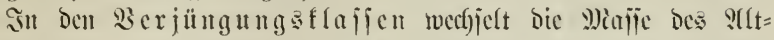

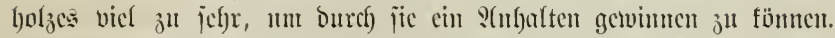
Effenhar verfiert aber bie lleferïicht ïber cill ganzes Revier, wem

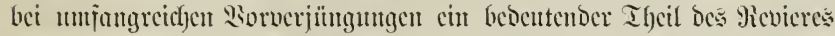

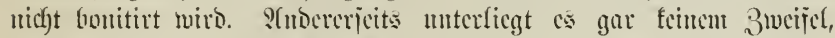

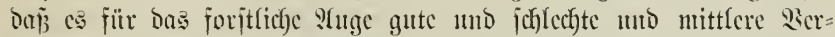

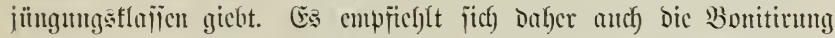
berjelfen. S(m bejten ijt es wohl, hier of)ne asciteres bic concrete

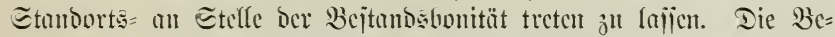

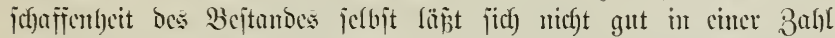
alsoriäfen, joll aber im Tarationsmamale burd) cinige Benterfungen

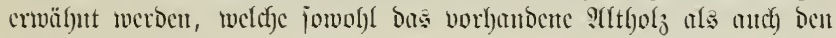
?achnutchs betrefien.

Bei bem Mittefwalde fünnte bic ans lluterfold und SGerfol,

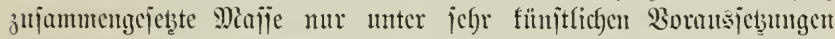

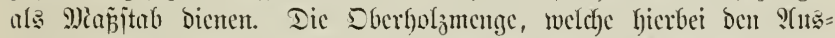

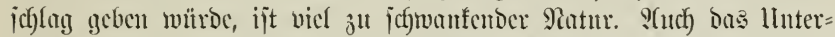

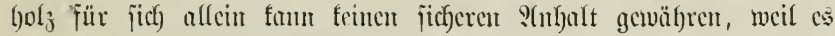
cbenfalls won ben verïnderlicf)en Eberfold abbängt. Lisir empfeflen beshalb, fier jene concrete Etendortsbonität ju mählen, wic ficf) bic=

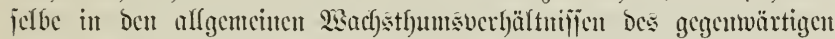
Bejtendes ansipricht. Sjt bies auch feine recht jichere 3 ald, jo boch immer bejier, als gar feinte, und müfïen wir es als cinen slangel

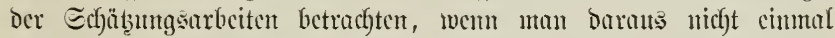

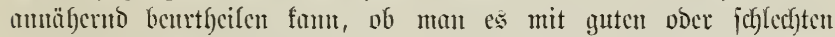
9)

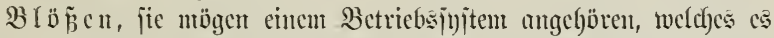
immer jei, fömen jelbjtocritünolich nur ciner @tandortšbunitirung unterficgen. Duí biejelbe ebenjo gut möglich ijt, wie bic eincr ein=

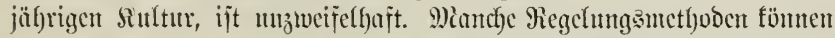

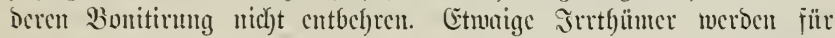

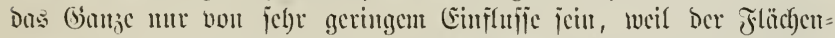

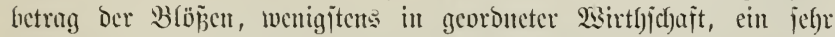

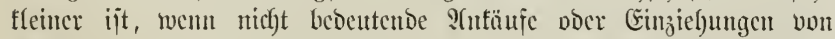

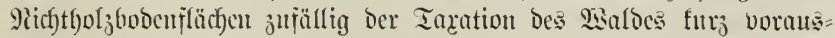
gingeut.

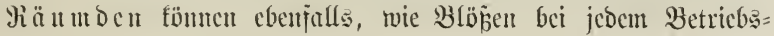




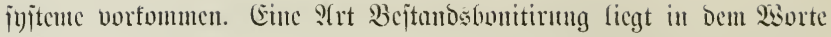

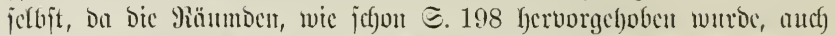

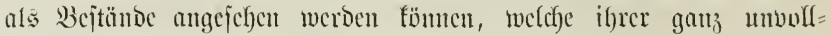
ftändigen Bejtocfung wegen nidgt cinmal bic Majīe ber nicbrigiten

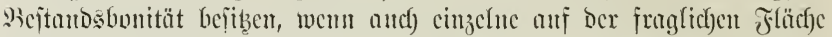

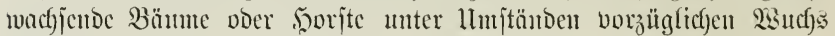
habent.

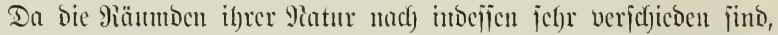

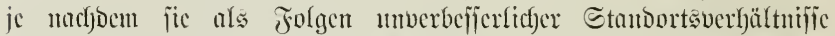

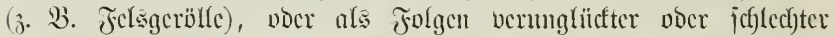

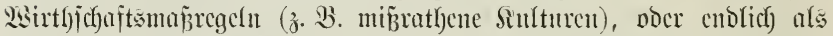

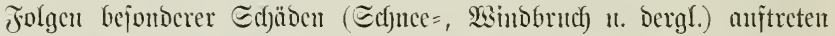

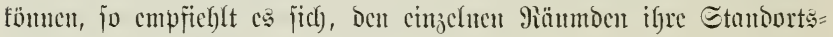
bonitüt beizujchreiben.

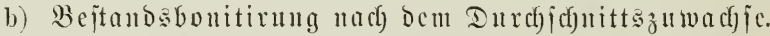

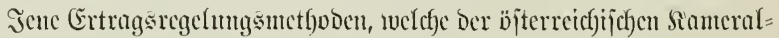

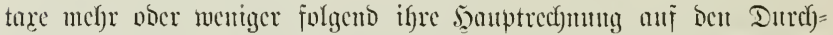

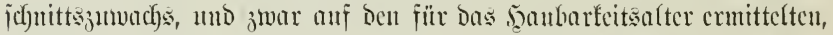

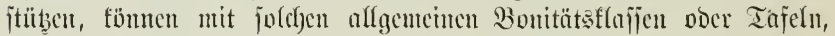
wic wir fie unter a im stuge haben, nicht viel anfangen. Sie be=

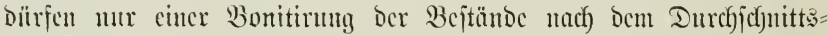

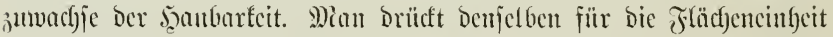

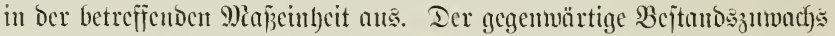
nocr beffen gegenwärtige Majje hat baun fecuen anderen Wiertly für bicje Bonitirung, als bou, cincm SAnfaltspuntt für Ermittelung bes cinjt ju crwartenden 5̧aubarfeitsertrages jul gewälyren.

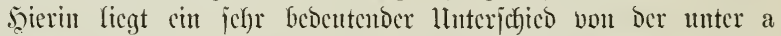

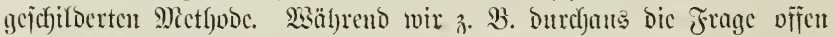

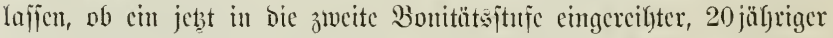

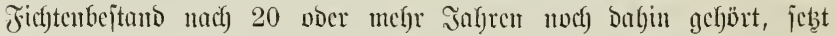

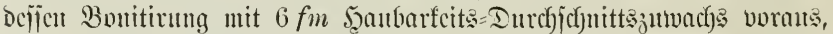

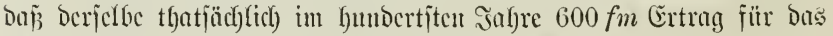
j)eftar getie.

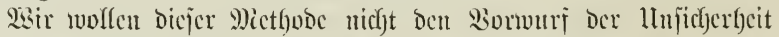

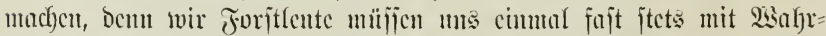

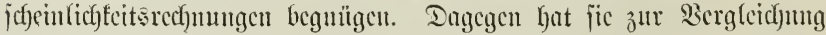

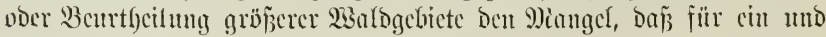

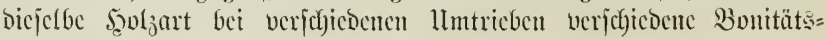




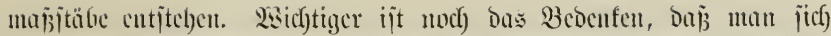

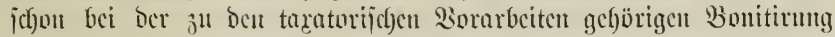
iiber das fiurptige joutharfeitsalter jedes cinjelnen Bejtundes cut=

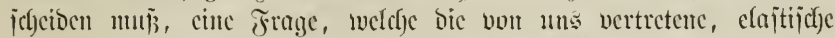
Bejtandsmirtljichajt für jüngere säblzer jedenfalls licher als offenc fictrachtet.

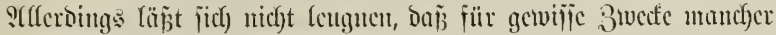

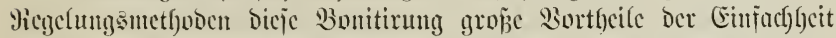
bictet, jo j. B. für bic Reduction ber Fläcf)en anf cint Bonitüt.

\section{s 77. \\ livourtim anf xine bonifät.}

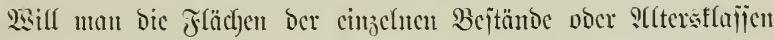

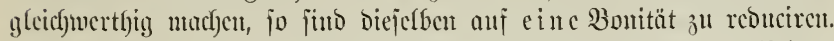

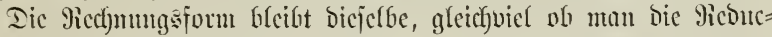
tivu anf bic normale voer auf bic coucrete Bontët vornimut. Erjteres gejeficht, wem man bic unter gegebenen Etmobrtsverfält=

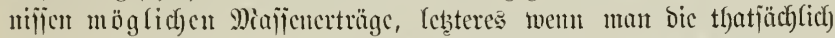

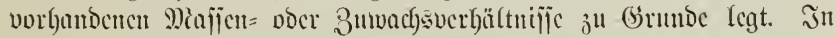
bicjem Einnc funn man and jtatt normaler Bonität jagen Etundorts =, aujtatt concreter Dagegen Bcitunds = Bonität.

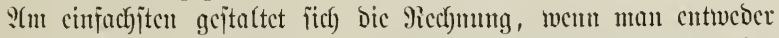

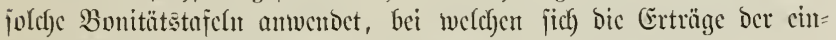
jefnen Silajīen verbalten, wie dic ifnen entipredfenden Biffern*), voer wentn man wou joldyen gans abjebeno, jofort ben cintem bejtimmten

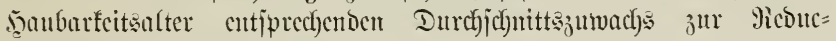
tîn woülylt.

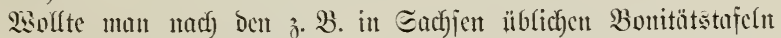
genau reduciren, jo dürfte dies mur mit jeilfe ber entiprect)enden

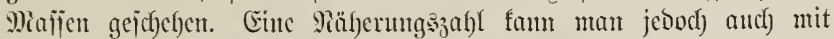

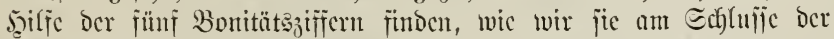

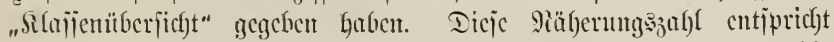

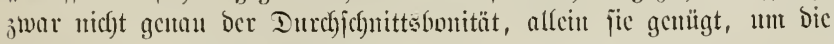

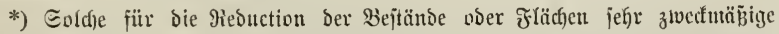

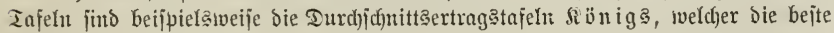
Bontït mit 1 bezeichnet, bie geringeren burd) Decintalen von 0,9 bis 0,1 aus=

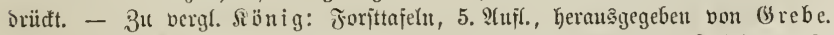

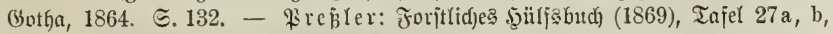
utto bejien metrifd)e ?(uffage (1874), Tafel 28. 


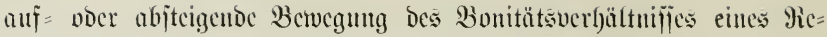
vieres oder einer Betricbsflafic überbant on mejien.

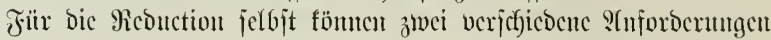
gejtellt werden:

a) Dic Emmme der cinzelnen, reducirten Flächen folf gleich)

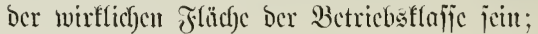

$\beta$ ) bic (bileichlycit bicjer Eumunc wird nicht gejorbert.

Jut Falle $\alpha$ uñ añ bie verglicfenc (geometrijod)=mittlere), im Fulle $\beta$ fum auf jeoc Gelicbige Bonität reducirt werocu.

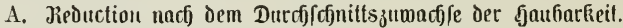

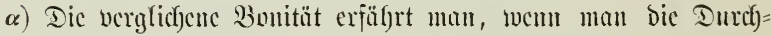

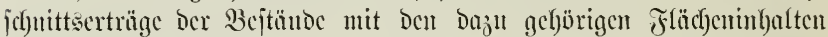

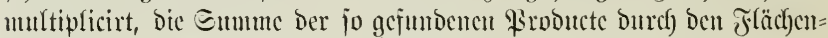
gefalt ber gauzen Betricbsflajic bividirt.

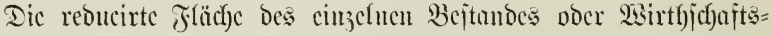

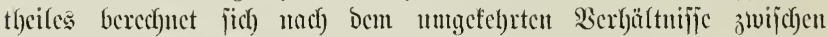
jener Bonität un Der verglicfenen.

Beifpiel. Eine Betriebeflajie von 1000 ha entfalte:

$$
\begin{aligned}
& \text { a) } 200 \mathrm{ha} \text { utit } 6 \mathrm{fm} \text { Dutd) j(d)nittşuwad)?, } \\
& \text { b) } 100, " 5 \text {, } \\
& \text { c) } 200, " 4 \text { " } \\
& \text { d) } 500 \text { " " } 3 \text { " }
\end{aligned}
$$

Sergliçene Bonität :

$$
\frac{200 \times 6+100 \times 5+200 \times 4+500 \times 3}{1000}=4,0 \mathrm{fm} .
$$

Betrig ber einjelnen, rebueirten F̌läd)en:

a) $4: 6=200: \mathrm{x}$, hierang $\frac{200 \times 6}{4}=300 \mathrm{ha}$,

b) $4: 5=100: \mathrm{x}, \quad$ " $\frac{100 \times 5}{4}=125$ "

c) $4: 4=200: x, \quad$ " $\frac{200 \times 4}{4}=200$ "

d) $4: 3=500: \mathrm{x} \quad$ " $\frac{500 \times 3}{4}=375$ "

\section{Sumue $1000 h a$.}

Bum Bwede einter proportionalen Sdylageintheilung betednet fid) bor Jahresfd) lag an wirflidyer Jlädye ebenfalls nad) Dem umgefebrten Berbält= niije ber Bonitätent.

Gejeb̧t einen 100 jährigen llmtrieb, jo Getrïgt Gier die reducirte Flädje Des Jahreşjulages $\frac{1000}{100}=10 \mathrm{ha}$. Die wirflidje Jläd)e Deljelben wäze fït: 

a) $6: 4=10: x$, hiernus $\mathrm{x}=\frac{40}{6}=6,667 \mathrm{ha}$,
b) $5: 4=10: x, \quad$ " $x=\frac{40}{5}=8,000$ "

II. i. w.

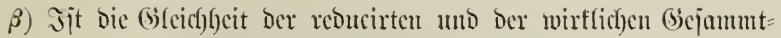

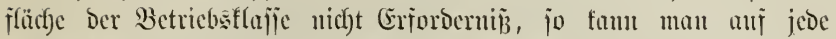

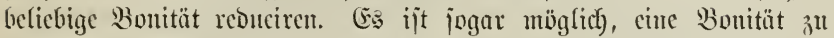
wählen, weldye auj bem betreffenden Sieviere gar nicht norfoumt,

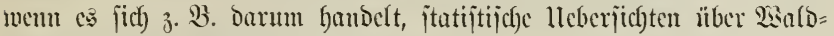
ungen gröjerer Ëebiete ju geben.

Dic reducirte (sejammtfläclye finbet man, indom man bic ver= jefbiedenen Sonitäten mit ifren Flächengelfalten multiplicirt, Dic Eumme

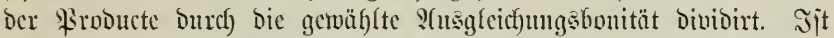
lebtere cine joldse, welche über ber verglichenen jtebt, fo wiro na= türfich) bie reducirte (sejammtyläct)e jtets fleiner, als bie wirfliche tveroen, andernjalls gröp̈er.

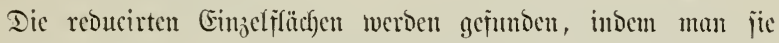

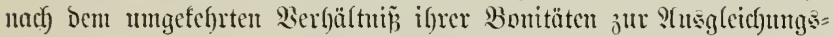
Gonität berechnet. Mit anderen Worten, man bivibirt burch leb̧tere alle ïbrigen Bonitäten umb multiplicirt mit ben Enotienten bic be= tref̈enden Fläcben.

Im vbigen Beifpiele joll auj bie B̧onität rebucirt werden, meldye $5 \mathrm{fm}$

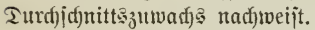

Sieducitte (Bejammtfläd)e:

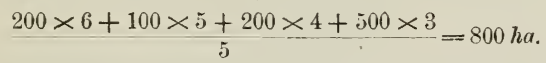

Betrag ber cinzelnen, reducirten F̦lächen:
a) $200 \times \frac{6}{5}=240 \mathrm{ha}$,
b) $100 \times \frac{5}{5}=100$,
c) $200 \times \frac{4}{5}=160$,
d) $500 \times \frac{3}{5}=300$,
Eumma $800 h a$.

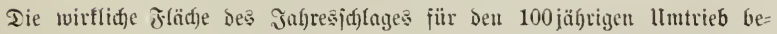
redgnet fid) ans ber rebucitten Frädhe nach ?(nalogie bes umiteheno (beiagten:

$$
\text { Riesucirter J̃afreşj(d) } \frac{800}{100}=8 \mathrm{ha} \text {. }
$$




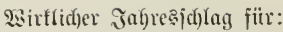
a) $6: 5=8: x$, gietaus $\mathrm{x}=\frac{40}{6}=6,667 h a$,
b) $5: 5=8: x, \quad$ " $x=\frac{40}{5}=8,000$,

B. Reouction mittefst Ertragstafén.

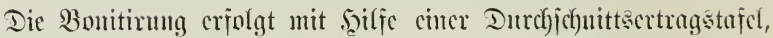

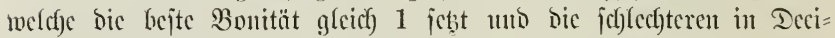
malen won 0,9 bis 0,1 ansoringtet.

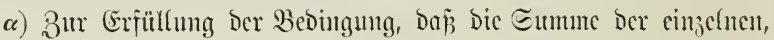

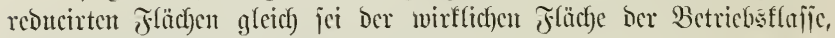
hat man zuntäcbjt bic verglicfene bonität ju berecfunen. C5 ge=

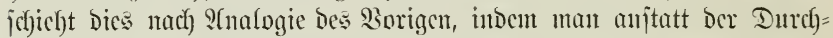

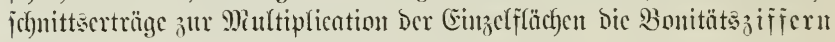
numenoct.

3eifpiel. Ein Miesermals von 160 ha im 20 jäfrigen llmtriele enthalte folgende Bontitäten :

Bergliçene Bonität:
a) $50 \mathrm{ha}, \mathfrak{B}$ onität 0,7 .
b) 40 " " 0,6 .
c) $70 "$ " 0,3 .

$$
\frac{50 \times 0,7+40 \times 0,6+70 \times 0,3}{160}=0,5 .
$$

Betrag Der einželnen, rebucirten Frlädyen:
a) $\frac{50 \times 0,7}{0,5}=70 \mathrm{ha}$,
b) $\frac{40 \times 0,6}{0,5}=48$,
c) $\frac{70 \times 0,3}{0,5}=42$,
Summe $160 h a$.

Reducirter Jafreșjulag:

$$
\frac{160}{20}=8 h a .
$$

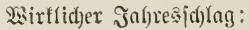
a) $0,7: 0,5=8: x$, hictans $x=\frac{4}{0,7}=5,71 \mathrm{ha}$,
h) $0,6: 0,5=8: x, \quad$ " $x=\frac{4}{0,6}=6,67$,
c) $0,3: 0,5=8: x, \quad$ " $x=\frac{4}{0,3}=13,33$ " 


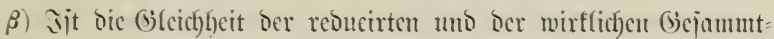

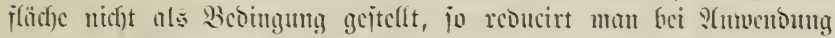
jolffer Iajeln am hejten auj 1, D. h. man multiplicirt bic Ëruzel=

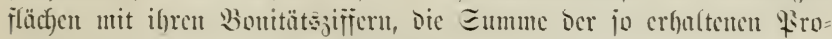
Ducte giebt Die reoucirte Gicjunmefläche, wie folgt:

a) $50 \times 0,7=35$ ha reducirte Flädic.

b) $40 \times 0,6=24$ " " "

c) $70 \times 0,3=21$,

Eumme: 80 ha reducirte (siejaunutïläd)e.

Resucitter Эahteąj(jlag:

$$
\frac{80}{20}=4 h a
$$

․ㅗirflid)e งิahreşid)läge:
a) $0,7: 1=4: x$, hiernus $x=\frac{4}{0,7}=5,71 h a$,
b) $0,6: 1=4: x, \quad$ " $x=\frac{4}{0,6}=6,67$,
c) $0,3: 1=4: x, \quad$ " $\quad x=\frac{4}{0,3} \cdot=13,33$,

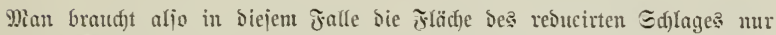

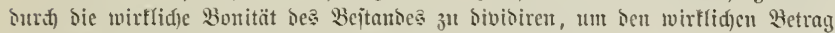
jeines Jafresijdlage? zu fintert.

\section{$\S 78$.

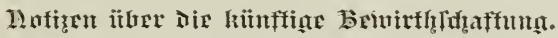

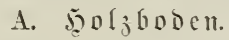

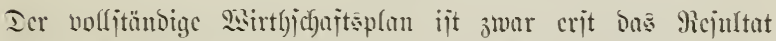

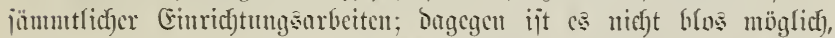

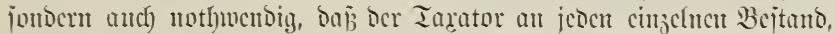
chemjo an jeoc 2Ealoblöje bie Frage itclle, mas banit im mirtljichajt=

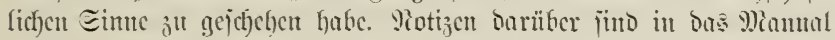
cinzutragen.

Eelbitucritümolich ijt cs, Daj Derartige Potijen um afs vor

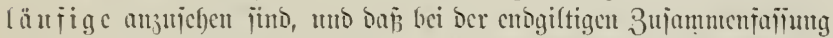

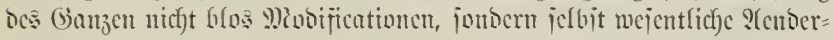

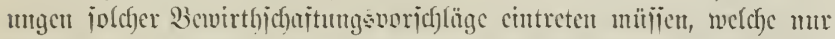

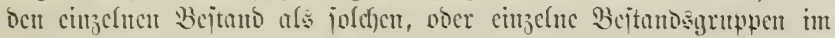

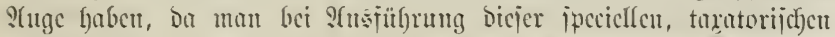

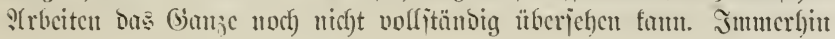




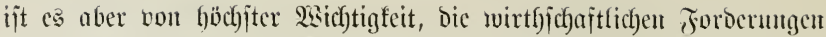

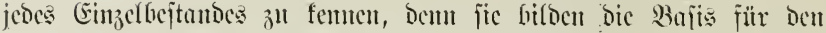

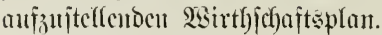

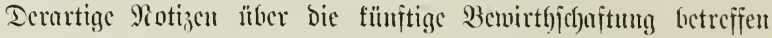
ben gaแz
a) Dic Ernte,
b) Dic Beitandspfifegc,
c) Dic Bcjtandsgrü\#Dung,
d) Forituerbeficrungen ïberbaupt.

Bur Erlähterung mögen mur eiutige Epecialitäten bcifpielšmeife hervorgebohen werben, da eine volljtünbige 3 wammenjeflumg alfer

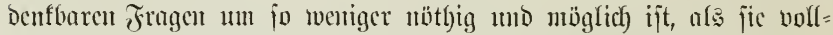

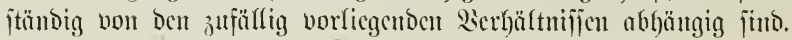

\section{a) BCöhglich Der Erutc.}

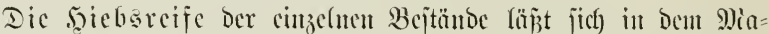

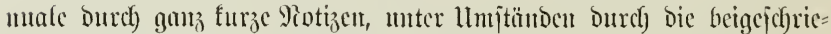

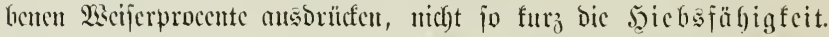

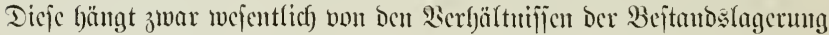

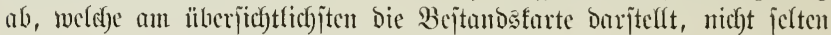

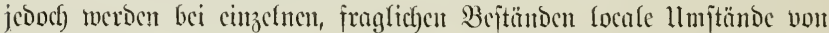

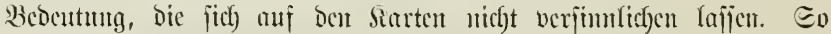

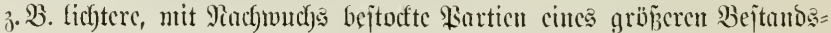

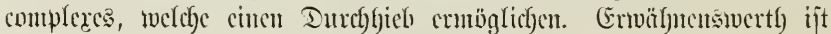

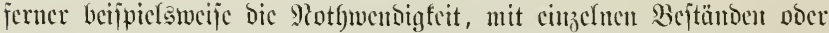

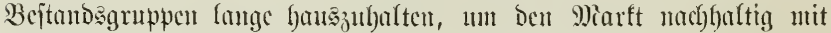
gewiffen Eortimenten 3 berfent.

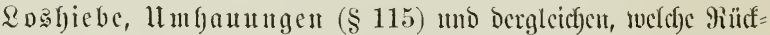

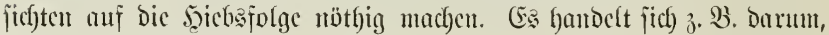

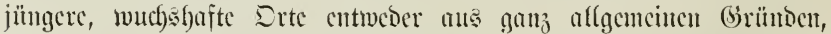
ober ipeciell jur Erziclyutn wertfyoller, itürferer Eortimente beim

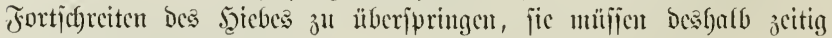
geuth jreigejtellt werben.

Durefopitungu im engeren Sime des 2isortes, alfo wom Etandpunfte der Rubutg ans betracftet. Bei alfen ficr in Fragc

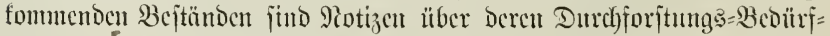
tigfeit ober säligfeit jil gुeben. 


\section{b) B̧ezüg(id) Der Bejtandspilege.}

Durffioritungen im wciteren Eimne, nämlich :(us:(id)tungen

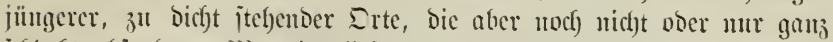
jeblecht abjetshares Diaterial licjern, Räuterungshiche.

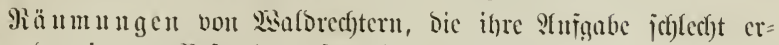
füllen, Den jungen Bejtumo ju jehr benud)tbeiligen.

Sf ufajtungeu cinzeluer, merthooller Baumexcuplare oder ganzer Bicjtüuถc.

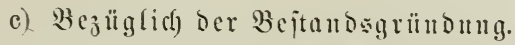

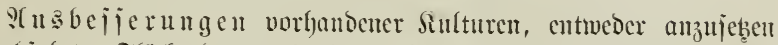

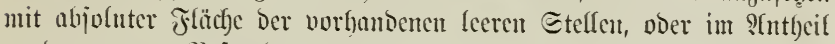

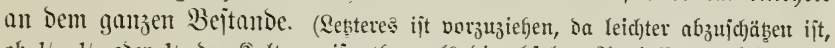

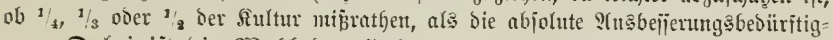
feit.) Dabci ijt bie 2 safl der sjolzarten ins ?age zu faffen.

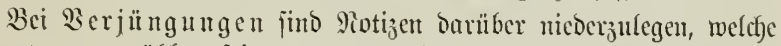

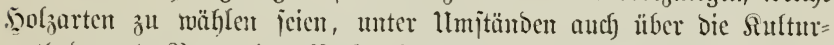

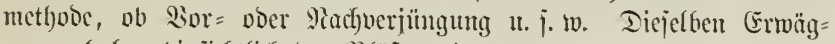

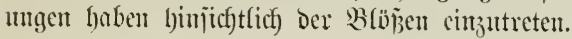

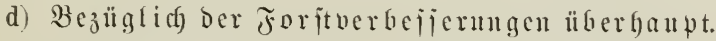

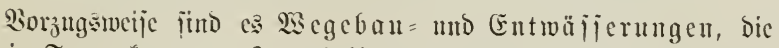
fier in Frage fommen. Imturbalb des Mafmens eines allgemeinen Sisebouplanes ober eines alfgemeinen Entwäjiermigsplanes blcibt überalf noch genug Ietail übrig, um bei ber Betract)tung Der cin= zeluten Bejtände bejondere Rith(fïchtnabune zu verbienen.

$$
\text { it. i. เw. }
$$

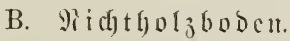

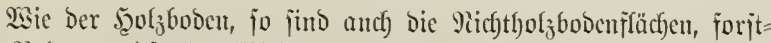

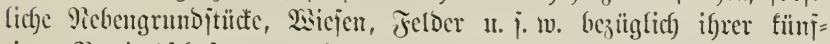

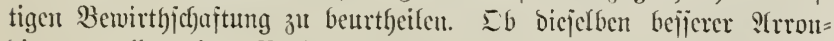

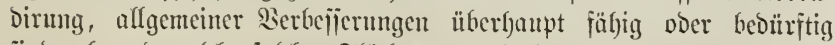

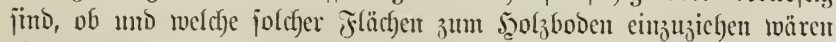
ober nicht.

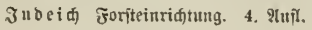


3. Ermittelung Der bisherigen Erträge un Siditen.

\section{\$ 79.}

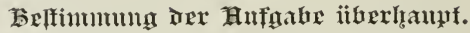

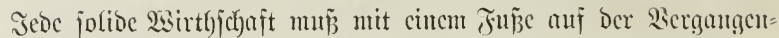

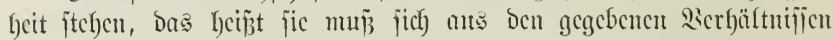
cutrvicfelu, bics jefbit bam, wem aud für bic Bufunft gamz ncucu

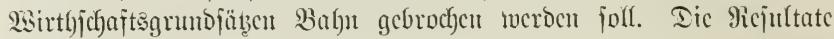

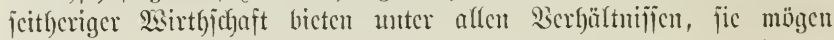
günjtige oder ungïnjtige getwejen jecit, wertfovolles Material jum 2Sciterbat. Es ijt baher hei jeber menen Einrichtung zu beflagen, wenu wegen mangelfafter, älterer buchfüf)rung mur wenig ober gar nich)t genügende lluterlagen gemonmen werocn fömnen. 2ỉo leştere aber irgend wic geboten fint, berjäume man nicht, jie zll fammefn,

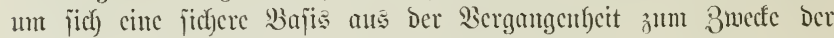

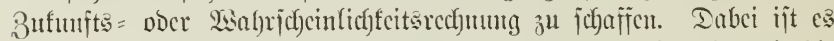

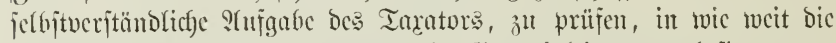

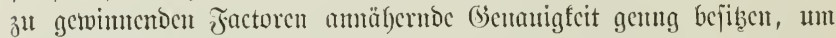

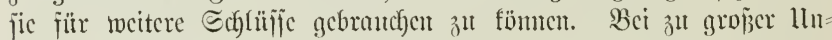

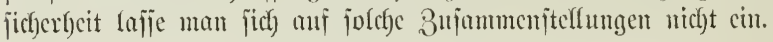

gisir haben es fick zut thum mit

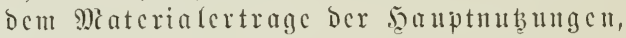

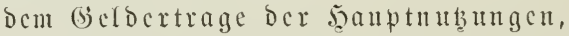

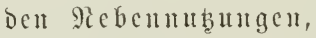

Den Siofitur.

Tnmerfung 1. Derartige Bufaumenjtelfungen für ganze Malocomplexe,

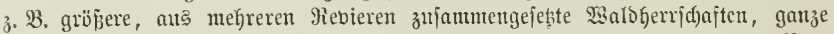

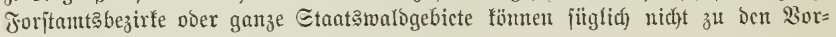
arbciten gerechnet werden.

Itmerfung 2. Sisir geben bier nidyt ipecielle Iabellenjuemas, weil fich

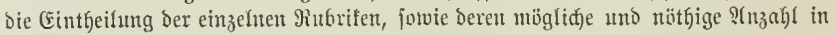
Den verjudiedenen fällen verjdjiçen gejtalten muj.

$\$ 80$.

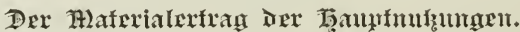

a) Erträgc dcs gauzen Revicres oder eiuzclucr betricbs=

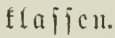

Fït jich allein betrachtet geben berartige jummarijobe Bujanmen=

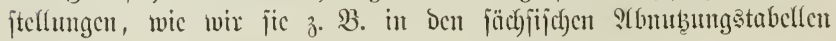


(\$ 104) fintoen, menig s(ub)ult, $4 m$ fo mentyer, je fürzere 3citräunte

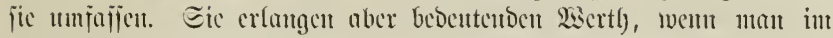

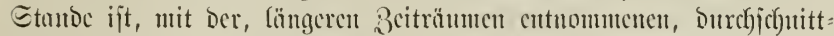

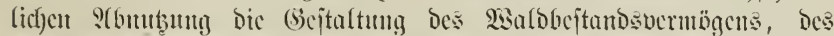

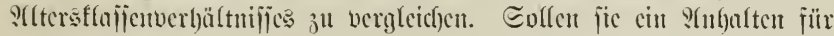

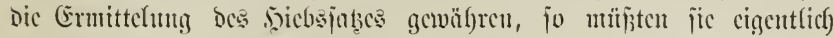

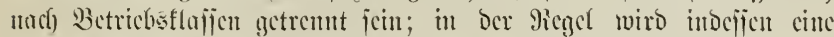

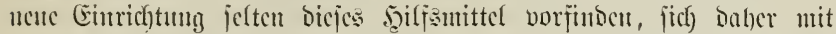

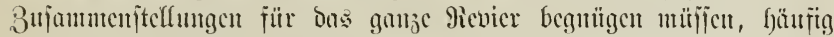

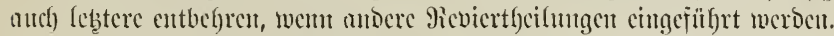

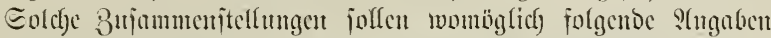

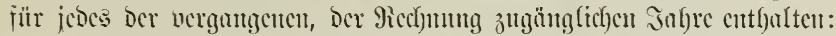

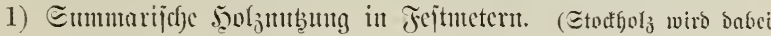

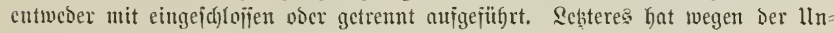
regefmäß̄igfeit biejer 9iuถ̨ung viefes für jidd.)

2) Iremung bicjer 5ुofzmajfe nach) ben 5ुolzarten, wonigjtens in

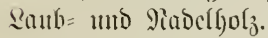

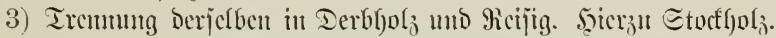

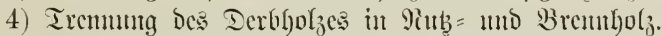

5) Tremung Der gejamunten Ernte in S(btricbs = und 3mijech

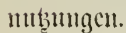

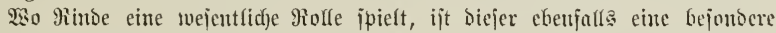
Minbrif zu wibmen.

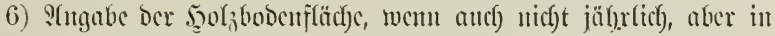
gemilicn 3eitubjontenten.

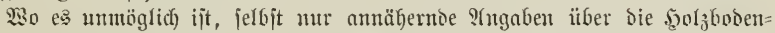
jläche zu gesvinnen, haben jelgjtverjtänorich) afle bieje 8 mammenjteflungen wenig

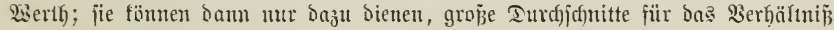
Der einzehten Sortintente unter fich zu gewimnen.

Den wič)tigiten Der unter 1 bis 5 genounten Pojten ijt cinc

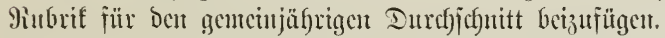

b) Materiaferträge Der cinzelnen Bejtä ube.

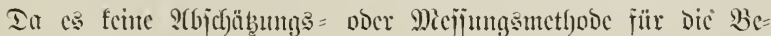

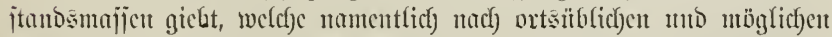

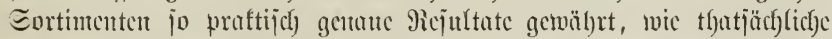

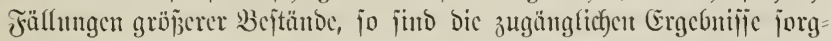
fältigjt zu jumntı. Dabci bandelt ce jich wejentlich mit unt bic

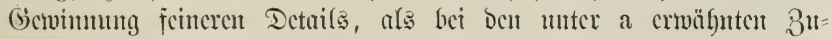
jummenjtellutgen fïr bas ganze Revier: 
a) $\mathfrak{A} \mathfrak{b}$ triebsnuถูน

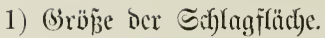

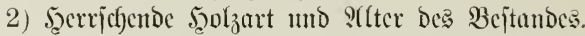

3) Summarifec) Mubung, ausgebrüdt in Fejtmetern (crel. Etodf=

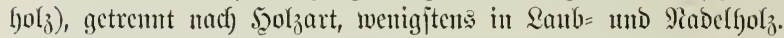

4) Trenmung Des Gejammtertages in Derblyol and Meifig. -

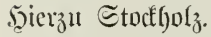

5) Trenmung Des Derblyolzes in 9ublgolz und Bremulyola unter Stugabe Der vericficoenen Gortimente.

B) 3 wijagenut thugen.

1) Brïßje und Ertrag Der Durcl)jorjteten, cinjelnen Bejtände.

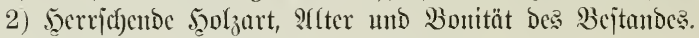

3) Summurijche 9hubuty in Fejtmetern.

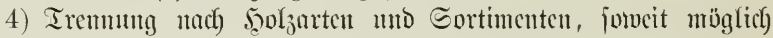
แno nöthig, wie bei ber s(btriebsnubutg.

\section{$\S 81$.}

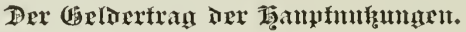

a) Ertrag des gauzen Sicviercs oder cinzetucr Betricbs= flajfen.

Dieje SAngaben laujen paralfel Denen ̈̈ber Den Materialertag.

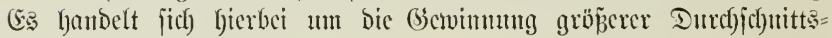
zab)(en, und fonmen in Betracht:

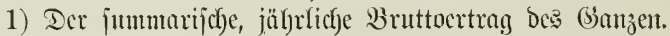

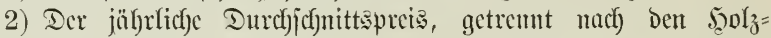
arten, unter llmitünoen audf) jummarijch) für bas Fejtmeter (Derb= $\mathfrak{l} \mathfrak{o l} \mathfrak{l}_{\mathfrak{z}}$ uto Tieijig).

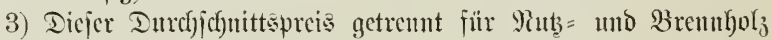

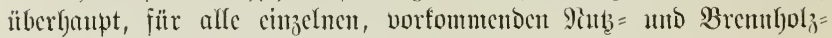
Sortimente, für dic Mimbc, cndlict) für das Etodffolz.

b) Ertrag cinzclucr Bejtändc.

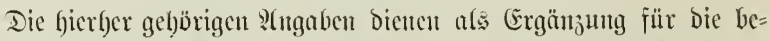
treffende Вujammenjteflung Der Miaterialerträge cinzelner Bejtünde an S(btricbs= und 3rifchemutbungent.

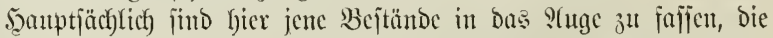
ägnliche Cegaraftere baben ober batten, wie joldhe, bic fitch in ber 


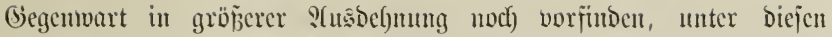

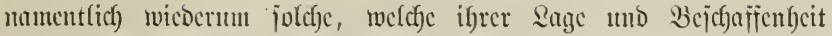

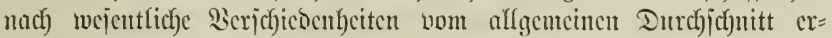

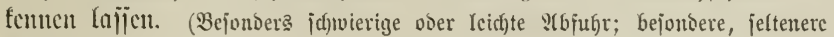
Eortimente u. โ. w.)

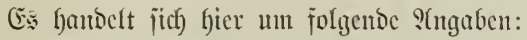

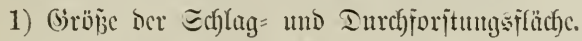

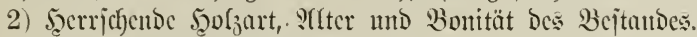

3) Eummarijcher Bruttocrtrag.

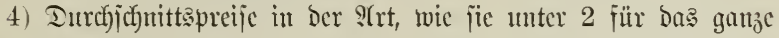
Sicvicr angegeben twurben.

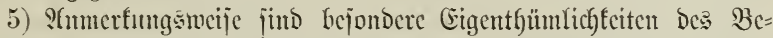

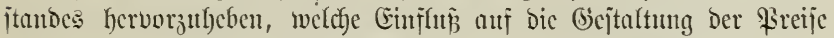

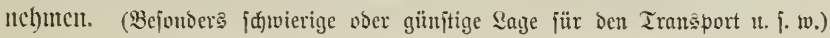

\section{$\$ 82$.}

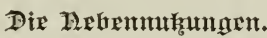

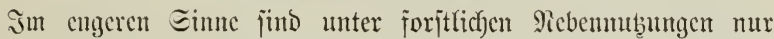

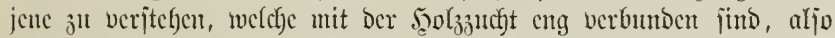

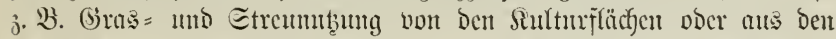
Wejtäntoen, von Den Echucijen ル. j. w. - Erträge jogentumter 9ieben=

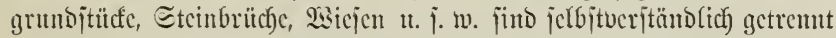

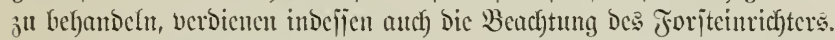

Die jorjtlichen Piebenmtbungserträge hat ber Tarator wic bie 5̧uljertrïge ju betract)ten

a) fïr Das gange 9icuicr ober cinzelne Betrichsflaĩfen,

b) für cinzelnc Bejtände.

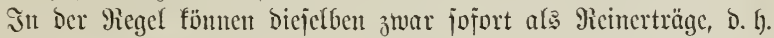
bejreit von Den Gicwinnumgsfojten in Rechnung fommen, Doch ijt cs

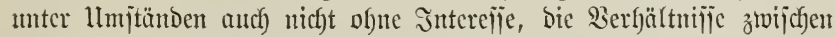
Bruttoertrag und Siojten żu crmitteln.

Die Stugaben für bas ganje Revier haben Den Brwef, bic Gic= jammerträge bes leţteren fennen ju fernen, während bic ber cinjelnen

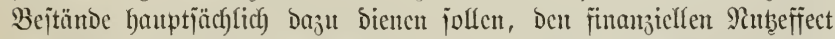

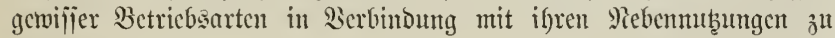
crmitteln.

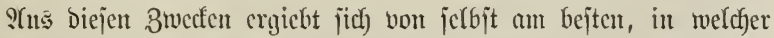
2iseije für ben gegebente Jall betrefienoc 9iotijen zu fammeln jün. 
\$ 83.

\section{Die Tiulfen.}

Dicje zerfallen in allgemeine und bejondere jowoht für die Jeaut $=$, als für bic Nebemutbutugen.

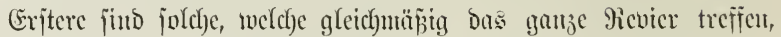

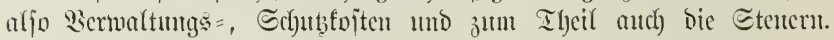

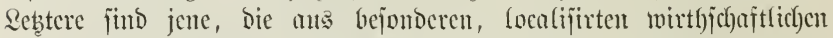

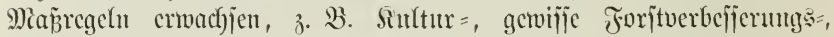

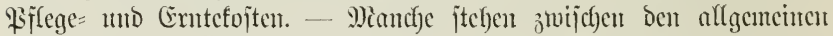
umb bejonderen Sojten, bifben gewifijerntajen cimen llebergang, jo

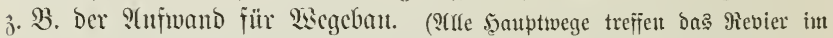
(Şauzen, währento mand)er Rebenuveg unt fïr bie Cinte einzefner Bejtände gebaut

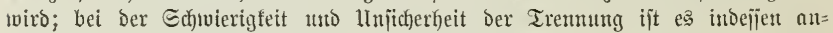

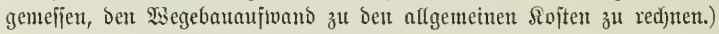

a) Jür das gauze gevier oder eiuzelue betriebstlajjen.

Seier fommen it erjer Pieife bic alfgemeinen Siojten in Betracht.

Der Tarator hat jie getrent fïr bie einzelnen Fubrifen, jum=

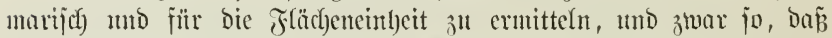

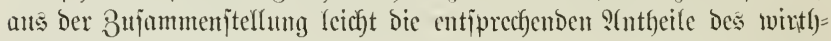
jafaftlichen Grumbcapitates (\$15), jomic bie Factoren zur Bered)umty Der $\mathfrak{B a}$ aldorente gefutudon weroen fömucu.

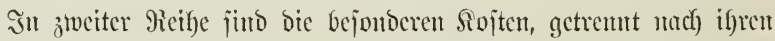

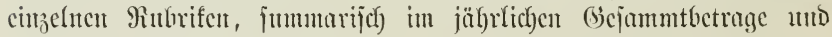

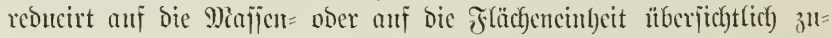

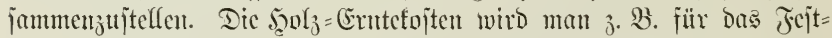

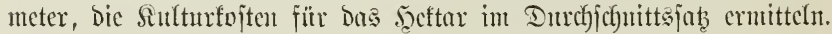

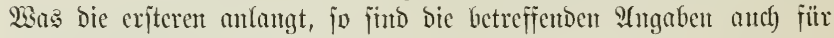
bie einzelnen Eortimtente zu jammelı.

b) Fïr den einjelucu Bejtand.

Der satheil, weldyer bou ben allgenemen Sivitent ben Eüzel= bejtand trifft, crgiebt jicl) ants der 31 jammenjelfutg unter a. Die

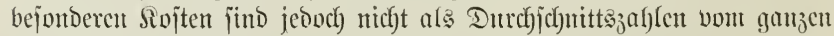
Reviere, jombern in wirflicf)er, Dem fraglichent Eim jefbejtande gehöriger

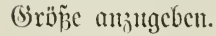

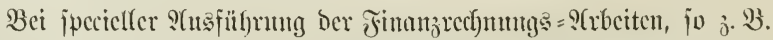

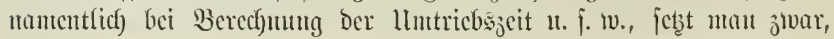


wic wir jrüber bervorfoben, alle Erträge fofort crntefoitenfrei an, 1 m

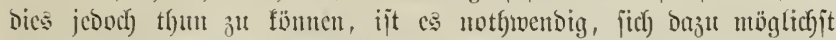
gentue lluterlagen ju verjadafïen.

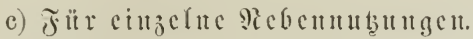

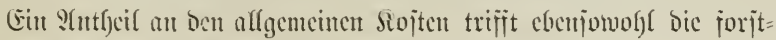

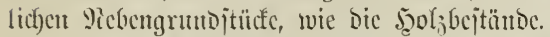

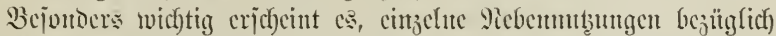
igrer Siojten z"l unterjuchen, um ans Dem Bergleiche mit Dem Ertrage

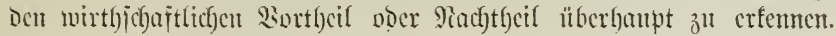

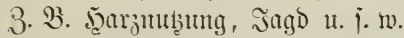

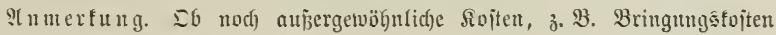

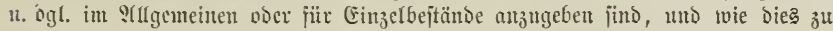
gejd)eben babe, mü jïh aus ben vorliegenden Rocalverbältnijien ergeben.

\section{s 84 .}

\section{W) xifere furgeruman.}

Dic nach den $\$ s 79$ bis 83 gejammelten Piotijen ermöglichen

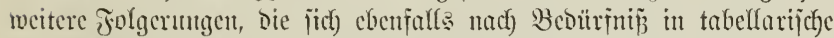

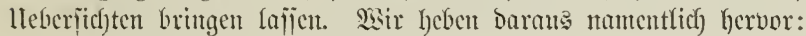

\section{Dic gialorente.}

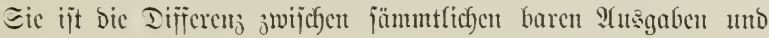

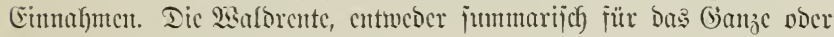
für bic Flächeneinfeit ansgebrütft, ijt jwar mur cit wou ben birecten Baranslagen bejreiter Piohertrag, allein inmerbin hat jic mejentliche

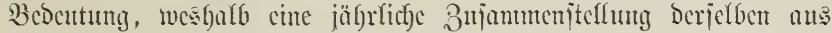
ifren pojitiven und negativen 5auptelementen ju fertigen ijt. 2Bir

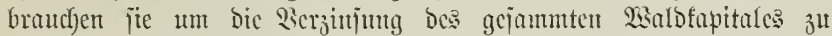

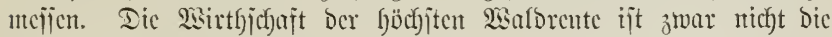
brite, unter Boransjebung gleich hohen Bobenrcintertages ijt aber

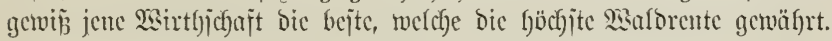

\section{Ias 2ialdefatal.}

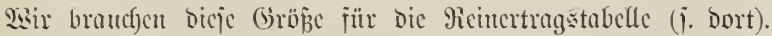

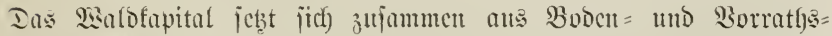
fapital, 2isirtbjchajtsgebäubcu, Gerätfent und antocren Inventargegen= jtämben. Erjtere betben Factoren fund in ber Siegel bie wejentlicfjent. 
a) Das Bobenfapital.

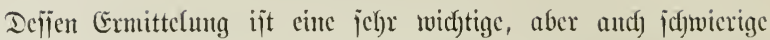
SGujgabe Der Şorarbeiten. Die für bic Einzelfejtänoc gejammelten Motizen üf

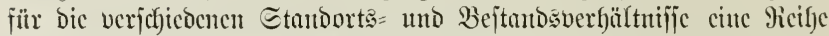

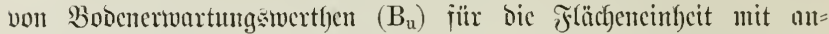

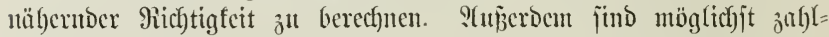

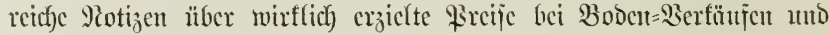

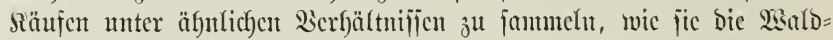
wirtbjechaft bictet.

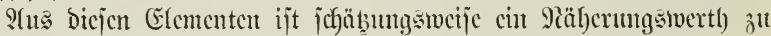
bejtimmen, weldfer ala Bodenfapital (B) in bic Picincrtrags̆tabelle cint= gejtellt werden faum.

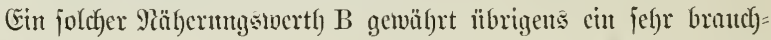

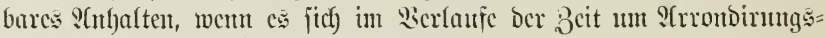

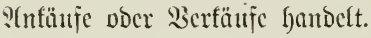

\section{b) Daร 5ูolzoorathalapital.}

Dic Ermittclung Des Sorrathstapitules foll und famt bci Deut

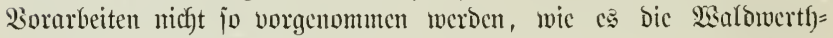

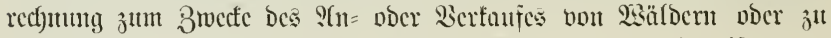

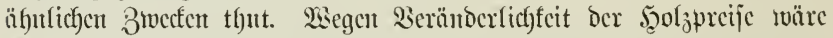

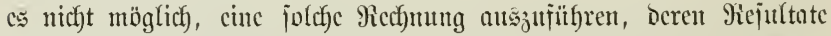

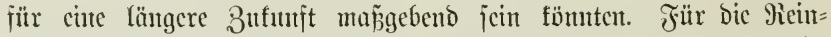

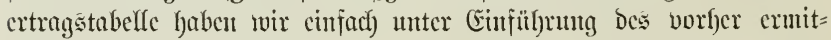

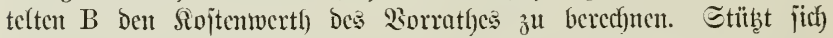

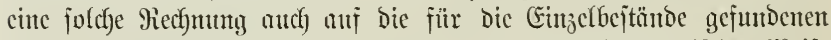

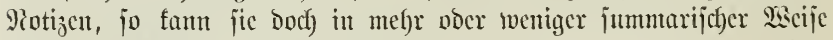

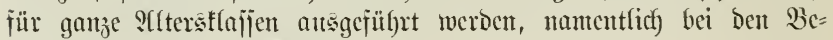
¡täuben Der jüngeren Slafjen.*)

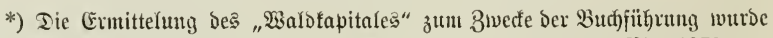

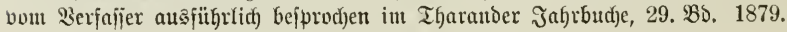




\title{
III. A $\mathfrak{f}$ F $\mathfrak{f t g} \mathfrak{H} \mathfrak{i} \mathfrak{t} \mathfrak{t}$.
}

\section{Ermittelung ber alfgemeinen แแb äuß̨eren Forituerbältuifie.}

\author{
$\$ 85$.
}

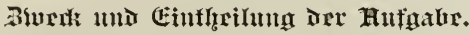

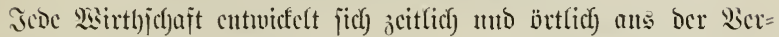

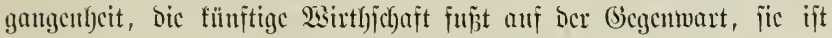
ferner abfjätgig won Den rect)t(ichen, nautentlich Den Eigentfums= Berbältuiffēe, unter benen fie bejtebt, fernter von bem allgementen,

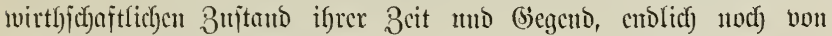
mandéc) anderen llmitämben.

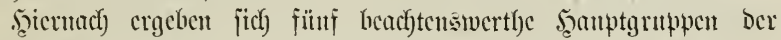

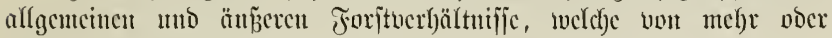
tweniger mafigcbender Bedeutung für bic Eimrichtung und Ertrags:

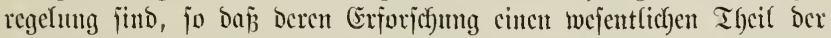
Sorarbeiten mit 9icedyt billoct.

1) Topographijdyc Bicrbältuijic.

2) (bejdidjte des Joritcs.

3) Ėigcuthumsvcrhältuijje.

4) S(llgemein wirthjedaftlidger 3 uftand ber (jegend.

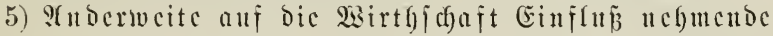
Bacrlältuific.

Einjelue widhtige Epecialitäten, weldye fice in Frage fommen,

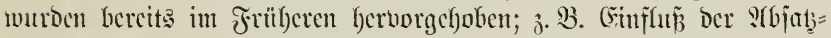
verbältnific แ. j. to. Dic folgenden Paragraphen haben die hetreffen=

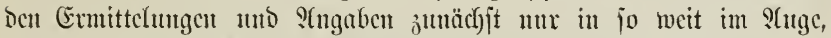

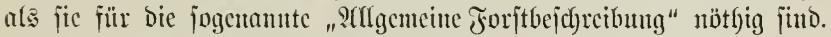

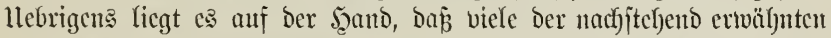

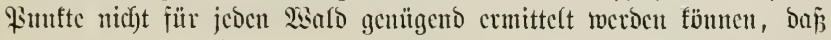

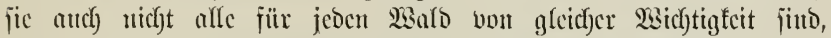

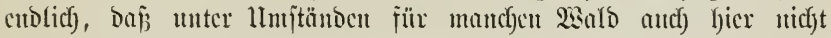
genaunte Dinge widetig werocn.

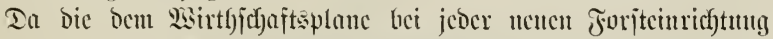

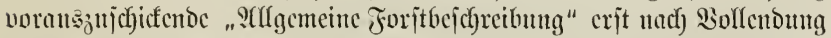

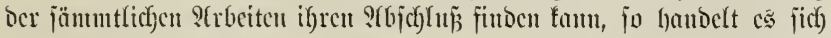
bei ben Sorarbciten nur um dic Eammlung des Etoffes und nu 


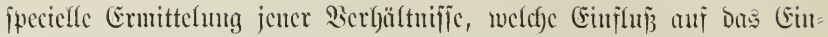
ricftungswerf in majgebentor 2 secife birect nefmen.

\section{$\$ 86$.}

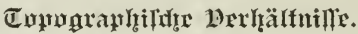

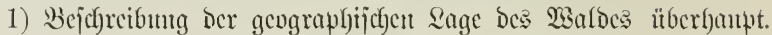

2) S(llgentente flimatijache Sicrfältniije.

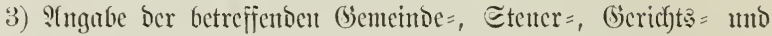

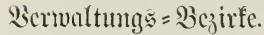

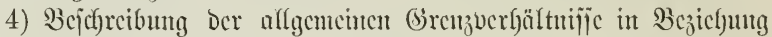

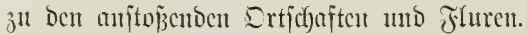

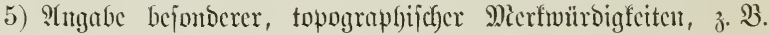
bervorragenoer Berge, von Sieifenden oft bejucteter Drte u. j. w.

Epecialitäten über 1. uno 2. ergiebt bie Etandortsbeid)reibung ber einjelnen Waldojtrecfen, über 4. Daล (3renzregifter.

\section{$\$ 87$.}

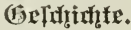

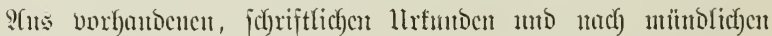
Erfundigungen ijt alles bas zu crörteru, was ans ber 2 sergangenlycit

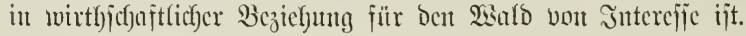

Worjugsucije fantoclt cs fich babci um Folgendes:

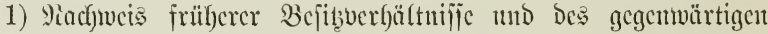
Bejitititcls.

2) Fritfere Betricbart. Etwaiger Wsedjel ber Betricbsinjteme

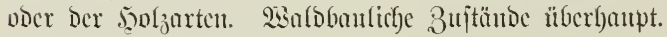

3) Siscen ber frïferen Forjtciurictutung.

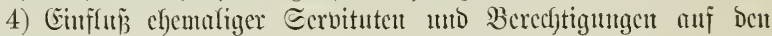
Misulozujtano.

5) Frïl)ere sfbjąucrbältnific, Preije.

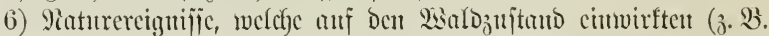

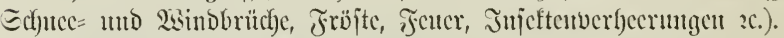

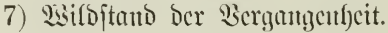

8) Ebenulige Foritfrevel und beren Einflitifc.

\section{$\$ 88$.}

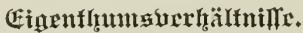

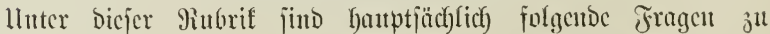
crledigent: 
1. Stut des Eigentlumes.

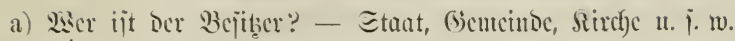

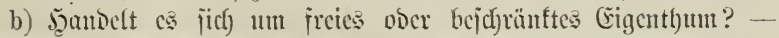

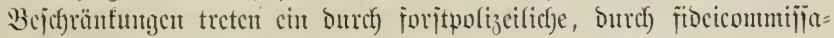

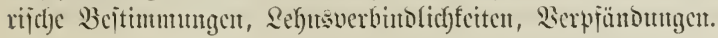

2. Belajungen DCs Jor jes.

a) Dutref) Ecruituteu,

b) Durdf Bcyüutitigutgen,

c) Durcl) Icputate.

\section{Picthe bes jor ites.}

a) Irijt $=$ obcr $\tilde{\Im}$ (öjercirecht

b) Siscycberechtigung aüerfalb des 2isalocs.

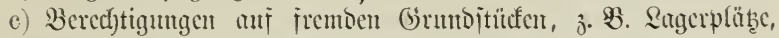
Dicilerītütten แ. $j$. w.

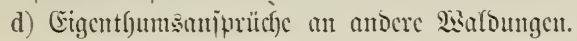

\section{u. j. w.}

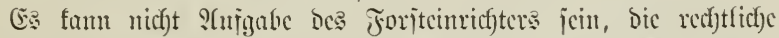

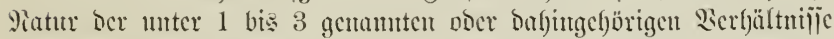

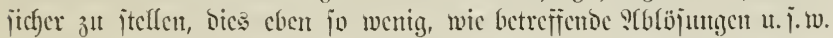

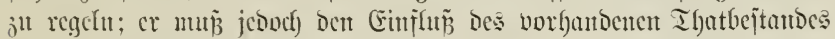
aui Dic 2isirtfjichajt jelbjt wïroigen.

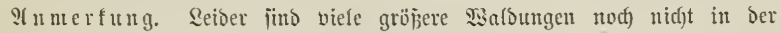

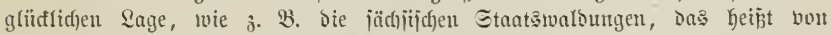

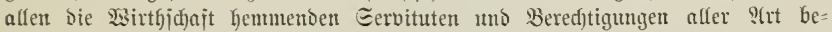

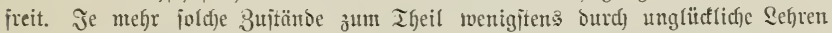
ciner veralteten Etanteforjtwirthichajt

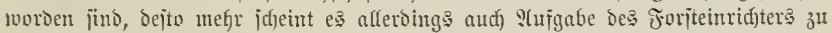

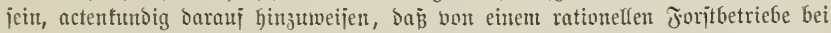

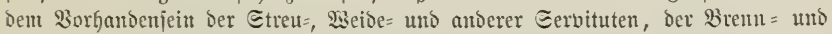
Baufoloberedtigungen gar feine rebe jein fantr.

\section{$\$ 89$.}

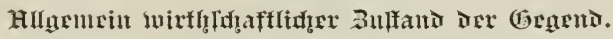

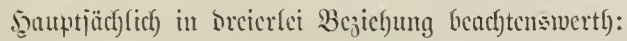

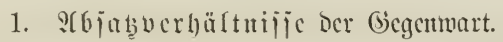

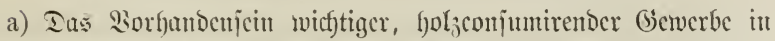

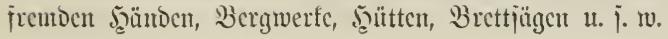




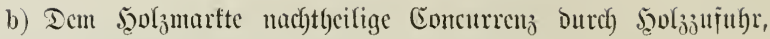
Durch) Bremtgolajurrogate, wic Eteintol)(cn, Torf.

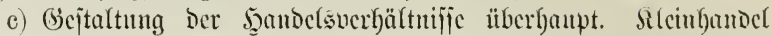

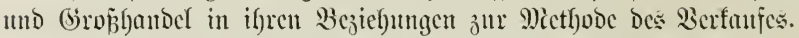

d) $\mathfrak{g}_{2} \mathfrak{2} 3$ bedarf

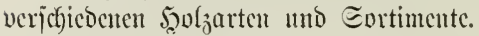

e) Der Bujtand Der vorhandencu Iransportmittel: Etraß̈en,

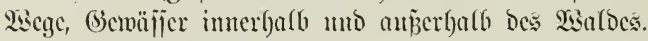

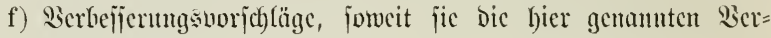
bältniīje nötf)ig machent.

21. j. 10.

2. Sirbeiterverlyältuifje.

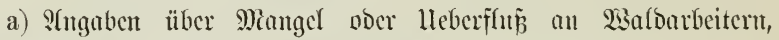
llrjachen bes gegenwärtigen Berljältmijies.

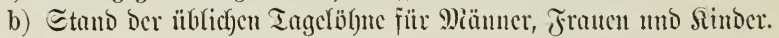

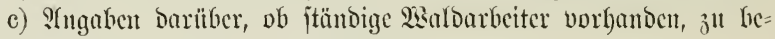
j(f)affen jino vocr midyt.

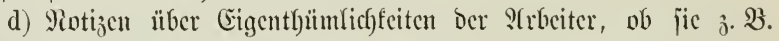

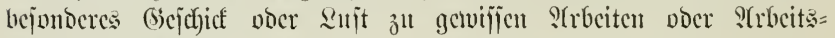
metboden baben.

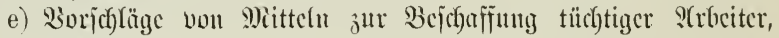
two bicic jeljlen.

11. ז. I1.

3. Foritirevel.

a) llmiang ber Foritfreud überbant.

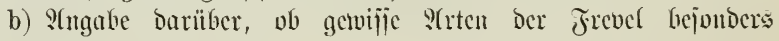
bäufigg finto.

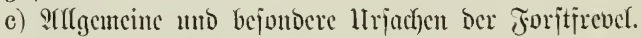

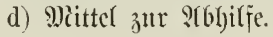

11. โ. Iv.

$\$ 90$.

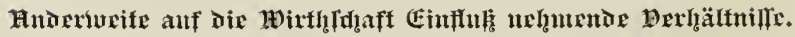

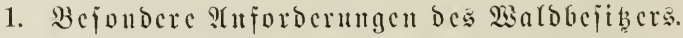

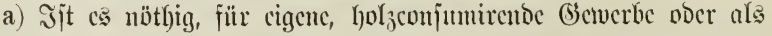
Ieputate bejtimmte Ricferungen utad) Miajic un Eortimente ciujubalten? 


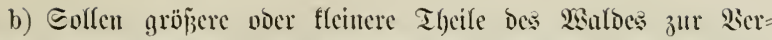

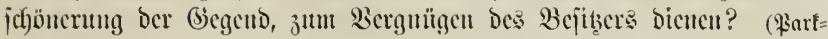
ivirtffidjaft.)

c) Forbert ber Bcjilizer bie Errfaltung cincs gropen 2 sillo=,

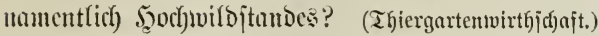

2. Finunziclle Rräfte bes Bejibers.

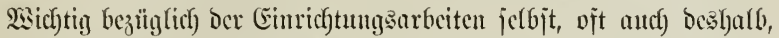

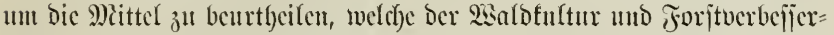

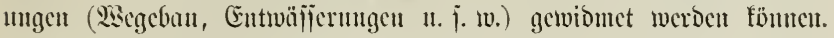

3. Dic Möglichfeit, vortheilhafte Secänderungen mit bem Forjtgrube jelbjt vorjucl)ucu.

Tfrronbirungen burch) Taufich ober Stauf. Llmwandfungen in Feld poer 2isicje.

4. Serjoualuerbältuifje.

a) Dienteinricftutgen überfoupt.

b) Bildungigrad Des Rerjouales.

11. โ. 10.

\section{Alrfdnniff.}

\section{Sarten und Edjriften.}

\section{\$ 91. \\ $\mathbb{3} \mathfrak{i} \mathfrak{x} \mathfrak{c}$.}

Dic jut Den Borarbeiten gefyörigen Sarten und Ecfriften faben

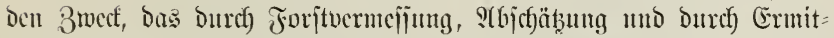

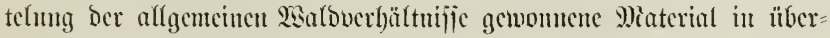

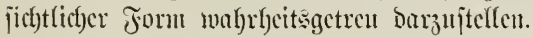

1. Die Siartin.

$\S 92$.

Liarfen iilurtḩautyt.

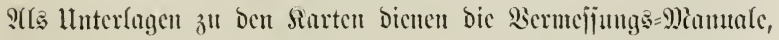

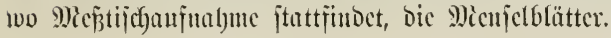


Tür Die Sorarbeiten fann ce jidf) cigentlict) mur um bic SAtlage vorlänfiger Bcjtands: แmঠ Terrainfarten handelı, wäl)rend an

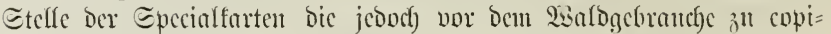
renden Pienjelblätter bienen. Bur nollitändigen Siartirung ijt dic

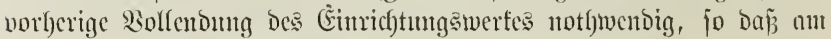

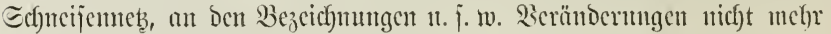
norgenomunen werden. Llm jedoch bic in anderer Bezicl)ung cin $z^{\mathfrak{t h}=}$

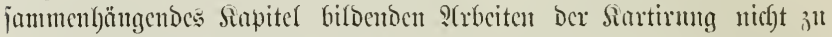

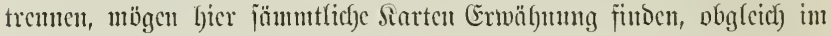
Evjtem Der Darjtelfung Doburd) Dcm Folgenden ctmas borgegriffen

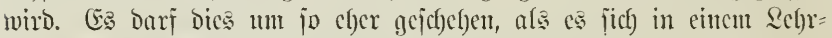

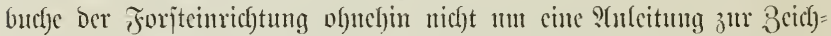

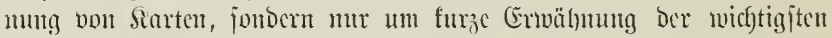
P) Bomente hamdelu famm.

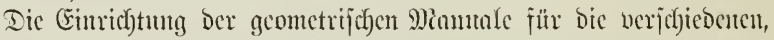

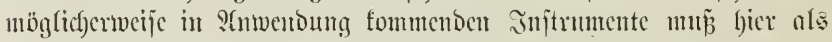
befamt vorauggejetst merben.

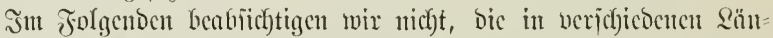

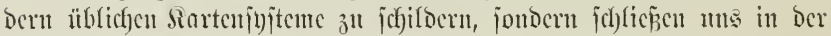

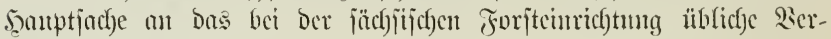
fal)ren all. *)

Bejonbers jei mur herworgethohen, dẩ auj allen Sarten dic 9iorbnabel und bic mal)re Mittagshinie anjutbringen find. Hebrigens müfīen bic Siarten, wenn irgend möglich), jo gezcichnet werben, bap Dic Mittags finie parallel Der Eeite Des Bilattes nad) Sben zeigt, ober

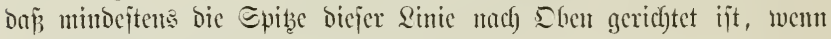
aud melre ober weniger gencigt.

Eämmetliche Siarten zerfallen in folgento Formen:
a) Epecialfarten.
d) Bobenforten.
b) Bcitundsforter.
e) Şicbşugsfarten.
c) Tcrrainforten.
f) Pictbfarten.

\section{$\$ 93$.}

\section{Die sperialkatie.}

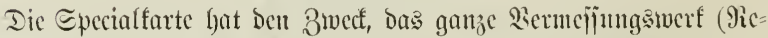

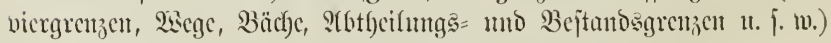

*) In Eadjien erjobien 1879: S(nweijung zum Fertigen Der von ber fönigl.

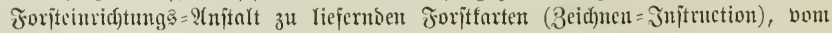
28. Jebrun: 1878. 


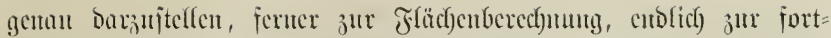

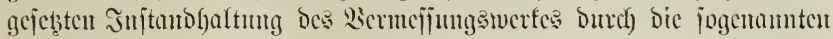

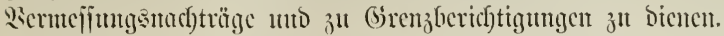

Fïr bic forjtficfen Buccfe gentight cin Miapjitab von $\left.1: 5000 .{ }^{*}\right)$ Dic Epeciulfurten cuthulten:

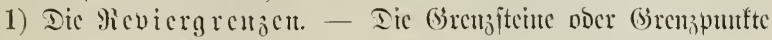

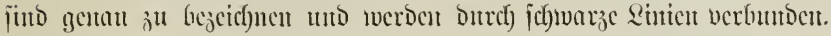

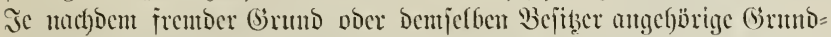

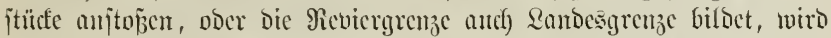

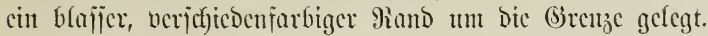

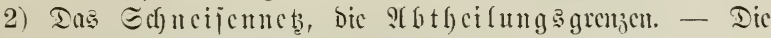

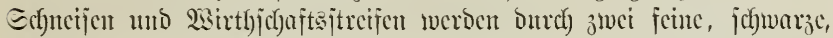

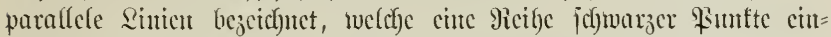

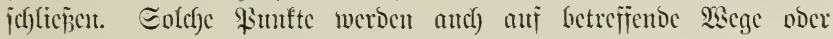
Bäche gelegt, wo bieje s(bthcifmuggrenzen bilbent.

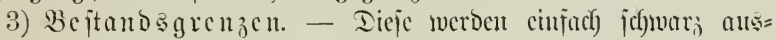
gezogcut.

4) Die Sidferbeitsitciut. (\$ 112.)

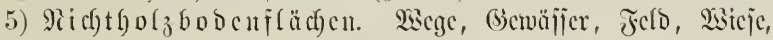

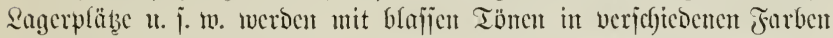
angelegt.

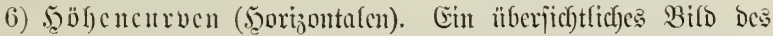

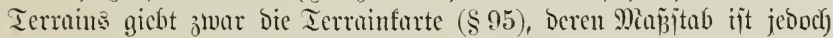

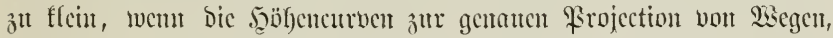

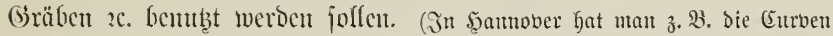
auf Siarten im Miaj̧itab von $1: 5000$, in Baben $1: 4000$.)

Dic Carven werden mit blauter poer brauner Farbe als feinte

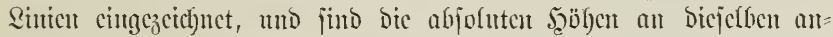
zuijoreiben.

Dex Berticalabjtand Der Curven bürfte cigentlich) bei mentg ge= neigtem Terrain nich)t über $5 \mathrm{~m}$, fïr fteile Şänge fütute or bis $10 \mathrm{~m}$

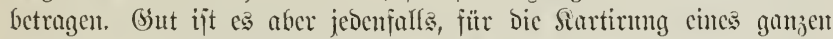

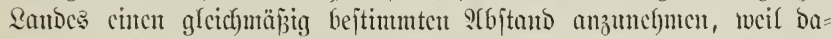

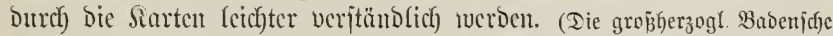

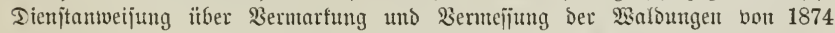
idfeibt alfgemein einen Sertiealabjtand bon $6 \mathrm{~m}$ vor. - In Eadjen iit bisher Das

*) Die färdjitjon Spectalfarten wuroen iut Majpitabe von $1: 4853^{1} / 3 \quad(80$ Piutgen gleid 3 Dresoner 3oll) gezeidynet. Bei nenen ?(tfuahnen wendet man jeit 1871 den Miaß̄jtab $1: 5000$ an. 


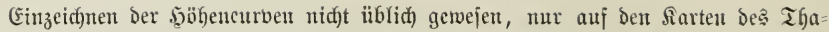

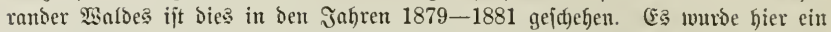

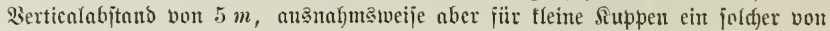
$2,5 \mathrm{~m}$ gewö̈glt.)

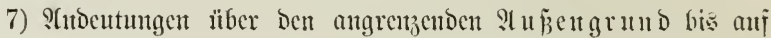

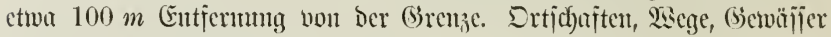
It. i. w. („lteberarbeitung“.)

8) ๔d)rift.

a) Titel: Mame bes Revieres. - Gröjéc beffellon ober bes auf

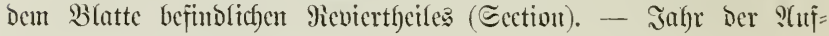
nafme oder Berichtigutg.

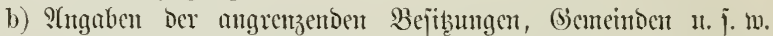
unto Siufturgattungen buref llmjchreibutg an ben Brenzen. - Niuneru Der (3rrenjzeicben.

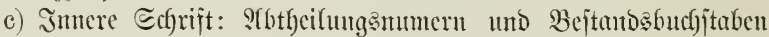
j(f)warz. - Nichtholzbodenfläcben mit rotben Bucfjtaben. - Nimern

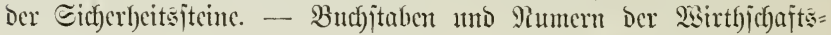
ftreifen und Echuteijen. - Benenmutg Der Wege, (Betwäfifer unb be= jonbers widftiger Siunfte น. j. w. - Breite oer, mit Fläche ôt ver= recfutentom, "gebauten" 2isege.

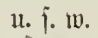

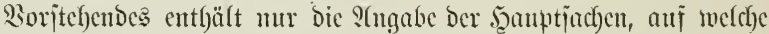

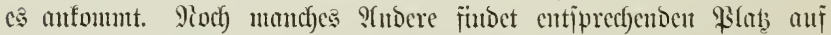
Den Epecialfartent, doch hüte man fich nor bem 3uviel, meil badured)

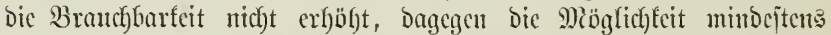
crjejwert mird, alfe Berämberungen, welde im Saufe ber 3eit nor=

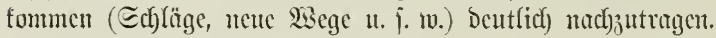

Die Epecialfarte mird in zwei Eremplaren angejertigt: Der Brouillou und dic Eopic.

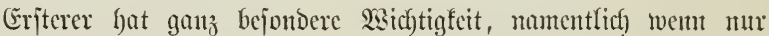

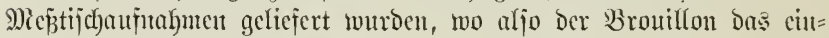
zige, braud)bare Jeiffsmittel ijt, um neue Sarten anjertigen zu fömen. Ein bejontorer Borjug Der trigonometrijefen ?(ujnafmen bejtebt eben

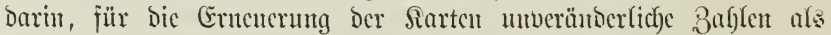

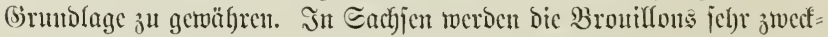
mäpig nicft anj mit Seimmano, jondern mit bünner, aber jejter Pappe 1!nterzogenes \$apier gezeicfnet, und um bic Durch) das Cülaujen ent=

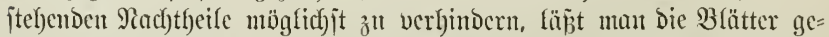
möbnlich längere Beit in Borrath) liegen, che fie zur Benthung gelangen. 
Bon ben Broutllons weroen bie Copient für Den (Sebrauch Der

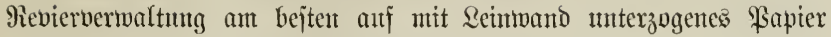
übertragen.

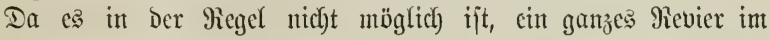
Maßjitabe von $1: 5000$ auf cin Błlatt zu bringen, fo theilt man eriferes in melyr oder weniger "Sectionen", Derent jede cine be= jonbere Epecialfarte bilbet. (Eine weitergehende Bedeutung hat bieje Sec= tionstheilung nid)t.)

Recht empfeblentwerth futo bie in \$reupen üblichen Special= Eouponfarten.

\section{$\S 94$. \\ Die Belfantograrte.}

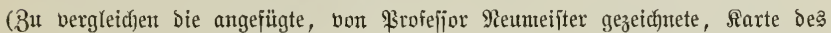

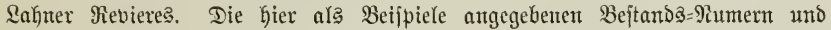
Buchitaben beziehen ïh) auf Sieje Sarte.)

Die Bejtandsfarte hat ben Bwedt, ein überjichtriches Bild von

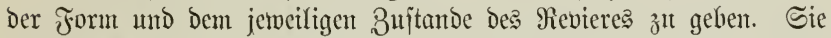

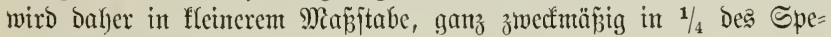
cialfartenmafjitabes, aljo $1: 20000$ gezeiçntet.

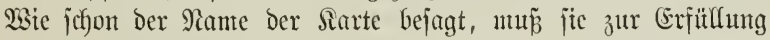
ibres 8 wedtes enthalten:

1) Das Bild der vorlyandenen 5evr

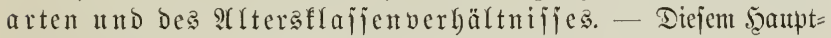

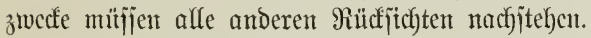

Für den feochtwald erfalten die verjojiedenen, bejtandbildenden

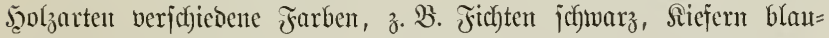
j(b)

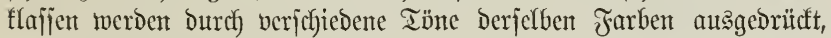

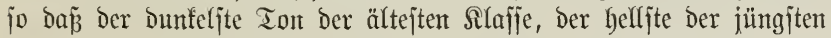
gegeben wird.

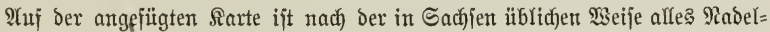

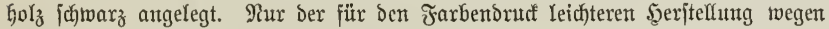
find bie veridiedenen Iöne burd) S(d)raffinung erzeugt worben; Diefe S(hraffirung

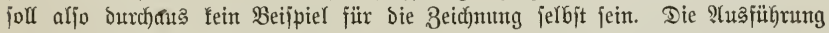

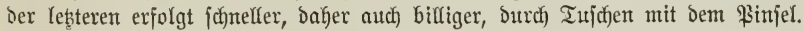

WSie das beigegebene Sdjema zeigt, ijt 3 . B. 1a ein Radelfolzbejtant I., D. h.

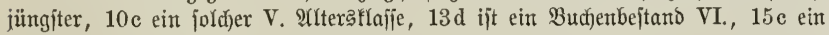
Eirfenbejtand III. Atrterstlajie.

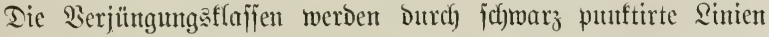

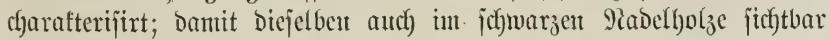


merden, giebt man ben helferen Ton cinter jüngeren Sllterstlaffe als Unterlage.

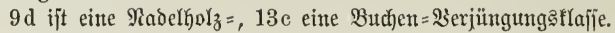

Rieder = und Mittelmald werden grïn angelegt, eriterer ofne, lebs= terer mit eingezeichneten Bäımd cen.

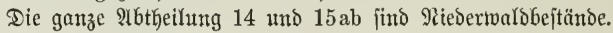

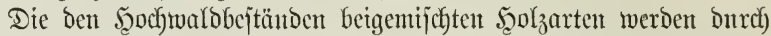
Einzcichnung fleiner Bäume verjđdicdener Farbe ntmb Form angegeben.

Dex Bejtand $10 \mathrm{~b}$ hat eine ftarfe Einmiffutng bon Birfen, Der Bejtand $12 \mathrm{c}$

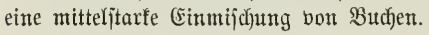

Plentertwald mürde die Farbe jeiner Şauptfolzart erfalten, Duref) Einzeidfyung fleiner, weiper 3cidfen in bejtimmter Form, viefleidyt

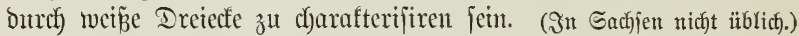

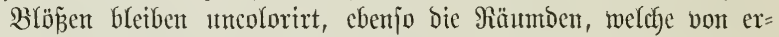
iteren Durch Einzeicfnutg von Bäumchen unterfefjeden werben.

Blöpen find $1 \mathrm{~b}, 9 \mathrm{e}$ und $10 \mathrm{a}$.

Dic Şiebsorte bes nächjten Sahrzehntes werden als Nachverjüng=

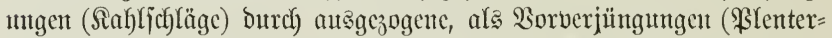

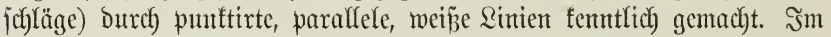

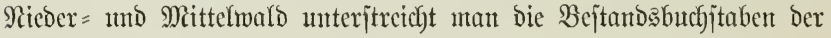
ઝॄicbsorte rweī.

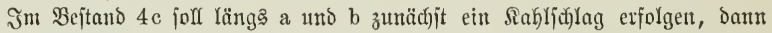
aber Borberjüngung eintreten. Die Roshiebe (§ 115) 3ab, 4e, $6 \mathrm{~d}$ ttho $7 \mathrm{~b}$ werden

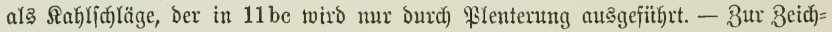

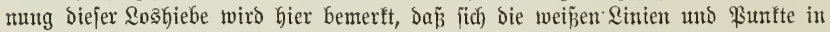

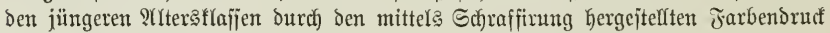

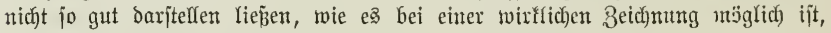

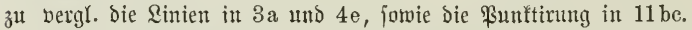

Der meiß̈punftirte Theil des Buchenbejtandę $13 \mathrm{~d}$ bedentet einte Sorverjïng= ung, Der Eidfenbejtand 15e forf fahl abgetrieben twerden. 14abe und 15 ab jitno Siebso

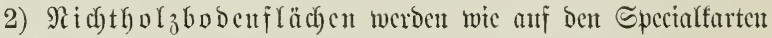
Durch verjchiedene Farben angelegt.

Lit, a eine $\mathfrak{B}$ ieje, lit. b ein Fels.

3) $\mathfrak{B}$ ege und (Semäjicr. Soweit biejelben nicht mit Fläclye

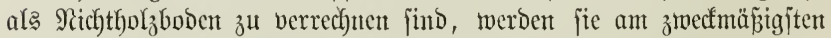
mit Dedfarben als einfacke rotbe mo blaue Sinient gezeidfnet, Fur jteige rotf) punftirt. Pit Fläche zu verred)nende breite $\mathfrak{B c g e}$ erl)alten einen lichten, rotbgelben Ton no jeitliche Einfajīntg mit einfad)en,

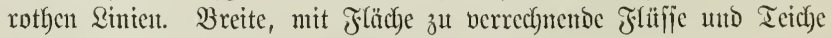
werben lichtblau angelegt mit feiten, blauen Sinfajpungslinien und 
blautem Schatten. Ebenjo werben Wege und Bewäjier auf den Epe= cialfarten gezeichnet.

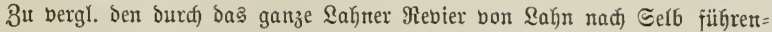

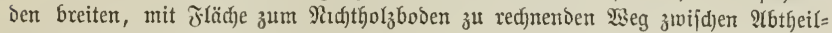

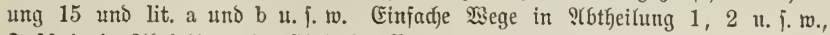
Fupiteig in Stbtheilung 6. (Einjacher Bad) in 6,7 น. j. w.

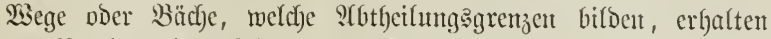
jaymarze Puntte, wie auf der Specialfarte. 10 und 11

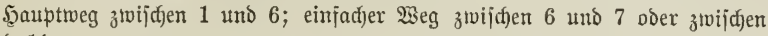

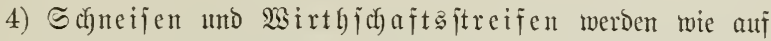
Den Epectalfarten Durch zwei jeine, ichwarze, parallele Sinien bezeidynet,

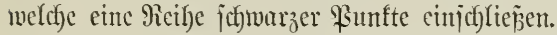

5) Die Reviergrenzen merben burd) veridjedente Farben bar= gejtellt.

6) Diantherlei andere Dbjecte, wie Torfitiche, Jeljen, Şäujer 1t. . w. erbalten beitimmte Beichen.

Jeljen in 10a und 14 abc. Forjtgaus in lit. b.

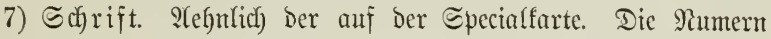
Der S(btl)eilungen und Bucfjitaben Der einzelnen Bejtänbe find bie

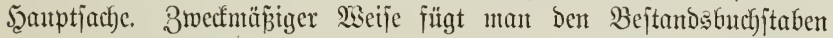

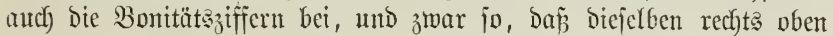
neben ben Budfjtaben gejchrieben werden, went ber Bejtutb Der jün= geren Jृälfte ber Durch Den Farbenton dyarafterifirten SUtersffafje angefört, bagegen rechts unten uteben Den Bejtantoshudjitaben, wenn Der Bejtand zutr älteren Şälfte Der ?(ltersflajje zäl)lt.*)

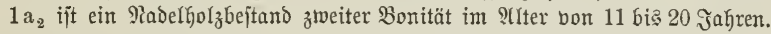
$6 \mathrm{c}^{2}$ ijt ein 21 bia 30 jähriger $\mathfrak{R a b e l g o l} z$ bejtand ztweiter Bonität.

Ulebrigens jind auj Der Sarte bie Bonitäten nad) der in Gadjjen üblidjen

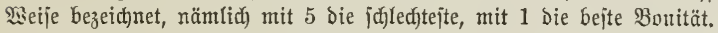

Schneijen und Wirthjofaftsitreifen erfalten ifyre Paunern, bezie= heritlich Buchjtaben wie auf Der Epecialfarte.

Grenz= und Sicherbeitsjteine werden nicht eingezeichnet, folglich audf) Derent Pamern nicht eingejdyrieben.

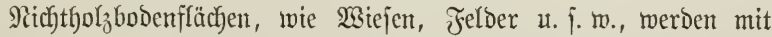
fleinen, rotfen Butfjtaben bezeicfnet, wie auf Der Specialfarte.

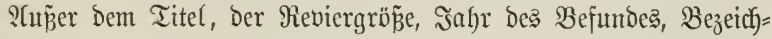

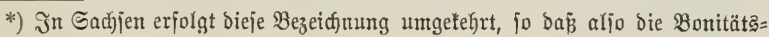
zifïer oben neben bem Bejtand引budjitaben bie ältere, unten bie jüngere ఏälfte ber

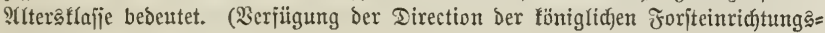
Injtalt bom 8. Samuar 1884.) 
mung ber angrenzenocn Flächen u. j. w. finden recht zmectmäp̈ig neben,

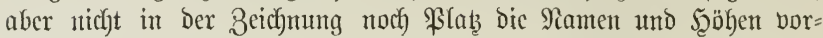
tommender Berge oder fonjt hervortretender $\mathfrak{B u n f t e}$

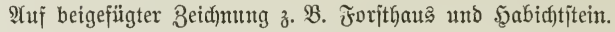

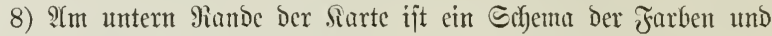
eine Erflärung Der Beichen zu geben.

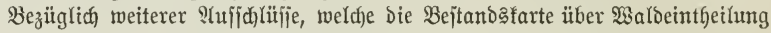

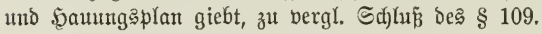

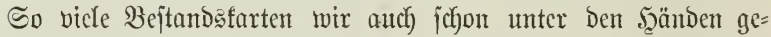
habt haben, es ifit uns noch teine vorgefoumen, welche cin jo beut=

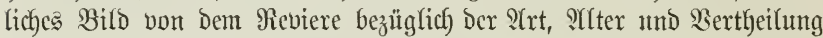

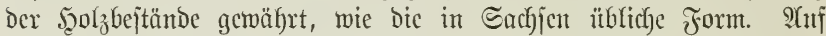

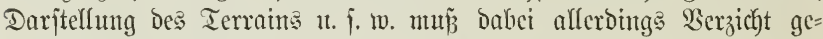
leijtet werden, weil bic bunfeln Töne ber selthölzer bies nicht gut geftatten. Un Terrainzeichnungen Deutfich herbortreten zu lafien, hat

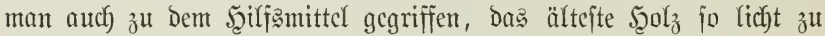
halten, Dẩ es die unterliegende Schraffirung ober bic Scorizontalen

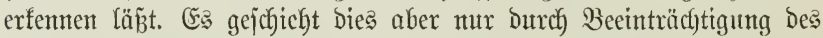

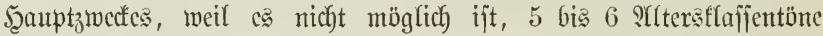
jobarf genug marfirt zut geben, wenn man nicht für bie ältejte Sllaffe einen ganz Dunfelı Ton wäh)lt.

Die in mebreren Excmplaren*) anzufertigenden Bejtandsfarten jüt bei jeder 10 jährigen Revifiton ju ernetern, ç empfichlt fitch

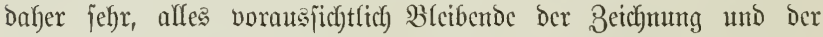
Sdyrift litbographiren zu lafjen, woburch bie arbeit Der Copic we= jentlich erleichtert wiro.

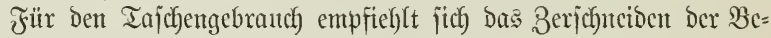
ftantosfarten, um cin handriches Jormat zut crbalten.

\section{$\S 95$.}

\section{Die Terrainkarte.}

Der Brwed Diejer Sarte ijt bie Darjtellıng Der Icrrainverfält=

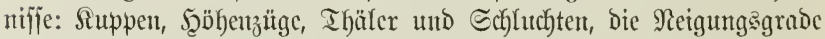
Der Bergfänge. - Scauptjächliç, Dient jie zum Entwurfe bes Schncijen= und Des Wegentęcs.

*) In હadjen werden 3 (Exremplare gezeidjnet, eines für die Revicrbermalt= ung, cines jür Die Dberforjtmeijterei unt eines für bie Forjteinridjtungsanjtalt. Dą leb̧tere Exemplar bient zugleid) zum (bebrauche für bas Minijterium. 


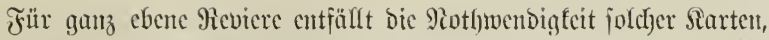
jie fint aber umentbefreticl) für (bechirgstualdoungen.

Der Maājitab von $1: 20000$ genügt für bie Terraintarten.

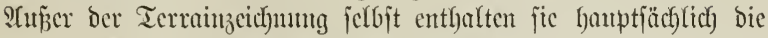

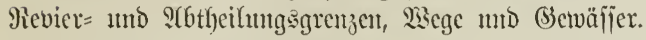

In Eachjen werben bieje Surten nad) Der jogenanten Refymant=

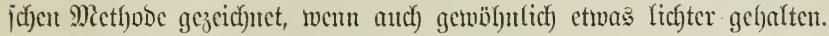

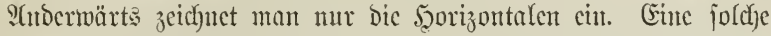

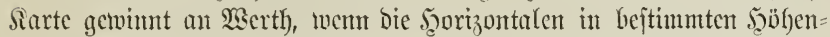
abjtufungen aufgetragen, and bie şöhen ferbjt angejefricben werben.

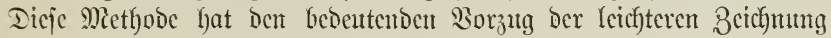

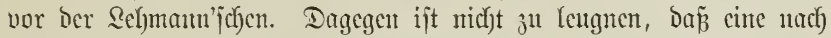

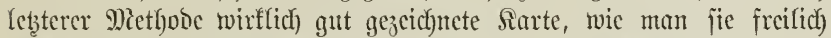

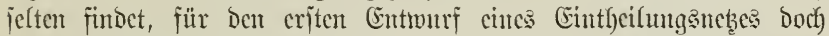
cinen flareren Gicjammtüberblicf über bic Ierrainverfältnifje gewälyrt, als bie Şorizontalen alleint.

Dic Ierrainfarte wiro nicht überflüifitg, went aud bic ફ̧ori= zontalen auf Dor Epccialfarte cingetragen jütD; Ieştere fam Des

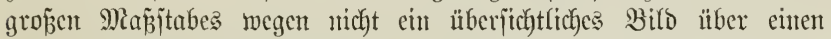

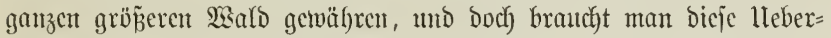

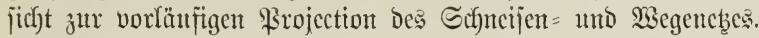

$\S 96$.

\section{Die fontrukate.}

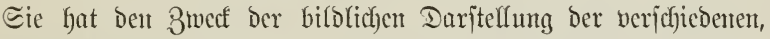
vorfommenten Bjebirgsarten. Da es genügt, diefelben in licht ge= haltenen Farben zu untericheiden, fo läp̧t jidd) bieje Siarte mit ber Terrainfarte rect)t gut vercinigen, ofme Der Deutflicbfeit leb̧terer Ein= trag jut thun; autd) cine graphifiche Darjtelfung ber Etandort șbonitäten

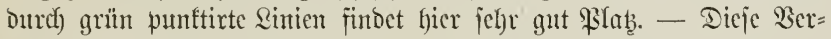
cinigung erfolgt z. $\mathfrak{B}$. in Sachjen.

\section{$\S 97$.}

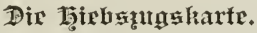

Dicje Siarte hat Den 3wect, Dent projectitten Bang Des Şiebes

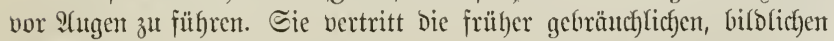

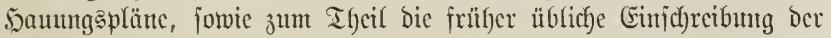
Aieriodenzahlen. 
Sie enthält mur Revier = uno Afbtheilungsgrenzen, 2Gege uno

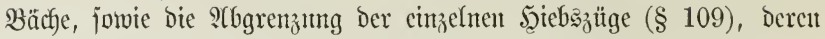
Richtung Durch einen grünen oder rothen \$ffeil angebeutet wird.

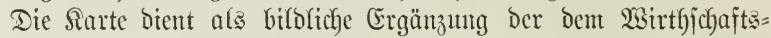

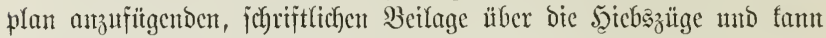

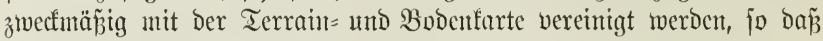
biejc Drei Sarten ein einjiges Blatt bildon. ITndernfalls wählt man ১az̆ eine der für bie Sejtandsfarten angefertigten Rithographien.

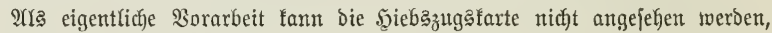

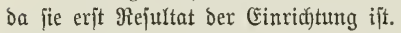

\section{$\S 98$.}

\section{Die Defrltarte.}

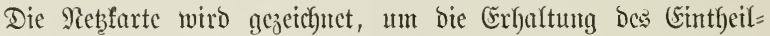

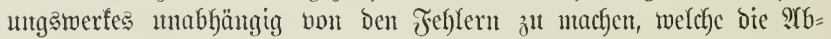
nahme ber Maß̃e vou ber Specialfarte unbermeiolich) mit jich bringt, went es gilt, verloren gegangene Sidjerbeitsfteinc zu erjeşen, ver= wachjene Schneijen wieder aujazhauen.

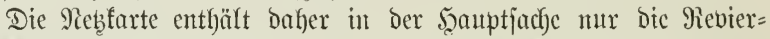
แn 9(btheilungsgrenzen und bie Sicherbeitsiteine. S(ngejurteben werden die Maje ber Gorizontalen Entfernung von einem Steine zum anderen und die $\mathfrak{W}$ infel, welche bic Gerhneijen bildon.*)

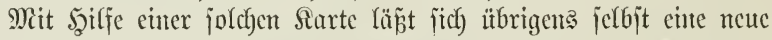

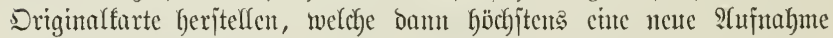
Des Details nüthig mad)t.

Sit bas Schneijenteb trigonometrifich alfgenonmen, bant braucht man allerdings bicje Siarte nicht, jie ijt aber immerfin cin gittes,

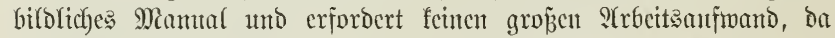
man recht gut cine Bejtandsfarten = Sitfographic bazu verwenton fann.

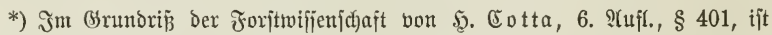

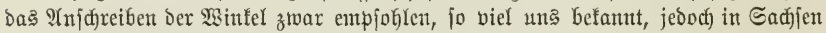

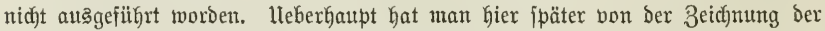

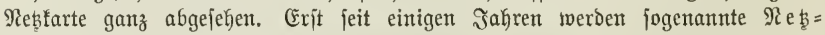

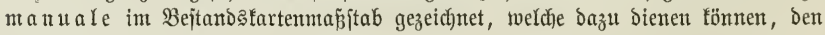
Broulllon im ₹ralle eines $\mathfrak{B}$ erłujtes z̆

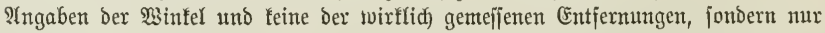

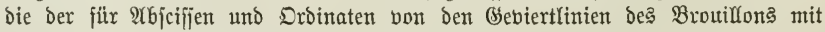

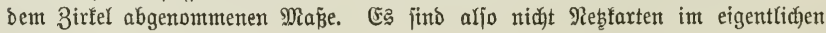
Sinne beకె $\mathfrak{3 0 r t e s . ~}$ 


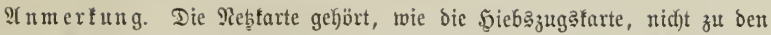
eigentlidjen Borarbeiten, wir erwähnen biejelbe fier nur, un bas Sapitel von ben Rarten nid)t auseinanberzureiß̈en.

\section{Die Shriftet.}

\section{$\S 99$.}

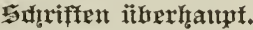

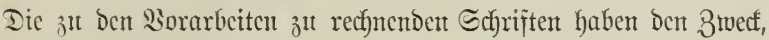
Das Dutref) eritere gemomente Material in (cicht brauch)barer, überjitcht= licher $\mathfrak{B a j e i j}$ jujammenzuitellen.

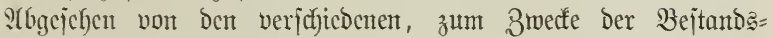

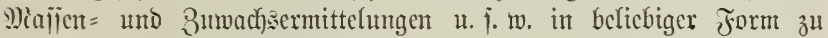
fül)renden Mantalen, añ bie fier nicht näber einjugefoen ijt, f̈nto folgende Ecfyriftitüdéc zu nenten:

a) Ias Taxationsmanual.

b) Die Bejtands= ̊llajientabelle.

c) Die Silajientïberjtcht.

d) Die Etamoorts= Rlajīentabclle.

e) Die Ŝfnutbungstabelle.

f) Das Grrmaregijter.

\section{$\S 100$.}

\section{Das Caxafinmmantal.}

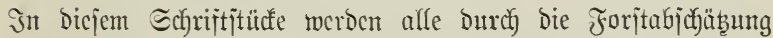
gemonnenen Plotizen ïber Den forjtlichen Thatbejtand uno über fün $\tilde{f}=$

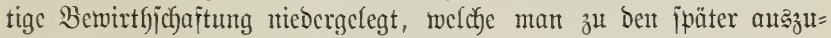
füfrenden $\mathfrak{A}$ rbeiten zu brauclyen gedentt.

Das Tarationsmanual fann jwar in ganz freier Form gefüfrt

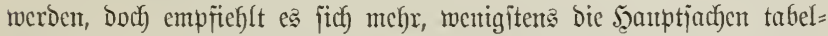
larijch zu vroncm, um baburch crjtens llebcritchtlicffeit zu gewimen,

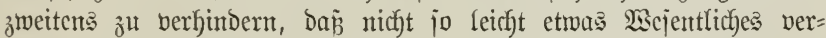
gejien wirs.

Dic Gişger in Sachjent übliche Tabcllentorm entipricht nicht rectyt, weshalb wir cinc andere, etwa folgende, empfehlen: 


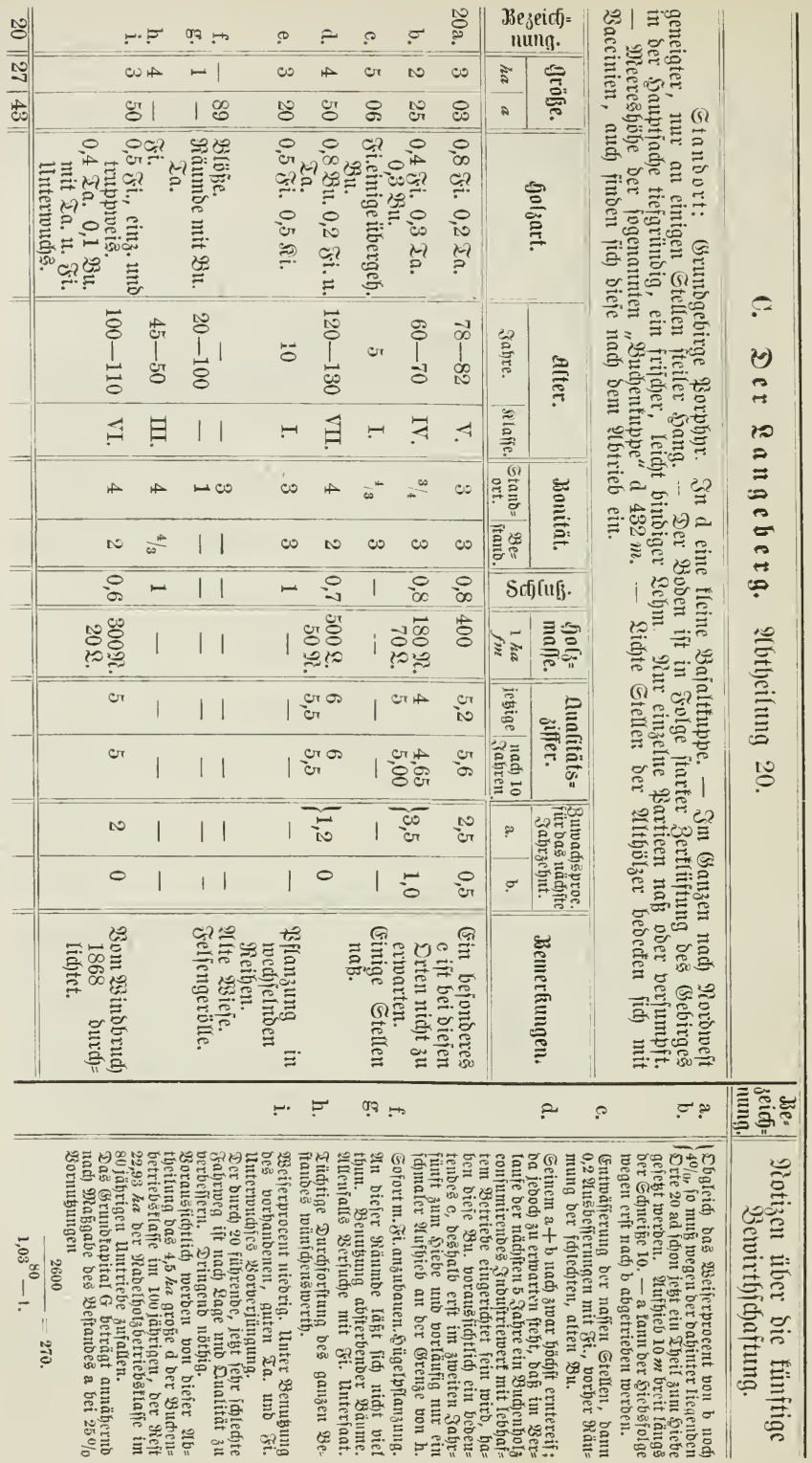




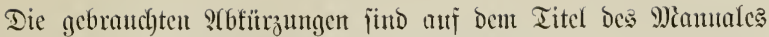
ju verzeichuen. Siach Dem, was in Den fritheren \&aragraphen itfor

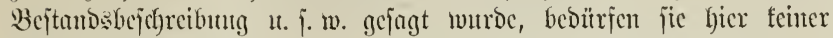
wciteren Erlänterung.

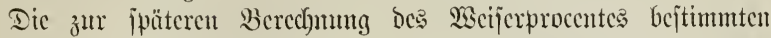

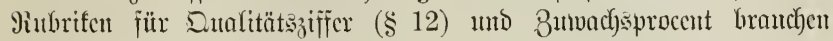

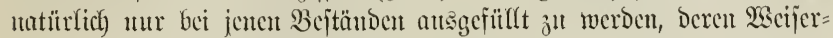
procent ilberfantpt in Frage fonmen famm.

Bei joldsecu Drten, Deren Bonttätsjiffer für Standort oder Bejtand 3weifclhaft ijt, 3. B. Kier bei $20 \mathrm{~h}$, ijt Dics im Manual baburd) ant=

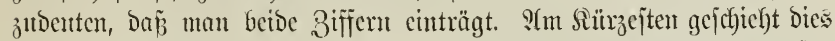

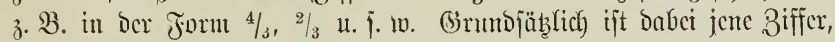
welche für bie weiteren S(rbciten gelten joll, zuterjt, o. h). an oberjter

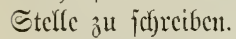

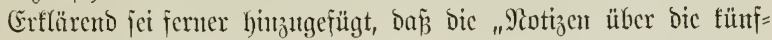

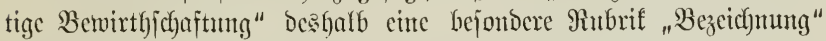
crbalten, aljo nicht in fortlaufentor Beile ber erjten Bezeichnumg gegen= ïbergeitefft werden, weil afle SYugnben Der liufen Seite bes Manuales in Der Regel auf ciner Duterzeile Plab funden, Die Notizen Dagegent für viele Bejtünde oft ganz entbebrefich) jüno, für andere vielzeiligent

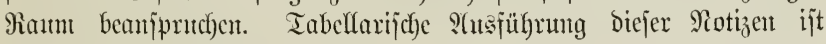
niḑ)t gutt möglich).*)

Das Mamual wird am beften in gropem Dutartformat angelegt.

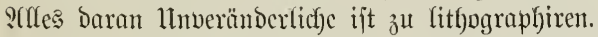

Jebe : (botheilung erbält mindejtens cinte Seite.

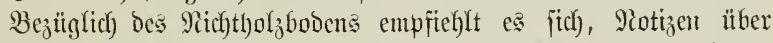

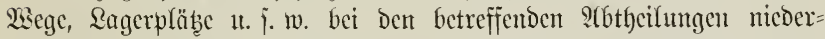
zuichreiben, ferner am Echlujic bes Mamuales Bemerfungen baritber

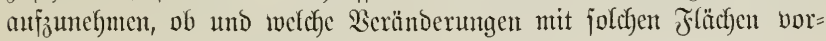
genomment werden möchten.

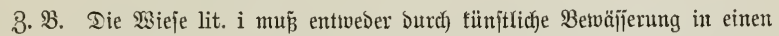
beïeren Zujtand gebrad)t werden, oder ijt einjt zum ફुol

*) Jür Das gegebene Sdhema wurben andere Bahlen uno Berfältnifije ge=

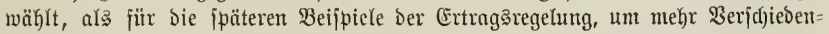

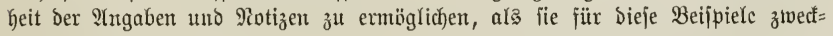
mäß̄ig erichien. 


\section{$\S 101$.}

\section{Die Beffantio- Silantahelle.}

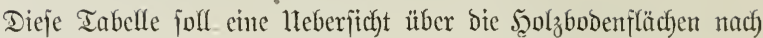

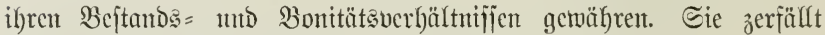
aljo in jo viele Scaupttheile, als bejtandbildonde 5ुolzarten und $\mathfrak{B} e=$ tricbsarten vorfantoen find. S(m richtigiten ijt es, für jeoc Bctriebs= Hlafie cute bejonbere Rlafjentabelle anzufertigen.

In ifrer Summe bictet fic eine volljtändige 1leberjid barüber, wie viel Fläche von jeder ?lltersflajfe vorlfanden ift.

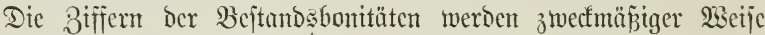
mit rother Tinte eitgejdrichen.

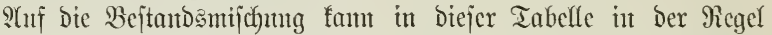

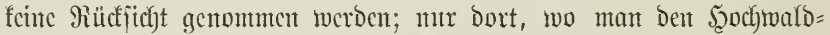

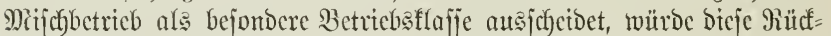
jicht jutmmarijch genommen.

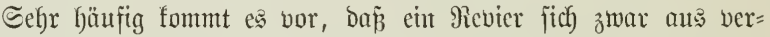

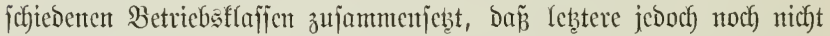
rein bertreten fint. (5s wird $3 . \mathfrak{B}$. cine Betricbsflajje für $\mathfrak{B u c h e n t =}$

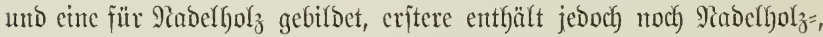

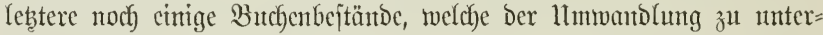
liegent habent, fobald fie ber Şicb trifft. In joldfent Falle mü die

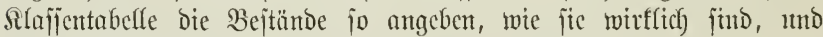

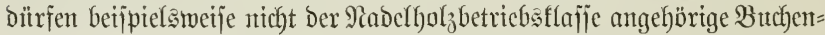

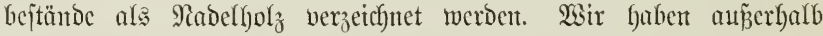
Gachjens mehrmals gefumben, Daj lebteres geidjeht. Gs ijt bics

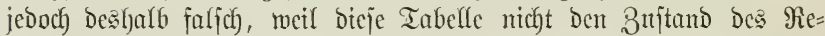
vieres wiederzugeben hat, welchem man zuftrebt, fondern einfach Den wirffich vorbantoenten, forjtfichen Thatbejtanto. - Dajjelbe gilt audf) für bie int $\$ 102$ ju fofjildocmbe Silafjentuberficfyt.

Folgentes Schema bedarf einter weiteren Erläuterung nicht, eS enthält j̧wei ?lbthcilungen tmo die Summte des $\S 121$ u. f. für die Ertragsregelutg bentbiten, einfachen Beifpieles. 
Sdifriften. § 101.

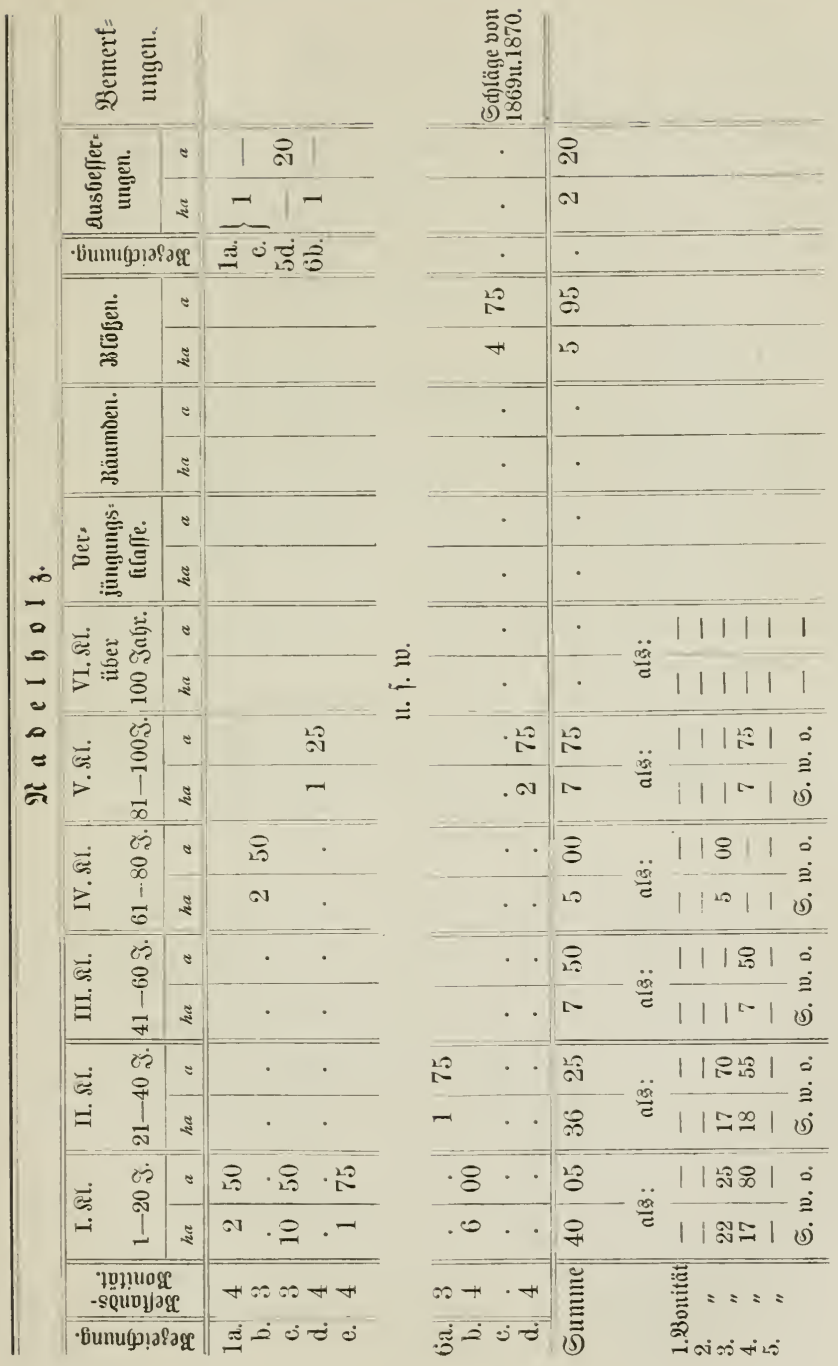




\section{$\S 102$.}

\section{Dix Elallemilfrerltutit.}

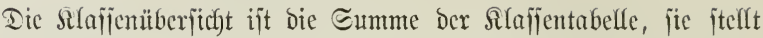

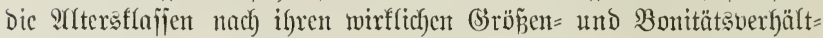
niffen zufammen. Für jede Betrichälaffe ift eine gejonderte Silafīen=

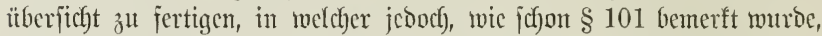

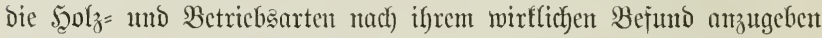

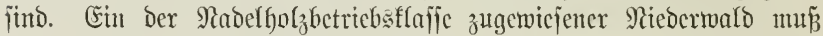
3. B. jo lange afs joldyer fortgefïfyrt werden, bis dic llmwandolung wirffich erfolgt ift.

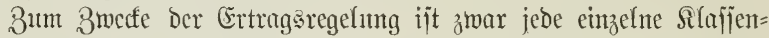
überfitcht der getrennten Betriebs= ober Wirtbjefafitsflajien von be=

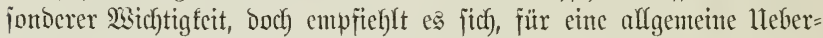
jitcht bes Bamzen dic einjeluten Betriebsflajfen zu fummiren.

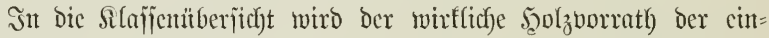
zelnen Betricbsflaffen แnd Des ganzen Mevictes cingetragen.

Dieje Tabelle wird won Salyrzelnt zu Jahychnt fortgefüfret, am

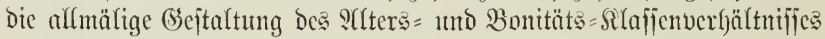
beurtbeilen zat fönnent.

Umitchendes Schema giebt Den Befumb des Sifafjentuerbältnifjes des im \$ 121 u. j. w. bemubeten Beijpicles zu Sufang des Jafhres 1871. RUfgabe der alle zehn Jah)re ricoerfehrenden Revijionen ijt es, die Tabelle in derjelben $\mathfrak{B e i f e}$ fïr 1881, 1891 u. f. to. วu er= günzen.

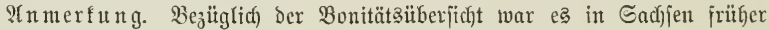

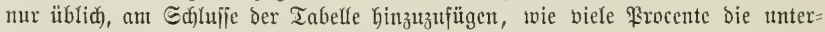

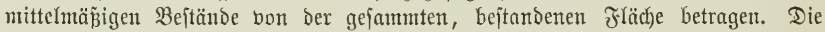

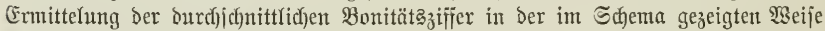
ijt jedoch entiprechender, meil bie Beräntoerung der betreffenton Bahlen bon Эahr=

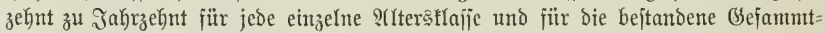

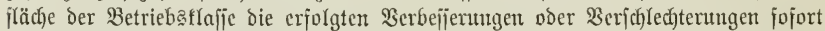
erfenten 【ajien. Geit einigen Iahren twerben beşhalb audy in ben Rlafienïber=

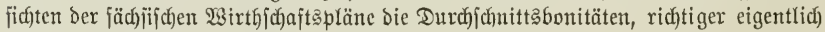
burdfidntittlidje Bonitätşziffern, beigefügt.

Wie aus ber Entwiffelung im $\$ 77$ herborgeht, ift die im Schema bei=

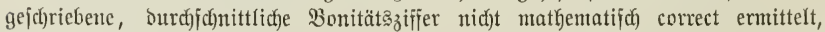
da fie 3. 23. Fïr die Sitmme cinfad) Geredfnet inurbe $\frac{44,95 \times 3+51,60 \times 4}{96,55}$

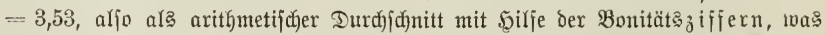
nutr Dann ganz ridytig wäre, wenn bie ben lețteren entipredfendent Erträge fid hier berbielten wie $3: 4$, unter 2 (nntagme bon 5 Bonitätąflajien allgemein 
Sdriften. $§ 102$.

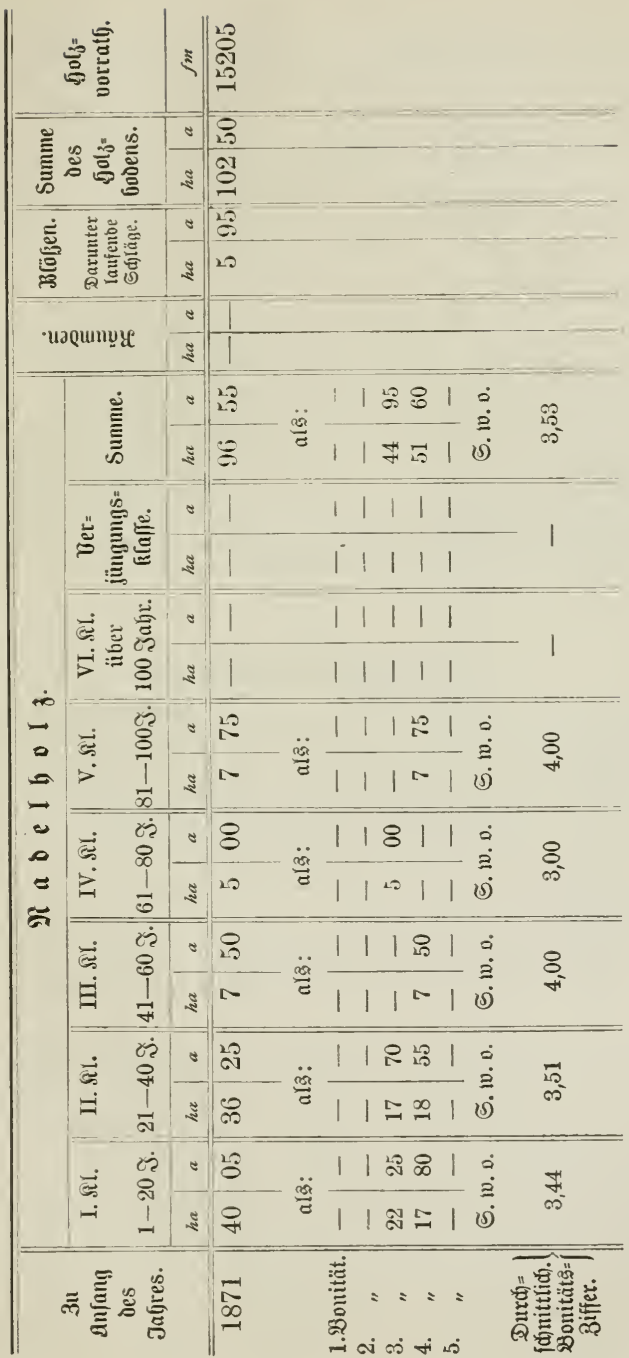




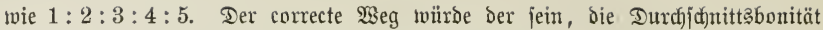

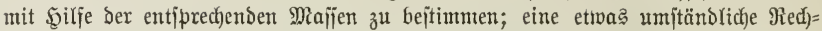
nung, weshalb wir jie zu bem 3.wede nicht empfeblen, welcher hier borliegt. Diefer ift fein anderer, als in furzer $3 \mathrm{ahl}$ die auf = ober abjteigende Betwegung

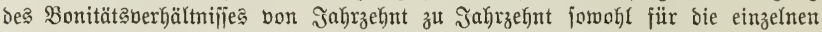

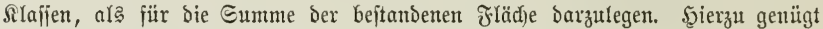
Der bon uns eingeid)lagene $\mathfrak{G e g . ~ - ~} \mathfrak{S O}_{0}$ zur Bonitirung bie älteren Tafeln von (Eotta oder תönig angetwendet werden, entfällt diejes Bedenfen jelbjitberitündlid)

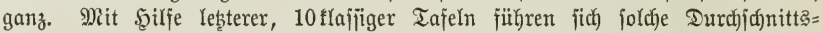
red) nutgen am leidhtejten aน⿰纟.

Würbe man eine Reduction der Bejtände auf eine Bonität (\$ 77) vor= nehmen, jo brauchte die Durdhjd)nittliche Bonitätsziffer nidjt beigejdrieben zu merden, bie rebucirten Jlächen treten Dann fulbj̈t an beren Stelfe. Da indofjen

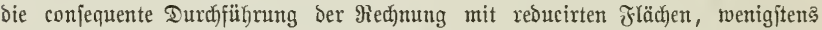

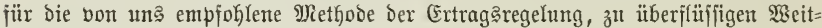

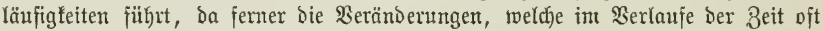
mit ber abjoluten Bejammtfläche, jteţ aber mit den abjoluten Flächen ber ein=

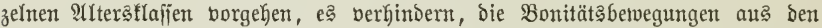

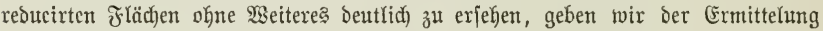

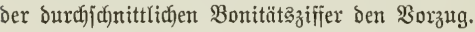

\section{$\S 103$.}

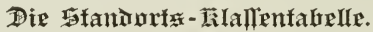

Sbgleidy wir meniger (Setwid)t auf bie Bonitirung Des Stand= ortes, ales auf bie bes Bçtandes legen, jo erfemen wir boch an, Daj einte überjichtliçe Bujammenjtellung Der Standortsbonitäten wejentlich mit zur flaren Darlegung bes forftlichen Thatbeftandes bient.

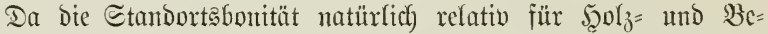

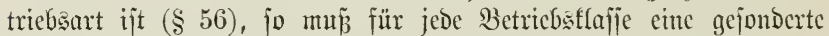
Derartige lleberjicht gefertigt werben. Daburch ijt indefjen cine Sum= mirung für bas (Sanze nicht autsgejchlofien, jedoch mutí man jich)

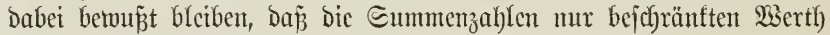
haben.

Für bie Tabclle felbjt empfechlen wix folgendes Seljema. $3 \mathfrak{3}$

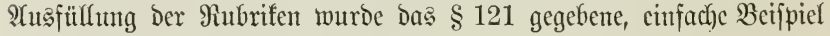

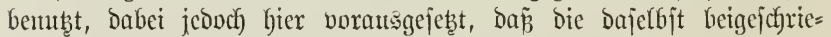
benen Bonitätæziffern für Den Standort gelten jollen.

Anmerfung. Da Den berichiedenen Standortşbonitäten bejtimmte Duanti=

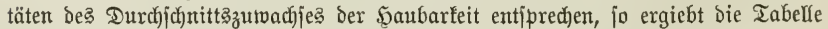

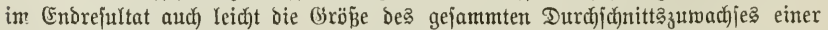

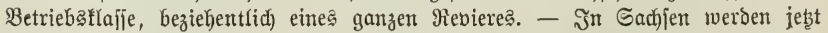

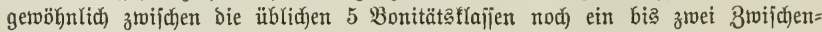

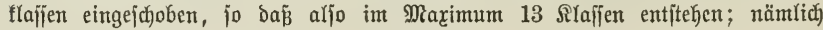
1., $1 / 2,2 / 1,2 ., 2 / 8,3 / 2,3$. u. $\{$. w. 
Schriften. \& 103.

\begin{tabular}{|c|c|c|c|c|c|}
\hline \multirow{4}{*}{ 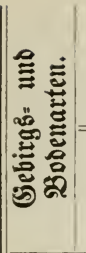 } & & 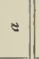 & 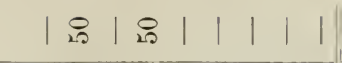 & 11 & \multirow{6}{*}{ 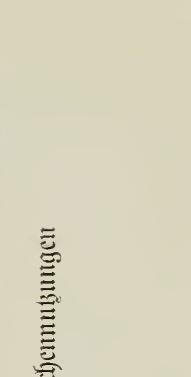 } \\
\hline & Е & 3 & | a d a d a $\left|\begin{array}{l|l|}\mid & \mid\end{array}\right|$ & 20 & \\
\hline & \multirow{2}{*}{ 氞 } & " & 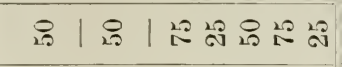 & 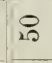 & \\
\hline & & si & or $|\leadsto| \Theta \approx 20.20$ & $\Xi$ & \\
\hline \multirow{2}{*}{\multicolumn{2}{|c|}{ 棓 }} & 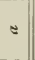 & 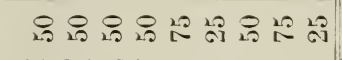 & $\therefore$ & \\
\hline & & sil & 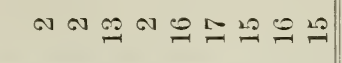 & @ & \\
\hline \multirow{10}{*}{ 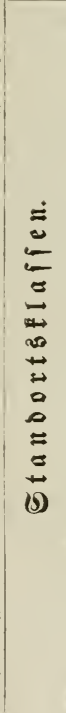 } & $\therefore$ & $\because$ & 111111111 & 1 & 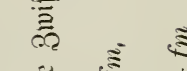 \\
\hline & 5 & sil & $|1| 1|1| 1 \mid$ & 1 & 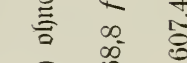 \\
\hline & & * & 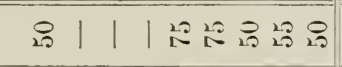 & 18 & 莺 \\
\hline & $\equiv$ & 5 & 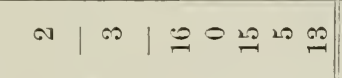 & 120 & 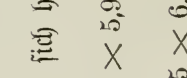 \\
\hline & & $\cdot$ & | & 28 & \\
\hline & & $\approx$ & $\mid a @ \infty)$ & Ұ & \\
\hline & $\Xi$ & * & 1111111111111 & 1 & \\
\hline & & 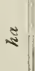 & 1111111111 & 1 & 害 \\
\hline & 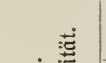 & " & 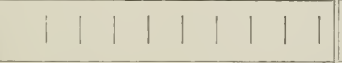 & 1 & 言莒 \\
\hline & & \& & $1|1| 1|1| 1$ & | & 美㪯 \\
\hline & ॠ & & 害言 & & $\dot{\widetilde{Q}}$ \\
\hline & ఊँ & & 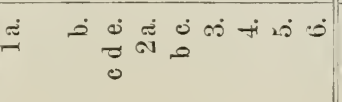 & $=$ & \\
\hline
\end{tabular}




\section{$\S 104$.}

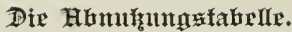

Dic Sfonub̧ungstabelle entfält eine Bnjammenjtellung ber fum= marifchen Materialmubuıng für bie einzelnen Salyre und ben daraus berechneten Durchidynttsertrag. Die S(ngaben find nad) $2 a u b=$ แnd

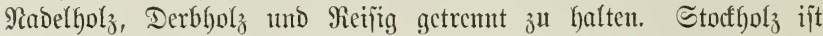
gejondert beiznjabreiben. Dieje Tabelle, meldbe übrigens nur her= geitellt werocn fam, mo cine längere, vrocntliche Bucbjülfrung vor= ausging, ijt um. jo mertfboller, je längere Beiträume jie umf́ắt.

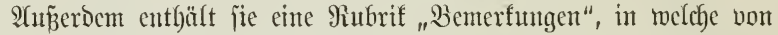
5 z̆ 5 Jahren - bei längeren 9ievifionszeiträumen in gröperen $\mathfrak{Q} \mathfrak{b}=$

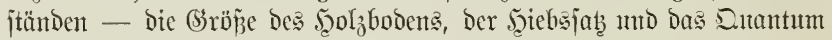
eingetragen twerden, 1 m weld)es lebुterer jäf)rfich überjobritten ober แn= exīitllt gelajjen murbe.

Bu münjchen wäre cinte Tremung Der 2 (btriebs = von ben $3 \mathrm{mi}=$

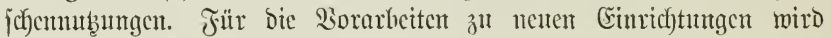
man indeffen getwöfnlich jodjon jebr zufrieben jein müfjen, went utan Itnterlagen für ène, wenn auch nur furzen Beitraum umfajjente, jummariface Tabelle in obiger Form erlangen fumt.

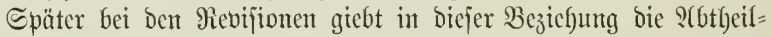

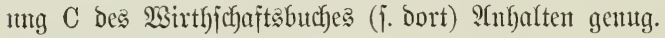

Die in Sachjen übliche Form der P(bnubungstabelle ijt folgende: 
S(frifter. $\$ 104$.

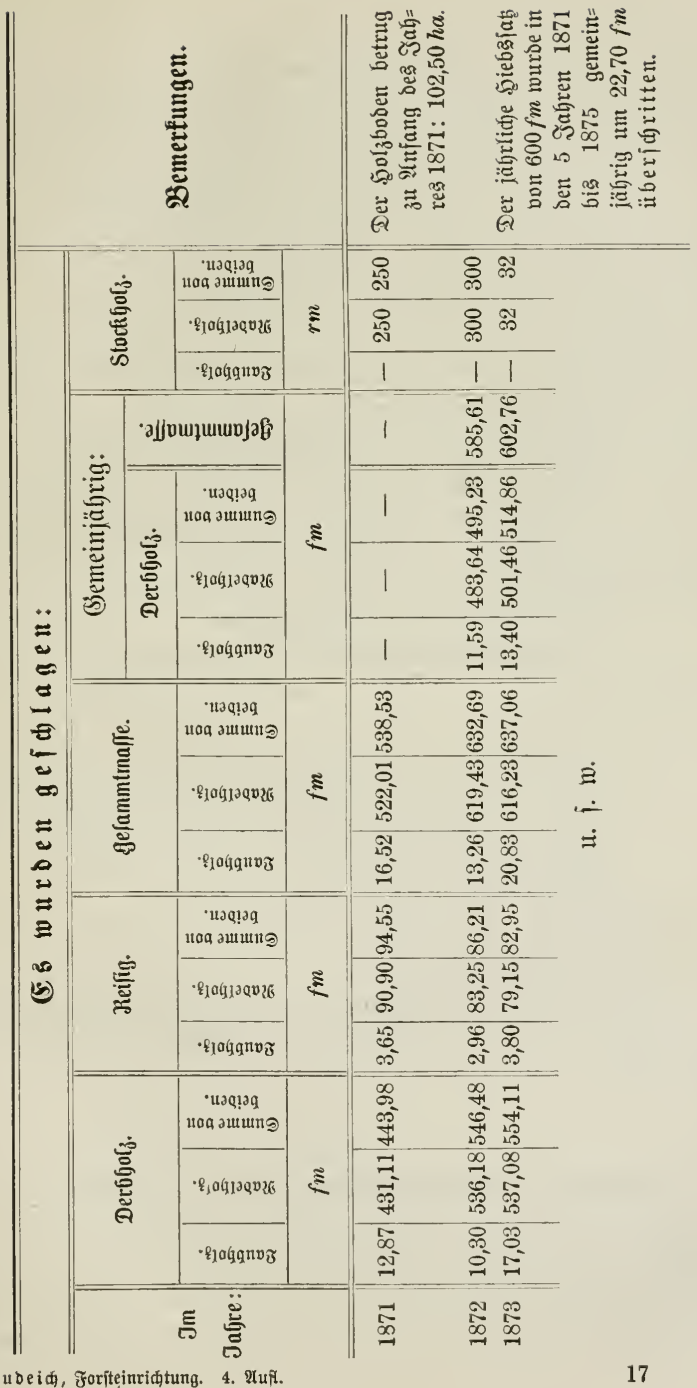




\section{$\S 105$. \\ Das forenzenilfar.}

Ein Bitlo der Brenze jelbjt und der jie jajarf bejtimmenden Brenz= zeichen (Steine 2c.) gieft zwar die Epccialfarte, um jich jeboch) zum

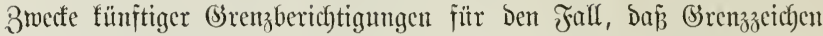
verloren geben follten, von ben ber graphifichen Darjtellung unver= meiblich anbaftenden Feblern freizulalten, ftellt man in tabelfarijch überjiçtlicher Form ein jogenanntes "(Srenzregijter" jujammen. Das= jelbe hat folgende Rubrifen zu enthalten:

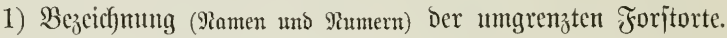

2) Şorizontale Entfermungen.

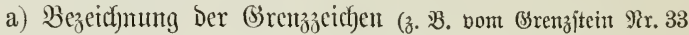
nach) Brenzitein $\Re r .34$ ).

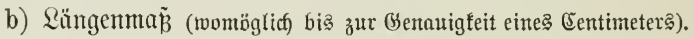

3) Brrentzintel.

a) Bezeichnung Des (Sirenzzeichens.

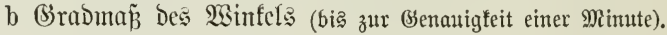

4) STmerfungen.

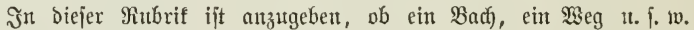
Brenze bilden, wo bie Brenzlinie nicht gerade bon dem Mittelpuntt eines Steines zu Dem des anderen ไäuft; db Grrenzgräben, Brenz= mauern poer bergl. borbanden; bei welchen Entfermungen zwijd)en

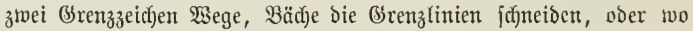
S(i)neifen leţtere betühren; it. j. tw.

5) Siamen ber angrenzenden Brundjtüfe uns beren Bejizer. (2Ingabe, ob Feld, Sileje, Wald u. f. w.)

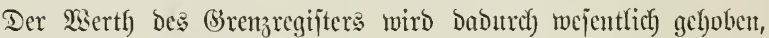

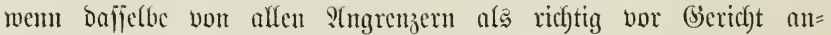
erfannt witro.

\section{$\S 106$.}

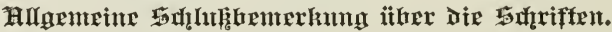

Wir haben in ben vorlergehenden Faragraphen bie haupt=

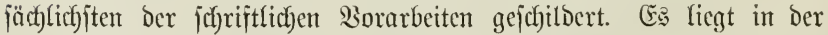

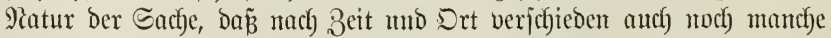

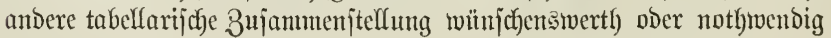


fein faum. Beifpielweife für Den Eichenffhältwald Material = und

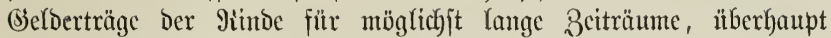

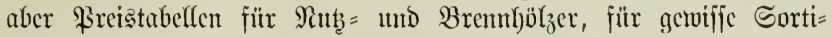
mente und bergleichen mefre. Aftgemein giltige Tabelfenformulare

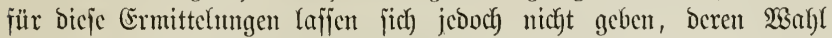

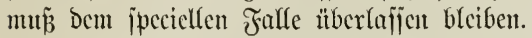

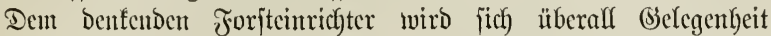

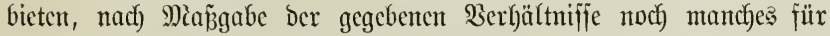
bie weiteren 9 (rbeiten 2 sidftige zu ermitteln und in überifichtlichen Bufammenftelfungen zu orbuten. Sint wir audh feine Freunde bes

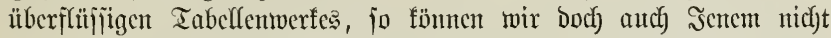

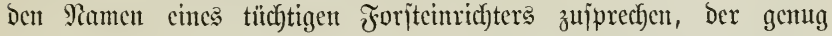
gethan zu lyaben glaubt, went er alfe inftructionsmääpig vorgefchrie=

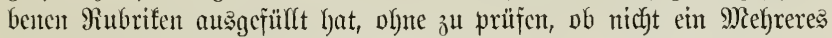
nothruentig jei.

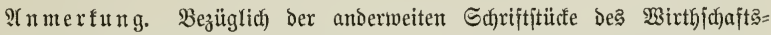

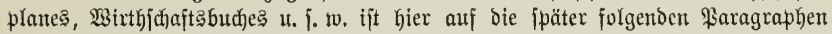
孔t verweifent. 


\section{B. Warbeintheilung.}

\section{$\S 107$.

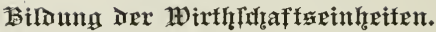

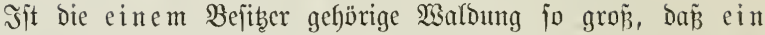
Bermaltungšbeamter allein bafür nicht genügt, jo mun cine Theilung

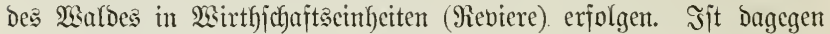

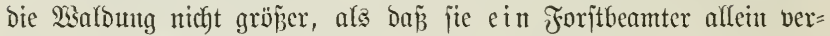
walten fönnte (unter llmitäntoen ber Befitzer jelbjt), jo bitbet fie an umD für jich eine Wirthjochajtseintbeit.

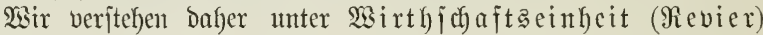
einen $\mathfrak{B a l d}$, Der cinem Bejiber gehört und einem Wirthjuafts=

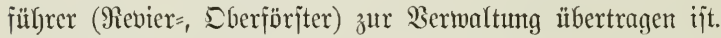

2(utge

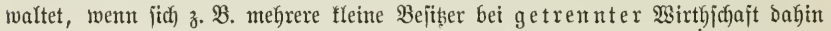

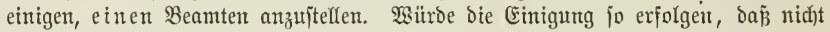

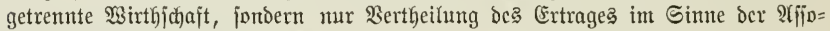

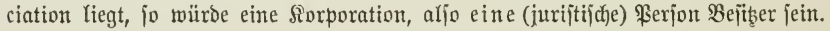

Die (S) allgemein nidft bejtimmen.

Dic niebrigite Grenze Der Ffächentusbefunutg wiro Dutch ben fleinjten Umiang des jelbititändig jür jïch bejtefyenden Warbeigentfumes bebingt, unter IImitänoen auch burch) ifolirte Sage einzelner Theile cines größzeren Walbförpers.

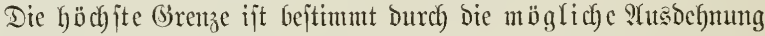

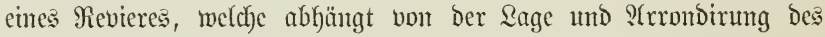
Waldes, jomie vout ber Intenjität ber Winthjchajt an Srbeit. Sie

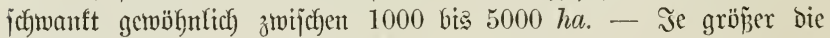

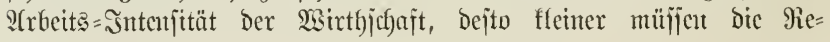
viere jein.

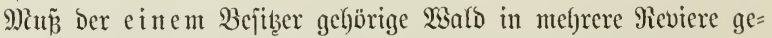
theilt merben, jo wirb es nöthig, eine ttach IImftänden verjchicbente

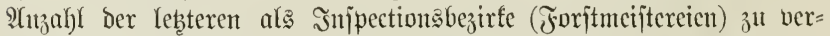

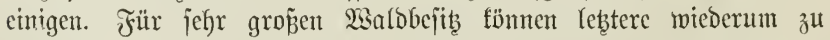
verjchiedenen Directionsbezirfen zujammengefajpt werden. (\$rovinzen grö̈̈erer Stanten.) - Dicje tweiter gehende Drganijation ber ge= 


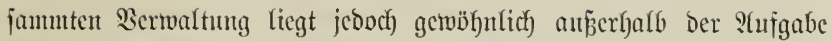
Des Forfteintidfters, er hat es in Der Ricgel nur mit Der Bitbung

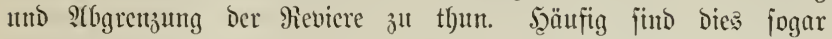
gegebene Briö̈̈ent.

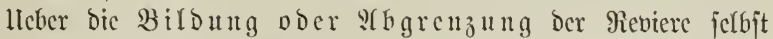
lafijen jidf) alfgemein giltige borichriften nicht geben. - Die Söjung

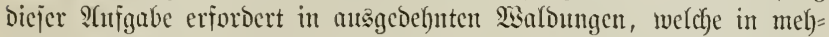

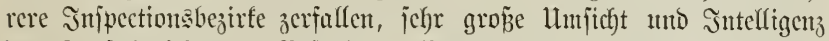

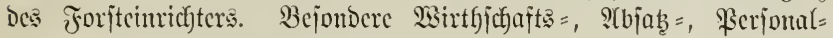
werfältnifje werden maj̄geheno.

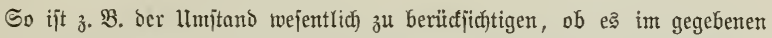

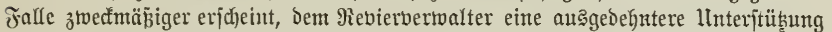

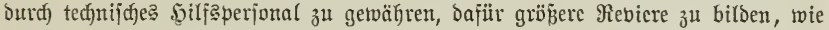

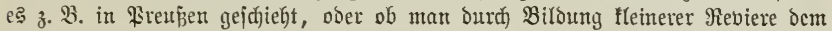

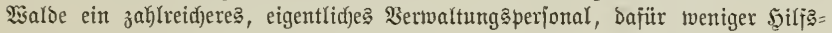
perjonal geben will $\mathfrak{u}$. j. w.

Sft werben bie Reviergrenzen burdf Ierrainverbältniffie bejtimmt

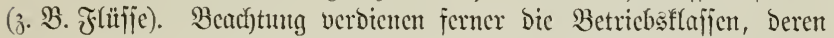
(birenzen, wein und fo weit ce möglich, gern audch als Revicrgrenzen bemub̧t werben.

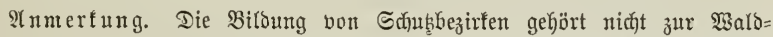

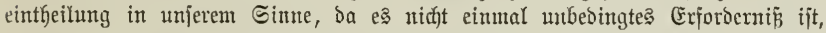

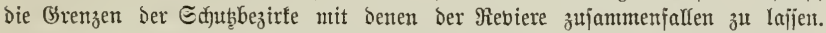

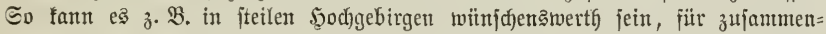
Gärgende, obere Fartieen einen ভđutțbejirf zu birben, aud wem derjelbe ver= idjiesene Mieviere trifift.

\section{$\S 108$.}

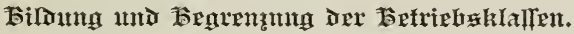

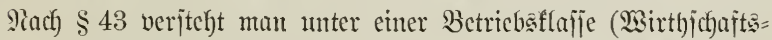
flafie) alle einer unt Derjelben Sefflagorbnung zugewiejenen 2 sald =

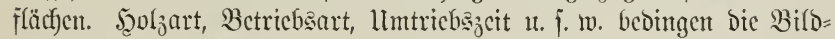
ung von Betricbesflajicn.

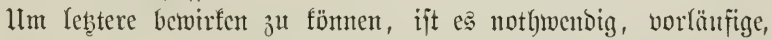

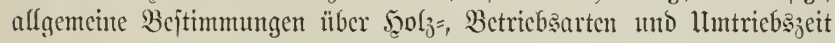

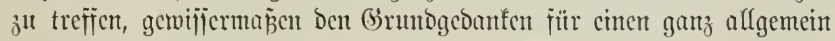

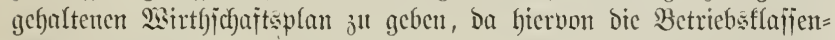
biloung abjängt.

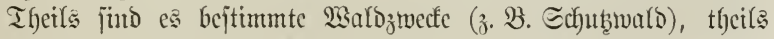

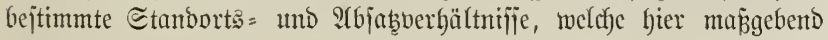


werden. Wir berweifen hier auj bie betreffenden Refren des $\mathfrak{\text { Sald }}=$

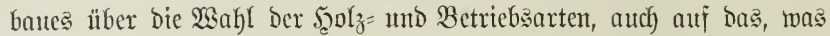
wir itber bie finanzielle Ilmtriebszeit im allgementen Theile unferes

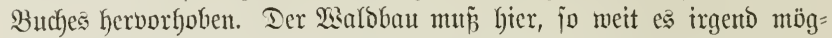

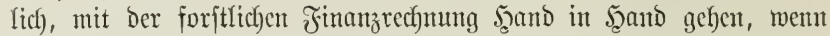
er ein rationeller jein mill. Daun miro er auch bem Forjtenticfter Stühpunfte gentrg geben, um bie fier vorliegenden Fragen zu Ł̈̈jen.

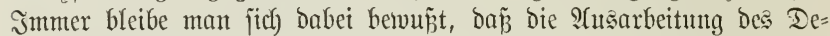
tails noch manche Seränderungen ber vorläufigen Bejtimmungen beranlafien wiro.

Scat man bie Betriebsflaf̣en im Sllgemeinen gewählt, fo jind bie $=$ jelben im $\mathfrak{W a l b e}$ abzugrenzen. Sft merden Terraintwerbältnifje (Thal=

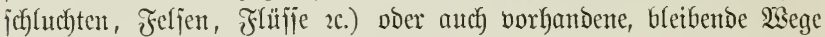

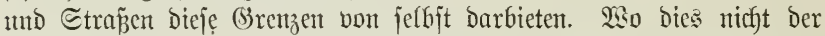
Fall, müfijen leb̧tere fünjtlich hergejtellt werden, und zwar in ber Regel jo, Da $\tilde{\beta}$ eine volljtändige Selbitjtändigfeit einer jeden Betriebs=

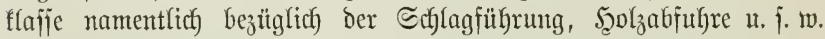
crzielt miro. Bejonders ifjt die Bemubutg bleibender $\mathfrak{W}$ ege zu $\mathfrak{B} e=$ triebsflajiengrenzen zu empfeblen.

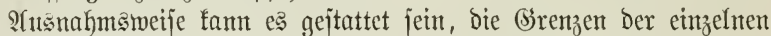
Betricbiffajien im Walde nicht bejonders on marfiren, bies aber eigent= lich nur bant, wenn aus anderen Siründen bie Selbjtjtändigfeit ber

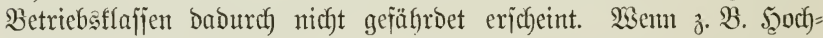
mald = und Piederwaldflajie an einander grenzen, namentlich went

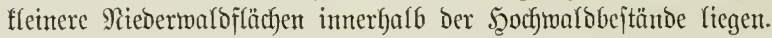

Bei Der B̉enubung vorbandener Edyneifemtebe zu teuen Einricht= untgen ober bei Saenderungen Der Betriebsflaffentheifung im Berlaufe

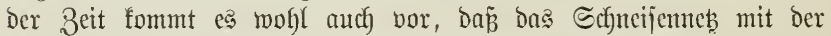
Silafjentbeilung nidft überall harmonirt; es ijt bies indeijen etwas nur Gejtattetes, feinesmegs aber etwas $\mathfrak{W}$ simjchenswerthes.

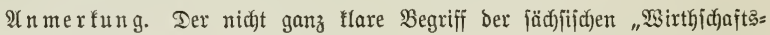

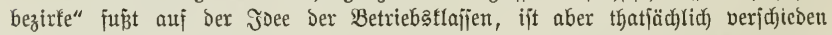

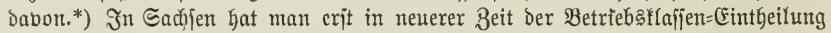
Redjutng getragen, bezeidnnete Die betreffenden swaldtheile eine Beit lang mit Dem

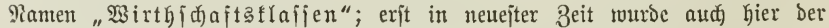

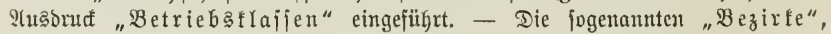
welche man in fädsfiffden Edjriften und Rarten genaunt fintoct, haben mit Der

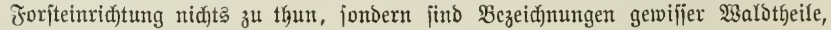
Waldjtreden, bie man aus alter Beit übernommen umb beibehnlten hat, weil fic volfsttjümlid) geworben. Streng genommen jino jie überflüfifig.

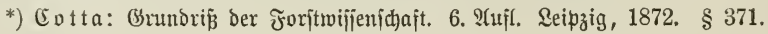




\section{$\S 109$.}

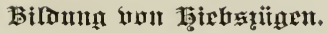

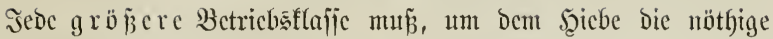
Dromung und Berweglichfeit zut geben, noct) weiter in Schlagparticen

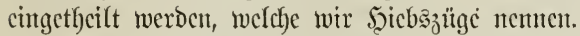

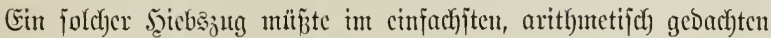
Pormalzultande bic cincm bejtimunten Umtrieb cutiprechento normale Schlagrethe einmal jo enthalten, baż bie Schlägc lifber feinc ganze

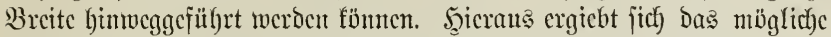

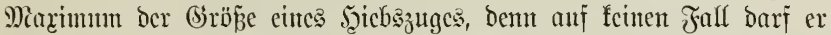

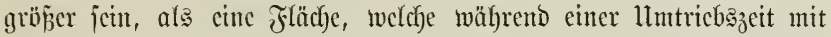

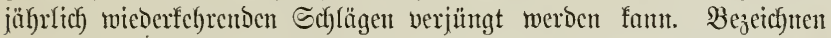

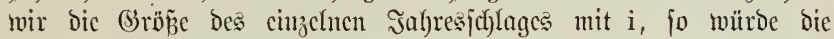

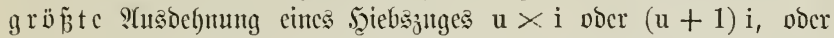
$(u+2)$ i u. $\{$. w. Getragen, jenach) bem bie Schlägc fofort antgebaut werben, ober ein, zwei ober mebrere Sabre ala Blößjen liegen bleiben.

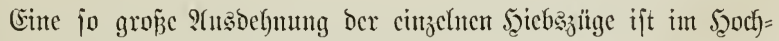

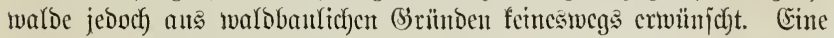

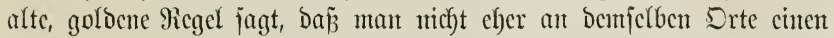
neuen Echlag anlegen jolle, bis utcht ber jullebt gefü̈brte fitcher it $\mathfrak{B} e=$ itand gebrad)t, b. h. bis auf ihm uicht bie fiultur volljtändig gelungen jci. Dieje Regel fann abcr nur Dann befolgt werden, wem man burch

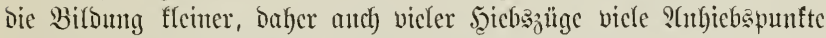
gejchaffen hat, meldye cinen ijteren $\mathfrak{B}$ echjel ber Schläge crmb̈glichen. In biejem Simte fant nitht blos, jonbern foll jogar der cinzelne

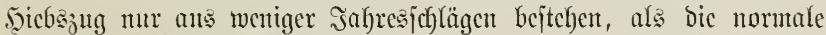

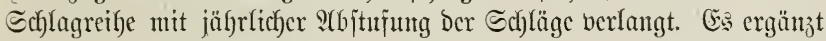

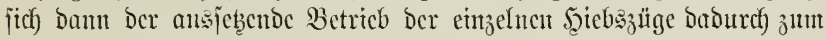

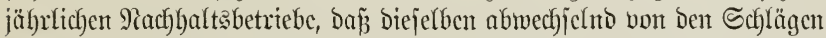
getroffen werocn.

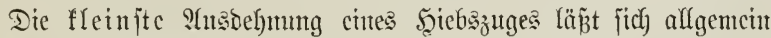
nicht bejtimmen, benn jic fängt nicht blos von ber Girößje, jonbern

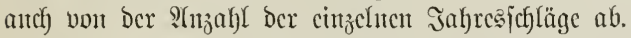

Meijt wird matt im Şoct)malde, Der hauptjädylich hice in Frage

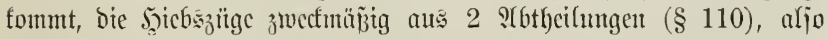
nicht über 30 bis 60 ha groj biloct. Unter Umitämben fam es aber

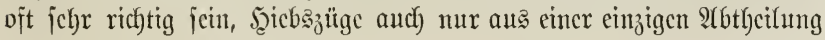




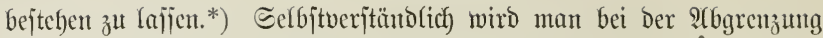

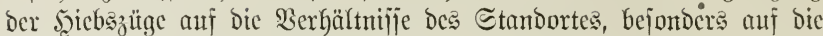

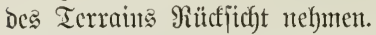

Dic fleinen Şicbszüge Gebingen feintesncgs unwirthjuchaftlich fleine

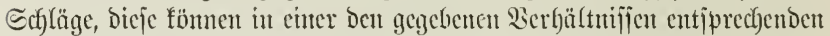

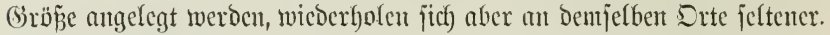
Bei Safhlichlagbetricb wäre Darnach) zu itreben, in einem Safyrzelynt

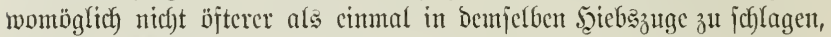
Damit Den Sinlturen bic jo jehr nötfjige 9iulfe gegöntt werben fann.

Die Eintheilung Des Revicres oder Der Betriebsflajfen in fleine

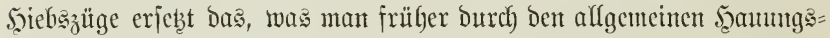
plan mit jeiner \$eriobeneintfycilutg ju erreichen judfte. Sie bifoct bie Brunblage ber feinen Bufunftswirtljichaft mit jreier Bewegung,

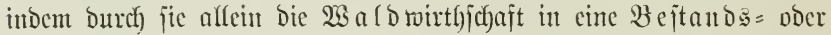
Bejtand scomplexwirthjchaft bermandelt werden fanm. In ihr liegt

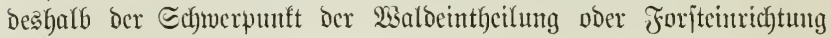
im engeren Simte.

Durch Das Streben utad) möglichjter Eclbjtitäudigfeit Eleiner

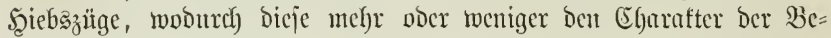
triebsflajien, menn aud nicht für bas Яicchnungswerf, annchmen,

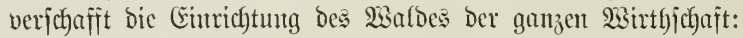

eritens bie Mëglichteit, ben Etandortsbedingungen auch im Silcincu in ausgebchntejter Weije Sicchmung zu tragen ;

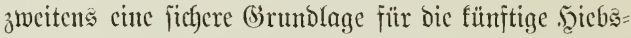

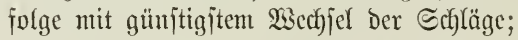

Dritten jente mohlthätige Elajticität, welche es ber Zufunft möglich macht, in cinzelnen Bejtandsgruppen

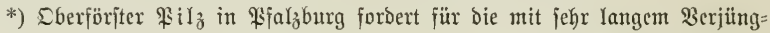

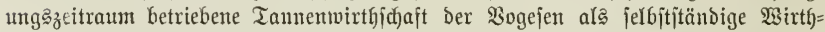

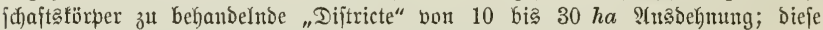

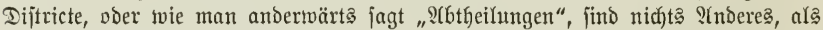
fleinc Şiebszüge in unjerem Sinne. - 3u vergl. \$ił Die AYtersłlajien= Berreipung", Jorjtliche Blätter, 1882, ․ 168 u. f. - Derjelbe:

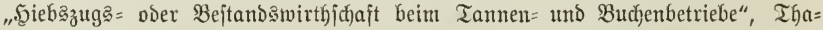
ranber Jahrbudf), 33. BD. (1883), S. 193 u. f. - Яeume ifter: "Die Bebeutung

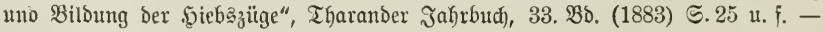

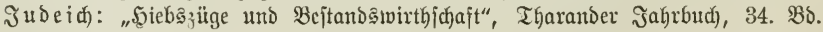
(1884), §. 44 น. f. 
rajefer, in anderen langiumer mit Dem 5̧icbe vorzugeben,

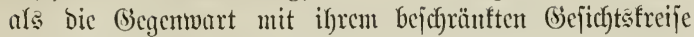
vorusbejtimmen funn;

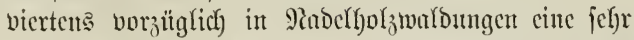

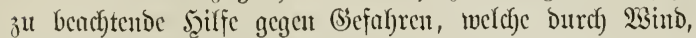
Injeften*) utno Feuer Drohen;

füuftens entolich cinc beachtentsuertbe llnterjtüb̨ung des localen Szolzabjabes.

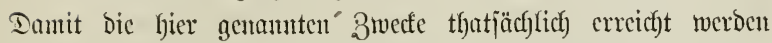
föntucn, hat Dic Forjtcinrichtung für Folgendes ju jorgen:

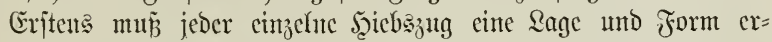

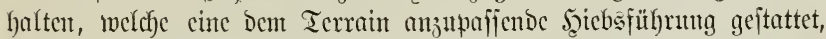
möglicbjte Sicherbeit gegen Eturmgefahtent uno möglichjte (Erleichterung bejüglich Des f̧oljtransportes gewährt.

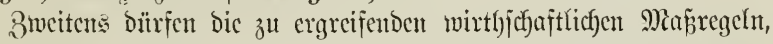

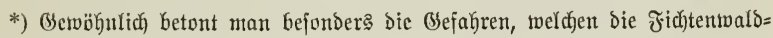
ungen Durch Sturm atıgefest find, in Riefernvaldungen bie Befahren, welche das

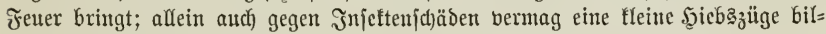
Dende Foriteinriditung wejentlich mit zu helfer.

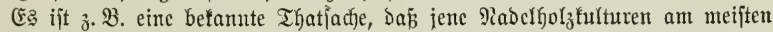

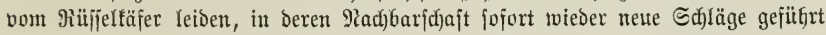
twerden.

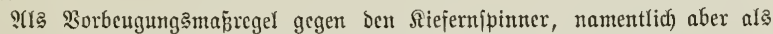
Gelfende Maß̈regel bei der Befämpfung Delielben, empfiehlt fid bie Unterbred)ung

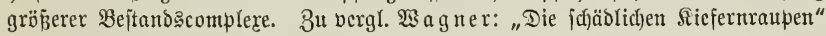
u. \{. เพ., Tharanter Jafrbuch, 23. Bb. (1873) S. 182 น. โ.

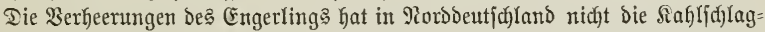

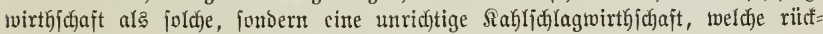

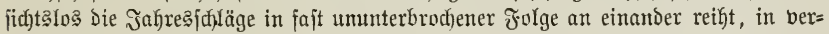

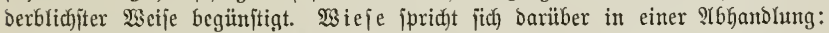

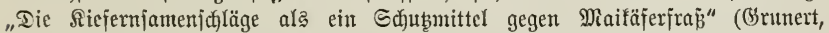

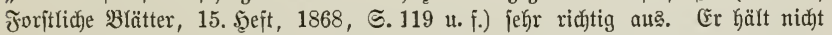

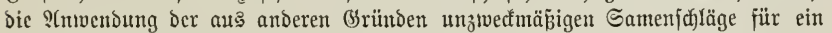

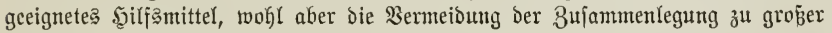
Sđjotungsfläd)en.

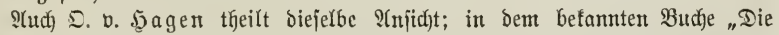

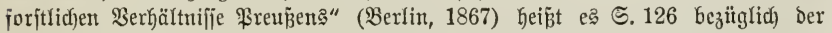

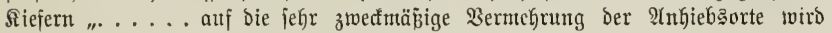
Durd) Biloung fleiner Betriebeffiguren (J̃gen) von nidyt über 110 Morgen (28 ha)

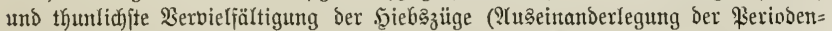

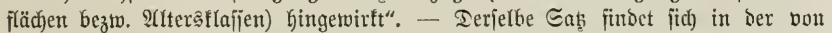

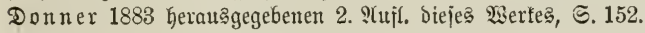




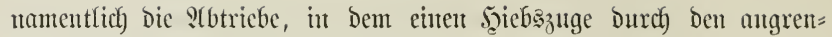
zenden anderen nicht beengt werben.

Sind bicje Biele fäufig auch) nicht vollftändig zu erreidfen, fo foll man jie boch als leitenden (Ssedanten für bie Waldeintheiluntg im Stuge befalten.

Wo bie cinzelnen Sciebszz̈̈ge jeitlich ancinander grenzen, erreicht mant bie Selfitjtändigfeit jedes cinzeluten, jomeit bieje überhaupt mög=

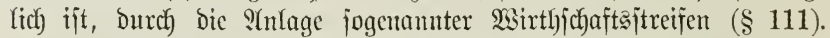

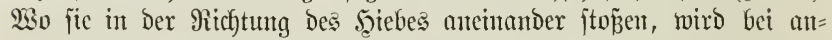

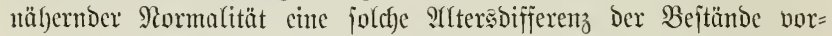

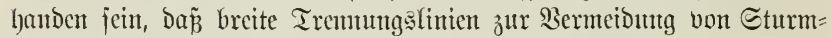
¡chäben nicht nötbig jint. Bei einem abuormen şltersflajjenverbält= nifje hat ber Forjteinrict)ter burch entpprechend augelegte Soshiebe (\$ 112) Den Şauntugen Die nöthige Berveglichfeit zu veridhaffen.

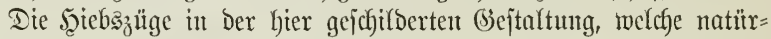

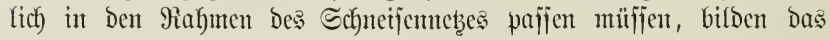
Biel, Dcjien Erreichung und Erbaltung Dic auf cine gute Forjteinricht=

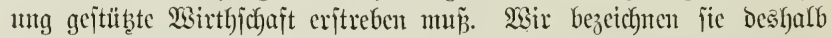
mit Dem ?fusbrutf: bleibente Şicbszüge. Bei Der meift abuormen

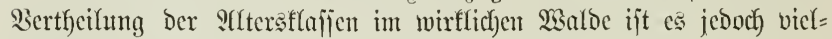
fach) unmöglich), bie Echläge obne 2Beiteres nach Den Stuforderungen Der bleibenden ober bleiben follenden betebszüge ju bejtinmen, man wird im Segentheil auf bic vorfandente, unregelmäp̈ige Bejtands=

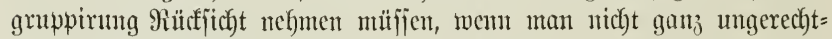
fertigte Spfer bringen will. Mian wird Deshalb, aflerbings unter Beachtuntg Der einftigen Beitaltung ber bleibenden şiebsjüge, oft Sciebsbejtimmungen treffen müfijen, weldye angentblicflich) nicht ber ge= troffenen (Eintheilung entipredyen. Daburely entiteben vor übergeben de 5̧icbşüge, in ber Fegel nur flemere Echlagpartien innerhalb blei=

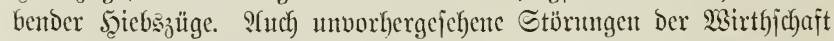

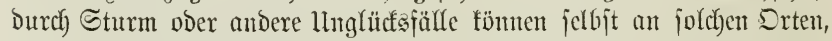

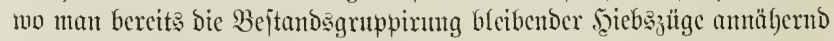
erreicht hatte, bie Bildung vorïbergebentoer J̨iebszüge wieber nöthig

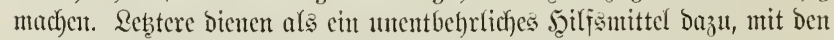

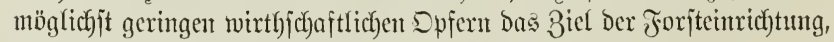
Die Bildutg und Erbultutg Der bleibenden şiebszitge zu erreichen. *)

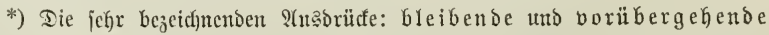

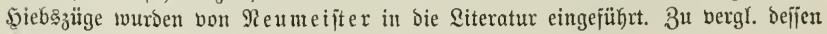

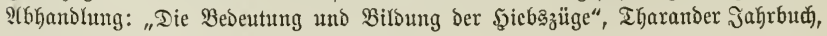
33. B๖. (1883) ธ. 25 น. f., ipeciell S. 32. 
Saum hedarf es Der Ermälnung, Daj für Rieder = und Mittel=

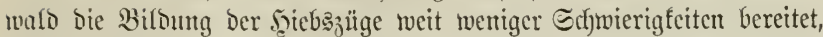

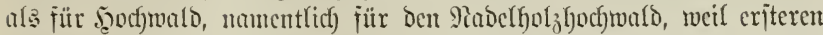

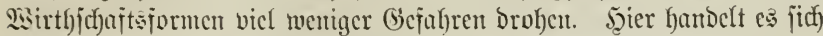

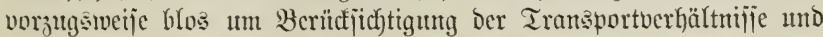

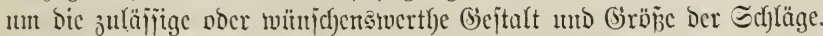

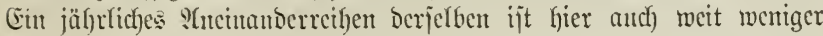

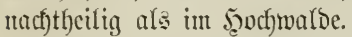

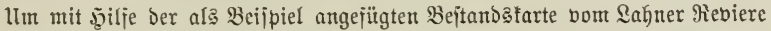

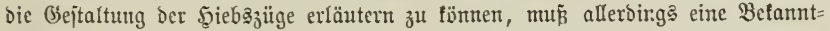
ichajt mit ben folgenden, bie \$albeintheilung betreffenten \$aragraphen, jowie mit

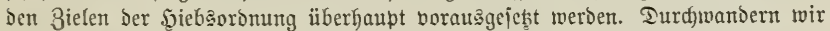

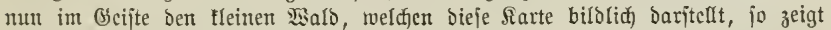
uns bieje bejüglid) Ser Bildung Der Şiebşüge uno ber im engiten 3ujammen=

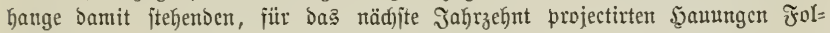
gettoes:

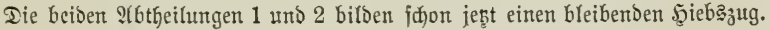

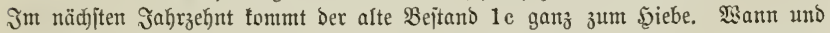
wie lesterer in श(btheifung 2 fortidreiten wiro, barüber enticheibet bie 3ufunft.

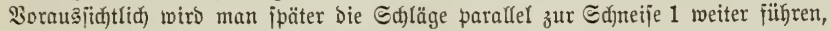
wegen ber Jugento ber vorliegenden Bejtände aber nur jefr langjam fortjđdreiten laịjen.

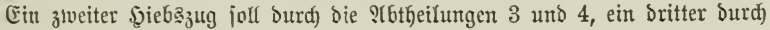

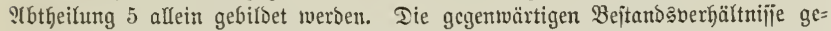

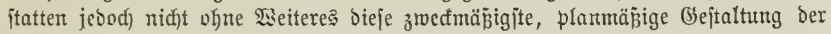

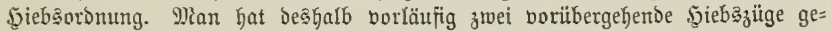
billbet. Der eire bejteht auß Der stbtheilung 3 uno ben beiben Beitänden 4 ab.

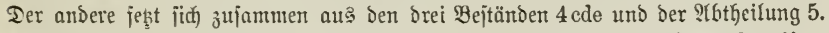

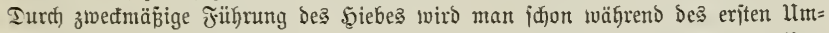

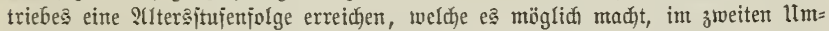

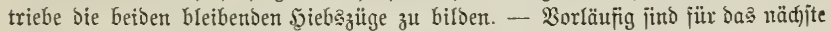

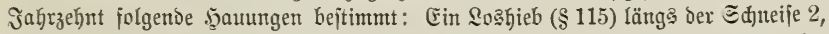
weldjer 2 cd zeitig genug an ben freien Stanto gegen Sitoweit gewöbnt, um im

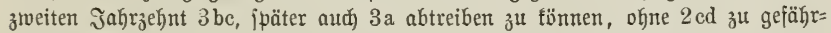

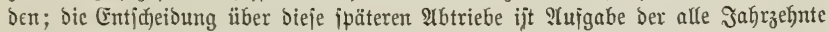

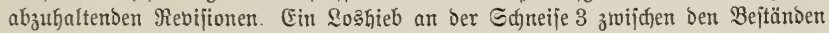

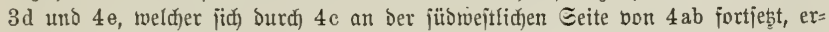

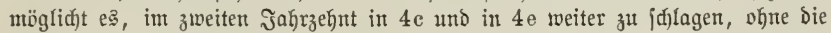

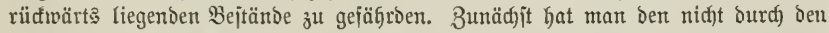

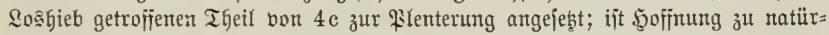

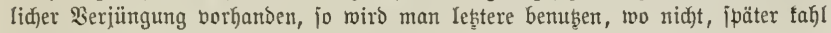

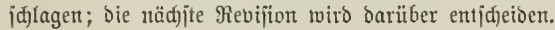

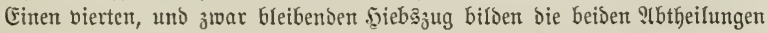
6 und 7. Ter ફ̧ieb liegt jeşt fajt in der Mitte deijelben. Die llmbaunng von $6 \mathrm{c}$,

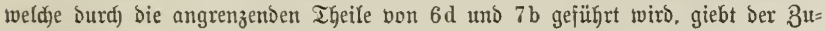




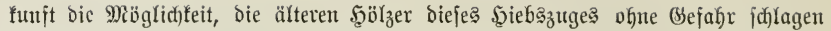

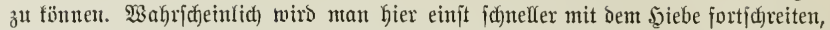
als in ber nad) Rorbweiten vorliegenden 2(btheilung 2. (Exjt nach Dem Durdibiebe von 7 be fangen einjt bie Sd)läge in 6 ac an; ob man bann bie beiden jüngeren Drte 7 de, ober mentigitens $d$, für ben z'veiten limtrieb itberhalten fanu voer nidjt,

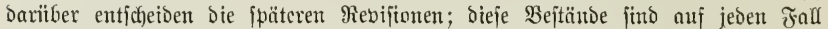

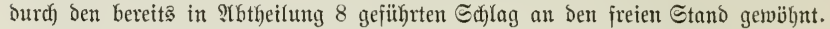

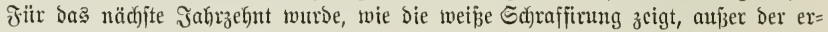
wähnten llmbaunng nur $6 \mathrm{~d}$ z̆m j̧iebe gejețt.

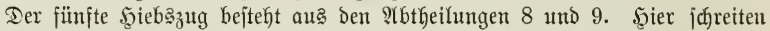

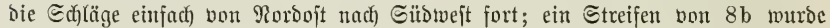

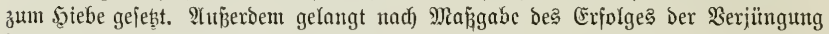

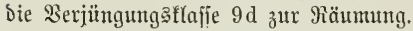

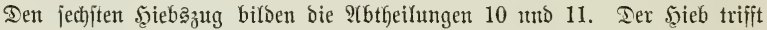

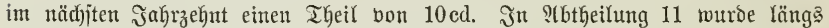
ber Schneife 2 ein etwa $20 \mathrm{~m}$ breiter Streifen zu jtarfer Durdfforjtung ober \$lenterung angejebst. Ylter und Bujtanto ber betreffenden Beitände, Yiē̄en cinen

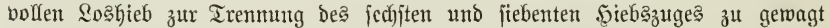

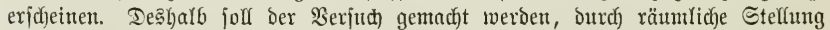

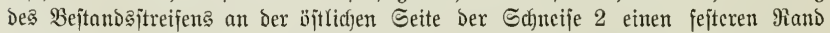
zu bilben, weldher enit vielleidyt Sdaläge in 12 geitattet. Die nädjite Revifinn

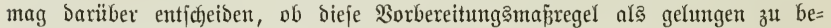
trad)ten jein wirb. Man hofft bies un jo mebr, als bie Budhermijuchung in 12

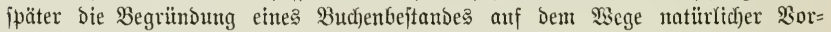
verjüngung erntöglichen bïrite, wodurd) bie für 11 immerfin etwas gefäbrlidbe

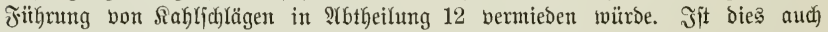
nidht ohne Seiteres Direct aus Der Siarte zut erjehen, jo legt and Dieje allein eine

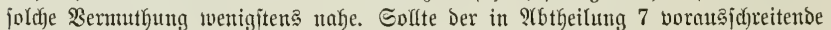

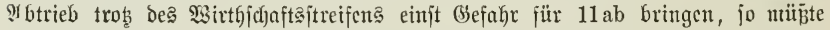

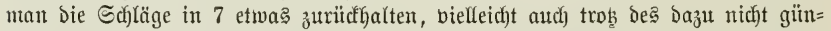

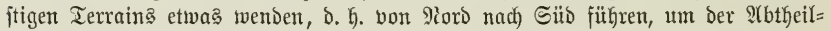

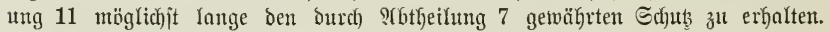
Rïnftige Revifitonen entidjeiben bartiber.

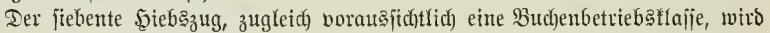

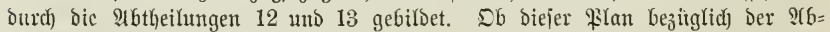
theilung 12 เvirflid) jejtgehalten werben jolf uns fann, Darüber mögen erff fpätere

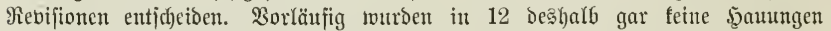

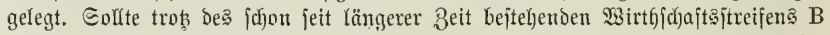

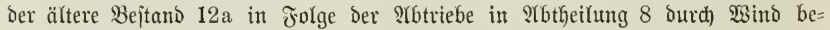

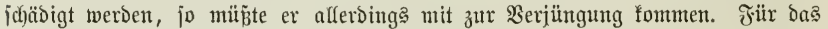

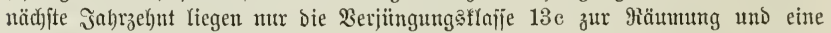

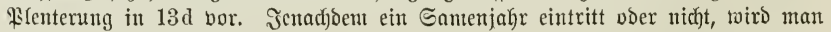
utit Diejer \&lenterung ftärter ober jd)twäd)er vorgehen. - Sollte man übrigenţ

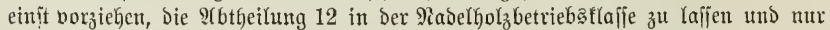
aus 13 eine Buchenbetriebstlaffe zu bilden, fo zerfiele Diejer 7 te 5̧icbsjug in zwei fleinere şiebszüge, beren jeder nur eine eimige 9rbtheilung enthalten witrde.

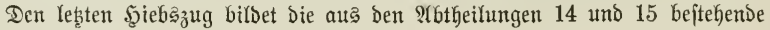




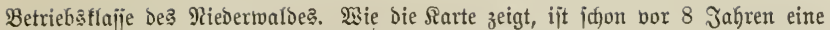
Edjlageintheilung berjelben jür 15 jährigen $l$ mtrieb bewirft toorben, weăhalb bie eirzelnen 8 Echläge in Stbtheilung 14 beveita Gejondere Bejtandsbudjitaben er= Gielten. Ifbtheilung 15 zeigt nod) feine foldhe \$(Gjtujung, jondern nur 3 Bejtände

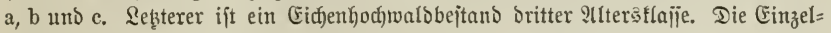

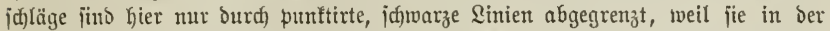

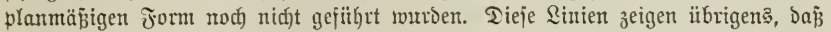
Der Eidjenbejtand e in Mieberwald umgemantoelt werben jofl. Derjelbe ijt zum

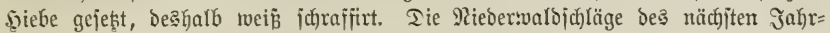

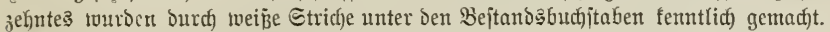

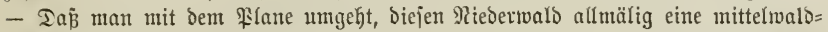

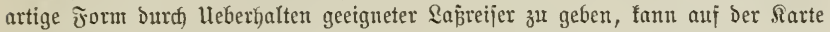

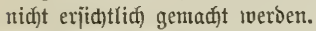

\section{§ 110 .}

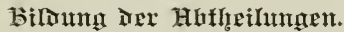

3u Dem 3wecfe Der Drientirung im Salbe, Der Drontung ber

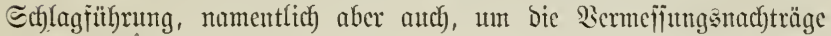

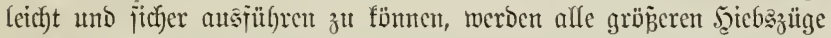

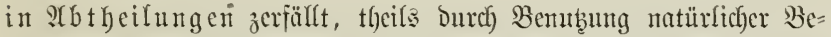

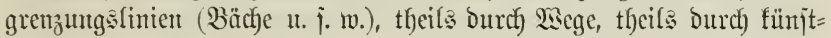
licfje Ecínteijent.

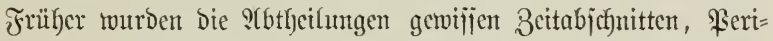

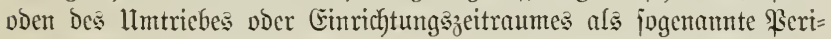

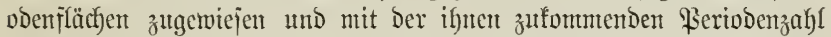

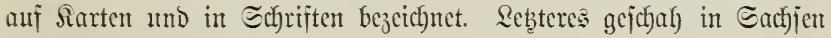
noch) lange, nactybem bereits bie Praxis ber Periobencintbeilung jelbjt (§ 121) einen $\mathfrak{s e r t f ) ~ n i c f t t ~ m e f r r ~ b e i l e g t e . * ) ~}$

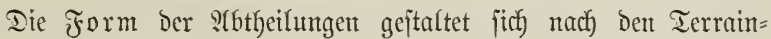

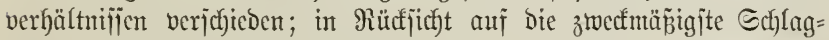

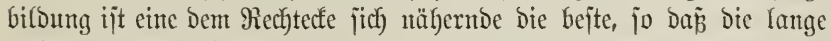

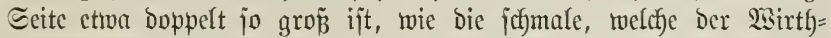
ichajtejtreifen begrenzt.

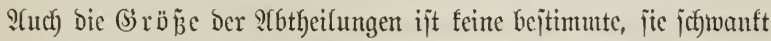
für Den Şocthwald meritens zrwijchen 15 bis $30 \mathrm{ha}$. 9iod) gröpere

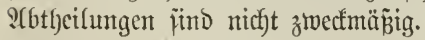

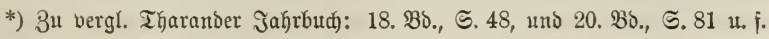




\section{$\S 111$.}

\section{Day sogneifenner.}

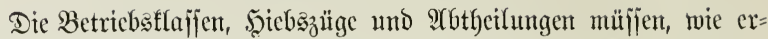

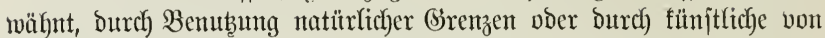
einander getrent werben. Namentlich madyen bie erjteren beiden joldse Trenmungen noth)wendig, weldhe bic Selbititändigfeit ber betreffenden, fleinen $\mathfrak{x}$ irtbjichajten ermöglichen.

Sebr midftig ït es hierbei, auj vorhandene bleibende Wege

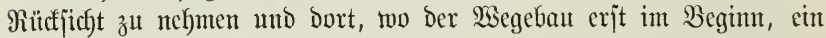

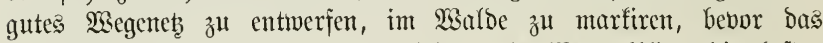
Schneifemeb befintitio bejtimmt wiro. Die $\mathfrak{W e g e}$ bilben bie bejten

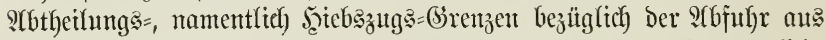

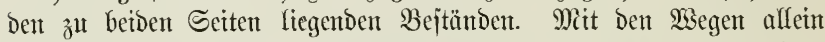
fann man jedoch nicht atsfommen, denn bas Wegeneb hat andere

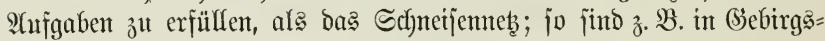

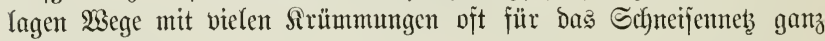
unbrautblbar.*)

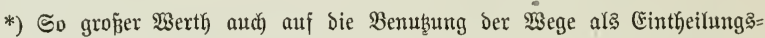
Yinien zu legen ijt, jo glauben wir Dod) hier, in einem Lebrbudje Der Forjteinridjtung,

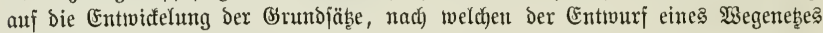
exfolgen muю́, niđgt näher eingeben zu bürfen. Sn ebenem Terrain Gereitet bie

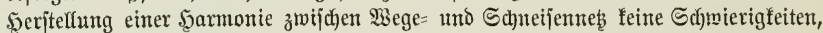

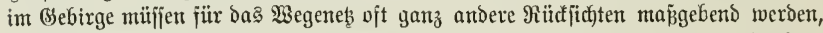

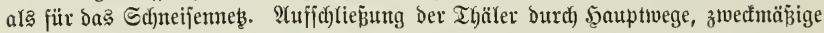

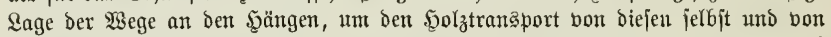

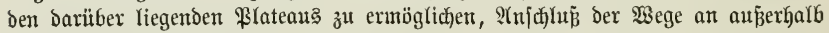

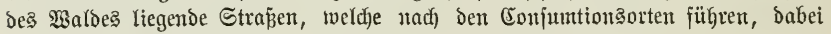
Bead)tung des möglichen Gefälles, das fint bie Şauptrü̈ffichten, weldye beim

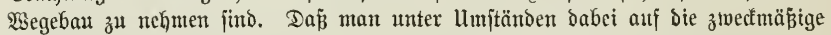

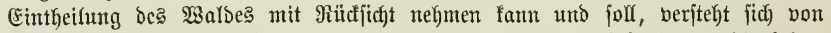

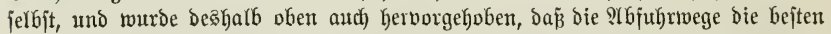

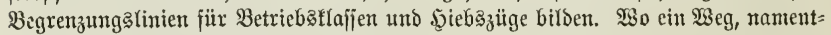

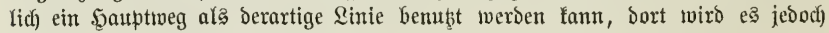
wohl meift ridftiger fein, bieje (Grenzlinie nad) den Anforderungen des

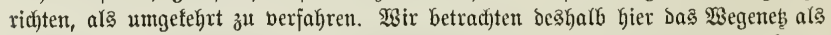
etwas (Ssegebenes; Die $\mathfrak{3 a l d e i n t b e i l u n g ~ h a t ~ ( i d ) ~ i n ~ f o ~ w e i t ~ n a c h ~ o e n ~ g e g e b e n e n ~}$

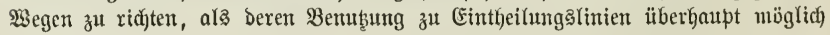
erjojeint.

Ebenjo glauben wir nidyt, daß̄ Gier auf eine (Erörterung der namentlich bom Forjtmeifter $\mathfrak{R}$ aifer behandelten, jehr widftigen Frage einzugehen ijt, in wie tweit

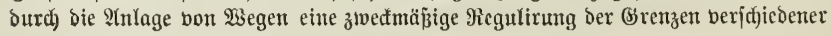




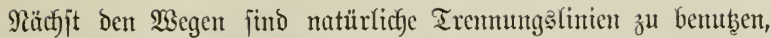

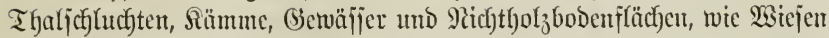

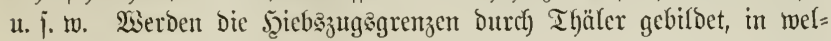
chen, wie es oft vorfonmt, ein Bact) und bleibender $\mathfrak{S e c g}$ vorfanden,

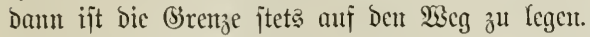

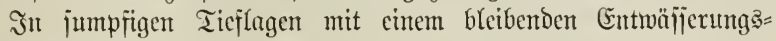

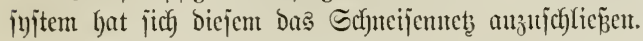

\section{a) Wirthjefaftajtreifen.}

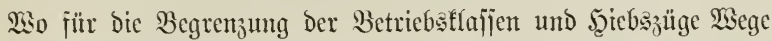
undfft gewählt merben fömen, wo ferner auch bie vorbandenten, natür=

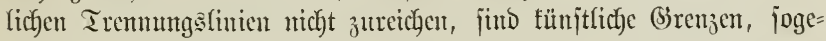

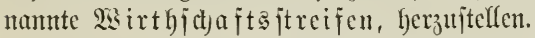

llnter leşteren verjtef) man bleibend holaleer zu erfaltende Streifen. Sie haben Den Zwect, bie jie begrenzenden Bejtände an

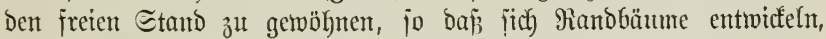

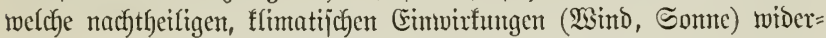
jteben, went audf ber neben = ober vorliegende Bejtant abge= trieben wirb.

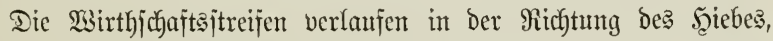
bei uns gemöhnlich fonach von Dit nach 2 sejt, wo nicht das Terrain eine andere Risftung bebingt. Die einzelnen Echlaglinien fallen methr

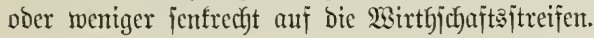

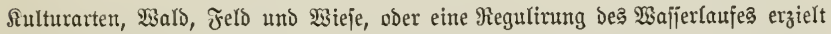
werben fann.

Bu vergleidyen hierüber namentlid):

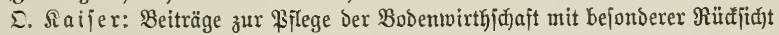
auf bie Waijeritandăfrage. Berlin, 1883.

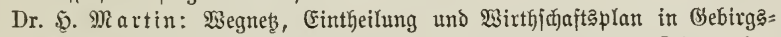

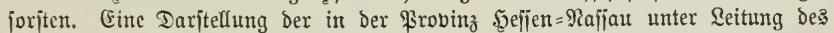

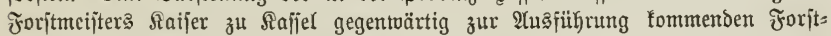

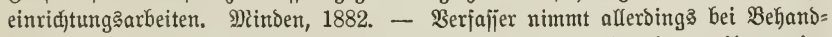
lung biejer Frage einen etroa anderen Standpunft ein, als wir, weil er eine

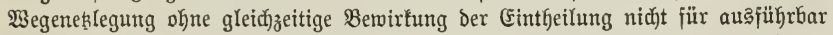

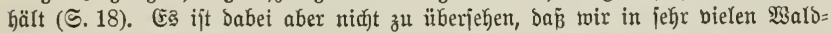

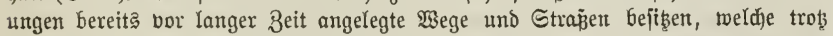

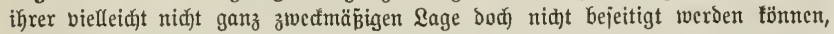

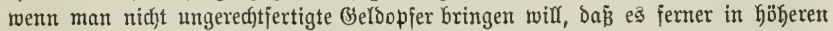
Bebirgen mit jehr jđjwierigem Terrain mitunter ganz unmöglid) iiłt, ein jo betail=

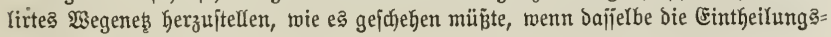
linien ganz erjeşen joll. 
Die Breite Der Yesteren richtet fich nach) Dem Standorte, folvie

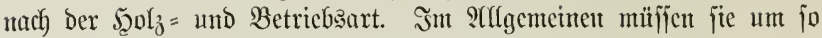
breiter jein, je längeres $\mathfrak{J g l}_{z}$ man zur Beit ber \$̧aubarfeit zu er= marten bat.

Nieber = und Mittelwald bcoïrfen mutr jummaler, etwa $2,5 \mathrm{~m}$

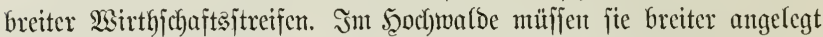

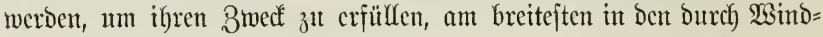
brude) gefübrocten Fichtenwaldungen, ober in ben ber Feuersgefafr jel)r ausgcjebten Sitefentmälbern ber (sbente; man giebt ibnen bort eine Breitc von 10 bis $12 \mathrm{~m}$.

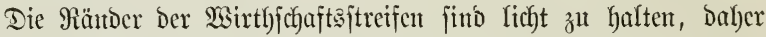
von früher Sugend an jtarf ju burchforften, bamit fich tiefbeajtete und

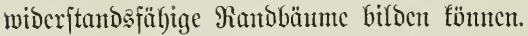

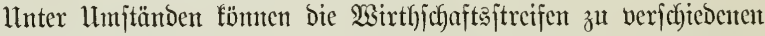

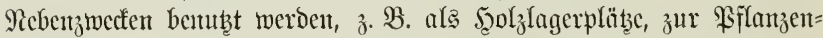

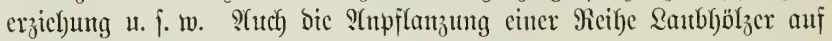
dic Mitte des Streifens empfichlt fich mitunter.

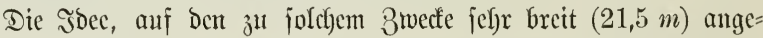

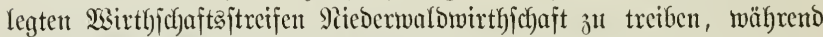
Die autgrenzenden Bejtände Dem Sood)wald angebören, ift in Sachjen früber viclfach praftij(h) burctggefül)rt worden, hat jich jedoch nirgends

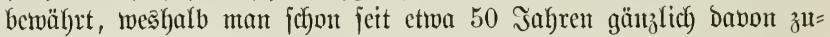
rüdfofommen ijt.

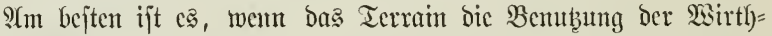

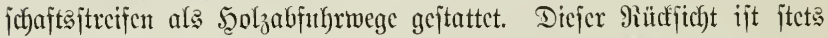

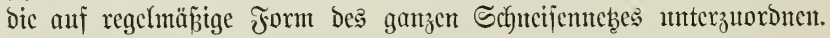

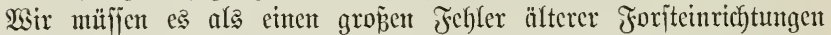

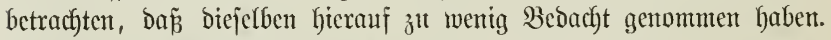

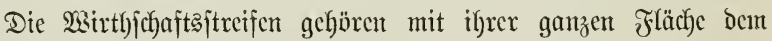

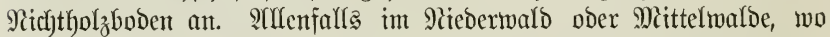
jie gantz ichmal gebalten werben fömten, läßzt es jich rechtfertigen, ilgre

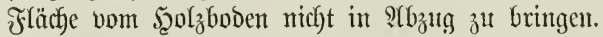

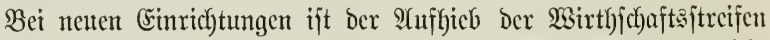

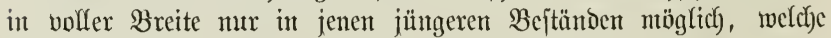

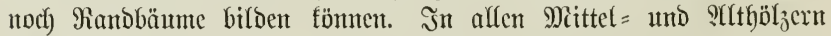
beģnügt man fich), bicfe Streifen vorläufig nur 2 bis $3 \mathrm{~m}$ breit burd)= zufauen, und überläp̈t cs Der 3uffurft, gelegentlich) betreffender Sabläge dic volle Breite herzujtellen. 
b) Sdyneifen.

Barallel den Edylaglinien, aljo meljr ober meniger jenfred

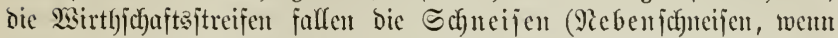

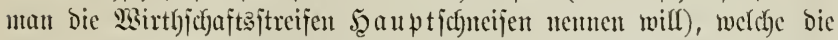

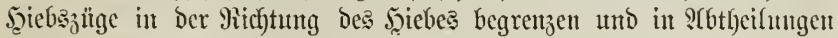
zerfüllent.

Da bie Echneijen nicht ben Brwedf haben, Riunbbäume zu bilden, geniigt für fie cinte Breite von etwa $2,5 \mathrm{~m}$.

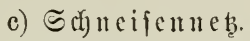

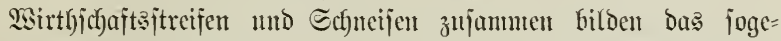

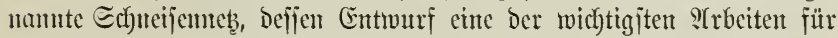
den Forjteinricter ijt, Dem es joll und fann etwas Bleibendes jein, jo lange nicht gauz bejondere umjtände, wie ber Bau einer ben

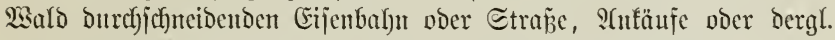
it 3ufunft Penderungen bebingen, während alle jonjtigen $\mathfrak{S a l D =}$ verbältniffe mehr verämberliç)er Ratur jïno.

Puf Die Sullage des Schneijentebes hat namentlich in Bebirge

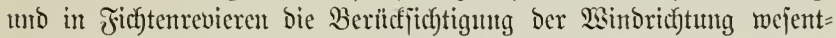

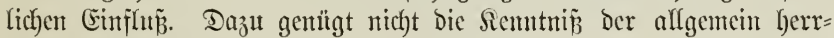

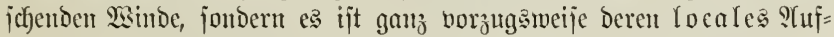

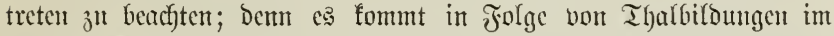

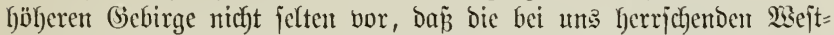

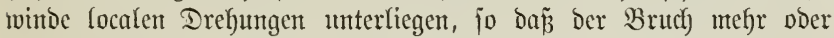
iweniger birect von ?ard ober Sild, jelbjt von 9lorb = ober Sübojt her erfolgt. - Sorjefriften von alfgemeiner Biltigfeit lafjen jich bier=

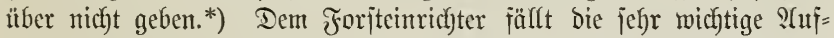

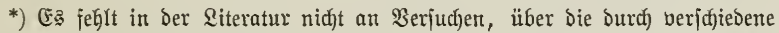

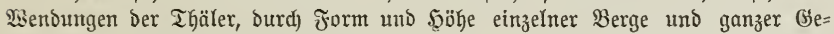

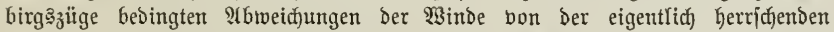

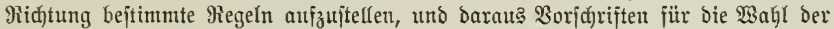

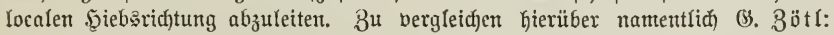

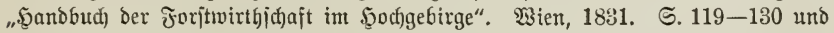

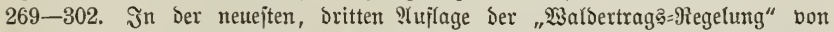

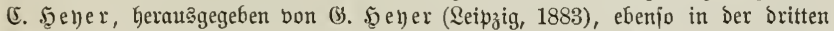

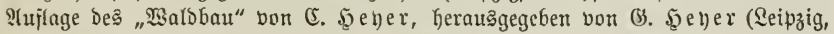

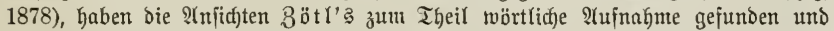
jind burd) erläuternbe friguren ergänzt worbert. Wiir berzichten Gier auj beren Mit=

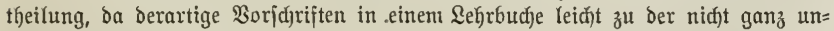

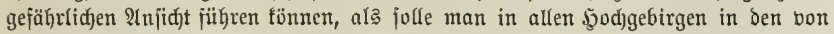

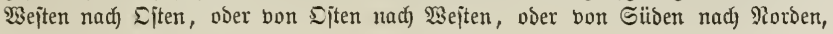




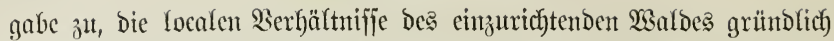

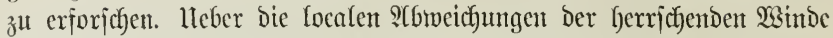

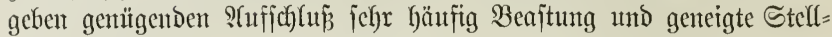
ung Der Bäume an Den Bejtantsan̈nocrn, fowie bie Lage ber von

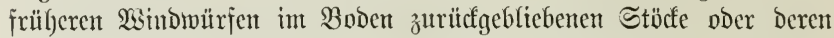
Eroballent. Sehr bift miro man aber aud bon älteren, mit ben ört= lichen Berbältnifjen vertrauten Forjtbeamten, jelbjt audb von $\mathfrak{B a l d}=$ arbeitern braudbbare Notizen barïber crbalten fb̈men, in welcher Richtung vorzugameife Windwürfe jtattgefunden lonben. Man hüte jich aber vorjichtigit Davor, ans Den Durch jtarfe, ungemölntiche Stürme, 3. 3. Durch bon (Semittern begleitete Drfane, hervorgernfenen Erjechein= ungen Schlüfie auf locale Drefuntugen der gewölynlich herrfachenden Winde zut zichen.

\section{Die Bebcutung cines Echneijennebes ift eine Dreifache. Eriftens}

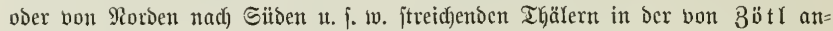

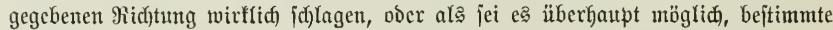

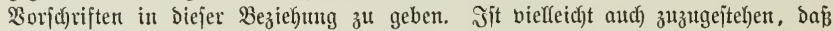

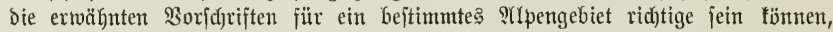
obgleidh fie mitunter etwas wunderlid) exfdeinen, jo ift Deren alfgemeinere biltig=

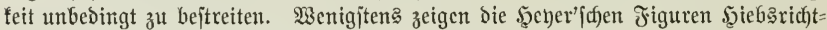
ungen, weld)e wir nad) unjeren (Erfahrungen zum Theil für unridjtige balten mïjjen.

Reuterbings wurbe dieje widftige Frage bon Foritmeipter F. Ba

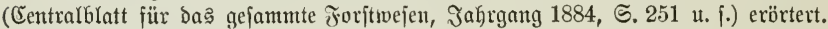

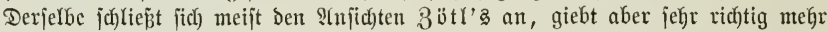

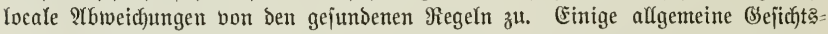
punfte lafien fidj) ja geminnen, bas ijt nidft zu Yetgnen, und je twidytiger bie Stumfrage ijt, bejto willtommenter mitijen Derartige, Durd)bad)te (Erörterungen in

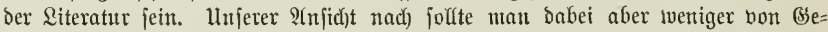

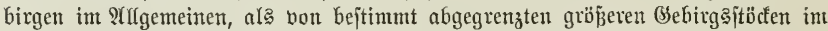
Einzelnen fpredjen. Die berjdjiedenen (bebirge berbalten fid eben beridfieden. So

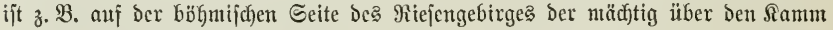

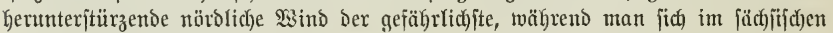
Erzgebirge weit wentger bor bem Morbwind zu fürd)ten brautd)t. Mir find ferner

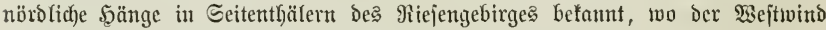

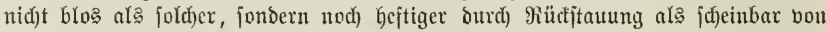

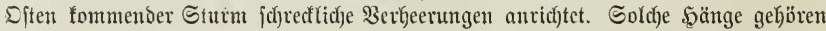

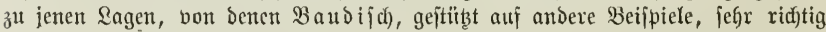

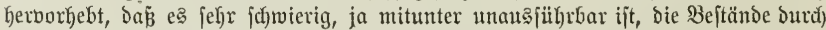

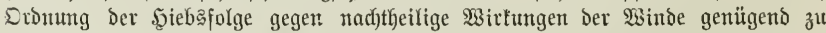

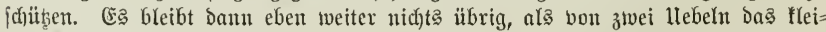

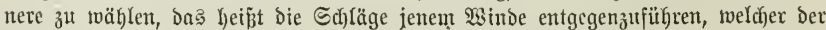

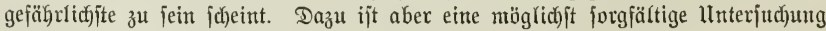
ber ïrtlidfen $\mathfrak{B}$ erbältnifie unbedingt nüthig. 


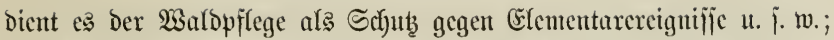

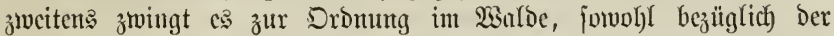
Erute, als ber Siultur; Drittens erlect)tert es alle Bermejiungararbeiten, bie neuten jowohl, wie bie Padfträge, indem es viele jitfere Stnbinde= punfte gemährt. Der burdy bie Edyeifen in ?(njprutch genommente Bobentaum fan baher gar nidyt in Betrad)t fommen, itm fo weniger,

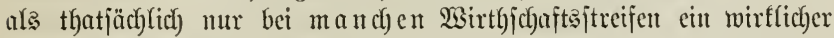
Berlujt ftattfindet, uno als ja überbaupt bie Piente vom $23 a l d b o b e n$

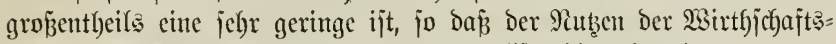
itreifen ben fleinen Entgang an Jiente vollitändig überwiegt.

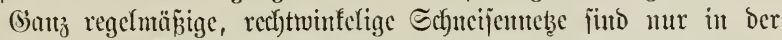

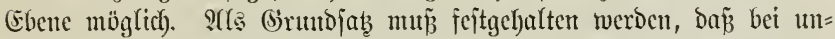
ebenem Terrain bas Schneijenneb jich möglichjt bicjem atjupajien hat.

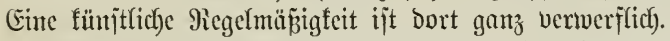

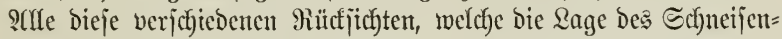
nebes bebingen, rufen utzäblige Berjobiedent)eiten beffelben hervor.

SCla erläuternbe Beijpiele mögen ferner folgende zıei Figurent bienen:

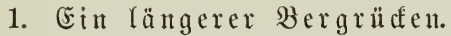

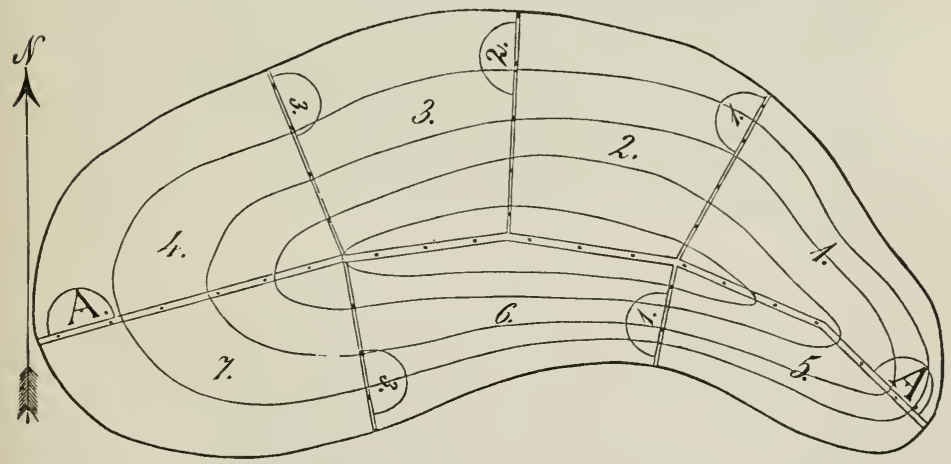

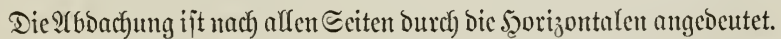

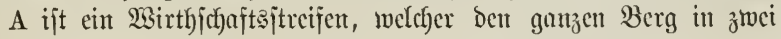
Theile jucheibet

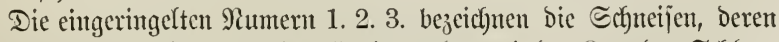

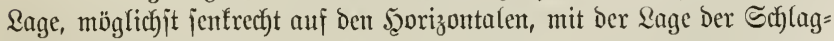
linien übereinjtimmt. 
Der Şieb hat in Der Şauptjache von Dit nack Wejt fortzujachreiten,

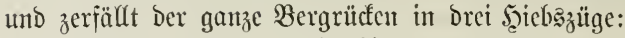

Ifotheilung 1. 2.

3. 4.

5. 6.7 .

Die Numerfolge ber Afbtheilungen entipricft fier ber Şiebsrichtung. Wären bei ähnlichem Terrain bie Scänge zu hoch, um bie Schläge

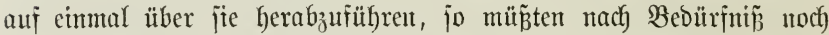

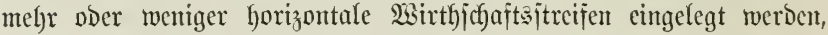

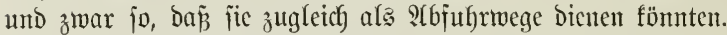

शidcht jelten funbet man in jolchen Fällen horizontale Echläge geführt, welche alfmälig von oben nach unten fortichreiten. Einte ber= artige Schlagorbmung wärbe bie am 5̧ange herablaufenden Echneijen

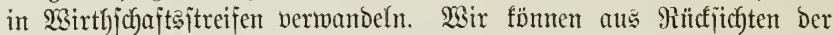
Bejtandspflege namentlich bei jteilem Terrain bieje f̧orizontaljchläge nicht billigen, möchten fie baher nur auf $\mathfrak{A}$ uลnahmefülle verweijen, wo jeljr ungümjtiges Terrain baż zmingt, ober nur vorübergehend gejtatten, wo eine fehlerbajte, frühere Echlagführung nicht plöblich) geänbert, fon= bern nur allmälig verbejfert werben fann. Fajt noch feblerfafter als bie von oben nach unten fortiç)reitende 5̧orizontalichlägen find joldche, welche in umgefelyter Michtung von unten nach oben fortjchreiten, weil Dann bie jungen Bejtände Der (Bicjahr von Bejchäbigungen Durch Den $\mathfrak{S e d}_{\mathfrak{z}}=$ transport, Durch gerabrollende Etcine, locrabrutjchende Sanghölzer we= nigitens an jteilen ફ̧äntgen fortwährento ausgejeb̧t jünd. Seider findet man Derartige Safläge in höheren (Sebirgen vielfach aus alter Beit jtumment.

\section{Ein ifolirter Segel.}

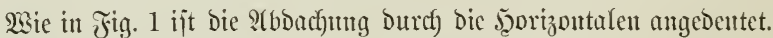

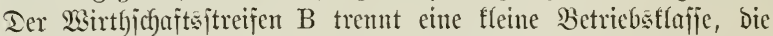
Epibe Des Bergfegcls, melche plenterweije betwirtbichaftet werden joll, vou ben bem feflagmeijen Betriebe zugehörigen 2 fbtheilungen. Dieje

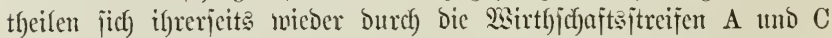

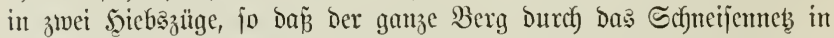
Drei ber leb̧teren zerfällt:

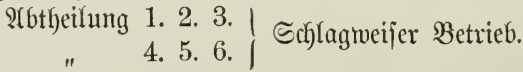

$$
\begin{aligned}
& \text { 7. Plenterivalo. }
\end{aligned}
$$

Die Pumerfolge ber 2(btfeilungen entiprict)t ber Şiebsfolge. 


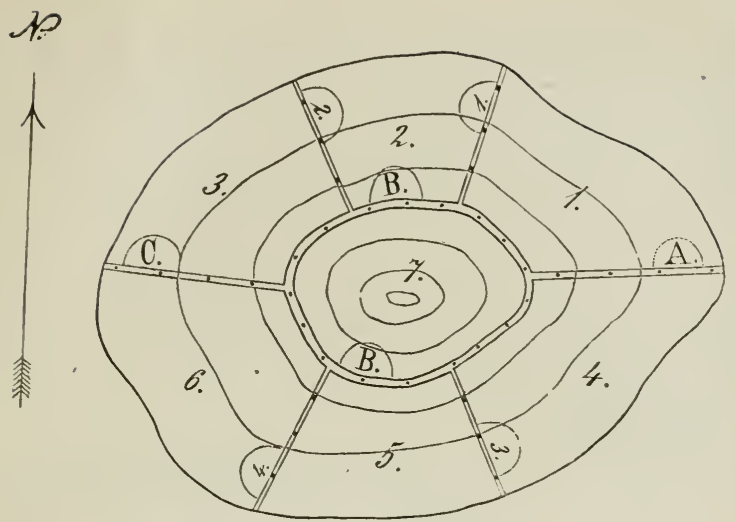

2Bäre ein ähnticher Siegel fo hoch, daz̃ bie jich von dem 2 sirth $=$ jchajtajtreifen B bis an bie Grrenze eritrectenden Schlaglinien zu lang

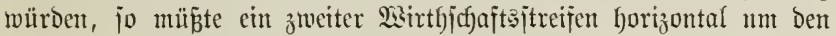

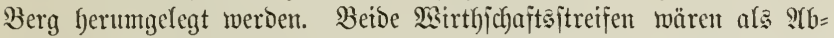
fubrwege herzujtellen und an geeigneten Etellen unter fich und mit Der Berggrenze Durch langjam aufjteigende 2 kege in Berbinbung zu jeb̧en.

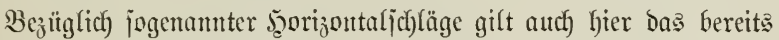
Giejugte, jie find mur Dort anzulegen, two bie gegebenen Terrain= ober

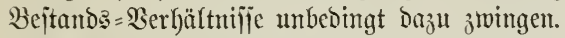

Ein lefr cinfades Beijpiel einer $\mathfrak{s}$ aldeintheilung bietet bie angefïgte $\mathfrak{B} e=$ itandsfarte. (Einen nad) Sïbojt abfallenden 5ang bilben bie, Aftheilungen $1-5$,

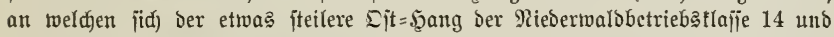

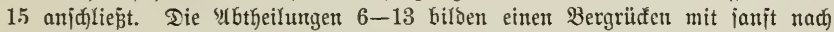

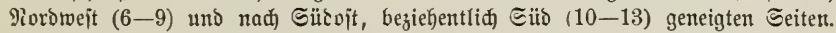

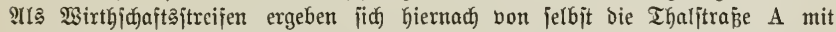

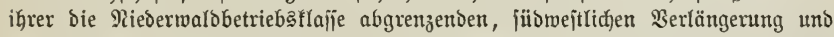

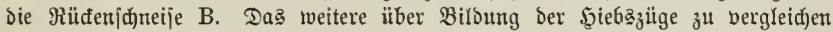
ธ. 267 น. f.

\section{$\S 112$.}

\section{5iuftrexhyeifelfeine.}

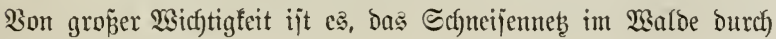

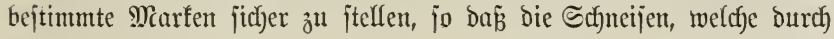




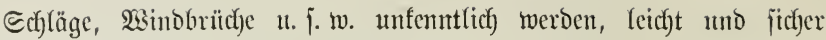

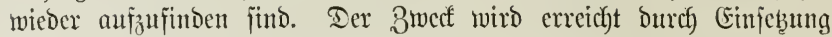

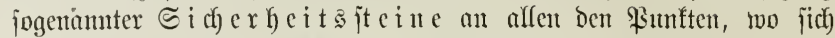
ङdyneifen frenzen Doer bred)ent, ferner aud anf allen geraben Sedfueijen, wenn bie Entfernung Der Rrenjungspunte jebr weit ift. Mindeitens alle 100 bis $150 \mathrm{~m}$ foll cin Cicberbeitsjtein gejebzt werben.*)

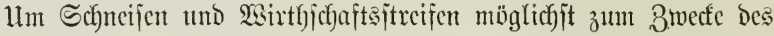
ร̧olztrantsportes bemuben zu fömten, find bic Sicherbeitsjtente nicht auf bie Mittelfinie, jontoern an bie Scite zu jteflen. Die jpätere Drientir= ung wirb erleichtert, wenn man bie Stcine ftets auf eine bejtimmte Ecite, z. B. auf bie Ditjeite Der Ecfuneijen und anf bie Porojeite ber Wirthjchafteftreifen ftelft.

8um Beijpiel:

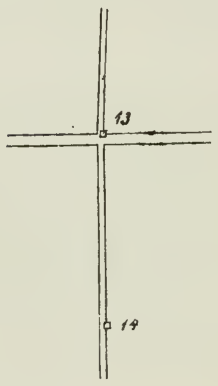

13 bcoutet einen Sidyerbeitsftein anf Dem Sireuzpunfte des 23 irthjichaftsitreifens mit Der Sefyneije, 14 einen joldyen, weldyex einfach bie \&äugerichtıng ber leb̧teren marfirt.

शuß̈er ber Sicherftelfung des Schneijen= neţes haben bieje Steinte noch Den żweiten 3 wed zu erfüllen, für alle Racf)tragsmefjungen in jpäterer 8cit gute Ŝnbindepunte ju gewäh)ren.

Ramentfich) ats leb̧terem (sirunde find aud alfe als ?lbtheilungsgrenzen bienenden Bäche

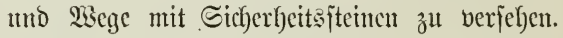
Diejelben fommen fertmärts von ber eigent=

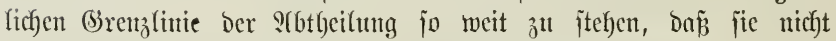
burch) bas $23 a f j e r$ ober Durch bie S(bfubre Bejchäbigungen erlciben fönnen.

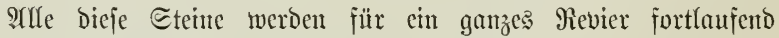
numerirt, die Numern eingefauen und gefärbt.

Bum Yeichten und fidjeren 2 (uffitiden ber Standpunfte verloren gegangener

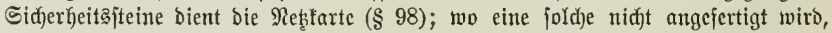
ift es nothwendig, in eitter Tabelle bie horizontalen Entfermungen ber einzelnten

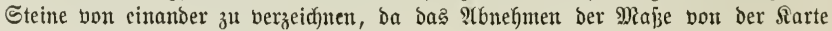
um jo unfiłherer wirb, je älter biejetbe ijt.

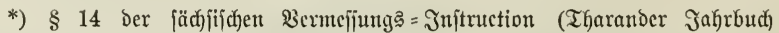

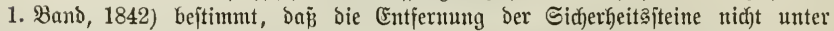

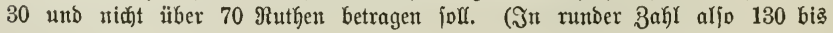
$200 \mathrm{~m}$.) 


\section{$\S 113$.}

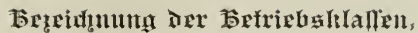

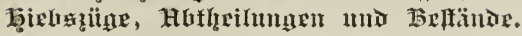

Berfällt ein gievier in melgrere Betriebstafjen, jo futo dicje

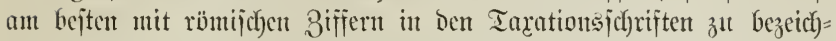
nen, ว. 3. aljo I., II., ı. j. w. Betriebsflafic. 2iothmendig ijt es nicht, bieje Biffern aud) auf bie Siarte zu übertragen. Rebteres würde nux bann einen Sint haben, wenn die Brenzent Der Betriebsflajfen Durch bejondere Beichen (Sirenze oder Dergl.) marfint werden.

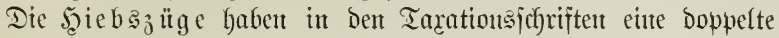

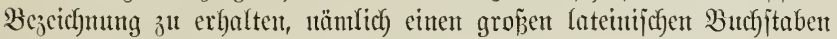
und cinen Paumen, Der Dem ortsïblichen (Siebrautche entipricht.

Dieje $\mathfrak{B}_{\mathfrak{e}}$ eidjunngen würben an Stelfe Der $\mathfrak{z}$. $\mathfrak{B}$. in Sadjfen für bie "Be=

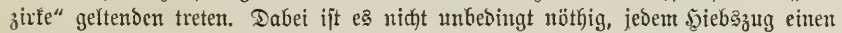
bejonderen Ramen z̆u geben, jobalb er mur einen bejonderen Butdjitaben erbält;

3. B. : $\mathfrak{A}$ Mautrhammer $A$.

S(m Miaterfammer $B$.

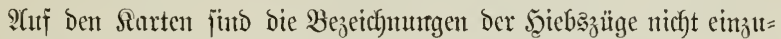
tragen, am menigiten bie Namen, höchjtents die Buchjitaben.

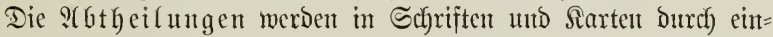
fache bentiche Bahlen: 1. 2. 3. u. \%. w. bezeichntet. (3u vergl. bie Fig. Des § 111.) - Die Pumerfolge hat jith Der leicften Drientirung im Malde wegen nicht nadf den Eectionen (\$ 93), wie es 3.93 . in

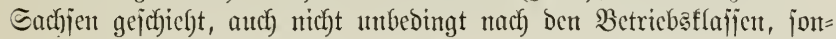
Dern möglicfjit nach Der J̧iebsfolge ju richten, fo ba fortlanfenden s(btheilungsinumern ber Bang bes beicbes angebentet ijt, wie Durch die \$ifeile der Satebsjugifarten (\$ 97).

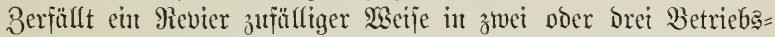
flajien, beten jede für jüch örtlich im Bujammentange liegt, bant

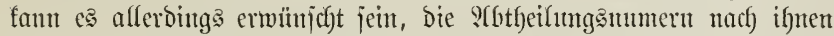

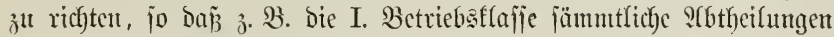
von 1 bis 30, bic II. jene won 31 bis 45 i. j. w. cutfielte.

Die Ilnterabtheifungen oder Beftände werben für jede s(b= theilung mit fortlaufendon, fleinen latcinijchen Budfjitaben bezeichnet, 子. $\mathfrak{B}, 1 \mathrm{a}, 1 \mathrm{~b}$ it. $\lceil$. w.

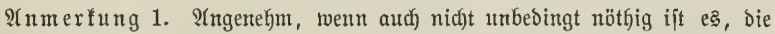

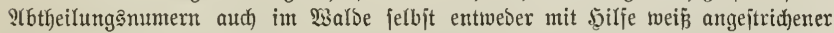
f̧ol $z_{z}=$ ober Binfbledtäfeldyen, ober burd mumerirte Steine fentutlid) zu madten.

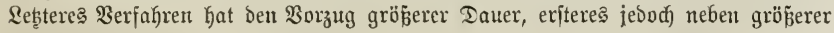
Bifligfeit nod) ben, aud im $\mathfrak{W}_{3 i n t e r}$ bei hohem Ed)nee Dienjte zu leiften. 


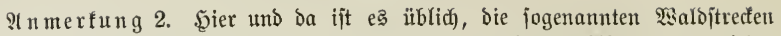
ober Bezirfe mit orts̈̈blidjen Ranten zu bezeidnen und ben 21bthcilungen jeder einzelnen $\mathfrak{B}$ aldjtrecte eine bejonber, bon $I$, anfangende Rumerjolge zu geben.

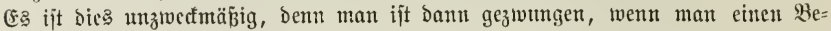
itand neunen will, brei Bezeiduungen anzuwenden. Wäbrend bei ber vou uns

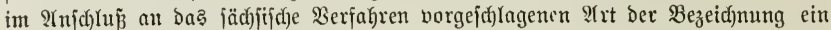

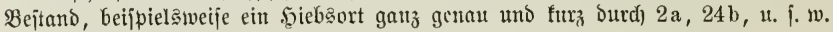
bejtimmt ijt, muг man nach obiger Metfode nod) Den mefr oder weniger langen

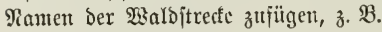

Der fleine Budhenberg IIa.

QIm gebrannten Stein Ie.

u. $\{$. w.

\section{§ 114.}

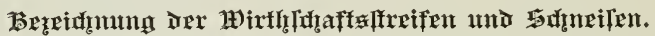

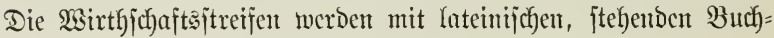
jtaben (A., B., u. j. w.), Die E(f)ncijen mit beutjchen Bahlen auf Den Sarten und in ben Sdyriften bejeichnet.

Itm bieje Bezeicfmungen, namentfich bie ber Schneijen von anderen anf Der Sarte, z. $\mathfrak{B}$. von den S(bthcilungsmumern zu untericheiden, wählt man für ibre Etellung als \$ajis die Schneijen jelbjt mo

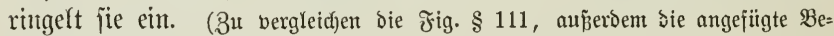
itantosfarte.)

Dabei erhalten ein Wirthjofaftsjtreifen oder eine Echneife, die munterbrod)en, wem aud nicht geradlinig fortlaufen, ci ts n Buck)= jtaben voer eine গiumer.

Richt felten bemirft man bic Bezcichnungen Des Schncifentebes

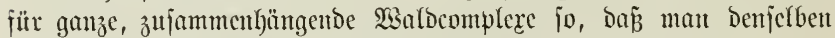

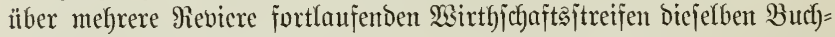
jtuben, analog den mebrere Reviere berübrenden Schneifen diejelben

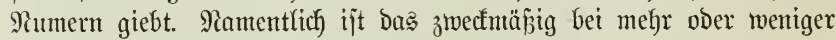

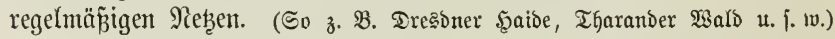

\section{$\S 115$.}

\section{Iothyiele.}

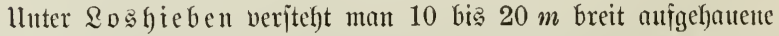
Etreifen, Durd) melche man Beftände in Der Richtung des Sciches Dort trenut, wo jpäter şanungen eingelegt werdon follen.

Fine folche vor ïbergchende Mapregel wiro in Madctfolz= 
foriten, namentlich in Fichtenwaldungen, nicht jeften nothwenbig. (Es lyandelt jich Dabei Darum, Bejtandsgrmppen, cinzelıe Bejtände voer Sejtandstheile au ben freien Stund zu gewölynen, jo baj fünjtig Schläge an ber gefübroten Ecite berjelben feinen Echaden verurjachen. (2indo, Somute 2c.)

Entweber fömuen größ̄ere, gleichalterige ober nah) zu gleichalterige Bejtandsemplexe butch joldce Streifen getrent werben ober audf un= gleichalterige. Rebeterer Fall tritt o. B. Damn cin, ment cin bald hiebs= reifer Drt a jo an cinen jumgen, jetst crit 20 bis 30 jübriger bejtand b

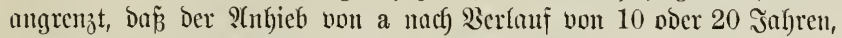

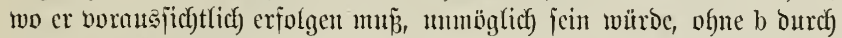
2Bind ôn gefübrocn.

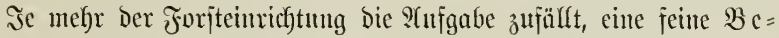

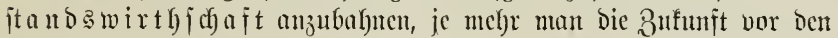
Siad)theilen zu bewabren hat, Der Şicbegfolge wegen zumachsarme $\mathfrak{B e}=$ jtänto jtef)en lajjen zu müjicu, Dejto wichtiger erjefeint es, burch) viçe S(nf)icbsränme eine möglichjt grof̧e Bemeglichteit in bic ganje 2sirtbjed)aft zll bringen.

Wo es bic Bejtunda = ober jonjtigen Berfältnijje gcjtatten, z. 3. bei nothwenbiger Tremung gleichalteriger Bejtände, legt man bie $\Omega_{0}:$ biebe gern an bic Edfucijen. Rotfyendig ijt es indefien nicht, jie fömen jich auch winfelig um bie Beptände hermmjieben.

Bezüglict) ber Benemung ber 20 shiebe fam man folgenden llnter= jajicd madyen:

1. Soshbebe, welche durd) gleichalterige nocr nafyezu gleichalterige Bejtände, und zwar in Der Piegel an Echneijen, angelegt werben, nenut man Sicherbeitsitreifen ober Durdybiebe.

Beijpiel:

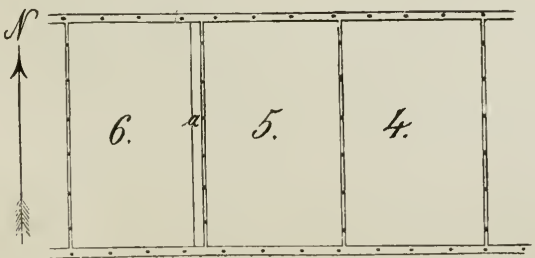


Der Şieb jadreitet von Dit nack $\mathfrak{B c j t}$ vor. S(btheilung 4 cutfält

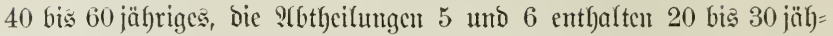

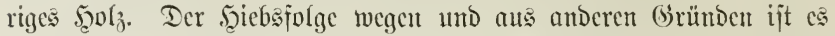

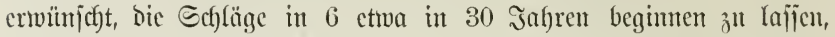

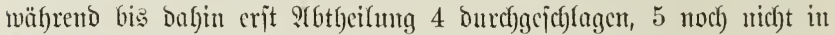

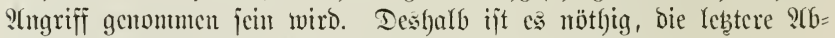

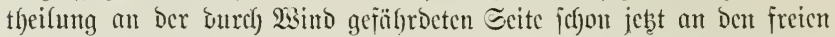
Stand zu gemöbnen, und bedentet a Den $10 \mathrm{~m}$ breiten, aus bicjem Grunbe angelegten Sicherbeitsjtrcifen.

2. Soshiebc, weldye fich winfelig um einzclue jum lleberbalten beitimmte Bejtände ober Beitandsgruppen herumzichen, nemut man ll $\mathfrak{n} \mathfrak{h a} u \mathfrak{u g} \mathfrak{u}$.

Beipipicl:

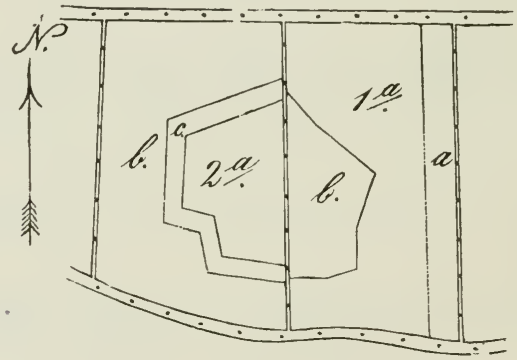

Der Şieb jefreitet won Dit nach Wejt vor. Der größ̄ere Theil

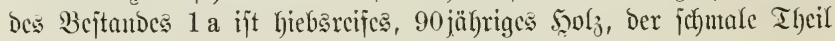
an ber Echncije ijt butreh) bie lanfenden Echläge gebilbet.

1b uno 2a find 20 jährige Beitände, bie nicht nit jum J̧icbe gebracht, fondern übergehalten werden joflen.

2 b cin 80 bis 90 jähriger Bcitanto.

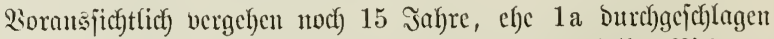

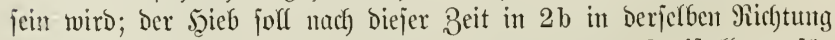
fortichreiten. IIm un ben Befalgren Der fïnftigen Freijtellumg fïr 2a vorzubengen, wirb jefon jeb̧t cin Theil von b, uäulich ber $20 \mathrm{~m}$ 
breite Etreifen e abgetrieben. Er bilbet cinen Soshieb, cime Umbau=

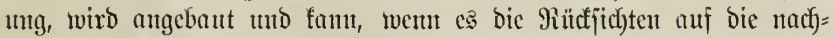
wachjende Siultur erforderu, allmälig breiter gemadht werben, noch che die laufenden Echläge $2 b$ treffen.

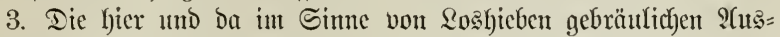

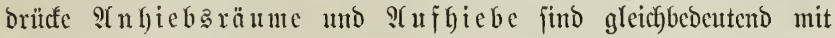
eritereut.

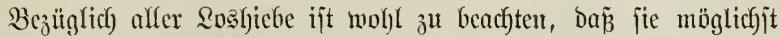
zeitig im jugenoficlfen ?flter Der Bejtände, und jwar breit genug an= gelegt merben, un jie jofort burch) Pffanjung ober Soat anbauen ju fömuen, Danit fie berciţ cinen mintejten 10 jälyrigen bejtand tragen, wenn cinjt bic cigentlichen Echläge an ber betreffenton Etelle fort= jchreiten jolfen. Nian wählt aljo cinte Breite von ungefähr 15 bis $20 \mathrm{~m}$. WHill man bei ber erjten Shnlage eines Loshiebes aus irgend

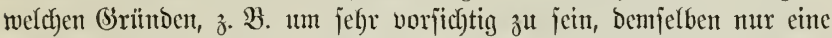
fo geringe Breite geben, etwa bis zu $10 \mathrm{~m}$, bá ein jofortiger S(nbau nicht thunlich erjecheint, jo mun nach etwa 5 bis 10 Jafren eine 2 ser=

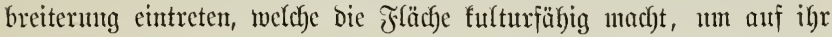
cinen jungen bejtund ju Gaben, ebe bie eigentlichen Schläge begiunen. Stets jund bie Roshiebe in jenem Bejtande zu fübren, weldyer eimpt juerft abgetrieben toerden joll, an Echneijen alpo in ber hegel an

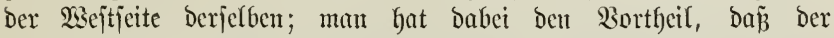
jtehen bleibende Bejtand an Dem frei werbenden Piande jadyon einige Bemantelung bejibzt. Eebenjo ift es bei lumbaunngen, weldbe jefre ber= jchieden alte Bejtände tremmen; fie merben nicht blos beshalb im alten, zlterit zum afbtriebe bejtimmten scolze gefübrt, weil maut bort bas nub̧barere Material gewinnt, jondern weil ber babinter liegende junge Beftand bereits wenigitens cinigermañen an freieren Stand gewöhnt ijt, eiute tiefere Beajtung hat.

(S) Weges, gewiffe Bejchaffenteit Der Bejtände, j. B. ungemölynlich lichter

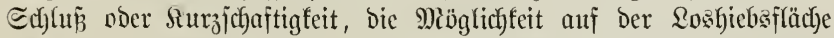

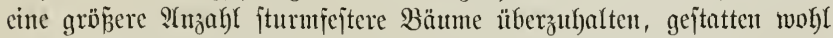
aucf in älteren Beptänoen \&oshicbe, allein mentigitens in Den iturm=

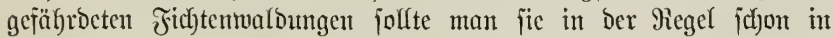
ober an 20 bis 30 jährigen, nicht mebr aber in ober an ïber 40 jäh $=$ rigen Bejtänben anlegen. Qebzteres ijt mintoftens jeflur getwagt.

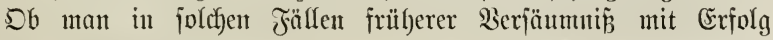

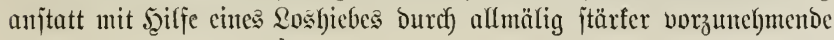




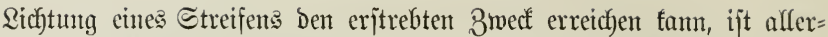
bings im Allfgemeinen zweifelfaft, allein immerhin etwas jidjerer, als ein gewagter Sosfieb. Ein joldher Sifunjitreifen ijt, wenn er an einer Echneije liegt, natïrlich nicht auf Der $29 e j t=$ jonbern auf Der Ditjeite Derjelben, überbaupt aber in ben von Den fünftigen Schlägen rünf́=

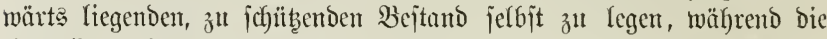

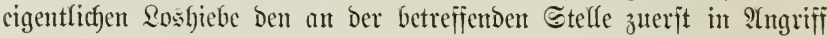
3t nehmenton Bejtand treffen.

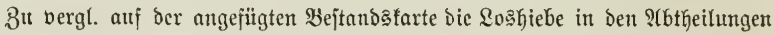
3, 4, 6 unD 7 , forvie ben zut lidstenden Sdjubjtreifen in 11 .

Die Roshiebe, auch wenn man fie im obigen Gimme als Sicher= hcitsjtreifen bezeidynet, unterjcheiden jich von Den Wirtbjichajtsjtrcifen

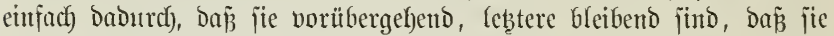
entweder jofort ober inentgitens nach einigen Sufren angebaut werden, mährend bie 23irtbfchaftsjttreifen untultivirt bleiben; antsnafmšmeije fann man auf bicjen höchjtens eine lieife \&anbyölzer ampflanzen

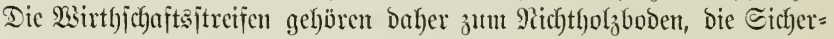

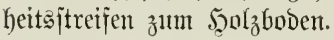

\section{$\S 116$.}

\section{Referven.}

Bitele der älteren Einridftungsmetlonden legten ein bejonderes

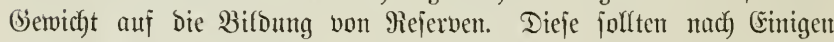
dazu bientu, eine in Folge unvermeidlidyer Srrungen bei ben erjten

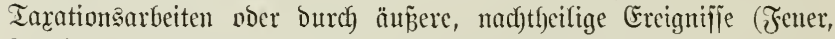
Injeften u. f. w.) etwa eintretende Störung Dor ftrengften Rachbaltig= feit zu becfen. S(ndere wollten bie Rejerven Deshalb haben, um Dem Waldbejiber int Siotbfall eimmal einte auperorbentfiche jăaung zu ge=

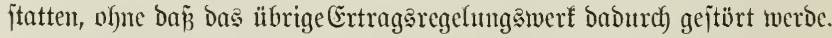

Man unterjechet jtehende and friegende Mejerven.

Erjtere werben Durth abgejonderte Bejtände gefilbet, melche man

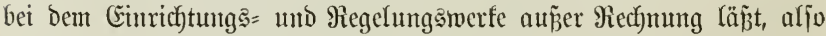

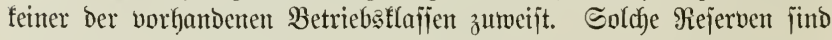
ganz verwerflich). (Earl Şeyer jagt Darüber jehr richtig: „Bejtimmt man

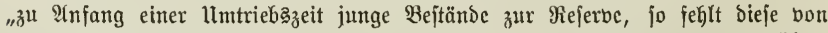
"born herein; wählt man baz̧u alte Bejtänbe, uno will man bieje nidjt über= "ftäntig werden lajfen, jonbern früher berjïngen, jo entbehrt man der Rejerbc

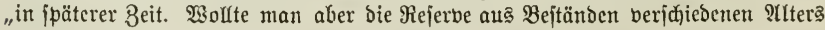

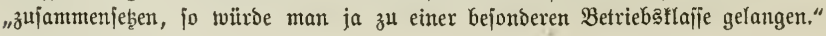




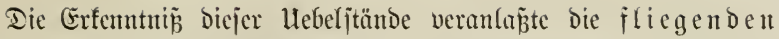

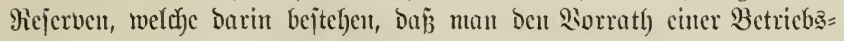

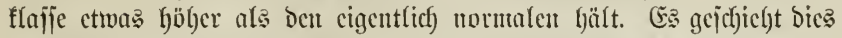

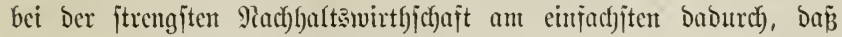

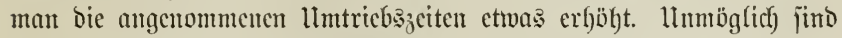

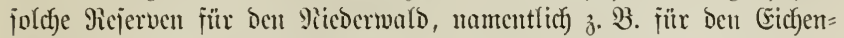

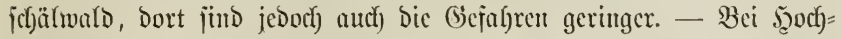

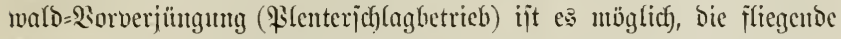

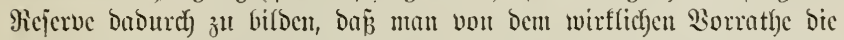

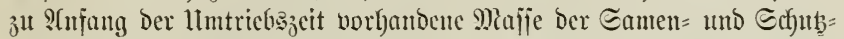

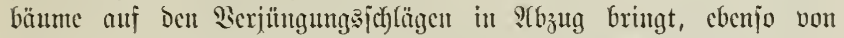
Periobe ju Periobe, wemt auch in anderen Bejtänden überträgt, bis einjt bic Piubzutug tiötfig wiro.*)

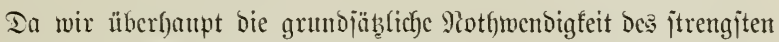

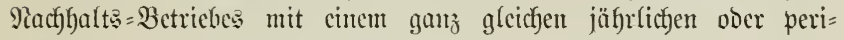

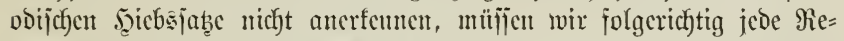
jerve, itehenbe fowohl, wie flicgende, nidft blos für überfiüjifig,

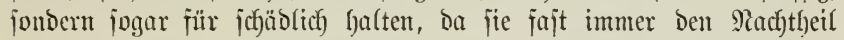

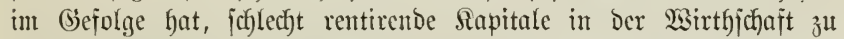

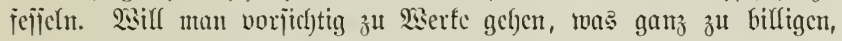

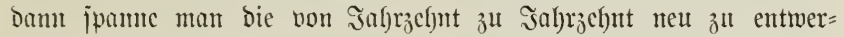

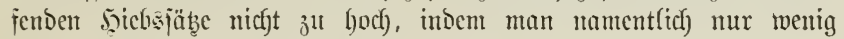

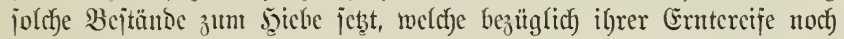
fraglicfer Patutu jüro.

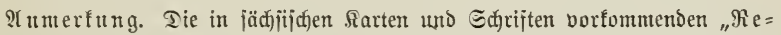

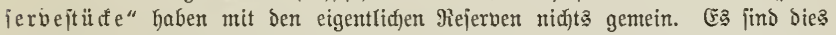

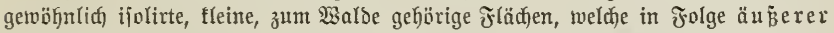

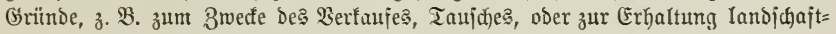
lidber Edjönkeit u. j. rv. aujer Eintheilung gelajien wurben.

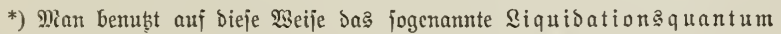
(nad) v. Sisedefind) zur Bildung ber fliegendent Rejerve. Die früher üblid)e, bis zur Epiełerei ausartende Red)nung, welche für jebe Reriode ber llntriebszzeit oder für jede llmtricbszeit gejondert gefüfrt unb bilanzirt wurbe, madjte es nöthig,

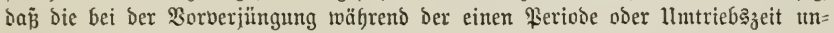

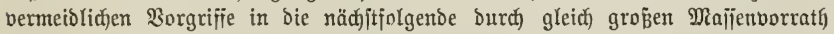
auf Den Yaufenden Berjüngungşjđlägen gedectt twurben. Ter ßorgrifi twurbe jo

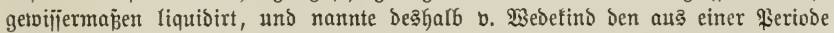

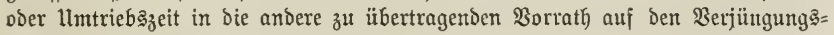

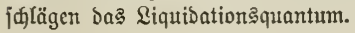




\section{\& 117.}

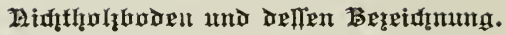

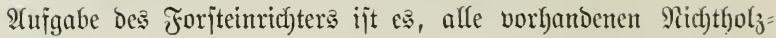

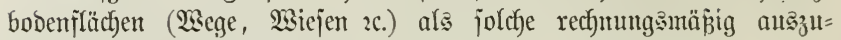
jheiben, beziebungąweije mit Eicherbeitsjtemen zu begrenzen.

Wege und Flüjpe werden mit den ifnen zufommenden शamen, die Wirtbj(c)ajtajtreifen mit ifren Budfjtaben in Sarten uno Sdjriften bezeichnet.

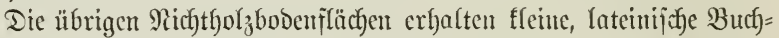
jtaben, bie zum llnterichiede von Den Bezeidfunngen Der Unterabtheil= ungen $($ Bejtände) mit rotfer Tinte gejedrieben werben.

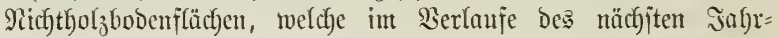
zefntes jicher zum jolzhoden falfen, beshalb auch in ben Sultur= plan aufgenommen werden, verlieren ifyren anfünglichen Égarafter und

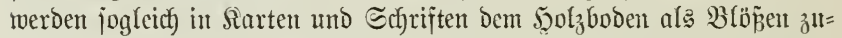
geid)rieben, wemu auth bie anderweite Benubung berjelben noch einige Jahre Dauert, wie es z. B. Gei verpact)teten Feldern, SBiejen, Teichen 2 . nicht jelten vorfommt.

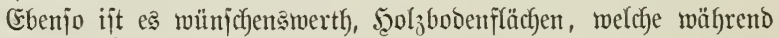

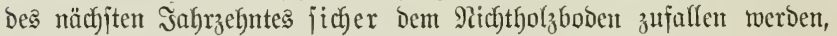
jofort als jolchen zu behandeln. 


\section{Ertragsocitimumun.}

\$ 118.

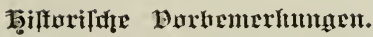

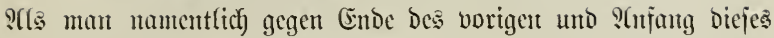
Jahrlfutnderts anfing, bic Frage viclfach ju erörtern, welchen ntad)=

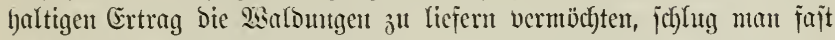

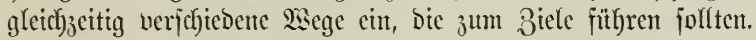

Es entwidfelten fich hiernach principicll werichtedente Metgoden, bie wir in vier Scauptgruppen orbnen fïment.

I. Die Echlageintfycilung.

II. Die Fact)werts = Miethodent.

III. Die Rormaluorraths = Miethodent.

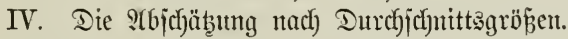

Der ipecielferen B̉etrachtunt Der einzelnen Methoden jei ein

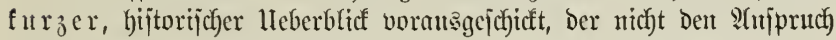
erbebt, einte (Siejchidc)te des Tarationsmejens bedeuten zu wollen.

1. Die ङchlageintheilung und Reime Der ipäteren Methoben.

Die Echlageintheilung (i. § 119), welche bie ganze Fläche bes $23 a$ aldes in jo viel cinzelne Echläge theilt, als bie llmtriebszeit sahre umfäat, ijt ohne Zweifel bie ältejte unt vor Berfmann wafyricheinlich bie cinjige Micthode, erhielt fich auth noch lange über bie Zeit beß leb̧teren ljunas. Dic mansfelder Forjtoromung wou 1585 fübrt bereits in Dem Sorberfarz eisten regelmäß̄igen llmtrieb mit Schlageintbeiluntg

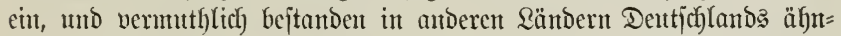
liche (Ëurtichtungen auts noch weit älterer Beit, namentlich in ben

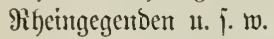

Dieje ältejte Methode wurbe verjefjeden auggebildet, do man bald

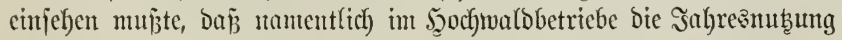

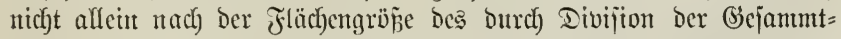

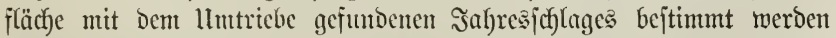
fonnte, jonbern baj bie Werjefjedenfeit Der Stantorts= und Bejtands= verfjältnijīe Berücfjichtigutg veroiente. 


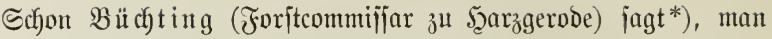

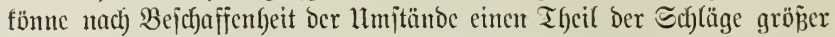

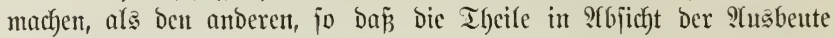

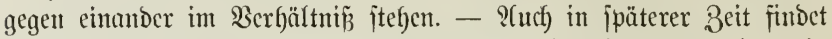
Büchting noch) Den leitenden (Brrtmogedanfen Der Evtragšregefung in

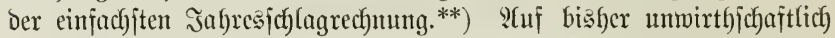

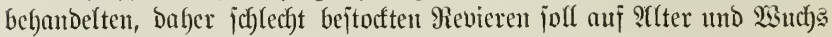

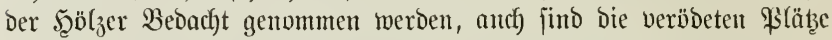

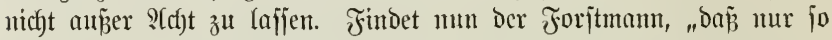

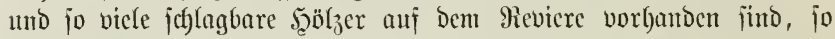
theilet er bicje bergeftult ein, Da ż jie jo lange zureicfen, bis bie fol= genten mieder joblagbar gemorben find. So verfägrt er burch alfe

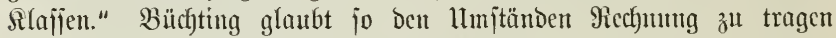
und Das gievier Durch) Sclyomung und S(nbau einem 3utande entgegen=

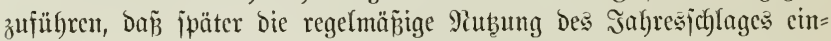
treten faun. Saie bas müglich jein foll, bleibt unflar, ba cr (1. c.

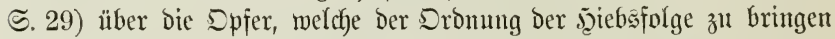

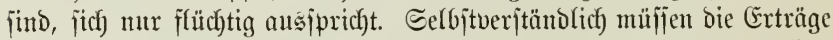
wenigitens wäh)reno bes eriten llmtriebes um fo unglcicher ausfalfen,

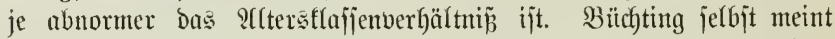

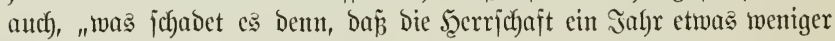
(Einfünfte aus Dem Yevicr hat; ween Dadurch) Dajficlbe in einen guten

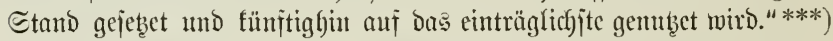

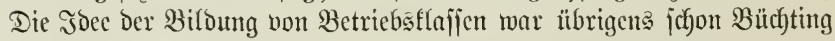
nicfyt fremo, tocrigitens beutet er jie an für Ricvicre, welche aus ver=

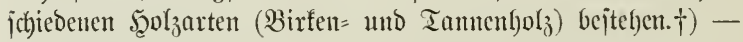

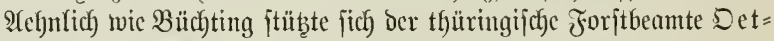

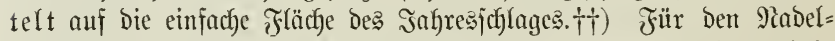

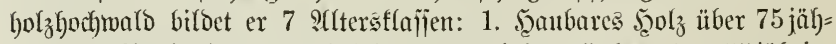

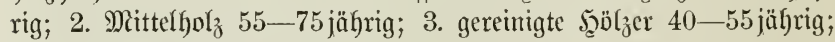
4. Єtangentyolz 24-40jäbrig; 5. junges Diffidyt 12-24jäf)rig; 6. juntger

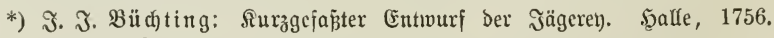
2. 2uflage 1768 . ธ. 241.

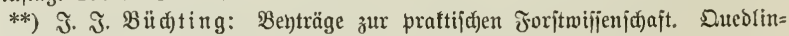

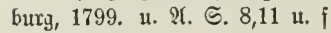

***) Entwurf ber Эägerel). (. 242.

†) Dajelbjt S. 245 .

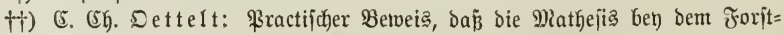
wejen unentbegrlide Dienjte thue. Eijenad), 1765. 3. 2(uplage 1786. - ફூicrzu

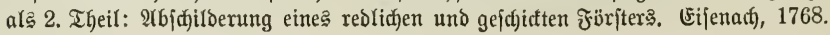




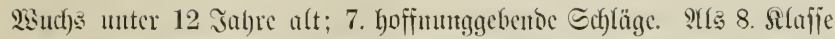

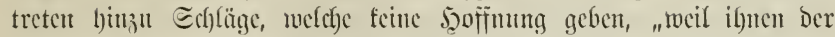
Rorjtand von Eamen benommen, ober tocil fic von Triften nicht

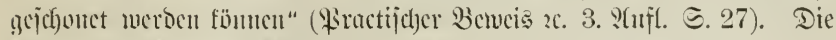
(S) jaumutfläcl)e ber 7 silafjen theilt er mit 7 mo findet in bem Dun=

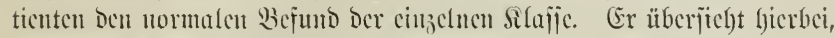

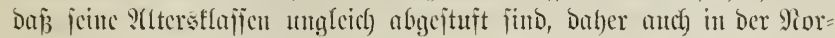

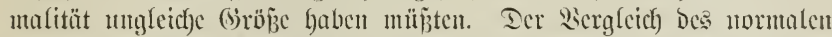

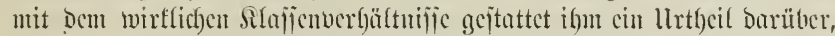

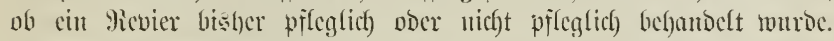

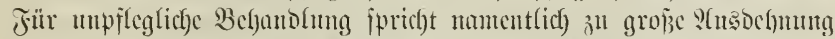
ber 7. und 8., ju geringe ?(usbelyum Der 1. Silafic.

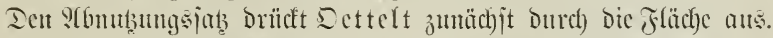

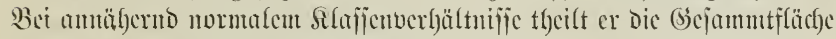
Der 7 Sifafien Durch bic Summe ans llmtricb mo jener 3cit, melef) bic Echläge ungçäly) bis zur vollen Bejtoctung liegen bleiben (Proce

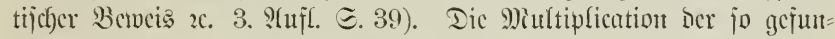

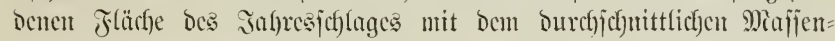

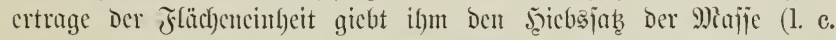
ङ. 42). Iabci Dari mun aber bic Echläge, meldbe jührlich abjumejien

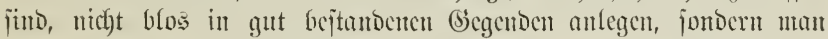

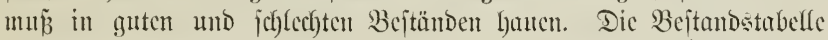
joll Drei Bonitüten, gut, mittefmäßjig uno jed lecht unterichciocn. -

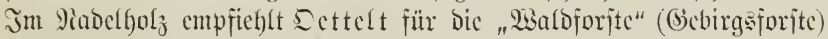

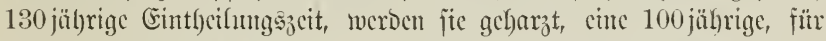
Dic "Zambjorjte" (23älocr Der (Ebcne) cinc 100 jübrigc.

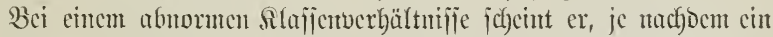

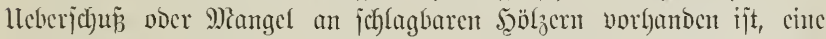

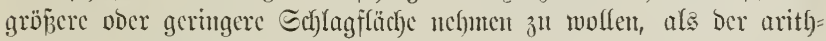
metijace Durchjönitt crgiebt.

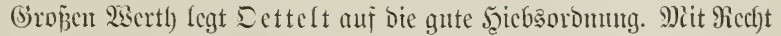

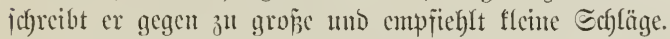

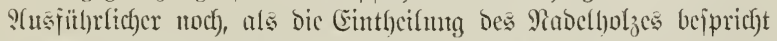
cr bic Des Zanbbolzes, bat aber babei nur ben zu feiner Beit im

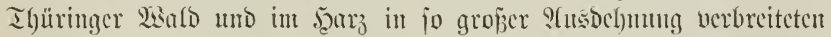

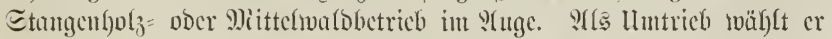

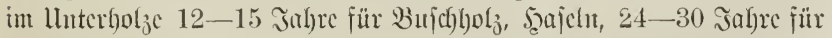
Birfen und $\mathfrak{A}$ spen, 36-40 Jafre für Buchen und Eichen. Der $\mathfrak{B C}=$

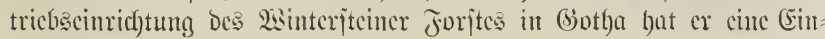

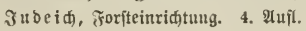


theitung nach ungefähren \$roportionaljchlägen zu (Srumbe gelegt (1. c.

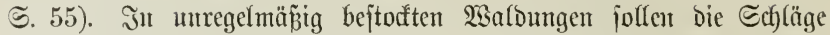
nicht zuerjt in bie ältejten Drte gelegt werben, meldfe ohnefin feinen

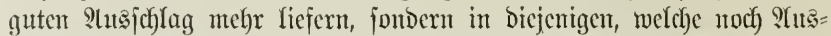
ichlug veriprechen; bie bereits zut alt gemordenen Beftäutbe fommen erjt

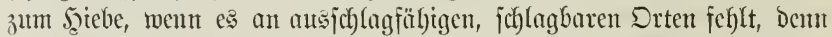

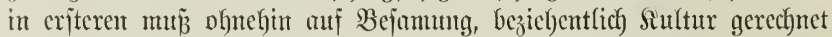
werden. Bei einem Mautgel in Der hanbaren Slafje vertheilt er biefelbe burch) geringe Qf(butbung auf fo lange Beit, bis bie nächit jüngere Silaffe haubar gemorben. Er verläß̈t bier vollftändig bie gleiche Safbres= muß̧ung. In cinem SBeijpiel*) mit 30jähriger Eintbeifung werden im eriten Sabrzebntte jäfrlich) 12 s(cter, in ben beiden folgenden jährlich) 42 :afer gejallagen.

Doglcidy Dettelt fich Den Pormalzujtand Des Waldes noch nicht volljtändig flar macht, hat er jich Doch um bie Entroidefumg ber Forjt= cinrichtung Serbienjt erworben, weil er noch gründlicher als Büchting

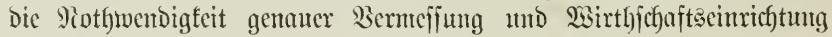
betonte. -

Der F̈̈ritlich fchönburgijche Forjtinjpector Johanu (Sottlicb Scufmann verwarf bic Echlageintlbeilung nollftändig, meil jie in

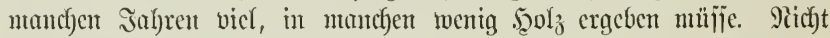
bie Echlagflächen, jondern bie şolzerträge jollen tach ifm antggeglichen werden, **)

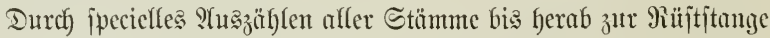

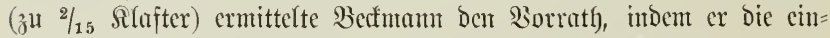
zelnen $\mathfrak{B a r b o i j t r i c t e ~ m i t ~ B i n b f a d o n ~ u m z g g ~ u n d ~ b i e ~ B a ̈ u m e ~ m i t ~ S c i l f e ~}$

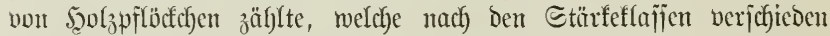
gefürbt maren. Den Bumachs veranjhlagte er je nach) Der Bodengüte mit 1,5 pder 2,0 pder 2,5 Procent. Die Sunme aus bent vorfandenent Borrath) und Dent an ifm erfolgenden, in Folge ber :(btricbe alfmälig fleiner werdenden Bumachje vertbeilt er (wabricheinfich ourch mübjames Srobiren) anf bie einzelnen Jufre cines Beitraumes, welther ifm

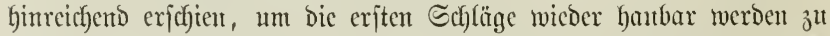
lafjen. Da er übrigens ben Borrath Der jungen, noch) uticht bie Stärfe Der Riujtitangen erreicht habenden Beptünde nicht nit in Rechnung

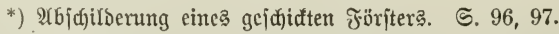

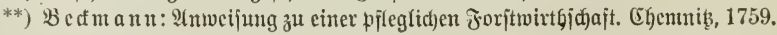
2. Afuffage, 1766 , 
itellt, jo müifien eigentfich) bicic bic eriten Crträge Des zmeiten llm= triebes geben.*)

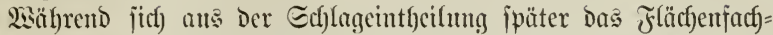

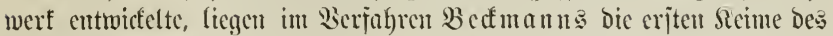
Diajienjactywerfes. -

Ein eigentbünliches Serjaf)ren menbete Der Ranbjügermeijter von 2Bebell an, unter befĩen Seitung vom Jahne 1777 an groje $23 a l d=$

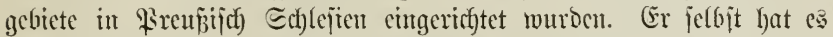

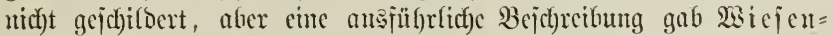
f) a vern, einer jeinter Jeilfsarbeiter, im Jafre $\left.1794 .^{* *}\right)$

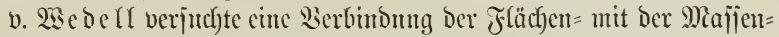
theilung ourdl) \$roportionalichläge. 23ic fein Forjtmann vor ifm, betont Wiejentavern in jeinem Buche bie Pothmendigfcit ber Bildung vou Betriebsflajien, weld)e er "5ुaupttheile" naunte. Seber jautpttbeil joll

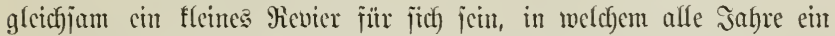

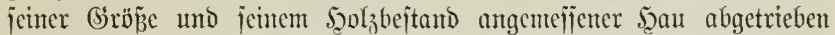

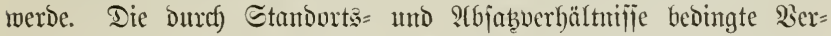

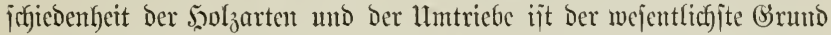
zur Theilung eines Reviers in "şaupttheile." (5)röbere, glcicfartige $\mathfrak{W a l d =}$

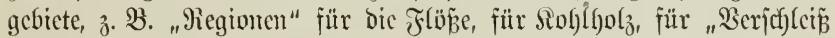
anfs ฉand", werden beshalb in şaupttheile jerlegt, um theils bie

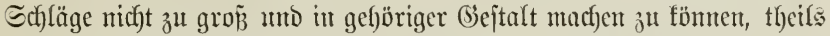

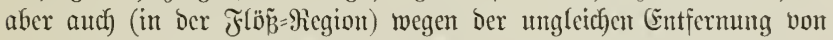

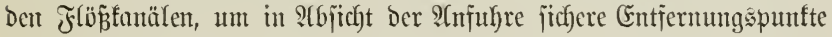
ju haben (1. c. ๔. 17, 18).

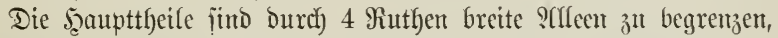
weldje beim Pabelfolge möglichjt gerabe von Djten nach 2 sejten, beim

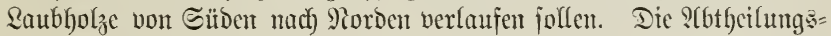
linien ber einzelnen Şaue verlaufen rechtwinflig ober jehräg von biejen

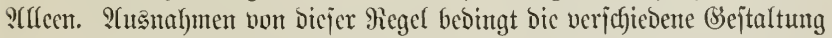

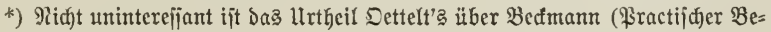
weis 2c. 3. श(ujl. S. 15): „Bectmann toürbe ein nod einmal fo grober Forjtmann

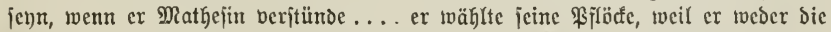
(Seometrie beritunde, nod) fid) jonjt auf eine andere 2(rt zu belfen twujte."

**) $\mathfrak{B}$ iejenkabern: Tnleitung zu ber neuen auj \$hyjif uto Dathematif

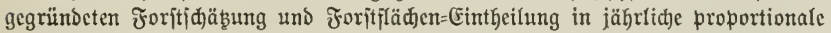

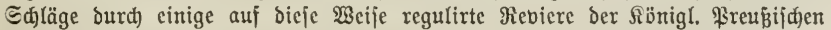
Forjen Sd)lep̣iens nebjt Denen Dazu geb̈̈rigen brei illuminirten Supfertafeln be=

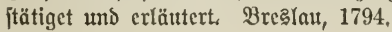




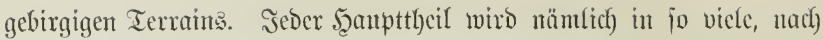

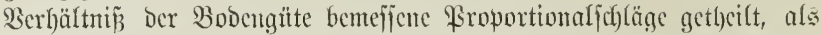
Der llmtricb Sabre zählt. Dieje Echlagcintbeifung gicbt bent Siabmen

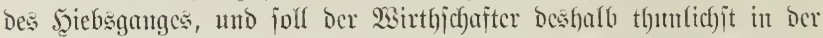

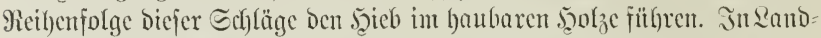

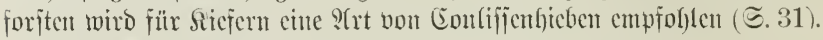

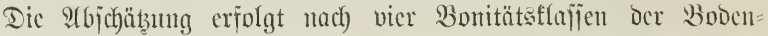

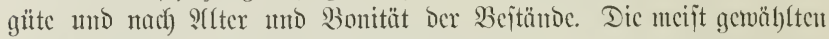

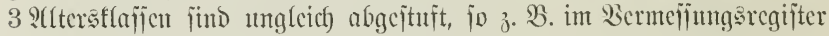
Des Reubujcfer Forftes für bas Padelf)olz: 1) Bau= und Patblgolz (oder

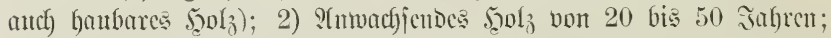

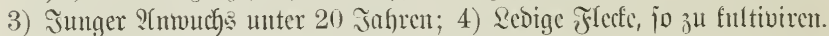

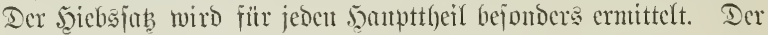

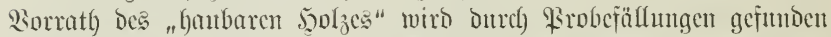

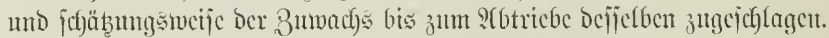

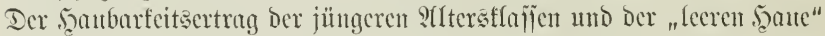

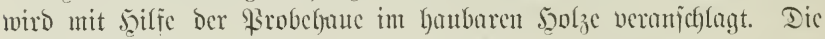
Summe biejer Şauborteitserträge afler Silaffen und leeren şane wiro

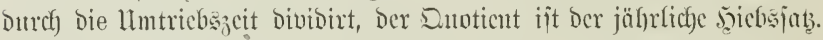

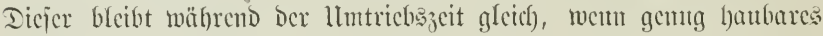

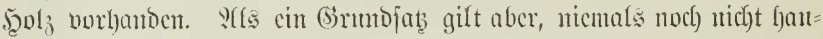

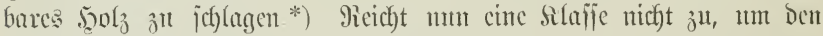

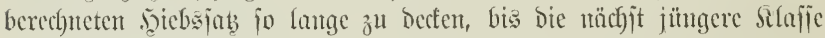

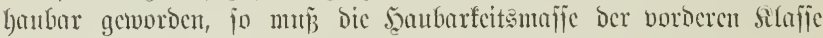
Dutref) Dic 3eit Divibirt werben, welche bic nächjt jüngere braucht, ım hantfor zu werden (ङ. 20).**)

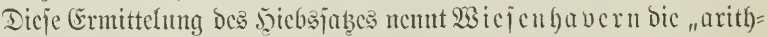

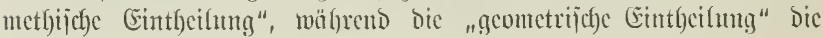
Şicbsoronung begrïnoct.

Das Sicrfabren ift ciu fïr bic bamalige Beit hervorragentos und

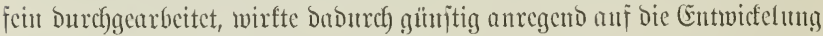

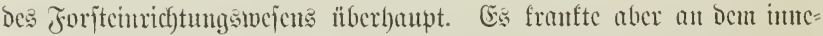

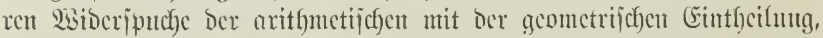

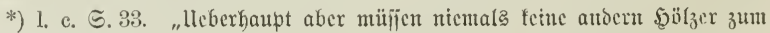

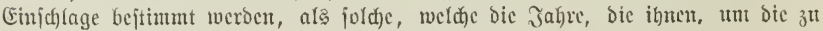

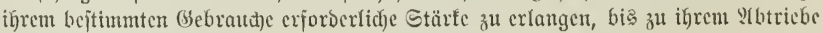
auझgejełset jïnd, völlig erreifjet haben."

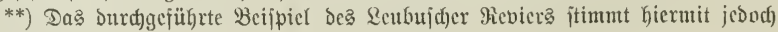
nidjt ganz ïberein. 


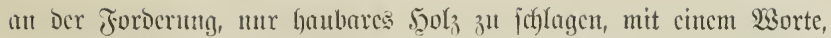

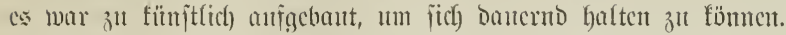

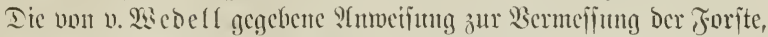

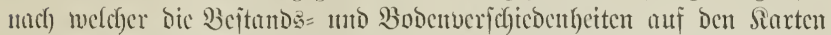

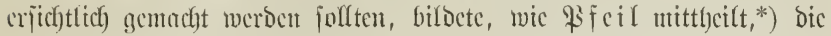

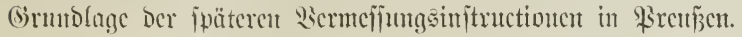

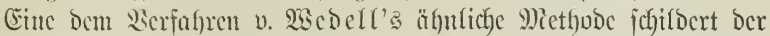
prenfifiche (sichciutc Forftrath, Dircetor Der Forjtarten=Sammer,

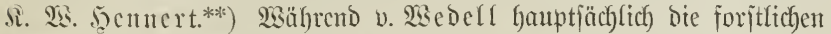

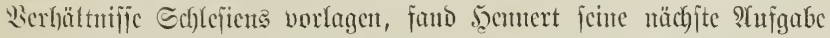
it Den Sicfernforjten Dex Marf. Seţtere waren bereits zu Jagozmecten itt ingentamte "Jagen" cingetfeilt, unD Dicje bemutbte 5̧ennert zur

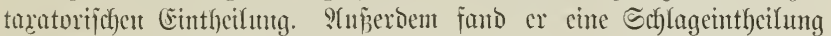

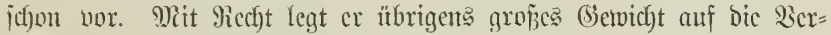

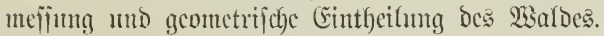

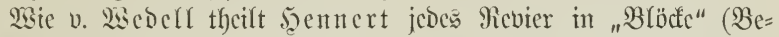

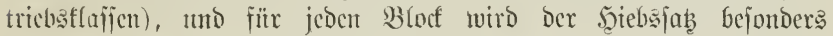

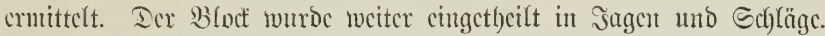

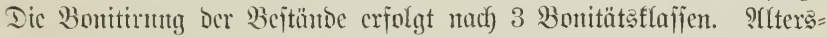

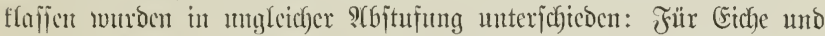
Budçc I. Sifajic 100 jäbrig mo Darüber, II. 30-100 jüfrig, III. 1-30=

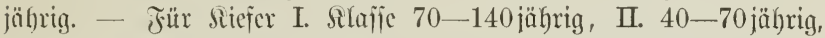
III. 15-40jährig, IV. แnter 15 jährig. - Für Birfe แ⿰亻 Erle I. Silajic $12-35$ jührig, II. bis 12 jälrig.

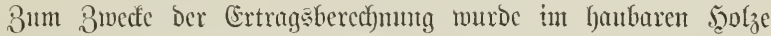
jedes Bloctes in jeocr Bontiät cin Frobemorgen gefällt oder $a \mathfrak{b}=$

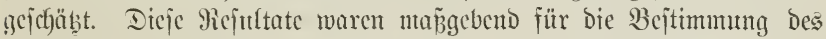

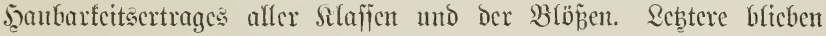

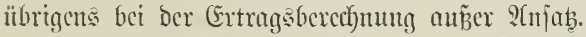

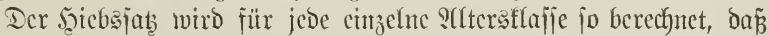
man Deren Saubarfcitscrtrag Durff bie 3 ahl Der Safre bividirt,

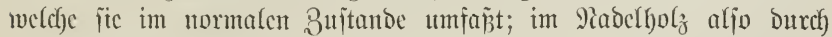
$70,30,25$ ItID 15.

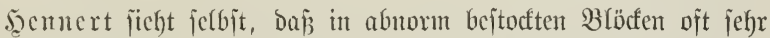
ungleiche Jaf)reserträge Jolge biejes Berfalgrens fein müljen. Unter

*) Bfeil: a. a. D. Die forjttaration. 2. Auflage, Berlin, 1833. - 3 Auflage, Reipjig, 1858.

**) Dennert: 9(nweijung jur Taçatinn ber forften. 1. Theil, Berlin, 1791. 2. Igcil, Bertin, 1795 . 


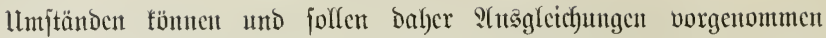
werben. Man theilt bie Enmme aller während cincr llmtricbszeit

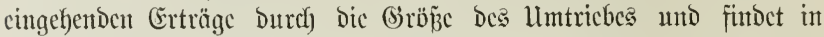
Dem Quntienten Den Jiebsab. Mit Diejem bivibirt man in ben Ertrag Der cinzelnen ?altersflajfen und erfäbrt fo Den Zeitraum, fïr

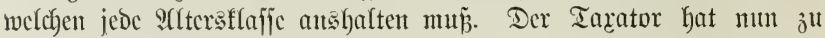
beurtheilen, ob nach Dem Slbtriebe Der älteren Silafie in Der näd)jt= folgenton genug haubares 5̧olz vorlyanden fein wirs, um Den Bebarf

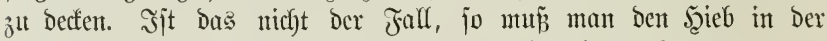

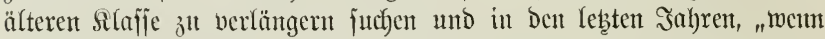
in Der folgenden Silajfe Silein = und M)ittelbaulyolz zu hoffen ijt, cin

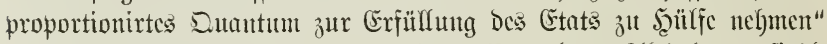

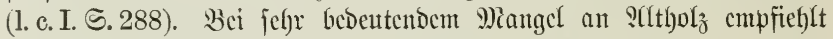

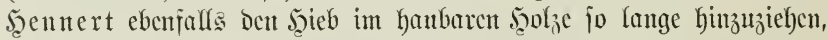

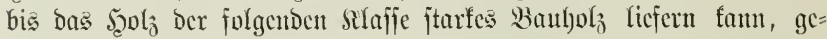
ftattet aber, um nich)t einen gar ju geringen (5tat ju erbalten, glcich)= jeitige Şauungen in Der nächjtjüngeren Silajic, beren ältejte Bçtänoc

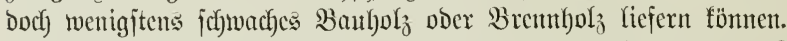

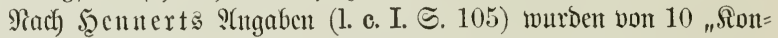
Duftetten in ber 3cit vom Frübjabr 1789 bis (Ende 1790 in verfochic= Denen \$̊rovinzen 191622 ha Staatäforjten vermeffen. Dic Dherffäcl)= lichfeit ber S(rbeiten geht hieraus von felbjt hervor; unt fo melgr, wenn

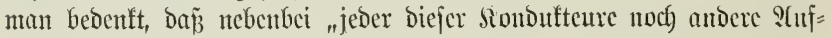
träge gchabt." Die Edfwicrigfeit, mit ber gcometrijct)en Echlagg=

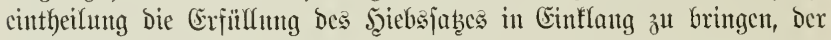

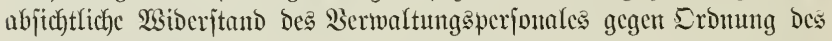

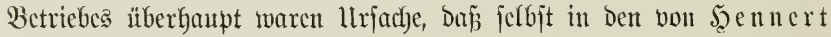

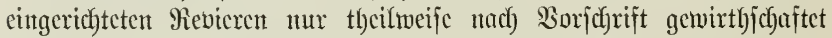

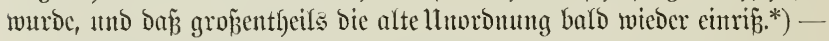

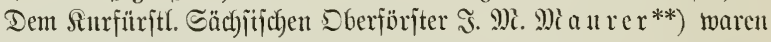
jedenfalls bie :Grbeiten jeiner Sorgänger nicht unbefannt. Er jefjildort auf rocuig Sciten jefr unvollfommen brei M)iethoden Der Ertrugs= bcitimmung.

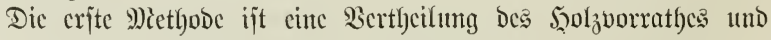

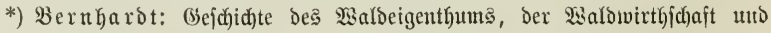
Jorjtmififentjdaft in Deutid)land. 2. Bant. Berlin, 1874. S. 138.

**) Diaurer: Betrad)tungen über eintge fid neuerlid) in bie Forjtwiffenfdaft

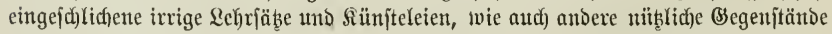

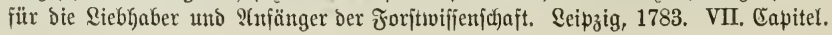




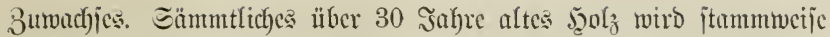

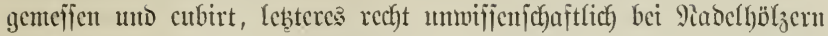
aus Dem Eutabrate bes arthmetijch mittleren, bci Saubhölzern ans

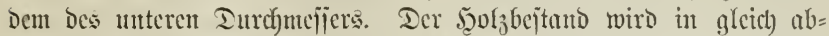

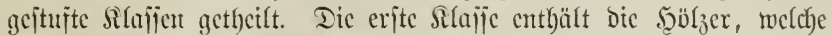
im erjten Jafry

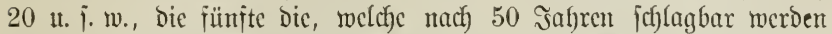

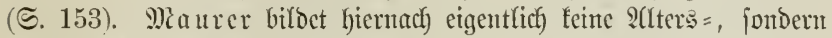

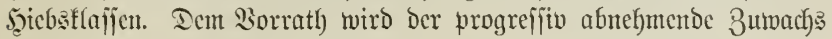

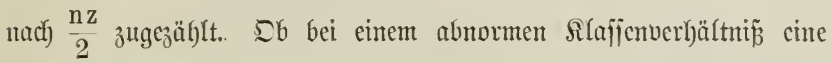
S(ttsgleicfung itattfinden foll ober nicht, Darüber ijt nichts gejagt. Sicic es jecheint, ijt bies nicht ber Fall, benn Maurer betont uirgends

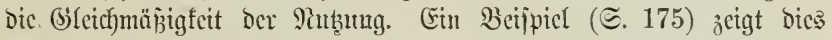

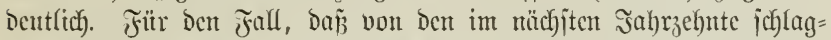

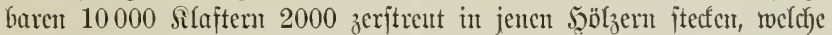
crit in 30 ober 40 Jabren jum Şicbe fommen, ift ber Wirthjchajter

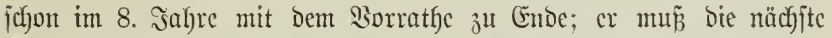

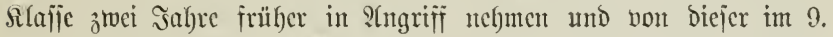

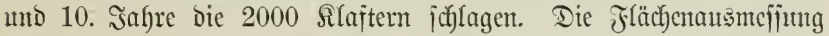
fann bei bicjer Miethode unterbletben, da alle 50 bis 60 Jahre das

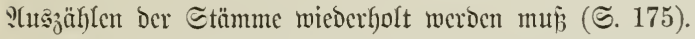

Die zweite Methode Maurers ijt cine Enombination von Flächen=

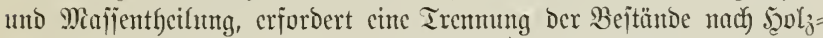

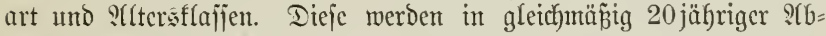

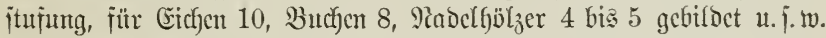

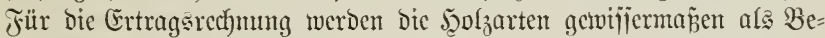

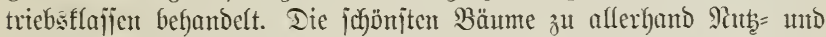

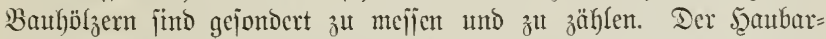
feitsertrag wird als aritfmetijches Plittel aus \$robefülfungen in guten

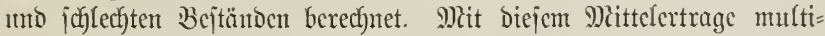

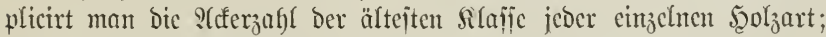

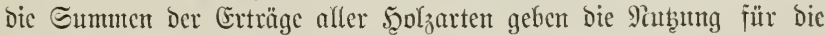

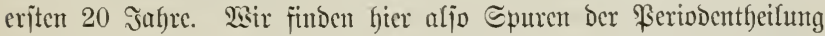
Der Fachwerfsmethoden. Son eiter Staghleichung ber periobijchen Ěrträgc ift aber nicht bie Rede. Dagegen hält $\mathfrak{D}$ a u rer, "wenn ber

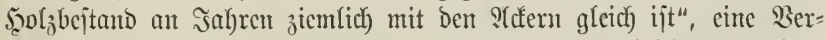
theilung Der gejammten Seantbarfeit:majfen burch Divifion mit bem Umtriebe auf bie cinzchten Salyre für Dejto jicherer. 


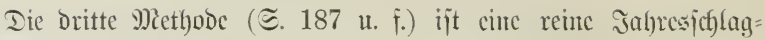

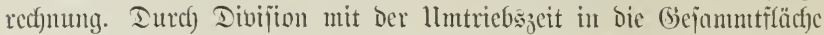

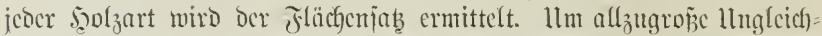
heiten des Ertrages ju vermeiden, biloct Miaure cine Fläcflentejerve

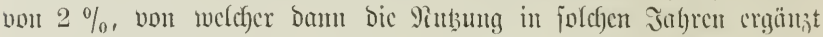
merocu joll, beren Ecfläge bcjonders jefledfte Bejtände treffen.

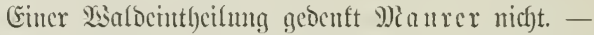

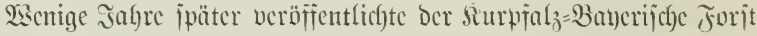

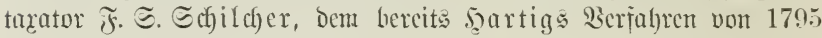

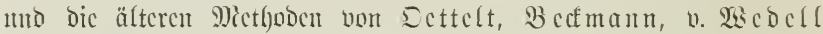
1. \{. w. befaunt waren, cinc bejondere 9)ictlyodc.*) (Er fefort jur reinen Ed)lageintheilung zurücf, legt aber bejonderes (sicmicht auj bas "Tcr=

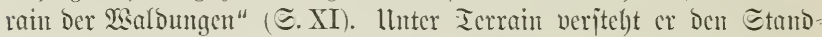

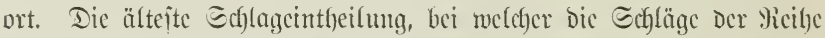

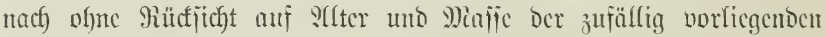
Beitände geïüfrt weroen follen, verwirịt er. 2̧ou ber "Inzation",

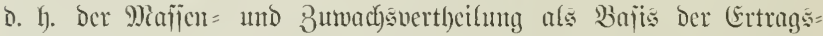

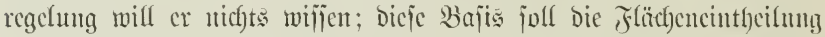

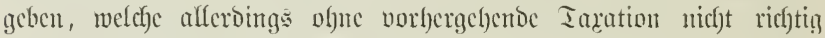
werden fann (ङ. 100), da einc Bonitirung Des Etandortes und Der Bcjtändo geforocrt wird.

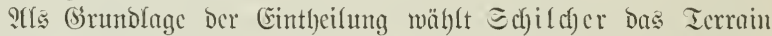
(Etmoort) und bildoct aus Den gleidfuerthigen Flächen "?(bt)cilungen" (cine Sat von Betricbsflajicn). Ia aber ber Ertrag eincs Dijtrictes

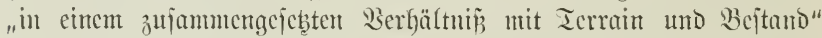

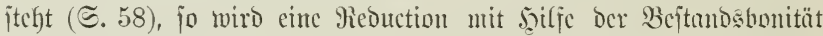
vorgenommen. Er gelangt jomit ju cince Picouction nach gemijefter

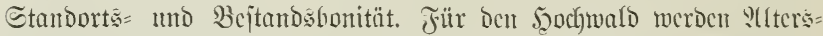
klajien ("Perioben") won ïber 70 Jal)ren, wout $40-70$, wou $20-40$,

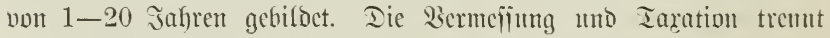

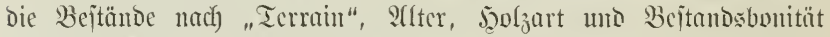

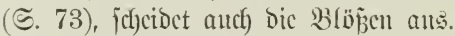

Für jede Unterabtbcilung wird dic Ierrainflafic bcjtimmt, jür bicje nach gut bejtocften Bejtänocn ber normale şaubarteitsertrag.

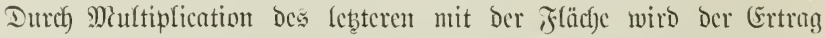
jeder normal bejtoften Ilnterabtheifung crutitelt; bei abnurmer $\mathfrak{B C}_{\mathrm{C}}=$ jtocfutg wird ber Ertrag gejumben Dutref) Fedouction mit ber vor=

*) Sdilcher: lleber die żmedmäpigite Metfode, Den Ertrag ber Warbutgen

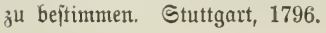




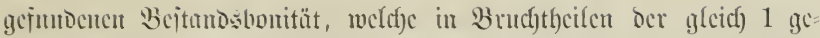

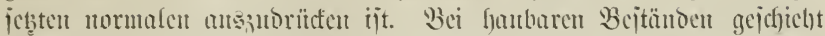
Dies ofye 2isciteres, bei jungen unter ñujuredfumy bes 3 unad)jes.

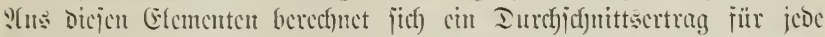

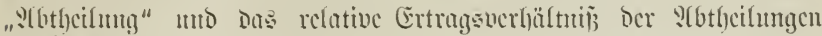
Inter cimbunder.

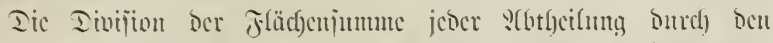

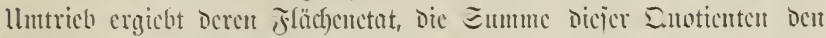
Jlächentetat Des Heviercs.

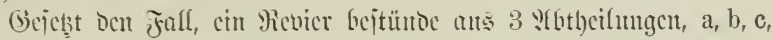
Dereu (Erträge jich uerficlten wic $1: 0,8: 0,5$, a jei 4000 , b 2000 , e 5000 Niorgen groj, jo müroc ber Flädfenctat bei 100 jähr. IImtricbe

$$
\frac{4000}{100}+\frac{2000}{100}+\frac{5000}{100}=40+20+50=110 .
$$

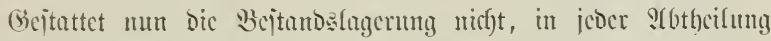

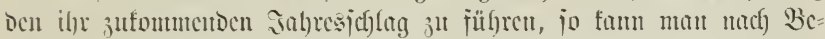

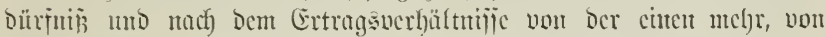
Der anteren meniger rechnen. 3. B. Dic s(butheifunty a geitatte im

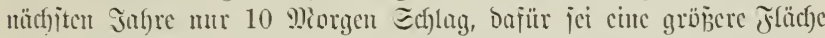

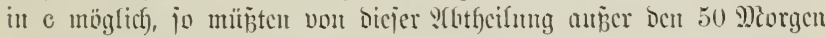

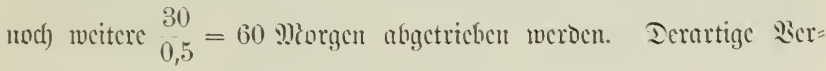

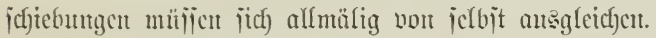

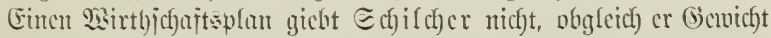

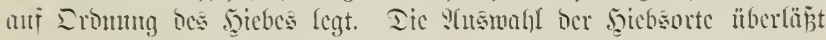

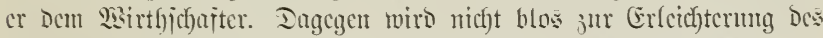

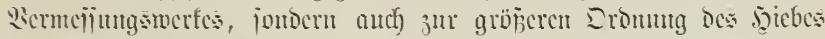
cin Echneijenncts ("Iiagonalen") empjohlen.

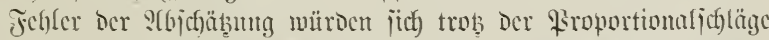

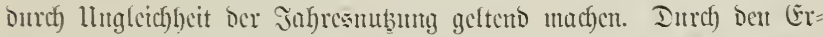
trag wird aljo bic Bichtigfeit Des Berfah)ens controlirt. Sleine Bicr=

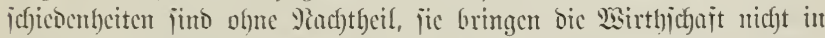

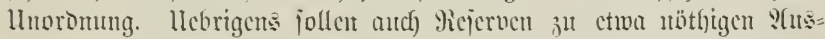

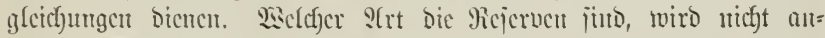
geyeben (ङ. 36).

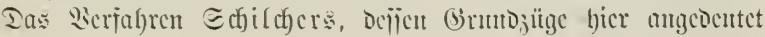

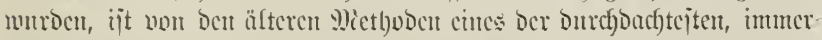
bin aber jefmen ans

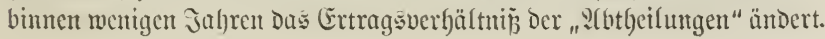




\section{Dic Fad wertamethoden.}

Eimen widftigen Fortidyritt fïr feine Beit bahnte noch fur vor

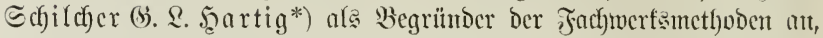
indem er bie llmtriebszeit in \$erioden tycilte, dic er mit antäberno gleichen ober fteigenden Maffen ausitattete. Fït bie eichenen Baum=

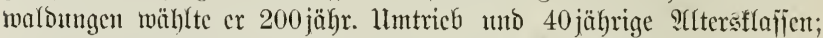

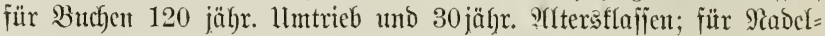

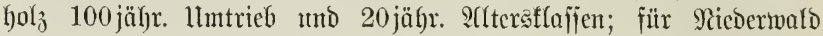

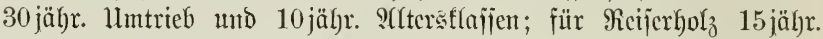

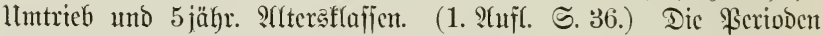
bilbet er, wie aus den angejügten Beifpielen hervorgebt, in Derjelben

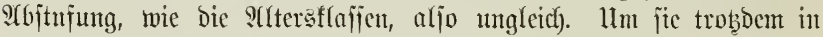
eine Bieneral= Iabelfe cinfügen zut tömen, wählt er in biejer jtatt Der Fierioden Jaljrachnte, in weldye jidy die Rerioden zerfällen lajjen (ङ. 170). In Der 2. SAtflage jobeint er für alle Şolzartent glcidy abgefufte 30 jährige s(ltersflafjen und audf) Pscrioben anjutelymen.

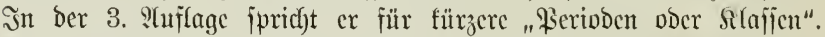

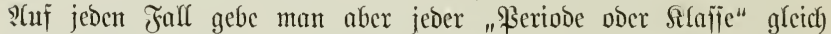

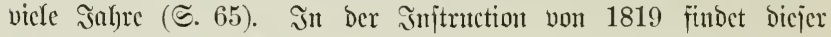

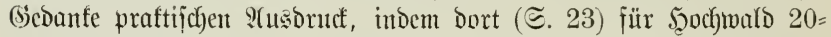

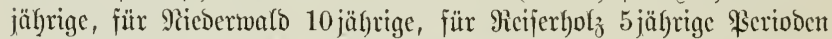
uno cbenjo abgejtujte Saltersflajien gebildet merben.

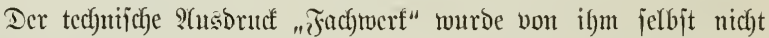
gebraudyt, jeine Methode jebod), bie er allnälig weiter ausbilbete, und bic 1819 ber preupififjen forjttaxation ju Sirmonde gelegt wurbe, ijt

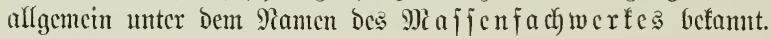

In Der erjen Suf̣lage jeites Buches ïber Taration ber Forjte (1795) fpricht 5̧artig won einem bie J̧icbsfolge oronenden Wirth)= ichaftsplane noch nicht. (Er betrachtet bas biebict einer jeden joblzart

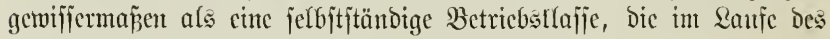
fïr fic bejtimmten limtricbes

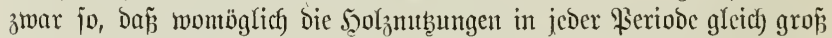
jeien. Den jägrlichen Şiebsjab̧ findet er Durch Divifinn mit ber

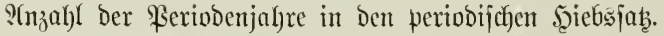

Der (Ertragaberedynung jelbit geht bie S(upnahme cines Taxationts=

*) 65. 2. Şartig: Anmeijung zur Taçation ber forjte ober zur Bejtimmung

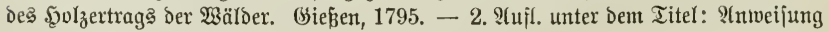

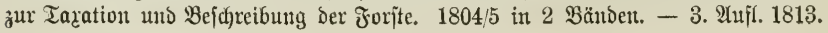


protocolles vorans, mobci jedc s(bthcilutg (Bejtand), wic Deujin*) ferb richtig herworbebt, jo behandelt wird, als ob fic im antsfebenden Betricbe bemirthichaftet mitroc. Dic S(btricbsjeiten twerocu nach bem normalen janbarfeitanlter bejtimmt, von ciner Situdficht auj bie DrD=

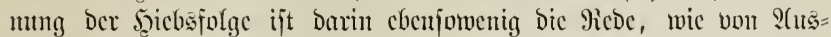

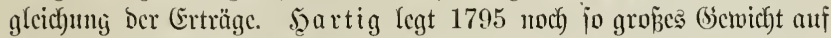
bie Einfaltumg normaler stbtriebsalter, Daj er mit jeiter forberung

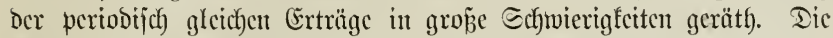
S(usgleichung Der Erträgc will cr Dcsfalb hauptjächlich mit Şilfe ber "Durchpplänterungen" (Durchforĩtutgen) bewirfen. Fällt in bic leb̧ten

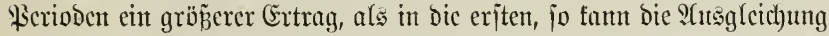
unterbleiben, weil in Folgc bes $\mathfrak{B a c h j e t s ~ D e r ~ B e v o ̈ l f e r u n g ~ u n d ~ b e s ~}$

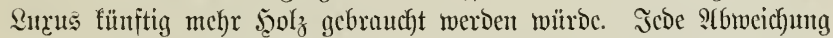
vou normalen Şaubarfcitsalter ijt natürlich) mit Ertragsverfujt ver=

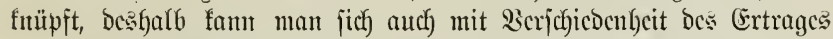
Der cüzelnen f̧olzarten begutïgen, wem utur Dic Ertragsjummen jiemlich) gleich werden. Sa cr gejtattet jelbjt bie : Susgleichuntug ber Erträge

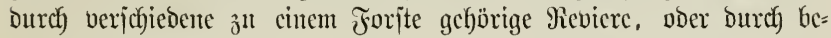
nachbarte Forite.

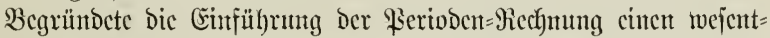

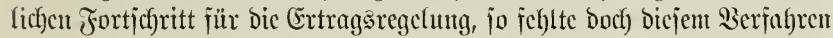

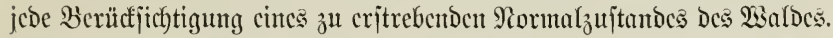
ben ce fich) überbaupt gar nicht flar macht.

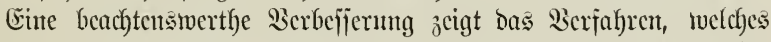

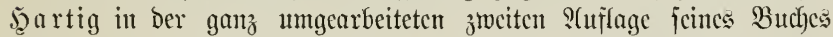
1804 und 1805 veröffentlichtc, gegenïber Dem Berfabren von 1795.

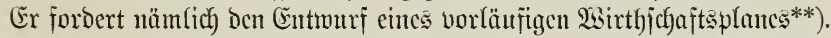
Sicineswegs ijt aber unter biejen \$ilan ein joldfer ju verjtchen, welcher

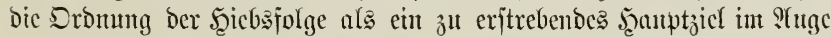
fechalten möchte, ment biejelfe Darin auch nicht gand überjefgen miro.

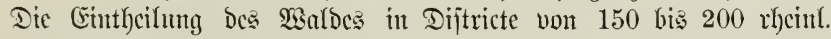
Mergen burch Echneijen - ijt ausorühflich) cmpjohlen. Dicje Eintlycilung

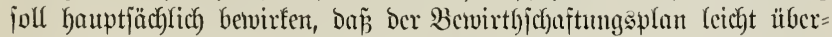
jeben, genau bejolgt und jets in jeiner Bulljtändigfeit crhalten werben

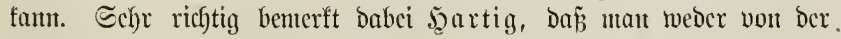

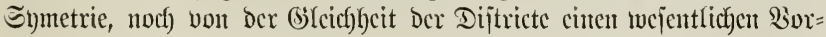

*) Denzin in: algenteine For

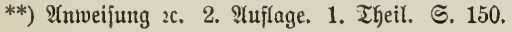




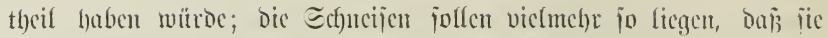

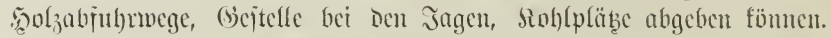

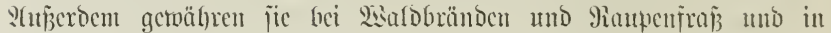

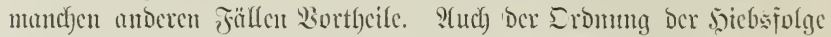
mird alleroings babei gedacht, und fehlt aljo bas Etreben nach) ber

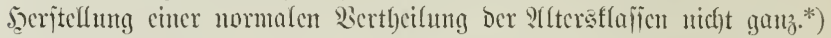
Inmerbin tritt aber biejes Streben gegerïber ber Siüfjicht auf bic (S)leich)feit, bejichentfich) alfmëlige Eteigerung ber periobijhen Erträge ichr zurït. Dicje Rïcfjicht wird von şartig 1804 nod) jejärfer betont, als 1795, und jwar nicht blos für das banzc, fondocrn für bic cinzelnen 5̧olzarten, jogar bcjonders für bas Bantholz.

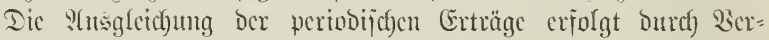

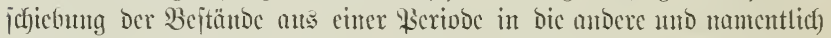
Dutch bic Bwij(hemutbutugen.

Für bie Sicheritellung Des hei Der Taxation ausgemorfenen Şiebs=

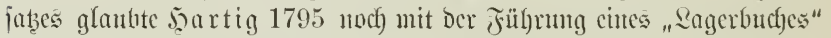

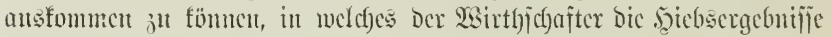
abtfcilungsweije jäfrlich) cinträgt. Eclbit ppäter (1813) glanbt cr noch)

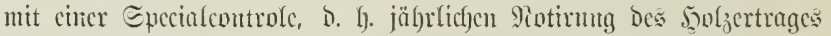

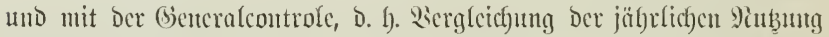

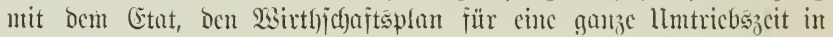
(Sicftung crfoulten żt fömnen.

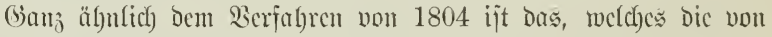

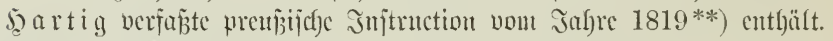

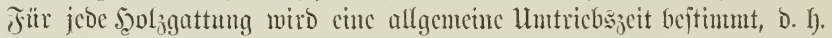

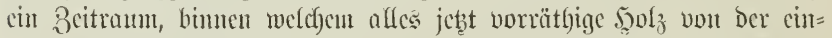
jäl)rigen \$iflanze an, conjumirt merben fam und jolf, um nach ?(blauf Dicjes Beitranmes anj ben zuerjt verjüntgten Dijtricten wieder gen̈̈geno

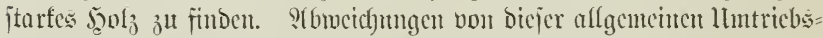

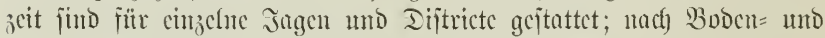

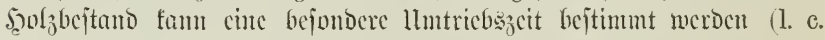
๔. 16). Beim vorlänfigen Entmuric des Tarationsplanes ijt Davon

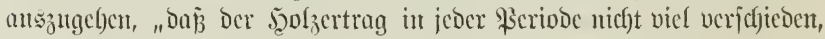

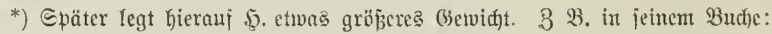

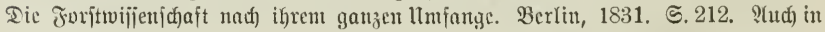
Den Jnjtructionen von 1819 u. 1836 pprid)t $\mathfrak{c r}$ in ber Etulcitutg und im 7. $\mathfrak{a b}=$

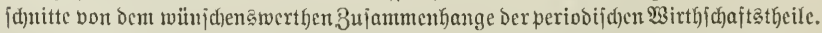

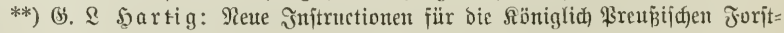

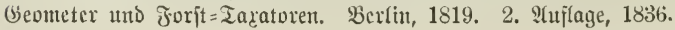




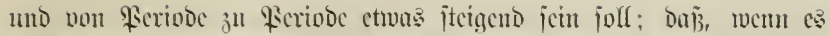

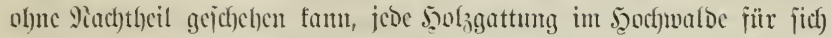
periobijch fajt gleiclen Ertrag geben joll; baj aber in bem falle bou

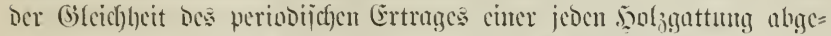

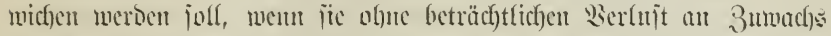

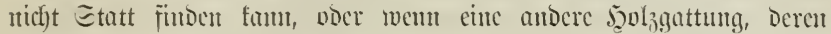

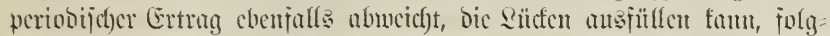

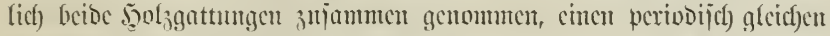

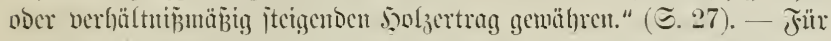

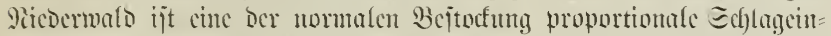
tbciluttg vorigcjejrichen (こ. 13).

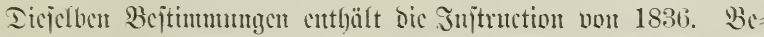
jonders interejijant ijt $s$, baj jogar modh in letsterer beijpielsmeije nicht blos bic Jeaubarfetts=, jombern and) Dic Iurd)foritnugserträge getremut

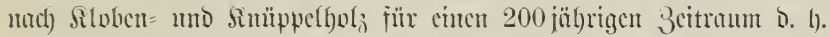

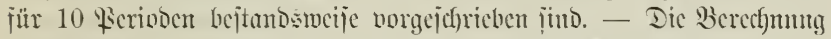
Des progreijiu abnclumenden Buwachies criolgt nach Procenten. -

Die llüicherbeit aller "Saration" für Ferne Bufunft, melde nament (ich) jef)on Echilcher betont hatte, war in Der Prexis befumnt gemty,

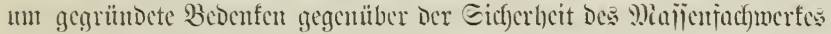

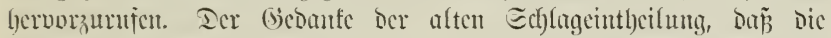

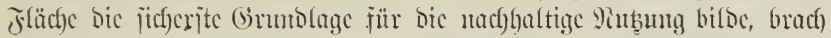

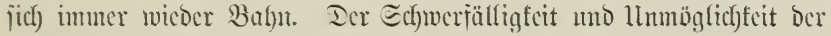

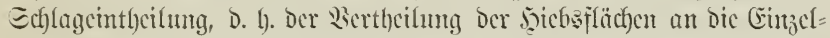

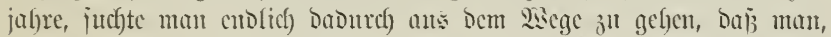

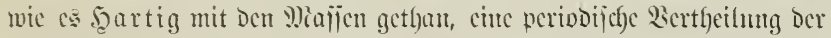

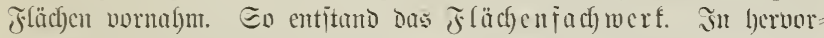
ragenter und cinflürecicher Siscije gah bicjem (jebanten numentlich) jecinrich (5otta*) G(usorut.

Cotta war aber nicft Der D) iann eintes jtarren Principes, er moll te

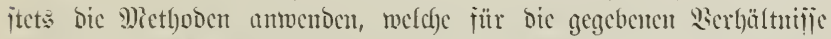

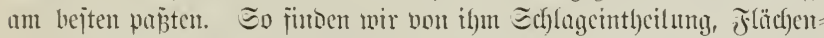

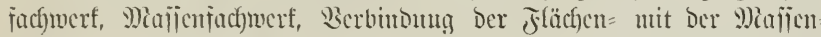
meth)ode cmpioblen. Einen (Sirmogedanfen hat er aber nic anjgegeben,

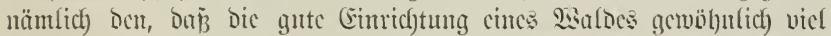

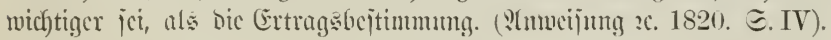

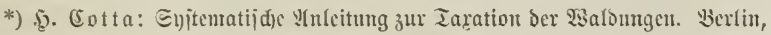

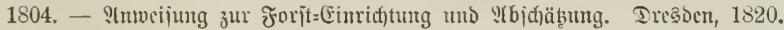




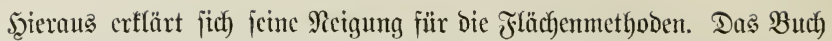

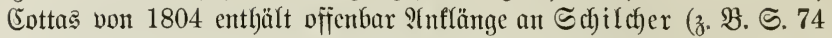
und 75), wo cr fïtr bic Serbindung von Flächen= und Miafientlycilung

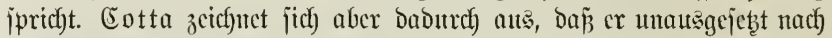
gröperer Sicreinfachung Des Serfafyrens jtrebt. Dic Bcit theilt Eotta bei 60 jährigem unb höherem llmtricb in \$erioden von 20 Jahren, hei 40-60jährigem llmtrieb in. jolche von 10 Jahren, bei 40 jährigem unס niebrigerem llmtricb in jolche von 5 Jahren. Bei 20 jährigen \$eripden wird jtets bic erite uicder in 2 Jafyrzefnte getheilt und bicje Theilung jo oft wiederfyolt, als eine Periode Gecndigt ijt. Dicje Perioben ver= gleicf)t ex mit "F̈ächern", "und in bieje Fä̌cher müfjen bei Der Forft= cutrichtung Dic Salbabtheilungen gebradyt merben". (ङ. 43.) :(uf

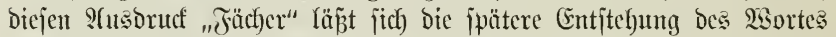

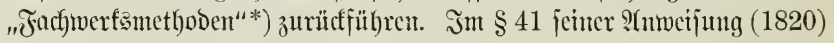
bebandelt (5 otta cin Beifpiel rein nach bem cinfachjten Flächenfach)werf, indem er bie cinzetnen Perioden mit gleichen Flächenjätzen ausjtattet.

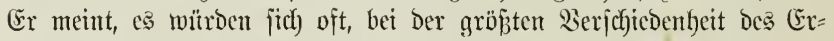
trages einzelner Drte, Die periobifchen (Erträge Dennoch) ausgleichen, ıenn bie \$iserioben ber Fläche nach) glecthgejtellt merben, Da mur jelten

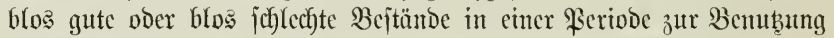
foumen. Im Weiteren (\$ 44) gebenft Cotta Der Miöglichfeit, wo bieje S(usglcichung uicht jtattfinoct, nach ber verjchicdenen Bejtandsgüte

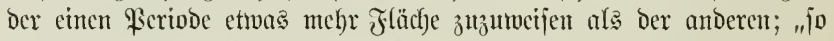

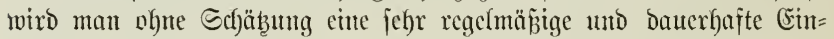

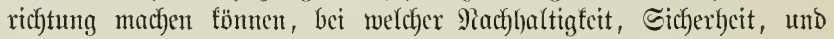

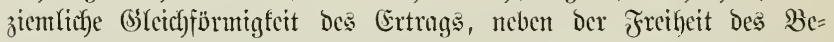
tricbes und ber ungelähmten 2ierwaltung aufs Bejte beiteben fönnen." Es wird aljo bier ein Flächenfact)werf mit annäbernder Rebucirntug

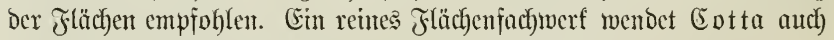

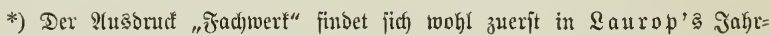

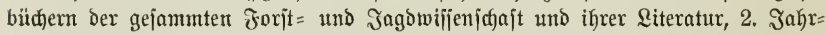

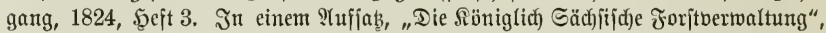

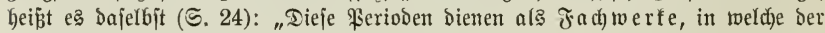

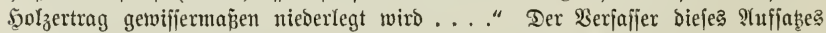

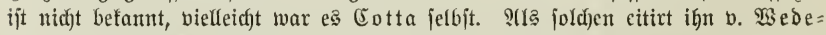

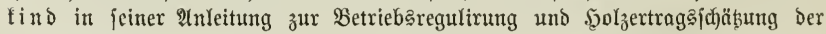

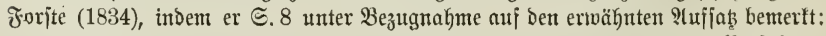

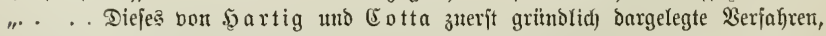

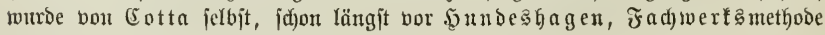
genannt." 


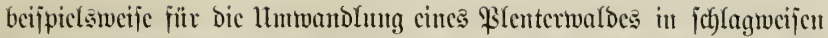
5̧od)malsbetrich an; cine betreffende Tahefle E am Schlufje jeiner

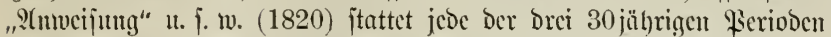
mit volljtümbig gleichen Flächen, aber mit ungleidl)en Ertrïgen แus.

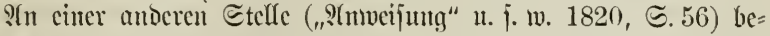
merft Eotta gegenüber ber cinfachen, borber von ifm empfoflenen

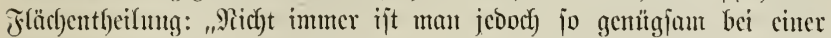

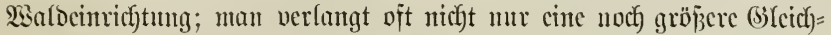
förmigfeit bes (Ertrags, als hicrourch) crrcicht wird, fondern man will

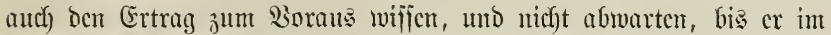

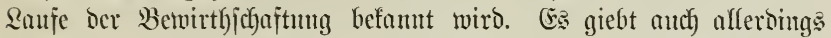
Fälle, wo biejes nöthig obcr wenigitens gut ijt". Fïr joldhe Fälle lefyrt or (ङ. 58 bis 63) cine ipeciclle Foritertragsbeitimmung nach) gutachtlicher Scfäbung und wendet babei cin Mafjenfacfuwerf an, indem

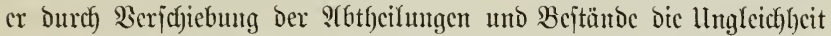

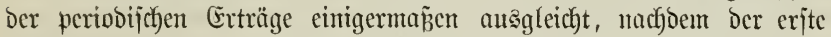
S(njab burch cinen mit Rücfficf)t anj bie Bcjtandslagernng cutworfenen

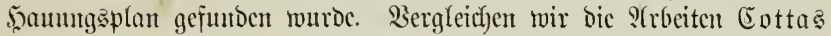

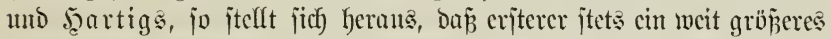
(Siemicht auf bie secritellung einer guten Bejtunbsorbuntug legte, als

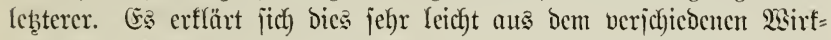
ungşfreije beider Meämter. Wäbrend Čotta hauptjüchlich zuerit bic

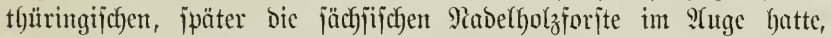

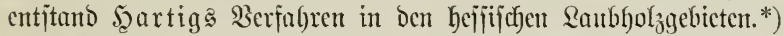

Die Joee Der Betricbaflajienbiloung ijt Evtta nicht jremo ge= wejen, er gicbt baribber indejien feine fajarf bejtimmten Borjedriften.

Eotta ermarb jich ein bejonders Sierdienjt baburch), baj cr jeflon

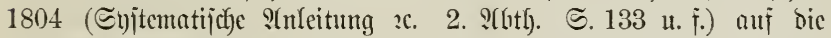

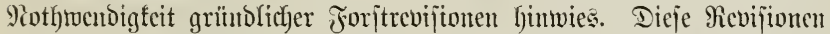

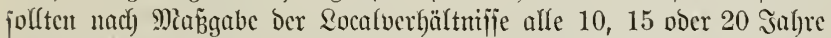

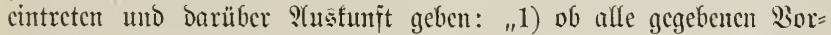
jedriften biazher gehörig befolgt worben fund? 2) weldyes ber Erjolg

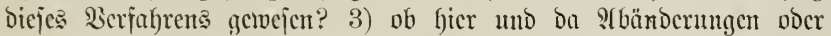

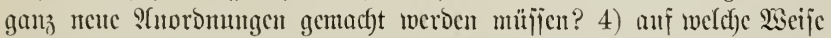

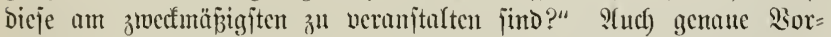

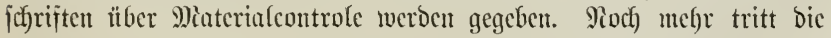

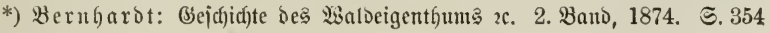
นกญ 355. 


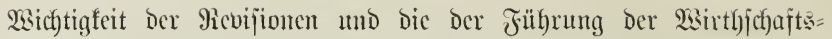
bücher in Der 9fnteijutg vou 1820 fervor (3. 9(bth). ङ. 141 น. f.). In Sachjen mutdo bie erite Mievifion 1824 abgeljalten.

Wo Eotta cin Majjenjachwerf anwendet, bergin̈t er uicht ber

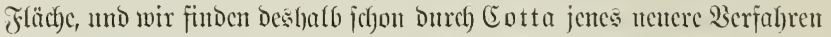
angebaf)nt, weldyes man combinites Faclyerf neme, ba Dafjelbe

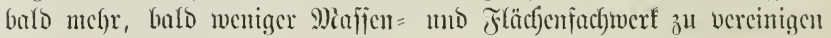
itrebt.

Ed)on zu Enttas Bcit, als Derjelbe noch Das Foriteinrichtungs: wejen in Sachjen leitete, cutwicfelte jich Das hicr angemendete Sier=

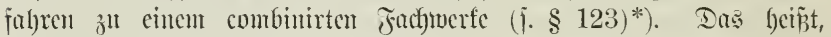
man hielt in Snterefie Der Serjteffung cincr guten Bejtandosorbung am Flüd)enfacfunerfe fejt und mies ben cinzeluen, 20 jül)rigen \$erivoen

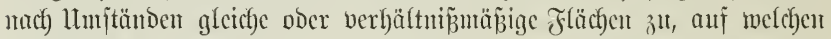
Die Şiebszat Der cinzeluen Drte jich utchr ober meniger nach Derjenigen richten follte, in weldyer bie ganzen A(btheifungen ber fïmftigen $\mathfrak{B}^{\mathrm{e}}=$ jtandefolge halber zur Bemtbung fommen jollten.**) Dabei verjuchte

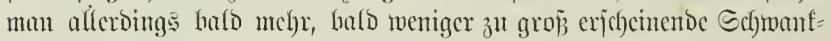

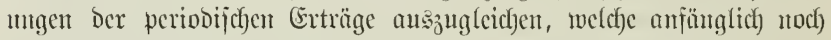

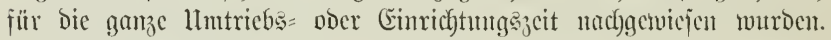

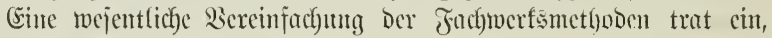

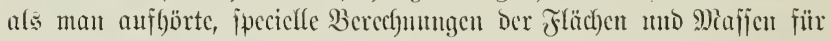
dic ganze llmtricbszeit anzuitellen. Buerjt war es woff nament(ich)

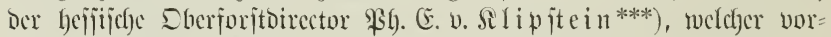

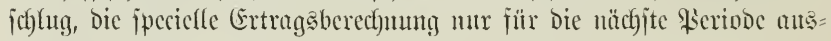
zufüthren, dic folgenden Perioben Dagegen mur ganz jummarij(d) z" beritefjidftigen. Ex geitattetc bis $20 \%$ Differenjen. S(ud) in Eadjjen

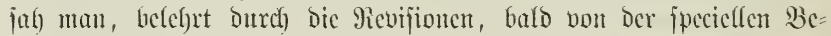
Ftimmung Der Bufunft afler Drte ab, und furze Zeit auf cincm $40=$

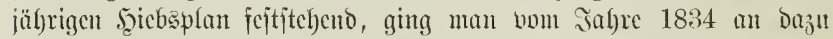

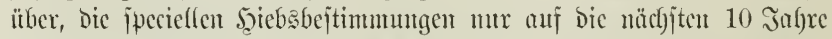

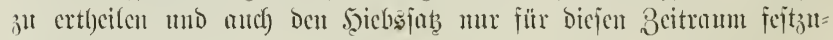
itclen. $\dagger$ )

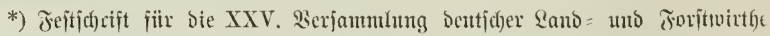
วน Dresoen. 1865. 2. Theil, S. 21 u. Ғ.

**) Feitichrift zc. 2. Theif, S. 22.

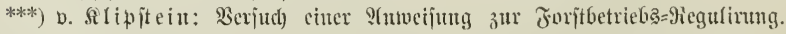

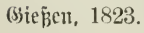

†) Feitid)rift 2., 2. Theil, હ. 24 . 
III. Dic Piormaluorratges= Mictloden.

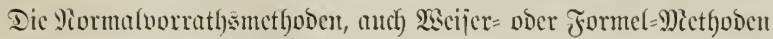

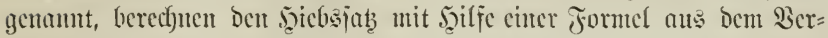

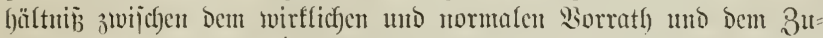

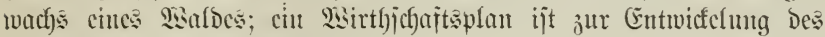
Jeicbsjabes nicht Siormejictung.

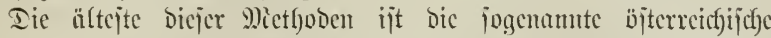

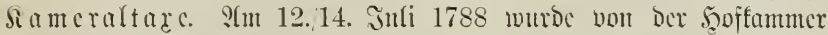
in 2isien cin Decret crlajien, wefches cin bejtimmtes Bierfalgren für

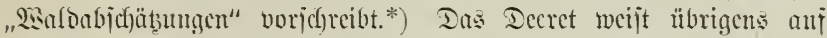

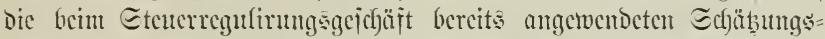

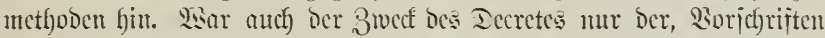

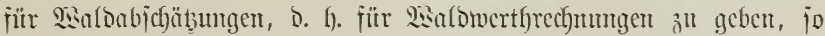

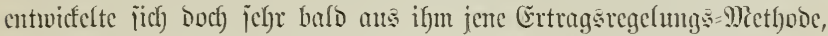

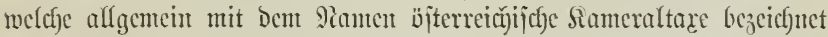

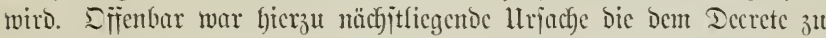

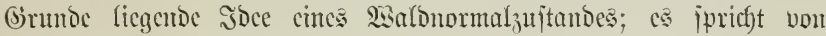

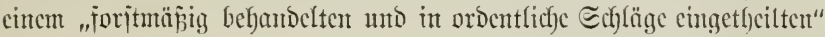
WafDe, Dem gegentiber von einem „über jeine Siräte bergenommenen"

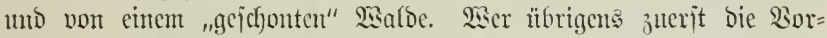
jâtriften Des Iecretes zu Zwecten ber Ertrags regefung anmendete,

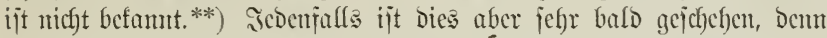

*) Der Sortlaut biejes Decretes mar in ber jorjtlichen Siteratur lange Beit

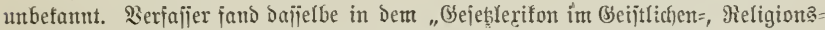

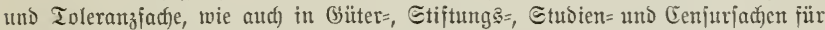

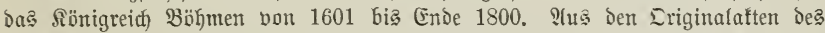

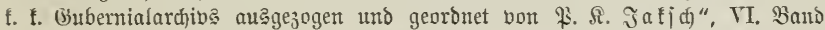
(1818), und theilte es im Tharander Jafrbuche, 19. Band (1869), ङ. 78 u. f. mit.

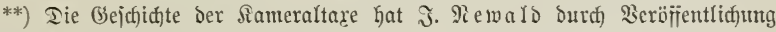
Der amtlidjen Berfandungen, weld)e zum Erlaj des Normales ober Iecretes ge= fïhrt Gaben, neuerdinge gejdildert. 3u bergl. \$ewals: Bur Gejuidte ber

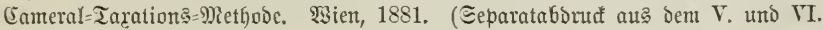

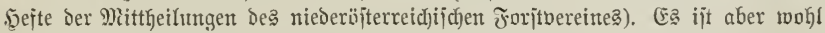

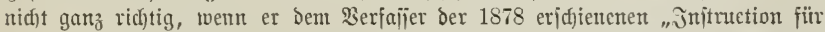

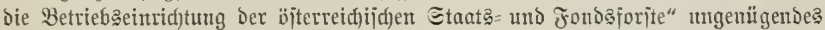
Bertrautjein mit bem Sejen Diejer Mietgode bormirft, und wenn er Das Tharander

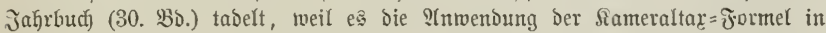

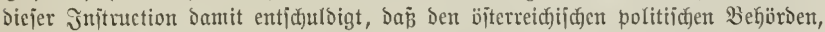

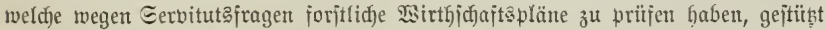

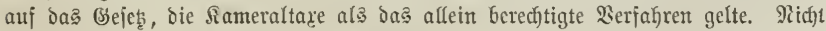

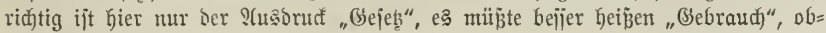

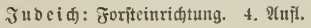




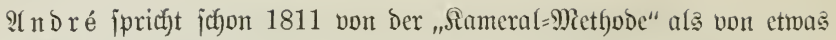
bercits Befauntem. Dieje Methode lebrt Den Sormalvorrath) (,fundus

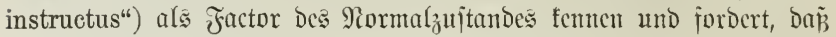

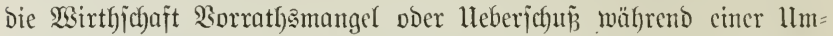
triebszeit ausgleiche. Dic Betricbstlajīen= (Eintbeilung mar bom Ber= fahren jedenfalls nicht jremb, Dagegen ijt bei ifm von cutem $23 i r t h=$

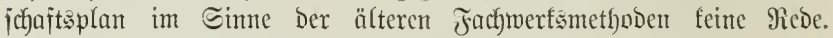
(jenauer geichildert murbe die Sameraltaxe zuerjt von (E). CEarl SA noré und namentlich non jeinem Eofne, Dem Forjtrath Emil $\mathfrak{A}$. S(noré*). Qebterer findet cinen Sorzug Der Sameraltade gegenüber

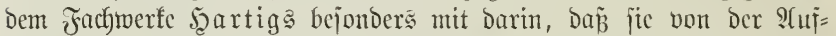

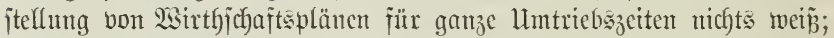

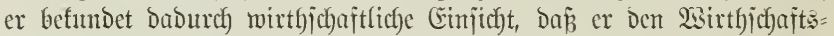

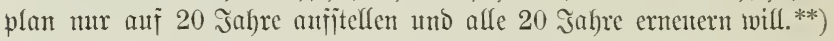

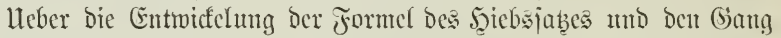

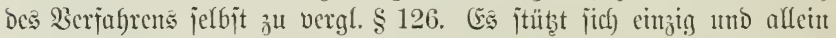

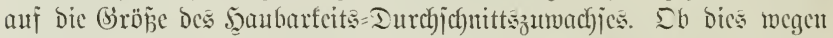
Mangcls an Ertragstafeln ober gruntojäblich gejcheben, läp̃t jitch mit Sicherbeit nicht entjubeidon. -

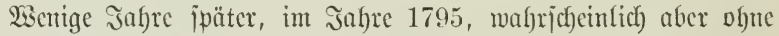

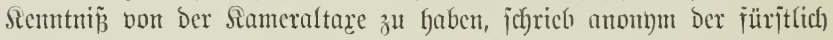
Sippejche Dberiöriter \$auljen jcine ,Surze, praftijche ?fmbeijung

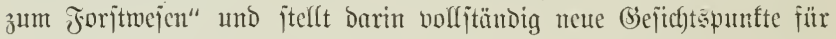
die Ertragšregelung aui***). Er entmicfelt bas Berbältnii bes

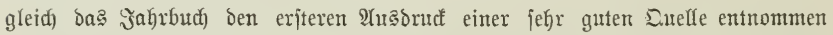

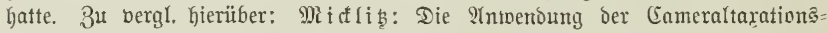

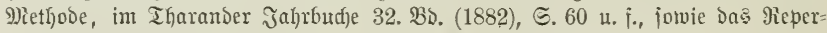
torium Des Tharander Jafrbudes 33. BD., S. 254 und 25.

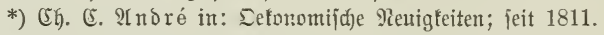

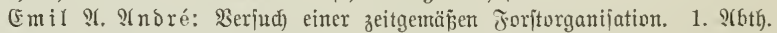
Innere Foritorganifation, enthaltend bie vollfommenjte Eideritellung Der Siad)= Galtigfeit. Frag, 1823. (2. 2uffl. 1830.)

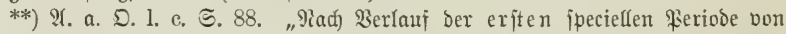

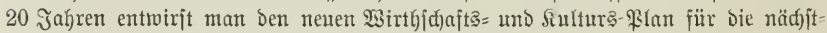

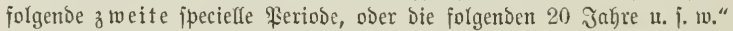

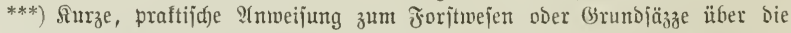

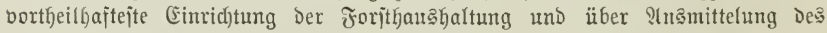
Wertfs vom Foritgrumbe, bejonders auf Die Brafidajt Lippe angewendet, ver= fafiet bon einem forftmanne und herauggegeben bon Beorg Ferbinand

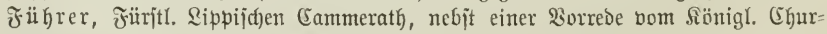

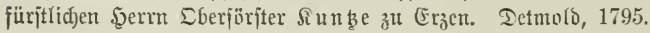


(Iaufenton) 3umadjjes ju Dem Ertrage des Forjtgruntes im voll=

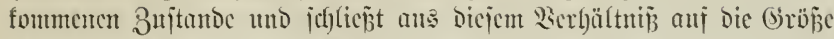

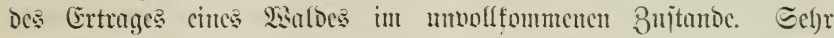

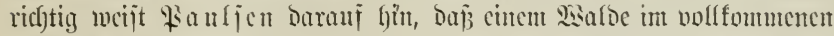

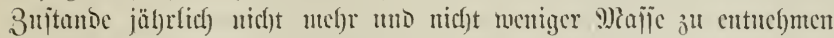

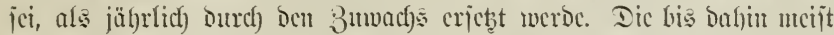

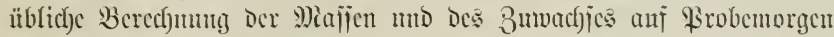

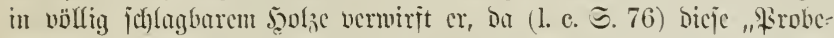

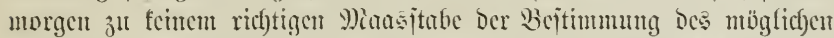
Ertrage vom forjtgrumbe Dicnen fümen, weil fic faum dic seälfte dce

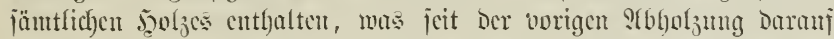

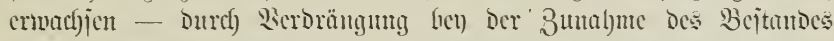
aber won ber Patur idfou nurfin abgeworien ijt, nod) was ferner bor

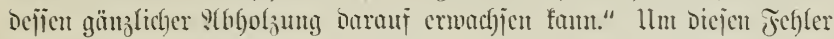

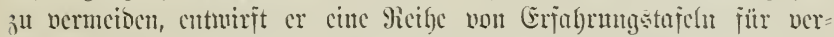
ichicbene 5edzarten und Bonitäten. Iieje Iafeln cothalten nicht blos bic joubarfeits, jombern aud bie 3mijchemubungen. Iaburch wiro

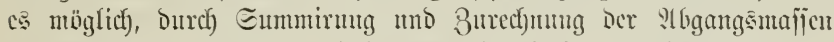

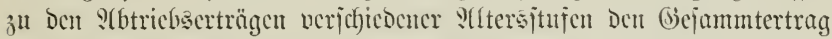

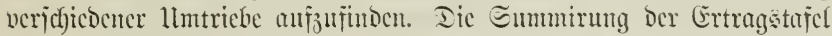
gicht Den Piorutalvorratl), uit bicjem wird in bie Eumme ans bem

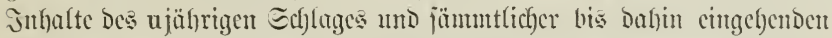

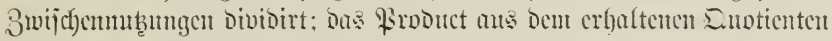
unit bent wirffichen Borrath cinte Der Bonität ber Ertragstajel cut=

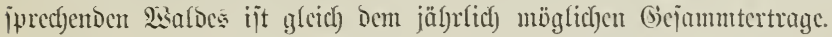

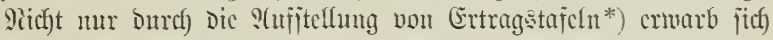

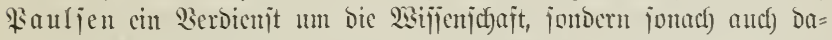

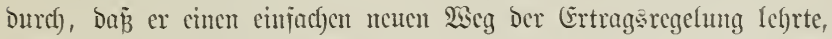

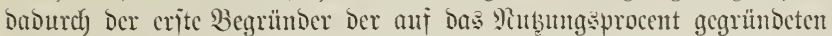
:(bjc)äbungstflecoricn murde**).

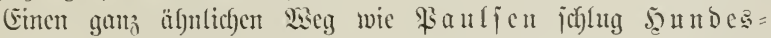

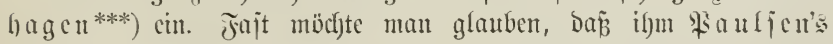

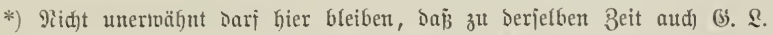
D)artig Erfahrungstafeln in ieiner 2(nmeifung zur Taration ber Jurite ver= ijïentlid)te.

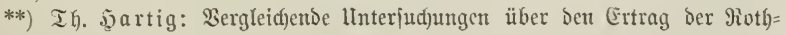
buche. Berlin, 1847. S. IV - Ih. Sartig hat hier Die Berbienjte Pauljen's zuerit in weiteren fireijen betaunt gemad)t.

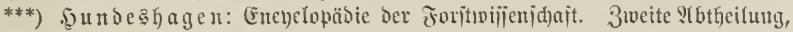


STrbeit nicft fremo geblicben fei, ohgleid) er bicjelbe, jo vief une be=

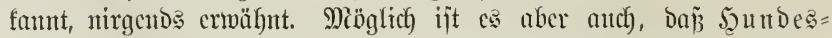
hageut gatz jelbjtitündig zu berjelben Ertragsformel gelangte. Wise

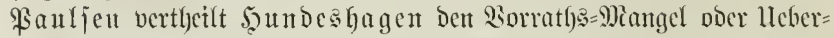

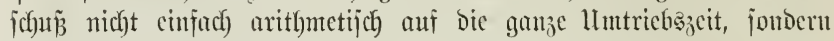
multiplicirt mit bem Duptienten ans Pormulvorrath) in ben normalen

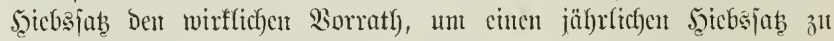

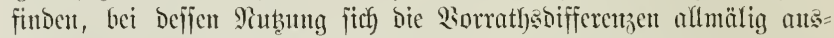
gleichen. Diejen Dupticuten naunte er bus Sinţutgsprocent, obgleicl)

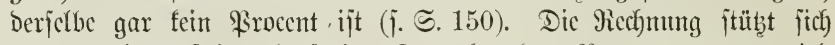

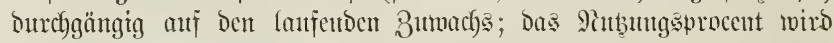
nur mit Şilfe bes s(btrieberertrages bered)utet, wäl)rento : Fauljen bic

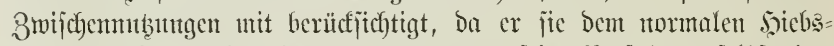

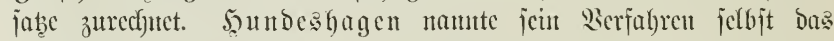
"xat ionelle", modurch fich ber Name "rationelte Methoden" jpäter auf afle 2iormafvorratbs= Miethoden alfmülig übertrug. Dic

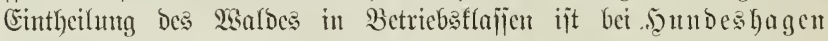

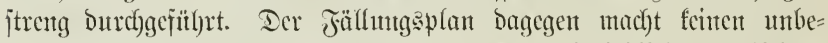

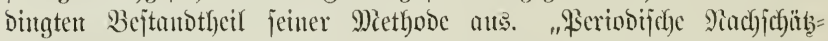

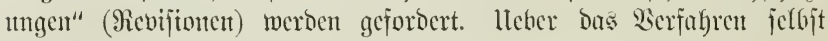
zt vergl. § 127 .

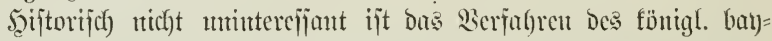

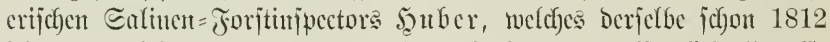
jeister Dienjtbebüroc mittlyeilte, aber crjt jpäter veröffentlichte*). Er

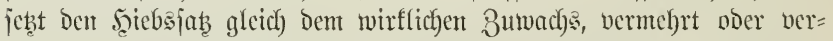
minbert un cinen entiprechenden Theil Der pojitiven ober negativen

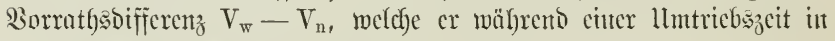

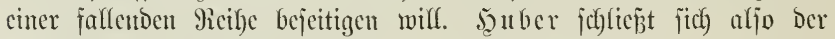

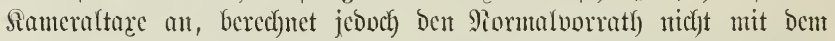

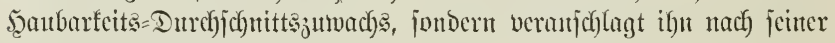

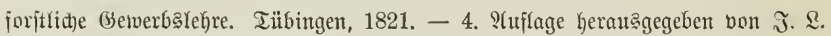
Rraupredift. Tübingen, 1843.

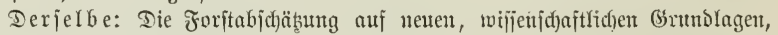

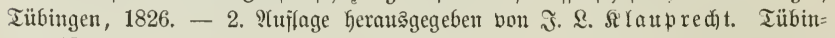
gen, 1848 .

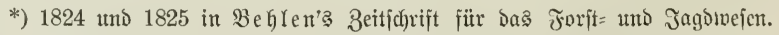
2. uno 4. Bans. - Später, 1832 uno 1833 lieferte er in ber Qtffgemeinen forit=

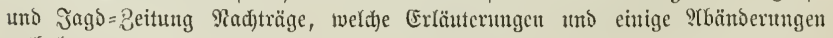
entibarten. 


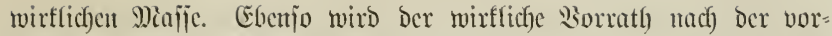

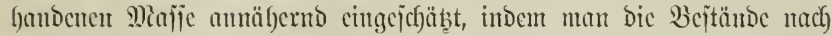

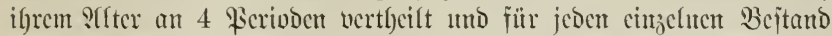
aunimmt, baj er bas mittlere sfler jeiner Feriode babe. Piur in

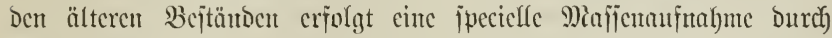
Pirobcriäclucn.

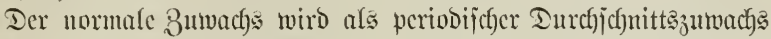

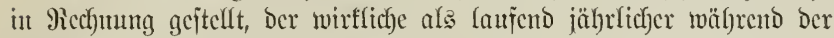

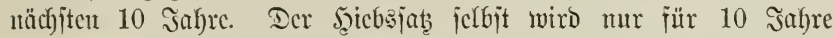
bejtimmt. Einc praftijde Bebcutung hat bas S3erfahren heute wohl nit(f)t melys.

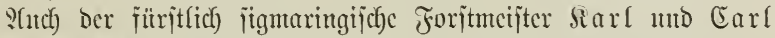

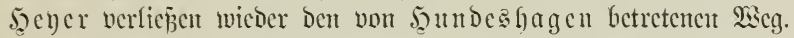

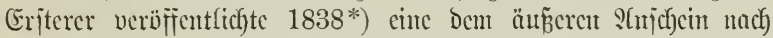

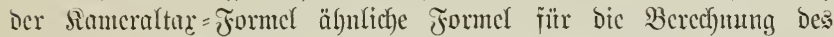
juicbsiates. Die cinzeluen Factoren werden aber uicht mit Şilfe des

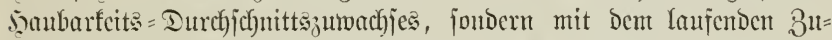
wachje berechutet, und an Etclle Des für bie S(usggleich)ung ber Differenz jwijchen Dem wirflichen und Dem normalen Borrathe von Der Sameraf= taxe milffürlich getwäh(ten Uuntriebes bejtimmt Siarl cinen nach) ben

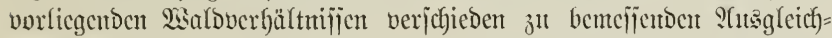

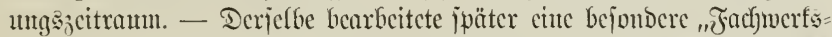
methodc", weldfe in Berbinoung mit einer ifre cigenthüntlicfen Borraths und 3umadjsberedfuning iteft**). Bu vergl. \$ 129.

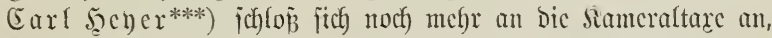

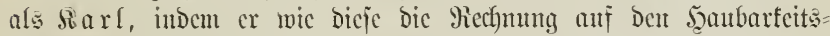

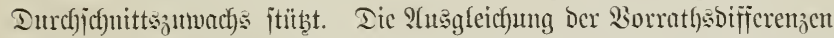

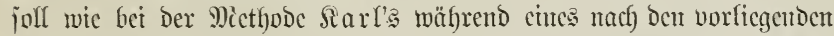

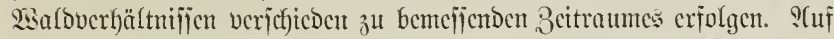

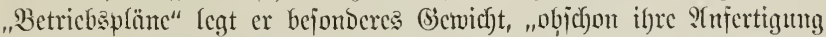

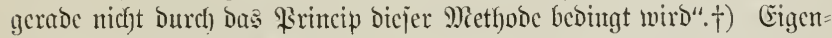

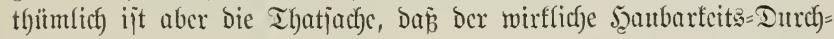

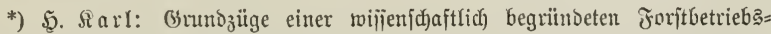
Regutimugs: Miethode. Sigmaxingen, 1838.

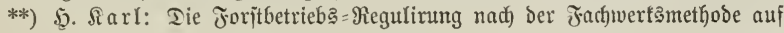
wijienjad)aftlid)en Snmblagen. Stuttgart, 1851.

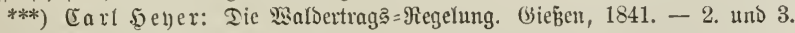

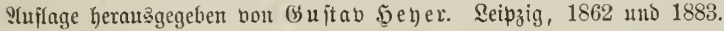

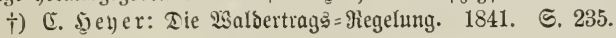




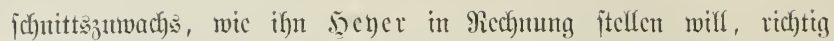

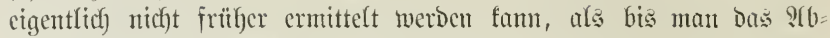

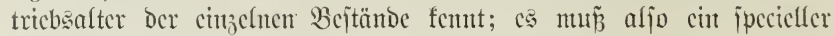

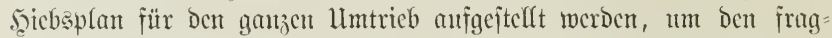

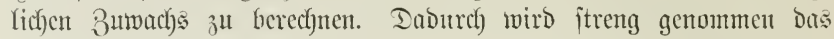

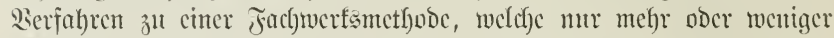

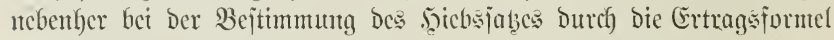

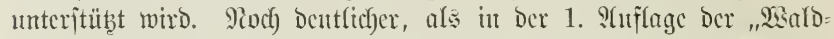

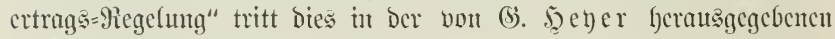

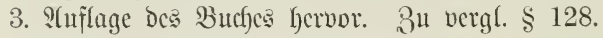

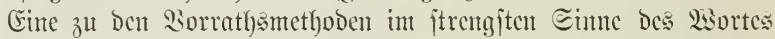

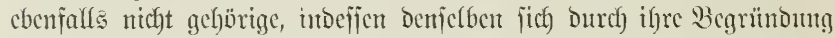

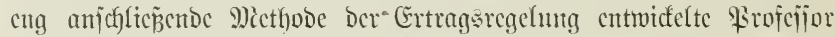
Breymann in Miariabrunn, indom er fich anj ben Factor cints ber

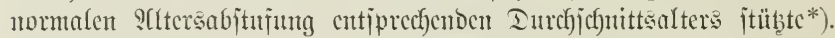
$3 \mathfrak{l l}$ vergl. § 130.

Endich verbient fjer nodh jenes Serfaf)ren Errwäf)mung, weldyes

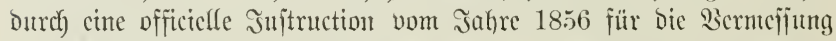

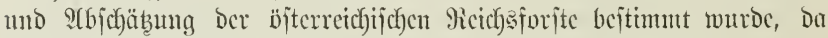

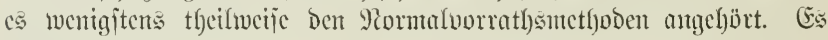

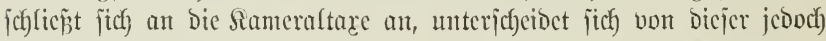
twejentlich) in Der Ermitteluntg Der cinjechent Factoren. S(n Etelfe Dicjer Initruction trat cinc neue im Jabre 1878. 3n vergl. \$ 131.

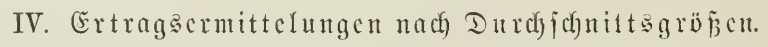

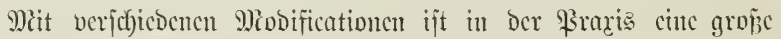

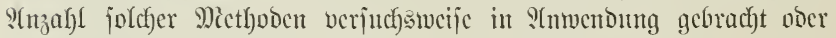
and ) nur cmpfohlen morden.

Wisir fönnen bicjelben in folgenoc Sategorien theilen:

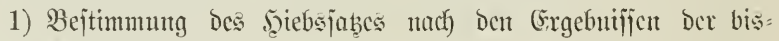
berigen S(bnuţutrg.

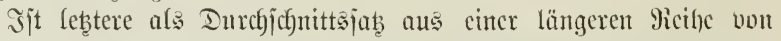

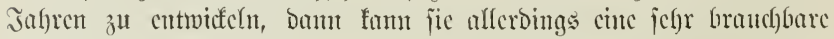

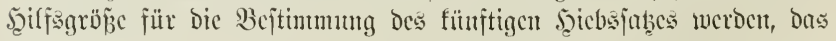

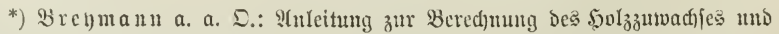

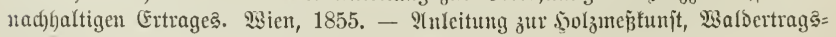
beftimmung und $\mathfrak{s a l d w e r t h b e r e d ) n u n g . ~ W i e n , ~} 1868$. 


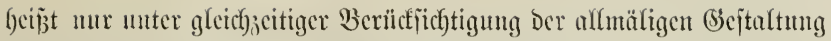

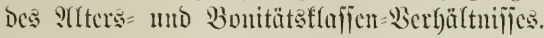

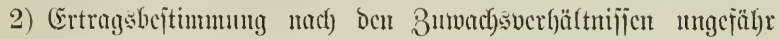
paifertoer (Erfahlumigstafcht.

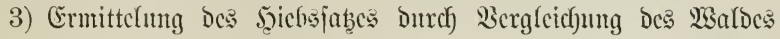

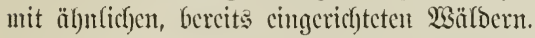

Dic unter 2 unb 3 genumten Metfloden find mur Dam von

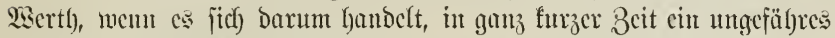
Itrtheil abzugebent.

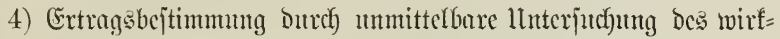

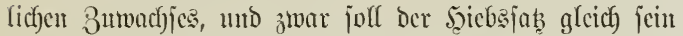

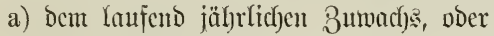

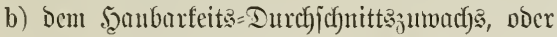

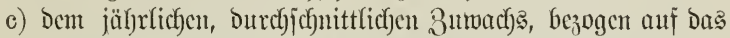
gegentwärtige SYlter unt bic vorfandene Mafije Der Bejtände.*)

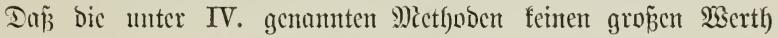

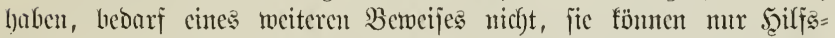
grö̌̃en für bic Bejtimmuntg bes Miaterialertrages bicten. Wir werden jie baljer im Folgentoen nicht näger bejprecf)en.

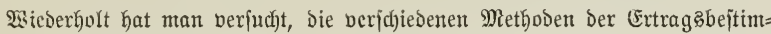

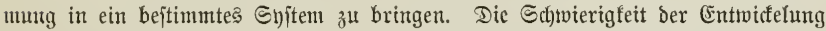
eitte joldhen Sujtemes liegt in bett zah)(reid)en Uebergangagormen, weld)e bie ver=

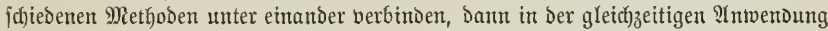
veridfieduner Methoden, aljo in ciner Bermengung Derjelben. In Der \$raxis

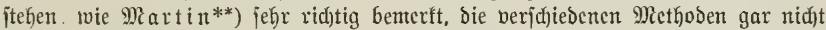

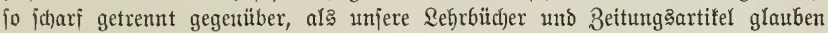
madhen. \$sir haben es beşfalb vorgezogen, in den folgenden ş nur einige tnpifde

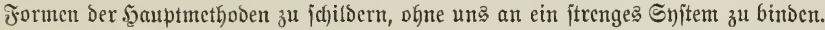

Foritmeifiter Dr. Etüşer***) hat in neuefter 3eit ein Syjtem ber Methoden

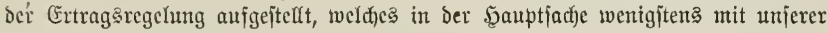
Anorbmung derfelben ïbereinjtinmt.

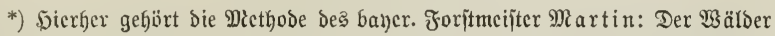

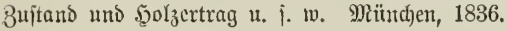

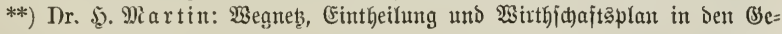
birgşoriten. Münden, 1882 .

***) Jorittwiffenj(d)aftliç)es (Eentralblatt. 1884. S. 522 u. f.: Einige Bemer=

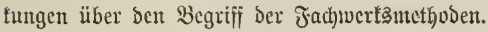


Dajpelbe Iautet mörtfich wie folgt:

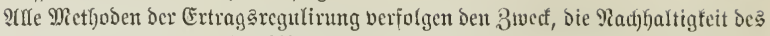

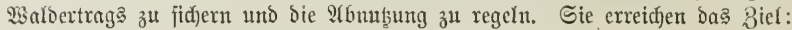

I. Durd) ïrtliche (Eintfeilung des $\mathfrak{B a l}=$

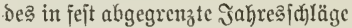

II. Mit Şilf̣e und anf Gritnd einces in

A. Fläd)en= obor Sdygeintbei= I

Fädjer cingetheilten tabellarifoct ßiantes .

1. mit Ælächenfachen

B. Fachueriamethoden.

2. " Majientiad)en . . . .

a) Jläcf)enfach)wert.

b) Maijenfachtwerf́.

3. " Fläd)en= 11. Diajientiad)en

a) Für Den ganzen Einrid)= tunģzetratm.

c) Combinirtes ฐadjwerf.

b) für cinten Theil defielben

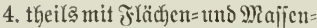

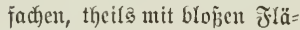
d)enjactyen

๔) vollç combinirtes Jachiverf.

B) unvolffoumenes combinites Jact)=

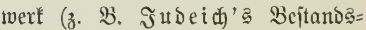
wirthjchaft).

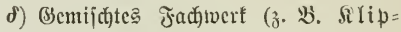

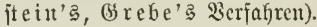

III. Durch T(bleitungen auţ ben aritf)=

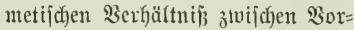
rath und 3utwachs, zu Deren Ent=

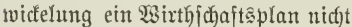
Boraugfțung, jondern mur eventuelles Darjtellungênttittel ijt . .

C. Tormalooratgs= Do. Formel = methoden.

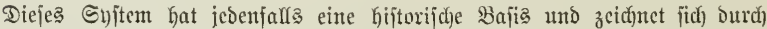

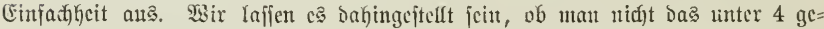

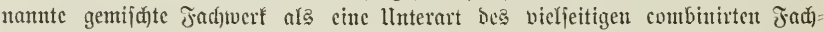
unerfes betrad)teu łinture.

II

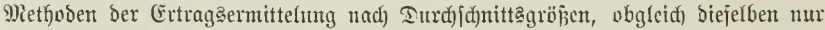

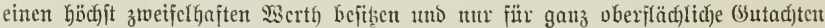
Anwentung finton föntuen.

\section{$\$ 119$.}

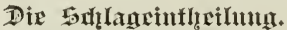

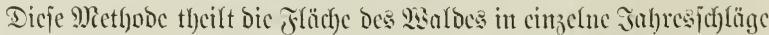
und grenjt lebetcic örtlid) fejt ab; Der Ertray jebes cinzelucu Echlages

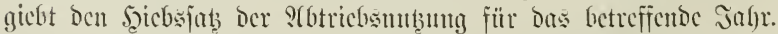

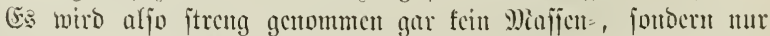

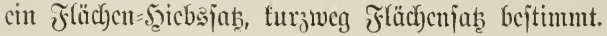




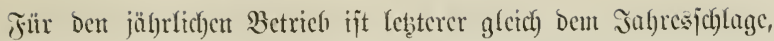

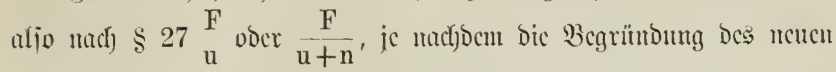

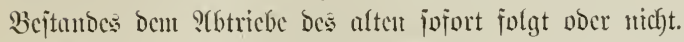

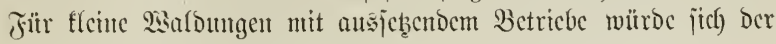

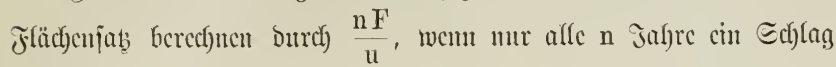
gefüiflyt werben joll.

Mian unteridjecibet:

a) Dic cinfache obcr geometrijche Esblagcinthcilung,

b) Dic Eäntheilung in Froportionalichläge.

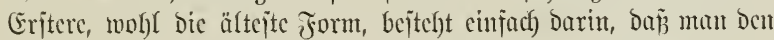

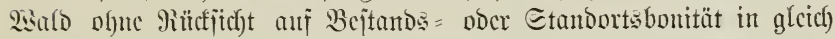
groje Safresifgläge theilt. Se nad) ben Terrain= mo jomitigen $2 i c r=$

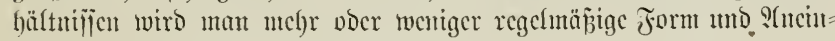
anderreifumg Der lebeteren erlangen fömen.

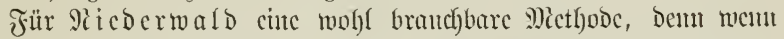

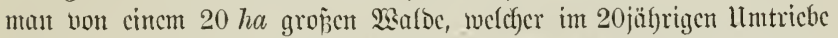

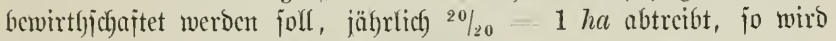

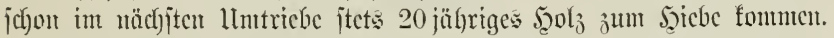

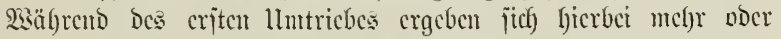

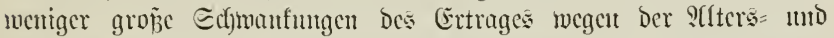

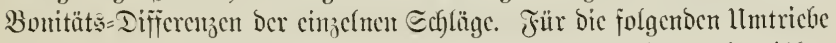

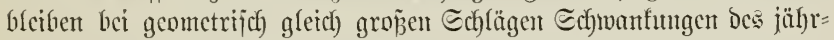

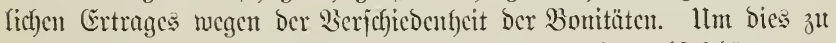
vermeiden, wutrde bic Eintlecilung in Froportioutafichläge an=

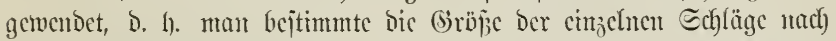

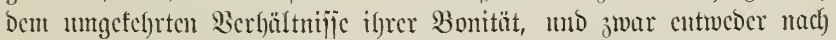
Der Standorta = ober nach Der Beitand

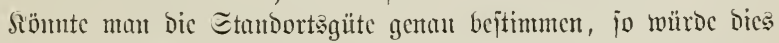

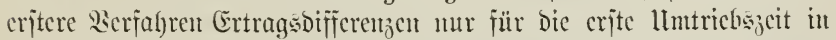

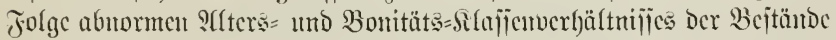

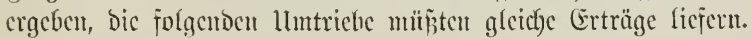

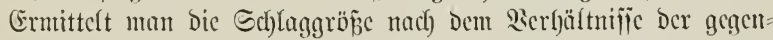

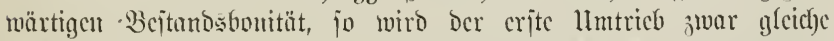

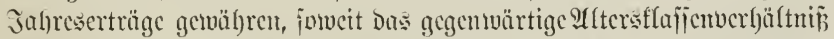

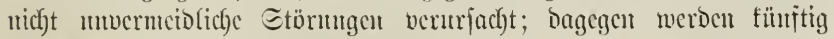
Dic Differenjen ber Etandortsbonitüten anf bic Iancr Ech)wattutgen Des Şiebsjabes Gervorrufert. 


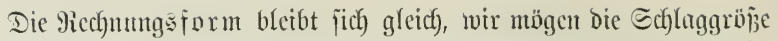
nad) Der Bontität Des Standortes oder nach Der bes Beftandes crmittelıt. Bcijpiel. Ein 100 ha grojer 9iederwald mit 20jübrigem Um= tricbe entbalte dret verichiedene Standortsffajfen:

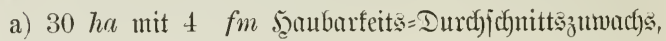

b) 50 " " 3 "

c) $20 "$ " 2,5 "

9)iittlere Bonitüt:

$$
\frac{30 \cdot 4+50 \cdot 3+20 \cdot 2,5}{100}=3,2 \mathrm{fm} .
$$

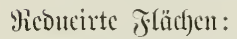

$$
\begin{gathered}
\text { a) } 30 \times \frac{4}{3,2}=37,5 \mathrm{ha}, \\
\text { b) } 50 \times \frac{3}{3,2}=46,9 \text { " } \\
\text { e) } 20 \times \frac{2,5}{3,2}=15,6 \text { " } \\
\text { 3ujaumten } 100 \mathrm{ha} . \\
\text { Jabresjatag } \frac{100}{20}=5 \mathrm{ha} .
\end{gathered}
$$

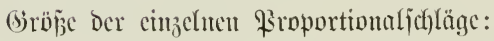

$$
\begin{aligned}
& \text { Wint a) } 4: 3,2=5: \mathrm{x} \text {, ficrats } \mathrm{x}=\frac{3,2.5}{4}=4,00 \mathrm{ha}, \\
& \text { " b) } 3: 3,2-5: \mathrm{x}, \quad \text { " } \quad \mathrm{x}-\frac{3,2.5}{3}=5,33 \text { " } \\
& \text { " c) } 2,5: 3,2 \quad 5: \mathrm{x}, \quad \text { " } \quad \mathrm{x}-\frac{3,2.5}{2,5}=6,40 "
\end{aligned}
$$

Jeterutach) mïtrde liejern:
a) $\frac{30}{4}=7,50$ Jaf)resjchlägc,

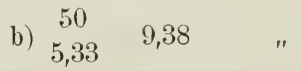

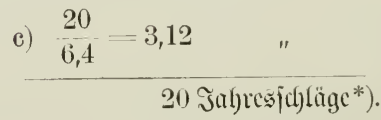

*) 3u vergl. $\$ 77$. 


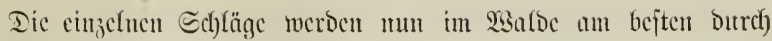

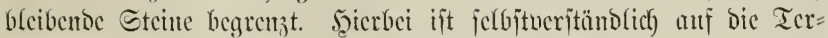

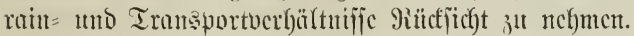

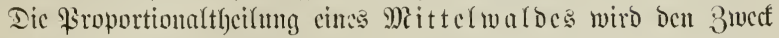

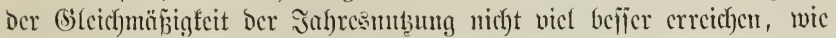
bic cinfuct)e genmetrifd)e Ech)(agcintheilung, weil bie (Erträge bes Dher=

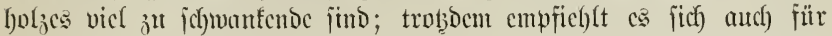

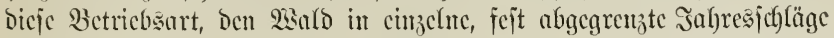

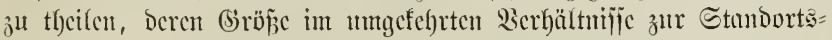
Eonitüt itcht.

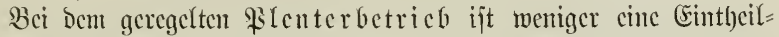
mug bes 23 ald

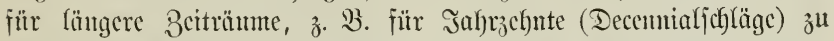
cmpichlen. (Es bildot bicje Form Der Ech)lngeintheilung Den Heber=

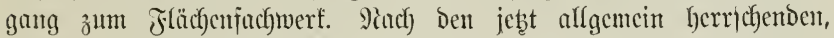

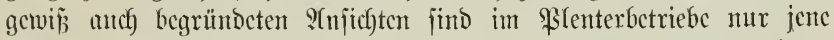

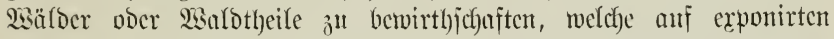
Sumpen, Bergrücfen, Rüften 2 . als cigentliche Echuţwaldutgen ju

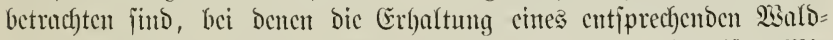

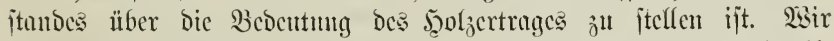

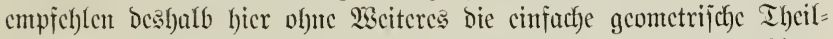

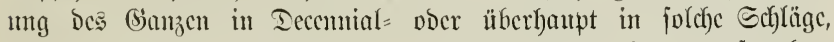

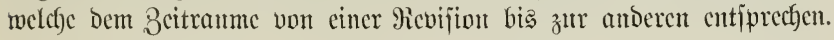

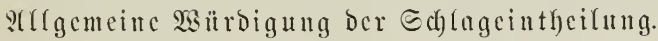

Dic Edflagcintf)cilung ijt unter aflen Miethodon Der Errtrage: regelung biejentige, weldye am meiften birect ber Secritellung Des nor=

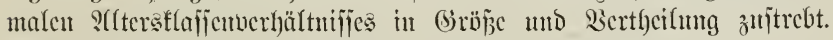

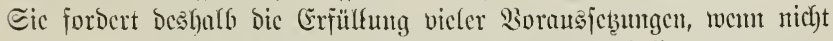

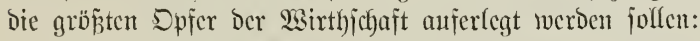

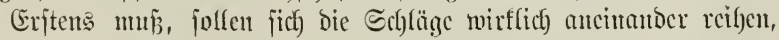

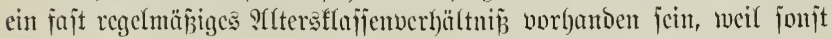
ganz ungerechffertigte Spfer Durd) Den şicb unreifer und burch Das

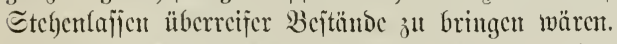

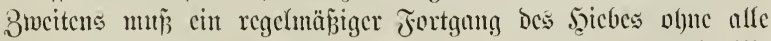

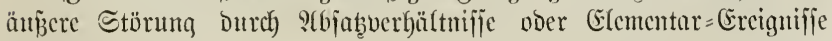
fittfïnocn fömusn.

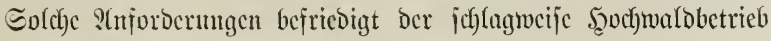


felbjt für bie blof̧e Dhaterial=Ertragseregefung nicmuls, um fo weniger,

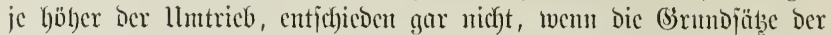

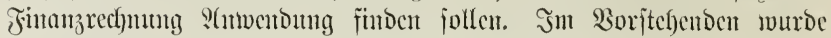
Desflyalb bicjer Betricb nicht bejonders crwäl)nt.

S(nwentbar ift bic Meth)ode für Den Piedermald atno Mittelwald,

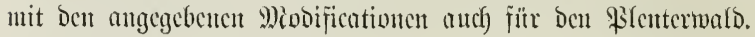

\section{$\$ 120$.}

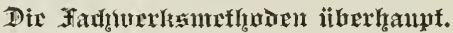

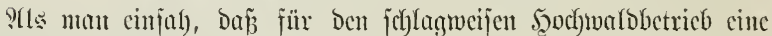
Echlageintheilung nicht Durchfitt)rbar war, um jo wentiger, je höber Der Ilmtrieb, vocr je melyr man S(ujpruch machte auf cute gemific

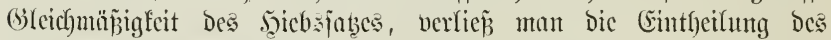
(Sanjen für bie Eimzeljalne nno juchte fie anf längere Beitnbjobntte (Ferioben, Fächer) jut begrüuton. Man cutwarf cinen fïr ben

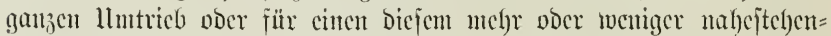

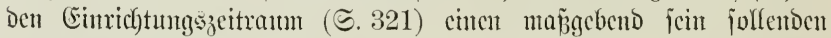

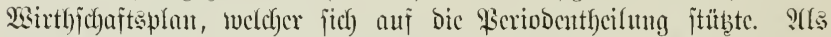

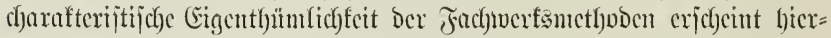

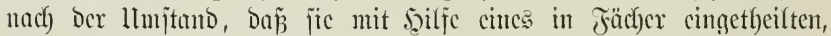

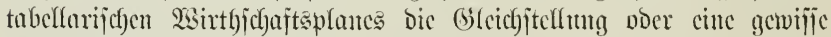

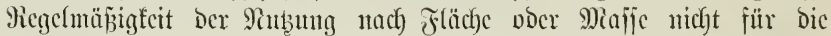

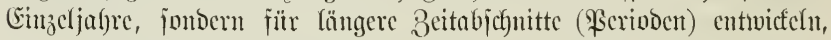

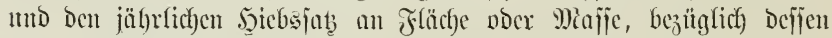

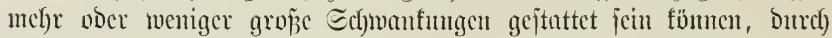

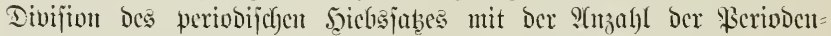
julye finton.

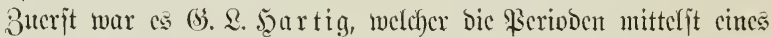

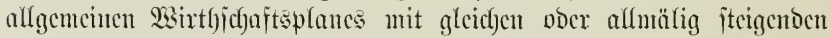

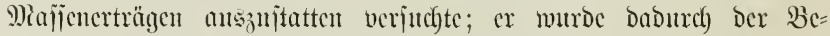

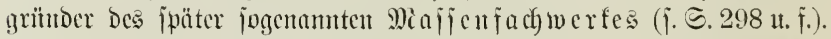

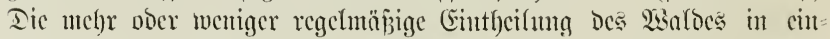

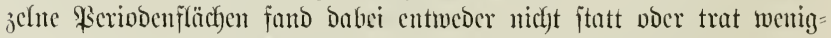

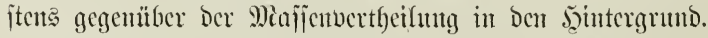

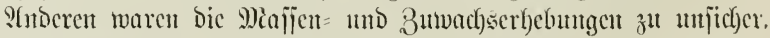
fie theilten Deshalb den cinzelnen Beitabjenten (Perioden) bejtimmtc,

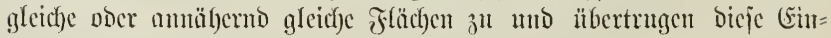

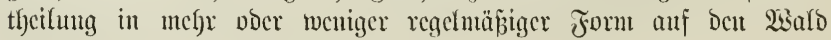




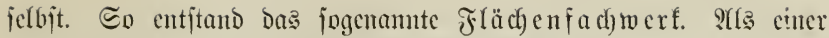

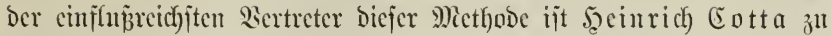

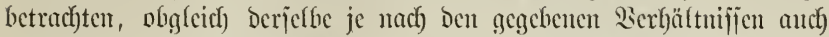

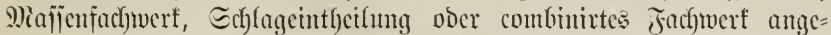

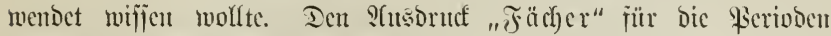
hat Cotta zuerjt gebraud)t (i. ङ. 302).

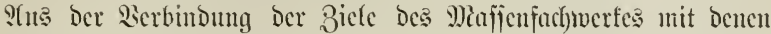

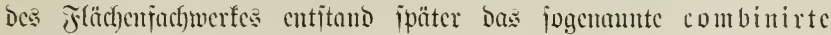
Fach werf.

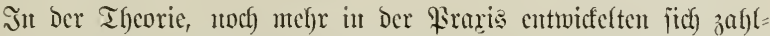

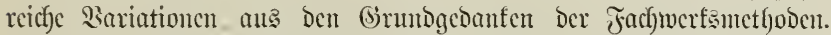

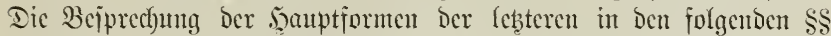
fant mur einjelner bicjer Sartationen nebentyer gebenfen, fie joll junächjt bic Jauptmethoden in cinfachjter Form idfiltern. Sint audf bicje cin= fachjten (Sintubformen woh) niemals, mentgitens nicht fïr längere Beit

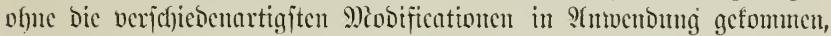
jo tritt bei ifnen bas (eitento \$rincip Dod) an jefärfĩten Gervor.

Sn netejter 3eit hat Dberföriter Denzin ein Enjtem Der Fachwertsmethoden aujigcitellt. ${ }^{*}$ ) (Ex veriteht unter leşteren "Diejenigen Miethoden ber Waldertragä=

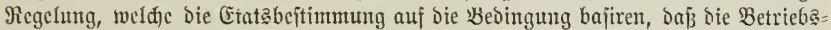

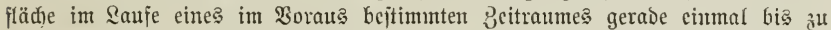

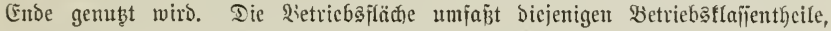

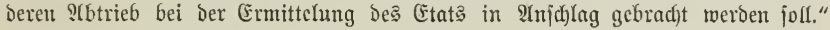
Sein Evitem lautet:

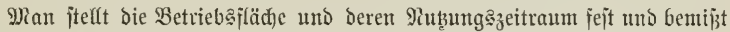

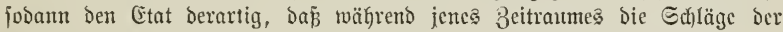
Einzeljafre

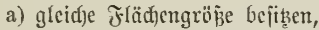

b) Flä̌d)en von gleidjer norntaler Extragąfäbigfeit bejitzent, $\mathfrak{F} l a ̈$ d) $\mathfrak{e} \mathfrak{n}$ fach wert.

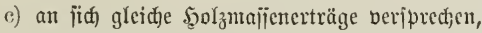

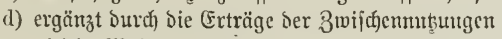
gleidje Majienterträge veriprechen,

Ma i i $\mathfrak{n}$ fad wert

e) partienweije (etrwa zu 20) in Sumna an= näherno fowohl gleide Flächengröß̉e bejț̦en, als aud gleidgro iprechen und in biejen qiartien einzeln gleid)e Majijenerträge erivarten laijen,
Sombinirtes

Fa d duert.

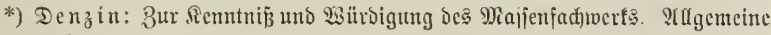

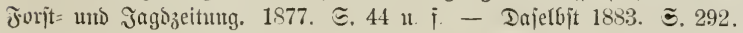


f) partienmeife (etra zи 20 oder 40 ) in Summa

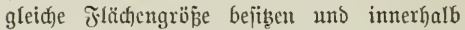
Diejer Rartien cinzeln gleidje Miajienerträge veriprechen,

Fartielles

§lä (h)en =

fa d) wert.

g) innerfalb von $\mathfrak{B a r t i e n , ~ D i e ~ a l l e ~} \mathfrak{B}$ citände Det= jelben Materaflajie rmfajien und jobiel Salägc

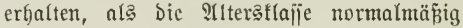

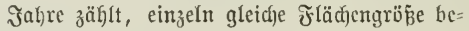
jitben,

h) gIciđgc Miajienerträge veripred)en.

llnoollfom= menes $\mathfrak{l}$ läden= fachive $r$ f. $\left\{\begin{array}{c}\text { Inoolfiom }= \\ \text { menes Majien } \\ \text { fadroert. }\end{array}\right.$

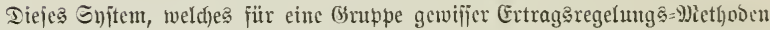

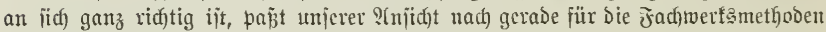

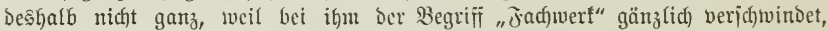

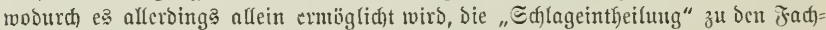
werfen zu rectinen.

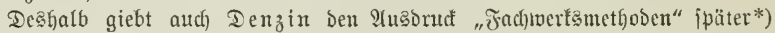

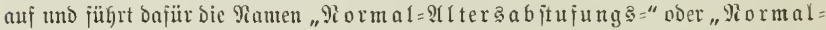

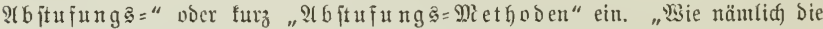
Tormalvnrtatbametgoden bie Iembenz verfolgen, Den Normalvortath herbeizuführen,

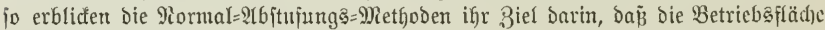

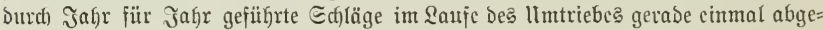
trieben uno wicber angebaut wiro und mithin auf ifr eine Stujenjolge bon $1-u$ jähr.

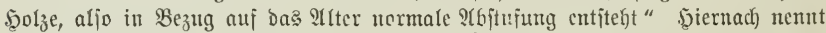

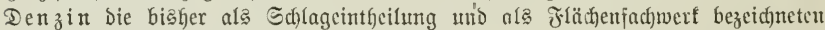

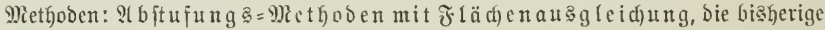

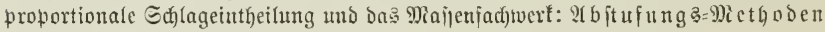

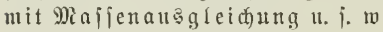

\section{\$ 121.}

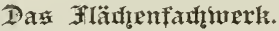

Unter Flächenfach)werf weritehen wir biejenige Riegchungsmethode,

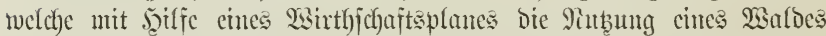

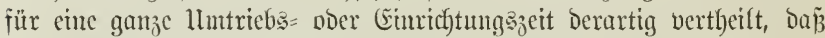

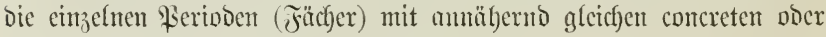
redueirten Flächen ausgejtattet werden.

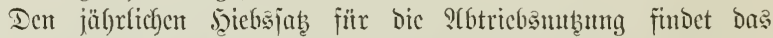

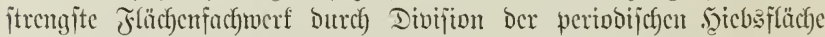

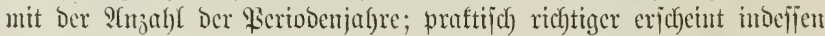

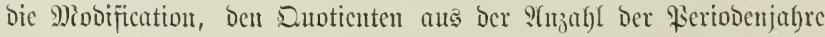

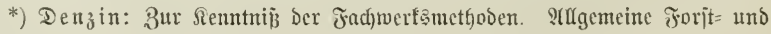
Jagozeitung. 1883. Є. 289 น. Ғ. 


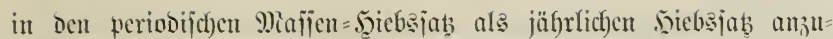

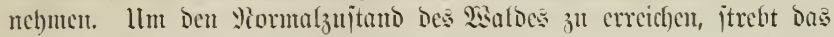

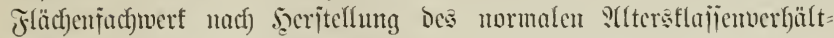

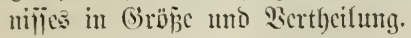

Mian theilte jede 9 (btheifung $(\$ 110)$ cinter bejtinmten Periode im

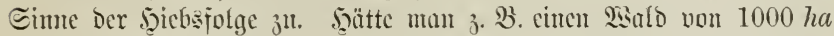
im 100 jührigen llmtricbe ju bewirthichajten, jo witrocn jich 5 Fierioben in 20 jühriger sebjufuntg ergehen, und bic Eumme ber cincr joldfen

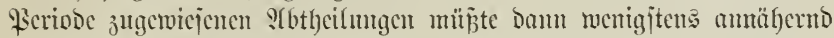
gleid) fein $\frac{1000}{5}-200$ ha. Diejen Pachweis findet man in älteren,

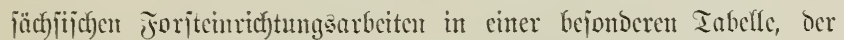
jogenannten "Bujummenjteflung Der Pieriobenfläcl)en" geliejert.

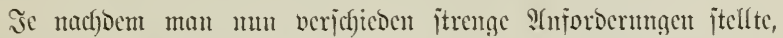
verlangte man amähernoc Gileicf)heit blos ber contereten fäläche, die

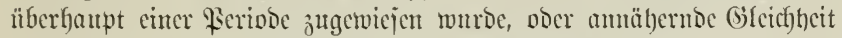

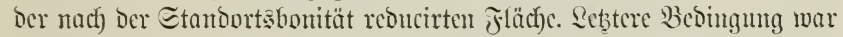
praftijch jajt ganj muereinbar mit Der Iendenj Des Flächenjachmertes, bic Periobentbeilung auch auf ben 2 anlo jelbit in einer ber bejten

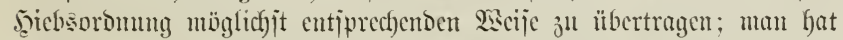

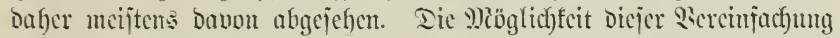

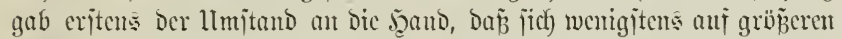

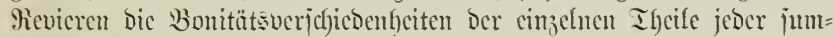
marijchen Pistobenfläche von jelbjt gegenjeitig annäherno ausgleidyen,

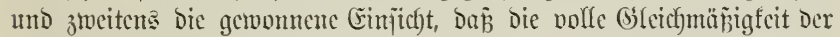

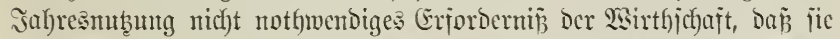

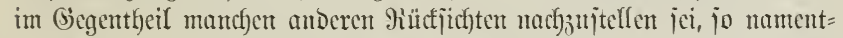
(ich) Denen anf cinc mohlgcoronete siebsifolge.

Reţtere wurde bei Der llebertragung Der Eintheilung auf ben

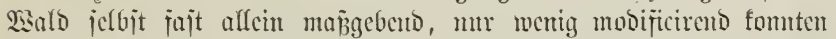

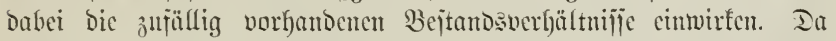

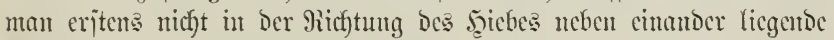
SLbtbeifungen cincr und Derjelben Reriode jumeijen burîte, meil man jonjt zu breite Echläge erfalten fättc, Da man zrwcitems bics eben jo

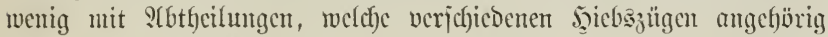
an Den Wirtbjidfaftsitreifen ancinander grenzen, thun fomte, meil bics

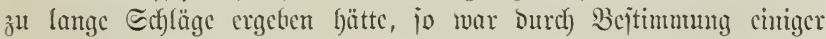
meniger Periodenflächent die gauje übrige Eintbcilung gegeben. 
Die Betracftung folgender Figur mag Das (sejagte erläuteru.

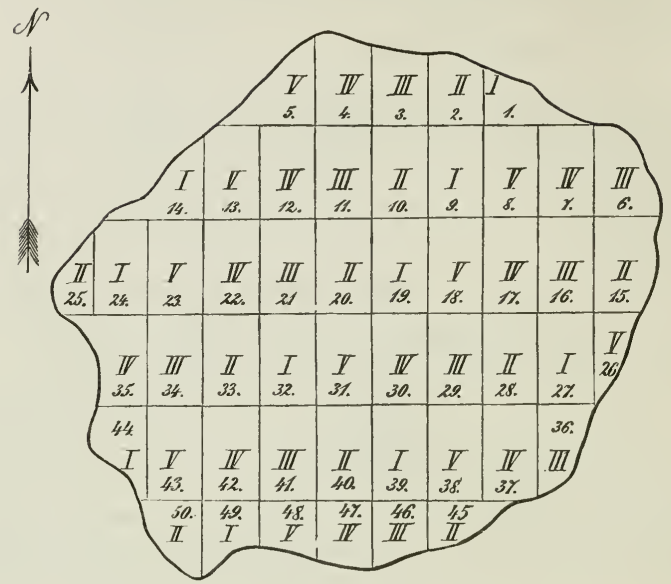

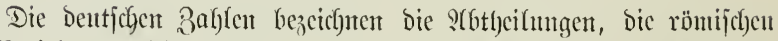

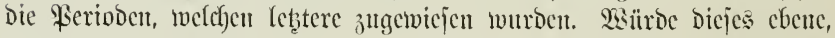
baber cine regelmäß̄ig rechtwintelige Eintbeifung nertragende Fevier im 100 jäfrigen Ilntriebe bemirtbjefaftet, fo entiprïchen bic Pierioben=

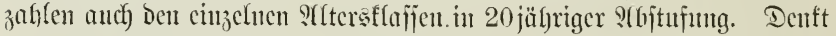

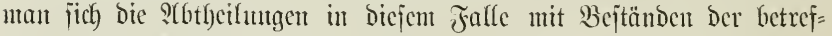
fenten sflereftafien bejtodt, jo bietet bic Fignr bas Bild bes vom J(äc)enfact)merf eritrebten 9ormalzujtandes*).

(5) ift aus Der Figur erjichtlich), Daj z. B. bic uebeneinander

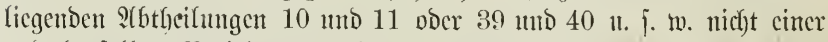

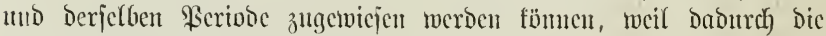
Salyesjechläge boppelt jo breit werden milipten, als fic ber eritrebte Tiormalzujtand forbert. Eben jo wenig fant man bic slbtfeilungen 3 und 10 Derjelben Feriode zutheilen, Denn in Folge dejien würden die einzelnen Echläge dic Doppelte Sänge crljalten. Pioch uturichtiger

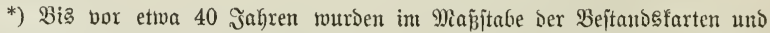
mit Denjelben Farbentönen Derartige Sarten zur birblichen Darjtellung bes er=

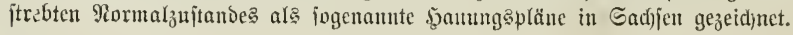




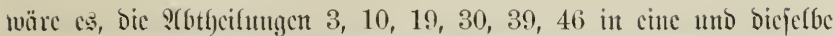

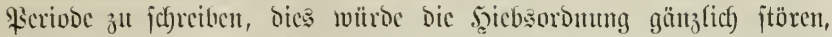

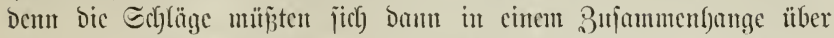

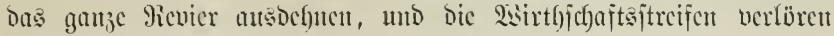
jede B̉cbcututug.

Bei ber Beptimmung Der \$eriobenffäclyen crforberte namentficf)

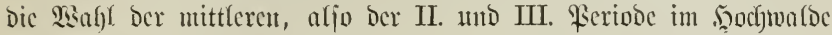

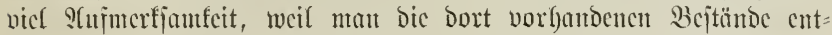
weder fo zum stbtriebe bringen mujte, wie fie gerabe vorlagen, ober

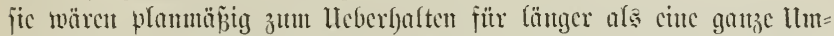

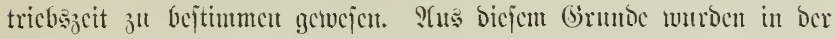

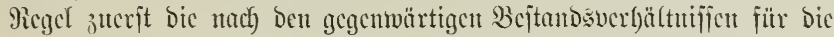

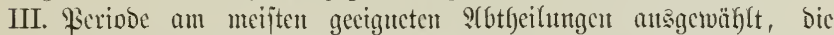

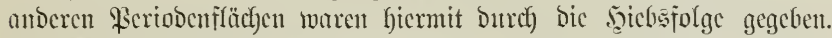

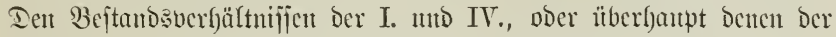

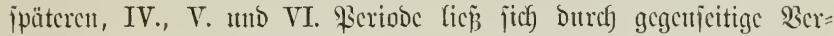
jefjicbungen und ?tusgleichutugen lecichter Picchumug tragen.

Einc eigentlicl) Betricbsflafjeneintl)eilung wor bcm F(äcl)enfach) werf anfüutglich fremb, obglecich fie ganz gut mit bicjer Ricgeluntge metfobe verbutocn werben fam und autf) fpäter verfunden morden ift*). Dic verjeficocuen Betriebsarten wurben jwar itets getrennt

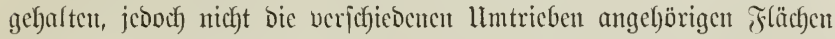

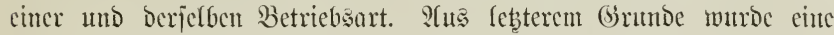
Beranjtaltung nötbig, mittelit Deren man bic verjechiesenen llmtriebe

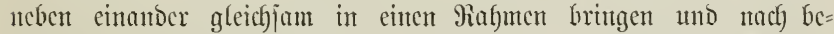
jtimunten Beitabjefnitten überjeben funt. Man naunte bicjen Mabmen Den Einrichtungszeitraum und theilte biejen, aber nicht Den

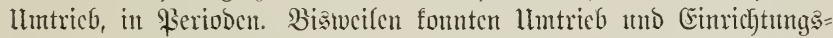

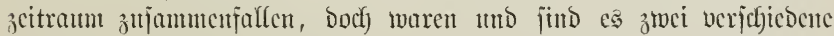
Begriffe.

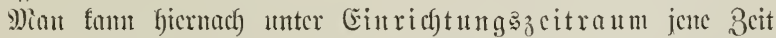
verjtefon, binnen weldfer man cinmal mit bent şicbe bos ganze Revier burchlaufen will, um twenigitens cinige Drontutg in bic bejtants: verbältnij̄ic zu bringen.

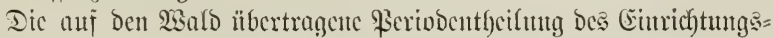
zcitraumes ober unter getwifjen Sierbältuijīen auch bes llutriebes gab

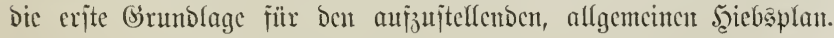

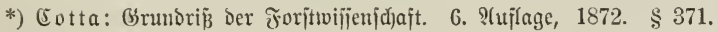

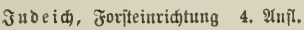


Dejien Bejtreben muñte vorzugšweije Dabin gerichtet jein, bie Bertheil=

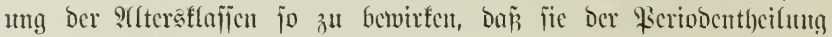
fïc) alfuälig mebr uto mel)r anpap̃te.

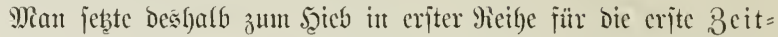
periode dic abtriebsbedürftigen Bejtände der leb̧ten, IV., V. It. ¡. w.

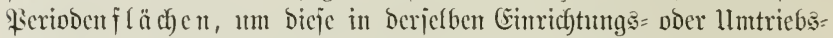

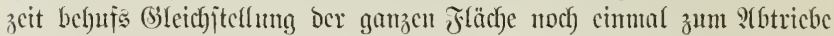

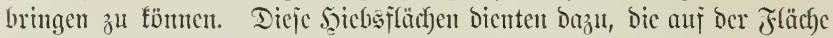
Der I. Feriobe nicht baubaren Drte zu erjeben. Für bie II. IntD III.

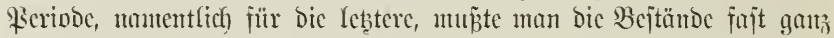
jo nefmen, wic fic famten, weil hier ber Doppelte ?(btrieb nicht möglicf),

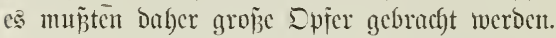

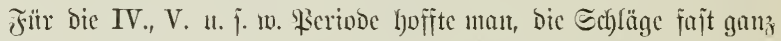

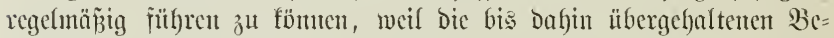
jtände entichicben abjab̧fäfiges s)iaterial entfalten muñten, weil ferner Dic it ber erjten 3eitperiobe abgebof mabricheinfich machtent.

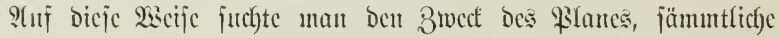
Ferioden mit gleichen Şiebsiflächen ju verjeben, möglichjt ju errectchen, Im bamn nach ?tblanf Des criten llmtriebes oder (Eimrichtungszeitraumes

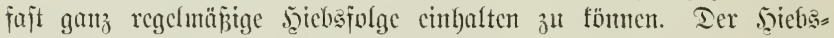

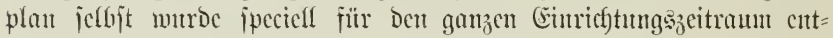
wovifen.

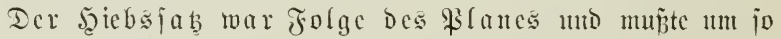
ungleicher ansfallen, je mef)r man ber stuforderung des fläcl)en-

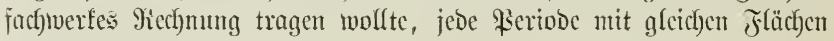
anṡzujtatten.

Den Bumachs berechnete man, wic bereits früber ertwäbnt, jo, Dá

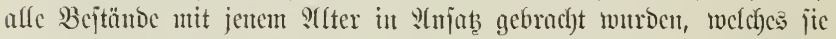
it ber Mitte jener Weriode erreicht habent würden, ber fie zum Jeicbe

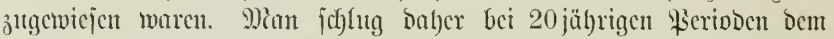
gegenvärtigen :(lter Der Bejtünde jut: Für bie I. Ficriobe 10, für bic

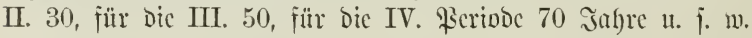

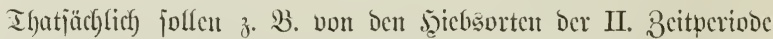
Dic erijten nach) 20 Jabren, bie lebzten nach) 40 Jaf)ren abgetricbent

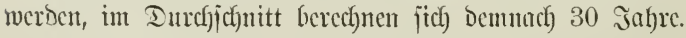

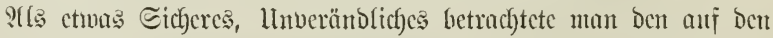

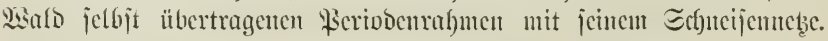




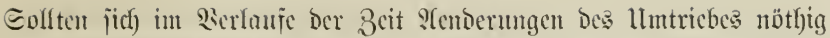
mad)en, jo blicben bieje jeberzeit obne jtörenten Einfluj. Im obigent, Durch) bie Beidymug verbeutlichten Beijpicle war nan vom 100jäfrigen

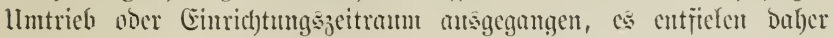
bei 5 anf cinamber folgenden Feriobenflächen fïr jede Durchjict)nittlich) 20 Safre; molfte mun mu jpäter senjelben Eintf)eilungstabment für

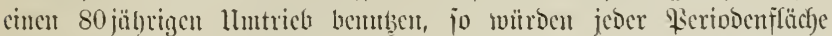

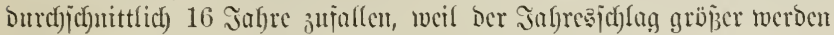
mïīte.

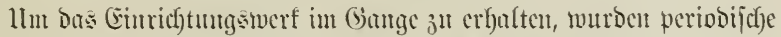

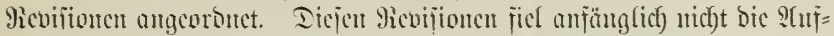
gabe ju, neue \$iläne ju entmerfen, fonbern uur bie, ben alten fïr ben

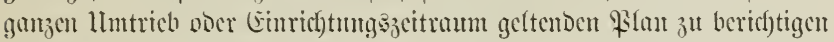

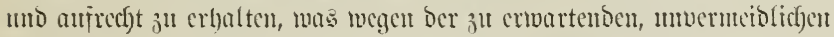
Etörmugen burch) Ėlementurereignijic sc. ntothwendig erjefien.

Für bic crite Feriobe, nach Einfülnung 10 jäbriger Pievifions: jeiträunte, wurbe innerhalb Der Girenzen Des affgementen Pilanes ein ipecieller jeicbsplan angejertigt un biejem ein Sinturplan jur Ecite gejtcllt.

Pnmerfung. Bon ben zahlreiden Mobificationen, weldye bas faläd)enjadfwerf erfitten, verbient hier bejonders bie eine Ermäfrung, weldhe von einer rationellen

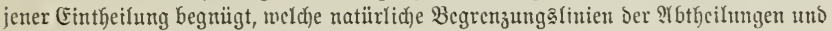

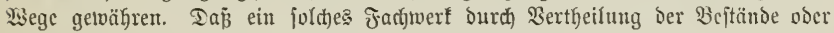
Bejtanosgruppen an bie ciltzelnen Beitperioben ebenfalla einen Fläch)et= ober \$iajīn=

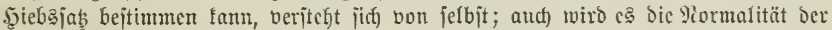

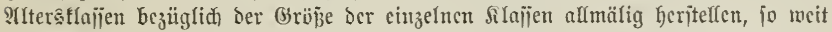

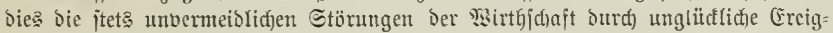

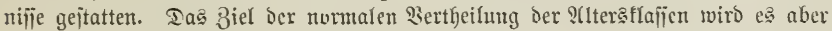
nie auch nur annäferno erreidhen, weil ç fid bicjes Biel nidft flar madt, uno

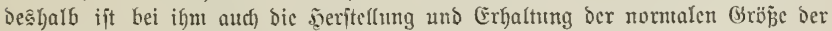

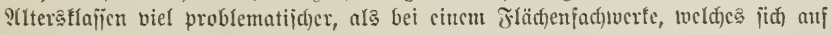
cine gute sildocinthcilung itüișt.

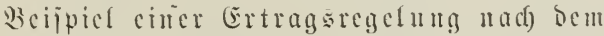

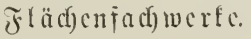

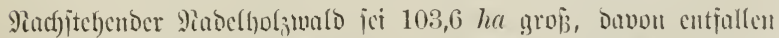

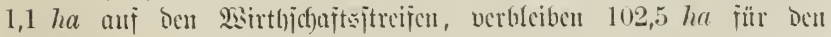
गु) 


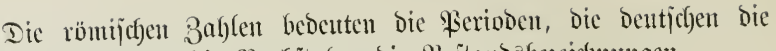

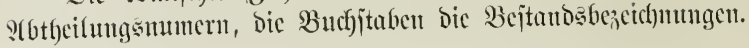

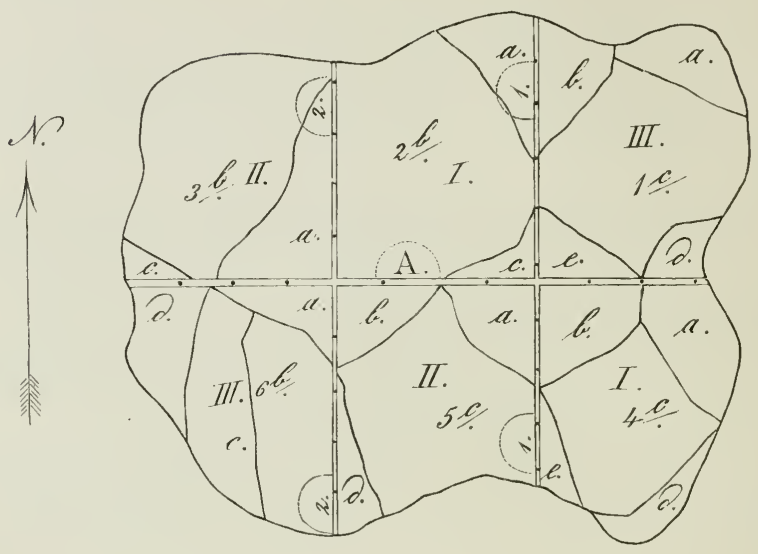

Die Bejtandsbonität ijt zum Theil die ber $\$ 11$ mitgetfeilten Erfabrumgstajel, zum Theil cime niebrigere. Bejecichnen wir critere mit 4., leb̧tere mit 3. Bonität, uno geben bicjer folgendo Erträge:

\begin{tabular}{|c|c|c|c|c|c|}
\hline Alter. & Ertrag. & $\begin{array}{l}\text { Periodifdjer } \\
\text { 3umadjs. }\end{array}$ & alter. & Ertrag. & $\begin{array}{l}\text { fritiodiffjer } \\
\text { 3umadfs. }\end{array}$ \\
\hline Jahte. & \multicolumn{2}{|c|}{ Fe ft meter. } & Jahre. & \multicolumn{2}{|c|}{ Feft meter. } \\
\hline 5 & 5 & 7 & 55 & 271 & 35 \\
\hline 10 & 12 & 15 & 60 & 306 & 34 \\
\hline 15 & 27 & $\begin{array}{l}10 \\
22\end{array}$ & 65 & 340 & 32 \\
\hline 20 & 49 & 25 & 70 & 372 & 30 \\
\hline * 25 & 74 & 28 & 75 & 402 & 28 \\
\hline 30 & 102 & $\begin{array}{l}20 \\
31\end{array}$ & 80 & 430 & 25 \\
\hline 35 & 133 & $\begin{array}{l}01 \\
33\end{array}$ & 85 & 455 & 22 \\
\hline 40 & 166 & 34 & 90 & 477 & 19 \\
\hline 45 & 200 & $\begin{array}{l}54 \\
35\end{array}$ & 95 & 496 & 16 \\
\hline 50 & 23.5 & 36 & 100 & 512 & \\
\hline
\end{tabular}




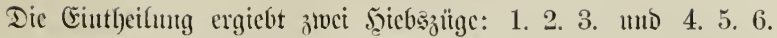
Die Bejtände \%)

1 a. $\quad 2,50$ ha 5 jährig. 4. Bonitüt.

b. 2,50 " 75 " 3 . "

c. $10,50 " 5$ " 5 . "

d. 1,25 " 90 " 4.

e. 1,75 " 20 " 4 . "

9(bt). 1. 18,50 ha. III. Periobe.

2a. 2,50 ha 75 jäf)rig. 3. Bonität.

b. 15,75 " 40 " 4 . "

c. $1,00 " 20,4$ "

9(bth). 2. 19,25 ha. I. \$eriode.

3a. $\quad 4,75$ ha 40 jüfrig. 3. Bontitüt.

b. $11,75,15, " 3$

e. 0,75 " 100 " 4 . "

?(btf). 3. 17,25 ha. II. Periodc.

4a. $\quad 3,00$ ha 90 jäfrig. 4. Bontitüt.

b. 2,80 " 20 " 4 . "

c. 7,50 " 50 " 4 . "

d. 1,20 " 3 ใöณ̃e.

e. $1,00,30$ jährig. 4 . "

:(bth). 4, 15,50 ha. I. \$eriode.

5 a. 2,25 ha 20 jäfrig. 4. 3buntät.

b. 1,80 " 40 " 4 . "

c. 11,20 " 25 " 3.

d. $1,50,5$ " 5 "

9(btf). 5. 16,75 ha. II. 距riode.

6 a. 1,75 ha 40 jä̈rrig. 3. Bonitüt.

b. $6,00 " 5$ " 5 . "

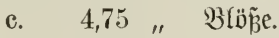

d. 2,75 " 100 jäfrig. 4 .

9(bth) 6. 15,25 ha. III. Periode.

Iic feläche ber I. Ferivod beträgt fieruach) 34,75 ha,

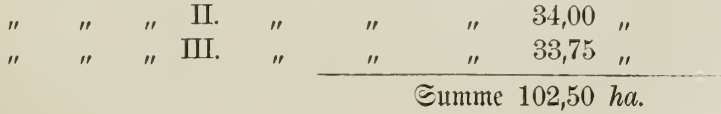


Der Sïrze wegen jei cin unr 60 jähriger IImtrieb angenommen,

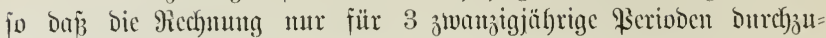
fïbrent ijt.

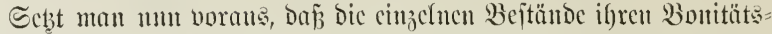
c)arafter bis jum Dereintigen S(btriebe bebalten, und bercebuet beren

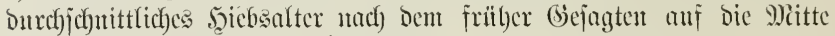
ber betreffenten Feriode, jo daz̃ aljo der I. 10, ber II. 30, Der III.

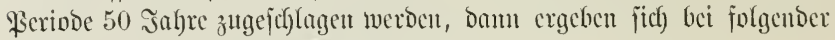
Sertbeilung nachjtebende :(btricbserträge:

\begin{tabular}{|c|c|c|c|c|c|}
\hline \multirow[t]{2}{*}{ зезеicfinung. } & \multirow{2}{*}{ fläcfje. } & \multirow{2}{*}{ 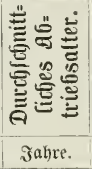 } & \multicolumn{2}{|c|}{$\begin{array}{l}\text { Ertray } \\
\text { in } \\
\text { feftntetern }\end{array}$} & \multirow[t]{2}{*}{ Bemerḱungu. } \\
\hline & & & I ha. & Summe. & \\
\hline \multicolumn{5}{|c|}{ I. Beriode. } & \multirow[b]{2}{*}{ 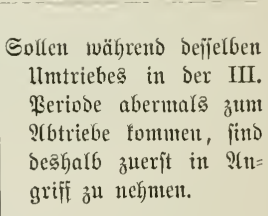 } \\
\hline $\begin{array}{r}1 \mathrm{~b} . \\
\mathrm{d} .\end{array}$ & $\begin{array}{l}2,50 \\
1,25\end{array}$ & $\begin{array}{r}85 \\
100\end{array}$ & $\begin{array}{l}455 \\
630\end{array}$ & $\left.\begin{array}{r}1138 \\
787\end{array}\right\}$ & \\
\hline $2 a$ & 2,50 & 85 & 455 & $11: 37$ & \\
\hline $\left.\begin{array}{r}\text { b. } \\
\text { Davout }\end{array}\right\}$ & 12,00 & 50 & 275 & 3300 & \\
\hline $3 \mathrm{c}$. & 0,75 & 110 & 670 & 502 & \\
\hline 4 a. & 3,00 & 100 & 630 & 1890 & \\
\hline c. & 7,50 & 60 & 354 & 2655 & \\
\hline $\left.\begin{array}{r}5 c . \\
\text { Dับขอก }\end{array}\right\}$ & 1,00 & 35 & 133 & 133 & $\begin{array}{l}\text { Roşbieb, um 5a zum 8wede } \\
\text { bes lleberbaltens an Den } \\
\text { freienStand zugeivöhnen. }\end{array}$ \\
\hline $6 \mathrm{~d}$. & 2,75 & 110 & 670 & 1843 & Wic $1 \mathrm{bd}$. \\
\hline ธuntntc & 33,25 & & & 13385 & \\
\hline
\end{tabular}




\begin{tabular}{|c|c|c|c|c|c|}
\hline \multirow[t]{2}{*}{ зъезеiсfnung. } & \multirow{2}{*}{$\begin{array}{l}\text { f(üc)je. } \\
\text { ha. }\end{array}$} & \multirow{2}{*}{ 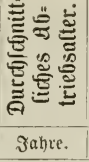 } & \multicolumn{2}{|c|}{$\begin{array}{c}\text { Ertrag } \\
\text { in } \\
\text { feftmetern. }\end{array}$} & \multirow[t]{2}{*}{ bemerkungen. } \\
\hline & & & tha. & Summe. & \\
\hline \multicolumn{5}{|c|}{ II. Beriode. } & \\
\hline $\left.\begin{array}{r}2 b . \\
\text { Dex Eicit }\end{array}\right\}$ & 3,75 & 70 & 433 & 1624 & \\
\hline $3 a$. & 4,75 & 70 & 372 & 1767 & \\
\hline b. & 11,75 & 45 & 200 & 2350 & \\
\hline $4 \mathrm{e}$. & 1,00 & 60 & 354 & 354 & \\
\hline $5 \mathrm{~b}$. & 1,80 & 70 & 433 & 779 & \\
\hline 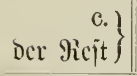 & 10,20 & 55 & 271 & 2764 & \\
\hline Eumme & $\begin{array}{r}33,25 \\
\text { III. }\end{array}$ & geriode & & 9638 & \\
\hline 1 a. & 2,50 & 55 & 314 & 785 & \\
\hline b. & 2,50 & 40 & 200 & 500 & 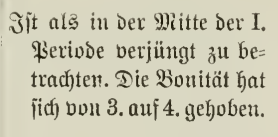 \\
\hline c. & 10,50 & 55 & 271 & 2846 & \\
\hline d. & 1,25 & 40 & 200 & 250 & QIter ermittelt, wie bei $1 \mathrm{~b}$. \\
\hline e. & 1,75 & 70 & 433 & 758 & \\
\hline $5 \mathrm{~d}$. & 1,50 & 55 & 314 & 471 & \\
\hline $6 \mathrm{a}$. & 1,75 & 90 & 477 & 835 & \\
\hline b. & 6,00 & 55 & 314 & 1884 & \\
\hline c. & 4,75 & 50 & 275 & 1306 & \\
\hline d. & 2,75 & 40 & 200 & 550 & Wic $1 \mathrm{~b}$. \\
\hline Eumme & 35,25 & & & 10185 & \\
\hline
\end{tabular}

2Bicderl)olung Der Eummen:

jeicbsfläcfe der I. Herivde 33,25 ha mit 13385 fm Ertrag.

\begin{tabular}{|c|c|c|c|c|c|c|c|c|}
\hline & $" \quad$ II. & " & 33,25 & $"$ & " & 9638 & $"$ & $"$ \\
\hline " & " III. & $"$ & 35,25 & $"$ & $"$ & 10185 & $"$ & $"$ \\
\hline
\end{tabular}

$\left.\begin{array}{c}\text { Eumme währent des } \\ \text { ganzen Beitraumes }\end{array}\right\} \quad 101,75$ ha mit $33208 \mathrm{fm}$ Ertrag. 


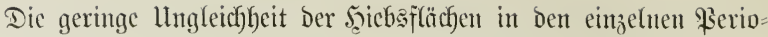
den während des erjten llmtriebes wiebcrjpricht bem Principe des Jlächenfachmerfes nicht.

Für ben zweiten llmtricb wäre ç un möglich), olyte irgend nen=

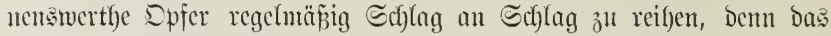

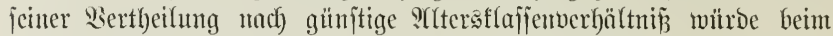
Beginne bicjes llmtriebes folgende (3rö̈zen nachweijen:
I. Silaffe 1-20jälyrig
$\begin{array}{ll}\text { II. " } & 21-40 \text { " } \\ \text { III. " } & 41-60 \text { " }\end{array}$
35,25 ha
33,25
34,00
(5̨iebgfläd)e ১cr III. 3citpcriode.)
(nämนlidx):
II. " )
26,75 von Der 5̧iebaffäd)e bex $I$. 3eitperiode.
7,25 über 60jährig: 2c., 4bd., 5a.)

Summe 102,50 ha.

Ilm ben gejammten Şiebsjab Des 9ieviers 3n beitimmen, twäre nock) Der Betrag Der Bwijchenumbungen Den oben crmittelten S(btriebs=

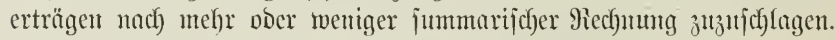
Dies geichieht in Der Regel mur für bie erite Reriode.

Dic Ilngleichycit Der Erträge ift nach Dem Wrincipe des Flächen-

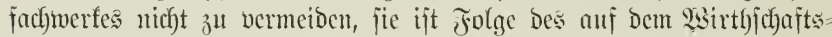
plane fußenden Etrebens, bie normale J̧ichsoronung in fürzejter 3cit

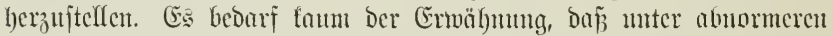
Serbältnifjen, als bie des vorliegenden Beijpieles find, nod) recit

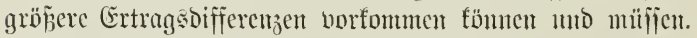

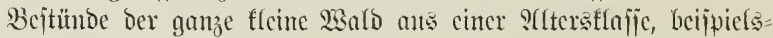

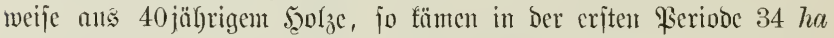
50 jälyriges, it Der II. 34 ha 70 jülyriges, แmo in Der III. แur

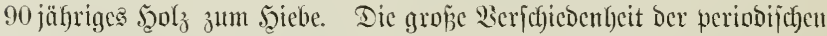
Erträge läge fyier auf Der şand.

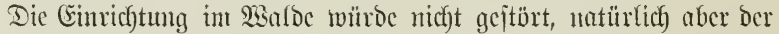
23irthjchaftsplan jelbjt Beränderungen crleiden, wenn man fich fpäter

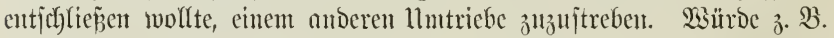
fünfitig ein 90jähriger llmtricb gemählt, fo würocn, anjtatt baj jeß̧t jeder Feriodenfläche 20 Jal)re angebören, 30 Jah)re bafür cutfallen,

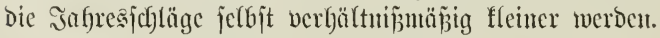

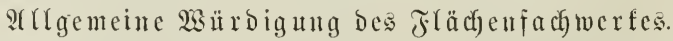

Dicje Mietlyode zeichnet jich Daburd) ants, Daß̉ jie, wic bic Schlagein= theilutg, Ginnen fürzejter Beit, wem nicht jtörende ělementarereigniīfe 


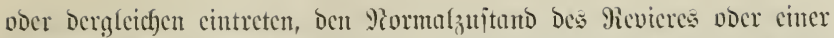

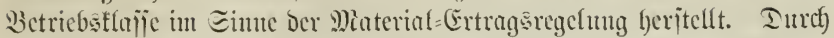

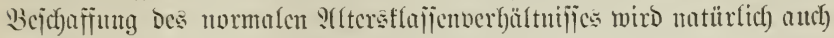

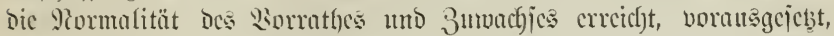

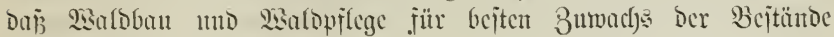
Eorge tragert.

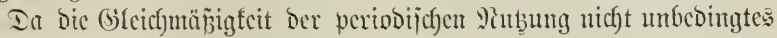

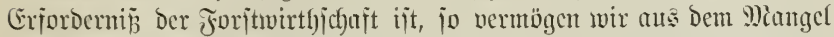

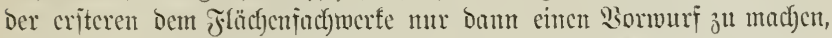
wenn jene (j)renzen Der Differenjen überichritten werden, weldhe ber

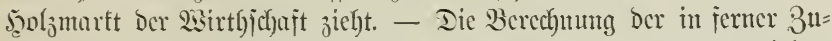
funft licgenoen, perindijc)en Erträgc, numentlicf) bei holyen Umtrichen, iit juar ctmas llnjchäbliches, um jo meljr aber etwas lleberiflitifiges,

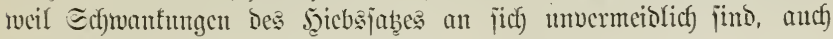
gar nicht bem \$rincipe Der Methode wiberjprechen.

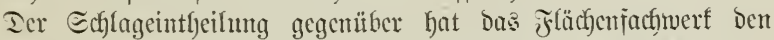

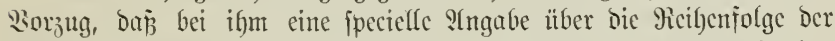

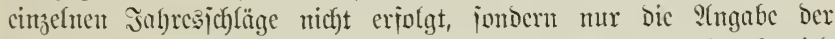

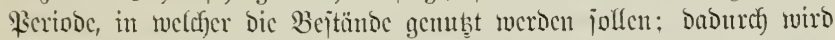

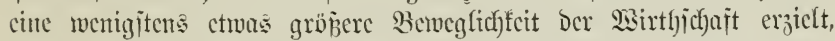

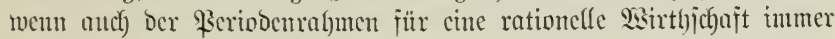

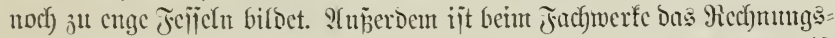
werf weit cimfacfer, Detin bie Berechum Der periobijchen Erträge ijt

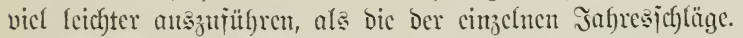

Enticfiedenc Sachtheile für Den Erfolg ber 2sirtbjefjajt rujt Das

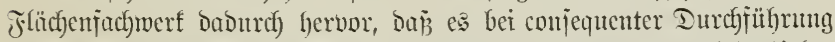
oft unbegrünocte Epjer fordert, wetche theils im Stbtricbe nicht biebs= reijer, theils in langen llcberbalten cutjedjicben biebsreifer Erte be= iteben. Iicje Epjer werocn um jo gröp̈er, je abnormer bas wirtliche

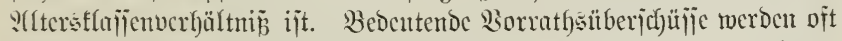

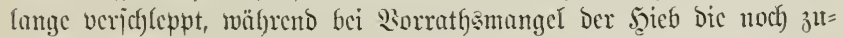
wachsreichjten Bcjtänoc trifït.

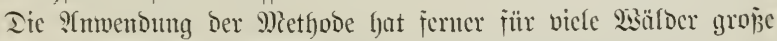

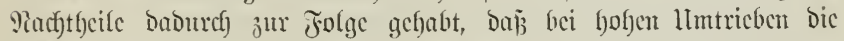

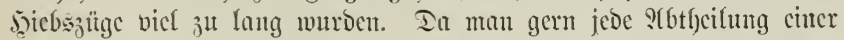
einjigen Periode zumies, cntitanden 3 . B. bei 100 jüfrigem llmtricbe

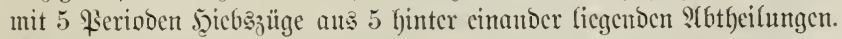

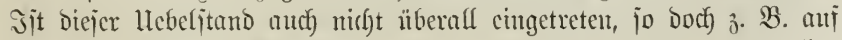
einigen Revieren in Eachjen; wo er aber eintrat, ijt er unter allen 


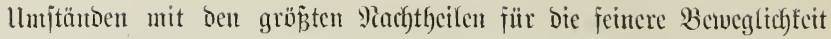

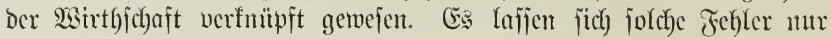

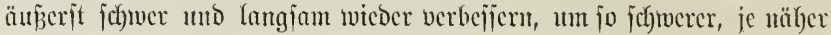

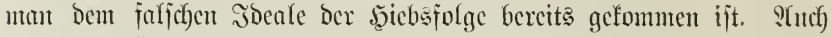

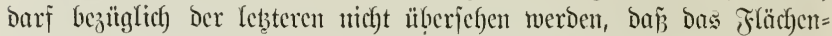

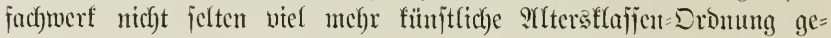

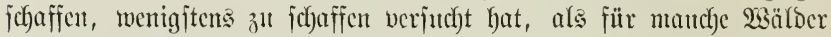
gut ijt, nautentlich gilt bies für Gicbirgsivaldungen. Wirt wiederfolen jeovech, baf biejer Fehler nicht in Frincipe Der Miethode, fondern nur in Der 2 fnwentoung Derjeffen hervortritt.

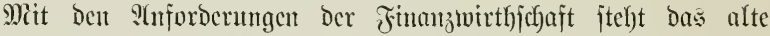
Flächenfactbwerf unr joweit in Scarmonie, als ç bie Tenoenj verfolgt, Dronnng it ben biang bes Şiebes zu bringen.

\section{$\$ 122$.}

\section{Das 2t2alliefartrumerk.}

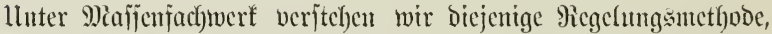

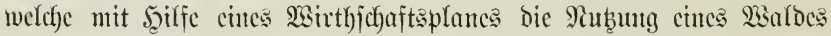
fitr einc ganjc llmtriebs= ober Einrichtungsocit berartig vertbeilt,

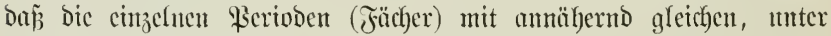
Ilmjtänden mit jteigenden, felten mit allmälig jüfenden Miafjen bebacht werben.

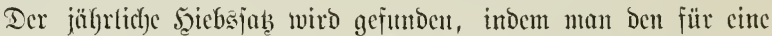

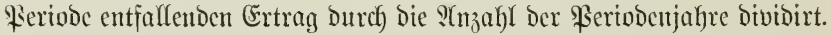

Begrünocr bicjes Sierfahrens ijt (5). 2. Şartig (†. ๔. 298 u. f.).

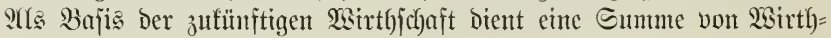

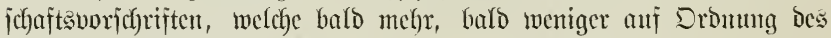

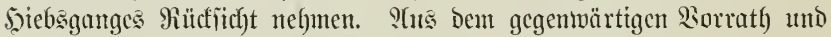
Zumacfje ber cinzelnen Bejtände berechnen fich bie ju cruvartenden

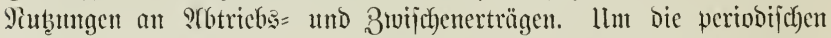
Schwanfungen berjefben $j^{\text {th }}$ vermeiden, werben bie Bejtände jo lange

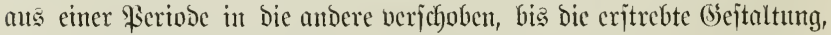

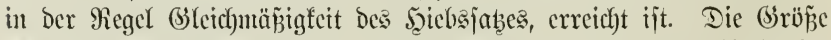

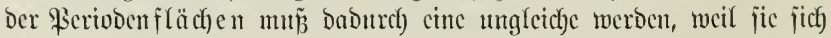

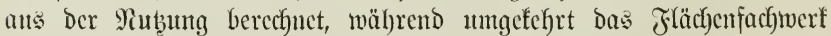

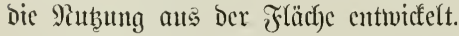

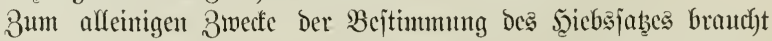
Das Mafjenfadywerf eine Betriebsflafjeneintbeilung nicht, weil jid 
criptere ats der Eumme bes Qiorrathes und Bumadbies ber einjelnen

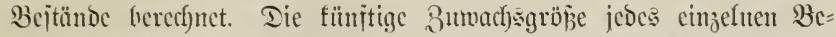

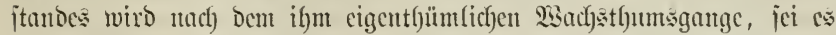

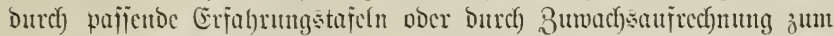
vorbantocnen Sorratbe gefumben. In Folge befien fann man benjelben

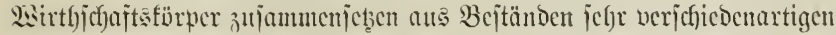

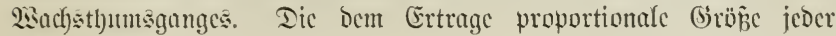

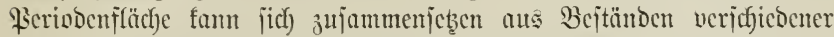

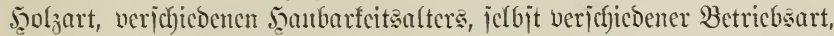

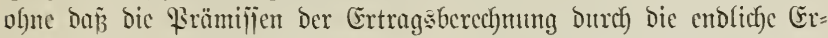
tragserbelung werleb̧t merden.

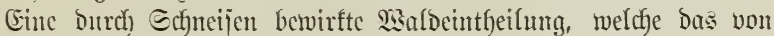

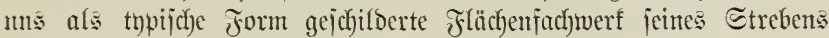
nach) gcorbneter Sjiebsojolge wegen gar nicht entbebren famn, braucht Das Majlenfacfwerf วแ Ermittelung des Sciebsjabes nicft unbedingt.

Die Betricbstlajienbildung ijt aber bereits (3). \&. Şartig nicht fremo, indom er jefon 1795 wenigitens das Gébiet einer jeden 50 ba zart als jelbitjtändige Betriebsflajic hetrad)tet. Epäter in Der 2. S(ufl. jeinter "Ŝtuncijung" (1804) verbejijerte er jein Berjahren mejentliç Daburch),

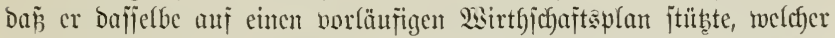
nicht mefre blos das jojebsalter ber cinjefnen Bejtände, jondern, wenn autch in unvollfommenter 2iscije, auth Dic Dronumg Der S(ltersflajien=

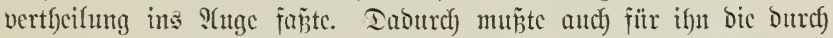

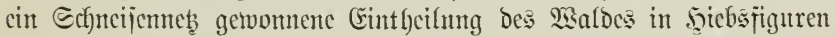
Bedcutung gewinneut.

Dic S(usgleich)ung Der periobijchen Erträge erfolgte burch) wieder= holtes 2ierjefieben ber verjefiedenen Bejtände ans cinter Periode in bic antore. Jुartig jelbjt legte ganz mejentliches (Gemicht Darauf, zu biejer S(usgleidfung bie 3mijcfenmub̧ungen ju benuben, um nicht Durch bie Sierjchicbutugen ber Jeiebsorte gezmungen z̆t werden, biejelben vicl vor

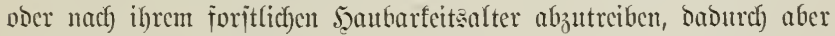
3uvachsverlujte $z^{\text {nt }}$ erlciben.

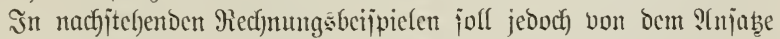
Der 3mijchenub̧utgen ber Einfach) weit wegen abgejeben werden.

\section{Red)ungabeipict.}

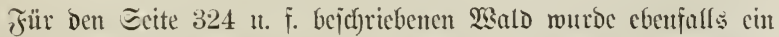

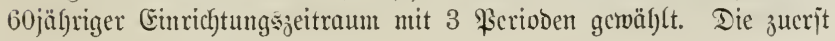


veriud)te Ertragsherechnung habe ähntiche Aicultate ergeben, twie das

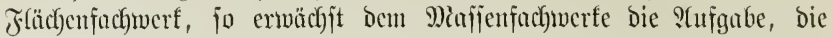

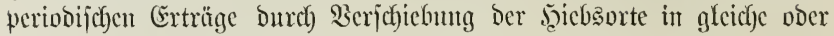
alfmälig jteigenoc zu vermantocı.

Saffen wir ber Sïrze wegen bic Soverträge fier untherüdfictbtigt, jo funn nach wiederboltem Berichicben und \$robiren chtolich folgende

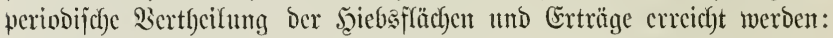

\begin{tabular}{|c|c|c|c|c|c|}
\hline \multirow[t]{2}{*}{ ßезеicfmung. } & fläcfje. & 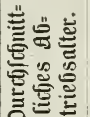 & \multicolumn{2}{|c|}{$\begin{array}{c}\text { Ertrag } \\
\text { ill } \\
\text { feftmetern. }\end{array}$} & \multirow[t]{2}{*}{ Bemerfiungen. } \\
\hline & $h a$. & รabre. & I ha. & Summe. & \\
\hline
\end{tabular}

I. Periode.

\begin{tabular}{|c|c|c|c|c|c|}
\hline $1 \mathrm{~b}$. & 2,50 & 85 & 455 & 1138 & \multirow{9}{*}{ Lostieb. } \\
\hline d.. & 1,25 & 100 & 630 & 787 & \\
\hline $2 a$. & 2,50 & 85 & 455 & 1137 & \\
\hline $\left.\begin{array}{r}\text { b. } \\
\text { Davon }\end{array}\right\}$ & 4,75 & 50 & 275 & 1306 & \\
\hline $3 \mathrm{c}$. & 0,75 & 110 & 670 & 502 & \\
\hline $4 \mathrm{a}$. & 3,00 & 100 & 630 & 1890 & \\
\hline c. & 7,50 & 60 & 354 & 2655 & \\
\hline $\left.\begin{array}{r}5 \mathrm{c} . \\
\text { Davoll }\end{array}\right\}$ & 1,00 & 35 & 133 & 133 & \\
\hline $6 \mathrm{~d}$. & 2,75 & 110 & 670 & 1843 & \\
\hline Summe & 26,00 & & & 11391 & \\
\hline \multicolumn{5}{|c|}{ II. Periode. } & \\
\hline $\left.\begin{array}{rr} & 2 b . \\
\text { Der } & 9 e j t\end{array}\right\}$ & 11,00 & 70 & 433 & 4763 & \\
\hline $3 a$. & 4,75 & 70 & 372 & 1767 & \\
\hline $\left.\begin{array}{r}3 \mathrm{~b} . \\
\text { Dawont }\end{array}\right\}$ & 5,00 & 45 & 200 & 1000 & \\
\hline $4 \mathrm{e}$. & 1,00 & 60 & 354 & 354 & \\
\hline $5 \mathrm{~b}$. & 1,80 & 70 & 433 & 779 & \\
\hline $\left.\begin{array}{r}\text { c. } \\
\text { Der } \Re \text { iejt }\end{array}\right\}$ & 10,20 & 55 & 271 & 2764 & \\
\hline Summe & 33,75 & & & 11427 & \\
\hline
\end{tabular}




\begin{tabular}{|c|c|c|c|c|c|}
\hline \multirow[t]{2}{*}{ Bezeidjnung. } & \multirow{2}{*}{$\begin{array}{l}\text { fläcfje. } \\
\text { ha. }\end{array}$} & \multirow{2}{*}{ 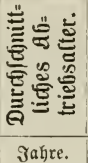 } & \multicolumn{2}{|c|}{$\begin{array}{l}\text { Ertrag } \\
\text { in } \\
\text { feftmetern. }\end{array}$} & \multirow{2}{*}{ 3emerfinngen. } \\
\hline & & & I ha. & Sumut. & \\
\hline
\end{tabular}

III. Periode.

\begin{tabular}{|c|c|c|c|c|c|}
\hline $1 \mathrm{a}$. & 2,50 & 55 & 314 & 785 & \\
\hline c. & 10,50 & 55 & 271 & 2846 & \\
\hline d. & 1,25 & 40 & 200 & 250 & 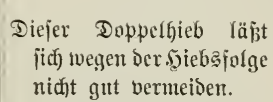 \\
\hline e. & 1,75 & 70 & 433 & 758 & \\
\hline 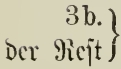 & 6,75 & 65 & 340 & 2295 & \\
\hline $5 \mathrm{~d}$ & 1,50 & 55 & 314 & 471 & \\
\hline 6 a. & 1,75 & 90 & 477 & 835 & \\
\hline b. & 6,00 & 55 & 314 & 1884 & \\
\hline c. & 4,75 & 50 & 275 & 1306 & \\
\hline Eımume & 36,75 & & & 11430 & \\
\hline
\end{tabular}

Eummarijache 8ujaumenjtellung:

Scicbsfläctye der I. Periode 26,00 ha mit $11391 \mathrm{fm}$ Ertrag.

" " II. " III. " 33,75 " " 36,75 " 11427 " 11430 " "

Eumme mährent des ganzen 3eitraunes

$96,50 \mathrm{ha}$ mit $34248 \mathrm{fm}$ Ertrag.

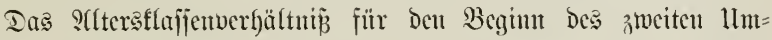
tricbes müroc biernady jein:

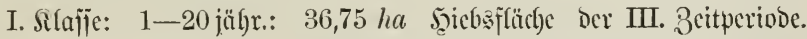
II. " $21-40$ " " 33,75 " II. " III. " $41-60$ " 24,75 " v. D. " " I. " ïber $60 " 7,25$ "2c, 4 bd, 5 a.

\section{Eumme 102,50 ha.}

Bei Der Bertheifung nacf) dom Majjenfact)werfe beträgt bic jumma=

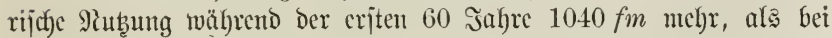

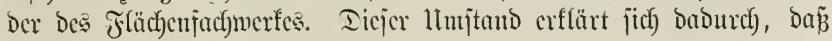


Bejtandstfycile ans der I. Periobe in dic II. und aus bicjer in bic III. verjefoben mutrocu, weshalb am vorbanocnen Borrathe melyr $31 t=$ wachs erjolgte. 3weitens ift aber autch in Folge bicjes 9)ielgr=

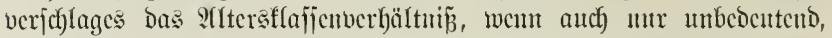

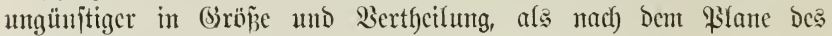
Fläch)enfact)mertes.

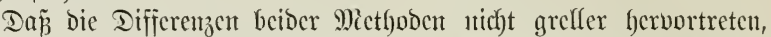

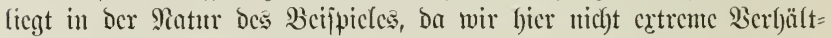
mîje wälylten.

2. Redfnugabeifpict.

Der Deutlichfeit wegen jei noch ein Bahlenteifpict gegeben, bei

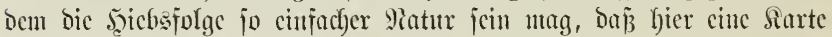
entbefrlich ijt:

Eiu für ben 80 jälfrigen llmtricb bejtinunter $23 a l b$ bon 96 ha

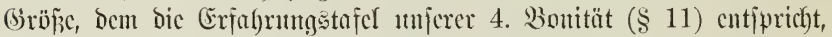

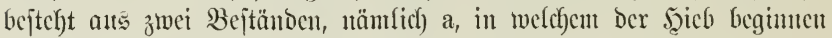
fann, 56 ha 60 jäl)rig und b 40 ha 40 jäl)rig.

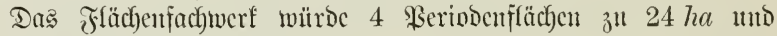
folgenoc Ertragsejulttate geben:

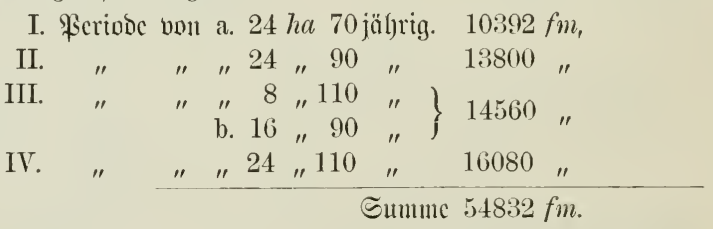

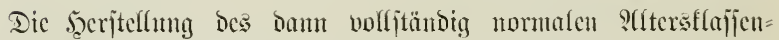

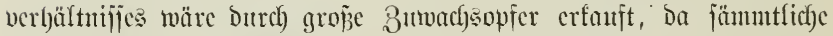

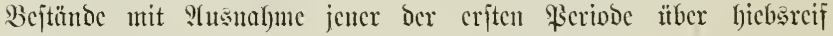
merden müfjen.

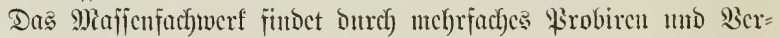

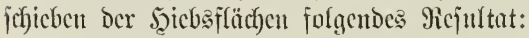

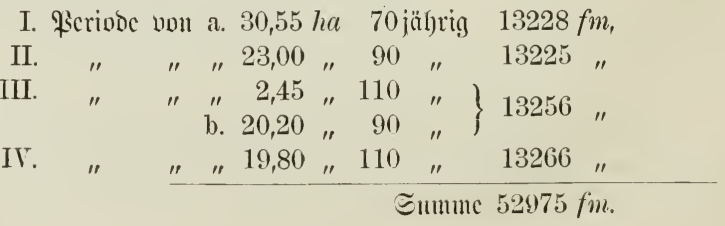




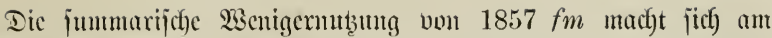

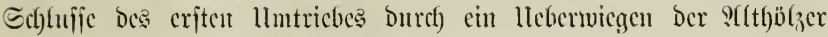
geltent. Es jind baun nämlich) vorl)antoen:

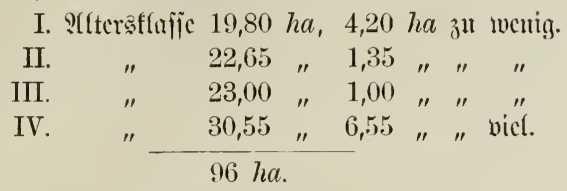

Beide Mietfyoden juäbigen in joldyem Falle bas Snterefic bes

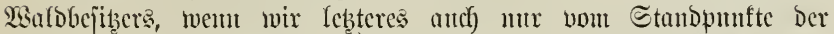

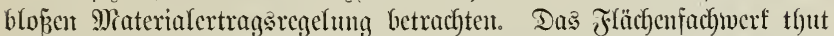

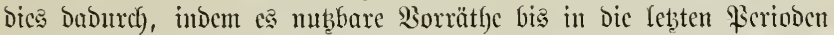

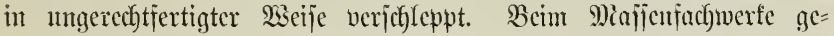
fechicht bies eincs Theiles meniger, indem es bie crjte Rerivde ctwas reichlicher bedenft, anderen Theiles aber $m$ fo mehr, da cs pogar Dem

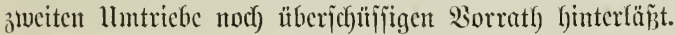

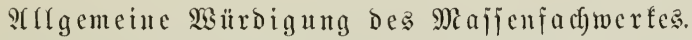

Der B̉orzug Dicjer Metflode gegenüber Dem flächenfactberfe be= jteht grumbjäb̧lich Darin, Daj es etwas mefr Den ?(uforbcrungen bes Eunzelbejtandes Riüfficht tragen famm. Dies that z. B. Şartig, namentlicf) in bem zuterịt (1795) bon ifyn gejujilderten 2ierfabren be=

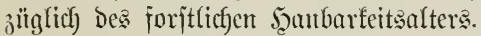

Unbegründete Ertragsopfer fordern Geibe Fachwerfe non ber Wirtl)=

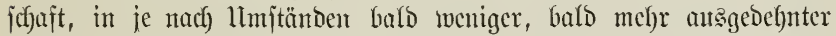

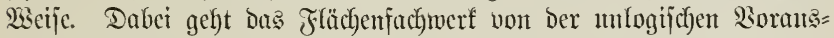

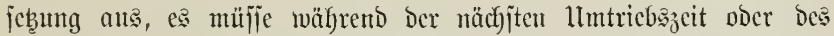
nächjten Einrichtungşzeitraumes ber Normalzujtumb des Waldes mög= lichjt hergeitellt werben, währeno jich bas Majienfact)werf anf cine anbere irrige Bajis jtïbt, nüm(ich) anf bie Bsfeichmäßjigfeit ber peri=

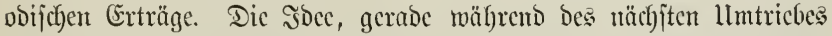

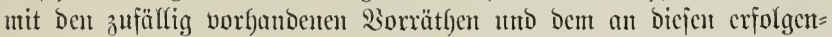

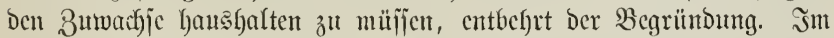
(Bcijte Şartigs und Der Bertreter Des Majjenfactucrfes fpielt bic Sorge für bie fortbauernoc Befricbigung Des Bebarfes ber Eonjumenten

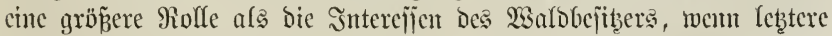

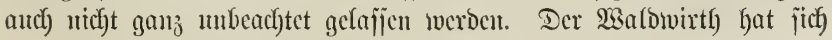




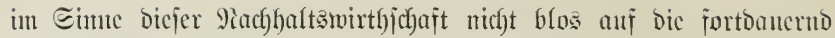
gleichmäp̧ige Bejricdigung Des bisherigen Bedarfes. Der Conjuntenten zu bejchränfen, nicht blos auf dic grumbjäbliche llnantajtbarfeit ocr=

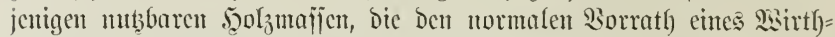
jefaftsförpers bilden, jontoern cr muz jelbjt da, wo bic Sicrbejicrung

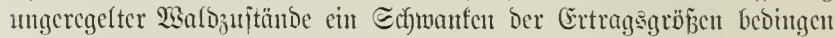

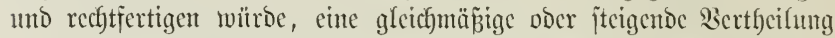

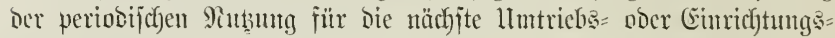

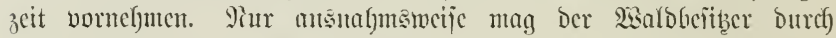

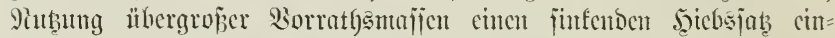
halten.*)

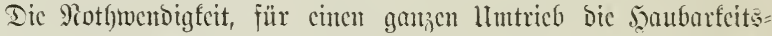

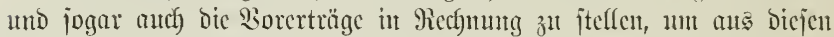

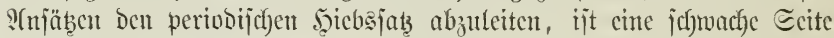

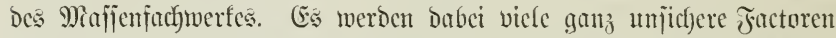

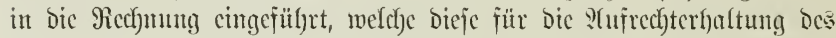

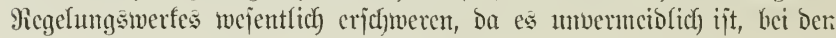
Revijionen immer wieder neue 3 citundsucriffiebungen norzunchmen, un erfolgte હtörungen aนšzugleichen.

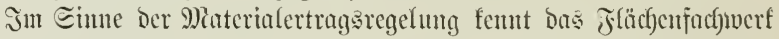

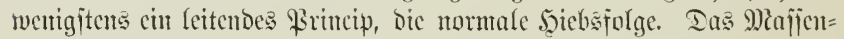

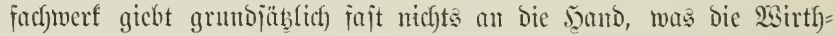

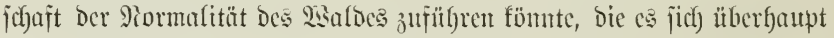
nicht flar macht. Ilnter ber Soransjeb̧ung ber richtigen Bildung von

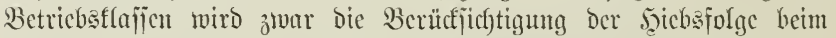

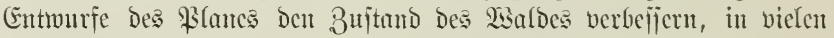

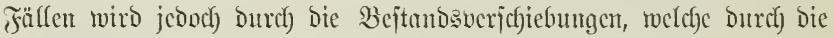

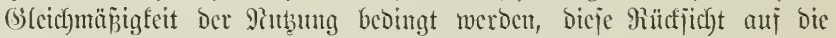

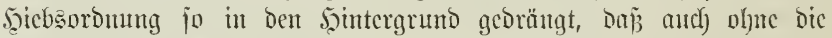

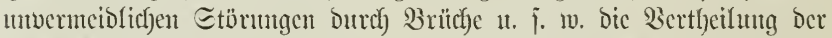

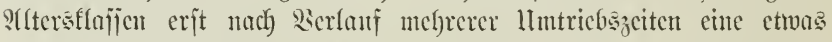

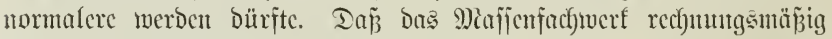

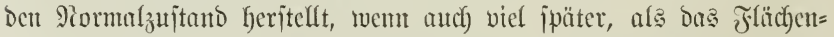

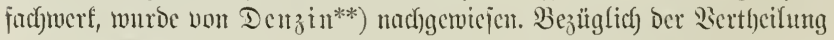

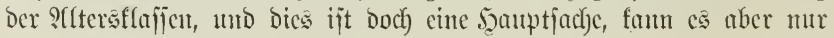

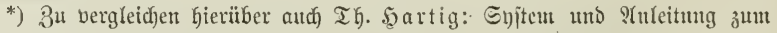

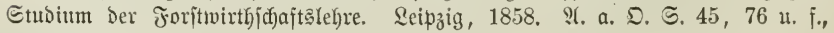
厄. 311 .

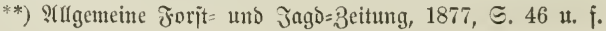




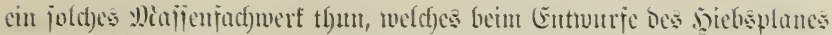

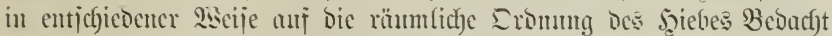
nimmt.

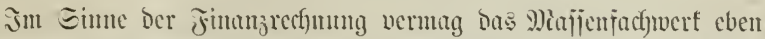

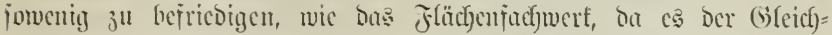

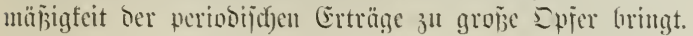

\section{$\$ 123$.}

\section{Das vombinite Fadţmerk.}

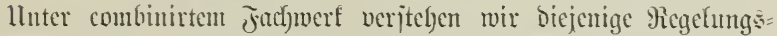

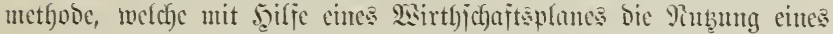

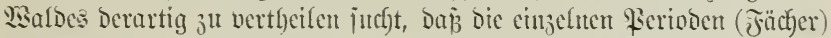

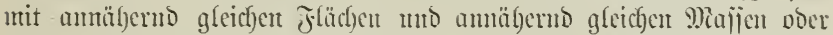
theils mit annäherno gleit)en Miajjen, theils mit annäbermo gleidfen Ffäc)en ausigejtattet meroen.

Sic Bertfeilung jelbjt erfolgt:

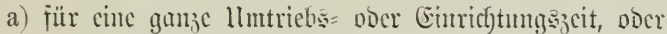

b) für cincu fïržeren 3citrum.

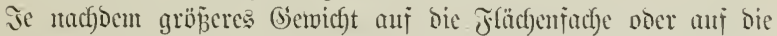

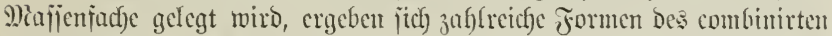

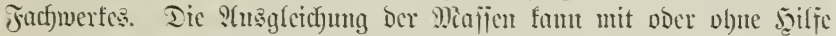

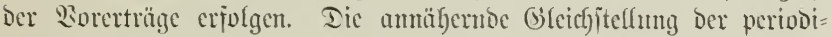

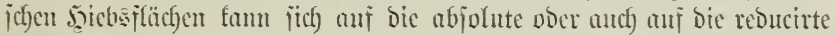
Jläche bejichen.

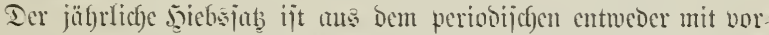

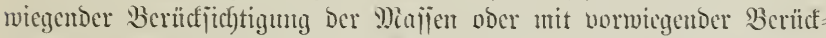

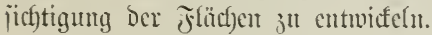

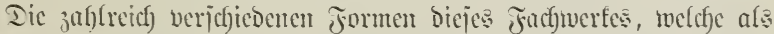

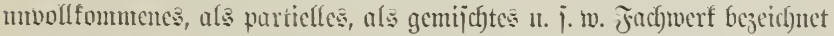

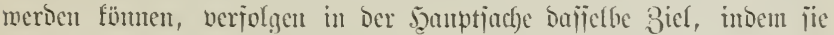

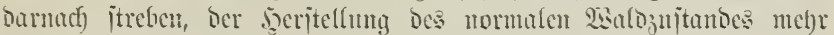

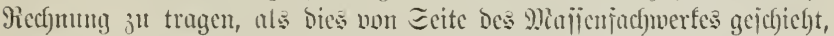

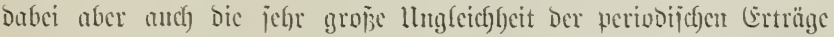

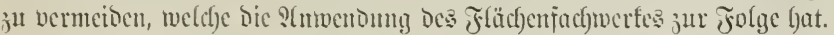

Iic llmmöglichteit, Das iocale Biel Des combinirten Jad)merfes ju

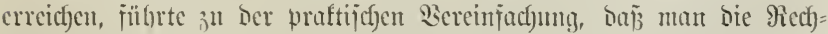
mutg nicht mefre jïr fämuntliche Ficrioden des llutriebes ober bes

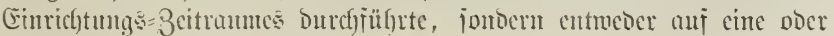


auf bie beiden erjten Beitperioben bejchränfte, Den fpäteren aber Durch) den allgemeinen Şiebsplan annäherno gleiche Flächen zunies.*) Şierin lag cin entichiedener Fortichritt, Denn man gab bas Ctreben nach ftreugiter Rachbaltigfeit baburd auf und hegnïgte firf bamit, plan= mäßjig Der ferneren Bufunft eine genügento ?(nzah) von Bejtënden 子um Sciebe zu übergeben.

Das Berfahren jelbft bedarf nach Den für bas glächen= und Dajien=

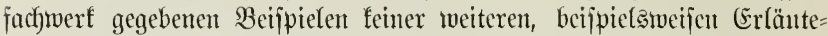

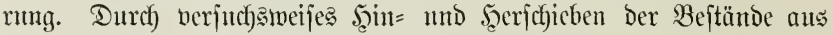
einer $\mathfrak{P e r i o b e}$ in bie andere ftrebte man nach möglicbjter (Erreichung des obengenannten 3ieles. Se nachdem man hierbei ein größz̈eres (be=

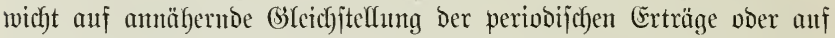
bie ber periodijchen Sciebsflächen legt, je nachocm man bie Dromung

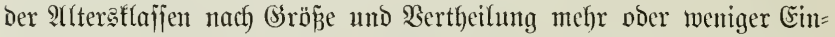

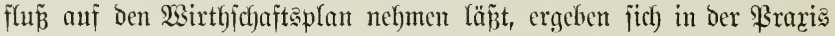
die veridfiedenften Modificationen bes Berjahrens. -

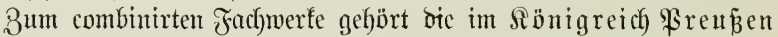
übliche Methode ber Forjteinricftung und Ertraggregefung.**) (Sejtüb̨t

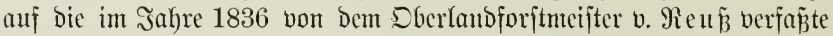
"श्Amweifung zur Erbaltung, Berichtigung und Ergänzıng Der Forjt=

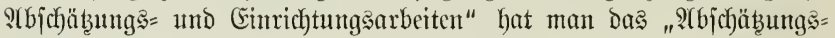
Berfahren" ipäter mehr und mehr vereinfacht, je melyr bie Erfahrung

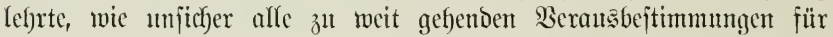

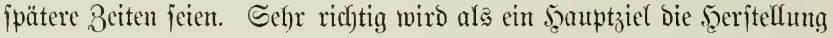

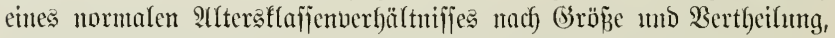

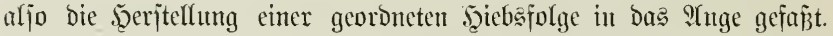
Dabei jucht man grundfäbzlich jene Spfer möglichjt zu vermeiden, welche

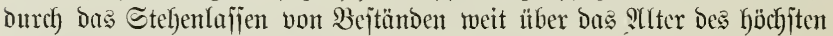

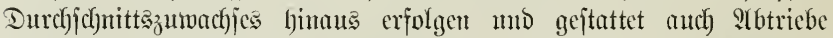
jüngerer Drte, wenn bie ?(brweichungen won bem für Den Beitanto an

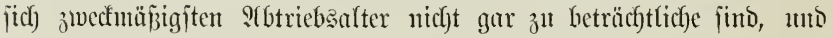

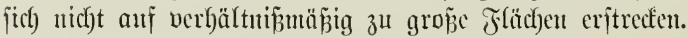

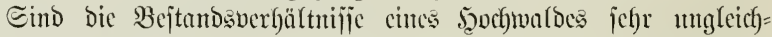

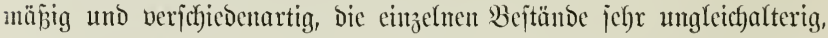

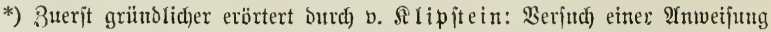
zur Forjtbctriebäregulirung. Bjiēenen, 1823.

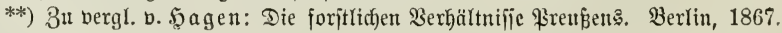
5. 131 u. f. - 2. Pufl., herauggegeben von Donner, Berlin, 1883. 1. Bano, ธ. $16: 2$ ก. $\mathrm{f}$ 


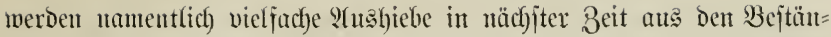
ben ber jpäteren Fieriode nïthig, jo wirb bic Ertragsberechnumg, weldye jich) immer mur auf bas Derbholz bejchränft, für mef)rere ober alfe Perioben Der Bered)nungszeit (bes (Einrich)tungszeitraumes) Durchgefül)rt. Etelfen jüch Darnach) Dic (Erträge ber cinzelnen \$erioden fechr ungleidf),

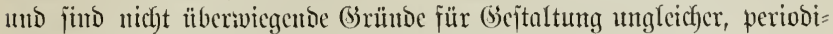

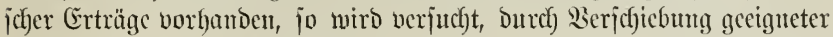
Bejtände aus eincr S(btriebsperiobe in bic andere bic llugleich geit zu

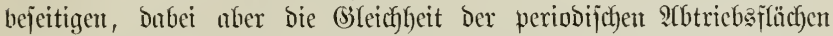

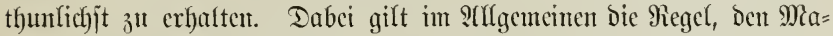

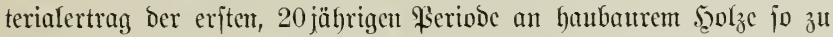

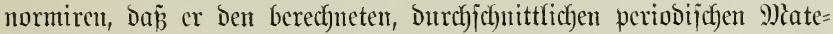
rialertrag Der IIntriebs= oder Berect)mungşzcit ammäl)ernd erreicht, mährend üher bie Ungleich)citen Des Materialertrages ber ipäteren

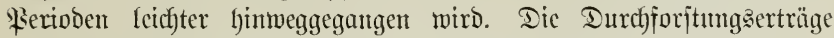
werben nur für bie 1. Peridde in :

Sinto die Bejtundsverbältnifje regelmäp̃iger, jo bejchränft jich bie Ertragsberechuntg mur auj bie erjte \$eriobe, und werben zum शach)=

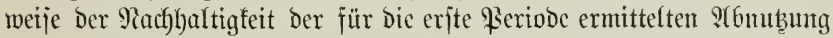
mur die Den einzelnen Perioden Der Berechnungszeit zum Stbtricbe über= wiejeren Bejtandsflächen nach ifyer burch Die Bobenqualität bebingten Ertragsfähigfeit auf eine, Der (Ertragsfähigfeit Der bejten oder auch Der im Reviere überwiegend vortommenden $\mathfrak{B}$ bdentlajie entjprechente Fläche reducirt. Ergiebt bie Summirung biejer reducirten Flächent für bie ein=

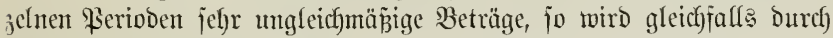

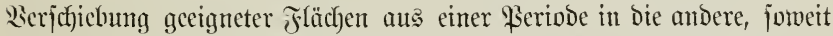

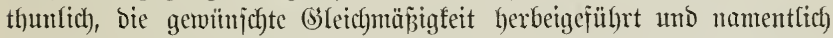

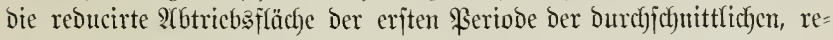

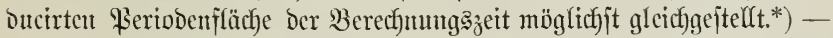

Bum combinirten Fachwerfe ijt jene Methode zu rectunen, weldec ber herzogl. anbaltijche Foritimpector $\mathfrak{F}$ it $j$ chel eingerübrt mo ver=

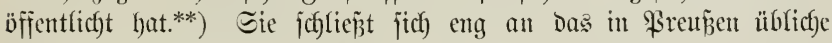
Berjahren an, indom fie bajiclbe noch weiter bercimfacht.

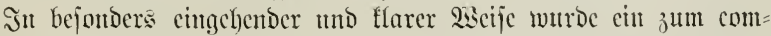

*) v. Şagen, 1. c. S. 142 und 143, - 2. Aquffage von Donner, 1. Band, ธ. 170 .

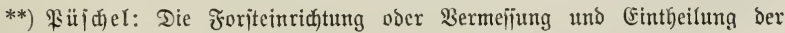

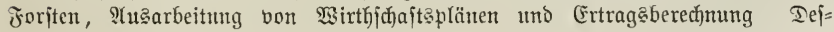
jau, 1869. 。 
binirten Fadymerf geböriges Berfabren nenerer 3cit non (3) rebe ge= j(f)ildert. *)

\section{PUlgemcinc 2 ürdigung Der Methode.}

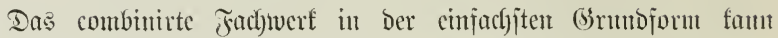
jeinen ibealen Buect jerbjtuerjtänolid) nur Durel) günjtiges Bujammen=

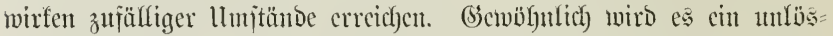

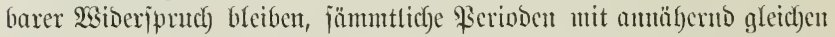

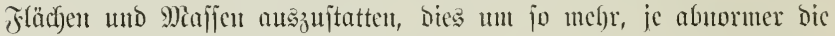

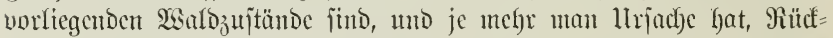

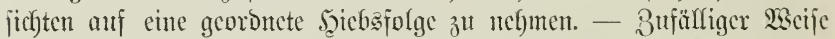

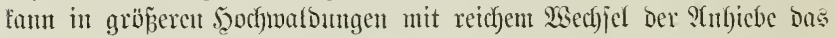

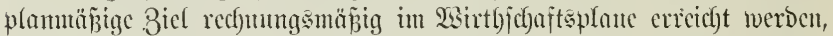
Den thatjürdflich erfolgenden Etörungen bleibt bicie M)ethode ebenjo ausgejebzt, wie jedc andere. Sleinere, irgend abnorm bejtorfte gsälder

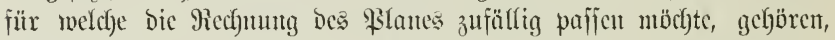
jebenfalls zu ben gropien Eeltenbeiten, weshalb mix auth auf eine weitere, beijpielsmcije Erlüuterung verzichten. In Den Eeite 324 und Seite 334 gegebencn Fällen ijt bie itrengite Durd)fül)rung Des $n=$ iprünglichen Principes des combuinten Fachuertes nicht möglich. Der

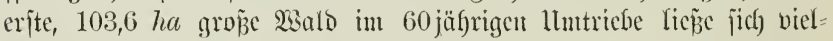

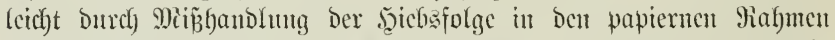

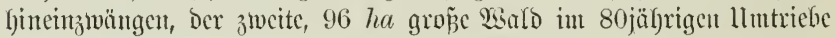
Dürfte ivol) jeder Derartigen Bemilfung frotten.

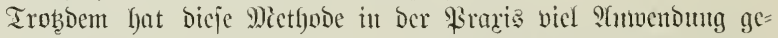

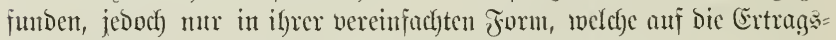
berechnung für bic ipäteren Perioben $\mathfrak{B}$ erzicht leijtet. Siur Darf mat

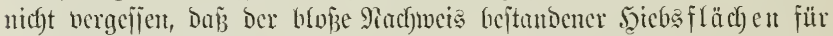

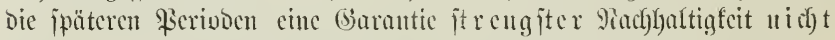
bictet. Sicht man aber won lebgterer ah, was woir bollitändig billigen,

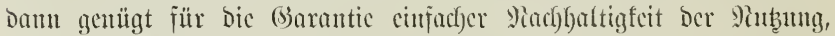

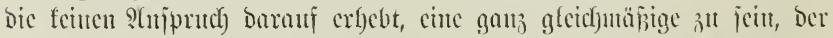

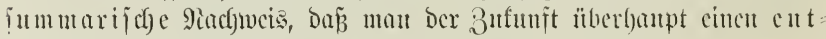

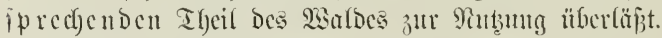

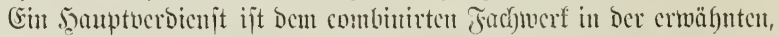

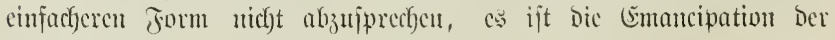

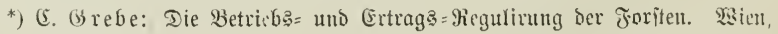
1866. - 2. शแflage, Wien, 1879. 


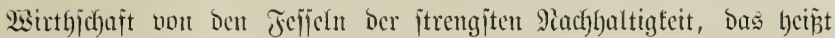

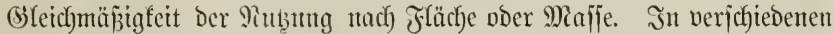
Miodificationen hat mohl numentlich Deshalb bie Praxis mit richtigem

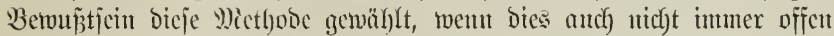
jugejtanton wirb. Ferner lag in Diejem Serfafjen entes numtebr

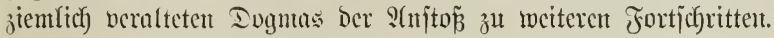

\section{$\S 124$.}

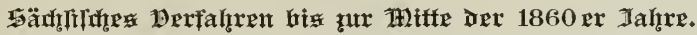

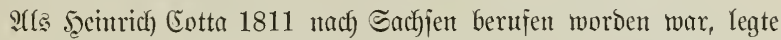

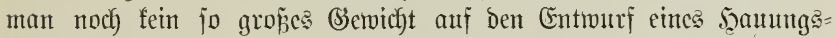
planes, wie ipäter, jondorn betract)tete bie Ertragsermittelung als bie

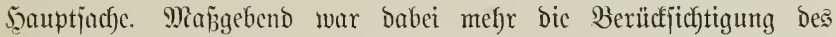

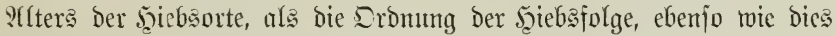
bei J̧artig anfünglich ber Fall war. Man brachte einfach Das ältejte

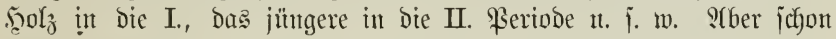

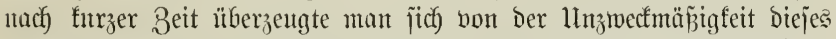

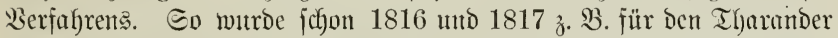
Wald eine llmarbeitung Der ?(bjc)äb̨utg von 1811 antsgefüh)rt, indem man eine periobijche, fejt begrenţ Flächeneintheilung entwarf. Es war bies bas crite Mial, daj in Eachjen derartig verfalyren murbe. Das urjprünglich) angewendete Mafjenfachwerf wurde burch bas Flächen= facf)werf ergänzt. Dicje Ergänzung litt noch as Dem gropenen Febler,

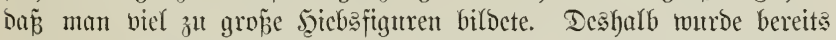
1827 Der Tharamber Wald anjtatt einer 10 jäfrigen Revijion einer

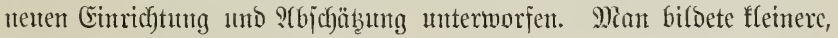

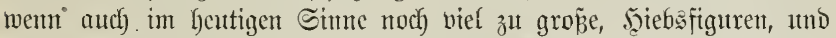
für bie verjdicbenen Betriebsarten Betricbstafjen. Co murbe Rabel=

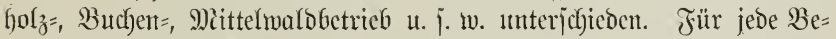
triebsflajie*) wnroe zunächjt eine $\mathfrak{B}$ eriobentheilung entworfen, weldye

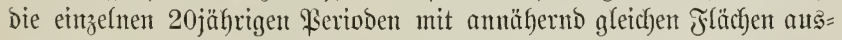
itattete, joweit bies möglich war, indem jede ber einzelsten im Walde gebildeten und fejt begrenzten ?tbtheilungett im Simte ber b̧iebs= vronung einer beftumten Beitperiobe zugetheift wurde, wie es bas Jlächenfact)merf verlantgt. Dagegen murde fütr Den 100 jäf)rigen Ein=

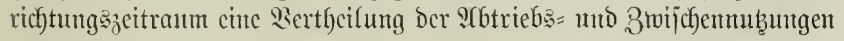

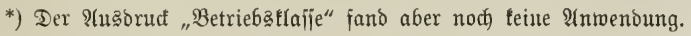


in Summe jämmtlicher Betriebstlajien im Sinne eines Majfenfact)= werfes vorgenommen, die crite Periode babei in zoei Jahrzebnte ge= theilt. Bei biejer Bertheilung wurbe allerbings nach Maßjgabe ber vorliegenden $\mathfrak{B e r b a ̈ l t n i j j e ~ w e g e n ~ l l e b e r j a d u j e s ~ a n ~ a ̈ l t e r e n ~ u n d ~ j u ̈ n g e r e n ~}$ abtriebsbediurfigen Bejtänden bie erjte łeriode mit wejentlich größ̄erer Sziebsfläct)e und Maaje ansgejtattet, als bie librigen vier \$erioben. SUf Deu Bejtandsfarten wurben Die einzelnen S(btheilungen mit ihren entiprect)enden \$eriobenziffern bezeichnet.

2isin funden aljo hier cine Serbindung bes Fläd)enfachnerfes mit Dem Mafjenfacfmerf, eine Sat combinirtes Fadjwerf. Der Brumbjab Eottas, Daj bie gute Einrichtutíg Des Waldes widftiger jei, als bie Ertragsbejtimmung, trat 1828 nod) jechärfer hervor als 1816.

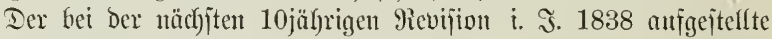
2Girtffid)aftsplan Des Tharamber Revieres nummt nod) Beznt anf bie 1828 Den einzelnen Perioden bis zum Jaf)re 1927 zugewiejenen Majjen, während in bem das Jahrzef)nt 1848/57 betreffenden Pilane nichts mehr bavon zau lejen ijt.

Mechr und mefre gemann nämlich bei ben itets regelmäp̈ig abge=

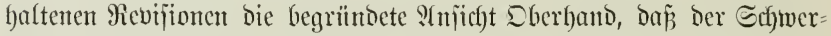
punft Der ganzen Ertragsregeluntg nicht in bem beim ?fufange Der

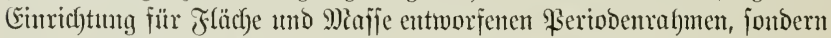
in ben Revijionen jelbit zu jucfen jei. Sebtere nafmen baburd einen amberen Egarafter an, als ifnen bie älteren Fachiwerfismethoden ge= geben hatten, fie murben zu periobijchen Fortjeb̧ungen bes (Ëmricht= ungswerfes, namentlich Der Ertragsregelung jelbjt. Die uriprüngliche

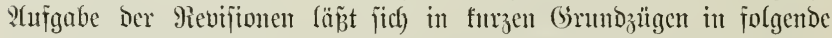

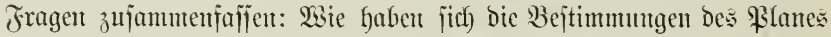

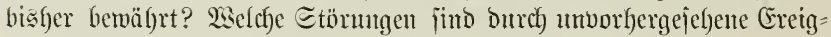
niffe cingetreten? Wie lajjen jich bie Folgen biejer Etörungen ober jonjt etwa nöthige Beränderungen mit Dem bereits gegebenen, fer tigen Wsirthjchaftsplane nereinigent? - Den Rievijionen ber ipäteren, neueren Zeit Glieben von Den genumten Drei Fragen bie beiden erjten ebenfalls zur Beantivortung übrig, bic lebete jedoch, welche für bie আ= iprünglicken Revifionen bie şautjacte war, entfiel bagegen bis auf

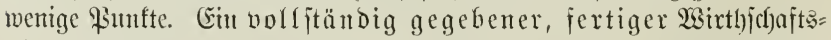
plan licgt nicht vor, lebeterer rebucirt fich vielmel)r mur auj bie burd)

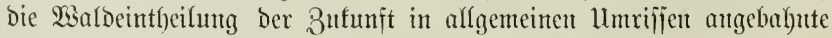
Eromung ber Şiebsfolge. Eine Berichtigung Des frütheren \$llanes im Sinne der älteren Borjoriften fanm aljo nidft erfolgen. Dagegen 
jragt die Revifion jeden eitzelnen Bejtand, forwohl vom Sejefchtspunte

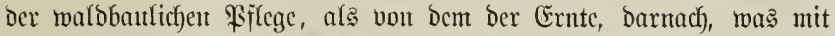

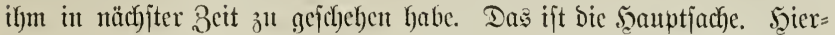

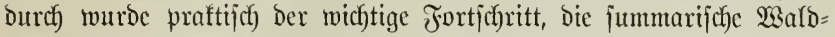
mirtbjchaft in bie feinere Beftandsmirtljichaft umzumandeln, bereits vor langer Beit augebalynt, che bie Theorie jich biejen (Srundfab voll= jtünoig flar machte.

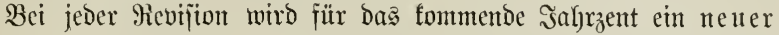
\$lan entworfen, für ben nur ctmas ?llgemeines, bie mit ber gegcbenen

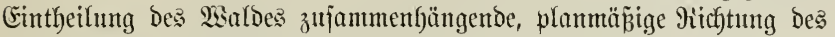

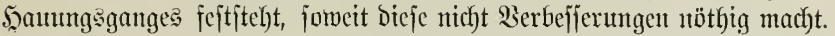

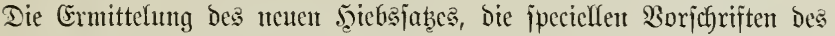
teuen Flanes für bas fommende Jahrzelynt benuben bie burch ver=

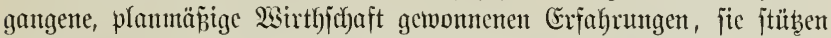
fich aber nicft unbebingt auf bie frülger gegebenen Borjchriften, Deren

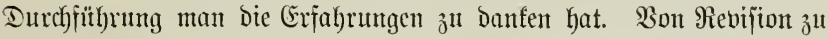
Fevifion geminnt Deshalf das ganze Werf ber Eimrichtung und Er:

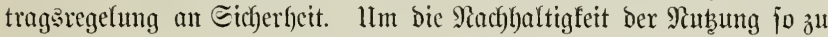

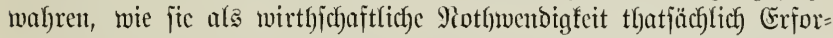

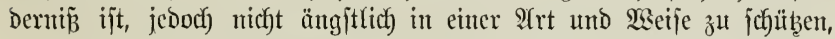

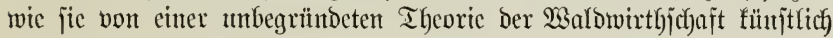

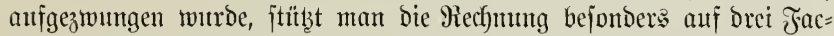

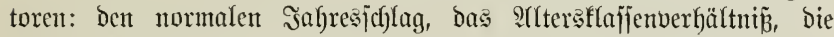

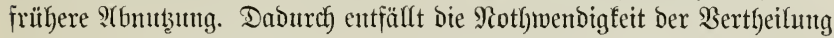
ber einzclnen Bejtünde auf jämmtliche Perioden Der Bufunft.

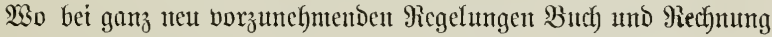

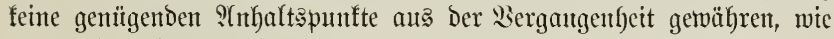
es woh) vorfommen fann, bleibt jreilid) nichts anderes übrig, als bent

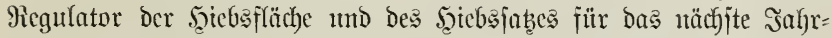
3chnt burch cine etwas weiter gefonde Betrachtung ber 3ufunft zu ge= wiunen. F̧ajt imuter genügt zu biejem Bweef ein Şaunngaplan für 3 bis hödfjtens 4 Jabrzel)nte; von ber Tabellcuppielerei, vier bis ject)

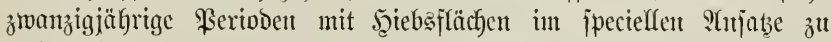

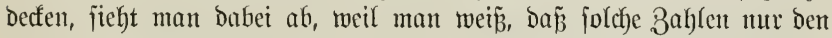
forjtlidben Qaien blenden.

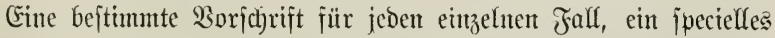

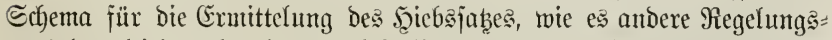

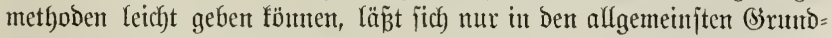
zügen entwerfen, da in jedem vorliegenden Falle nach Maß̃gabe ver= 


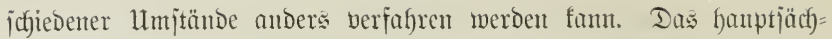
lichjte Etreben Der Eimrichtung bleibt auf Serjtelfung Der annähento

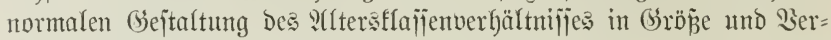
theilung gerichtet. Der 2 sege, bie zum Biele fübrent, giebt es viele, IntD ijt ę Dem cinzelnen Falle vorbehalten, jelbjt für Den eimzujd)lagen=

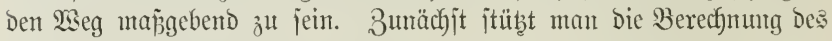

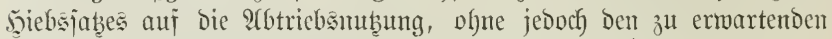
Ertrag Der Bornuţungen unbedingt einflup̃los auf Bcjtimmung Der Giröß̄e erjterer bleiben zu lafien.

Schon jeit fanger Beit wurbe mun barauf Bedacht genommen, Dem

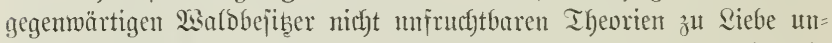
gerechtjertigte Epfer aujabürden, Deshalb aber immer mefr und mehx

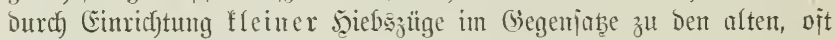
viel zu langen Reriobentonren, nach ciner größ̈eren Bemeglichfeit Des şiebes gejtrebt.

₹ui Dieje Beije entwicfelte jitch in jteten praftijchen Fortict)ritte allmälig ein Cinricftungšmejen, meldes mir nod) geringer Modiji= cationen bedurite, um jener jreien Methode Der Bejtandswirtfjichait Epielraum zu gemähren, weldfe wir nad) Beipred)ıng Der 9ormal= vorratbs= Methoden ipecieller jefildern wollen, weshalb von eingehenden Ietails hier abgejehen werben fann.

3ur Errläuterung jeien mur bie in Den ş 121 mo 122 gegebenten Beijpiele bier jo behandelt, wie man jie nuch diejer jächjïichen Netfode

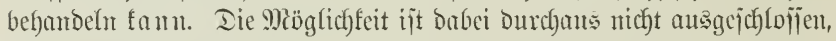

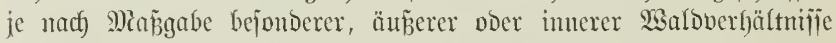
anders zu verfahren, ohne gegen bie Grumbjätze der Miethode zu ver=

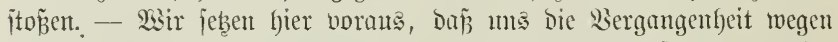

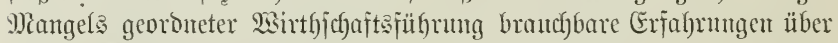

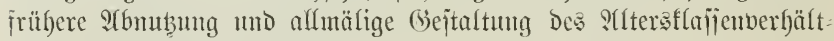
nijies nicht ïberficfert habe.

\section{Rect)nungabeipię.}

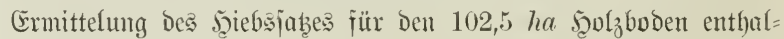

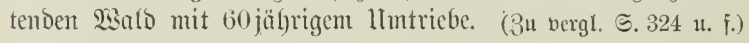

Die $\mathfrak{B a l b c i n t b e i l u n g ~ i f t ~ j o ~ a n s z u f u ̈ h r e n , ~ w i e ~ w i r ~ j i e ~ b e i ~ B c i p r e c t ) = ~}$ ung bes Jlächenfachmerfes gegeben, ber 2isato jelbjit zerfällt hiernach)

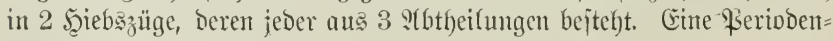
theifung wird nicht vorgenommen. 


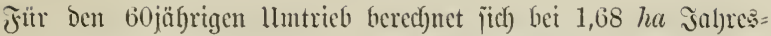
jeflag als normaler Blößje cinter jeden SIltersflajie cine Fläche von 33,60 bis 33,61 ha. Die Bergleidyung des ivirflichen Silajienverbält= uififes mit bem ntormalen ergiebt folgentes giejultat:

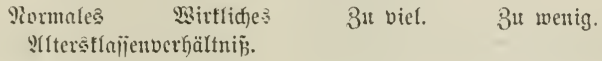

\begin{tabular}{|c|c|c|c|c|}
\hline 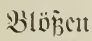 & 1,68 & 5,95 & 4,27 & - \\
\hline I. $\Re$ II. & 33,60 & 40,05 & 6,45 & - \\
\hline II. " & 33,61 & 36,25 & 2,64 & - \\
\hline III. " & 33,61 & 7,50 & & \\
\hline IV. " & - & 5,00 & - & 13,36 \\
\hline T. " & - & 7,75 & & \\
\hline
\end{tabular}

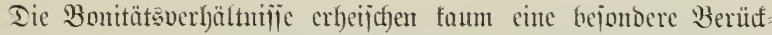

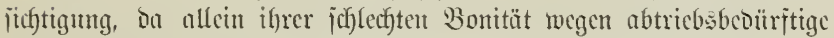

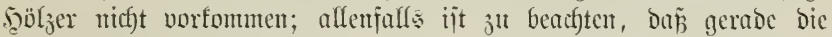

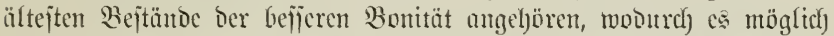
miro, ben Manget an Fläd)e berictben burch) beren gräßjere Erträge jit erjeţeut.

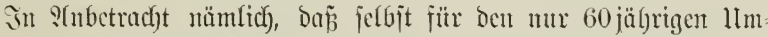

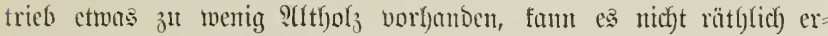

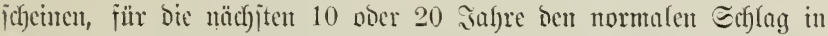
Natjab̧ jut bringen. (E⿱

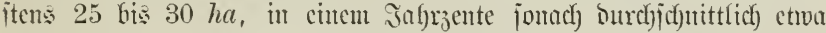

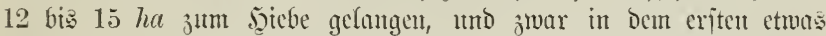
mentiger, als in bem jubeiten, weil cs mit Den ältejten, ertrugesreichjten Bejtünden autsgejtattet ijt.

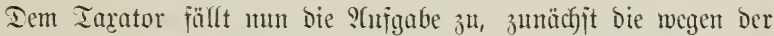

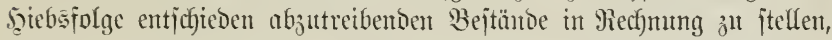

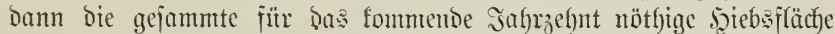

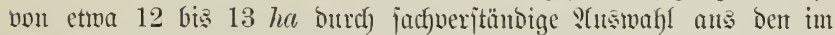

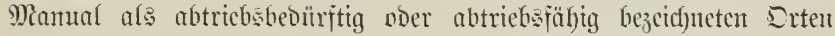

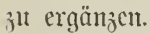

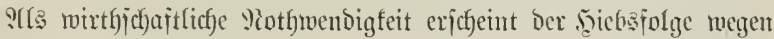

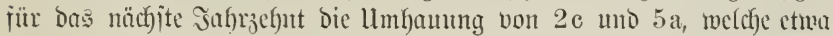
0,75 ha von $2 \mathrm{~b}$ und 1 ha von $5 \mathrm{e}$ beanjprutd)t. Wion ben befieren

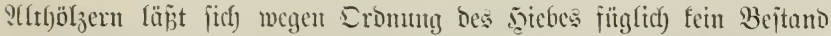

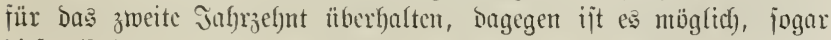

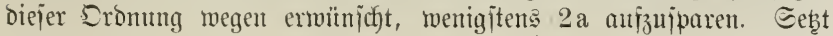




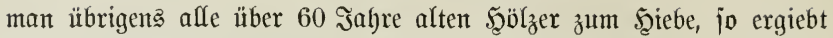

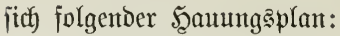

\begin{tabular}{|c|c|c|c|c|c|}
\hline \multirow[t]{2}{*}{ ßезегісбnung. } & \multirow{2}{*}{$\begin{array}{c}\text { ffäcf)e. } \\
\text { ha. }\end{array}$} & \multirow{2}{*}{ 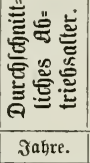 } & \multicolumn{2}{|c|}{$\begin{array}{c}\text { Ertray } \\
\text { ili } \\
\text { feftmetern. }\end{array}$} & \multirow[t]{2}{*}{ Bemerkungeu. } \\
\hline & & & I ha. & Summe. & \\
\hline $1 \mathrm{~b}$. & 2,50 & $\left.80^{*}\right)$ & 430 & 1075 & \\
\hline d. & 1,25 & 95 & 604 & 755 & \\
\hline $\left.\begin{array}{r}2 \mathrm{~b} . \\
\text { dabon }\end{array}\right\}$ & 0,75 & 45 & 237 & 178 & 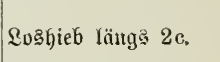 \\
\hline $3 c$. & 0,75 & 105 & 653 & 490 & \\
\hline $4 a$. & 3,00 & 95 & 604 & 1812 & \\
\hline $\left.\begin{array}{r}5 c . \\
\text { Davon }\end{array}\right\}$ & 1,00 & 30 & 102 & 102 & 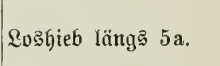 \\
\hline $6 \mathrm{~d}$. & 2,75 & 105 & 653 & 1796 & \\
\hline 巨ummte & 12,00 & & & 6208 & \\
\hline
\end{tabular}

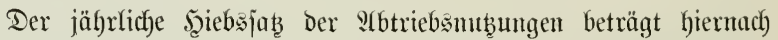
$620,8 \mathrm{fm}$. Demielben jüto nun nicht nach jpecieller Schäbung, jon= Dern nach) jummarifcker \$eranjeflagung bic zu erwartenden 3wijchen=

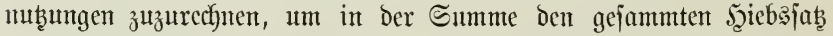
zu erfalten. Die zur Durdfforitung vorliegenden Bejtände werben einzeln mit Fläche im \&lane verzcichntet, jene, aus venen Räımungen von Walbrect)tern oder Dergleichen z"l erfolgen baben, nur genannt,

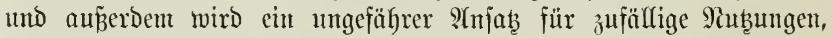
子. $\mathfrak{B}$. Wind, Schneebruch)örzer 2 . gegeben. .

Will man bei Dem Mangel an llnterlagen aus ber $\mathfrak{B}$ ergangenteit vorfitchtig zu Werfe gel)en, fo wäre noch für bas zmeite Jahrzelhnt cin

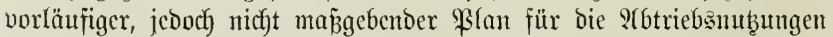

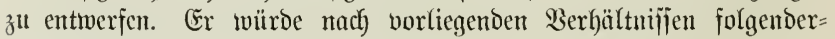
mafien lauten:

*) Wir haben hier in Conjequenz der Thcorie einen durchjdntittlidjen $8 \mathfrak{t =}$

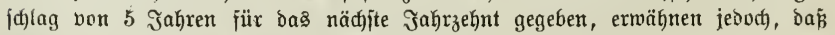
man fid) in ber \$raxis bierauf nicht einzulafien pflegt. 


\begin{tabular}{|c|c|c|c|c|c|}
\hline \multirow[t]{2}{*}{ Bejeitfnumg. } & \multirow{2}{*}{$\begin{array}{c}\text { fläcfie. } \\
\text { ha. }\end{array}$} & \multirow{2}{*}{ 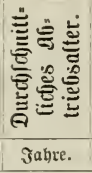 } & \multicolumn{2}{|c|}{$\begin{array}{l}\text { Ertrag } \\
\text { in } \\
\text { feftmetern. }\end{array}$} & \multirow[t]{2}{*}{ Bomerkungen. } \\
\hline & & & I ha. & Eumme. & \\
\hline $2 a$. & 2,50 & 90 & 477 & 1193 & \\
\hline $\begin{array}{r}2 \mathrm{~b} . \\
\text { Davon }\end{array}$ & 5,00 & 55 & 314 & 1570 & \\
\hline $4 \mathrm{c}$. & 7,50 & 65 & 394 & 2955 & \\
\hline Eumme & 15,00 & & & 5718 & \\
\hline
\end{tabular}

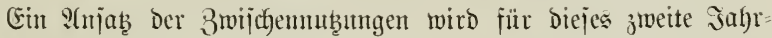
jebnt nicht gegeben.

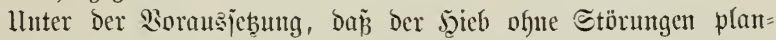
mäp̃ig erfolgen fonnte, würbe am :Henange des britten Jahrzehntes bas Slltersflajjenverbältnin folgendes jein:

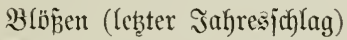

$1,50 \mathrm{ha}$.

I. Slafje 5,95 fultivirte, alte Blö̈̄en,

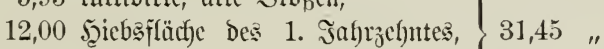
13,50

2.

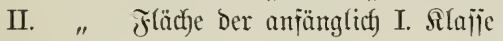

40,05 "

III

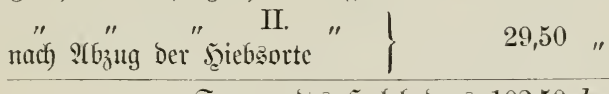

Eumme Des 5ुoljboden 102,50 ha.

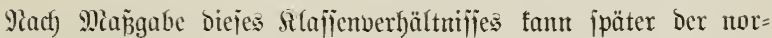

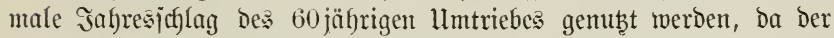
ältcjten Silajfe mur noch 4 ha jehlen. $D$ b aud bieje Rleinigfeit im britten und vierten Jahrzebnt erjpart merden joll ober nicft, barüber fann bie Bufunit enticheiben. Borläurig bedari es eines anberen $B e=$

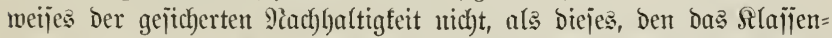

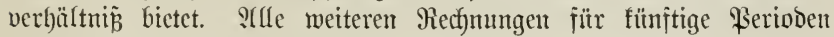

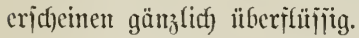

\section{Recfnungabeijpief.}

Ermittelung Des Şicbsjabes für dent 96 ha grok̉en $23 a$ ald mit 80 jährigem Itmtriebe. (ङ. 334 แ. i.) 
Iie Bergleichung Des normalen mit bem mirffichen :Itersflajןen= nerbältnifí crgielot folgentes Rejultat:

\begin{tabular}{|c|c|c|c|}
\hline \multicolumn{4}{|c|}{ Silaj̄enverbältmí̄. } \\
\hline 1,19 & - & - & 1,19 \\
\hline 23,70 & - & - & 23,70 \\
\hline 23,70 & 40,00 & 16,30 & - \\
\hline 23,70 & 56,00 & 32,30 & - \\
\hline 23,71 & - & - & 23,71 \\
\hline
\end{tabular}

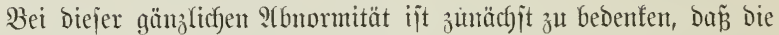
40 ha II., jomie die 56 ha III. Den höchjten Stufen ifrer betreffenden Slaijen angehören, mithin jchon im $\mathfrak{B}$ erlaufe bor nächjten Sahre in bie folgenden Silajien ïfertreten. Will man baher bieje Bejtünde nicht zu alt merden lajjen, jo ijt es nötfig, etwas mehr als bic Fläche des normalen Echlages zum Şiebe zu ftellen. Im Banzen mird es jich) Deşfalb rechtfertigen, mit Den Sauungen in etwa 60 bis 70 Jaf)ren cimmal ben ganzen 2 ald ju burchlaufen, jo baj für ben Jabreğchlag 1,4 bis 1,6 ha entfaflen würden. Ta mun Der şieb wälgrento bcs

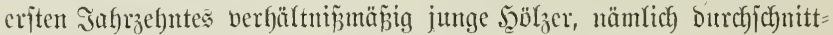

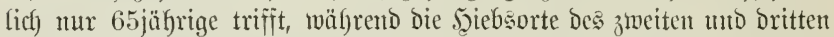
Jahrzebntes amtäbcrnoer int 5̧aubarfeitsalter zur Berjüngung gelangen,

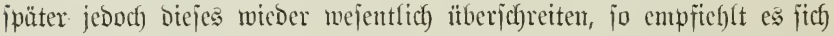

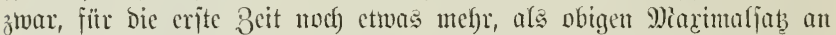

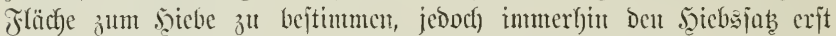
vom ziveiten Jahrzefnte an itcigen zu laffen, um nicht im erjen zu viele der zumadjsteichen Srte abtreiben zu müfijen.

Der vorläufige Şaumngsplan fömute balger lauten:

1. Jaforgel)ut:

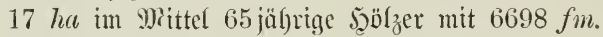

2. Jaf)rzefint:

16 ha im M)ittel 75 jül)rige \$ูölzer mit $7552 \mathrm{fm}$.

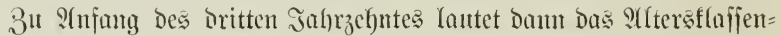
verbältuí̧, wemn feine Etöruntyen cintreten:

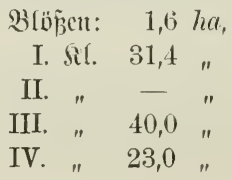




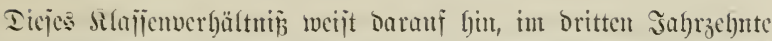

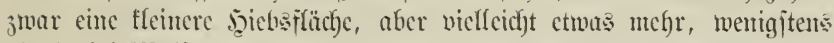

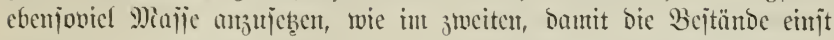
nicht zll alt merben. Erjt vom vierten ober jünften Jalyrzellnte au

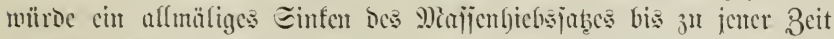

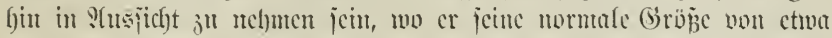

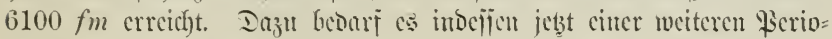

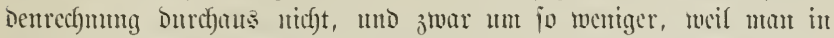

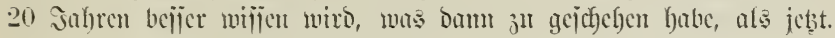

\section{Plfgenteine Wëtroigung ocr Methode.}

Echon Eingangs bes Paragraphen hohen mir feruor, baż eit

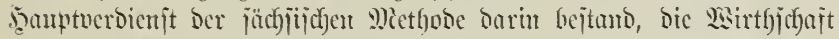

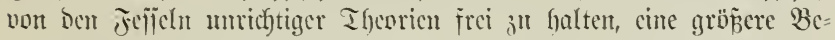

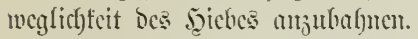

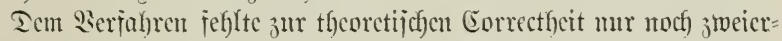

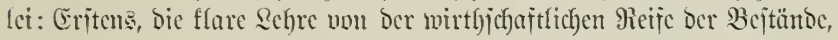

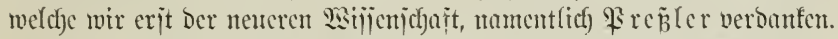

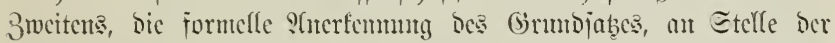

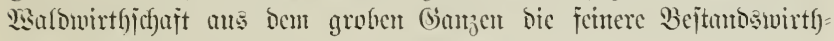

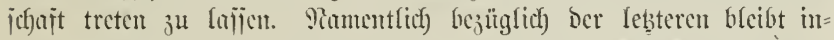

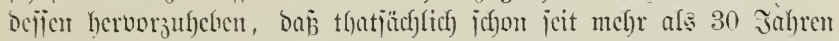

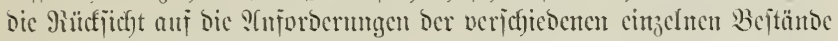
inmer melyr Boben gewam, fo baj wir mit ber forberung einer Bejtandswirthichajt feine nene Methobe begrïmocu, fondern mur bic

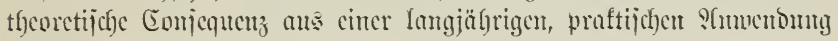
jieljen.

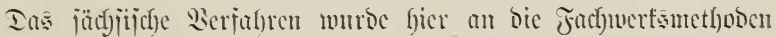

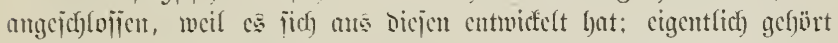
es ibnen aber nou jener 3eit nicht ganj jtreng melyr an, als cs bic

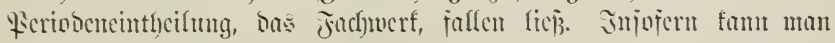

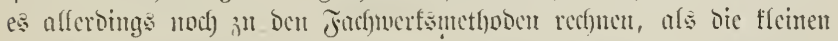

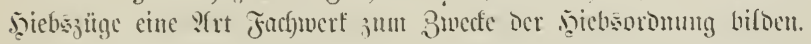

\section{$\S 125$.}

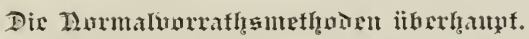

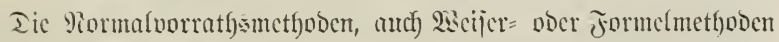

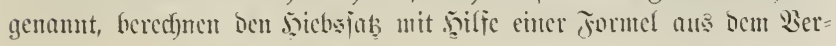


bältnī̄ zmijchen bem wirflichen umo bem normalen Sorratl) uno bem

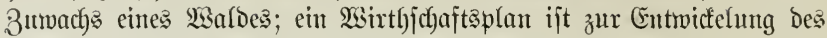
Seiebsjabes nicht Sorausjebzung.

Bon den Fachwerfsmethoden, von ber Schlageintheilung, fowie von

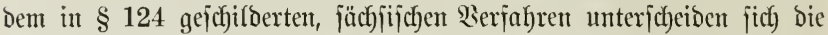

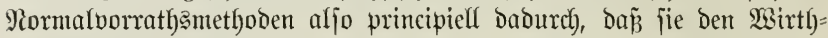
jchaftsplan entweder nicht femten ober mur mobificirento auf ben aus einer Formel entwidfelten Ђูiebsją̧ einwirfen lafjen.

Während Dent reinen Majfenfactywerf in einfactyjter Form feinte (Sirumbbebingung Des normalen 2 aldozujtandes flar wiro, wäfreno bie Echlageintheilung, bas Flächen= und das combinite Fach)werf, jo auch) Das ältere jächjifiche 2ierjahren, mit melgr ober meniger Dpfent

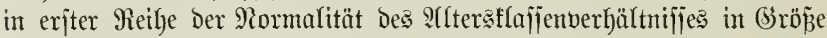
und Bertheilung zujtreben, finden bie Normalvorrathemethoden in ber

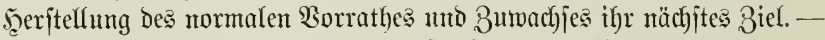

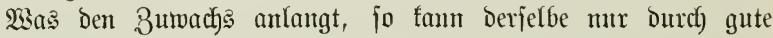
Sultur, Bejtandspflege und Dromung bes Jetebsganges, namentlich autf)

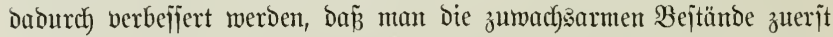

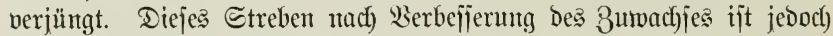
alfen Methoden gemeinjam, wem jie es auth nicht birect ausjprechen, fondern ganz jelbjtuerjtändich finden. Der (E)arafter der Rormal= vorrathsmethoden ijt aljo vorzugsmeije Durch) bie Eimfülfrung des Rormalvorrathes als eines birect wirfenden Rechunumgactors in bie

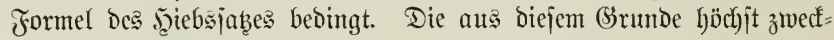

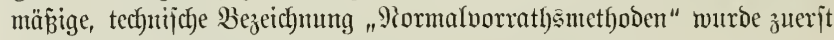
vom Foritmeifter $\Omega \mathfrak{r a f t}$ ) in S(umenoung geloracht.

Int Folgenden follen bie widhtigiten Gruntormen Der Pormal= vorrathsmethoden bejprochen mernen.

\section{$\$ 126$.}

\section{Die Lanteralfaxe.}

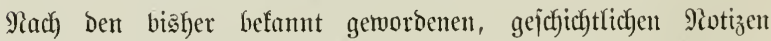

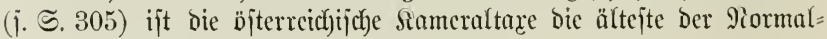
vorrathsmethoden, menu cs auch möglich ift, baji ipütere Theoricu

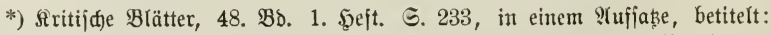

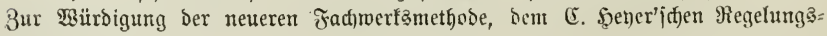
verfahren gegeniiber (ङ. 222-240). 


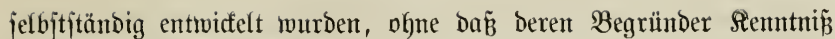
von ber Sameraltace hatten.

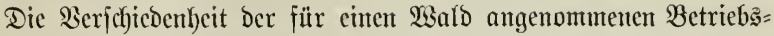
ivfteme und Ilmtriebszeiten bedingt fïx bie Sameraltare bie Bildung won Betriebselajīen.

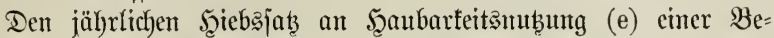
triebstlajfe finbet bicje Methode in ber Summe aus bem jährlichen (Sejammtzumachje (Z) und Dem Duotienten aus Der llmtriebszeit (u) in bie pojitive ober negative Differenz zroijchen Dem rwirffichen $\left(V_{w}\right)$ und bem normalen $\left(V_{n}\right)$ Borrathe.

Die Formel des F̧iebşą̧es lautet hiernach):

$$
\mathrm{e}=\mathrm{Z}+\frac{\mathrm{V}_{\mathrm{w}}-\mathrm{V}_{\mathrm{n}}}{\mathrm{u}}
$$

Die Rameraltare jtreft aljo Damach), Durch (Eriparung bei einem

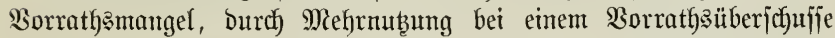
Den wirffichen Borrath twährent einter Umtriebszeit bem normalen gleidf 子"it jellen.

Die Borräthe werden mitteljt Des wirflichen Scaubarfeit: Durch)=

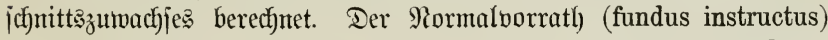
miro gefunden, indem man Durff) $\mathfrak{A}$ mwendung ber Formel $\frac{\mathrm{uZ}}{2}$ Den

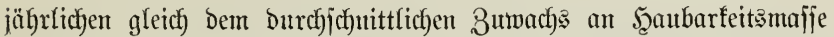
jeţt. Dadurch wird $V_{n}$ gleich der Şälfte jenter \$̧olzmaije, weldye die Betriebstlafje bejäß̨e, wonn fie ganz mit $\mathfrak{f c o l}_{z}$ im normalen

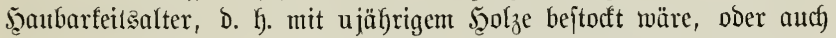
gleich jener Majfe, weldye eine Betriebsflajie bejißzt, bie burchgängig mit $\frac{u}{2}$ jährigem $\mathfrak{b} \mathfrak{o l z e}$ bejtanden ijt.

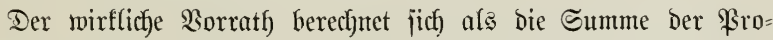

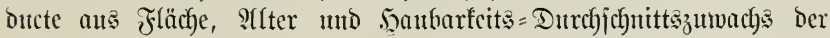
einzelnen Bejtände.

Durch) bieje Berectumung von $\mathrm{V}_{\mathrm{w}}$ gleicht jich, menn bie Bejtunds= verbältnijife nicht zu abnorm j̈mb, Der Febler genügend aus, welcher

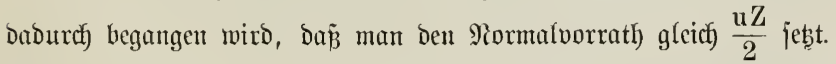
Beide $\mathfrak{B}$ orräthe werdon in Der Regel zu grof́. Da es in Der Formel bes Shebsjabes jedoch mur auf bie Differenz, auf bas arith= metijche Serbältnīi zwijchen $V_{w}$ unt $V_{n}$ anfommt, nicht anf bic 
abjolute Größze oex lebteren, jo bleibt biejer gemeinjame fahler obne

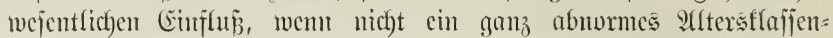
verbältnî̄ vorliegt.

Der 3umadys wird fait itets als wirf(id)er berecfunet und nicht als normaler. Itns ijt cin cinziger Falt won Bebentung befannt ge=

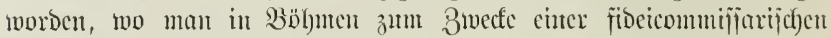

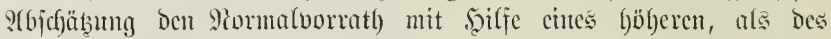
mirflicfen 3uwachjes ermittelte, weil ber Wiald ntachweisbar Durch

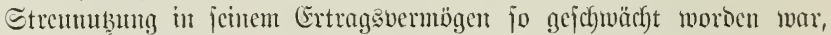

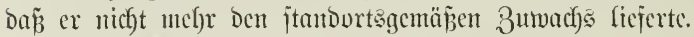

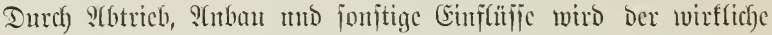

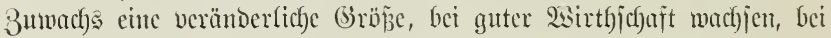

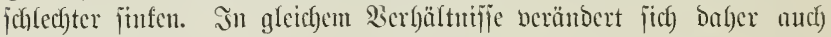
ber Siontalvorratl). Dicjer IImptand macht principiell auch für bic

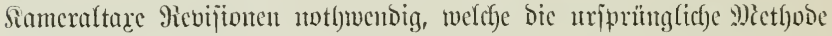
jedod) nicht fement.

Euten Wirthjichaftsplan fordert bic alte Siameraltage nicht. Da

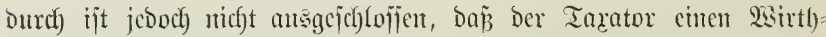

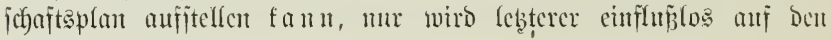
şiebojat, bleiben.

\section{Rechnutge be ijpiel.}

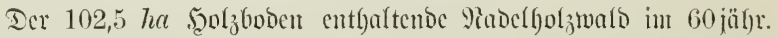

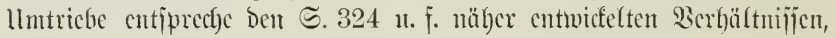

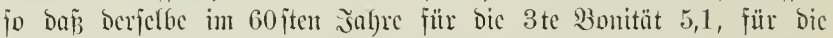

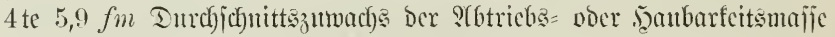

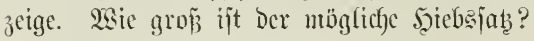

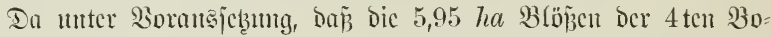

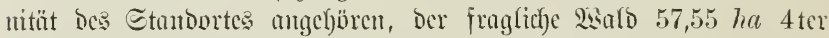

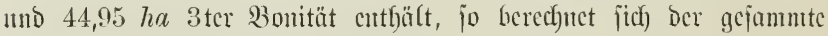

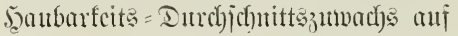

$$
57,55 \times 5,9+44,85 \times 5,1=568,8 \mathrm{fm},
$$

mo ber Piormalvorrath auf

$$
\mathrm{V}_{\mathrm{n}}=568,8 \times \frac{60}{2}=17064 \mathrm{fm} .
$$

Socr แach $\$ 77$ betrügc bic gcometrif(f) mittlere Bontiät 5,549 fm, jolylicic)

$$
\mathrm{V}_{\mathrm{n}}=\frac{5,549 \times 102,5 \div 60}{2}=17063 \mathrm{fm}
$$


Der wirffiche Worrath wird mun, wie ohen herworgefoben, nidgt nach) Der abjuluten, gegentwärtigen Miafie Der vorbandenen Bejtänoc

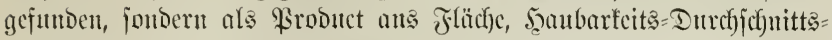

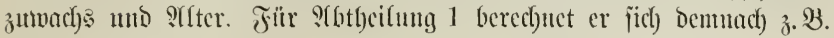
jolgendermajen:

$$
\begin{aligned}
\mathrm{V}_{\mathrm{w}} 1 \mathrm{a} & =2,50 \times 5,9 \times 5=73,75 \mathrm{fm}, \\
\mathrm{b} & =2,50 \times 5,1 \times 75=956,25 \quad " \\
\mathrm{c} & =10,50 \times 5,1 \times 5=267,75 \quad " \\
\mathrm{~d} & =1,25 \times 5,9 \times 90=663,75 \quad " \\
\mathrm{e} & =\frac{1,75 \times 5,9 \times 20=206,50 "}{\text { Summe } 2168,00 \mathrm{fm} .}
\end{aligned}
$$

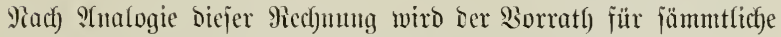
Bejtänoe gefunten, und jellt jich) Dant in Enmme

$$
\mathrm{V}^{\mathrm{w}}=17902 \mathrm{fm} \text {. }
$$

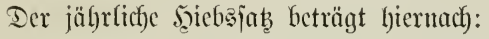

$$
\mathrm{e}=568,8+\frac{17902-17064}{60}=582,8 \mathrm{fm} .
$$

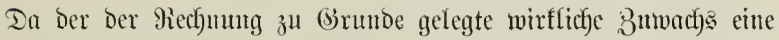

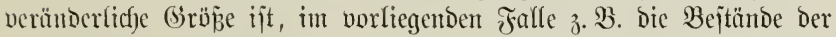

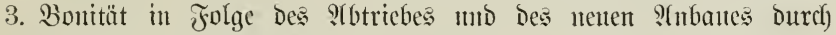

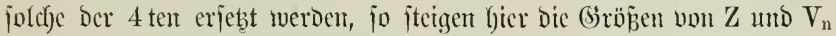

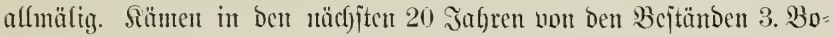
nität $1 \mathrm{~b}$ mit 2,5 , von 5 e $1,0,2$ a mit 2,5 , zujamuten aljo 6 ha zum

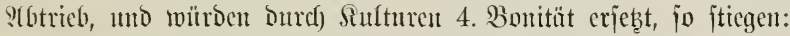

$$
\begin{aligned}
& \mathrm{Z} \text { ani } 63,55 \times 5,9+38,95 \times 5,1=573,6 \mathrm{fm}, \\
& \mathrm{V}_{\mathrm{n}} \text { " } \frac{573,6 \times 60}{2}=17208 \mathrm{fm} .
\end{aligned}
$$

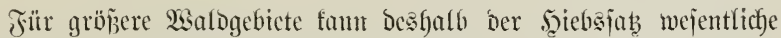

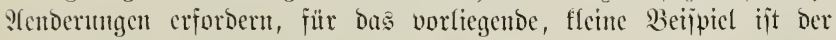

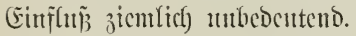

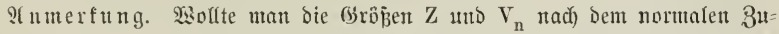
wachs, afjo nad) ber Etandortsbonität obigen Saldes ermittelt, jo witrde

$$
\mathrm{e}=604,75+\frac{17902-18142}{60}=600,75 \mathrm{fm} .
$$

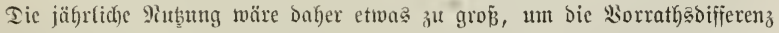

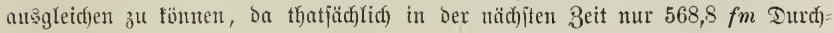

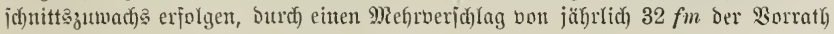




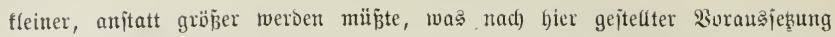
eigentrich body gefibehen jorlte.

Wolte man Dagegen nur $V_{n}$ nadh Dem normalen Buwadje bejtimmen, Den

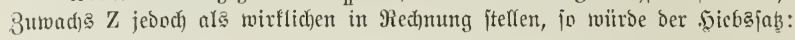

$$
\mathrm{e}=568,8+\frac{17902-18142}{60}=564,8 \mathrm{fm},
$$

mitfin zn flein, oder die Eriparniß̧ unüthig groj.

\section{Rechuug ga beifpiel.}

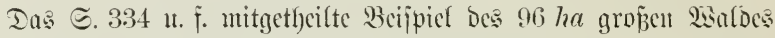

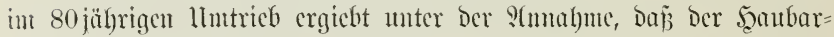
fcits = Durdjichnittszmmad) abgermoct 6,4 fm für bas ha betrage, iolgeubes Ricjultat:

$$
\begin{aligned}
\mathrm{Z} & =6,4 \times 96=614,4 \mathrm{fm} . \\
\mathrm{V}_{\mathrm{n}} & =\frac{614,4 \times 80}{2}=24576 \mathrm{fm} . \\
\mathrm{V}_{\mathrm{w}} & =56 \times 6,4 \times 60+40 \times 6,4 \times 40=31744 \mathrm{fm} . \\
\mathrm{e} & =614,4+\frac{31744-24576}{80}=704 \mathrm{fm} .
\end{aligned}
$$

jü̈r 20 Sabre entfallen Demuad) $704 \times 20=14080 \mathrm{fm}$, und ge $=$ itultet jich Dic \$crtfocilung Des şicbes, wic folgt:

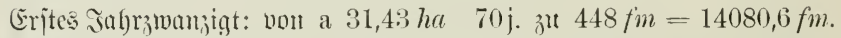
Bucites Drittes

$$
\begin{aligned}
& \text { " " 24,44, } \\
& \left.\begin{array}{lrrrrr}
\text { " } & 0,13 \text { " } 110 j . & 704 \text { " } \\
\text { " b } 24,29, & 90 j . & 576 & \text { " }
\end{array}\right\}=14082,5 \text { " }
\end{aligned}
$$

"

Wicrtes "

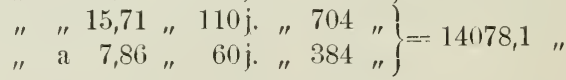

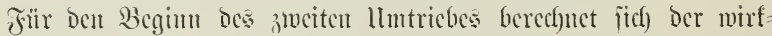
(iche Siorratl):

23,57 ha, im 9littel 10 jährị̣: $10 \times 6,4 \times 23,57=1508,5 \mathrm{fm}$,

$$
\begin{aligned}
& 24,42 \text { " " " } " 30 \text { " : } 30 \times 6,4 \times 24,42=4688,6 \text { " } \\
& 24,44 \text { " " " } 50 \text { " : } 50 \times 6,4 \times 24,44=7820,8 \text { " } \\
& 23,57 " \text { " " } \quad 70 \quad: \quad 70 \times 6,4 \times 23,57=105.59,4 \text { " }
\end{aligned}
$$

Er hat jüch aljo bis anf cine verichmindend fleine Differenz ber

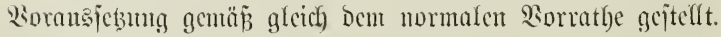

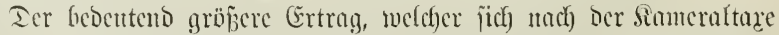
in vorficgenden Beifpiele gegenïber ben Pejultuten Der Fachwerfe be= 


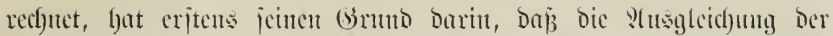

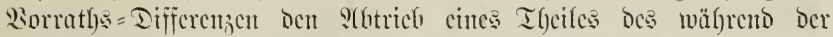
criten llmtriebseit crwachicnen, nenen Siorrathes gejtattet, jweiten in

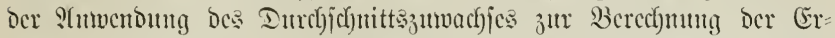

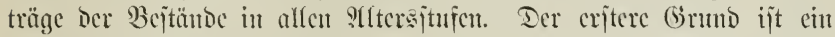

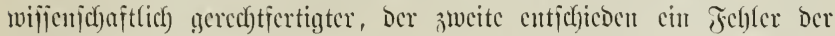

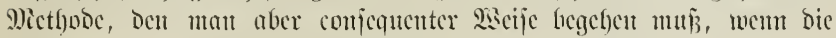
Siechnuting fimmen jolf.

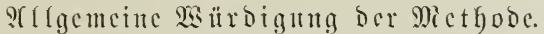

Ier Sianteraltace ijt junächjt vom Etambpunfte Der Miaterial

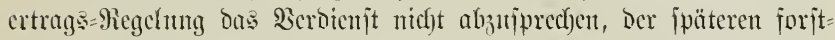
licken Ifecoric eine Bajis für bic Joce bes Pormalmalbes gejchaffen

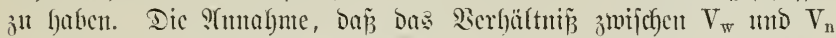

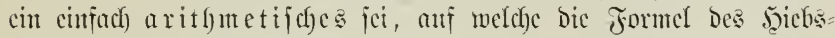

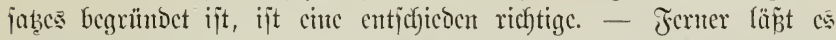

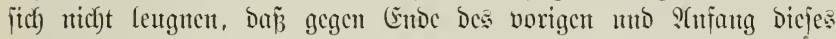

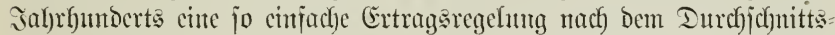

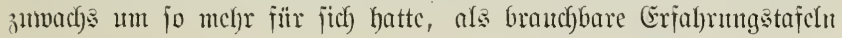
nod) f(l)itent.

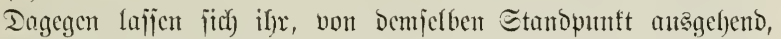
folgende Q⿻orwilrfe madhen:

Der Felfler bei ber Ermittchung bes Pormafuorrathes butrel bic

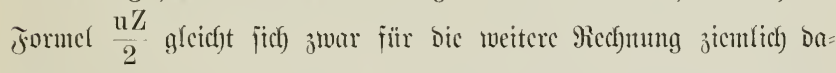

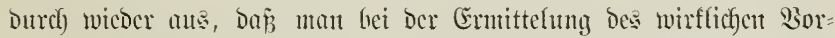

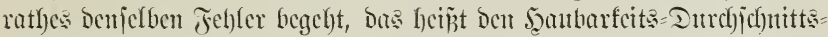
jumachs in allen Rebensaltern ber Bejtände gleich Dem laufenden jeb̧t,

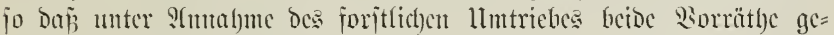

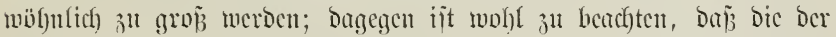

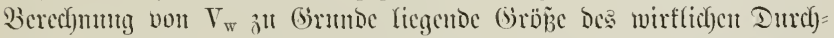

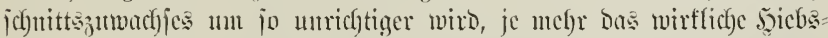

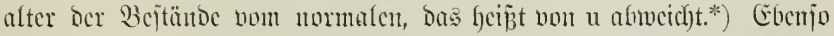

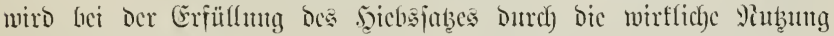

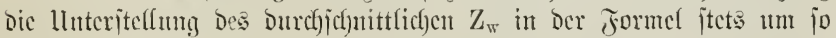

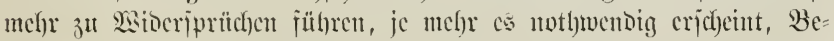

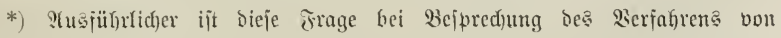
(5. 5e rex erörtert. 3u vergl \$ 128 . 
jtände weit unter oder erjt weit über Dem angentommenen Jुaubarteits= alter z̆ пuß̧еп.

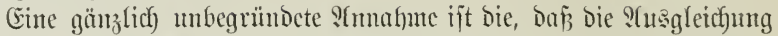
Der Sorratbsoffferenjen gerabe imterfalb ciner llmtriebszeit erfolgen müjie, wäfrend es je nach Den vorliegenton berbältnifjen oft vick

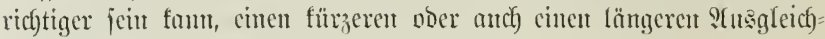

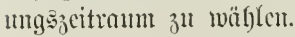

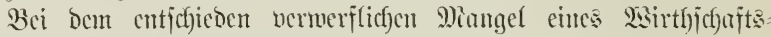
plantes miro bie Sameraltaze auch für jenen 2sald cinten, wem andf)

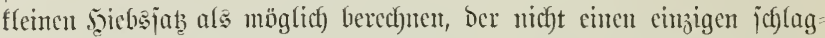
baren Baum anfanweijen hat. Bejtünto in Dem żveiten Rechnutgs:

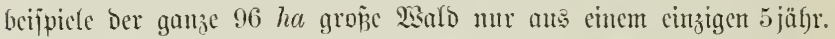
Bejtante, jo wïrde $V_{w}=3072 \mathrm{fm}$. Icr jübrlicl)e fyichajab betrïge nach) Der Jormel:

$$
614,4+\frac{3072-24576}{80}=345,6 \mathrm{fm}
$$

während thatjächlich vor :(blauf vieler Jahre nicht ein citziger Baum

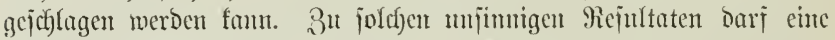

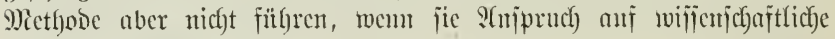
Eorrectheit erbeben will.

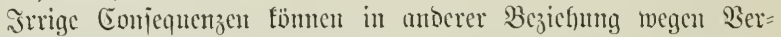

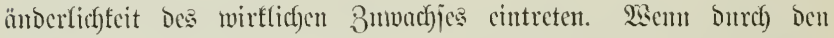

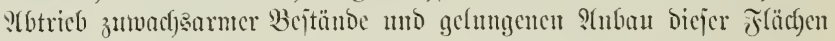
$Z_{\mathrm{w}}$ bedeuteno gefoben wird, jo wird jich zwar cin ctwas gröjerer Diebsjab berechnen, als vorber, alleit jelbjt für ben Fall, baj an= fänglich) Der Tiormalnorratf) gleich) Dem wirkficlen war, cinte ntegative Iifferenz jwijchen beiden Sorrätben entïtefen und bleiben, bis ber

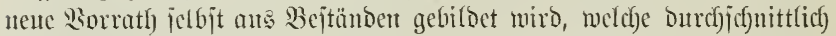
bas balbe llmtriebsalter crereidyt baben, bis er aljo jerbit gleidy bem ttormalen gemorber. Betrad)ten wir cuen ganz cinfact)en, beshalb fünjtlichen Fall. Ein 100 ha groper 2 sald bejtele ans cincm $50=$

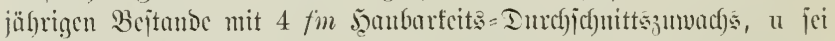

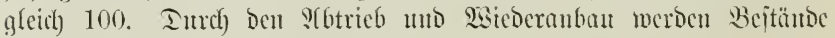

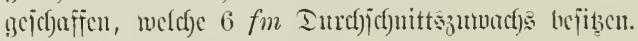

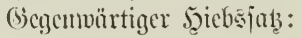

$$
400+\frac{20000-20000}{100}=400 \mathrm{fm} \text {. }
$$

2:ähreno Der nächiten 20 Jahre werocn aljo gemubt $8000 \mathrm{fm}$, InD gefbören Dajn 33,33 ha Des im Mittel bicjer Zeit 60jäfrigent Beitandes mit $60.4=240 \mathrm{fm}$ Ertrag. 
Gejebst mun, in 21 jen Jahre criolge cine neute Ermttelung Des

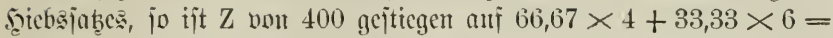
466,$66 ; V_{\mathrm{n}}$ beträgt $\frac{466,66: \times 100}{2}=23333 ; V_{\mathrm{w}}$ Dagegen $66,67 \times$ $4 \times 70+33,33 \times 6 \times 10=20667,4 \mathrm{fm}$. Ier füntige 5iebsjab miro uunt jwar jteigen:

$$
\mathrm{e}=466,66+\frac{20667,4-23333}{100}=440 \mathrm{fm},
$$

Dagegen jtellt jich cinc Sorratlgsifferent von $2665,6 \mathrm{fm}$ herants. Wo

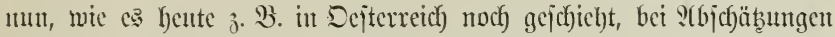

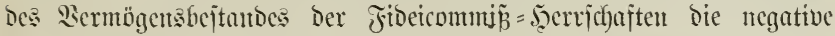
Differenz jwijchen bem fundus instructus tund Dem wirflichen Worrath

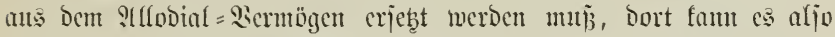

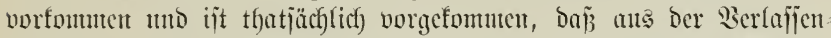
jofajt cines Fitocicommin = Infabers cut Durch vorzüglicfe finlturen

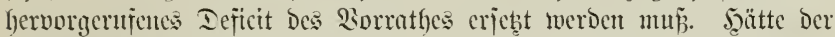

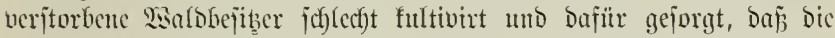

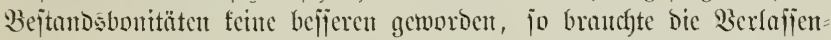
ichajt in obigen Falle s. B. nicht $2665,6 \mathrm{fm}$ in (Gelbwerth) zँ crjeben.

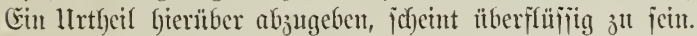

3om wirthjefaftlidfen oder mit anberen 2 sorten, vou fütüjiellen Etanopunte ans betrachtet, fat entich bicje Dietfode nur negativen

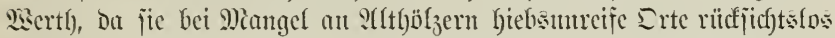

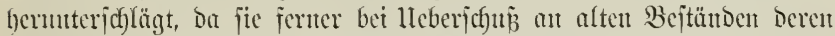

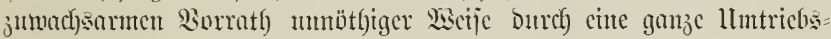

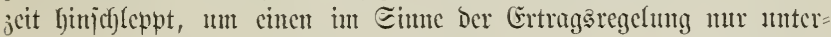
georbnetent Factor, Den Siorntufoorrath, zll erreidfen. Dieje Sormürfe treffen bic Miethode jelbjt Dant, wem fie ifrer Rechnung den funan-

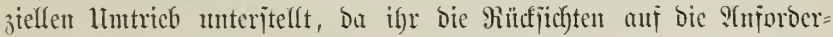
ungen des Einjelbejtundes fremb bleiben.

\section{$\$ 127$.}

\section{5ึนnญ}

J) meraltaxc, Den Iurchicfnittsumachs allen ?(ltersitujen als cinen gleicf)en

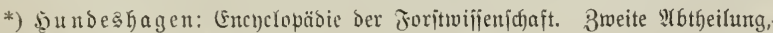

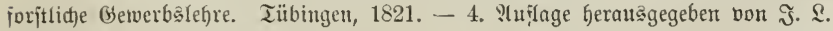
I Iauprec)t. Tübingen, 1843.

Dexfelbe: Die Forjtabjă̈bung auj neuen, wifjenjdajtlichen Grund= 
$3_{\text {ll }}$ unteriteffen, berechnete beshalf ben nomalen 2orrath mittelit Er fafrungstajeln, Den mirflicfen jo, wic ifn bie Bejtünbe thatjächlich)

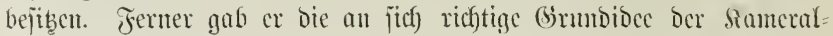

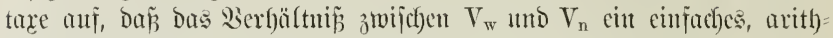

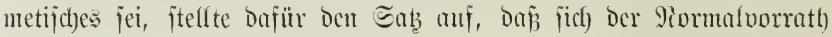
z $\mathrm{mm}$ normalen şicbsjabe verbalte, wic ocr mirffiche Sorrath jum

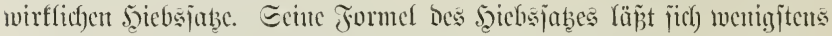

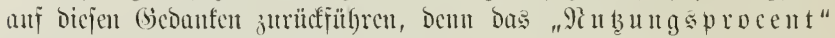
entwicfelt jith ans ber Proportion

bievaus

$$
\mathrm{V}_{\mathrm{n}}: \mathrm{e}_{\mathrm{n}}=\mathrm{V}_{\mathrm{w}}: \mathrm{e}_{\mathrm{w}} \text {, }
$$

$$
\mathrm{e}_{\mathrm{w}}=\mathrm{V}_{\mathrm{w}} \times \frac{\mathrm{e}_{\mathrm{n}}}{\mathrm{V}_{\mathrm{n}}} .
$$

Der Factor $\frac{\mathrm{e}_{\mathrm{n}}}{\mathrm{V}_{\mathrm{n}}}$ ijt Das jogename Viubutugsproccut.

Ier Piormalvorratf) beredfuct jith als Eumme einer (Ertragstajel, melche Den betrefienocn Etandorts = mo Betricbsocrbältnijien cutjpricht.

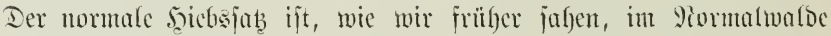
gleid) dem älteiten (Silicbe ber betreffenden Ertrugstafel woer autd)

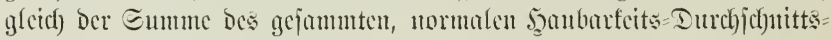

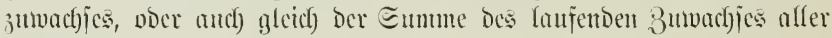
Beitänoc.

Eince Directen Ermittelung Des mirflichen Bunarbjes bedorf ca bei biejer Mietl)obe cigentlid) nicht, höchjtens für bic in ben nüchjten

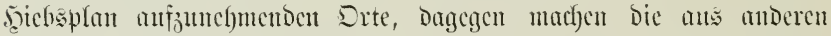

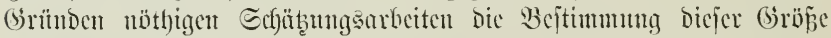
(et)r lectet.

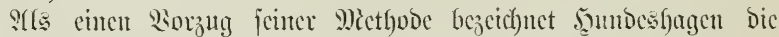

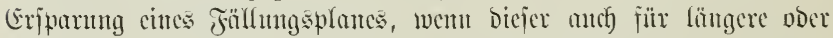
fïrzere 3eit gejtattet ici. Deshalb, namentlich aber, weil ber wirflicke

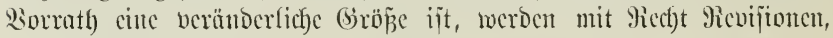

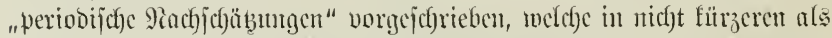
10 jügrigen, unter IImîtünden in läıtgeren Beitrüunen cinzutteten haben.

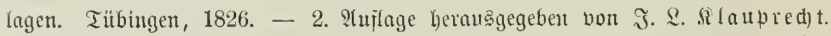

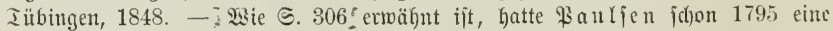
ähnliche Mietfode Der Ertragsregelung entwictelt, wie şundeshagen, jo Dab man

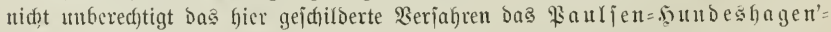
iche nentuen fönnte. Da jeond) leţterer bie Methode grimolid)er und weit aus=

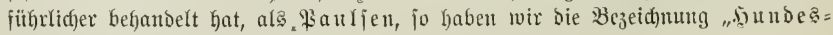
h) agen's Beriakren" beibehalten. 


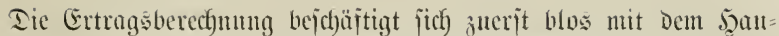
barfeitsertrag; ijt bicier reguliet, jo werben bic 3roijchenmub̧ungen

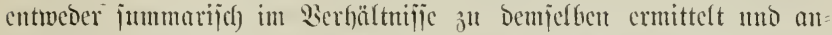

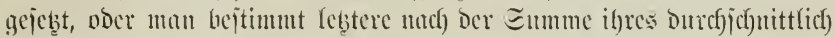

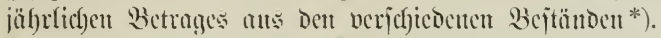

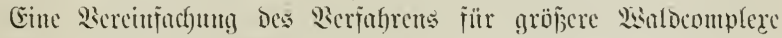

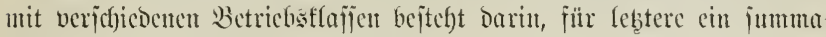

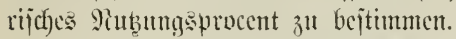

Endtich wiro als abgetür

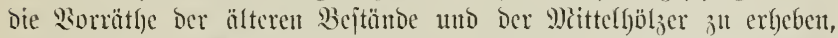

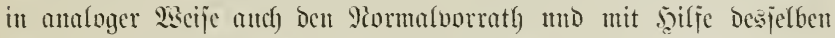
cin "particlles Pitbeutysprocent" jull berect)nen.

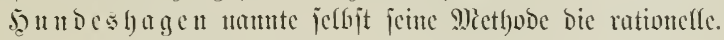

\section{Sicchungabcipiel.}

Für Den im 60 jüfrigen llmtricbe jut bewirthjichaftenden 2 sald

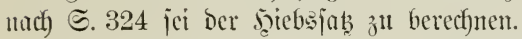

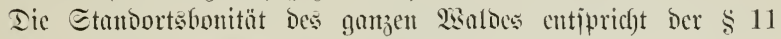

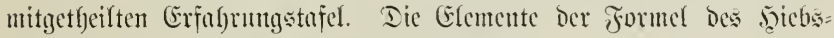

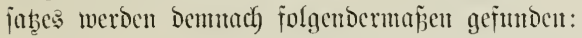

$$
\begin{aligned}
\mathrm{V}_{\mathrm{n}} & =\left(6+20+40+65+96+129+164+200+237+275+314+\frac{354}{2}\right)^{5} \\
& =8615 \mathrm{fm}, \text { D. h). für } 60 \mathrm{ha} . \\
\mathrm{e}_{\mathrm{n}} & =354 \text { " " " } 60 \text { " }
\end{aligned}
$$

Sierau:

$$
\text { Piuţıungsprocent } \frac{354}{8615}=0,0411 \text {. }
$$

2sirfficfer 2iorratf):

$$
\begin{aligned}
1 \text { a } 2,5 \times 6 & =15,0 \mathrm{fm}, \\
\text { b } 2,5 \times 402 & =1005,0 " \\
\text { c } 10,5 \times 5 & =52,5 " \\
\text { d } 1,25 \times 575 & =718,75 " \\
\text { e } 1,75 \times 65 & =113,75 "
\end{aligned}
$$

u. $\lceil$. w.

$$
\text { Eumme } 15204,8 \mathrm{fm} \text {. }
$$

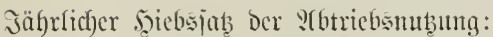

$$
15204,8 \times 0,0411=624,92 \mathrm{fm} .
$$

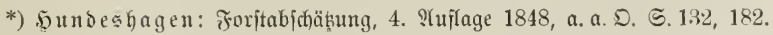


Diejer ફ̧iebsjał̧ ijt jtreng genommen bereits im nächjten Эabre ein anderer, ba jich) $V_{w}$ fortbauerno ändert.

Siommen in Den nädfiten 10 Sabren jente 12 ha mit $6208 \mathrm{fm}$, melche im Speciellen S. 346 nadhgemiejen jün, anferdom zur Er:

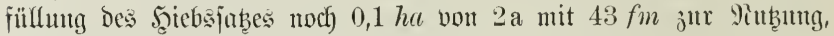

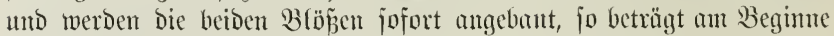
Des zweiten Sabrzefuntes Der ivirfliche Sorratfy nur noch $14202 \mathrm{fm}$, aljo weniger, als Der normale, obgleich er anfänglich ïber leb̧terem

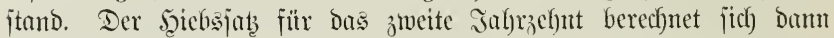
auf $14202 \times 0,0411=583,70 \mathrm{fm}$. Ђeätte utan erjten Jafrzefntes feine gievijion cintreten lajjen, jondorn bic $625 \mathrm{fm}$

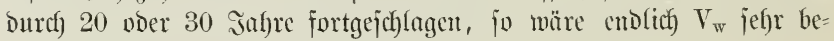
bentento ffeiner als $V_{n}$ gemorben.

$\mathfrak{A m e r f u n g . ~ S o ̈ m u t e ~ m a n ~ i n ~ D e m ~ v o r l i e g e n d e n ~ B e i f p i e l e ~ v o u ~ D e r ~ S o r a u s : ~}$

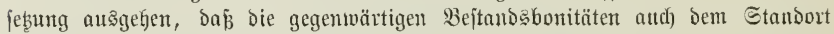
entipräd)en, fo hätte man Pormalborrath und norntalen Jeiebsjats des ganzen SalDes nad) Den Fläd)en Der beiben Bonitäten zu bered)nen.

Jür 57,55 ha 4 ter und 44,95 ha 3 ter Bunität ift $\mathrm{V}_{\mathrm{n}}=13608,5 \mathrm{fm}$ แm $\mathrm{e}_{\mathrm{n}}=568,79 \mathrm{fm}, 9$ ubuugaprocent baher:

$$
\frac{568,79}{13608,5}=0,0418 \text {. }
$$

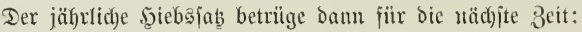

$$
15205 \times 0,0418=635,56 \mathrm{fm} \text {. }
$$

Dieje fleme Differenz fommt Daher, weil nad) Der vorausgefetsten (Ertraga: tajel bei Dem 60 jährigen llmtriebe das 9ußumgsprocent fïr bie Dritte Bonität etwas größjer ijt, als fïr die vierte.

\section{Siednumgobeifpict.}

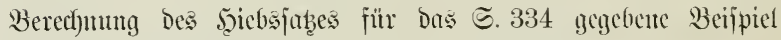
cines 96 ha grofen $26 a l d e s$ im 80 jährigen llntriebe.

Rormalvorrath:

$$
\begin{gathered}
5\left(6+20+40+65+96+129+164+200+237+275+314+354+394+433+472+\frac{509}{2}\right) \\
=17267,5 \mathrm{fm} \text { für } 80 \mathrm{ha} .
\end{gathered}
$$

Mormaler 5̧iebsjab $=509 \mathrm{fm}$ für $80 \mathrm{ha}$.

$$
\text { Nubutugprocent }=\frac{509}{17267,5}=0,0295 .
$$

Wirflicher 2orratl):

$$
\begin{array}{r}
\text { a) } 56 \times 354=19824 \mathrm{fm} \\
\text { b) } 40 \times 200=8000 \prime \prime \\
\text { Summe } 27824 \mathrm{fm} .
\end{array}
$$




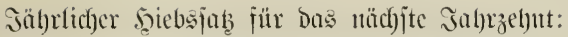

$$
27824 \times 0,0295=820,81 \mathrm{fm} .
$$

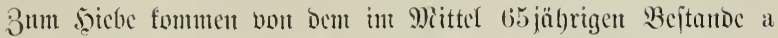

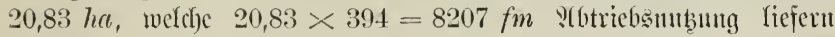
mürbett.

Siach) S(blauf bicjer Beit beträgt Der wirfliche 2iorrath), da bic

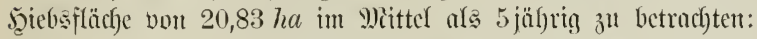

$$
\text { a) }\left\{\begin{array}{l}
20,83 \times 6=124,98 \mathrm{fm}, \\
35,17 \times 433=15228,61 " \\
40,00 \times 275=11000,00 \mathrm{\prime \prime}
\end{array}\right.
$$

Situme $26353,59 \mathrm{fm}$.

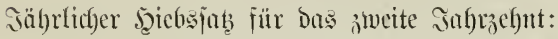

$$
26353,6 \times 0,0295=777,43 \mathrm{fm} .
$$

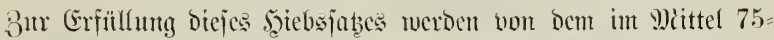

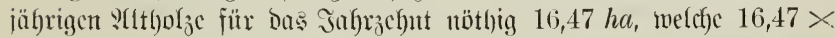
$472=7773,84 \mathrm{fm}$ 9(btricbsertrag gevuä)tcet.

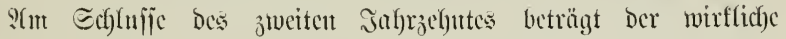
Worratf):

$20,83 \mathrm{ha}$ im Mrittel 15 jährig, jontad) $20,83 \times 40=833,20 \mathrm{fm}$,

$$
\begin{aligned}
& 16,47 \text { " " } 5 \text { " " } " 16,47 \times 6=98,82 \text { " } \\
& 18,70 \text { " " " " } 80 \quad \text { " " } \quad 18,70-509=9518,30 \text { " " } \\
& 40,00 " \text { " " " } 60 \text { " " } \\
& \text { Sunume 24610,32 fm. }
\end{aligned}
$$

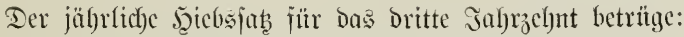

$$
24610,32 \times 0,0295=726 \mathrm{fm} .
$$

it. ๆ. เo.

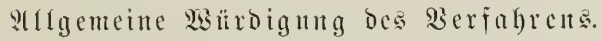

Etellt man fich zutüchjit auf Den Standpunft ber Theorie Der

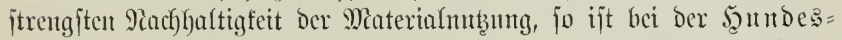

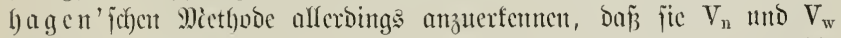
richfiger beredfuct, als bic alte Simmeraltaxe, bugegen entbel)nt bic

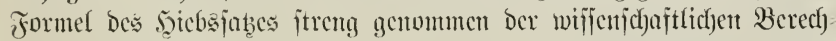

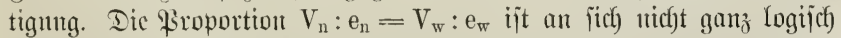

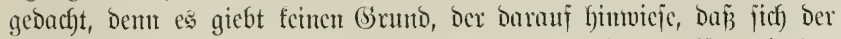

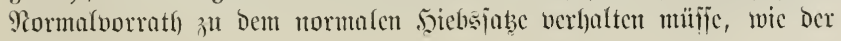




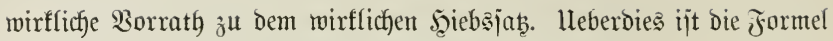
$V_{\mathrm{w}} \times \frac{e_{n}}{V_{n}}$ im rein mathematijchen Sinne cine irrationale, da cine ganz

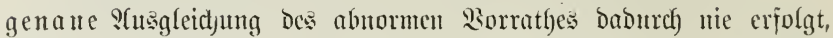

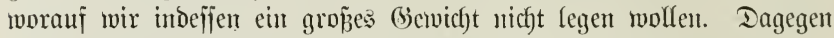

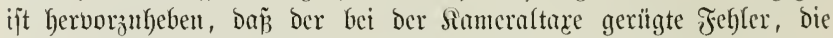

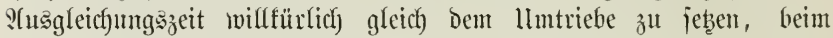

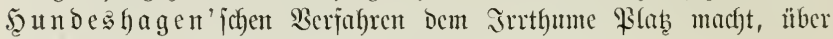

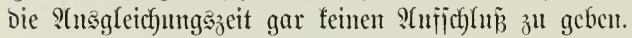

Mit Der Rameraftare lgat übrigens bieje Methobe Den Fefbler ge-

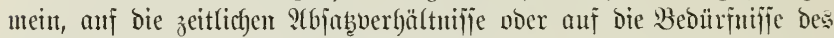

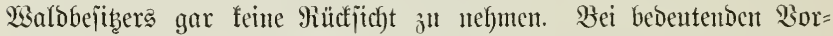

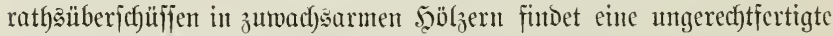

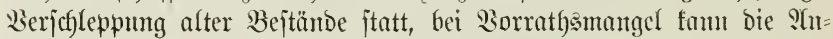
menoung Der Formel Des joiegafabes zum Stbtriebe gantz untreifer Drte

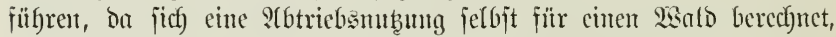

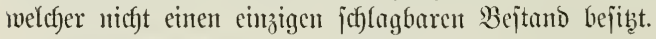

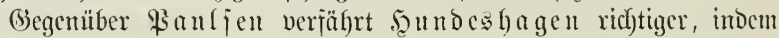

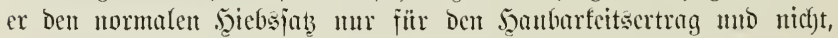

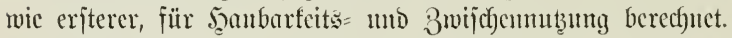

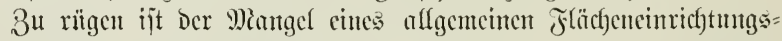

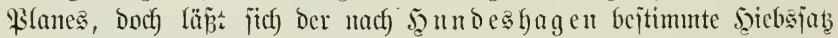
mit einem joldenen $\mathfrak{P l a n e}$ vereinigen.

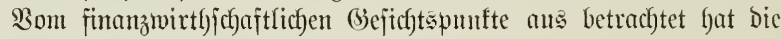

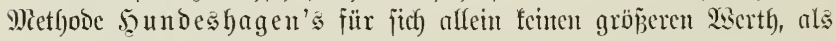

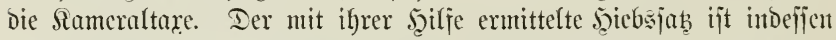

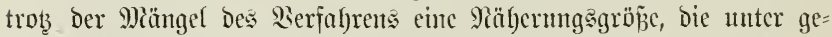
wififen Sierbältmiffen gut zu, verwerthen ift. Erifens fann man ben=

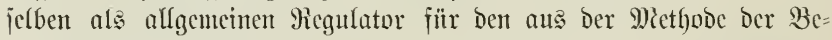

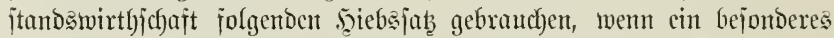

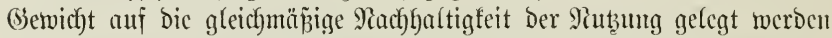

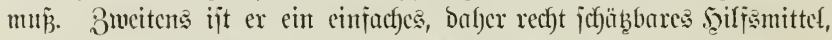

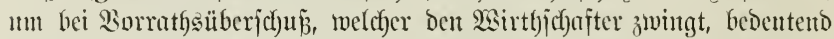

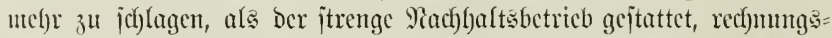

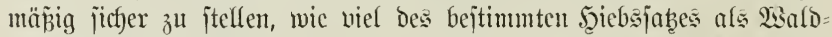
rente zu betractente und wie viel Der Miajic mur aus ber Wisirthjchaft

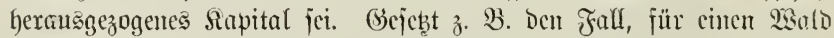

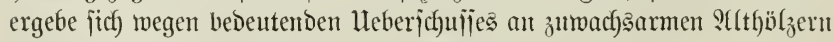

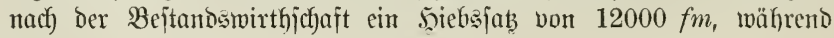




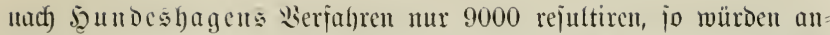

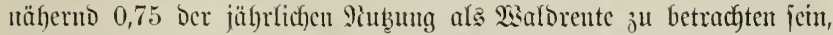

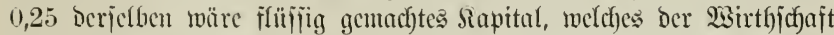

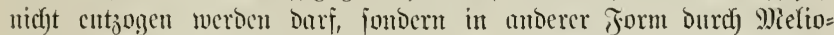

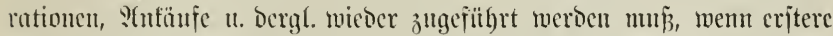

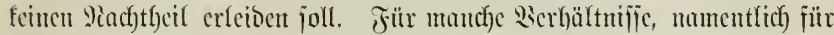

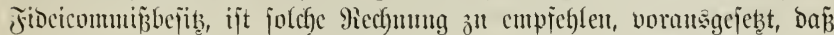
fie mindocitens alle 10 , noch) befier alle 5 Jafyre ermeuert wirb, und

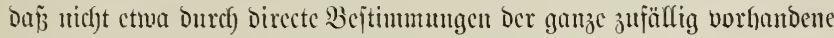

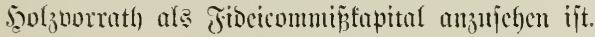

\section{\$ 128.}

\section{Carl Finer' Dexfalgreut.}

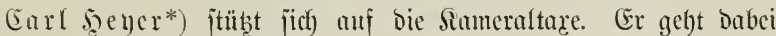
טun folgenden (Bejichtspunften aแs:

Sit cine Betriebsflafje in Pormal3uitande, D. h. juto beren 811 machs, Lorruth uno S(ltersitujenfolge normal, bum läpt fich ber bem

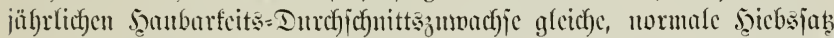
io) lange fortmuben, als feine obiger (5imbledingungen gejtört wiro.

Wäre Der Siormalvorrath be cintem abnormen ?(Itersflajfenverbält= mific vorfantocu, io itelft fich lebteres von jelbjt normal, wem man

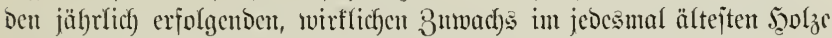

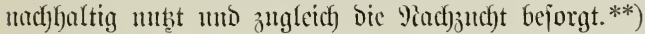

Bei S(bnormität bes Borratbes ijt bicjer baburef) anf jeinen nor= malen Stuno zll bringen, Daj man entweder ipart, weun cr ju flein,

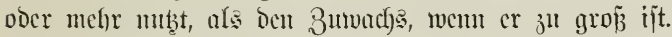

Ein betrïchtlicf)es 3umachsmanto an altem Sorratbe fum zur

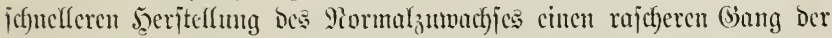
Berjüngung erforbe:n, jefbjt went baburef) bie Scerjtellung bes normalen

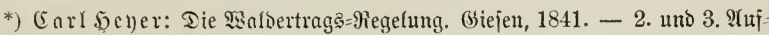

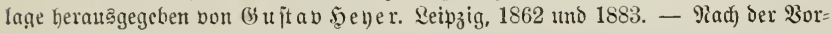

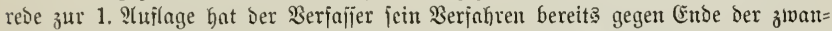
ziger Jahre in Mamujcript zun Druffe bollendet gehabt. Es ijt aljo feinesmegs eine blop̧e Seränderung Dor im jolgenden Raragraphen zu juildernden Sarl'jdjen Micthodc.

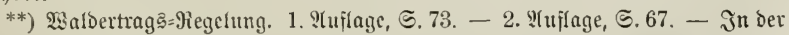

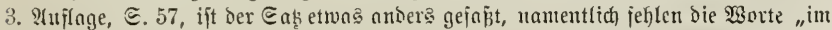
jedesntal älteiten Solze", won beren llutidtigfeit fid wohl (sult a b seyer ïber= zengt hatte. 
3orrathes verzögert, oder jogar leb̧terer, menn er z̆ufällig vorfanden, gệtört miro.

Der 3eitraum, biunen weldyem cin abnormer Borrath auf bent

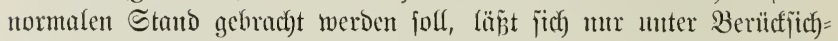

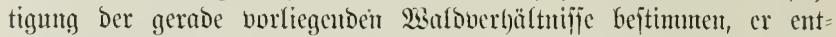

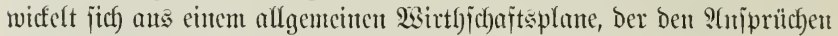
Des $\mathfrak{W a l d b e j t b e r s ~ m o ̈ g l i c h j t ~ \Re e c h m u n g ~ t r a ̈ g t . ~}$

Ier Rormalvorratl) mird für jede Betricbsflafje nach ber Formel $\frac{\mathrm{uZ}}{2}$ Gerechnet, moriut Z Den gejammiten normalen Scanbarfeits= Durch) ichnittรณunachs bedcutet.

Der wirffiche Siorrath wird, wie bei ber Sameraltace, fït alle

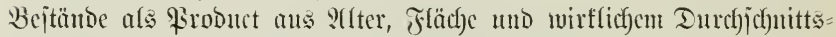

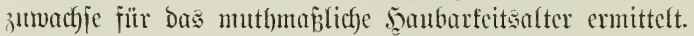

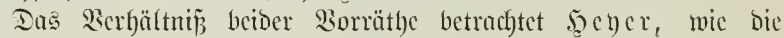
Siameraltare, als cin cinfaches, arithmetijches.

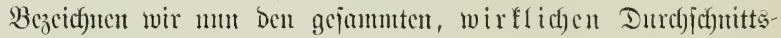

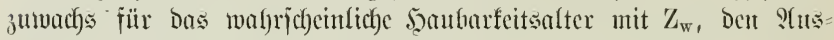
gleidfungszeitran mit a, jo lantet bie Formel bes jeichsjabes:

$$
\mathrm{e}=\frac{\mathrm{V}_{\mathrm{w}}+\mathrm{Z}_{\mathrm{w}} \times \mathrm{a}-\mathrm{V}_{\mathrm{n}},}{\mathrm{a}}
$$

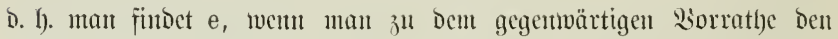
jummurifchen Buwachs möbrend a abbirt, bon bicjer Summe den

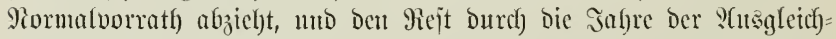

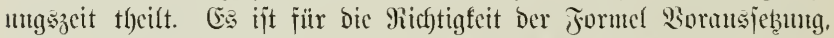

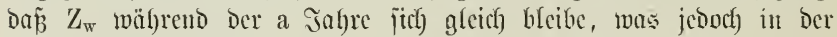
regel nicht Der Falf ijt.

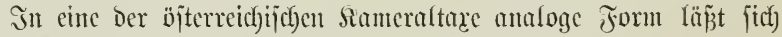

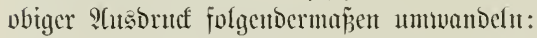

$$
\mathrm{e}=\mathrm{Z}_{\mathrm{w}}+\frac{\mathrm{V}_{\mathrm{w}}-\mathrm{V}_{\mathrm{n}}}{\mathrm{a}} .
$$

Der 9 usgleid) ungszeitraum a ijt mur in Dem Fatle Durd)

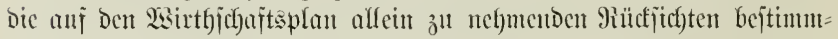
har, ment $V_{w}$ grö̈jer, als $V_{n}$. S)ian hat es bam in ber Syand, a be

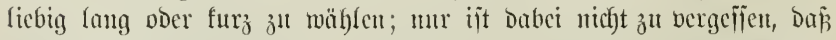

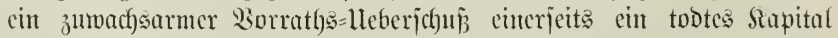

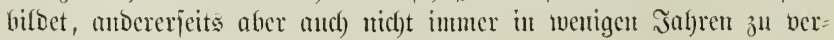
rerthen ij̄t. 
Sit bagegen $V_{w}$ fleiner als $V_{n}$, bandelt es jict) aljo um eine Er=

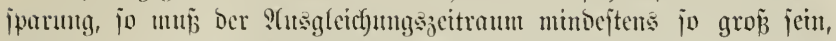

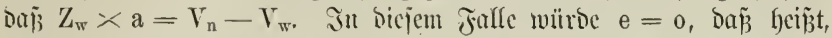
mäl)rent des 3eitrumes a fünde gar feite s(btricbsunţung jtatt,

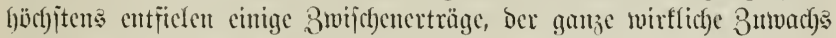

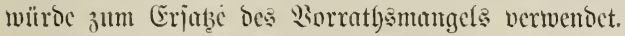

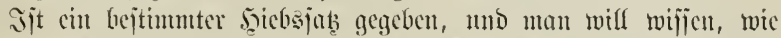

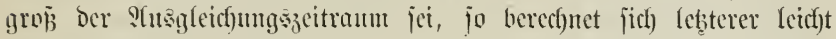
ats Der Formel

$$
\mathrm{e}=\mathrm{Z}_{\mathrm{w}}+\frac{\mathrm{V}_{\mathrm{w}}-\mathrm{V}_{\mathrm{n}}}{\mathrm{a}}
$$

Бุicrats

$$
a=\frac{V_{w}-V_{n}}{e-Z_{w}} \text { oder in anderer form } \frac{V_{n}-V_{w}}{Z_{w}-e} \text {. }
$$

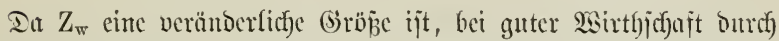

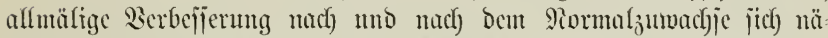

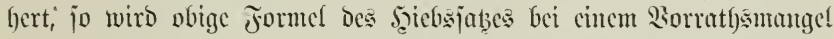

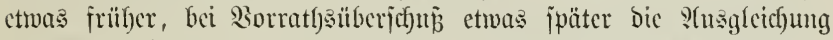

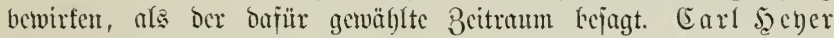

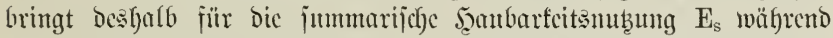
cinces angenommenten 3citrumes a, an befien Ende ber 9iormulvorrath

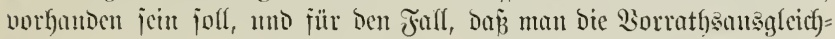

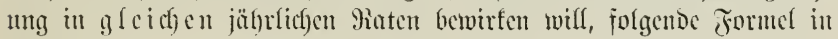
Sorichlog:

$$
\mathrm{E}_{\mathrm{s}}=\mathrm{V}_{\mathrm{w}}+\mathrm{Z}_{\mathrm{ws}}-\mathrm{V}_{\mathrm{n}}
$$

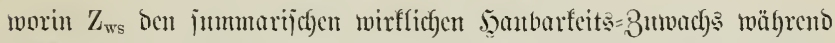

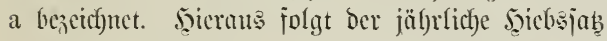

$$
\mathrm{e}=\frac{\mathrm{V}_{\mathrm{w}}+\mathrm{Z}_{\mathrm{ws}}-\mathrm{V}_{\mathrm{n}}}{\mathrm{a}}
$$

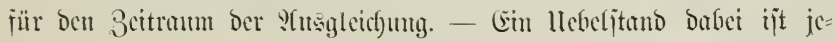

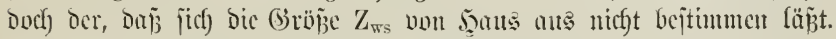

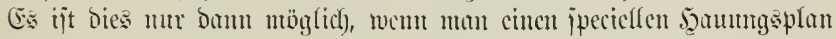

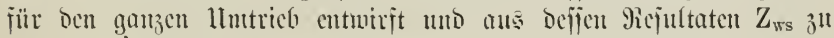
crmitteln verjuctft. *)

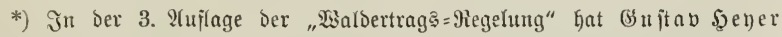

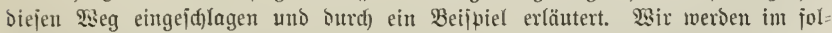
genten Redyumgsbeijpicl ebenjo verfahren, sa man ftreng genommen $\mathrm{Z}_{\mathrm{w}}$ ̈̈ber= haupt nicht anders ganz ridftig finben tann. 


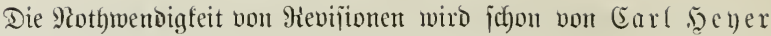
atsoritaflich) betont, ebenjo von (3) ujtav jecyer.

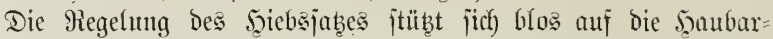

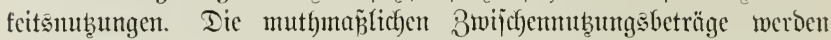
gewöhnlicf) mur für bic nüchjte \$eriobe ober bei langen \$erioden für

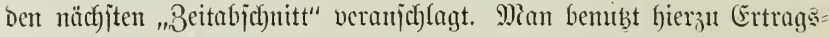

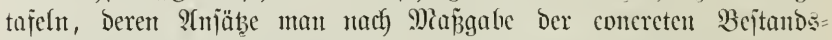
verhältnifje mobificirt. Cind bie zu Durchjorjtenden Bejtände von den=

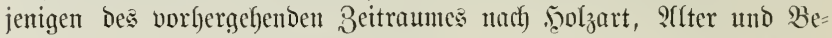

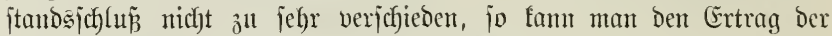
3wijchennubzungen nach bem Berhältniē berechnen, in welchem biejelben jeither zur Şaubarfeitsmakng jtomben. Man wirft fie alsomu in Summe für jümmtliche Bejtändc, welche einer Dumchjorjtuty bebürjen, aนเร.*)

\section{Rechnung sbei ipiel.}

Für Den Seite 324 beijpielsmeije gejchilderten 102,5 ha 5̧ol 3 boden

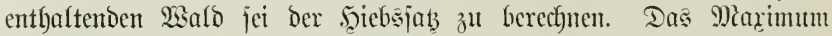

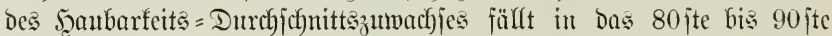
Jahr, wir wählen deșhalb einen 80 jährigen Umtrieb, zcriällen diejen in 4 ßeribden zu 20 Jahren. Die einzchten Bejtünde werden unt

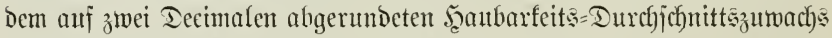

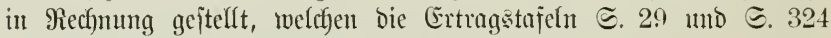
für bie verjofjedenen Bonitäten ergehen.

a) Berectutug des normalen 3 uwadjes.

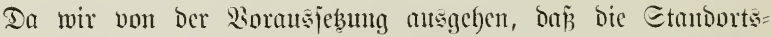
Gonität Durchgängig Die Der Ertragstufel \$ 11 cntiprechentoc 4 te jei,

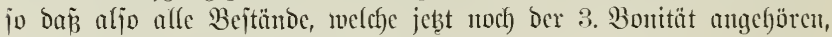

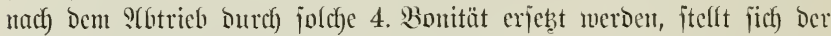

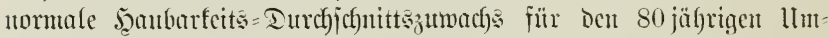
tricb anj

$$
6,36 \times 102,5=651,9 \mathrm{fm} .
$$

b) Berechung des normalen Sorrathes.

Pach Der Formel $\frac{\mathrm{u} \mathrm{Z}_{\mathrm{n}}}{2}=\frac{80 \times 651,9}{2}=26076 \mathrm{fm}$.

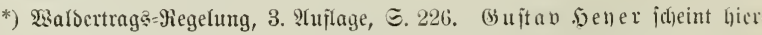

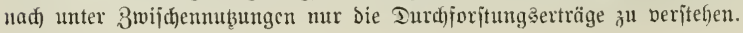




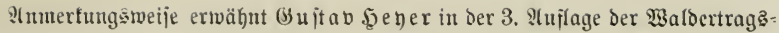

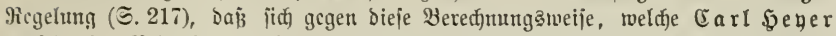

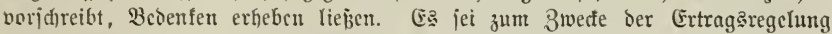

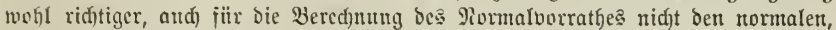

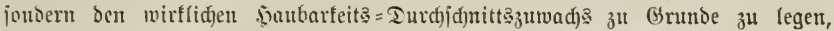

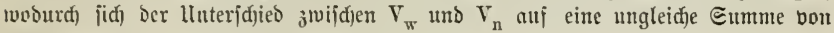
"IItterseinheiten" rebuciren würbe.

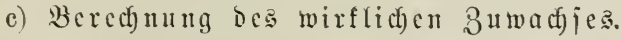

Bereits bei Bejprect)ung ber fameraltare machten wir Darauf

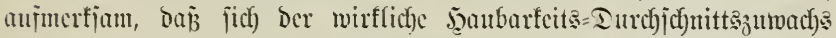

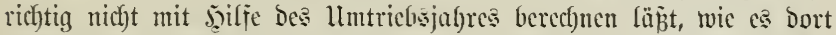

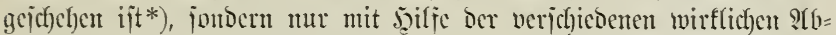
trichsjabre ber cinzelnen Bejtände. Lm Dies thum ju fönnen, wiro ç nothwendig, emen ipecicllet J̧icbsplan für bie ganze llmtriebszeit

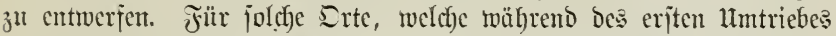

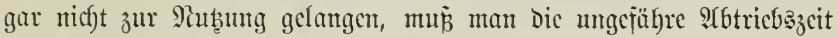
mäbrend bcs zweiten llntriebes crmittcln.

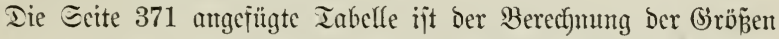
$\mathrm{Z}_{\mathrm{w}}$ und $\mathrm{V}_{\mathrm{w}}$ gewiomet. Bejüglich Der Butwad)sbered)nung biente noch) Jolgendes jux Erläuterung:

1. Findet ber : Sbtrich cines Bejtandes erjt nach Irblauf bes

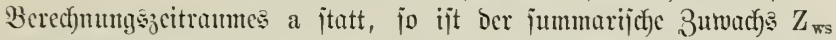
bicjes Bejtandes mährend ber a Jabre gleich Dem Probuct aus a uno

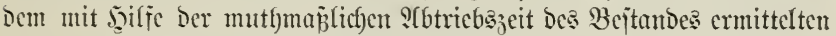
รู)

Beifpiel: Bon Dem 10,50 ha gropen Bejtand 1e fommen uad Der Tabelfe

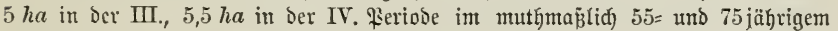

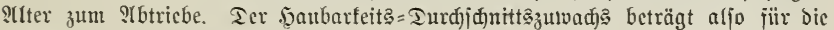
$5 \mathrm{ha}$, went $\mathrm{a}=40$, fïr biejen Beitraum $5 \times 4,93 \times 40=9 \mathrm{\varepsilon} \mathrm{fm}$, fïr bie $5,5 \mathrm{ha}$ Dagcgen $5,5 \times 5,36 \times 40=1179 \mathrm{fm}$.

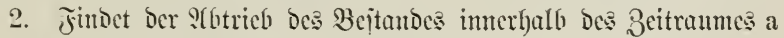
itutt, jo jutb jwei Fälll ju unterjacheiden.

a) Ier Bejtand ijt normal bejchaffen uno fommt in bem näm=

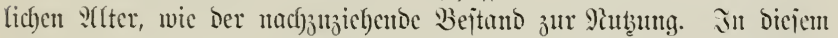

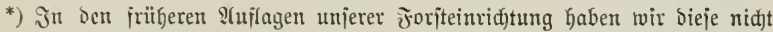

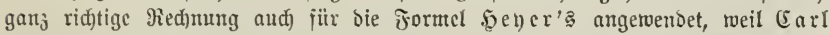

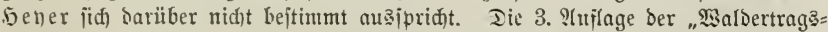

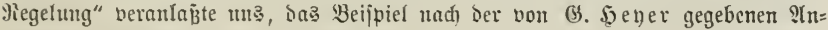
reitung $3 \mathfrak{u}$ beredhnen. 
Falle ift der fummarifde Buwad)s wälyreno ber Beit a gleidf bem

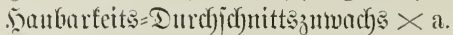

Diejer Fall fonmt bei Der eriten Sertbeilung in unjerem Re(f)ungabeifpiele nifft vor, Dagegen mit 3 ha bon $4 \mathrm{c}$ in ber jweiten Bered)unn (S. 375). Es twiro fier cinfach Der normale Bnwad) s in bie gicchnung eingejtellt.

b) Der Bejtand ijt abnorm bejefoffen ober foum in cincen an=

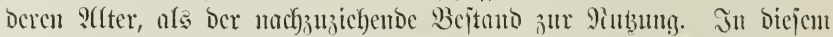

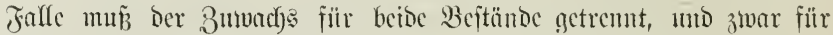

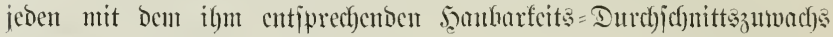
berectuet werden.

Beifpiełe. Der 2,5 ha grope Beitano 2a, weldjer ocr 3. Bejtandsennität

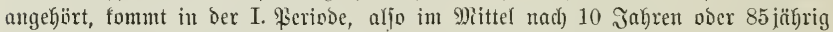

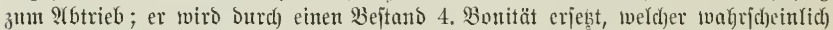

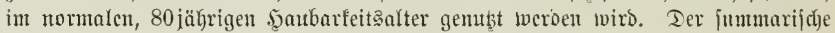

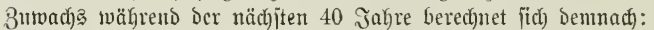

$$
2,5 \times 5,35 \times 10+2,5 \times 6,36 \times 30=133,75+477=611 \mathrm{fm} .
$$

Bon Dem 23 citande 5 e foumen 6 ha in ber II. Feriose im Mittel 55 jägrig

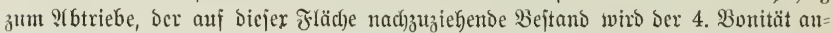
gefören uno bermutglich im normalen seanbarfeitsalter von 80 safren genubzt wocrbent, fo beträgt Der \{umtmarijd) 3utwact)

$$
6 \times 4,93 \times 30+6 \times 6,36 \times 10=887,4+381,6=1269 \mathrm{fm} .
$$

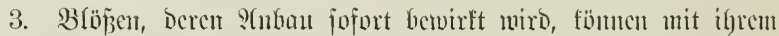

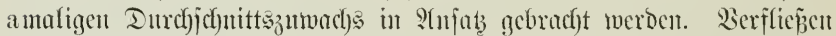

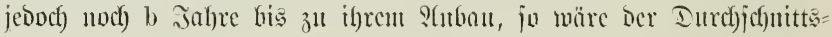
zแmad)

Beifpicl. Die Biöpe $4 \mathrm{~d}$ wiro jojort angebaut, Der nen begründete Bejtand

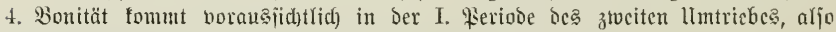

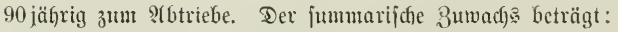

$$
1,2 \times 6,39 \times 40=307 \mathrm{fm} \text {. }
$$

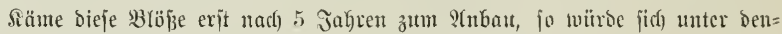

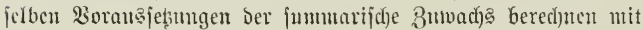

$$
1,2 \times 6,4 \times(40-5)=269 \mathrm{fm} .
$$

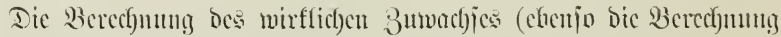

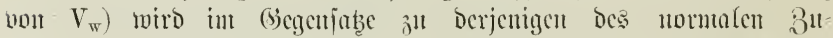
wachjes (und $V_{n}$ ) getrenut für jeden Bejtanto, unto twemt dicjer ant

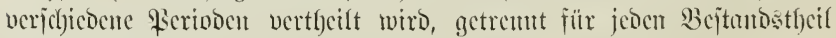
borgettomuter.

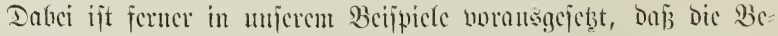

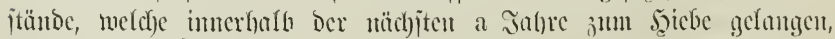

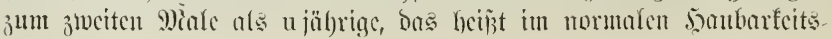




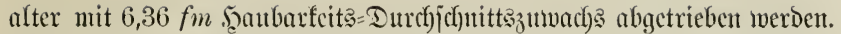
Wollte man bicje Borantsjeţung nicht unterjtellen, weil Deren Michtig=

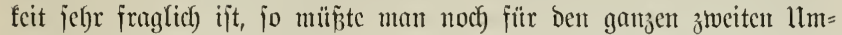

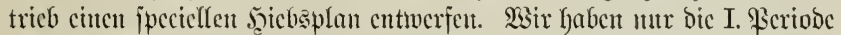
Des zweiten llutriebes poneit berindfictigt, als cinige ber bereits vor=

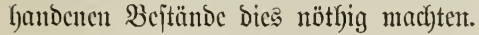

\section{d) Berednung des wirflichen Borratbes.}

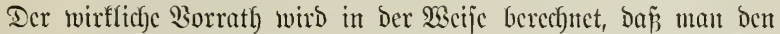

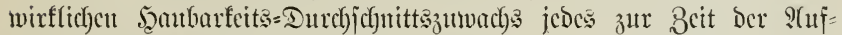
jtelfutg Des J̧icbsjabes vorbandenen Bejtandes mit bejien gegentwärtigem ?clter multiplicirt.

Der wirfliche Borrath jeder Blößj̧e, aud ment bicjelbe jofort beim $\mathfrak{B c g i n u t}$ dcs Beitraumes a angebaut wiro, ijt $=0$.

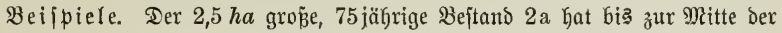

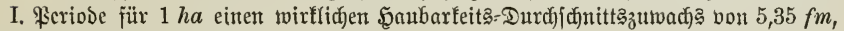
jeiı $\mathrm{V}_{\mathrm{w}}$ beträgt bałer:

$$
2,5 \times 5,35 \times 75=1003 \mathrm{fm} .
$$

Der 2futheil an Dem jeşt 25 jährigen Bejtande $5 \mathrm{c}$, weld)er mit 6 ha in ber

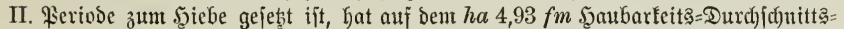
zuwachs. Gein $\mathrm{V}_{\mathrm{w}}$ beträgt baher:

$$
6 \times 4,93 \times 25=739 \mathrm{fm} .
$$

e) Tabelle zur Berednung bes wirflidgen 3 uwadbjes und Borrathes.

Nach) Den unter e und d gegebenen (Erläuterungen Dürfte bie $\mathfrak{B e}=$ rechmung Dcs jummarijed)en Scaubarfeit wirffichen Borrathes in nachjtebenter Tabefle leicht verjtündficf jein. Bur Erflärung Der Bertheilung ber Şiebsorte und ber $\mathfrak{W a b l}$ des

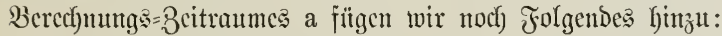

S(uch) ofne Beredfunng Der Borrüthe zeigt bas S(ltersflafijenver=

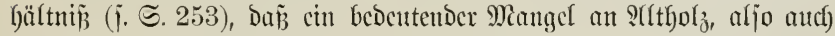

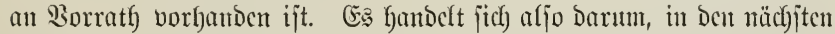
Jabrzchnten jparjam zu wirthjochaften, aljo nitcht Den ganzen jüfrlichen

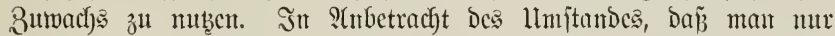
burch grofe Eparjamfeit vermeiben fann, mit bem S(btricbe viel volf=

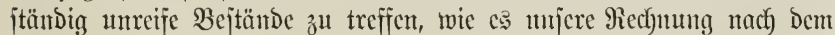
Flächen= untD nach Dem Majijenfachmerfe namentfich) Deshalfb thut, weil beijpielsmeije ein nur 60 jäbriger llntricb angenoumen wurbe, unter 


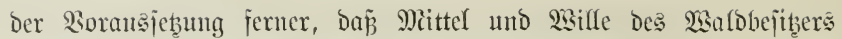

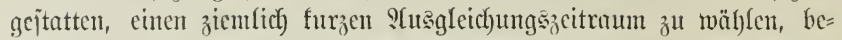

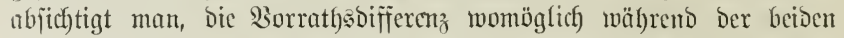

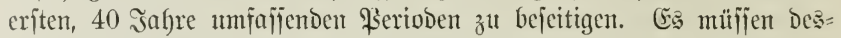
balb bicje beiden Perioben mit weniger ffläche und Mafje ausgejtattet werden, als bie ipäteren und jeb̧en wir vorläufig Den ? zeitraum gleidf Dem bered)mungszeitraum, aljo gleich jenem, fïr weldyen $\mathrm{Z}_{\mathrm{w}}$ ermittelt wiro, nämlich gleich) 40 . Errläuternd jei hierz̆ noch) $\mathfrak{b e}=$

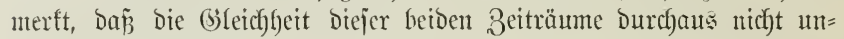
bebingt nöthig ift.

In bicjem Einne wurbe die Siertheifung Der Bejtände jo bemirft,

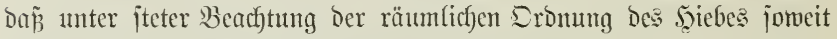
als thunlich bie ältejten Bejtände juterit zum Sabtriebe bejtimmt wurben. Irobecm war es nicht zu vermeiden, aud abgejelen von ben $20 s=$ hieben, mit bent Seiebe einige Bejtände zu treffen, beren mittleres Jjicbsalter fich crit auf 55 Jahre beläuft.

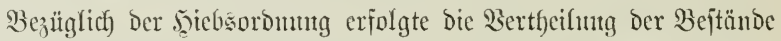

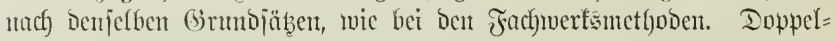
aftricbe fommen bier jebod) nicht vor, ba fie ber fier gewählte höblere, nämlich 80 jäf)rige llmtrieb nicht nöthig madhte.

Die theilmeije Berütjichtigung Der I. Fertode Des zmeiten llm= triebes erfolgte, wie jdjon crwähnt murbe, injoweit, als in berjelfou Die jebt bereits vorfyandenen Bejtände $2 \mathrm{c}, 4 \mathrm{~b}$ und $5 \mathrm{a}$, jowie ber im eriten Jabre zu begrïndende Bejtand $4 \mathrm{~d}$ höchjt wafricheintich jum

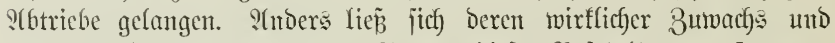
Borrath uicht berechmen. Die Eumme bicjer bejtünoc, 7,25 ha, um=

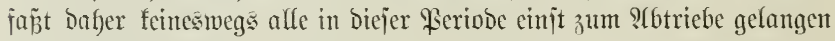
joflemben jeiebsorte. 


\begin{tabular}{|c|c|c|c|c|c|c|c|c|c|c|c|c|c|c|}
\hline \multirow{3}{*}{$\begin{array}{c}3 e= \\
\text { zeicf }= \\
\text { nusty. }\end{array}$} & \multirow[b]{2}{*}{ ffïctie. } & \multirow{3}{*}{$\begin{array}{l}\text { jof } \\
\text { att. }\end{array}$} & \multirow{3}{*}{ 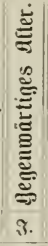 } & \multirow{3}{*}{ 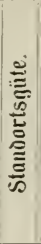 } & \multirow{3}{*}{ 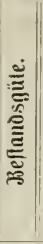 } & \multicolumn{5}{|c|}{ Jjerioden јu 20 Jagren. } & \multirow{2}{*}{ 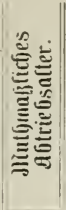 } & \multirow{2}{*}{ 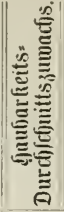 } & \multirow{2}{*}{ 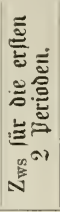 } & \multirow[b]{2}{*}{$\begin{array}{l}\text { Wirfi- } \\
\text { ficfer } \\
\text { Dor- } \\
\text { ratf. }\end{array}$} \\
\hline & & & & & & I. & II. & III. & IV. & $\begin{array}{l}\text { bes } \\
2 . \\
\text { um }= \\
\text { trie }= \\
\text { bes. }\end{array}$ & & & & \\
\hline & $h a$ & & & & & \multicolumn{5}{|c|}{ ha. } & \multicolumn{2}{|c|}{ כahr. } & \multicolumn{2}{|l|}{$\mathrm{fm}}$. \\
\hline $1 \mathrm{a}$. & 2,50 & Fi. & 5 & IV. & 4 & - & - & 2,50 & - & - & 55 & 5,71 & 571 & 71 \\
\hline b. & 2,50 & $"$ & 75 & " & 3 & 2,50 & - & - & - & - & 85 & $\begin{array}{l}5,35 \\
6,36\end{array}$ & $\begin{array}{l}134 \\
477\end{array}$ & 1003 \\
\hline c. & 10,50 & " & 5 & " & 3 & - & - & $\begin{array}{c}5,00 \\
-\end{array}$ & 5,50 & - & $\begin{array}{l}55 \\
75\end{array}$ & $\begin{array}{l}4,93 \\
5,36\end{array}$ & $\begin{array}{r}986 \\
1179\end{array}$ & $\begin{array}{l}123 \\
147\end{array}$ \\
\hline d. & 1,25 & " & 90 & " & 4 & 1,25 & - & - & - & - & 100 & $\begin{array}{l}6,30 \\
6,36\end{array}$ & $\begin{array}{r}79 \\
238\end{array}$ & 709 \\
\hline e. & 1,75 & " & 20 & " & 4 & - & - & - & 1,75 & - & 90 & 6,39 & 447 & 224 \\
\hline $2 \mathrm{a}$. & 2,50 & " & 75 & " & 3 & 2,50 & - & - & - & - & 85 & $\begin{array}{l}5,35 \\
6,36\end{array}$ & $\begin{array}{l}134 \\
477\end{array}$ & 1003 \\
\hline b. & 15,75 & $"$ & 40 & " & 4 & 1,00 & - & - & - & - & 50 & $\begin{array}{l}5,50 \\
6,36\end{array}$ & $\begin{array}{r}55 \\
191\end{array}$ & 220 \\
\hline c. & 1,00 & $"$ & 20 & " & 4 & - & 14,75 & - & - & 1,00 & 110 & $\begin{array}{l}6,19 \\
6,36 \\
6,10\end{array}$ & $\begin{array}{r}2739 \\
938 \\
244\end{array}$ & 3652 \\
\hline $3 \mathrm{a}$. & 4,75 & " & 40 & " & 3 & - & - & 4,75 & - & - & 90 & 5,30 & 1007 & 1007 \\
\hline b. & 11,75 & $"$ & 15 & $"$ & 3 & - & - & 6,00 & $\overline{5,75}$ & - & $\begin{array}{l}65 \\
85\end{array}$ & $\begin{array}{l}5,23 \\
5,35\end{array}$ & $\begin{array}{l}1255 \\
1231\end{array}$ & $\begin{array}{l}471 \\
461\end{array}$ \\
\hline c. & 0,75 & " & 100 & " & 4 & 0,75 & - & - & - & - & 110 & $\begin{array}{l}6,10 \\
6,36\end{array}$ & $\begin{array}{r}46 \\
143\end{array}$ & 458 \\
\hline 4a. & 3,00 & " & 90 & " & 4 & 3,00 & - & - & - & - & 100 & $\begin{array}{l}6,30 \\
6,36\end{array}$ & $\begin{array}{l}189 \\
572\end{array}$ & 1701 \\
\hline b. & 2,80 & " & 20 & $\prime \prime$ & 4 & - & - & - & - & 2,80 & 110 & 6,10 & 683 & 342 \\
\hline c. & 7,50 & " & 50 & $"$ & 4 & 7,50 & - & - & - & - & 60 & $\begin{array}{l}5,90 \\
6,36\end{array}$ & $\begin{array}{r}442 \\
1431\end{array}$ & 2212 \\
\hline d. & 1,20 & - & 0 & " & - & - & - & - & - & 1,20 & 90 & 6,39 & 307 & - \\
\hline e. & 1,00 & Ji. & 30 & $"$ & 4 & - & 1,00 & - & - & - & 60 & $\begin{array}{l}5,90 \\
6,36\end{array}$ & $\begin{array}{r}177 \\
64\end{array}$ & 177 \\
\hline
\end{tabular}




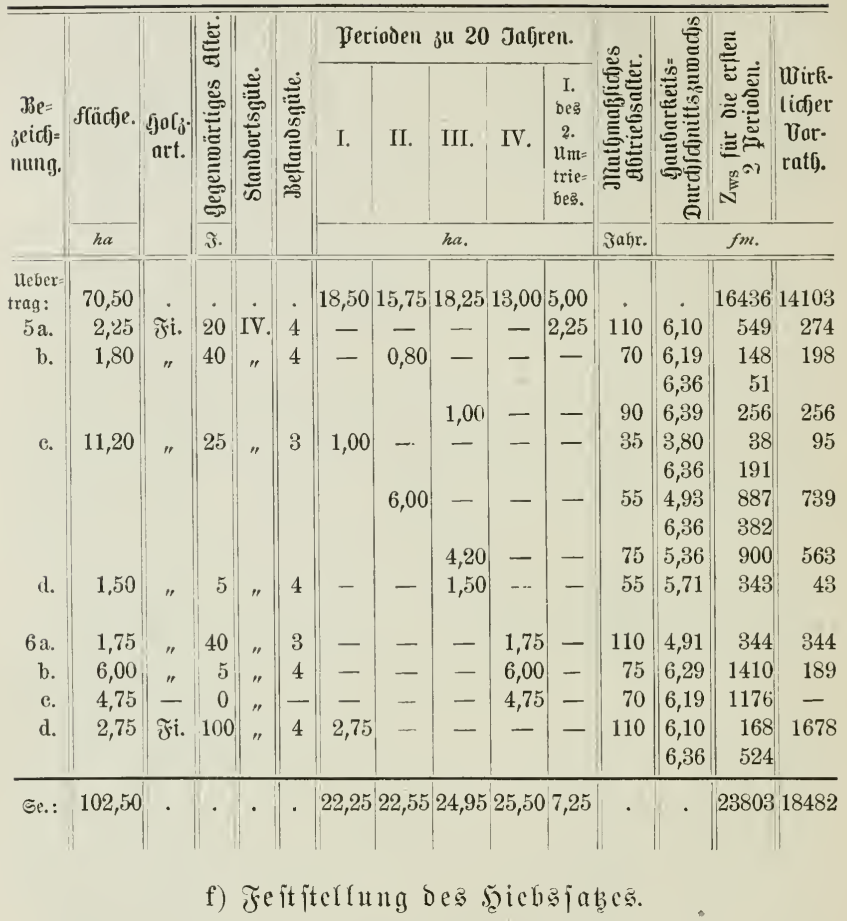

Der Sjebsjab einer Feriobe jebt jich znjammen ans bent inner= falb berjelben erfolgentoen wirflidfen Buwachs unt ben zu nubentoen voer zu crjparenden Theil ber Borratgs= Differenz.

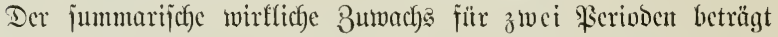
23803, für eine Periode aljo $\frac{23803}{2}=11901 \mathrm{fm}$.

Der IInterjichico zrvijchen $V_{w}$ und $V_{n}$ betrïgt

$$
18482-26076=-7594 \mathrm{fm} \text {. }
$$

S(us Den früber hervorgebobenen (Sründen beabjichtigt man bicien

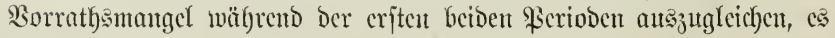
jind aljo in jeder Periode zu erjparen $\frac{7594}{2}=3797 \mathrm{fm}$. 


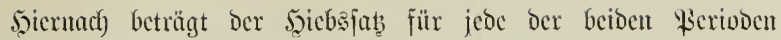
$11901-3797=8104 \mathrm{fm}$.

Dafielde Mejultat crbält man nach ber Formel

$$
\begin{gathered}
\mathrm{E}_{\mathrm{s}}=\mathrm{V}_{\mathrm{w}}+\mathrm{Z}_{\mathrm{ws}}-\mathrm{V}_{\mathrm{n}} \\
\mathrm{E}_{\mathrm{s}}=18482+23803-26076=16209 \mathrm{fm},
\end{gathered}
$$

fït cinc Fieriode jontach) $\frac{16209}{2}=8104 \mathrm{fm}$.

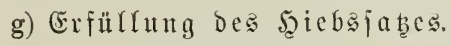

๙) (Exfte Beredrumg.

I. Feriobe.

Die ber I. Beriode vorläufig zugcwiejenen Beftände lajijen folgende 9 (ftricbšerträge erwarten:

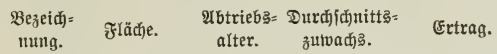

$$
\begin{aligned}
& \text { 1b. } 2,50 \times 85 \times 5,35=1137 \\
& \text { d. } 1,25 \times 100 \times 6,30=787 \\
& 2 \text { a. } 2,50 \times 85 \times 5,35=1137 \\
& \text { von } 2 \text { b. } \quad 1,00 \times 50 \times 5,50=275 \\
& \text { 3c. } 0,75 \times 110 \times 6,10=503 \\
& \text { 4a. } 3,00 \times 100 \times 6,30=1890 \\
& \text { c. } 7,50 \times 60 \times 5,90=2655 \\
& \text { wou } 5 \text { c. } 1,00 \times 35 \times 3,80=133 \\
& \text { 6d. } \frac{2,75 \times 110 \times 6,10=\frac{1845}{22,25 h a}}{10362 \mathrm{fm} .}
\end{aligned}
$$

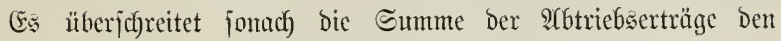
Ђૃicbs̄a tm $10362-8104=2258 \mathrm{fm}$.

Dbgleich Durrch eine wejentliche weitere Eriparung bie Şicbsfläche

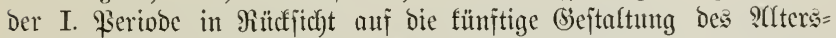

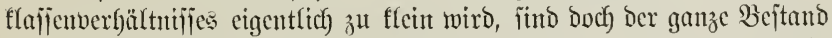
2a und von $4 \mathrm{e}$ etwa 3 ha ber II. \$eriobe jutzumeijen, jo ba $\tilde{\beta}$ aljo noch) $5,50 \mathrm{ha}$ und $2199 \mathrm{fm}$ eripart werden.

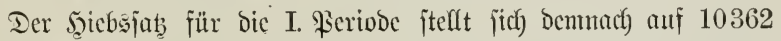

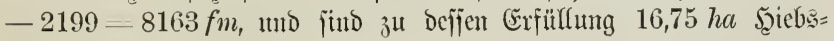
fläche nötthig.

Bon biejem Şicbşą̧e wären $11901 \mathrm{fm}$ auj ben Zumachs und als Eriparung, folglich regativ $3738 \mathrm{fm}$ auf Den 3orratfesmangel ju recthren. 
II. \$eriode.

Da it Der I. Reriode 3738 fm cripart wurben, reducirt fich bic

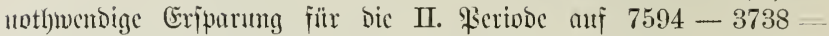
$3856 \mathrm{fm}$.

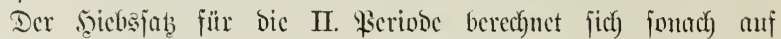
$11901-3856=8045 \mathrm{fm}$.

Die biejer Fieriode vorlä ufig in der Tabelfe und aus ber $I$. Pie=

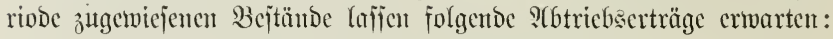

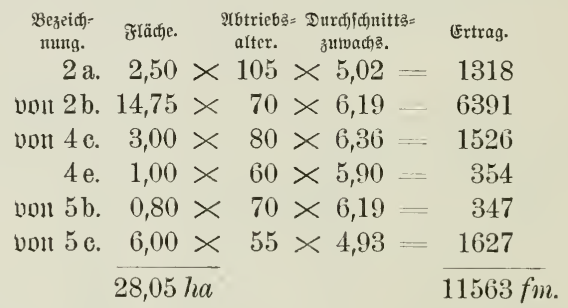

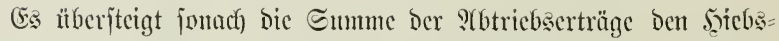

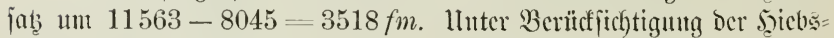

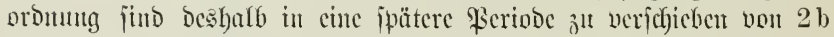
5 ha mit $2166 \mathrm{fm}, 5 \mathrm{~b} 0,80 \mathrm{ha}$ mit $347 \mathrm{fm}$ und von $5 \mathrm{e} 3,5 \mathrm{ha}$ mit

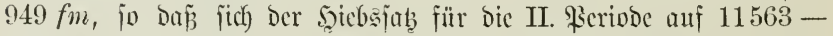

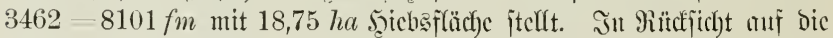

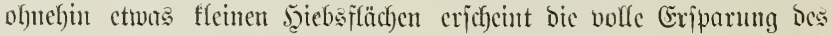

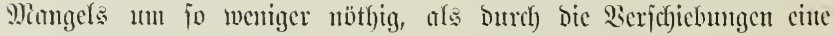

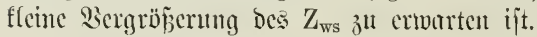

\section{ß) Bweite Beredjmung.}

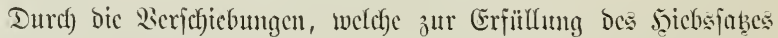
Der beiben erften Perioben jtattgefunden haben, wurben bic s(btricbs=

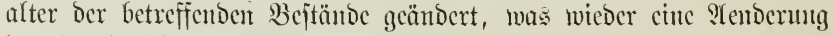

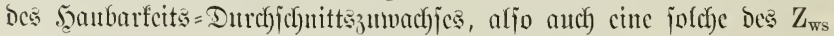

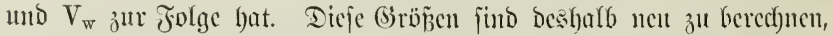

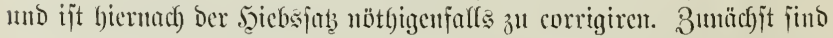

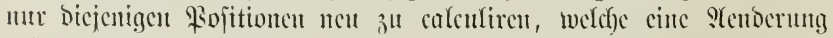

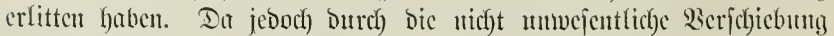

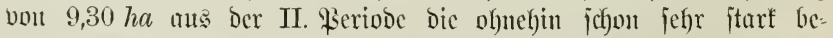

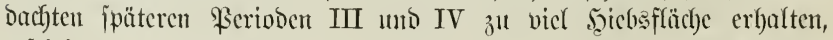

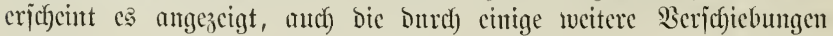

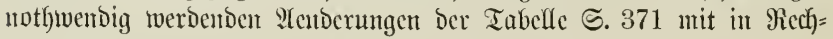


Iung ju jtellen. Dieje werden wenigjtens darin bejteben, baj 1 a แnt $1 \mathrm{c}$ ans ber III. ut bic IV. Ferivde, fowic 1 e mb $6 \mathrm{c}$ ats ber IV. Pieriobe in bic I. Des jweiten Umtriebes verjedjoben werdent.

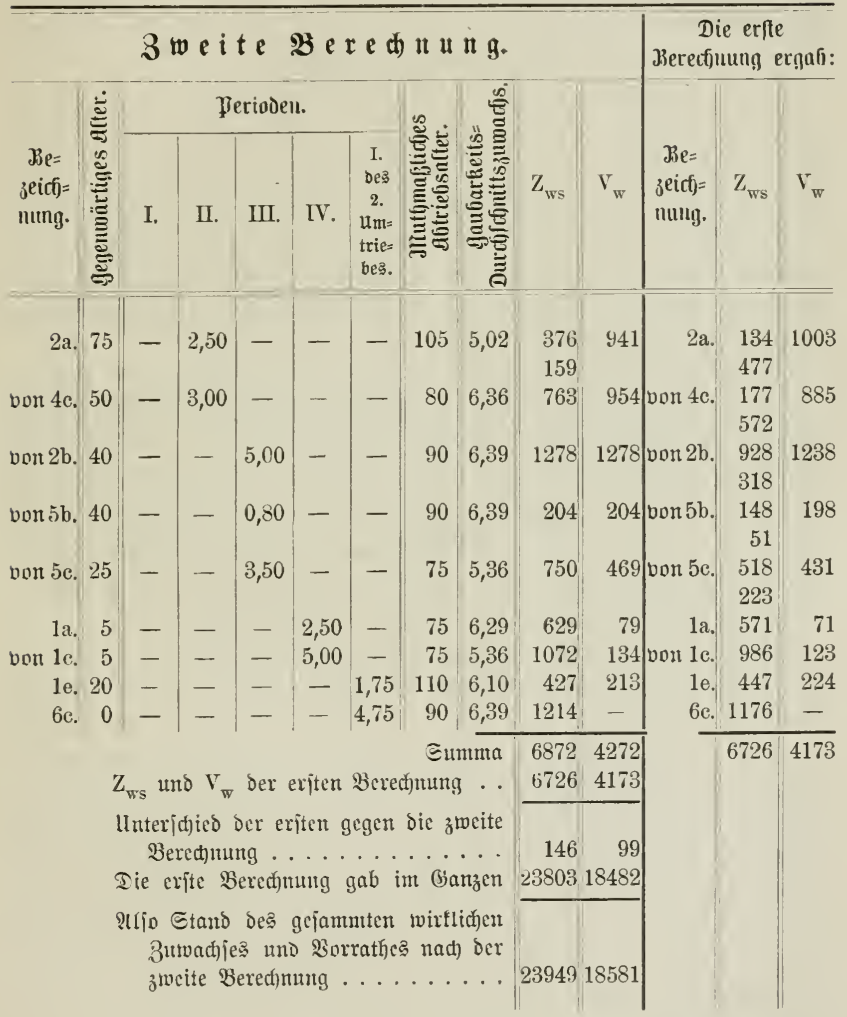

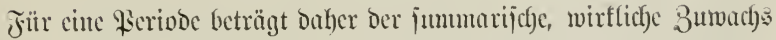
23949 $2=11974 \mathrm{fm}$.

Die zน cripparende Siorraths sifferenz beträgt im Ganjen 18581 $26070^{\circ}-7495$. 
Bertheift man biejelbe auf zroci \&erioden, fo entfallen auf jede $\frac{-7495}{2}=-3747$.

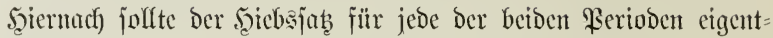
(id) betragen:

$$
11974-3747=8227 \mathrm{fm} .
$$

Der Iluterjechicd zwijchen bem nach ber erjten und Dem nach Dex

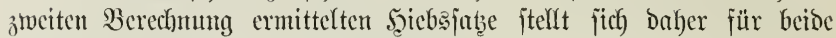
Berioden auf $16454-16264=190 \mathrm{fm}$. Err ift jo unbedeutend, jährlick

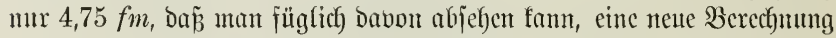

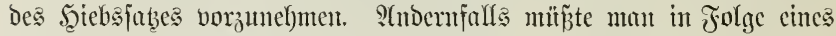

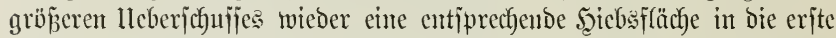

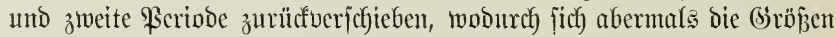
$\mathrm{Z}_{\mathrm{ws}}$ und $\mathrm{V}_{\mathrm{w}}$ etwas c̈ndern würden.

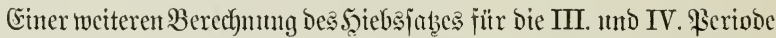
bedarf es nicht, ba man ofynefin nicht erwarten faun, bar affe Sioraus= feb̧ungen in Erfüllfung geben werden, anf welche fith bie fütr bie beiben

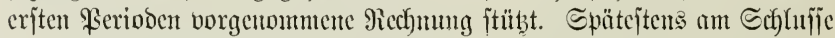

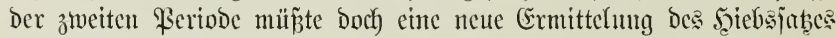
ftattfinden. Wair verjict)ten Daber Darauf, dus Beifpicl weiter Durd)=

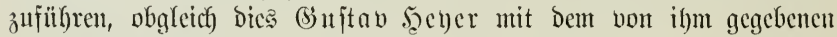

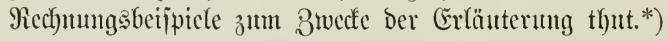

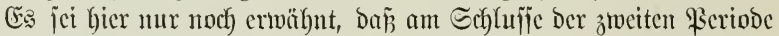

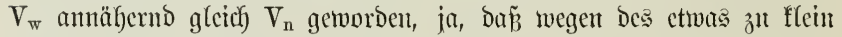

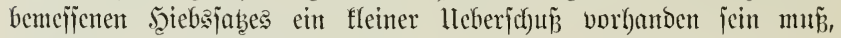

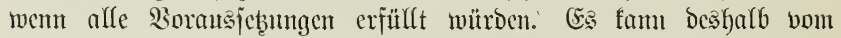

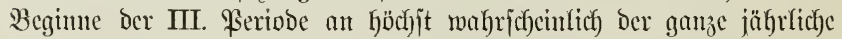

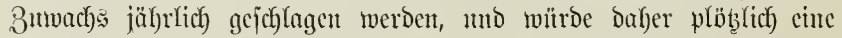
bedcutende Errböhung des şicbsjab̧es cintreten. Will man bies ver=

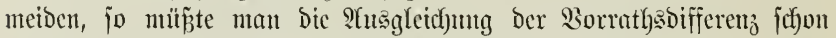
jebzt auf mehr als zwei ßeribden vertheilen. Für bie betoen erjten

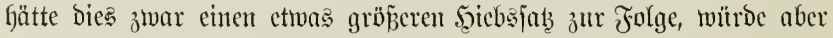

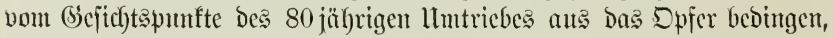

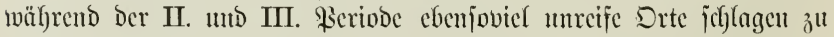

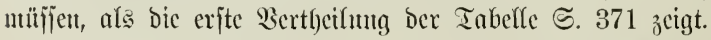

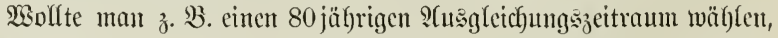
jo fëmnte man auf unjere erjte Bertbeilung zurüdgreifen. In jeber

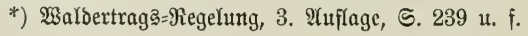




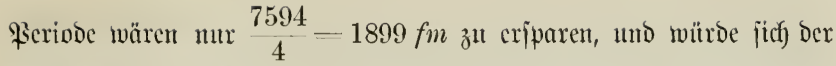

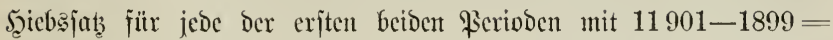
$10002 \mathrm{fm}$, für beioe jujammen mit $20004 \mathrm{fm}$ berectynen. Da nun

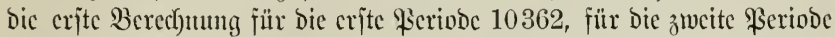

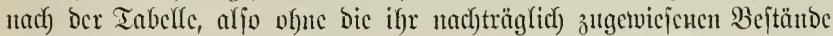
2a und $4 \mathrm{c}, 8719 \mathrm{fm}$, für beide zujammen $19081 \mathrm{fm}$ ergicbt, jo wären umr ivenige Bierjefiefuntgen nothwendig, weldye auf $Z_{w s}$ und $V_{w}$ nur eincu ganz geringen Einflü baben fönnten. Man wäroc aus

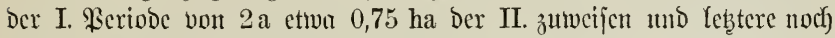

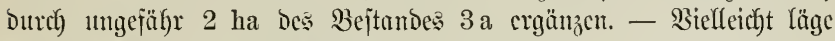

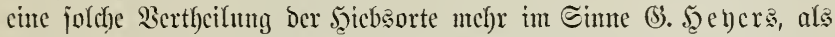

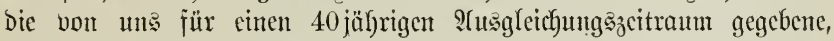

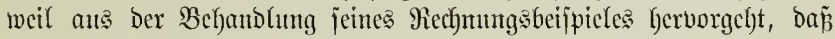

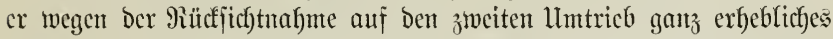

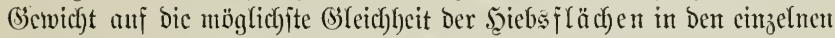

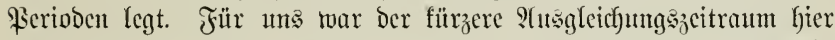

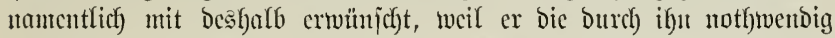

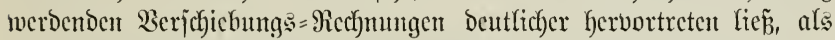
ç ein 80 jäl)riger Zcitraum gethan hättc.

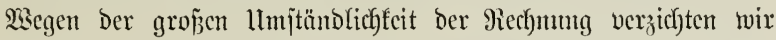

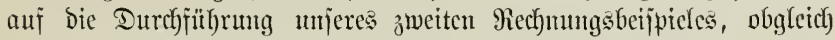

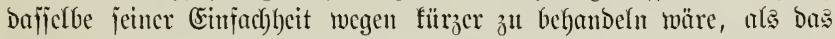
crịte von uns gerwäflte. Diejes war aber gceigneter, Dent (biang ber

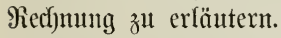

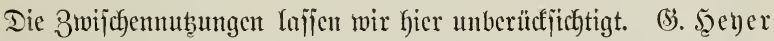

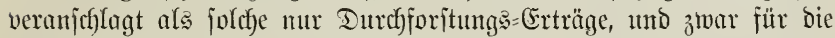
einzechen Bejtände auf Den Beitraum ber I. Feriode. Dubei barf

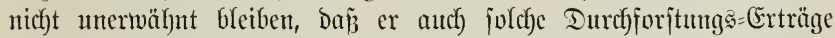

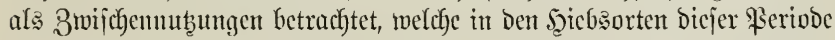
ausfallen.

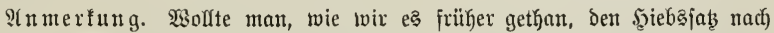

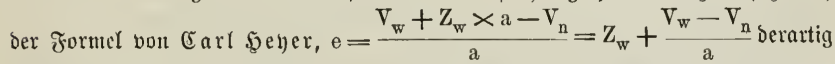

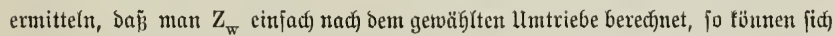

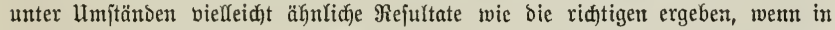

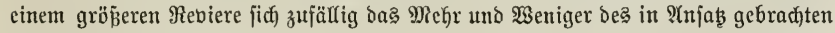
$\mathrm{Z}_{\mathrm{w}}$ ausgleidft. (Getwöhnlid, wirb aber ber Fehler erheblich herbortreten, weil derjelbe

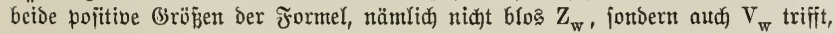




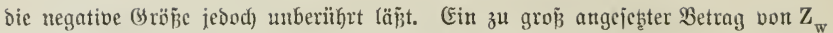

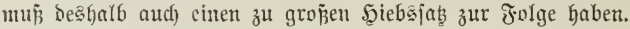

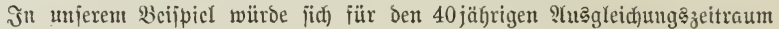
bie giechnung fiellen wie folgt:

$$
\mathrm{Z}_{\mathrm{w}}=44,95 \times 5,38+57,55 \times 6,36-607,8 .
$$

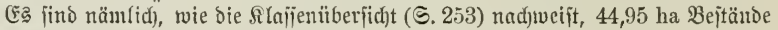
3. unb 51,60 ha Beịtände 4. Bonität vorfanden, und müij̈en bie 5,95 ha Blör̈en ifres Standortes wegen bor 4. Bonität zugerechnet werden.

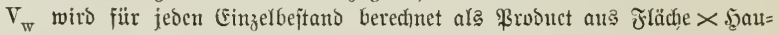

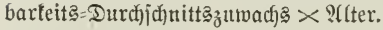

2xljo 3. $\mathfrak{B}$.

$$
\begin{gathered}
\text { 1a } 2,5 \times 6,36 \times 5=79,5, \\
\text { b } 2,5 \times 5,38 \times 75=1008,75, \\
\text { c } 10,5 \times 5,38 \times 5=283,45, \\
\text { d } 1,25 \times 6,36 \times 90=715,5 \\
\text { u. } \text { i. w. jür alle } \mathfrak{B e i t a ̈ n d e . ~}
\end{gathered}
$$

Summe bon $\mathrm{V}_{\mathrm{w}}=19163 \mathrm{fm}$.

$\mathrm{V}_{\mathrm{n}}$ Gleibt, wic Seite 368 nachgetwiefen, $26076 \mathrm{fm}$.

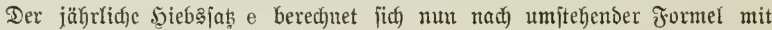
$608+\frac{19163-26076}{40}=435 \mathrm{fm}$.

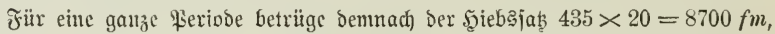
aljo $537 \mathrm{fm}$ mefr, ala ifn bie ridgtige Michnung für bie I. \$Periode ergicbt.

\section{SUlfgemeine 9 ü roigung Des 3 erfahreus.}

Som Ctanopunfte ber blopien Materialertragsregelung betrachtet,

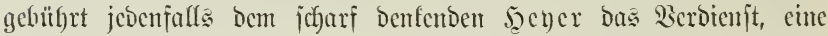
Siegelungsmetfode mit logijcher Eonfequenz durthgefüf)rt zu haben, welche jict) von ben bisher bejprochenen 9iormaforrathismethoden

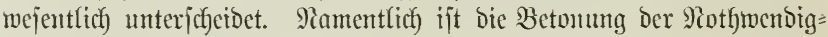

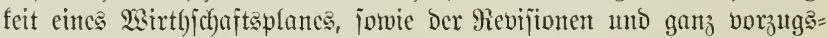

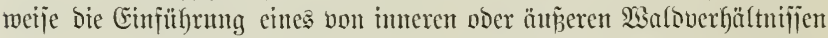

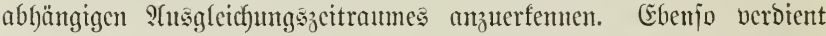

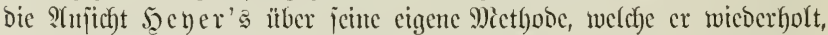

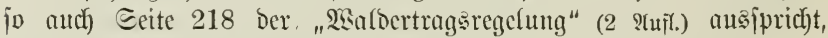
volle Beadtung, weshall wir fie hier wörtlidf wiedergeben:

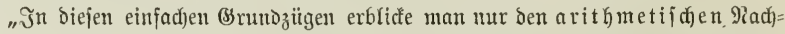

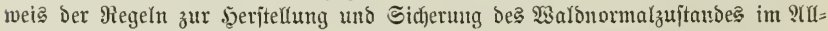
gemeinen - aber feinešnegs die Miöglidjfeit einer jederzeitigen ganz itrengen

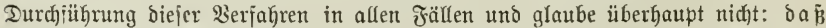
bie praftifde Etaţoronung mit gutem Erfolge in bicengen Đoränzen einer mathematifacn formel fid einztwängen lafie. Wir wieberbolen 


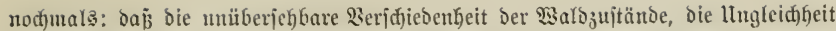

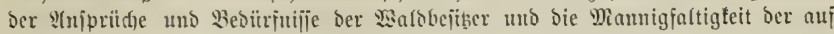

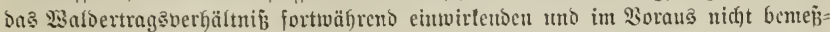

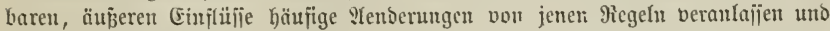
mitunter jelbit smingen, Den ¡đon mühjam ermugenen Normaljujtand einer voer

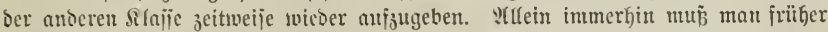
noer ipäter bie bezeid)neten Wisge wieber einjolagen, wenn man mit ben geringiten Dpjern ber hödjiten Stuje ber \$isthjdajt - Dem Sădonormaljujtande - jid) an= näbern เvill."

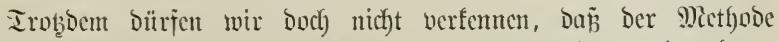
Dever's chenfalls nidgt volfe Correctheit zugejprochen werben fann.

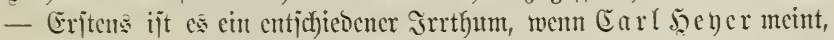

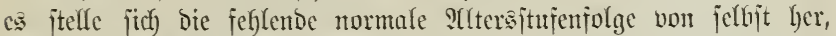
johulb heim siorfandenjein bes siormalvorrathes entweber ber normale

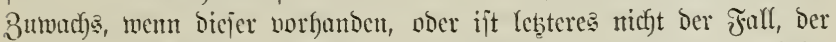

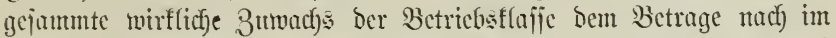

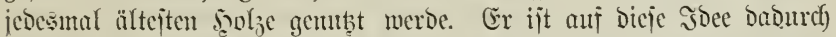

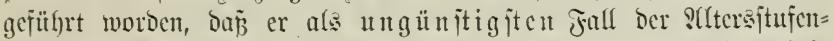

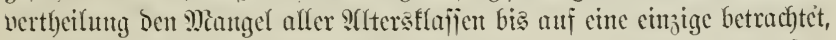

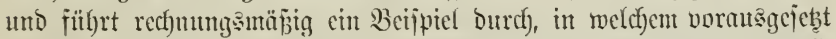

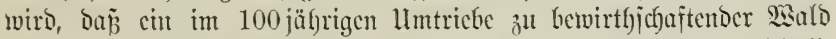

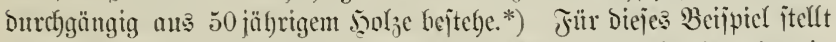

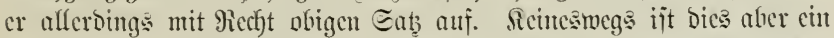

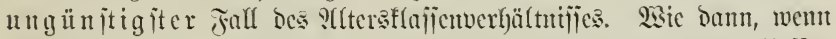

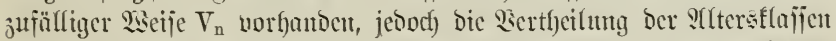

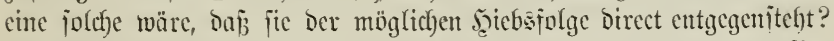

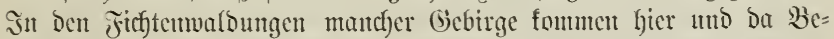

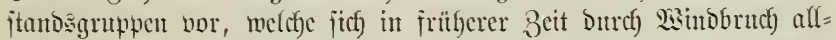
mälig verjüngten unto in Folge bejien eine jo untgünitige Bestlfeiluty

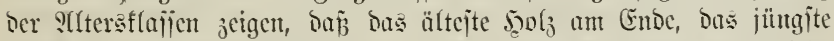

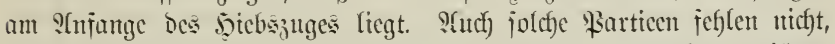
mo unjere 2 sorfaf)ren an Berghängen von unten ntad) vbent verjüntgt

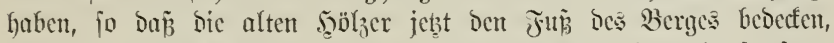
mährend oben bie jüngiten zu finden jint. Bect abermula jo fort=

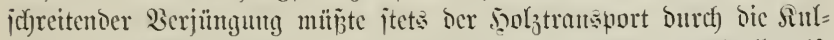

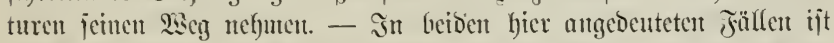

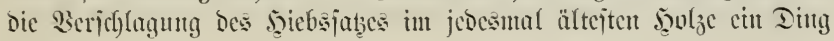

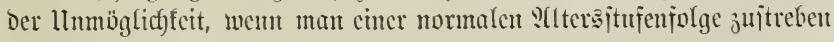

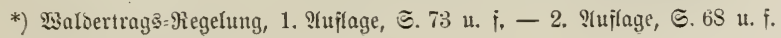




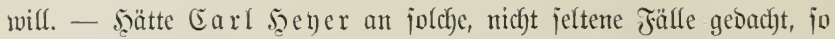

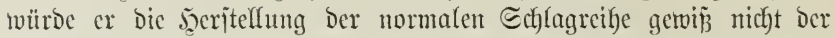
Des Rormaluorratges jo untergeoronet haben. - $23 i r$ behaupten im Gegentbeil, Daż leb̧terer non jelbjt jich entwicfelt, wenn man ben beiden

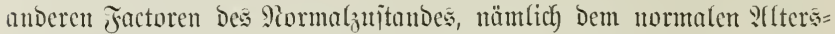

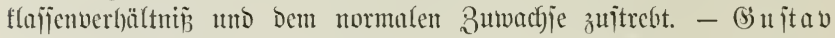

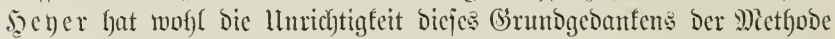
ipäter crfannt und jugt beshalb*): "sjt Der normale Borratf umb Der normale Bumadfs vorfanden, io jtellt jich bie normale sflers= itufenfolge mit normalen Flächenantheilen ber einzelnen Etufen allmälig ganz von jelbjt her, wenn man jälyrlich ober periobijch Den normalen Etat (welcher gleich) Dem normalen Buwachje ijt) muţt und zugleich) für jofortige গacfjudfyt Der abgetriebenen Bejtände forgt." Ģanj richtig ijt ber Sats aber immerfin nicht, Denn bie ?iotfmentigfeit eitter richtigen Bertheilung Der :Aterseflaijen ijt Darin nicht ermähnt.

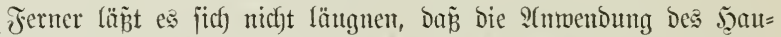

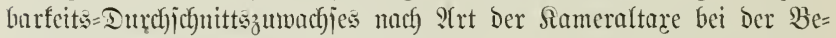
rechnung Des Jjiebsjates biejen jelbjt unrichtig macht. Bezühlich Der

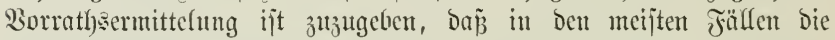

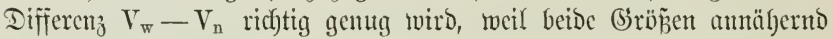
von Demielben Fefler berïfrt merben. S(nders ijt es aber mit bem berechneten joiebsjabe uno Der wirflich erfolgenden Pubung, Deren

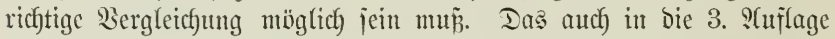

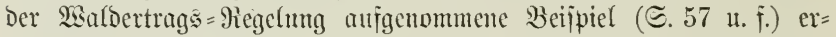

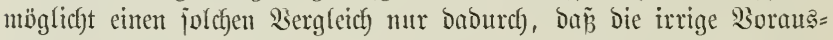

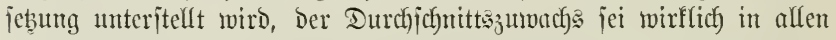
Rebensaltern Des Bejtandes Derjelbe, näm(ich) gleicf) Dem Scaubarfeits= Durchichnittşzumachs des ujäbrigen Bejtandes. In bem jpäter (1. e. ङ. 227 น. i.) ausgefülrten Rechmungšbeijpiele, nach weldfem wir aucf) Das unjrige berectuet baben, ijt $\mathrm{Z}_{\mathrm{W}}$ mit Scilfe cincs für einen ganjen

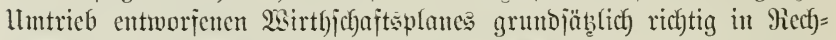
nung geitellt, allein es fann Dody Sicmano erwarten, baj wirflich alle

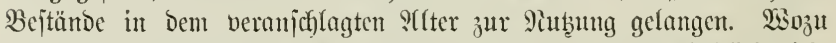

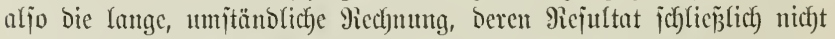

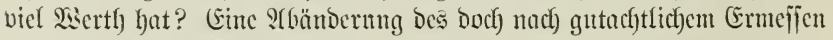

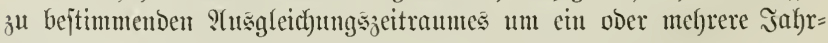

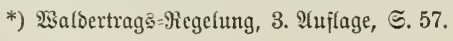




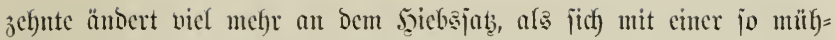
jam anf gunze llmtriebe hinantsgejüf)rten Piecfunmg bertrïgt.

lleberhaupt famn es jef)r jweifelfajt jein, ob man nach) ben in

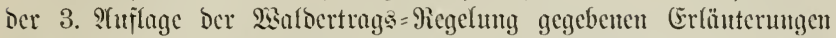

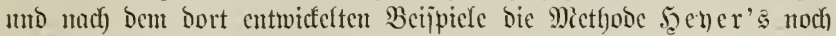

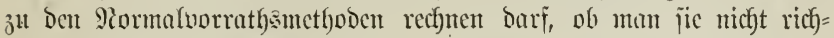
tiger zu Den Fach)werfsmetljoben zäh)

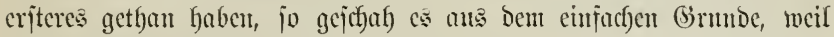

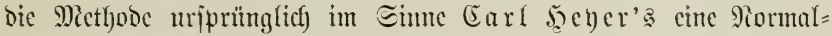

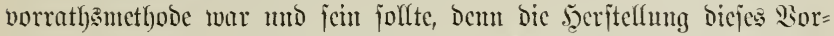
rathes bilbet ifyr nächjtes Biel. Stuch erfemt (5. Şeyer jwar bie

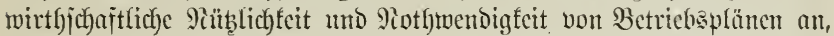
meint aber Doch, Daj bicje nicht Durch) Das Rrincip ber Miethode be=

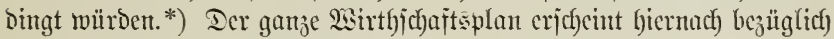

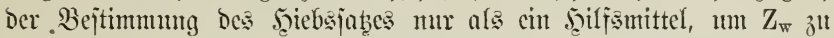
funben; man wendet es mur notbgebrungen an, weil man bicje (3iróp̃e leider micht anders ermitteln famm. Die Miethode Ear 1 jecler's ijt Deshalb ifyer fijtorijden (Entwidelung mad) allersinge cine Plormal= vorratgemethode.

PAtbers ijt es freilich mit ber weiter ausgebildeten Metfode

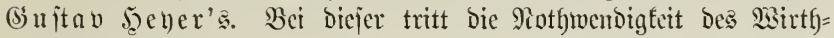

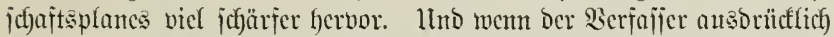

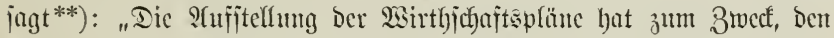
Etat cines ganzen $\mathfrak{s u l d e s}$ zu bejtimmen .....", fo crichemt unter Berïffictbtigung Der gegebenen Errlänterungen uno Becijpicle die

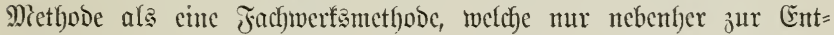

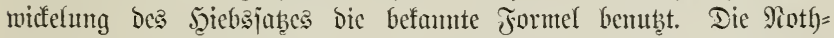
mentigfeit leb̧terer entfällt cigentlich) volljtändig, Dent chenjogut wie

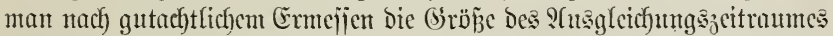

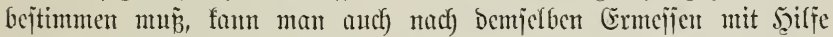

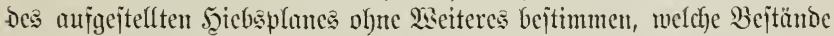
unto twelche Mafjen in Den eriten Rerioben zum Jiebe fommen jollen.

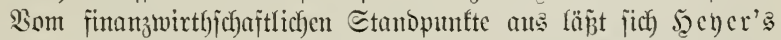

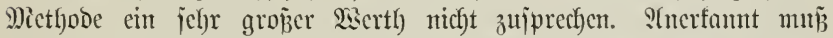

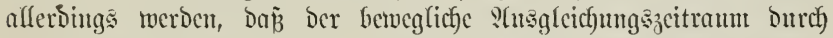

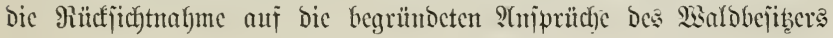

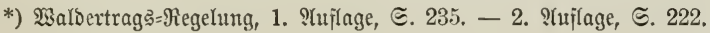

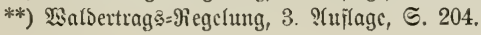


Diejelbe über bic Sameraltaxe und auch ïber Seundeshagens Ser=

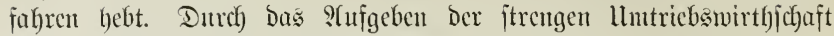

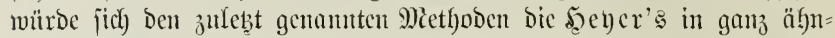

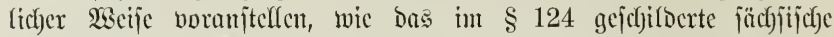

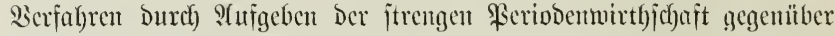
ben älteren Fachlverfen, wenn nicht bie richtige Ermittelung $\mathrm{Des} \mathrm{Z}_{\mathrm{W}}$

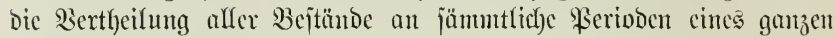
Ilutriebes nöthig machte.

(Enolich) ijt aber bie ganze Rechnung nad) Dem Durchjodnittşu=

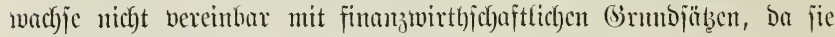

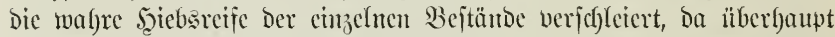

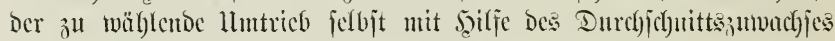
niclyt ermittelt werden fann.

\section{$\S 129$.}

\section{Tiarl' Dexfalgren.}

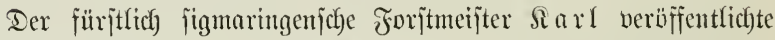

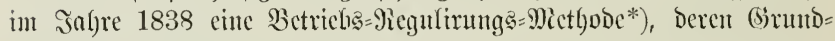
gedanten wolyl aud burd) bie öfterreichijche Slameralture antgeregt wor=

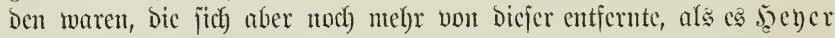

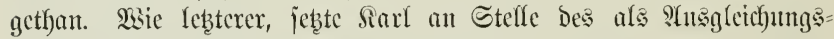
zeitraun von Der Sameraltaxe gewäh)lten llutricbes eincu anderen,

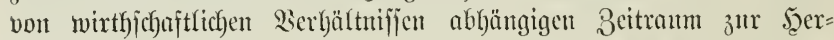
jteflung Des Romalzuftandes. Ferner ermittelte er Den Rormalvor= ratl) mit Sziffe von Ertragstajeln, Den wirflidben Durch Erbebung Der

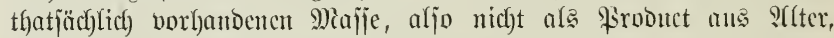

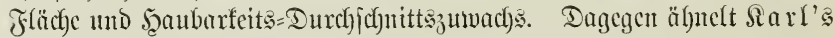
Ertragsformel ber sel)ex's in po fert, als barin chenfalls bie popitive voer negative Differenz zwijchen normalem und wirflichem Borratlyc

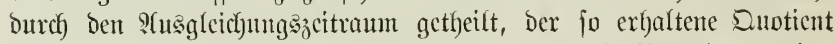

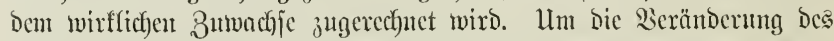
leb̧teren, ס. h. Defĩen allmälige S(nnäberung an ben normalen in Fiech)=

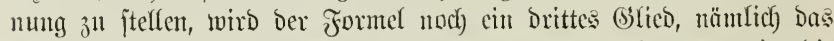

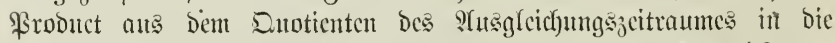

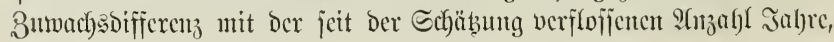

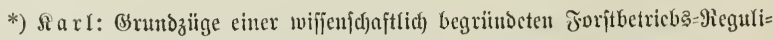
rung: Miethode u. \{. w. Signtaringen, 1838. 


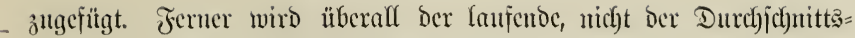
zuwachs der Pieclumung zu (Sirumbe gelegt.

Sarl's Formel für Den Şiebsjab lautet:

$$
\mathrm{e}=\mathrm{Z}_{\mathrm{w}}+\frac{\mathrm{D}_{\mathrm{m}}}{\mathrm{a}}+\frac{\mathrm{D}_{\mathrm{z}}}{\mathrm{a}} \times \mathrm{n} \text {. }
$$

ES bedcuten barin:

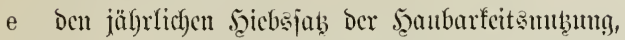

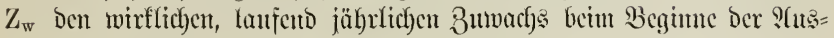
glcichumgszcit,

$\mathrm{D}_{\mathrm{m}}$ bic Differenz zwijchen wirf(ichem und nomalem Sourathe, aljo entrocoer $V_{\mathrm{w}}-\mathrm{V}_{\mathrm{n}}$ oder $V_{\mathrm{n}}-\mathrm{V}_{\mathrm{w}}$,

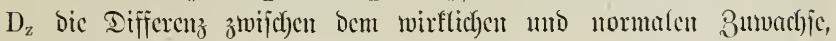
aljo entroeder $Z_{w}-Z_{n}$ oder $Z_{n}-Z_{w}$,

a bic ?.usgleicl)ung

n Dic : S(nzahl Der feit Der Schäßzung verflofifenen Sabre.

Für ben ?anfang Des eriten Jahres, aljo beim Beginne Der Schäbutug ift $\mathrm{n}=0$. - Da nun itreng genonmen in Folge bcs

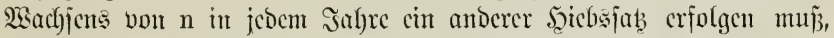

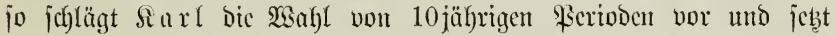

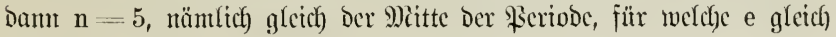
grof́ jein joll.

Bor Der Mafjendifferenz $\left(D_{m}\right)$ wird jtets bas 3erdfen + beizu= bebalten jein, wenn $V_{w}>V_{n}$, im entgegengejebten Falle tritt bas Bcidyen - ein. Dic Buwadysdifferenz $\left(D_{z}\right)$ erfält jtets das entgegen= gejebte Borzechen Der Majfendifferenz. Sit Iebtere pofitiv, jo wiro critere negativ und ungefelyrt.

Der Witrthfchaftsplan joll mux ganz allgemein gebalten werden,

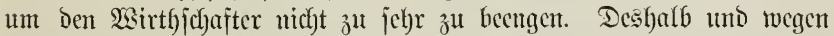

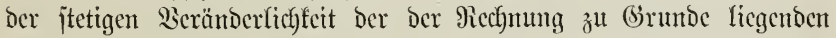

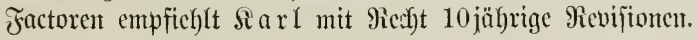

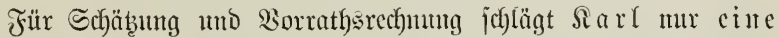

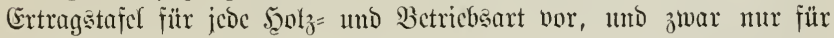
bic bejte Etandortsflafic. Dicje wirb gleicl) 1 gejetzt, und merden bei ber Bonitirung bie geringeren Böüteflajicn jomohl bes Etandortes als Dce Beitandes in Decimalen ausgedrïcft. Dic Stantovts = Bontiät nennt Siarl "Ertragsfähigfeit", Die Des Bcitandes "Ertragsvermiögcu“.

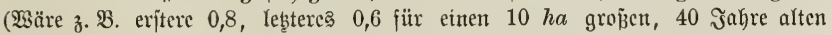

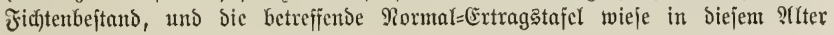


$200 \mathrm{fm}$ (Ertrag nad), io würbe jeine jeţige Majie $10 \times 0,8 \times 0,6 \times 200=960 \mathrm{fm}$

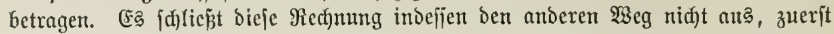

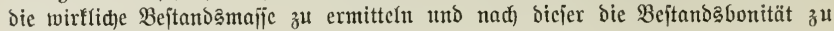
beziffern.)

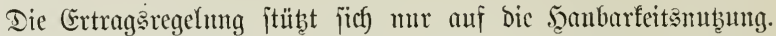
Der Ertrag Der Durchforjtungen wird jummarijch crmittelt und $\mathrm{D} c \mathrm{~cm}$ J̧iebsjabe zugejchlagen. Man zicht von ber gcjammten Fläche des 5ुoljhodens bie jüngiten, itets auber ber Durchforjtung liegenden

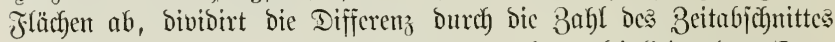
Der Wiederfyolung Der Durchforjtungen uto multiplicirt ben Dun=

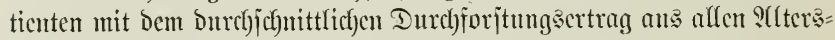

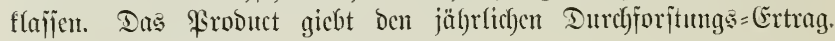
(1. c. § 13 uा 39.)

\section{アedtungabeipiel.}

Beptimmung Des Sciebsjabes für ben 102,5 ha f̧olzhoden ent= haltenden $\mathfrak{B a l d}$ im 60 jährigen Umtriebe. -

Ceben wir Dor Sï̈ze wegen voraus, dic Fläche von 102,5 ha jei bereits auf cinc Etandortsgüte rcoucirt, welcher bie Erfabrung:

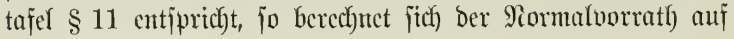

$$
8615 \times \frac{102,5}{60}=14717,3 \mathrm{fm} .
$$

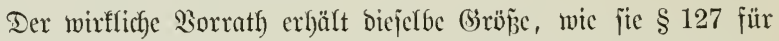
Die গุundeshagen'iche Mietfode mittbeilt, nämlich 15204,8 fm.

Icr normale 8 uwachs ijt

$$
354 \times \frac{102,5}{60}=604,75 \mathrm{fm} .
$$

Der mirflict)e Bumact) wird als laufender für bie cinzelnen $\mathfrak{B} e=$

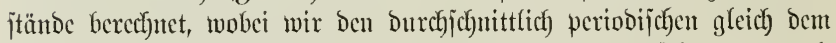
laufend jährlichen jeben fönnen, da bic betreffenton Tafeln (\$ 11 uto

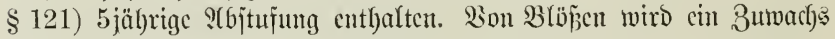
uicht angcjebst. 
23inflidyer 3uwachs:

1 a. $2,5 \times 2,8=7,00 \mathrm{fm}$,
b. $2,5 \times 5,6=14,00 "$
c. $10,5 \times 1,4=14,70 "$
d. $1,25 \times 5,8=7,25 "$
e. $1,75 \times 6,2=10,85 "$
u. j. w.

6a. $1,75 \times 6,8=11,90$,

b. $6,00 \times 2,8=16,80$,

c. 4,75

d. $2,75 \times 4,6=12,65$ "

Sumute $102,50 \mathrm{ha} . \quad 513,80 \mathrm{fm}$ wirffict)er 3 umadj).

Scicruach Der Şiebsfab für bic folgendent 10 Sahre, twenn wir

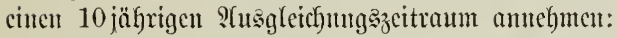

$$
\begin{aligned}
& \mathrm{D}_{\mathrm{m}}=15204,8-14717,3=+487,5 . \\
& \mathrm{D}_{\mathrm{z}}=604,75-513,80=-90,95 . \\
& \mathrm{e}=513,8+\frac{487,5}{10}-\frac{90,95}{10} \times 5=517 \mathrm{fm} .
\end{aligned}
$$

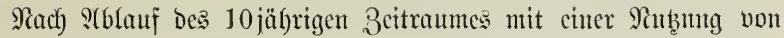
$5170 \mathrm{fm}$, weldye aus Den $\subseteq .346$ nachgewiejenten Bejtänden, mit Der

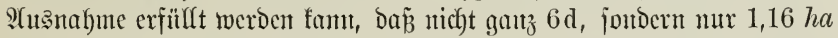

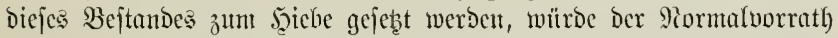

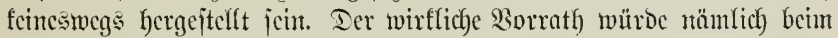
Beginne Des zweiten Jahrzehntes $15303 \mathrm{fm}$ betragen und wäte bicr=

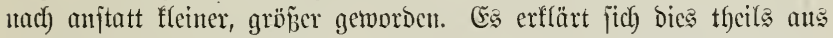
Den während Des Jahrzechntes erfolgenden 3uwachsveränderungen, da Der wirfliche 3uwachs vou 513,8 auf 597,1 gejtiegen ijt, theil: ba=

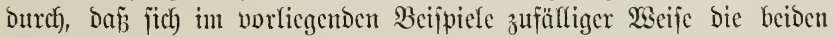
(ślieder ber Formel, weldye $\mathrm{D}_{\mathrm{m}}$ und $\mathrm{D}_{\mathrm{z}}$ betreffen, gegenjeitig bis auf bie geringe Differenz bon 3,28 ausgleteffent.

Wollte man, was un jedoch Der 2orjefyrift Sarl's zuwiber zu

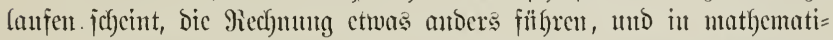
icher (Eorjequenz Die Borzeichen nicht für $\mathrm{D}_{\mathrm{m}}$ und $\mathrm{D}_{\mathrm{z}}$ entgegenge\{ebt geben, fontoern für $\left(V_{w}-V_{n}\right)$ und für $\left(Z_{w}-Z_{n}\right)$, dic Formel aljo unter alleı Umîtänden folgendermaj̧en faffen:

$$
\mathrm{e}=\mathrm{Z}_{\mathrm{w}}+\frac{\mathrm{V}_{\mathrm{w}}-\mathrm{V}_{\mathrm{n}}}{\mathrm{a}}-\frac{\mathrm{Z}_{\mathrm{w}}-\mathrm{Z}_{\mathrm{n}}}{\mathrm{a}} \times \mathrm{n},
$$




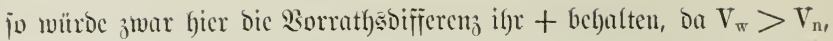

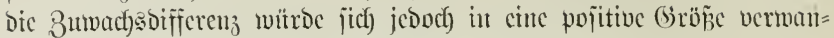

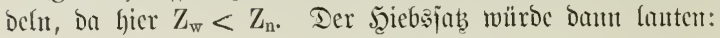

$$
\begin{aligned}
e & =513,8+\frac{15204,8-14717,3}{10}-\frac{513,8-604,75}{10} \times 5 \\
& =513,8+48,75+45,47-608 \mathrm{fm} .
\end{aligned}
$$

Berect)nen wir ficrnach abermals Den mirfliffen Borratl) unter

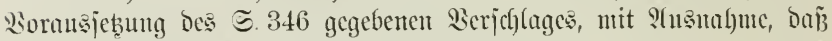
wou $6 \mathrm{~d}$ mtr 2,56 ha zum Şiebe foutmen, um den Jुicbsjats vou 6080)

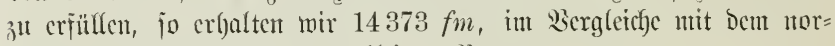
malen Borrath aljo cinen zit flciucu Betrag.

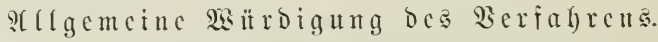

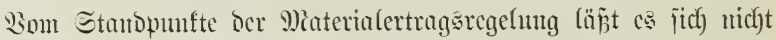

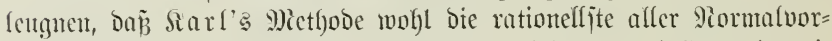

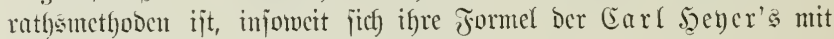

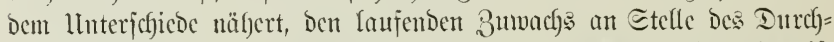

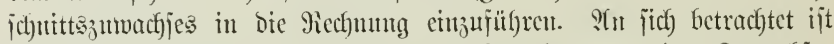

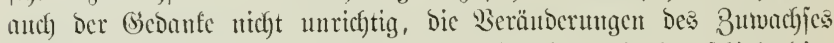

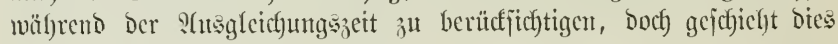
Durch Dic Sfunchoung Des lebten Formelgliedes $\frac{\mathrm{D}_{z}}{\mathrm{a}} \times \mathrm{n}$ uicht iut cut=

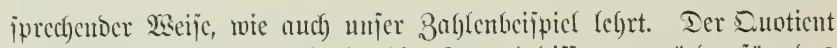

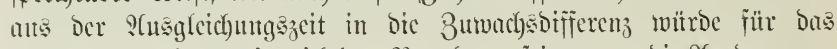
Mejultat un bam cin ridftiger Fiegutfator jein, wem bie ?enberungen

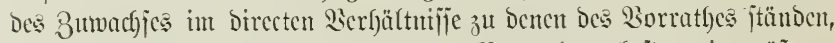

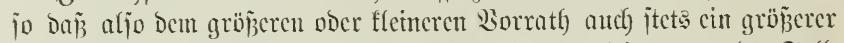

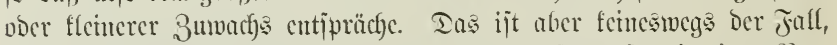

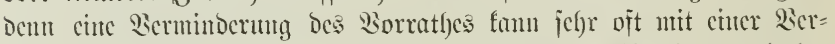

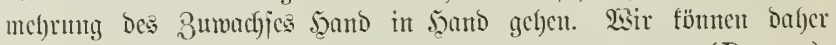

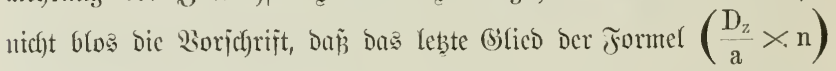

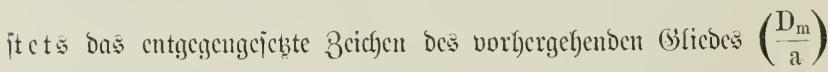

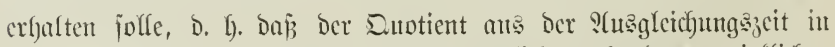

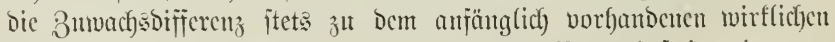
Buwach) aboirt werben mitfice, fo lange ber Bortuth itcigt, iut um= 
gefebrten Falle abor abujichen jei, wem ber Siorrath jich vermutbert,

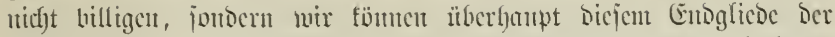

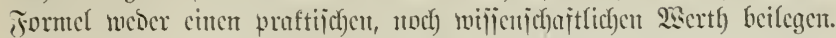

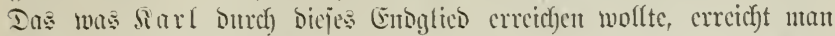

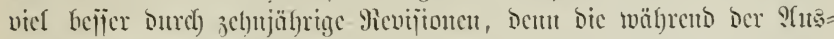

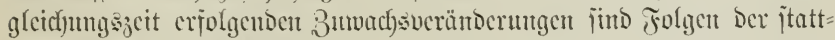

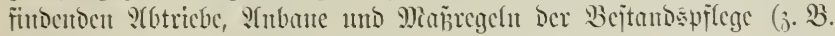

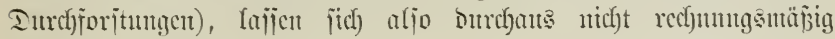
voratsiscjtument.

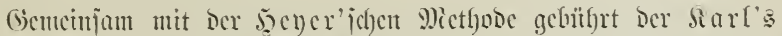

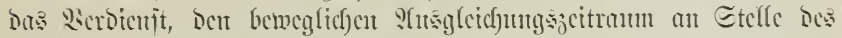

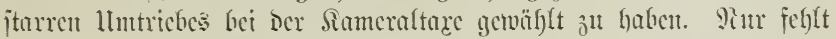

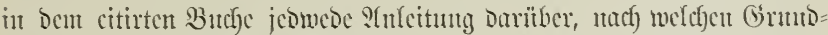

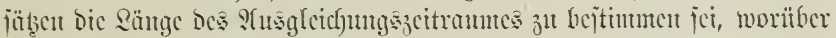

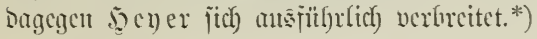

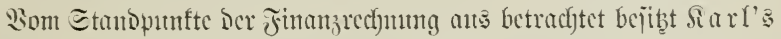

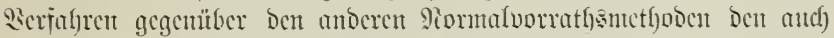

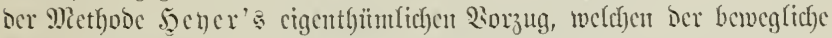

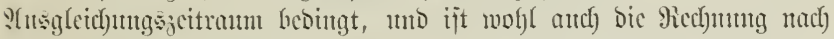

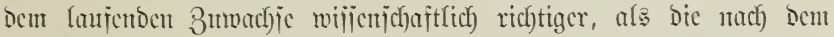

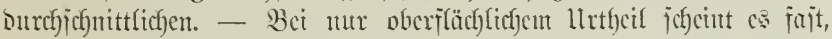

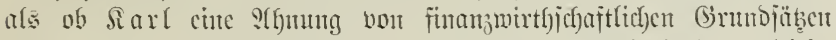

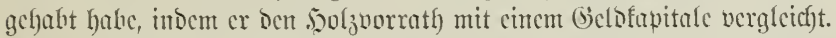

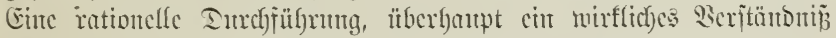

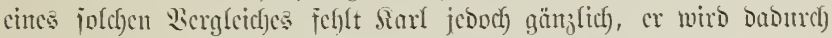

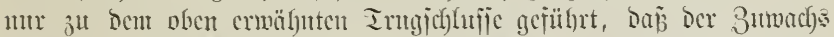

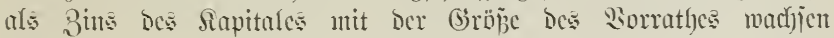

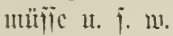

Eincu Lesertf) hat ficunad) Siarl's Ertragseregelutug für ben

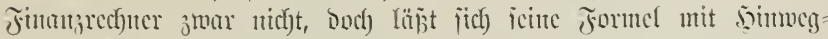

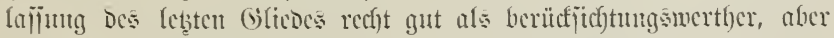

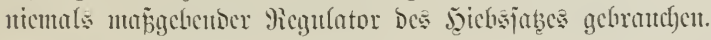

*) Ia wir bieien Fragen ber reinen Materialertragŝtegelnutg mad) itrengitem

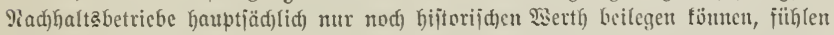
wir uns nid)t beranlajt, Gierauf nod) ipecieller cinzugehen un vermeijen beshalb

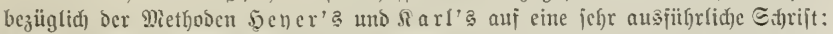

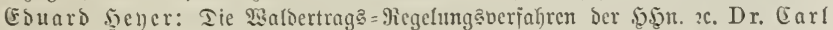

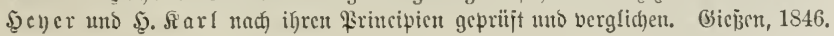




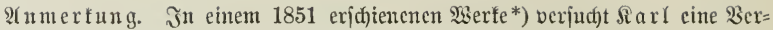

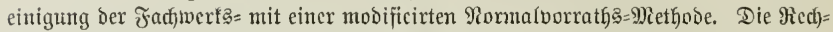
mung jtübst jich ant Durd)jonittşumad) einzclncn Salstfeile gefunden wirb, went man bie Flädje mit Dem Bejtandsaltev multiplicirt. Die Summe ber jo gejundenen einzelnen Probucte giebt ben Bejammt=

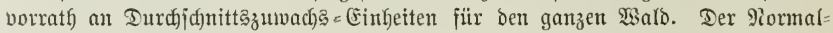
vorrath an foldjen Finfoiten wiro ermittelt, indem man bie Flädje ciner normalen

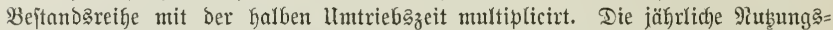

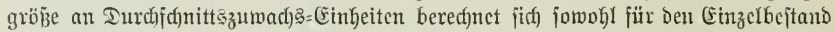

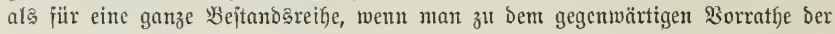
Durfjj(d)

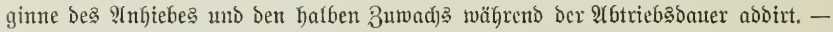

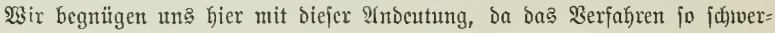

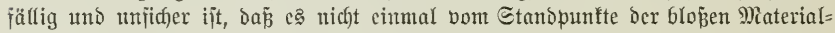
ertrags̆regelung irgend welche Empfehlung verdient. - Jür Den Finanzrectner

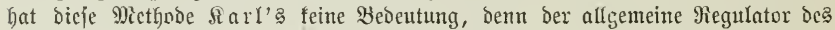

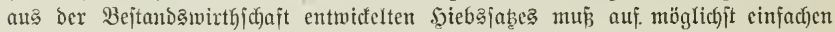
(Grunbjäben betuthen.

\section{$\S 130$.}

\section{Exemuan' Dexfaḩนen.**)}

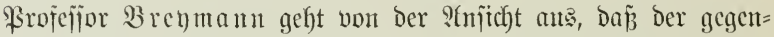

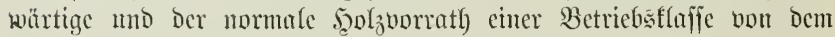

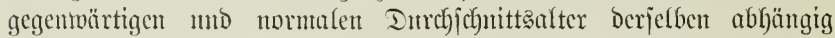

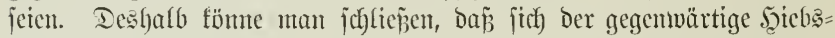
jab ciner Betriebsflajie zu ifrem gegenwärtigen Durchjebnittsalter ver= balte, wie ber normale J̧iebsjah zum normalen Durchjechnittsalter.

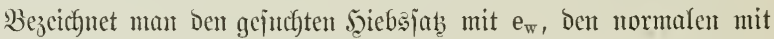

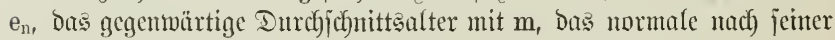
(sirïñ mit $\frac{u}{2}$, jo lautet die entiprechende \$roportion:

$$
\mathrm{e}_{\mathrm{w}}: \mathrm{m}=\mathrm{e}_{\mathrm{n}}: \frac{\mathrm{u}}{2}
$$

bierans

$$
\mathrm{e}_{\mathrm{w}}=\mathrm{e}_{\mathrm{n}} \times \frac{2 \mathrm{~m}}{\mathrm{u}}
$$

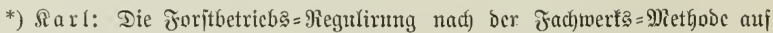

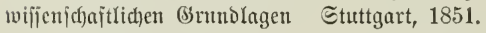

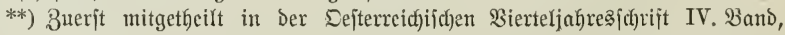

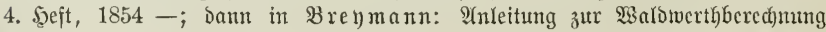

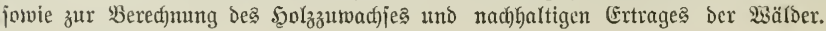


Das geyenwärtige Durd) f(d) Betricbstafic wird gefunden, went man bic auj cine Bonität redu=

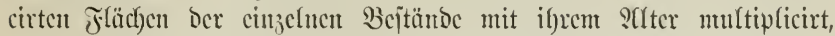
bicje Firobucte aboirt und bic Riroductemjume burch bic reducirte (B) jaumnträläcl)e bividirt.

Das Durchjectnittanter cincr normal bejtucten Betricbstajic be= redfuct jich) auf bicje $\mathfrak{R}$ cij̄e mit $\frac{\mathrm{u}}{2}$.

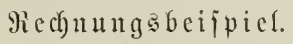

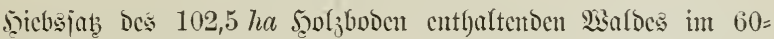
jül)rigen lintriebs.

Echt man bic Dajelbit angenommente 4. Bontität gleidf) 1 , fo $\mathrm{re}=$ Duciren fich) bic Bejtandsflächen ber 3. Bontität Duref) M)ultiplication mit $\frac{5,1}{5,9}=0,864$. Die reducirte (ścjammtfläche beträgt bicrnad):

$$
\begin{aligned}
\text { 4. Bonität } 57,55 \times 1 & =57,55 \\
\text { 3. " } \quad 44,95 \times 0,864 & =38,84 \\
\mathrm{~F}_{\mathrm{r}} & =96,39 \mathrm{ha},
\end{aligned}
$$

Das gegenwärtige Durchj)

$$
\begin{aligned}
1 \text { a. } & 2,50 \times 1 \times 5=12,50 \\
\text { b. } 2,50 \times 0,864 \times 75 & =162,00 \\
\text { c. } 10,50 \times 0,864 \times 5 & =45,36 \\
\text { d. } 1,25 \times 1 \times 90 & =112,50 \\
\text { e. } 1,75 \times 1 \times 20 & =35,00
\end{aligned}
$$

it. โ. เo.

$\begin{array}{rlr}\text { ba. } & 1,75 \times 0,864 \times 40= & 60,40 \\ \text { b. } \quad 6,00 \times 1 \times 5= & \times, 00 \\ \text { c. } \quad 4,75 \times 1 \times 0 & \times 0,00 \\ \text { d. } 2,75 \times 1 \times 100= & =275,00 \\ \text { Summe } 102,50 & 3033,70 .\end{array}$

joicrnad):

$$
\mathrm{m}=\frac{3033,7}{96,39}=31,47 \text { ฐal)re. }
$$

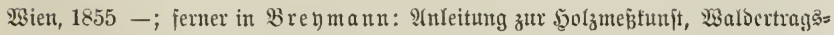
bejtimmung uno waldwerthberechnung. Wien, 1868. 
(5s ift ferner

$$
\left.\mathrm{e}_{\mathrm{n}}=96,39 \times 5,9=568,7 \text { * }^{*}\right)
$$

Die betreffenden 2 scrthe in bic formel cingcjebt, ergiebt:

$$
\mathrm{e}_{\mathrm{w}}=568,7 \times \frac{2.31,47}{60}=596,6 \mathrm{fm} \text {. }
$$

Sumerfung. Brenmann verfteft unter Bontiät bic Des Bejtandes, Dutch weld)e er aber autch bie zeitlidge Standortabonität auşbrïtent will, ba ber gegen= wärtige 5 golzbeitanto \$roduct icines Standortes jei; wir founten baher im vbigen Beifpiele bie Rieductionen nad) den gegebenen Beítandsbonitäten vornefimen.

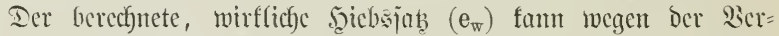
ïmberfichteit ber iln bedingenton Factoren, namentlicf) wegen Sier=

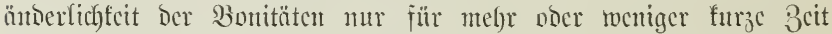

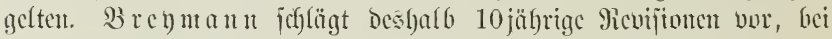
Denen itcts cure neue, herichtigenoc Emttelung ocs joicbsjabes jtatt= วufintón lout.

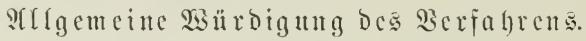

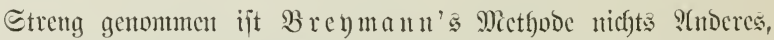

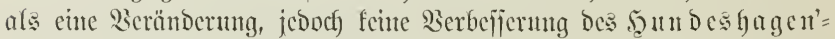
jefen Berfafyrens. Der Boransjebung, es muijfe fich ber-normale Soiebsjał zn Dem normalen Durdjjednittsalter verbalten, wie oer wirt=

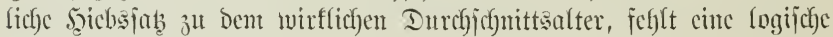
Bapị.

Wir brauchen uns Deshalb auf Specialitäten Der I4sfinfrumg

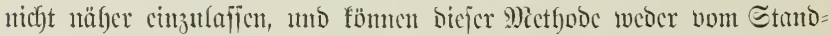
punfte Der Materialertragsegefung, unch weniger von bem ber Finan

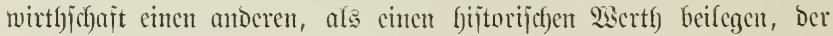
if)r indefïen nicht ganz abgejprodben werben mag.

\section{$\S 131$.}

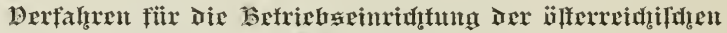 Reidtuxforlfe.}

1. Siad) Der Inftuction von 1856.

Das Serfafren**) jobließjt jich wenigitens theifweije ben Piormal=

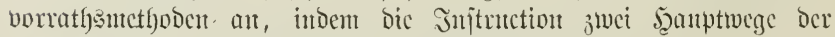

*) Die geringe Differenz biejes en mit bem $\subseteq .352$ bered)neten erfläut jïh

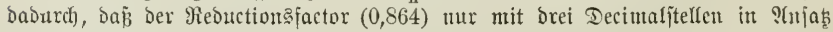
gebracht mutroe.

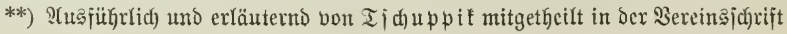


Ertruystegelung bem foriteinricfter frei gicfot. Der eine $2 \mathrm{seg}$ ijt

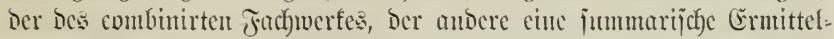

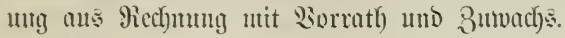

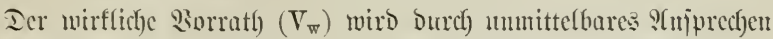

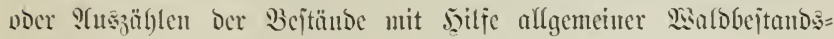
Tajelu ermittelt ( $\$ 30$ Der Sicrorbumg).

Fer normale Siorrath $\left(V_{n}\right)$ joheint chenjalls nach Ertragstafeht

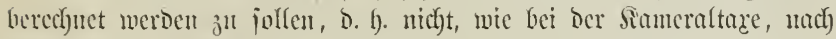

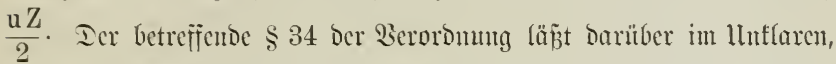

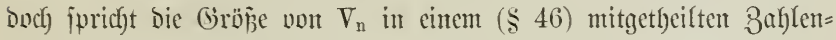

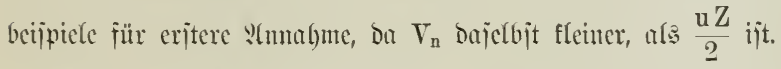

Eigenthüntlicf) ijt bic Bejtinmung Des $\$ 29$ übcr bic Ermtittefung

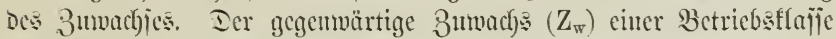

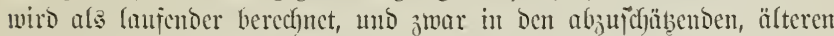

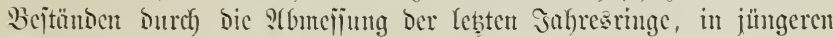
Erten burch) 9(nmendung von Errtragstafch. Der fünftige Bumachs $\left(Z_{\mathrm{k}}\right)$ jolf zum Theil afs bुaubarfeits = Durchidgnitts =, zum Theil afs

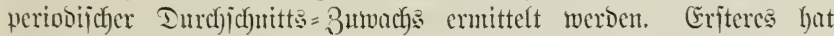

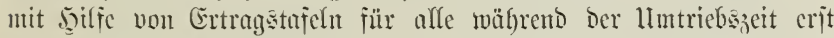

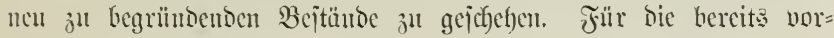

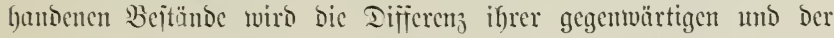

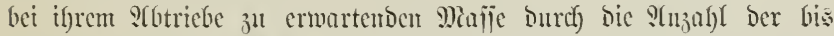

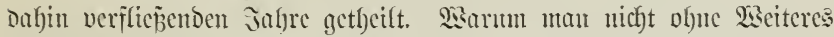

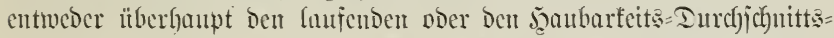
zumact) gewählt hat, becibt unflar. Is geringer indejien bic betrej= fenden Differenjen in Der Regel jein werben, Itm jo mefgr hat woht B̧reyman giecht (1.c. C. 137), Leţteren ein jür alfemal als $Z_{\mathrm{k}}$ an=

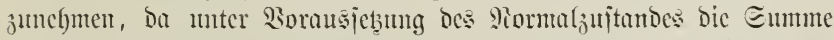

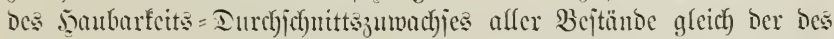

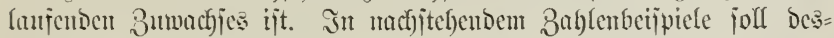
hatb chenjo verfafuen werben.

Etimmen $V_{\mathrm{w}}$ und $\nabla_{\mathrm{n}}$ nabejn überein, fo werben auch) $Z_{\mathrm{w}}$ umb $Z_{\mathrm{k}}$

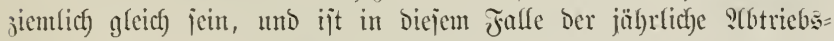

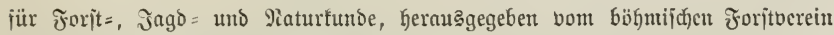
1857 unt 1858 (Nicue jolge 14. unt 16. 5ुeft).

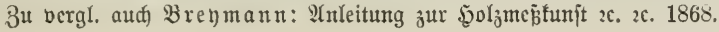




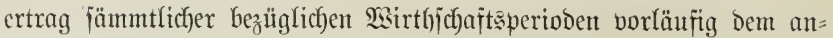

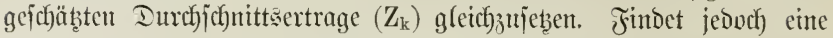

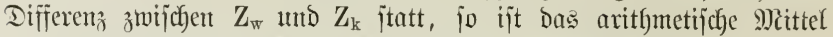

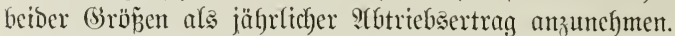

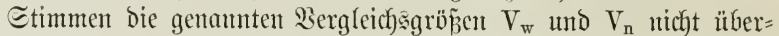

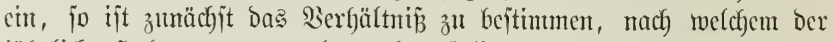

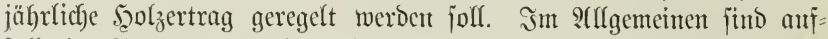
fallende Eprünge von Dem bišger bezogenen zn Dem fünftig bejich=

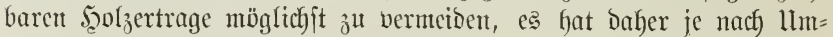
itäntoen cutmeder eine allmälige હteigeruntg ober ein allmäliges હinfen des Sciebsjabes eintzutreten. In ber 9iegel follen jedoch währent bes Bertaufes enter llmtriebszeit etwaige Borratbs= Differen;en fo aus=

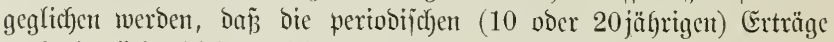
nach) jiemlich gleidyen Differenjen jteigen noer fallen.

Die Formel bes Sciefsjabes lautet nun:

$$
\mathrm{e}=\frac{\mathrm{Z}_{\mathrm{w}}+\mathrm{Z}_{\mathrm{k}}}{2}+\frac{\mathrm{V}_{\mathrm{w}}-\mathrm{V}_{\mathrm{n}}}{\mathrm{u}} .
$$

Red) un gabeijpiel.

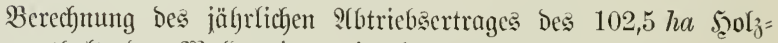
boden entfaltenden $\mathfrak{s a l d e s ~ i m ~} 60$ jährigen lluntriebe (ङ. 324).

$\mathrm{Z}_{\mathrm{w}}$ nach) $\$ 129$ wie für Siarl's Methode berectunet ijt $=513,8 \mathrm{fm}$.

$\mathrm{Z}_{\mathrm{k}}$ ebenfalls wie dort $5,9 \times 102,5=604,75 \mathrm{fm}$.

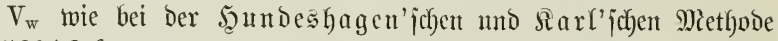
$=15204,8 \mathrm{fm}$.

$\mathrm{V}_{\mathrm{n}}$ еъепір $=14717,3 \mathrm{fm}$.

$\mathrm{e}=\frac{513,8+604,75}{2}+\frac{15204,8-14717,3}{60}=640,5 \mathrm{fm}$.

Da mut nach Scerjteflung Des Miormalzujtandes $605 \mathrm{fm}$ ge= jublagen werben fömten, io ijt bie Differenz fanm erfeblich genug,

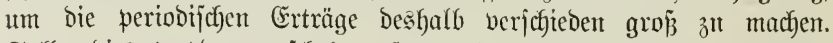
Sollte bies trobzocm gejuchen, jo würben viclleicht folgende s(njäbe entiprechen:

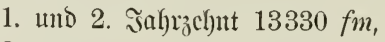

3. "4. " 12800 "

5. " $6 . \quad$ " 12300 "

Summe $38430 \mathrm{fm}=640,5 \times 60$. 


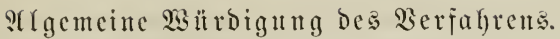

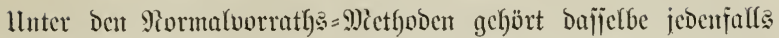
ju Den befieren, numentfich) vom Stambpunfte Der blojen Miaterial= Ertragsiregelung aus betrachtet. Ias crite Gilied ber Ertragsformel $\left(\frac{\mathrm{Z}_{\mathrm{w}}+\mathrm{Z}_{\mathrm{k}}}{2}\right)$

trägt Den mafrejchcinlichen 3 umachsäntocrungen cigentlick)

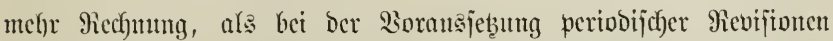

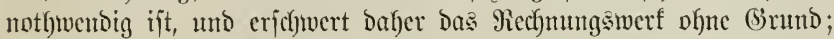

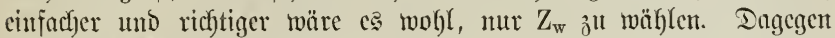

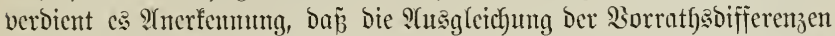
nur in ber Regel, aljo nicht jtets während Des Bcitranmes bes erjten

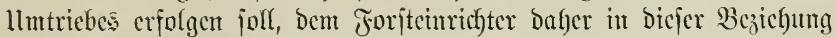
nach) Den vorliegenton Serbältniffen ein getwiffer Epielraum gefafjen wiro. *)

Rebzterer Ilmjtand gejtattet ïbrigens anch), ähnlich wie bei ben

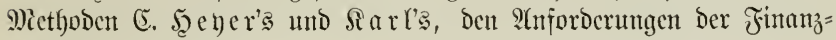
rechuutg twenigitens etwas mefre Piechnutg zu tragen, als bies nach ber Siameraltaxe, nach f̧undeshagen nder nad breymann mäglich ijt. Einen bejonderen $\mathfrak{W e r t h}$ föunen wir Der Metljode indeffen von bicjem (Sejichtspunte aus cbenfalls nicht aujprechen. WSir theilen jie untr beshalb mit, weil fie für bie Entwidfelıng Der Sebre von ber Ertragsregelung ïberbaupt nicht obne bijtorifches Interejie ijt.

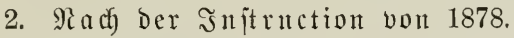

Bez̧üglidy Der $\mathfrak{B a l b c i n t l y c i l u n g ~ g e l t e n ~ n a c h ) ~ b i e j e r ~ I n j t r u c t i o n * * ) ~}$

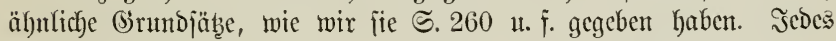

*) Die Beredjunng von $\mathrm{V}_{\mathrm{n}}$ nach $\frac{\mathrm{u} \mathrm{Z}}{2}$ ijt bem (Seijte bes Berfafyrens zuwibcr, würbe aud) niat anwendbar jein, bcun baburd) wïrbe ber Jactor $V_{n}$ gauz aนక Der Formel berjatwinben, ebenjo $\mathrm{Z}_{\mathrm{k}}$ jelbjt:

$$
\begin{aligned}
& \mathrm{e}=\frac{\mathrm{Z}_{\mathrm{w}}+\mathrm{Z}_{\mathrm{k}}}{2}+\frac{\mathrm{V}_{\mathrm{w}}-\mathrm{Z}_{\mathrm{k}} \frac{\mathrm{u}}{2}}{\mathrm{u}} ; \\
& \mathrm{u} e=\mathrm{Z}_{\mathrm{w}} \frac{\mathrm{u}}{2}+\mathrm{Z}_{\mathrm{k}} \frac{\mathrm{u}}{2}+\mathrm{V}_{\mathrm{w}}-\mathrm{Z}_{\mathrm{k}} \frac{\mathrm{u}}{2} ; \\
& \mathrm{e}=\frac{\mathrm{Z}_{\mathrm{w}}}{2}+\frac{\mathrm{V}_{\mathrm{w}}}{\mathrm{u}} .
\end{aligned}
$$

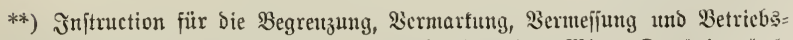

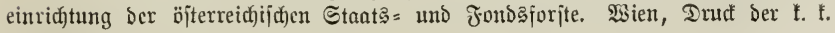
Sgof= und Stanţ̧orudferei, 1878. 


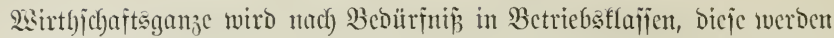

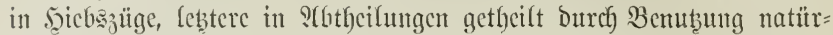

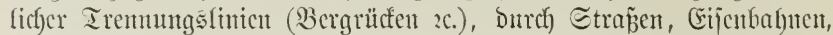

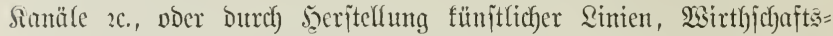

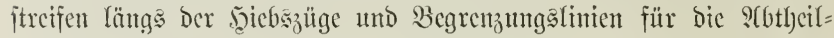
migen.

In bie atbtfeifungen merden anf Der Siarte Reriobennumern eingetragen, um ben Gang Des jeiches anjchaulich ju utacken, pder

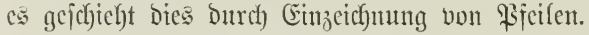

Für die taratorijchen Sorarbeiten werden Socal=Ertragstafelı gefordert.

Die "Betriebsorichläge" haben ut. 9. cin Socal für bic fünftige

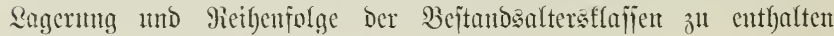
(1. c. ๔. 76 ).

Der Ertrag wird getremnt für bic 5aubarfeits = แnd für bie Bwijh) idjicd nicht itatt.

Für bie Beredfuntug Des Scaubarfeitsertrages gelten folgentoc (Be= jithtspunfte (1. c. Є. 80 น. f.):

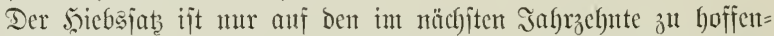

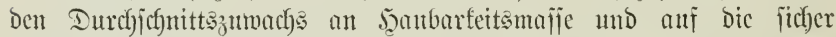

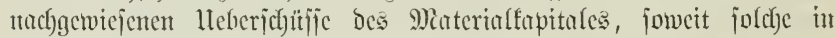
Dicjem Jahrzefut anfgezchrt werden bürfen, ju bajiren. - Etrwa ein= tretende Buwachserhöhutgen finden von felbjt Beachtung Dutred) bic regelmäfig twiederfehrenden 10jährigen Revijionen, bei weldyen jtets neue Ertragsermittefungen vorgenommen werden. - Sit ber wirffiche $\mathfrak{B o r}=$

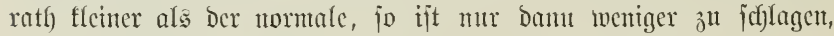

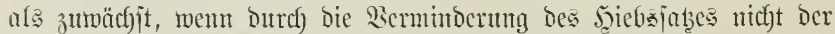

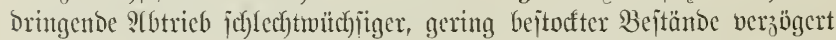

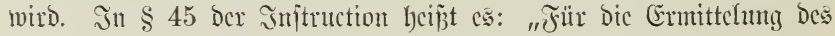

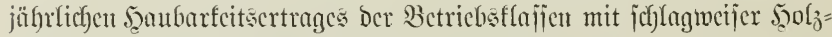
Intutu, und jwar für jebe Betriebsflajfe fpeciell, bient bic Formel ber

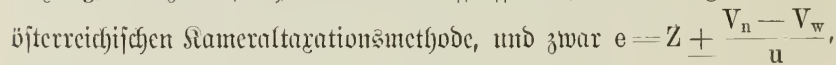
wobet $Z=$ Der in Der Bejtundstabelfe zu findenden Eumme des

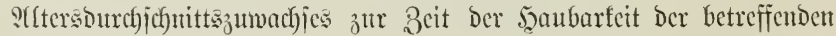
Betricbsflaific;

$\mathrm{V}_{\mathrm{n}}$ - Derjenigen (Sirößje, welche ber ftoctende Majjenvorrath be=

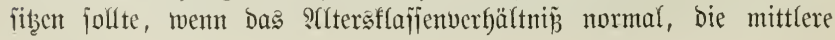




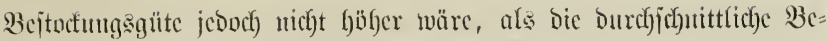
ftocfung Des gegenwärtigen voer mittleren Miajicnvorratfes;

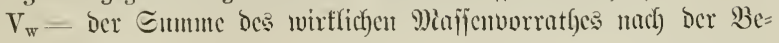
jtutbstabefle;

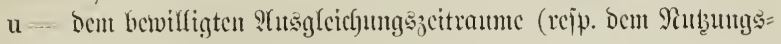
tırmuts) jut jesticu ijt." -

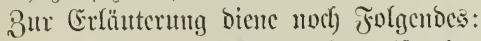

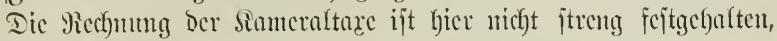

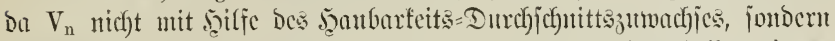
anf Grumb ciner Socal=Ertragstafel berecthet weroen foll, wic ces Şundeshagen und Siarl thum. Tabei tritt nod) Die Modification

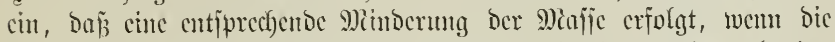

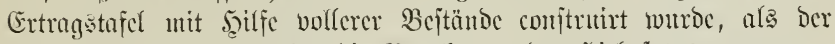

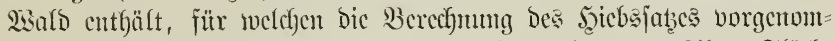

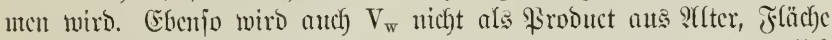

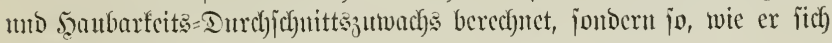
wirtfich vorfiubct.

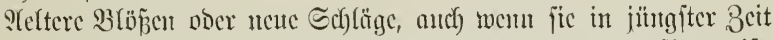
angebant murocn, oder itellenweife anf ifuen Samen angeflogen ijt,

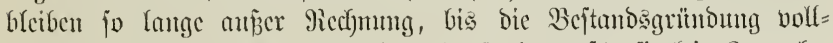

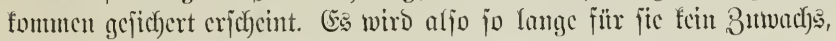

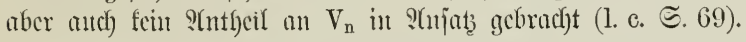

Redunngabeifpiel.

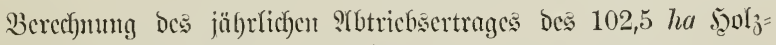
boden enthaltenton Rialdes (\$. 324).

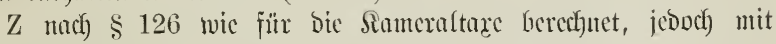

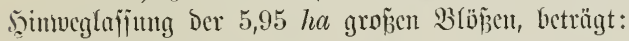

$$
51,60 \times 5,9+44,95>5,1=533,7 \mathrm{fm} .
$$

$\mathrm{V}_{\mathrm{n}}$ wiro, chenfalls unter şismeglafiung ber ber 4. Etandorts:

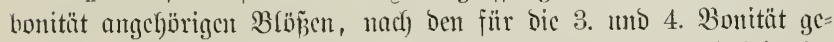
gebenen Ertragstafeln bejtimut, weldye wir hice als 2ocaltafelu be= tracf)ten wollen. Paach Summinumg Der letsteren beträgt $V_{\mathrm{n}}$ fïr 1 ha

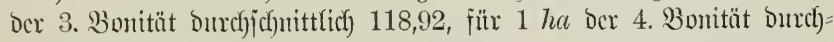
idfuttlicf) $143,58 \mathrm{fm}$; in Summe jonad)

$$
V_{n}=51,60 \times 143,58+44,95 \times 118,92=12754,2 \mathrm{fm} \text {. }
$$

$V_{w}$ bereclunet jich wie bei f̧undeshagen und Siarl auf $15204,8 \mathrm{fm}$. 


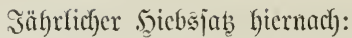

$$
\mathrm{e}=533,7+\frac{12754,2-15204,8}{60}=574,5 \mathrm{fm} .
$$

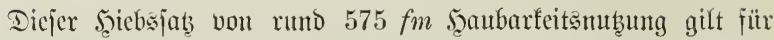

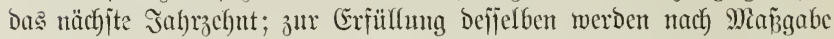

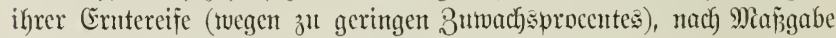

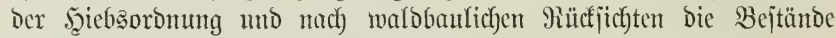
ansgewählt, in welchen gejd)lagen werden foll.

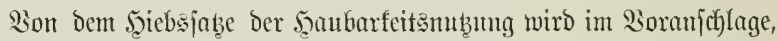
wie beim Bergleidye Dee Erfolges, Der Der Zwifchenu gefalten. Diejer wiro ebenfalls mur für bas nächjte Jafrzelynt $\mathfrak{b} c=$

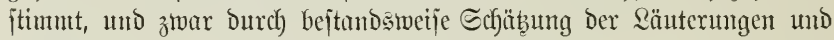

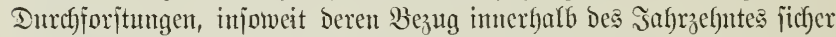

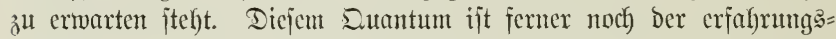

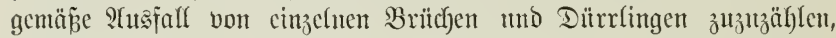

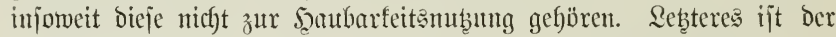
Fall, wenn fie in Şiebsorten erfolgen voer anbaufühige B̉löß̄en, von

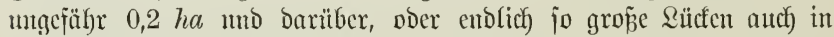

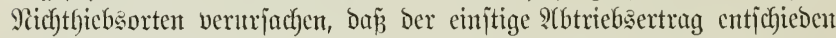

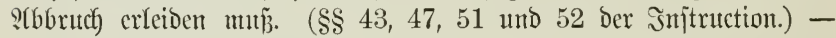

Der Ertrag des Pilcuterwaldes, forvie ber des Sherholzes im Mittelwalde, wirb mittelit entiprect)ender Natbutugaprocente, welche in

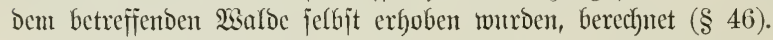

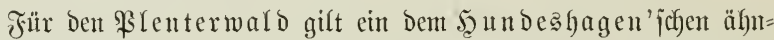
liches Berfahren. *) $\mathrm{V}_{\mathrm{w}}$ wiro unter Berüneffichtigung Der orei Şaupt=

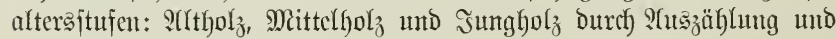
gecigntete $\mathfrak{B r o b e f}(\ddot{a} \mathrm{c}) \mathrm{en}$ ermittelt; beim Jungholze bleiben bie nicht

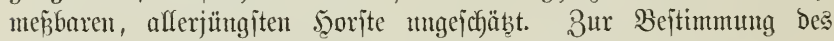
Sinbungâprocentes wählt man für jede wejentlich abweichende Form,

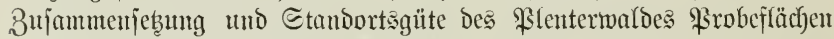

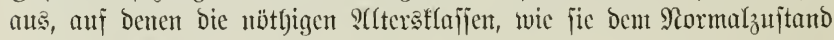

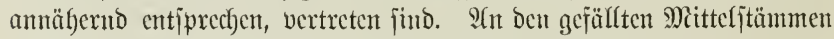

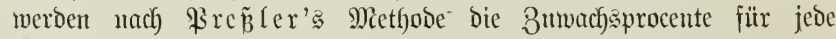

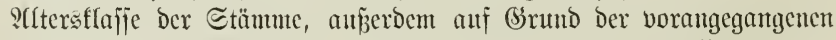
flafjentweijen S(ušfluppirung auch bic gegentwärtigen Maffenvorräthe

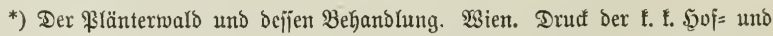
Staateobruterei. 1878. S. 8. 


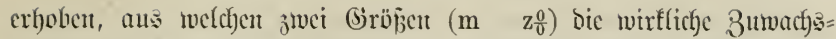
majie jeder S(Ytersflajie hervorgeht. Die Summe bes Majienvorrathes alfer ?fltersflajien verglichen mit der Eumme des 3umachjes der

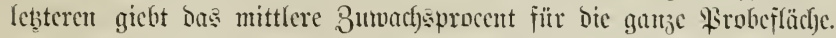

3um Bcifpiel auj 1ha Probefläclye befinden jitd):

\begin{tabular}{|c|c|c|c|}
\hline Miaijenvorrath. & 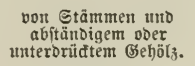 & 3ีtuad) $\%$. & $\begin{array}{c}\text { baher Buwactses= } \\
\text { maije. }\end{array}$ \\
\hline $32,5 \mathrm{fm}$ & 160 jährig & 0,50 & $0,162 \mathrm{fm}$ \\
\hline 26 & 140 & 1,00 & 0,260 \\
\hline 22 & 120 & 1,25 & 0,275 " \\
\hline 18 & 100 & 1,50 & 0,270 \\
\hline 15 & $80 "$ & 1,75 & 0,262 " \\
\hline 10 & $60 "$ & 2,00 & $0,200 \quad$ " \\
\hline 8 & $40 "$ & 5,10 & 0,400 \\
\hline
\end{tabular}

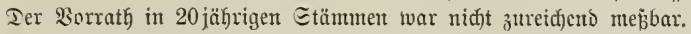

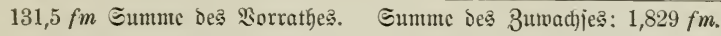

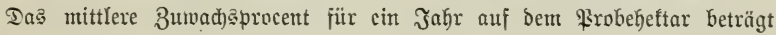
hiernađi $\frac{1,829 \times 100}{131,5}=1,39$.

Durch) Muttipfication Des $\mathrm{V}_{\mathrm{w}}$ mit Dem aus Dem 3umarfsprocente

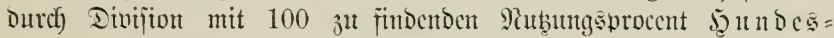

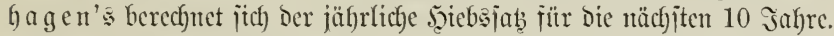
Eine Tremung von jुatbarfeits= 1 mo 3 wijchenutbung findet nicht itatt.

Bur Eriüllung biejes Jुicbjabes werden bie am meijten biebs: ober plenterungšbcoürfitigen bocr fäbigen Site im Einne cincr guten geicboorbunt ausgejucht.

\section{Nefgemeinc :}

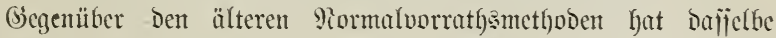

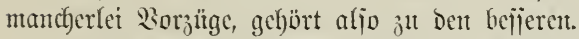

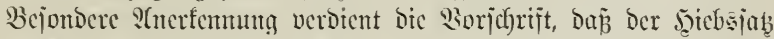
grumbjäblich nur für Die näctjiten 10 Jabre bejtimmt, in Folge befien

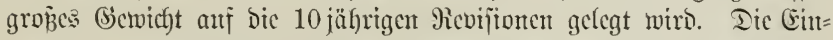
füf)rung von 5 jäfrigen Brvijchenrevijtonen gejtattet übrigens berichtig= untgen, wenn bicje bringend nothuendig erjchetnen.

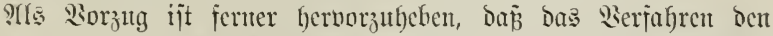

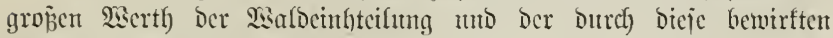
jobeboronung ricftig crfenut.

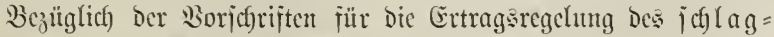
weijen Betricbes ijt bejontore Jolgendes zut cruähnten: 


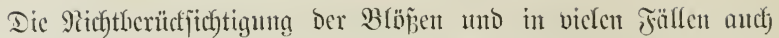

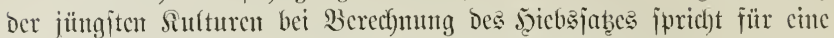

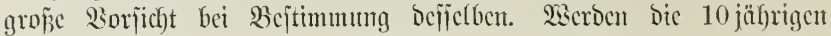

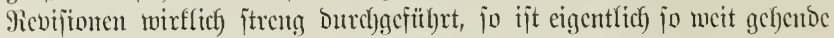
Borficht nicht nothyentig.

Formell jebeen wir an Der Ertragsformel aus, baj man nidft lieber

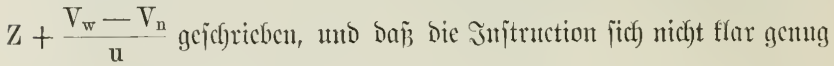

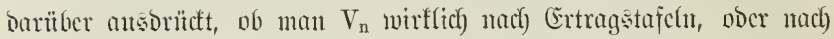

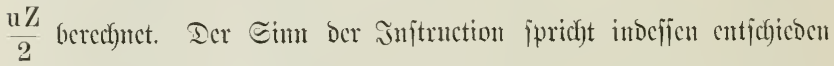
für critere. Der Gicoante, Den mit Jुilfe von localen Ertragstafelu

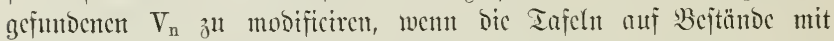

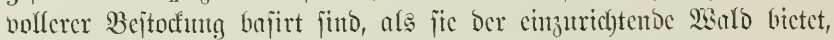

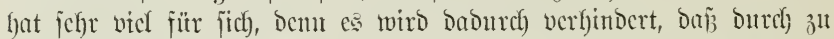

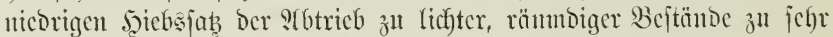

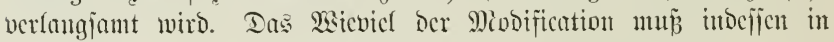

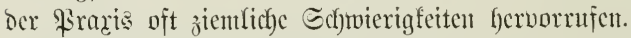

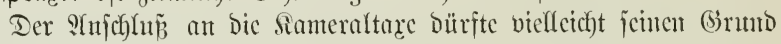

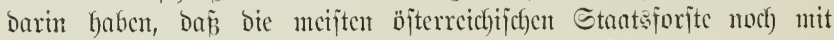

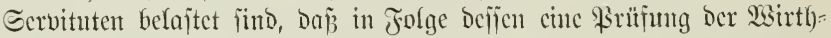

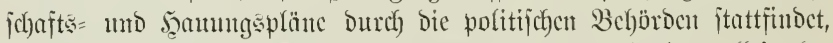

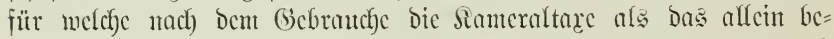

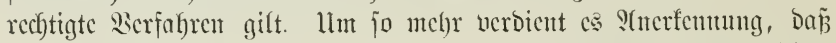

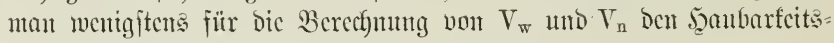

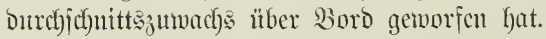

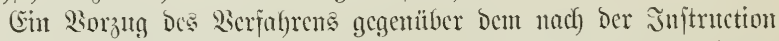

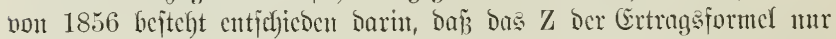

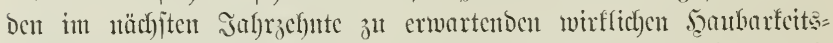

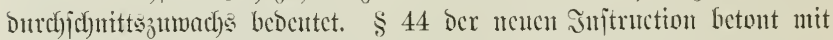

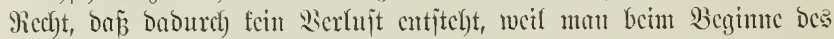
näcfjiten Decenniums bic Extrugsermittelung conencert und fout cincr

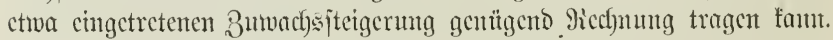

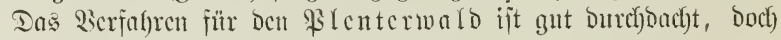

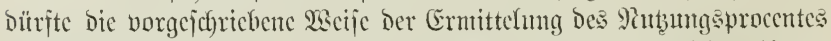
megen Miangels an normalen \$robeflächen groj̉e Echwierigfeiten Licten. (5e fragt fich, ob man auf cinfachere 2iscije nicht cbenjo richtige

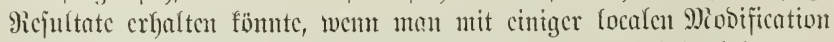

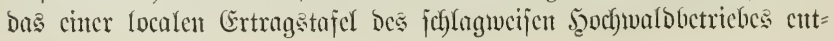




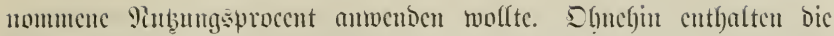

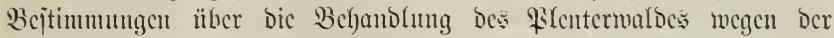
weitats überwiegenden ?fbnormität Der biejer Betrichsart jugeniejenen

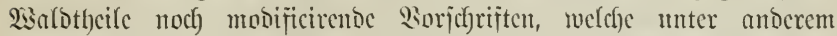

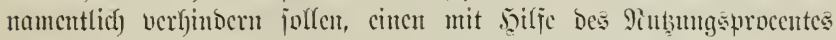

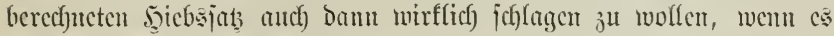
an mbbaren Etämmen fehlt. In Plenterwald = Betriebsflafien mit überwiegeno jüngeren, viclleidgt and amtäf)ento gleichalterigen $\mathfrak{B} c=$ jtänden, joll nux jür jente Erte, meldfe mit jeffon ljebsercifen und in

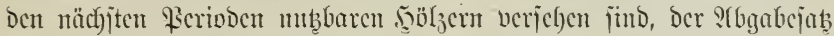

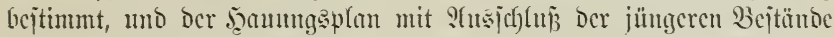

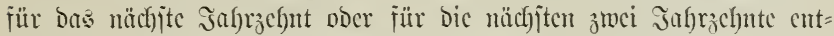
morfen merben.*) Dicje Siorjefrift ijt jedenfalls zu billigen. Ilnjerer

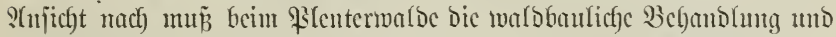

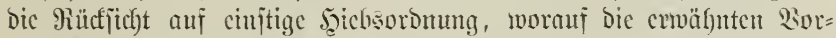

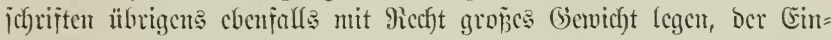
haltung cincs bejtimmten juicbsjabes meit voratsgchen, tweshalb

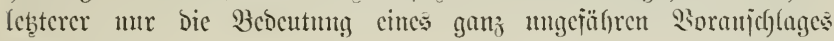
haben famt.

\section{\$ 132.}

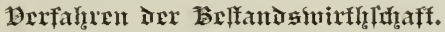

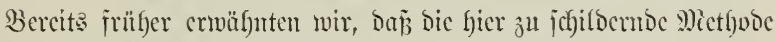
nicft mit bem S(njpruct) auftreten farm, etwas Reucs ju jein, joubern Daj jite nur bie theoretijche Eonjequenz aus ciner langjüfrigen, praf= tijchen sumenoung ijt, wie jie in $\$ 124$ mitgetfeilt wurbe. Dicjer fehfte zur theoretijuchen Correctheit nur noch) żmeicrlei, criptens bic flare

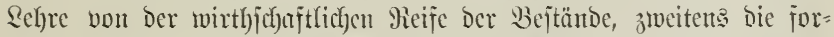

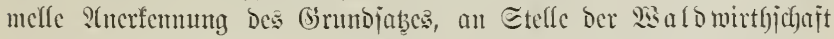
ans bem groben Ganjen, bic feinere $\mathfrak{B c}$ itand lajien.

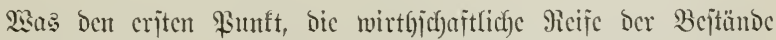
antangt, io verweijen mir hier anj bie betrefjenton Faragraphen 15 und 16 mijeres Butches.

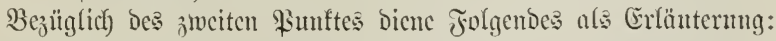

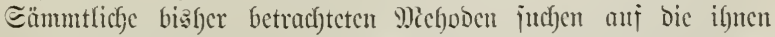

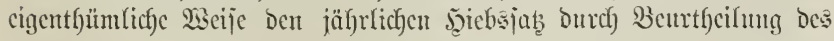

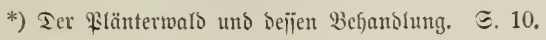




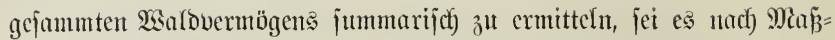

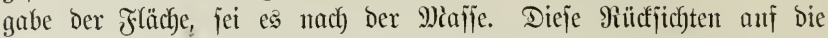

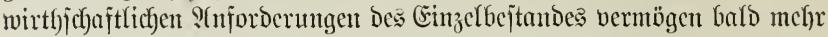
bald wentiger mobificirend auf Den fummarifech Siebsjab cinłunirfen,

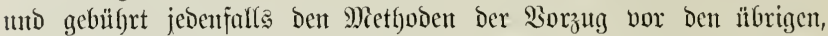

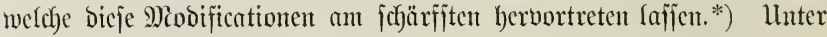

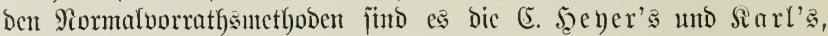
fowie Das öjterreidfijche Berfafhren nach ber Injtruction von 1878; unter Den Flächenmetfoden ijt es ofyne 3weifel das im $\$ 124$ ent=

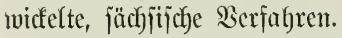

Die Methode Der Bejtandsmirthjichaft betritt, wie aus bem Folgen= Den näher hervorgelft, Deıt entgegentgejebaten $\mathfrak{W} \mathrm{eg}$.

\section{Dex af(gemeine Wirtbfofaftsp[an.}

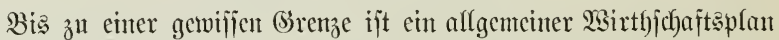
ebenjo (Brundlage ber Forjteinrichtung uno Ertraggregelung für bas Berfafyren Der Bejtandsmirtfjçchaft, wie für die älteren und netueren Jachwerfsmethoden, als wie für bas in $\$ 124$ gejchildoerte jächjtiche Berfagren.

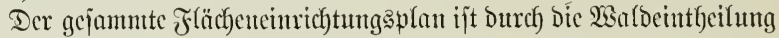

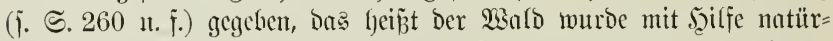

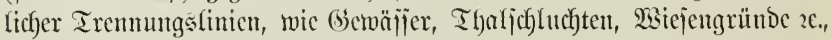

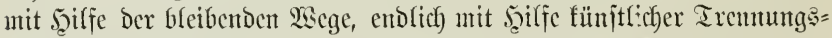

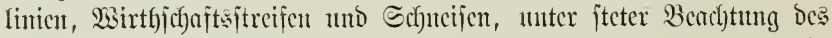
Terrains, Der Iransportuerfältnifje in Betriebstaffen (\$ 108) in Jejebszütge (\$ 109) und atbtheilungen (\$ 110) getheilt. Dicje Wald= cintheilung bahnt bic allgemeine Sromung des J̧iebsganges an, gicbt

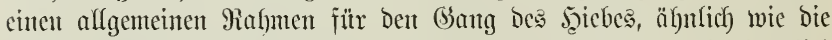

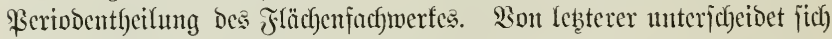
bicjer Rafymen mamentlich Durch Folgendes:

Erjten fordert dic Bejtandsmirtlfichaft vicl fürzere şiebszïge,

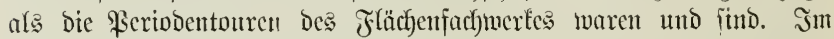

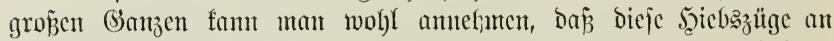

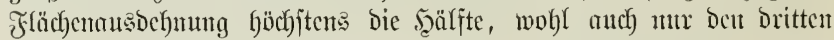
ober vierten Theil ber alten \$ieriobentouren umfajjen jollen.

*) Die älteren Methoden, wie 3. B. Das exite Berfahren (5. L. Şartig's

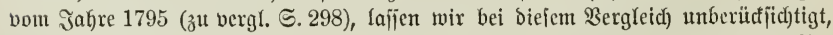
da fie nux nod) hijtorijchen siserth haben und für bie \$raris heute ohne alle $\mathfrak{b} e=$ beutung fino. 
3weitens jief)t bic Beftandsuirthjichaft, gectude fo wie Das im

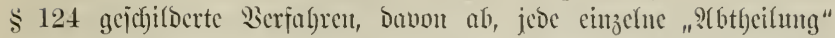
ciner bejtimutten Beitperivoc zuzutheilen. Dics wird ichon baburd)

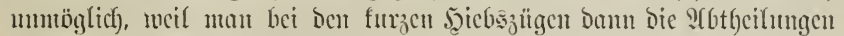

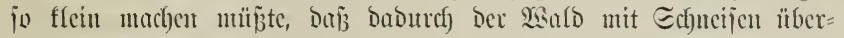
laben würde. Es ijt afer, wic cinte 30 bis 40 jührige, im Grojen

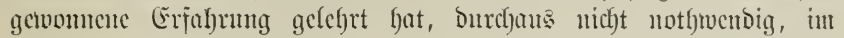

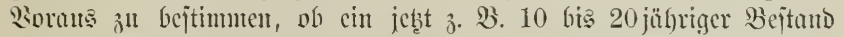
in 60 oder in 80 oder in 100 Jafreen jum 9 (btricbe jull bringen fein mirb. Dicje 9?otfwentigfeit verichwand mit ben langen Feriobentouren.

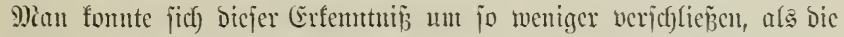
Errfahrung gelehrt hatte, Daj wenigitens in Den Eturm=, Snjecten= mo

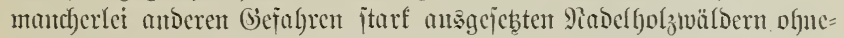
fjin alfe bic bis in grane 3ufunft reichenten Soransejtimmungen Sicbelbildoer waren.

Frcilid gicbt es fïr Den Foritteinrichter faum etma: Bepuemeres,

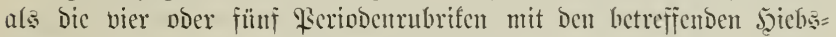

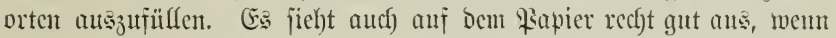

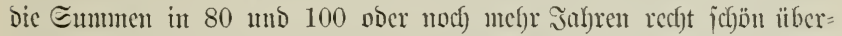

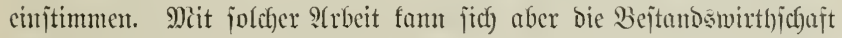

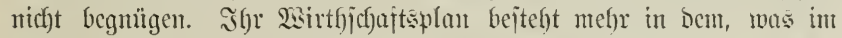

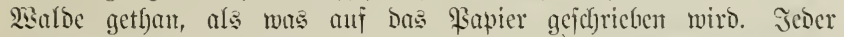

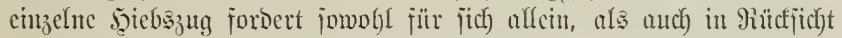

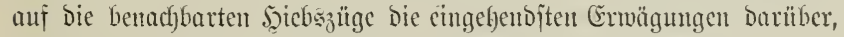

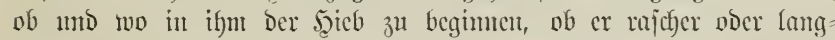

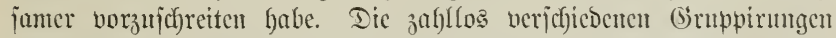

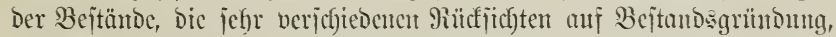

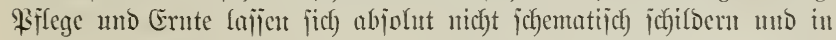

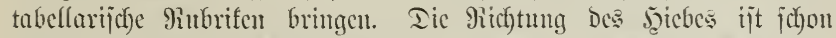

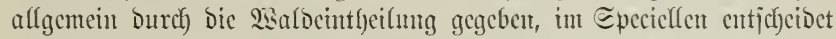

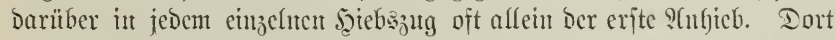

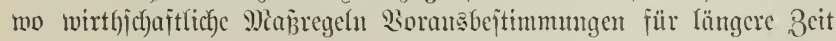

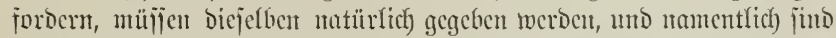

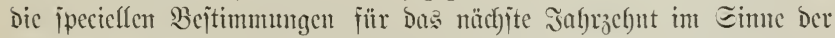

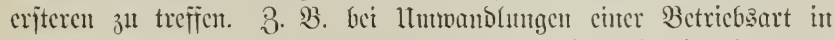

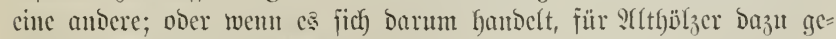

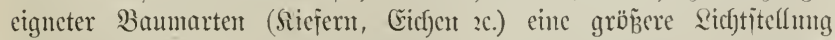

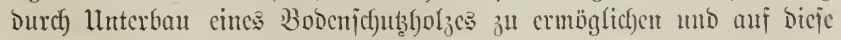

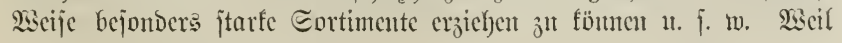




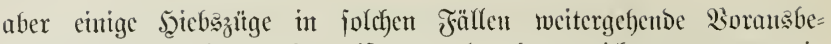
fimmungen nöthig machen, ift man burchane nicht gesmungen, in foldfen Şicbszügen, melde bies nicht forbern, Derartige Bufunftsnor ichriften $\jmath^{\mathfrak{l}}$ geben.

Go faffen wir den allgemeinen Wirthfichafteplan auf, wollen ihn alfo auf tcinen Fall entbelyen. In joldfent Sinne fönnte man autd)

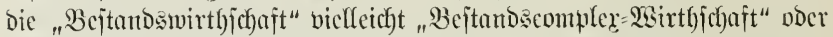

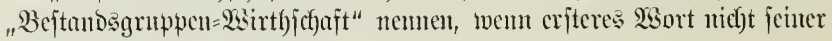
Sïrra und Form megen Den Sorzutg verbiente.

\section{B. Die A6triefsnub̧üngen.}

Dic $\mathfrak{W a l b c i n t b e i l u n g}$ ijt gegeben mo baburch) Dic allgemente Sord = mung Des Jeicbsganges bereits angebarnt.

Someit es nicht bercits bei ber Bejtimmung ber Betricbstlajien gejcheben, jutcht mu ber Tarator Den fintmjicllen Ilmtricb Durch Die Beredfnung Der Bodententen aus charafteriftifchen Bejtänden zu cr=

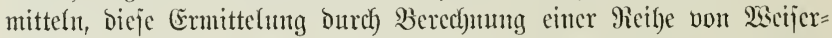

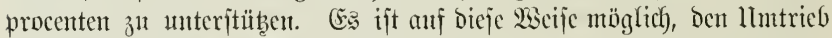

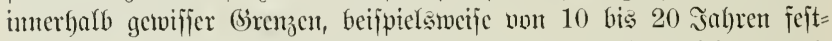

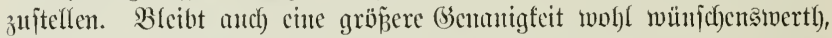

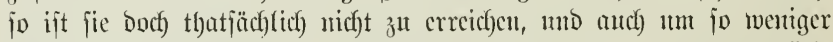
unbedingt nöthig, als ber funanjiefle llmtrich itets eine verïndorlick) (S) rïßje bleiben toiro.

Diejelbe giebt cinten alfgemeinen Shtbaltspunft Darïber, inmerbalf

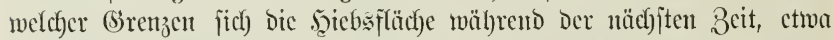
mäf)rento Der nächjten 10 bis 20 Jabre zu bewegen hat, foweit nicht

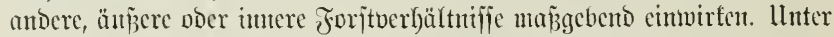

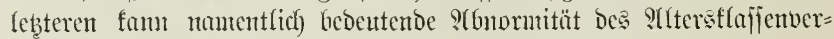

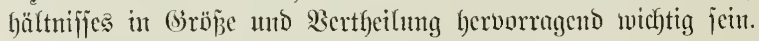

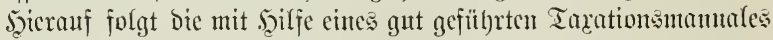

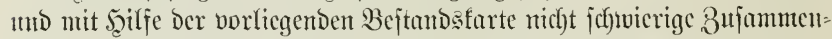

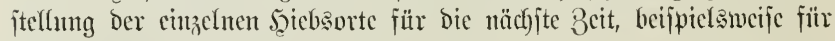
cin Jaf)rzebut.

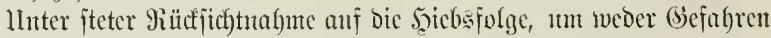

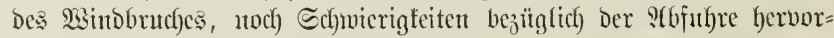

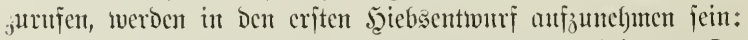

1. Sorfe wirthjefaftlichen Siothwendigfeiten. Dabin ge= hören alfe Roshicbe, fenter jenc Schfäge, melche zutr Serfürzutug Der Şiebşüge angelegt werben ntïfien. 
2. Alfe cutidyeden hicbsreifen Drte, ocren Weiferprocent

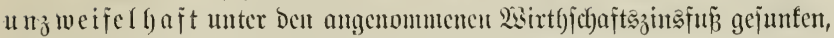
foweit es überbaupt möglich ijt, in biejen Beftänben unter Beacftung

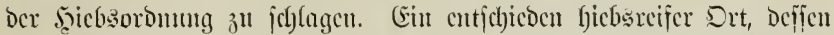

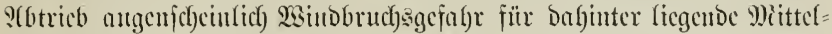

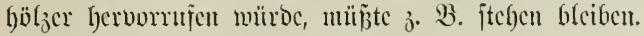

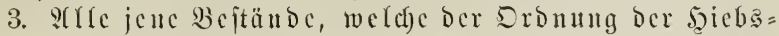
folge enticficden als Dpfer falfen müffen. 3. W. fleme

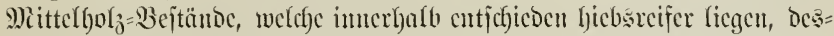
hafb nicht übergefalten werdon fömten; cin vou cinem 120 jäfrigen Bcjtande alljeitig umijchlofijenes, 60 jälgriges Fichtenjtangenlyolz u. j. w. -

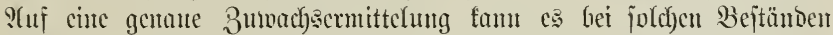
nicht anfommen, jobald fie notbwendiger $23 c i j e$ falfen mülïen, wemt man nicht zur Gisuinnung localer Erfaf)rungen überlyaut Derartige Ebjecte benthęcu will

Bezä̈glich Dex unter 2 und 3 genaunten Beftände fanm es jelbit= verjtämblich oft zlucifelfaft jein, weld)es Dpfer gröferer ijt, entweder bas

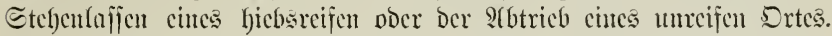

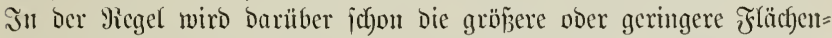
ansobfunutg bes eincu poer bes anderen Bejtandes ein ?hthalten ge= währen, da man z. 3. nicht citen 0,5 ha grojen, cntjchicden abtriebs: beoürftigen Bejtand abtreiben wird, wenn bicjer Stbtrieb für 20 ha

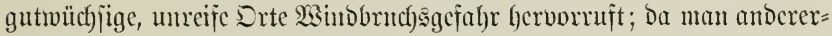
jeits nicht ein 20 ha umfajjendes, reifes :(ftholz jeffonch wirb, um eincn Darin gelegencu, unteifen 50-60jülyrigen Bcjtand ntoch länger Ïbcrhalten zu fömten, menn biejer nur 1 ha flädbe cntbält. - Ic

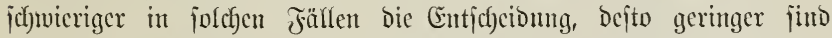

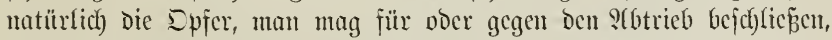

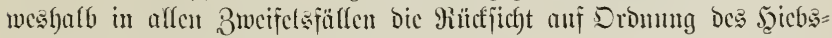
ganges majigebeno werden foum.

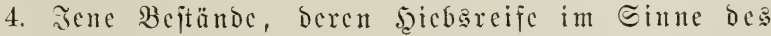
Weijerprocentes jweifelfaft ijt, fowcit bicje ïberfant vom

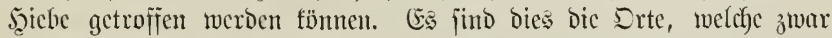

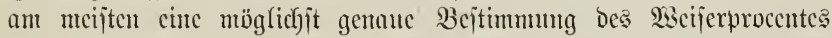
nötfig mad)en, bejüg(ich Deren jedoch cben wegen Der geringen Diffe= renjen etwaige Irthümer and mit ben geringiten wirthjichaftlichen Spfern verfun̈pft finto. -

Sind für bic nädhjten 10 oder 20 Jafgre die unter 1 bi 4 ge= naunten J̧icbsorte mit if)ren Erträgen zujammengcjtelft, Dann rejultirt 
in Der Summe Der Şiebşab für Fläche und Miafie als Folge ber ab= joluten Bejtandsmirthjichaft.

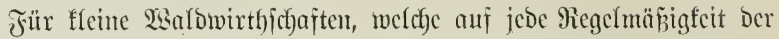

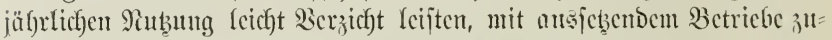
fricocu jein fönnen, bebarf es cutes weiteren Regulators nicht. Ethuas

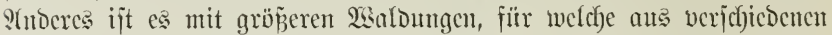

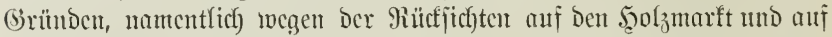
bie Walbarbeiter, Der ausjebente Betricb ummöglich) ijt, allzugroj̄e (Er=

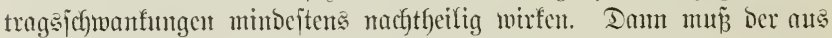

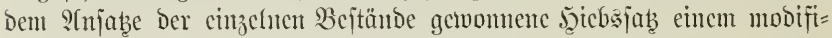
ciremben Piegulator unterliegen. S(ls joldcher ijt am einfachjten ber bem finanzielfen llmtrieb cntjprechendoc, normale Jabresichlag zu betrachten,

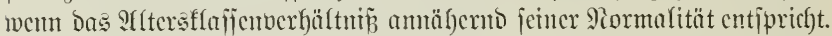

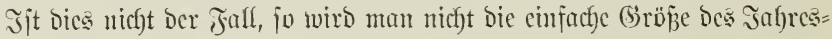

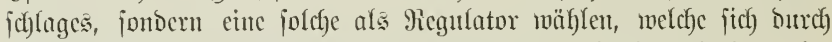
Berücfficftigung ber vorfantoenen ?f(bnormität ermittelt, bci einem $\mathfrak{b c}=$

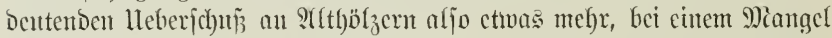
Derfelben etwns mentiger Fläche beträgt. (5s handelt jich hierbe burchaus

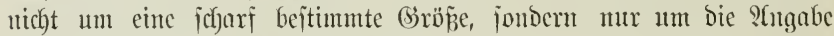

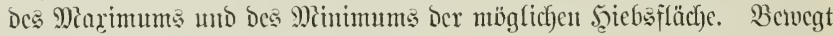

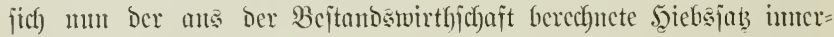

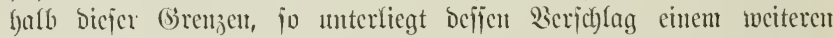

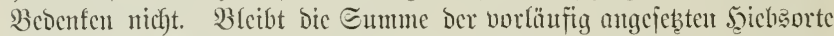

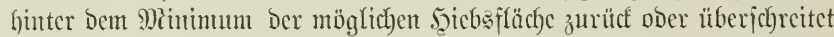
iic Deren Marimum, fo wiro eine Eortectur Derjeffoen notbwentig, weldye in ber Sicgel leidft mit Şilfe oer unter 4 bezeidfneten, fraglicfen Dite erfolgen fumn.

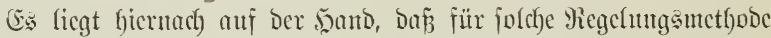
cbenjowenig cin Recept borgejefreben werden fanm, als für bas im

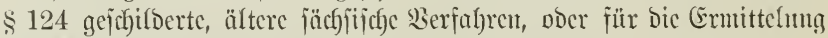

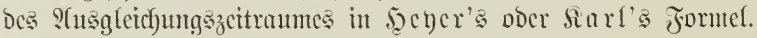

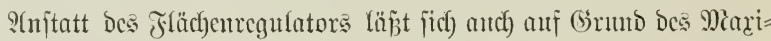

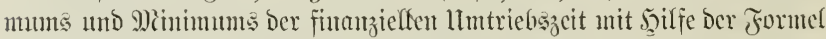

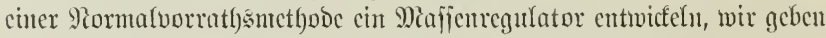

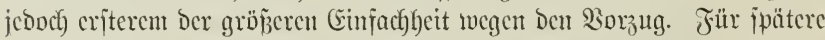
Picvijïnten gewimut Das Endrejultat Der S(futubungstabelfe (\$ 104) Den (Egarafter cincs ganz vorzüg(id)en, höd)jt cinfachen Regulators bes

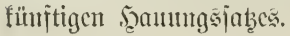




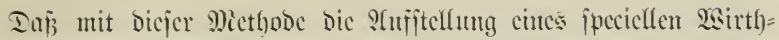

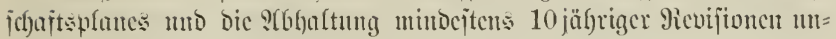
bebingt verbundon jein müfïen, verjteft ficf von jelfjt.

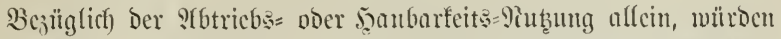

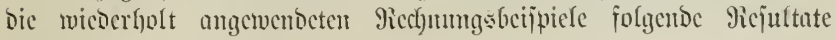
crgethen:

1. Rechnugą beifpiel.

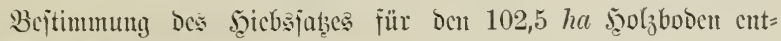
haltentoen 2 sald.

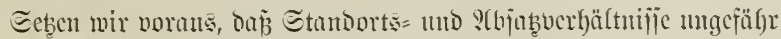

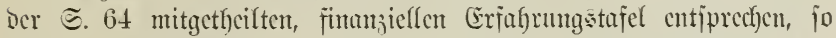

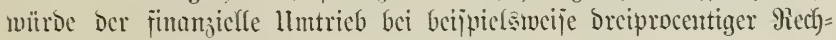
unty in Das 80 fte fis 90 fte Saffr fallen.

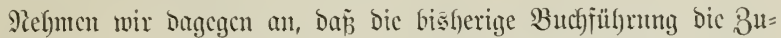
jammenjiteflung eincr julchen Tajel nicht gejtutte, weif über Den Ertrag

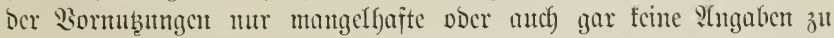
Fintocn jeien, jo veranjichlagen mir bicje Ẽrträge nad) ?(nalogie unberweit

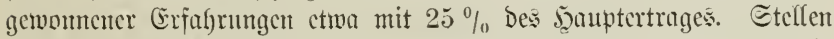
wir ferner bic Sulturfojten mit 30 fl. in Yiechmung, io crfalten wir

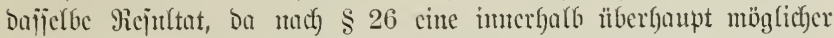

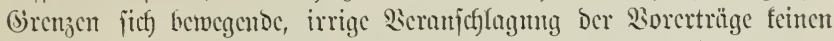

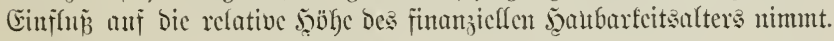

Siutturfojtenfreicr Bscjammtertrag:

$$
\begin{aligned}
\text { im } 70 \text { iten Jafre: } 1429+357-238=1548 . \\
" 80 " \quad \text { " } \quad 2036+509-319=2226 . \\
" 90 " \quad \text { " } \quad 2875+719-429=3165 . \\
" 100 \quad \text { " } \quad 3780+945-577=4148 .
\end{aligned}
$$

Bobenbruttorente:

$$
\begin{aligned}
& \text { im 70iten Эafre } \frac{1548}{230,59}=6,71 \text {. } \\
& \text { " } 80 \text { " " } \frac{2226}{321,36}=6,93 \text {. } \\
& \text { " } 90 \text { " " } \frac{3165}{443,35}=7,14 \text {. } \\
& \text { "100 " " } \quad \frac{4148}{607,29}=6,83 \text {. }
\end{aligned}
$$


Comeit wir Don Umtrieb für bie Rechnung brandfen, genügt es

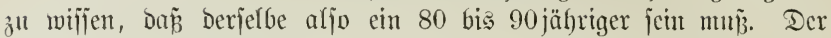
normale Jahresjichlag itellt fich hiernach anf 1,13 bis 1,26 ha.

Die Bergleichung bes wirffichen mit bem normalen :Gterstlaijen= verbältnifĩe giebt folgendes Rejultat:

\begin{tabular}{|c|c|c|c|c|c|c|c|}
\hline \multirow{3}{*}{ 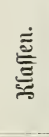 } & \multicolumn{3}{|c|}{ Alterskfarrenverfältnik. } & \multicolumn{2}{|c|}{ für dell 80 j. $\mathfrak{u}$. } & \multicolumn{2}{|c|}{ für den $90 \mathrm{j} . \mathfrak{\text { l. }}$} \\
\hline & \multirow[t]{2}{*}{ mirfáticjes } & \multicolumn{2}{|c|}{ normales } & \multirow{2}{*}{ zll biel } & \multirow{2}{*}{ z̆l wenig } & \multirow{2}{*}{ zu biel } & \multirow{2}{*}{ zu wentig } \\
\hline & & $80 \mathrm{j} .1 \mathrm{ll}$. & $90 \mathrm{j.}$ II. & & & & \\
\hline BI & 5,95 & 1,26 & 1,13 & 4,69 & - & 4,82 & - \\
\hline I & 40,05 & 25,31 & 22,53 & 14,74 & - & 17,52 & -- \\
\hline II & 36,25 & 25,31 & 22,53 & 10,94 & - & 13,72 & - \\
\hline III & 7,50 & 25,31 & 22,53 & - & 17,81 & - & 15,03 \\
\hline IV & 5,00 & 25,31 & 22,52 & - & 12 & - & 17,52 \\
\hline $\mathrm{V}$ & 7,75 & - & 11,26 & - & & - & 3,51 \\
\hline
\end{tabular}

Bei Dem entichicbenen Heberwiegen ber jungen, oder bei Dem

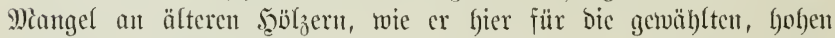
Itmtriebe unzweifelfaft zu Tage tritt, erjckcint natïrlich möglichjte

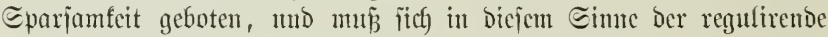
Einfluz des jummarijh crmittelten Flächenjabes auj bie ber reinen

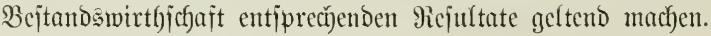

Rebtere find folgende:

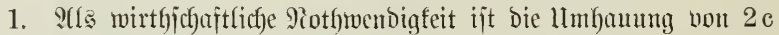
und 5 a ju betrachten, แ⿰亻⿻ fommen Deshalf in S(njab von 2 b 0,75 und von $5 \mathrm{c} 1 \mathrm{ha}$.

2. Entjucheden Giebsreife Drte, Deren Weijerprocent unter Den

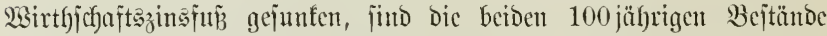
$3 \mathrm{c}$ und $6 \mathrm{~d}$. In zweiter Beige ericheinen afs joldhe bie beiben $90=$ jährigen Srte $1 \mathrm{~d}$ und $4 \mathrm{a}$.

3. Bejtände, reldye nur Der Dromung Der şicbsfolge entichieden

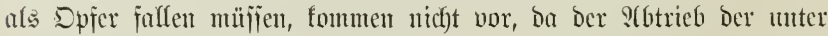
2 genaunten Srte in biejem Sinne jwar notgrwendig, jeboch nicht mit Spfern verfmüpit ijt.

4. 3mcifelfafter शatur find 1b und 2 a. - Dicjelben gebören 


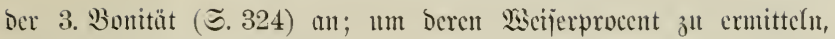
jei Jolgendes vorausgejest.

Ier 2arrath Des 75 jäbrigen Bejtandes beträgt 402, Der maf)r=

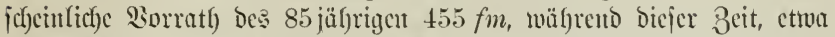

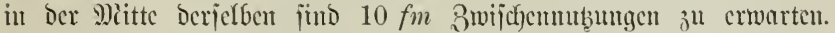
Der crutefojtenfreic Prets des 75 jährigen Jolyes ijt 3,5 , Der bes 85 jälrigen 4,2, Der bes Worertrages 2,6 fil.

Duantitäts $=3$ umachsprocent.

$$
\begin{aligned}
& a=\frac{465-402}{465+402}+\frac{200}{10}=1,45 \% \text {. } \\
& \text { Sunalitäts }=8 \text { mwach):sprocent. } \\
& \mathrm{Q}=\frac{455 \cdot 4,2+10 \cdot 2,6 \cdot 1,03^{5}}{455+10}=4,175 \text {. } \\
& q-3,5 . \\
& \text { b }-\frac{4,175-3,5}{4,175+3,5} \times \frac{200}{10}=1,76 \% \text {. }
\end{aligned}
$$

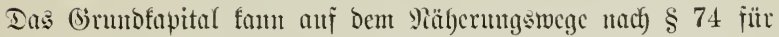
Den 80 un 90 jübrigen llmtricb berechnet werben, mo fund Majien mie Preije Der 4. Bonität in STmentung ju bringen, da ber Etand= ort jolcher Bonitüt cutipricht:

$$
\begin{aligned}
& 0,13 \mathrm{H}_{80}=0,13 \times 509 \times 4=264,7 . \\
& 0,09 \mathrm{H}_{90}=0,09 \times 575 \times 5=258,8 .
\end{aligned}
$$

In runder 3 affl fönnen 260 fl. angenumenten werden.*)

Der mittlere jeoljoorratfsimerth betrïgt

$$
\frac{1941+1407}{2}=1674 \mathrm{fr} \text {. }
$$

Reductionsbrud) (jternad)

$$
\frac{1674}{1674+260}=0,87 \text {. }
$$

*) פ̧ollte man ১aร (Snumbfapital unter Sntwendung ber 3. Bonität be=

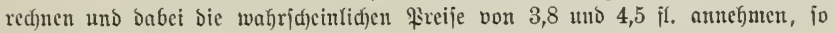
erthält unau

$$
\begin{aligned}
& 0,13 \mathrm{H}_{80}=0,13 \times 430 \times 3,8=212,4 \mathfrak{i l} . \\
& 0,09 \mathrm{H}_{90}=0,09 \times 477 \times 4,5=193,2, "
\end{aligned}
$$

in abgerumocter 3 ahl jonad 200 il.

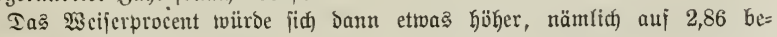
ved)ntn. 
Weijerprocent

$$
\begin{aligned}
& (a+b) 0,87 ; \\
& (1,45+1,76) 0,87=2,79 \% .
\end{aligned}
$$

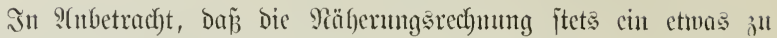
fleines Siefultat liefert, fam man abgerundet jonady Das 2iscifer=

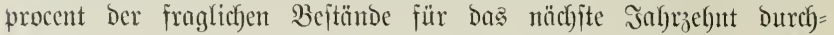

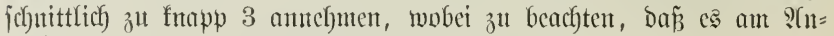
fange Dicjes Beitranmes höher jteht, als am Siblujje Defijelforn.

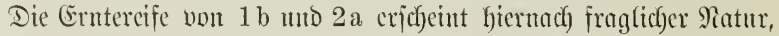

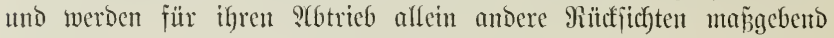
feitt fönnen.

Die Flächenfumme der in ben vorläufigen Entmurf anfgenom= menen Soicborte ift mm:

$$
\begin{aligned}
& \text { unter 1) won } 2 \mathrm{~b} \quad 0,75 \mathrm{ha} \text {, }
\end{aligned}
$$

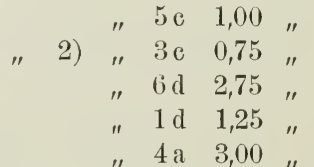

$$
\begin{aligned}
& \begin{array}{llllll}
\text { " 3) " } & \text { 4) } & -1 \mathrm{~b} & 2,50 &
\end{array} \\
& \text { " } 2 \text { a } 2,50 \text {, } \\
& \text { 8ujammen 14,50 ha. }
\end{aligned}
$$

Die als alfgemeiner Regulator aus Der Siergleidfung bes wirt=

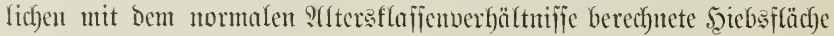

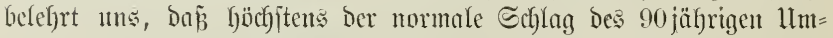
tricbes mit 11,3 ha, wommög(id) jedoch noch etwos weniger jum f̧iebe gejebzt werben möchte, wiorigen falles man fonft (scfal)r laufen würbe, im zweiten Jabrzelbnte zu jebr in bie unreifen Drte greifen, aljo

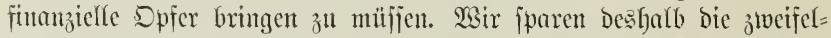

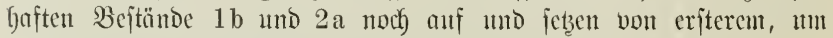
Den Jjieb bartin anzubalyuen, mur etron 0,5 ha mit ant.

Dic s(btriebsumbung von ben auf bicje ?(rt jum Şicbe bejtinmten 10 ha wätro $5348 \mathrm{fm}$ betragen. (3n vergl. ธ. 346.)

Die am Beginne Des zlveiten Jafrzelfntes cintretende Revifion

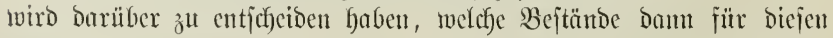
Beitraum zum şiebe ju jtellen jeien. Die Forjteinridytung hat ben Sang befielben foweit georbnet und angebabnt, afs es nothtwendig 


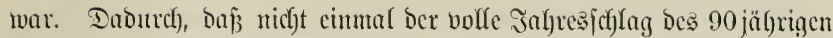

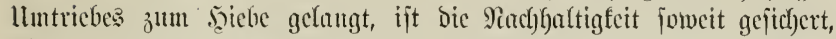

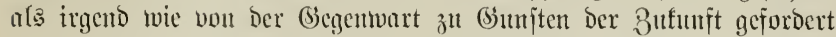

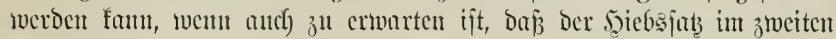
Jalyrjelfute nod) ctwas juffen mo erjt fpüter wieder iteigen bürfte.

\section{Redunugefijpicl.}

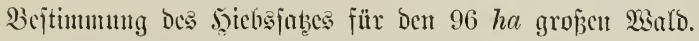

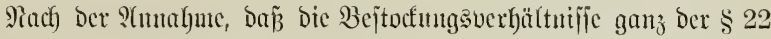

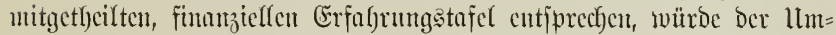

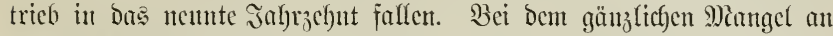
Gichereifen Bejtünton, da bas 2 scijerprocent bes 60 jührigen Drtes a nod) auf fnapp $4 \%$ lautet, ijt jeber stbtrieb in Siunc ocr Fintunz=

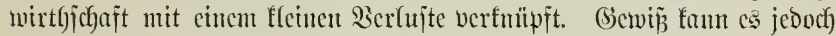

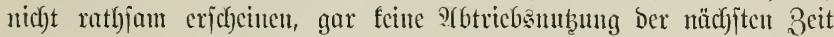

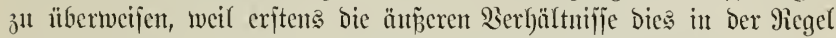

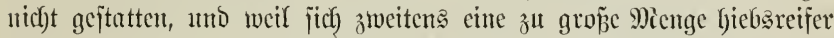

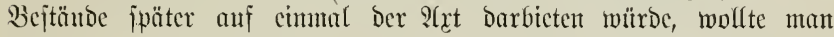

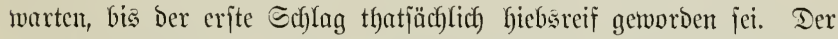

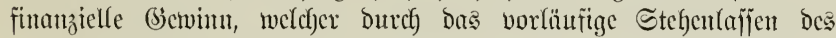

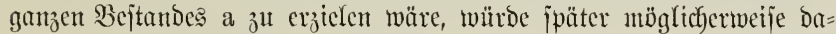
Durch gänjlich paralyfirt, vielleicht jogar in Berlujt vermandelt, wenn man bie ju grojęen Mafjen beu local jebr bejchrünften Marfte nicht bicten fönnte.

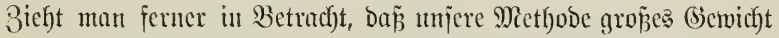

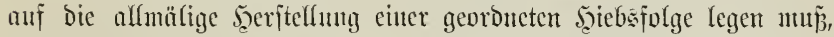
jo rechtfertigt fiff ber s(btrieb cines mäp̈igen Echlages im unteifen

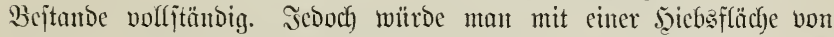
$10 \mathrm{ha}$, jonach) mit ciner ?(btricbsmubutng von $10 \times 394 \ldots 3940 \mathrm{fm}$ wohl zltrieden jein fömten, worauf freilich eine bebentende Etcigerung

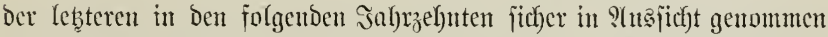

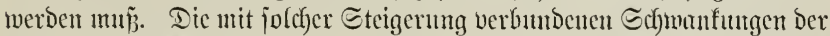

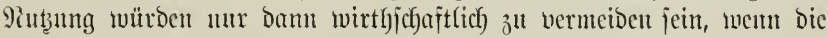

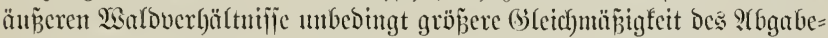

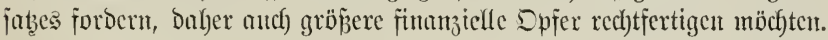

\section{Die 3misctjennutzungen.}

Im Einne des Pormalwaldes werden 3 wijithen= ober Bornutbungen fajt nur als Durchforjtungen (im weiteiten Begriffe bes Wortes) er= 
folgen. Dic Wirtljoj)aftsfüfrung forbert Dagegen cine formelle :(b= grenżug, weldye den Begrif̈ ocr Bwijchenmutumgen etwas crweitert,

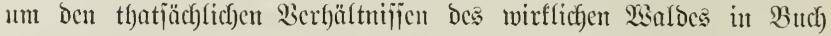

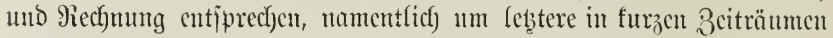
abjülię̧en zu fömnen.

3ur Erflärung bes Begriffes "Bwijchenutbutugen“ gehen wir von

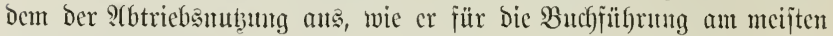
pajiend erficheint.

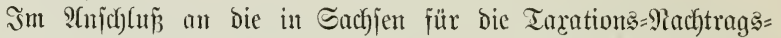
arbeiten 1873 erlafjene Injtruction betract)ten wir als $\mathfrak{A} \mathfrak{b}$ triebs= กt $u$ b $\{\mathfrak{n} \mathfrak{g}$

1. alle Erträge, weldfe in Bejtänden oder in Bejtandestlyeifen ausfallen, bie jutr Serjüngung bejtimut j̈mb,

2. hei anberen Drten Diejenigen Erträge, meldbe in Folge von

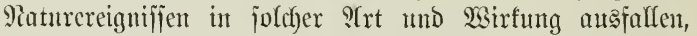
Dá baburch die Berjüngung Des betreffenden Bcjtundes oder Bejtandstyeiles geboten erjcheint, gleichviel ob ber :(btrieb in nächjter Beit wirflich erfolgen fanm oder nicf)t.

:(f 3 wijd)ennubungen gelten alle übrigen bei uno bebuf: der $\mathfrak{B}$ cjtandspflege ansfallenben Maj̃en, jowie bie zufällig cingebenden

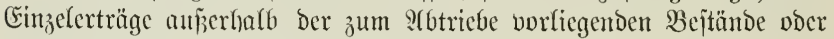
Bejtandstheile. $3 u$ ifnen gehören aljo:

1. Die Erträge Der Durchjorjitungen,

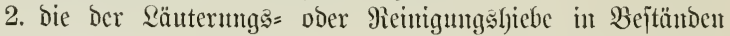
ber jütugiten ?lltersflajie,

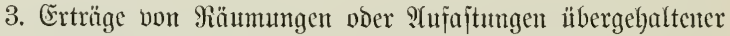
Walorect)ter 4. $\uparrow$. เw.,

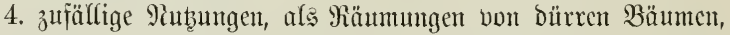

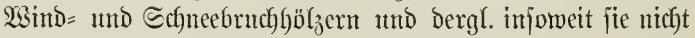
in şiebsorten erjolgen.

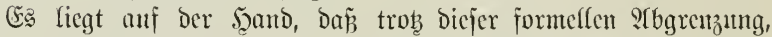
nament(ic) bezüg(iç) Der unter 4 gentumten, zuf̈̈̈lfigen (Erträge mautc)= mal 3meifel barïber entitehen fömren, ob jie zut ben 2 fbtricbs $=$ oder

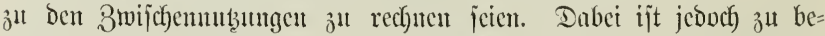

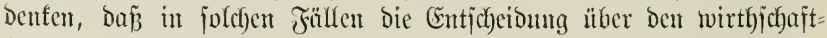
lichen Charufter Der Pubungen cine weitgehende, möglichermeije bas

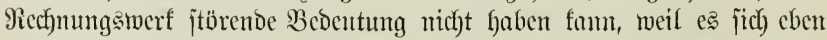

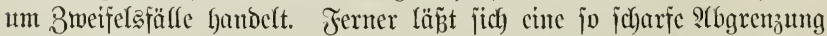

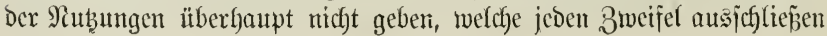




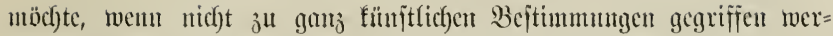
Den joll.*)

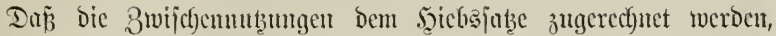
Gulten wir für ridgtig, baygegen ijt nur won Fall zu Fall Daritber z"t

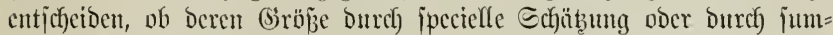

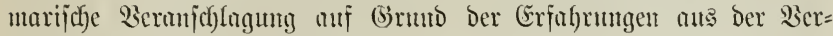
gangenfeit bejtimnt werden joll, ferter ob Deren zu erwartender $\mathfrak{B} e=$

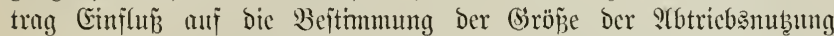

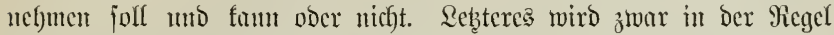

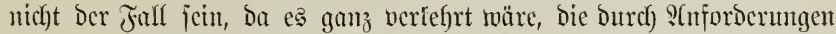

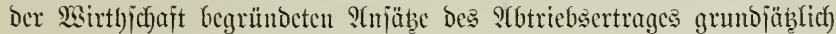

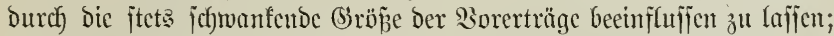

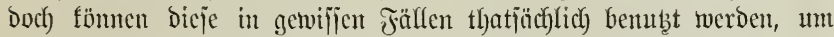

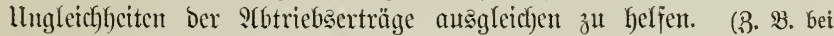
Llmwandluntgen, vorläufigent Miangel Giebşreifer Beitäıbe u. j. w..)**)

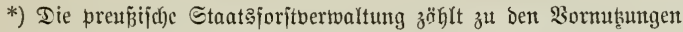

1. "Die Durdfforfinngen, weldhe den Yebenbejtano betreffen;

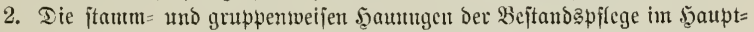

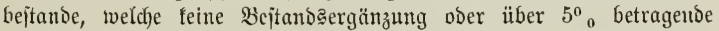

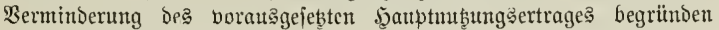

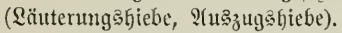

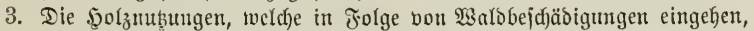

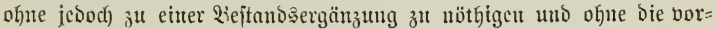

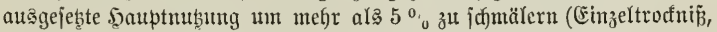
Einzelbritd) Durd, $\mathfrak{S}_{3}$ into $2 \mathrm{e}$.).

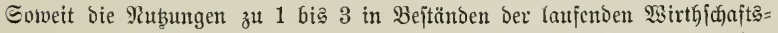

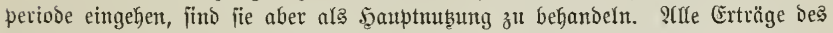

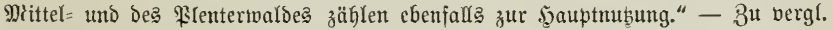

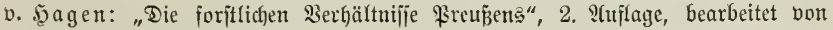
Donner, 1883. 1. Bd. S. 178 . -

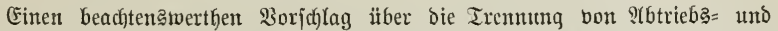

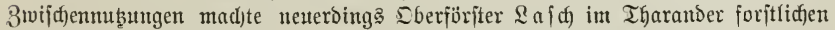

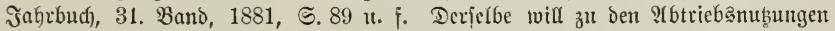
alle (Entnafmen red)uen, weldye ein bejtano nad) feinem (Eintritt in bas baubar=

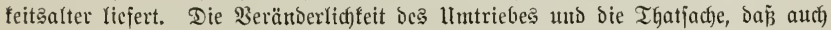

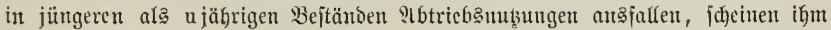

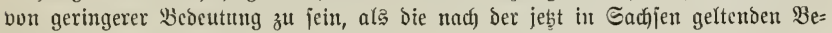
ftimunung vielfach möglid)en 8 meifel.

**) Schmieriger ijt bie (Erlesigung Der Frage, in wie weit ber $\mathfrak{B i r t b j d a j t e r ~}$

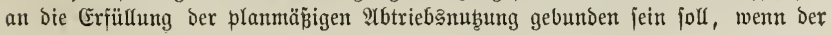




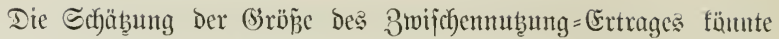
ıur bei Den eigentlichen Durchjorjłtungen und bei Den Räumungen bon TSaldred)tern und bergl. bejtandsweife erfolgen, Dent ber Ertrag ber Qäuterungshiebe ift in ber 9iegel zu unbebeutend, und bie zufïlligen Piubzungen lajien fich im (Einzelnen im Soraus nicht bejtimmen. Des=

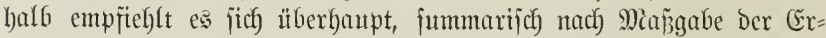
fabrungen aus ber Bergangenfeit vorzugeben, bieje alferoings mit

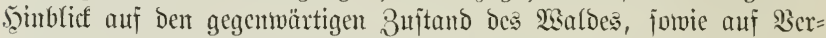

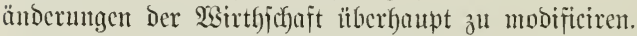

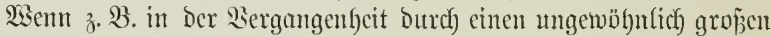
Echuectond ber Betrag ber Bwijhenmubutgen ein bejonders hoher war, fo faum er nicht ofne burch biejen Imitand bebingte Mobifi=

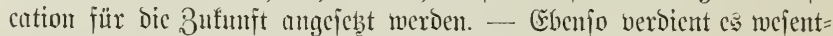
(ich) Beachtutg, ob bie Durchforjtungen früber bejonders ftarf betricben murben, ober of fie zurücfblicben น. $\uparrow$. เ

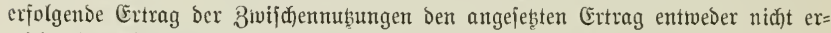
reidst oder überif(f)eitet.

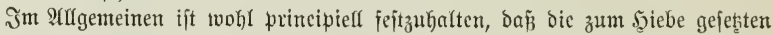

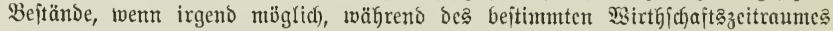

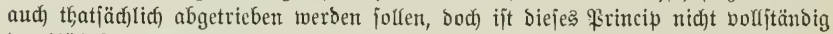

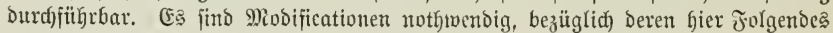
zu bemerfen ijt:

1. Erreid)t ber twirflidie (Extrag Der 3wijd)enmutbungen den angejebten

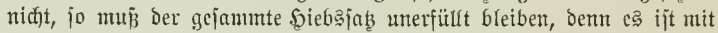

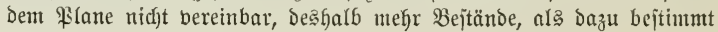
twurden, zum 2(btriebe zu bringen.

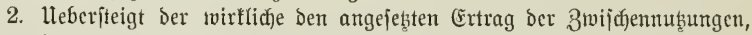

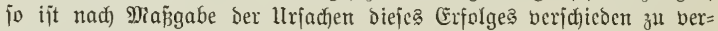
fahren:

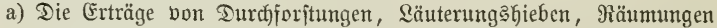

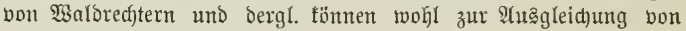

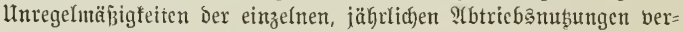

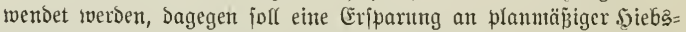
fläd) niemala ₹o folge Derartiger Mehrerträgc jein.

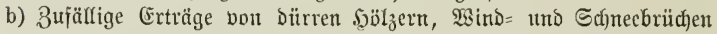

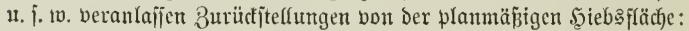

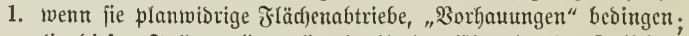
(in Diejem Falle berlieren fic ofnefin ben (Égarafter Der 3wija)en=

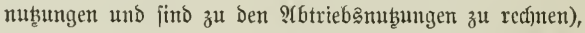

2. wenn fie nacfiweisbar, und zwar mit bebeutendem Betrage auf Sojten ber fünftigen $\mathfrak{A}$ btriebącrträge einzelner Bejtämbe erfolgen,

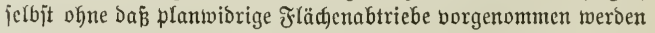
tömnen oder nü̈fjen. 


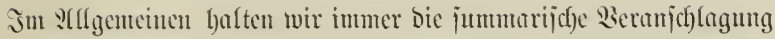

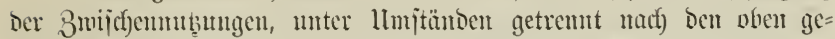

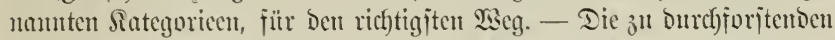

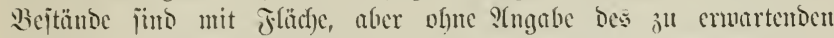

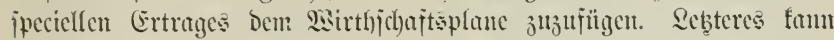

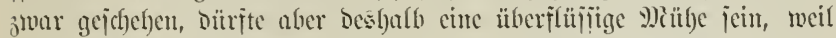

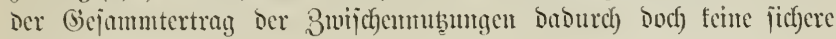
(Sröß̄อ miro.

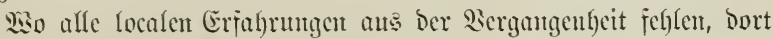
werben bem geülten Forjteinrichter wohl fajt immer vout atberen,

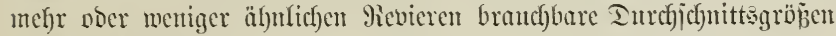
$3^{\text {hl }}$ Gebote jtehent.

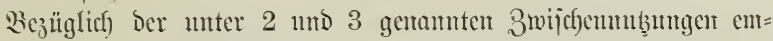

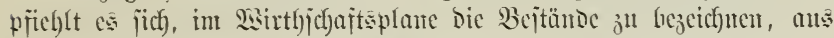

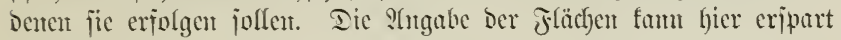
werbent.

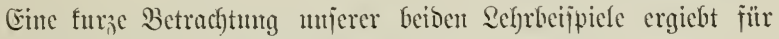

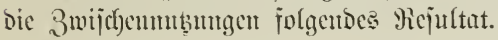

\section{Rechnutugacijptef.}

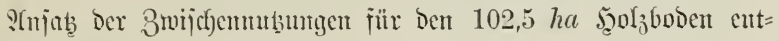
loultentoen sialo.

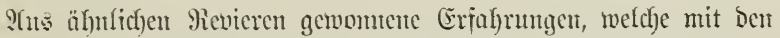

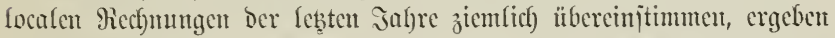

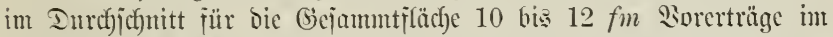

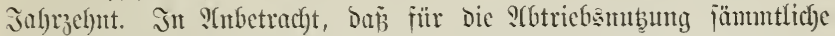

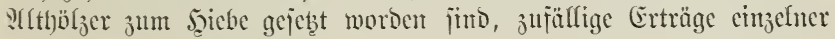

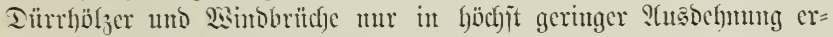

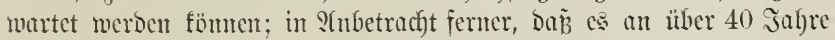

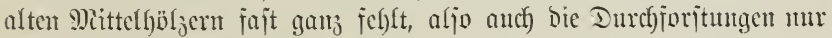

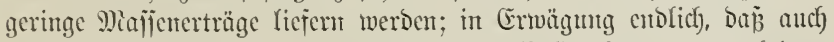

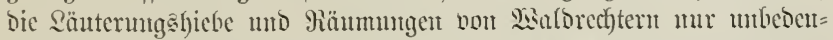

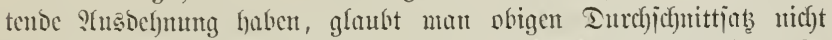

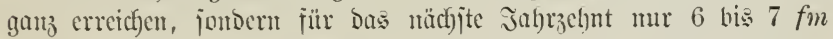

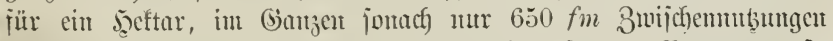

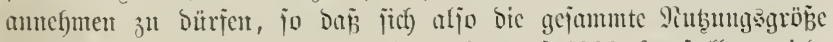
auf $5348+650=5998$ ober alygerundet anf $6000 \mathrm{fm}$ itelfen wirb.

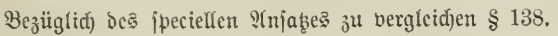




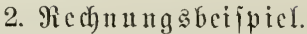

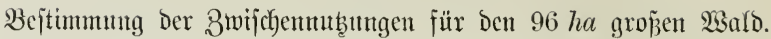

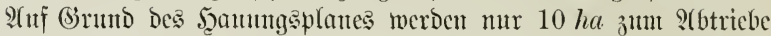

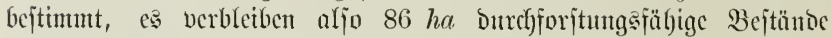

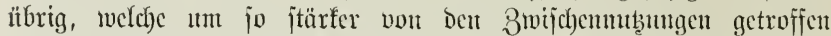

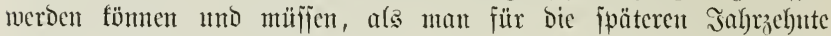
wojentlich) böbere S(botriebentbungen mit Sidyerbeit crwarten fann. Durchforjtet man bic nicht zum Szicbe gejtell te Fläche int nächjiten Jahrachnte zwcimal, jo fömuen füglich vom Sceftar im Durchjichnitt 30 bis 33, im banzen ponad) circa 2600 bis $2800 \mathrm{fm}$ 3wijichen= utb̧utgen erwartet merden.

Dic S(btriebsnubung beträgt 3940, und ijt hiernach) bie gejamnte

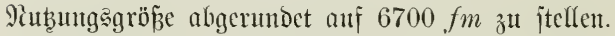

Je mehr es gerechtfertigt ijt, bejüglich Der Bejtummutg bicjes Sciebsjabes jummarijch, ohnte ängitliche benautgfeit vor

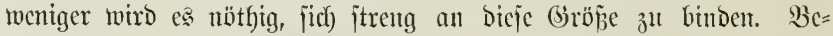

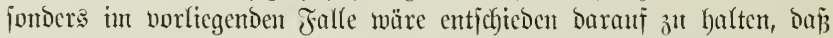
bie angejebzte Fläche wirffich) abgeholzt weroc, fowie da baulich und nach Den Marftuerbältnifïen mïglichen Durchjorjtutgen เvitflich) zux S(usfülyrung gelangen, jelbjt twemt beren Miaterialertrag llebexichreitungen des j̧iebsjabes jur Folge hätte.

\section{Stockifor r $_{3}$.}

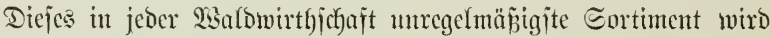

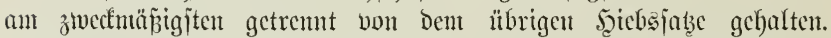

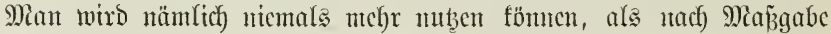

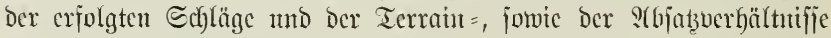
utöglich ijt. Es genẗgt baher ein ganz ungefübrer Soranj(b)lag ent=

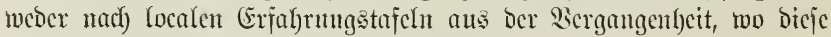

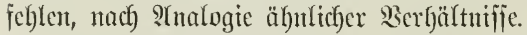

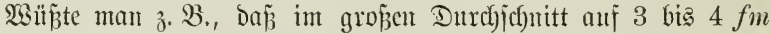
Derfigolz $1 \mathrm{rm}$ Stodfholz entficle, jo twitroen für Den 102,5 ha grofien

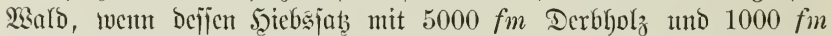

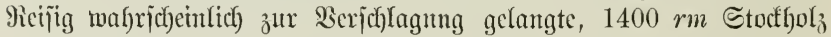
anzujeben jein.

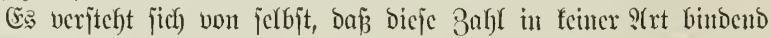
jein fantu. 
St nuerfung. Das hier für Dent Saldlichlagbetrieb crläuterte Berfabren bedarf für bie S(nwendung auf ben \$ilenterjed lagbetrieb untr geriuger, und jowar utr formelfer Miodificationen. SUls 9iegutlator Des ants Der Bejtandswirthichaft ermittelten biebsjabes exjefeint chen jo gut bie unter Beach)tung Des S(ltersflafjenverfältniffis berech)nete

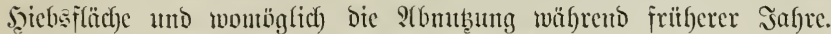
- Bei Bejprechututy Des "2sirtljidfaftsplantes" merden wir auf cute

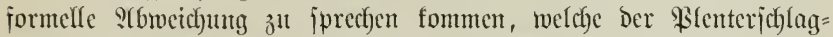

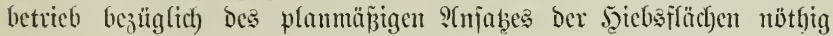
ntacht.

\section{$\S 133$.}

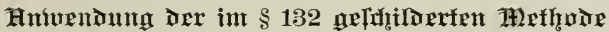

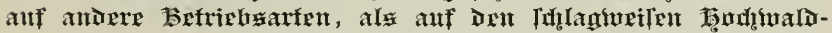
betrielr.

\section{A. Jiedermato.}

Für bicje curfache Betriebsform ijt mur ber finanjielle llmtricb jll crmitteln und cine auf biejem bajitte geometrijede, bei wejentlid

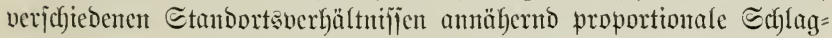
cintbcilung Durtfzufüfren. Der aus leb̧terer folgende Şiebsją̧ bedarf

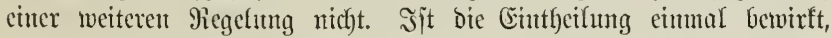

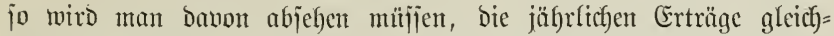
mäp̈iger gejtalten zu wollen, aļ fic bic Echlagcintbeilung giebt, bent jeder 2orgriff an einem Echlage in Den anderen, ebenjo jedes Burüct=

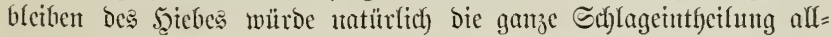
mälig zerītören.

31wijchemmbungen von Bedeutung wird Der Piebermald mur bei

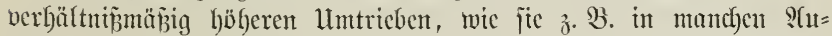

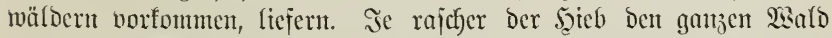
Durch)fäuft, Dejto jefferer werden bie aus Der Dergangentheit zu getwin=

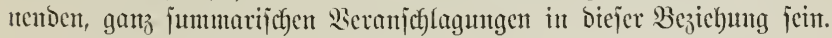

\section{B. Mittefmato.}

In Der Şauptjache berutyt bie Dicjer Betriebsart nötfige Piegefung zunächjt anf ciner für bas Interbolz, wie beim litedertwalde, zu tref= fenten Echlagemtheilung. 2abaricheinlich bürfte wohl in ben meijten Fällen ber finanjielle llmtricb Des llnterholzes etwas niebriger liegen,

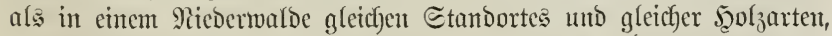

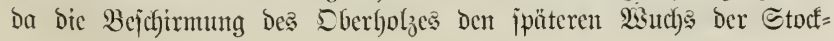


ausifchläge mel)r becinträchtigt, als wic es in Den jüngeren 9 (ftern Der Fall ijt. Ulbrigens geftattet audf) Der niebrigere llmtrieb bes llnter= holzes cine feinere sberfiglapfege, als ber höbere.

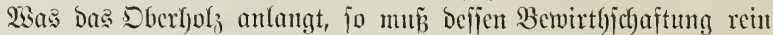
in bas Gebiet Der Foritgärtnerei fallen, weshalf eritens ber Mittel=

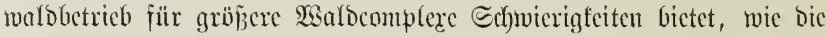
Erfalyrung wicberfolt gelefret fat, weshatb cs aber zmeitens ein ver= fehltes Beginnen wäre, hier cincn amberen, als ganz clajtijchen, unge=

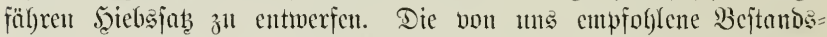
wirtbichaft wird in Dem an 2 (rbeit intenjiwen Mittelwaldobetricbe zux Banmmirthichaft, wenn man wirflich rationdl verfabren will.

$11 \mathrm{~m}$ Den Şicbsjat jut beftinmen, bleibt nichts anderes itbrig, als nach) bewirfter Echlageintbeilung eine je nach Bedürfuiiß mel)r oder

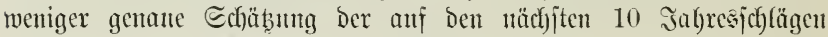

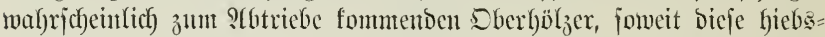

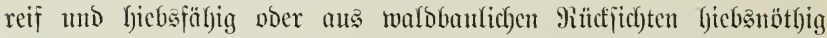

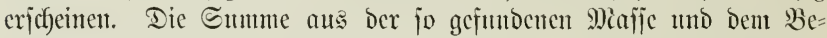

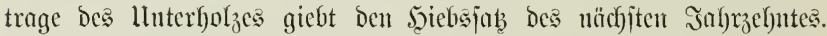
Dent 23irthjechafter wird es aber nicht in ben Sinn fonmen ditrfen,

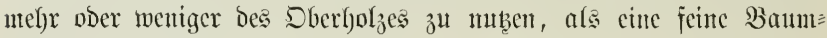

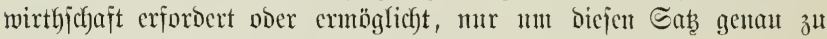

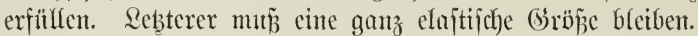

Yuf bicje Weije allein mird es möglicl), Den Mittelwald that= jächlich in entfpred)entom Bujtande ju erfalten. Ferner wirb baburech aber anch jedc voransgefende Beptimunng eincs llutricbes für bas Sberfyolz expart.

Bei irgento niedrigem IImtriebe bes lluterljolzes von 10 bis 20 Sabren werben in ber 9iegel 3roijchentubatugen entweder gar nicht ober mut it geringen Beträgen z" crmarten jeit. Siommen jic für

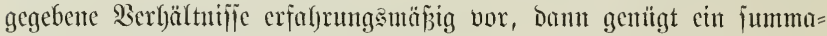
rijcher Soranjchlag nach (Erfaf)rungen aus ber Bergangenlyeit.

Scalten wix ç in 9flfgemeinen für cinen Febler, bie annäl)ernoc

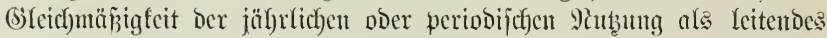
Frincip an bie Epitze Der Ertragsregelung z" itelfen, fo müfjen wir bies ganz bejonders beim Mrittelwalbe hetonen, ber nutr bei gröjter Freibeit Der Bewegung gedeifen fant. Sollen größzere Saldocompleçe thatjäcblich in bicjer Betriebsart erfalten werben, fo wirb alfmälig

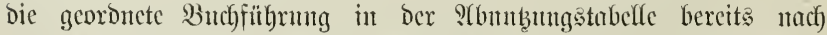

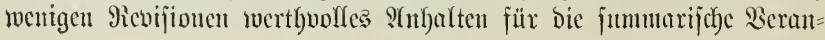




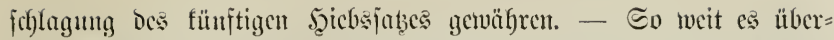
Gaupt möglich ift, wird Der Pachbaltigfeit Durch bic crwälgnte Eaflag= cintbcilung Piechumng getragen.

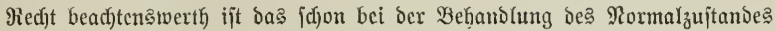

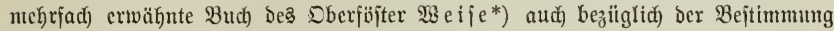

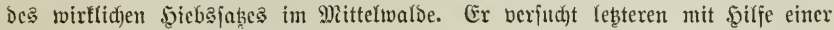
Normalborrathsmethode zu crmitteln.

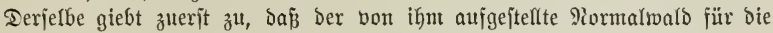

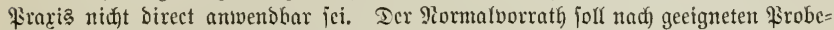

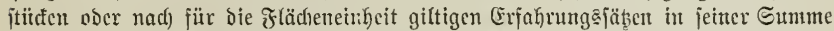

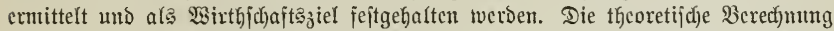

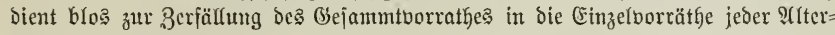
flafie (1. c. ๔. 31).

Der wirflidje 3orrath twirs Durd bejtandsaufnakme, wie aud ber normale,

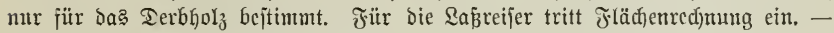

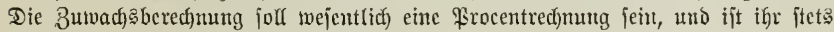

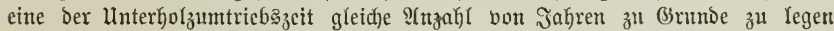

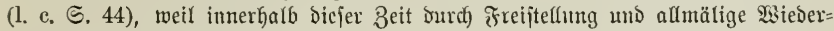
heritellung Des Echlujię Dic Jahrestringbreitent in ber Yiegel am brcitejten uno wieber am jdjmäliten werden.

Dffentar bereitet die Bejtimmung aller biejer Factoren bie allergröp̈ten Sd)wicrigfeiten. Sind jic aber eimmal ermittelt, fo wiro für einen $S_{d}$ lag

$$
\mathrm{e}=\mathrm{Z}_{\mathrm{w}}+\frac{\mathrm{V}_{\mathrm{w}}-\mathrm{V}_{\mathrm{n}}}{\mathrm{a}},
$$

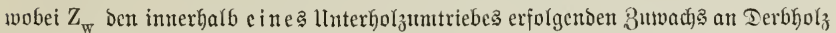

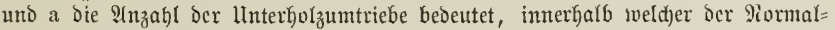
vorrath hergeitellt werden joff.

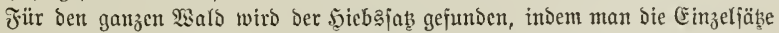

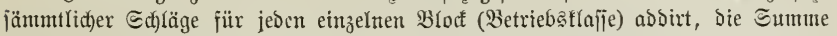

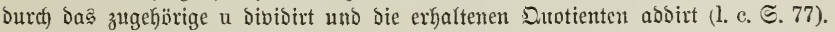

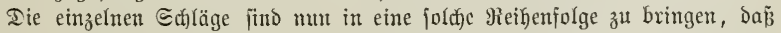

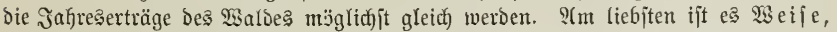

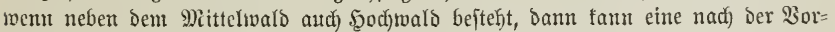

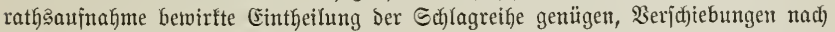
Der (Ertragębered)nung łönnen unterbleiben; man haut im Mittelmalbe lebiglidf)

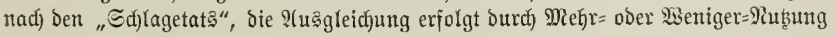
im 5oodfiwalbe (1. c. S. 79).

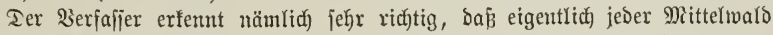

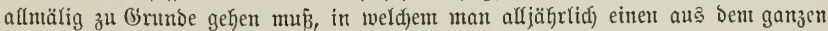

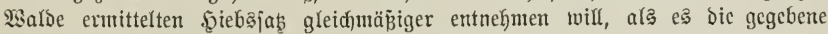

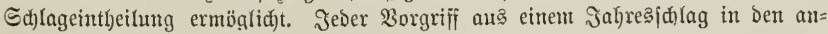

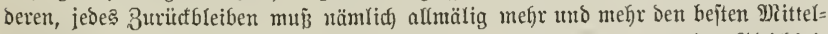

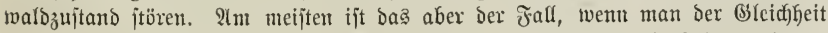

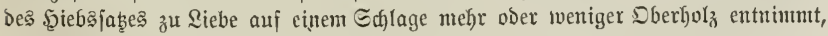

*) W. Weife: Die Laxation dç Mittelwalscs. Berliu, 1878.

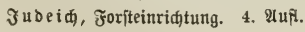




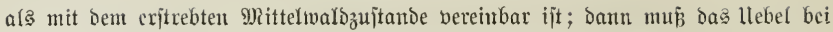

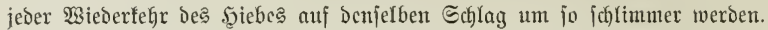

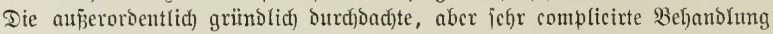

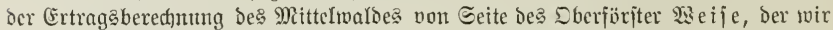

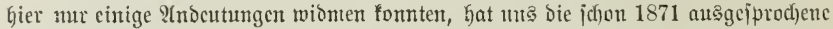

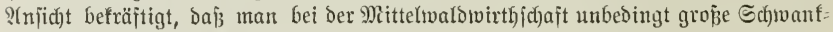

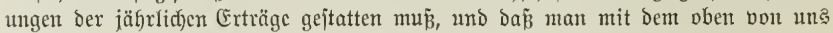
empfoblencn Serfahren auşutommen bat.

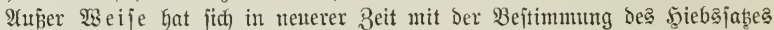

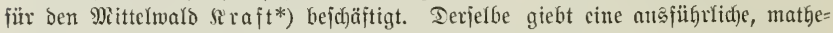

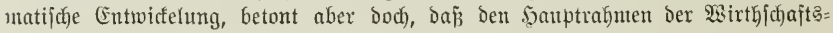

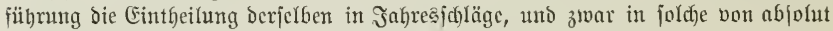

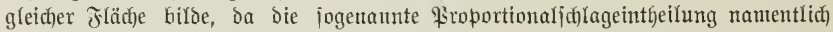

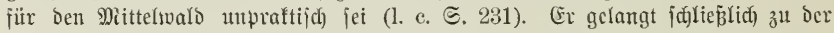

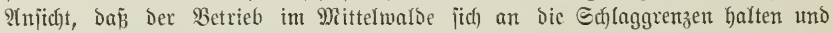

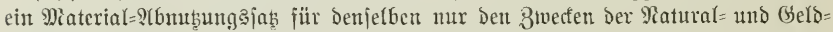

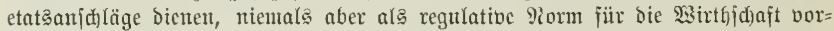
gejchrieben werben jollte (1. c. S. 232). WHix find ganz Damit eimberjtanden,

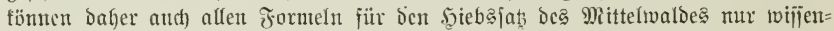

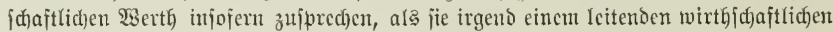

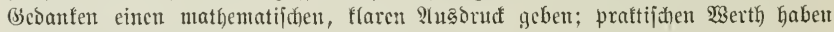
fie nicht.

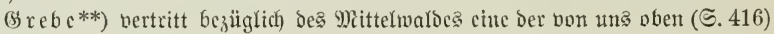
ausgeiprochenen ganz ähnlidbe Strficht. Nur will er bie Ertragsanjätse auj einte llm=

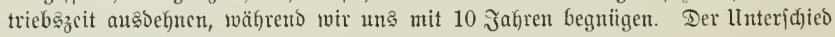
ijt bei bem meift fleinten u bes Mittelwaloce nidjt jefr groj.

\section{C. plentermalo.}

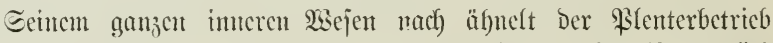

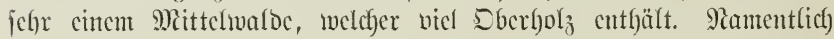
ijt Dics Der Fall beim Sanbholze. Intzweifclfaft füb bie älteren Mittefraloungen nidfyt auj Grumb vorangegangener Meberlegung fïmit=

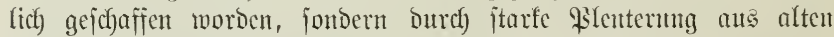

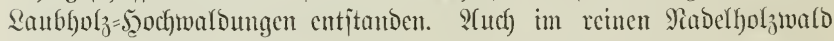

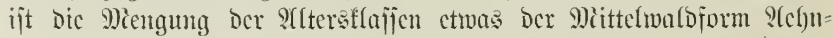

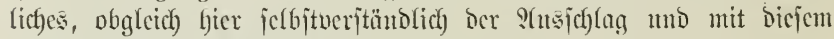

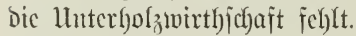

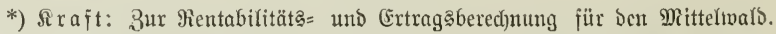

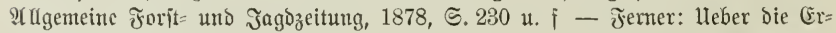

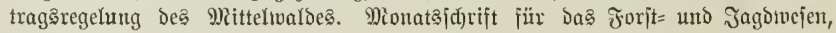
1868 , ङ. 165 u. ๆ.

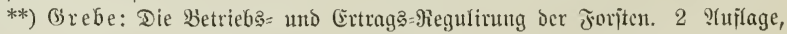
mien, 1879, ๔. 306. 
Se melkr wir ben Pilentermalo in jene Sagen verweijen müjïen,

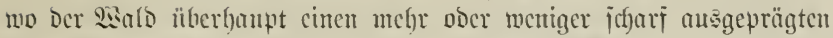

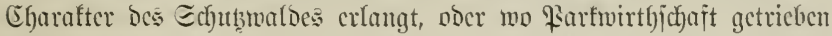

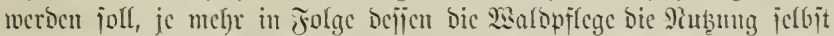

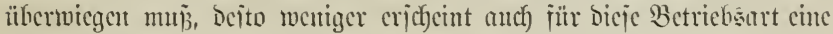

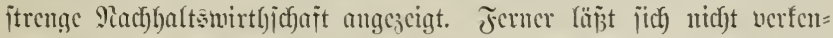
nen, Daj3 in Folge bicjes Den Rilentermalo cigentfüm(icf)en Ebarafters

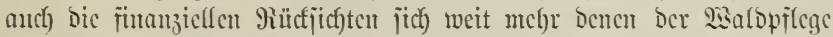
unterorbuen müjīen, als bei irgend meldfem anderen Betricbe.

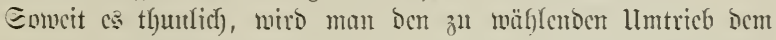

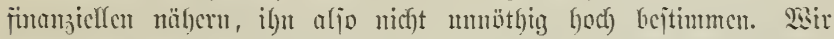
gehen aber gern ju, baj mobl in ben jeltenjten Fällen cine lleberein=

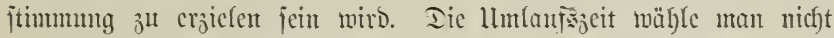

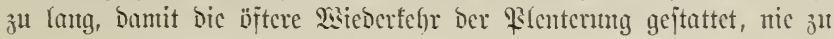
vicl auf cimmal ans cinem Erte ju jollagen.

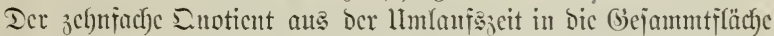

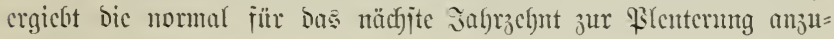
jeţende Flädbe.

Bezüglich ches iscalen Bifocs in bicjer Bejichnutg nermeijen wir auf ङ. 102, bemerfen bicrä jedod), Daj zum 3mecte der Erientirung mo jut Ertechterung Des Transportes Durch mehr odcr mentiger

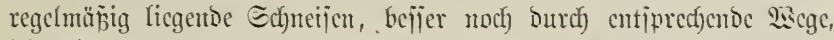

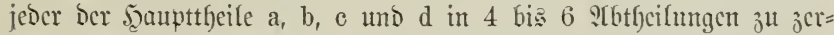

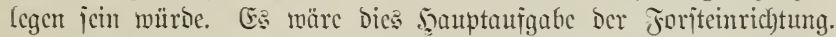

sielymen wir an, ber bout in ibealer Jorm gejdjilberte \$ifenter= wald von 600 ha Giröje jei gegenmärtig vollitänbig umregelmäp̈ig bcjtocft, wcil jrüher planlos auj Der ganjen Jläche herumgeplentert murbe, jo würbe man für bie nüchjten 10 safre ben 2isalotgcil a

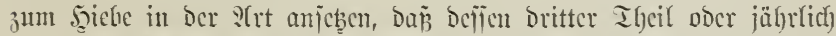
$1 / 30$, aljo 5 ha, in fleinen seoriten heraușenommen mürocn. Tic

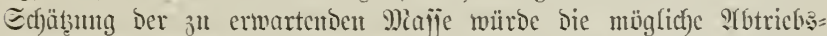

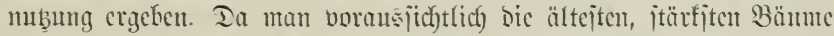

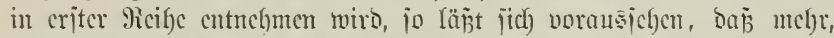
als Der britte I geil bes in a überfaupt vorbandenen Piorrathes jut Peubutg gelautgen wirb. Betrüge ber mirfliche Borrath in a fïr 1 ha 400, im Ganjen jontach $60000 \mathrm{fm}$, jo büriten leidyt gegen

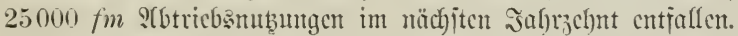

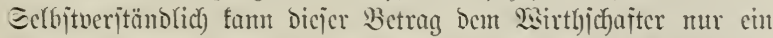

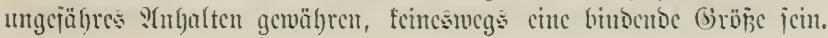




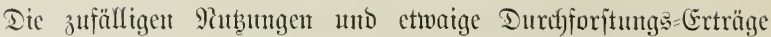

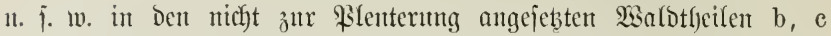
utro d mitroen ben Charafter von 3wijchen= und Bornubutgen an= nelomen.

Einer weiter gehenden Ertragsregelung bedarf ein joldyer \$Blenter= wald nicht.

\section{Jimmandumgen.}

llmmantolungen ans cincr Betrichart in bie andere giebt es jefr

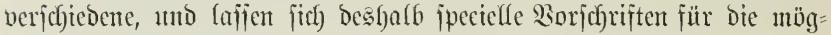

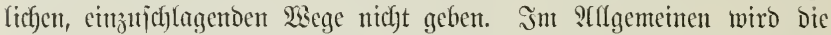
Bajis ber Errtragsregefung in ber möglichen Sciebsfläche zu juct)en jein.

9ehmen wir beifpiefweije den Fall an, es jolle cin unregelmäp̃iger

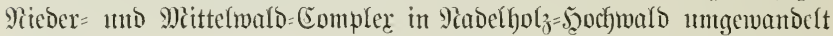
werbent.

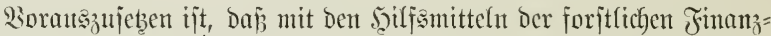
rechnung zunäcd)jt bie Sortbeile Der llmwandung in fo weit flar Dar=

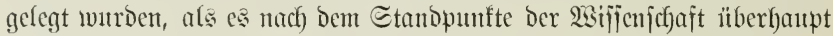
mïglich ijt.

Die erite und wichtigite SUfugabe ijt bie Crintfycilung Des Banzen

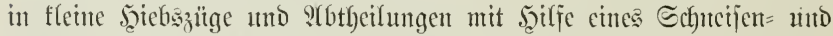

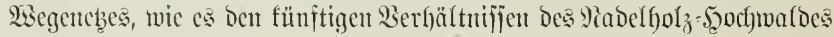
entipricht.

Solweit bie llntricbsfrage bejüglich bes leţteren nicht zur Ent= icheidunt; barüber gebraudf)t wurde, ob itberfaupt umgewandelt werden jolf ober nicht, fan fie gewöbntlich fait gan aujer sacht gefajjen merben.

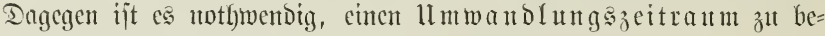

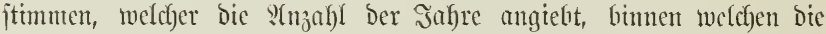

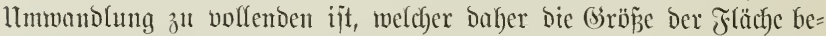

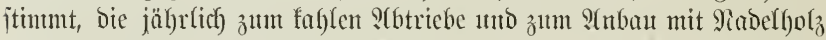
gelangen joll. - Diejer Untwantolungszeitraum beinegt jich) zwijchen zwei Grenzen. SAm längiten wïroe er bauern, wenn ex mit bem für bos

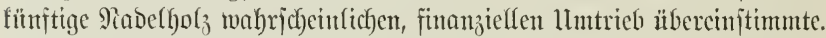
Sn ber Regel wirb man aber cine jo lange Dauer bes exiteren nicht

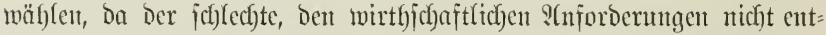
iprect)ende Bujtand bes Mittel= ober 9iedertwaldes bie 23eranlajiung 3ur

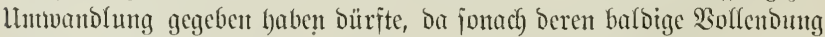

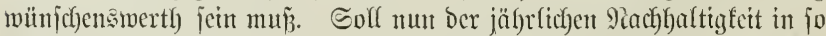

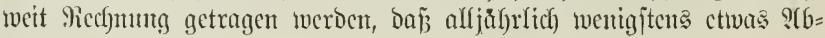




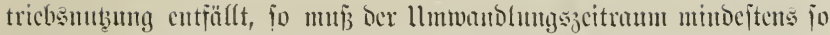
lang jein, baj bas juerit angebaute Pabdholz his jur Bollendung ocr

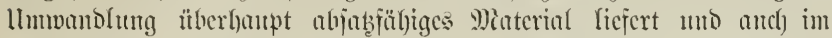

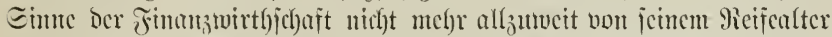

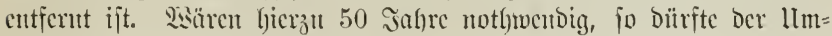
watolungşzitrum auch nicht fürzer gemählt merocu.

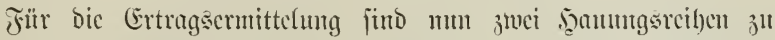
unterjcheion: Eritens bie Sicife Der Siafljchlägc, żweitens bic allmälig

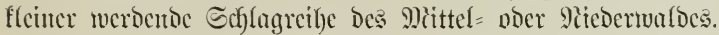

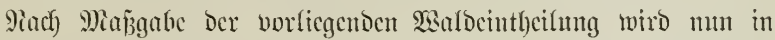

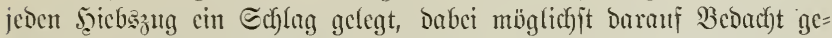

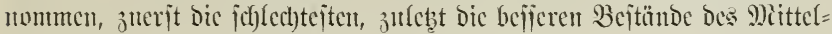
obcr Siedecrmaldes jur llmmandmug zu brimgen.

\section{Jiechnungbeippict.}

Ein 1000 ha grofier Mittelmalo joll in Piadelfolz binuten 50 Jabren umgewandelt werden, io ergiebt jich für bas nächjte Jaf)rzebut aus ber Sicife Der Sitrllichläge eine Şiebsfläche von $\frac{1000}{5}=200 \mathrm{ha}$.

Dic Sicrtheilung Der Echläge wirb nach Den oben angedeuteten Sï̈dfichten crfolgen. Bcitimmte bie Einthcilung für Den fraglichen

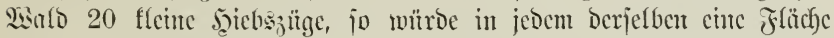
von 10 ha anjujetsen jein. Den 2 sirthjefajter bleibt es überfajjen, für gehb̈rigen Siectj)

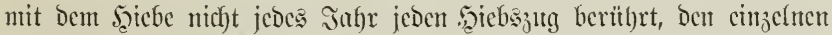
Echlägen aljo cine ?Hobefmung vont etwa 2 bis 3 ha giebt. Der ab=

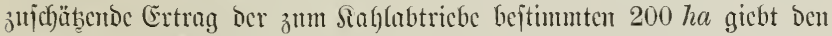
cincn Theil Der S(htriebsmubung für bas näch)ite Saf)rzehnt.

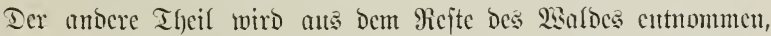

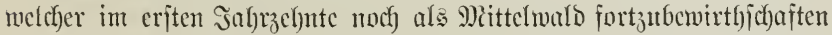
ijt. Im gegebenen Falle bileiben $1000-200=800$ ha Mittefwald

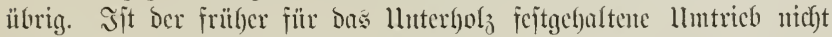

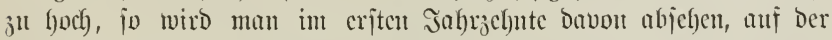

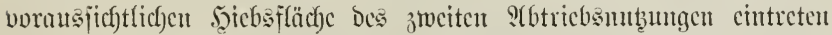

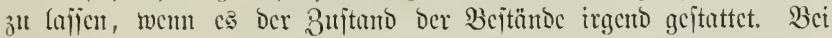
15 jül)rigem llntriebe müroc bas ältcite llnterf)oly, uno zwar auf Dem

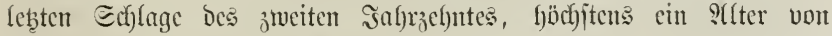
$15+20=35$ Jabren errechen, was bei angemejiener Durdyforjungs: 
pfflege wohl möglich ijt; bies um jo mefr, als bie Erhaltung Der

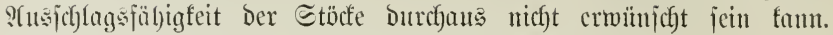
Dic im criten Jahrzefut als Mittelwald zu bemirthichaftemben $\mathfrak{B} e=$ itäube reduciren jict) jontach) auf $3 / 5$ Der Gejammtf̃läche, anf $600 h a$.

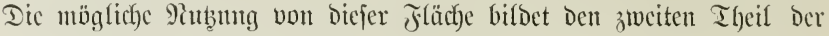
: (btrichsertrïge.

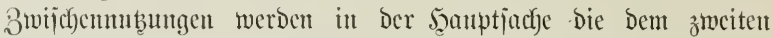

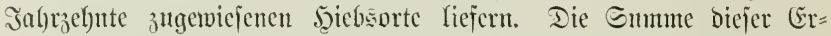
träge und Der etwa ansfallenden, geringen 3rijefennubungen Der als Mittelmald fortzuberwirthjefaftenden 600 ha giebt Den betreffenden รૃiebsjab.

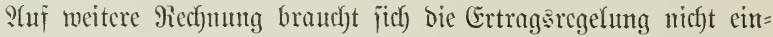
zulafien.

Deflien wir jeboct) beijpiefsmeije unjere Betradftung bis jum Ende des llmwandhutgs= Beitraumes aus, fo ergiebt jich folgendes. Rejultat:

Im żwciten Safyrzchnte betragen bie Siabljchläge abermala $200 \mathrm{ha}$,

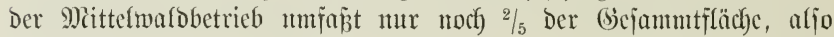
$400 \mathrm{ha}$.

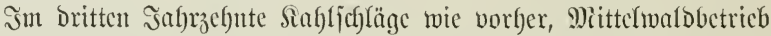
$200 h a$.

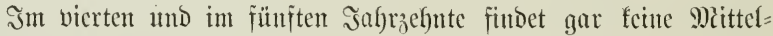
walbwirthjefjaft mebr jtatt, jonbern cs ijt jedes berjeffoen nur mit 200 ha Sabljchlägen autgegcjtattet.

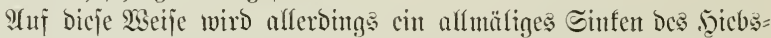
jabes bis jum vierten Jafrjelyut unvermeidich jeit. Dem wird it=

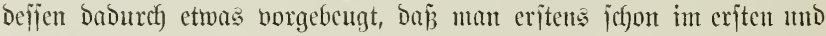
jwciten Sabrjefnte Darauf Bebacht nimut, die Pubung bes Dher=

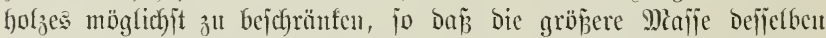

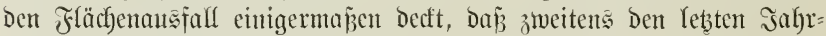

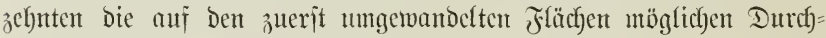
foritungserträge zu Şilfe fommen.

\section{\$ 134.}

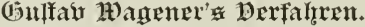

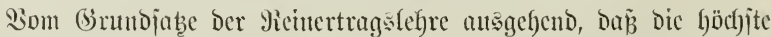

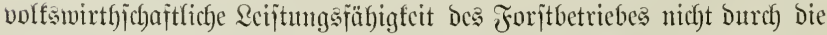
Fortjebung Der bisherigen Bunbungart Der Waldungen, aud nict)t 


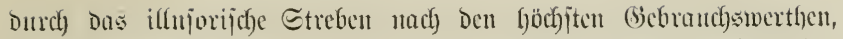

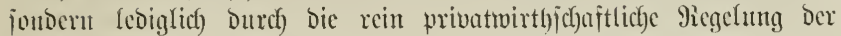
Foritmirtfjechaft berbeigefülyrt merden füme, verjucht (5). Wagener cin bejoutoces Serfal)ren oer Piegelung Des Forjtbetricbs ju entwicfeln.*)

Er nertangt Eintheilung Des 2 saldes in Betrichsflajfen, fïr Fichten=

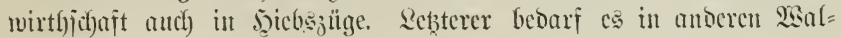

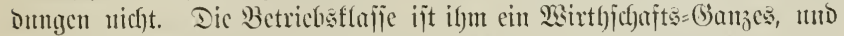

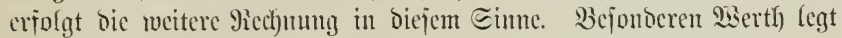

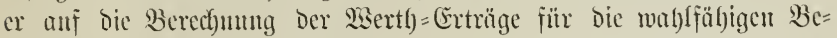

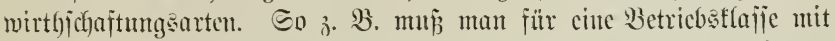
nörolicher ober jjtticher S(boad)ung anf gutem Boben it Rechnung

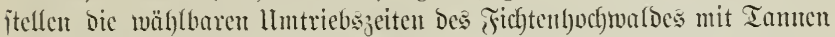
แmb Rärchen แmD Błuchen, Dic Sicfermumtriebszeiten, Den M)ittelmald=

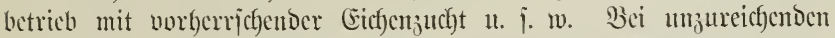

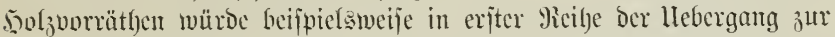

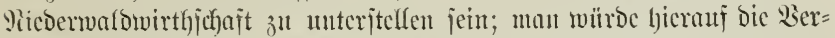

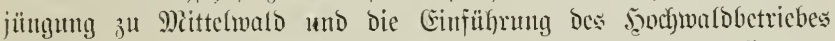
mit ben örtlich wäh(baren llmtriebşzeiten betrachten. Dabei jollen an=

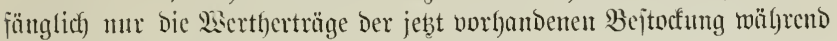
DCs Cinrichtumgszeitraumes beachtet merden.

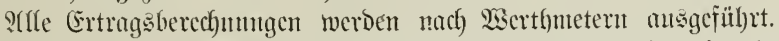

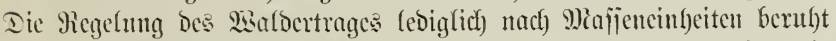

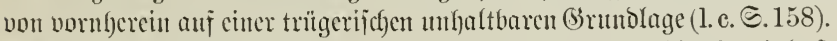

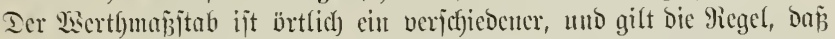

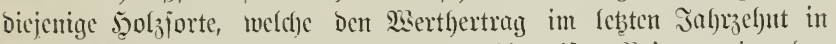
erjter heife geliefert bat, Daju ju wählen ift. Bet normiegender

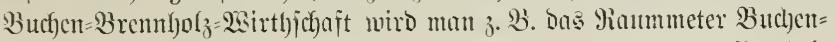

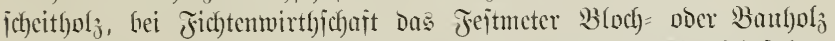

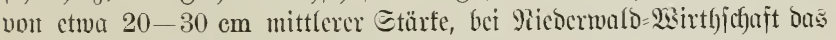

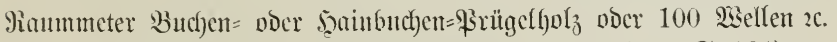

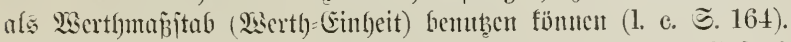

Sit bic cinträglicfjite Benubung Der jeb̧t vorfandonen Bejtocfung

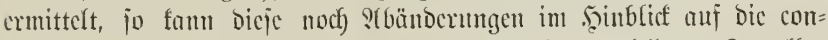
creten Erträge Der normalen Slltersflajfen

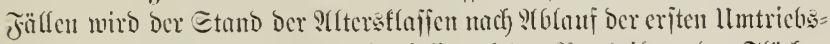

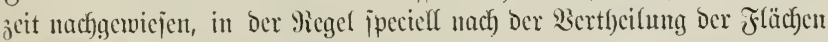

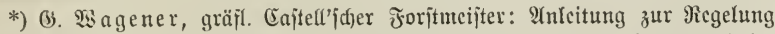

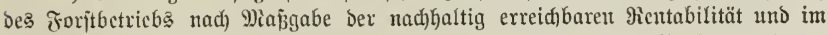

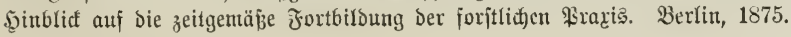




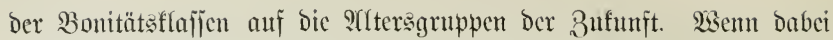
nicht gau auffalfende und abjolut unzuläfïige Ulıgleichbeiten crifbeinen,

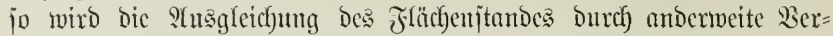
theilung Der 2 sertf)-Erträge unterlafjen, benn bicje Dispojitionen, dic Der forjtliçen Ertragsorbnung im jwanjigiten und cimundzwanjigiten

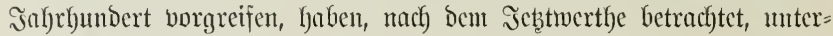
gcorbucte Bedeutung.

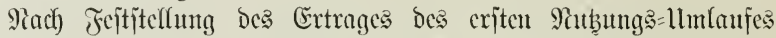
werben bie Erträge ber nackjubauenden Bcjtoctung bejtummt, bie joge= namnten Pormal=erträge. Deshalb ijt jefon bei ber Bonitirung ledig=

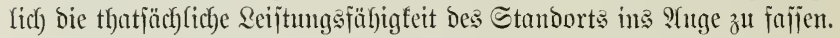

Das Enojiel ber ganzen Piechunng gipfelt in bem fogenannten

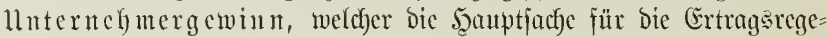
lung ijt.

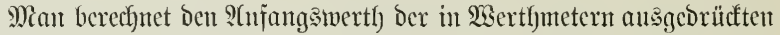

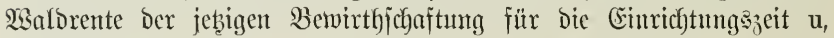

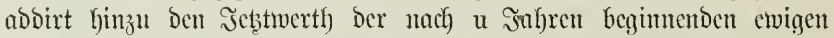
Waldorente für bie erjtrebte llmtriebszeit; für bieje Summe des "Wsald=

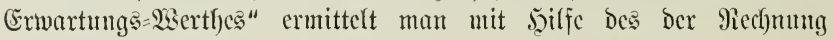

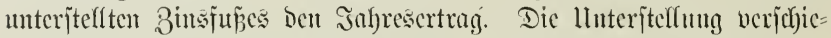

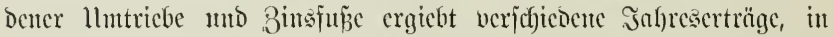

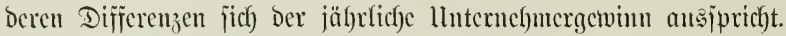

Wiir wagen nicd)t, dic Fïlle won Biffern ju crmitteln und mit= zutbeilen, weldye micr fleine Beifpiel ber Ertrageregelung für ben 103,6 ha grofen Wald ergeben wïrde, wenn wir ifn nach bicjem

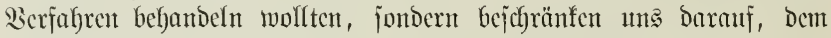
'Buche felbit (ङ. 318) folgendes Beifpiel zu entrebmen.

(Finc Buchenbetriebaflajie ergiebt bei fortgejester \$udhenwirtbjđaft im $80=$ jäbrigen llmtriebe:

Jahresertrag bon ber vorbandenen Beittociung 2150 Werthuncter.

$$
\text { "aljo beginnend nach } 80 \text { Jabren). " }
$$

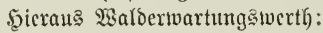

$$
\begin{aligned}
\text { mit } 5 \% & =2150 \times \frac{1,05^{80}-1}{1,05^{80} \cdot 0,05}+\frac{3000}{1,05^{80} \cdot 0,05}=43343 \\
" 3 \% & =2150 \times \frac{1,03^{80}-1}{1,03^{80} \cdot 0,03}+\frac{3000}{1,03^{80} \cdot 0,03}=74330
\end{aligned}
$$

Şiemnd) Jahresertrag ber jețigen Bewirtfidchajtuntg. mit $5 \%=43343 \times 0,05=2167$, " $3 \%=74330 \times 0,03=2230$. 


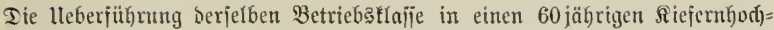
walo würbe ergeben:

Jahresertrag bon ber borkandenen Beituctung 2360 serthmeter

" " nact)zujiebenden " 3050 "

(aljo beginnento nad) 60 Jahren).

gierauล :

$$
\begin{aligned}
\text { mit } 5 \% & =2360 \times \frac{1,05^{60}-1}{1,05^{60} \cdot 0,05}+\frac{3050}{1,05^{60} \cdot 0,05}=47939 \\
" 3 \%_{0} & =2360 \times \frac{1,03^{60}-1}{1,03^{60} \cdot 0,03}+\frac{3050}{1,03^{60} \cdot 0,03}=\$ 2570
\end{aligned}
$$

ģiemad) Jahresertrag ber neuen llnternehmung:

$$
\begin{aligned}
\text { mit } 5 \%{ }_{0}=47939 \times 0,05=2397, \\
\text { " } 3 \%=82570 \times 0,03=2477 .
\end{aligned}
$$

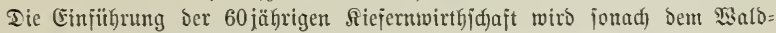
bejitser jäbrliden lInternchmergetwinn gewäfren:

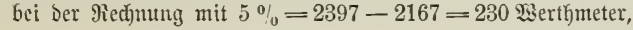

$$
\text { " } 3 \%=2477-2230=247
$$

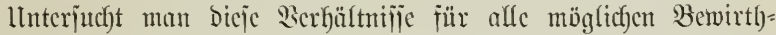

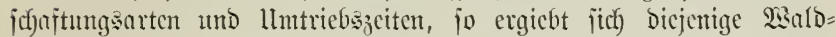

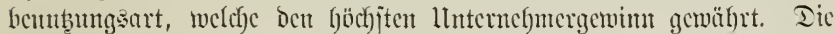

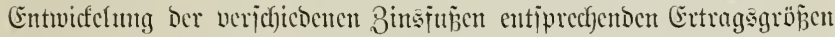

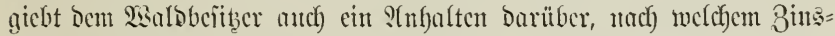

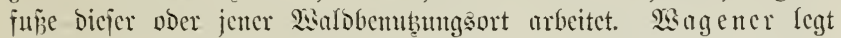

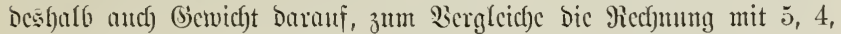

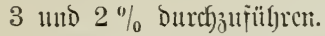

SUT bic Ecfildorung Des von ifm empfohlenen Berfahrens zur Ermittelung Der Stbtrichareifonjolge für bie cinzelnen Bejtünde ver=

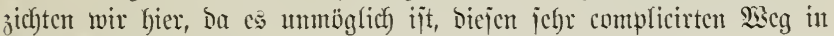
Sï̌ze flar zu jtellen.

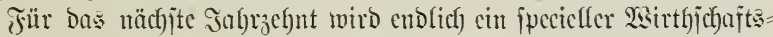
plan aufgeitellt, won ju erwartenden Mlajienerträgen ijt barin chen= jowenig Dic Redc, wie im allgemeinen Rlane für Den ganzen Einticht= ungs

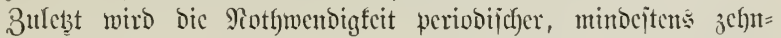
jäbriger Revijionen betont.

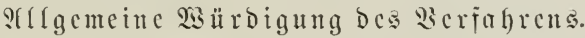

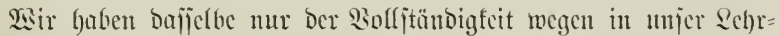

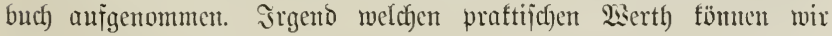

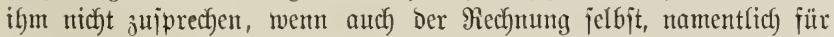


Qöfung Der Frage von llmmandungen einer Betriebsart in bic andere, cin richtiger Gedanfe ju Brumbe liegt.

23eil wir bie forjtlichen Ertragsechnungen unter alfen llmitünoen

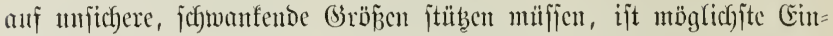
fachbeit gefoten. Dieje erjtrebt bas von um empjoblente Berfahren.

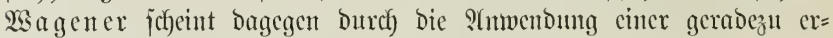

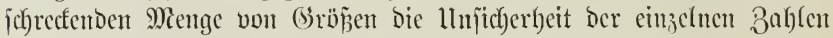
verichleiern zll woolfen llumb̈glicfes foll man aber nicht critreben.

Ein wejentlicher Fortichritt Des Forjteinrichtungswojens Der ucueren 3 cit beitegt in ber Emancipation von tüntitlicken, zu weit getricbenen

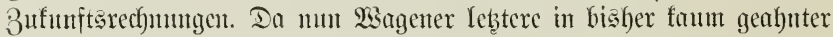
S(usbel)nung wieber anwenden will, würde jein Berfabren auf bem wichtigen Sebiete Der Foritemrichtung einen Siücfichritt bebenten, wenn

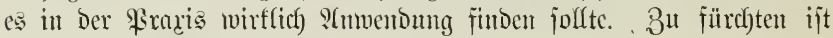
dice jeood) j(b)werlich.

Wir Galten es beshalb audb nicht f̈̈r notfowenbig, fier auf weitere

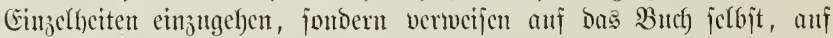
unjere Bejpredfung Deffelben in Tharantor Jahrbuche*), jowie auf bic zaflreidfen Journalartifel $\left.\mathfrak{W a g e n e r} \xi^{* *}\right)$. Sebtere Gaben unfere

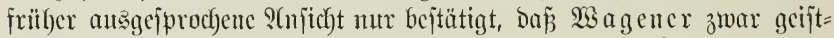

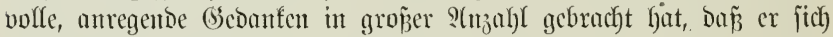

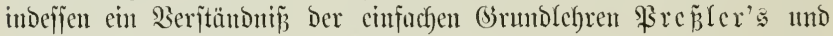
unicre Bejtandsmirthj(c)ajt nicht ermorben hat.

Siaum bedarf ce bejonderer Erwähnung, baj̃ mir es für entichicocn

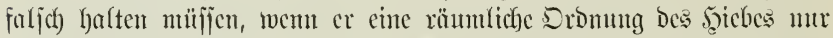

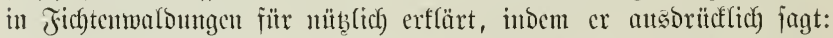

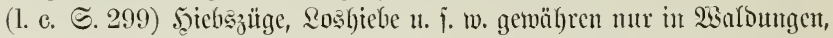
in benen ber Fichten=2Betrieb heimifach ijt ober cingebintgert werben joll, entiprechenden 9iubcu.

*) Tharander foriflidees Jafhrbudf. 27. Band, 1877. ๔. 265 .

**) Centralblatt fïr dą gejaunnte Forftwejen. 4. Jahrgang, 1878. ङ. 483. 536. - 5. Jafrg., 1879. ङ. 229 - 6. Jahrg., 1880. ङ. 160 . - 7. Jahrg., 1881. ङ. 55. - 8. Jahtg., 1882 . ङ. 113.

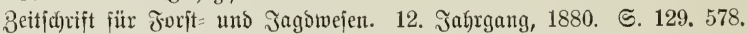

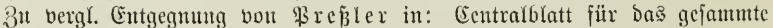

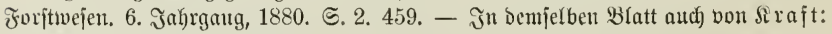
7. Jafrgang, 1881 . ङ. 366 . 


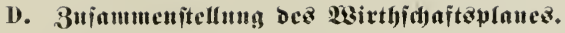

\section{$\S 135$.}

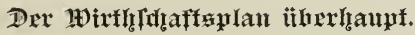

$23 i$ veritefon bicr unter "Wirthjof aftsplau" jentes ?(eten= jtücf, in weldbem bic wejentlicbjten Rejultate Der Sorarbeiten, Der

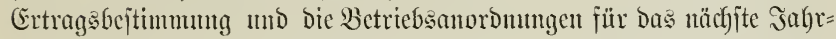

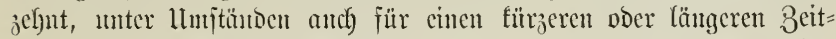
raum, jo georonet jujammengejteflt werden, Daj c

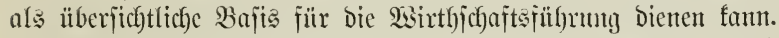

In Den folgenden Faragraphen mögen bie cinjelnen Theile Des "2sirtbjchaftsplants" täber bejprodjen werden.

\section{$\$ 136$.}

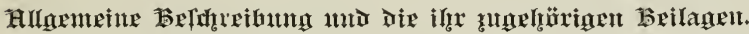

Dic jogenannte allgemeine Bejchreibunty hat Den 8 werf, cine furze lleberjidft ïber Den foritlichen Thatbejtand und cine Begrïndung Der Einrichtung ïberbant, fonic Der Ertragsbeftimmung im Epeciellen $a^{\mathrm{H}}$ geben. Sie foll ferner den Simn mo Geijt Darlegen, in weldfem die

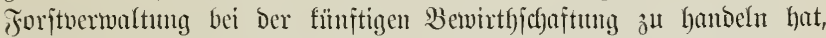

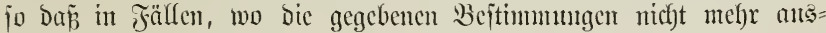

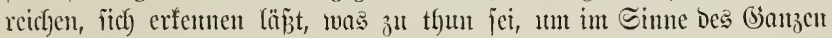
ju verfabren. *)

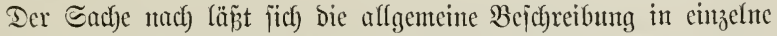
S(bjofnitte ober Sinpitel zerfällen, weldhe jtch zum Theil jwar gegen=

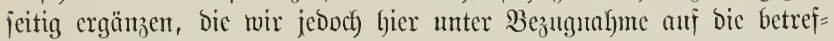
fenoen, bereits bejprochenen \$aragraphen getremt, Imo juar furz

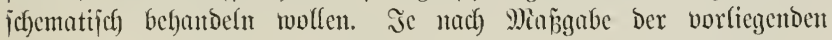
Sierhältnifije wird die eine ober bic antocre Frage bald metre, bald

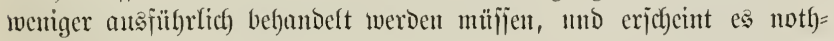

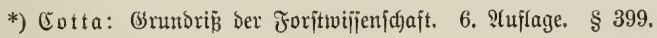




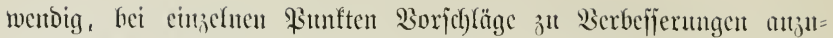

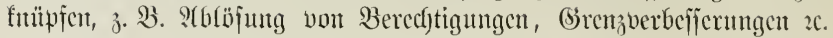

\section{Sapitel. Der foritliche Igatbejtand.}

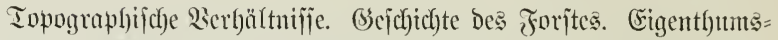

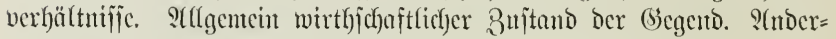

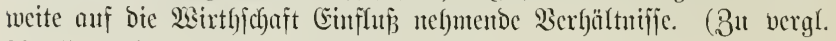
$\S \S 85-90$.)

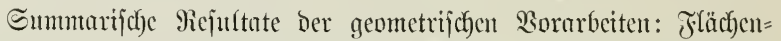

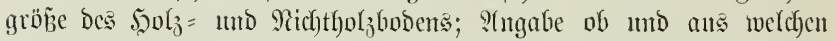

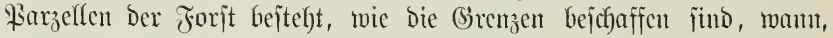

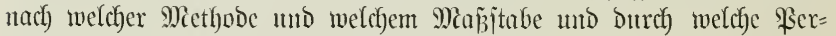
fonen bic Sermefijutg und ?fufnahme erfolgte.

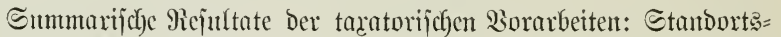

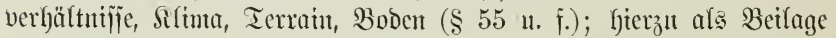

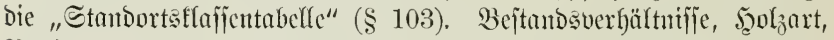

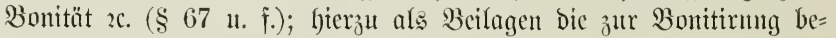

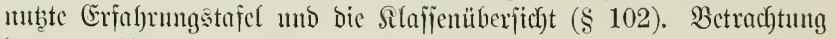
Der ortêüblichen Siaummañe ( $\$ 65)$.

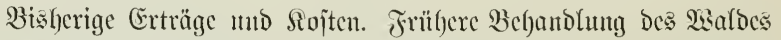

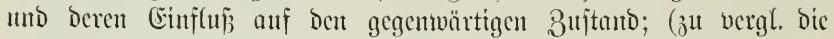

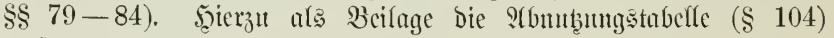

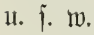

\section{Sapitel. Die 2 aldeituthcilutg.}

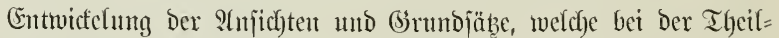

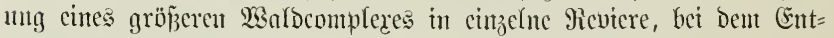
twurfe bes Schneifenucbes, bei Der Billoung Der Betriebsflaffen und

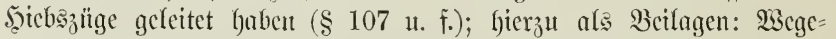
bauplan, wo berieffe cutweder ganz tuen zu entmerfen ober two cin

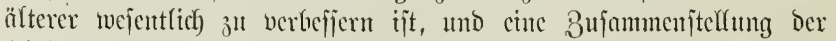

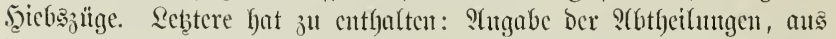

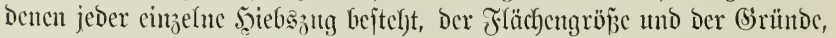

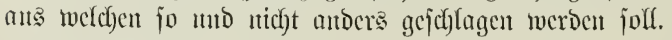

Mamentlich bei febr mugïnjtigen Ierrainverbältnifficn, wo bie Bejtinunung

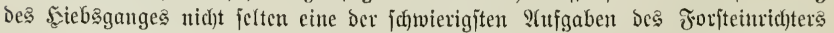

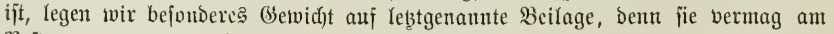

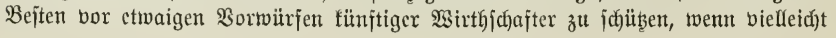


wohldurdbad)te ?(norbnungen jïh) nidjt ganz betwähren jolften. Dieje Bujammen= jtellung giebt ben leitenden Gebanten eines jogenanten genereflen ober alfgemeinen

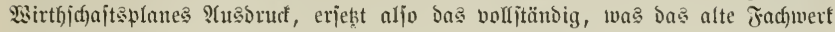
ourch jeine Feriodentheilung bezwedte.

\section{Sapitel. Ertragabcitimmug.}

Surrze Begrïndung Der $\mathfrak{B a h}$ ) Des vorläufigen llmtriches, forwie

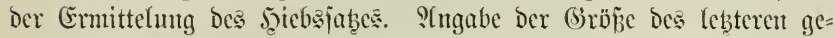

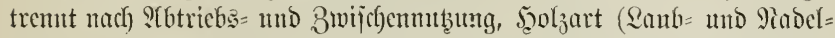
hol $(z)$, nach Eortimenten, jo weit bics nötfig. Betrachtumgen allgemeiner Patur über Den wabridfeinlicfen, fünftigen S(bgabejab, ob Derielbe

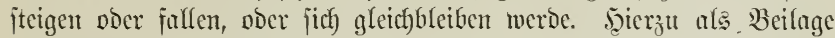
cine begrimbete Berechumtg des llmtricbes, Des Jetcbsjabes nuch) Fläche Into Miaj̃e.

Sir verweijen leb̆tere Einzelfeiten in ente bejondere Betlage, weil anderen= falls biejer Theil ber allgemeinten Bejureibung zu unfangreidf) werben unb $b a=$

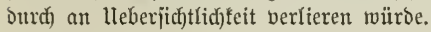

\section{Sapitel. Sïnftige $\mathfrak{B a l b b e h a n d}$ lung.}

In ganz allgemeinten (Brundjügen füt leitente (Bejüchtspunfte

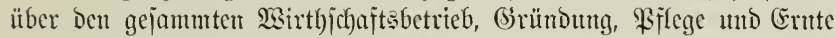
Der Beptänto zu geben. Epeciefl ijt fier ber Siulturen, Entröjijer= ungen, Der Durchjoritungen zu gedenten. S(ngabe Der S(usdefmung

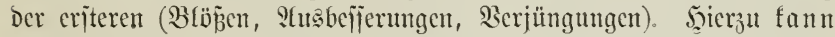
eine beilage unter Dem Titel: "Yllgemeine Wirthjchaftsvorjd)riften"

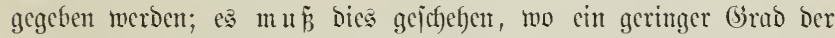
Fachbildoung bes Sermaltungsperionales ipecielle Sorjefriften utent= bel)rlicf) macht.

\section{Sipitel, Beridiedenes.}

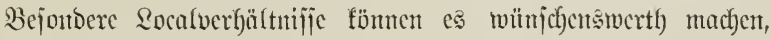

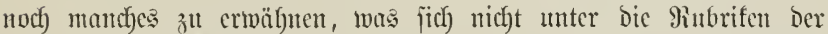
genannten vier Slapitel bringen (ä̈̈t, es mirb bics bier nad)träglich) bemerft. S(m Echlujie ijt anzugeben, Durch) wen und mann bie Ein=

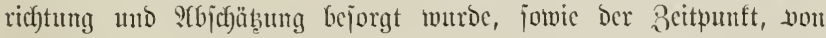

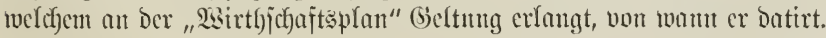




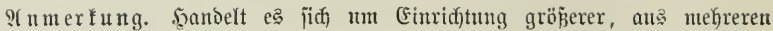

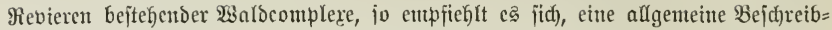

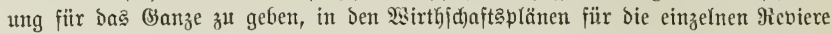

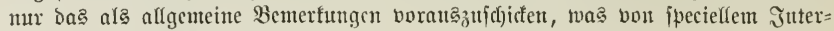

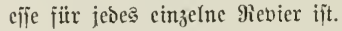

\section{$\S 137$.}

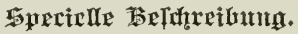

(Flächen = und Bejtumbs=9iegifter.)

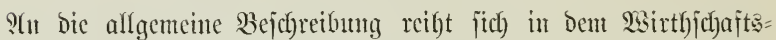
plane bic jogenannte fpecidle Bejefreibung. Cic hat für jeden cin= zelnen Bejtand (llnterabtheilung) genane SAngaben ïber Flächentingalt,

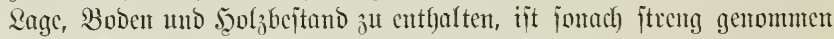

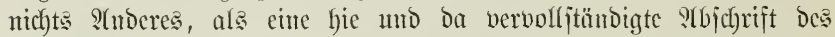
Taxationsmtanuales $(\$ 100)$. Wo lebeteres in cutpprechentor form gefülyrt und anfbewabrt wird, fam man baher bic fpeciefle Bejchreib= ung eriparen und an Deren Etelle ein jogenamtes $F l a ̈ c h c n=4 n d$

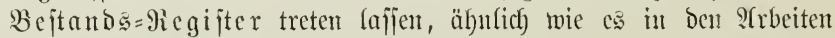

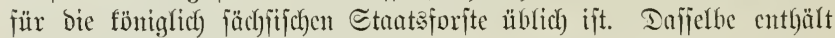
mur eine ganz furze Bejchreifung jedes einzeluen Bcitandes, und zwar

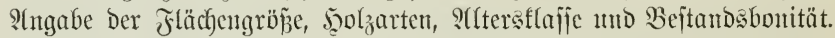

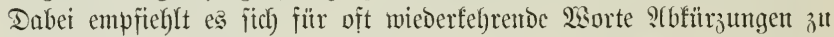

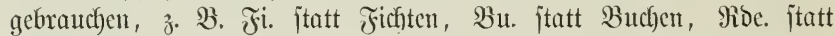

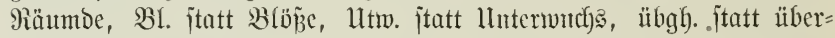
gehaltene, hjitw. jtatt horjtweis, eiz. jtatt einzcln, ei. jtatt einige ı. j. w., ferner ein für allemal die 9l(tersflajfen mit römijchen,

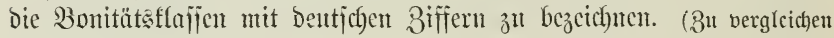
iibrigens \$ 67.)

3ur Erlüutterung mag folgendes Beifpicl bicnen: 


\begin{tabular}{|c|c|c|c|c|c|}
\hline \multirow{2}{*}{ 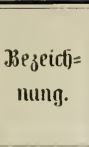 } & \multicolumn{2}{|c|}{ größe. } & \multirow{2}{*}{ 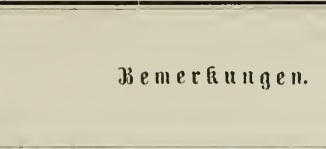 } & \multirow{2}{*}{ 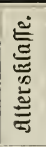 } & \multirow{2}{*}{ 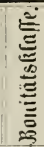 } \\
\hline & $h a$ & $"$ & & & \\
\hline \multicolumn{6}{|c|}{ A. Das Sifenthola. } \\
\hline $1 \mathrm{a}$. & 2 & 50 & Fi. ci. ïbgh). $\mathfrak{B}$ & I. & 4. \\
\hline $\mathrm{b}$. & 2 & 50 & 0,7 รi. 0,3 Ta. ci. $\mathfrak{B} ı$. & IV. & 3. \\
\hline c. & 10 & 50 & ร̃i. ci. tïbgh. Ta. mit $\mathfrak{B}$ i. & I. & 3. \\
\hline d. & 1 & 25 & 0,5 Ti. 0,5 Ta. ci. 311. & V. & 4. \\
\hline e. & 1 & 75 & 0,8 Jii. 0,2 গii. & I. & 4. \\
\hline 1. & 18 & 50 & & & \\
\hline
\end{tabular}

$$
\text { II. i. w. }
$$

B. $\mathfrak{P}$ itcincruen $\mathfrak{B}$ ild.

\begin{tabular}{|c|c|c|c|c|}
\hline $4 a$. & 3 & - & 0,5 Ta. 0,4 Fi. 0,1 hjtms. Bit. & V. \\
\hline b. & 2 & 80 & 0,7 Fi. 0,2 Sit. 0,1 Ta. & I. \\
\hline c. & 7 & 50 & 0,8 ริi. 0,1 Bu. 0,1 Ta. & III. \\
\hline d. & 1 & 20 & 3ł. ci. übgh. Ta. & - \\
\hline e. & 1 & - & Ji. & II. \\
\hline 4. & 15 & 50 & & \\
\hline
\end{tabular}

$$
\text { 1t. i. to. }
$$

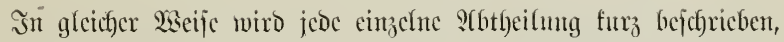
Daun bic Summe vnu fämmtlidyen ?fotheilungen gczogen.

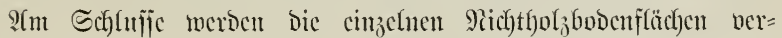

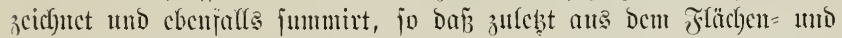

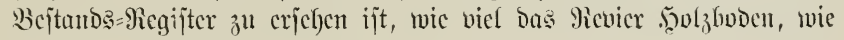

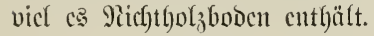




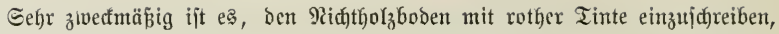
baunt fidh oie ifm zugeförigen Bezeichnungen uto 3iffern in bie 2 (ugen faffens

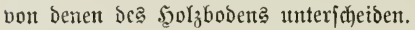

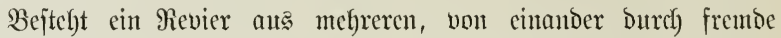

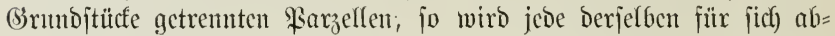

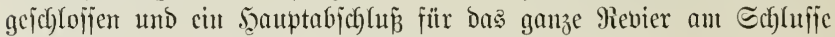
tics Flächen= unt Bejtands= Regijters gegcben.

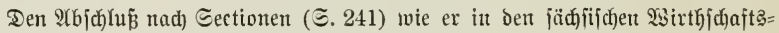
plänent üblić), Galten wir für überfiłiffïg, fogar für ftörento, ba bie Sectionen feine Bcoeutung fïr bas Einricftungąwerf baben.

\section{$\S 138$.}

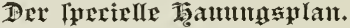

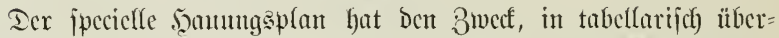

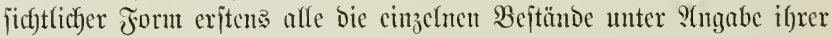

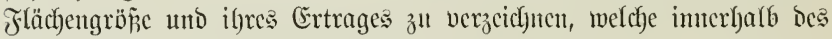

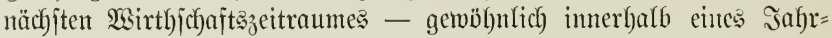

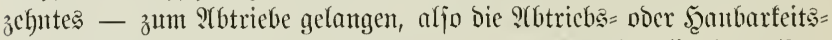
mub̧utg gewäl)ren jolfen; jweiten hat er eine ?Ingabe aller jener Drte

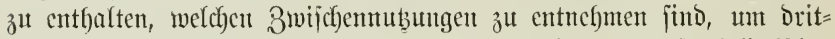

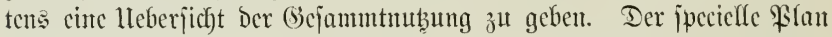

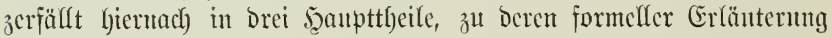

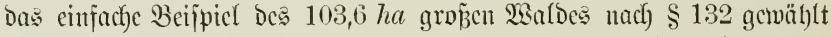
werben mag. Dicjes Beifpiel jofl jedoch) Dutchante nicht als giecept

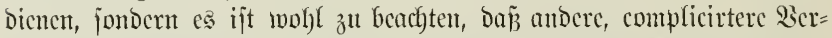

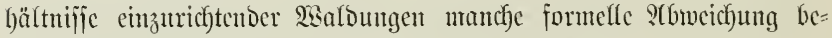

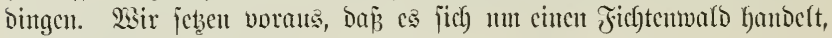

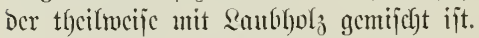




\section{Abtriebsnufungen.}

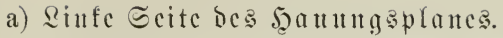

\begin{tabular}{|c|c|c|c|c|c|c|c|c|c|}
\hline \multicolumn{10}{|c|}{ aftriegsun } \\
\hline \multirow{3}{*}{ 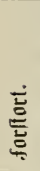 } & \multirow{3}{*}{ 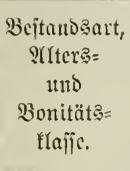 } & \multirow{3}{*}{ 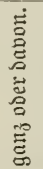 } & \multirow{2}{*}{\multicolumn{2}{|c|}{ fläcfie. }} & \multicolumn{4}{|c|}{ Jin $\int e$ in feftmetern. } & \multirow{3}{*}{ Bemerkungen. } \\
\hline & & & & & \multicolumn{2}{|c|}{ ¿nubgolz. } & \multicolumn{2}{|c|}{ Paadelyolz. } & \\
\hline & & & $h a$ & $a$ & $1 \mathrm{ha}$ & $\begin{array}{l}\text { über } \\
\text { fyaupt. }\end{array}$ & $1 h a$ & $\begin{array}{c}\text { 藏er= } \\
\text { l)autpt. }\end{array}$ & \\
\hline
\end{tabular}

A. Das $S \mathfrak{d} \mathfrak{d} \mathfrak{f} \mathfrak{l}) \mathfrak{l}$.

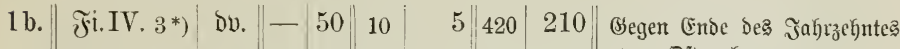 \begin{tabular}{l|l|l|ll|l|l|l|l|l|l} 
d. & Ji. V. 4. & 93. & 1 & 25 & 24 & 30 & 580 & 725 & $\begin{array}{c}\text { bon Diten her. } \\
\text { Barb abzutreiben. }\end{array}$
\end{tabular}

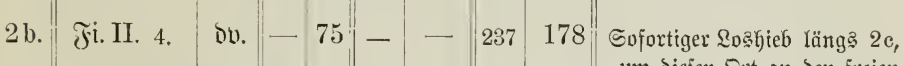 utm biejen Dit an Den freien Stanઠ วิน getwöfnet.

\begin{tabular}{l|l|l|l|l|l|l|l|l|l|}
3 c. & Fi. V. 4. & 9 & 9 & 0 & -75 & 20 & 15 & 633 & 475
\end{tabular}

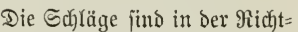
uttg von Dit nad) Seit gleid)= zeitig in $3 \mathrm{c}$ unb $6 \mathrm{~d}$ zu füfyren. Sorberjïngutg mter Bemtz= ung bes vorfandenen, natür= richent IInterwudfica.

B. SI fteinernen Bilb.

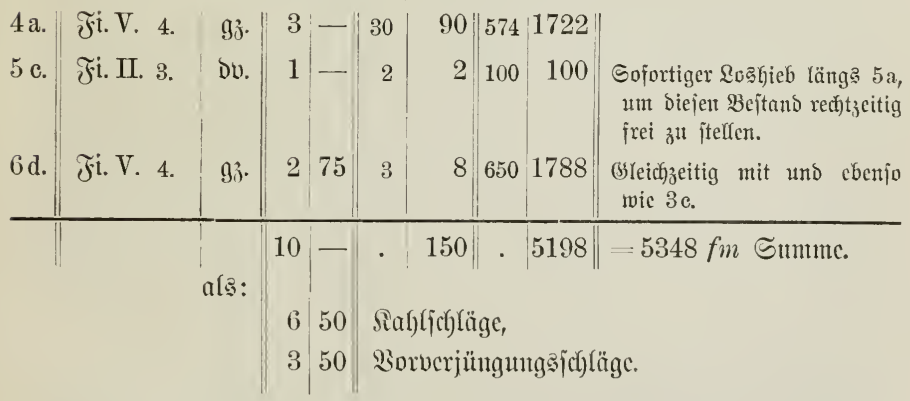

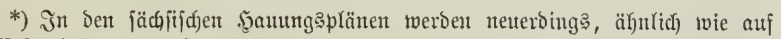

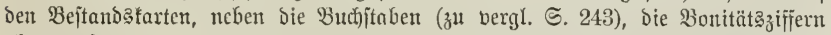

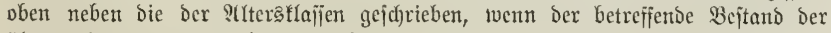

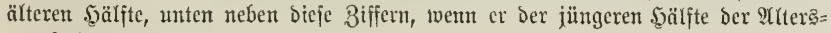


In ber Regel pflegt man bie Ertragsangaben abzurumben, Da ç

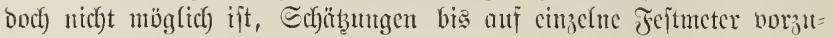
nef)men. 2Bsir fjaben bicje Stbrundung ljier abjichtlich unterlajjen, um

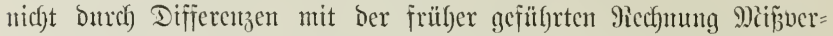

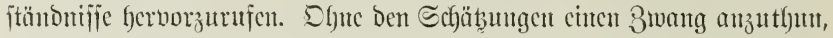

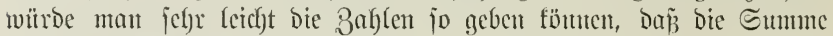
arf $5350 \mathrm{fm}$ (5200 Sabelgolz und 150 \&aubfol $)$ fauten möcfte. Pioch) weit fitürfere sforumbungen fint jull gejtatten.

2Senn bic Echläge in ciner S(btheilung glcidbzeitig ïber mefyrere

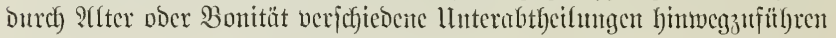
find, fam es a ustallus weije gejtattet jein, fomohl im Snjabe des \$lanes, als bei Den Rechmungsnachträgen (§ 144 it. f.) Die Erträge

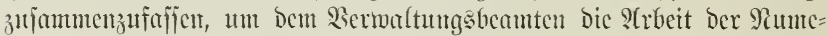

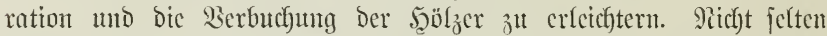

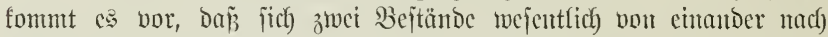

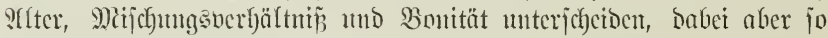

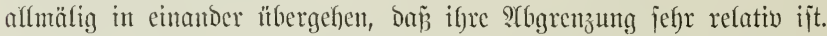

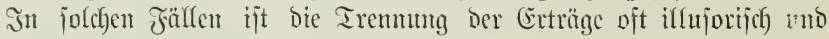
bahei jef)r zeitranbent. - Dic Flächen jelbjt müfjen jedonch) immer ge=

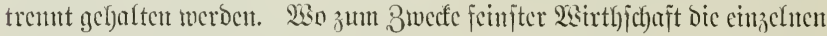

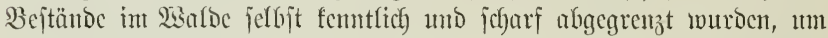

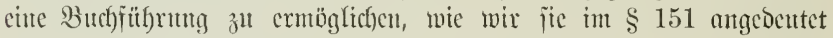

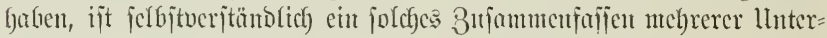
abtbeilungen unjtattbajt.

Im umptebend gegebenten Beifpicle handelt es jict) um bie einfact)jte

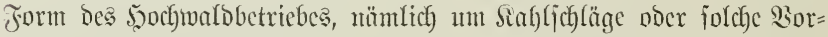

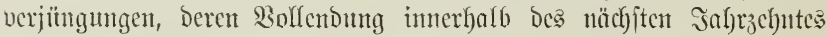

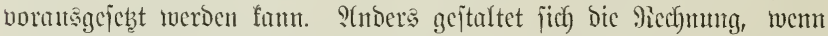
ans Bejtänoen zum Buecfe natürficher nder fünjt(icher Borberjüngutg mur cist Theil ber Mafje zum Şieb angejetzt wirs. Da wir bie fum=

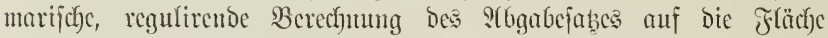
jtübsen, jo ijt in folchen Fällen mur bic nach Dem Miajfenverfältniffic

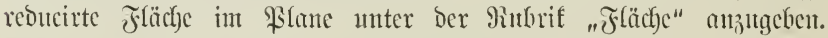

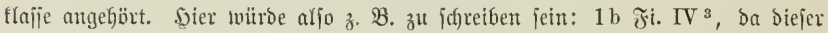

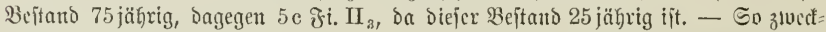

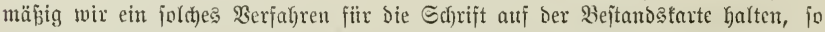

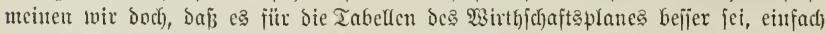
cine Rubrif fït bie ?lltersangabe zuzufügen, um biejelbe genaucr zแ haben, als

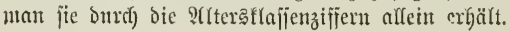




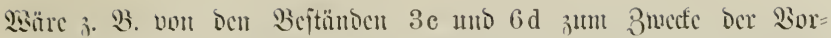

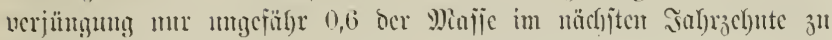

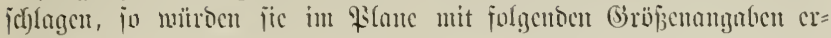
jd)cincu:

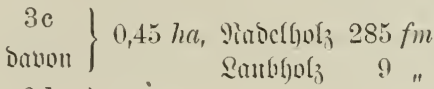

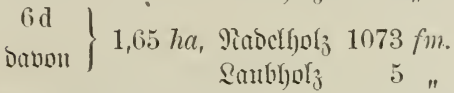

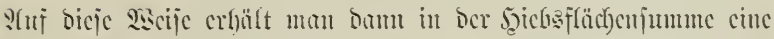

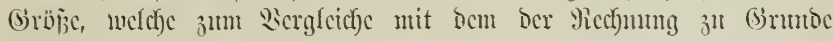

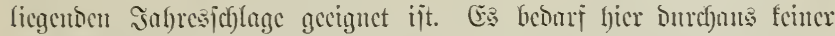

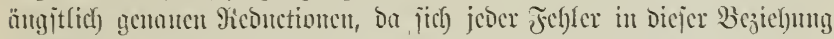

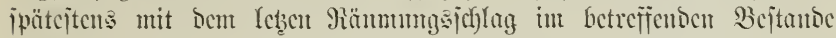

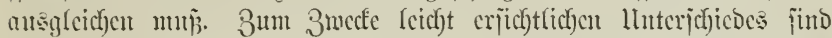

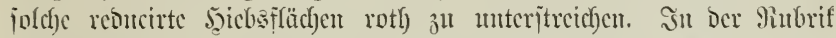

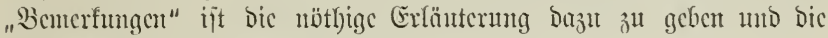

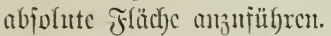

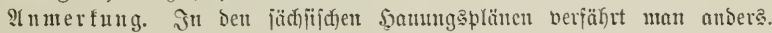

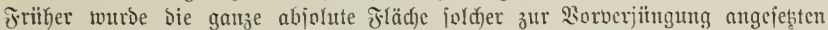

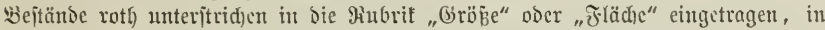

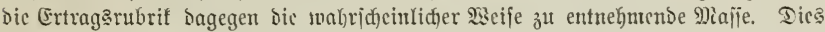

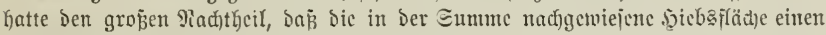

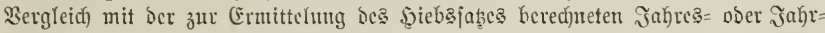

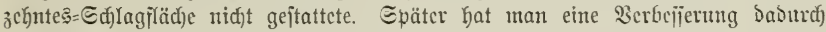
vorgenommen, Daß̃ man bei Derartigen Şiebsorten bie nad) Der Mialie rebucirte

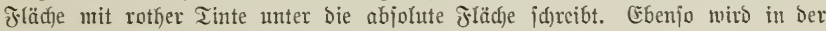

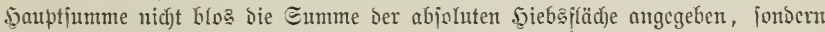
mit rother Iinte audf jenc Enume, weldhe jid̆ bered)net aus ben vnllitänbigen

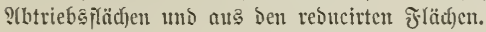

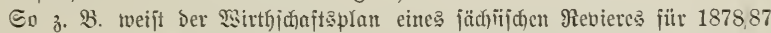

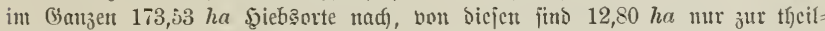

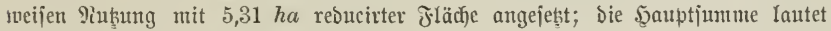

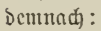

\section{3,53 ha}

ober 166,04 " rcoucirte Fläche.

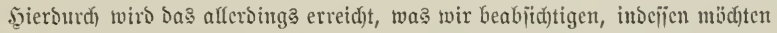

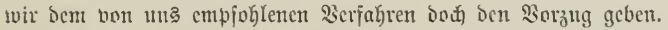

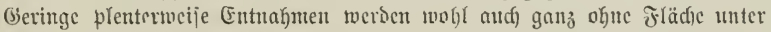

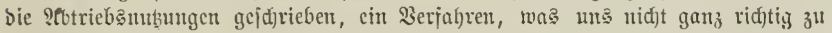
jein idjeint.

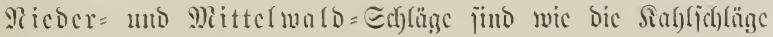

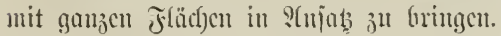


In $\mathfrak{B}$ lenterwalde bient als jummarijhes ?nfalten für

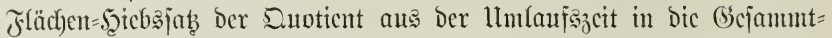

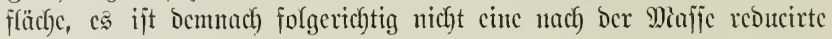
Flächc, jondern bic abjolute im Scaunngplane ju verzeichucn. WSic vid Bejtandsmafje entnonment merden foll, giebt bic Ertragsrubrif

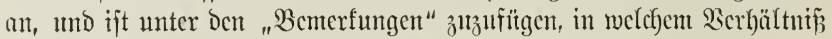
ungcfälyr bie zu entnelyntende zu Dex vorbandenen Majfe jtcht.

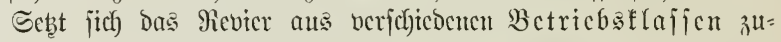
jammen, fo ift entweder ein jpecieller Seauntugplan jür jede cinzclute

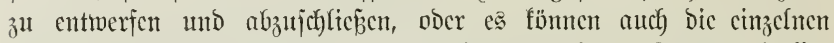

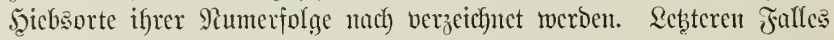
ijt jedoch bie Endjumme ber Flächen und Maffen in Den Den cinzeluen

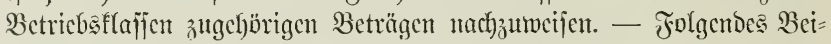

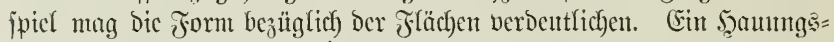
plan entbalte in Der Summe Der Flächenrubrif 325,5 ha, jo ijt $3^{11}$ bemerfen: $\mathfrak{B}$ on obigen 325,5 ha gel)ören:

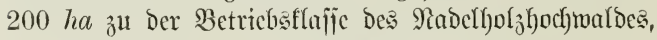

1.. ว. 150 ha Siablichläge,

50 " auf Den Siollfeitano reducirte Sior= verjïngıngsichläge, bercu abjolute Fläche 100 ha beträgt.

100 ha jut Der Betrichatfafic bes Butchenfochnaldes,

น. ว. 40 ha auf Den Bollejejtand reducirte Berjüngungミflaj̄en, Deren ab= folute. Fläche gleich $120 \mathrm{ha}$.

60 " reducirte S3orverjüngungs: ichläge in Sallbcitünden, be= ren abjolute Flïche gleich) $150 \mathrm{ha}$.

5,5 " зи Der Betricbsflajic des Mittchwaldes. 20,0 " za Der Betricbsflaijie des Plentermaldes. ธ. $\mathfrak{1} . \mathfrak{0}$.

Die Trenmutg ber jugebörigen Majjen bedarf feiner Gejonderen Erläıterung.

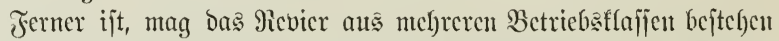
ober nicht, erjteren Falfes getrennt, lebeteren Falfes mutr für dic (Şe= jammtjumme nachzumeijen, aus weldhen sflecsflajien แn Bejtands=

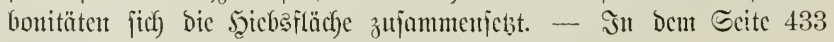

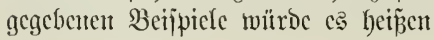


2ion Den jun ?fbtriebe beftimmten 10 ha gebören an:

1,00 ha Den $21-30$ jährigen Bejtänoen Der I. 2tItersflajje,

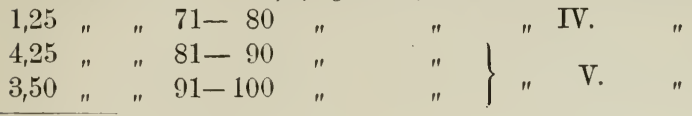

ธ. เพ. v.

jowic

$$
\begin{aligned}
& 1,5 \text { ha bcr. 3. Bonität, } \\
& \frac{8,5 \text { " } "}{\text { S. wo. } \mathbf{~ . ~}}
\end{aligned}
$$

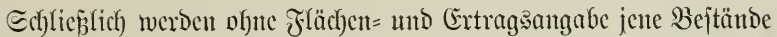

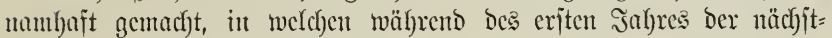

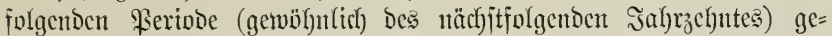

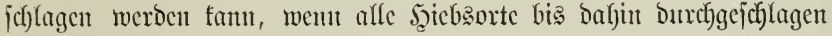

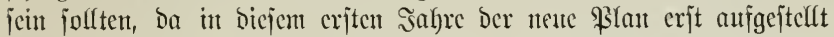
wirb, aljo noch nicht fertig vorlicgt. - Für unjer flemes Beijpiel

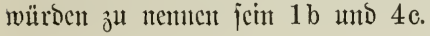

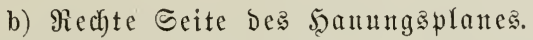

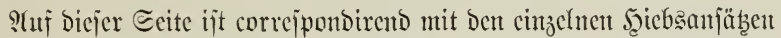
Der linfen Seite Dcs Plantes Der Pachneis über bic in jedem Jabre

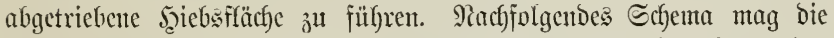

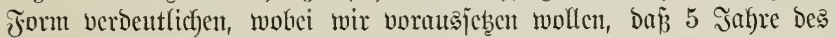
2isirthjofjaftszeitraumes 1871/80 verflofjen jeicu. 


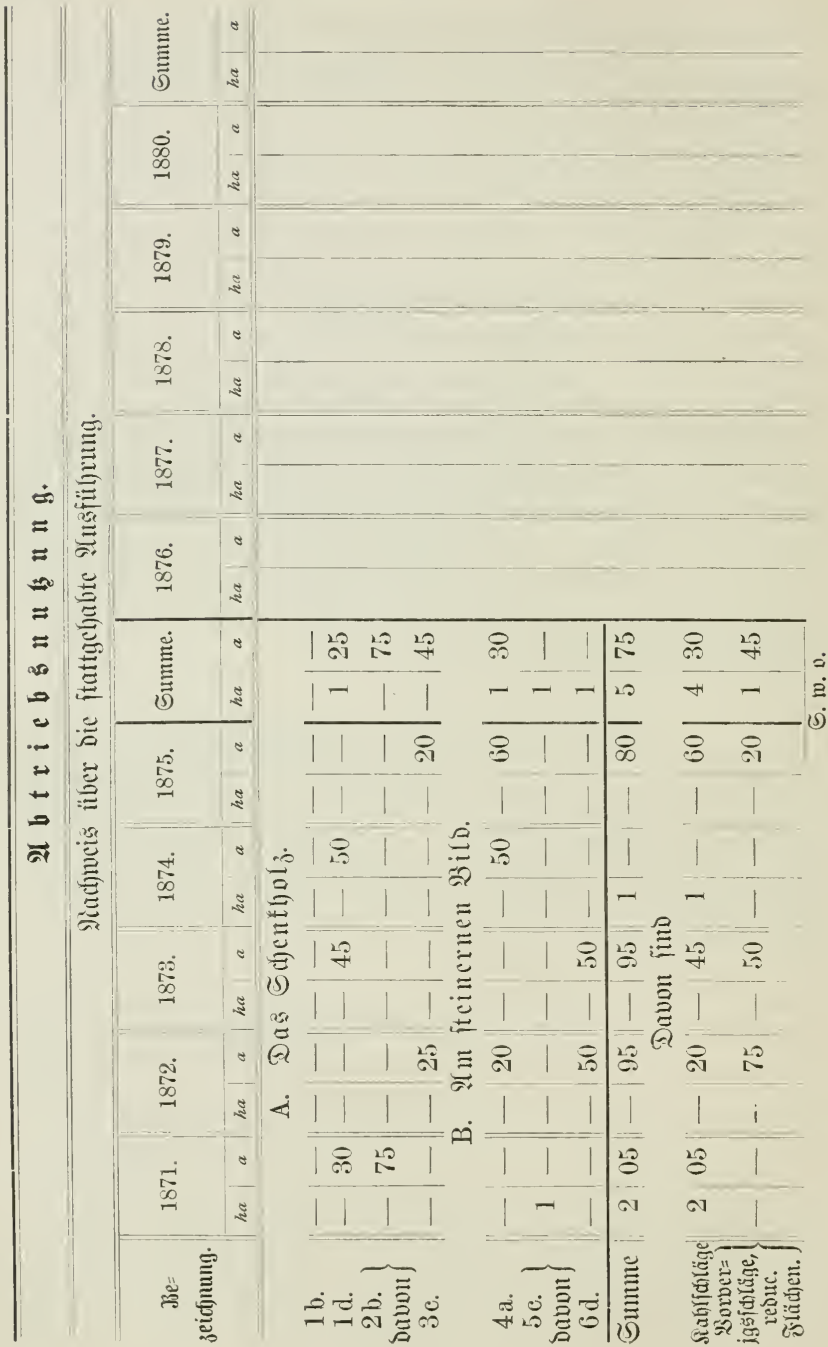




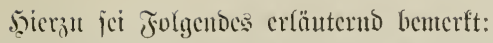

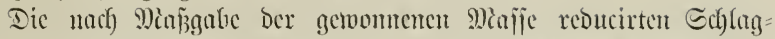
flüchen in $3 \mathrm{e}$ und $6 \mathrm{~d}$ fut roth jul unteritucichen. Dic 9ieduction crfolgt ıur ungefüly, Denu jede Damit zufammentäugende Differenz

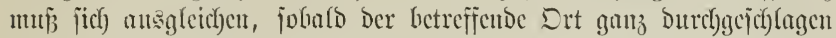
ift, weil zu bicjen Zeitpunte bie reducirte Fläche gleid) ber abjoluteu

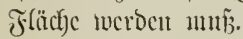

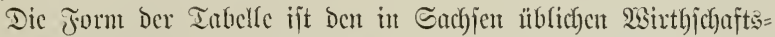

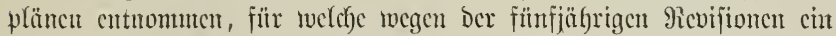

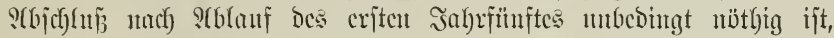
Daber bic Doppclte ginbrif "Sumnte". Scalten wir aud bieje fünf= jüfrigen Sevifionen nicht für unbebingt notfowenbig, jo ift boch jeden=

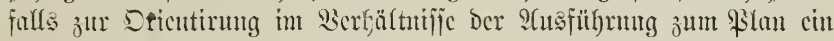

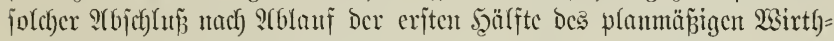

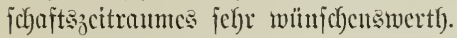

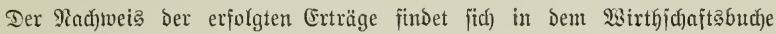
(\$ 144 u. f.), ebenjo die Ingabe über planvidrige Şaumgen (3orfaunngen).

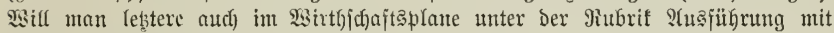

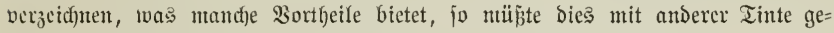

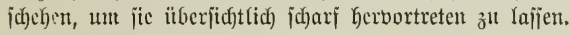

\section{3wi(cjemututungen, *)}

Dic Bwiffenutbungen wurben mit

$$
650 \mathrm{fm} \text {, }
$$

$$
\text { als: }
$$

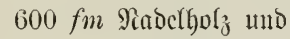

$$
\begin{aligned}
& \frac{50 " \text { 'anblyoly }}{\text { S. w. D. }}
\end{aligned}
$$

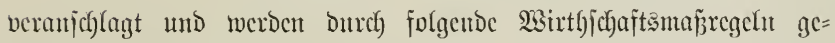
Ivorncit:

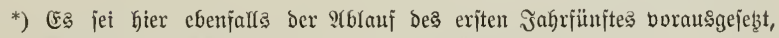

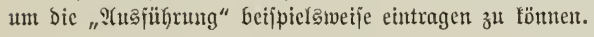




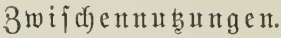

1. Durdjoritungen.

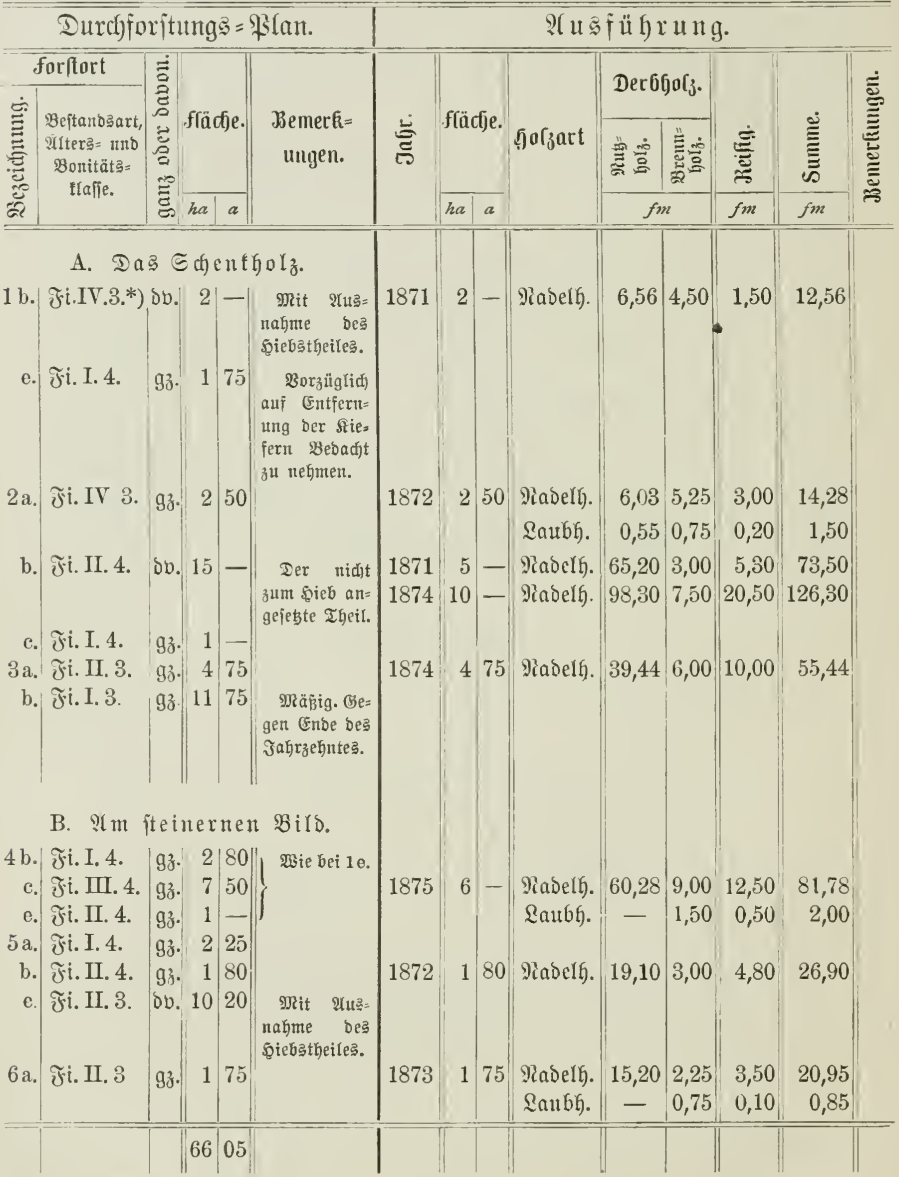

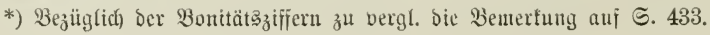




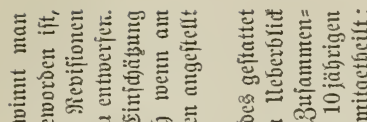

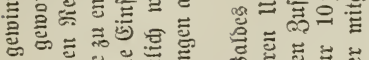

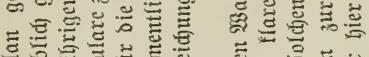

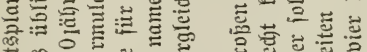

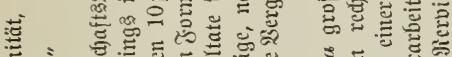
बे

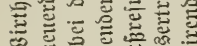

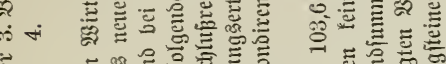

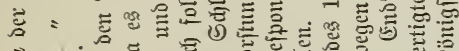

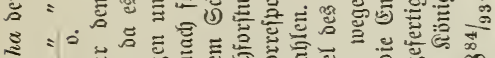

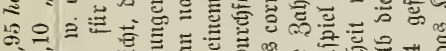

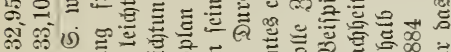

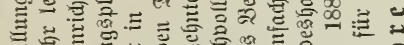

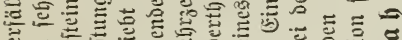

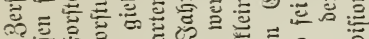

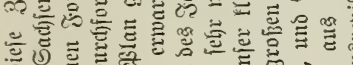

号

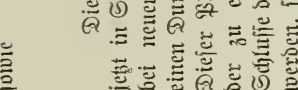
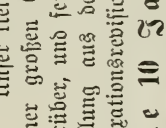

22

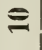

$\therefore$

\section{$\bar{\Xi}$}

莺

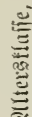

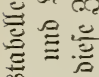

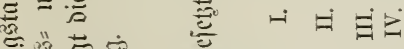

运至总焉

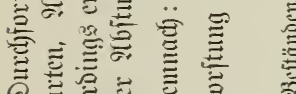

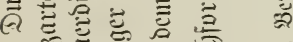

¿

들

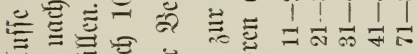

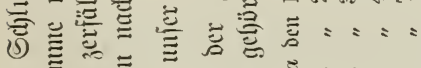

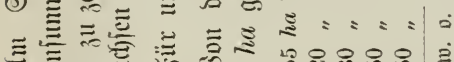

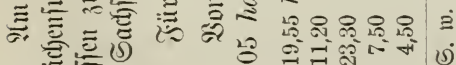

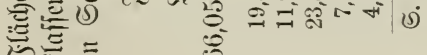

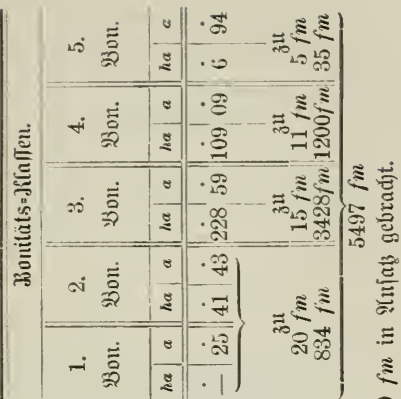

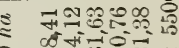

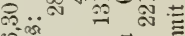

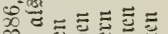

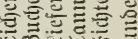

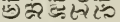

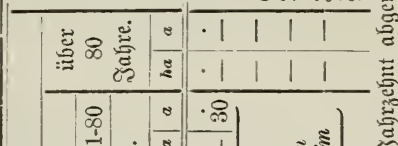

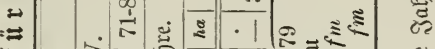

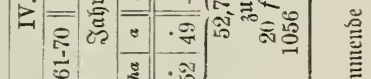

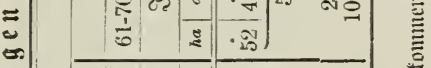

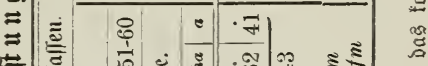

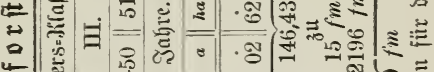

E

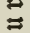

A 
2. Sïumungen übergegalteucr Maforedter, Sänterungs= voer Reingungshicbe in Bejtänben jüngiter itterstajic.

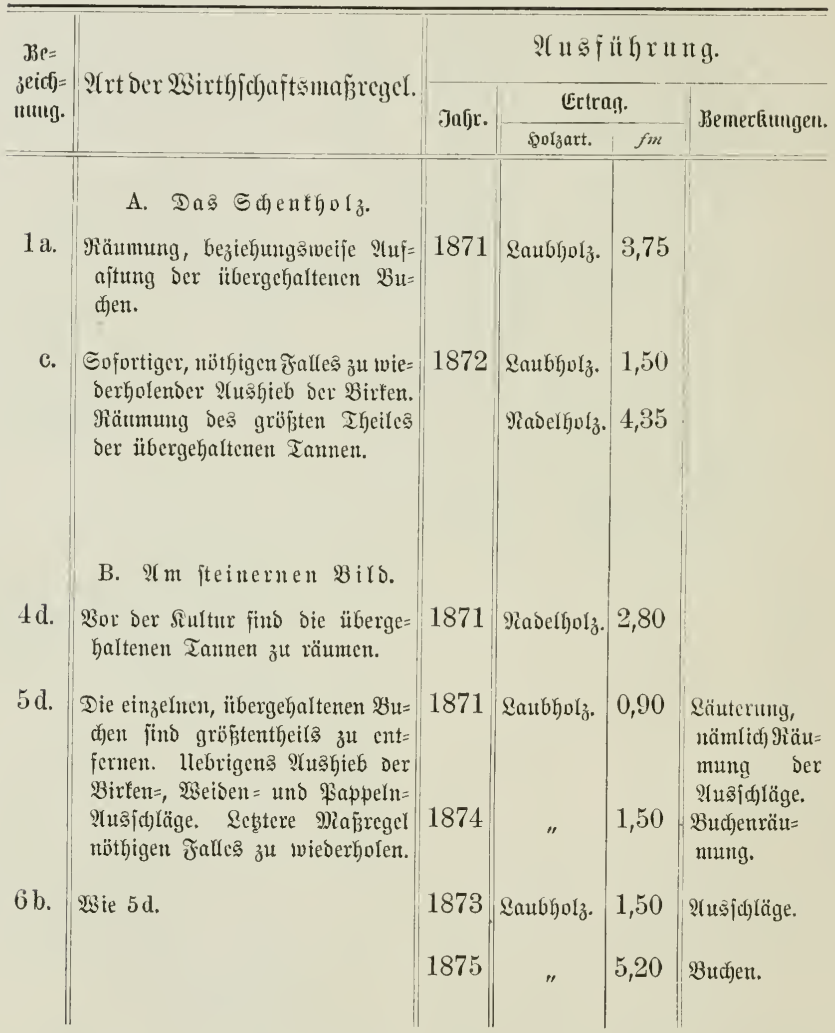

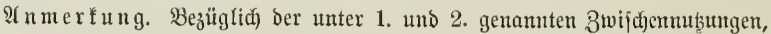

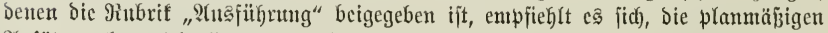

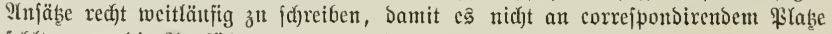
fehlt, wenn bie $\mathfrak{T}$ (t)

Beide Tabeflen ftimmen mit Der jeb̧t in Sadjjen üblidjen Form überein, mit

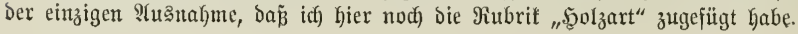




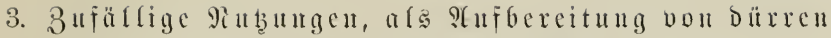

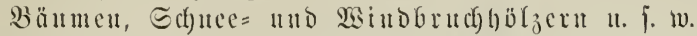

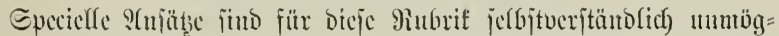

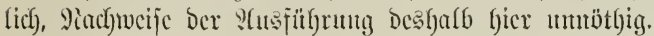

III. gefammtnuţuแ̣̣.

\section{(5) $\mathfrak{e} \mathfrak{a} \mathfrak{m} \mathfrak{m} \mathfrak{t} \mathfrak{n} \mathfrak{a} \mathfrak{a} \mathfrak{u} \mathfrak{g}$.}

\begin{tabular}{|c|c|c|c|c|c|}
\hline \multicolumn{2}{|c|}{$\begin{array}{l}\text { Jröfje der jul ver= } \\
\text { jïnģenden f(ürf)e. }\end{array}$} & \multicolumn{3}{|c|}{ Jliaffe in feltmetern. } & \multirow[t]{2}{*}{ ふететкйgen. } \\
\hline$h a$ & $a$ & Eaubhot & Rabelfyol & Ueberbaupt. & \\
\hline 10 & - & $\begin{array}{r}150 \\
50\end{array}$ & $\begin{array}{r}5200 \\
600\end{array}$ & $\begin{array}{r}5350 \\
650\end{array}$ & 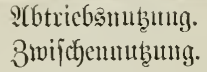 \\
\hline & & 200 & 5800 & 6000 & Sicjuntmtnub̧ung. \\
\hline
\end{tabular}

IV. 3ufammenffeftung des gie6sfatjes.

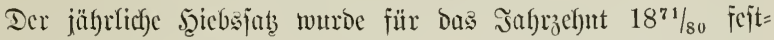
geitcllt auf: $600 \mathrm{fm}$

$$
\text { als: }
$$

$580 \mathrm{fm}$ शabelfol ${ }_{3}$,

20, Saubljols.

๔. เพ. 1).

Deren Berjed lag wird zu gejofyef)en haben mit:

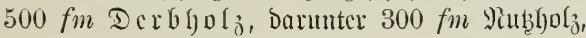
IIID 3war,

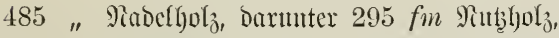

15 " 2aublobl " " 5 " "

ธ. 1w. D.

IIII):

$$
\begin{aligned}
& 100 \mathrm{fm} \text { Rcijig. } \\
& \text { Itno jwar: } \\
& 95 \mathrm{fm} \text { Nadellyol? } \\
& 5 \text { " হaublyolz. } \\
& \text { ङ. w. } 0 \text {. }
\end{aligned}
$$

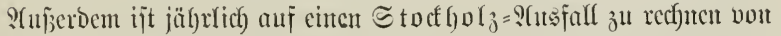

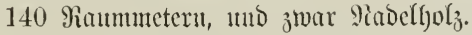

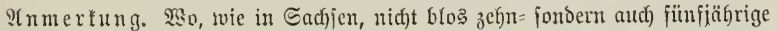

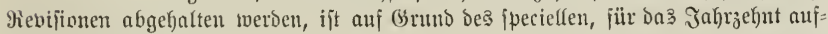

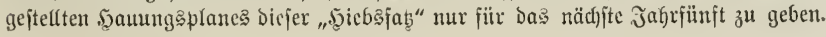




\section{\$ 139 .}

\section{Der ligeciefle tulfurulan.}

Der fpecielle Sinturplan joll in tubellarijch georoneter Form cine

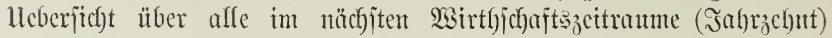

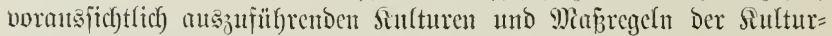
und $\mathfrak{B}$ çtuntopflege gewährent.

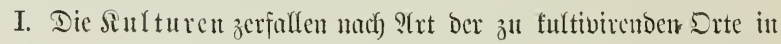

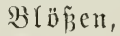

Sasbefferungen und

Berjünguıge t.

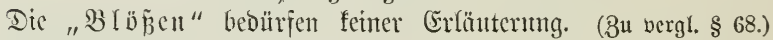
Bezüglich) Der "S(

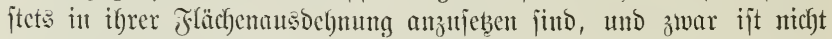
dic Sicjammtfläche des auszuthefijernden Bejtandes, fondern unt die

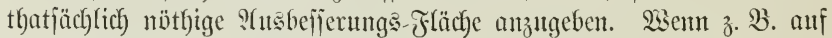
ciner 2 ha grojent Sitlturfläche Der vierte Theil Der Pffanzen cint= gegangen ift, io werben zur : (usbefierung 0,5 ha vorgejchrieben. Ilnter "Berjü

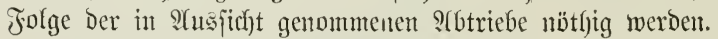

Wie für bie sfbtriebsmubung wirb auf bie linfe Geite bes \$llancs

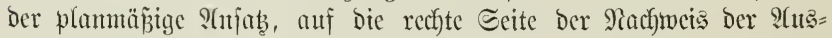
fïl)rung gejdyrichen. Ilu lebteren int folgenton Echenta geben z"

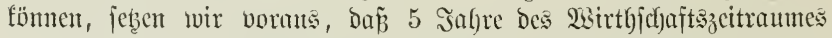
$18^{71} / s_{0}$, welcher für ben 103,6 ha grojen $23 a l d$ getvälglt murbe, ver= itricken feien.

Durch bejondere Anmerfungen find am Seflujic, wo cs nötfig, mögliche Differenzen zu erläutern, welche bie im Siulturplane nach)=

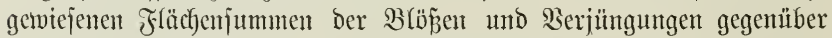
Denen ber Silaffenitherjïcht, bezief)untgsmeije bes şanung splanes crgeben. Soldye Differenzen fommen in unjerem einfachen Beifpicle nicht vor, fie fömnen aber baburch entjteben, daj Roshjebe (Sicherbeitsftreifen) ifrer geringen Breite wegen, andere Blö̈en ifrer Lage twegen vor= läufig ganj unangebaut liegen blciben follen. Ferner jind jolche

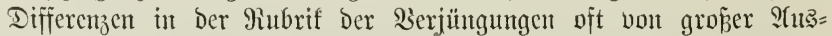

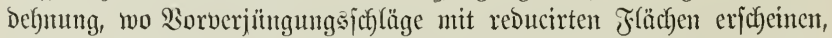

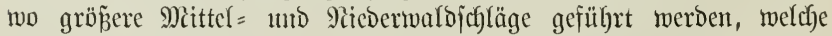

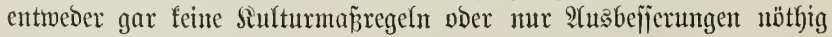
madjen. 


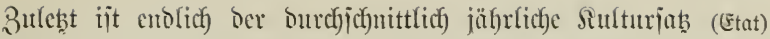
aujugchen. Nan rechuet babci, baj im uächjten Jabrzehnt alle an=

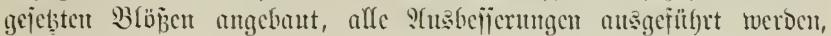

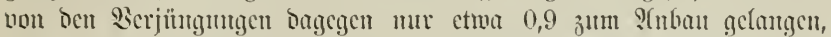

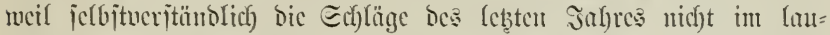
fenden 2sirthjchaftszeitraunte fultiuirt werden fömen. In unjerem

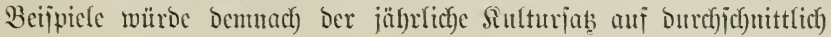
1,7 ha lanten.

Erläuterno jei hicrall noch Jolgendes benterft:

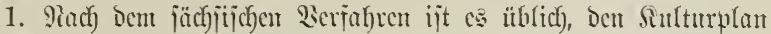

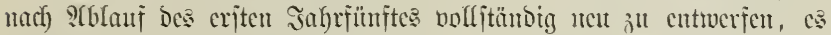

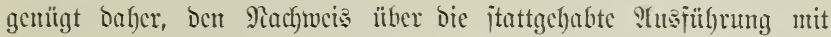

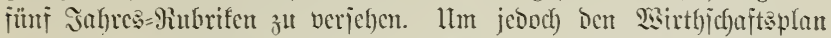
überbaut, aljo auch joweit or bic fulturen betrifft, fït bas ganje

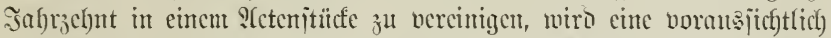
entiprect)endo :

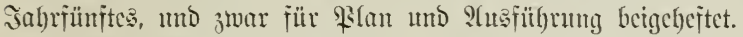

2. Tư alle Fälle cmprichlt cs jitch, bie linfe Ecite Des Planes rech)t meitläntïg ju ichreiben, Damit es nicht an correjponbirentom

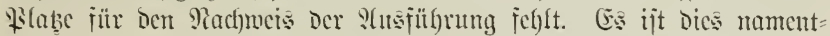
(ich) Daun nötf́g, menn man auch nicht planmäã mit cintragen roill, was mit hervortretentor, j. B. rother Tinte ge=

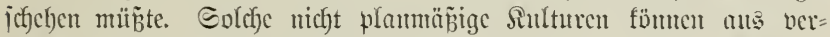

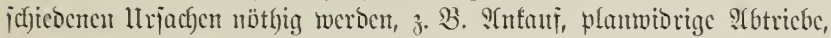
Tushejīerungen, Dic nicht vorauszujefen maren u. j. เv.

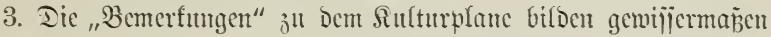

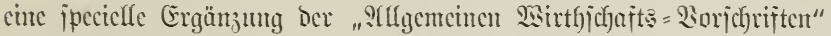
(§ 136, 4. Sapitel), jowcit bicic ben Sulturbetrich betreffen. Sc geringer Der (Srad Der Fachbiloung Dcs ausfüfrenden Bermaltumgsperjoutales

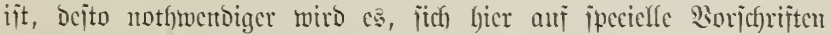

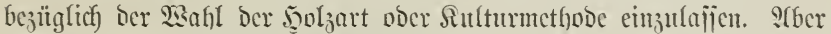

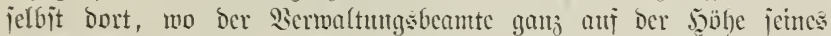
Faches jteht, ijt bies in eingelnen, iffwicrigen und fraglicfen Falle

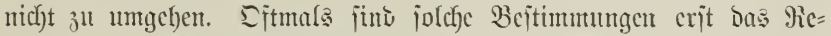

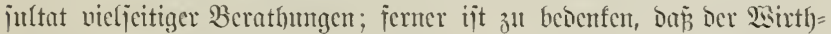

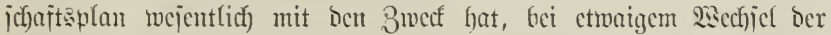

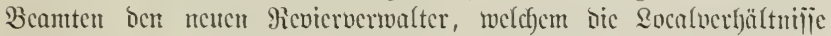

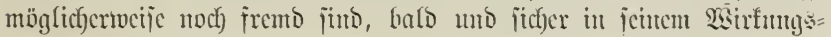
freije zu orientiren. 


\section{a) Sinfe Seite des Siulturplancs.}

\section{Sulturen.}

\begin{tabular}{|c|c|c|c|c|}
\hline \multirow{2}{*}{$\begin{array}{l}\text { ße }= \\
\text { zeidf)= } \\
\text { nu1!y. }\end{array}$} & Збӧқен. & $\begin{array}{l}\text { Alts= } \\
\text { beffer= } \\
\text { ungen. }\end{array}$ & $\begin{array}{l}\text { Verjüng: } \\
\text { tııgen. }\end{array}$ & \multirow[t]{2}{*}{ Bemerkungen. } \\
\hline & $h a$ & $h a$ & $h a$ & \\
\hline
\end{tabular}

A. Dab Enentigulz.

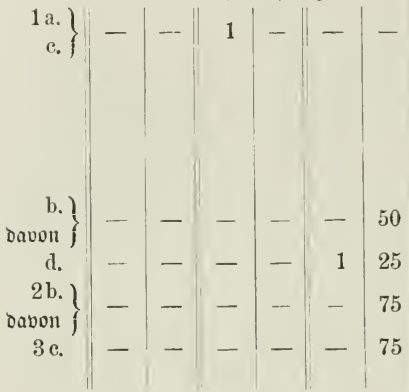

B. 2 m itcinernen birs.

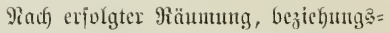
weije 9ufajtutg ber alten Buchen unb Tamnen, fomie ber Birfen. Theilmeis, auf Den trocfteren Stellen wenigitens, mit niejern auszufïhren.

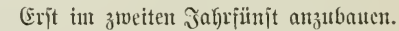
Der vorf)andene, natïr(id)e Madjuwt(f) ijt zut benutsen.

Pach Entfermung ber alten Tammen. Der feudbte, juiblidje Igeil ijt durd Fid)ten = Shigelpflanzung in Weitano zu bringen. (ङ (d)lag vou 1869.)

\section{2sie $2 \mathrm{~b}$.}

Erjaț Der durch Deu Riüjelfäjer getös= teten \$ff́nzen.

Sd)läge von 1869 unto 1870. Mact) erfolgter Stodrodung.

פite 3c. 94uศ den bejonbers guten Etandort zeigenden Partient nad) Dent

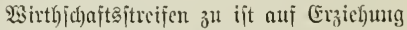
einiger 5orîte von Gid)en, gïijtern unt Budben Bedadyt zu nebmen.

18,15 ha Gefanmtbetrag. 
b) Micdite Scite ocs Siulturplanes.

Raducis über bie ftattgebabte ?a

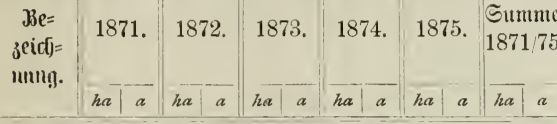

A. DaZs $S$ dientholz.

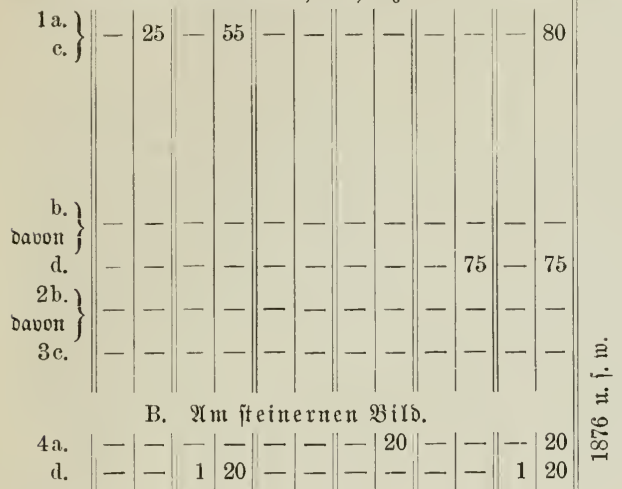

Bemerkang̣en.

$20 a$ Wenigetbebari.

$\left.\begin{array}{c}5 \mathrm{c} . \\ \text { davon } \\ \text { d. }\end{array}\right\}-20-1$
6b.

6b. $-50--50--1-$

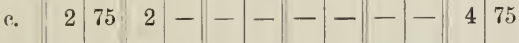

d.

$$
\begin{array}{lll|l|l|}
3 & 70 & 3 & 75|-50|-|20-75| & 890
\end{array}
$$




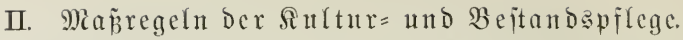

Sierfer fut biejentgen D perationen und ? (nlagen zu recfunen*), weldfe von Der Begrüntung Der $\mathfrak{B c j t a ̈ n চ c ~ a n ~ m a ̈ b r e n t ~ D e r ~ g a n z e n ~ U m t r i e b s z e i t ~}$

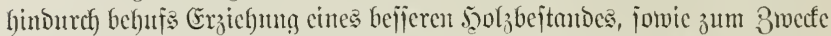
Der Erbaltung und Mefrung Der Bobentraft ausgcfüfrt werben, fofern

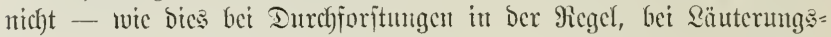
Gieben meijt ober Doch vieffach Der Fall iit - Der entjprechende Sioiten= anfmand Durch Den fummarifichen Betrag Der tarifmäżigen Echläger=

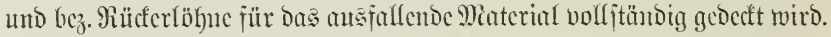
- Maj̄nafymen gegen Injeftenichäben geförent nicht fierf)er. Bejonders

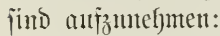

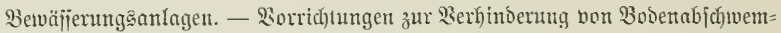

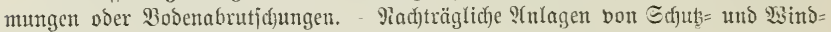

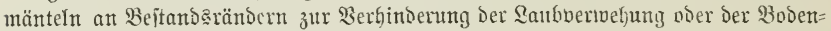

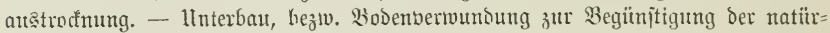

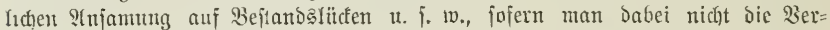

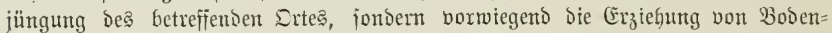

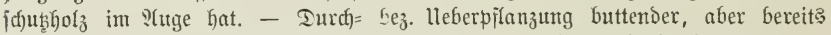

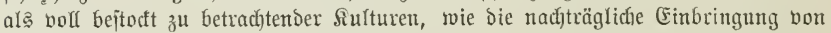

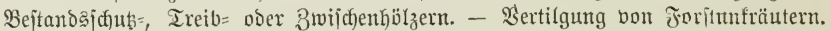

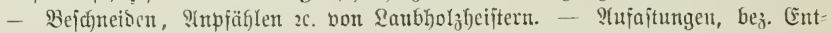

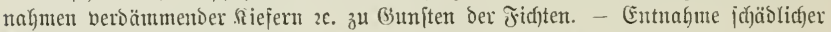

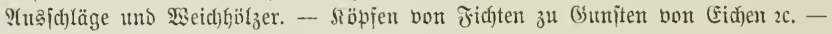

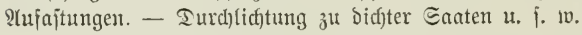

Dieje Maß̃regcln gehören z̧war nicht cigentlich zur Forjteinrichtung,

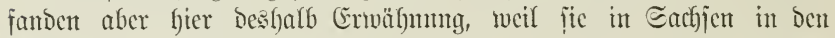
Sutturplan neuerdings bejonders aufgenonmen werden. Wisir begnügen

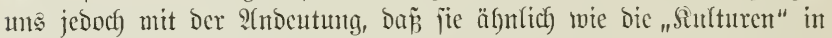

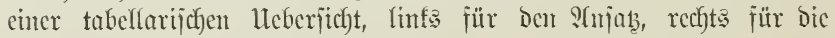

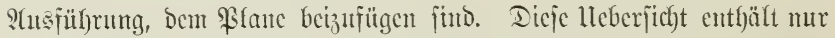

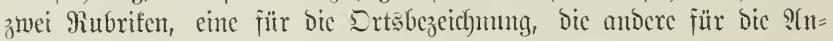
gabe Der Maßjregel.

Entwäjierungen werben in @adjen unter Den Foritberbeiferungen bejonbers,

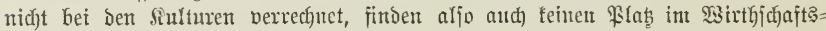
płane. STleinere Entıäijerungen fömnten allenfalls in Den "Benterfungen" zum

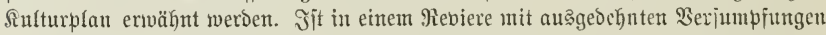
ein umfaîjender Entwäjīerungąplan nöthig, fo wïrde biejer in gecigneter form bem Siulturplan als 21nfang beizufügen fein.

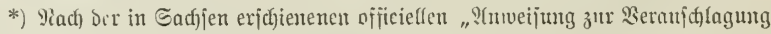

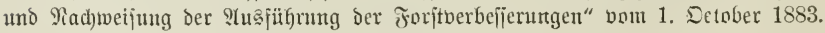




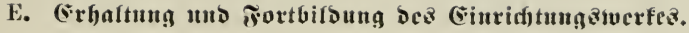

\author{
$\$ 140$. \\ HIfnentrints.
}

Pidcht blos ber gejunde Mienjefenverjtatb, jontern audf bie viel=

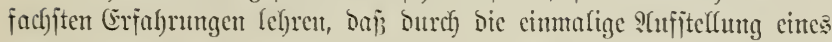
Mirthjchajtsplanes, Durd) bic cimmalige Berectumg eintes Seiebsjabes

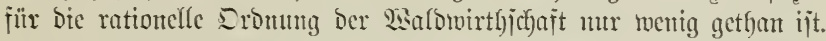

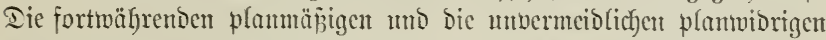

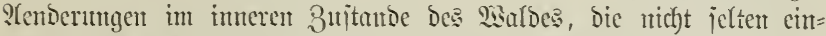

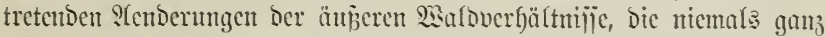

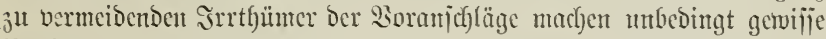
Sorfebrungen nötlgig, weldfe das Eintrichtumgswerf ergänjen uno lebens= fräftig erbalten. SEer heute noch glaulben wollte, burch : Surfitellung

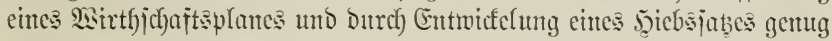

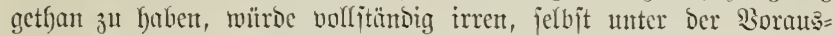

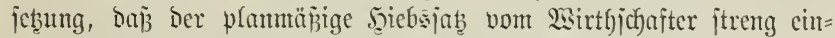
gefalten und bic Sulturen gewijienfajtejt atsgejülyrt wütroen.

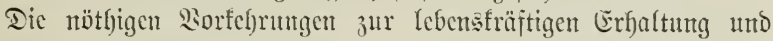

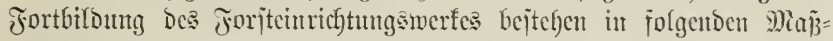
regeln:

I. Siermeijungena chträge.

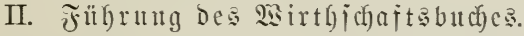

III. Revifionen.

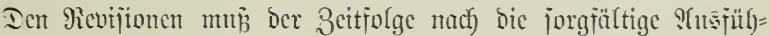
rung ber unter I. und II. genanten Shrbeiten vorausgenen, meshalf wir bicje alterit betradjten wollen.

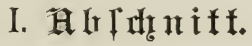

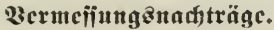

\$ 141.

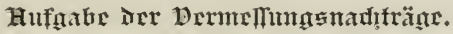

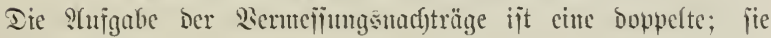
faben ç วu thut: 


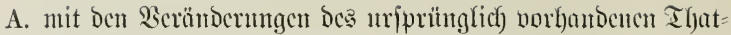
beitalloce,

B. mit Berichtigung oder Bejeitigung in Berlante ber 3eit ent= itebender Miängcl.

Bur Erläutcrung bicjer ?ufgabe bicue Madjitchendes:

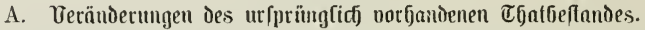

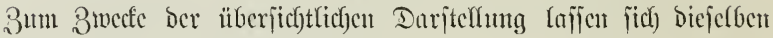

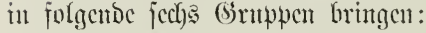

1) 2 endorungen ber Biejamuntfläthe.

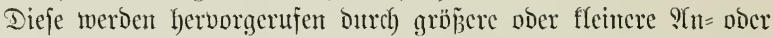
Serfüufe, Durd) Bertauj(b)ugen, Durch (Sirenzberichtigutgen.

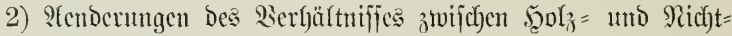
h) $0 \mathfrak{l}_{2}$ bod

Dafjin gehören: Dic ?(nlage von breiten, mit Ffläcl) ju vertech)=

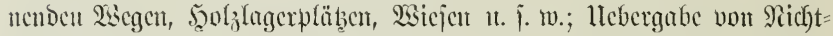
hol,

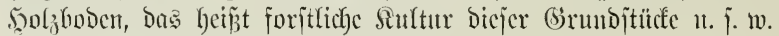

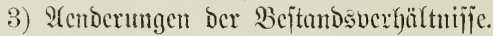

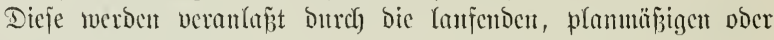
planmibrigen Ech)!äge.

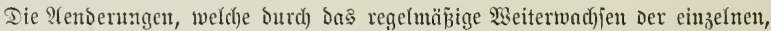

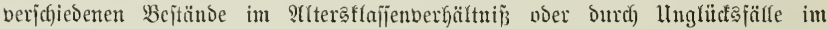

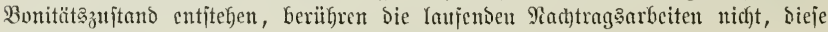

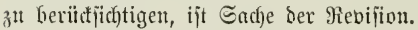

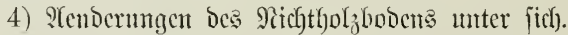

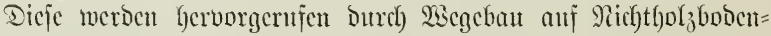

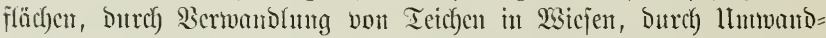

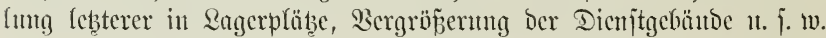

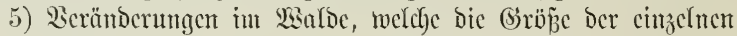

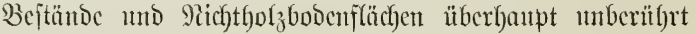
Iaĩjert.

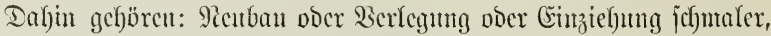

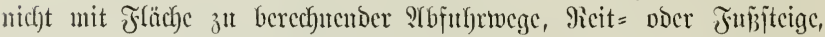

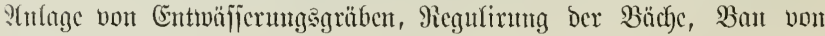
Briticfest t. $\{$. w.

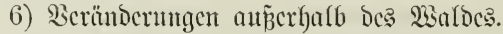

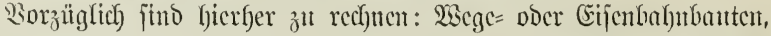

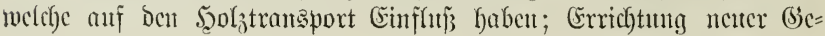




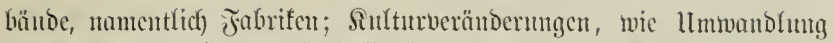

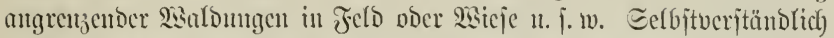
foumen bicje Beränderuntgen mux jo weit in Betrad)t, als jie fïr bas Bereich Der für bie Siarten nothwendigen, jogenumten "Ileber= urbeitumg" foullen.

Sflfe unter unter 1 bis 6 gentaunten ?(enderumgen müfïen, jowveit

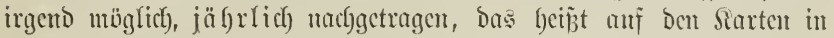

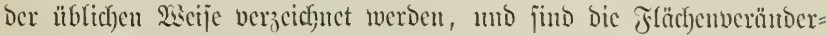

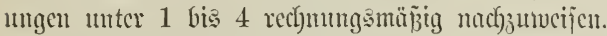

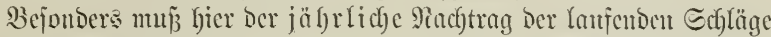
herworgelyohen merden, ba ex vou fo mandfen Forjtucrwaltungen nod)

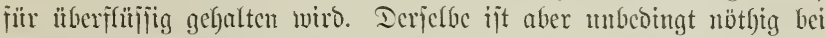

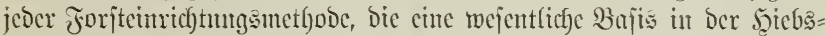
fläche fintot, weil man ganz iun Iunfeln tappt, menn man nicht ein=

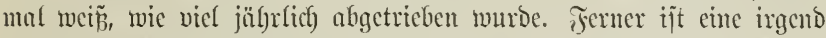
brauchbore Sinlturrect)ung unmög(ich), wenn utut nicht cimmal bic

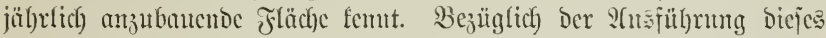

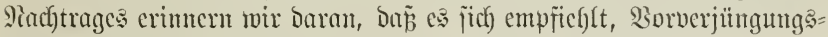

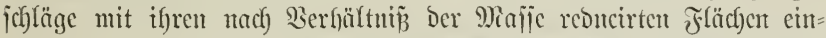
3utragen. Der Machtrag Der Echläge auf Den Siarten felbjit erfolgt

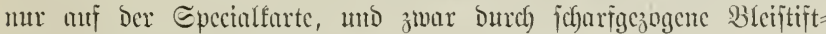
linten; bie Ketreffende sah)reszahl ift ebcrifalls mit Bleijtift dajelbjt еinzuj d)reibert.

\section{B. Bericfitigung oder ßefeitigung im Caufe der Beit entfefjender Jiänģef.}

Dic richtige Erfhaltung bes Sermefijungswertes beruft im Wejent= lichen auf ber Erbaltung aller (sirenzzeidfen, fowie bes Eefucifantebes und Der Sicferfeitsitcine.

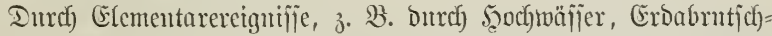

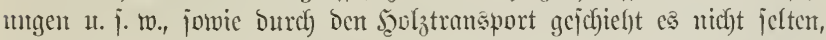

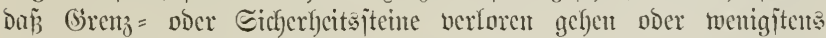
berausgerifjen uto von iffen cigentlicfen Etmopunft entfernt werden.

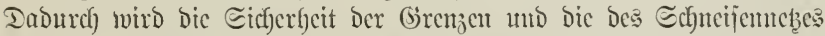

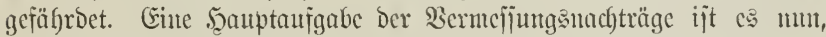
joldye Mängel möglichjit bald zu bejeitigent.

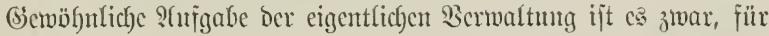
Sifenthaltung Der Echncijen zu jorgen, ebemjo wie für bic ber Girenz=

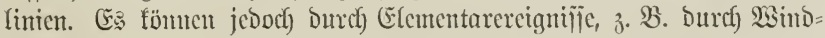

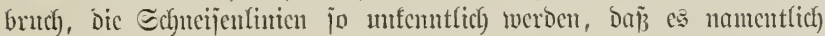




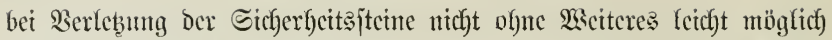

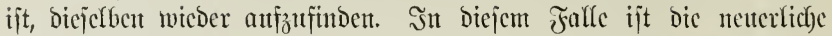

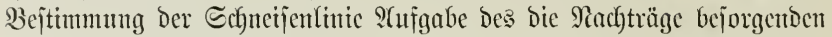

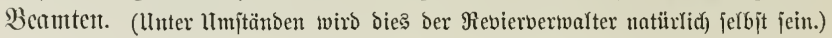

\section{§ 142.}

\section{Dag Mofiżentudt.}

Dic unter A genaunten Beränberuntgen treten żm Theil แtur allmälig cin, oder bie Bertidytigung Der unter B crwälnten Miängel

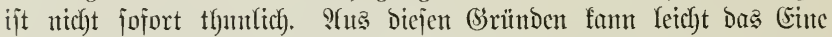
ober bas sfndere in Bergeffenfeit geratfen. Der Sicvierberwalter fat

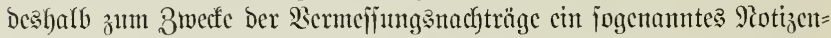

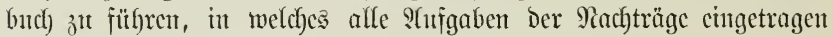
meroen.

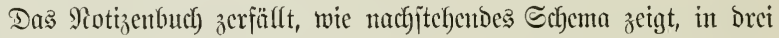
Rubrifen.*)

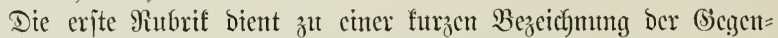

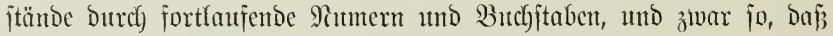

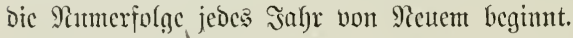

In bic zmeite Siubrif hat Der Revierwerwalter alle bic unter A

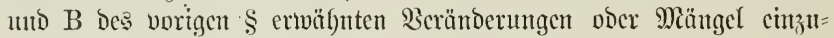

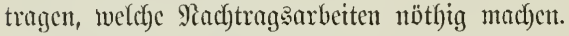

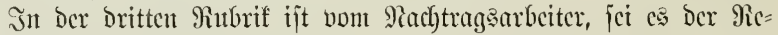

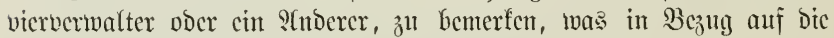
antgegebenten (Siegenijtändo von ifm gejebchen ijt.

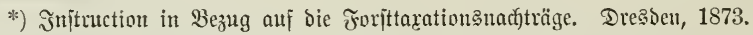

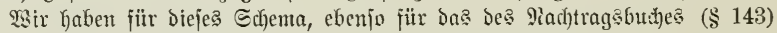

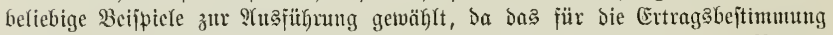

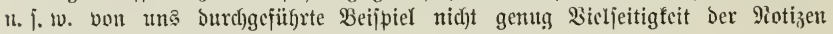
crmöglidgt. 
Jix. Alıgafe der nactjultagenden gegenftände.

3emerfiunģen

über Den Erfolg.

\section{Forịtahr 1877.}

1. Schläge.

a. Som Forjtjahre 1876.

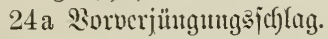

b. Som Foritjahre 1877.

2 b Rablicklag.

25d Desgleicfen (Durct)gejd)lagen).

40a b Desglcichcu (a Durd)gejchfagen).

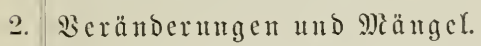

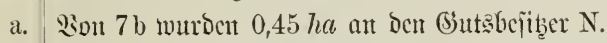
verfanft, bic amtliche Berainumg crfolgte am 20. P(pril 1877.

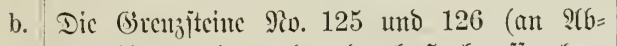

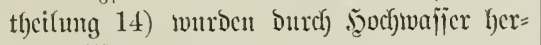
ausgcrifien.

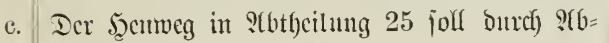
theilung 24 verlängert merocn, uno ijt ber Bau bis 24 a asgcfübrt.

Ract)getragen am 29. Ecpt. 1877.

Dic Sircuspunfte wurben Durd) itarfe \$rzäble wicder bejtimmt. :Tu 30. Stuguit 1877.

Dic :(nfuahme unterblicb, joll im fünftigen ฐal)r cróolger, ment ber $13 \mathrm{seg}$ fertig ijt.

d. Bon Der Sohtwicje (lit. m) murde bie fadymale nöroliche Epibe mit Fichten zugcpf́lanzt.

Padjgetragen am 30. Ecpt. 1877.

e. Dic Edfucije 12 umuróc $3,5 \mathrm{~m}$ breit planirt, Desglciclyen.

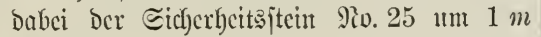
vericţt.

$$
\text { แ. } \text { i. } \mathfrak{w} \text {. }
$$




\section{$\$ 143$.}

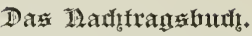

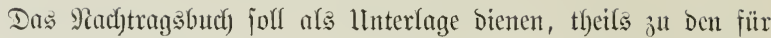

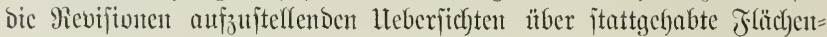
veräntoerungen ("Flächenauffitellungen"), theils zux Beridytigung unto Ergänzแng Der Sarten und (Sirenzregijter, überhaupt follen barin afle bie Bemertungen niedergelegt werben, welde jowohl für Don Bctrieb

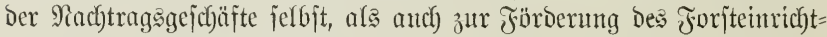
ungsmerfes nöthig erficheinen.

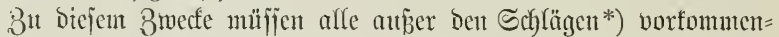

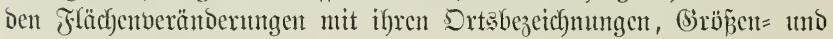

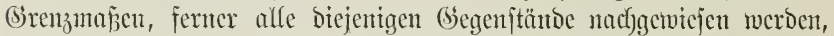

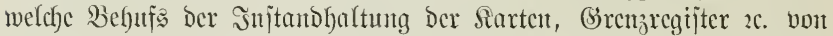

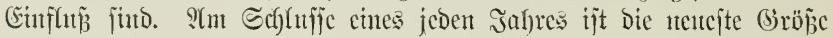

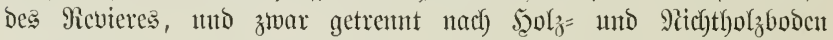
anzugebent.

Bur Crrläuterung biente nachfolgendes Echema, für welches vor=

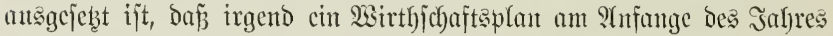
1879 beginnt.

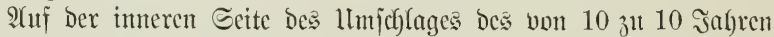
neu anjulegenden Rachtragsbutches wird ber jeiebsjab fitr bas nächjte

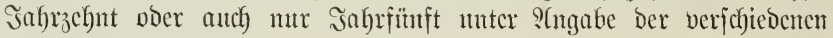
Eortimente bemerft.

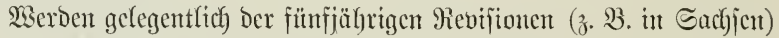

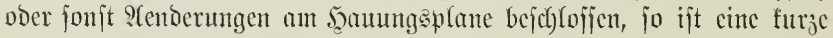
Potiz aus bem betreffenten \$rotofoll und ber nette Şiebjab juzufügen.

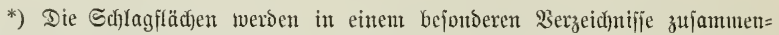

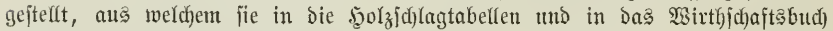

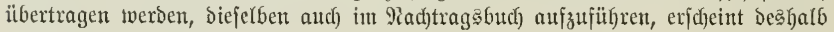
untiotbig. 


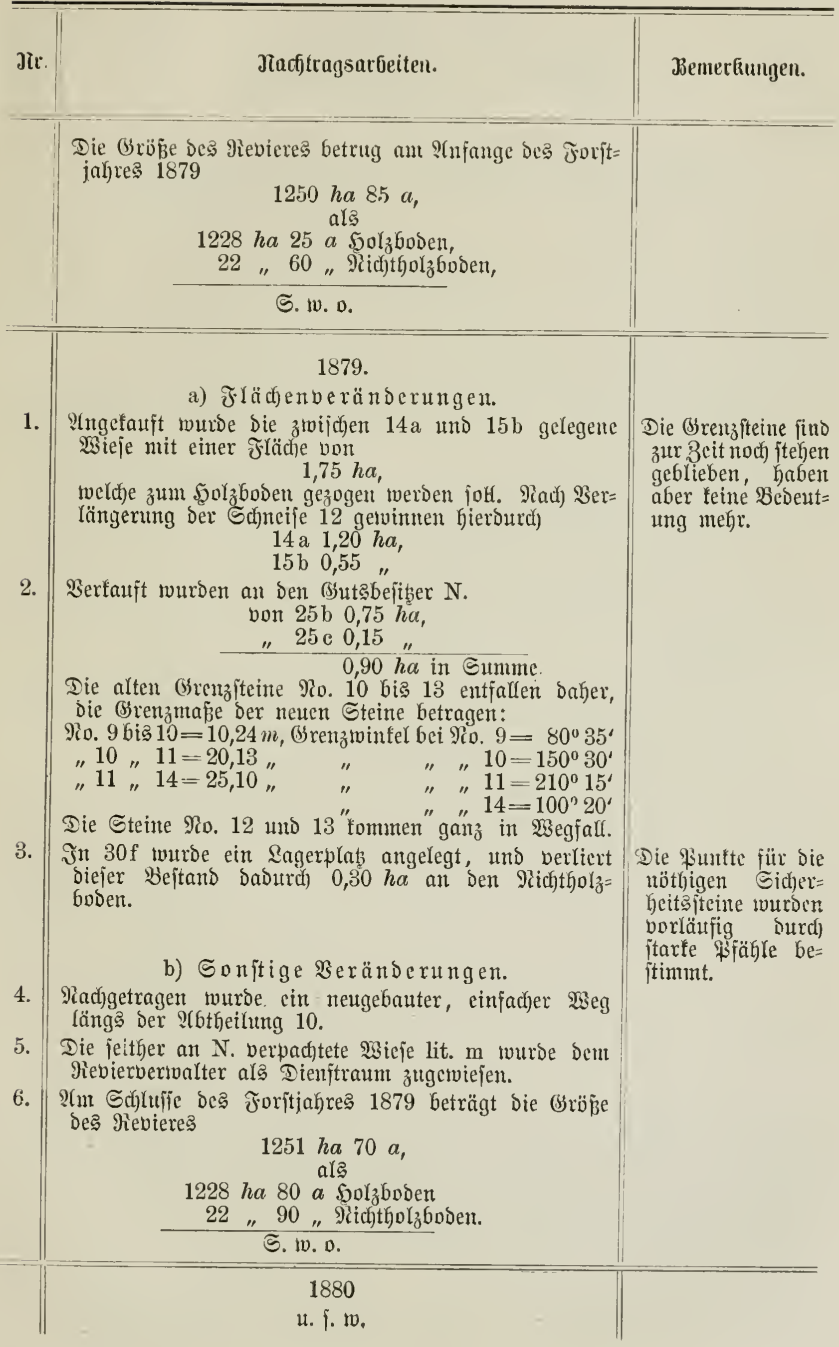




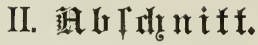

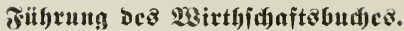

\section{$\$ 144$.}

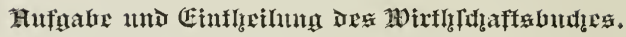

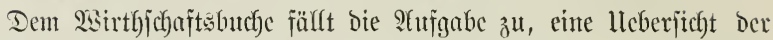
Deu $\mathfrak{B}$ ald

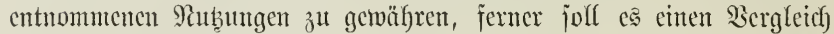

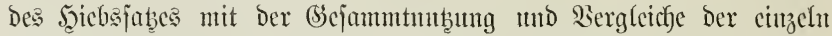
geichäb̧ten Miaterialerträge mit Den wirflichen Errträgen geben.

Das Wirtlyjchaf̌tsbuch zerfällt in 6 sfbtheilungen:

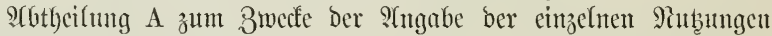
nach) Material mo (Beld.

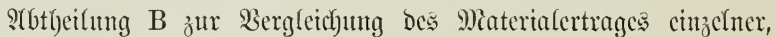
Durd)gejchlagener Sciebsorte mit Der Sdjäbung.

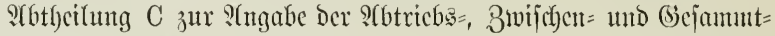
mubung nach Material und (Geld.

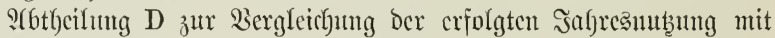
dem J̧icbsjabe.

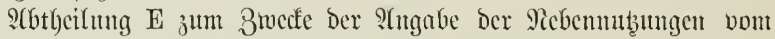

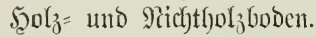

stotheilung F bie Ricinertragstabelle.

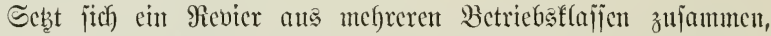

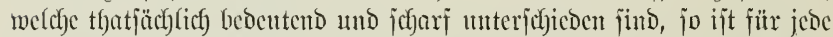

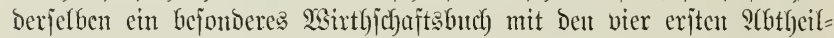
ungen 3u fül)ren, ebenjo twic aud für jebc cin bejonderer jeicbsjab u. \{. w. crmittelt wurbc. Dic giemertragstabelfe und ?fbtheilung E

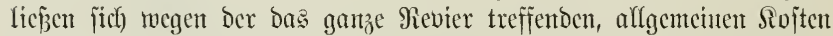

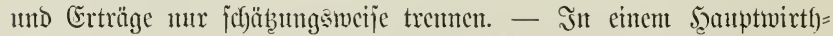

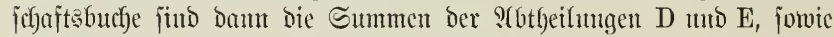
dic sabtycilung F für Das (Banze zu geben.

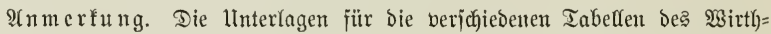

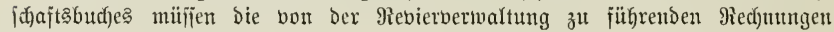

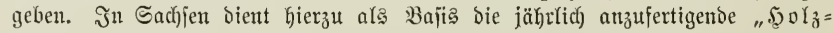

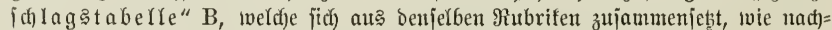

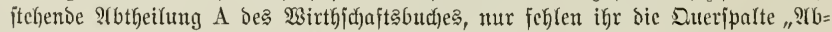
theilung 9ir." unt die Miubrifen "Ioritjagr" uno "Bemerfungen". Durch Ber=

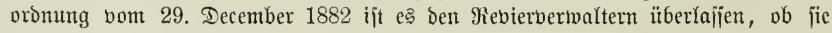

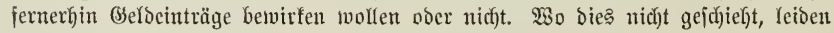

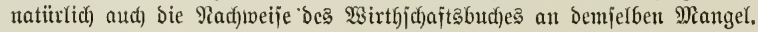


พirthj(hafts

\begin{tabular}{|c|c|c|c|c|c|c|c|c|}
\hline \multirow{3}{*}{ 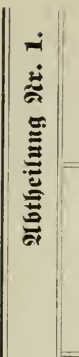 } & \multicolumn{3}{|c|}{ 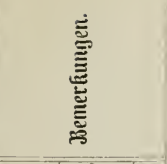 } & \multicolumn{2}{|c|}{ 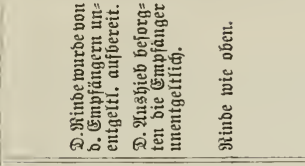 } & \multicolumn{2}{|l|}{ 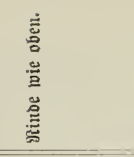 } & \multirow[b]{2}{*}{$\begin{array}{l}8 \\
\stackrel{8}{\infty} \\
\stackrel{-}{\circ}\end{array}$} \\
\hline & \multirow{2}{*}{ 苋 } & 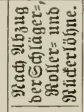 & \multirow{2}{*}{ 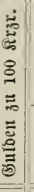 } & 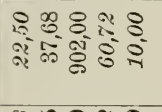 & 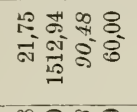 & 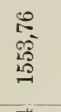 & 栗 & \\
\hline & & 总 & & 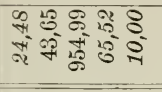 & 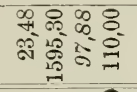 & 荡 & 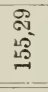 & 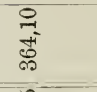 \\
\hline & \multicolumn{2}{|c|}{ 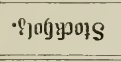 } & $\Sigma$ & 11111 & 1118 & 1 & 1 & 20 \\
\hline & \multirow{2}{*}{ 苛 } & \multirow{2}{*}{ 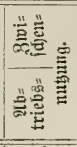 } & \multirow{10}{*}{ 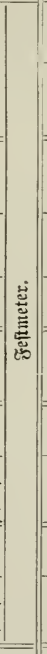 } & 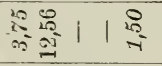 & $\begin{array}{llll}40 & 1 & 1\end{array}$ & 1 & 1 & 1 \\
\hline & & & & 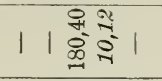 & 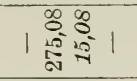 & $\begin{array}{l}\infty \\
\infty \\
\infty \\
\infty\end{array}$ & 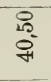 & 1 \\
\hline & \multirow{3}{*}{$\begin{array}{l}\stackrel{\dot{\Xi}}{\Xi} \\
\text { वे }\end{array}$} & 氖 & & 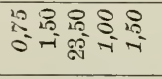 & 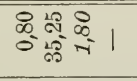 & 点 & $\begin{array}{l}20 \\
20^{\circ}\end{array}$ & 1 \\
\hline & & 产总 & & 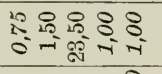 & 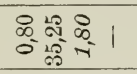 & 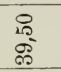 & $\begin{array}{l}250 \\
250 \\
20\end{array}$ & 1 \\
\hline & & 萢 & & 111180 & 1111 & 1 & 1 & 1 \\
\hline & \multirow{5}{*}{$\begin{array}{l}\dot{0} \\
\vdots \\
\dot{0} \\
\dot{0} \\
\stackrel{0}{0}\end{array}$} & 昱 & & 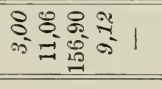 & 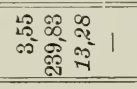 & 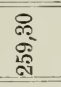 & $\begin{array}{l}8 \\
20 \\
65\end{array}$ & 1 \\
\hline & & 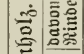 & & 11111 & 1111 & & 1 & 1 \\
\hline & & 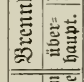 & & 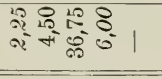 & 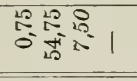 & $\begin{array}{l}29 \\
29 \\
9\end{array}$ & $\begin{array}{l}29 \\
25 \\
21\end{array}$ & 1 \\
\hline & & 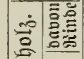 & & $\begin{array}{llll}1 & 8 & 8 & 1 \\
0 & 1 & 1\end{array}$ & $\begin{array}{lll}10 \\
4\end{array}$ & 8 & 1 & 1 \\
\hline & & 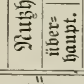 & & $\begin{array}{ll}0 \\
0 \\
0 \\
0\end{array}$ & 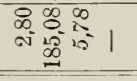 & $\begin{array}{l}20 \\
25 \\
125 \\
12 \\
\end{array}$ & 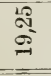 & 1 \\
\hline & \multicolumn{3}{|c|}{ 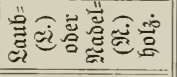 } & $\alpha i \dot{z}=\alpha \dot{c}=$ & $\ddot{n}=c i \dot{\pi}$ & $=$ & 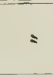 & $=$ \\
\hline & \multicolumn{3}{|c|}{ 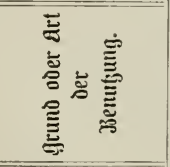 } & \multicolumn{5}{|c|}{ 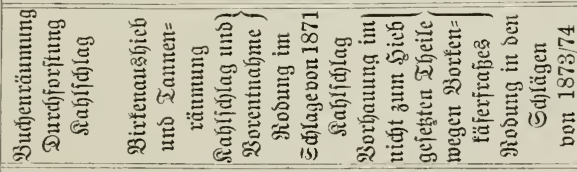 } \\
\hline & 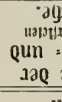 & 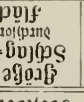 & $\Xi$ & 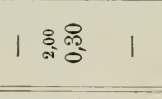 & 年 & 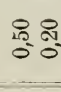 & & 1 \\
\hline \multirow[b]{2}{*}{4} & \multicolumn{3}{|c|}{ ploy } & $\therefore \dot{0}$ & $\dot{0} \dot{-}$ & $\dot{0}$ & & $-\dot{0}$ \\
\hline & \multicolumn{3}{|c|}{ - } & 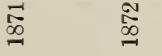 & $\underset{\substack{\infty \\
\sim}}{\stackrel{m}{\infty}}$ & 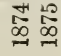 & & \\
\hline
\end{tabular}




\section{\$ 145 .}

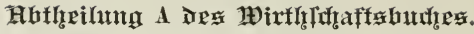

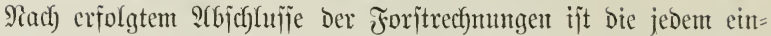
zelnen Bejtande entnomment \$̧olzmafie und Deren Beldertrag in bicjer

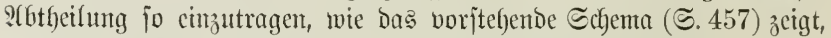
welches zn Dent im \$ 132 u. $\tilde{f}$. gegebenen Beijpicle gehört.

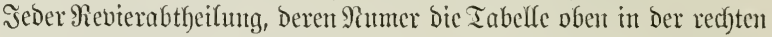
Ecfe entl)ält, finto in Der Regel 2 Seiten für Don allmälig erfolgenten Eintrag zu wiomen. Fü bejonders fleine ?fbtheilungen genügt eine Seite.

Ynmerfung. Borftehendes Sdjema gleidft ber in ber fagl. fädff. Staat: =

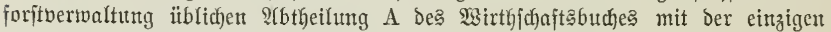

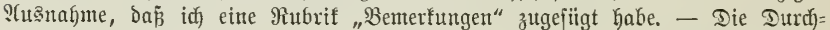

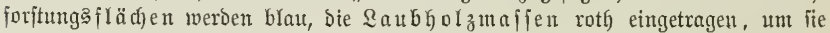

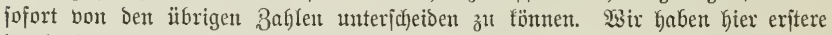

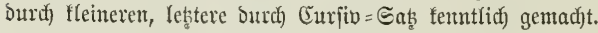

\section{$\$ 146$.}

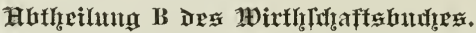

Dieje Stbthciluntg enthält die Bergleichung Des Materialertrages cinzelner, Durdfycidylagener Şiebsorte mit ber Schäbung.

Eit Drt ift Dam als "Durchgejchlagen" zu betrachten, went Defien Shlrieb vollentoet ift, weut cr aljo entweder ganz abgetrieben wurbe, voer wenn auf ber fläche mur emzelne jeorjte und Bämme in der

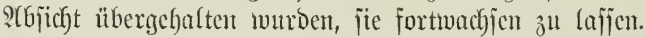

Das nadffolgende 巨dbema bcdarf teiter näferen Crrläuterung.

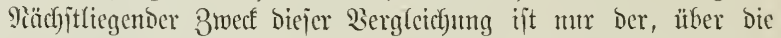

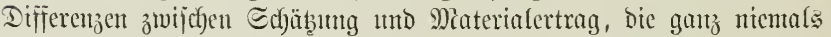
fehlen, einten Rachweis zll geminnen, Der einte lleberichreitung ober

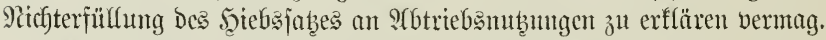

2f nmerfung 1. Wolfte man biefe Tabelfe zur Erfüllung weiterer Bwecte

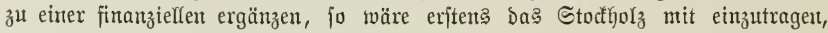
z'weitens ber Geldertrag. Iabei ijt aber wohl zu beachten, Daß bie gewomenen

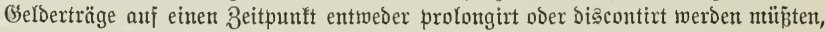

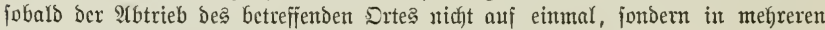
Jahreşj)lägen exfolgte. Für joldjen 3wedf cmpfeblen wir indefien mehr, gclegent=

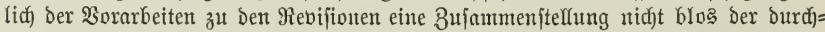

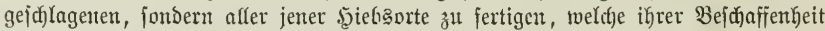

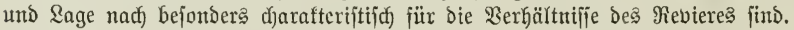

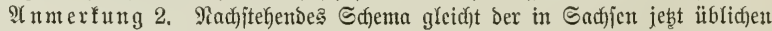

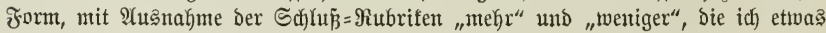
ïberfidgtlidjer gegeben habe. 


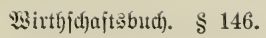

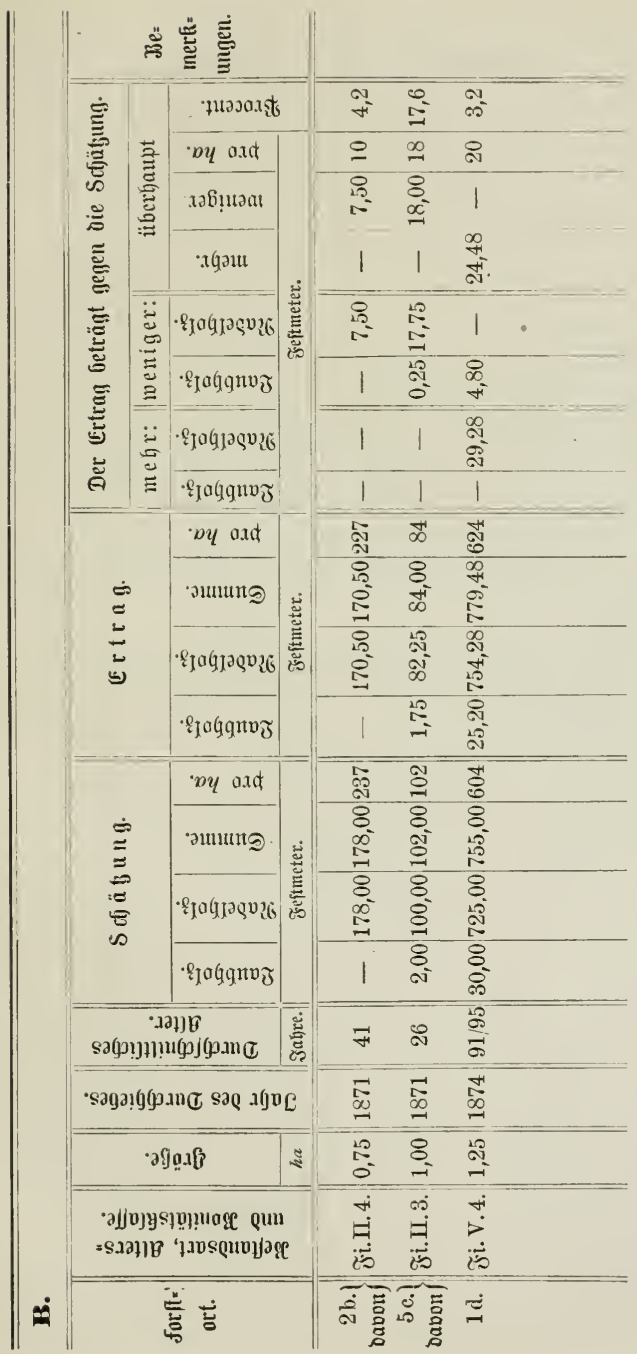




\section{$\$ 147$.

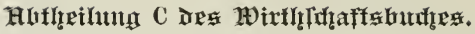

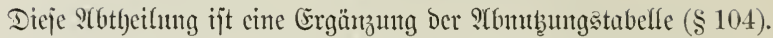

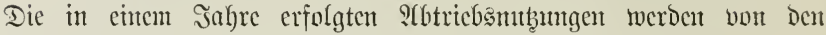

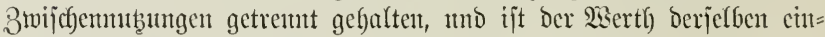

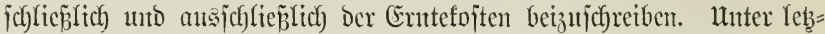

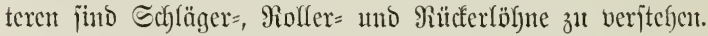

llm bie s(btheilung $\mathrm{C}$ nicht zu breit machen und baburch bie

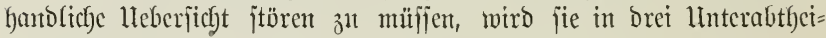
lungen getrent.

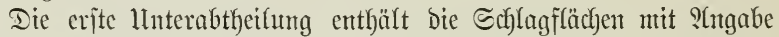

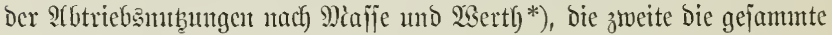

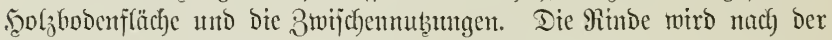

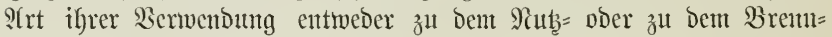
holze gerechntet.

Die Dritte Ilnterabtbcilung gicbt cinte Heberjicht ber Bicjammt=

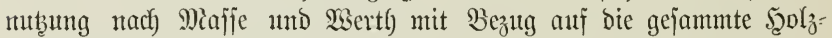
bodenfläctye.

Der Belderlös faum erjt dann cingetragen twerden, twent fämmt=

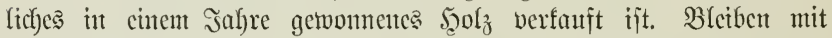

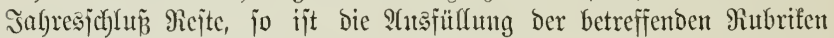
bis nach erfolgtem Serfaufe zat verichieben.

Umjtchendos, bie brei Эahre 1871/73 unjercs Beijpicles betreffen= Des Schenta mag bie Form der Tabelle verbentfichen.

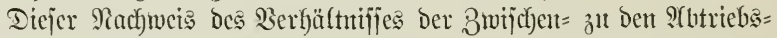

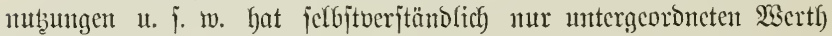

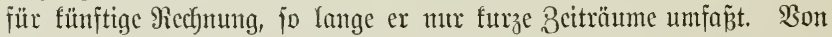

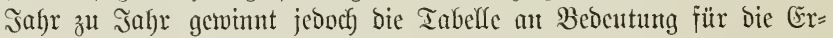
mitteluntg Der fünftigen Sciebsfäbc. Deshalb ijt aud am Schlujic

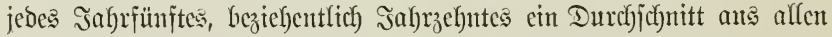

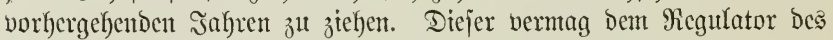

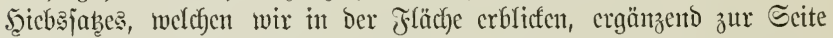

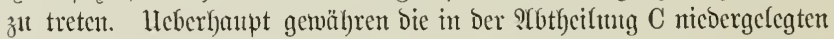

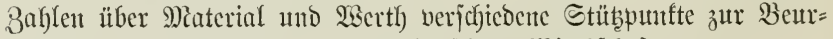
theilung Der vergangenen wie Der fünfitigen Wirthjichajt.

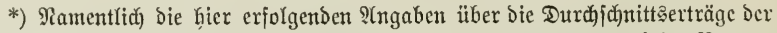

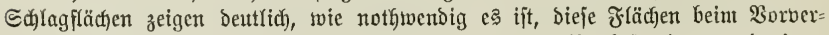
jüngungabetriebe nad) der Miafie bes ifnen entipredenden $\mathfrak{B}_{0}$ Ubejtandes z̆ reduciren. 


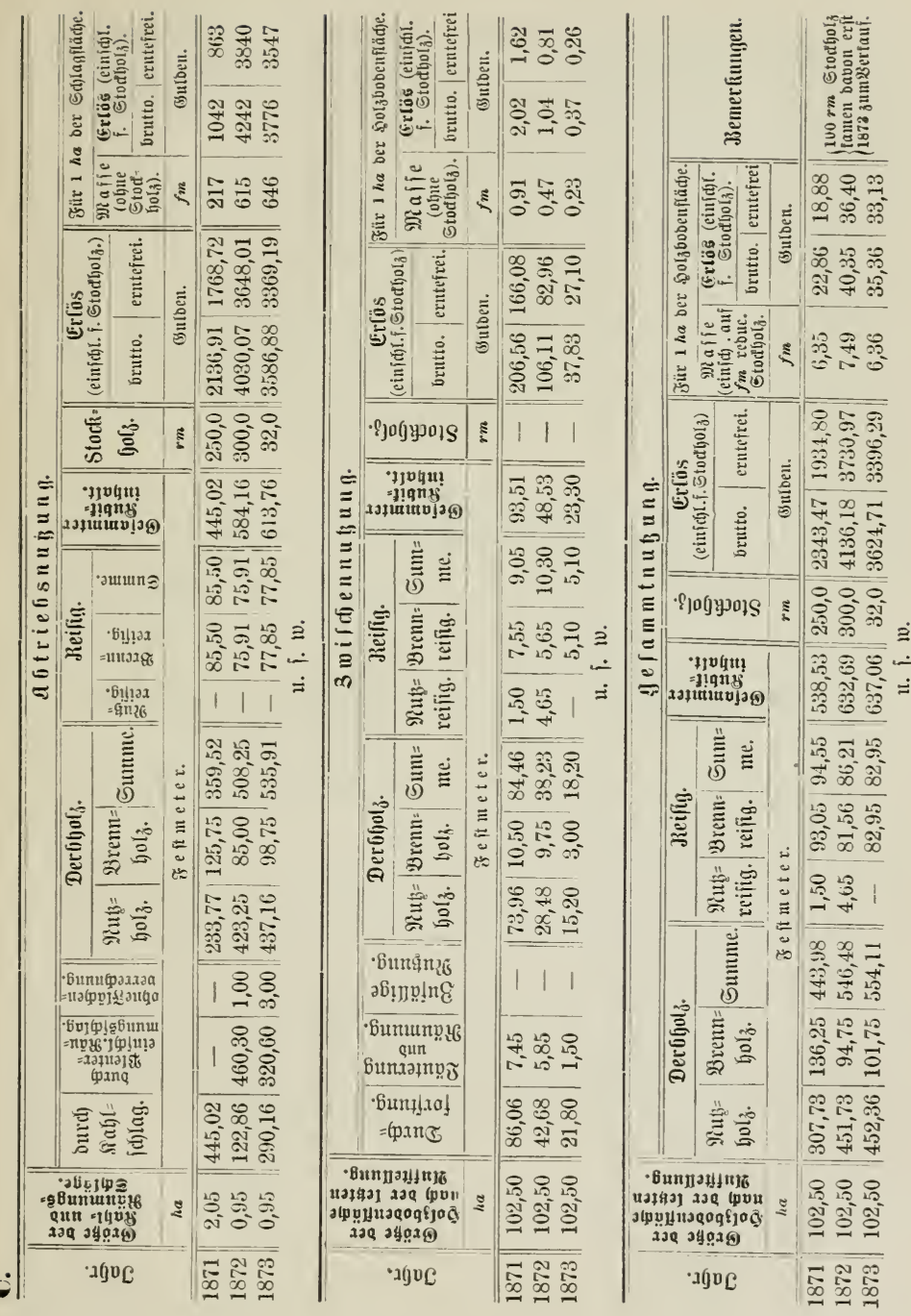




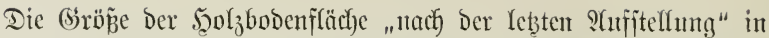
Den bic Bwijchemubutg und bie Bejammtnuţung betreffenden Tabelfen

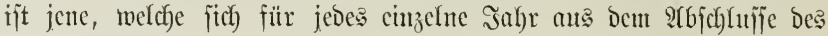
Nachtragsbutches (j. ๔. 455) ergiebt.

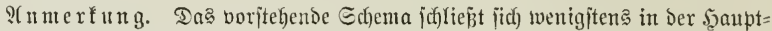

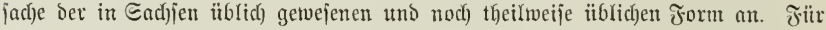

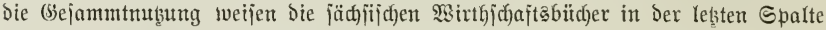

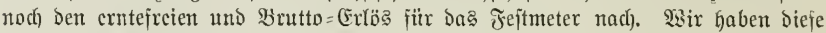

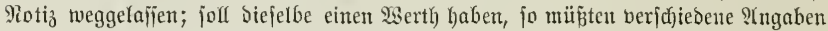
zufammengejtellt werden, je nadjoem man in Material und im Gels mu bas Derbbolz ober die bejammtntbung mit voer obne Etortholz berïffichtigt. Be=

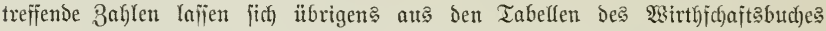
leicht ermittcln. - WBie aus Der Bemerfung auj 5 . 456 bervorgeht, fehlt jeșt auf vielen Revieren Der Rachtwcis des Geldertrages ïberbaupt.

\section{§ 148.}

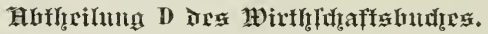

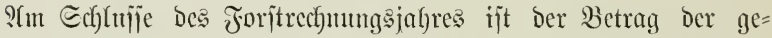

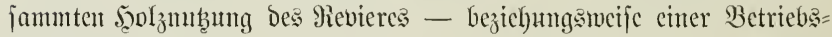
flajfe - zujammenjuftellen 1 mo mit Dem seichsjabe zu vergleidfen. 万̧ier

Die Beurtheiluntg, of im Sanzen ein Riüfitand vorlyanden oder ob ein Sorgriff erfolgt ijt, fam mur unter Berïffictftigung Der vor=

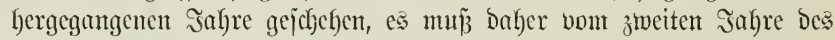

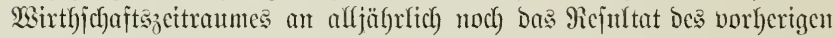
Sal)res mit in Rechnung geitellt worbent.

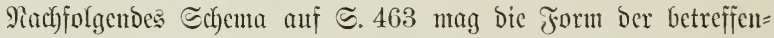

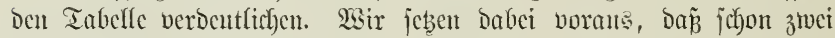

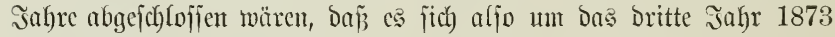
unjeres Beifpieles handele.

Jebem Jafre ift cute bejondere Ecite zll mioment.

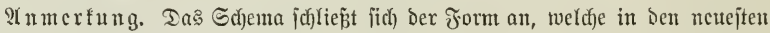

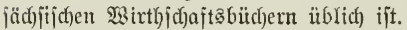

Dor leichteren lleberiidyt wegen trägt man Dą Laubhol ein; wir haben Daffelbe Gier ourd) (Euriju= Saț fenttlich) gematht. 
密䓂 ₹

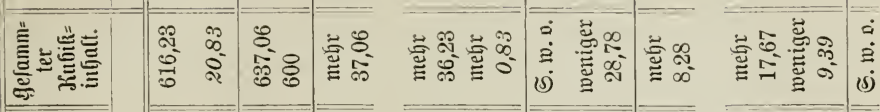

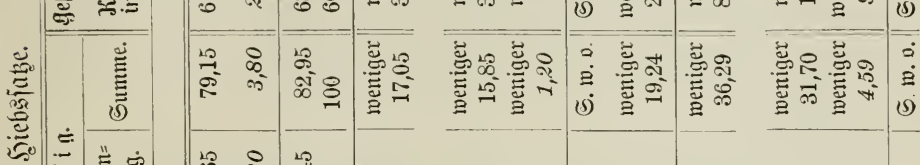

焉焉

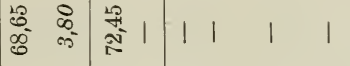

芯

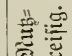

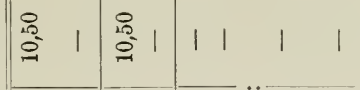

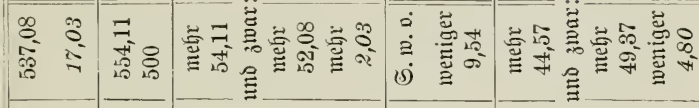

氞

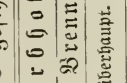

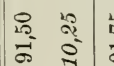

is

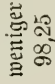

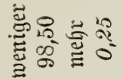

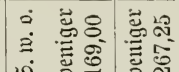

๒

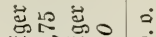

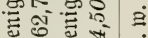

0

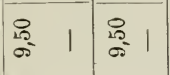

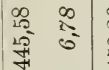

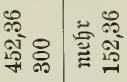

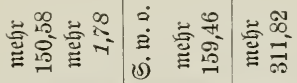

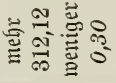

$=$

蒙

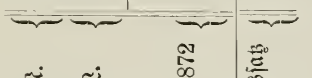

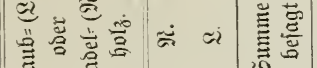

हैं

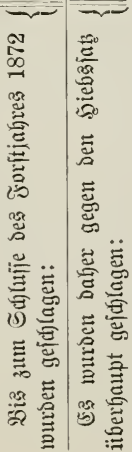




\section{$\S 149$.}

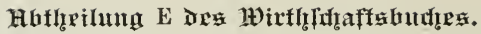

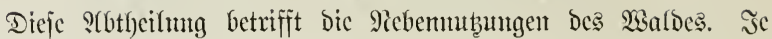

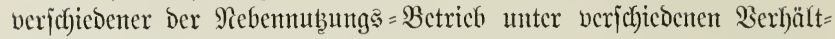

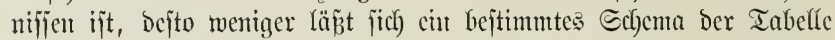
für bie $\mathfrak{B}$ errect)unng biejer Nitbungen geben. WBir erimtent beifpicts=

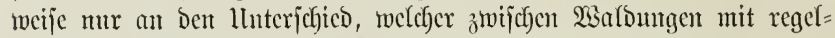

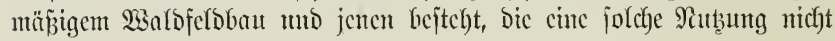
gewwäl)rent.

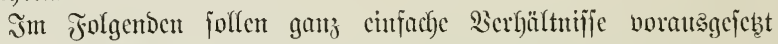

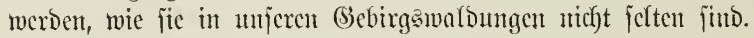

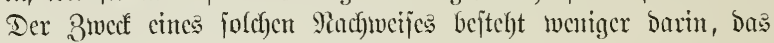

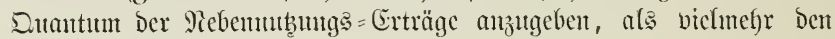

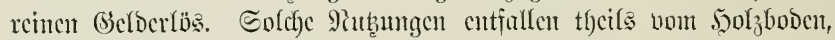

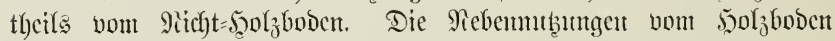
gebären entweber bejtimmten Bejtünton oder bem (Sanzen an, fo z. B.

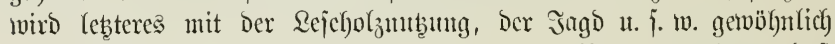

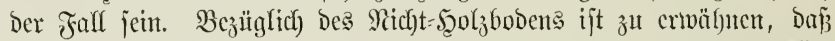

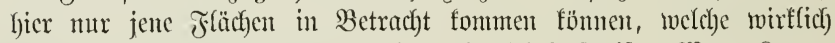

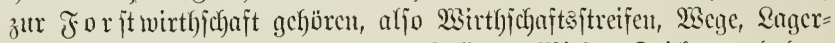

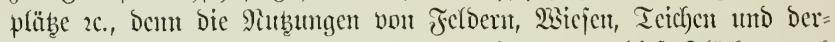

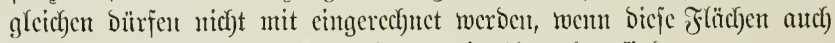

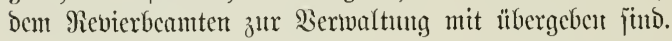

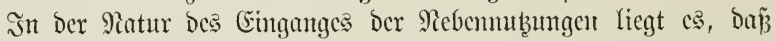

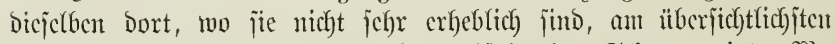
nach) Jahlen gcoromet werben, wie nadffolgendes Schema zeigt. 230

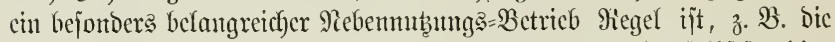

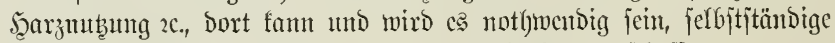

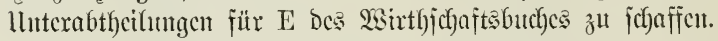

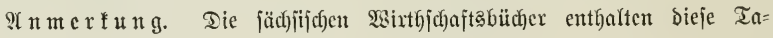
befle nidyt. 


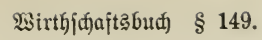

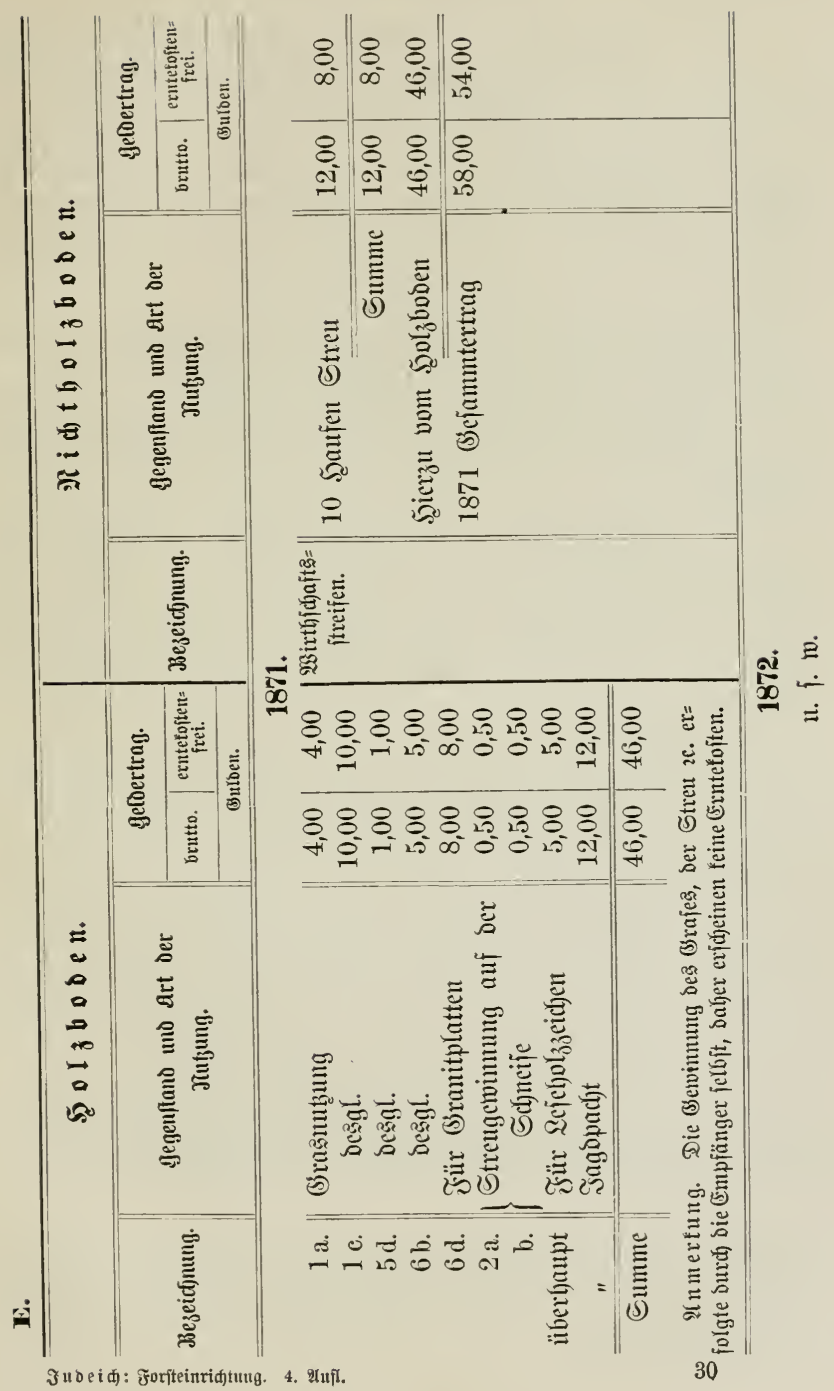




\section{\$ 150.

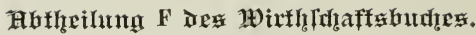

(Die Reinertragstabelle.)

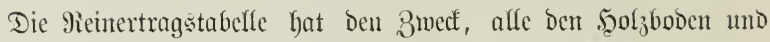
forjtlichen Richtholzboden treffenden Eimnahnen und STsgaben fum= marifa) nachzumeifen, bie Differenz bciber, aljo bie reine Waldrente, in Bergleich mit Dem Waldfapitale zu itellen, D. h. anzugeben, zu welchem Procentiatse ficf Diejes Sapital verzinjt.

Rachjtehendes Echema mit Ricjultaten Der Jahre 1871, 72 mb 73 unjeres Beifpieles verbentlicht bie Form Der Tabelle.

Die Fläche Des j̧oljbodens und des forjtlichen Ridjthol $\mathfrak{l}_{3}$ boden ift itets nach bem neueiten Befund in Dem betreffenden Jabre cin= zitragen.

llm bie :Ats̊gabepojten für Forjtuerbejjenutgen, Bermaltung und Schnb, ßerjchiedentes แt. j. w. im Einzelnen angeben z̆ fönnen, wie wir es im Folgenden nur angedentet haben, empfichlt es jith), jebcm Jahre cine ganze Eeite zu wiomen, Dafür an Echlujfe des Jahrs= zchntes auf einer Seite eine jummarijac Wiederholung Der Einzeljahre z't geben.

Das Maldfapital ijt gleich) ber Summe aus Boben= und $520 l z=$

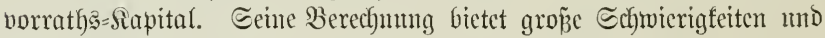

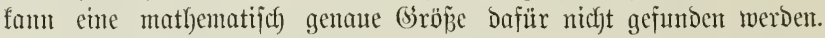
Bum Bwedfe ber Budffüfrung mun bic Ermittelıng bejfelben von an=

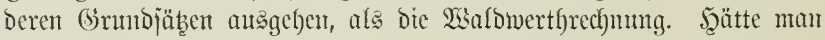
cinen Warlo gefauft, fo wäre ber gezalylte Sianfichilling bas für bic Sucffüfrung einzutelfende Sapital. Micijt liegt bie Sache aber nicht

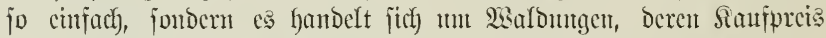
gar nicht bejtimmt werben fanm, ober in fo längit vergantgener Beit crlegt wurde, bajz er abjolut nicht melyr majgebend ift. *)

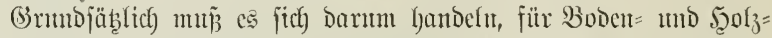

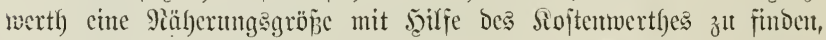

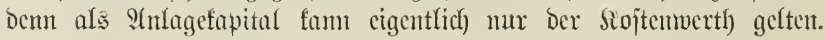

*) Wir Gaben unţ ausfüfrlid verbreitet ïber bie Ermittelung Des $\mathfrak{W a l d =}$

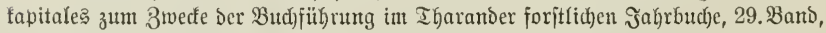

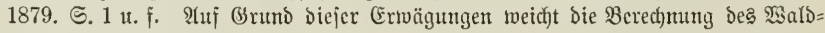

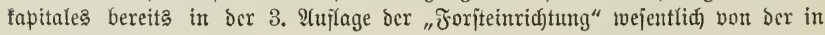
Den früberen $\mathfrak{A}$ trflagen $a \mathfrak{b}$. 


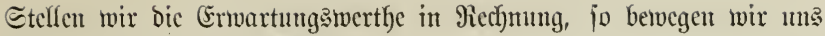
mefyr ober reniger im Sircije.

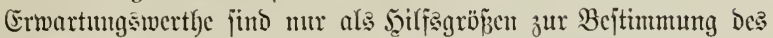

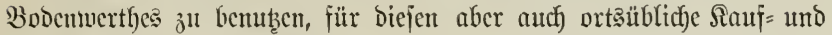

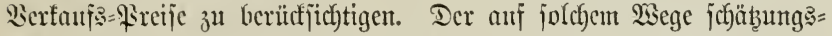

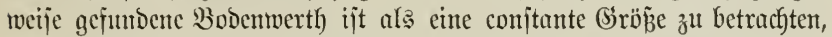

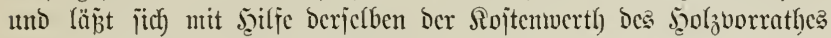

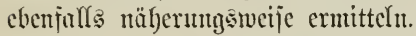

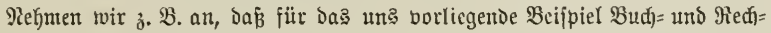

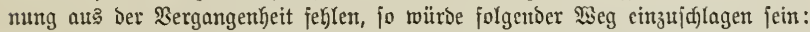

3ur Ermittelung des Bobentwerthes benthen wir Erfahrungen aus ähntliden

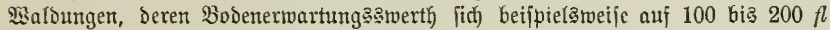

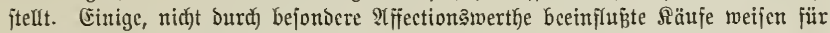

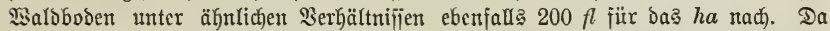

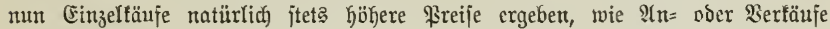

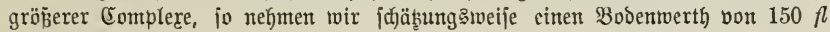
für bas $h a$ an.

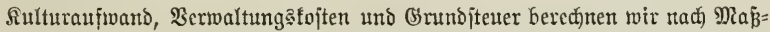

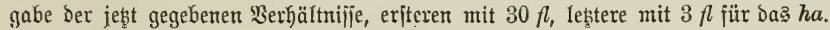

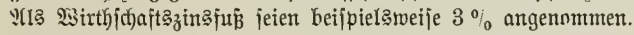

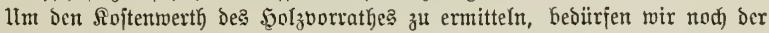

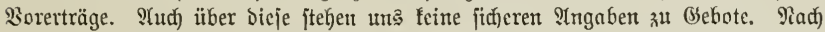

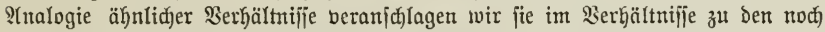

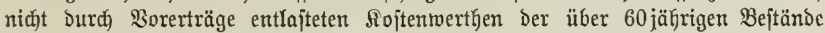
mit $25 \%$ Der 51 bis 60 jägrigen mit 20 , ber 41 bis 50 jährigen mit 15 , ber 31 Gỉ 40 jähriger mit 10 , Der 20 bis 30 jäfrigen Beftänte mit $5^{\circ}{ }_{\omega}$.

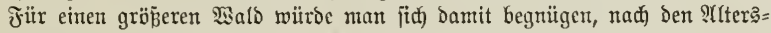

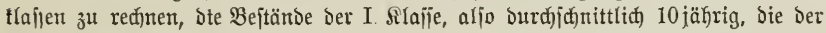

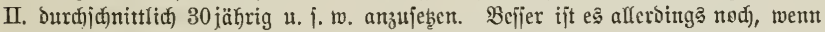

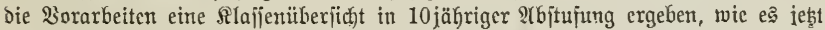

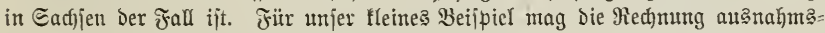

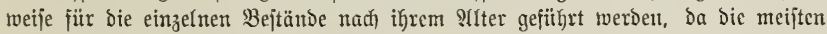

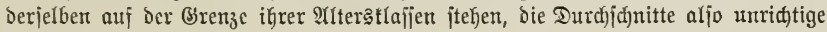
Rejultate geben unüßten.

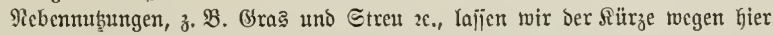
unbeadjtet ober denfen fie uns mit in obigen \$rocentfäz̧ent entfalten.

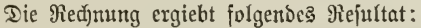

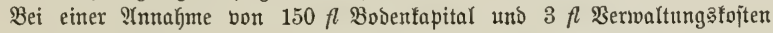
und Eteuern beredgnet fïd für $1 \mathrm{ha}$ Dic Bobenbruttorente auf $7,5 \mathrm{f}$. 
1. 3 e und $6 \mathrm{~d}, 3,50$ ha 100 jährige $B$ citände.

100 j. Endwerth der $\mathfrak{B}$ odenbruttorente $7,5 \times \frac{1,03^{100}-1}{0,03}=4554,66$ 100j. Radjwerth Der Siulturf́piten. . $\quad 30 \times 1,03^{100}=576,56$ Summe 5131,22 ફ̧ierbon ab $25 \%$ ßornuţungen . . . . . . . . . 1282,81 Siojtentwerth bes 1 ha grop̈en Beftandes . . . . . . 3848,41 $3,50 \times 3848,41=13469 \mathrm{fl}$.

2. $1 \mathrm{~d}$ unঠ $4 \mathrm{a}, 4,25$ ha $90 \mathrm{jährige} B$ citänbe.

90 j. (Endwerth Der Bodentruttorente $7,5 \times \frac{1,03^{9 \prime \prime}-1}{0,03}=3325,12$ 90 j. Nadjwerth Der Sulturfojten . . $30 \times 1,03^{90}=429,01$ Summe 3754,13

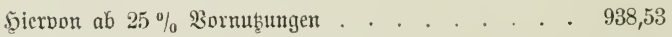
Iojtennerth Des 1 ha gropien Bejtandes . . . . . . 2815,60 $4,25 \times 2815,60=11966 \mathrm{fl}$.

3. 1 b und $2 \mathrm{a}, 5,00$ ha 75 jährige Bejtände.

75j. Enowerth Der Bodentbruttorente $7,5 \times \frac{1,03^{75}-1}{0,03}=2044,73$

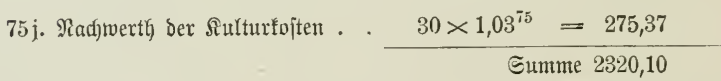

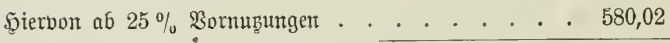
Sioftenwerth des 1 ha grop̃en Bẹtandes . . . . . . 1740,08 $5 \times 1740,08=8700 \mathrm{fl}$.

4. $4 \mathrm{c}, 7,50$ ha 50 jähriger $\mathfrak{B}$ ejtand.

50 j. Cnnbiverth der Bodenbruttorente $7,5 \times \frac{1,03^{50}-1}{0,03}=845,98$ 50j. Radjwertf Der Siulturfoíten . . $30 \times 1,03^{50}=131,52$ Summe 977,50

Şierbon ab $15 \%$ Borerträge . . . . . . . . . . 146,62

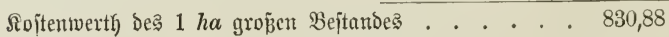


5. $2 \mathrm{~b}, 3 \mathrm{a}, 5 \mathrm{~b}$ tmo $6 \mathrm{a}, 24,05$ ha 40 jährigc $\mathfrak{B} \mathrm{c}$ tändc. 40j. Endowerth Der 3odenbruttorente $7,5 \times \frac{1,03^{40}-1}{0,03}=565,51$ 40j. Radjwerth Der fiulturtoften . . $30 \times 1,03^{40}=97,86$ Summe 663,37 S̨ierbon ab $10 \%$ :ormtsungen . . . . . . . . . 66,34

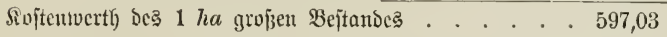
$24,05 \times 597,03=14359 \mathrm{fl}$.

6. 40, 1 ha 30 jälriger Beftand.

30 j. Endwerth der Bodenbruttorente $7,5 \times \frac{1,03^{30}-1}{0,03}=356,81$ 30j. Radywertf Der Sulturfojten . . $30 \times 1,03^{30}=72,82$ Summe 429,63 Siervon ab $5 \%$ :ornubungen . . . . . . . . . 21,48 Siopitentwertf Des 1 ha grof̧en Bej̆tandes . . . . . . 408,15 $1 \times 408,15=408 \mathrm{fl}$.

7. 5e, 11,20 ha 25jähriger Ẇełtano.

25 j. Enowerth ser Bobenbruttorente $7,5 \times \frac{1,03^{25}-1}{0,03}=273,44$ 25j. Rad)wertf Der Sulturfoiten . . $30 \times 1,03^{25}=62, \mathrm{S1}$ Summe 336,25

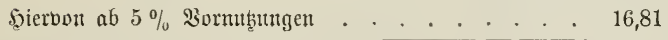
Sivitenwerth des 1 ha grop̧en Bejtandes . . . . . . 319,44 $11,2 \times 319,44=3578 \mathrm{fl}$.

8. 1e, 2e, 4 b und $5 \mathrm{a}, 7,80$ ha 20 jährigc Bej̆ände.

20j. Endwerth Der Bodenbruttorente $7,5 \times \frac{1,03^{20}-1}{0,03}=201,53$ 20j. Rad)werth Der Siulturfoiten . . $30 \times 1,03^{20}=54,18$ Summe 255,71 Điervon ab $5 \%$ :ormb̨ungen . . . . . . . . . 12,78 Ropitenwerth des 1 ha grojenen Bcjtandes . . . . . 242,93 $7,8 \times 242,93=1895 \mathrm{fl}$. 
9. $3 \mathrm{~b}, 11,75$ ha 15 jähriger Beftand.

15j. Enowerth Ser $\mathfrak{B o b e n f o r u t t o r e n t e} 7,5 \times \frac{1,03^{15}-1}{0,03}=139,49$

15 j. Radjwerth Der Siulturtojten . . $30 \times 1,03^{15}=46,74$

Siojtenwerth des 1 ha groṕen Bejtantę . . . . . . . 186,23

$$
11,75 \times 186,23=2188 \mathrm{fl} \text {. }
$$

10. $1 \mathrm{a}, \mathrm{c}, 5 \mathrm{~d}$ uno $6 \mathrm{~b}, 20,50$ ha 5 jährige Beftände.

5j. Endwerth Der Bodenbruttorente $7,5 \times \frac{1,03^{5}-1}{0,03}=39,82$

5j. Radiwerth Der Rulturtojten . . $30 \times 1,03^{5}=34,78$

תoftenwerth des 1 ha groṕen Beítandes . . . . . . 74,60

$$
20,5 \times 74,6=1529 \mathrm{fl} \text {. }
$$

Summe des f̧olzoorrathąapitales überhaupt:

3,50 ha 100 jährige Beịtände: $13469 \mathrm{fl}$.

4,25 " 90 " 11966 "

$5,00 " 75 "$ " $" 8700$ "

7,50 " 50 " " 6232 "

24,05 " 40 " 14359 "

1,00 " 30 " 408 "

11,20 " $25 \quad 3578$ "

7,80 " 20 " " 1895 "

$11,75,15$ " " 2188 "

20,50 " 5 " " 1529 ,

5,95 " Blö̈̈еn -

102,50 ha 5ुolzboden mit $64324 \mathrm{fl}$. Borrathätapital.

Şierz̆ Bodentapital 103,60 ha $\times 150 \mathrm{fl}$.= 15540 "

Summe des $\mathfrak{B a l d f a p i t a l e s ~ . ~ . ~ . ~ . ~} 79864 \mathrm{fl}$. 


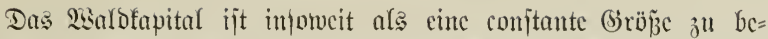

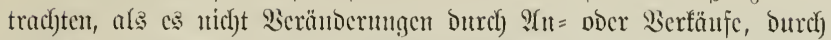

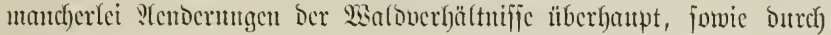

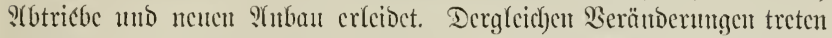

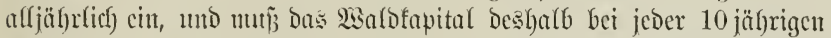
gicvifitum neu crmtittelt meròn. Mian bebält ben cinmal angenoumenen

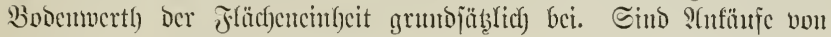

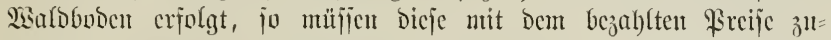
gerect)uct meroen; Gaben Berfäufe jtattgejunden, jo ijt mit ben be=

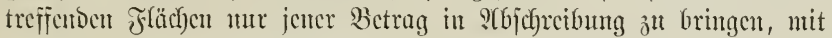

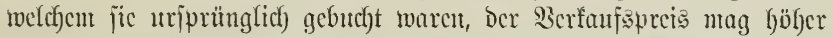
ober nicbriger Yanten. Die abgetriebenen B̉ejtäutoe meroen mit bem

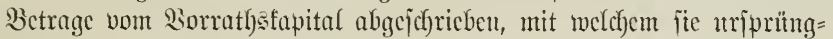

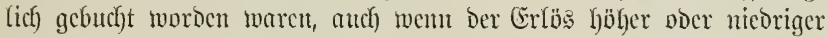

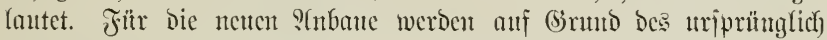

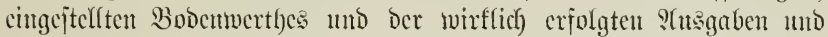
(Eimrafgnien Dic Siojtemberthe crmittelt. Der verblicbene alte Sorrath)

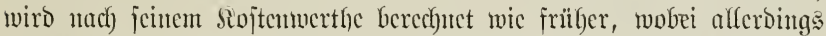

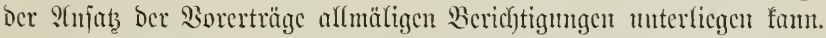

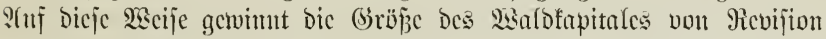

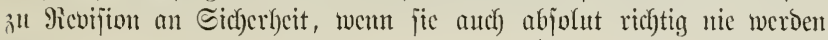
tumn. 
F.

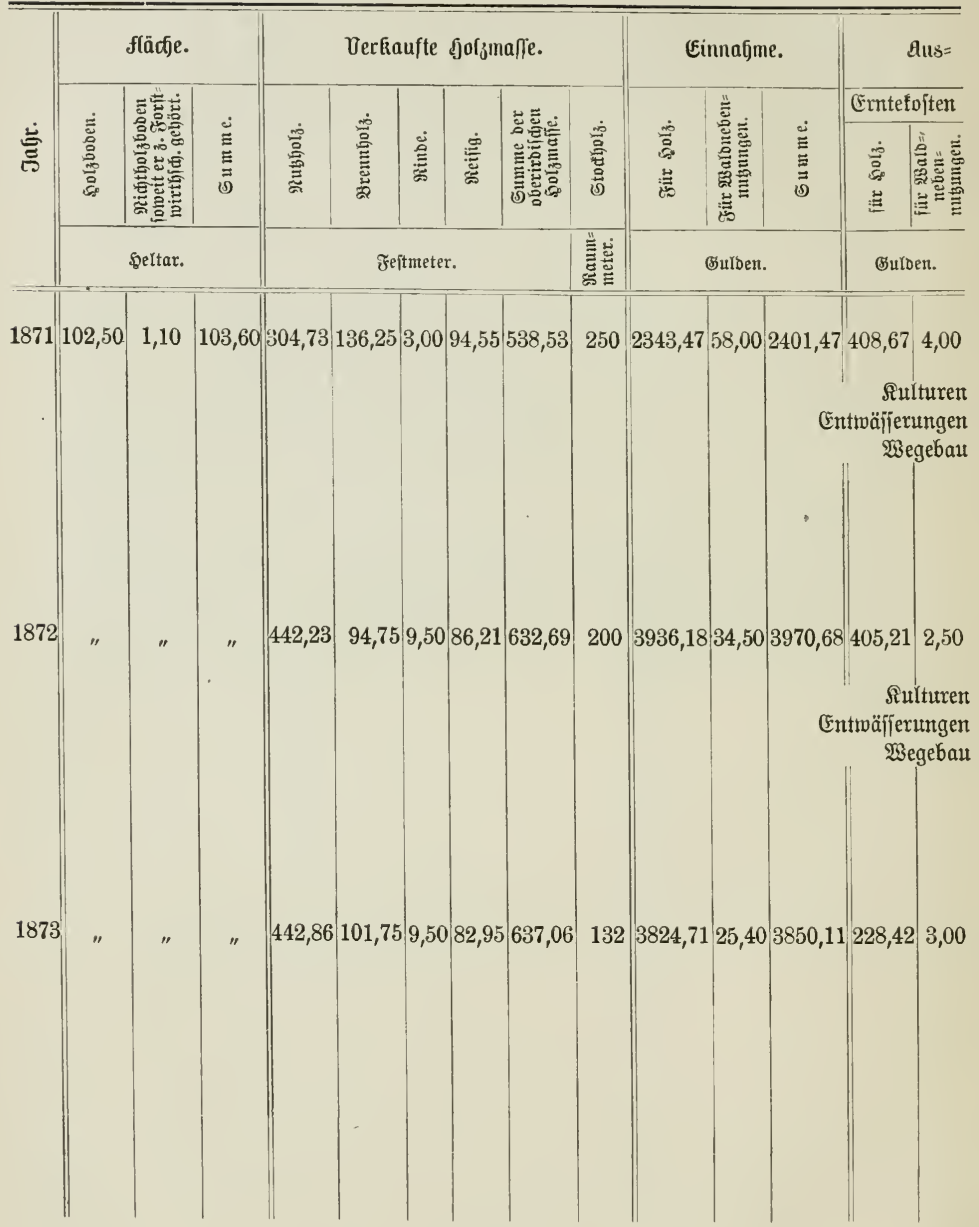

u. $\{$. w. 


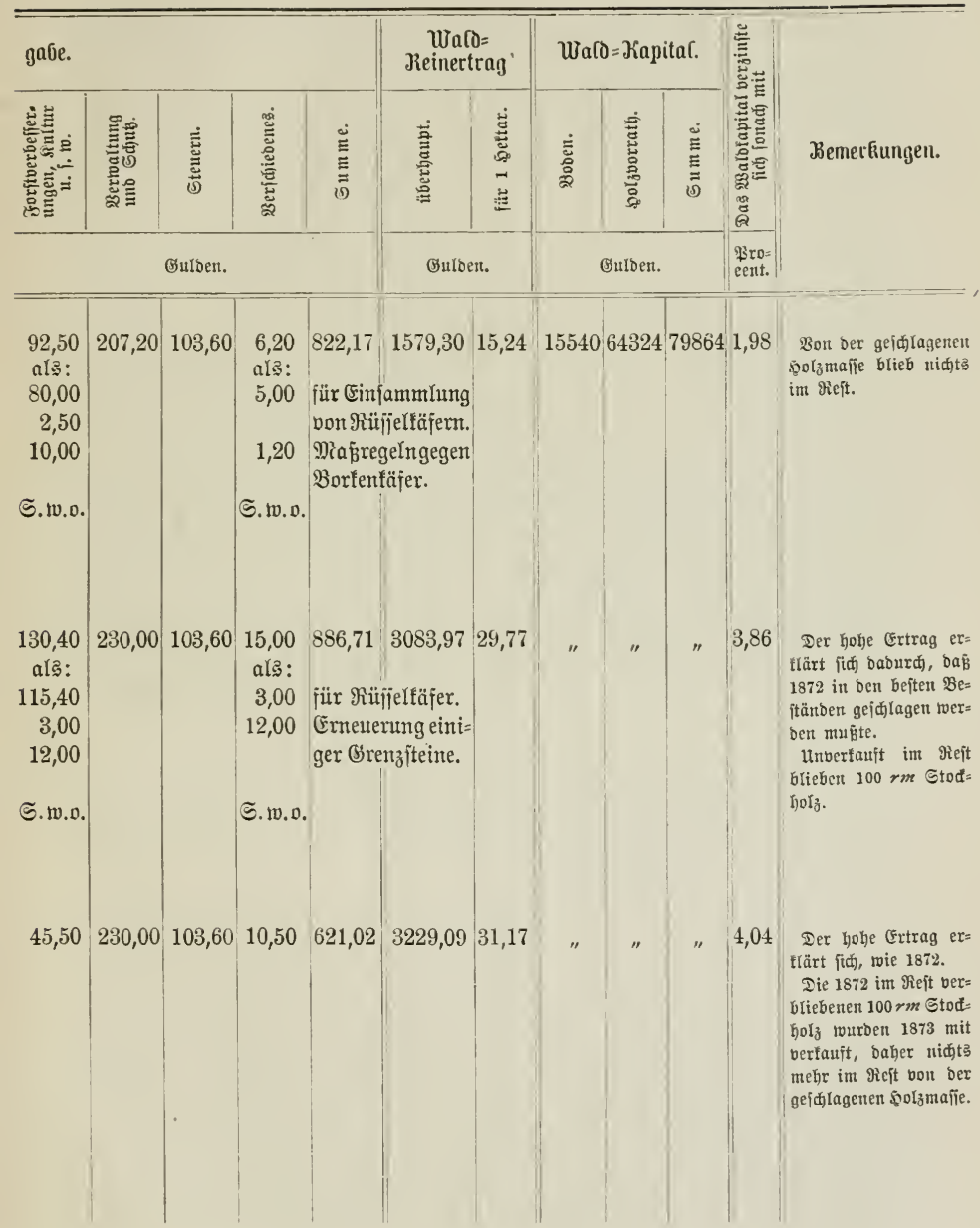

4. โ. แ1. 
S(m richtigiten twäre es natïrliç), bas 2 sarbfapital alljährfich) nen 3t bejtimmen. (Es ijt bies aber beshalb nidyt möglich, weil mur alle

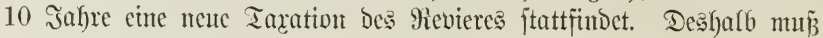

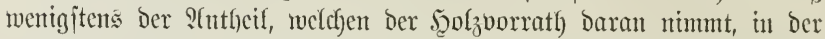

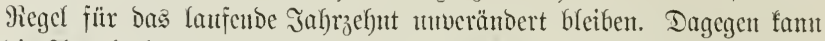

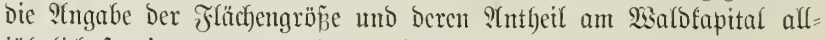

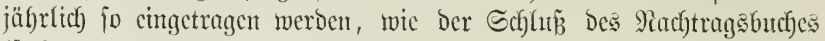

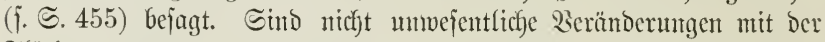
Fläche vorgegangen, jo verliert bie an jich ganz fichere SHgabe Der

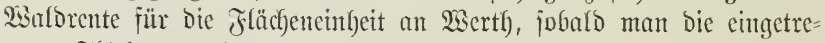
tenen Flächenverüntocrungen unberücfjüchtigt läp̃t.

Ganz gewié ijt es, trob Der Ilnjicherfeit der Mnterlagen, won Bebentung, went mir von euter Betriebstaffe, von cintem ßeviere jagen fömen: Inter S(muabme eines gewifjen Bobenwerthes, nuter

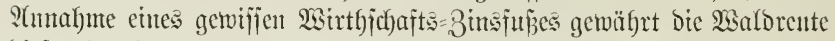

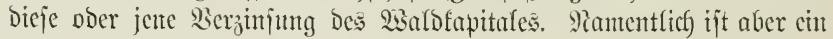

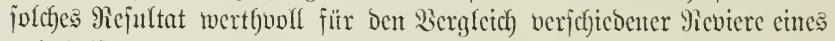
und beffelben grojen 2\$alogebietes, wenn bic Rechnung fït alfe nach)

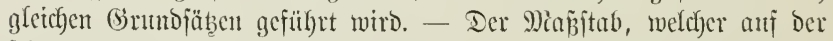

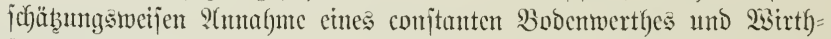

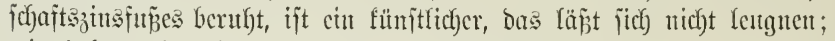
wir halten aber einen füntlifchen Majpjtab inmer noch für bejjer, als gar feincn.

3u bemerfen bleibt nod), daj̃ an Echlufie Des Beitraumes, für

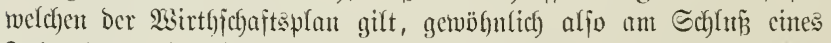
Jabrachntes, ber jummarifche Durchjefnitt ats Den Rejultaten affer eimzelnen Jahtre zul jêten ift.

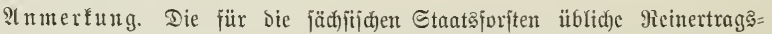
tabelle hat etroas anderen Juthalt. Statt Der von uns gegebenten "verfauften Solzmajie" enthält biejulbe bic "gefchlagene Derbholzmajie" und Den

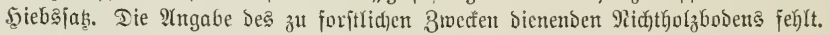

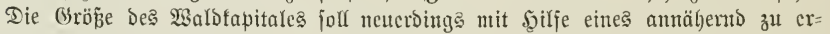

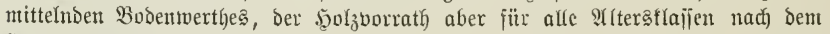

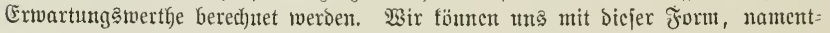
(ich) mit der Berect)unng der (Erwartungşwerthe, nicht cinverjtandent erflären. -

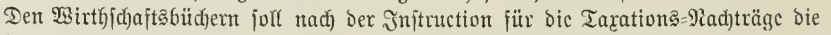

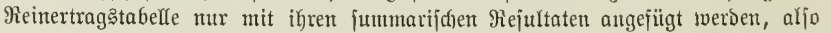

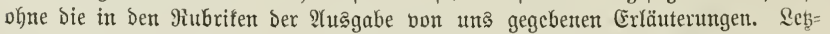
teré fint jebody in ber Sicinertragstabelle enthalten, welche zu Den S(cten Der gie= vicrverwaltung gehört. 
$\$ 151$.

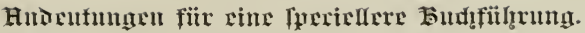

Sistr faben in ben Paragraphen 144 bis 150 cine Form Des

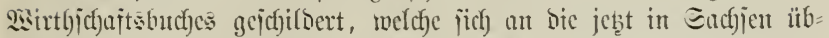

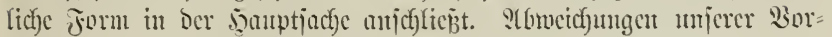
ichläge mutrocu anterfung șeije ermähnt.*)

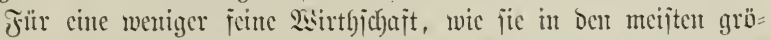

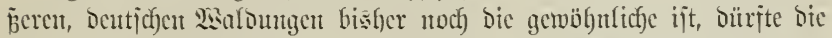
gegebente, jummarijebe Form Des ?achmeijes von Siojten und Erträgen

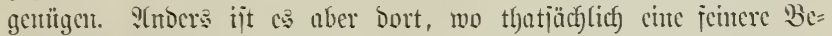

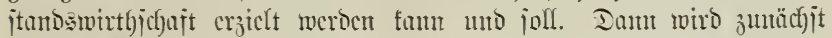

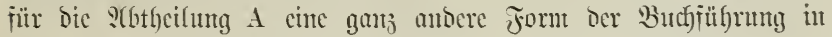
?(ntwentung getoract)t merben miijient.

Seocr cinjelute Bejtutto (linterabtbeiluntg) ijt als ein ntelyr ober

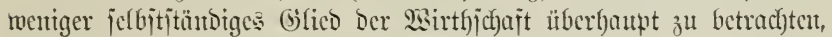
unD fint ifum Dafer jmei Eeiten bes Buthes ju wiomen, auf weldyen

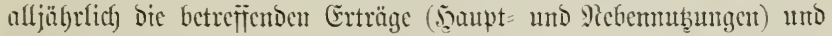
Siojten cinzutragen jün. Bon lebteren fömnen mur jene beim Jahres= eintrag im Einjelten mbeachtet bleiben, meldye ben ganjen 2isto

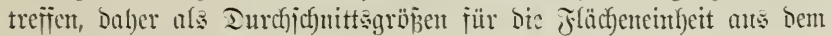
Gianzen berechnet merden.

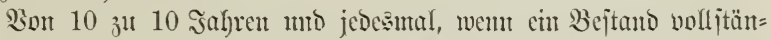

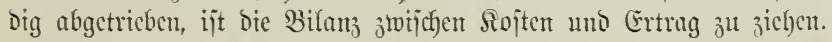

llebrigens ijt ber Machweis über bie entefojtenfreien Erträge Der

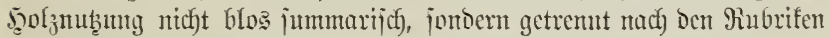

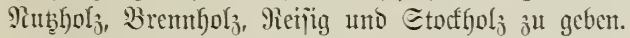

Mit şilfe einer berartigen Budff̈̈̈brung wären wir im Etanoc, cinjt vou jedem Bejtanto nachjumeijen, was er gefojtet uno mas er bereits getragen habe. Daj cin joldher ?adymet: jür ente mirflich) feine :

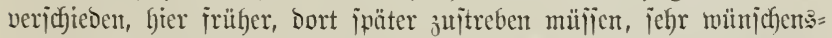

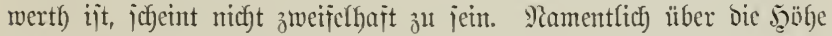

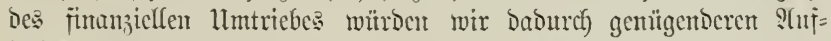

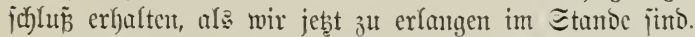

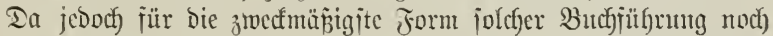
ber praftijuc Berjuch fehlt, uns ijt menigitens noch feiner befunnt

*) Die im Jahre 1873 erlajiene Injtruction gilt bezüglid) ber form Des wirthjdaftabudes nid)t mehr. 


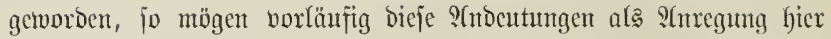
genügen.

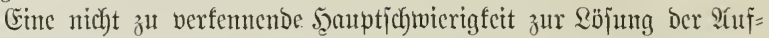
gabe licgt in Der Beränderlichfeit Der Flächengröß̄̋ cinzelner Bejtände.

Dicje Edywierigfeit jollte aber twenigitens nicht Davon abhalten,

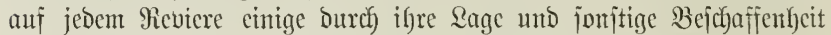
Dazu bejonders geignete Bejtände, namentlich joldse Dex III. und IV.

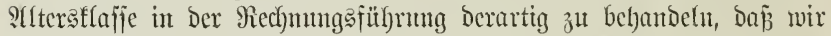
von Revijion zat Revifion allmälig fichereres stubalten über Den

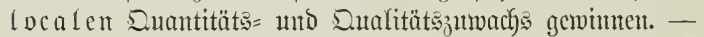

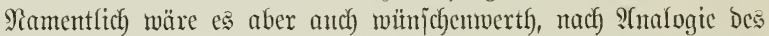

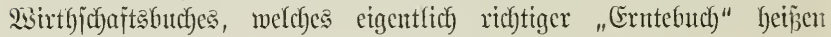

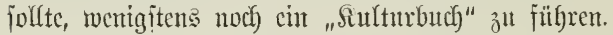

\section{Abrifnniff.}

\section{Dic Mevifinnen.}

\section{\$ 152.}

\section{Allgenteinex.}

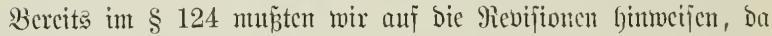
jich Das für bie Ertragsregelung in ben fünigl. fächj. Etaatsiforitent üb=

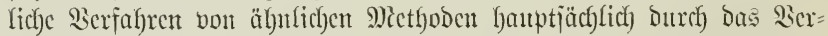

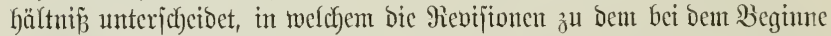
Der Einridbtung aufgejtelften \$3lane jteben. (5) jei unter Sginmeijung

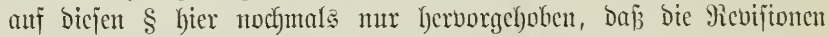

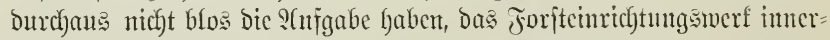

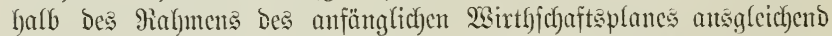
uno berichtigend jicher zu jtellen, jondocrn Dẩ jie periobijche Fortjesungent Der Forjtcintidytung, namentlicl) ber Ertragsregelung finb, bie zwar

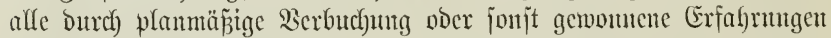

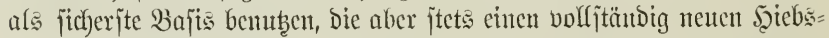
jab zu ermittcln haben. Das cinzige, wemt auch nicht unabänderfich)

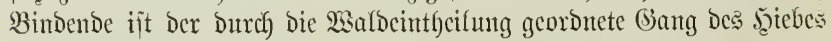
im Aflgenteinen. Sn Dicjem Sinne faffen wir menigitens bie fogenantent J̨auptrevifitonen auf, weldye in einem georoneten Forjthatshalte minde= jtens alfe 10 sabre wiebcrfebren müfien. Egarafterijtifd ijt für bicje

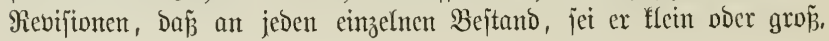


jutg ober alt, bie wirtbjchaftliclye Frage zu richten ijt, was mit ihm im nächjten Sabrachnte zu gejdyehen habe. Es ijt mit einem Worte ein

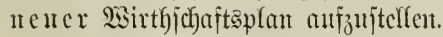

Se weniger fich biejer ber Form nach von bem bejprodjenen, alङ

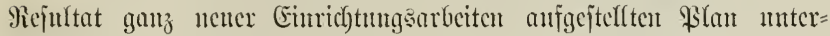
fcheibet, um jo fürzer verniögen wir mer bezugnabme auf bercits

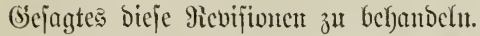

Den alle 10 Jabre wicderfebrenton Scauptrebifionten treten in Sachjen noch Die jogenannten fünfjälgrigen oder Bwijchentevifionen zur Ecite. Dicje loben fich allerbings mur immerfalb ber Brenzen cines

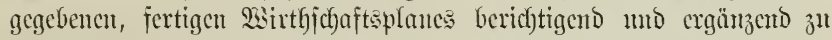
bemegen. Bei cinem in Der Jacuptjache ungejtört verfanfenten Betrieb

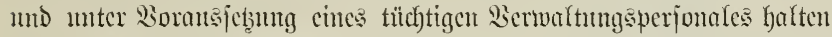

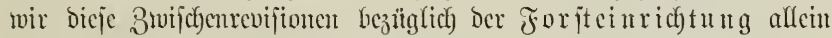
nticht, wie bic 10 jälgrigen Revijionen, für unbedingt notbrwendig, wer=

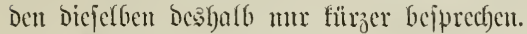

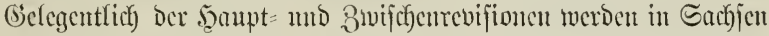

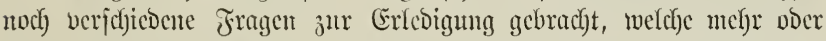
mentger in Das Gicbiet der Forjtuerwaltung in engeren Sinne dę

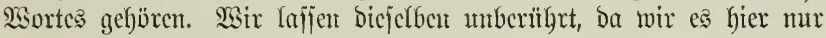
mit ber Forjtcinrichtung zu thun haben. Bejonders die fünfjährigen ober 3wijhyenrevifionen tragen Den Egarafter bon Betricbarevifionten,

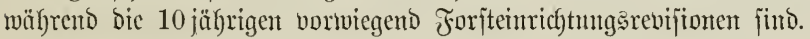

A. Die zelujägrigen oder 5 auptrevifionen.

\section{\$ 153.}

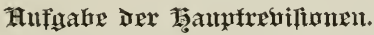

Dic SUfgabc Der Scantrevifionen, fomeit bicjelbc bas Forftein=

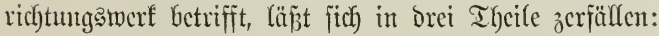

1) Unterfuchungen Darüber, wie bie Bejtimmungen bes abgelaufenen

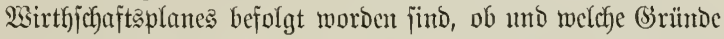
etwaige $\mathfrak{A}(b$ weichungen von biejen Beftimmungen rechtfertigen.

2) Unterfuchungen barüber, wic jich bie Bejtimmungen bes ß̊lanc: bemährt haben.

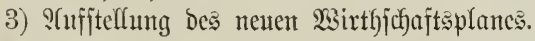

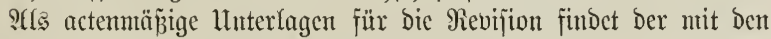
betreffentoen Béchäften betraute Beamte vor: 
a) Den vor 10 sabren aufgejtellten Wirthjchaftsplan mit Shtuabe Des Erfolges Der Şauungen und Sulturen ( $\$ \$ 135$ bis 139).

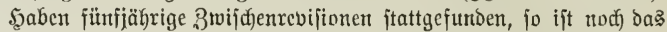

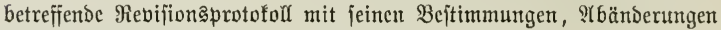

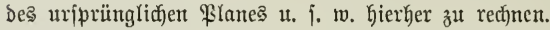

b) Die Durch jährlich exfolgte Bermefijungsnad)träge fortwährend bericftigten und ergänzten Epecialfarten, Dazu im Nachtrags = buckbe bic S(ngaben ïber alle ftattgehahten Flächenverände= rungen (\$§ 141 bis 143).

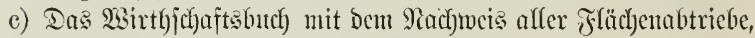
jorvic Der Material = und Belderträgc.

d) Die von Der Fevierverwaltung gefülyrten Forjtrechnungen über Fällungabetrieb unס "Forjtuerbejierungen" (Sulturen, Entwäjje= rungen, $\mathfrak{B}$ egebau).

Unter lumjtändon famt aus biejen actenmäß̈̈igen Unterlagen jelbjt

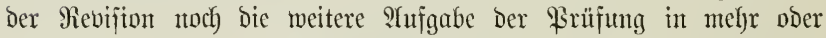
meniger antsgebchnter Weije erwachjen. (Es mag bies jeboch hier mn= berüsffitctigt bleiben.

1. Unteriudugen barüber, wic bic beitimmungen bes abgelaufenen Planes befolgt wurben, ob unb wel de (bründe etwaige $\{$ b weidungen re山tfertigen.

\section{$\S 154$.}

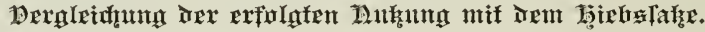

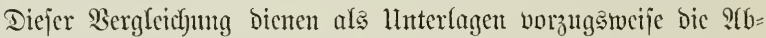

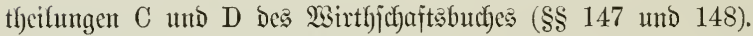

Man fann fitch fierbei begnügen, wic ç bis jebt in Sachjen ge=

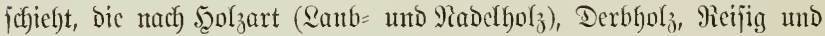

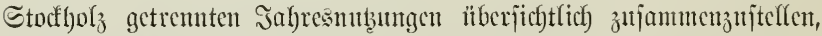

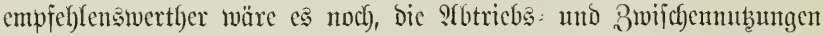

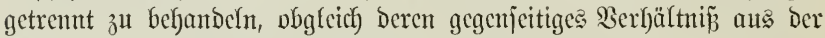
2(btheilung C Des Winthjochaftsbuches leicht zu erjeben ijt.

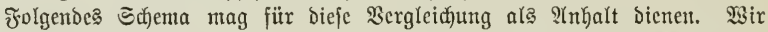

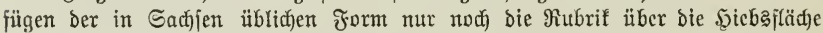

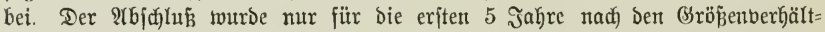
nifjen unjeres Beifpieles fïr bie Ertragäregelung gegeben. Diefes Schema ent=

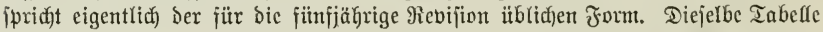

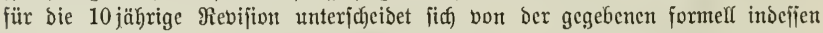

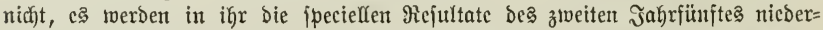

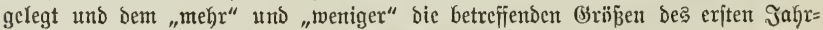

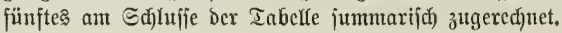




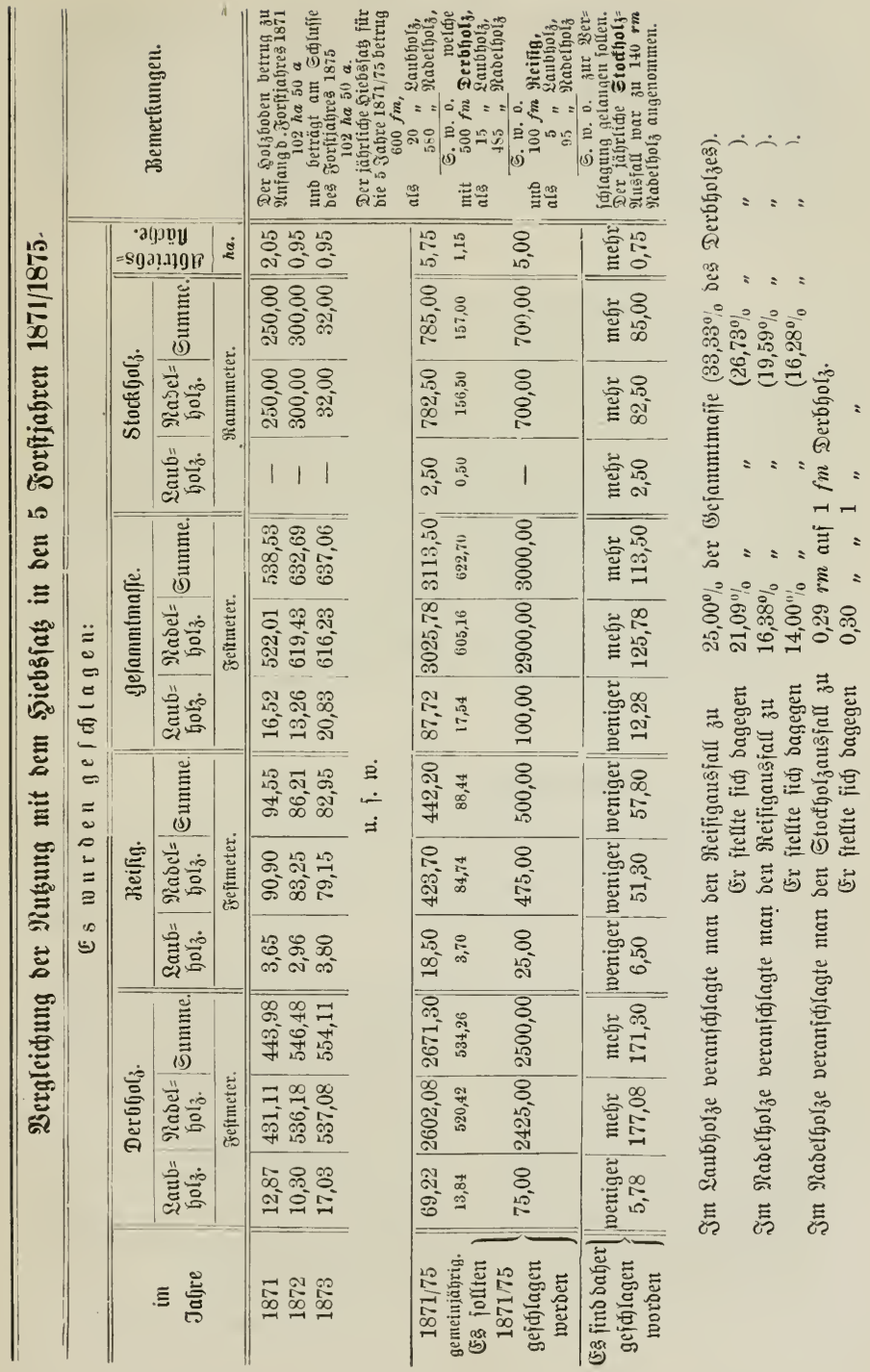




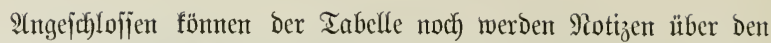
Betrag Des शabyolzes oder über andere nadh Den vorliegentben Rocal= verfältnifien bejonders wichtige Sortimente, z. B. Rinde im Ëicjen=

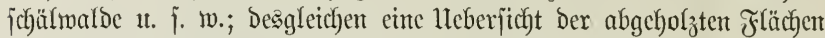
nach) Den Bejtandsbonttäten, went man $\mathfrak{e}$ nicht vorziegt, wie es jeb̨t in Sachjen gejujebt, bieje lleberjicht in einter bejonderen Tabelle zu= jammenzujtellen.

\section{$\S 155$.}

\section{3urammenlfeflumg จex plamwioxigen Taumngen.}

Planmibrige Scaungen ober fogenante "Borfaumgen"

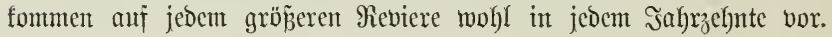
Sie werocn berurjacht burch Naturereignifje, nicht vorautsejebenen

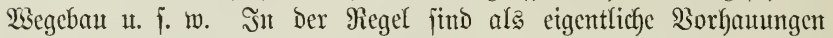
nur bie Flächenabtriebe zu betrachten, von ben 3rifchenmub̨ungen nux jene, meldye als zufällige Erträge entjabieden in bic Siategorie ber S(btriebsnuţung Deshalb gehören (j. ๔. 410), weil fie die Sciebs= bedürfitigfeit einzelner $\mathfrak{B}$ cjtände bedingen.

Die Borfaumnen find überjichtlich georonet zufammenzujtellen, und ijt bei jeber ber Brrund ber Beranlafjung anzugeben.

Ëines bejonderen Tabellenfhemas bebarf cs hierzu nicht.

\section{$\S 156$.

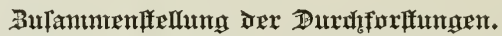

Se widftiger ber Durchforjtungsbetrieb als Maj̃regel ber Ernte

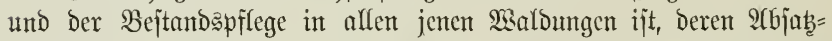
verbältniffie benjelben in entiprechender Weije gejtatten, Dejto noth=

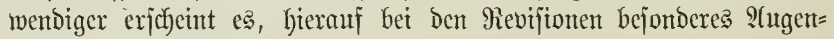
merf zu ridjten.

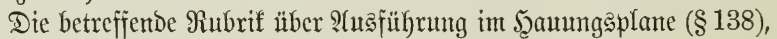

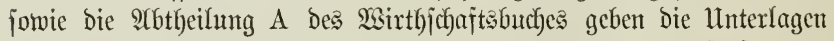
für eine 3ujammenjtellung ber wirffich exfolgten Durcfjorjtungen. Dieje fam nad) Dem anf Seite 481 gegebenen Echema erfolgen.

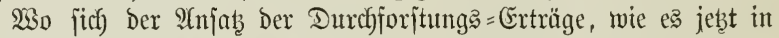
Sachfen geichiebt, zum Theil mit auf bie Tremung ber zu burch=

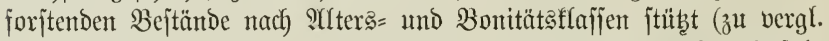

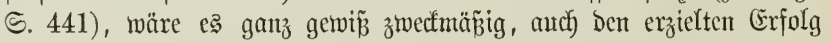

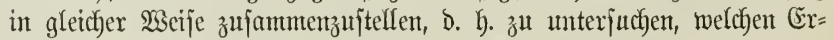
trag jede 2flters= ober Bonitätsffaffe wirflich gegeben bat. 
Revifionen. § 156 .

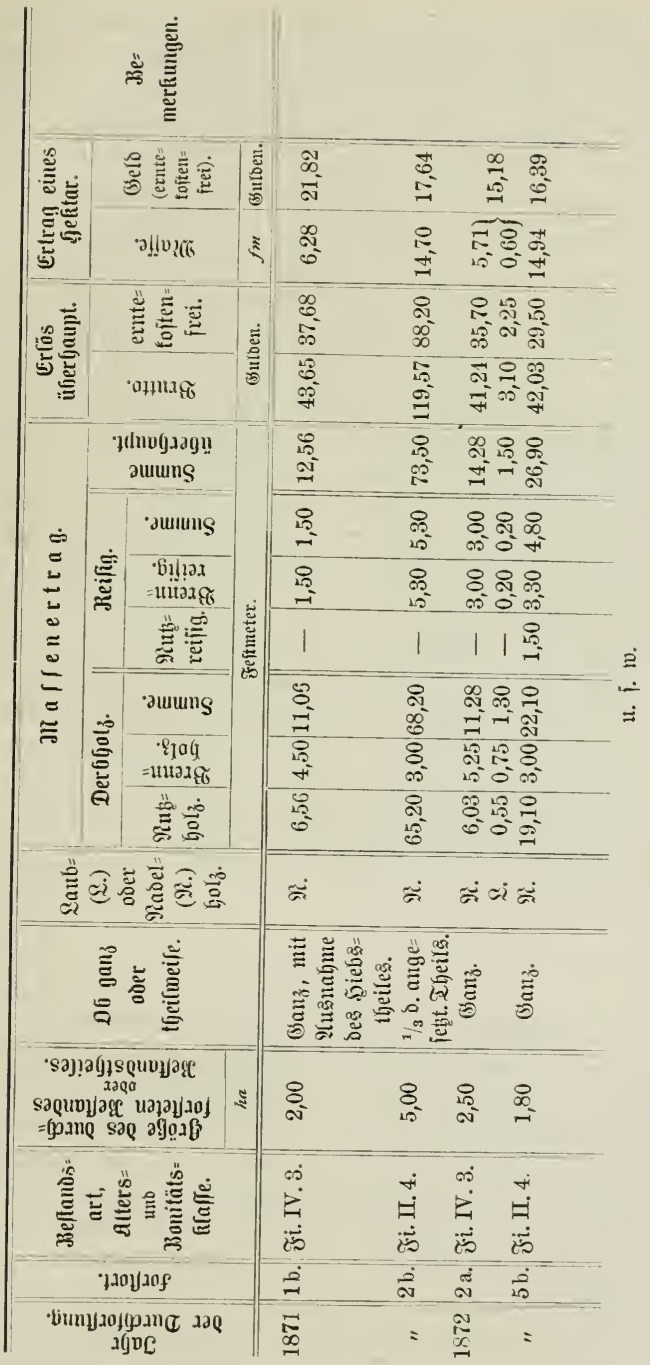


Die Miubrif "Db ganz ober theilmeije" ijt namentlich bann 4 = entbchrlich, wenn die Durchforjtung eines Bejtandes während meh)=

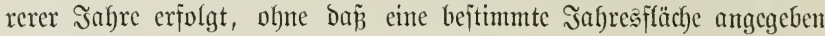
merden fann, weil die Mápregel in jedent einzelnen Jafhe den ganzen Bejtand trifft. Sn foldbem Falle märe bic Fläche nut beim eriten Jahre eimzutragen, und zwar nach ifrem ganjen Betrag, in ber ge= namten Subrif mürde "theifmeije" zu bemerfen jein. Dic ipäteren Durdfforjutungen in Demjelben Bejtande fund nur Ergänzıngen Der

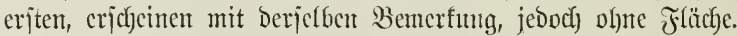

S(m Ech)lufje Der Tabelle ijt bie Summe für Fläche und Ertrag z"l zieben und ou ermitteln, welcher Maaterial = und Beldertrag im

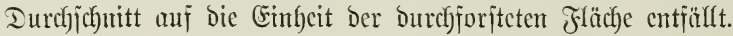

Endich) ijt ein bergleich) Der zutr Durchforjtung antgejebzten mit Der wirffich Durchjorjeten Fläche ju gebent. Bei wejentlichen Diffe=

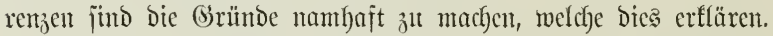

Fine weiter gebende Feintheit jür hochjtebende Wirthjchajt fönnte

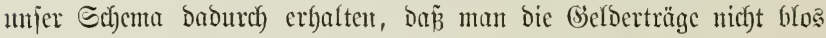
jummarij(f), jondern getremt nach ben einzelnen Sortimenten eintrïgt.

Die bei jeder 10 jäbrigen Revifiton unentbehrlichent, tą̧atorijchen

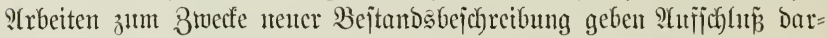
über, ob im Einne rationeller Bejtandsprfege bie Duredjforjtungen ent=

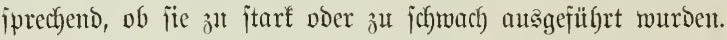

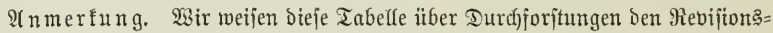

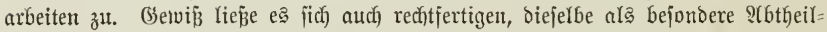

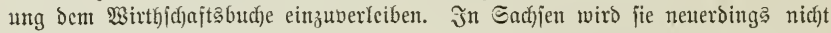
mehr, dafür aber eine Şaupttabelle fertigt (i. ธ. 483).

\section{$\S 157$.}

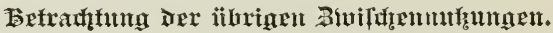

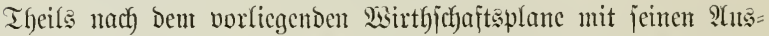

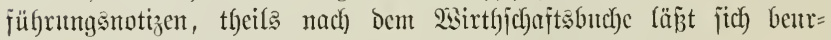
theilen, ob bie Bejtummungen ïber Zäuterungshiebe, Räumung von Waldrechtern und ocrgleichen bejolgt wutroen, bejichungsiveije befolgt werben fomten, ferner: welchen Betrug bie anfülligen Erträgc cr= reichten. Die neue Taration Des 9icvieres giebt Sarjichluti barüber,

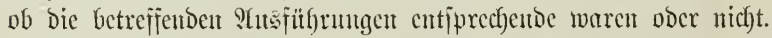

In Gadjen legt man jeit 1878 cinte "3wijchenuแbug tabelle" in nachjtebenoce Form ben Ricuijionsarbeiten bei: 
Meviïonten. § 157 .

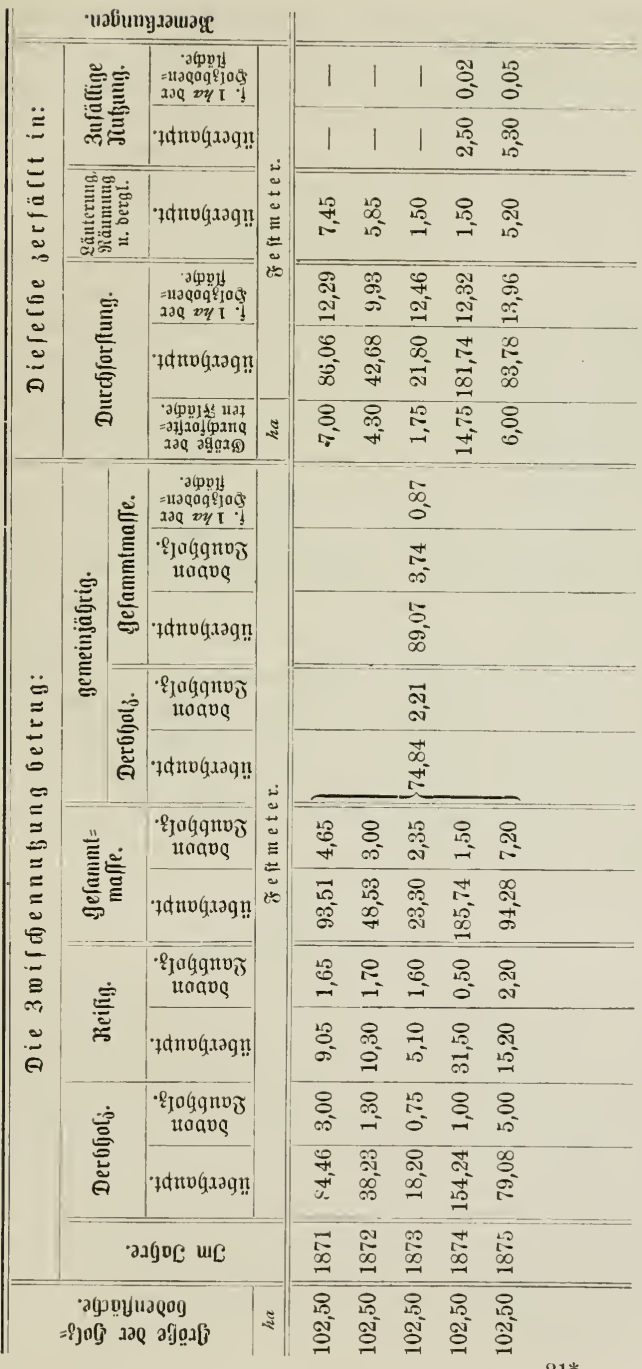




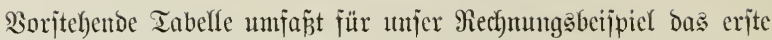

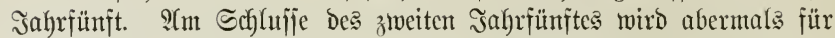

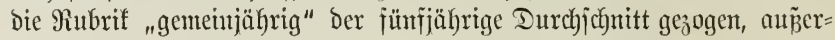
Dem aber bie Summe für bas ganze Jahrzebnt und ber hieraus be=

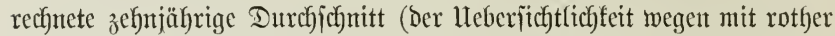
Tinte) zugejchrieben. Dicie Bwijchenutbutugstabelfe ijt baher eite noth=

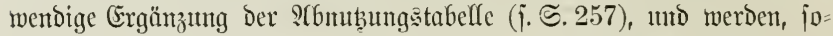

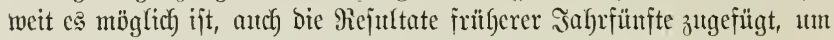

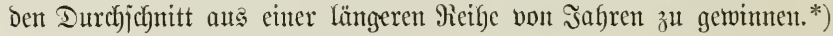

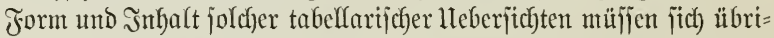

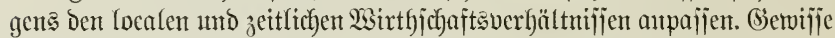

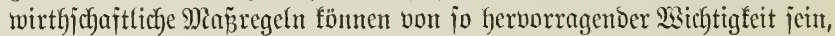

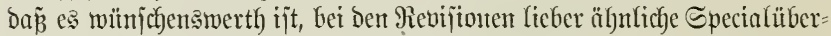
jichten aufzujtellen, wie wir jie jür bic Durchforjtungen empfoffen baben.

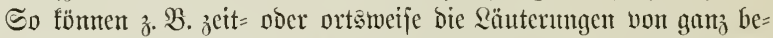

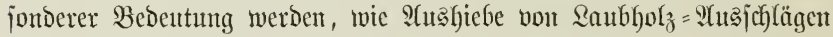

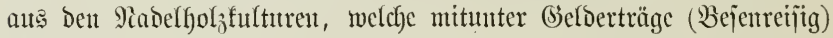
gerwähren, mitunter aber mur Sojten verurjadyen. EÉgenjo fömten bie

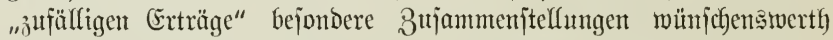

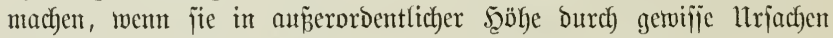

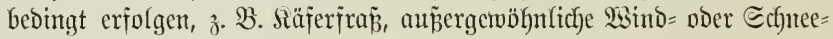

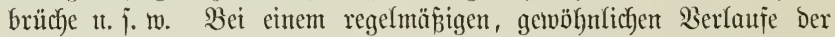

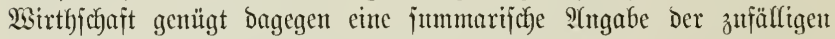
Ěrträge, um für ben neuen Bifan SYGthaltspunfte über ben betrag

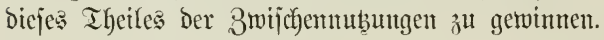

\section{$\$ 158$.}

\section{Die Delonuthunger.}

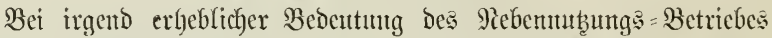

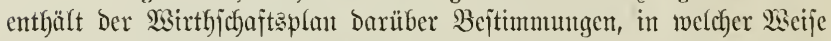

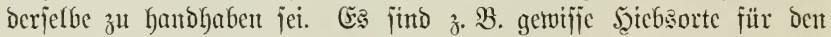

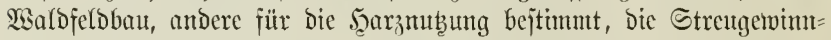

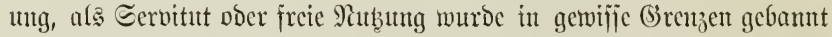

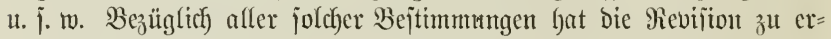

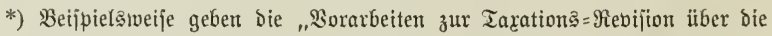

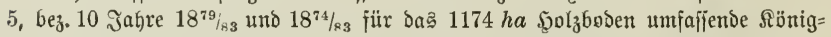

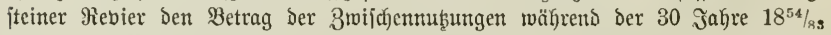
mit $33085 \mathrm{fm}$, gemeinjäbrig jontad) mit $1103 \mathrm{fm}$ ober für $1 \mathrm{ha}$ ber $5 \mathfrak{g l l}_{3}$ bodent= fläche mit $0,94 \mathrm{fm}$ ant. 
mitteln, ob jie eingebalten murben ober nidgt, Ieţteren Falles ju unter= judfen, medtye llrjad)en etmaige ?f(fweidfungen bebingten.

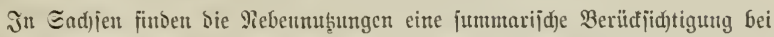

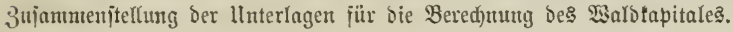

\section{$\$ 159$.}

\section{Forltuerbellerungen.}

a) Kultturbetrie6.

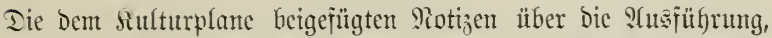
jowic bie weiteren von Der Bermaltung ju füfrenden Sulturrechmungen geben bie lluterlagen un bic j̧and, um zu ermittelı, ob bie planmǟ̄ig angejebste Fैäche mirflich fultivirt morden ijt, ob bic fulturen amper

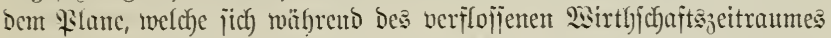

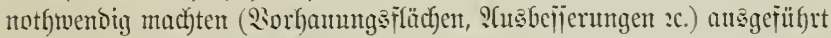

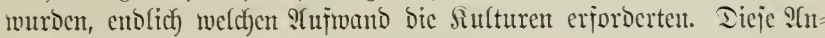
guben f̈nd überjithtlid) georonet jujammenjujtellen, babei vorfommen=

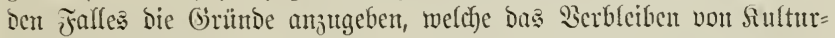
reiten veranlä̃ten.

lleber Die Dualität Der ausgeführten Sulturen fant crịt bic neue

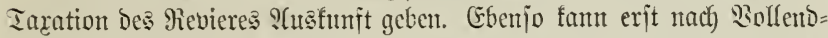
ung biejer 9 (rbeit beredynet merben, was ein Ђeftar in Bejtand ju bringen

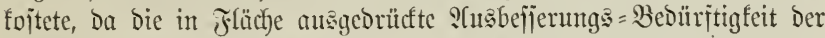

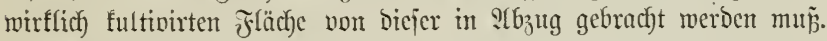

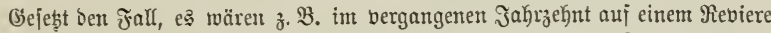
200 ha fultibirt, Dafür $5500 \mathrm{fl}$ ausigegeben morden; bei ber neuen Taration itellte

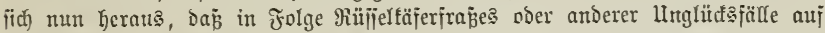

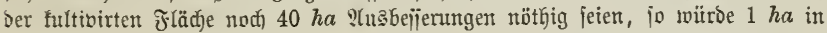
Bejtano zu Gringen nidht $\frac{5500}{200}=27,5 f l$ fojten, jondern $\frac{5500}{200-40}=34,38 f l . *$ )

*) Es ijt uns wiederbolt begegnet, in mandhen Foritwirthjđajten jobeinbar jehr billige Rulturen zu f̈nben, weil man faljd) rednet. Nidjt nur wirb bie hier gegebene

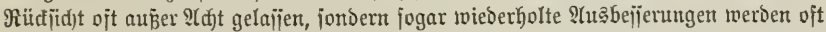

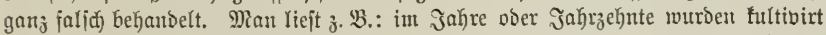

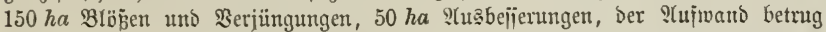
$5000 \mathrm{fl}$, folglich fojtet $1 \mathrm{ha} 25 \mathrm{fl}$. Tas ijt aber grumbjaljich, jobalo unter ben $50 \mathrm{ha}$

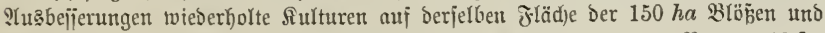

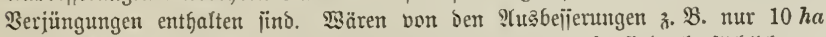
älteren Silturen angebörig, 4n ha aber \$sieberbolungen, jo jino thatïäditid mur 160 ha lultibirt woroen, uno 1 ha fojtet nidft $25 \mathrm{fl}$, fonbert $\frac{5000}{160}=31,25 \mathrm{fl}$. 


\section{b) Entmälferungen.}

Entweder wurben itber bie notfyentigen Entwäjjerumgen nur $\Re_{0}=$ tijen bent ipeciellen Sulturplante beigefügt, ober es murbe, twie wir Geite 448 andeuteten, ein bejonderer Entwuäjierungsplan aufgeitellt. Sn beiben Fällen hat bie Revifion die Frage $3^{\text {nt }}$ erlebigen, was in

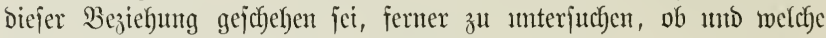
(S)rïnde etwaige s(bocidyungen vom Blane rechtfertigen.

c) Weṇefíau.

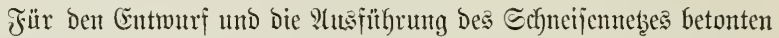
wir (§ 111) bie Roth)wendigfeit eines vorantsgel)enden, allgemeinen 2Begebauplanes. Der Revifion fällt auch ljer bie Sufgabe zu, zu unterfuchen, ob ber SSegebau bic nöthige $\mathfrak{B f f l e g e}$ und Beachtung im Simne bes gegebenten \$lantes, jomie im (Seijte einer rationdlen 2 sirth)= ichaft überbaupt fand.

2. Interjunugen barïber, wie fid bie Bejtimungen bes જlane bewägrt haben.

\section{$\S 160$.}

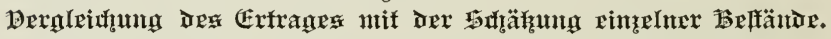

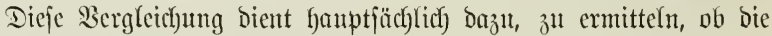

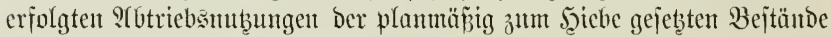

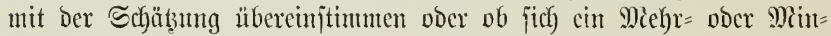
derertrag heransjtellt, meldyer Differenzen bes Sciebsfabes mit der Pub̨ung ütberbaupt erflärt.

(Sentuu tönnen allerding? mur bie Durchgejchlagenen Drte ver= glichen werden. Dic volljtäntoigen llnterlagen Gierzı giebt bic ?lftheilung B

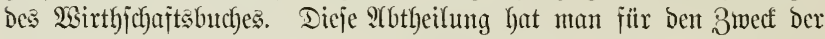
Revijton nur zu jummiren, um ein Durchjednittsrejultat zu geminten. Einer bejonderen Iabelle bebari es nicht.

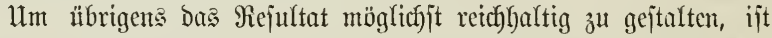

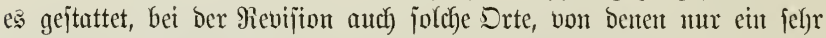

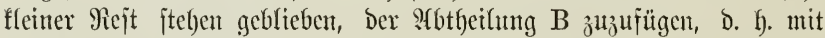

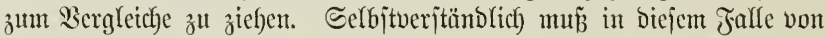
Dem gejefäbzten Ertrage cin Dem J̧iebsrejt entiprechender ?Antbeil in Stbjug gebrad)t werden. In Der Rubrif "Bemerfungen" Der genamten

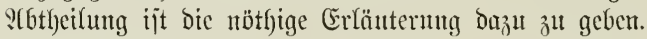

$\$ 161$.

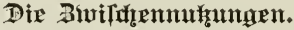

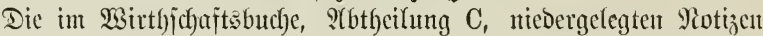
jomie bie it ben vorbergehenden, betreffenden \$aragraphen vorgefdyrie= 


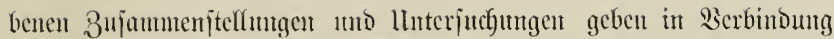
mit ber nenen Tagation bes Sicvieres Material gentrg an bie 5̧and,

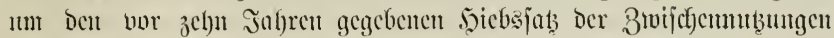

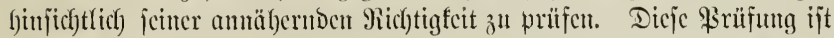

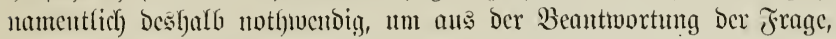
wie fick bie betreffenden bejtimmuntgen bes abgelanfenen Plance be=

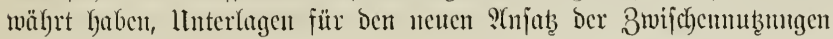
zat gewiunen.

\section{\$ 162.}

\section{Dix Thieb}

Bcfanntlid) legen wir anf Dic Serertelfıng cinex Den Rocalverbält=

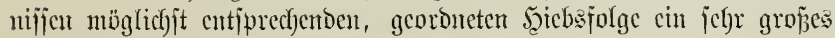

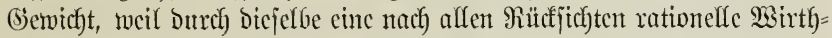

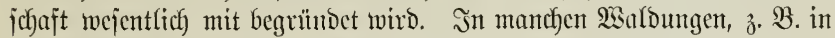

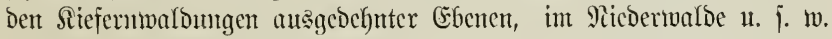
vermag wohl bic erjte Forjteinrichtunt immer bas giichtige zu treffen;

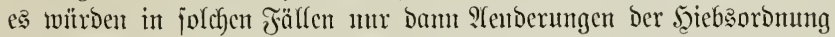

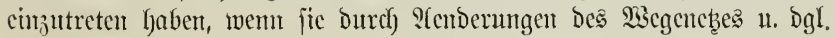

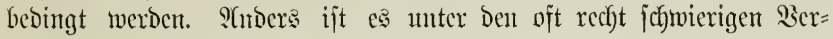

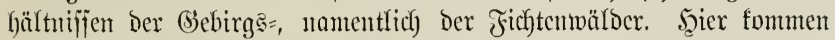

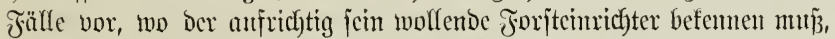

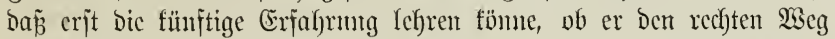

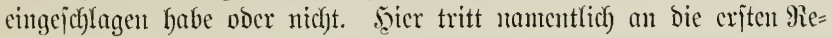

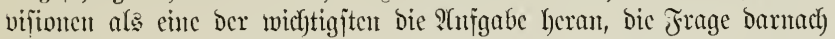

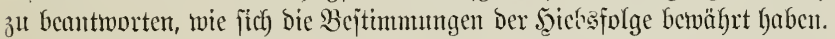

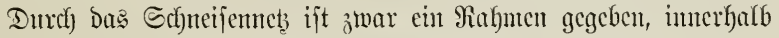

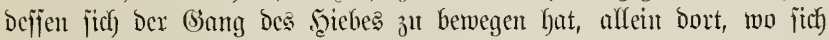
Die früferen Bscjtimmungen entichieden als irrthümlidbe fentzeichnen,

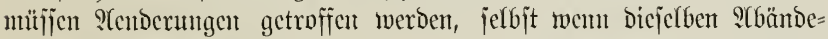
rungen cinzelner Theile bes Sifneijennebes unvermeidfich madyen jollten, waร itbrigen Durchaus nicht immer ber Fall ijt.

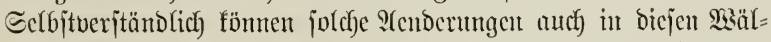

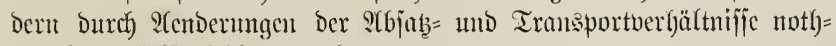
wentiger Weije bedingt werben.

\section{$\S 163$.}

\section{Dix Delommuthutuxn.}

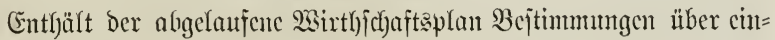

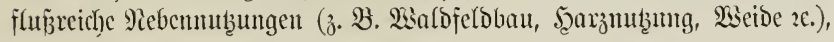




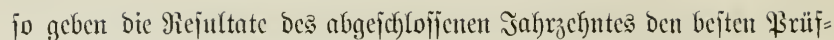
ftcin bafür, ob bicje Bejtimmungen ivirtbjchaftlich entiprectende waren

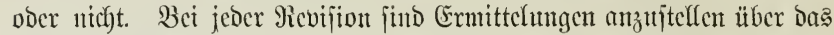

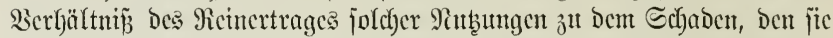

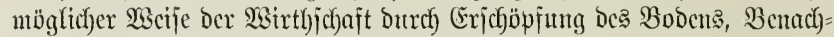

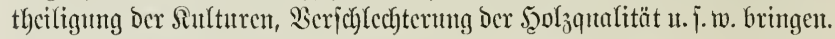

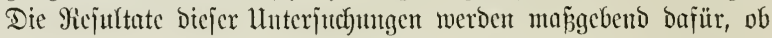

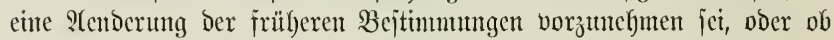
bicie in Siraft blciben follen.

\section{§ 164 .}

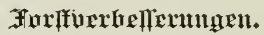

Sowneit Der 2 Sirtfjichajtsplan allgemeine und jpecielle Bejtimmungen über bic Forjtocrbejjerungen, aljo ̈̈ber Sulturbetricb, Entwäjjerungen

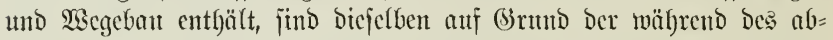
gefaufenen Wsirtfjichaftszeitraumes gewomenen Erfafrungen bejüglich)

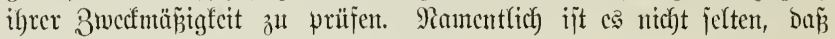

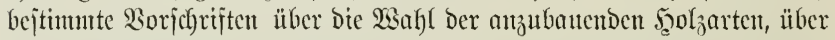

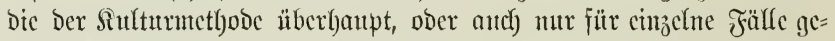
geben rutoden.

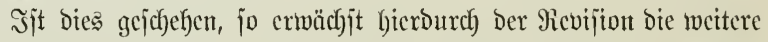

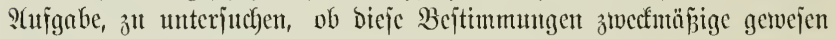

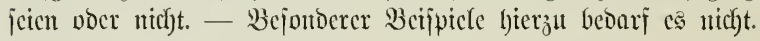

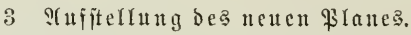

\section{$\S 165$.}

\section{Hagenxines.}

Dic Suffitellung bes nenen Wirtfjichaftsplance, fcincencys blos Dic Berichtigung ober Ergäntung Des alten, abgelanfencn, biloct Dic

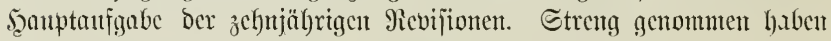
Die in ben $\$ \$ 154$ bis 164 vorgejchriebenen Interjuchungen und $31 \mathrm{t}=$

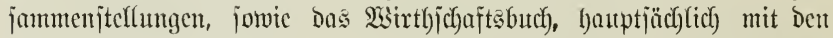

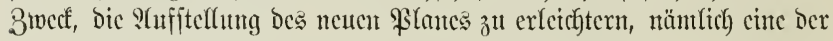

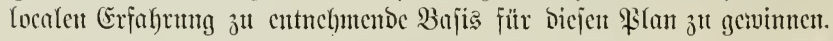

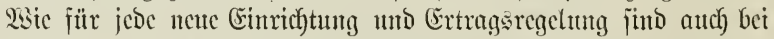

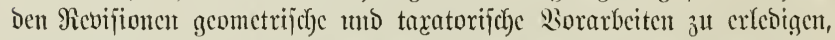
ehe zur Fejtitellung bes neten Flanes gejchritten werben faum. Bes züglich) Der erjteren ijt jedoch) ż bemerfen, Daj biejelben in Der Miegel 


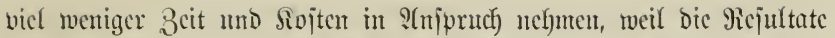

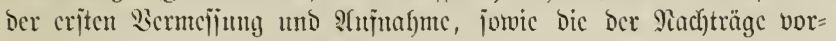

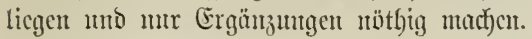

\section{$\$ 166$.}

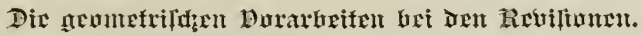

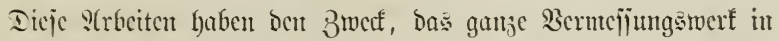

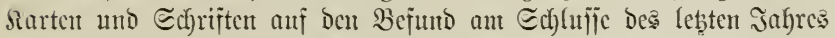

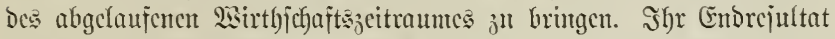

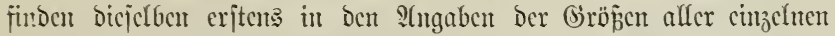

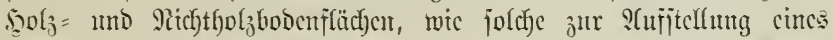

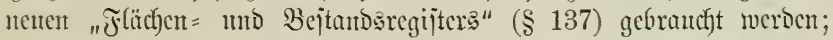

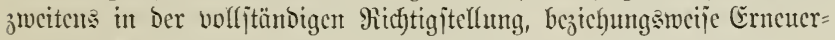
uny Der Sarten.

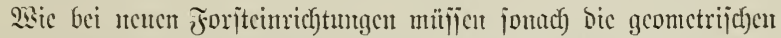
mit ben turatorijchen ?(rbeiten şand in Danto gehent, zutm Theil

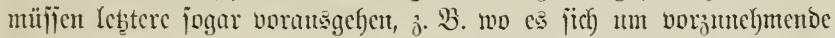

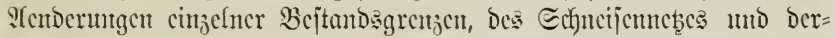
gleichen fjantoclt.

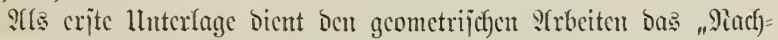

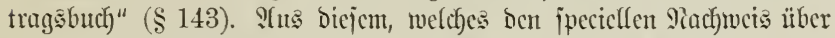

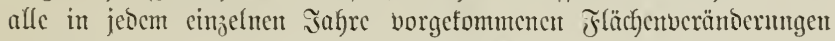

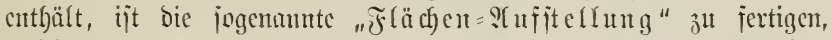

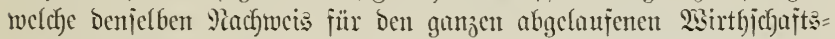
jeitraum licfert.

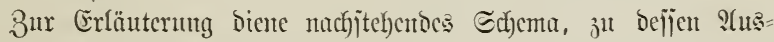

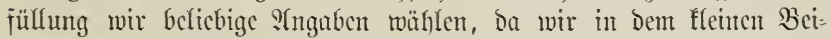

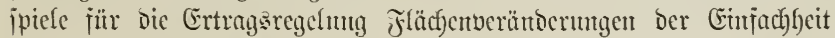
wegen vermetioen mollten.

Dic jweite llnterlage geben bic in sisintfjid)ajtesbutche verzeidfucten

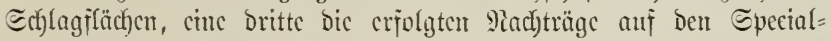
farten, bic vierte enditif Dic taratorijichen Borarbeiten, injoweit Durct) Diejelben Flächenverüttoerungen bebingt werben.

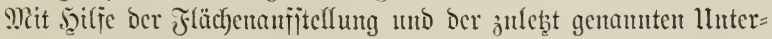

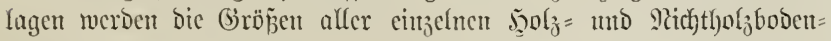

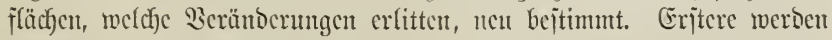

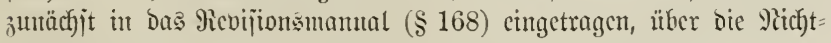

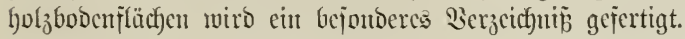


ร) ด

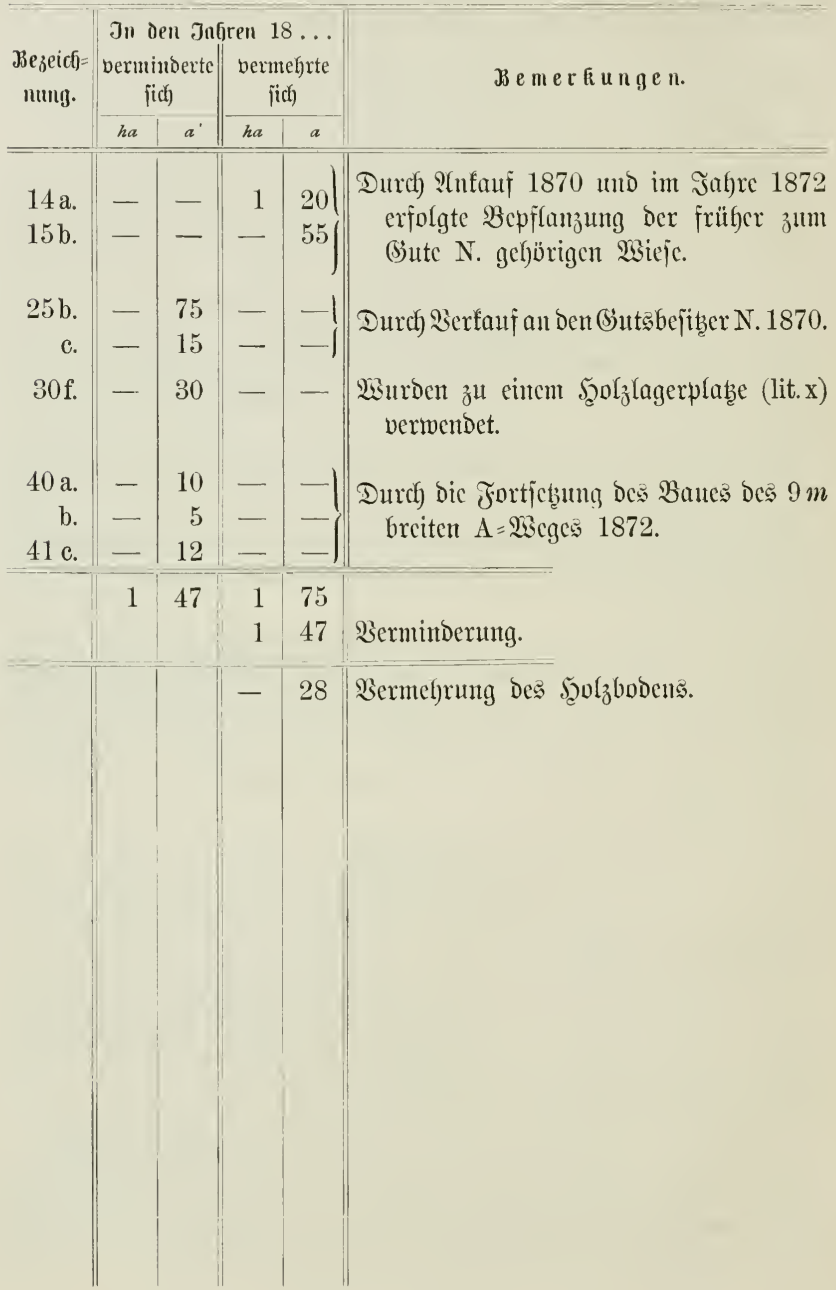




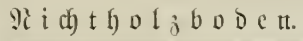

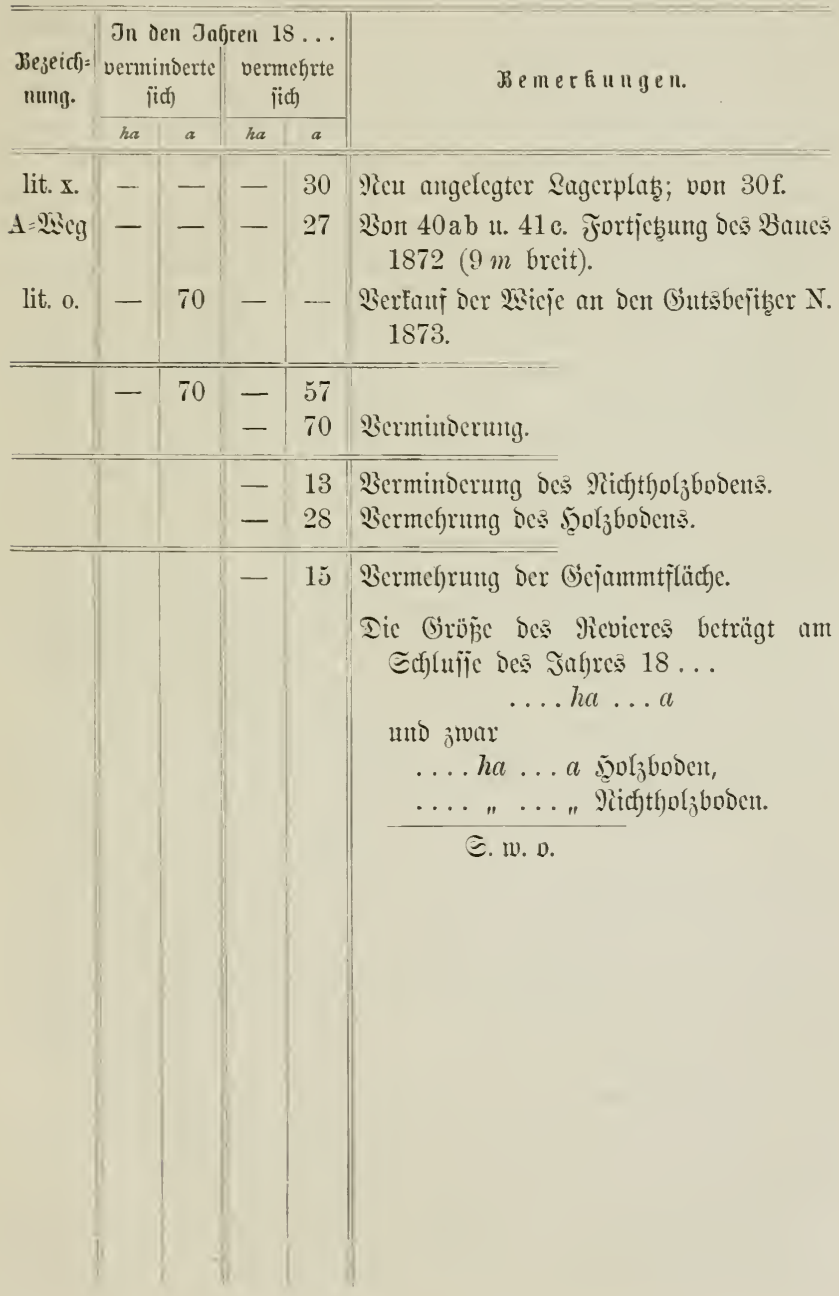




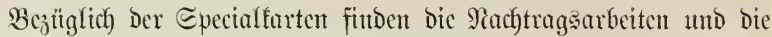

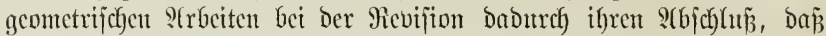
alle farbig fein follenden Sinten, welche von Dem Pact)träger vorläufitg oft mur mit $\mathfrak{B}$ leijtift eingezeidnet wurben (ð. B. WSege 2 .), mit ben ent iprechenden Farben angelegt werben. Ferner werben bie lebsten Echlag= linten in jencu Bejtändon, weldye nutr theilmeije zum S(btriebe gelangten,

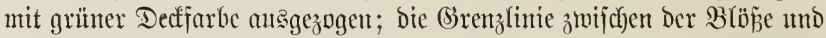
Der bereits fultivirten Fläche in einer und berjelben Itnterabtheilung ijt

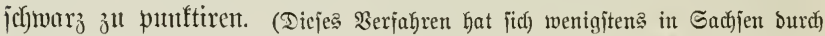
langjährige Erfafrunt jefrr gut benährt.) (Endlich finto Dort, wo :(bänderungen

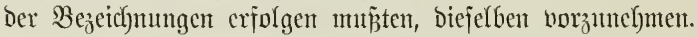

Wo ce irgent thunlich, jünd jedoch Senderungen der Bejtands=

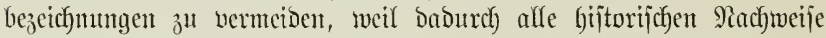

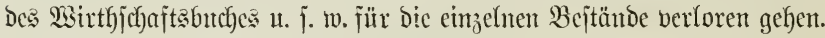

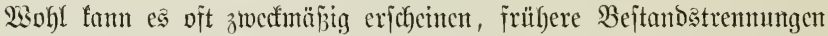
falfen zu lafjen ober audh neue zu bilden, woburch bie Butchjtabenfolge gejtört wirb. Zebterer aber bas Dpjer Durchgreifender $\mathscr{A}$ enderungen

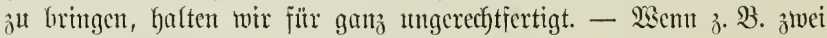
Bcitünde, a uno b, eriterer cin Buchen=, leţterer cin Fichtenort, gleich)= zeitig abgetrieben wutrden, tmo es findet fitch num an Etclle betoer cinc glcichmäß̄ig bejchaffene Fichtenpflanzung, jo fam jreilich cin Budbjtabc cutfallen, beffer ijt es aber Dam, Denjelben ber Getreffenden ?lbtheilung fünftig ganj fehlen z" lajfen, als die Budjitabenfolge Durch weitere

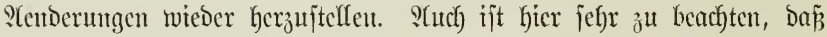

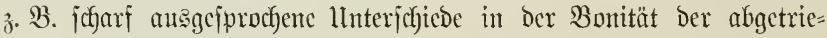
benen, alten Sejtände jwar im jungen, neu begrïndeten Bejtande an= fänglich) veriç̧wumben fein tömen, fpäter aber wieder lyerwortreten, in=

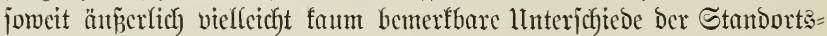

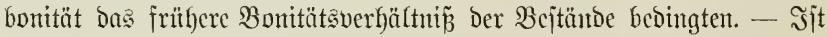

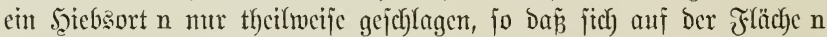

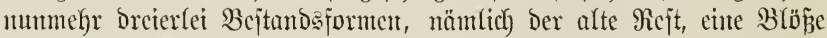

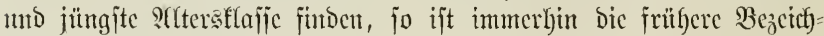
mung $n$ beizubchalten.

Q4f Der Bejtandsfarte werben S(enderutuen aticht nachgebrad)t, jon=

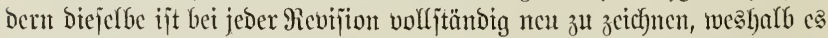

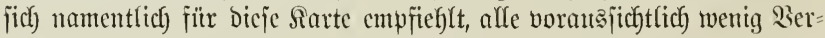
änverungen unterliegende Beidfmutg umb Echrift litfographiren zu lafjen.

Terrain $=$, Boden $=$ แmD Petzfarte tocroen jotweit berichtigt, als e

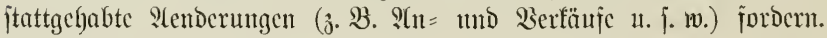




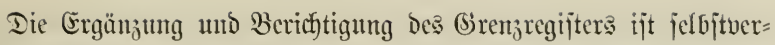

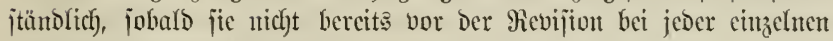
(3)renżveräıberung fitattfanto.

\section{$\S 167$.}

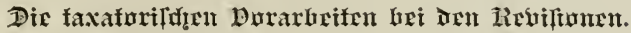

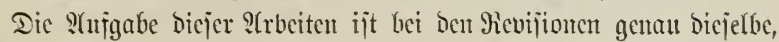
wie bei bent neten Cintidytungen. (Es Gandelt jich Daher um bie Etanto=

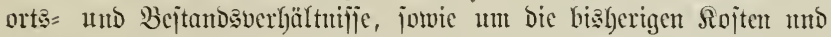

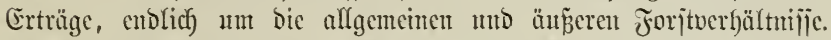

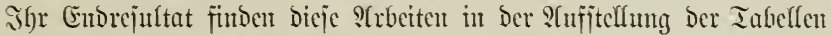

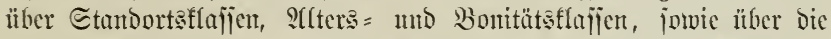

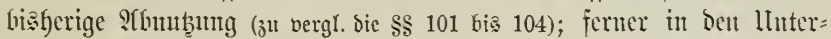
lagen, meldfe jie ben geometrijchent : Strbeiten für bie neuten Flächen=

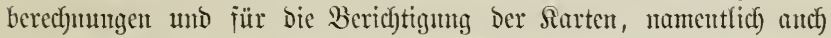

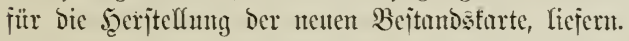

\section{a) Standortsucrhältnijjc.}

Snjoweit bicje unneründerfidyer Matur jüno ober üherhant feine

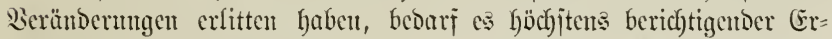

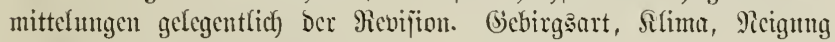
Der Şänge bleiben biejclben.

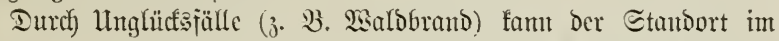

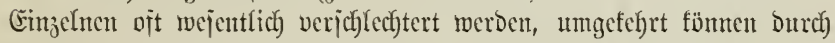

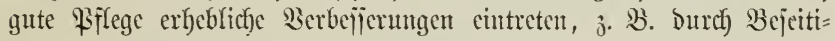
gung ber Etreumub̨ung, Durch) Entwäjijerungen, Durch bie Folgen bes ?(tubaues verangerter Raflffächen tr. j. w.

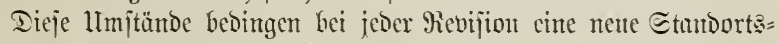

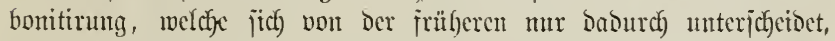

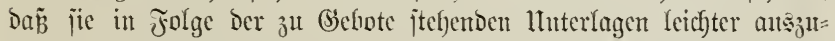

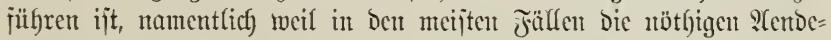

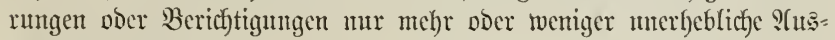
befmung haben.

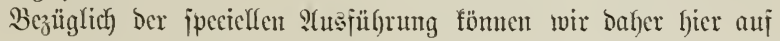
bie $\$ \S 54$ bis 57 vermeijert.

b) B̉ejtandaverhältnifje.

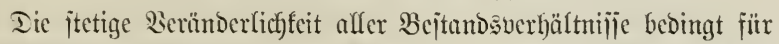

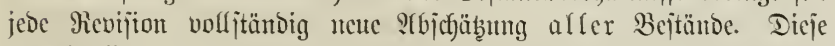
unterjcheibet jich) von ber bei ganz neuen Forjteinridytungen einjig 


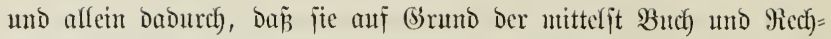
mung gewomenen localen Erfahrungen wejentlicf) an Sicherheit ge= minnt. Dic $\$ \S 66$ bis 76 , welche von Der "Bejtand\$bejchreibuntg" handefn, Gaben wolle (siltigfeit autd) für Die bei ben Şanptrevifitonen auşzufüfrenden, betreffenden : (rbeiten.

c) Bißgcrige Siołten und Erträge.

Ein gut gefülfrtes Wirtbjeffafteshuch verntag bie fier in Betracht fommentoen Ermittelumgen auperorbentlich) zut erfecchtern mo weit

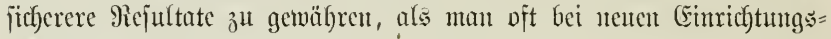

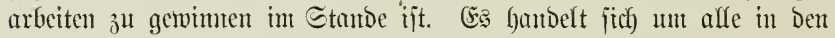

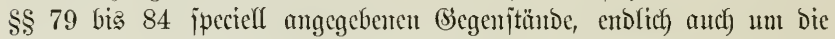
abermalige Berechnumg des $\mathfrak{S a l b f a p i t a l c s .}$

Sebr zu empfellen ijt eine 3ujammenjteflung ber cinzeluen, abge

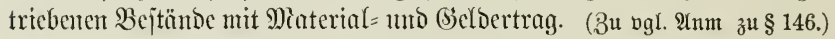

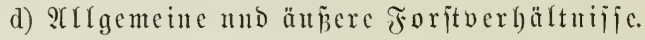

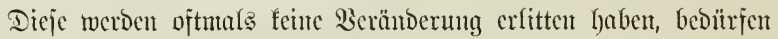

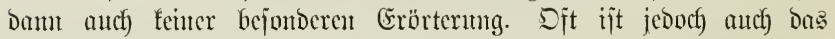
(B)egentgeil ber Fall.

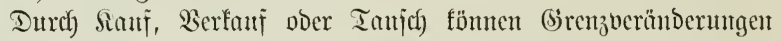

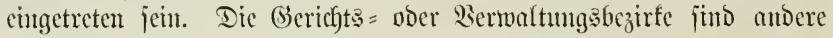
gewoorden. Für bie (bejchicfle des Forjtes fömen fervorragende Er=

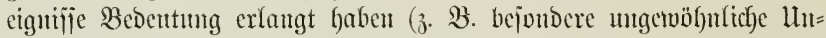

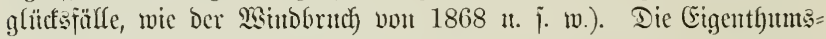

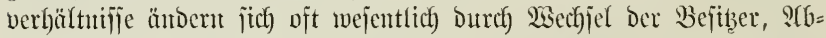

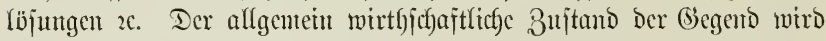

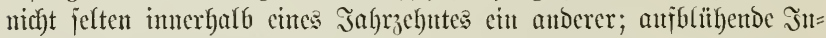

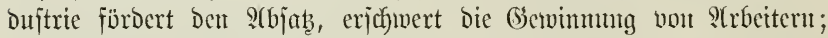
nene Eijenbahnent erweitern ben 5 gedzmarft, bringen jeboch) autch) (Con= curren; gewijie Foritfrevel verichwinden, antore treten an beren Stelle

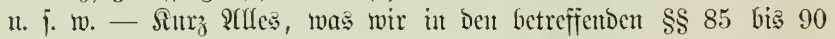
angedeutet haben, erforbert bie Beachtutug Der Bevifiton, bies um jo melyr,

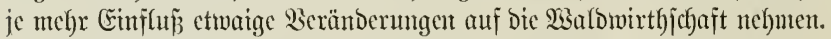

\section{$\S 168$.}

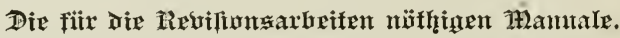

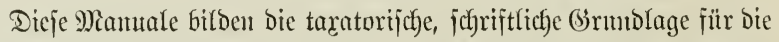

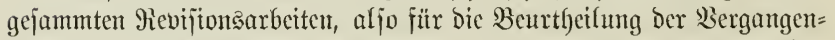

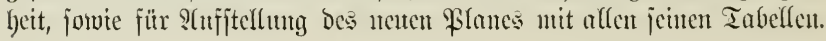




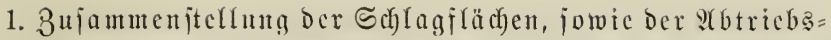

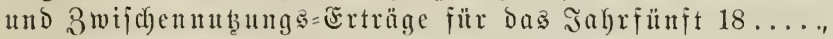

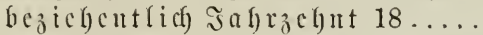

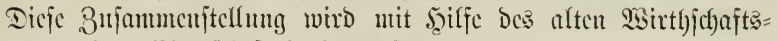
plantes und Des sistrtbichaftebuct)es gefertigt.

a) Für bie $\mathfrak{i} \mathfrak{b} \mathfrak{t} \mathfrak{i} \mathfrak{c} b$ stu Form üblict):

\begin{tabular}{|c|c|c|c|c|c|c|c|c|c|}
\hline \multirow{3}{*}{ ઝезеirffuming. } & \multirow{3}{*}{ Jajir. } & \multicolumn{2}{|c|}{ größje. } & \multirow{2}{*}{\multicolumn{2}{|c|}{ Ertrag. }} & \multicolumn{3}{|c|}{ ģiéistefte. } & \multirow{3}{*}{ Bemerkumgen } \\
\hline & & & & & & $B e=$ & $\begin{array}{c}\text { Eđä̈b= } \\
\text { mulg }\end{array}$ & (Grö & \\
\hline & & ma & $a$ & $f m$ & $h a$ & $\begin{array}{l}\text { narg. } \\
\text { nats } \\
\text { nathe }\end{array}$ & pro ha & $h a$ & \\
\hline
\end{tabular}

Die 3ujammenjtelfung erfolgt in Dicjem Manutale cinfach nad) Der Niumerfolge ber StGtheilungen. Sic iit bie beite Eontrole ber

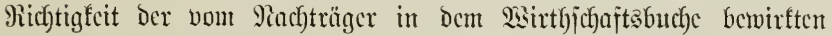

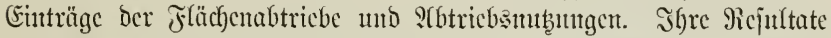

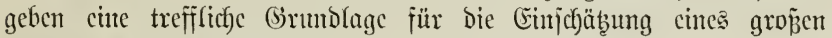
Theiles der neuen ઝ̧iebante.

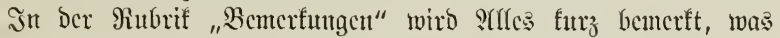

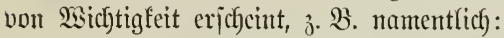

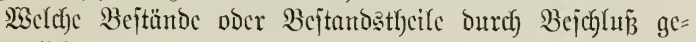

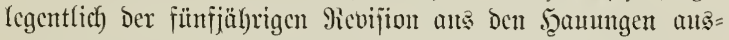
traten, welche hinz̧l famen.

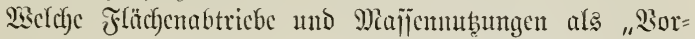

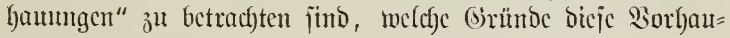
ImIgeıt veranlap̃ten.

Wiseldfe Drte "Durthgejofflagen" find und jur Bergleichung gelangen fönuten.

Db und weldye Eorrecturen Der Echlagflädyen vieflesefft nöthig wurden u. j. w.

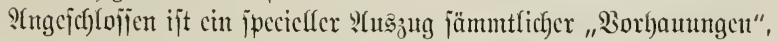
getremt nach) Den beiben verflofienen Jafurünften.

Ferner ijt jutgejügt bie $3 u$ jammenjtellung Der Flächenabtriebe nach) Bcitandşbonitäten, cbenfalls getrement nacd) beiben ฐaf)rfünften.

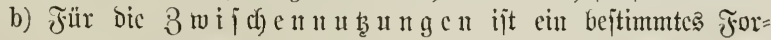
mular nicft borgcicffricben.

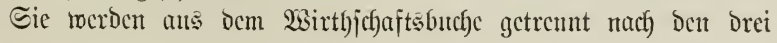




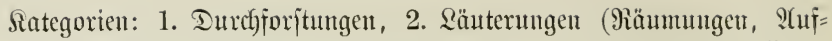

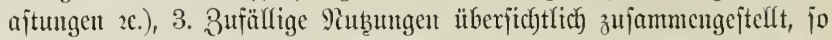

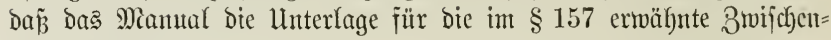
nub̧ungstabelle giebt.

\section{Das $\mathfrak{A} u \mathfrak{l}$ tumanual.}

YAs Den von ber Serwaltung gefïf)rten Rufturrectumugen und

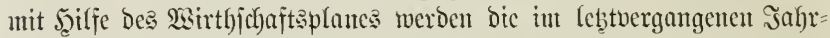
fünft ausgefülbrten Siulturen in Eachjen nach folgendent Edfenta $3^{11}$ jammengejtellt:

\begin{tabular}{|c|c|c|c|c|c|c|c|c|c|c|c|}
\hline \multirow{2}{*}{ 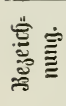 } & \multirow{2}{*}{ 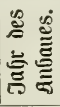 } & \multirow{2}{*}{ 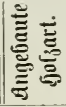 } & \multirow{2}{*}{ 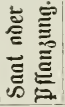 } & \multirow{2}{*}{ 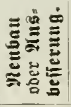 } & \multicolumn{3}{|c|}{ 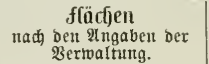 } & \multicolumn{4}{|c|}{ 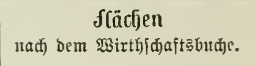 } \\
\hline & & & & & ha & $a$ & Bemerfungen. & Beзеid)ntng. & ha & $a$ & $\mathfrak{s a \mathfrak { h r } .}$ \\
\hline
\end{tabular}

Dieje Rubrifen fülfen die linfe Eeite Des in Duartjorm zแ füh)= renden Manuales aus. Die ganje gegenüberliegende redte Seite ijt beitiumt für "9lotizen über bie jtattgebabte :(usfübrung". Qebtere fönnen zum Theil jefon im 3imuter gegeben werden, joweit fie z. $\mathfrak{B}$. die Flächengröp̈en betreffen, zum Theil find jie erjt bei und nach er= folgter Beficftigung Der Sulturen int 2 Salde zuzufïtgen.

Sngefïgt werben biejem Miamuale noch ?(ngaben über Gaat = 4mo Plflanzfämpe, über Siojten น. j. w.

Ferner find nod) angej(blofien Bujammenjtellungen ber anderen

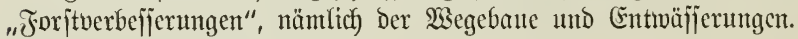

\section{Das Revifionsmaund.}

Diejes Mamual tritt bei Anjertigung Der Revijions= Sorarbeiten an Eteffe des Iaxationsmanuales für neue Foriteimichtungen. Wir

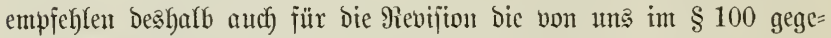
bene form, da es fich ja hier ebenfalls um volljtändig ncue Taxirung des Revieres handelt.

Die Brröß̧̧ Der Bejtände, weldye Beränderungen erlitten, liefern

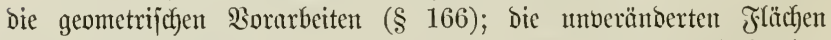
fömnen of)ne Weiteres aus dem früferen flächen = und bejtands= regịter eingetragen merden. Für bie ÂEfüllung ber übrigen Theile Des Mamuales ergeben bie taxatorijecten ?trbeiten (\$ 167) und bie hier unter 1 und 2 errähnten Mamuale das nötfige Material. 


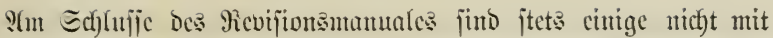
Piutbrifen verjebenc Eeiten bejufïtgen, ant weldye ganz furz alfe jente Piotijen nieberzulegen find, welche bejüglich) ber biablerigen Siojten uno

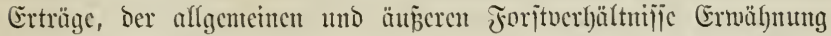

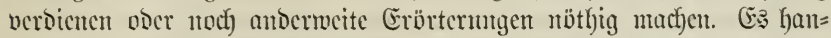

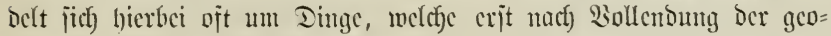

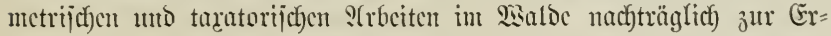

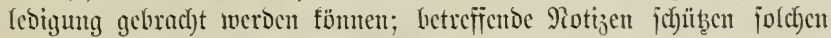
Jalles an bejten vor ben Viergejien.

Die in Eachjen übliche Form bes Micuijionsmunualcs ijt cinc antocre, und jwar folgende:

\begin{tabular}{|c|c|c|c|c|c|c|c|c|c|c|c|}
\hline \multirow{2}{*}{ 总 } & \multicolumn{2}{|c|}{ größje. } & \multirow{2}{*}{ jo[3arten. } & \multicolumn{2}{|c|}{ テrïhere } & \multicolumn{2}{|c|}{ Jestige } & \multicolumn{2}{|c|}{$\begin{array}{l}\text { @đ)äвung } \\
\text { nađ́ } f m t \\
\text { pro ha. }\end{array}$} & \multicolumn{2}{|c|}{ 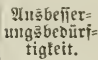 } \\
\hline & $h a$ & $a$ & & Elafie. & $\begin{array}{l}\text { tats= } \\
\text { flaîe. }\end{array}$ & Ilafie. & $\begin{array}{l}\text { tats } \\
\text { fla }\end{array}$ & $\begin{array}{l}\text { Saub } \\
\text { hol? }\end{array}$ & $\begin{array}{l}\text { Jabel }= \\
\text { hol }\end{array}$ & $h a$ & $a$ \\
\hline
\end{tabular}

Diejer linfu Ecite des in Euartformat zu fülyrenden D)iamules jteht rechts eine ganje Eeite für "गiotijen" gegenüber.

\$ 169.

\section{Der Wirfhlifhaftaplan.}

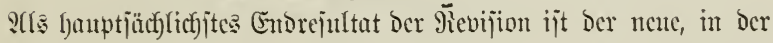
Regel abermals für 10 Jafre geltende SBirthjichaftaplan jn betrud)ten.*)

*) Sile bereits ftüher ervähnt, Yajjen wir jenen Theil Der Rebijionsarbeiten Gier entweder ganz unberü̈j̈̈dtigt ober Denten ifn nur an, welder nidft umittel=

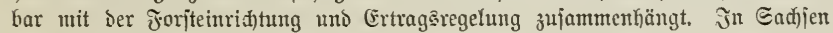

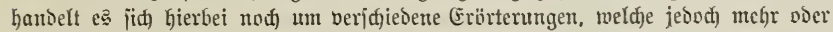

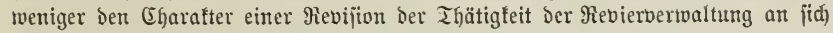
tragen, aljo nidgt hierher gehören.

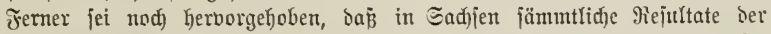
Rebifinnsarbeiten, weldhe theils bie Unterjud)ungen über bie Nergangentheit be= trefien, theils als llnterlagen für den neuen \$lan bienen, als jogenannte ",oor=

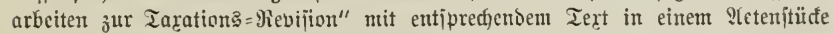

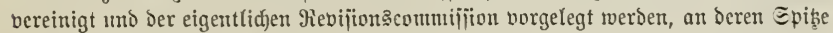

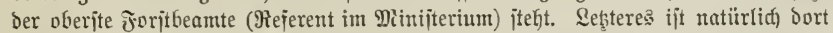
nid)t nothwentig, wo anbere Serhältnijie einen anberen Sejđäjtagang bebingen,

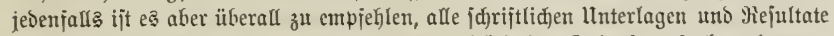

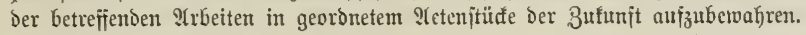

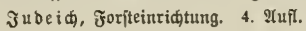




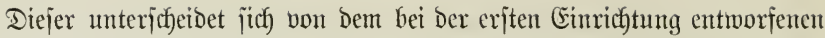
Plane Durch Die Şinweglaffung Der allgemeinen Bejchreiburg und even=

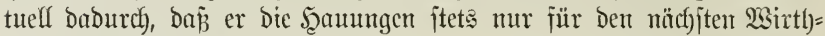

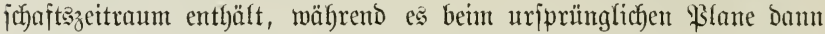

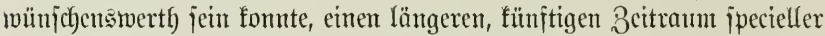

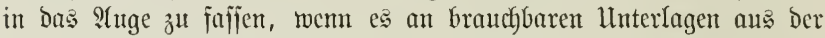

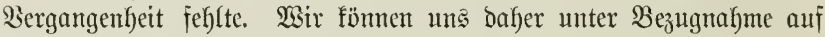
Das jrüher Bscjugte bier mit folgenden ?(ndentungen begnügen.

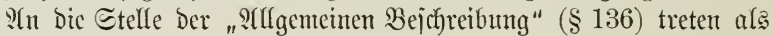

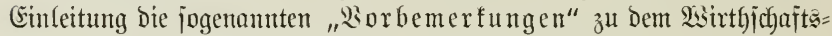
plane. Dieje fömen bejüglich) Des foritlicfen Thatbejtandes unto Der

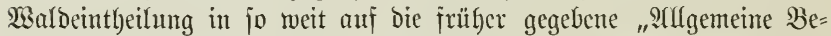

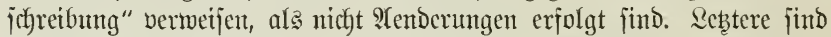

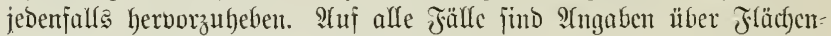

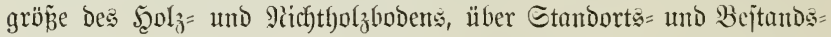
verbältnifje, jowie über bie bišherigen Mlafïen = und Gectderträge in

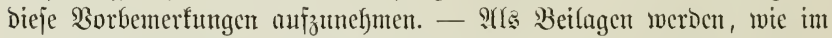

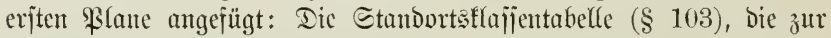
Bonitirung benub̧te Erfabrungştafel, Die Silajienüberjicht*) (§ 102), Die S(bnub̧ungstabelle (§ 104). Reb̧tere żwei Bcilagen enthalten, wie

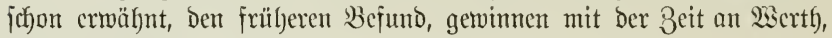

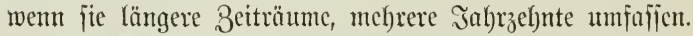

Daś Sapitel über bie (Ertragśbcitimmung tutericheidet jith formedl

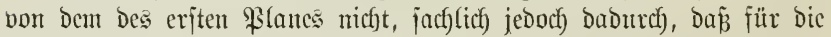

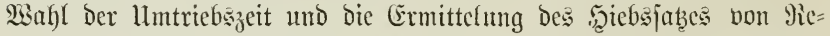
vifion zu Revifion braudfbarere Erfahrungen gewounen werden. WSic in Der ",Allgemeinen Beichreibung" Find audf) in Den "Borbemertungen"

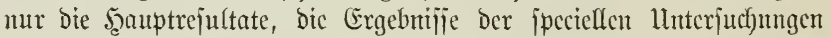
anz̧ugeben, bic ausfübrlicfe Mittbcilung Der leb̧teren jelbjt ijt in cine

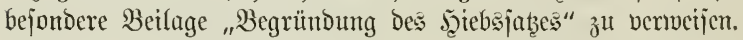

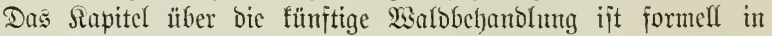
Derjelben oder wenigitens in äbnlicher Weife ju fajien, wic cs Der

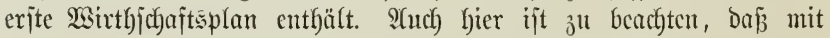
jeber weiteren Yicvition bie alfmälig gemonnencn, localen Errafgrungen

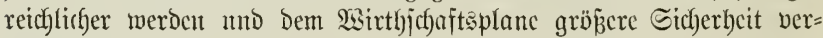
icfaifien.

*) Die Silafientabclle wird nidj)t Dem :

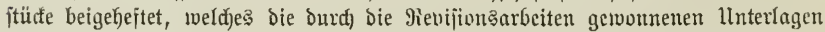
umfajist. 


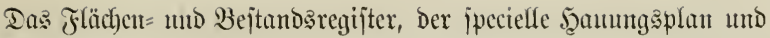
Der Sitlturplant werden für ben neten 23 irthjichaftsplan formell gettaut

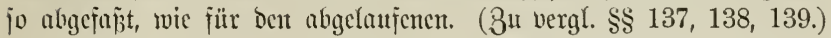

B. Die fünjiürrigen oder 3 wifdentrevijionent.

\section{$\$ 170$.

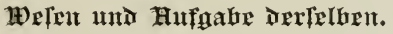

Dic Şufgahe, weldye man früher fajt allgentein Den Revifitonen

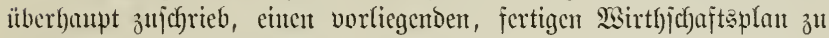
crgämzen und zu berichtigen, fällt im Simme bes in Eachjen ïblidfen Sierfafrens mer nod) Den jogenamten fünfiäfrigen ober 3mijd)en=

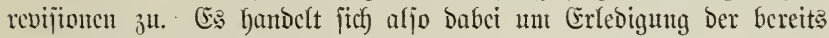
Eeite 342 angedeuteten Frtagen:

Wie haben fich bie bejtmnnungen des \$lantes bisher be= währt?

Wectcye Störungen jind Durch unvorfergejefene Ercignifife cingetreten?

Wie laffen fidy bie Folgen biejer Etörutgen ober bie jonjt

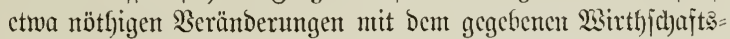
plane vereinigen?

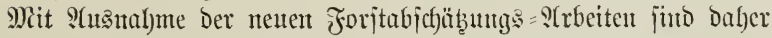

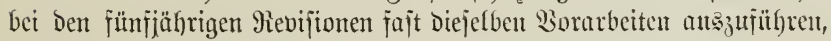
wie für bie zehnjährigen.

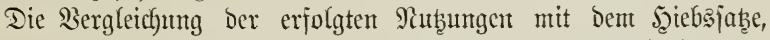
3ujaumenitellungen Der planwiorigen Şauutgen, Dor Durdjorjitungen, Der 3wifd)emutbungen überfant, cinte Bergletchung Des CErtrages Der Dutrdfgejd)lagenen Drte mit Der Echäbzung, IInterjuchungen ïber

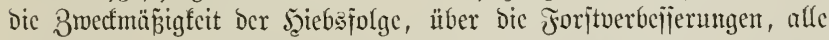
bieje Dinge hat auch bie fünfjährige 3 mijchenrevijion $3^{\mathfrak{h}}$ erörten

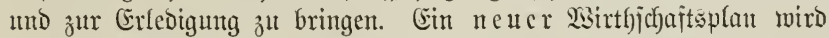

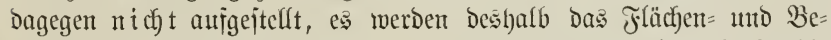
jtandsregijter, bie Standorts= und 9Gtersflajjentabelle, ebenjo bie Sitajienüberijicht nidf)t neu zujammengejtellt. Bejüglich) Der Flächen=

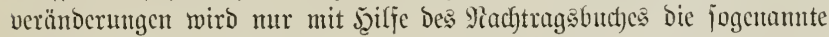

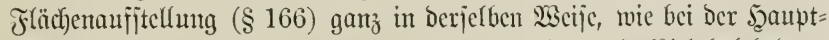

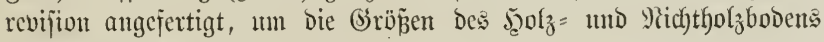
jitcher ju jtellen. 


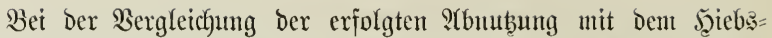

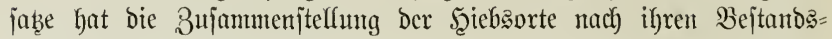
bonitäten Gejontore Bedeutung, Da jith auts ifre ergiebt, $\mathfrak{o b}$ in Dem

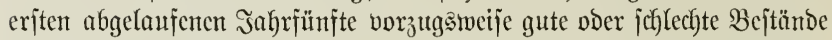

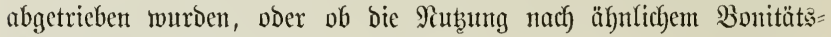

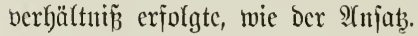

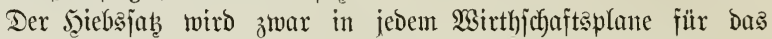

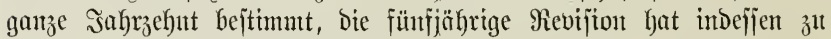

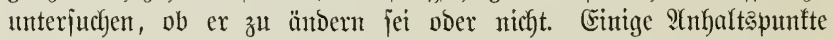

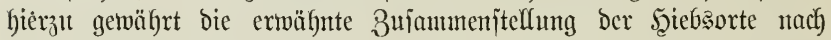

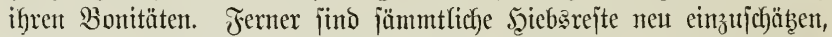

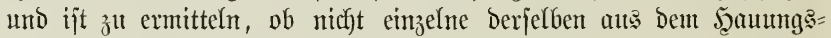

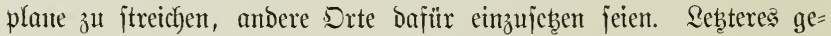

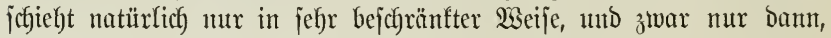

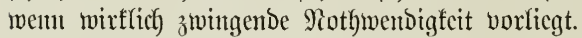

Die Unterlagen für bie Bejtimunutg bes nenen Şiebfabes weroen

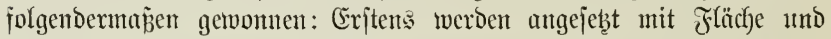

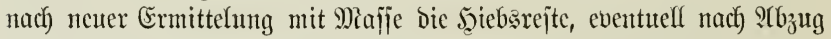
voer Butritt einzelter Drte, z'weitens werben von bem jo getwoutuenen

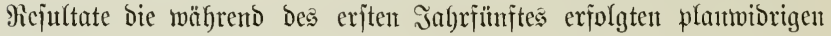

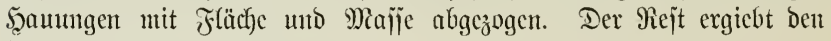

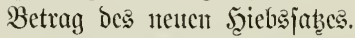

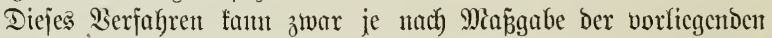
Berfältnifije verjeffiedenen Miobifieationen unterfiegen, ce ift jeboch bic

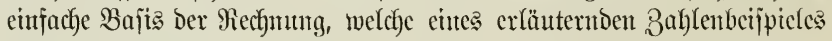
fier faum bebarí.

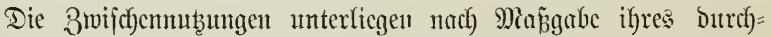

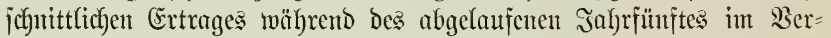

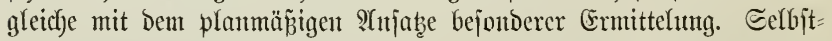

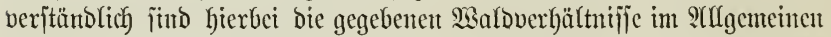

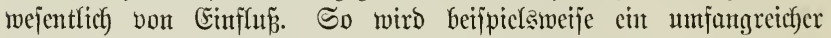

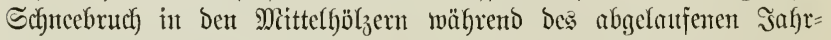

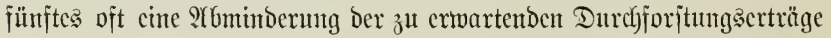

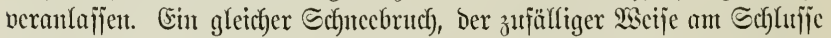

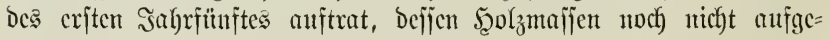

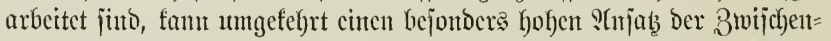

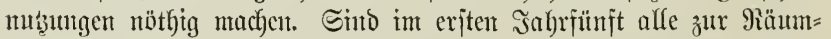

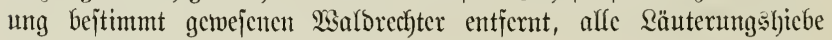
bejorgt worden, jo fann natürfliff für bieje Siategorie ber 3wijchen= 


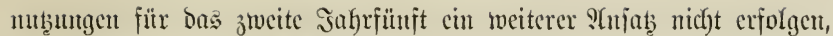

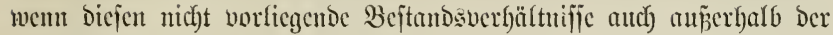
Grenzen bes alten Plantes bebingen.

Dic Cumme aus bem zu crwartenden Betrage Der Stbtricbs= und

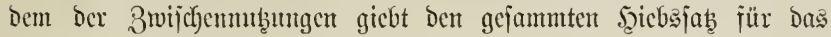

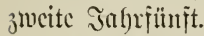

Ein netter, ipecielfer şanungsplan twird nicht angejertigt, ba es genügt, Die gelegentliç der fünfjährigen Picvifion vorgenommenen

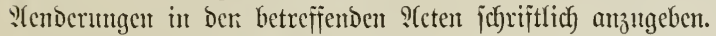

Shnchin wird man S(enderungen Des anfünglich) bejtimmten Sciebs:

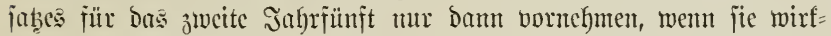
(ich) geboten erjcheinen.

Snocts verbält es jich mit Dem Sulturplane. Diejer wiro nach) vorausgegangener Bejuchtigung aller Siulturflächen jum Bmecte Des

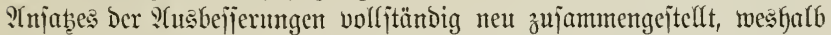

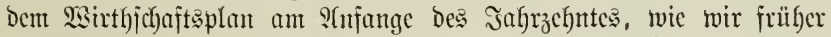
crmähnten, jo viele fithographirte Bogen bes Sulturplante lecr beige=

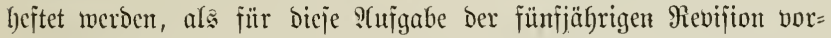

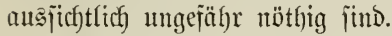

In Folge Der bei bicjen Bmifdenrevifionen borgenommenen $\mathfrak{Q}(\mathfrak{b}=$

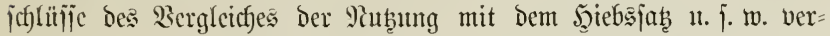
ändert fich Die Form Der betreffenden Iabellen fïr bie Scauptrevijion in jo jeut etwas, als dieje bant Epecialitäten mur fïr bas zweite

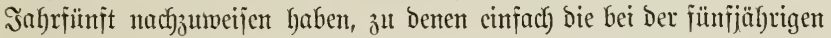
Revifion gemonnenen, jummarijchen Rejultate abdirt werden. -

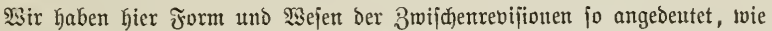

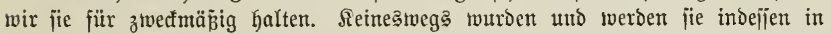

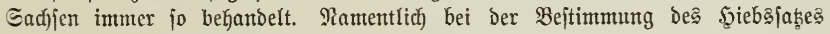
verfährt man etwas anders.

Bur Erläuterung Dieję \$erfahtens jei bier ein Beippiel Der "Etat

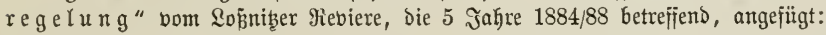




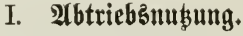

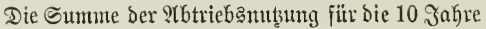
1879/88 ift im Şaungaplane angejetst mit . .

Rad) Den (Ergebnifijen, welche bie Bergfeicfung ber Durdfgejolagenen unb angefauenen Dite bes I. Jahriünftes gefiefert hat, ift bie Summe im Miabelfolze red)untgämäpig um $3{ }^{\circ}{ }_{0}$ z̆ er= höhen,

bagegen im $\mathfrak{a} a$ bholze rechumngamäp̃g um $16 \%$ z"l erniesrigen,

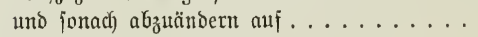

Dabon wurben im I. Jahrfiitsft geidlagen . .

(einfdyl. ber Borfaumngen an $-\ldots a$ mit - $f m$ (Ertrag.)

B(eiben bemuad) verfügbar für's II. Jahrfünft Im Durdfidgnitt jährliḑ). . . . . . . . জaren zeither angeję̧t. . . . . . . . .

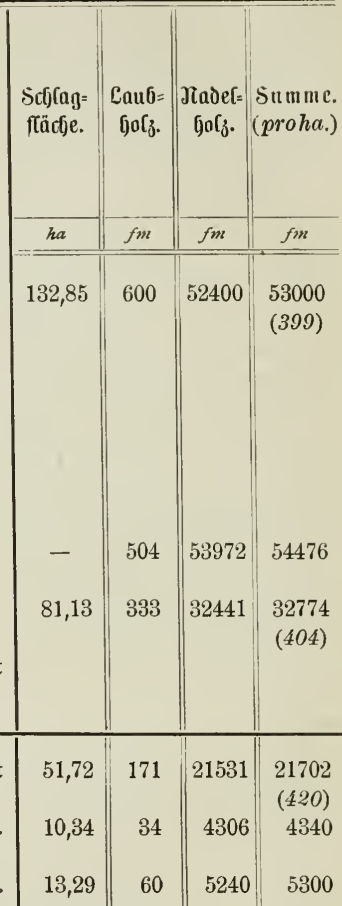




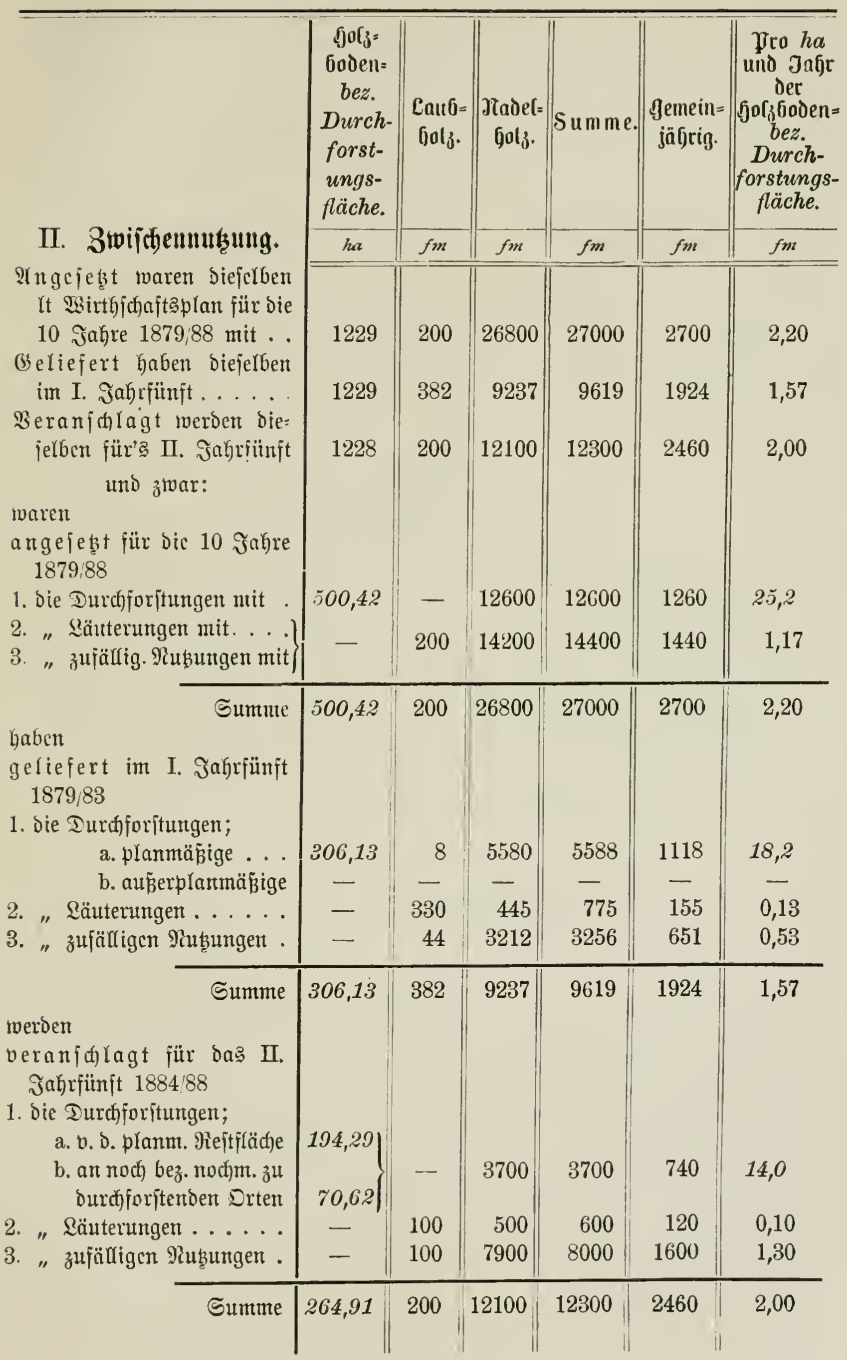


III. Gejammtıนฬżutg.

Diejelbe jteff jish nach) Borjtefendem fïr's z weite Jahriünit auf:

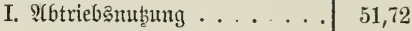

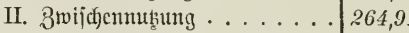

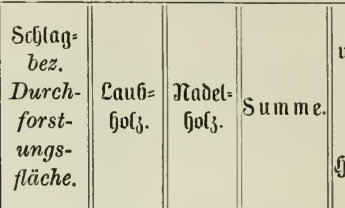

Pro $h a$

und Jafic

Der

$\operatorname{sdj}[\mathrm{ag}=$

$6 e_{3}$. Got $600 \mathrm{den}=$ fläcje.

\begin{tabular}{|r||c|c|c||c}
\hline \multicolumn{1}{|c|}{$h a$} & $f m$ & $f m$ & $f m$ & $f m$ \\
\hline & & & & \\
51,72 & 171 & 21531 & 21702 & 4,20 \\
264,91 & 200 & 12100 & 12300 & 2,00 \\
\hline
\end{tabular}

Hlebergaupt $\ldots \ldots \ldots \ldots$

Im Dur(c)j(h) jäfrlich)

$\left\{\begin{array}{l}\text { abgerundet } \\ .\end{array}\right.$

Darumter Derbgol $\ldots \ldots$.

(अieijigprocent bon ber Brejammt= maije zeitfor) .......

(Meiïgprocent bon der (bejammt= majic $f u ̈ u f t i g) \ldots \ldots \ldots$ -

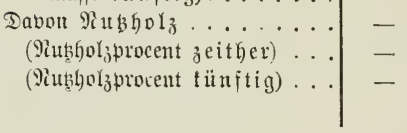

Beitheriger (Etat.

Gicjanntmajie...........

51,72

371

33631

34002

5,53

Darunter Derbhol

13,29

$50,04\}$

80

7920

8000

50

5750

5800

Der berechnte füuftige Etat beträgt ge=

gen ben zeitfcrigen in Derbholze:

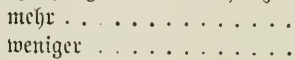

wentige 


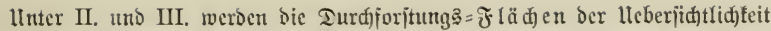
wegen mit blauer Tinte cingetragen. Wir haben bicjelfen Gier burd) Curfiv= હał femtlidf) gentadft.

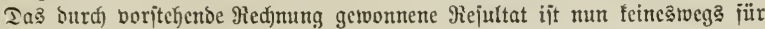

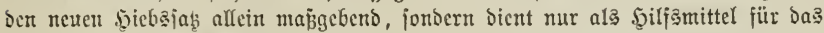

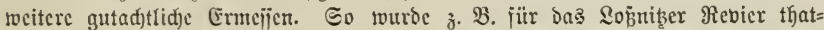

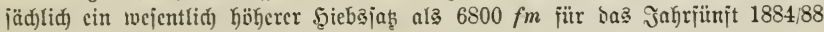
angenommen, weil

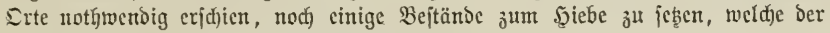
Saluungäplan fürt bie 10 Jahre $1879 ; 88$ nidjt mit entfielt.

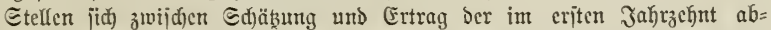
getricbenen Bejtände bebeutenbere Difïerenzen heraus, als ç fier ber fall war,

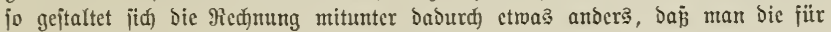

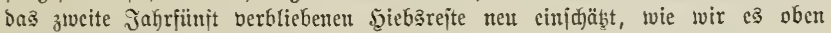
(ङ. 500) empfoflen haben.

\section{$\S 171$.}

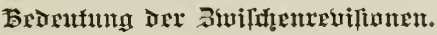

Die Meinung Darüber, ob Die jünjijäfrigen Mievijionent wirflich notthwentig jeien ober nicht, ijt Gier in Eachjen eine getheilte.

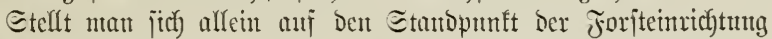

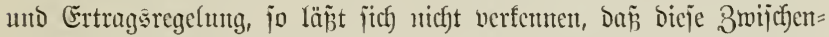

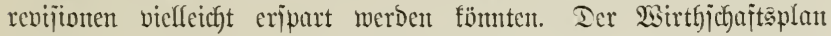

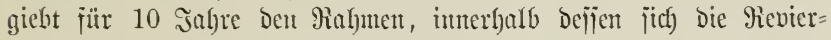
verwaltung ju beweget hat; Die ?tuggletefutg Der cinjelnen Jahre

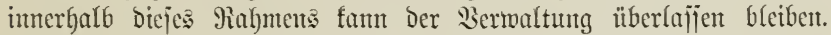
Sollen und müjïen Dagegen, unvorgergejehener ltmitänbe wegen, cin= zelne P(biveiffungen vom \$lane vorgenomment werben, jo wäre bei

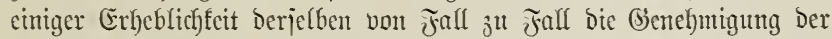

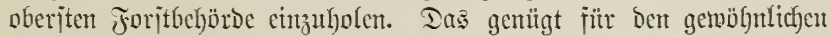

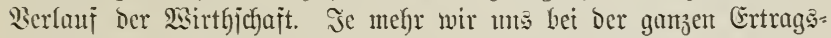

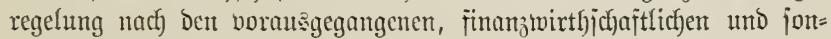
jtigent Errmägungen auf bie Ştebsfläche als auf einen allgemeinen

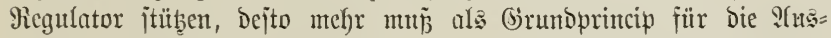

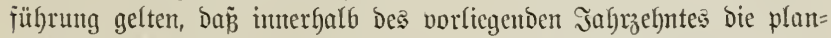

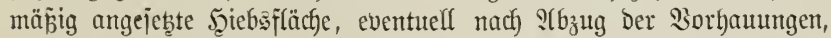

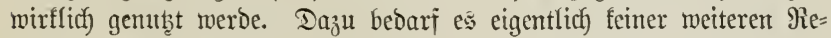

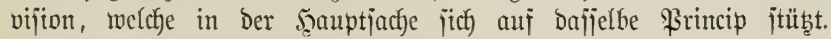

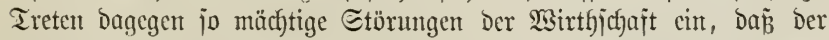
Filan abjolut nich)t mefyr pajpt, bann faun es vortheilfafter jein, ben=

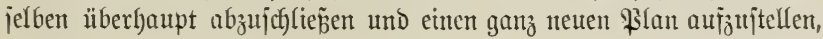




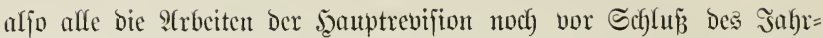
zchutes auรzufüfren. Solche Störungen hatte z. $\mathfrak{B}$. auf viclen $\mathfrak{R}=$ vicren Mittel = Deutfchlands Der Wintobrud) vom 7. December 1868 hervorgeruffen.

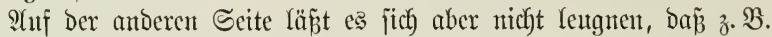

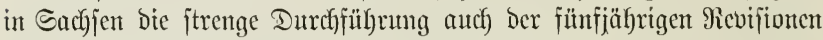
wejentlich mit bazu beigetragen bat, ba: gejammte Forjteintrichtung werf in Dronung ju halten. Pamentlid) gilt bies für bic \$rüfung

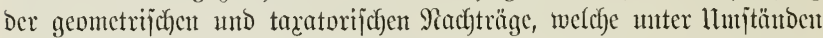
nicht mel)x in genẗgender Weije erfolgen faum, went eit Zeitraum von 10 safren bereits verf́lofien ift.

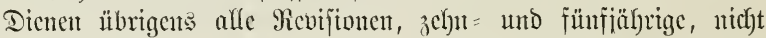
Glos Den Bwerfen Der Forjteinridftung, jondern merden fie von ber

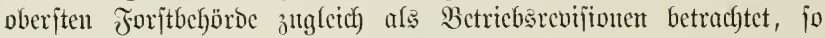
faben aud bie Brwijchentevijionen ifyen bejonderen Werth, weldyer Deren S(bbaltung jeldr winjichentwerth madyt. Die weitere Betrad)tung bicier Frage gebürt jedoch nicht in bas Bereid) utujerer :(trfgabc, fon= Dern in bas ber forjtficlyen Dienjteinrichtutut überbaupt. 


\section{AnItan}

\section{$\S 172$.}

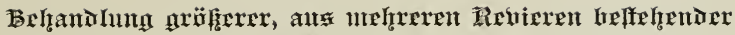 w)aldunen.}

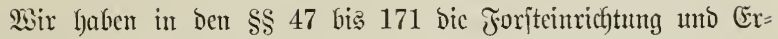
tragstegelung eincs cinjelncu, für fïch bejtehenden Pievieres betractet,

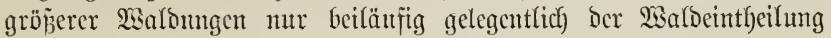
unto ber allgemeinen Bejd)reibung gedacht.

Aefnfich) twic fich einzelne Bejtänto vocr Bejtandsgruppen zu ben einzelnen Betriebsflafīen, bicje zu bem einjelnen Reviere verbalten, fo

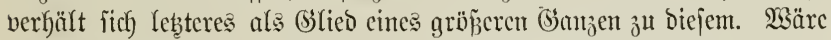

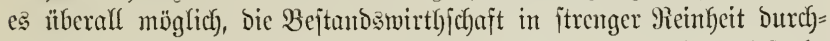

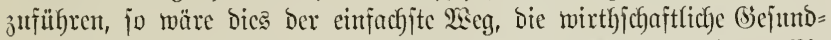
heit Des (Sanzen Durch dic jeiner cinzehten Theile herzutellen. Wir

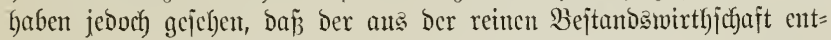
widfelte Sjiebsfab eines allgemcinten Ricgulators bcoarf, weil größ̈cre

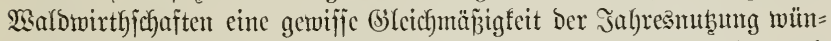

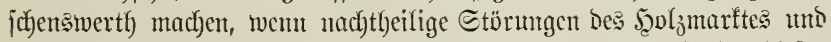

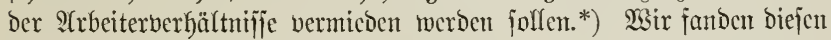
Miegulator unter Beachtung des S(ltersflafjenverbältnifjes hauptiächlich)

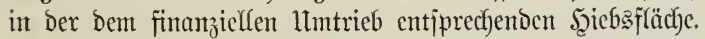

Ebenjo wie es nöthig ijt, cine Mobification DCs aus ber reinen

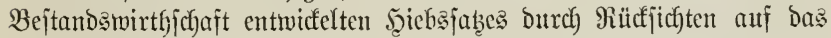

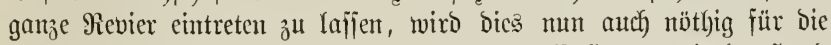

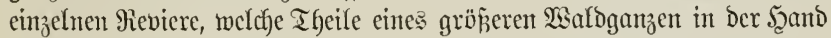
eines Befibers, namentlich Theile eines und defijelben Marftgebictes fint.

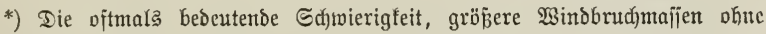

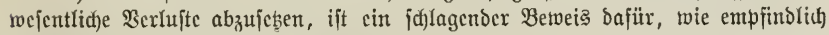
ber Şolzmarłt gegen plöb̨liđe lleberfüllung ijt. 
Bejtimute formelle Borjchriften hicrüber laffen fich nicht geben, cs licgt jedoch anj ber Şand, daj̃ man oft bie Echmantungen bes

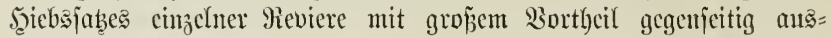
gleichen fann. Bejebst z. B. Den Fall, Das eine Revier habe einen

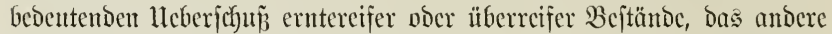
lcibe Mangel Daran, bejibe jeovd) Dafür enten lleberjachun an zuwachs: reichen Mittclhölzen, weldye Dem erjten Reviere fehlen. Pichts wäre

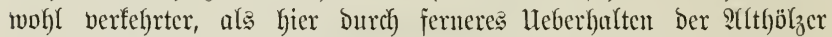

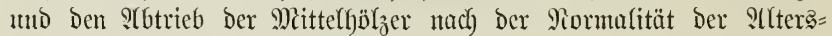

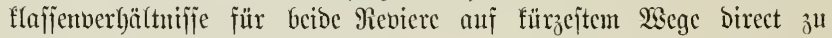

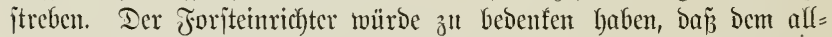

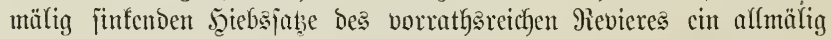
jteigenter DCs anderen ergänzend zur Eeite tritt.

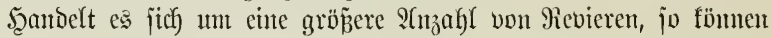
bcrartige Erroügungen von größ̈̈tem Einfufuje auf bic Bcjtimmung bes

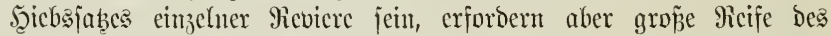
wirtbjichaftlichen Urtheiles.

Gelbjtwerjtünolich verbient bie Thatịache ber 3 ujammengehörigfeit

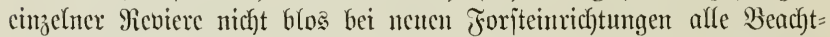
ung, fondern jtets auch bei Den Revijionen. Bun biejem (jejichts: punfte aus, wäre es am bejten, wonn leb̧tere für alle Feviere eintes

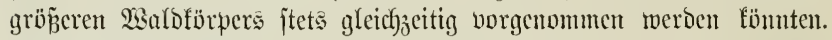

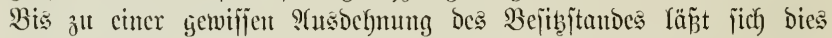

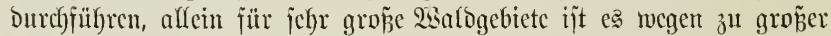

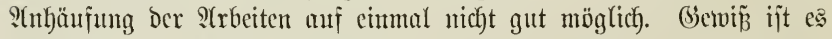

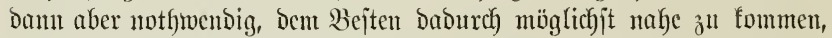
baj̃ man wenigitens bie Reviere cines Sulpectionsbezirfes gleidfzeitig Der Mevifion unterwirft. Sanu mun Dabei vermeisen, Die cinem Miarft=

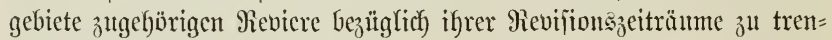
nen, Dejto befier.

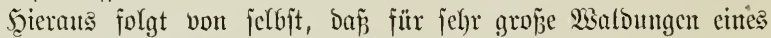
Bejtiers cin \$ilan barïber entworfen mo fejtgehalten werben muf́, in

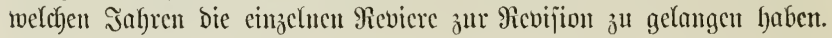

\section{§ 173.}

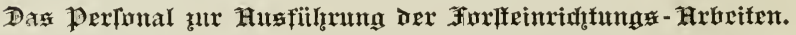

Dic Frage, wem bic Susführung Der Forjteinrichtungs=? ?rbeiten 3u ïbertragen jei, ijt viclfach in Der Qiteratur erörtert, von ber \$raçi 
werjefieden beantwortet worden. Dffenhar gebört fie ftreng genommen nicht ganz hierber, fombern zur Qcl)re ber forjtlichen Dienjteseinricht=

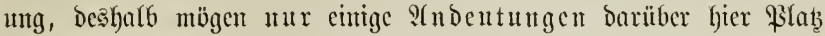
funben, bie wir jedoch nicht gan übergeben ju fömen glauben, weil

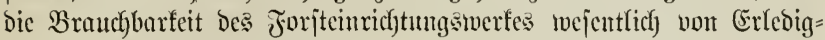
ıng biejer Frage mit abljäıgt.

Eine abjolute Entjcheidung Darüber, weldye Dienjtescintichtung überhaupt bic bejte jei, ‘äpt jich befamntlich nicht geben, Da bei ber

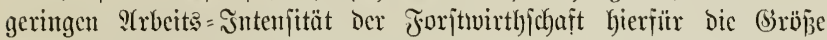

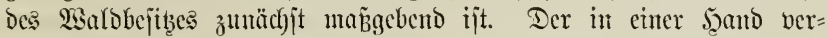

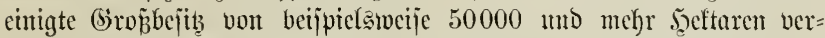
trägt nicht blos, fondern forbert einte andere (Einridftumg Des Dienjtes,

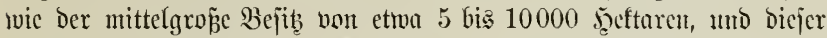
wieber eine andere, als ber Silcinhejitb. Irobsem ijt fïr alle Befitis= formen cin allgemein wirthjchaftliç)er (Sirumbjab vorfjanden, nämlicf)

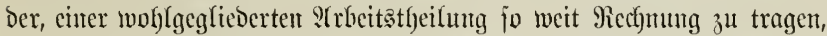

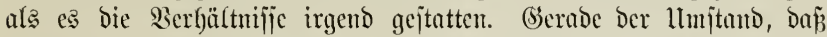
bei Der Waldwirtbjefajt mur ein grofer Befits bie Forderungen ber Srbeitstheifung befricoigen fann, begründet cinen wejentlichen $\mathfrak{B o r}_{\mathfrak{z}} \mathrm{ng}$ dej̈elben gegenüber den Sileinbeịibe.

Bei jeber Frage Der Dienjteseinrichtung, jo aud bei ber bier vorliegenden, müjfen bemjenigen, ber jie rationell entjcheiden will, bieje allgemeinen (Sejichtspunfte flar vor : :ugen jtełsen.

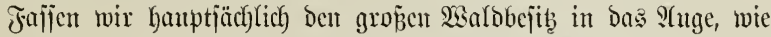
cr fich) etwa in Den J̧änden ber fleinen, Deutjchen Etnaten oder autd) in Denen einzehtuer, groper (5rundherren befundet, weil jich hier bie bejte Srganifation leicht burchjülyren läp̈t.

Richt blos die für jebe Forftemrichtıng nöthigen geometrijchen,

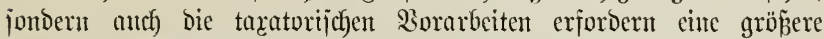

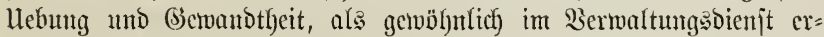
langt werben fönnen. Secfon Dicjer llmjtand allein begrïndet bie

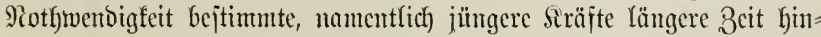

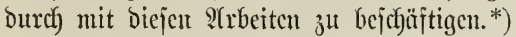

Bejondere, Durch vieljeitige Erfahrung gereifte Siemntnific,

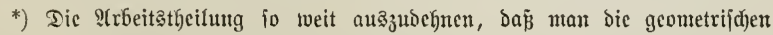

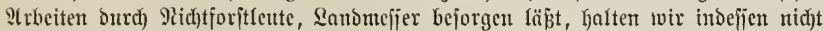

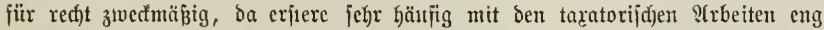

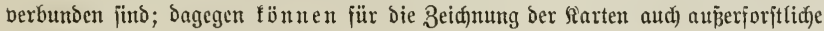
Iräjte mit zu গ̧ilfe genontnten weroen. 
melyr als jie jich im Berwaltungesdenjte allein crwerben fafjen, bebarf

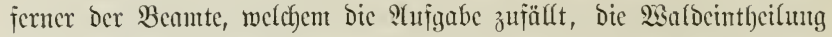

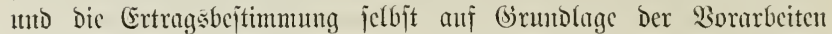
zunt entogittigen S(bjchlujic zu bringen. Eoll cin jolcher Beamter allen Shiforderungen entiprechen, jo mun er Durch dic Schule bes

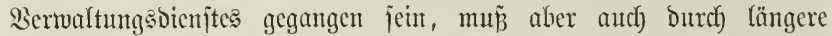
Thätigfeit im (Sebiete Der Forjteinrichtung jich) für jeine ipecielle 9 (uf= gabe bejondore Bejäligunt verjajafit haben.

Dieje Gründo iprechen cutjchieden Dafür, Das Foritcinrichtungs= wejen einer bejonderen Sechörbe jult übergeben, weldhe mehr voer meniger als Theil ber oberiten Foritoirection zu betrachten ijt.

Die Bortheile eigener Forjtentrichtunga = (Taxations:) Befüroen liegen auf Der Jeand und haben jïf) jtets Dort herautsgejtellt, wo man bie=

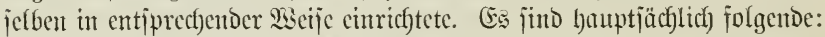

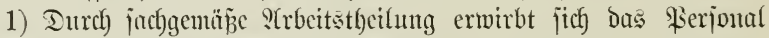

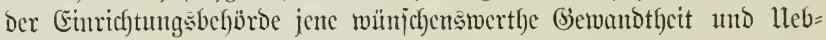

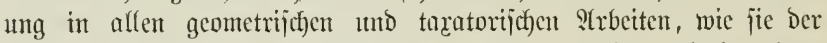
Revierberwalter als joldger nidyt leicht jich erwerben und bemalgren faun, meil ber gröp̈te Theil jeiner Beit burch andere s(rbeiten in P(tipprucf) genommen wiro.

2) Die Bonitirungen Des Standortes jowohl, wie bie ber $\mathfrak{B} e=$ jtände merben Durd) bas einer Behörde angehörige Perjonal vicl mebr nach eintem übersinjtimmenden Maj̄itabe erfolgen, als wenn fie für jcoes Revicr von cincm anderen Revicrverwalter vorgenommen merben, Dem bicjelbe Slujgabe vielleicht nux Drei= ober vicrmal in jeinem \&eben zufällt. SAuch bat es jeine begründeten Bedenfen, vom

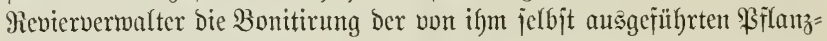
ungen u. $\tilde{\text {. }}$.. zu fordern.

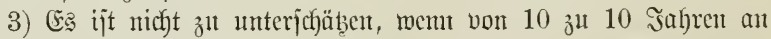
jeden einzetren Bejtand bie Frage, was mit ifm zu gejofeben habe,

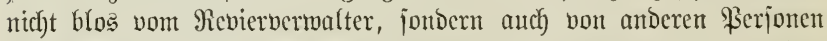
gejtellt wirb, bie mit Der ipeciellen Berwaltung jelbjt nichts zu thun loaben. Dadurch entjtelyt ein jrudftbarer Micinungsaustaujich.

4) Enolich vermag mur eine eigene Einrichtungšbchörbe fämmt= licke (Einrichtunggarbeiten nad) einem gemeinjamen \$̧lane mit über= cimptimmenden Formen Der Sarten und Echriften Durchzufülyren, ofne

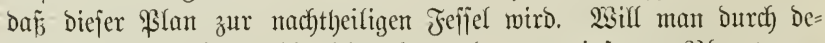
taillirte Injtructionen bie Ëinfaltung Des gemeinjamen \$lantes von Den Hicvierverwaltern crjiningen, fo artet berjelbe mur alljulcicht in 
eincn papierenen Echematismus aus, Der ben Fortichritt auf Dem michtigen Giebiete bes Jorjteinridytungswejens jwar nidyt unbedingt

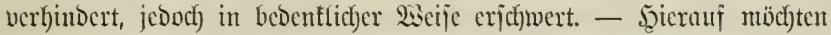
wir nach mandferlei Erifabrungen ganz bejonderes Givicht legen.

Sion anderer Ecite hat man namentlich Folgendes gelteno gentacht:

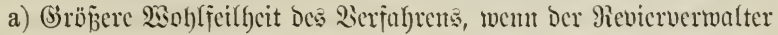
cinen grojen Theil Der Einrichtungs = oder Revijionsarbeiten nebenher jertigen fömue.

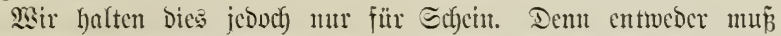
Der Berwalter, während er jich mit Dicjen $\mathfrak{Q}(r$ beiten bejchäftigt, jeine eigentlichen Berufisangaben zurüdtreten lajien, ober bie eriteren wer= Den Den Eharafter nebenther gelicferter :(rbeiten beutlich an Der Etim tragen; Deren Eualität wirb Daun andf) Der Sisobffeilbeit entiprechen.

b) Die Serwertfumg Der gründicf)eren Siemtnip aller bittichen Rerbältnijie madye den Roculbenuten bejonders geciguct für alle Ein= ridftungs: આmD Ricuijionsurbeiten.

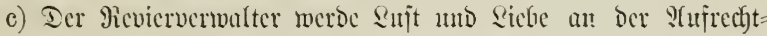

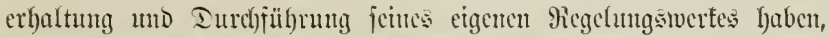

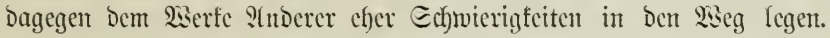

Die beiden fier unter b uto c erroähnten Bedonfen verjchwinden ganj Durd) cinten (Bejchäftsgang, wic wir benjelben in Sorjejlag bringen, verlieren ofnefin ifje Bebcutumg, went ber 2 sechjel bes Diemjtes an= Dere Beamte bem betreffenden Fieviere bringt. -

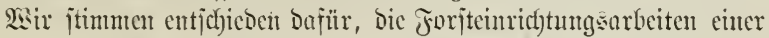

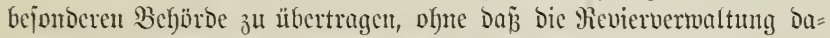
Durch) von ber Betheiligutg an biejen Sarbeiten angejchlofien werbe. Der (Sejchäjtsgang wäre in jeinen (Simnozügen etwa folgender:

\section{A. Jieue forfteinricftungen.}

Eämmtliche geometrijchen uno taratorijchen 3 orarbeiten werden von Den Rerjonale Der Cinrichtungsbehörbe bejorgt.

Die Saldocintheilung wiro Durch Den Sioritand Der lebteren in (S)emeinj(haft mit bcm bicrwaltung beamten fejtgejest. Bei Berjefjedentheit ber S(njichten enticheidet Die Jorjtbirection. Ias \$erjonal Der oben genannten Befjörbe füfort bie betreffenden ?trbeiten im 2 salde ats.

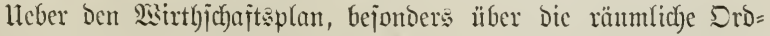

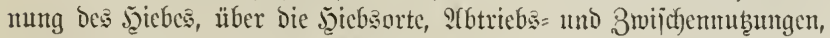

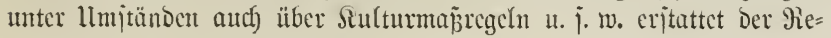


vierverwalter, foweit es ifym bie zu Bebote itehenden Unterlagen mög=

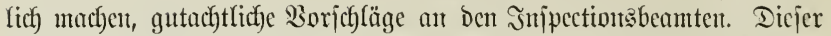

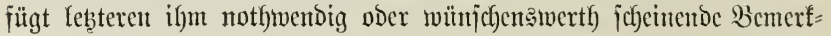
ungen zu und legt fie Dem Sorjtande Der Eitrictutungabljörde vor. Der bie taratorijchen STrbeiten bejorgentoe Beamte biejer Behörde itellt

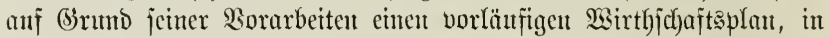
erjter Reiłe Şauungiplan auf, welchen er zunächjt Dem Sorjtande Der Befjörde unterbreitet und mit biejem an Srt und Eteffe bejpricht.

Der von Der Bermaltung vorgelegte und Der von Der Crumicht= ungsbehöroe vorläufig entworfene \$lan merden nun theits in Bimmer, theils an Drt und Stefle im $23 a l d e$ einer gemeinjamen Berathung Durch) Den Sorjtand ber (Sinrichtungsbehörde, Den auşührenden Be= amten Derjefben, Den Injpectionsbeamten und ben Gievierverwalter unterzogen. Meijt werben fich bei biejer Berathung Meinung:

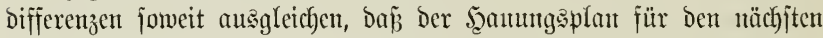

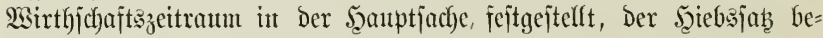
rechnet werben fann. Sflle nicht ausgeglidjenen Meimungsbifferenzen, jomie überbaupt bejonders wichtige und jweifelfajte Fragen werben einer jweiten, unter Borjţ eines Mitgliebes ber Forjtoirection vou Den oben gentunnten Beamten theils im Bimmer, theils im $2 a$ ald abzu=

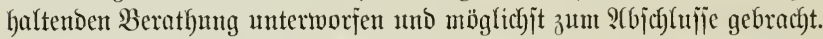

Sene Fraglichfeiten, über welche mant fich bei biejer zweiten $\mathfrak{B e}=$

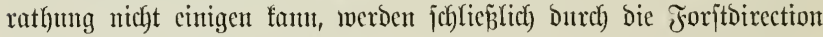
(Minijterium) entjufjeden.

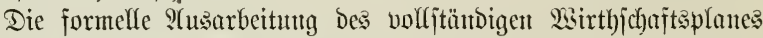
erfolgt Durdh bie Eintrichtutgabehörde allein.

B. gefcfäfte jur Alufrecfterfaltung uno fortfeģung Des Ciuricftungsmerkes.

a) $\mathfrak{i a d b} \operatorname{trag} \mathfrak{s} a \mathfrak{b}$ eiten.

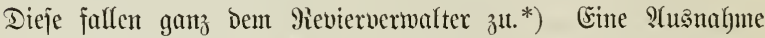

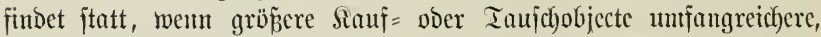

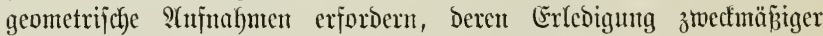
Durch einen Forjtgeometer ber Taxationsbeljörbe erfolgt.

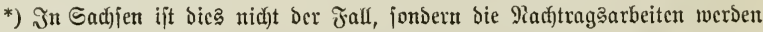

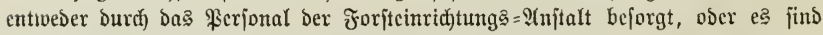

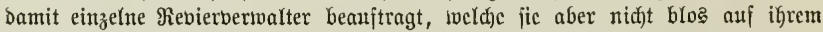

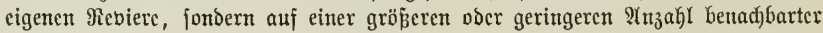
Rebiere alsfingren. 


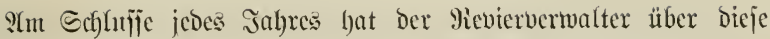
S(rbeiten entweder birect bem Siorjtande ber Iarationsbel)örde ober

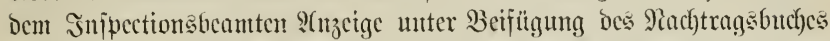
3lt erjtatten. Qebteren Falfes jammelt ber Injpectionsheante bic ein=

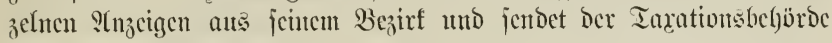
eincm soutptjafjresberidjt.

\section{в) Revijiouen.}

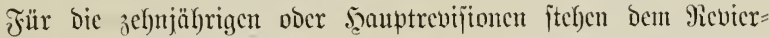
verwalter im (5ijijte bes ganzen Forjteinrichtınģwertes gezeicfnete

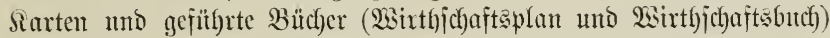

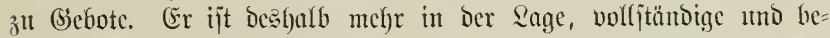

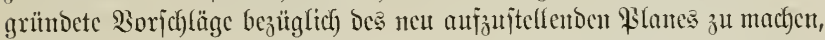
als bics bei neuen Eimrichtungen ber Falf ijt. Dicjen 2iorjeblägen bat ex namentlich auch bie ifm auf (S)rund jeincr Rocalerjab)rungen

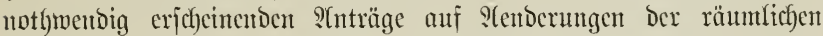
Dronung Des స̧iebes beiznfügen. Er fam unter llmitänden cinen fajt vollftünbigen, neutu, ment aude) formell nicht abgejchlofiencu

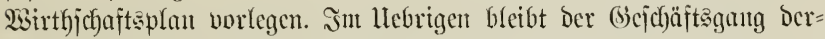
jelbe, wie wir ifnu unter A cmipjoblen haben.

Die fünfjäl)rigen ober Bmijchenrevifionen fönnten allenfalls in bic

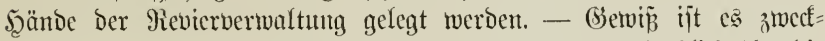

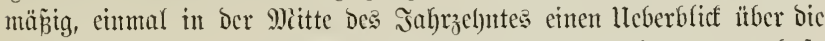
Sicjultate ber 2istrtfjichajt zu gewinten, um jich) zu "überzeugen, ob jo jortgemirthjichaftet werben fömne, wie bisher, ober ob utto welche 9(ent= berungen des \$lanes mïnjchentwerth crichcinen. Scierzu bedarf es feiner umfangreichen, geometrijchen und taratorijchen Rorarbeiten, Das

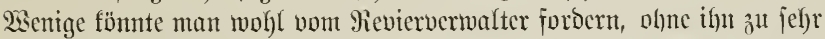

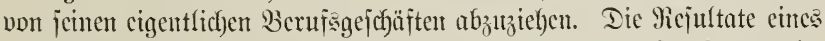

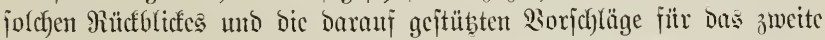

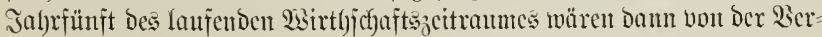
waltunt, bezief)ungsweije von Der Injpection, Der Forjtemrichtungabe=

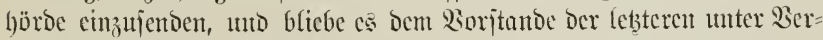

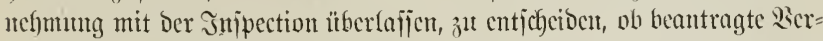
änDerungen Des \$\&lauts cine Socalbejichtigutg von feiner Ecite erforbern

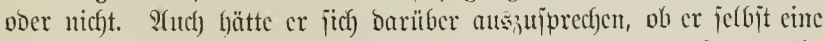

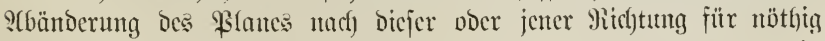

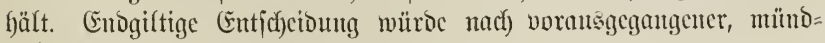
licker Beratflung bic Forjtbirection geben. 


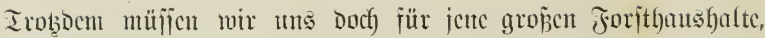

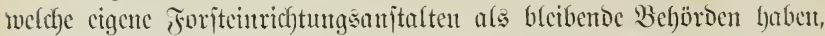

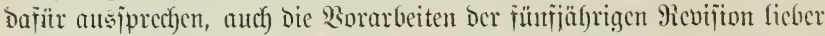

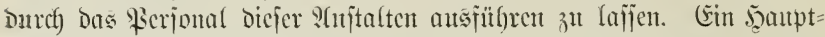

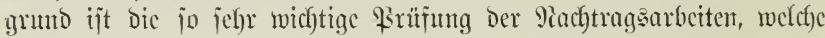

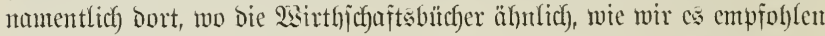
haben, audh) bic (siclderträge nach)weijen, ntach) meht afs s Jahren faum

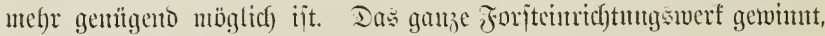

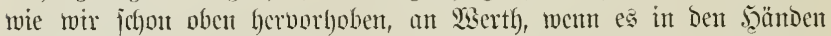

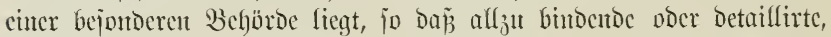

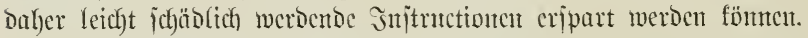

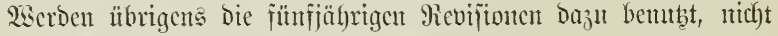

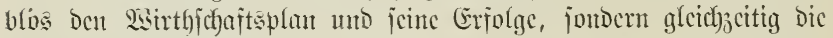

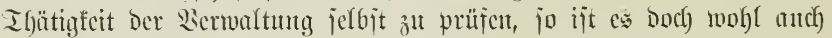
cmpfehlenswerther, die Daju nöthigen Siorarbeiten Durd) antoere Beante

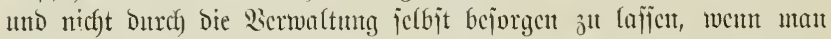
autch zugebon fann, da

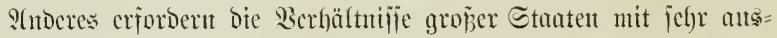

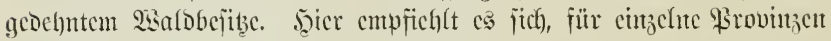

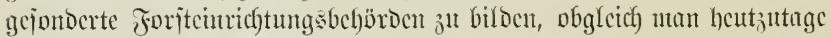

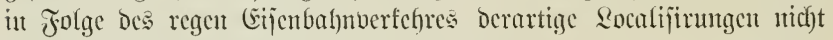
mef)r fo weit ausjubchnen braudft, als früber.

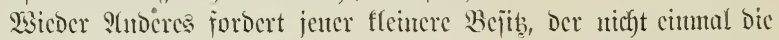
Iremuntg von Injpectiont mo Direction crmb̈glicfyt, uricht felten jogar wic es ani viclen Pribatherrichajten Der Fall iit, Den Ecfwerpunft Der

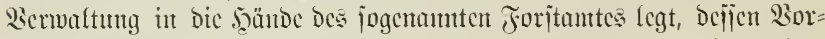

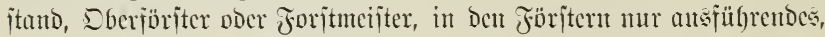

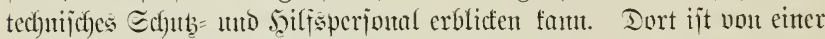
bejonderen Foritcumrichtungsbehörde ntatürlich feinc Picoc. Doch fant

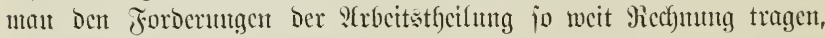
Daz wenigitens für alle geometrijchen mo taratorifchen Sorarbeiten, fïr Die Bejorgutg Der Padfträge Dem Forjtamt ein bejoutocrer Beanter

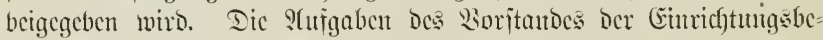
beförde crledigt ber Forituteijter jelbjt, und , jwar entuncoer alleut oder unter $3 u_{j}$ ichung cincs frentocn Eachucritündigen. 


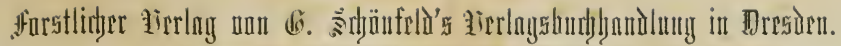

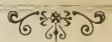

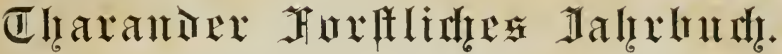

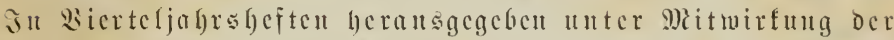
Zchrer an der Sïnigl. Sächj. Forjtatademic

(jec). Dberiorituth Dr. Judrid), Dircctor.

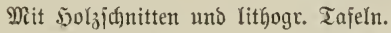

18-24. Banto (1868-1874). Wreis à ell 6.-.

25-35. Band (1875-1885). अreis à eth 8.-

Supplemente dazu:

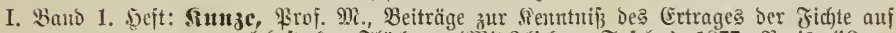
normal bejtodten ơlächen. (Mit 3 lithogr. Tafeln.) 1877. Freis $\mathscr{A l}$ 3.-.

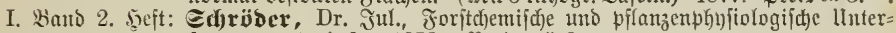
juthungen 1. Socft. 1878. Preis dl 3.-.

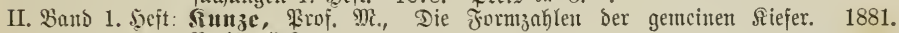
अreiz $\mathscr{M}$ 2.-.

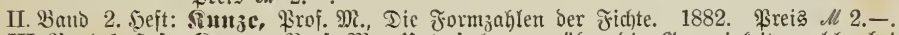

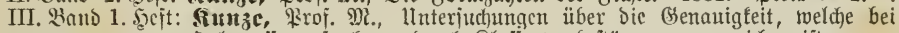

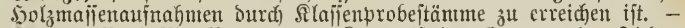

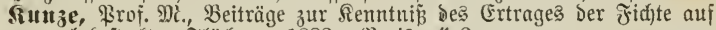

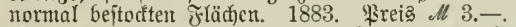

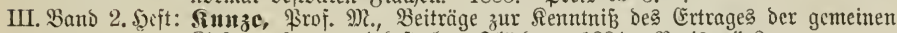

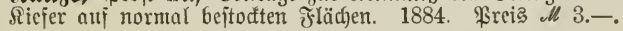

Siebenstellige Kreisflächen für alle Durehmesser von 0,1 bis 99,99. Von Max Friedrich Kumze. 4. geh. Preis $\mathscr{H}_{6}$ 3.-.

Alaflentafel für Iladelhuljklöłze nad) (I) berftärke. Son Mlax Siunze,

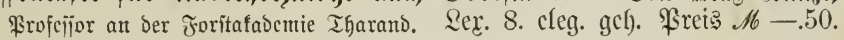

Die wichtigsten Formeln der Zins- und Rentenrechnung. Für das Bedürfniss des Forstwirthes zusammengestellt von Max Friedr.

Kunze, Professor an der Forstakademie zu Tharand. 8. eleg. geh. Preis $\mathscr{K}-.80$.

Meteorologische und hypsometrische Tafeln. Von Max Kunze, Professor in Tharand. gr. 8. eleg. geh. Preis 16 4.-.

Die wirth/dhaftlid) Bedentung der Aufaftung. Erntwurf cines Plancs

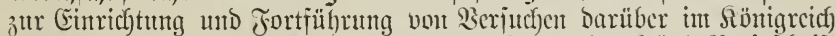

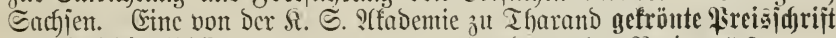

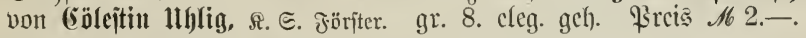

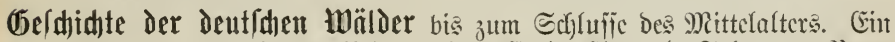

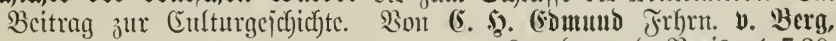

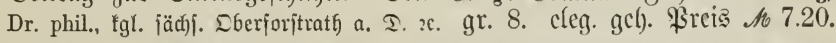

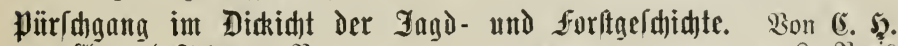

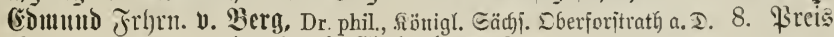

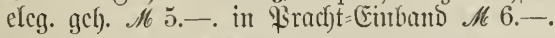

Ileber eine einfudhe Atethode nad) weldher naturaetreue Xbbildumaen

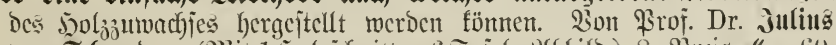

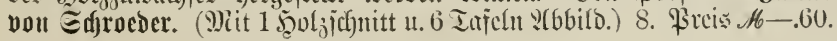




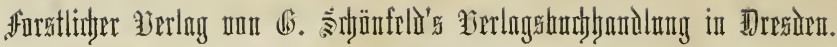

cos

Das fiolz Der Coniferen. Won Dr. Iuling Sdroeder. Mit 11 Fुglz= ¡chnitten. 8. eleg. geh). Freis 161.60.

Beiträge za der frage ïber die Mnturgefełliden und volkswirth-

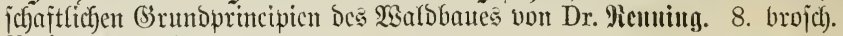
Sreis $\mathscr{1} 6-60$.

Iägerhörnlein. In̈gerlïgen. Iägerlieder. Thierzanber. Dę Эäger= Breviers anderer Theil. gr. 8. EEleg. gebunteet in Bantzlcintwand unit Scrgoldutug. Freis 16 4.- .

Darftelluna der ßagl. Süd). Stantsforftuerwaltung und ibrer Eraebniffe.

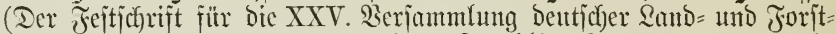
wirthe zu Dreaben 1865. 3wciter Their.) 2ex. 8. X. ut. 72 S.

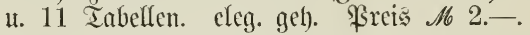

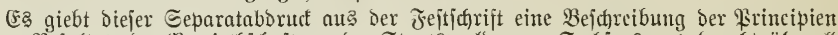

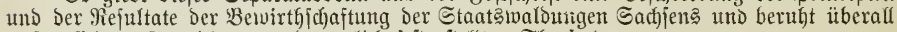
auf pojitiven (biundlagen uno amtlid) ieitgejtellten Thatjachen.

Die mikroskopischen Feinde des Waldes. Naturwissenschaftliche Beiträge zur Kenntniss der Baum- und Holzkrankheiten, für Forstmänner und Botaniker bearbeitet und in zwanglosen Heften herausgegeben von Dr. Moritz Willkomm, fr. Professor an der Königl. Sächs Akademie für Forst- und Landwirthe.

Das erste Heft (mit 4 Holzschnitten und 8 lithographirten Tafeln, wovon 4 in Buntdruck) enthält: Einleitung. I. Die Methode der Forschung. II. Die mikroskopischen Schmarotzer der Holzgewächse. - Zur Kenntniss der Roth- und Weissfäule. Erster Artikel - Der schwarze Brand der Rothbuchentriebe. Eine neue Baumkrankheit. Lex. 8. Eleg. geh. Preis $\mathscr{8} 8.40$.

Zweites $\mathrm{H}$ eft mit 3 Holzschnitten und 6 lithographirten Tafeln (wovon 2 in Buntdruck) nach Originalzeichnungen des Verfasser's. Inhalt: Die Rostpilze der Nadelhölzer und die durch sie verursachten Krankheiten. - Der Rindenkrebs der Lärche oder die Lärchenkrankheit. - Berichtigungen und Zusätze zum ersten Hefte. - Zur Abwehr und Verständigung. - Lex. 8. Eleg. geh. Preis $\mathscr{U} 6.60$.

Die Iloune, Der firefern/pinter und die łiefernblattwespe. Fopuläre

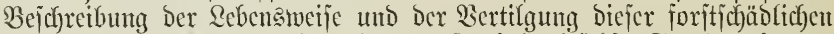

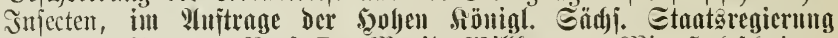

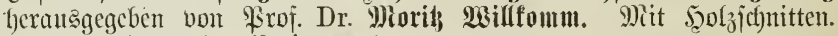
Sex. 8. cleg. get). Siseis 16 1.-.

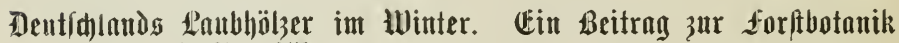

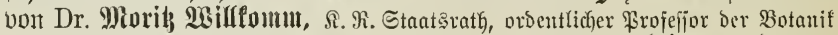
und Direftor bes Botanijøen (sartens in \$rag. 3. umgenrbeitete und ver= mef)rte P(usgabe. Mit 106 nach) Driginat=3eid)mungen Des Sicrfaficts

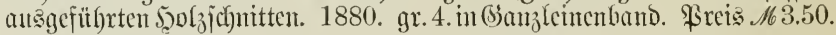

Die Riteratur bot bor (Erjheinen Der eriten \&uggabe Diejes vortrefflichen Budje

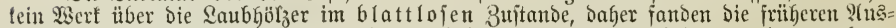

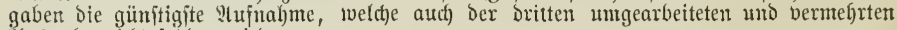
Shaggabe nid)t fehlen wiro. 



